National Water Quality Program

\title{
Data Analysis Considerations for Pesticides Determined by National Water Quality Laboratory Schedule 2437
}

Scientific Investigations Report 2018-5007 



\section{Data Analysis Considerations for Pesticides Determined by National Water Quality Laboratory Schedule 2437}

By Megan E. Shoda, Lisa H. Nowell, Wesley W. Stone, Mark W. Sandstrom, and Laura M. Bexfield

National Water Quality Program

Series Name 2018-5007 


\title{
U.S. Department of the Interior \\ RYAN K. ZINKE, Secretary
}

\section{U.S. Geological Survey William H. Werkheiser, Deputy Director exercising the authority of the Director}

\author{
U.S. Geological Survey, Reston, Virginia: 2018
}

For more information on the USGS - the Federal source for science about the Earth, its natural and living resources, natural hazards, and the environment-visit https://www.usgs.gov or call 1-888-ASK-USGS.

For an overview of USGS information products, including maps, imagery, and publications, visit https://store.usgs.gov.

Any use of trade, firm, or product names is for descriptive purposes only and does not imply endorsement by the U.S. Government.

Although this information product, for the most part, is in the public domain, it also may contain copyrighted materials as noted in the text. Permission to reproduce copyrighted items must be secured from the copyright owner.

Suggested citation:

Shoda, M.E., Nowell, L.H., Stone, W.W., Sandstrom, M.W., and Bexfield, L.M., 2018, Data analysis considerations for pesticides determined by National Water Quality Laboratory schedule 2437: U.S. Geological Survey Scientific Investigations Report 2018-5007, 458 p., https://doi.org/10.3133/sir20185007.

ISSN 2328-0328 (online) 


\section{Foreword}

Sustaining the quality of the Nation's water resources and the health of our diverse ecosystems depends on the availability of sound water-resources data and information to develop effective, science-based policies. Effective management of water resources also brings more certainty and efficiency to important economic sectors. Taken together, these actions lead to immediate and long-term economic, social, and environmental benefits that make a difference to the lives of the almost 400 million people projected to live in the United States by 2050.

In 1991, Congress established the National Water-Quality Assessment (NAWQA) to address where, when, why, and how the Nation's water quality has changed, or is likely to change in the future, in response to human activities and natural factors. Since then, NAWQA has been a leading source of scientific data and knowledge used by national, regional, state, and local agencies to develop science-based policies and management strategies to improve and protect water resources used for drinking water, recreation, irrigation, energy development, and ecosystem needs (https://water.usgs.gov/nawqa/applications/). Plans for the third decade of NAWQA (2013-23) address priority water-quality issues and science needs identified by NAWQA stakeholders, such as the Advisory Committee on Water Information and the National Research Council, and are designed to meet increasing challenges related to population growth, increasing needs for clean water, and changing land-use and weather patterns.

Understanding the occurrence and distribution of pesticides in the Nation's waters is a priority water-quality issue. Pesticides in drinking water and aquatic ecosystems have the potential to adversely affect human health and aquatic life. This report provides information necessary to interpret pesticide concentrations determined by a new analytical method developed by the U.S. Geological Survey National Water Quality Laboratory (NWQL). This new method, known as NWQL schedule 2437, has been used by NAWQA to determine pesticide concentrations in water-quality samples since 2013. A total of 1,323 quality-control samples and paired environmental samples collected in groundwater and surface water are analyzed in this report along with laboratory quality-control samples to document information critical to interpreting pesticide results and assessing analytical method performance in the laboratory.

We hope this publication will provide you with insights and information to meet your waterresource needs and will foster increased citizen awareness and involvement in the protection and restoration of our Nation's waters. The information in this report is intended primarily for those interested or involved in resource management and protection, conservation, regulation, and policymaking at the regional and national levels.

Dr. Donald W. Cline

Associate Director for Water

U.S. Geological Survey 


\section{Contents}

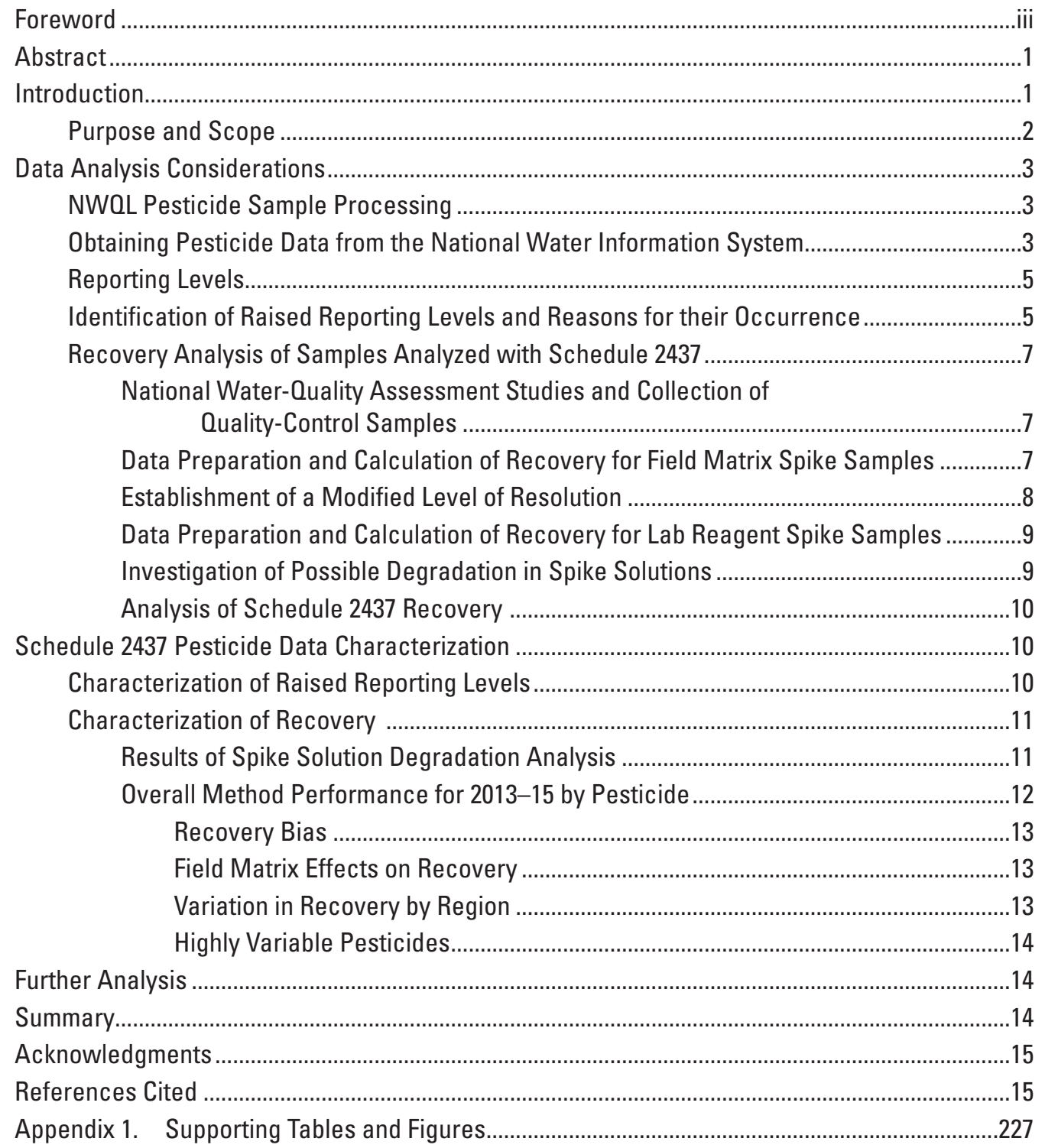

\section{Figures}

1. Image showing explanation of a boxplot that is used to depict the distribution of recovery.

2. Graph showing distributions of recovery for pesticides in schedule 2437 by analytical method group, matrix, spike lot, and spike lot age. Recovery values larger than 400 percent are not shown.

3. Graph showing distributions of recovery for pesticides in schedule 2437 by analytical method group, matrix, and spike lot. Recovery values larger than 400 percent are not shown. 
4. Graph showing distributions of recovery for pesticides in schedule 2437 by matrix. Dashed lines are at 130 percent and 70 percent.

Recovery values larger than 400 percent are not shown.

5. Graph showing distributions of recovery for pesticides in schedule 2437

in surface water by Major River Basin. Recovery values larger than

400 percent are not shown.

Tables

1. Sequence and purpose of samples analyzed in one batch by schedule 2437..................4

2. Common value qualifier codes (VQC) assigned by the National Water Quality Laboratory

3. Reasons for the occurrence of raised reporting levels, background information, value qualifier codes, example laboratory result comments, and guidance for how the raised reporting level value is determined corresponding to each reason

4. Summary statistics for the recovery of schedule 2437 pesticides in lab

reagent spikes, and groundwater and surface-water spike samples. .203

Table 4 is also available as a downloadable file at https://doi.org/10.3133/sir20185007.

\section{Conversion Factors}

International System of Units to U.S. customary units

\begin{tabular}{lcl}
\hline \multicolumn{1}{c}{ Multiply } & By & \multicolumn{1}{c}{ To obtain } \\
\hline liter (L) & Volume & \\
liter (L) & 33.82 & ounce, fluid (fl. oz) \\
liter (L) & 2.113 & pint (pt) \\
liter (L) & 1.057 & quart (qt) \\
liter (L) & 0.2642 & gallon (gal) \\
\hline & 61.02 & cubic inch (in $\left.{ }^{3}\right)$ \\
\hline gram (g) & Mass & \\
\hline
\end{tabular}

Temperature in degrees Celsius $\left({ }^{\circ} \mathrm{C}\right)$ may be converted to degrees Fahrenheit $\left({ }^{\circ} \mathrm{F}\right)$ as follows:

$$
{ }^{\circ} \mathrm{F}=\left(1.8 \times{ }^{\circ} \mathrm{C}\right)+32 .
$$

Temperature in degrees Fahrenheit $\left({ }^{\circ} \mathrm{F}\right)$ may be converted to degrees Celsius $\left({ }^{\circ} \mathrm{C}\right)$ as follows:

$$
{ }^{\circ} \mathrm{C}=\left({ }^{\circ} \mathrm{F}-32\right) / 1.8 \text {. }
$$

\section{Supplemental Information}

Concentrations of chemical constituents in water are given in nanograms per liter ( $\mathrm{ng} / \mathrm{L})$. 


\section{Abbreviations}

$\begin{array}{ll}\text { CSRL } & \text { calibration standard reporting level } \\ \text { FMS } & \text { field matrix spike } \\ \text { GC/MS } & \text { gas chromatography/mass spectrometry } \\ \text { HRL } & \text { higher reporting level } \\ \text { IRL } & \text { interim reporting level } \\ \text { LRS } & \text { laboratory reagent spike } \\ \text { MDL } & \text { method detection level } \\ \text { MRB } & \text { major river basin } \\ \text { NAWQA } & \text { National Water-Quality Assessment Project } \\ \text { NWQL } & \text { National Water Quality Laboratory } \\ \text { NWQN } & \text { National Water-Quality Network for Rivers and Streams } \\ \text { OBSP } & \text { Organic Blind Sample Project } \\ \text { PCODE } & \text { parameter code, a USGS 5-digit code unique to each parameter } \\ \text { OC } & \text { quality-control } \\ \text { RL } & \text { reporting level } \\ \text { RRL } & \text { raised reporting level } \\ \text { RLDQC } & \text { reporting level determined by DOCALC software } \\ \text { RSQA } & \text { Regional Stream Quality Assessment } \\ \text { USGS } & \text { U.S. Geological Survey } \\ \text { VOC } & \text { value qualifier code }\end{array}$




\title{
Data Analysis Considerations for Pesticides Determined by National Water Quality Laboratory Schedule 2437
}

\author{
By Megan E. Shoda, Lisa H. Nowell, Wesley W. Stone, Mark W. Sandstrom, and Laura M. Bexfield
}

\section{Abstract}

In 2013, the U.S. Geological Survey National Water Quality Laboratory (NWQL) made a new method available for the analysis of pesticides in filtered water samples: laboratory schedule 2437. Schedule 2437 is an improvement on previous analytical methods because it determines the concentrations of 225 fungicides, herbicides, insecticides, and associated degradates in one method at similar or lower concentrations than previously available methods. Additionally, the pesticides included in schedule 2437 were strategically identified in a prioritization analysis that assessed the likelihood of occurrence, prevalence of use, and potential toxicity. When the NWQL reports pesticide concentrations for analytes in schedule 2437, the laboratory also provides supplemental information useful to data users for assessing method performance and understanding data quality. That supplemental information is discussed in this report, along with an initial analysis of analytical recovery of pesticides in water-quality samples analyzed by schedule 2437 during 2013-2015. A total of 523 field matrix spike samples and their paired environmental samples and 277 laboratory reagent spike samples were analyzed for this report (1,323 samples total). These samples were collected in the field as part of the U.S. Geological Survey National Water-Quality Assessment groundwater and surface-water studies and as part of the NWQL quality-control program. This report reviews how pesticide samples are processed by the NWQL, addresses how to obtain all the data necessary to interpret pesticide concentrations, explains the circumstances that result in a reporting level change or the occurrence of a raised reporting level, and describes the calculation and assessment of recovery. This report also discusses reasons why a data user might choose to exclude data in an interpretive analysis and outlines the approach used to identify the potential for decreased data quality in the assessment of method recovery. The information provided in this report is essential to understanding pesticide data determined by schedule 2437 and should be reviewed before interpretation of these data.

\section{Introduction}

In 2013, the U.S. Geological Survey (USGS) National Water Quality Laboratory (NWQL) implemented a newly developed analytical method for routine determination of pesticide concentrations. Method O-2437-15, used for NWQL laboratory schedule 2437 (hereafter referred to as "schedule 2437 "), is a liquid chromatography-tandem mass spectrometry method developed in collaboration with the USGS National Water Quality Program National Water-Quality Assessment (NAWQA) Project. Schedule 2437 measures the concentration of 225 pesticide compounds, including 109 pesticides and 116 pesticide degradates (hereafter referred to as "pesticides") (Sandstrom and others, 2015). Pesticide usage has varied with changes in regulation, development of new chemicals, price, pest pressure, and other factors (Atwood and Paisley-Jones, 2017; U.S. Geological Survey, 2017a). The pesticides determined by schedule 2437 were chosen for inclusion in method development based on their probability of occurrence, prevalence of use, and potential toxicity (Norman and others, 2012). This method improves upon past analytical methods because it accurately determines the concentrations of more pesticides at low concentrations in a single sample. Schedule 2437 also includes many pesticides of interest to federal and state agencies, water resource managers, environmental groups, and other stakeholders.

The purpose of NAWQA is to characterize the quality of the Nation's streams and groundwater and to track changes in water quality over time. One goal of the project is to inform local, State, Tribal, and National stakeholders and the public on the status of the Nation's water (Rowe and others, 2013). NAWQA supports these goals with large-scale and temporally intensive water-quality sampling, including collection of samples for the analysis of pesticides. The pesticide concentrations measured in these samples are used in many ways: to determine overall occurrence and distribution of pesticides in the Nation's water (Deacon and others, 2015), for comparison to aquatic and human-health benchmarks (Nowell and others, 2018; 
Stone and others, 2014; Gilliom and others, 2006), to assess changes in surface-water and groundwater conditions over time (Oelsner and others, 2017; Ryberg and Gilliom, 2015, Toccalino and others, 2014), and to create models for predicting pesticide occurrence when direct measurements are not feasible (Stone and Bucknell, 2014; Stone and others, 2013).

An understanding of the supplemental data available for schedule 2437 results, the occurrence of raised reporting levels, and analytical recovery complement the use of data in each of these interpretive paths. Data users should refer to Sandstrom and others (2015) for information about the development and validation of the analytical method used to determine the pesticides in schedule 2437 . This report briefly reviews some of the information in Sandstrom and others (2015) and discusses issues specific to data interpretation that are not included in Sandstrom and others (2015).

A key concept for understanding pesticide concentrations determined by schedule 2437 is the analytical reporting level. A standard laboratory reporting level (RL) is the less-than $(<)$ value reported to data users when the pesticide is not detected (Childress and others, 1999). Typically, this value is set to twice the method detection level (MDL) and is used to control both false positive and false negative error (Childress and others, 1999). Pesticide concentrations in water-quality samples determined by schedule 2437 and other NWQL schedules are frequently less-than or near the RL. NWQL-reported waterquality data, regardless of the constituent, at least occasionally contain raised reporting levels (RRL). An RRL is a less-than value reported at a concentration higher than the RL. RRLs can vary in magnitude above the standard RL and can result from a variety of environmental and analytical circumstances. In pesticide analyses of previously used laboratory schedules, RRLs have been retained in the dataset as nondetects (Oelsner and others, 2017; Martin and Eberle, 2011), treated as missing values and not used in data interpretation (Ryberg and others, 2010), or screened at user-defined levels (Gilliom and others, 2006, Supplemental Technical Information). The treatment of RRLs has implications for data interpretation and should be considered before using schedule 2437 pesticide data. This report includes a discussion of changes to RLs and why they occur, why RRLs occur in schedule 2437 analysis, and describes one approach for screening RRLs in data interpretation.

Another important concept for understanding data quality is analytical recovery (hereafter referred to as "recovery"), which is defined as the measured amount of pesticide in a spiked quality-control (QC) sample expressed as a percentage of the amount spiked, ideally 100 percent (Martin and others, 2009; Martin and Eberle, 2011). Recovery can be calculated from field matrix spike (FMS) samples or measured directly in the analysis of laboratory reagent spike (LRS) samples.
Recovery in field matrices is calculated by pairing environmental samples with spiked QC samples. A spiked QC sample is an aliquot of the environmental sample that is spiked with a known quantity of pesticide(s) (Martin and others, 2009). In previous reports, recovery has been calculated and modeled to assess the impact of temporal changes in the performance of an analytical method on a long-term scale (Martin and others, 2009). These temporal changes are incorporated into the analysis of long-term trends in pesticide concentrations and are necessary to understand how water quality changes over time, one of the goals of the NAWQA project.

In addition to identifying temporal trends in method performance, recovery can be useful for determining the presence of analytical bias - that is, a systematic error, such as the consistent measurement of a pesticide at concentrations less than or greater than the known concentration (Sandstrom and others, 2015). Variation in recovery is also important, because the specific characteristics of a pesticide or the matrix of a water sample can increase or decrease the range in calculated recoveries. This report identifies results with the potential for decreased data quality that might affect recovery assessment and discusses reasons why a data user might choose to exclude these results in an interpretive analysis. When interpreting pesticide data determined by any analytical method, it is important to consider any deviations from the standard RL for each pesticide, and the reasons for such RRLs, as well as potential bias and variability in recovery for each pesticide.

\section{Purpose and Scope}

The purpose of this report is to review existing information about NWQL schedule 2437 and provide additional supporting information important for evaluating pesticide concentrations determined by this schedule. This report will review the processing steps in schedule 2437 analysis, discuss the presence of sample- and result-level comments and qualifiers, and explain how to obtain all the data necessary to interpret pesticide concentrations, the circumstances that result in an RL change, and the occurrence of an RRL. This report also calculates and assesses recovery for the pesticides determined by schedule 2437 in samples from three NAWQA studies during 2013-15: the National Water-Quality Network for Rivers and Streams (NWQN), Regional Stream Quality Assessment (RSQA) studies, and the NAWQA groundwater network studies. The majority of samples analyzed with schedule 2437 in 2013-15 were collected as part of these studies. It is outside of the scope of this report to provide guidance on how to interpret pesticide results determined using schedule 2437 or to conduct a comprehensive analysis of all QC samples analyzed with schedule 2437. 


\section{Data Analysis Considerations}

The following section describes how samples analyzed by schedule 2437 are processed in the laboratory, how to obtain the resulting data and supplemental information, and a review of how reporting levels are determined. In addition to this background information, a discussion is provided on how to identify RRLs and the reasons for their occurrence, then one approach is presented for interpreting RRLs through the use of a modified level of resolution. The screening process for using FMS and LRS samples in calculating recovery and the subsequent calculation is described and the approach used to investigate spike solution degradation is also provided.

\section{NWOL Pesticide Sample Processing}

One benefit of the method used to determine schedule 2437 over previous analytical methods is that sample filtration is the only preparation necessary prior to analytical determination. Additionally, the pesticide concentrations in up to 75 environmental samples can be determined in each batch processed by the analytical instrument. In addition to the environmental samples, each batch contains blanks, calibration standards, and other laboratory QC samples. The instrument analyzes one batch at a time, in a specific order (table 1). The first two samples analyzed are blanks, to verify the cleanliness of the instrument. These are followed by a series of calibration standards used to develop the calibration curve, which relates the instrument-measured peak intensity area response of each pesticide to concentration and will be applied to the environmental samples within the batch. Instrument blanks then verify that the high-level calibration standards have not contaminated the instrument prior to the analysis of a QC sample. A series of a continuing calibration verification standards, which verify the quantitation of an intermediate-level standard, an instrument blank, and about 15 environmental samples are then analyzed. This process repeats five times with a set of one LRS and one laboratory reagent blank following the first set of environmental samples. After the last set of environmental samples is analyzed, another calibration verification standard is analyzed, followed by an instrument blank, and then three standards to check instrument sensitivity at the end of the batch. The last sample analyzed is a blank for determining if extra cleaning procedures are required prior to analysis of the next batch.

The analysis of multiple QC samples throughout the course of the batch allows the laboratory analyst to check for contamination of the samples, instrument fouling, and overall instrument performance. These data are used by the NWQL to ascertain if laboratory blank contamination was detected and to what degree. In addition, the data are used to set RRLs for an entire batch or selected environmental samples within the batch. These data can also be used to evaluate method performance and to develop an understanding of how the operation of the instrument affects the interpretation of pesticide concentrations.

\section{Obtaining Pesticide Data from the National Water Information System}

Results for routine water-quality samples collected by the USGS and analyzed for pesticides are publicly available through the National Water Information System (U.S. Geological Survey, 2017b). Obtaining pesticide (and other analyte) data is best done by following these steps:

- Access the National Water Information System database online at https://waterdata.usgs.gov/nwis;

- Choose Water Quality under the Frequent Searches by Data Category heading;

- Choose to view data from Field/Lab samples;

- Choose the site or location information you wish to use for data retrieval and choose Parameter groupings under the Data Attribute heading;

- Provide the site details, such as site name, site number, or state of interest; and

- Choose Organics, pesticide from the Parameter groupings, and under the Retrieve Water-Quality Samples for Selected Sites heading, choose Tab-separated data with the settings One result per row and Expanded attributes.

The value qualifier code, or VQC (abbreviated "val_ qual_t $\mathrm{x}$ " in the data file) and laboratory result comment (abbreviated "result_lab_cm_tx") are especially important in data interpretation, including interpretation of schedule 2437 data. The VQC is assigned by NWQL analysts to communicate to data users circumstances unique to the determination of that sample or pesticide that might affect data interpretation (table 2). The laboratory result comment is an open text field available to NWQL analysts to provide standard comments or their own notes on the pesticide result reported.

In addition to routine water-quality samples, field QC samples are also collected as a regular component of all NAWQA studies. Field QC samples typically include spike, blank, and replicate samples. The FMS and LRS sample data and calculated recoveries that will be discussed in this report are provided in the accompanying data release (Shoda and others, 2017b). Field replicate data are also available for schedule 2437 results for 2013-15 samples (Shoda and others, 2017a). Other field QC data are available to the public by a request to the Water Data Support Team through NWIS. 
Table 1. Sequence and purpose of samples analyzed in one batch by schedule 2437.

[ng/L, nanograms per liter; QC, quality-control; See table 8 in Sandstrom and others (2015) for more information on analytical sequence]

\begin{tabular}{|c|c|c|}
\hline $\begin{array}{c}\text { Sequence } \\
\text { number }\end{array}$ & Sample type & Sample purpose \\
\hline 1 & Blank & Wash blank. \\
\hline 2 & Blank & Instrument blank to verify the wash. \\
\hline 3 & $1 \mathrm{ng} / \mathrm{L}$ calibration standard & Instrument calibration. \\
\hline 4 & $2.5 \mathrm{ng} / \mathrm{L}$ calibration standard & Instrument calibration. \\
\hline 5 & $5 \mathrm{ng} / \mathrm{L}$ calibration standard & Instrument calibration. \\
\hline 6 & $10 \mathrm{ng} / \mathrm{L}$ calibration standard & Instrument calibration. \\
\hline 7 & $25 \mathrm{ng} / \mathrm{L}$ calibration standard & Instrument calibration. \\
\hline 8 & $50 \mathrm{ng} / \mathrm{L}$ calibration standard & Instrument calibration. \\
\hline 9 & $100 \mathrm{ng} / \mathrm{L}$ calibration standard & Instrument calibration. \\
\hline 10 & $250 \mathrm{ng} / \mathrm{L}$ calibration standard & Instrument calibration. \\
\hline 11 & $500 \mathrm{ng} / \mathrm{L}$ calibration standard & Instrument calibration. \\
\hline 12 & $1,000 \mathrm{ng} / \mathrm{L}$ calibration standard & Instrument calibration. \\
\hline 13 & $5,000 \mathrm{ng} / \mathrm{L}$ calibration standard & Instrument calibration. \\
\hline 14 & $10,000 \mathrm{ng} / \mathrm{L}$ calibration standard & Instrument calibration. \\
\hline 15 & Blank & Instrument blank to check for cross-over contamination from high calibration standards. \\
\hline 16 & Blank & Instrument blank to check for cross-over contamination from high calibration standards. \\
\hline 17 & QC sample & Third-party check sample. \\
\hline 18 & 250 ng/L QC sample & Continuing calibration verification. \\
\hline 19 & Blank $^{1}$ & Instrument blank before analyzing environmental samples. \\
\hline $20-34$ & 15 environmental samples & Sample analysis. \\
\hline 35 & Laboratory reagent spike & Laboratory reagent spike. \\
\hline 36 & Laboratory reagent blank ${ }^{1}$ & Laboratory reagent blank. \\
\hline 37 & 250 ng/L QC sample & Continuing calibration verification. \\
\hline 38 & Blank $^{1}$ & Instrument blank before analyzing environmental samples. \\
\hline $39-53$ & 15 environmental samples & Sample analysis. \\
\hline 54 & $250 \mathrm{ng} / \mathrm{L}$ QC sample & Continuing calibration verification. \\
\hline 55 & Blank $^{1}$ & Instrument blank before analyzing environmental samples. \\
\hline $56-70$ & 15 environmental samples & Sample analysis. \\
\hline 71 & 250 ng/L QC sample & Continuing calibration verification. \\
\hline 72 & Blank $^{1}$ & Instrument blank before analyzing environmental samples. \\
\hline $73-87$ & 15 environmental samples & Sample analysis. \\
\hline 88 & 250 ng/L QC sample & Continuing calibration verification. \\
\hline 89 & Blank $^{1}$ & Instrument blank before analyzing environmental samples. \\
\hline $90-104$ & 15 environmental samples & Sample analysis. \\
\hline 105 & 250 ng/L QC sample & Continuing calibration verification. \\
\hline 106 & Blank $^{1}$ & Instrument blank after analyzing all environmental samples. \\
\hline 107 & $5 \mathrm{ng} / \mathrm{L}$ instrument detection level standard & Standard to check for instrument sensitivity. \\
\hline 108 & $10 \mathrm{ng} / \mathrm{L}$ instrument detection level standard & Standard to check for instrument sensitivity. \\
\hline 109 & $50 \mathrm{ng} / \mathrm{L}$ instrument detection level standard & Standard to check for instrument sensitivity. \\
\hline 110 & Blank & Instrument blank to check for any contamination at the end of the batch. \\
\hline
\end{tabular}

${ }^{1}$ used to assess for contamination of environmental samples 
Table 2. Common value qualifier codes (VOC) assigned by the National Water Quality Laboratory

\begin{tabular}{cl}
\hline VOC & \multicolumn{1}{c}{ Definition } \\
\hline c & See result comment. \\
d & Sample was diluted. \\
i & Result may be affected by interference. \\
m & Value is highly variable by this method. \\
n & Below the reporting level but at or above the detection level. \\
s & Instrument sensitivity problem. \\
t & Below the detection level. \\
v & Analyte detected in laboratory blank. \\
@ & Holding-time violation. \\
* & Warm when received. \\
\hline
\end{tabular}

\section{Reporting Levels}

Determination of an MDL is based on finding the concentration at which an analytical method can reliably confirm the presence of an analyte in a sample (Williams and others, 2015). As noted previously, the NWQL calculates the RL for an analytical method as two times the MDL (Childress and others, 1999). Pesticides that are not detected in water-quality samples are reported as "less-than" the RL; however, it is possible for pesticides to be detected in samples at concentrations less than the RL. For schedule 2437, results are reported as "estimated" when the measured concentration is less than the RL because the concentration value has an increased uncertainty. For estimated pesticide concentrations, the quantitative value, but not the qualitative nature of the detection, is uncertain (Sandstrom and others, 2015). If a pesticide is detected at or greater than the MDL and less the RL, it is noted with the VQC of " $n$ " (table 2). If the pesticide is detected at less than the MDL, the VQC is noted as " $\mathrm{t}$ " (table 2).

Beginning in water year 2013, each pesticide determined by schedule 2437 was assigned an RL known as the interim reporting level (IRL). The IRL is a temporary reporting level that was determined during original method validation. The RL has the potential to change every year as more data are obtained to quantify method detection capability (Childress and others, 1999). For schedule 2437 and beginning with samples analyzed in 2016, IRLs were updated with information from two sources: more data available from the routine operation of the method and a new procedure for the determination of laboratory detection (Williams and others, 2015). In addition to the use of data determined by schedule 2437 in an expanded time period (2013-15), a new software program known as DQCALC was implemented to determine updated IRLs (Williams and others, 2015; ASTM International, 2016). Results reported to the National Water Information System using the new software are identified with the RL type of RLDQC.

\section{Identification of Raised Reporting Levels and Reasons for their Occurrence}

The presence of RRLs can create a challenge for data interpretation, because RRLs represent greater uncertainty in the reported result for a pesticide than detections, or values censored at the RL. For example, a pesticide with an RL of 5 that is reported with an RRL of 10 has a range of values that might represent the true pesticide concentration between 0 and 10. A value less-than the RL narrows the potential range of true concentrations from 0 to 5 , although both examples represent circumstances in which the pesticide was not detected.

The incidence of RRLs was assessed in water-quality samples collected for NAWQA surface-water and groundwater studies in water years 2013-15, and the NWQL-provided VQCs and laboratory result comments associated with these RRLs were compiled. RRLs can be identified for schedule 2437 data by reviewing the RL associated with each pesticide result and identifying the results that have $\mathrm{a}<$ in the remark code field and a result value greater than the RL. The various reasons for these RRLs are summarized in table 3, along with the corresponding VQCs and typical laboratory comments associated with each RRL and guidance for how the RRL values were determined for each reason.

When contamination is detected in one or more laboratory blanks, the 15 environmental samples analyzed directly before and after the blank sample may be qualified with a VQC of "v," depending on the magnitude of the environmental sample detection in relation to the blank detection, as determined by the criteria specified in the USGS Office of Water Quality Technical Memorandum 2012.01 (Myers, 2011). Specifically, an RRL occurs when a pesticide is detected in a blank sample, and the pesticide concentration in the environmental sample is greater than the RL, but less than three times the concentration in the laboratory blank. In this case, the RRL is reported as less than the concentration of the environmental sample. An RRL can also occur due to blank contamination when the pesticide is not detected in the environmental sample, in which case the RRL is reported as less than the concentration detected in the blank sample. At the time of publication (2018), RRLs are also present due to dilution (VQC of "d"). The NWQL is currently reviewing these RRLs, which were reported in error. 
Table 3. Reasons for the occurrence of raised reporting levels, background information, value qualifier codes, example laboratory result comments, and guidance for how the raised reporting level value is determined corresponding to each reason.

[RRL, raised reporting level; VQC, value qualifier code; QC, quality control; LC-MS/MS, liquid chromatography-tandem mass spectrometry]

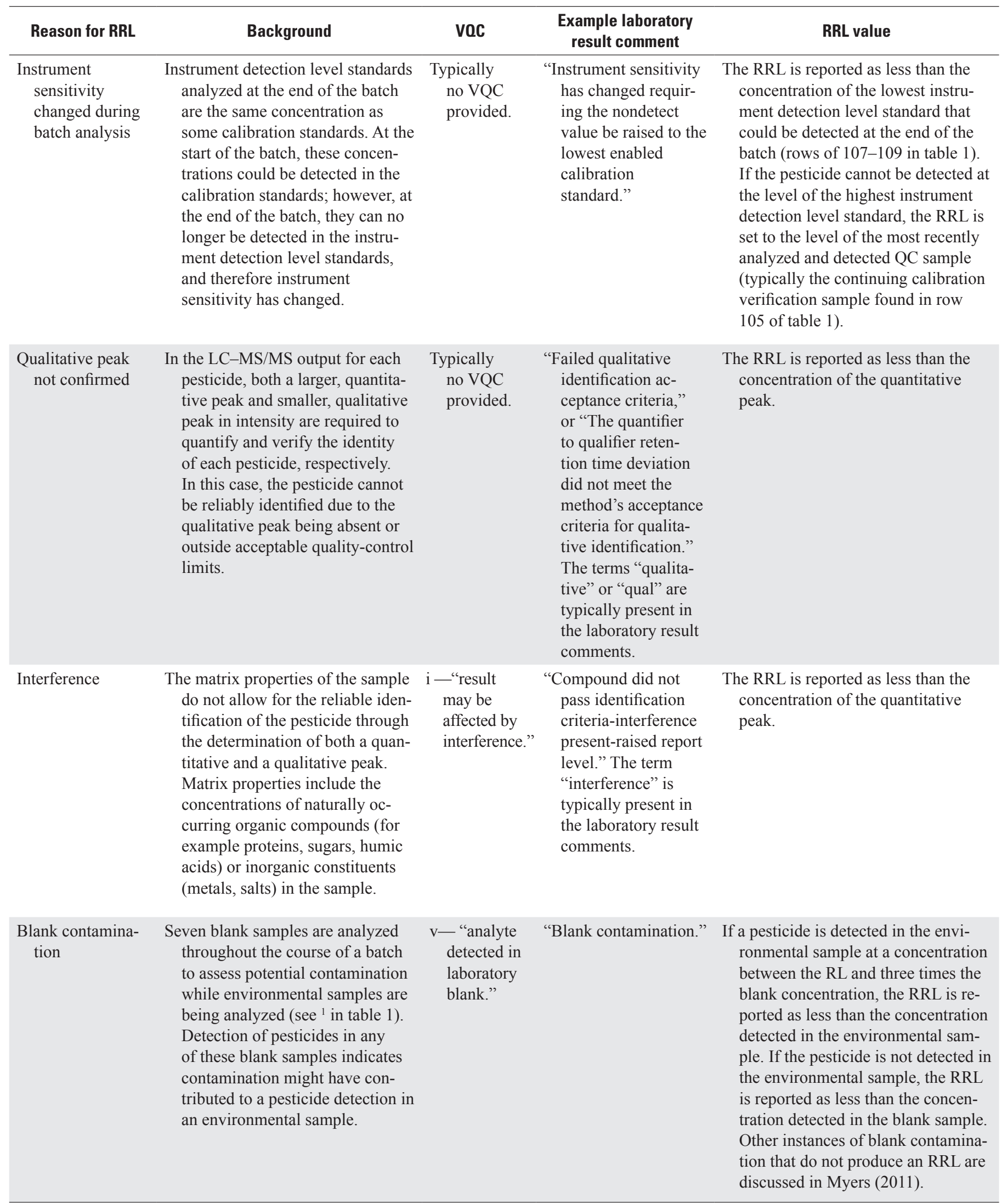




\section{Recovery Analysis of Samples Analyzed with Schedule 2437}

\section{National Water-Quality Assessment Studies and Collection of Quality-Control Samples}

Three NAWQA studies contributed data to this report. The NAWQA NWQN was established to determine the status and trends of the Nation's surface-water quality and is a long-term monitoring network consisting of approximately 76 sites, with the number of sites varying slightly each year during 2013-15. The NAWQA RSQA studies are temporally intensive, regionally focused short-term studies conducted in the summers of 2013 (Midwest), 2014 (Southeast), and 2015 (Pacific Northwest), with each region containing approximately 100 sites (U.S. Geological Survey, 2017d). The NAWQA groundwater sites are organized into studies and networks with varying objectives, including studies of principal aquifer, land use, major aquifer, groundwater flow paths, and the enhanced trends network. Across the different groundwater studies and networks, a total of 1,328 wells were sampled in water years 2013-15.

All of the NAWQA studies include collection of routine QC samples, including pesticide spike samples. A spike sample is a sample that has been spiked with known concentrations of analytes (Mueller and others, 2015). As stated in the introduction, FMS samples are regularly collected environmental samples to which a known volume of spike solution has been added. Spike samples are compared with environmental samples collected at the same time to calculate the recovery of an analyte in a field matrix. LRS samples are prepared in a laboratory using laboratory-grade blank water. LRS samples are used to assess analyte recovery in a sample under controlled settings, removing other potentially influential variables (such as matrix effects). Each spike solution is prepared by the NWQL and assigned a lot number; the concentrations of pesticides may vary slightly in different spike lots. The same lot of spike solution is typically used for both LRS samples and FMS samples prepared during the same time period. Data analyzed in this report include both LRS recovery and FMS recovery in groundwater and surface-water samples collected throughout the Nation during water years 2013-15.

\section{Data Preparation and Calculation of Recovery for Field Matrix Spike Samples}

Pesticide recovery in this report was determined as the concentration of a pesticide in a spiked sample, from which the concentration in the paired environmental (unspiked) sample has been subtracted, divided by the expected concentration. Recovery calculation is well documented in Sandstrom and others (2015), Mueller and others (2015), Martin and Eberle (2011), and Martin and others (2009), and the methods used to calculate recovery outlined in those reports were followed in this investigation. The 523 groundwater and surface-water paired environmental and FMS samples (1,046 samples total) available for the calculation of recovery for 2013-15 resulted in a total of 116,886 pesticide paired results (233,772 individual pesticide results) (Shoda and others, 2017b). The paired samples are the environmental sample and the FMS sample collected at the same site, on the same date, at the same time. The determination of 225 pesticides in each of the 523 paired samples would result in 117,675 pairs of results. The dataset used in this analysis was slightly smaller because analytical circumstances do not always allow for the determination of every pesticide in a sample. Prior to interpreting recoveries, this investigation omitted the use of some results due to the absence of information needed to calculate spike recovery, poor data quality, circumstances resulting in increased uncertainty of recovery, and other issues, as described in this section.

To calculate FMS sample recovery, a user must first calculate expected pesticide concentrations in an FMS sample. Expected pesticide concentrations are calculated using the concentration of each pesticide in the spike solution, the volume of the spike solution that was added to the sample, and the total sample volume after spiking (Martin and others, 2009; Martin and Eberle, 2011). The concentration of pesticides in the spike solution is referenced to the NWQL-assigned spike lot. For 81 of the 523 field matrix spike samples (15 percent), spike lot information was not provided by the field crew collecting the sample, but when possible, the lot used to spike a sample was assumed based on the sampling date and the spike lots available on that date. In most cases, there was high confidence in this assumption due to the use of standard field practices. However, it was not possible to make a reasonable assumption in all cases with missing spike lot information, due to the availability of multiple lots on the sampling date. Therefore, some paired samples were not included in the analysis due to lack of spike solution concentration information. When the volume of spike solution added to the sample was not provided or the value provided was not 0.1 milliliters, it was assumed to be 0.1 milliliters, based on standard field practices (Sandstrom and Lewis, 2009; Mueller and others, 1997). Upon review of recovery values, if samples with assumed spike solution volumes were found to have recoveries outside of a normal range (see criteria used for spiking error below), it was concluded that the volume assumption was false and the sample was not included in further analysis due to a lack of spike volume information. Expected FMS sample concentrations can be obtained in Shoda and others (2017b) and are usually greater than 100 nanograms per liter. Samples that were inadvertently spiked with an expired spike lot were not included in this analysis. Spike lots past their expiration date have potentially degraded, meaning that the concentrations of pesticides in the spike solution have decreased; therefore, recoveries calculated with these lots have an increased potential to be biased low. Lastly, paired samples were also excluded from analysis when total sample volume of the spiked sample was not provided. This can occur when a spike sample is submitted to the NWQL without being identified as such. Spike samples are the only samples analyzed with schedule 2437 to have their total sample volume determined. 
In some circumstances, the paired sample data were acceptable for calculating recovery, but there was reason to exclude certain pesticides within the pair because of increased uncertainty in the calculation of recovery. For example, paired results were not included in the analysis when the concentration in the environmental sample was higher than the expected concentration of the spike. Martin and others (2009, p.6) defined these samples as having "high background concentrations" and discussed the uncertainties they create in the analysis of recovery for pesticides determined by laboratory schedule 2033. For the pesticides in schedule 2437, Sandstrom and others (2015) found that recoveries for some pesticides were very high or very low when the background environmental concentrations were equal to or greater than the expected spike concentration. The eleven pesticides with the largest number of results flagged for high background concentrations comprise 85 percent of all results with this flag (Shoda and others, 2017b). They are: metolachlor (pcode 65090) and two of its degradates (pcodes 68650 and 68651), atrazine (pcode 65065 ) and three of its degradates (pcodes 68547, 68552, and 68660), three acetochlor degradates (pcodes 68522, 68523, and 68524), and 2,4-D (pcode 68500). High background concentrations of these pesticides in spike samples is expected due to their heavy agricultural use and frequent detection in water-quality samples in past analyses (U.S. Geological Survey, 2017a; Stone and others, 2014; Gilliom and others, 2006).

The presence of certain VQCs and laboratory result comments can indicate poor data quality. As such, environmental and FMS results were screened using VQC and laboratory comment criteria. If the environmental or paired spike result was an RRL due to laboratory blank contamination (noted with a " $\mathrm{v}$ " in the VQC field or stated in the laboratory comment), then the result was excluded. If the result was an RRL but there was no VQC to explain the rationale for the RRL, contamination could not be ruled out, and the result was not included in this analysis. There were 154 environmental sample results ( 0.1 percent of environmental sample results) and 34 spike sample results ( 0.03 percent of spike sample results) flagged because of an RRL due to blank contamination, and 2 results were flagged for the presence of an RRL with no explanatory VQC (Shoda and others, 2017b). Both of these circumstances were deemed to have increased the uncertainty in the quantitation of the reported result, and thus these results were excluded.

\section{Establishment of a Modified Level of Resolution}

In past pesticide studies, when RLs changed over the study period and RRLs were present, all data were screened using the maximum RL for the study period so that less-than values smaller than the maximum RL were accepted for analysis and less-than values larger than the maximum RL were removed from interpretive analysis (Gilliom and others, 2006, Supplemental Technical Information). The presence of a substantial amount of RRLs in this dataset and changes to RLs immediately following this study period due to the adoption of
RLDQCs warranted the use of a similar approach. This section describes a set of criteria to screen-out data with insufficient resolution to accurately assess recovery.

For each pesticide, a modified level of resolution in the form of an RL higher than the established IRL was determined and used to omit results from recovery analysis. Although RLDQCs were not used during laboratory analysis of samples collected during 2013-15, they represent an update to IRLs that reflects the most recent review of analytical performance and the implementation of the DQCALC software. In response to the changes in reporting level, a higher reporting level (HRL) was created for each pesticide, which is the larger of the two available reporting levels (IRL and RLDQC). This HRL defined a lower level of analytical resolution over the time period during which samples were analyzed and was used to determine another modified level of resolution: the calibration standard reporting level (CSRL). The CSRL is the value of the first calibration standard larger than the HRL and was used to incorporate more results in the final analysis. The values of the calibration standards are presented in rows 3 through 14 of table 1 .

The determination of the HRL and use of the CSRL as a modified level of resolution was adopted in order to increase the amount of meaningful information that could be included in the analysis of recovery. The authors determined that for the purposes of this report, data analysis did not require the highest level of resolution, which might have resulted in omitting all results reported with an RRL above the IRL or RLDQC. By screening data using the CSRL, low-level RRLs are included in data analysis and treated as nondetects. Uncertainty exists in the true determination of low-level RRLs, which might have been either nondetects or low-level detections. The use of low-level RRLs (RRLs below the CSRL) as nondetections in the calculation of recovery could bias results. Use of the CSRL as a threshold for the exclusion of data was considered acceptable, however, because although it allows for some increase in data uncertainty, it also expands the dataset to include more information.

To summarize, the process of determining and applying a suitable level of resolution for this dataset includes five steps: (1) determine the IRL reported for each pesticide; (2) identify the RLDQC, implemented in 2016, for each pesticide; (3) determine the higher of the IRL and RLDQC (HRL); (4) determine the value of the first calibration standard above the HRL; (5) consider RRLs at the level of the CSRL or below suitable to be considered in recovery analysis and omit from data interpretation any results with RRLs above the level of the CSRL for that pesticide.

For FMS samples, 3,954 environmental results (3.4 percent) and 519 spike results ( 0.4 percent) were flagged for the presence of an RRL above the CSRL and omitted from data interpretation (Shoda and others, 2017b). The pesticide with the most occurrences of RRLs above the CSRL was $1 \mathrm{H}-1,2,4-$ Triazole (pcode 68498), which comprised 9 percent and 17 percent of all RRL occurrences for environmental results and spike results, respectively. 
When the data needed to do so were available, recovery was calculated for each pair of environmental and spike results. Censored results (that were not omitted as described above) for either the environmental or spike sample were set to zero following Martin and others (2009). Some negative recoveries were calculated. Negative recoveries resulted from greater measured concentrations in environmental samples compared to spike samples. This can occur in the measurement of pesticides with high analytical variability, or when the environmental sample concentration is detected below the RL. In the latter case, the presence of a censored spike value (nondetection) and a noncensored value (detection) in the paired environmental sample result in a negative recovery. Because the pesticide was not detected in the spiked sample, each negative recovery was set to zero.

Before this dataset was used in further analysis, two final screening steps were completed. First, pesticides in which the HRL was greater than the expected spike concentration were excluded. In these circumstances, the method was unable to detect the spike concentration, and presenting these data as nondetects would bias recovery low. Second, samples in which more than half (113 out of 225) of the analytes had recoveries greater than 150 percent or less than 50 percent were assumed to be the result of a spiking error. Spiking errors can result when twice the volume of the spike solution is added to a spike sample (double-spiked), none of the spike solution is added (false spike), or other sample processing errors occur. Data screening based on missing sample information, expired spike lots, specific VQCs and laboratory result comments, the presence of an RRL above the modified level of resolution (CSRL), high background concentrations, HRLs greater than expected spike concentrations, and suspected spiking errors resulted in the removal of 22 percent of the data (Shoda and others, 2017b).

\section{Data Preparation and Calculation of Recovery for Lab Reagent Spike Samples}

Routine laboratory QC samples analyzed with schedule 2437 include instrument QC samples and batch QC samples. Instrument QC samples include instrument blanks, calibration standards, third-party check standards, continuing calibration verification standards, and instrument detection level standards (table 1; Sandstrom and others, 2015). The batch QC samples include LRS samples and laboratory reagent blanks and provide information about overall method performance in a control (clean) matrix (Sandstrom and others, 2015).

Recovery of LRS samples is determined differently from FMS recoveries because there is no need to collect paired samples. LRS samples consist of pesticide-free water that is spiked in a manner similar to a field spike, using the same spike solutions (and spike lots) that are used in the field. Because the reagent water is pesticide free, the recovery of an LRS can be determined directly from the concentration measured in the sample. In the review of LRS sample results, nondetection results were treated as zero recovery. It was not necessary to use the modified level of resolution (the CSRL) in the analysis of LRS samples, because RRLs did not occur for these data in water years 2013-15. The LRS dataset also includes results for samples in which it was not possible to measure the pesticide concentration, usually due to an analytical issue, and noted in the laboratory results comments. This resulted in a null value for recovery, which was removed from subsequent analysis.

\section{Investigation of Possible Degradation in Spike Solutions}

Beginning on October 1, 2015, the expiration dates for schedule 2437 spike solutions made and certified at the NWQL were reduced from one year to six months after certification. This change was implemented in response to lowered recoveries observed for many organophosphate and sulfonyl urea pesticides in LRS samples spiked with solutions aged more than six months. Additionally, LRS samples analyzed during May-August of 2013 showed decreased recoveries for 17 organophosphates and other pesticides spiked with NWQL spike lot 91219 during the later months of the spike lot's 1-year certification period. Recovery of these pesticides increased, largely meeting laboratory data-quality objectives, when samples were spiked with a freshly made spike solution (lot 91313) in August 2013. These observations, as described by the NWQL, indicated that there was possible degradation in spike solutions, either as a result of the age of the spike lot, or affecting one lot in particular. Before overall conclusions were developed about the performance of schedule 2437 during 2013-15 based on recovery calculations for FMS and LRS samples, this possible degradation was investigated to prevent bias in subsequent analyses.

To determine if aging spike solutions degraded, or if one lot specifically degraded, the recoveries of each pesticide were calculated and plotted by spike lot and spike lot age for each matrix (LRS, groundwater, and surface water). The ages of spike solutions were separated into the earlier time period after certification (12-6 months prior to expiration) and the later time period after certification (6-0 months prior to expiration). The number of individual pairwise tests between these groupings ( 2 ages of 8 spike lots for 225 pesticides in 3 matrices) resulted in 5,400 spike lot age pairs to compare, and thus performing pairwise comparisons of spike lot age for each spike lot, pesticide and matrix was rejected due to large number of tests. Therefore, plots were initially visually assessed for differences between the earlier and later periods. Because organophosphates were noted in particular as having reduced recoveries, pesticides were then separated by analytical method group and plotted by spike lot and spike lot age for each matrix. Sandstrom and others (2015) used analytical method groups to define pesticides of similar type; the groups primarily represent major groups of pesticides or major groups subdivided into chemical and other classes (table 1-1, appendix 1). Lastly, all pesticides analyzed by schedule 2437 were pooled and plotted by spike lot and spike lot age for each sample matrix. 
Differences between the spike lot ages, analytical method groups, and matrices were tested with Levene's test for homogeneity of variance followed by Welch's ANOVA and GamesHowell posthoc tests. All tests were done with the statistical software R (R Development Core Team, 2017). Levene and Games-Howell tests were done with the R package userfriendlyscience (Peters, 2017). The R package called "stats" and the function oneway.test were implemented for the Welch's ANOVA (R Development Core Team, 2017).

Levene's test assesses the equality of variance between groups (Levene, 1960). It was necessary to determine if significant differences existed in the variance between groups so that an appropriate test for differences in means between groups could be determined. The Welch's ANOVA is a global test for differences between means and is an alternative to the traditional ANOVA when Levene's test indicates a violation of the assumption of homogeneity of variance (Welch, 1938). Welch's ANOVA is robust to unequal sample sizes (Kohr and Games, 1974) and nonnormally distributed datasets (Algina and others, 1994). Lastly, when Welch's ANOVA indicated significant differences in the means of multiple groups, the Games-Howell posthoc test was used to determine which groups were significantly different from other groups. The Games-Howell test was designed for making multiple comparisons between groups with unequal variance, unequal sample sizes, and nonnormal distribution (Games and Howell, 1976).

The visual assessment results and the Games-Howell posthoc test results for recoveries grouped by matrix, analytical method group, spike lot, and spike lot age led to the pooling of the two ages and an analysis of differences in recovery and variation in recovery based on spike lot alone. Differences between lots were again visually assessed and tested with Levene's test, Welch's ANOVA, and Games-Howell posthoc tests.

\section{Analysis of Schedule 2437 Recovery}

After the data were reviewed and prepared as stated in the previous sections, overall assessment of method recovery was determined by calculating summary statistics for each pesticide, including relative standard deviation. Summary statistics were evaluated to identify pesticides that exhibited bias or high variation in recovery, using NWQL data-quality objectives for method development as a threshold (Sandstrom and others, 2015). Boxplots of recovery for each pesticide and matrix were used along with summary statistics to identify matrix differences. Lastly, bias and variation in recovery due to large-scale surface-water matrix effects were investigated by plotting surface-water recoveries by major river basin (MRB).

\section{Schedule 2437 Pesticide Data Characterization}

\section{Characterization of Raised Reporting Levels}

In the NWQN in 2013-15, there were 843,991 censored results, of which 105,053 were results reported with RRLs, comprising approximately 11 percent of all the data (censored and uncensored, all environmental samples). Of these 11 percent, RRLs ranged from 1.0001 times larger than the RL to 302 times larger than the RL. The number of RRLs and percentage of RRLs per pesticide also varied in the NWQN during 2013-15. The maximum percentage of RRLs per pesticide was for cyanazine (pcode 66592), with 72 percent of all results being RRLs, and the minimum was for alachlor sulfynilacetic acid (an alachlor degradate; pcode 68524), with 0.05 percent. Additionally, the occurrence of RRLs was unevenly distributed temporally; the maximum of 17,361 RRLs (16.5 percent) was in June and the minimum of 2,940 (2.8 percent) was in November. The treatment of these RRLs will affect data interpretation in different ways. In this example for the NWQN, excluding all RRLs above the RL would maximize the analytical resolution of the dataset, but it would also result in the loss of 11 percent of sample results, which would decrease the quantity of data available for some analytes and time periods and may bias recoveries high because many censored results would be deleted.

Although RRLs did not represent a large overall percentage of the data in either the recovery investigation or the NWQN during 2013-15, the timing of RRL occurrence, and the pesticides and sites affected, can have a significant influence on data interpretation. The magnitudes of RRLs above the RL is another factor that data users should consider. In the calculation of a pesticide detection frequency, for example, one might be more inclined to include all of the RRLs for a pesticide in which overall RRL occurrence was low and the magnitude of RRLs was low, whereas a pesticide in which RRLs were frequently reported with high magnitudes might cast doubt on the validity of using the RRL as a nondetect in a frequency analysis.

Circumstances that create RRLs can be classified into two different categories: cases in which there is uncertainty in the identification of the pesticide and cases in which there is uncertainty in the quantification of the pesticide. Interference, nonconfirmation of the qualitative peak, and change in instrument sensitivity during the batch are circumstances that belong in the uncertainty of identification category (table 3 ). In these circumstances, the analytical instrument was not able to reliably detect the pesticide due to the factors explained in table 3 . In contrast to identification uncertainty, blank contamination is a circumstance in which there is no uncertainty in the presence of a pesticide, but there is uncertainty in the source of the detection. Laboratory blanks can be contaminated, for example, when high concentrations of pesticides in environmental samples are not removed by the routine rinsing of the 
chromatography column, tubing, and other equipment between samples, so subsequent samples are contaminated. Uncertainty based on blank contamination can occur on a wide range of levels, from contamination being so small that it does not substantively affect the detected concentration, to blank contamination large enough to result in an RRL orders of magnitude higher than the RL.

The ultimate decision about how to treat results reported with RRLs and the uncertainty that a data user can accept in data interpretation will be dictated by specific study objectives. The use of VQCs and laboratory result comments allows the data user to screen out pesticide results that might have unacceptable uncertainty and (or) might not adequately represent true environmental conditions. The approach used in this report defined a modified level of analytical resolution to distinguish between RRLs that were acceptable for analysis and RRLs deemed to contain too much uncertainty.

\section{Characterization of Recovery}

Previous work has shown differences in groundwater and surface-water recovery of pesticides determined by gas chromatography/mass spectrometry (GC/MS) based on matrix effects (Martin and others, 2009). Due to the differences in sample preparation and matrix, LRS recoveries and field recoveries for groundwater and surface-water were assessed separately. The final, robust dataset was used to investigate overall method performance, recovery bias, differences in recovery between LRS and FMS samples, surface-water versus groundwater matrix effects, and regionally specific surface-water matrix effects.

From October 1, 2012, to September 30, 2015, eight spike lots were available; the first lot, 91211, was certified on June 6, 2012, and the last lot, 91518, was certified on July 20, 2015. Each of these lots expired 1 year after their certification (the change in certification period from 1 year to 6 months was implemented on October 1,2015). The spike samples in the dataset (Shoda and others, 2017b) were unevenly distributed with respect to sampling date, geographic location, sampling matrix, and spike lot. First, there were fewer groundwater samples than surface-water samples overall. The temporal and geographic distribution of spike samples in surface water was skewed, because the RSQA samples were collected only during the summer months in three specific regions of the country (U.S. Geological Survey, 2017d). Additionally, due to the timing of when spike lots were certified in relation to the field and laboratory QC schedules, some lots were used to determine a larger number of spike recoveries. For example, no LRS samples were spiked with lot 91518 between its certification and the end of the water year on September 30, 2015.

There is also variation in the number of recoveries reported for each pesticide. Analytical difficulties occasionally prevent the determination of every pesticide in a sample. Additionally, each spike lot is made independently, and for one spike lot, seven pesticides were not available at the time of production, and so not every pesticide determined by schedule
2437 is present for this one lot (lot 91518). Lastly, some results were dropped from the dataset during the screening process, as noted above, in the "Data Preparation and Calculation of Recovery for Field Matrix Spike Samples" section, and in Shoda and others (2017b).

\section{Results of Spike Solution Degradation Analysis}

Boxplots of recovery for each pesticide by spike lot, spike lot age, and matrix were largely inconclusive regarding the presence of, and the reasons for, pesticide degradation in a spike solution (fig. 1-1; appendix 1). Boxplots are explained in figure 1. A visual assessment of these plots did not reveal consistently lower recoveries in samples with spike lots aged more than 6 months. Further, pooling all pesticides into a single group and plotting recovery by spike lot age and matrix showed no consistent differences between the two ages in any matrix (fig. 1-2; appendix 1). The lack of a notable difference in the spike lot-age recovery boxplots in both of these groupings might be due to the high variation in recovery values for certain pesticides and matrices (see next section). Grouping pesticides by analytical method group was the most informative approach (fig. 2). From a visual assessment of boxplots of recovery by spike lot, spike lot age, matrix, and analytical method group, there is no evidence that spike lot age was associated with lower recoveries or more variation in recoveries, as a rule. For the organophosphate group, and the carbamate and thiocarbamate group in both groundwater and surface-water samples in particular, variation in recovery was increased for spike lot 91219, regardless of the time period in which the spike solution was used (fig. 2).

Levene's test for homogeneity of variance between spike lot and age groupings by matrix and analytical method group indicated significant differences in the variation of recovery for each spike lot and age group for every matrix and analytical method group at the level of $p<0.05$, with the exception of the acetanilide and amide group, and the acid group, in groundwater (fig. 2). Welch's ANOVA revealed that the means of recovery of each unique spike lot and age group were likewise significantly different $(\mathrm{p}<0.05)$ for every matrix and analytical method group when there was enough data to test each group, with the exception of miscellaneous pesticides in groundwater. These two global tests indicate that when recoveries are grouped by matrix, analytical method group, spike lot, and spike lot age, many of those groups had a significantly different variance and mean recovery than other groups (fig. 2). This conclusion is the basis for investigation into if those differences are due to the age of the spike lot.

When data for both spike lot ages were present, the difference between ages was tested for each spike lot, analytical group, and matrix using the Games-Howell post hoc test. Out of 48 possible comparisons between the 2 spike lot ages by analytical method group in laboratory reagent spikes, 14 comparisons ( 29 percent) were found to be significantly different at a level of $p<0.05$. One out of 27 comparisons (4 percent) was significantly different for groundwater, and 5 out of 
45 comparisons (11 percent) were significantly different for surface water. Overall, this statistical test did not show significant differences in recovery by spike lot age for most pesticide groups and matrices. Recoveries grouped by matrix, analytical method group, spike lot, and spike lot age were nonnormally distributed and had unequal variance and unequal sample sizes. These factors made conclusive, consistent testing of differences based on spike lot age challenging; however, the Games-Howell posthoc test is robust to these challenges and was supported by the visual assessments of differences based on age.

Next, spike lot ages were pooled and statistical tests were repeated to evaluate the effects of spike lot on recovery for the different analytical method groups and matrices, and in particular, to test whether the higher variability in spike recovery for selected lots and analytical method groups observed in figure 3 could be supported statistically. The Levene's test results for homogeneity of variance between spike lot, matrix, and analytical method group indicated significant differences in variation of spike lots for every matrix and analytical method group at the level of $\mathrm{p}<0.05$, with the exception of the acetanilide and amide pesticides in groundwater and pesticides with an acid functional group in laboratory reagent spikes and groundwater. Subsequent Welch's ANOVA tests for differences between the mean recoveries based on spike lot revealed significant differences in all matrices and analytical method groups. The differences between specific spike lots was tested with the Games-Howell post hoc test. Posthoc tests indicated many significant differences between specific lots in the different matrices. One meaningful result was that spike lot 91219 was significantly different from all other lots for the following analytical method groups and matrices: the organophosphate group and the carbamate and thiocarbamate group for surface water and groundwater; and the pyrethroid, organochlorine, and phenylpyrazine group in surface water. Although there were occasional other instances in which a single lot was significantly different from all other lots in a matrix, the investigation into spike lot age and spike lot 91219 in particular seemed to indicate that there was a specific issue with this lot. Degradation of this lot soon after certification, or preparation or handling error, might have contributed to its lowered recovery for certain analytical method groups.

A visual assessment of recovery boxplots, supported by the Games-Howell tests, formed the basis of the decisions (1) to pool spike lot ages and (2) to omit data for spike lot 91219 from further analysis. Although not all analytical method groups appeared to be affected, lower mean recoveries and higher variability for multiple analytical method groups indicate that spike lot 91219 was compromised. Without knowledge of the mechanism that caused the lowered recoveries and increased variation in lot 91219, the most conservative approach was adopted, and this lot was deemed to be invalid for assessment of analytical method performance. FMS and LRS samples spiked with lot 91219 were treated as unsuitable for interpretive analysis and removed from further analysis of recovery. These samples represent 22 percent of all FMS samples and 19 percent of all LRS samples for 2013-15.

\section{Overall Method Performance for 2013-15 by Pesticide}

The initial 116,886 paired field results available for this analysis, after the screening steps described in previous sections, narrowed the final dataset to 68,670 paired results (59 percent), including 32,443 pairs of FMS and environmental results collected in the NWQN; 27,719 pairs collected for the RSQA studies; and 8,508 pairs collected in NAWQA groundwater networks (Shoda and others, 2017b). After the lighter screening for LRS samples, 50,075 of the initial 62,343 result pairs were available for interpretation ( 80 percent) (Shoda and others, 2017b).

Not every pesticide analyzed by schedule 2437 had data available for laboratory reagent, groundwater, and surface water in the final dataset used for this recovery assessment (Shoda and others, 2017b). There are four pesticides - cisBifenthrin acid/cis-Cyhalothrin acid/cis-Tefluthrin acid (pcode 68553), dacthal monoacid (pcode 68560), dicamba (pcode 68571), and methomyl oxime (pcode 68646) - for which no valid field data were available to assess recovery following the data preparation steps listed above and in the "Data Preparation and Calculation of Recovery for Field Matrix Spike Samples" section, primarily due to HRLs greater than the expected spike concentration. Valid recoveries are available for these pesticides in LRS samples.

The recovery of each pesticide and matrix is displayed in figure 4. Schedule 2437 appears to perform reasonably well; most pesticides recovered near 100 percent and had good agreement in recovery among the matrices. Table 4 (also available as a downloadable file at https://doi.org/10.3133/ sir20185007) notes that the relative standard deviations for most pesticides and matrices are small, indicating low variability. There are notable exceptions, however. Sandstrom and others (2015) created data-quality objectives for analytical method development: mean recovery of less than 70 percent or greater than 130 percent (100 percent $+/-30$ percent) or variability of greater than 30 percent relative standard deviation was considered unacceptable performance in method validation experiments. These data-quality objectives were suitable for method validation and were applied to both LRS and FMS samples, but might be too conservative to assess the routine operation of this schedule, or the more widespread assessment of field spikes. Despite the potential conservativism of these criteria, the data-quality objectives provided by Sandstrom and others (2015) provide a reference for acceptable method performance. Table 4 highlights values for pesticides and matrices that did not meet these data-quality objectives.

Two pesticides ( 0.89 percent) in LRS samples, 23 pesticides (10.4 percent) in groundwater, and 24 pesticides (10.9 percent) in surface water had mean recoveries that did not meet data-quality objectives with recoveries less than 70 percent or greater than 130 percent. For all matrices, the bulk of these occurrences are for mean recoveries lower than 70 percent. Eighteen pesticides ( 8 percent), 50 pesticides (23 percent), and 46 pesticides ( 21 percent) did not meet 
data-quality objectives for relative standard deviation in laboratory reagent spikes, groundwater and surface water, respectively. Relative standard deviations outside of data-quality objectives ranged from 30 percent to 146 percent for all matrices. Overall, 2 (LRS), 20 (groundwater), and 21 (surface water) pesticides fell outside of data-quality objectives for both mean recovery and relative standard deviation. Regardless of matrix, many pesticides also had the presence of at least one outlier recovery value. Twenty-four pesticides in laboratory reagent spikes, 28 in groundwater, and 45 in surface water had at least one recovery greater than 200 percent.

\section{Recovery Bias}

Table 4 also highlights pesticides in laboratory reagent spikes, groundwater, and surface water in which the first quartile was greater than 100 percent recovery or the third quartile was less than 100 percent recovery. These distributions indicated that 75 percent of the recoveries calculated for these pesticides in these matrices were either above or below 100 percent recovery

(fig. 4). Although these results might not be outside of data-quality objectives, understanding the distribution of recoveries for each pesticide and matrix can be useful in assessing potential bias. Six pesticides ( 3 percent) in groundwater and 13 pesticides ( 6 percent) in surface water had a first quartile greater than 100 percent. No pesticides had a first quartile greater than 100 percent for laboratory reagent spikes. However, 45 pesticides ( 20 percent) in laboratory reagent spikes, 55 (24 percent) in groundwater, and 61 (27 percent) in surface water had a third quartile less than 100 percent. More pesticide recoveries were biased low than biased high in LRS and FMS samples, although many of these biases were small. Considering all matrices, of the 161 occurrences of third quartile values less than 100 percent, 97 of them (60 percent) were greater than 90 percent. The bias observed with the distribution of more mean recoveries less than 70 percent (compared to mean recoveries greater than 130 percent) discussed in the previous paragraph was present in this assessment of first and third quartiles as well; that is, many more pesticides had third quartiles less than 100 percent than had first quartiles above 100 percent, indicating overall that recoveries were biased low.

\section{Field Matrix Effects on Recovery}

Evidence of matrix effects (defined in table 3 ) on recovery can be seen in figure 4 and table 4 . Matrix effects can present as an increase or decrease in recovery of FMS samples compared to LRS samples, as seen with the pesticide asulam (fig. 4 AI, p. 45; pcode 68536) or as an increase in the variation in FMS samples, as seen with isoxaflutole (fig. 4 EA, p. 69; pcode 68632). Overall, there was evidence for matrix effects seen in the recoveries of many pesticides analyzed by schedule 2437 (fig. 4). LRS samples had the highest percentage (99 percent) of pesticides with mean recoveries within 70-130 percent and the highest percentage of pesticides ( 92 percent) with relative standard deviations less than 30 percent, compared to groundwater and surface-water matrix spikes, indicating better performance of the method in LRS samples.
A specific type of matrix effect is the difference in recovery between groundwater and surface-water spike samples. Martin and others (2009) found that recoveries of pesticides determined by GC/MS in surface water were consistently biased higher than recoveries in groundwater. For this analysis, there was a negligible difference in the number of pesticides with mean recoveries less than 70 percent (20 in groundwater and 21 in surface water; with 15 in each matrix represented by the same pesticides), and greater than 130 percent (the same 3 pesticides for each matrix) in groundwater and surface water. The quartile analysis showed that more pesticides in surface water consistently recover both higher (with more pesticides with first quartiles greater than 100 percent) and lower (with more pesticides with third quartiles less than 100 percent) compared to groundwater. There was, however, only a minimal difference in the number of pesticides with relative standard deviations greater than 30 percent, with 50 pesticides in groundwater compared to 46 in surface water. These results indicate a slight tendency towards less variation in recovery per pesticide, across a wider range of recoveries overall for surface water and perhaps higher variability in groundwater recovery. The difference in sample sizes between groundwater and surface-water recoveries made these comparisons difficult to assess and the collection of more groundwater QC data would be needed to investigate matrix effects.

\section{Variation in Recovery by Region}

Martin and others (2009) interpreted pesticide recoveries for samples analyzed by GC/MS and identified the potential for a bias in surface-water recoveries due to matrix effects and based on NAWQA study units. Study units are defined by MRBs and aquifers across the Nation (Gilliom and others, 2001) and were implemented in the first two decades of NAWQA. NAWQA sampling design did not incorporate sampling based on study unit in its third decade (beginning in 2013); however, a larger regional grouping can be achieved by analyzing recoveries based on MRB. MRBs are eight large geographical regions used as the basis for surface-water status and trends assessment (Crawford and others, 2006). Surfacewater sites in the NWQN and RSQA studies were each assigned to the appropriate MRB, and recovery data were plotted to determine if a matrix effect could be determined by this large-scale grouping. A strong effect of MRB on recoveries was not noticeable in plots of recovery by analytical method group (fig. 1-3; appendix 1). The lowest median recoveries for eight of the nine analytical method groups belonged to the MRBs located in California or Alaska; however, the sample sizes for these groups were one or two orders of magnitude lower than the other MRBs, so more data are needed in these regions to investigate potential matrix effects on recovery.

Schedule 2437 pesticide-specific matrix effects were documented in Sandstrom and others (2015), where low recoveries of some pesticides occurred in samples characterized by high $\mathrm{pH}$ values (8.4 to 9.6). Pesticide-specific matrix effects also seemed to occur between MRBs (fig. 5). For example, 
metolachlor (pcode 65090) had consistent recovery and variation among MRBs, while acetochlor sulfynilacetic acid (pcode 68524), another pesticide in the acetanilide and amide group, and a degradate of acetochlor (pcode 68520), had shifts in median recoveries and differences in variation between MRBs (fig. 5 EP, p. 162 and fig. 5 Z, p. 106, respectively). The findings of Martin and others (2009), Sandstrom and others (2015), and the apparent occurrence of pesticide-specific effects by MRB indicate that more investigation would be necessary to draw conclusions about how an environmental sample matrix might affect recovery of pesticides analyzed by schedule 2437 .

\section{Highly Variable Pesticides}

In addition to the pesticides that did not meet the dataquality objectives for recovery variability, some pesticide results were coded with a VQC of " $m$," meaning that the result is highly variable. The assignment of a VQC of " $m$ " was determined by criteria outlined in Sandstrom and others (2015) and reported by the NWQL for 19 pesticides, regardless of the relative standard deviation of recovery calculated by matrix for the pesticides for this report. These pesticides, listed in table 1-2 of appendix 1, do not have a VQC of " $m$ " associated with every result, but frequently when the result was not reported with a VQC of " $m$," it was censored at the IRL. More information about these pesticides can be obtained in Sandstrom and others (2015), where they are referred to as qualified analytes.

\section{Further Analysis}

There are sources of QC data associated with schedule 2437 that are not discussed in this report. One source is the surrogate data associated with each sample analyzed by schedule 2437 . Surrogates are analytes added to the sample to measure samplelevel method performance; they are not expected to be present in an environmental sample, but are chemically similar to expected analytes (Sandstrom and others, 2015). The recovery of surrogates is provided with the results for each sample and can act as a measure of analytical performance. Another source of QC data is the USGS Branch of Quality Systems Organic Blind Sample Project (OBSP). The purpose of the OBSP is to monitor, assess, and evaluate analytical results for organic laboratory methods, including schedule 2437, by using blind quality-assurance samples (U.S. Geological Survey, 2017c). Recovery over time, false positives and false negatives, and relations between recovery and concentration are assessed for each pesticide in schedule 2437 through the OBSP. One way the NWQL uses the results of the OBSP is to evaluate the presence of false negatives for each pesticide; these results can factor in the decision to change an RL. Laboratory blanks are another source of QC data that was briefly discussed in the "Identification of Raised Reporting Levels and Reasons for their Occurrence" section. Detections in laboratory blanks can indicate sample-, batch-, or method-level issues with contamination and might warrant the use of a raised censoring level (Nowell and others, 2013) or a minimum RL (Childress and others, 1999).
This report is not intended to be a comprehensive QC analysis of schedule 2437 and does not address the analysis of replicate, laboratory blank, or field blank samples. Replicate samples are two or more samples considered to be identical in composition and analysis (Mueller and others, 2015). Replicate data spanning 2013-15 in the NWQN, RSQA studies, and NAWQA groundwater networks can be obtained in Shoda and others (2017a), which provides an initial summary of replicate samples. Field blank samples are QC samples intended to be free of measureable concentrations of an analyte (Mueller and others, 2015). Laboratory and field blank data require a thorough analysis to determine the occurrence of pesticide detections in blank samples and their possible implications for data interpretation. Pesticide water-quality samples have been analyzed at the NWQL for decades using various other analytical methods. It is outside of the scope of this report to assess or compare those methods with schedule 2437; however, a detailed method comparison was done by Martin and others (2017).

One way that the recovery data discussed in this report can be used is in the development of a recovery correction model to adjust pesticide concentrations to account for temporal changes in performance of the analytical method (Martin and others, 2009). This report and accompanying data release (Shoda and others, 2017b) assess some aspects of method performance during the first 3 years of schedule 2437 routine analysis, however, this short time frame and the variation observed in this time period make it difficult to assess if these data are appropriate for the development of a recovery model. Method performance over time and possible recovery correction could be assessed when more data are available.

\section{Summary}

This report presents an investigation into factors that are important to understand when interpreting pesticide data from samples analyzed with National Water Quality Laboratory schedule 2437, including changing reporting levels, raised reporting levels, and spike recovery. The purpose of this report is to provide information on schedule 2437 so that data users know what supporting information is available for reported pesticide results and method performance and the implications of that information on future analyses. Figures and tables are provided that summarize laboratory batch processing, common value qualifier codes, the reasons for raised reporting level occurrence and the value qualifier codes and laboratory results comments associated with each reason, the investigation into the effect of spike lot and spike lot age on recovery, and the recovery of each pesticide by matrix. The information provided in this report is essential to understanding pesticide data determined by schedule 2437 and should be reviewed before interpretation of these data. 


\section{Acknowledgments}

The authors gratefully acknowledge Terri Arnold, Dan Button, and Casey Lee of the U.S. Geological Survey for providing data for this analysis.

\section{References Cited}

Algina, J., Oshima, T. C., and Lin, W., 1994, Type I error rates for Welch's Test and James's Second-Order Test under nonnormality and inequality of variance when there are two groups: Journal of Educational and Behavioral Statistics, v. 19 , no. 3, p. 275-291.

ASTM International, 2016, Standard practice for performing detection and quantitation estimation and data assessment utilizing DQCALC software, based on ASTM practices D6091 and D6512 of Committee D19 on water: Active Standard ASTM D7510, 2 p., accessed November 2, 2017, at http://www.astm.org/Standards/D7510.htm.

Atwood, D. and Paisley-Jones, C., 2017, Pesticides Industry Sales and Usage: U.S. Environmental Protection Agency Report, accessed November 29, 2017, at https://www.epa. gov/sites/production/files/2017-01/documents/pesticidesindustry-sales-usage-2016_0.pdf.

Childress, C.J., Foreman, W.T., Connor, B.F., and Maloney, T.J., 1999, New reporting procedures based on long-term method detection levels and some considerations for interpretations of water-quality data provided the U.S. Geological Survey National Water Quality Laboratory: U.S. Geological Survey Open-File Report 99-193, 19 p.

Crawford, C., Hamilton, P., and Hoos, A., 2006, National Water-Quality Assessment Program-Modifications to the status and trends network and assessments of streams and rivers: U.S. Geological Survey Fact Sheet, 6 p., accessed June 1, 2017, at https://water.usgs.gov/nawqa/studies/mrb/ mrb_factsheet.pdf.

Deacon, J.R., Lee, C.J., Toccalino, P.L., Warren, M.P., Baker, N.T., Crawford, C.G., Gilliom, R.G., and Woodside, M.D., 2015, Tracking water-quality of the Nation's rivers and streams: U.S. Geological Survey web page, accessed November 2, 2017, https://cida.usgs.gov/quality/rivers, doi:10.5066/F70G3H51.

Games, P.A., and Howell, J.F., 1976, Pairwise multiple comparison procedures with unequal n's and/or variances-A Monte Carlo study: Journal of Educational Statistics, v. 1, no. 2, p.113-125.
Gilliom, R.J., Hamilton, P.A., and Miller, T.L., 2001, The National Water-Quality Assessment Program-Entering a new decade of investigations: U.S. Geological Survey Fact Sheet $071-01,8 \mathrm{p}$.

Gilliom, R.J., Barbash, J.E., Crawford, C.G., Hamilton, P.A., Martin, J.D., Nakagaki, N., Nowell, L.H., Scott, J.C., Stackleberg, P.E., Thelin, G.P., and Wolock, D.M., 2006, The quality of our Nation's waters-Pesticides in the Nation's streams and ground water, 1992-2001: U.S. Geological Survey Circular 1291, 172 p., Supplemental Technical Information, available online, accessed November 2, 2017, at https://water.usgs.gov/nawqa/pnsp/pubs/circ1291/supporting_info.php.

Kohr, R.L., and Games, P.A., 1974, Robustness of the analysis of variance, the Welch Procedure and a Box Procedure to heterogeneous variances: The Journal of Experimental Education, v. 43, no.1, p.61-69.

Levene, H., 1960, Robust tests for equality of variances, in Olkin, Ingram, and Hotelling, Harold, eds., Contributions to probability and statistics-Essays in honor of Harold Hotelling: Stanford University Press, p. 278-292.

Martin, J.D., Norman, J.E., Sandstrom, M.W., and Rose, C.E., 2017, A field study of selected U.S. Geological Survey analytical methods for measuring pesticides in filtered stream water, June-September 2012: U.S. Geological Survey Scientific Investigations Report 2017-5049, 106 p., 7 app., accessed November 2, 2017, at https://doi.org/10.3133/ $\operatorname{sir} 20175049$.

Martin, J.D., and Eberle, M., 2011, Adjustment of pesticide concentrations for temporal changes in analytical recovery, 1992-2010: U.S. Geological Survey Data Series 630, 11 p., 5 app.

Martin, J.D., Stone, W.W, Wydoski, D.S., and Sandstrom, M.W., 2009, Adjustment of pesticide concentrations for temporal changes in analytical recovery, 1992-2006: U.S. Geological Survey Scientific Investigations Report 2009-5189, 24 p., plus appendixes.

Mueller, D.K., Martin, J.D., and Lopes, T.J., 1997, Qualitycontrol design for surface-water sampling in the National Water-Quality Assessment Program: U.S. Geological Survey Open-File Report 97-223, 17 p., 2 apps.

Mueller, D.K., Schertz, T.L., Martin, J.D., and Sandstrom, M.W., 2015, Design, analysis, and interpretation of field quality-control data for water-sampling projects: U.S. Geological Survey Techniques and Methods, book 4, chap. C4, 54 p., accessed November 2, 2017, at https://dx.doi. org/10.3133/tm4C4. 
Myers, D.N., 2011, Application of the result-level 'v' value qualifier code and ' $E$ ' remark code to selected organic results reported by the National Water Quality Laboratory (NWQL): Office of Water Quality Technical Memorandum 2012.01, accessed July 27, 2017, at https://water.usgs.gov/ $\mathrm{admin} / \mathrm{memo} / \mathrm{QW} / \mathrm{qw} 12.01 . \mathrm{pdf}$.

Norman, J.E., Kuivila, K.M., and Nowell, L.H., 2012, Prioritizing pesticide compounds for analytical methods development: U.S. Geological Survey Scientific Investigations Report 2012-5045, 206 p.

Nowell, L.H., Ludtke, A.S., Mueller, D.K., and Scott, J.C., 2013, Organic contaminants, trace and major elements, and nutrients in water and sediment sampled in response to the Deepwater Horizon oil spill: U.S. Geological Survey Scientific Investigations Report 2012-5228, 96 p, plus appendixes.

Nowell, L.H., Moran, P.W., Schmidt, T.S., Norman, J.E., Nakagaki, N., Shoda, M.E., Mahler, B.J., Van Metre, P.C., Stone, W.W., Sandstrom, M.E., and Hladik, M.L., 2018, Complex mixtures of dissolved pesticides show potential aquatic toxicity in a synoptic study of Midwestern U.S. streams: Science of the Total Environment, v. 613-614, p. 1469-1488, accessed November 2, 2017, at https://doi. org/10.1016/j.scitotenv.2017.06.156.

Oelsner, G.P., Sprague, L.A., Murphy, J.C., Zuellig, R.E., Johnson, H.M., Ryberg, K.R., Falcone, J.A., Stets, E.G., Vecchia, A.V., Riskin, M.L., De Cicco, L.A., Mills, T.J., and Farmer, W.H., 2017, Water-quality trends in the Nation's rivers and streams, 1972-2012-Data preparation, statistical methods, and trend results: U.S. Geological Survey Scientific Investigations Report 2017-5006, 136 p., accessed November 2, 2017, at https://doi.org/10.3133/sir20175006.

Peters, Gjalt-Jorn, 2017,_userfriendlyscience: Quantitative analysis made accessible_, R package version 0.6-1, accessed June 1, 2017, at https://cran.r-project.org/web/ packages/userfriendlyscience/userfriendlyscience.pdf [also available at https://doi.org/10.17605/OSF.IO/TXEQU].

R Development Core Team, 2017, R-A language and environment for statistical computing: Vienna, Austria, The R Project for Statistical Computing, accessed June 1, 2017, at http://www.R-project.org.

Rowe, G.L., Jr., Belitz, K., Demas, C.R., Essaid, H.I., Gilliom, R.J., Hamilton, P.A., Hoos, A.B., Lee, C.J., Munn, M.D., and Wolock, D.W., 2013, Design of Cycle 3 of the National Water-Quality Assessment Program, 2013-23 —Part 2Science plan for improved water-quality information and management: U.S. Geological Survey Open-File Report 2013-1160, 110 p., https://pubs.usgs.gov/of/2013/1160/.
Ryberg, K.R., Vecchia, A.V., Martin, J.D., and Gilliom, R.J., 2010, Trends in pesticide concentrations in urban streams in the United States, 1992-2008: U.S. Geological Survey Scientific Investigations Report 2010-5139, 101 p.

Ryberg, K.R., and Gilliom, R.J., 2015, Trends in pesticide concentrations and use for major rivers of the United States: Science of the Total Environment, v. 538, p.431-444, accessed November 2, 2017, at https://doi.org/10.1016/j. scitotenv.2015.06.095.

Sandstrom, M.W., and Lewis, J.A., 2009, Instructions for field use of spike solutions for organic-analyte samples: U.S. Geological Survey Techniques of Water-Resources Investigations, book 9, chap. A5, sec. 5.3.2, accessed June 1, 2017, at https://pubs.water.usgs.gov/twri9A5/.

Sandstrom, M.W., Kanagy, L.K., Anderson, C.A., and Kanagy, C.J., 2015, Determination of pesticides and pesticide degradates in filtered water by direct aqueous-injection liquid chromatography-tandem mass spectrometry: U.S. Geological Survey Techniques and Methods, book 5, chap. B11, 54 p., accessed November 2, 2017, at https://dx.doi. org $/ 10.3133 / \mathrm{tm} 5 \mathrm{~B} 11$.

Shoda, M.E., Nowell, L.H., and Bexfield, L.M., 2017a, National Water-Quality Assessment Project replicate surface water and groundwater pesticide data analyzed by the USGS National Water Quality Laboratory schedule 2437, water years 2013-15: U.S. Geological Survey data release, accessed November 2, 2017, at https://doi.org/10.5066/ F75H7DS8.

Shoda, M.E., Nowell, L.H., Stone, W.W., Sandstrom, M.W., and Bexfield, L.M., 2017b, Recovery data for surface water, groundwater and lab reagent samples analyzed by the USGS National Water Quality Laboratory schedule 2437, water years 2013-15: U.S. Geological Survey data release, accessed November 2, 2017, at https://doi.org/10.5066/ F7QZ28G4.

Stone, W.W., Gilliom, R.J., and Ryberg, K.R., 2014, Pesticides in U.S. streams and rivers - Occurrence and trends during 1992-2011: Environmental Science and Technology, v. 48, no.19, p.11025-11030, accessed November 2, 2017, at https://doi.org/10.1021/es5025367.

Stone, W.W., and Bucknell, M.S., 2014, Watershed regressions for pesticides (WARP): U.S. Geological Survey web page, accessed November 2, 2017, at https://dx.doi.org/10.5066/ F7R20ZD3.

Stone, W.W., Crawford, C.G., and Gilliom, R.J., 2013, Watershed regressions for pesticides (WARP) models for predicting stream concentrations of multiple pesticides: Journal of Environmental Quality, v. 42, no.6, p.1838-1851, accessed November 2, 2017, at https://dx.doi.org/10.2134/ jeq2013.05.0179. 
Toccalino, P.L., Gilliom, R.J., Lindsey, B.D., and Rupert, M.G., 2014, Pesticides in groundwater of the United States-Decadal-scale changes, 1993-2011: Groundwater, v. 52, p. 112-125, accessed November 1, 2017, at https:// dx.doi.org/10.1111/gwat.12176.

U.S. Geological Survey, 2017a, Estimated annual agricultural pesticide use: U.S. Geological Survey Pesticide National Synthesis Project web page, accessed November 28, 2017, at https://water.usgs.gov/nawqa/pnsp/usage/maps/index.php.

U.S. Geological Survey, 2017b, National Water Information System-Web interface: accessed June 1, 2017, at http:// dx.doi.org/10.5066/F7P55KJN.

U.S. Geological Survey, 2017c, Quality Systems BranchOBSP homepage: accessed June 1, 2017, at https://bqs.usgs. gov/obsp/.
U.S. Geological Survey, 2017d, Regional stream quality assessments - Study design: accessed December 28, 2017, at https://webapps.usgs.gov/RSQA/\#!/.

Welch, B.L., 1938, The significance of the difference between two means when the population variances are unequal:

Biometrika, v. 29, no. 3-4, p. 350-362.

Williams, T., Foreman, W.T., Decess, J., Reed-Parker, C., and Stevenson, D.L., 2015, Changes to National Water Quality Laboratory (NWQL) procedures used to establish and verify laboratory detection and reporting limits: National Water Quality Laboratory Technical Memorandum 15.02, accessed November 2, 2017, at https://nwql.usgs.gov/tech_memos/ nwq1.2015-02.pdf. 


\section{EXPLANATION}

$\times \quad$ Outlier - data value more than 1.5 times [p75th-p25th] distance from the 75 th percentile

Whisker-drawn to the largest data value not more than 1.5 times [p75th-p25th] distance from the 75th percentile

75th percentile (p75th)

Median

25th percentile (p25th)

Whisker-drawn to the smallest data value not more than 1.5 times [p75th-p25th] distance from the 25th percentile

$\times \quad$ Outlier-data value more than 1.5 times [p 75th-p25th] distance from the 25 th percentile

Figure 1. Image showing explanation of a boxplot that is used to depict the distribution of recovery. 
A. Organophosphate: laboratory reagent spikes

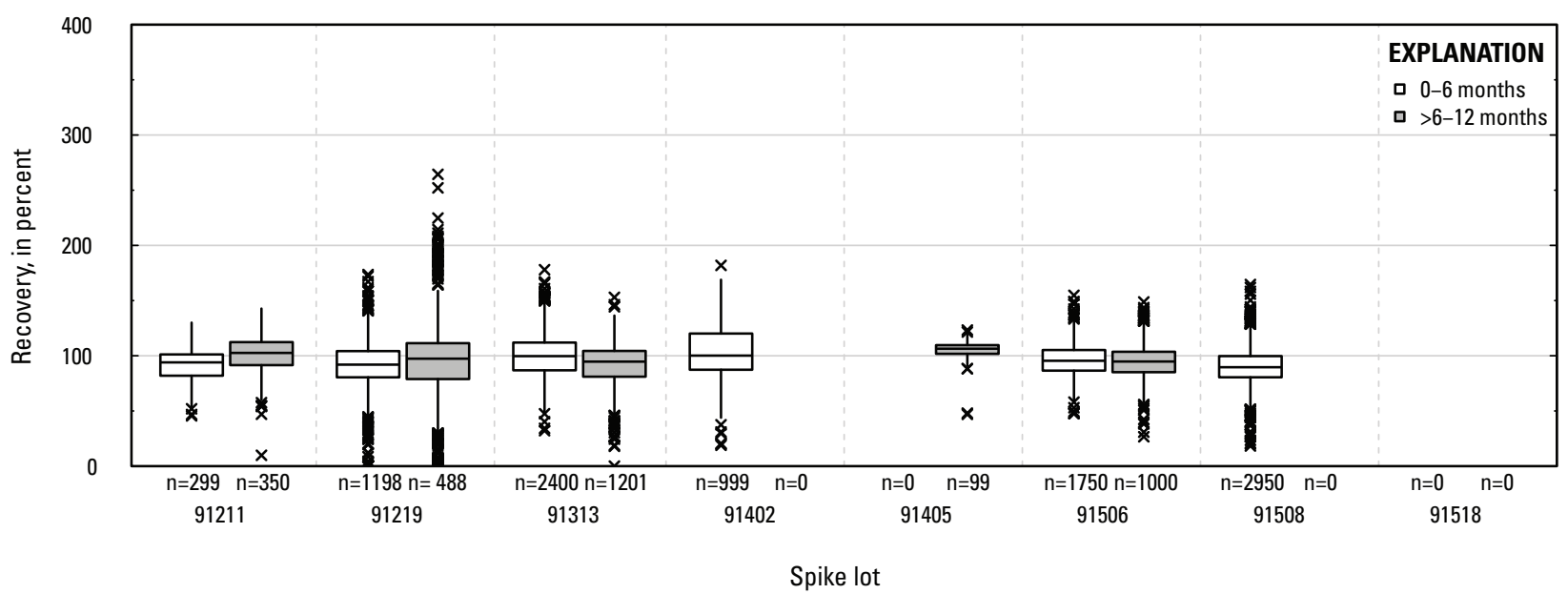

\section{B. Organophosphate : groundwater field matrix spikes}

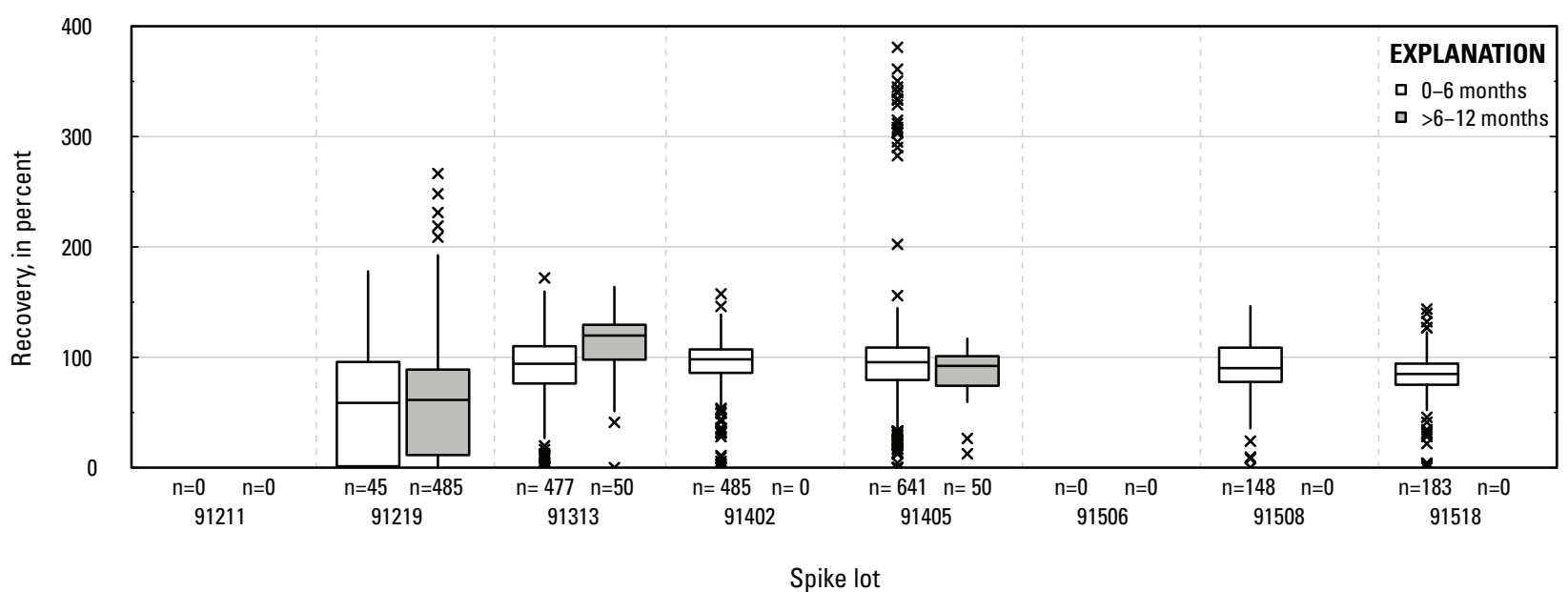

\section{Organophosphate : surface water field matrix spikes}

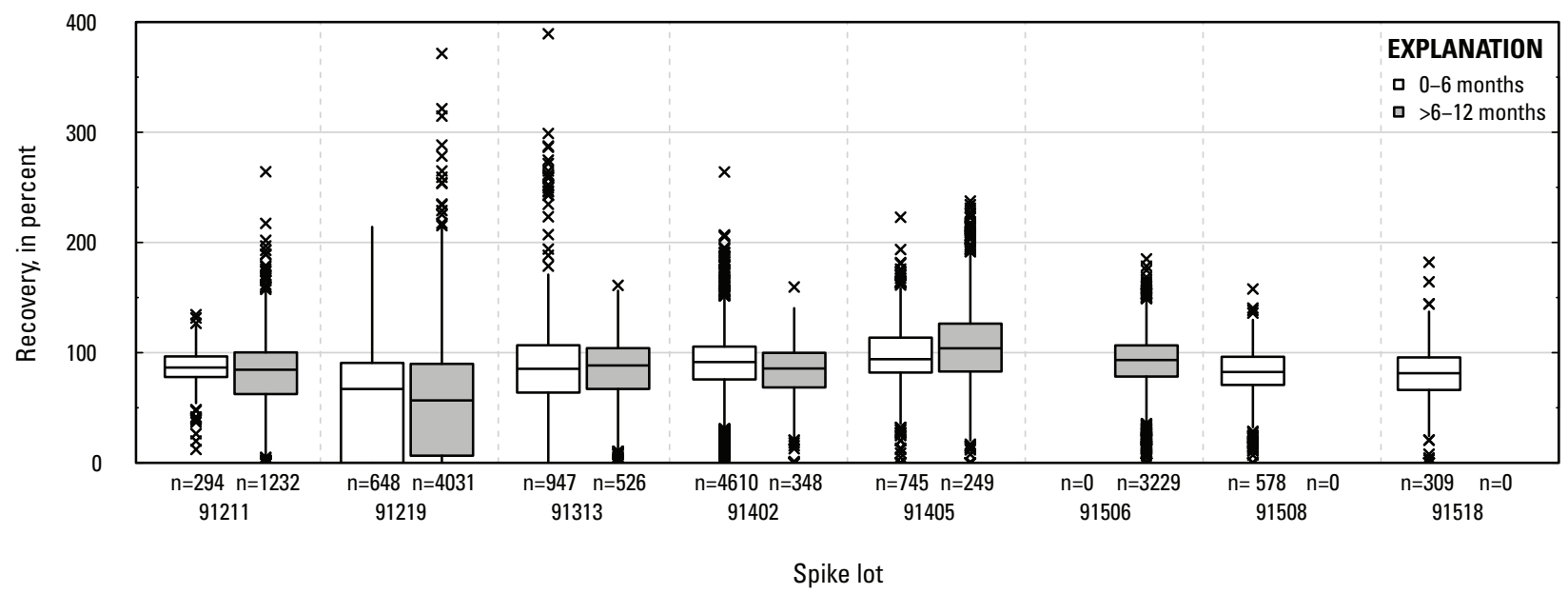

Figure 2. Graph showing distributions of recovery for pesticides in schedule 2437 by analytical method group, matrix, spike lot, and spike lot age. Recovery values larger than 400 percent are not shown. 


\section{Carbamate and thiocarbamate: laboratory reagent spikes}

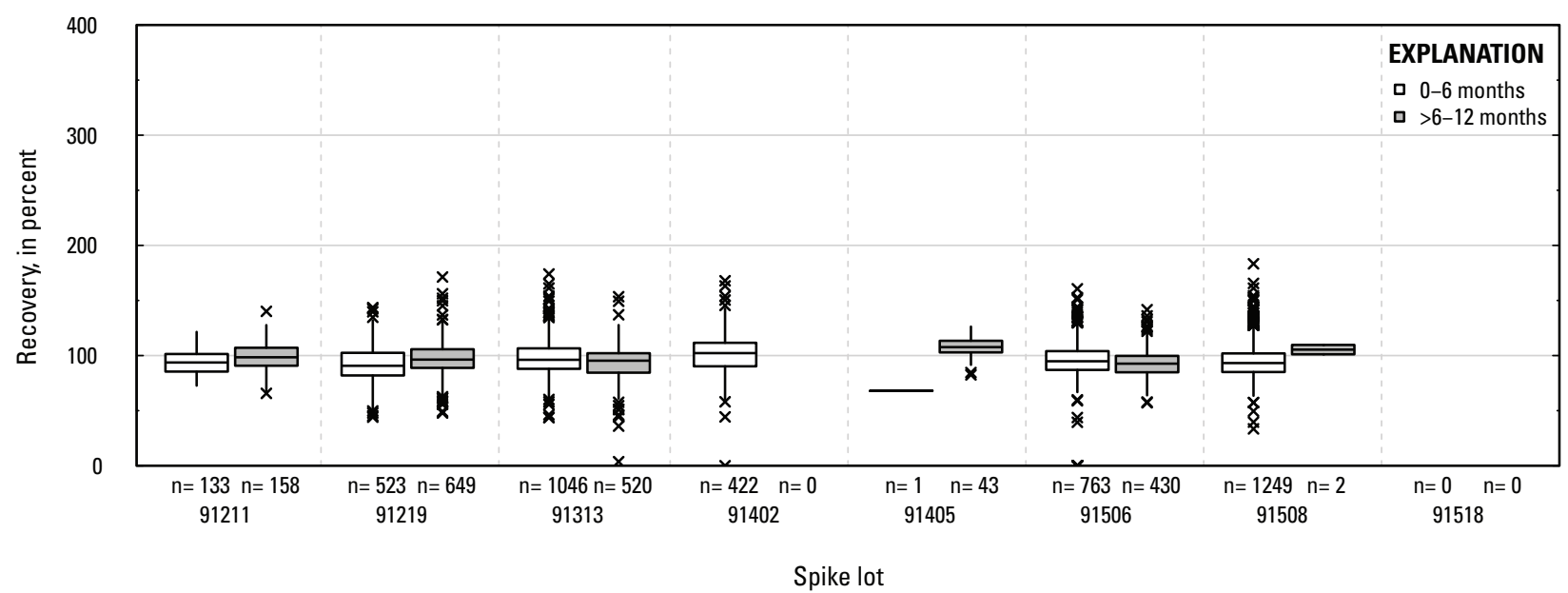

E. Carbamate and thiocarbamate: groundwater field matrix spikes

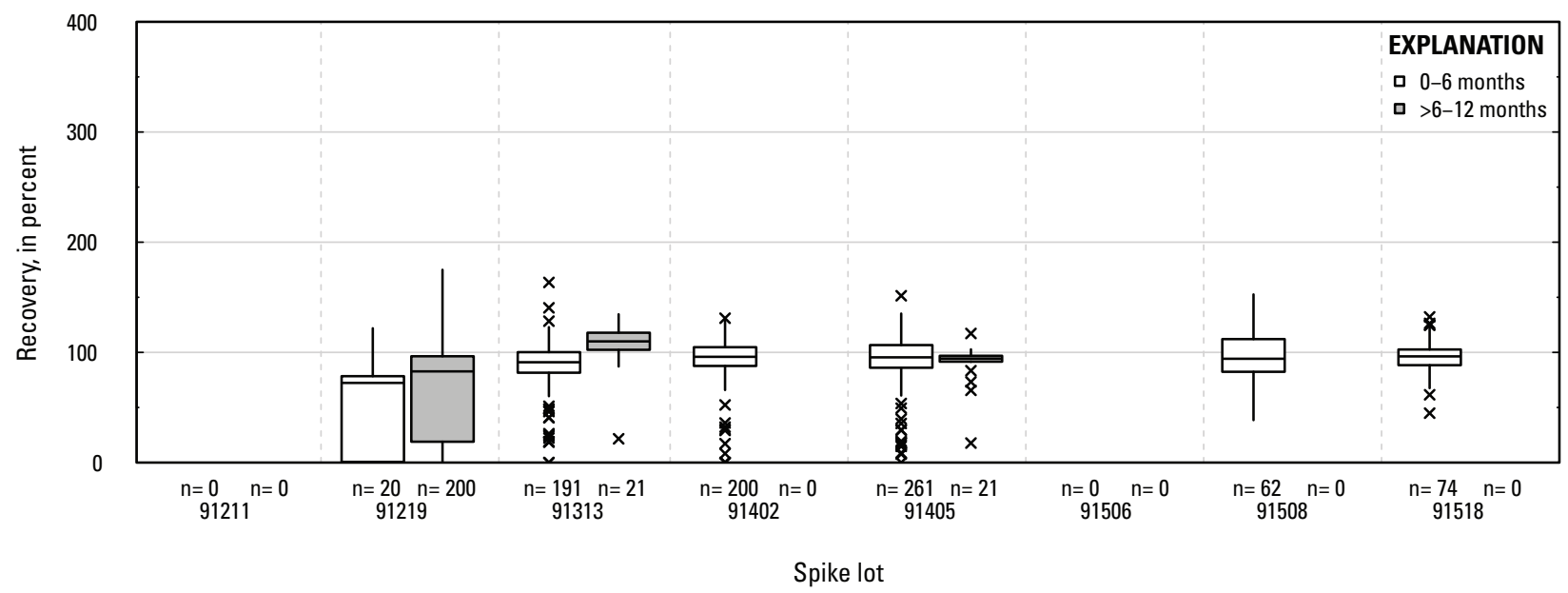

F. Carbamate and thiocarbamate: surface water field matrix spikes

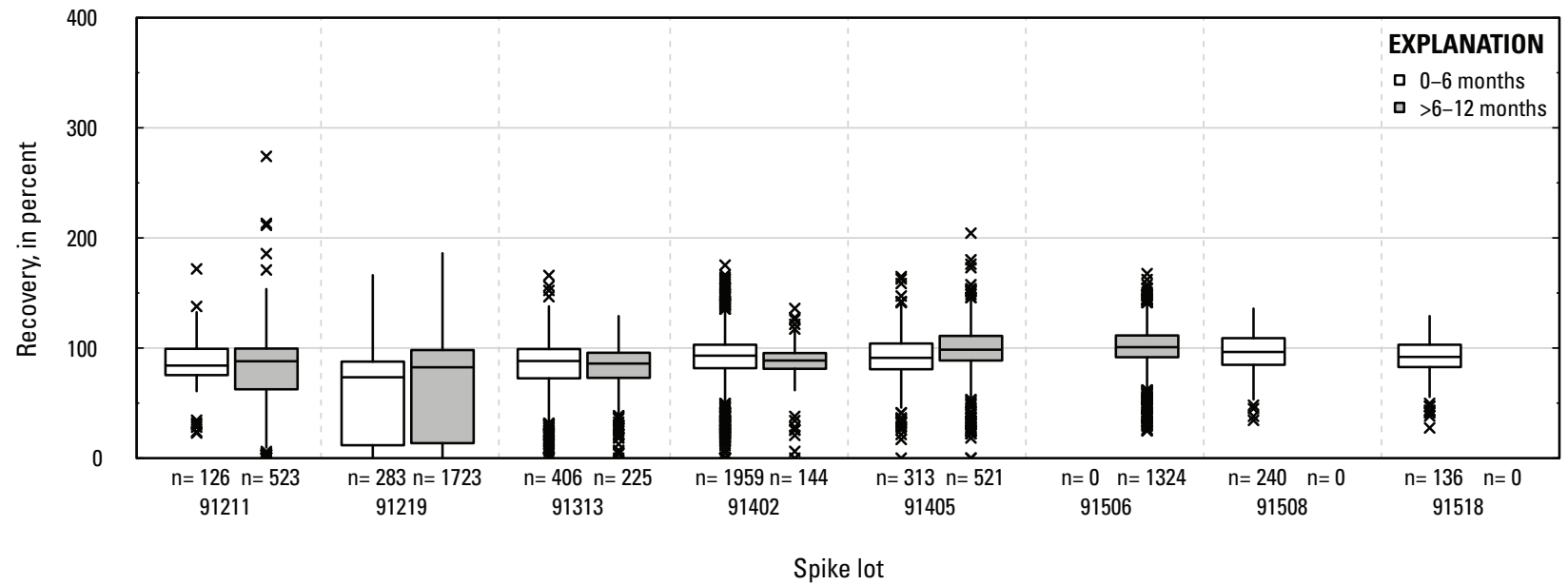

Figure 2. Graph showing distributions of recovery for pesticides in schedule 2437 by analytical method group, matrix, spike lot, and spike lot age. Recovery values larger than 400 percent are not shown.-Continued 
G. Pyrethroid, organochlorine and phenylpyrazine: laboratory reagent spikes

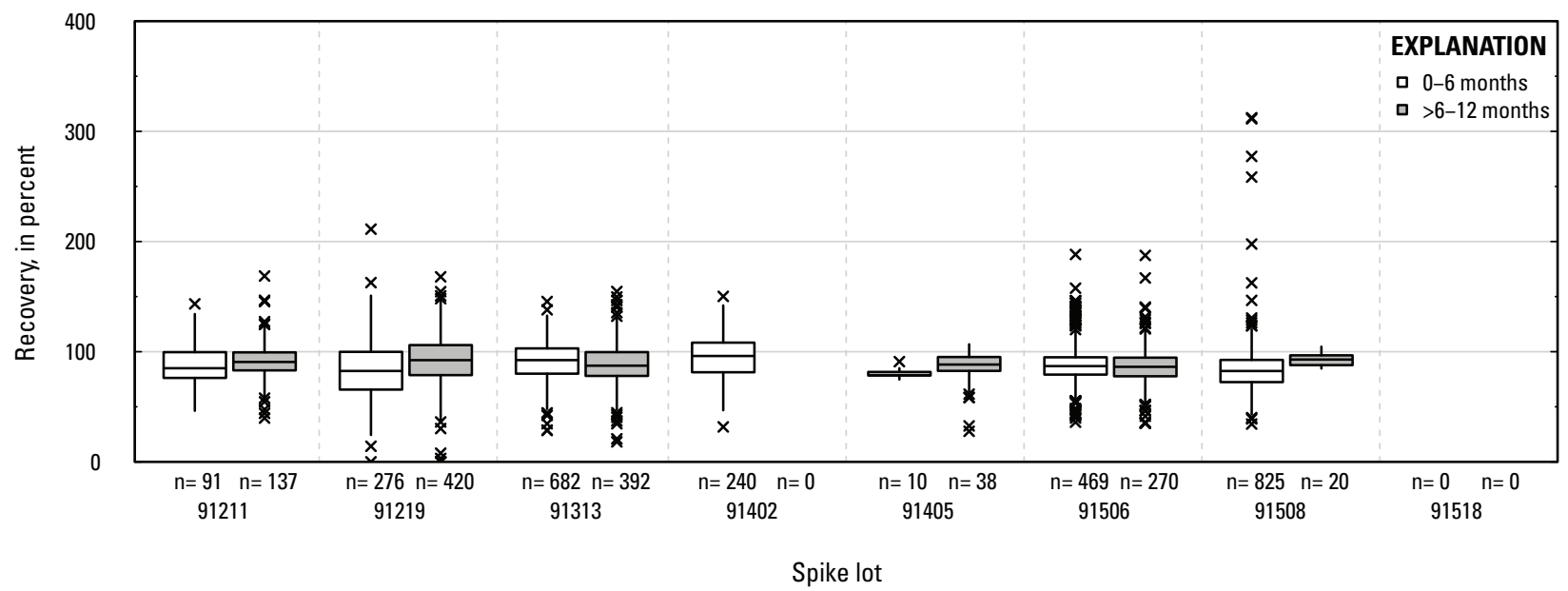

H. Pyrethroid, organochlorine and phenylpyrazine: groundwater field matrix spikes

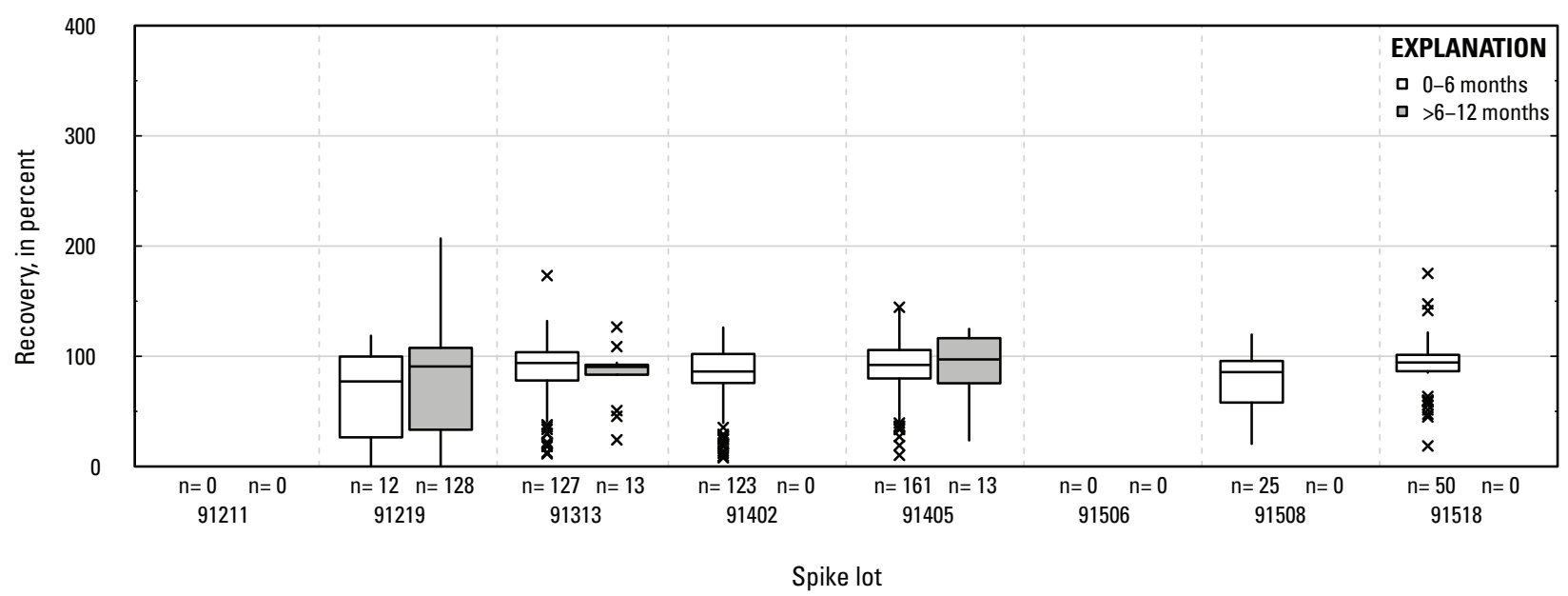

I. Pyrethroid, organochlorine and phenylpyrazine: surface water field matrix spikes

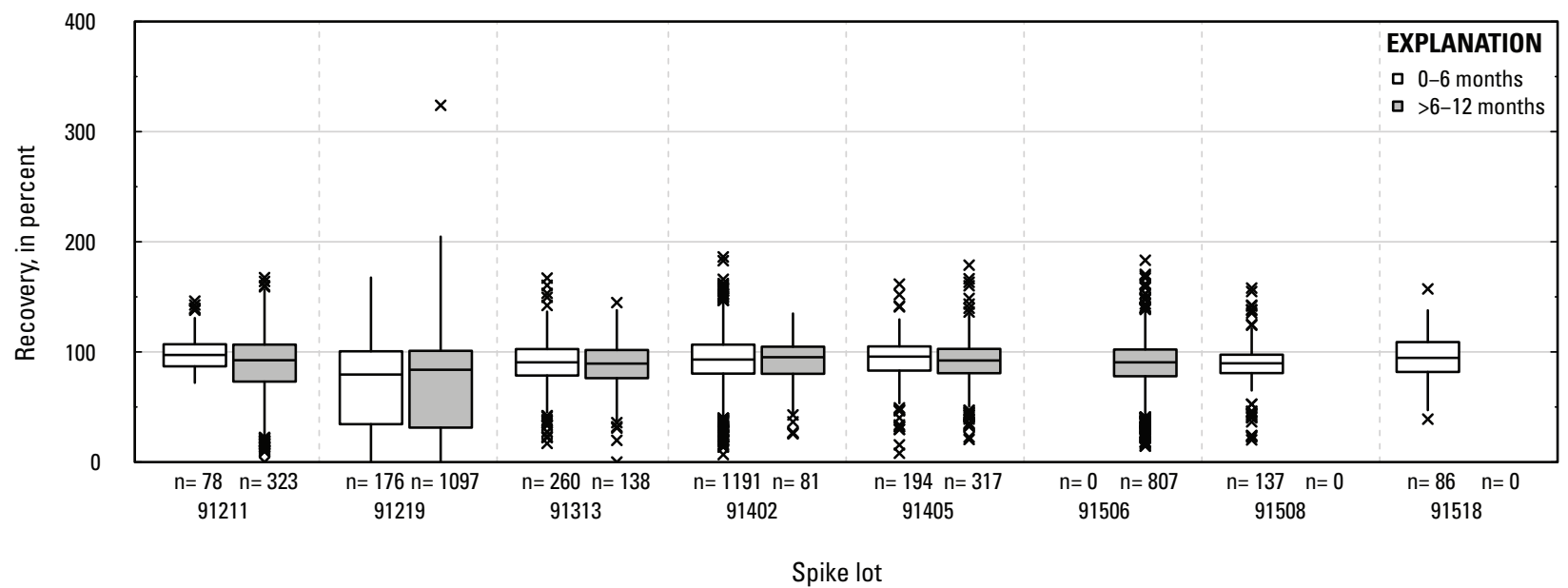

Figure 2. Graph showing distributions of recovery for pesticides in schedule 2437 by analytical method group, matrix, spike lot, and spike lot age. Recovery values larger than 400 percent are not shown.-Continued 
$J$. Triazine: laboratory reagent spikes

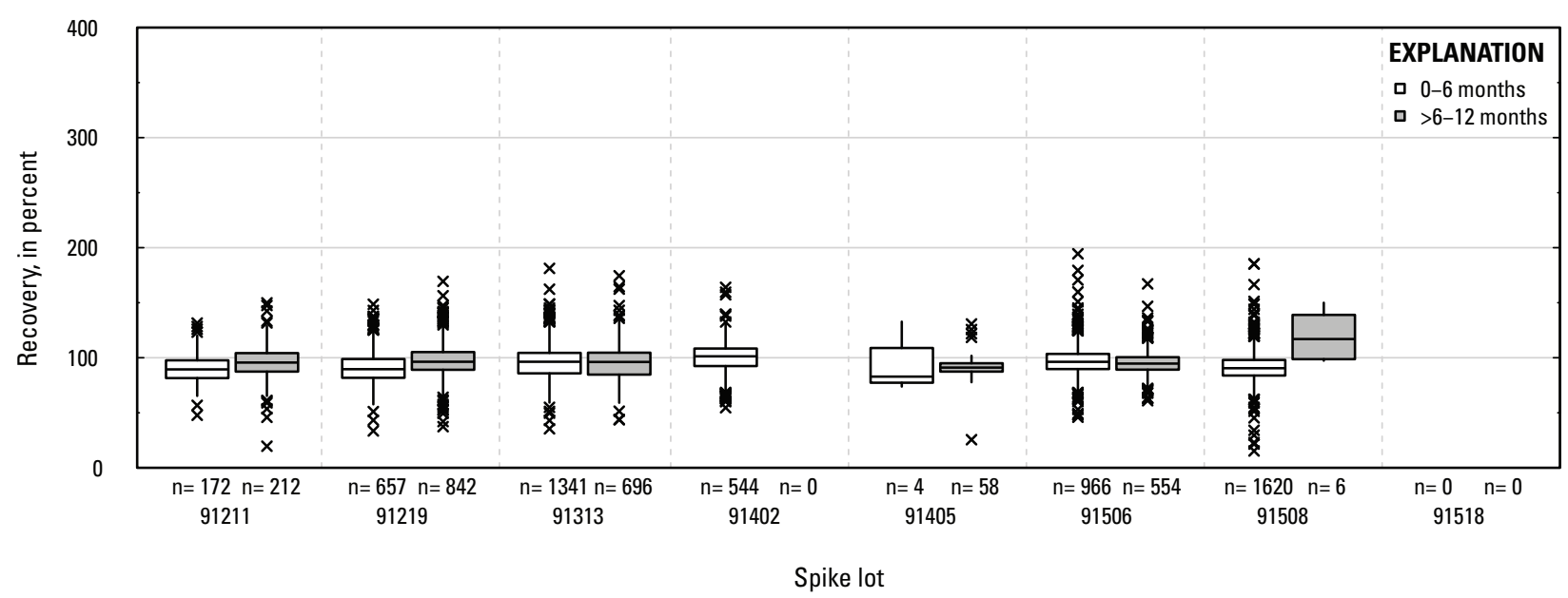

K. Triazine: groundwater field matrix spikes

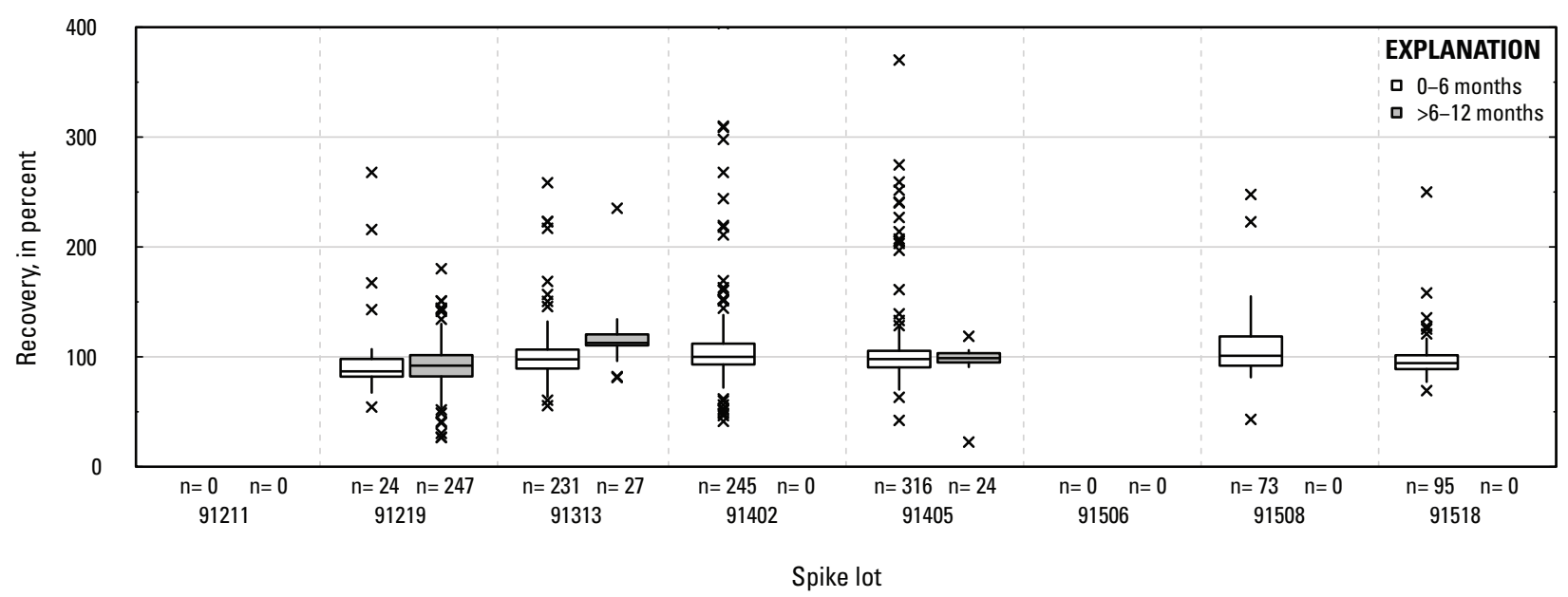

\section{Triazine: surface water field matrix spikes}

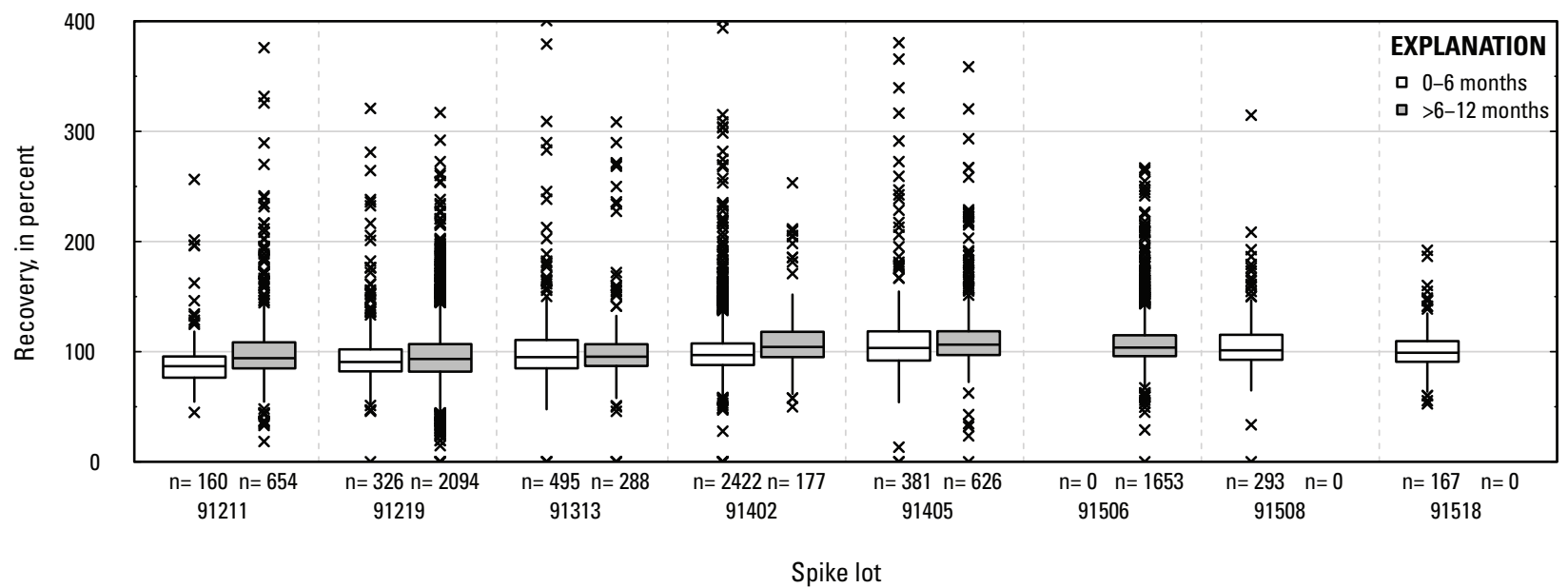

Figure 2. Graph showing distributions of recovery for pesticides in schedule 2437 by analytical method group, matrix, spike lot, and spike lot age. Recovery values larger than 400 percent are not shown._Continued 
M. Sulfonylurea and urea: laboratory reagent spikes

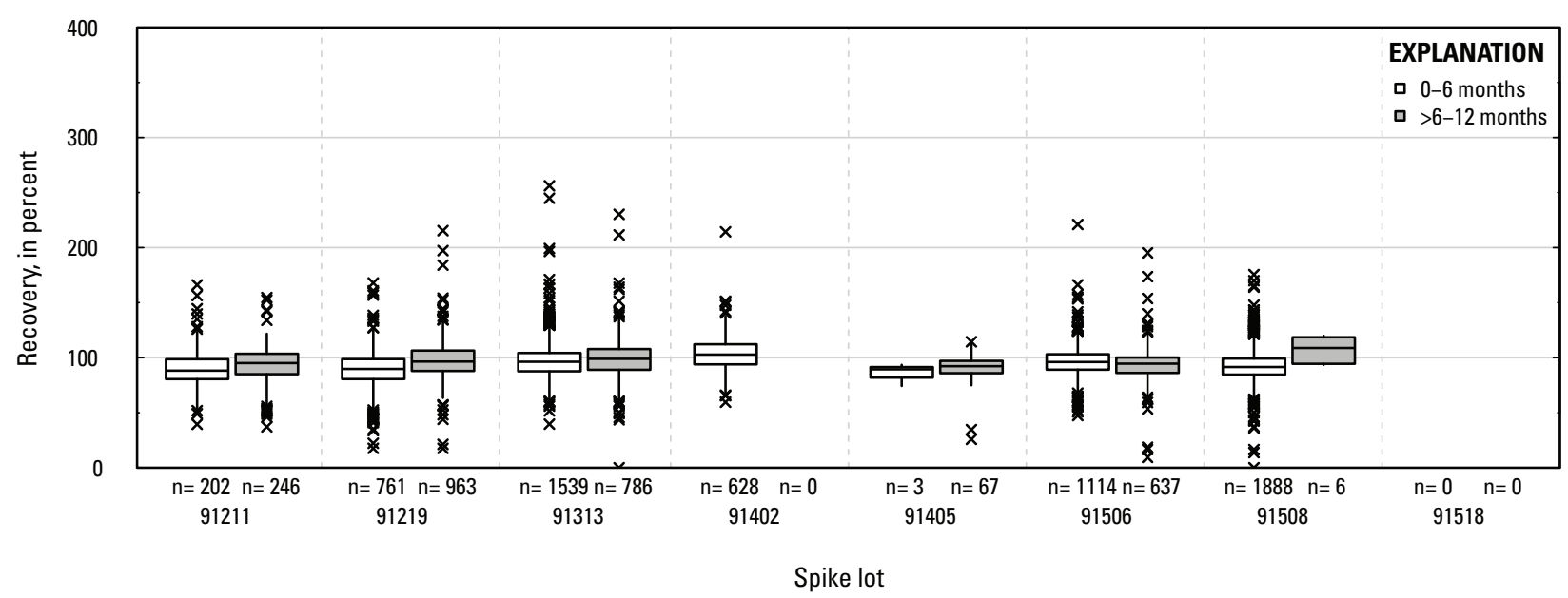

$N$. Sulfonylurea and urea: groundwater field matrix spikes

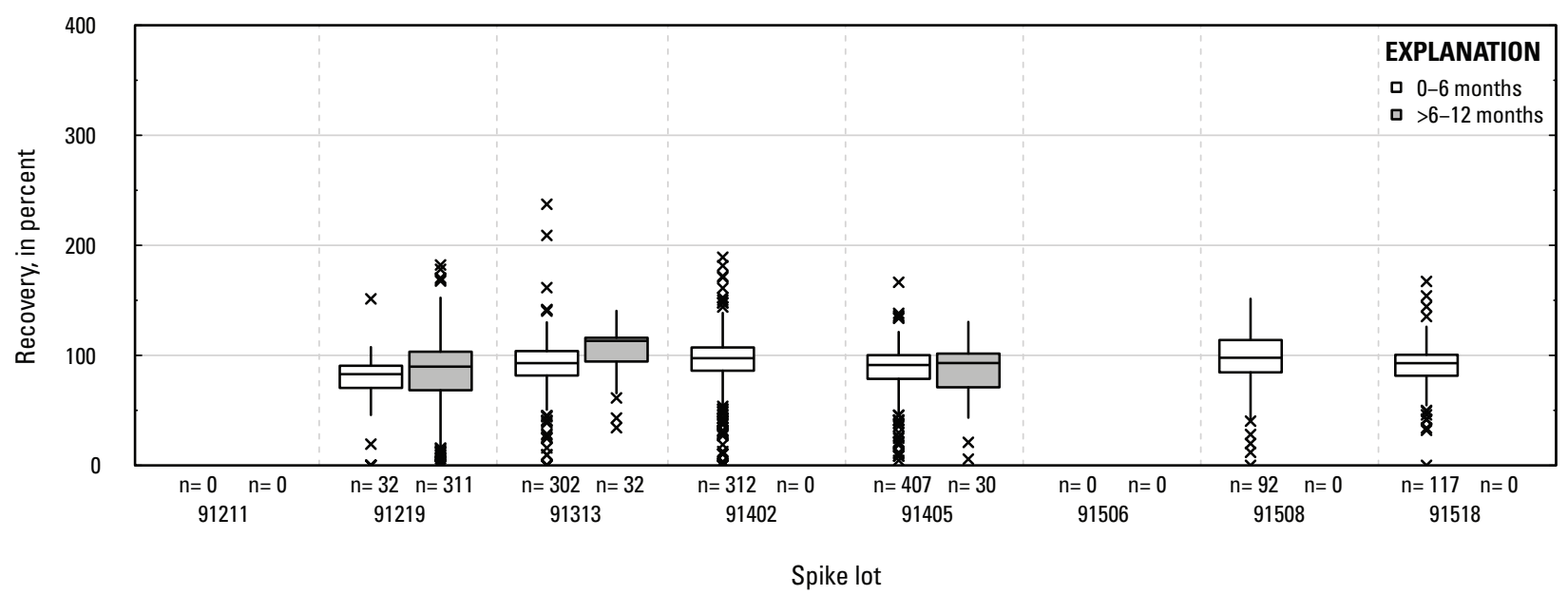

\section{0 . Sulfonylurea and urea: surface water field matrix spikes}

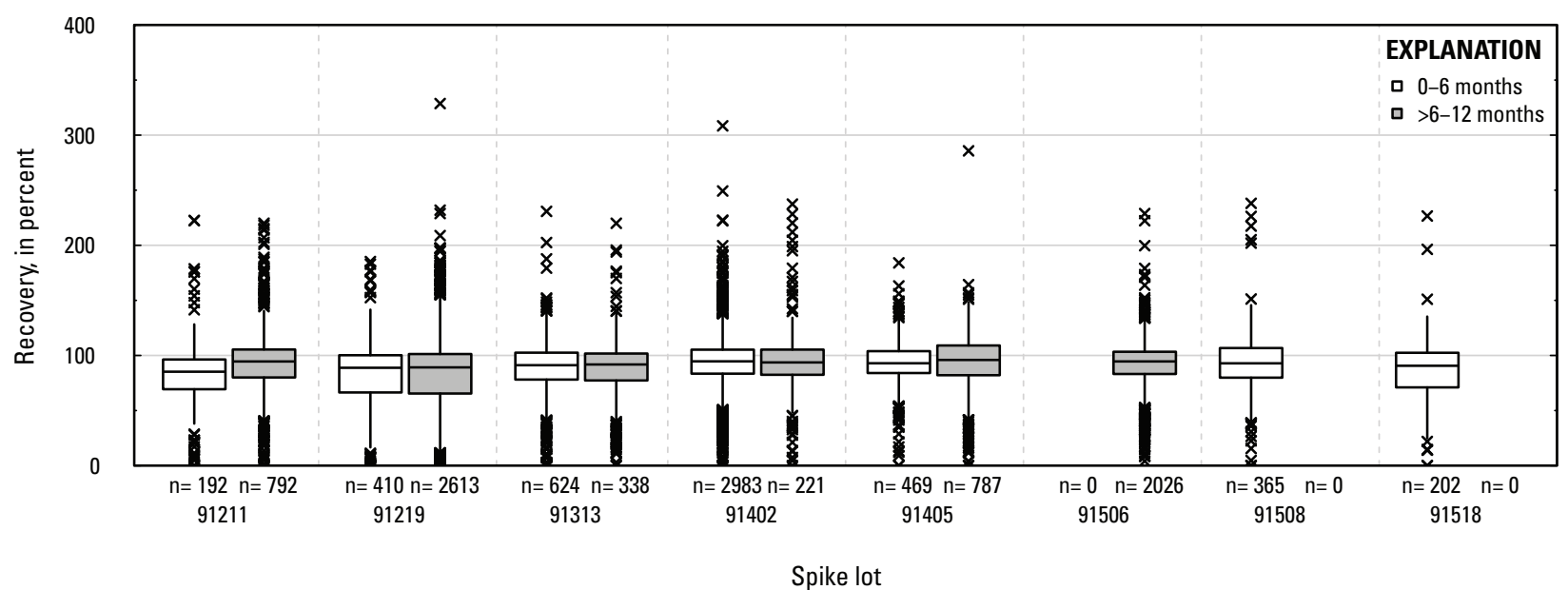

Figure 2. Graph showing distributions of recovery for pesticides in schedule 2437 by analytical method group, matrix, spike lot, and spike lot age. Recovery values larger than 400 percent are not shown.-Continued 
$P$. Acetanilide and amide: laboratory reagent spikes

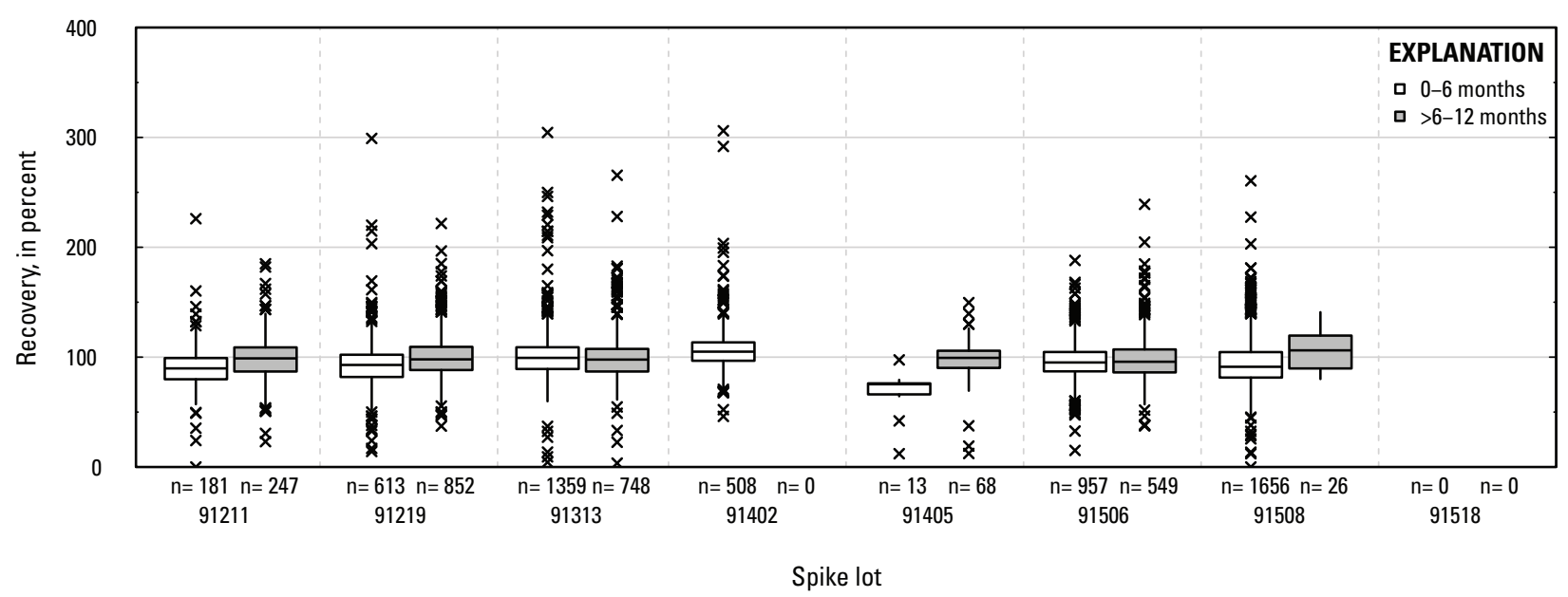

Q. Acetanilide and amide: groundwater field matrix spikes

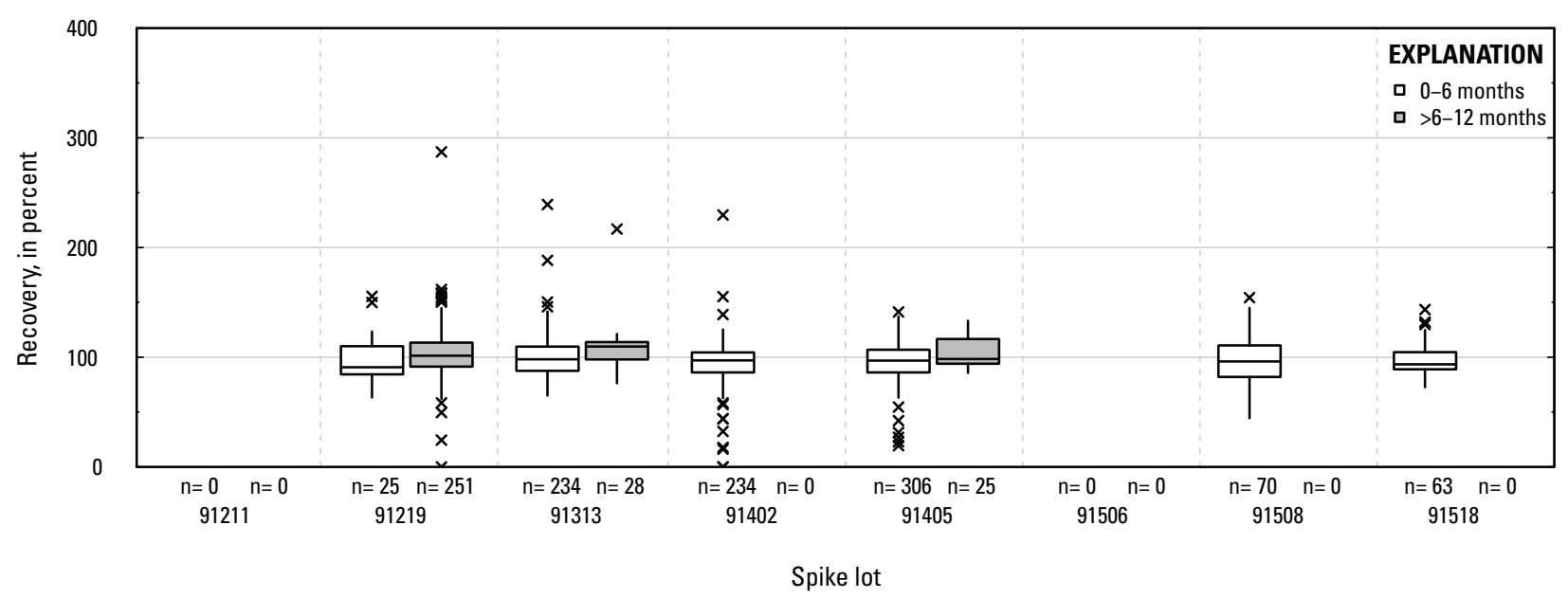

$\boldsymbol{R}$. Acetanilide and amide: surface water field matrix spikes

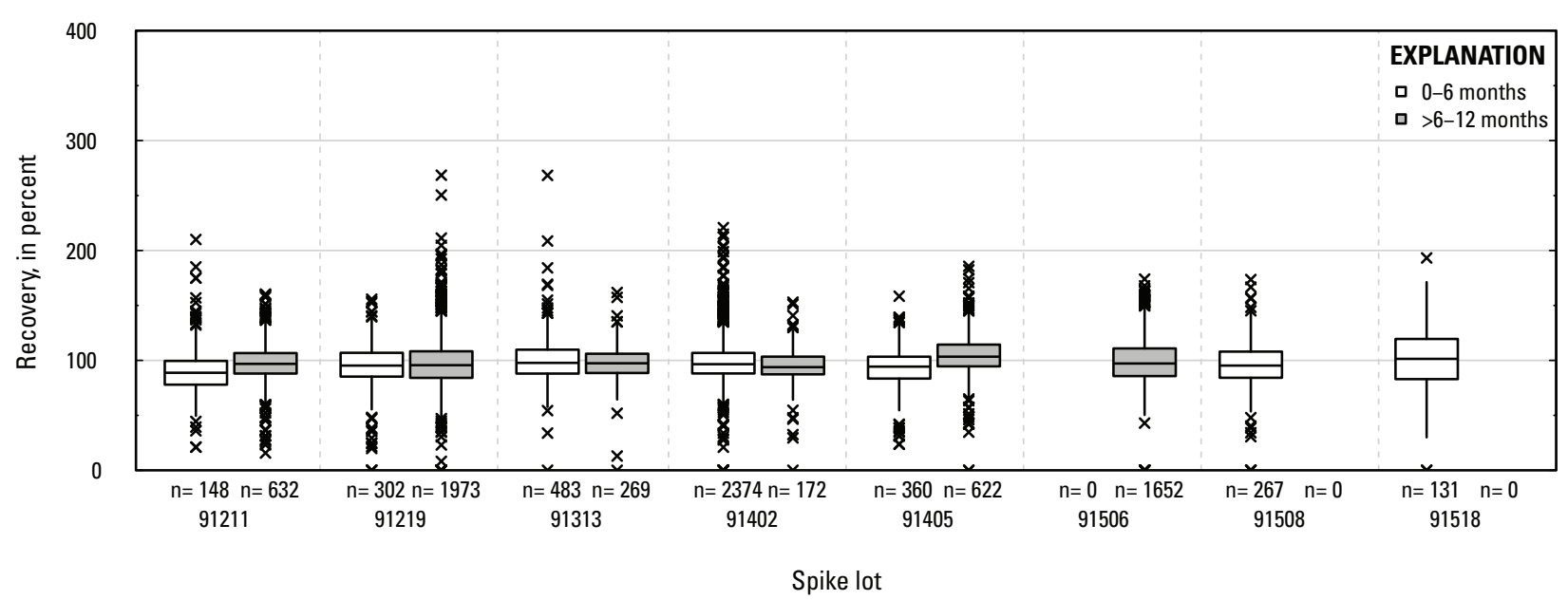

Figure 2. Graph showing distributions of recovery for pesticides in schedule 2437 by analytical method group, matrix, spike lot, and spike lot age. Recovery values larger than 400 percent are not shown.-Continued 


\section{$S$. Fungicide: laboratory reagent spikes}

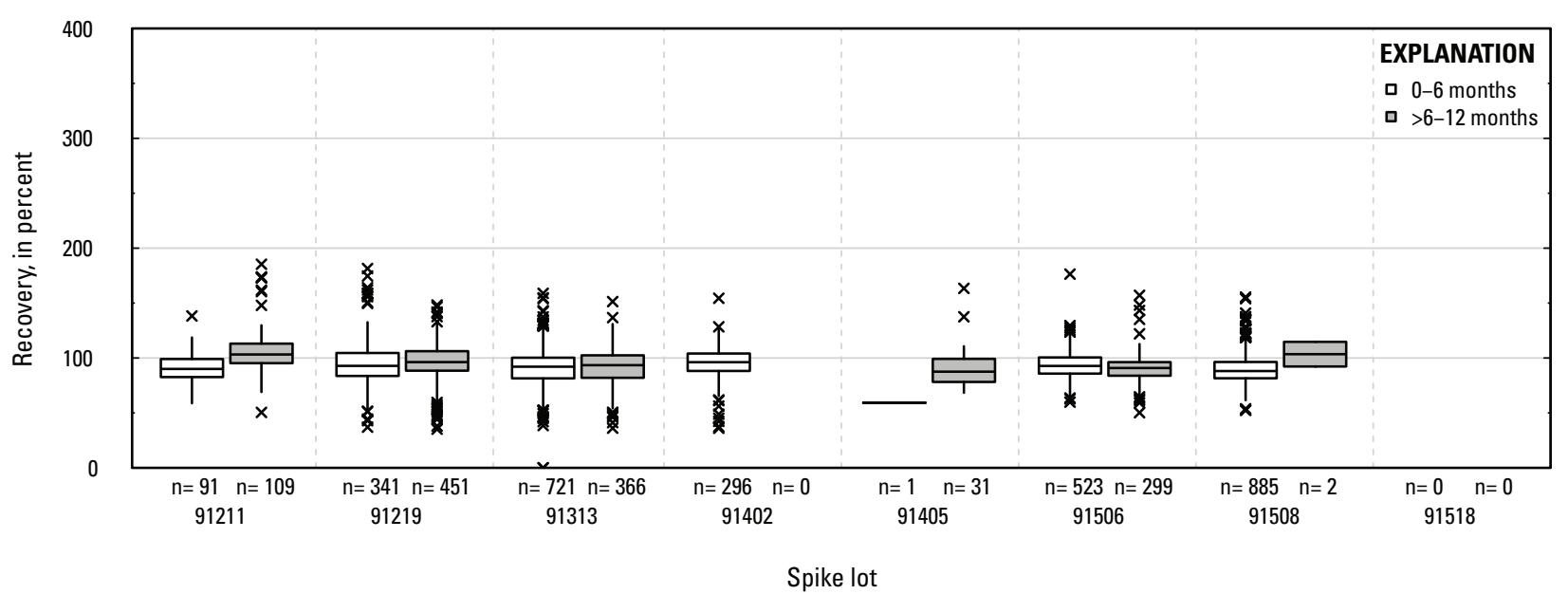

\section{$T$. Fungicide: groundwater field matrix spikes}

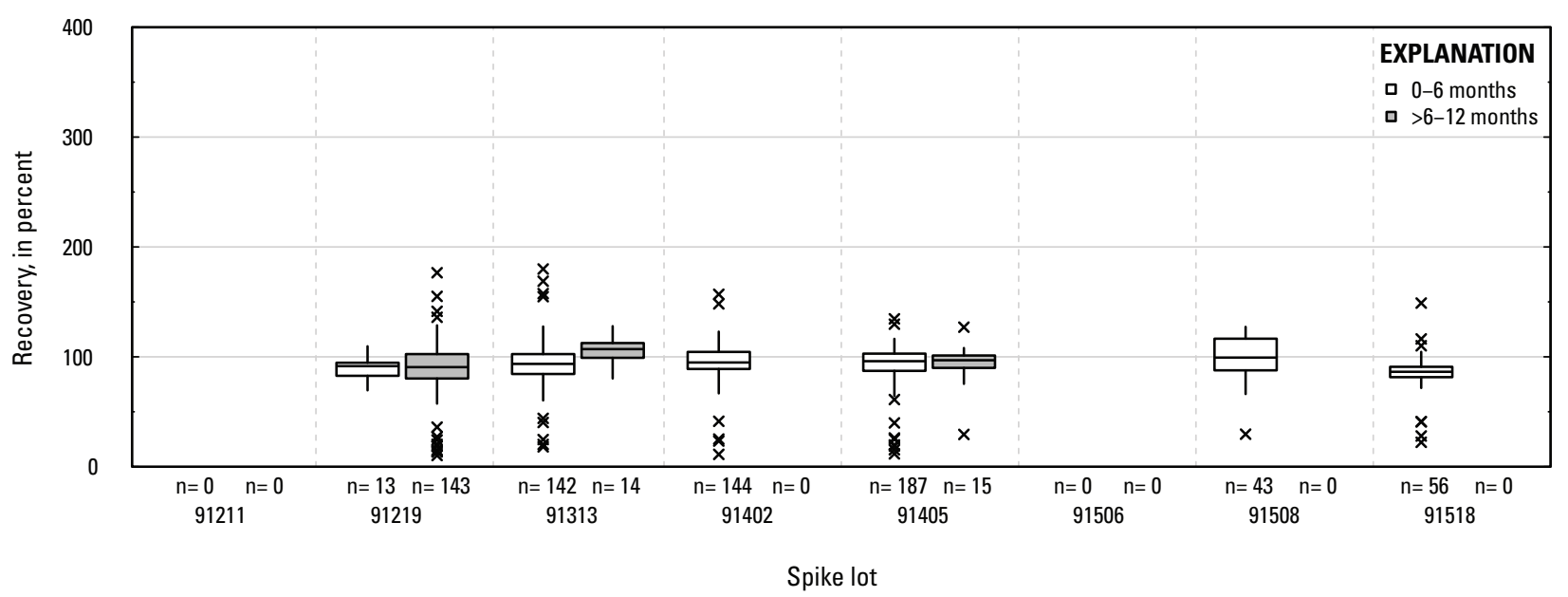

U. Fungicide: surface water field matrix spikes

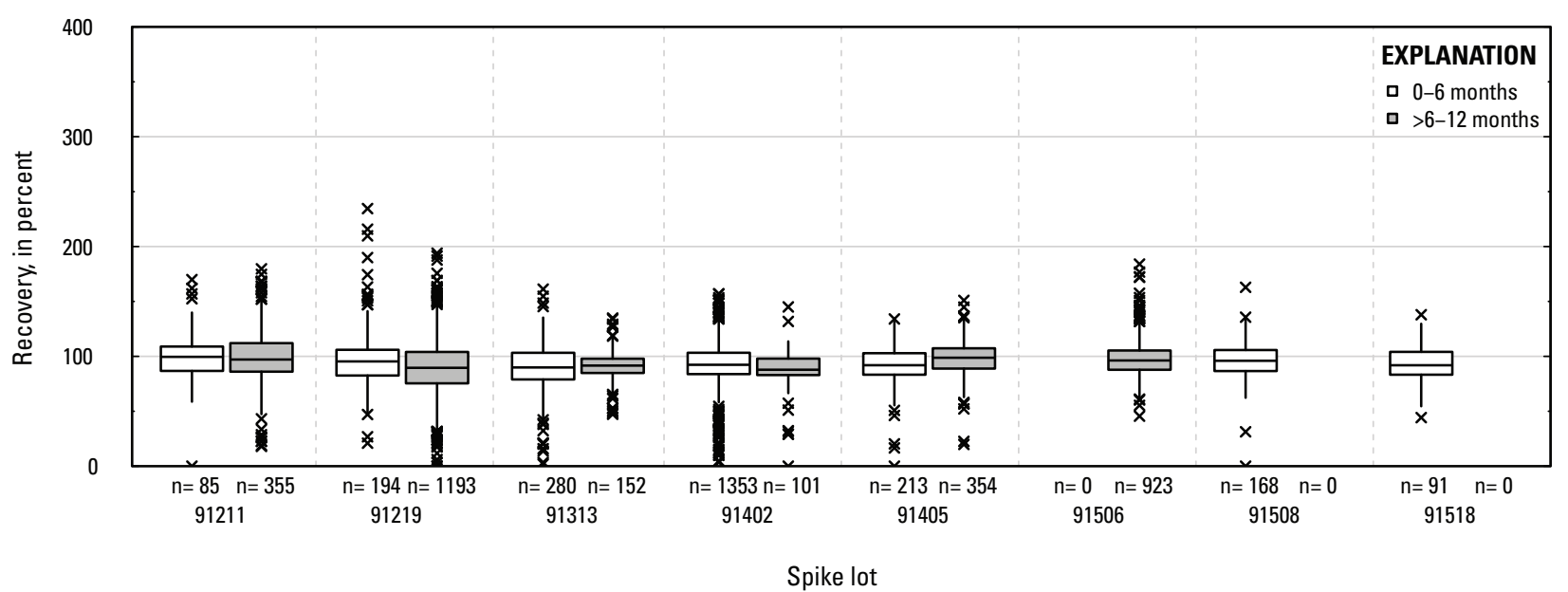

Figure 2. Graph showing distributions of recovery for pesticides in schedule 2437 by analytical method group, matrix, spike lot, and spike lot age. Recovery values larger than 400 percent are not shown.-Continued 
V. Acid: laboratory reagent spikes

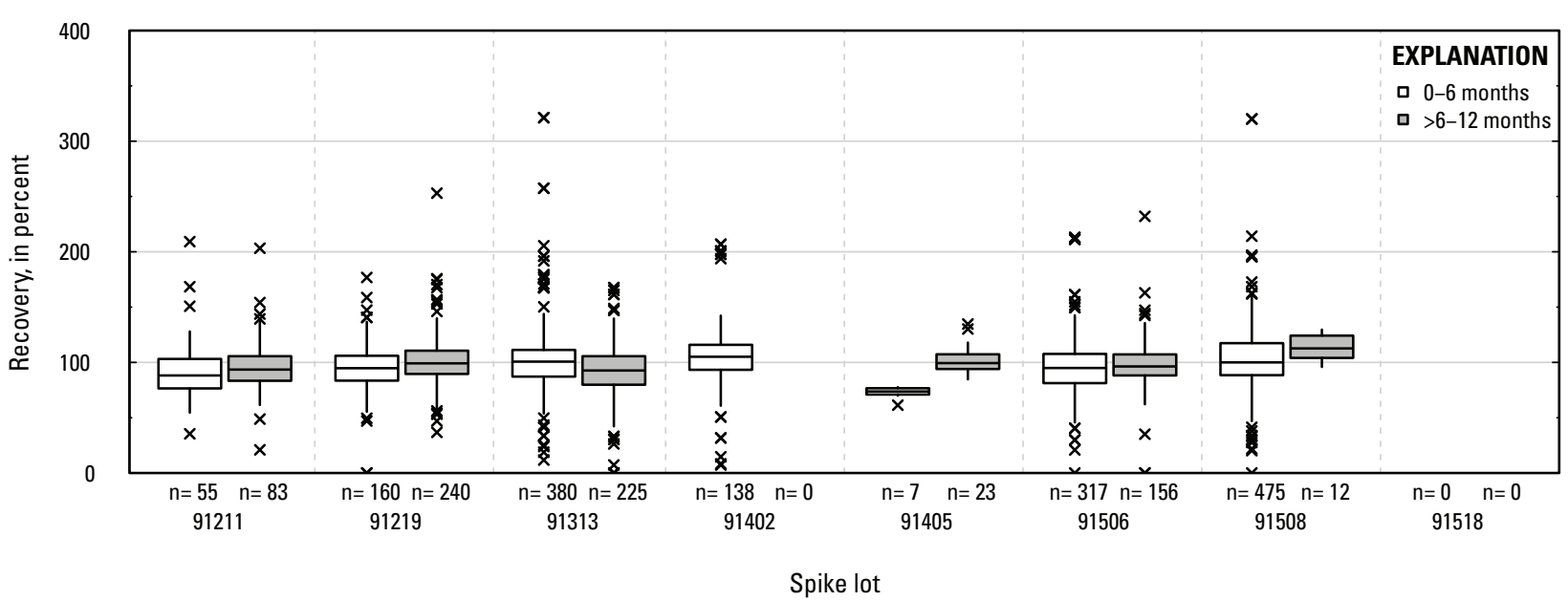

W. Acid: groundwater field matrix spikes

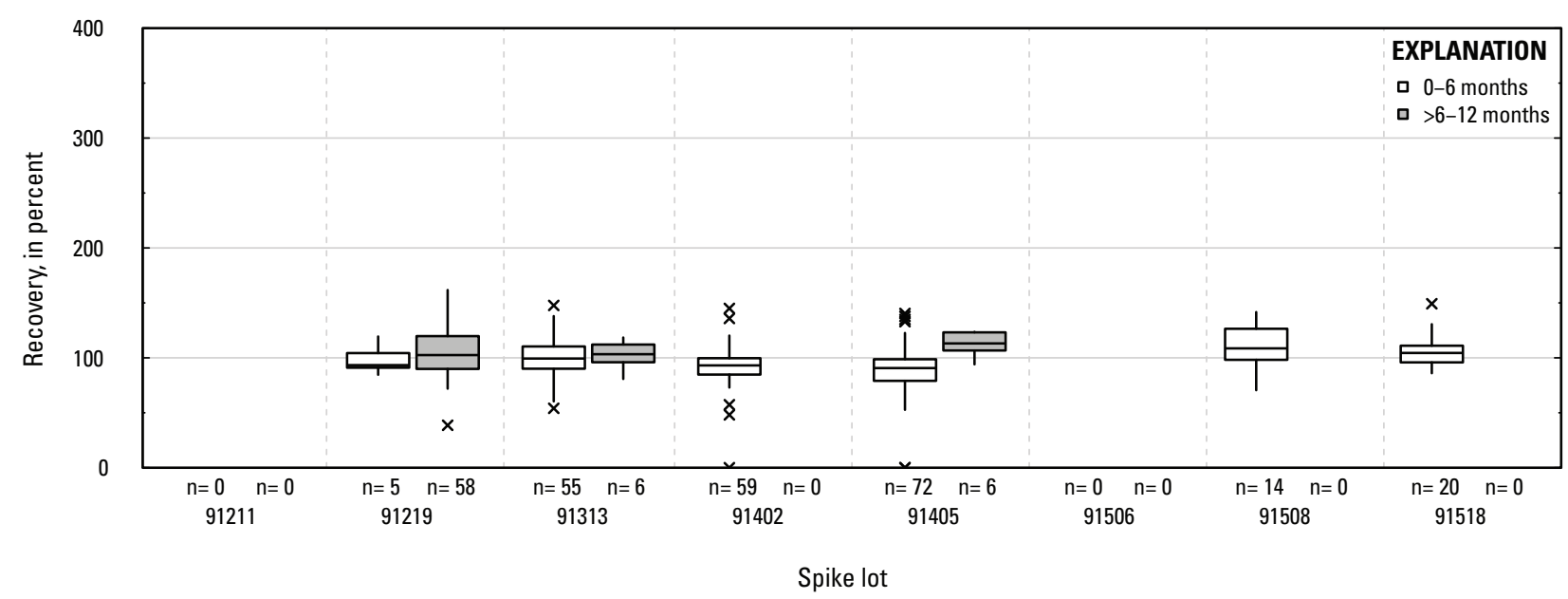

\section{$X$. Acid: surface water field matrix spikes}

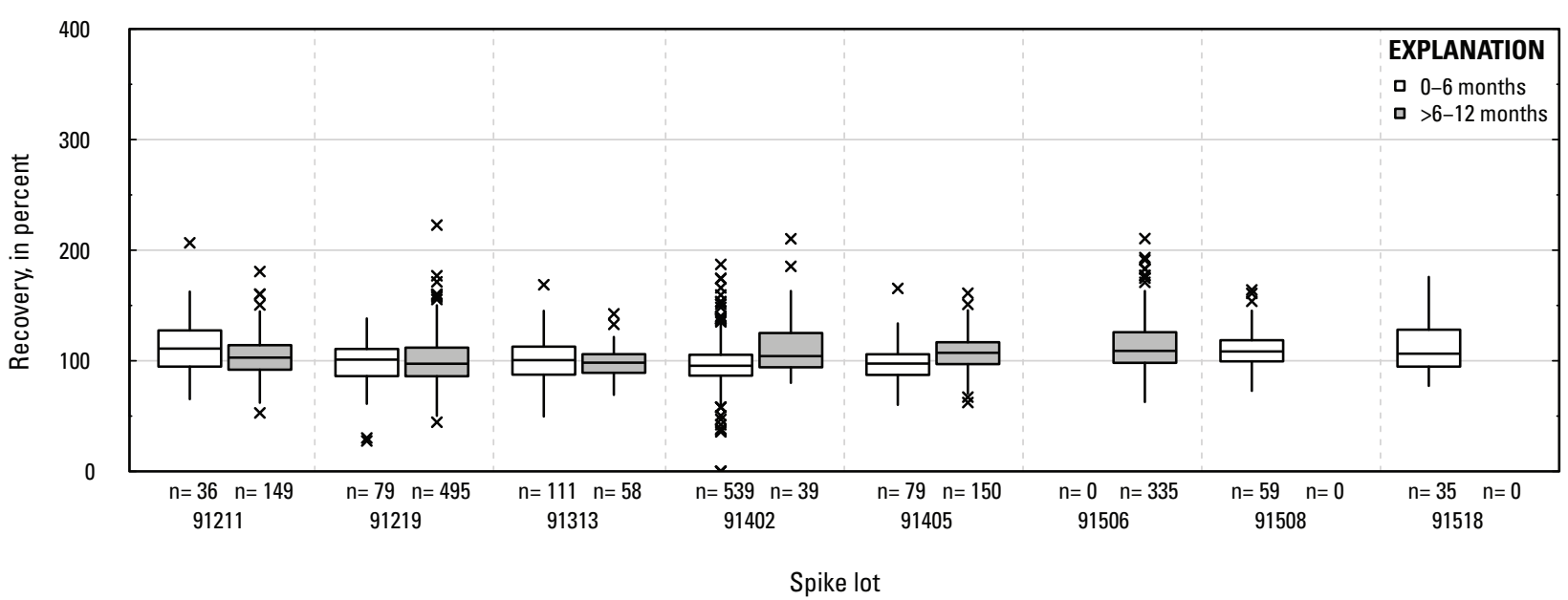

Figure 2. Graph showing distributions of recovery for pesticides in schedule 2437 by analytical method group, matrix, spike lot, and spike lot age. Recovery values larger than 400 percent are not shown.-Continued 
Y. Miscellaneous: laboratory reagent spikes

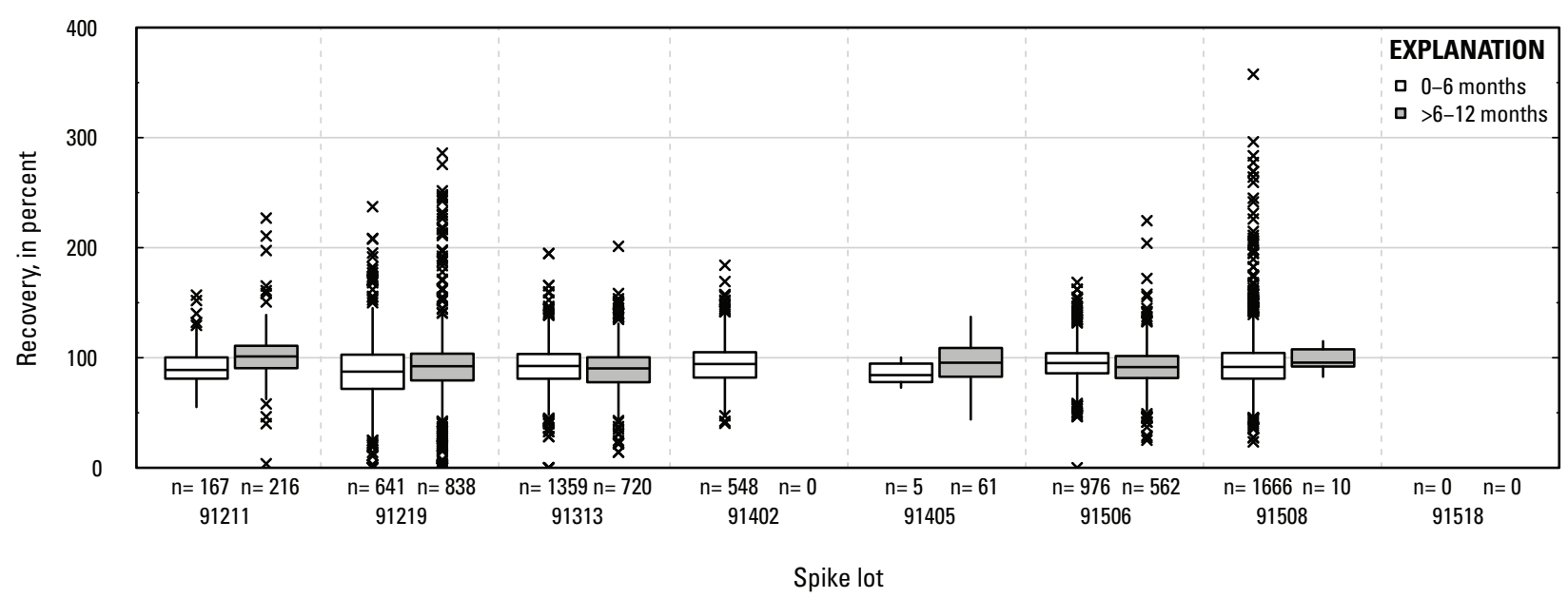

Z. Miscellaneous: ground water field matrix spikes

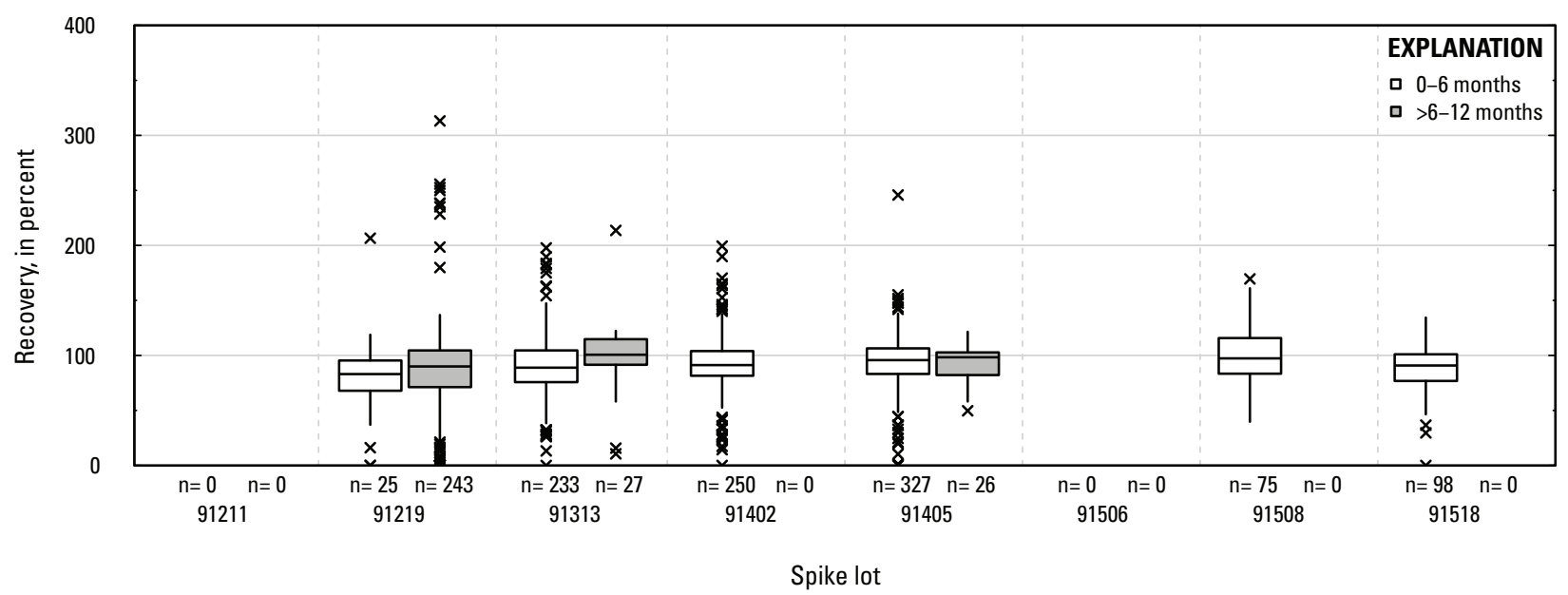

\section{$A A$. Miscellaneous: surface water field matrix spikes}

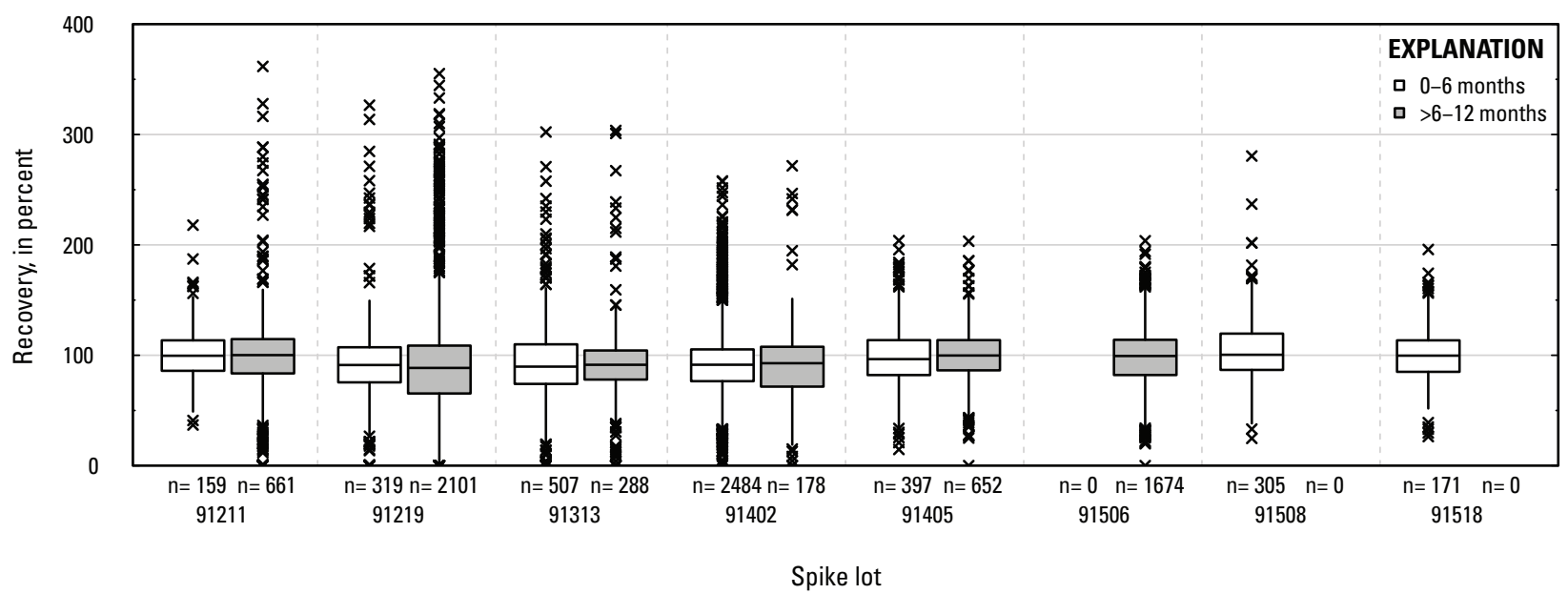

Figure 2. Graph showing distributions of recovery for pesticides in schedule 2437 by analytical method group, matrix, spike lot, and spike lot age. Recovery values larger than 400 percent are not shown.-Continued 


\section{A. Organophosphate: laboratory reagent spikes}

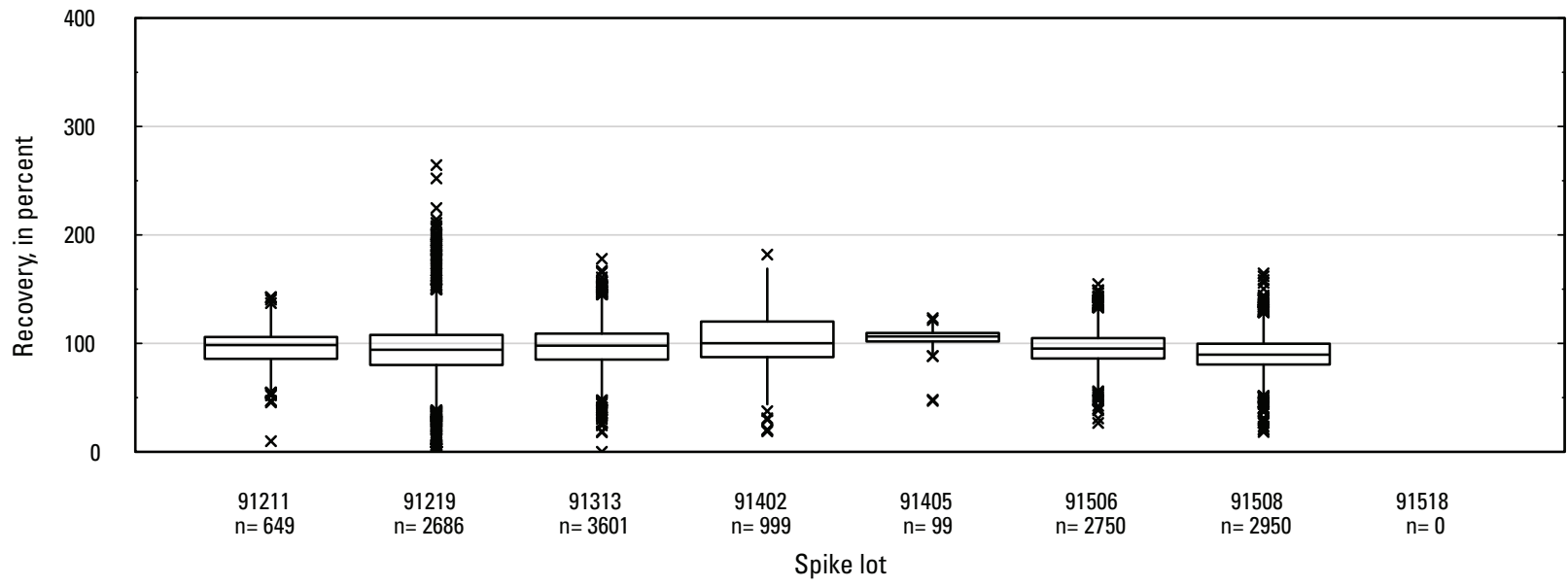

B. Organophosphate: groundwater field matrix spikes

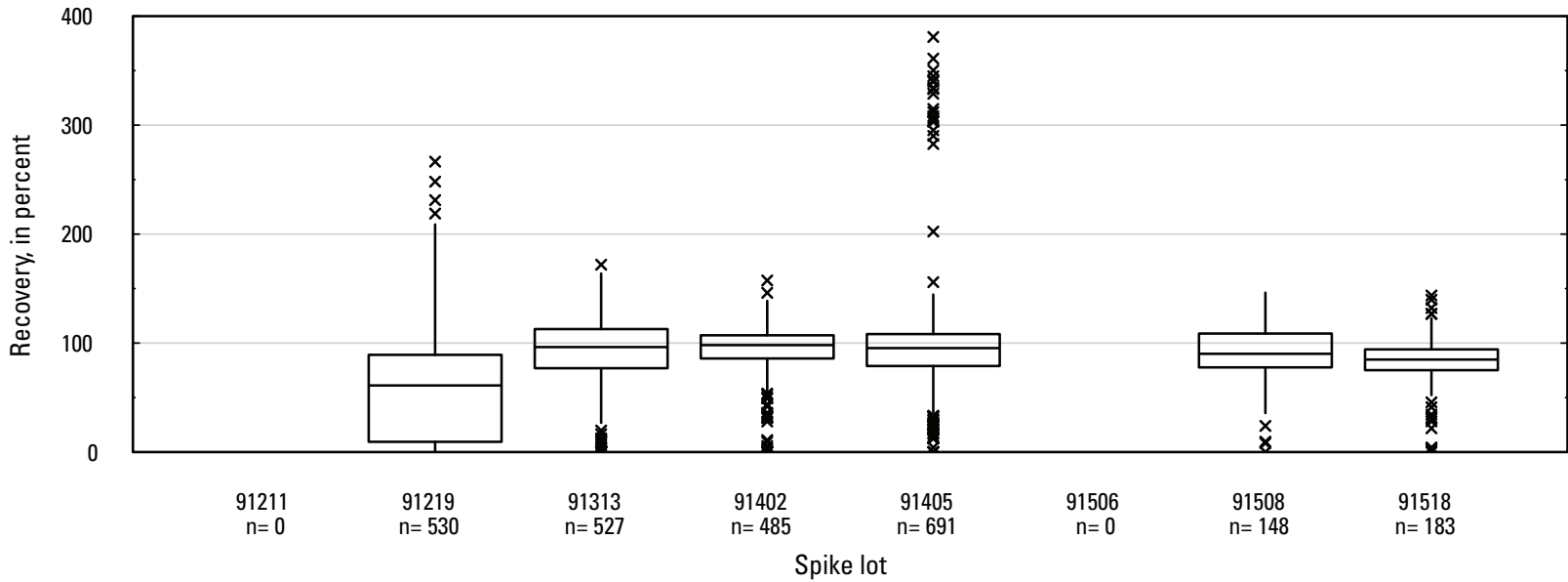

C. Organophosphate: surface water field matrix spikes

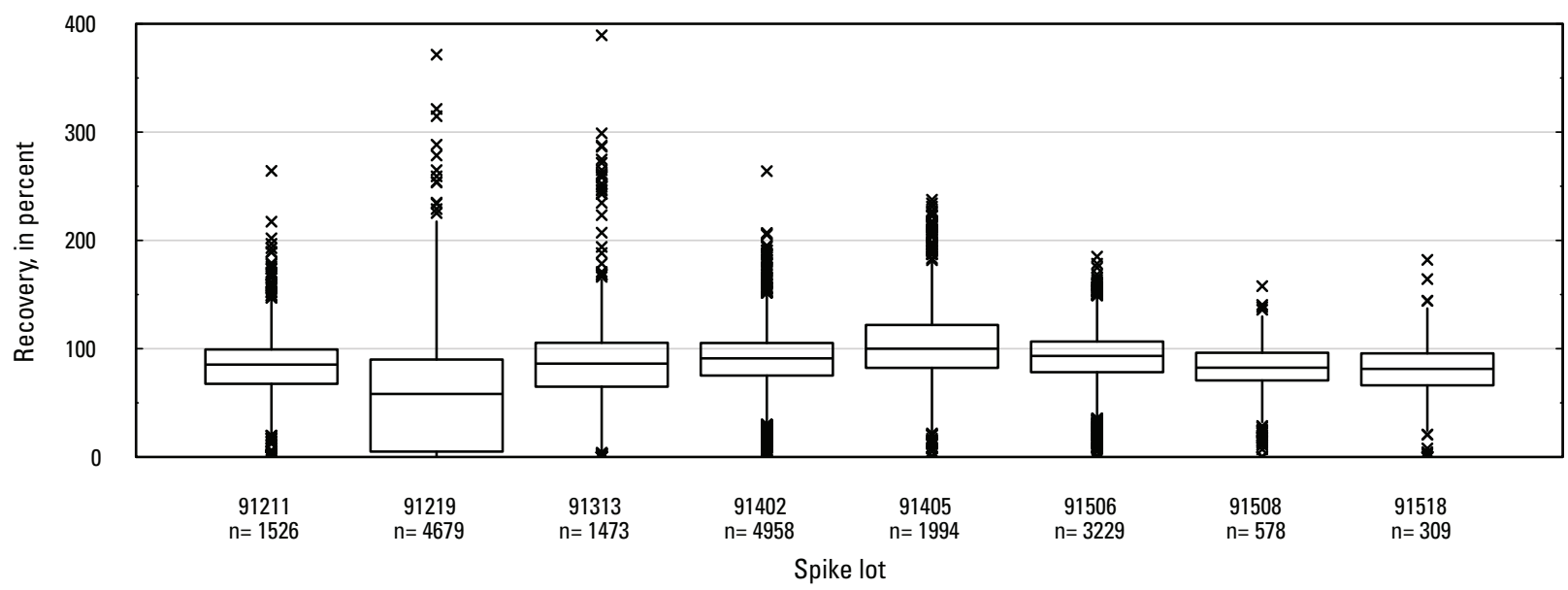

Figure 3. Graph showing distributions of recovery for pesticides in schedule 2437 by analytical method group, matrix, and spike lot. Recovery values larger than 400 percent are not shown. 


\section{Carbamate and thiocarbamate: laboratory reagent spikes}

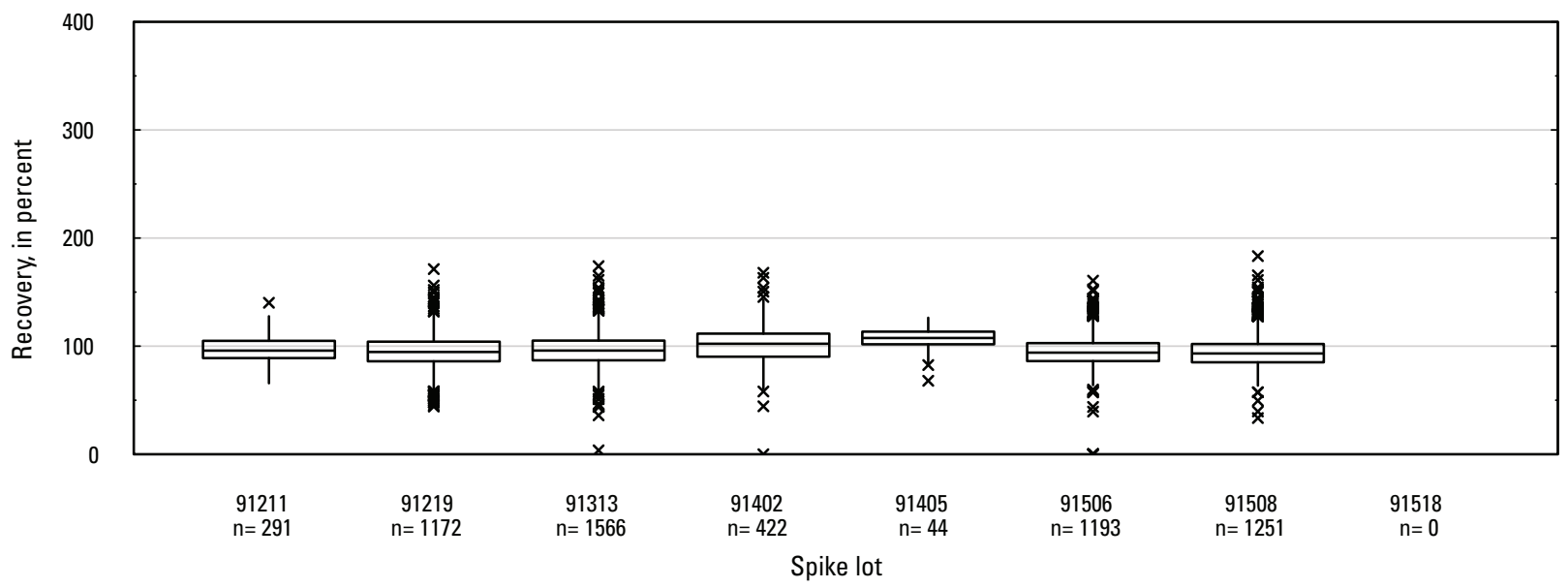

E. Carbamate and thiocarbamate: groundwater field matrix spikes

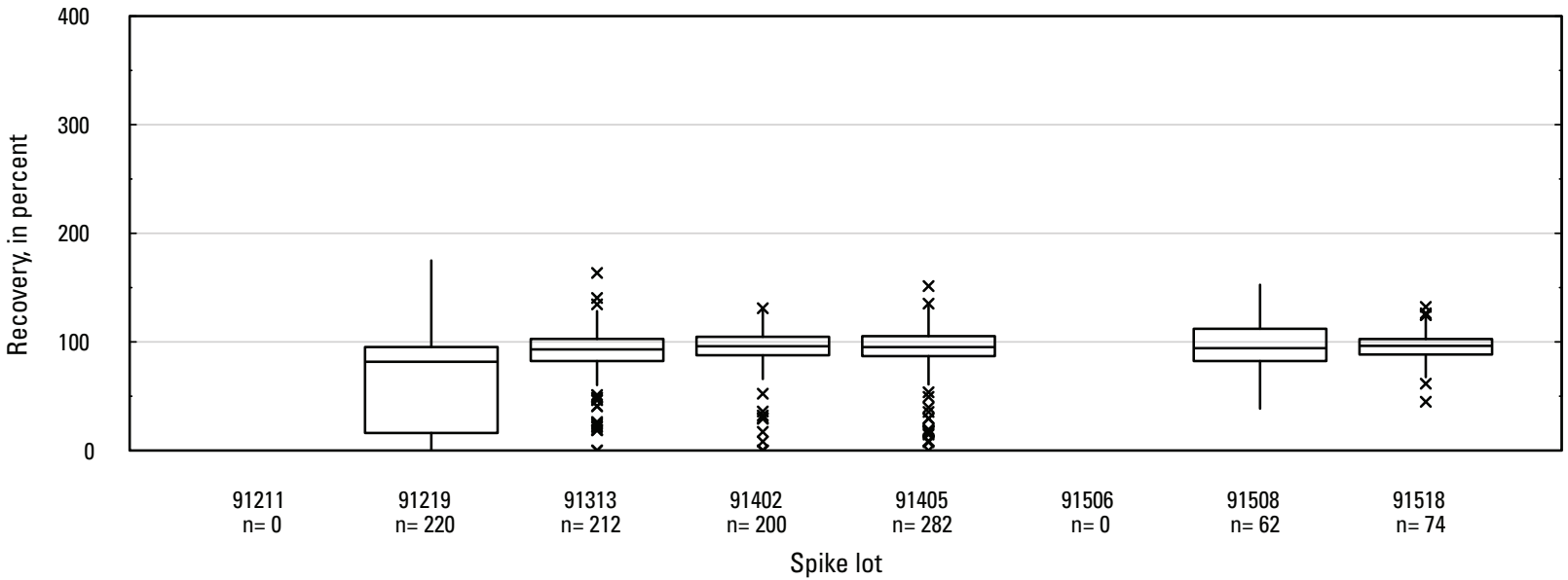

F. Carbamate and thiocarbamate: surface water field matrix spikes

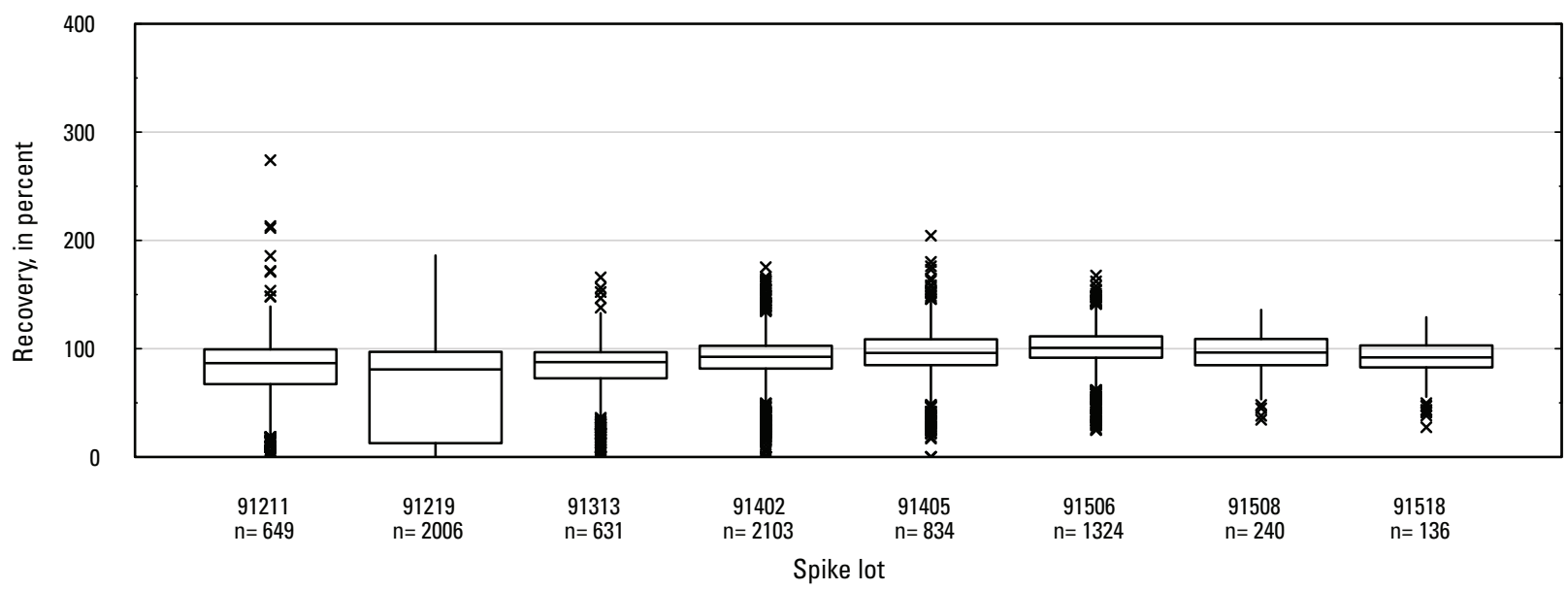

Figure 3. Graph showing distributions of recovery for pesticides in schedule 2437 by analytical method group, matrix, and spike lot. Recovery values larger than 400 percent are not shown.-Continued 


\section{G. Pyrethroid, organochlorine and phenylpyrazine: laboratory reagent spikes}

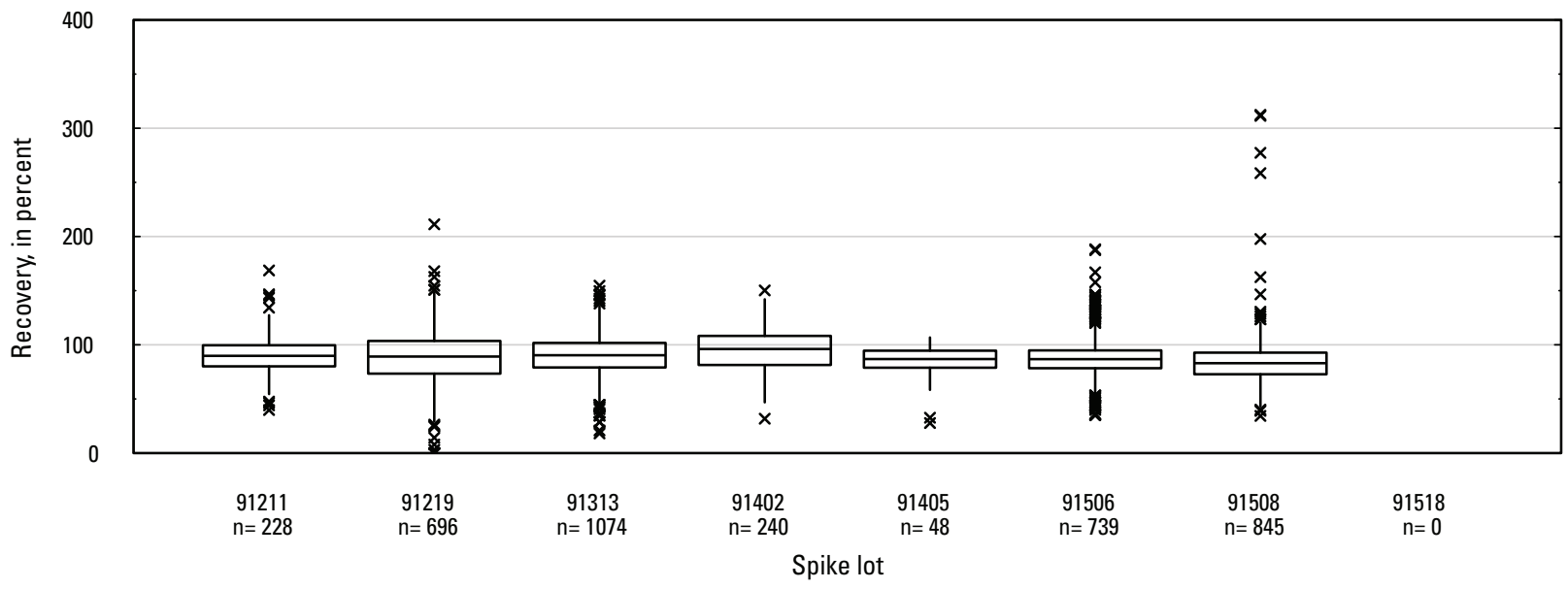

H. Pyrethroid, organochlorine and phenylpyrazine: groundwater field matrix spikes

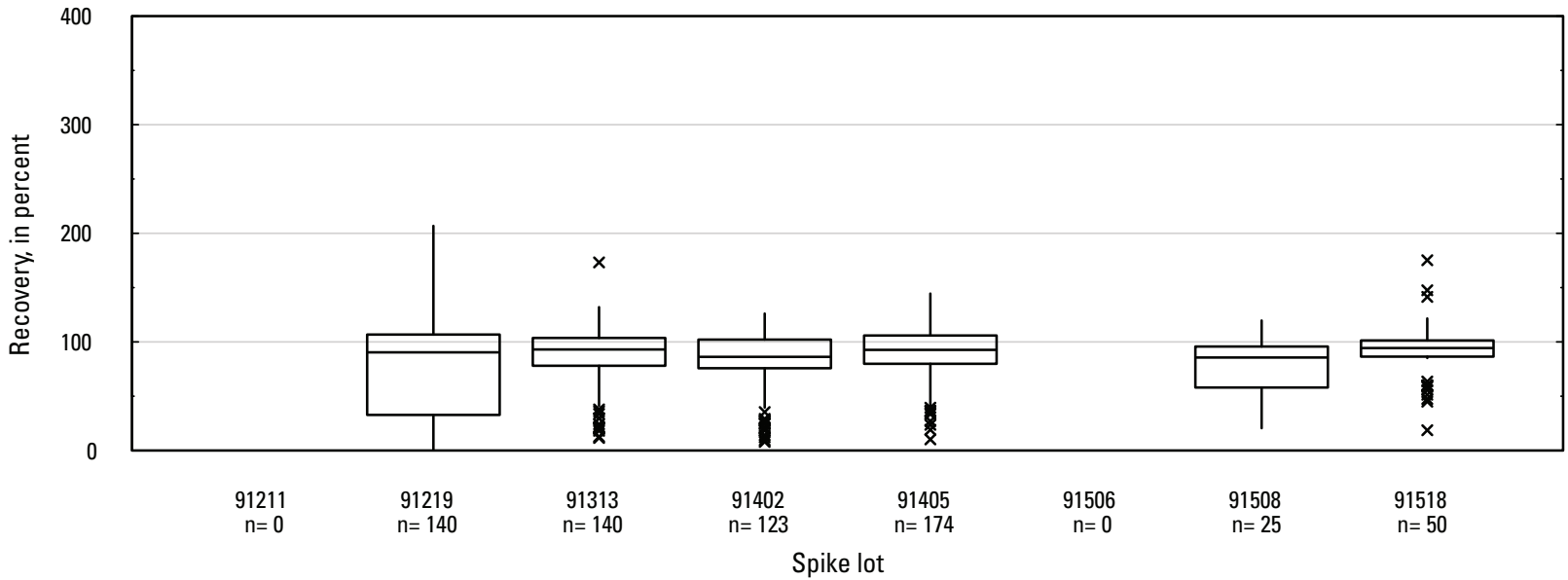

I. Pyrethroid, organochlorine and phenylpyrazine: surface water field matrix spikes

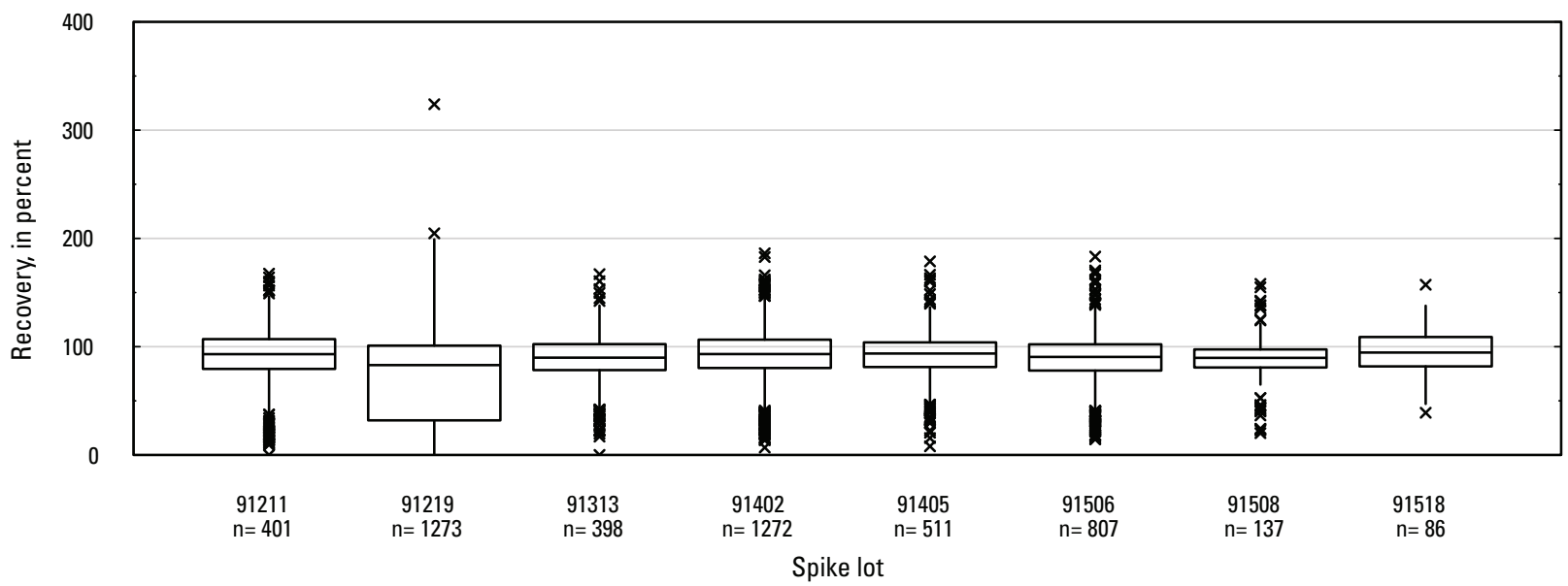

Figure 3. Graph showing distributions of recovery for pesticides in schedule 2437 by analytical method group, matrix, and spike lot. Recovery values larger than 400 percent are not shown.-Continued 
$J$. Triazine: laboratory reagent spikes

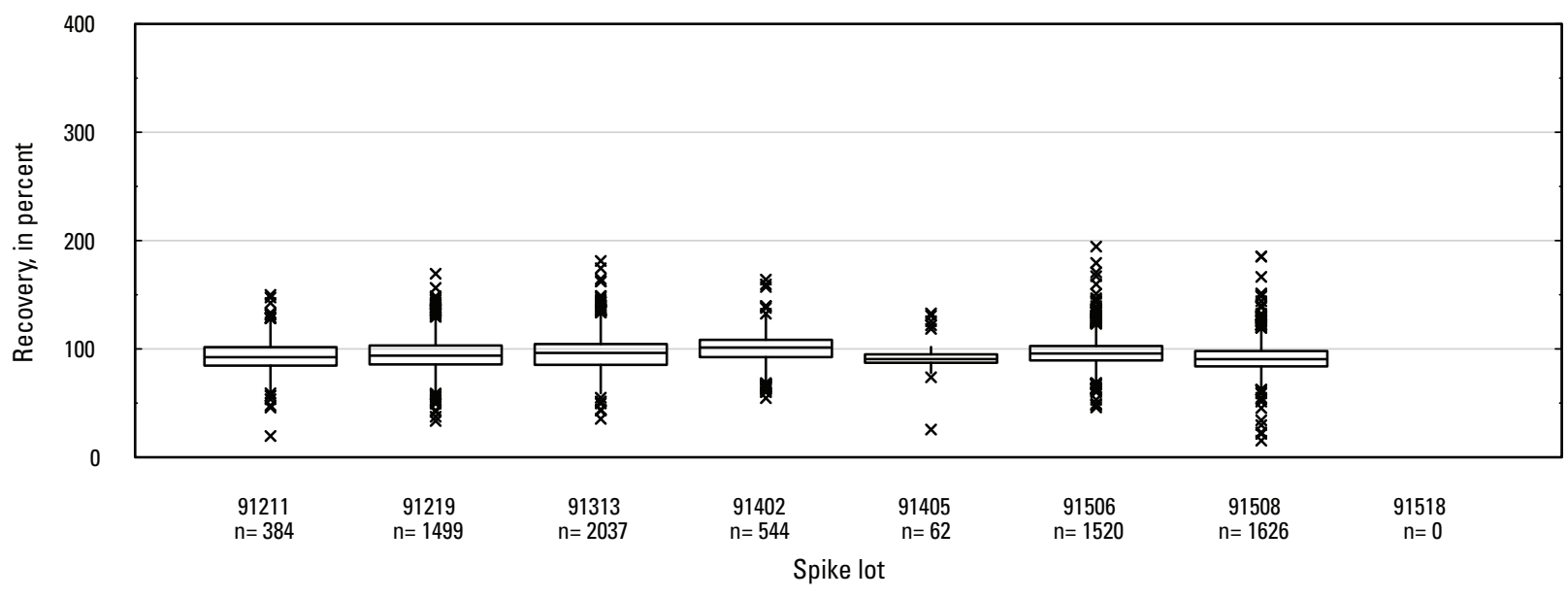

$K$. Triazine: groundwater field matrix spikes

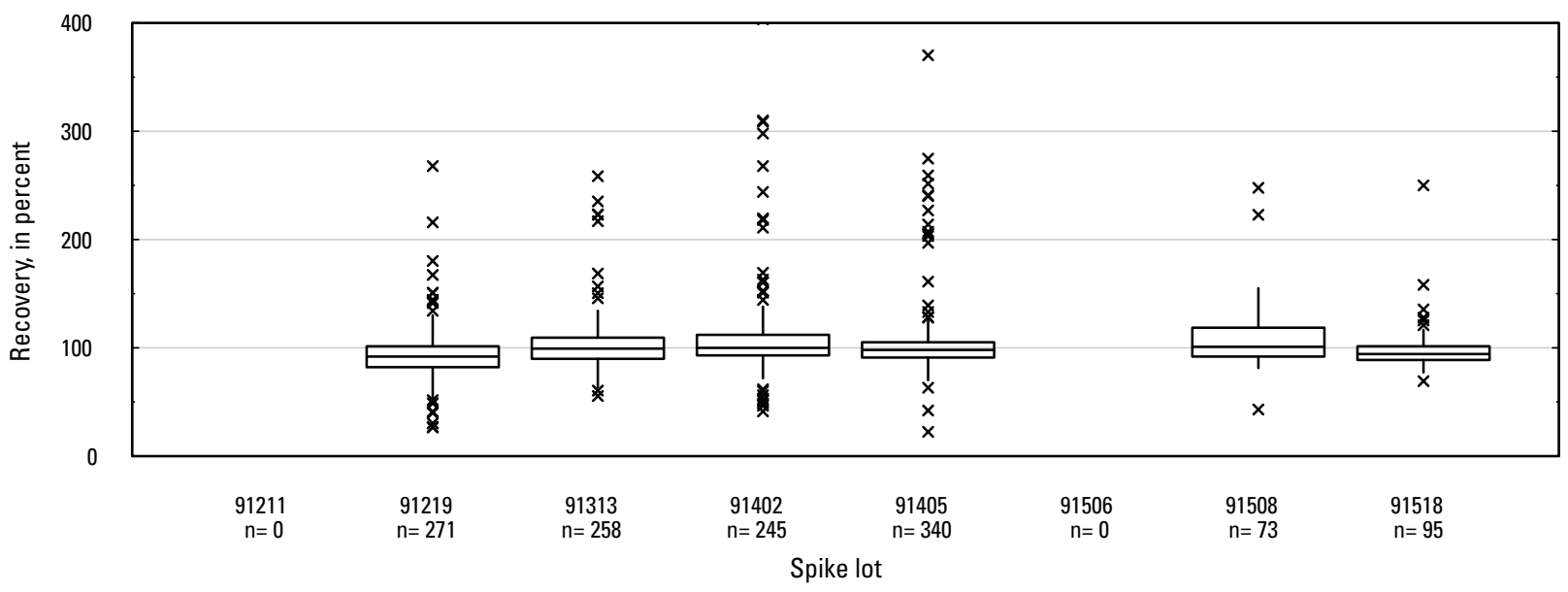

L. Triazine: surface water field matrix spikes

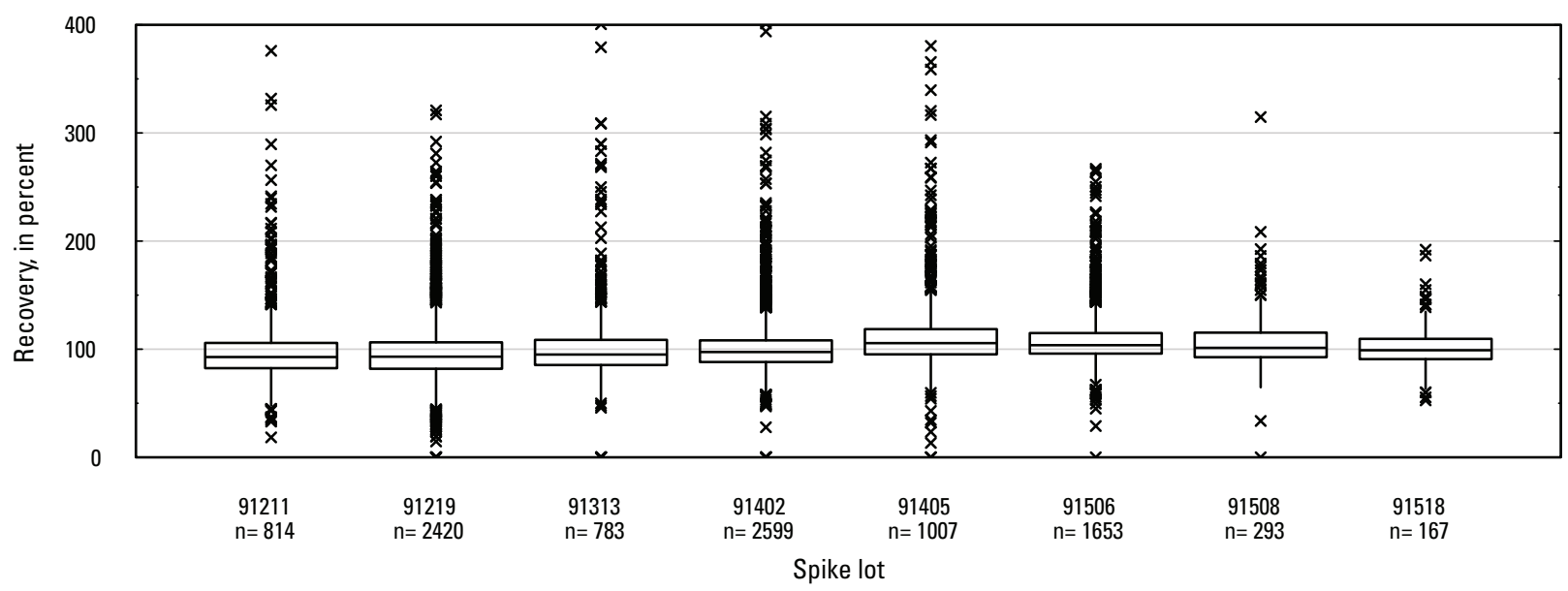

Figure 3. Graph showing distributions of recovery for pesticides in schedule 2437 by analytical method group, matrix, and spike lot. Recovery values larger than 400 percent are not shown.-Continued 
M. Sulfonylurea and urea: laboratory reagent spikes

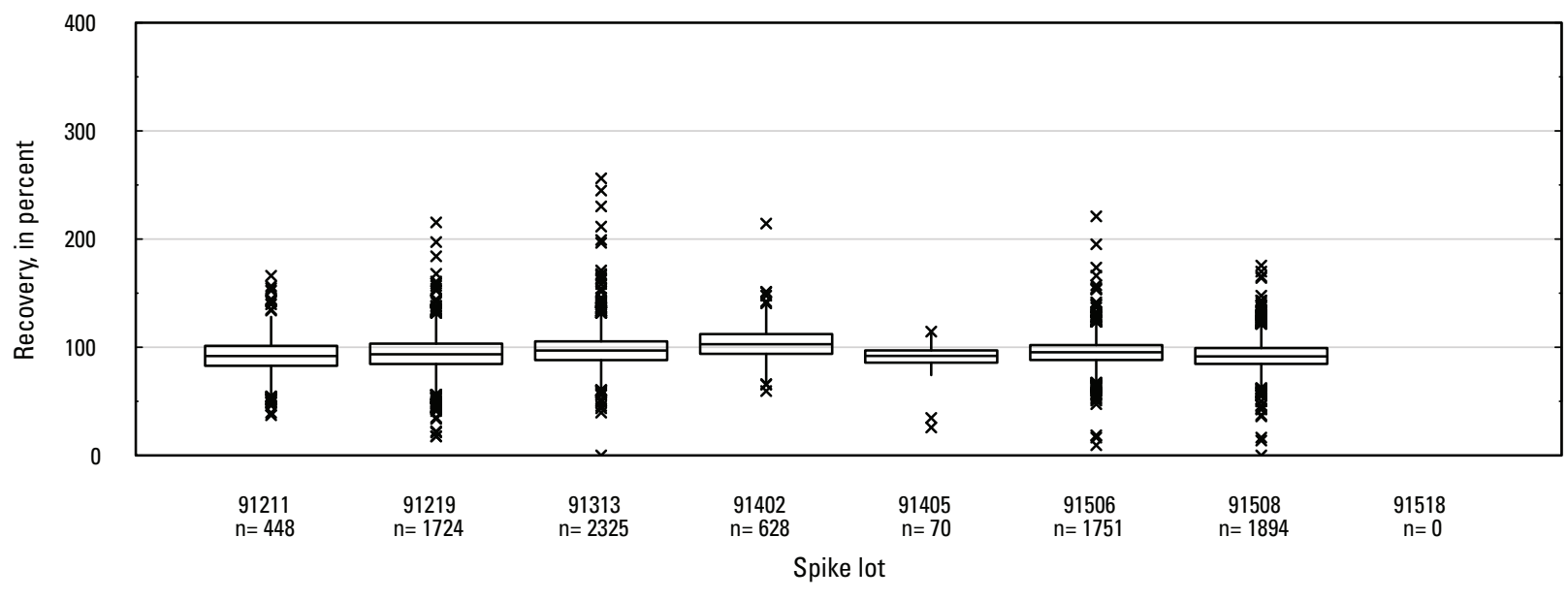

N. Sulfonylurea and urea: groundwater field matrix spikes

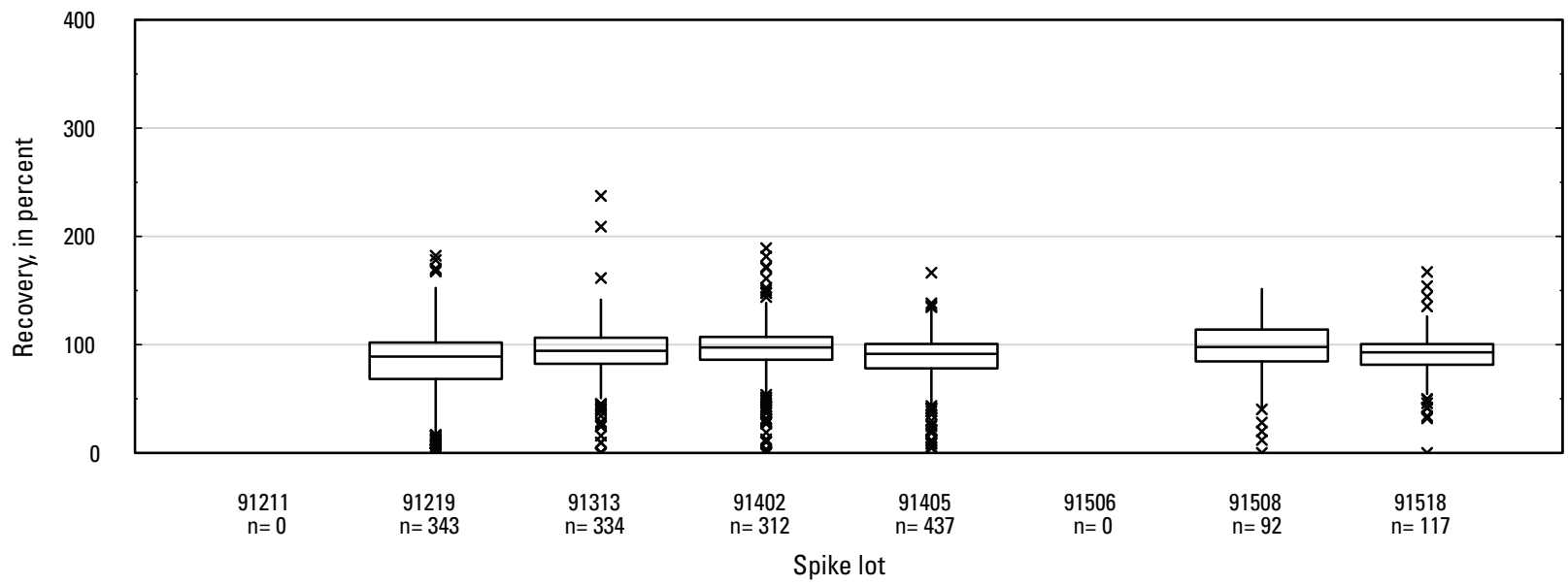

O. Sulfonylurea and urea: surface water field matrix spikes

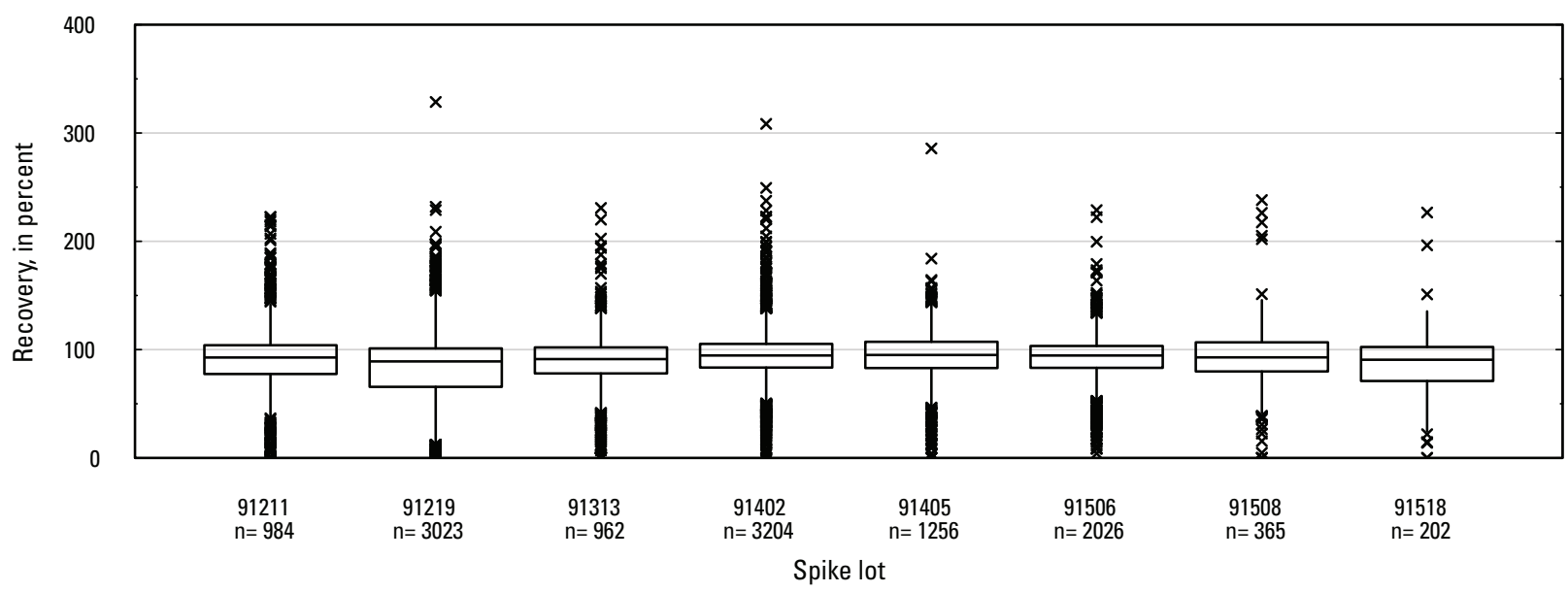

Figure 3. Graph showing distributions of recovery for pesticides in schedule 2437 by analytical method group, matrix, and spike lot. Recovery values larger than 400 percent are not shown.-Continued 
P. Acetanilide and amide: laboratory reagent spikes

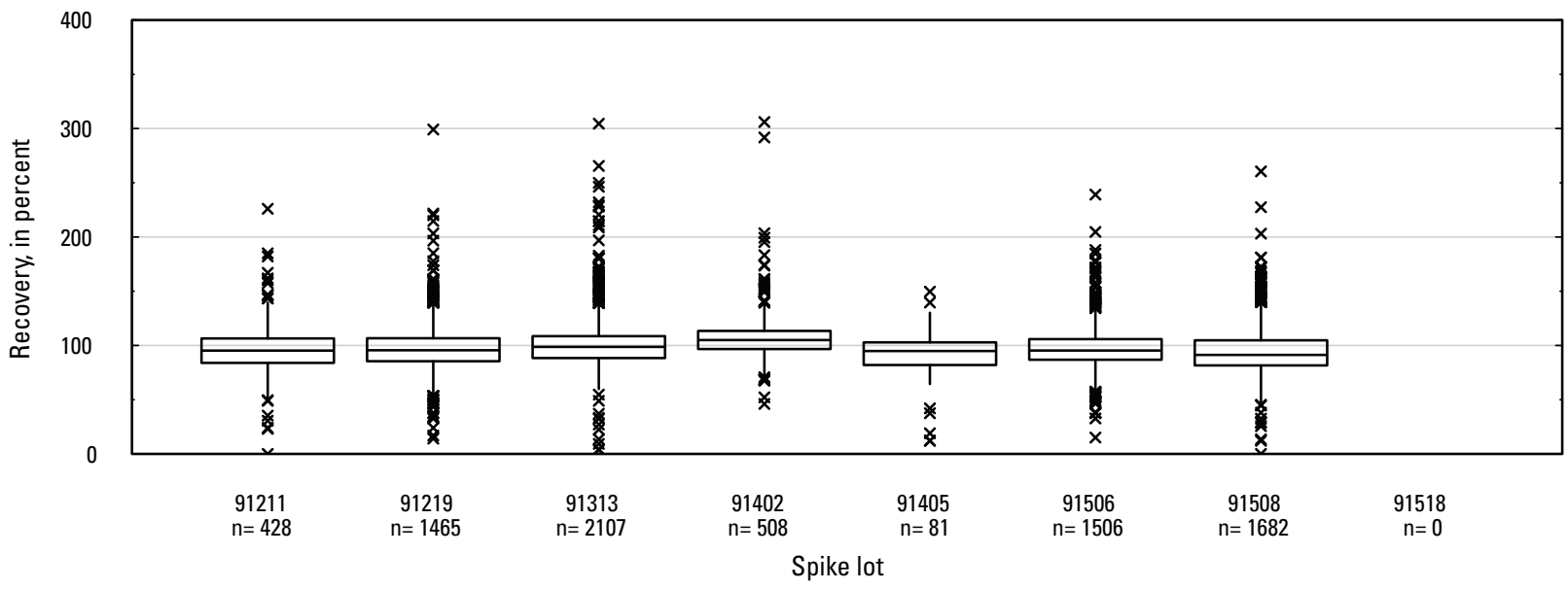

Q. Acetanilide and amide: groundwater field matrix spikes

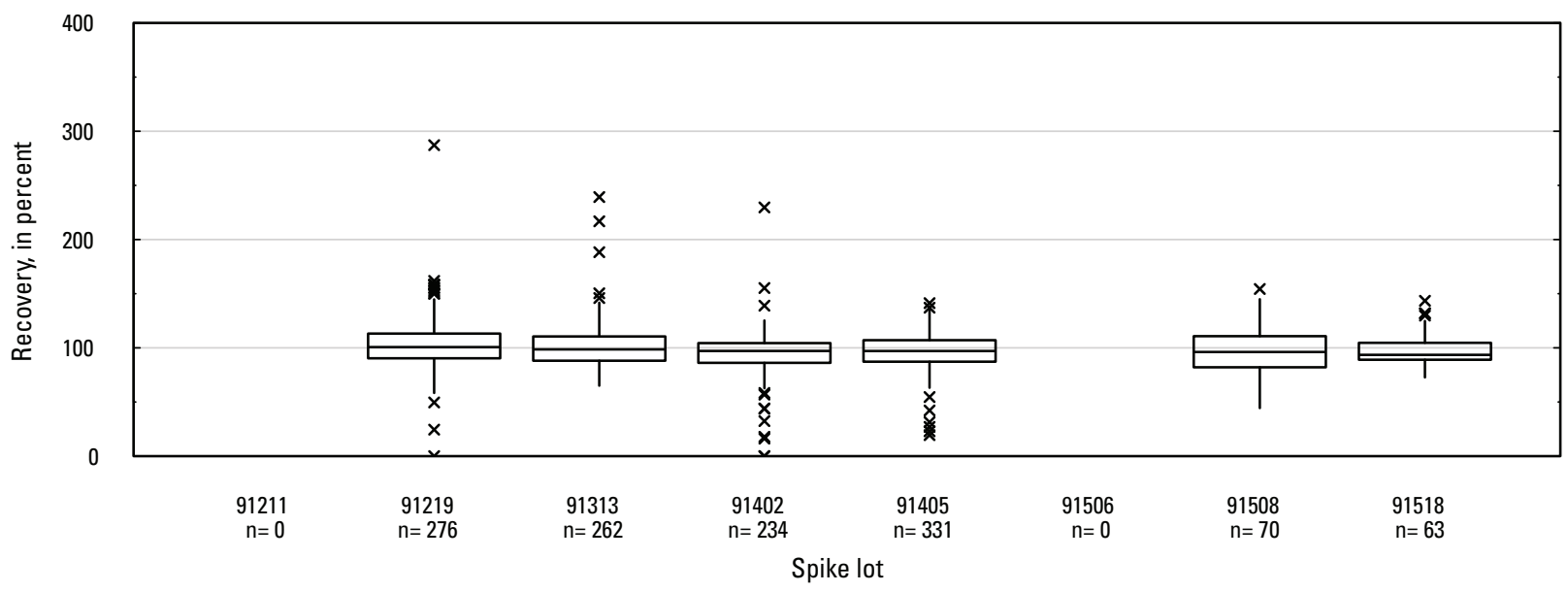

$R$. Acetanilide and amide: surface water field matrix spikes

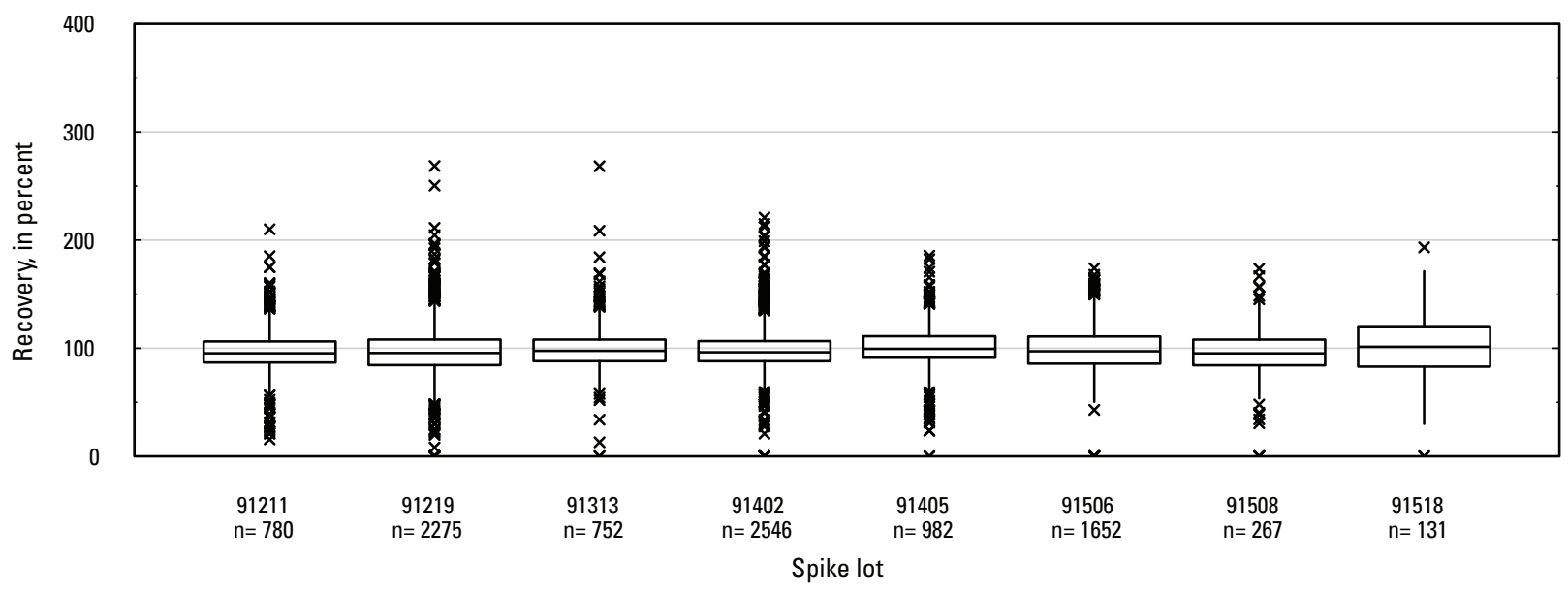

Figure 3. Graph showing distributions of recovery for pesticides in schedule 2437 by analytical method group, matrix, and spike lot. Recovery values larger than 400 percent are not shown.-Continued 


\section{S. Fungicides: laboratory reagent spikes}

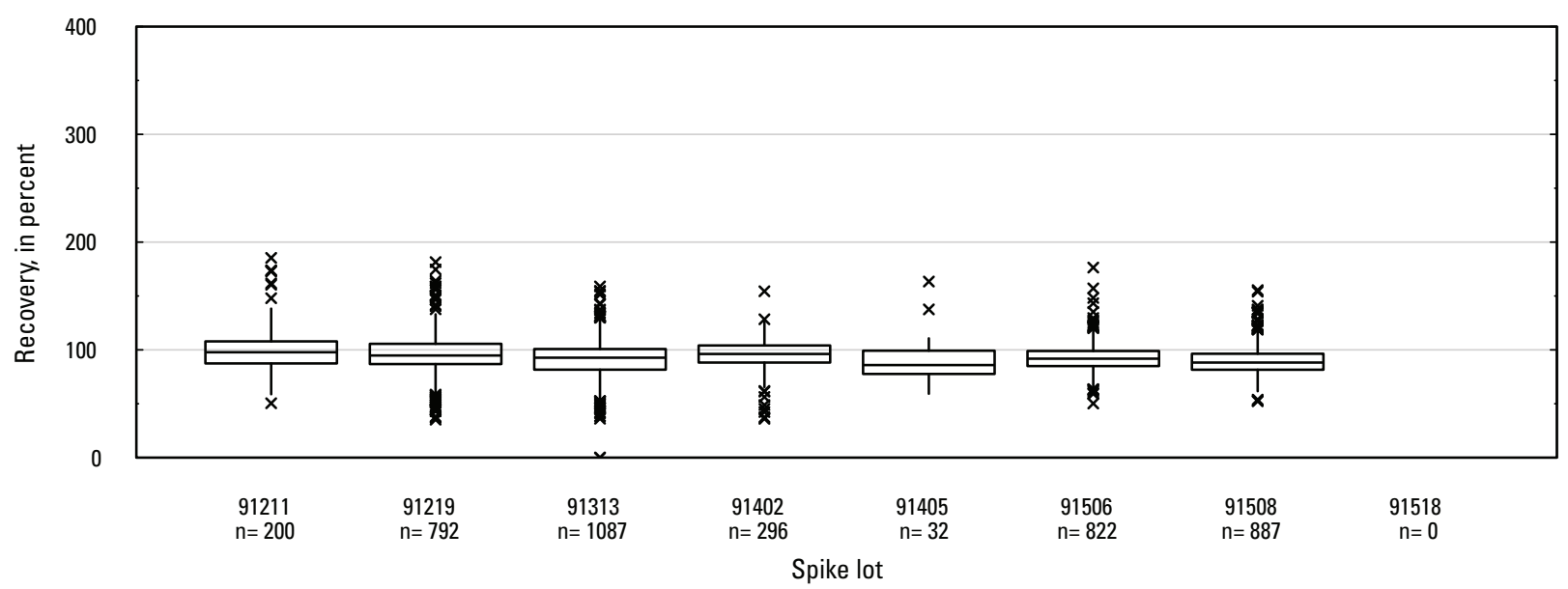

$T$. Fungicides: groundwater field matrix spikes

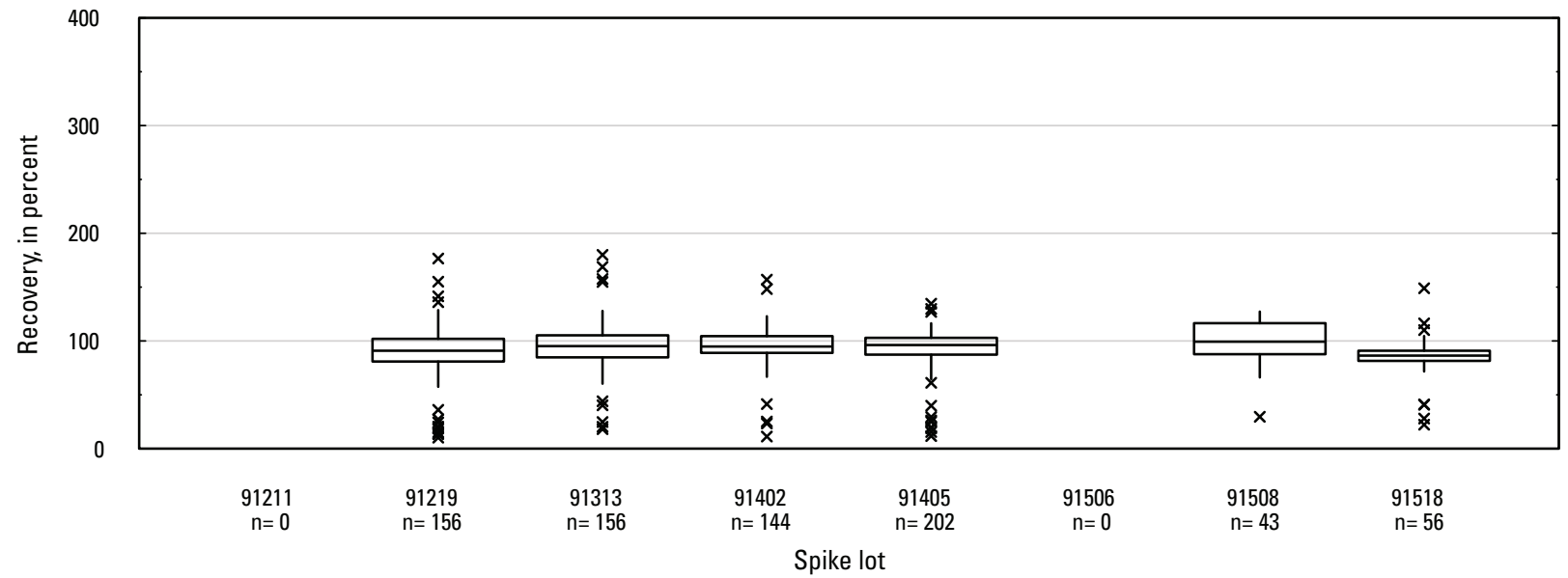

$U$. Fungicides: surface water field matrix spikes

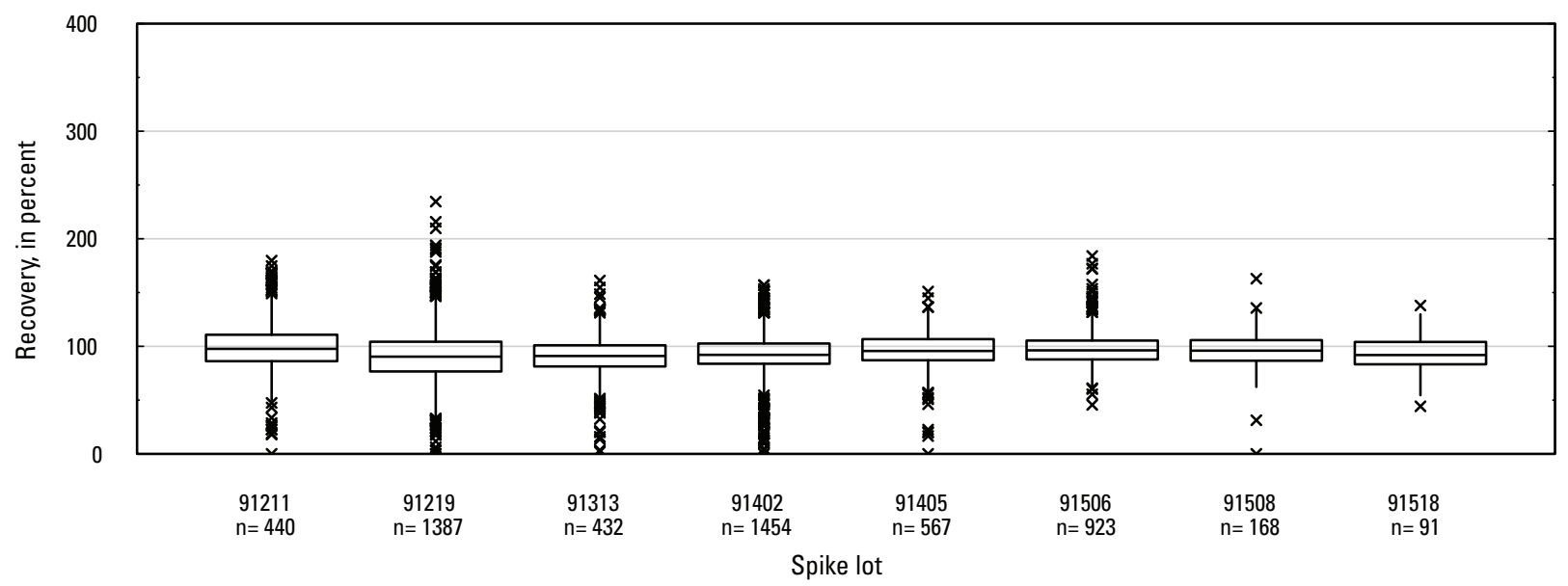

Figure 3. Graph showing distributions of recovery for pesticides in schedule 2437 by analytical method group, matrix, and spike lot. Recovery values larger than 400 percent are not shown.-Continued 
V. Acid: laboratory reagent spikes

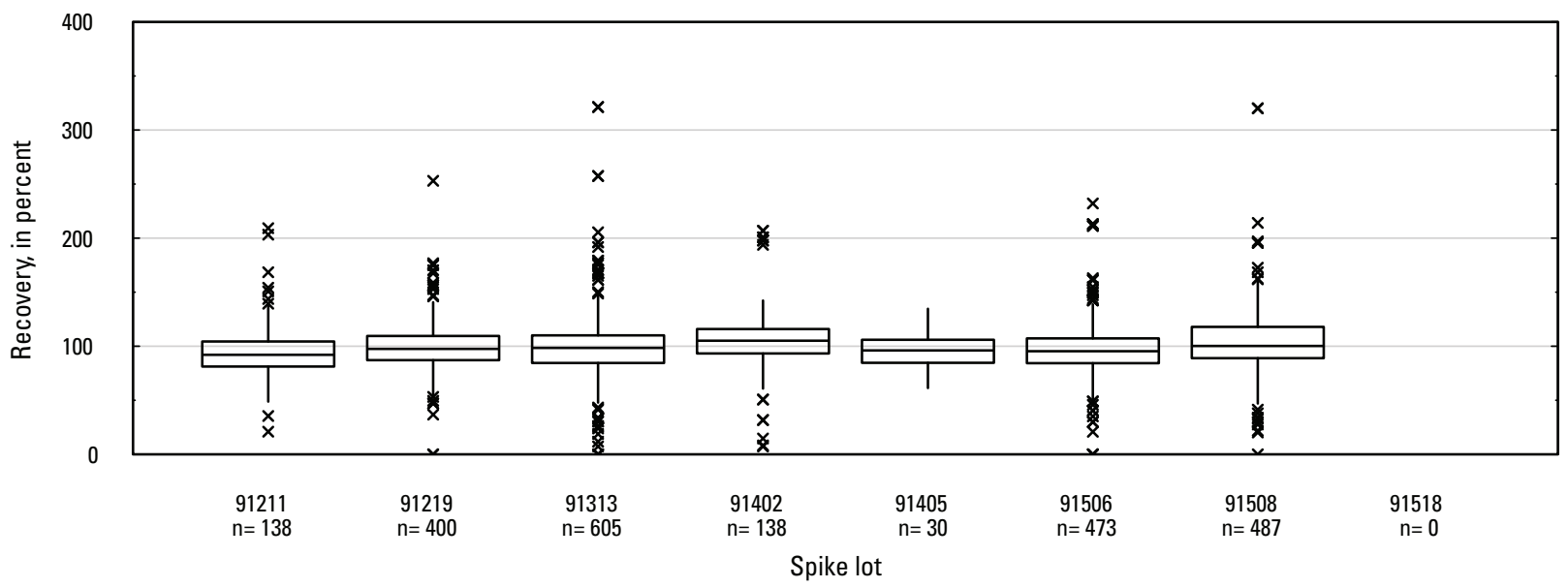

W. Acid: groundwater field matrix spikes

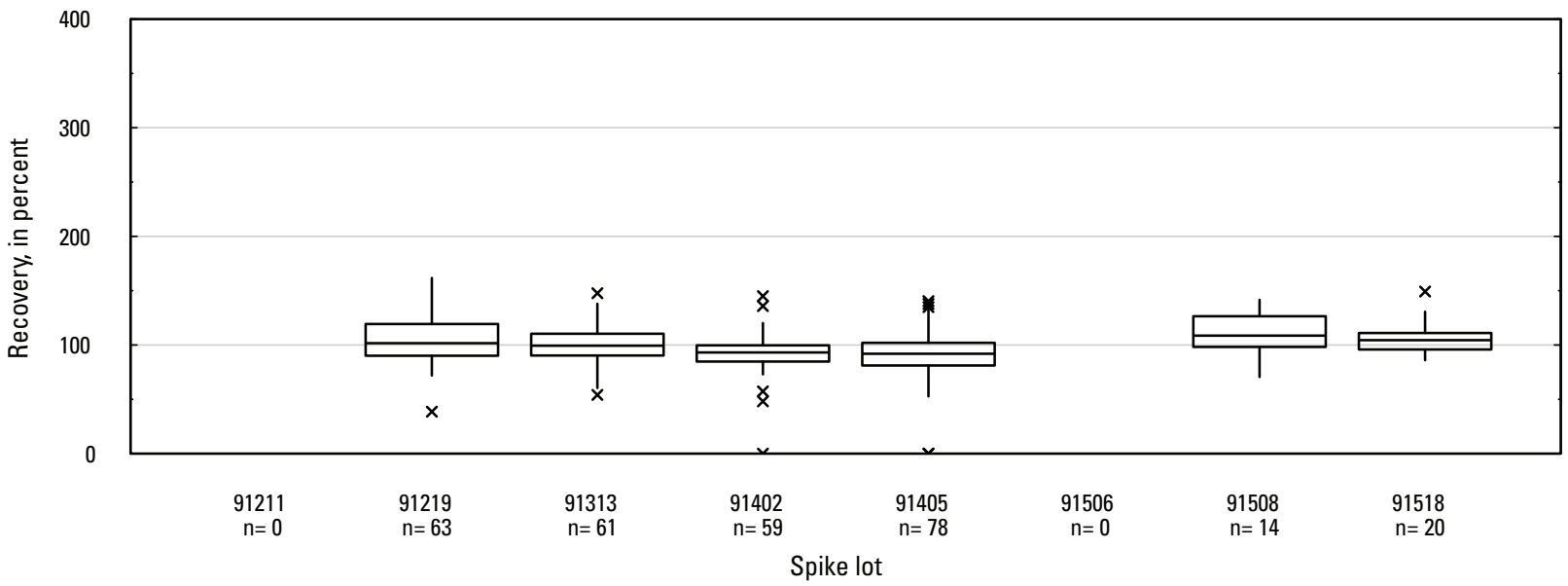

$X$. Acid: surface water field matrix spikes

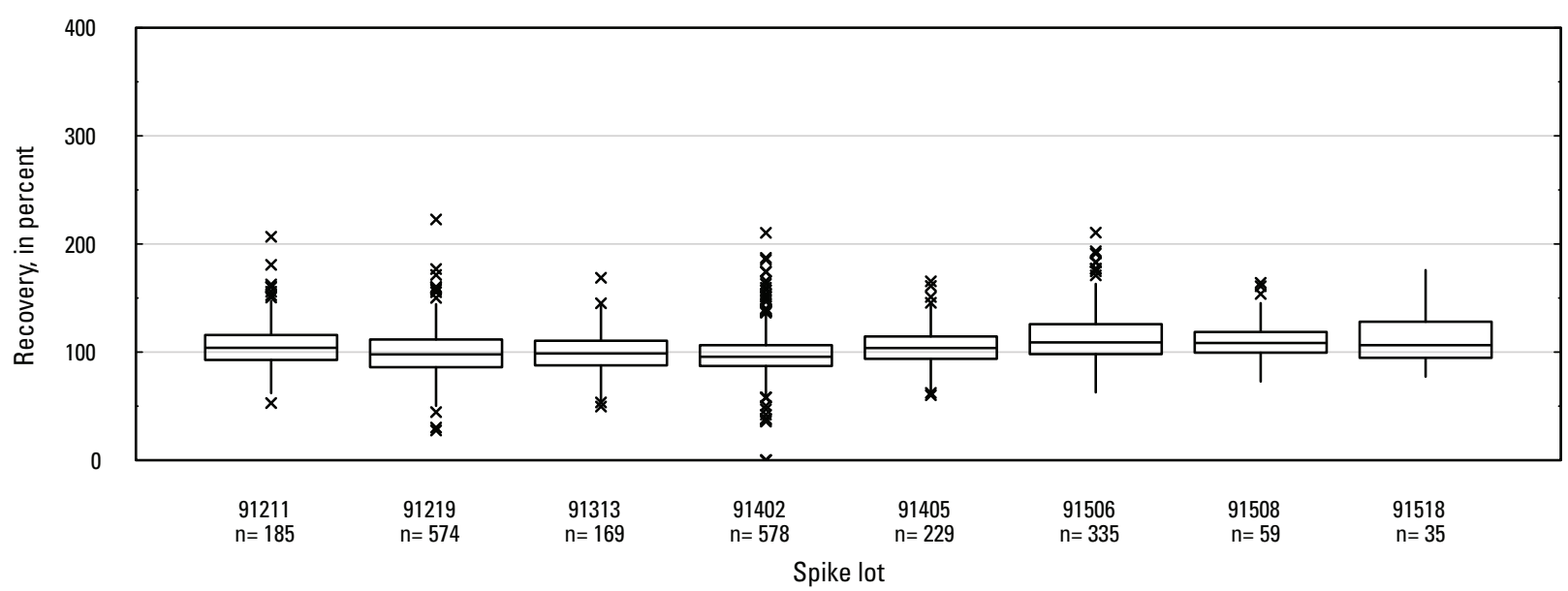

Figure 3. Graph showing distributions of recovery for pesticides in schedule 2437 by analytical method group, matrix, and spike lot. Recovery values larger than 400 percent are not shown.-Continued 


\section{Y. Miscellaneous: laboratory reagent spikes}

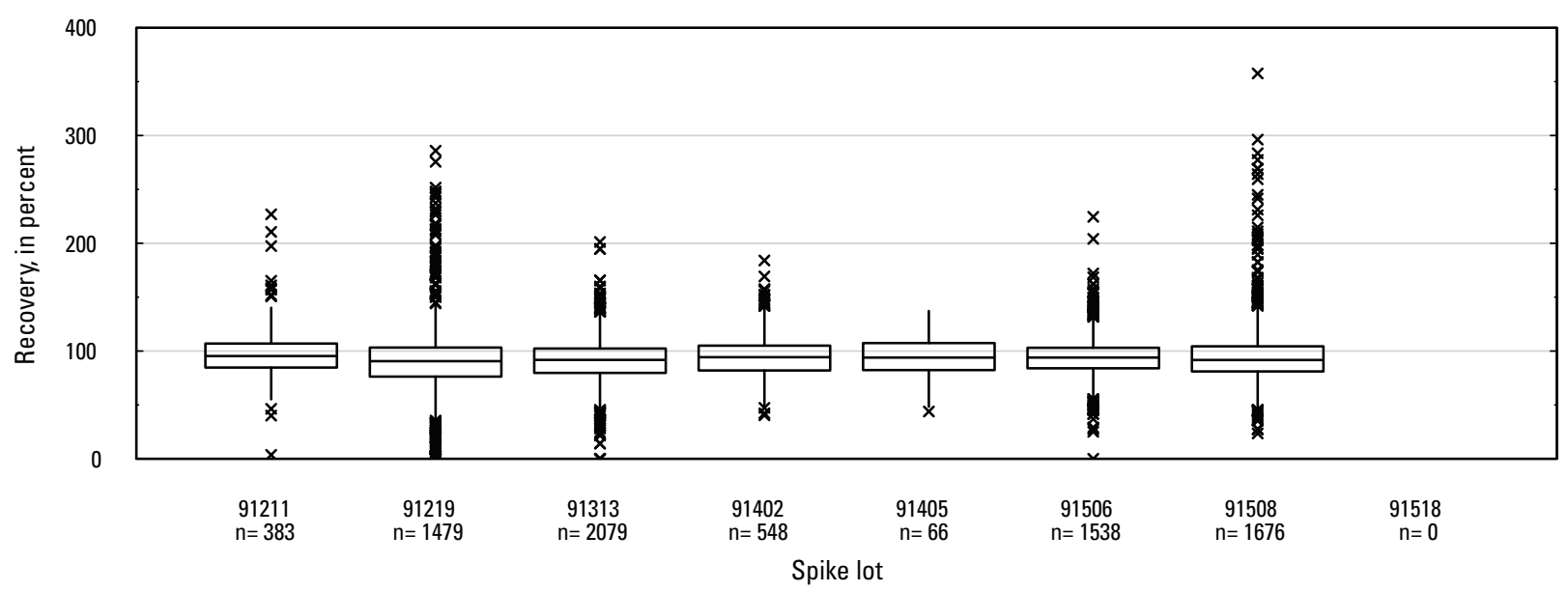

Z. Miscellaneous: groundwater field matrix spikes

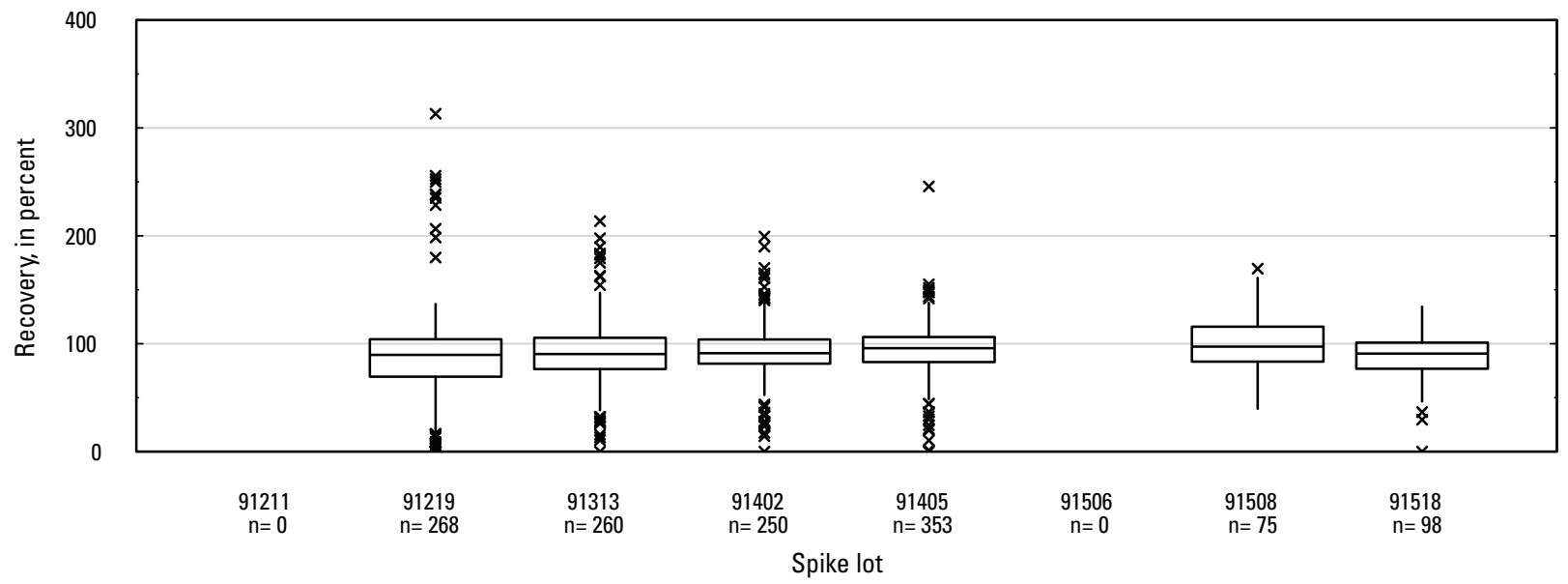

$A A$. Miscellaneous: surface water field matrix spikes

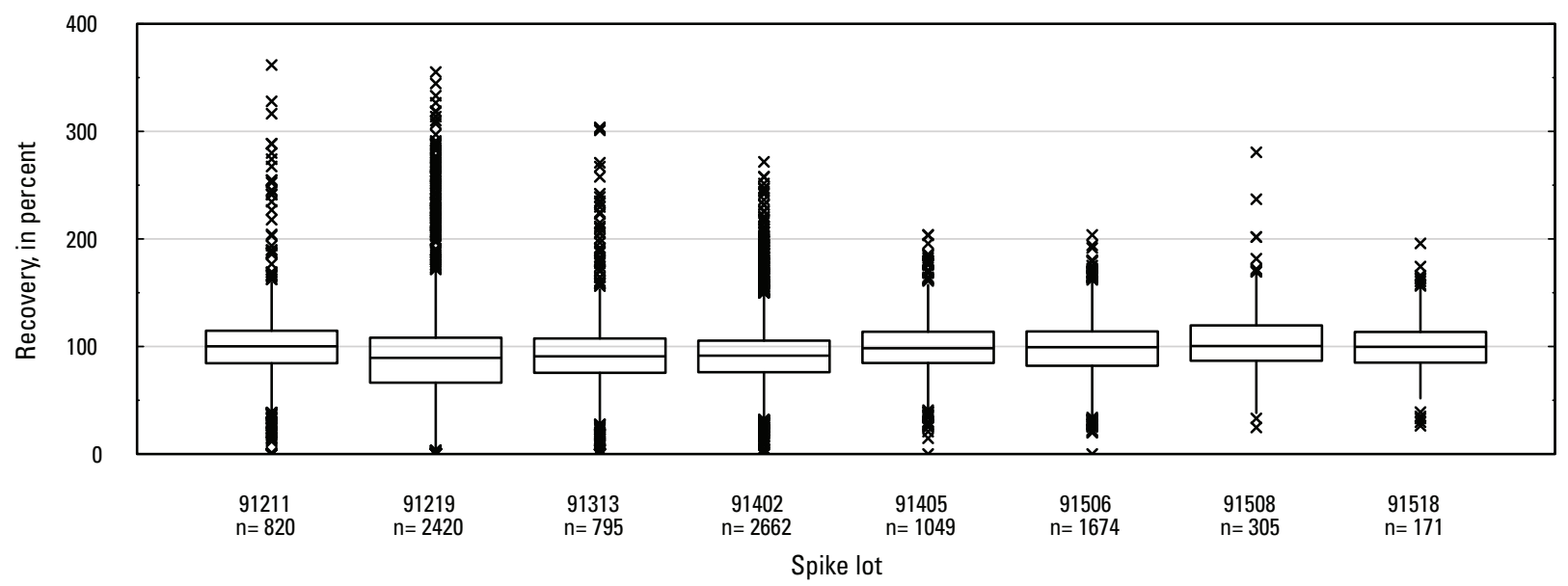

Figure 3. Graph showing distributions of recovery for pesticides in schedule 2437 by analytical method group, matrix, and spike lot. Recovery values larger than 400 percent are not shown.-Continued 
A. 1H-1,2,4-Triazole

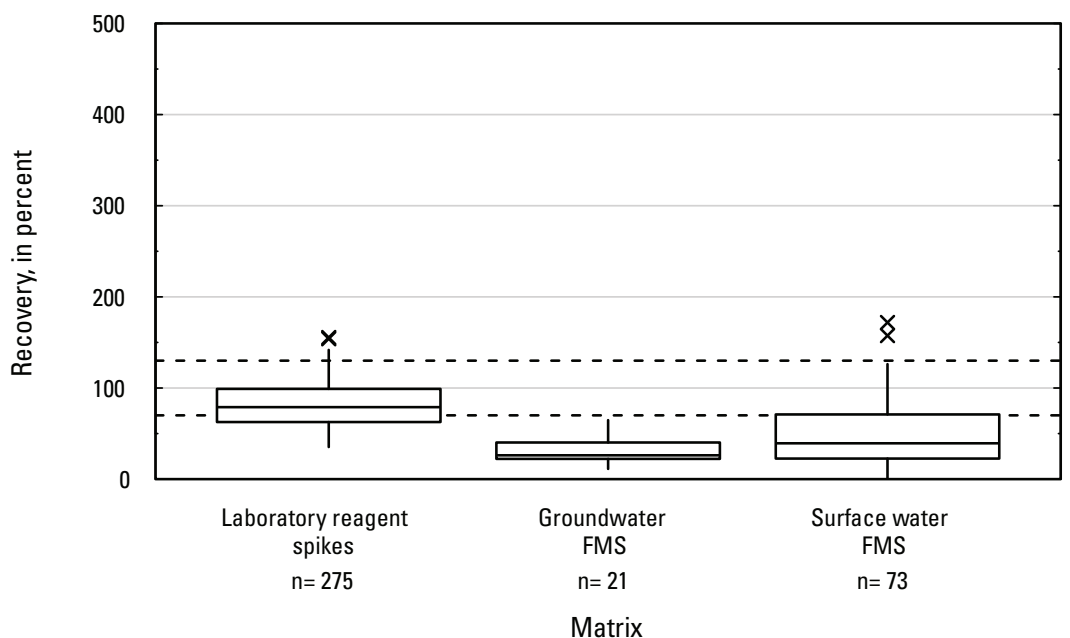

C. 2-[(2-Ethyl-6-methylphenyl)amino]-1-propanol

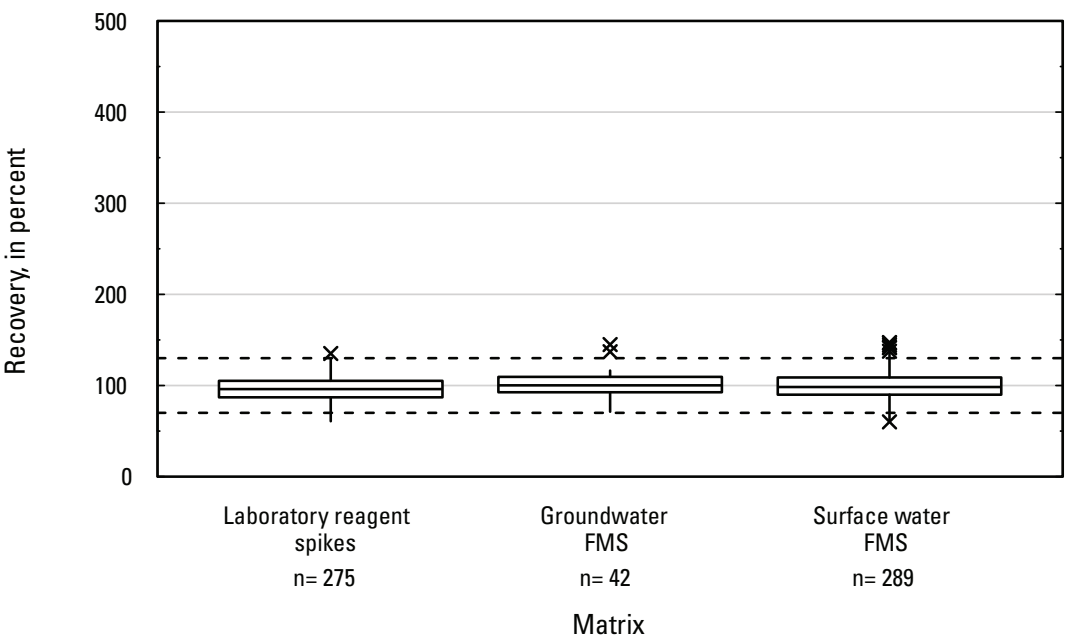

B. 2-(1-Hydroxyethyl)-6-methylaniline

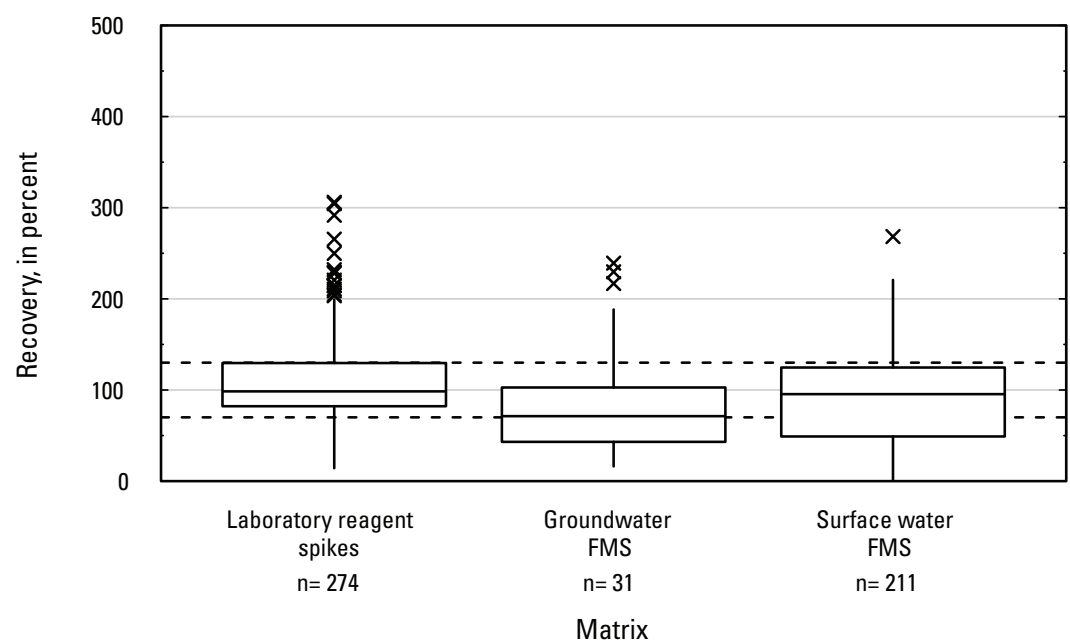

D. 2,3,3-Trichloro-2-propene-1-sulfonic acid (TCPSA)

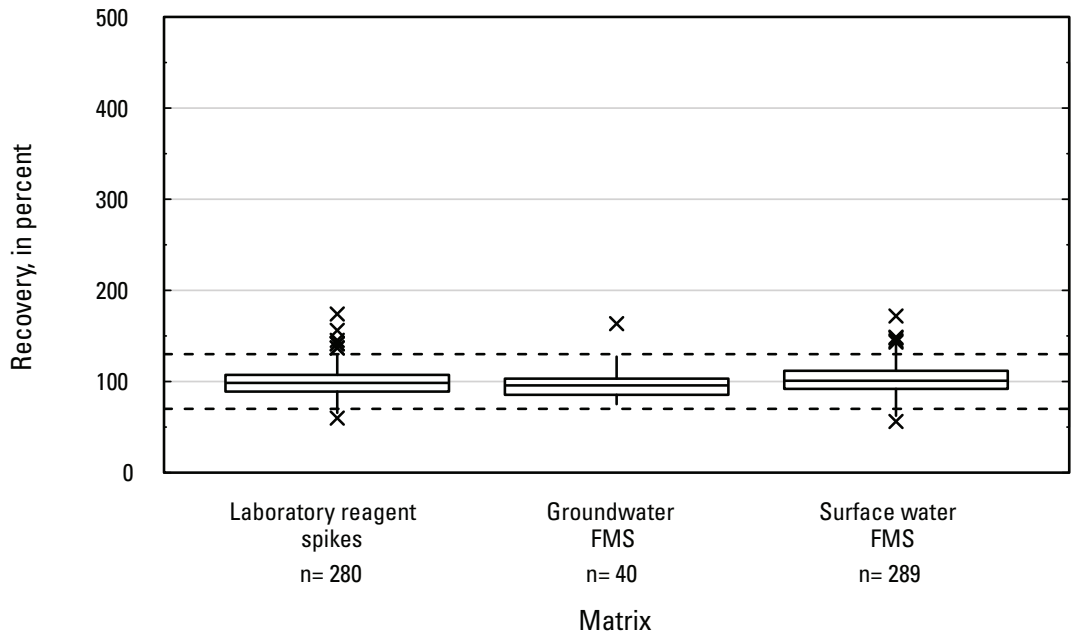

Figure 4. Graph showing distributions of recovery for pesticides in schedule 2437 by matrix. Dashed lines are at 130 percent and 70 percent. Recovery values larger than 400 percent are not shown. 
E. 2,4-D

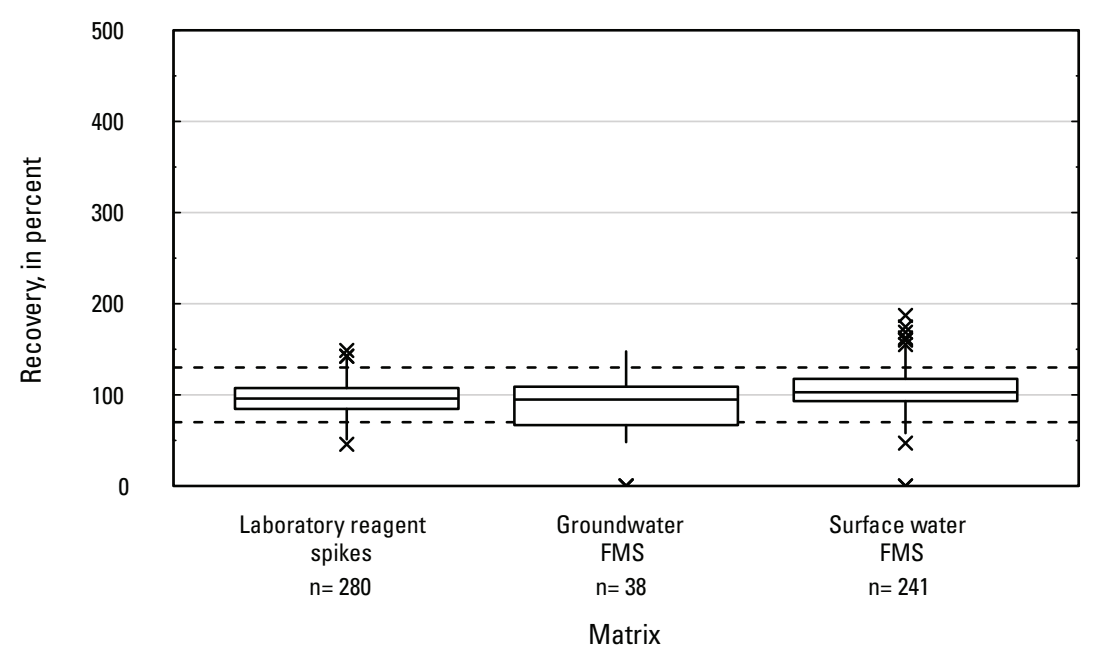

G. 2-Amino-N-isopropylbenzamide

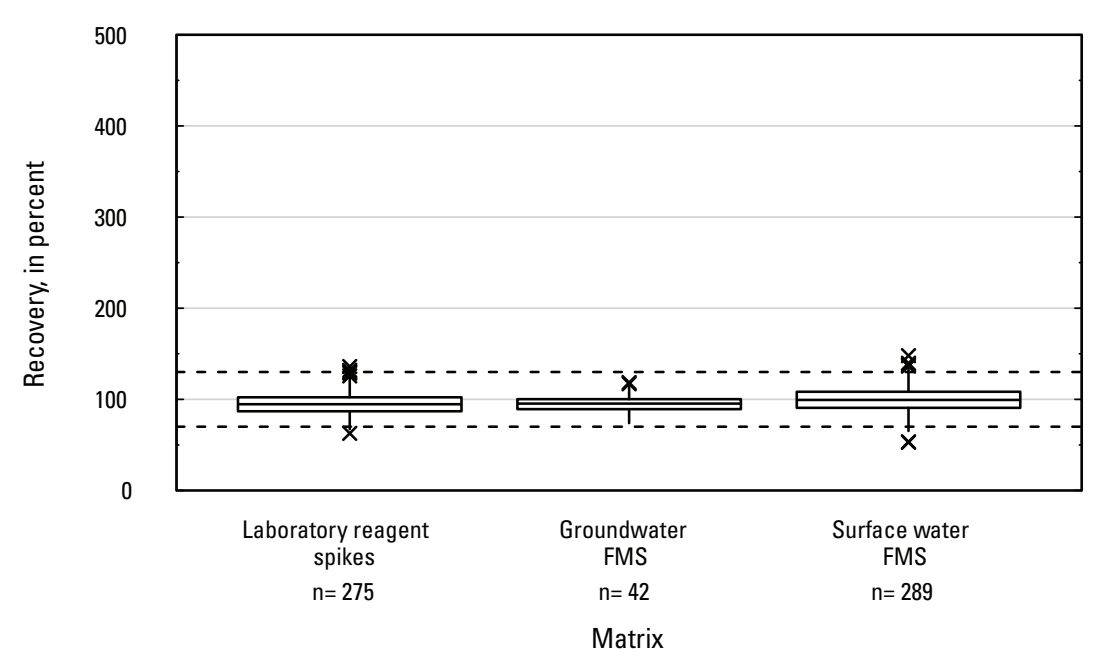

F. 2-Aminobenzimidazole

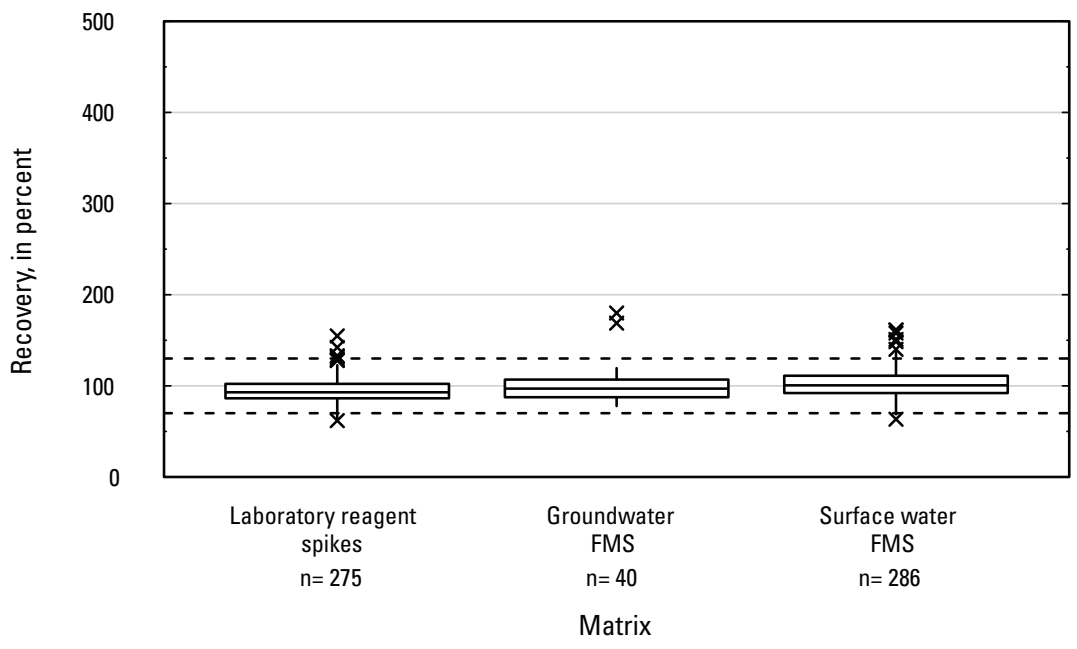

H. 2-Chloro-2',6'-diethylacetanilide

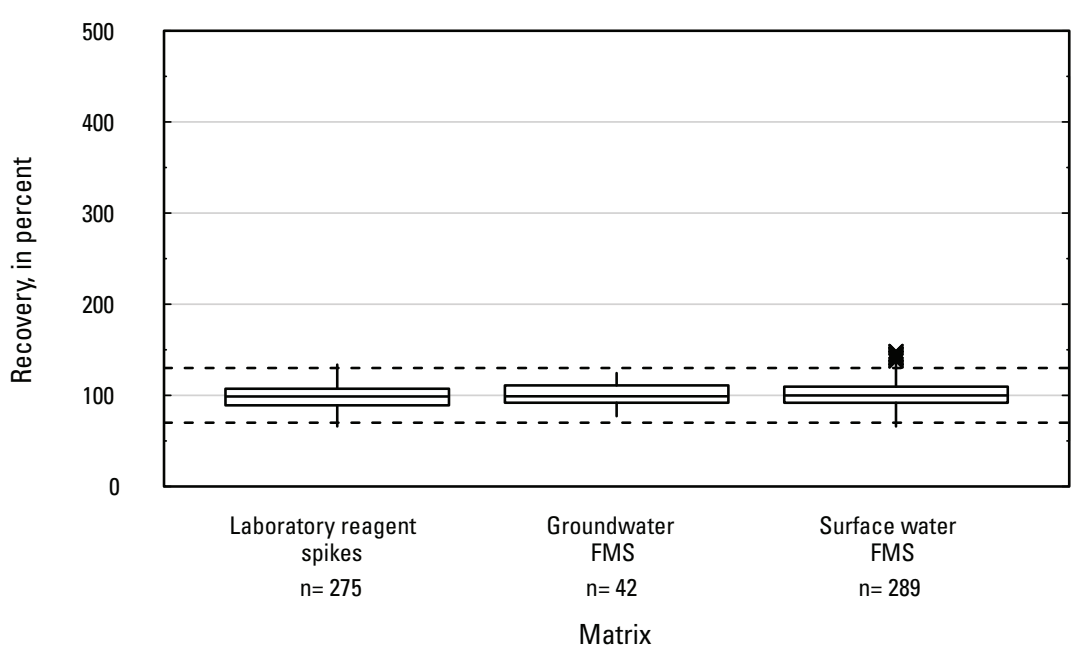

Figure 4. Graph showing distributions of recovery for pesticides in schedule 2437 by matrix. Dashed lines are at 130 percent and 70 percent. Recovery values larger than 400 percent are not shown.-Continued 
I. 2-Chloro-N-(2-ethyl-6-methylphenyl)acetamide

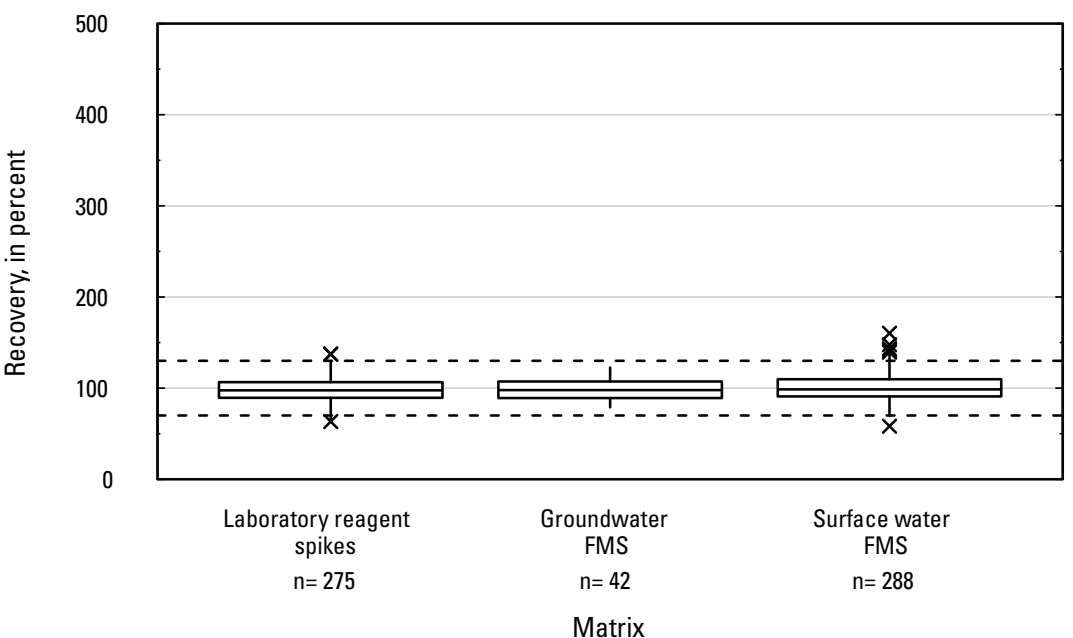

K. 2-Hydroxy-6-ethylamino-4-amino-s-triazine

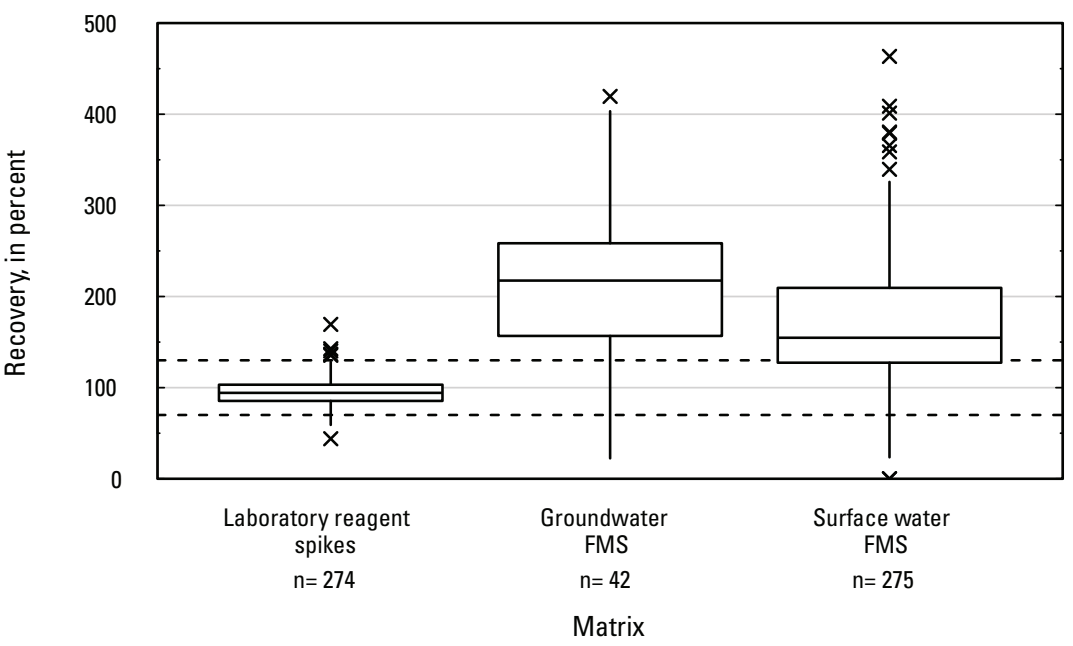

J.2-Hydroxy-4-isopropylamino-6-amino-s-triazine

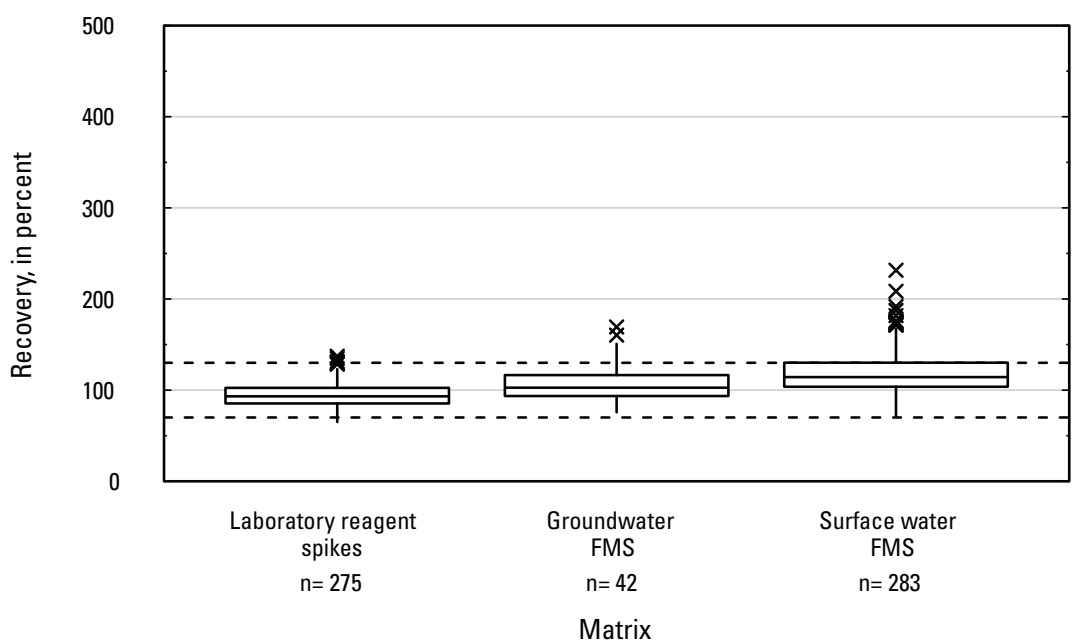

L. 2-Hydroxy-4-isopropylamino-6-ethylamino-s-triazine $\{0 I E T\}$

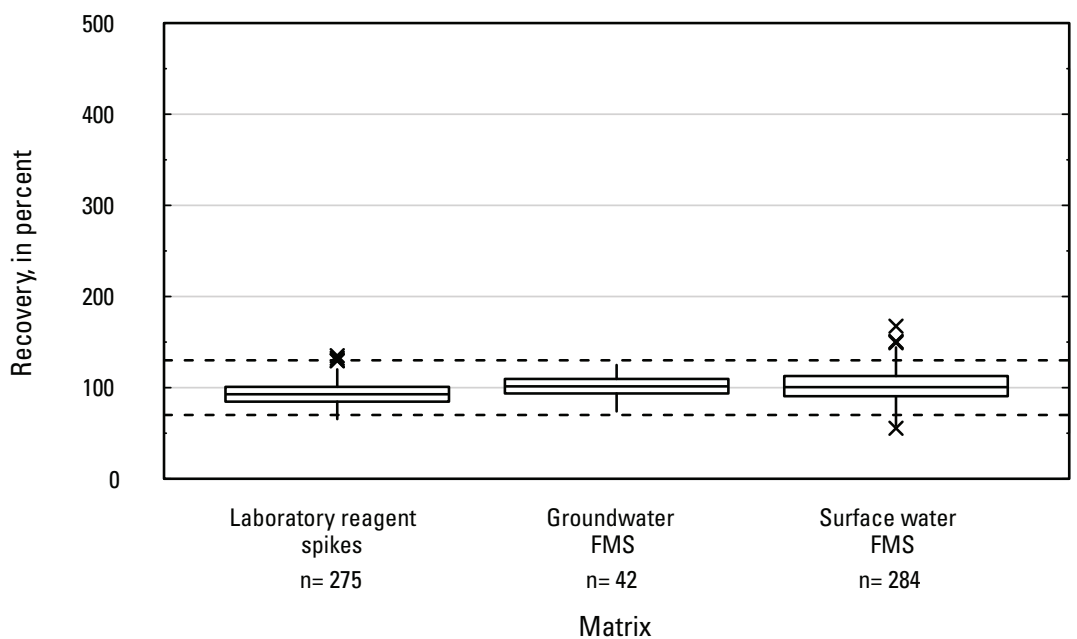

Figure 4. Graph showing distributions of recovery for pesticides in schedule 2437 by matrix. Dashed lines are at 130 percent and 70 percent. Recovery values larger than 400 percent are not shown.-Continued 
M.3,4-Dichlorophenylurea

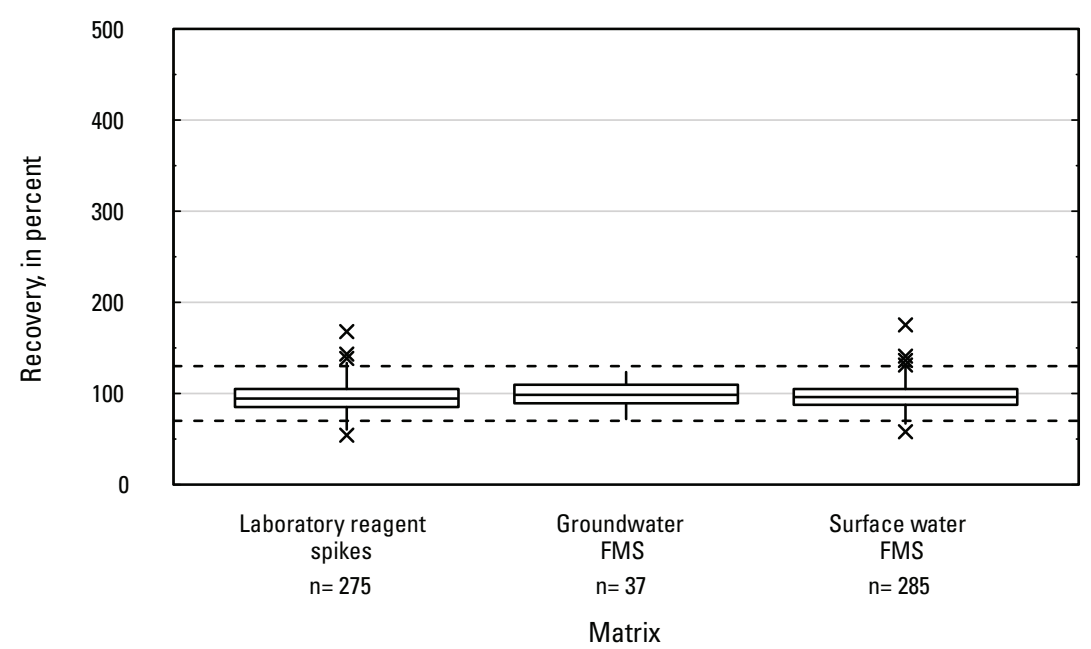

0. 3-Phenoxybenzoic acid

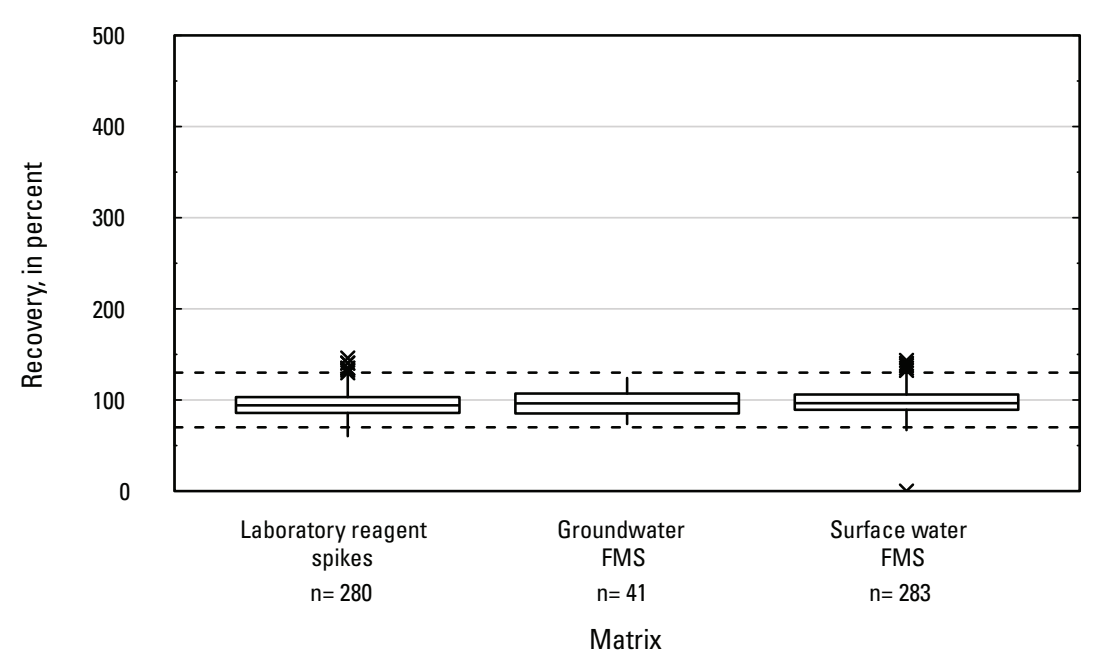
Matrix

Matrix
N. 3-Hydroxycarbofuran

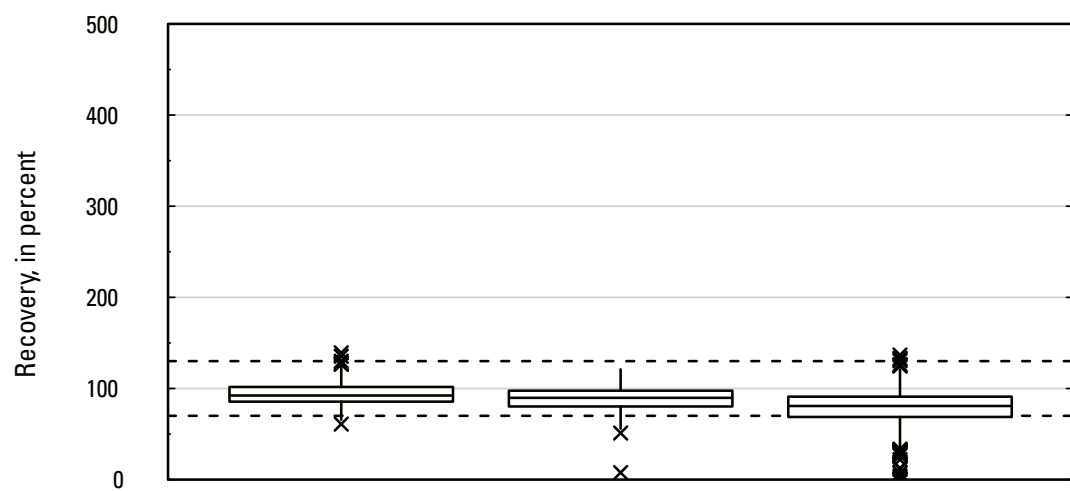

Laboratory reagent spikes
$n=275$ Groundwater
FMS Surface wate FMS

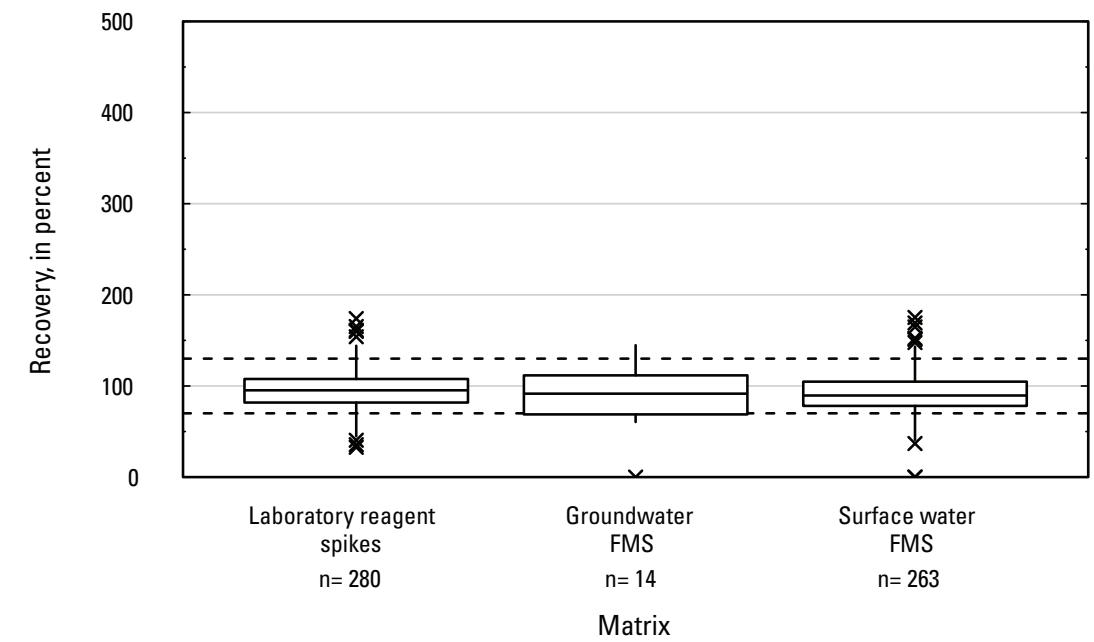

Figure 4. Graph showing distributions of recovery for pesticides in schedule 2437 by matrix. Dashed lines are at 130 percent and 70 percent. Recovery values larger than 400 percent are not shown.-Continued 
Q. 4-Chlorobenzylmethyl sulfoxide

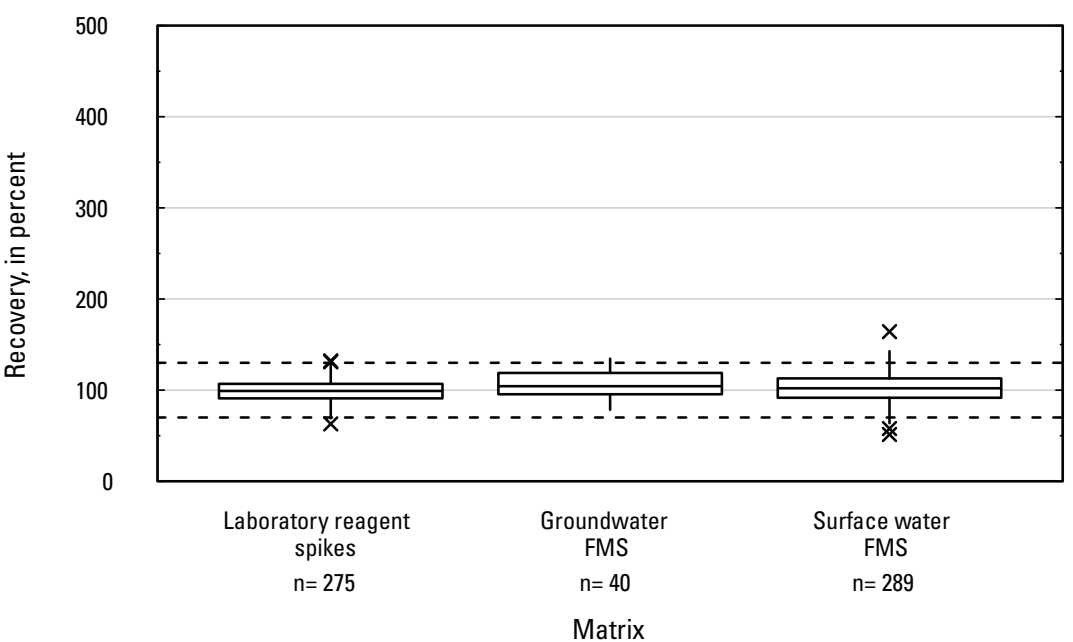

\section{S. 4-Hydroxychlorothalonil}

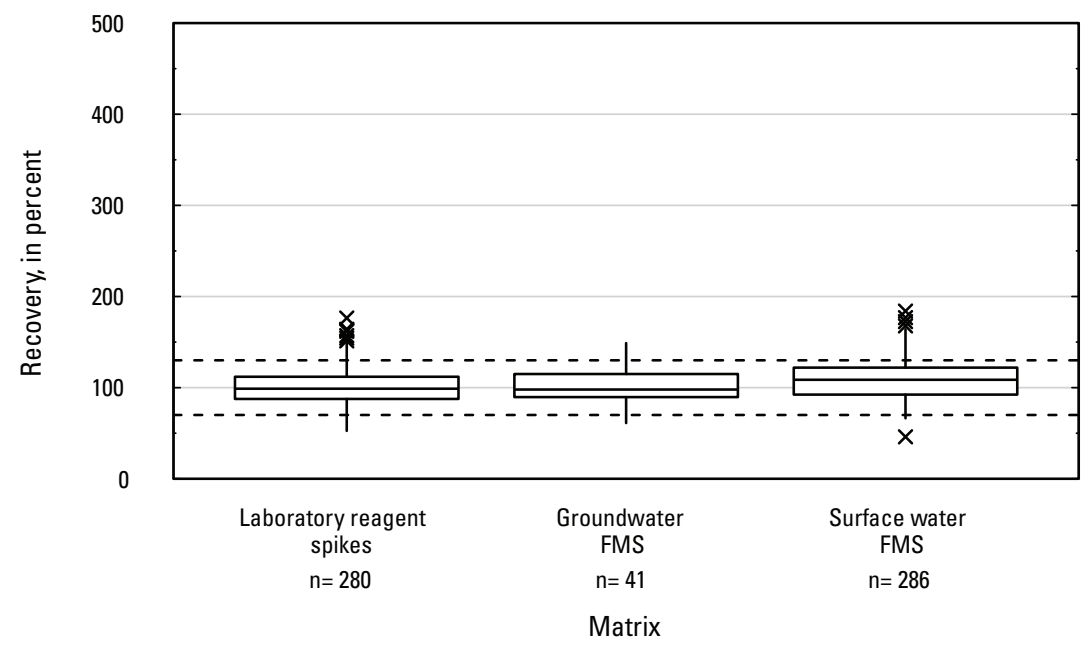

R. 4-Hydroxy molinate

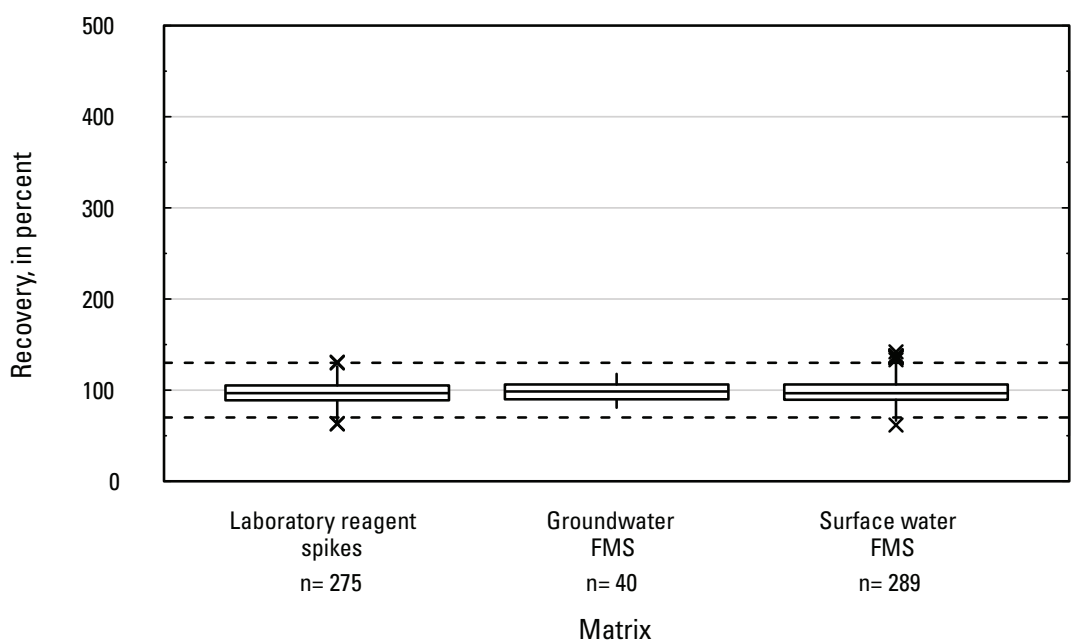

T. 4-Hydroxyhexazinone A

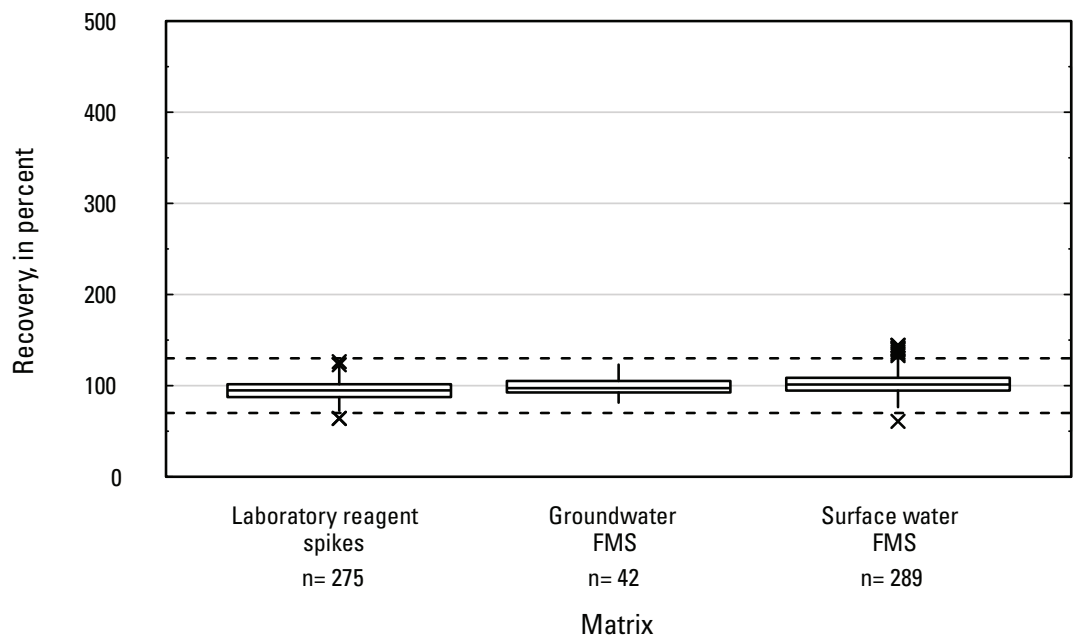

Figure 4. Graph showing distributions of recovery for pesticides in schedule 2437 by matrix. Dashed lines are at 130 percent and 70 percent. Recovery values larger than 400 percent are not shown.-Continued 
U. Hydroxy didemethyl fluometuron

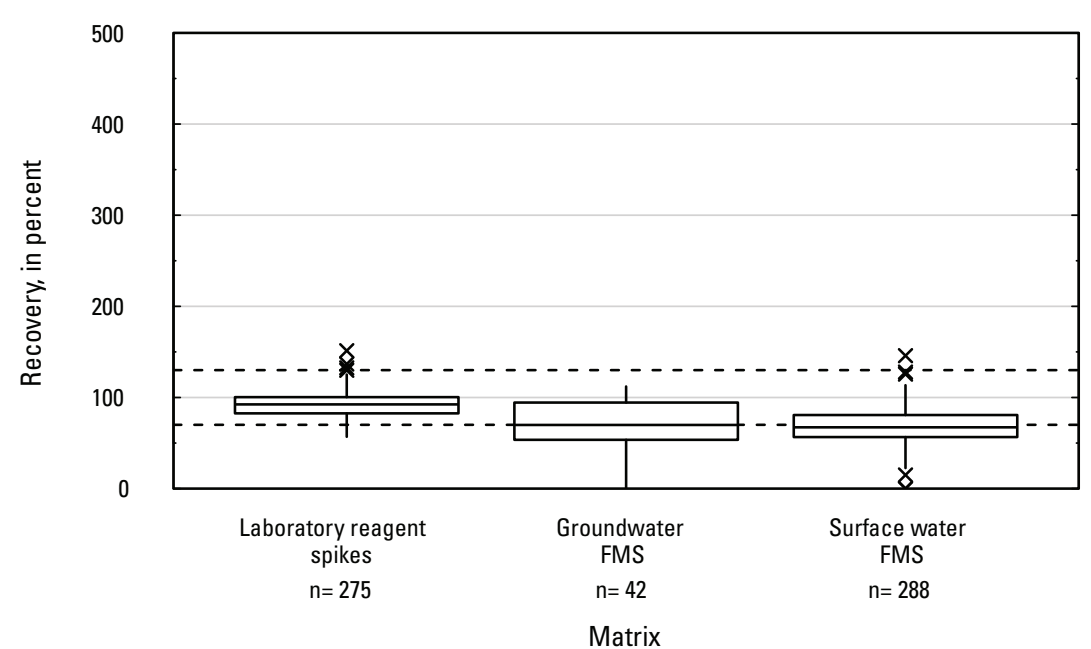

W. Acetochlor

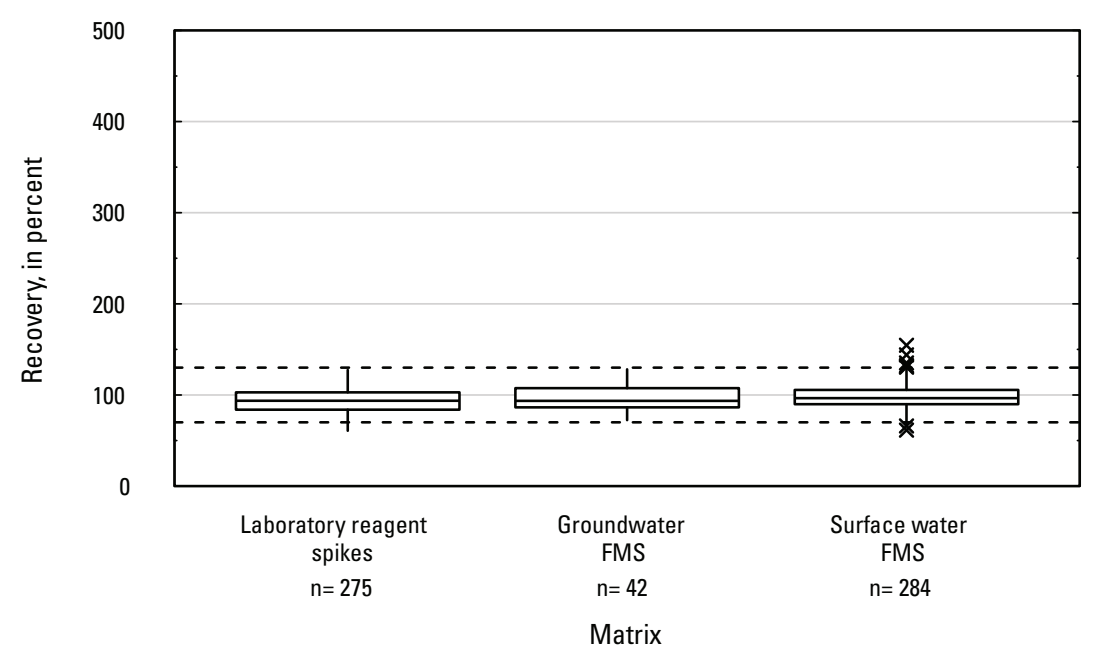

V. Acephate

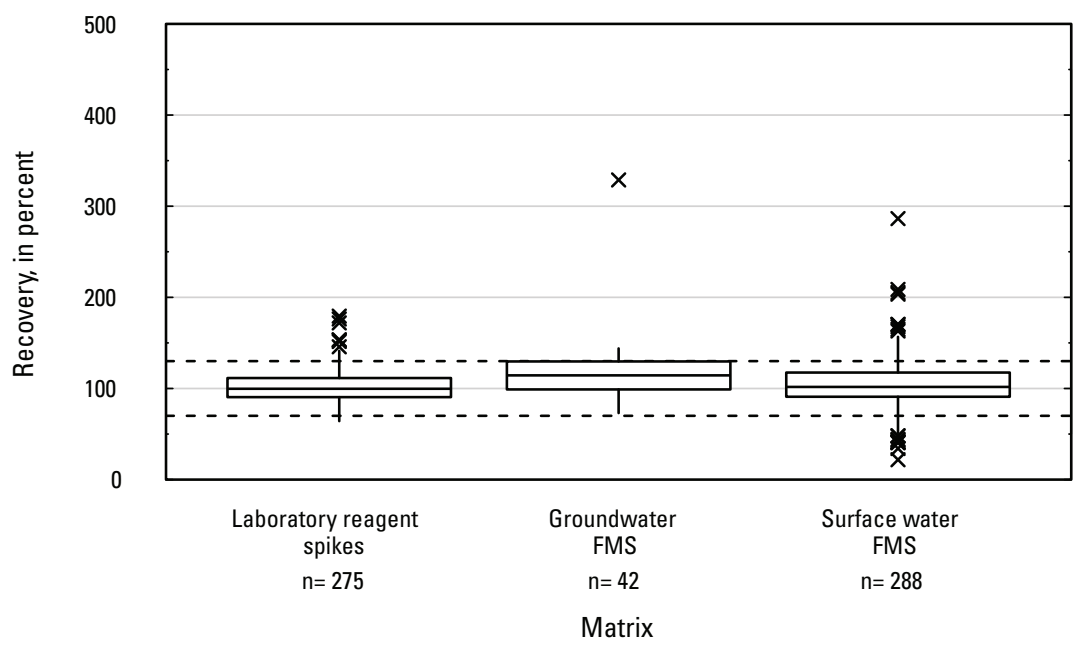

X. Acetochlor oxanilic acid

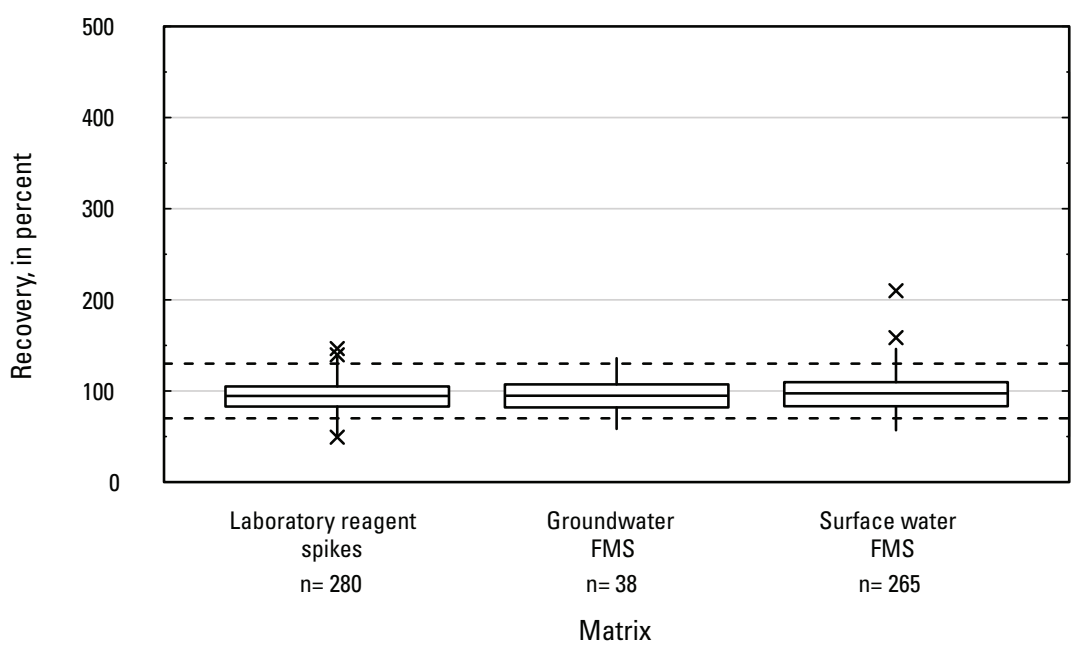

N

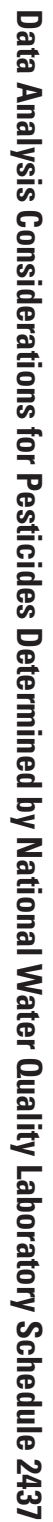

Figure 4. Graph showing distributions of recovery for pesticides in schedule 2437 by matrix. Dashed lines are at 130 percent and 70 percent. Recovery values larger than 400 percent are not shown.-Continued 
Y. Acetochlor sulfonic acid

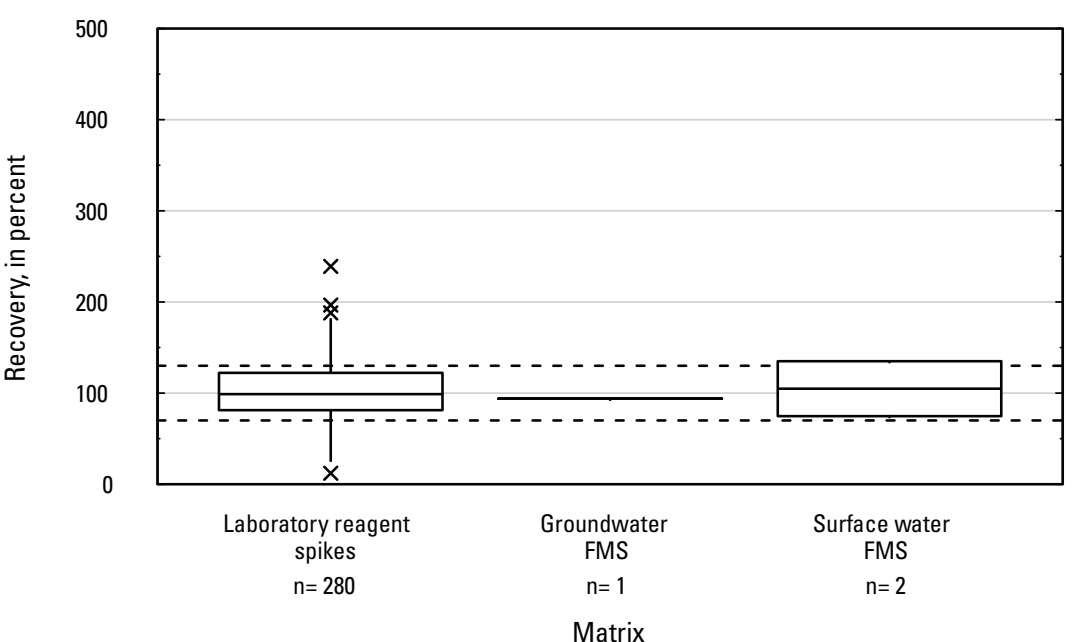

AA. Alachlor

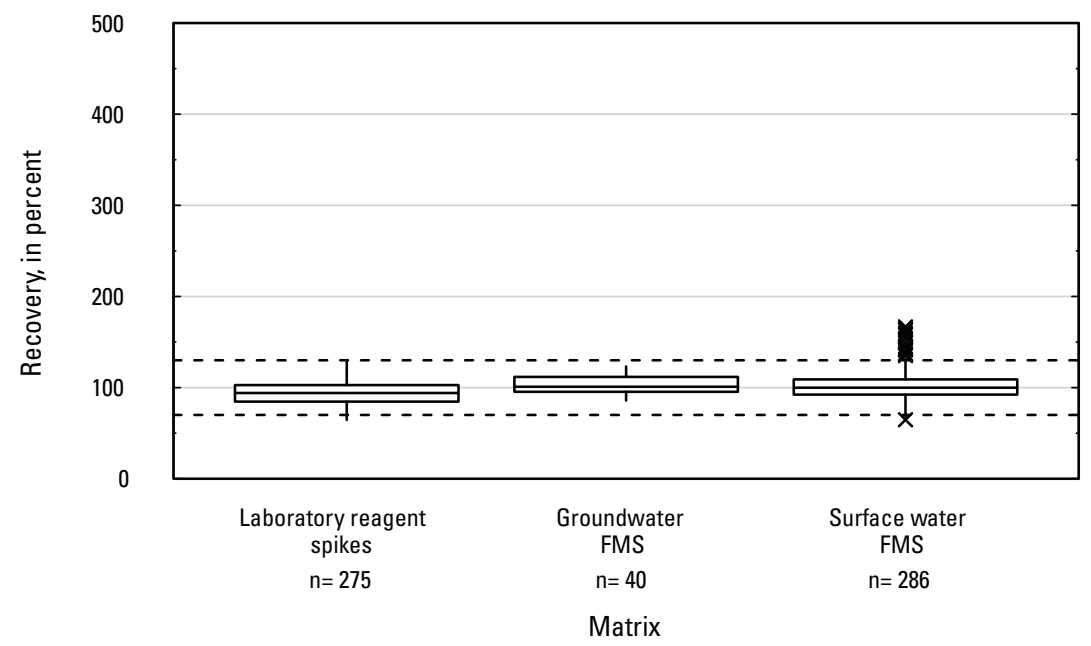

Z. Acetochlor sulfynilacetic acid

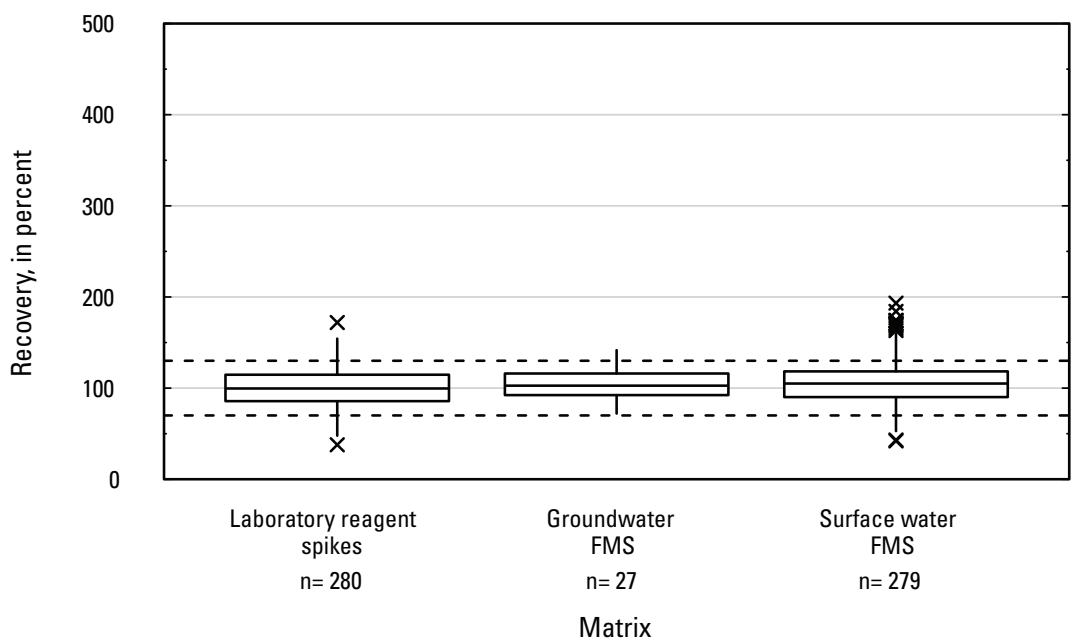

AB. Alachlor oxanilic acid

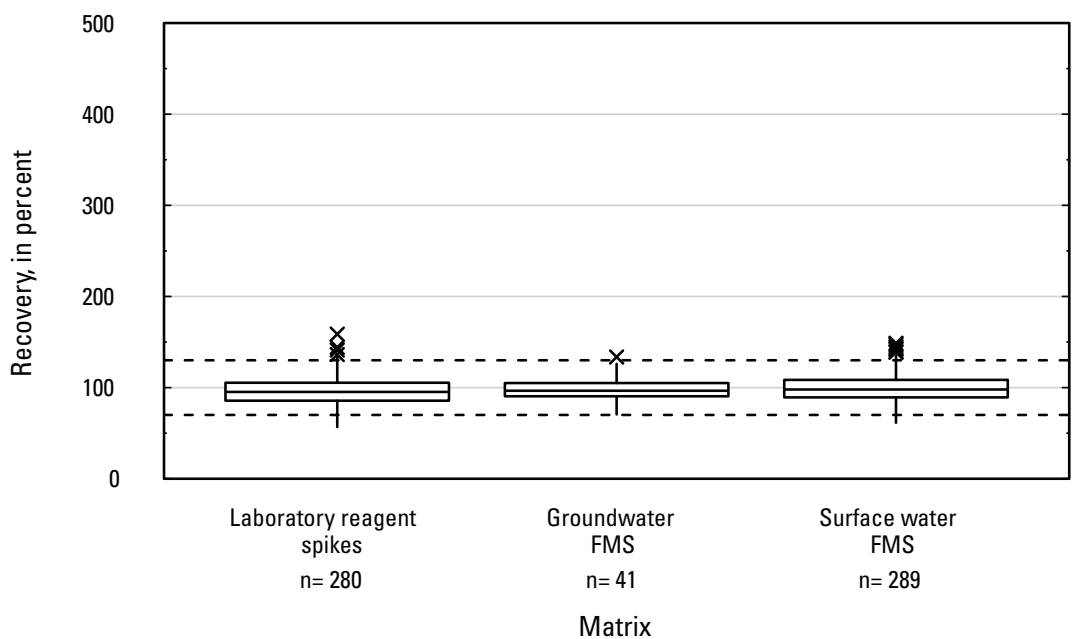

Figure 4. Graph showing distributions of recovery for pesticides in schedule 2437 by matrix. Dashed lines are at 130 percent and 70 percent. Recovery values larger than 400 percent are not shown.-Continued 
AC. Alachlor sulfonic acid

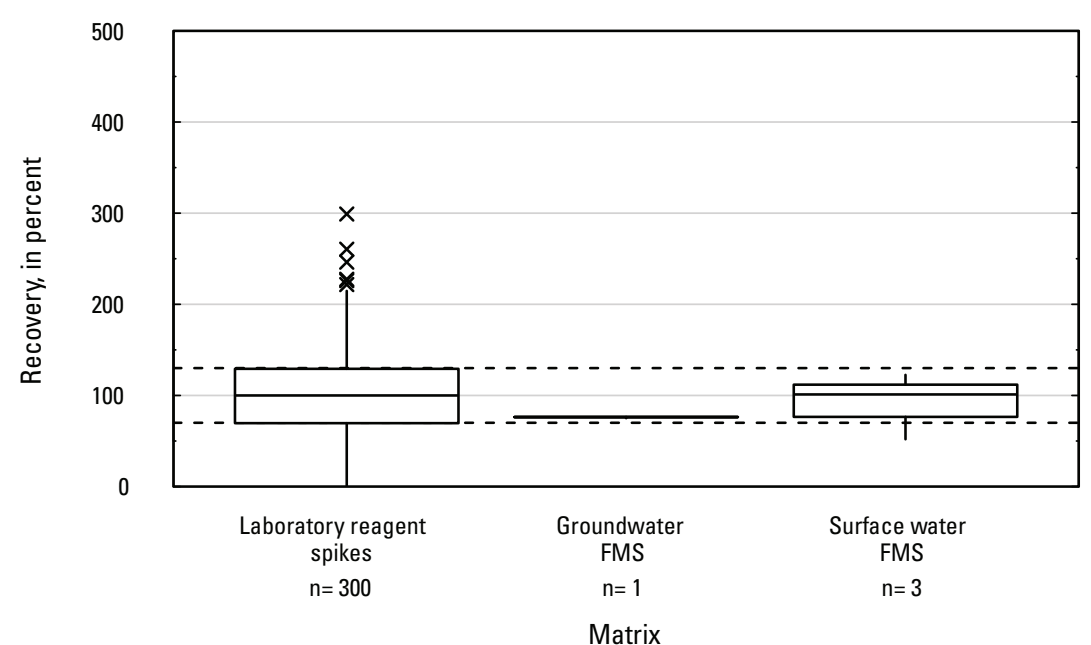

AE. Aldicarb

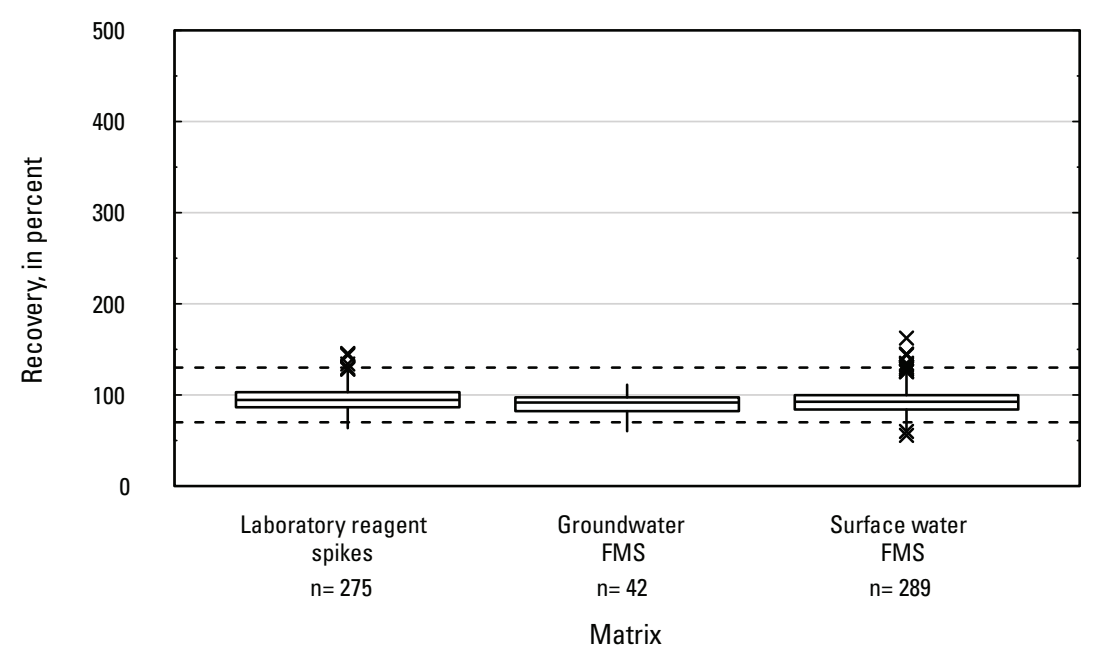

AD. Alachlor sulfynilacetic acid

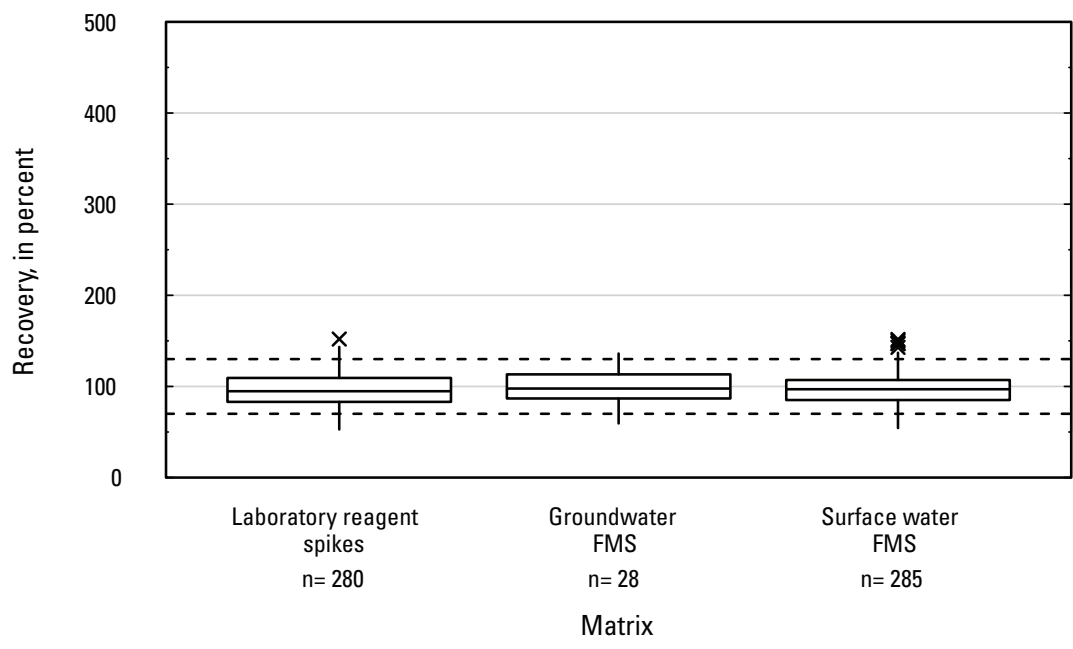

AF. Aldicarb sulfone

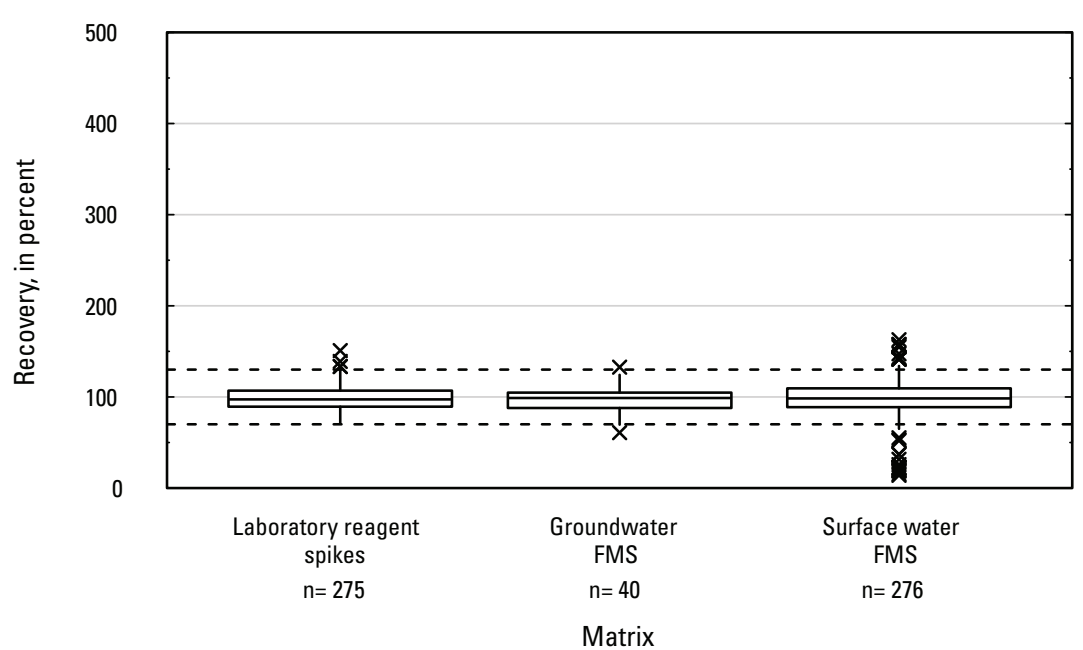

$\not$

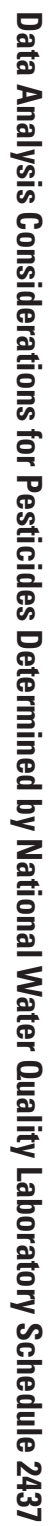

Figure 4. Graph showing distributions of recovery for pesticides in schedule 2437 by matrix. Dashed lines are at 130 percent and 70 percent. Recovery values larger than 400 percent are not shown.-Continued 
AG. Aldicarb sulfoxide

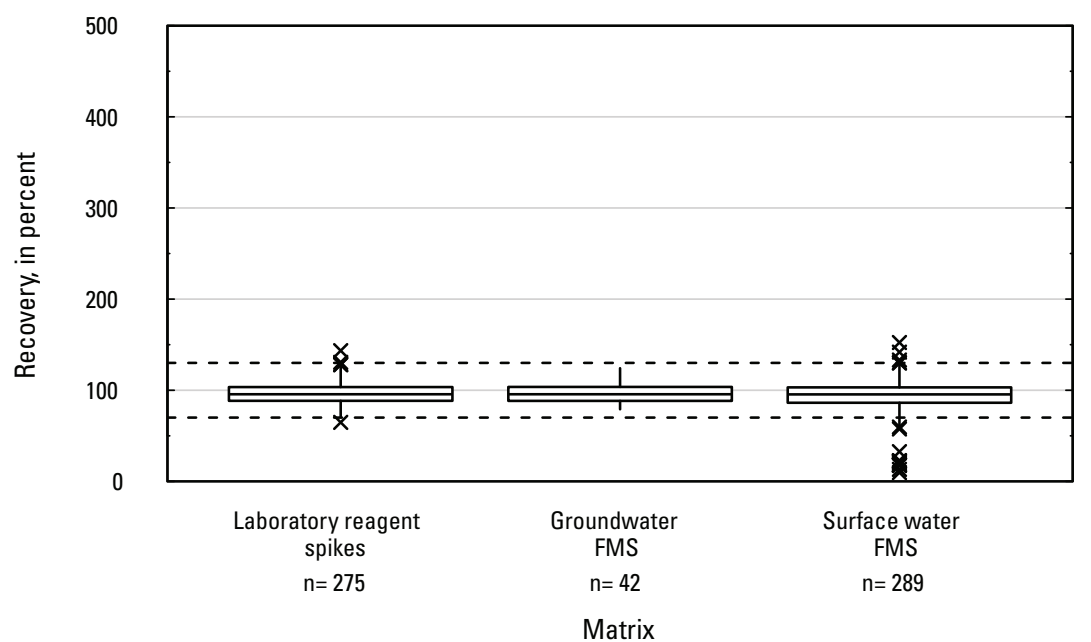

Al. Asulam

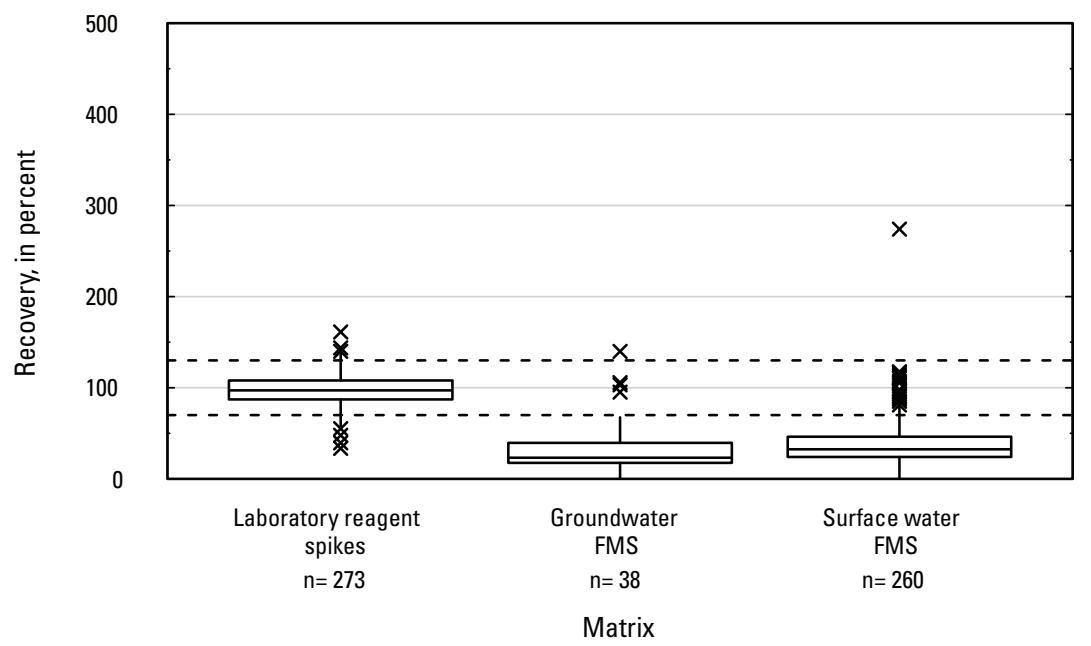

AH. Ametryn

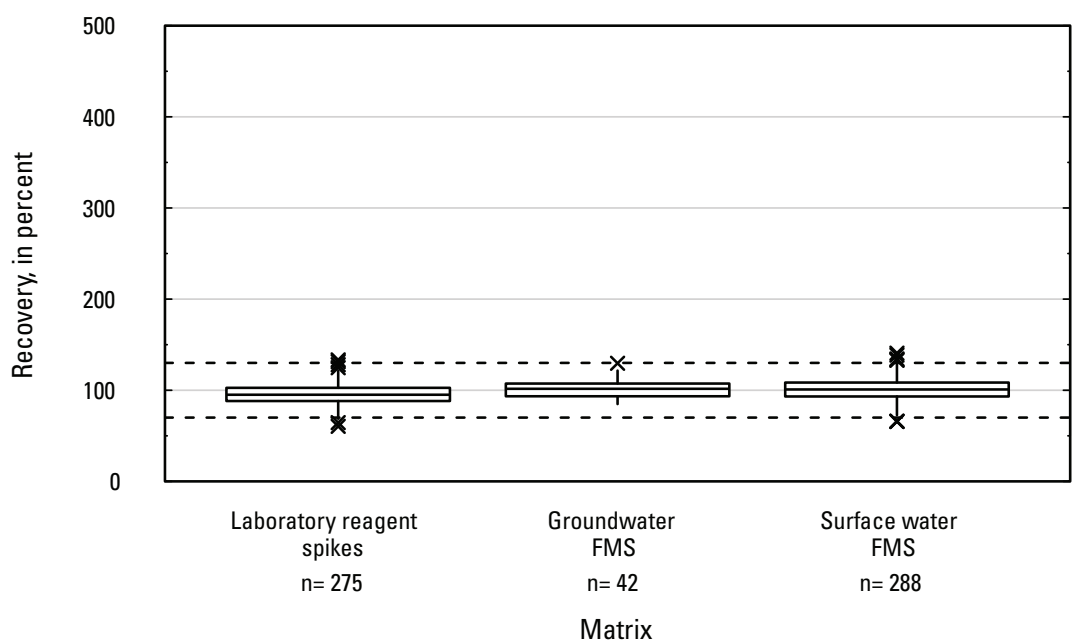

AJ. Atrazine

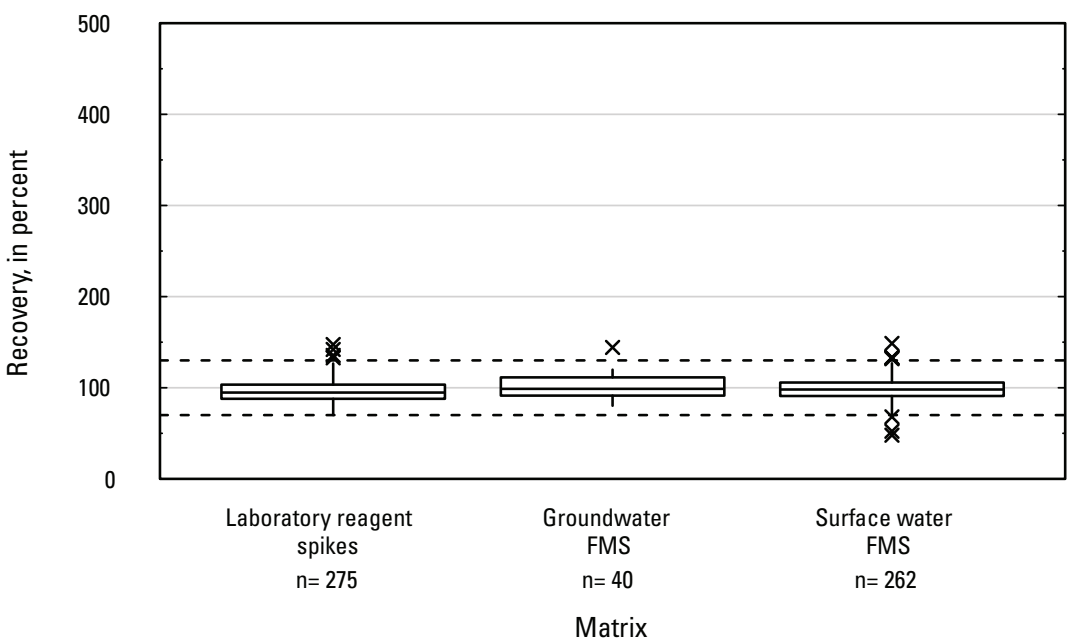

Figure 4. Graph showing distributions of recovery for pesticides in schedule 2437 by matrix. Dashed lines are at 130 percent and 70 percent. Recovery values larger 
AK. Azinphos-methyl

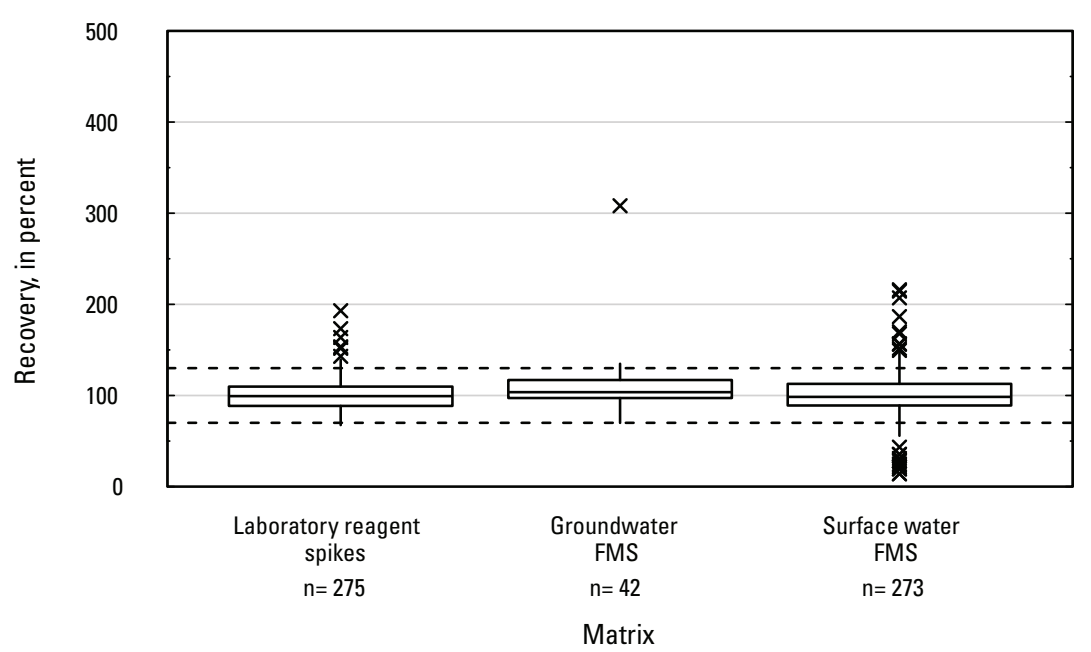

AM. Azoxystrobin

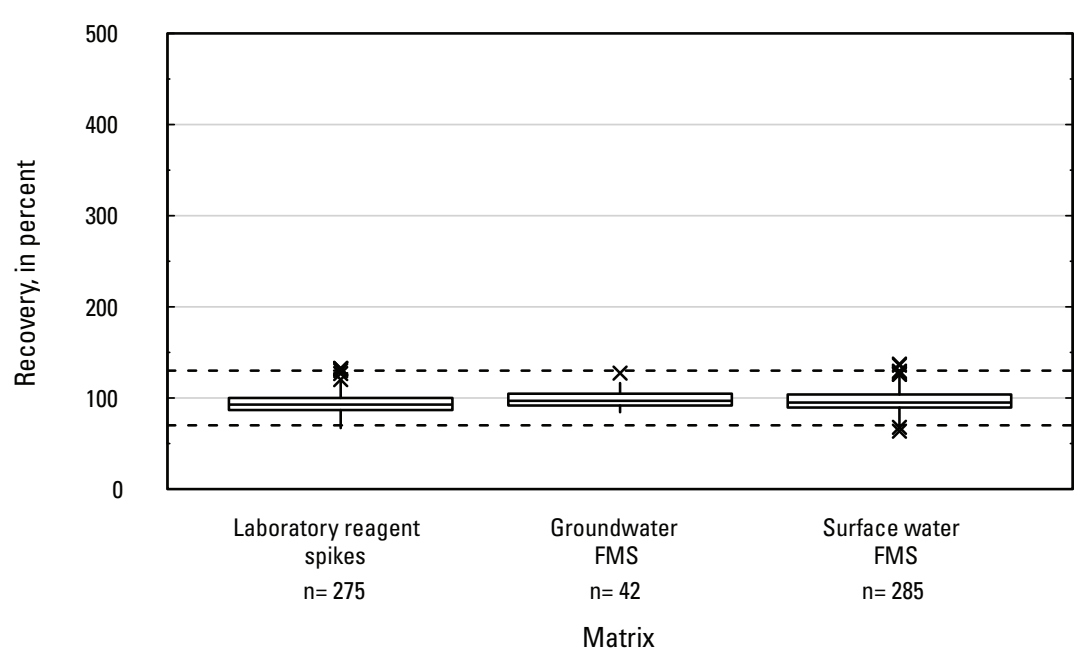

AL. Azinphos-methyl oxon

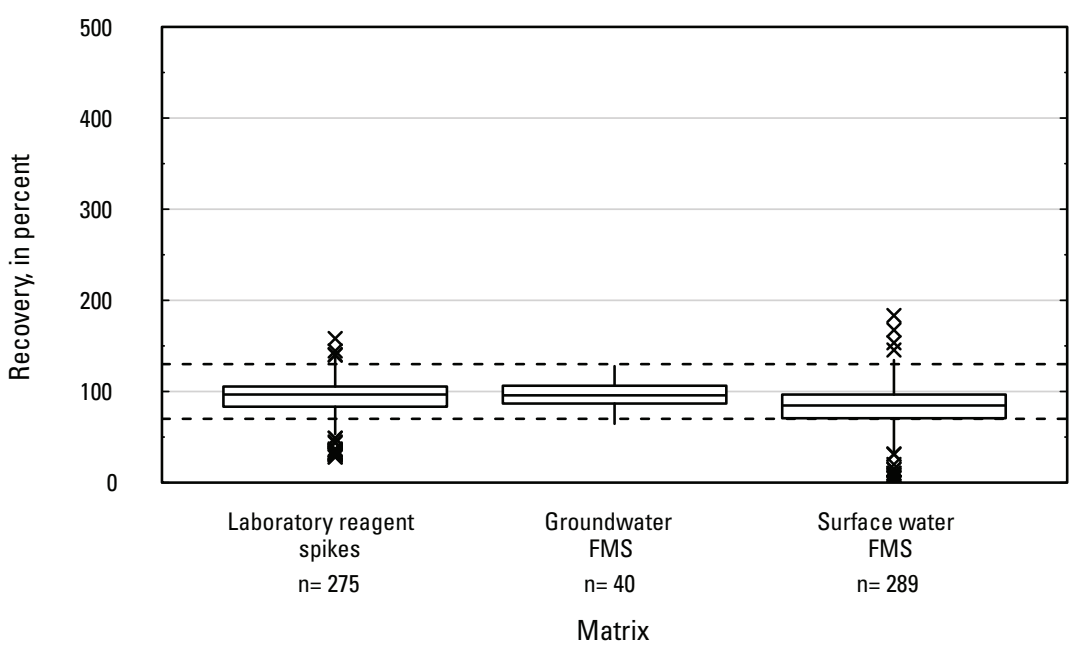

AN. Bentazone

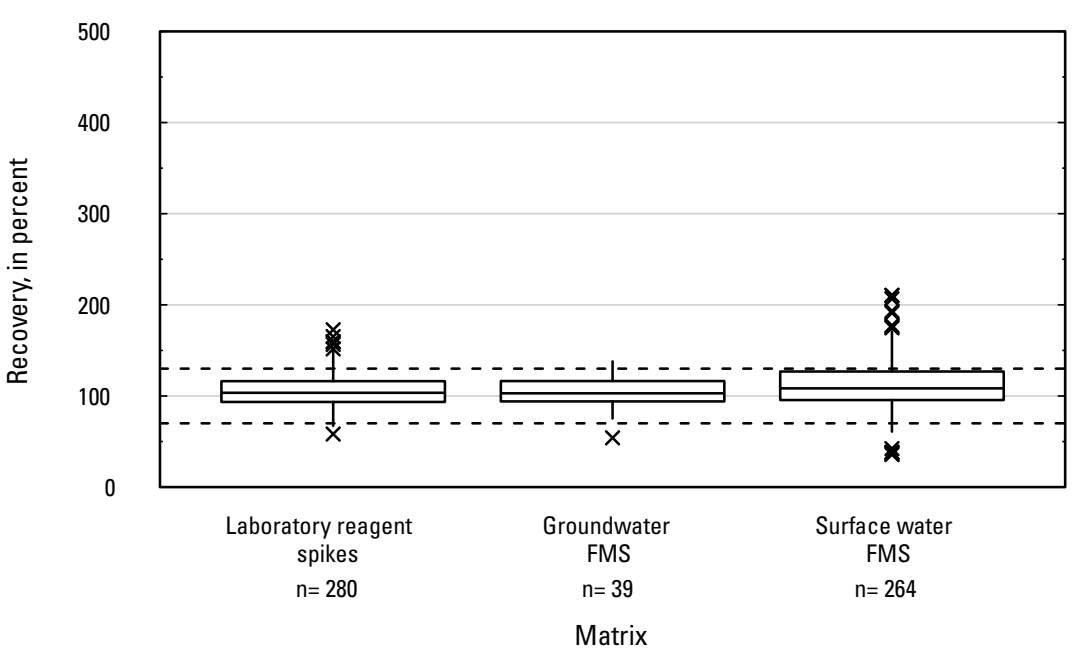

Figure 4. Graph showing distributions of recovery for pesticides in schedule 2437 by matrix. Dashed lines are at 130 percent and 70 percent. Recovery values larger than 400 percent are not shown.-Continued 
AO. Bifenthrin

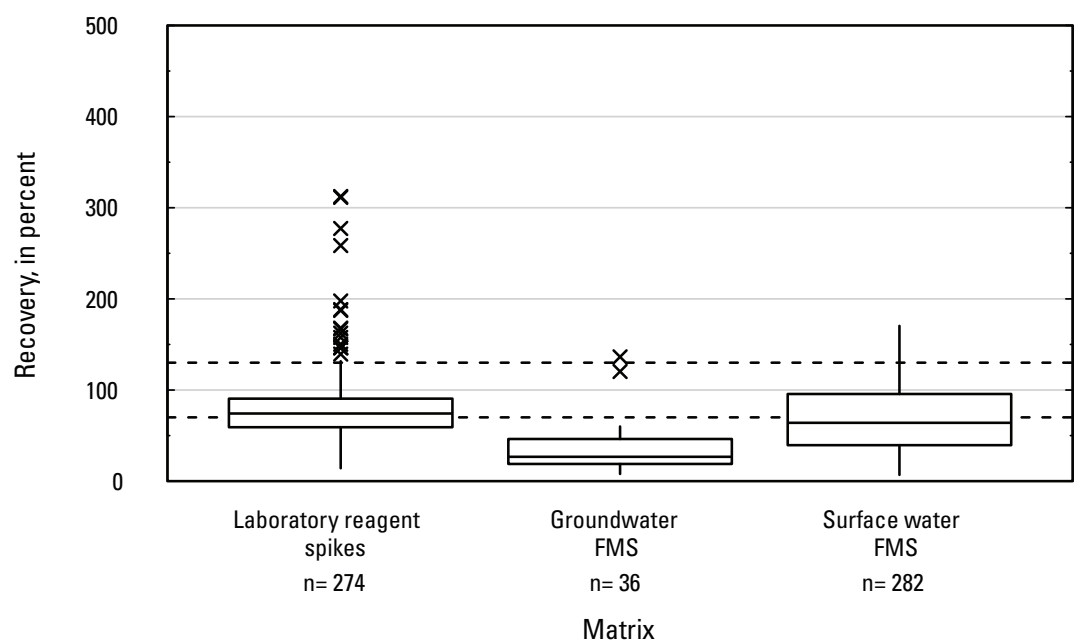

AQ. Bromoxynil

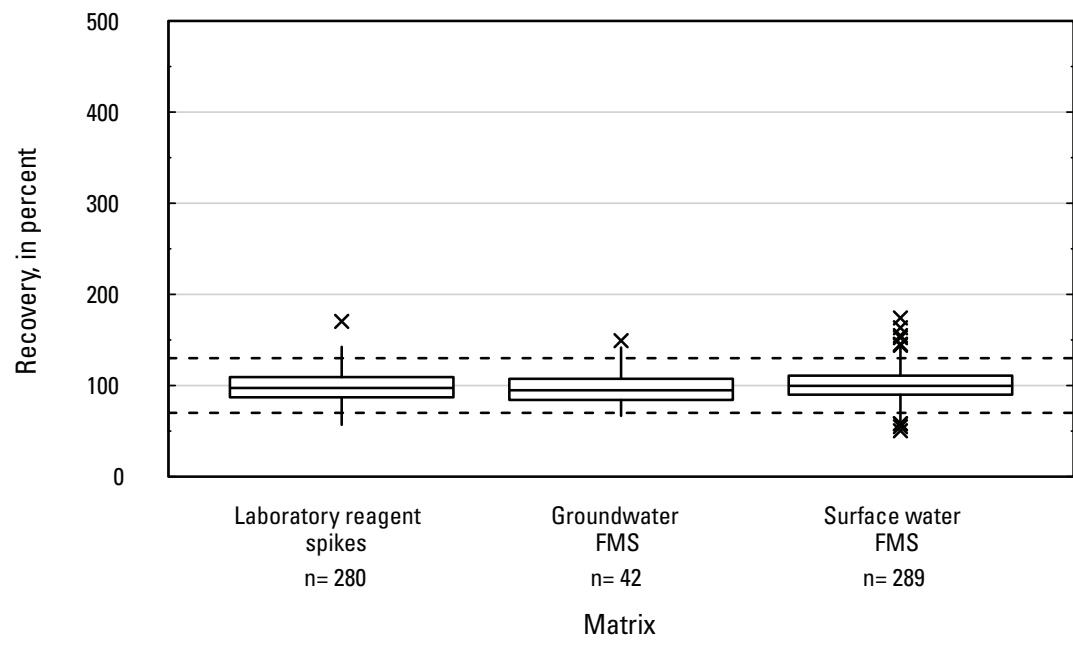

AP. Bromacil

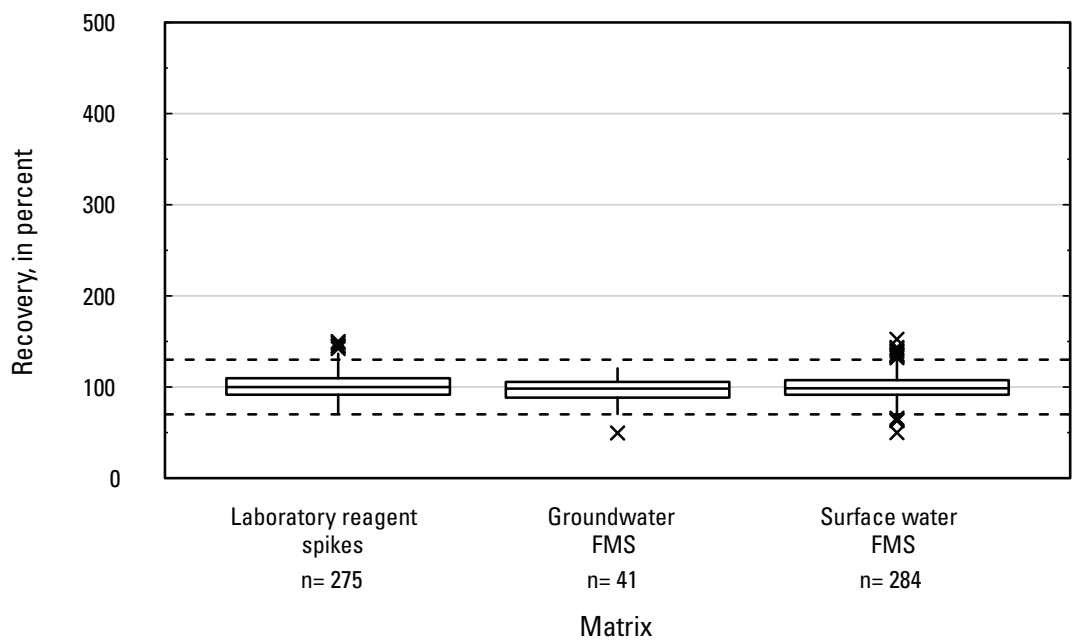

AR. Butralin

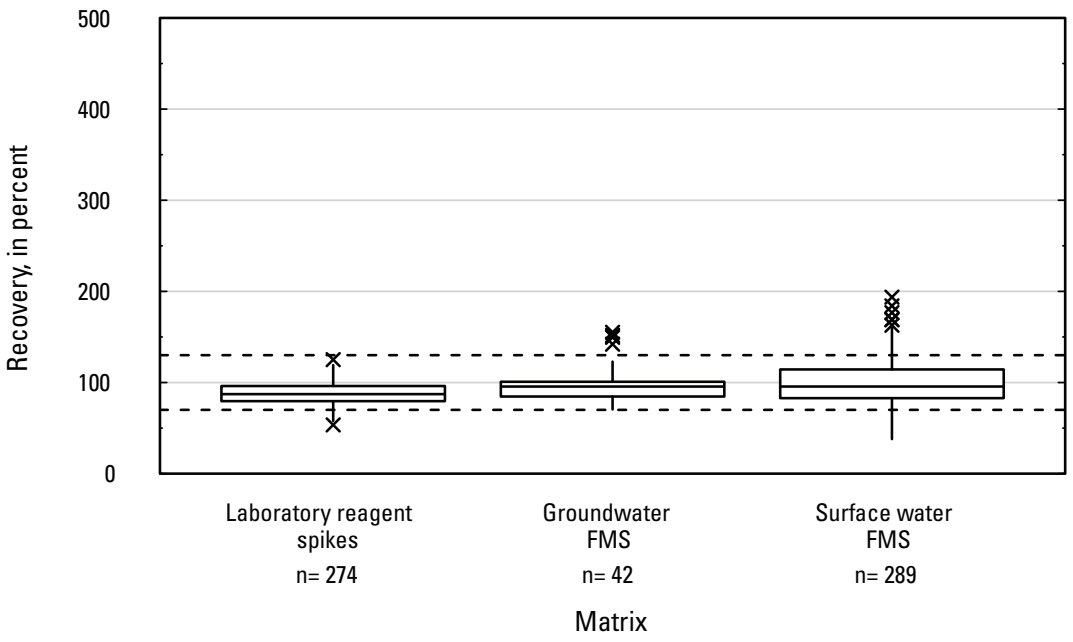

Figure 4. Graph showing distributions of recovery for pesticides in schedule 2437 by matrix. Dashed lines are at 130 percent and 70 percent. Recovery values larger than 400 percent are not shown.-Continued 
AS. Butylate

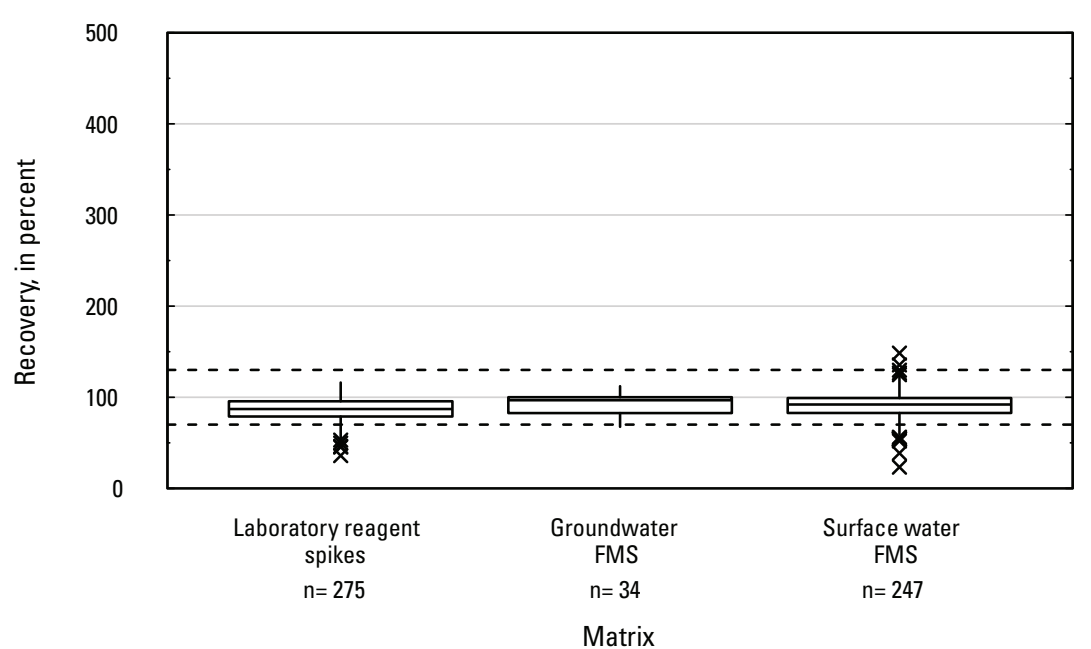

AU. Carbendazim

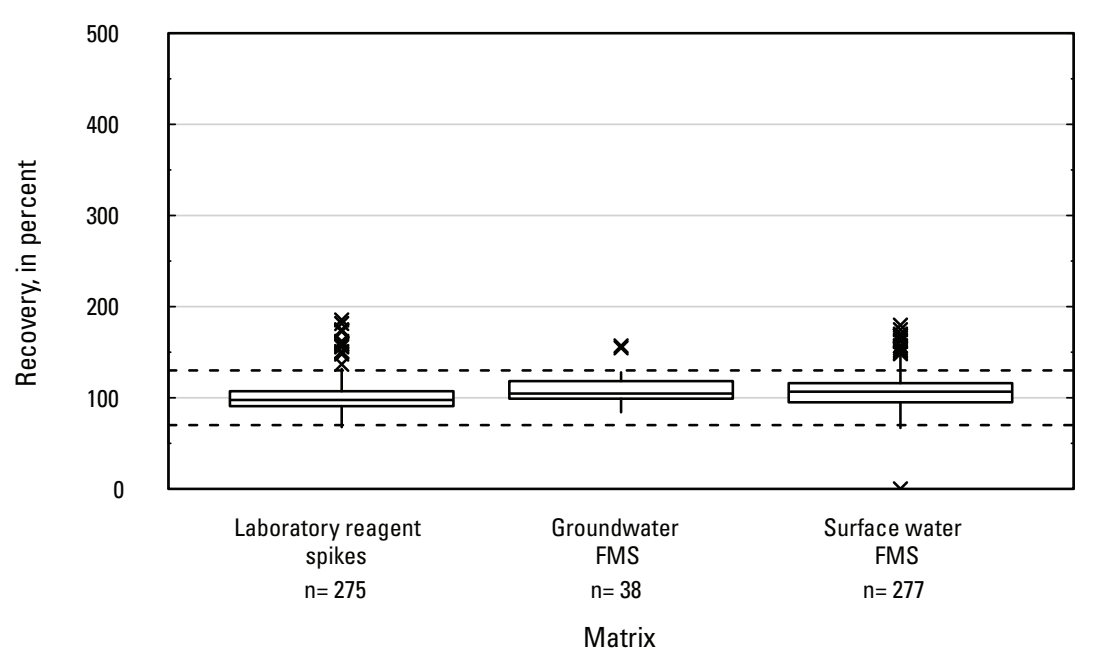

AT. Carbary

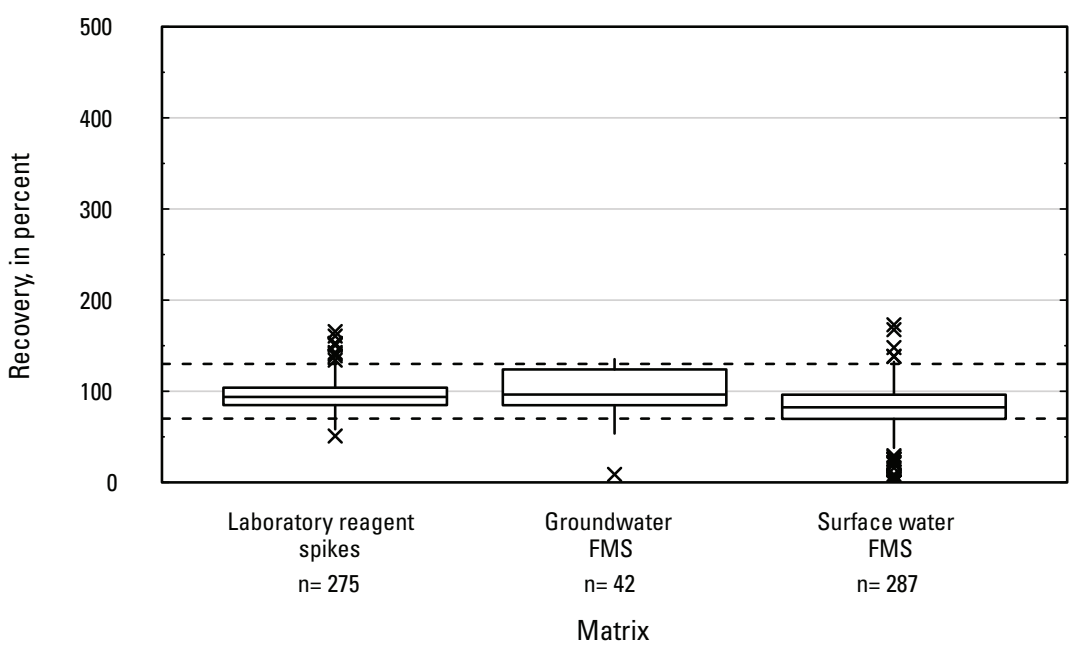

AV. Carbofuran

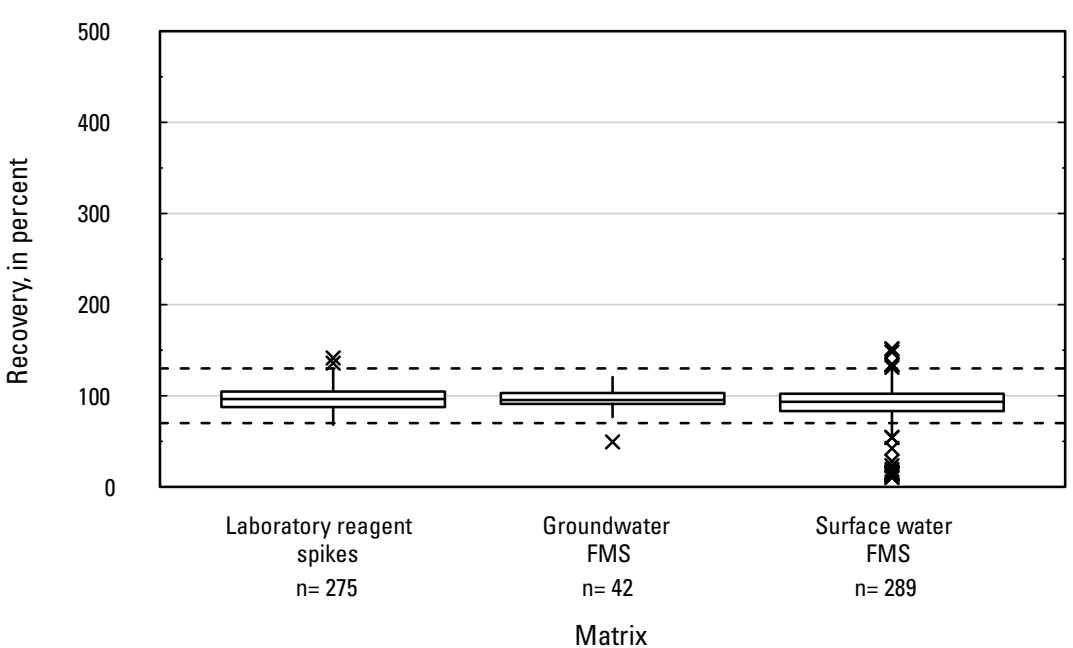

$\infty$

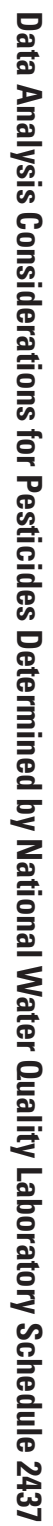

Figure 4. Graph showing distributions of recovery for pesticides in schedule 2437 by matrix. Dashed lines are at 130 percent and 70 percent. Recovery values larger than 400 percent are not shown.-Continued 
AW. Carboxy molinate

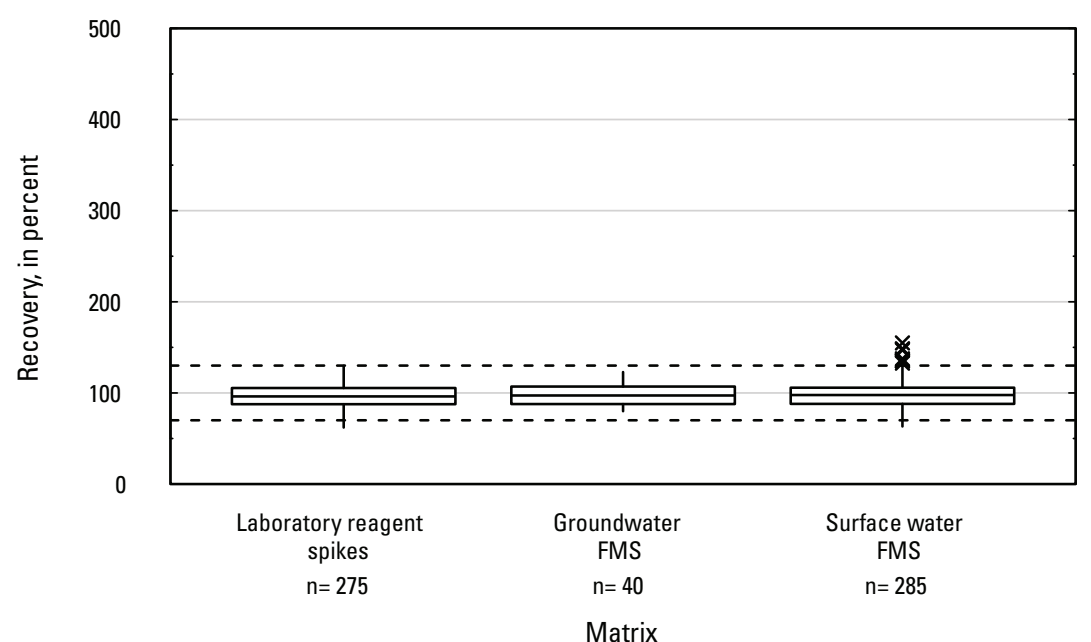

$A Y$. Chlorosulfonamide acid

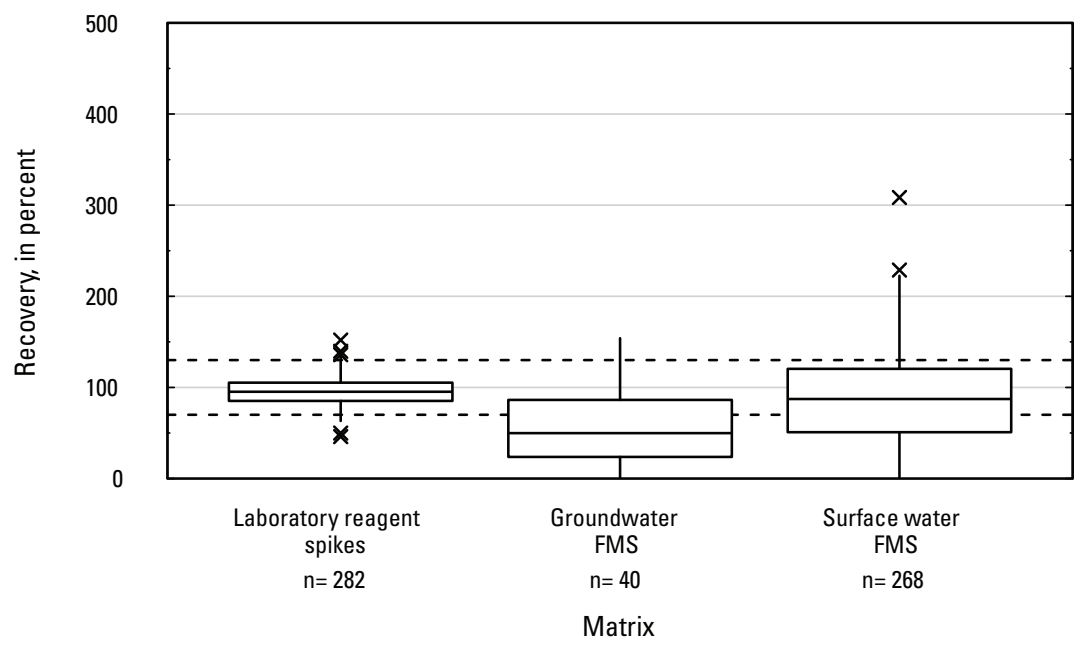

AX. Chlorimuron-ethyl

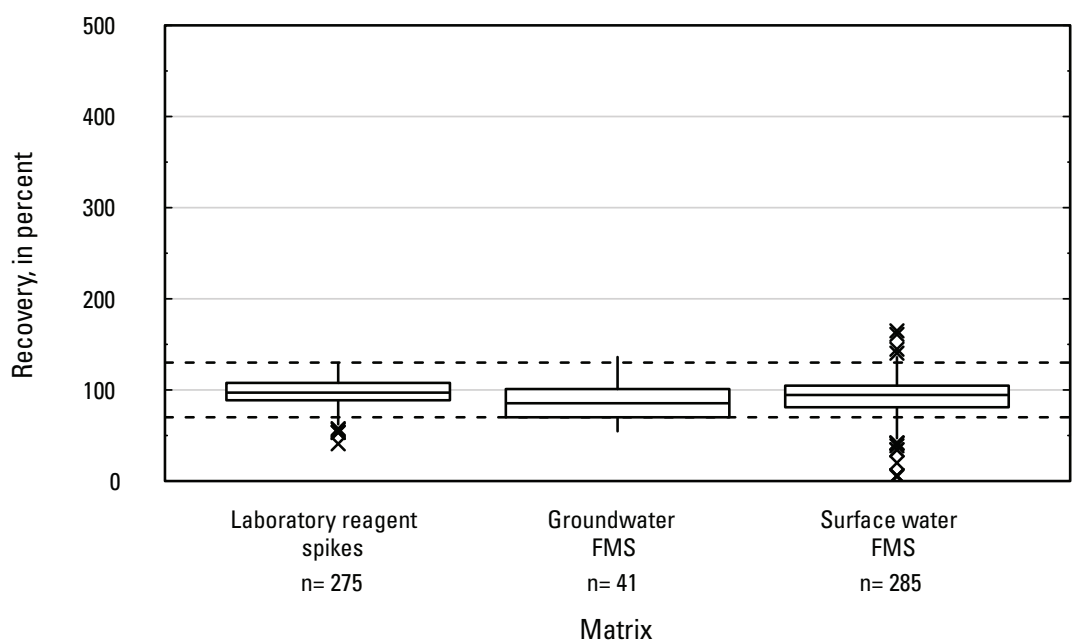

AZ. Chlorpyrifos

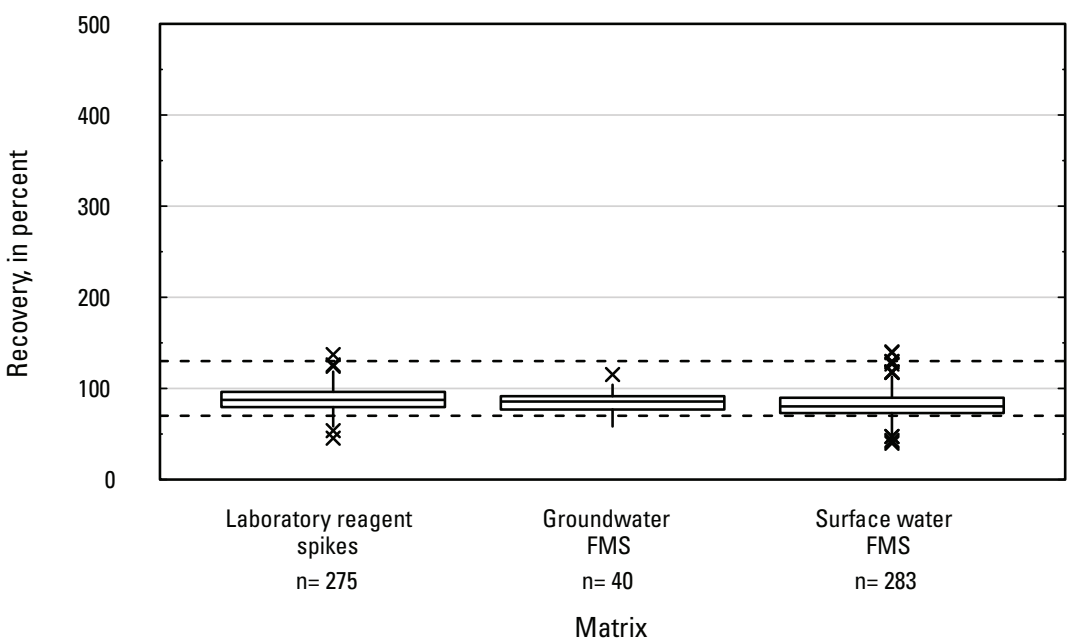

Figure 4. Graph showing distributions of recovery for pesticides in schedule 2437 by matrix. Dashed lines are at 130 percent and 70 percent. Recovery values larger than 400 percent are not shown.-Continued 
BA. Chlorpyrifos oxon

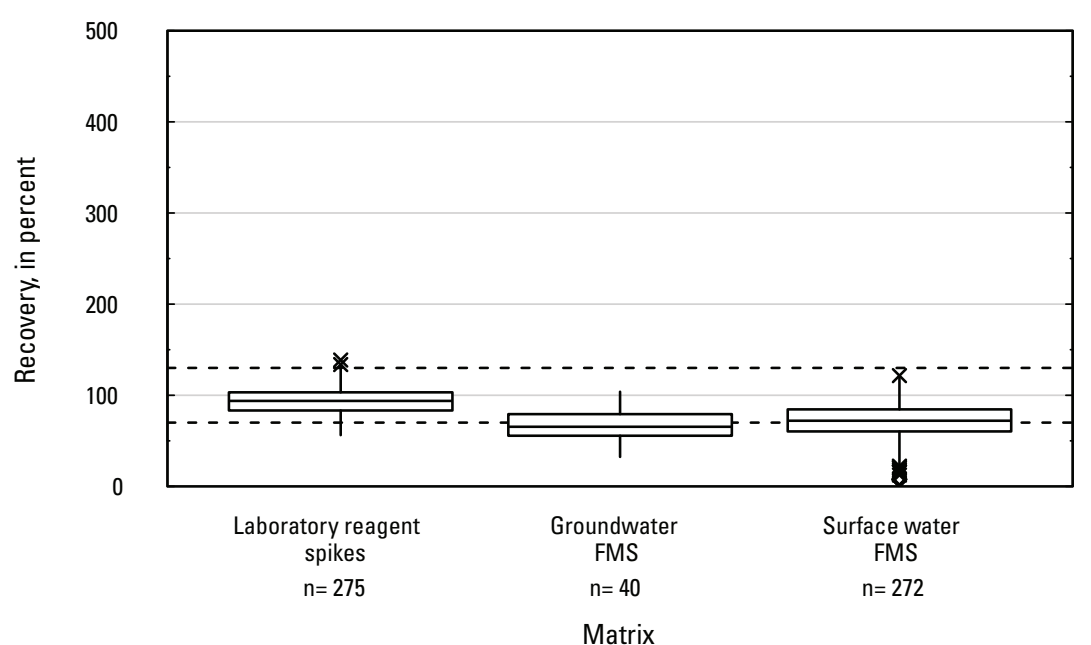

BC. Dacthal monoacid

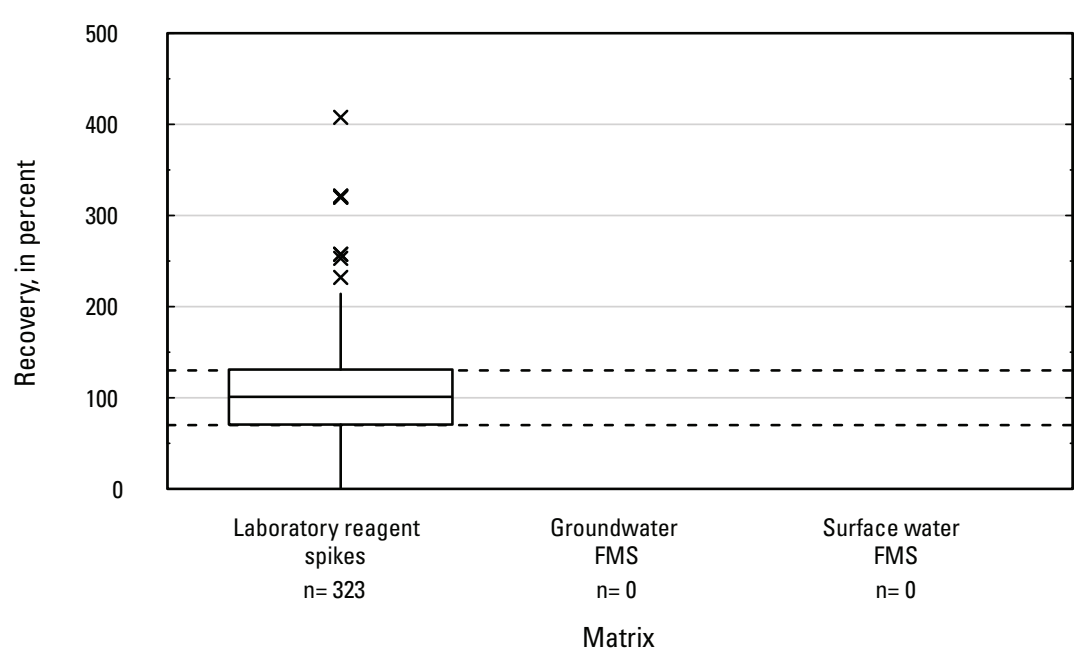

BB. Chlorsulfuron

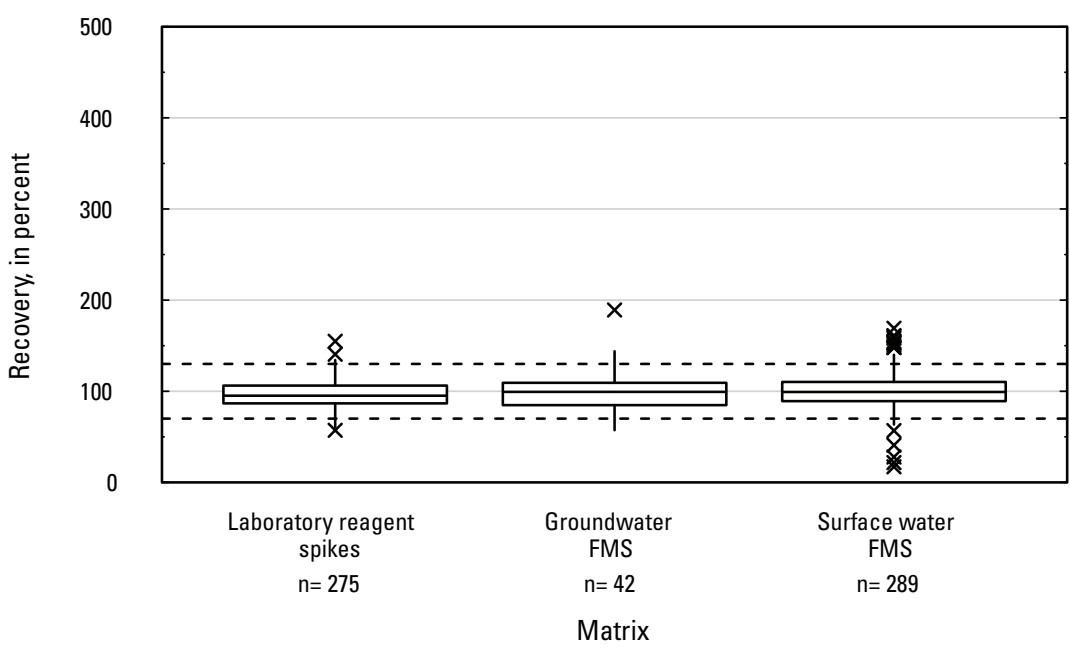

BD. cis-Bifenthrin acid/cis-Cyhalothrin acid/cis-Tefluthrin acid

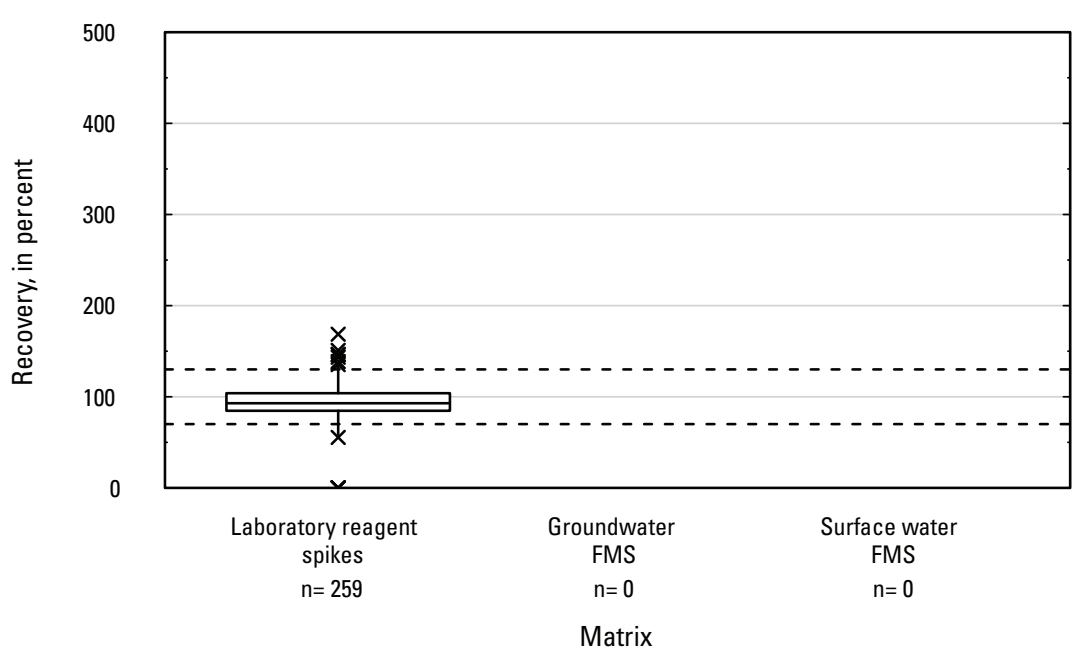

당

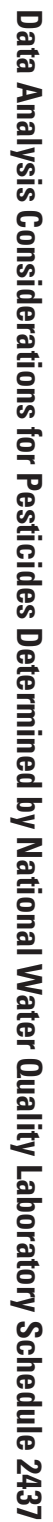

Figure 4. Graph showing distributions of recovery for pesticides in schedule 2437 by matrix. Dashed lines are at 130 percent and 70 percent. Recovery values larger than 400 percent are not shown.-Continued 
$B E$. cis-Permethrin

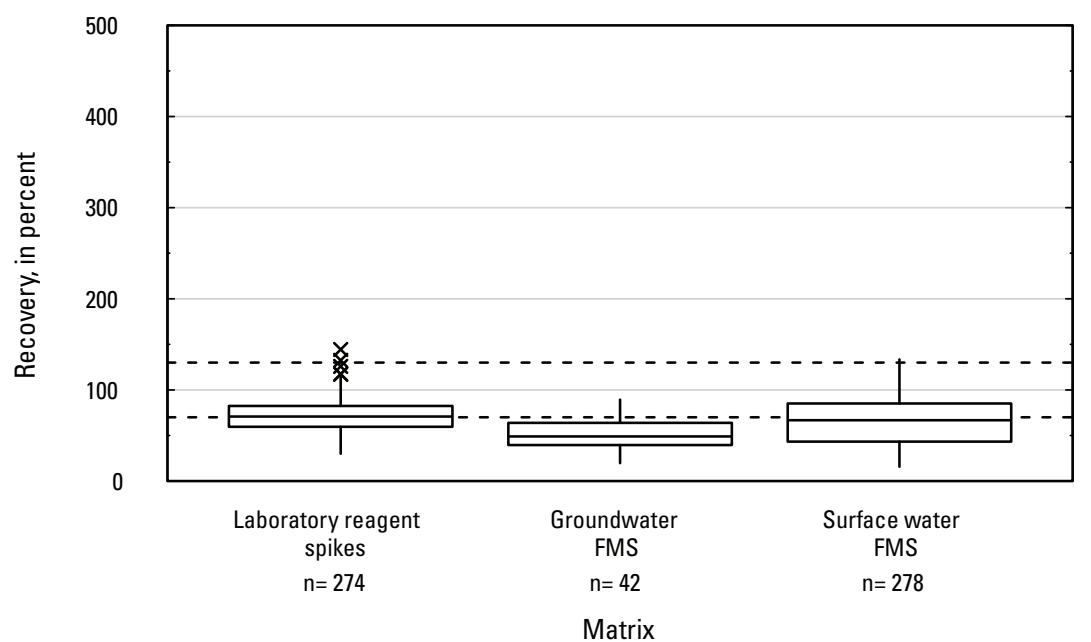

BG. Dechlorofipronil

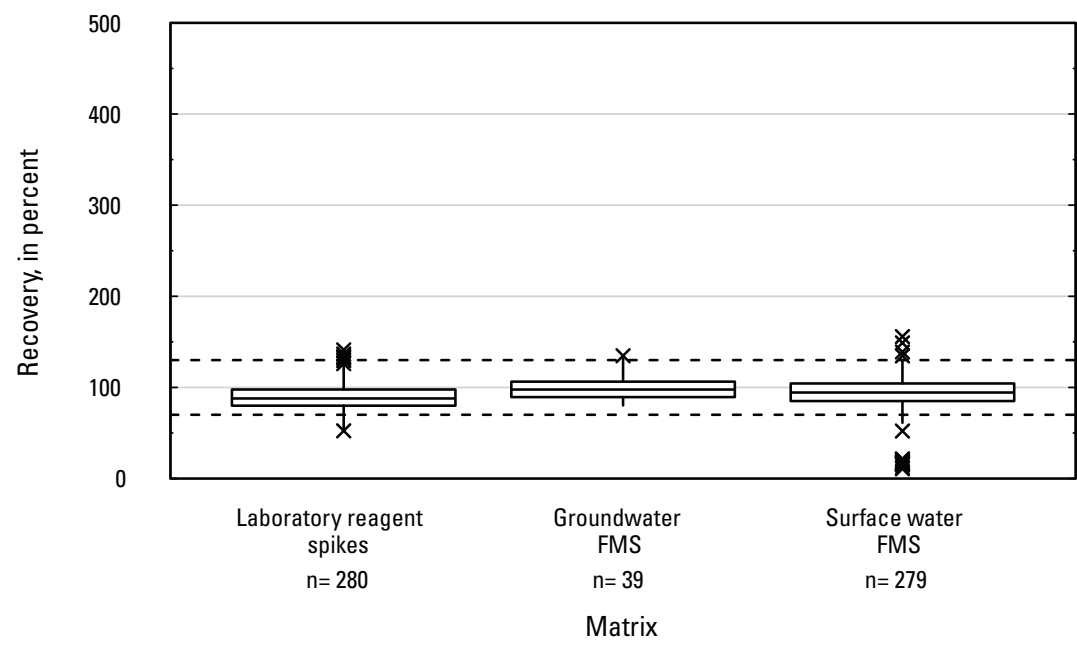

\section{BF. Cyanazine}

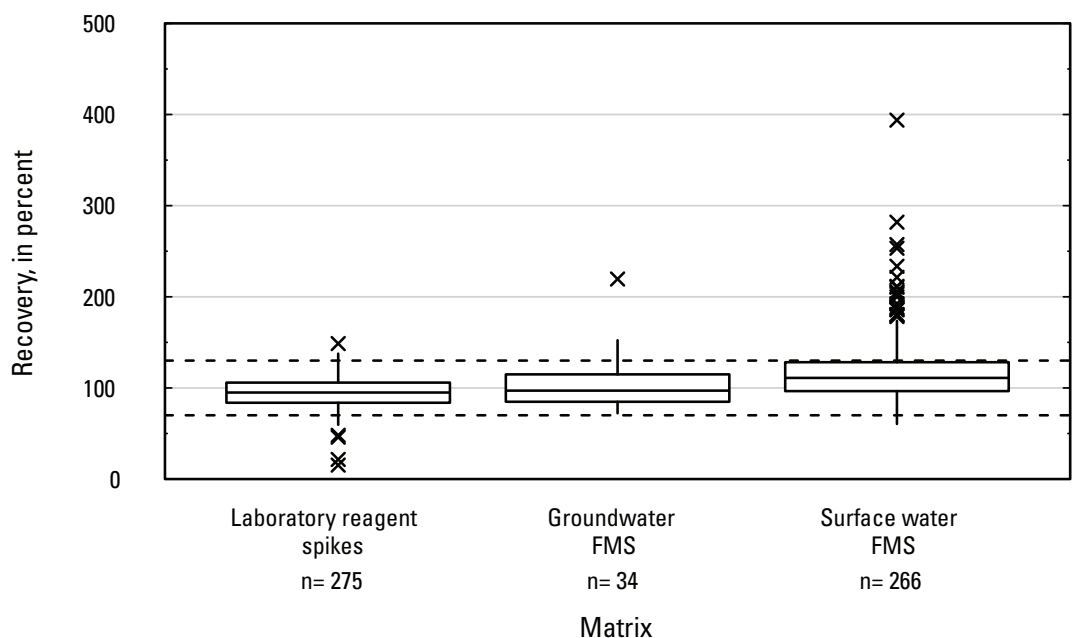

BH. Dechlorometolachlor

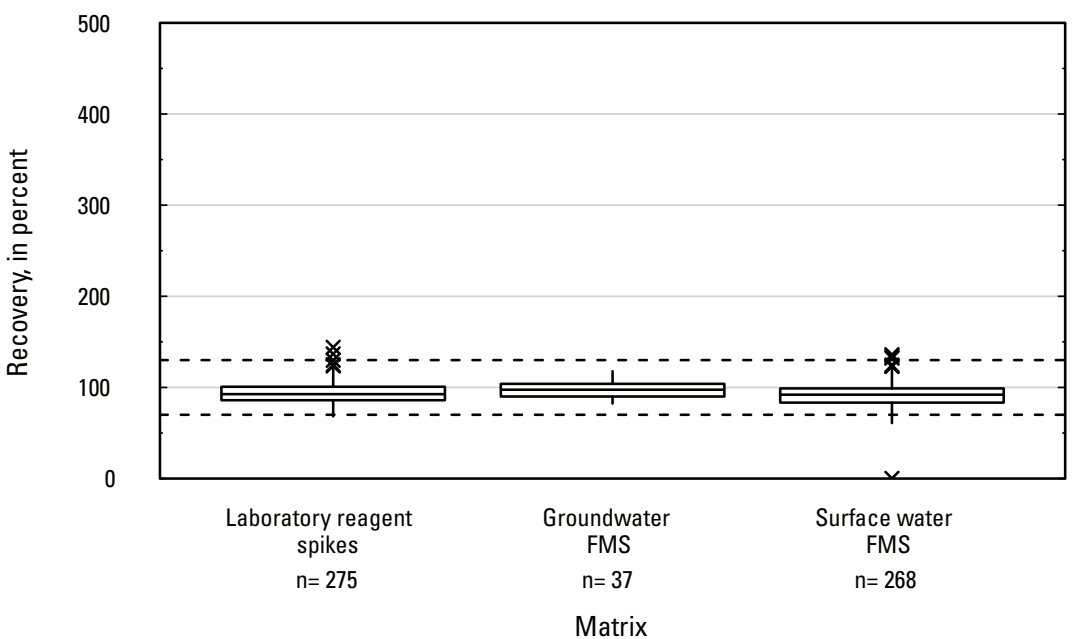

Figure 4. Graph showing distributions of recovery for pesticides in schedule 2437 by matrix. Dashed lines are at 130 percent and 70 percent. Recovery values larger than 400 percent are not shown.-Continued 
BI. 2-Chloro-4-isopropylamino-6-amino-s-triazine

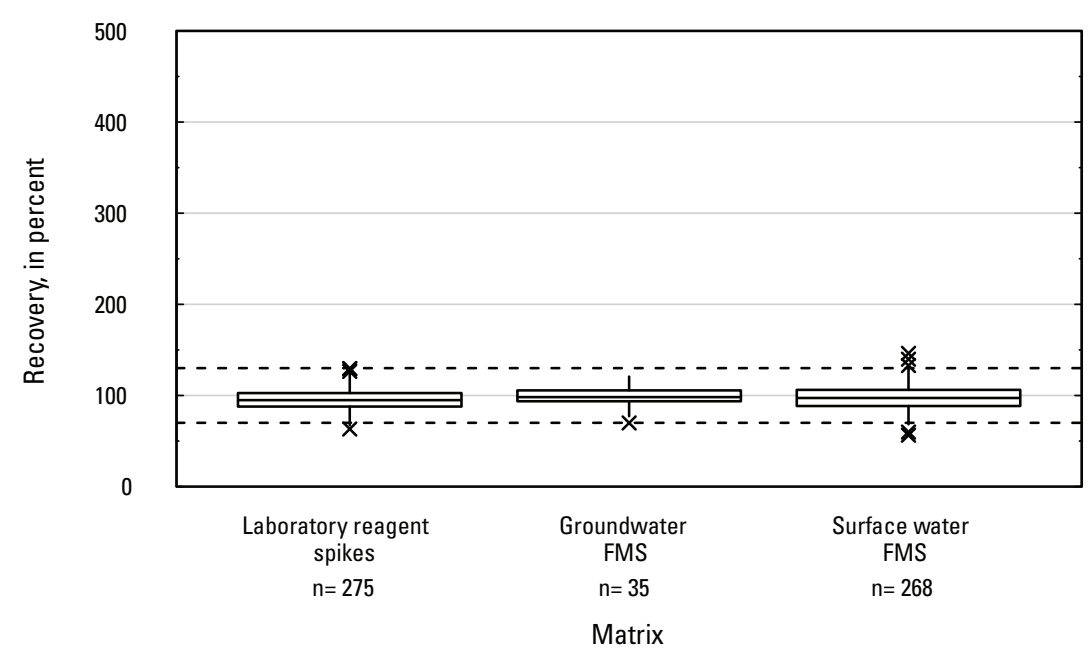

BK. 2-Chloro-6-ethylamino-4-amino-s-triazine \{CEAT\}

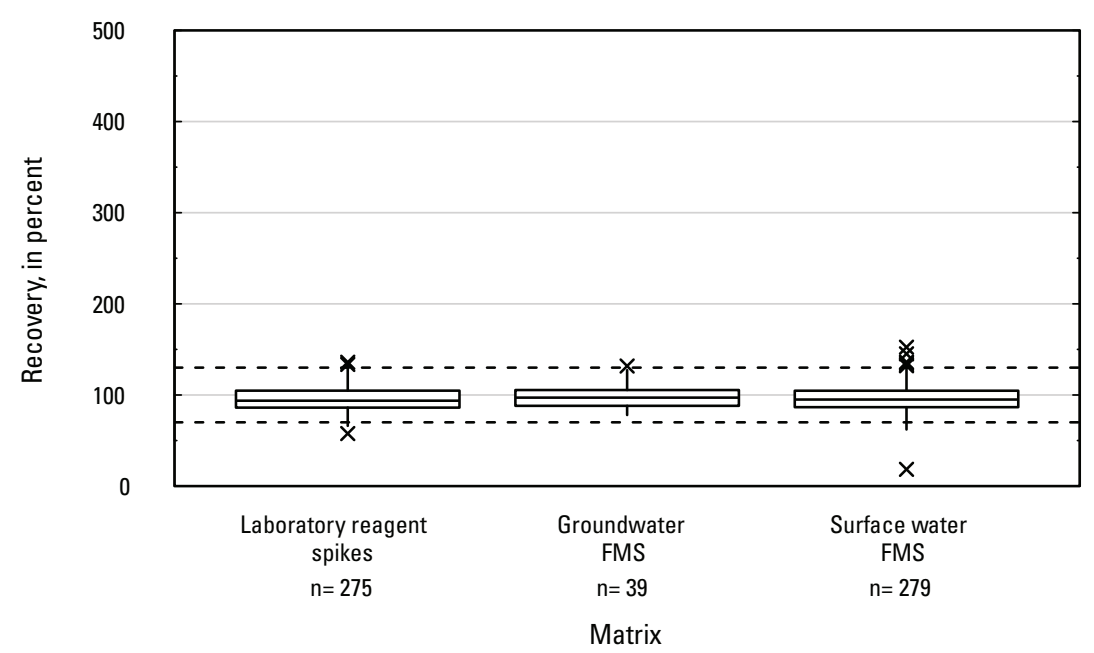

BJ. Deisopropyl prometryn

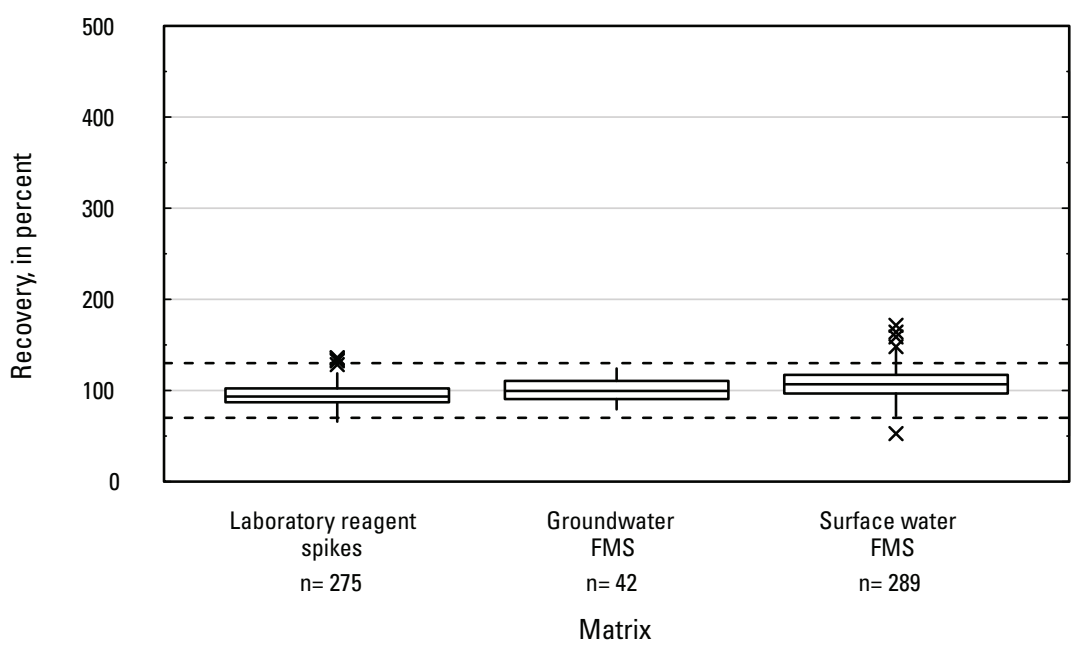

BL. Demethyl fluometuron

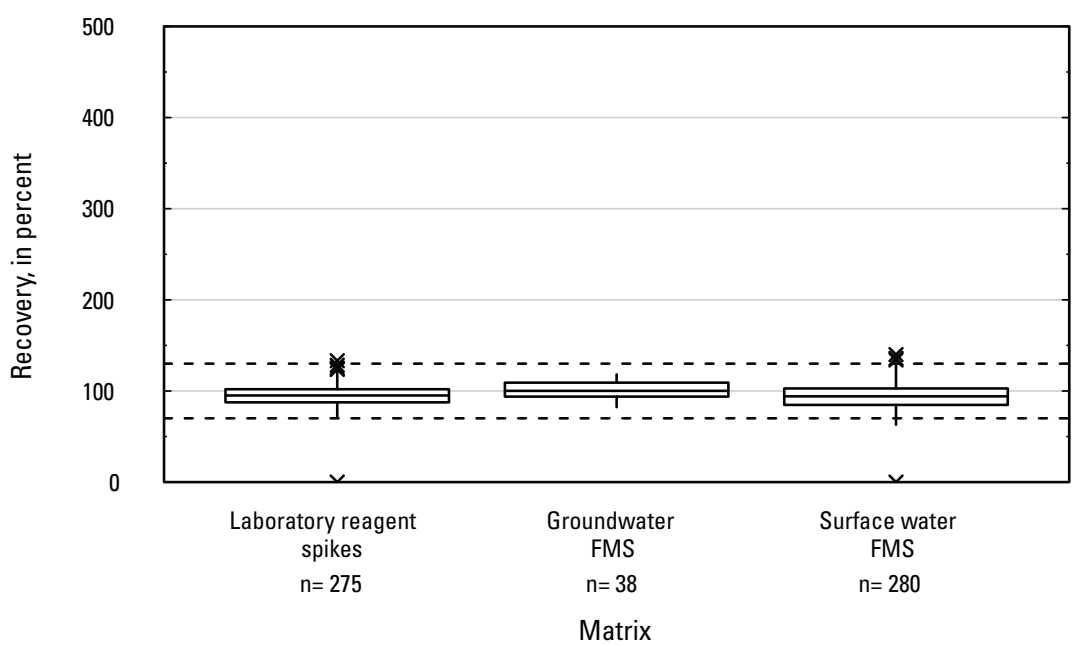

ज़

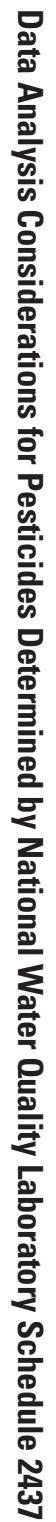

Figure 4. Graph showing distributions of recovery for pesticides in schedule 2437 by matrix. Dashed lines are at 130 percent and 70 percent. Recovery values larger than 400 percent are not shown.-Continued 
BM. Demethyl hexazinone B

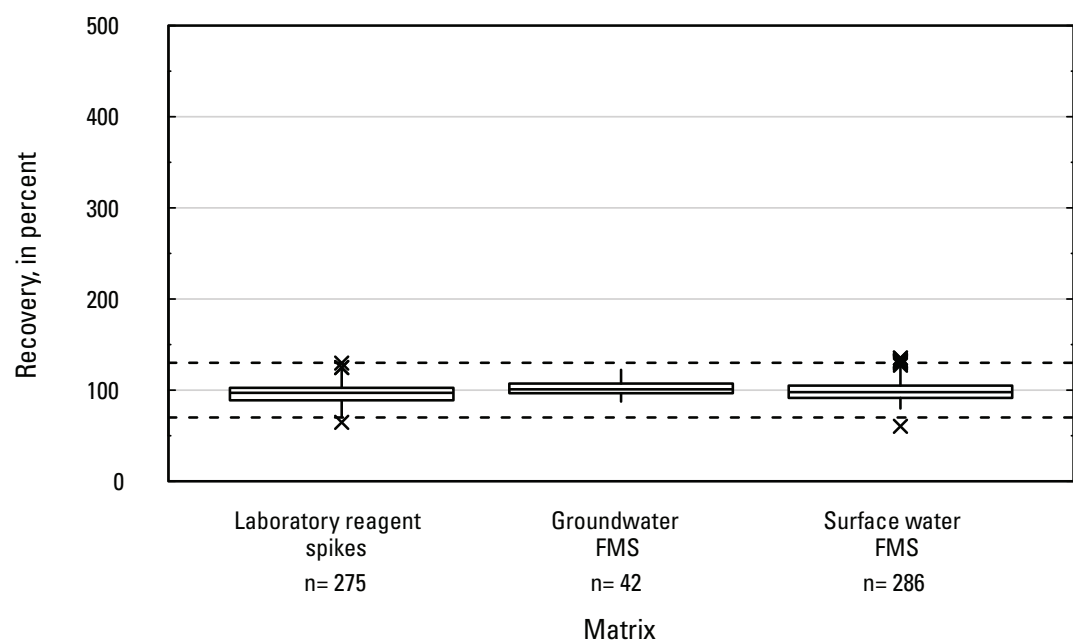

BO. Deiodo flubendiamide

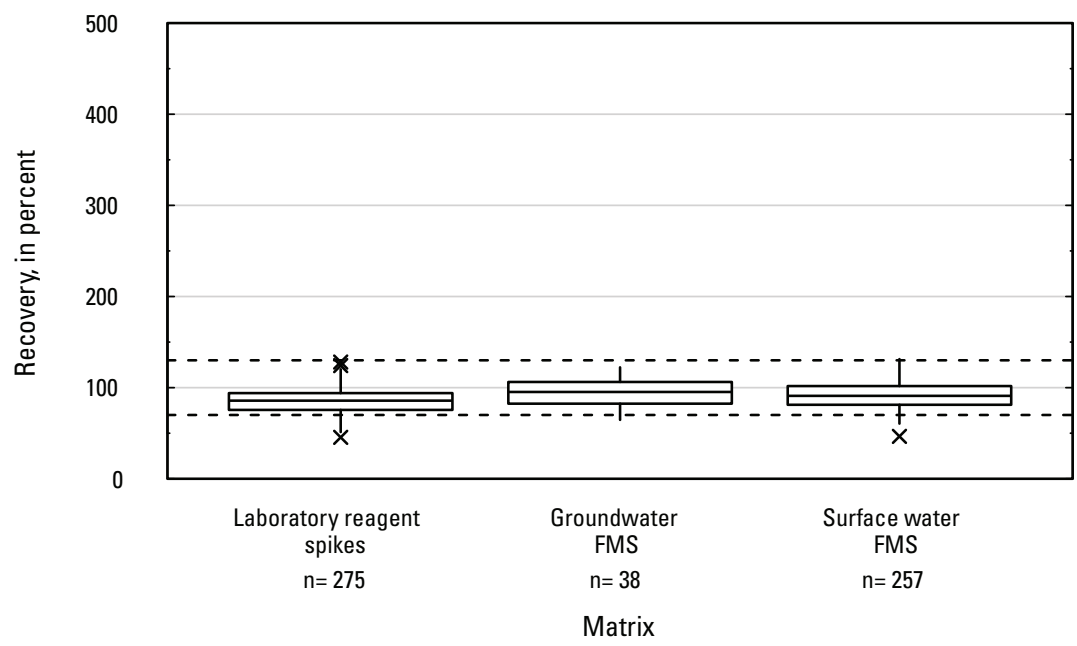

$B N$. Demethyl norflurazon

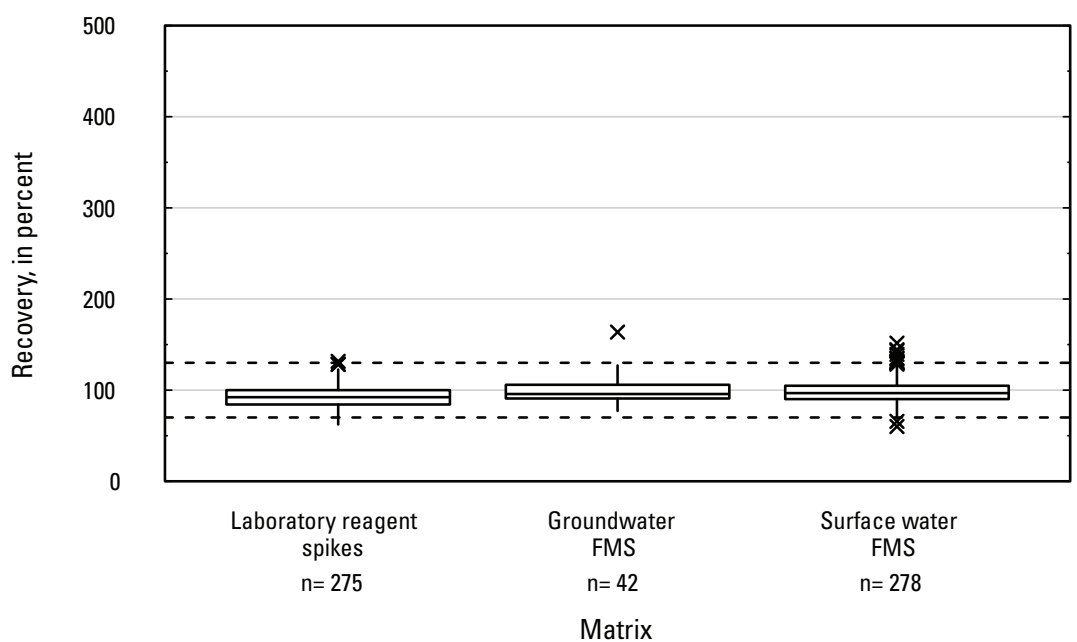

BP. Desulfinylfipronil

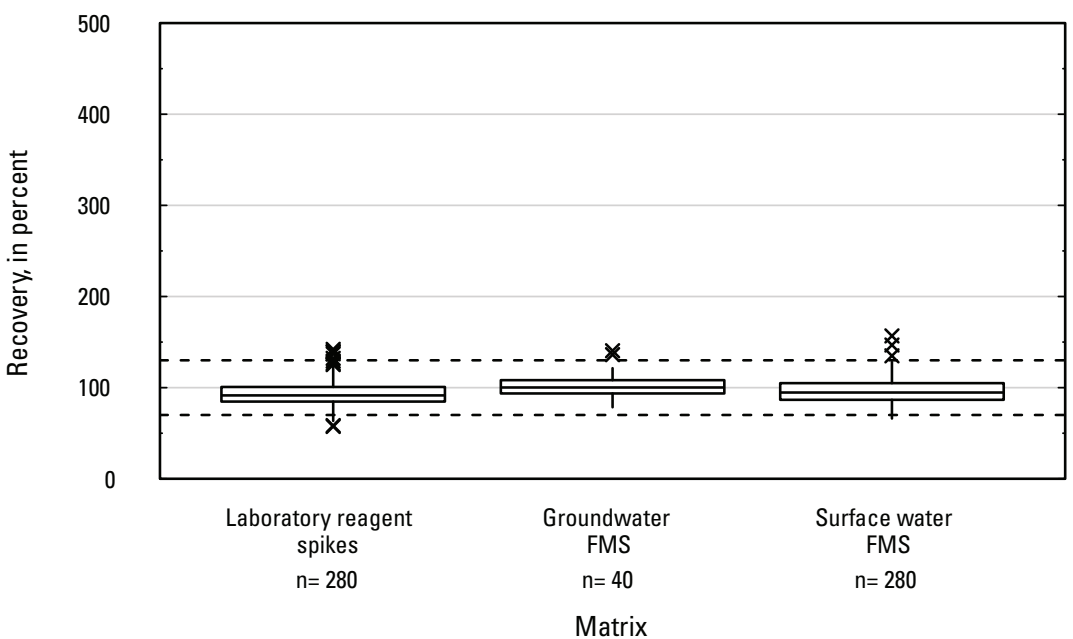

Figure 4. Graph showing distributions of recovery for pesticides in schedule 2437 by matrix. Dashed lines are at 130 percent and 70 percent. Recovery values larger than 400 percent are not shown.-Continued 
BO. Desulfinylfipronil amide

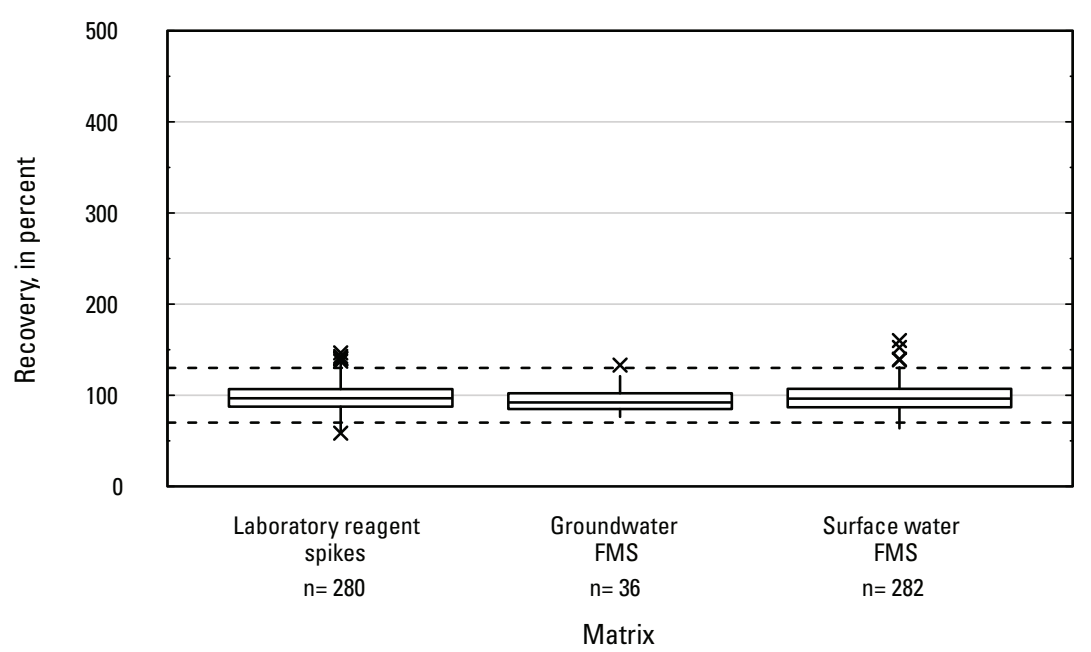

BS. Diazinon oxon

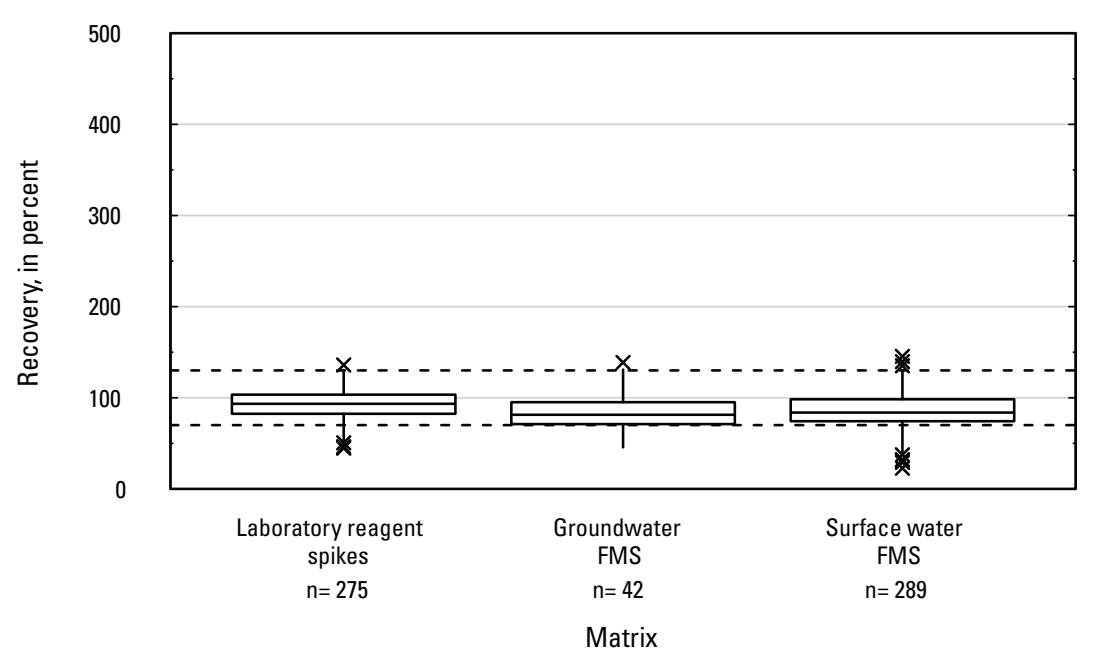

BR. Diazinon

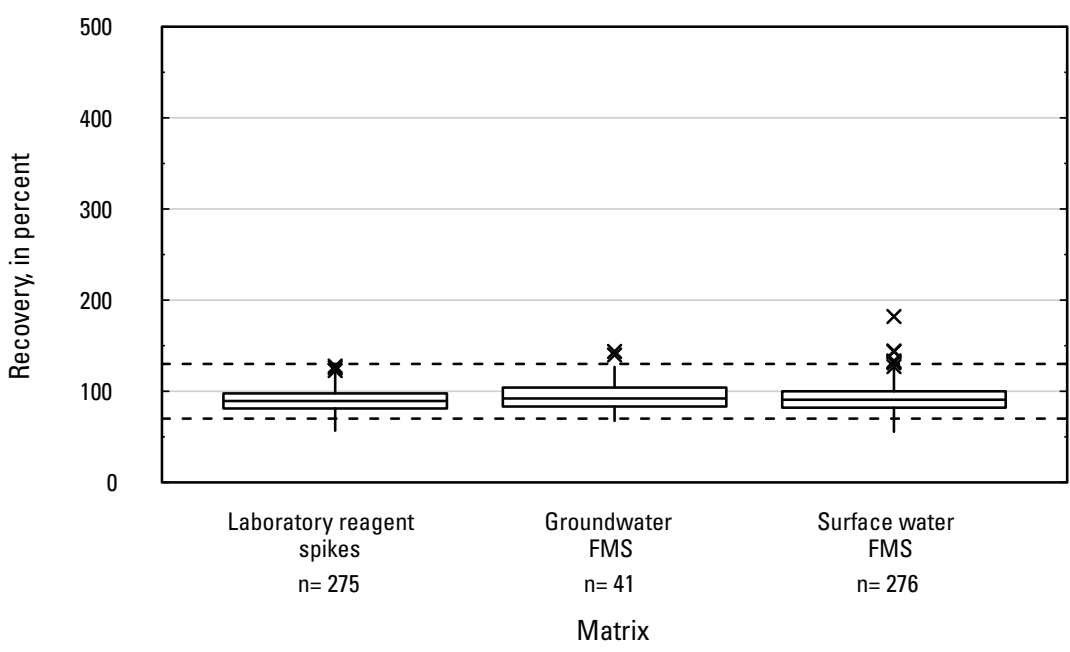

BT. Dicamba

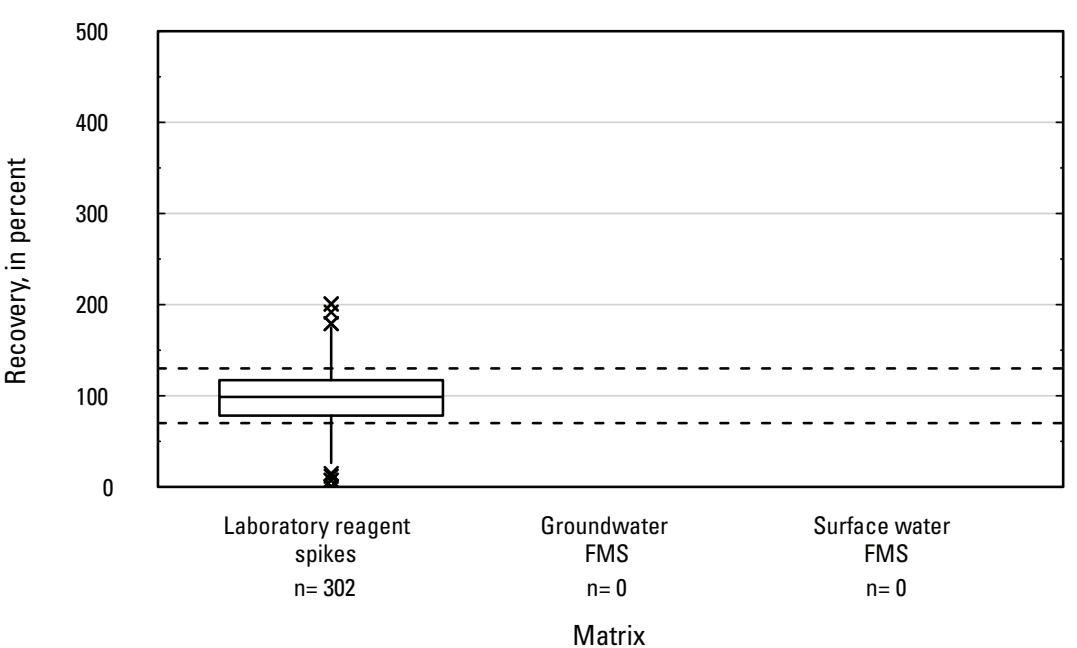

G

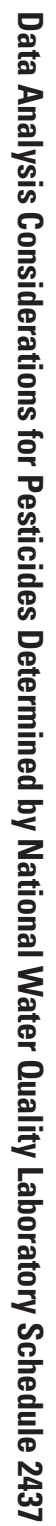

Figure 4. Graph showing distributions of recovery for pesticides in schedule 2437 by matrix. Dashed lines are at 130 percent and 70 percent. Recovery values larger than 400 percent are not shown.-Continued 
$B U$. Dichlorvos

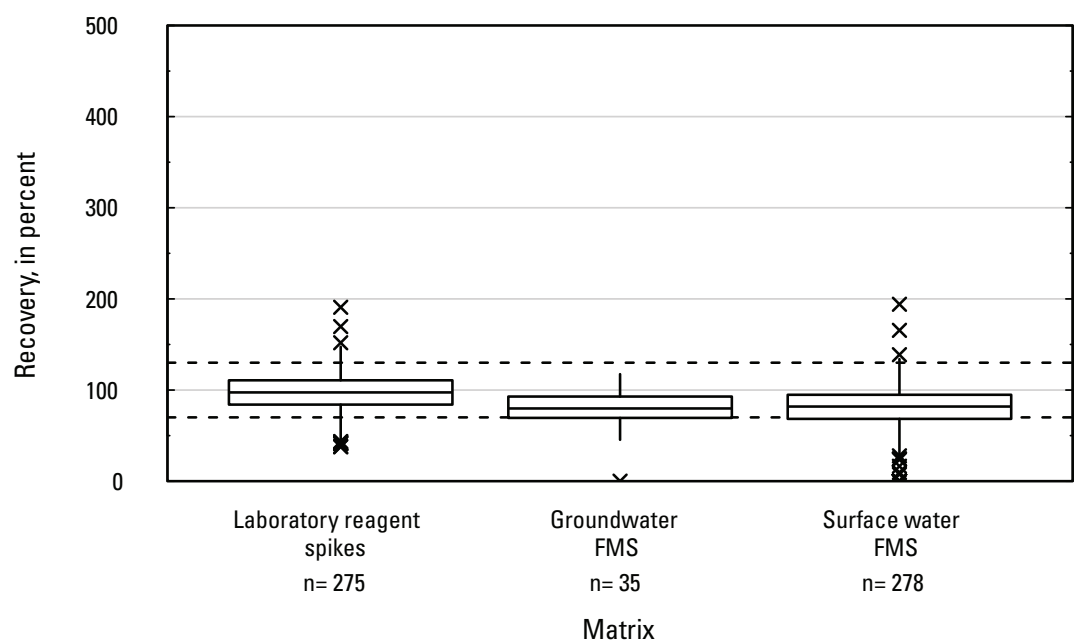

BW. 2-Chloro-4,6-diamino-s-triazine \{CAAT\} (Didealkylatrazine)

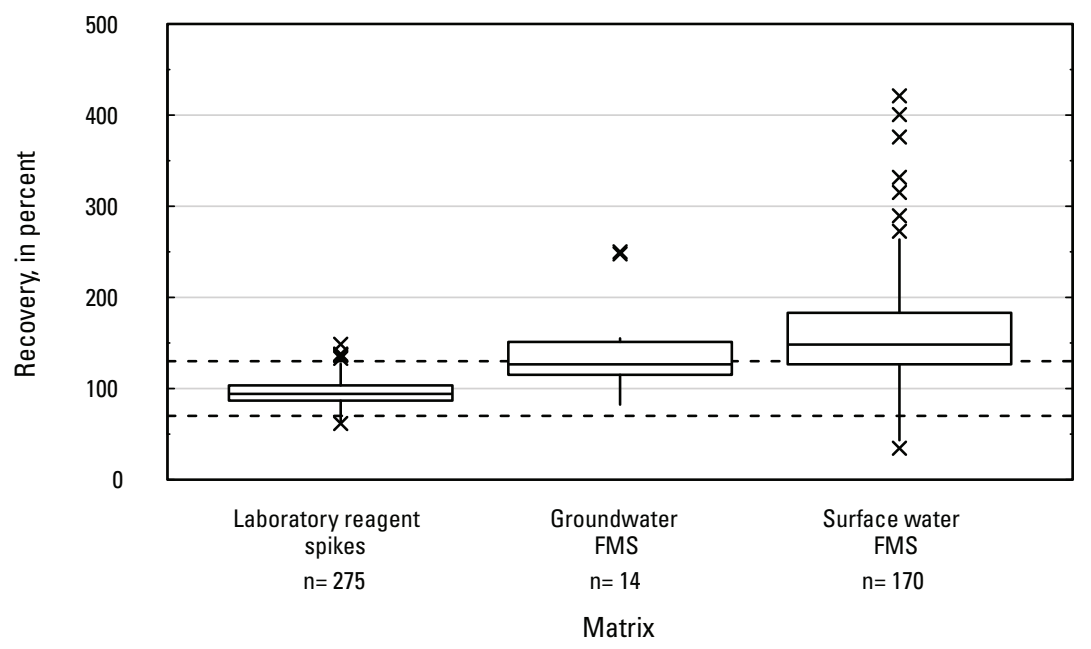

BV. Dicrotophos

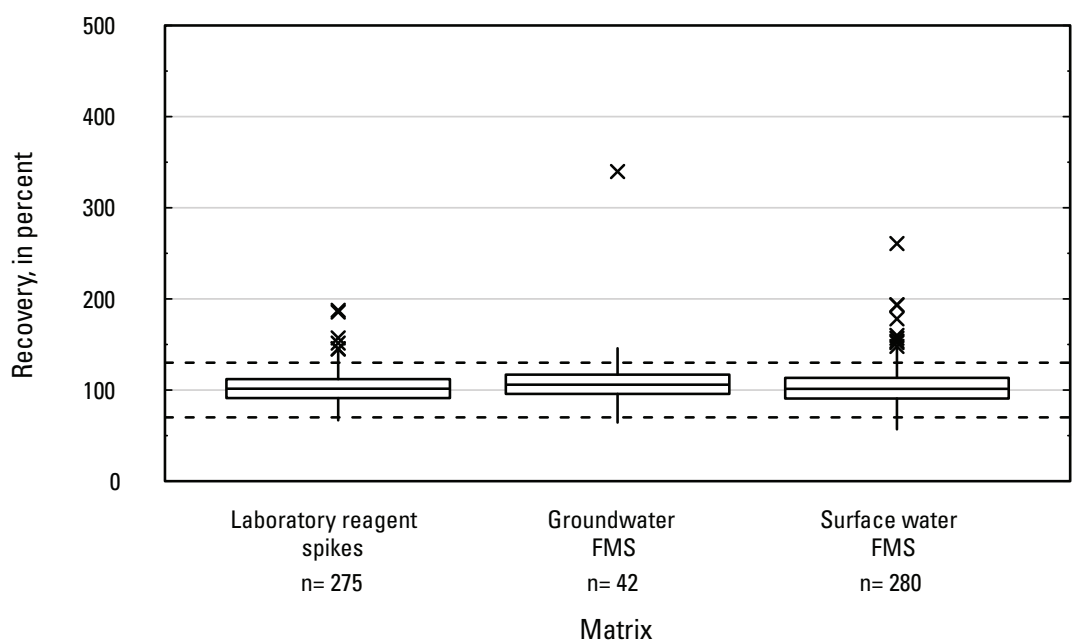

$B X$. Diflubenzuron

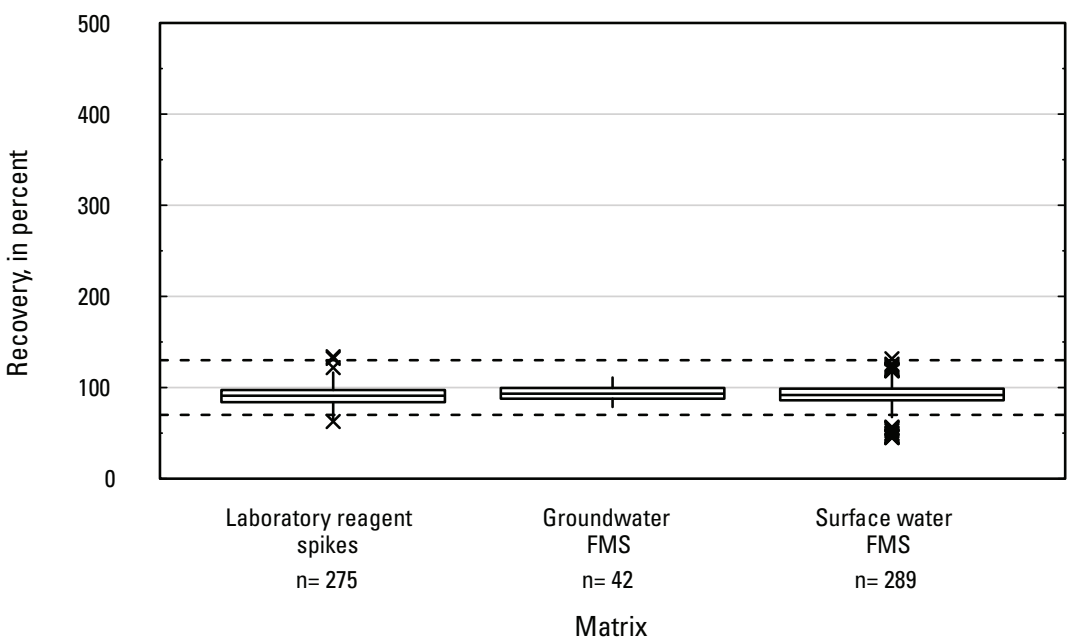

Figure 4. Graph showing distributions of recovery for pesticides in schedule 2437 by matrix. Dashed lines are at 130 percent and 70 percent. Recovery values larger than 400 percent are not shown.-Continued 
BY. Diflufenzopyr

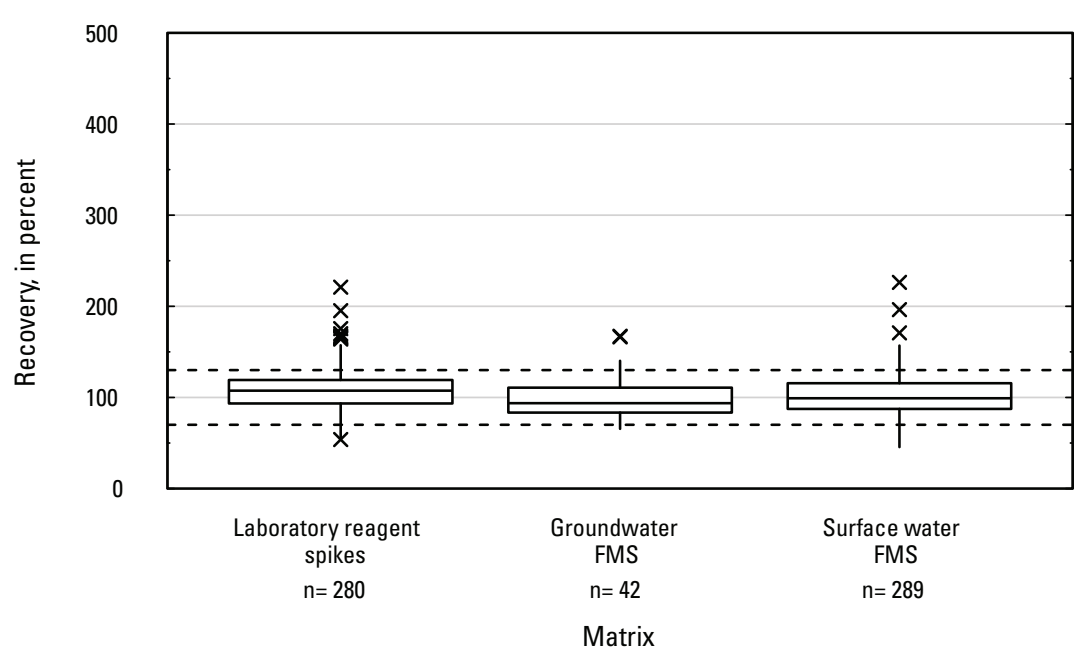

CA. Dimethenamid

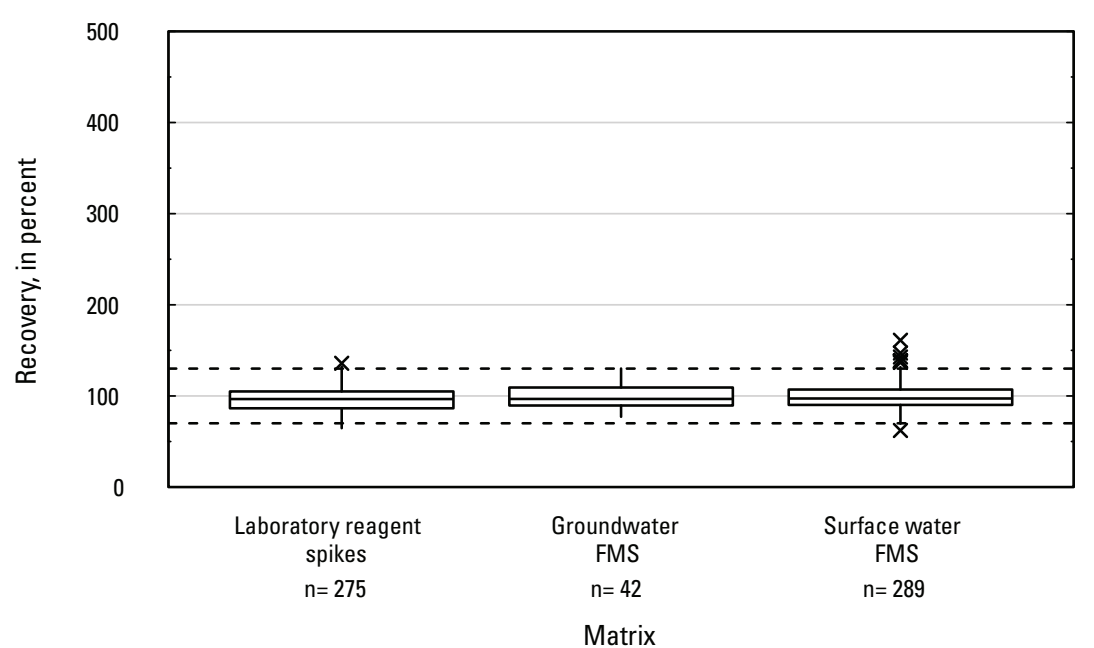

BZ. Diketonitrile-isoxaflutole

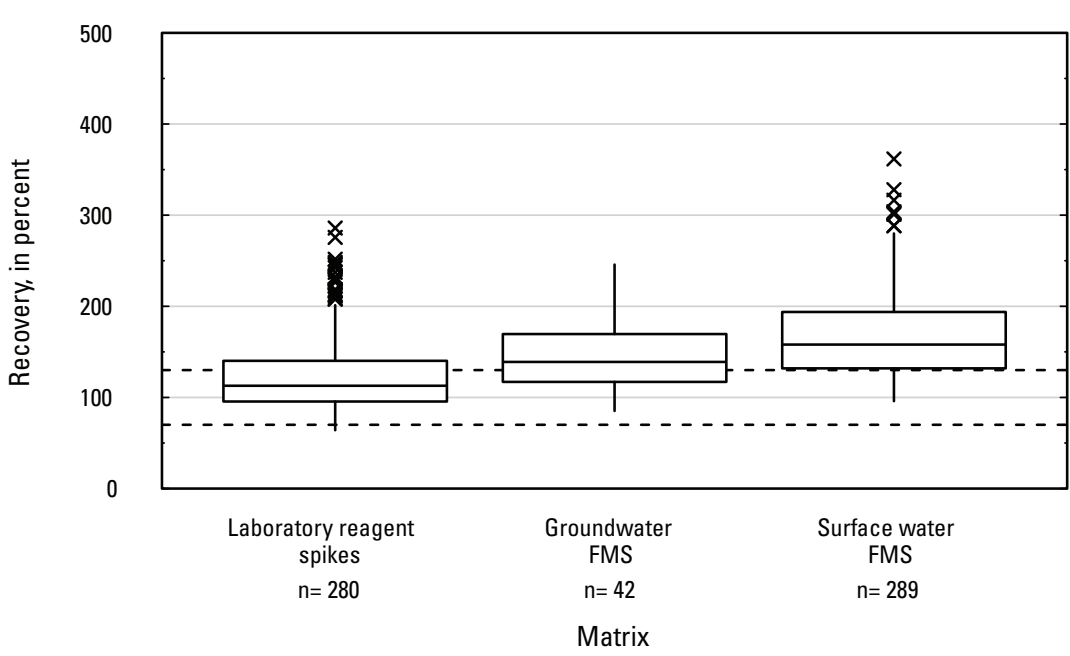

CB. Dimethenamid oxanilic acid

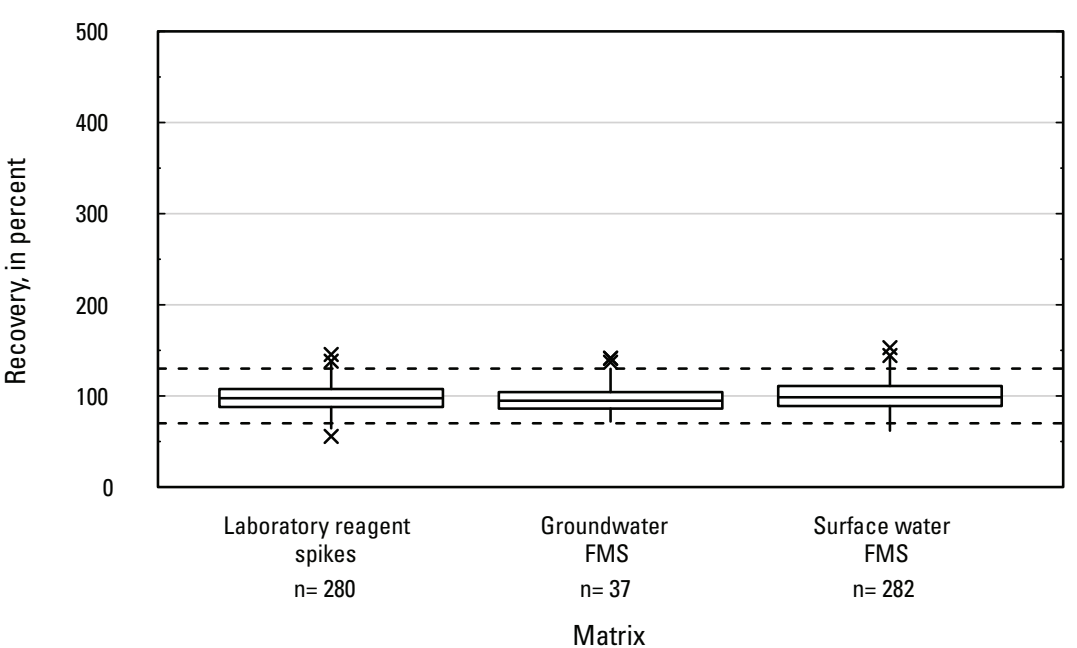

G

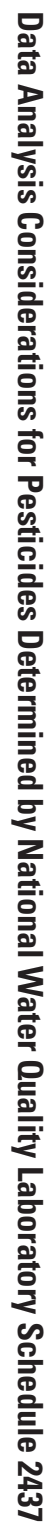

Figure 4. Graph showing distributions of recovery for pesticides in schedule 2437 by matrix. Dashed lines are at 130 percent and 70 percent. Recovery values larger than 400 percent are not shown.-Continued 
CC. Dimethenamid sulfonic acid

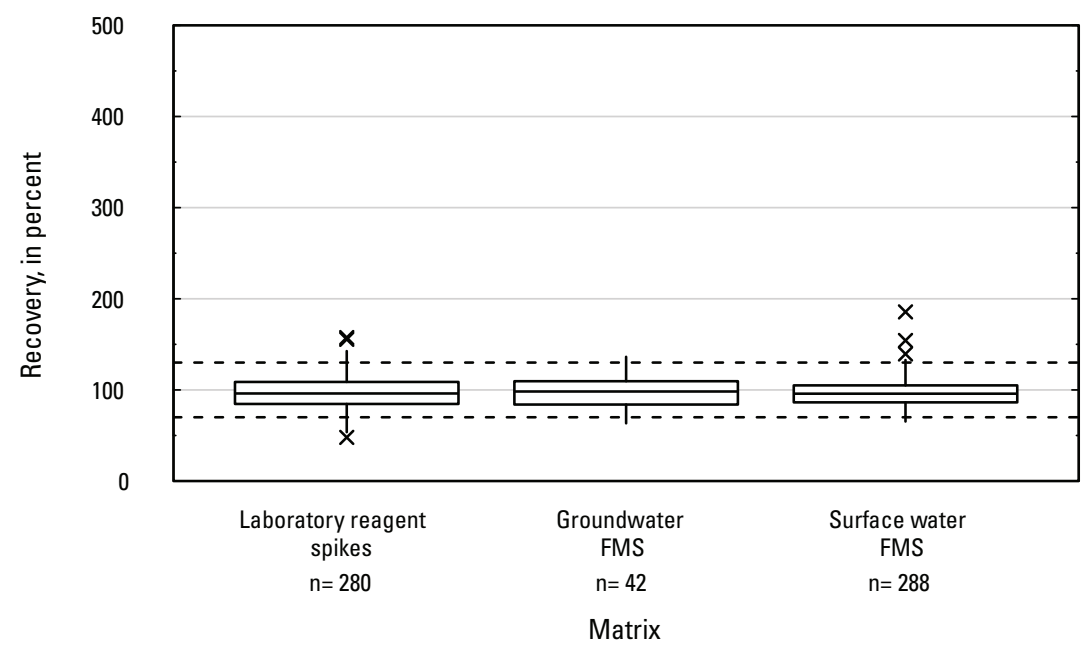

CE. Dimethoate

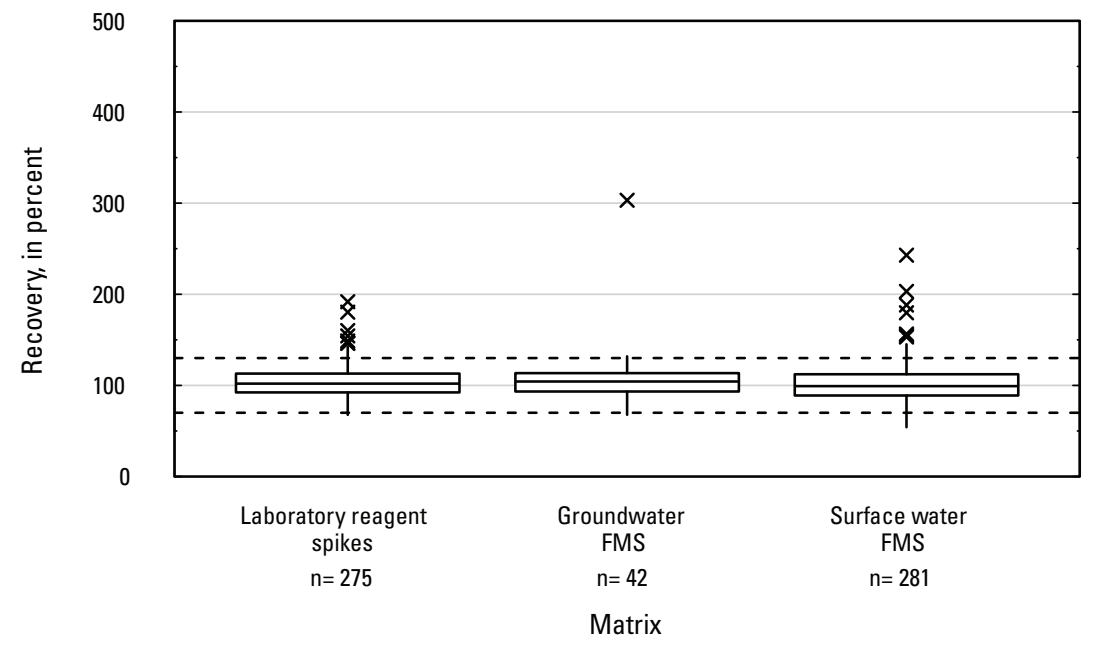

CD. Dimethenamid SAA

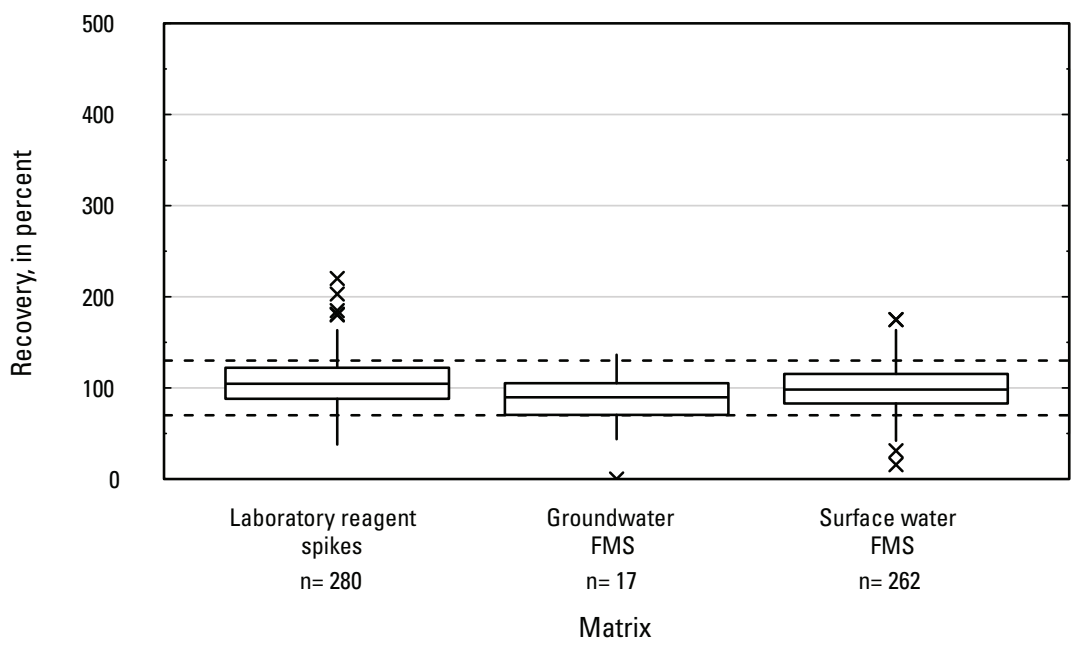

CF. Omethoate (Dimethoate oxon)

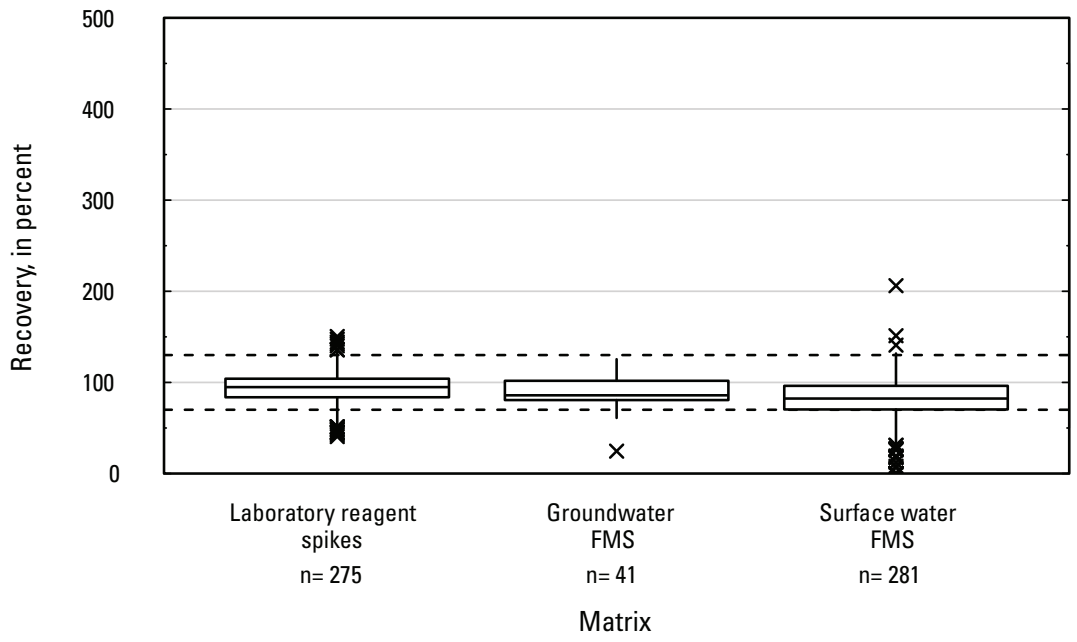

Figure 4. Graph showing distributions of recovery for pesticides in schedule 2437 by matrix. Dashed lines are at 130 percent and 70 percent. Recovery values larger than 400 percent are not shown.-Continued 
CG. Disulfoton

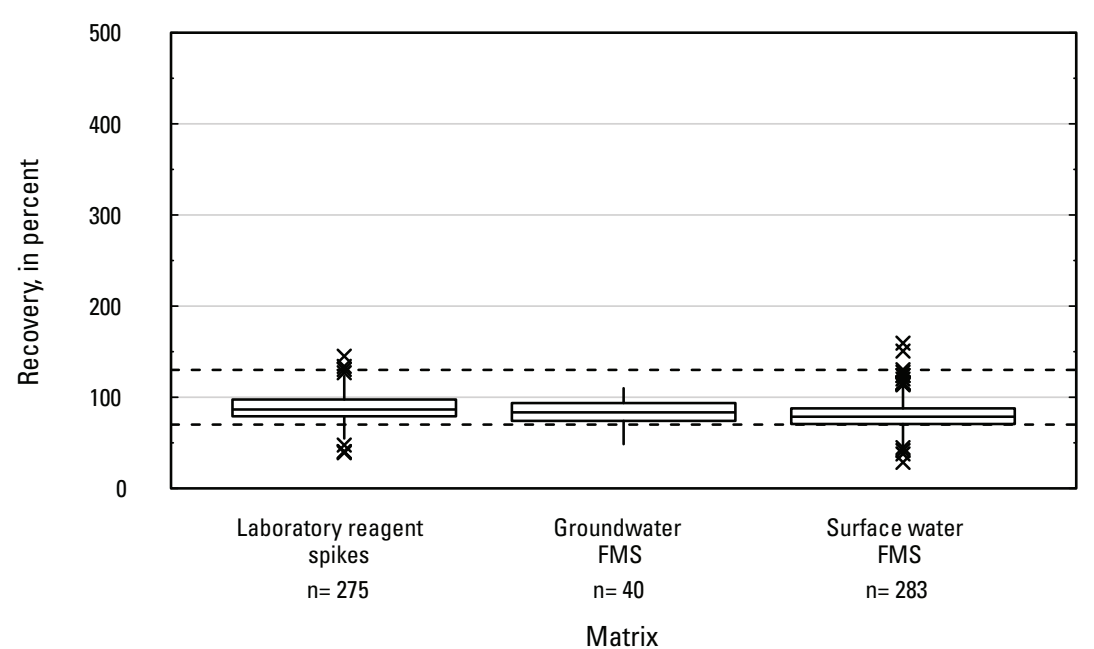

CI. Disulfoton oxon sulfone

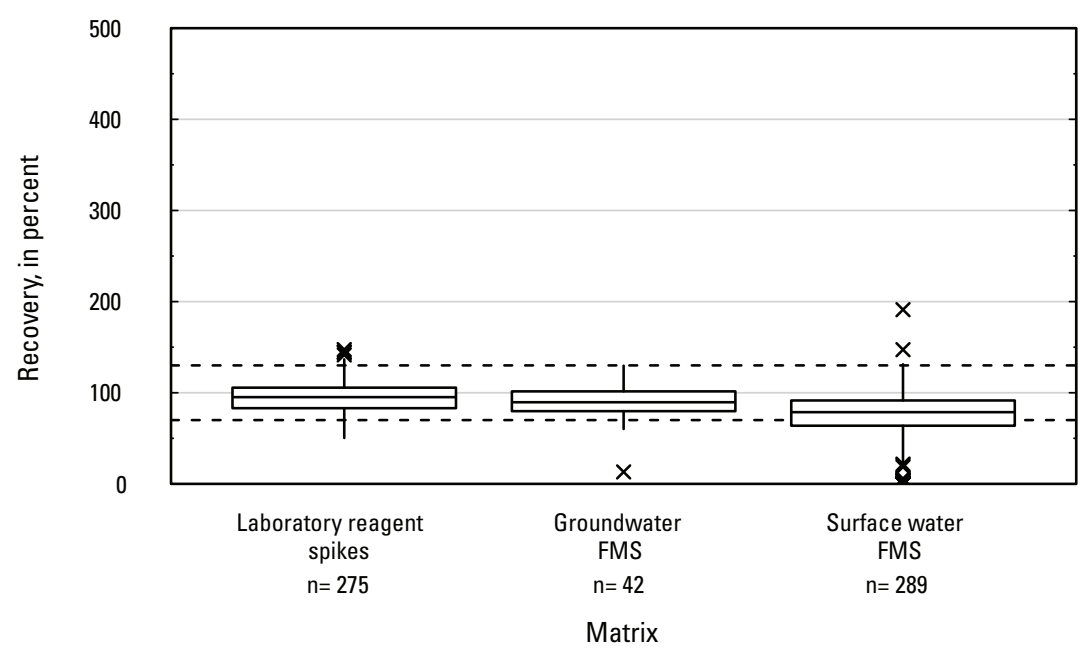

CH. Disulfoton oxon

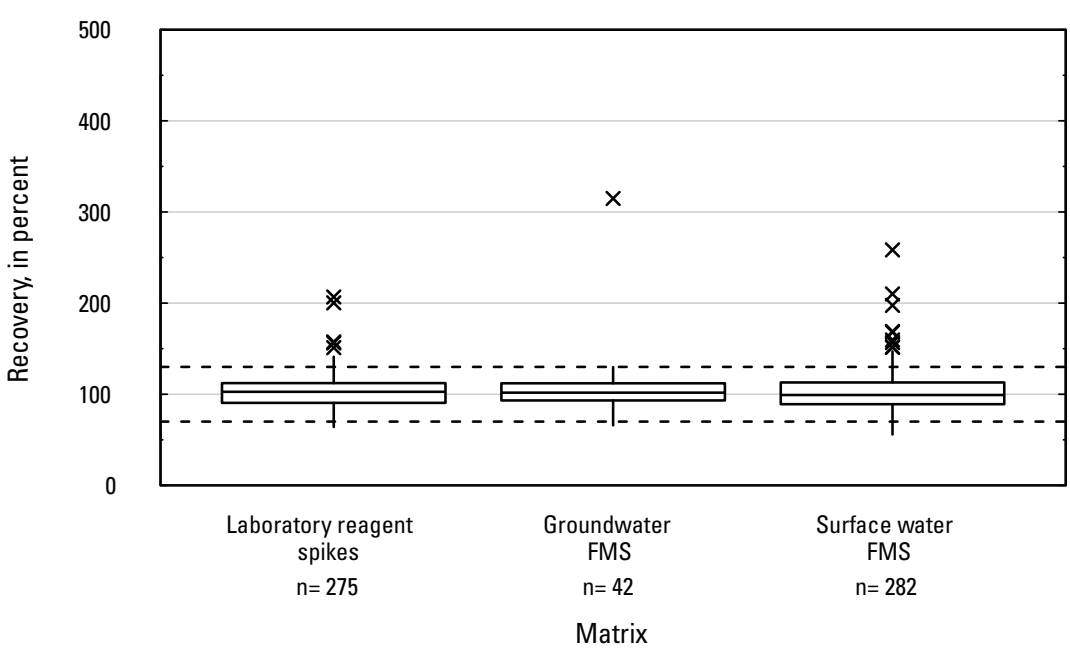

CJ. Disulfoton oxon sulfoxide

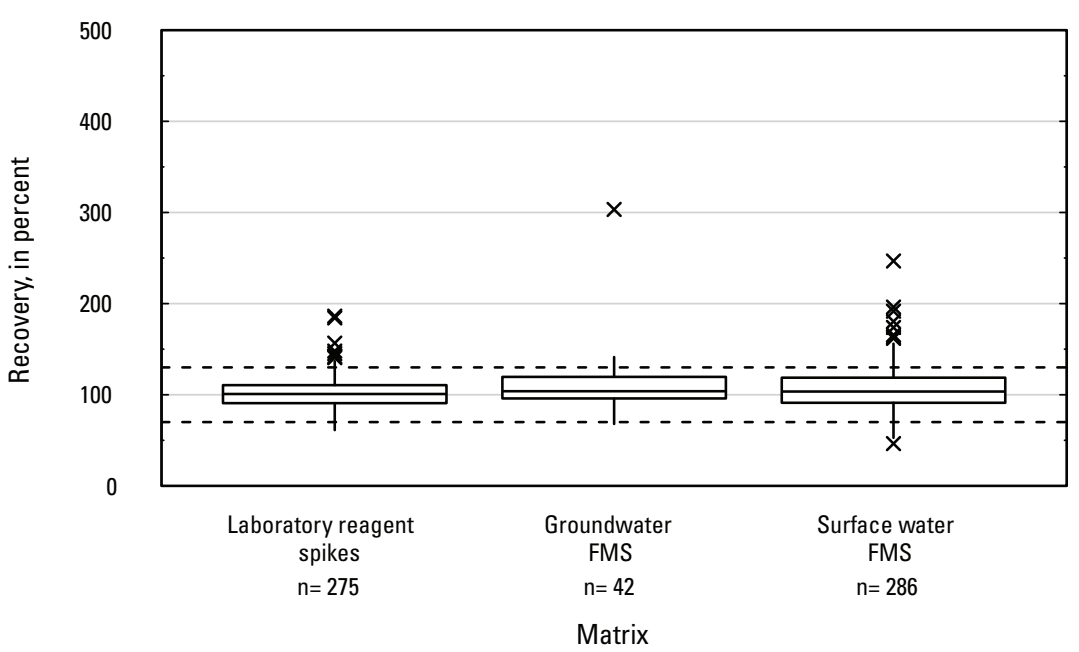

్

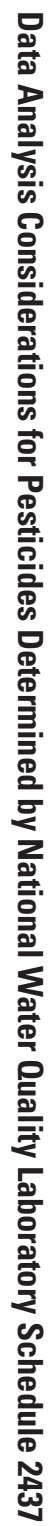

Figure 4. Graph showing distributions of recovery for pesticides in schedule 2437 by matrix. Dashed lines are at 130 percent and 70 percent. Recovery values larger than 400 percent are not shown.-Continued 
CK. Disulfoton sulfone

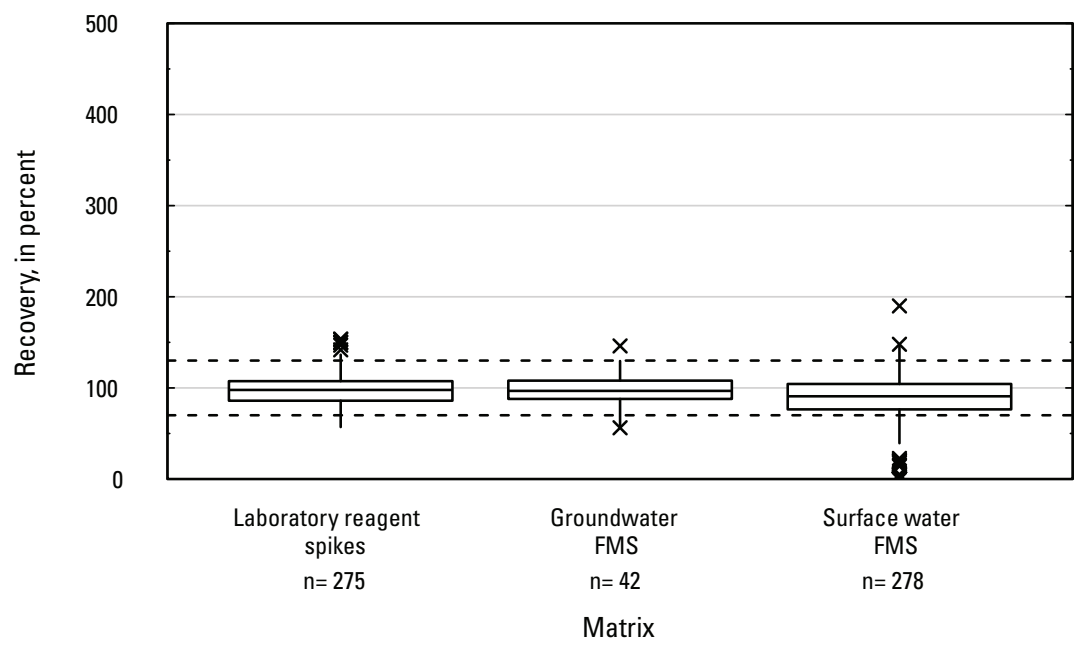

CM. Diuron

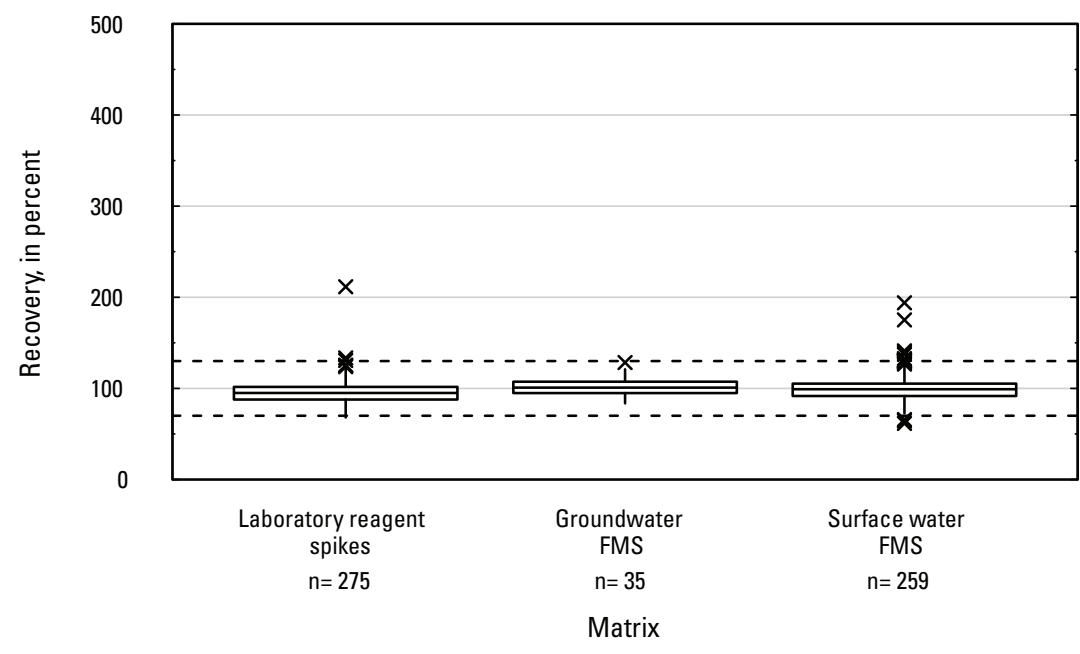

\section{Disulfoton sulfoxide}

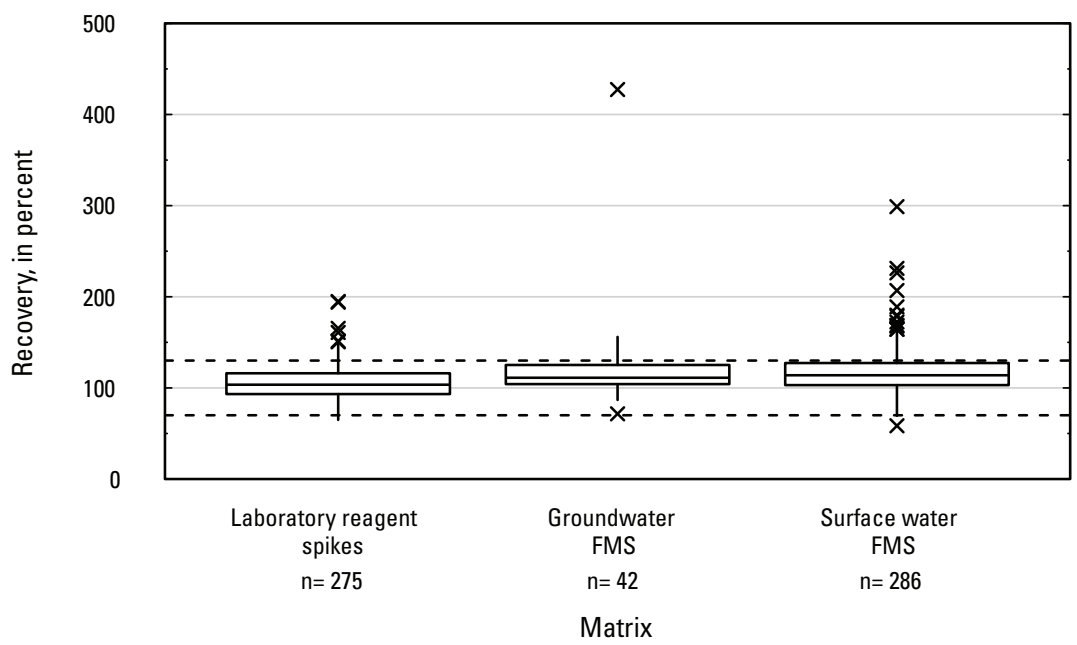

CN. EPTC

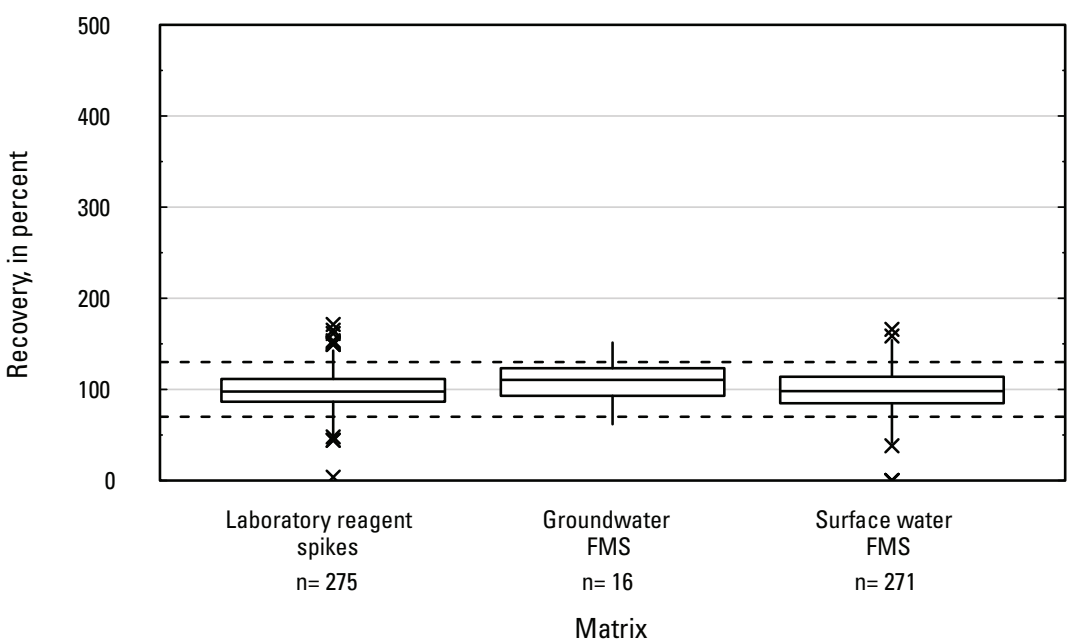

Figure 4. Graph showing distributions of recovery for pesticides in schedule 2437 by matrix. Dashed lines are at 130 percent and 70 percent. Recovery values larger than 400 percent are not shown.-Continued 
CO. EPTC degradate R248722

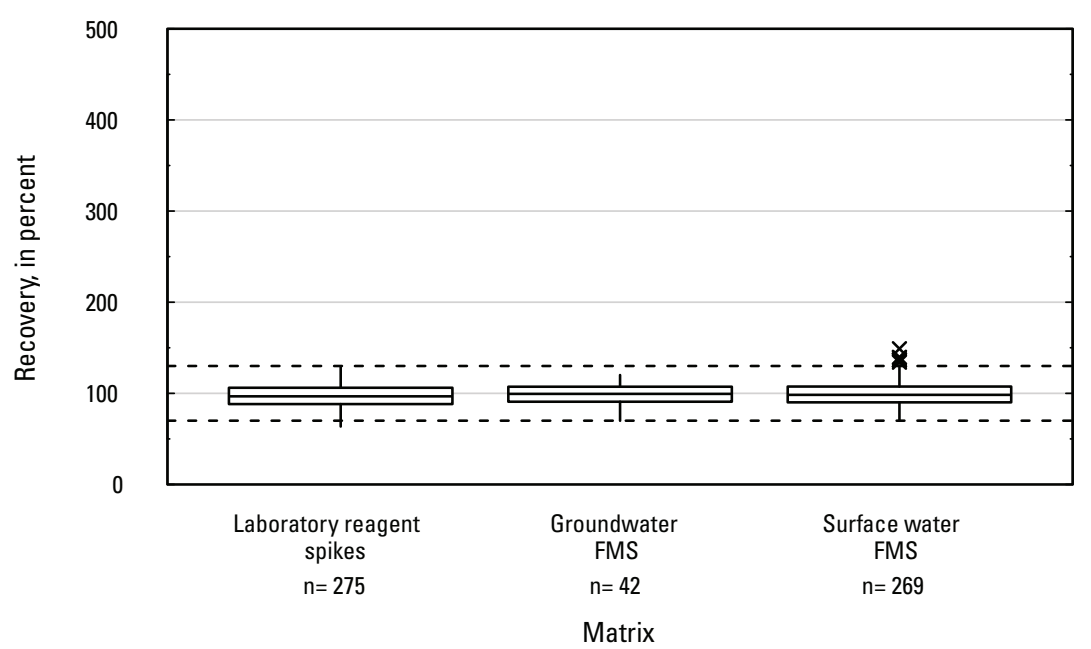

CQ. Etoxazole

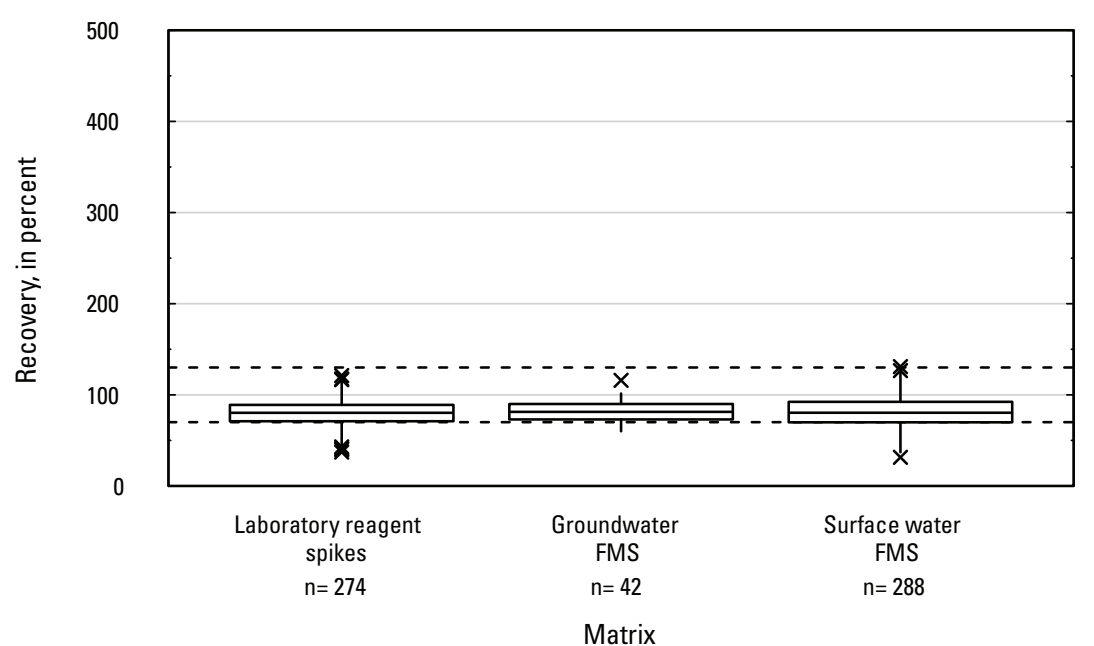

CP. Ethoprophos

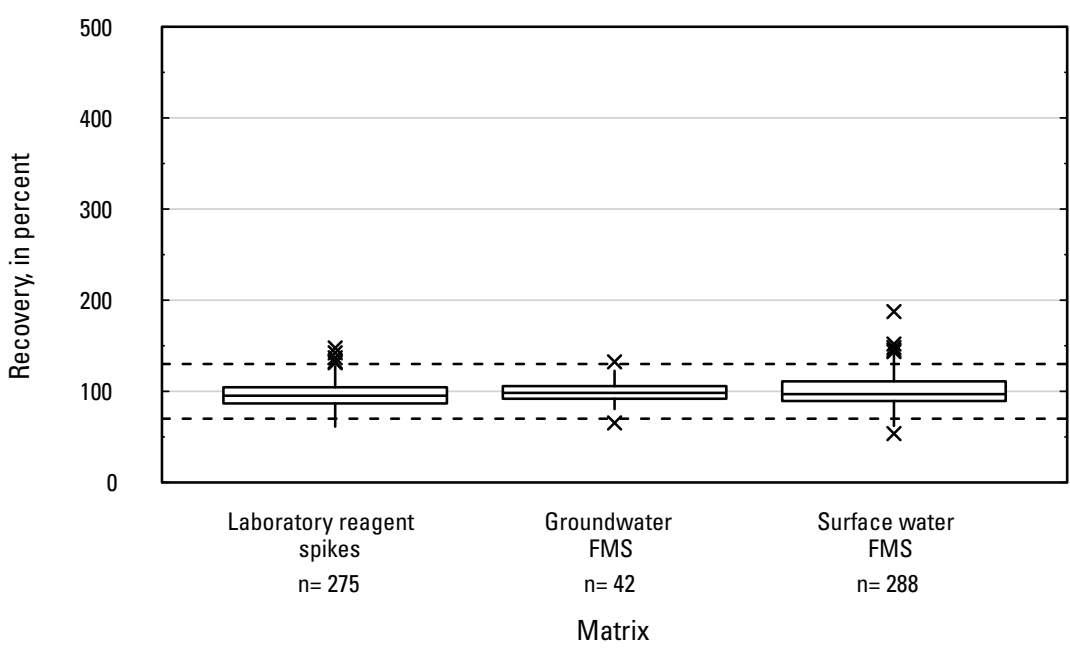

CR. Fenamiphos

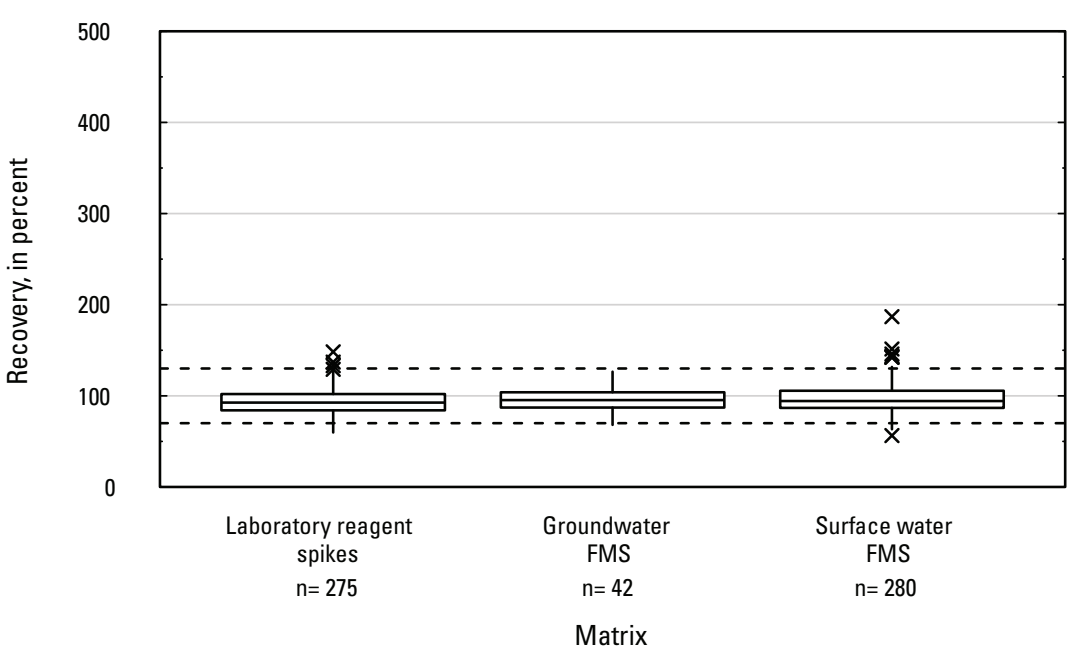

앙

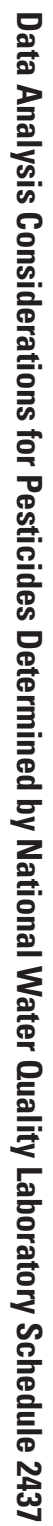

Figure 4. Graph showing distributions of recovery for pesticides in schedule 2437 by matrix. Dashed lines are at 130 percent and 70 percent. Recovery values larger than 400 percent are not shown.-Continued 
CS. Fenamiphos sulfone

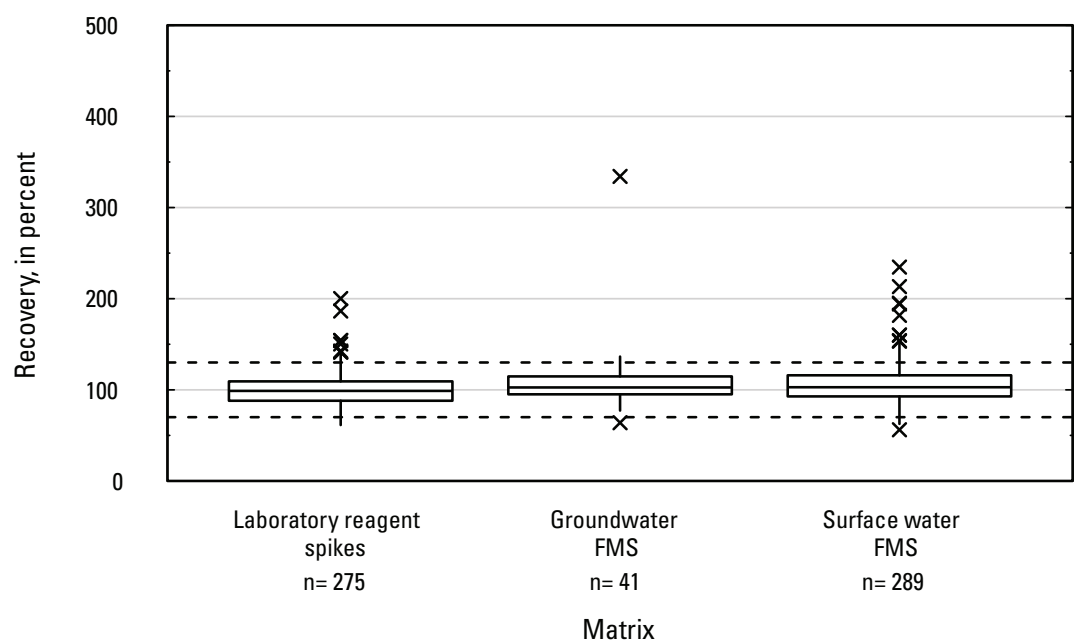

$C U$. Fenbutatin oxide

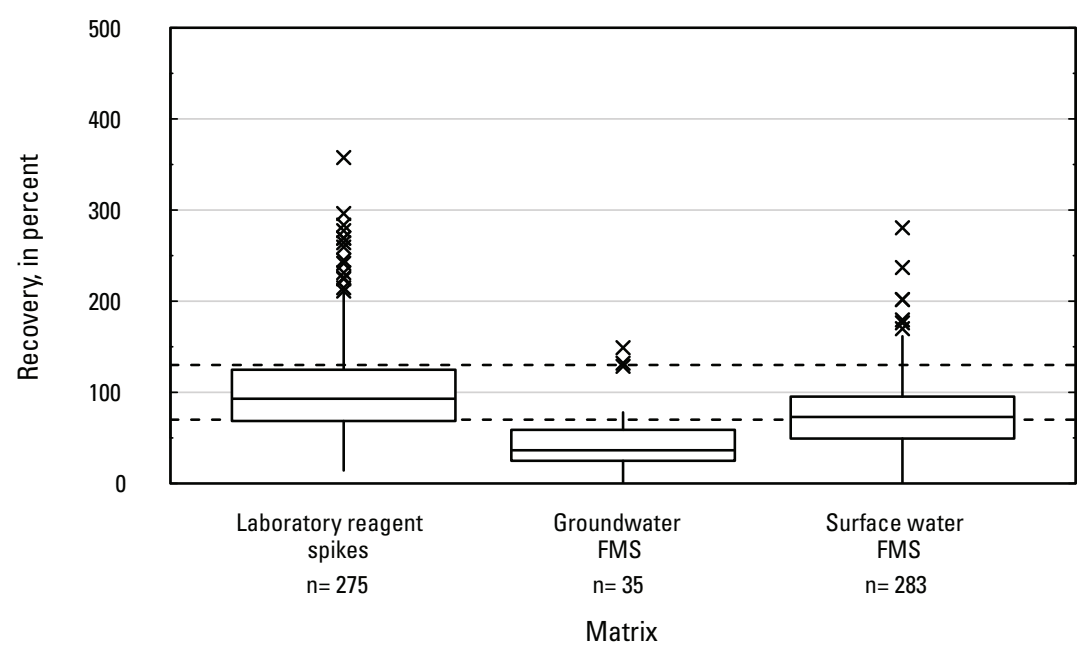

CT. Fenamiphos sulfoxide

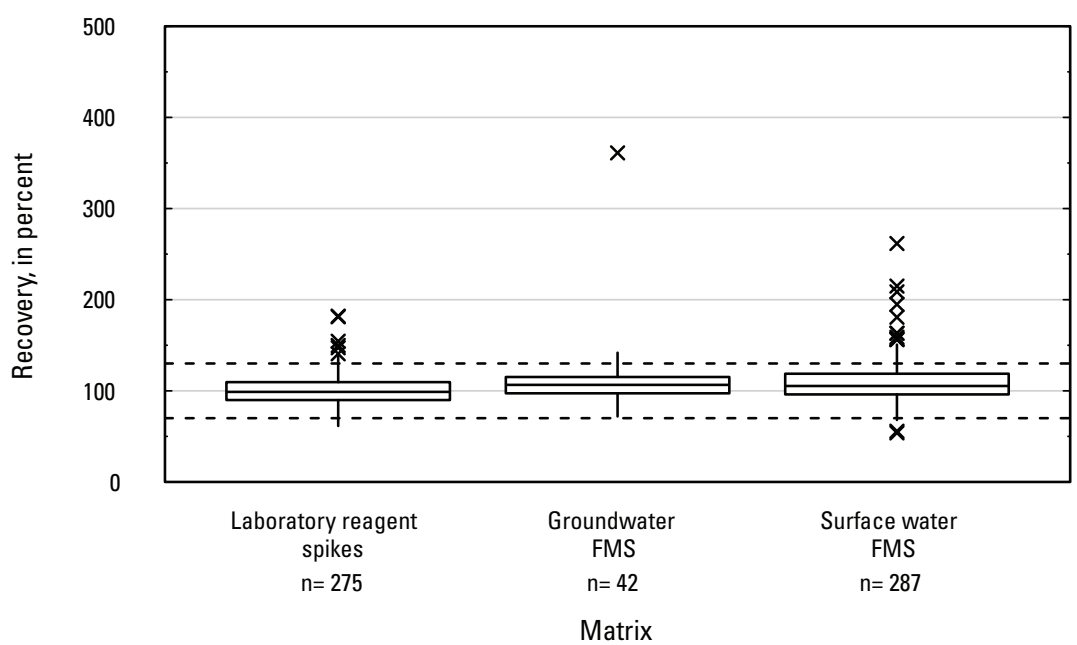

CV. Fentin

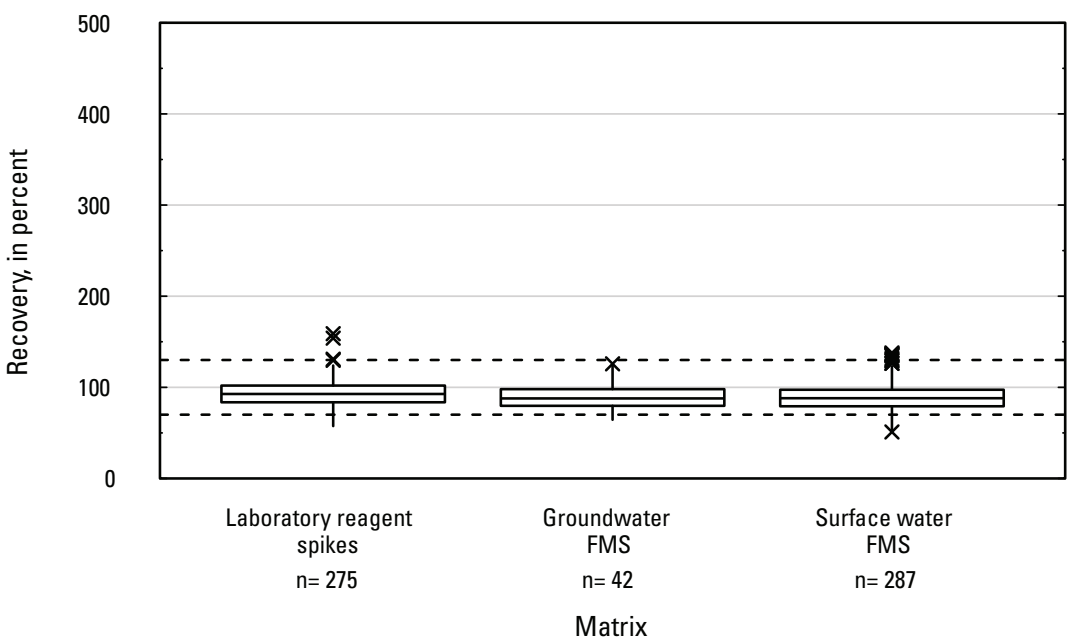

Figure 4. Graph showing distributions of recovery for pesticides in schedule 2437 by matrix. Dashed lines are at 130 percent and 70 percent. Recovery values larger than 400 percent are not shown.-Continued 
CW. Fipronil

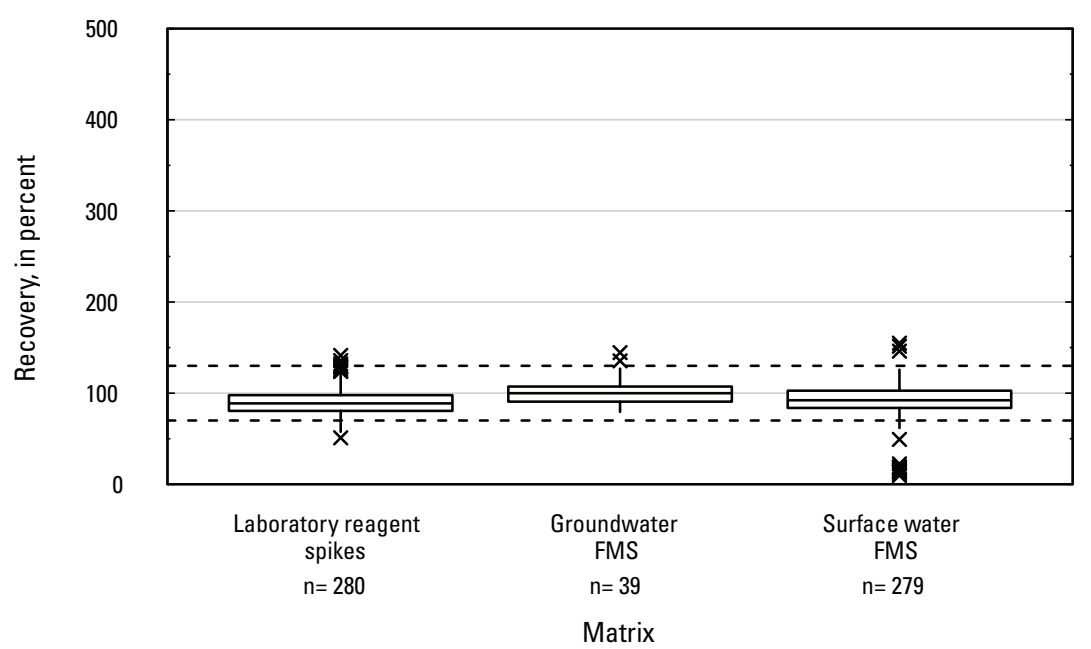

CY. Fipronil sulfide

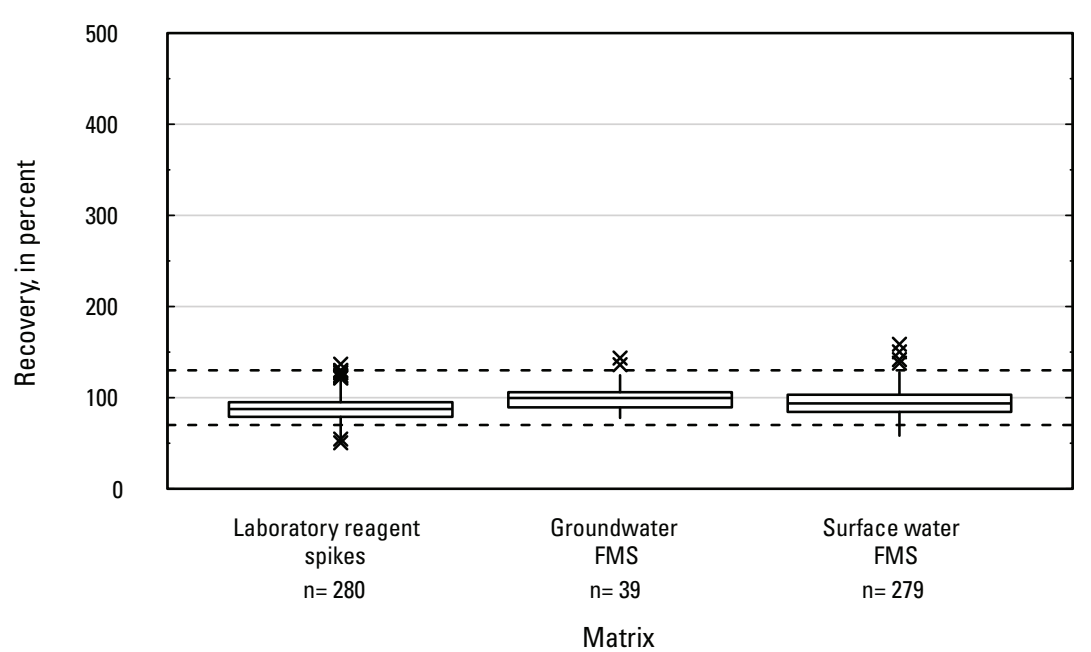

CX. Fipronil amide

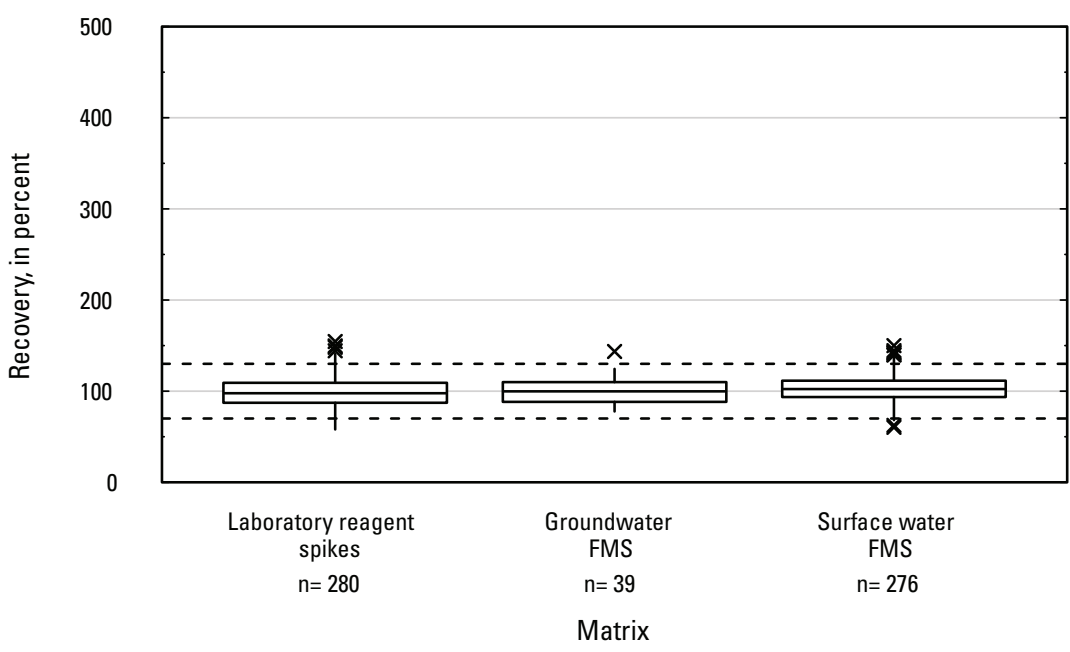

CZ. Fipronil sulfonate

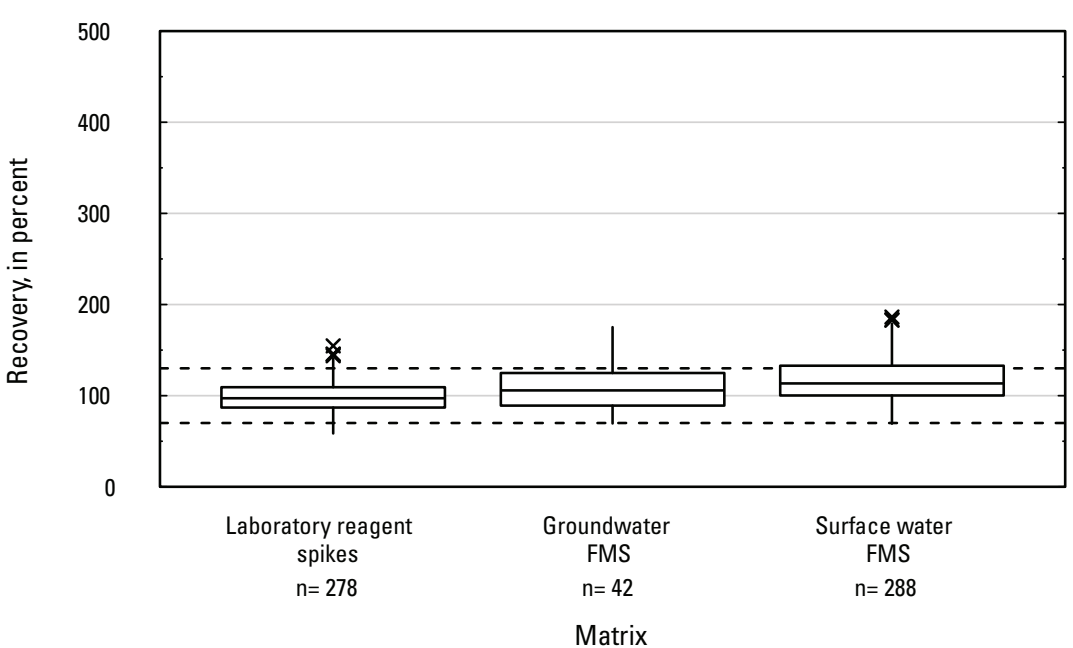

오

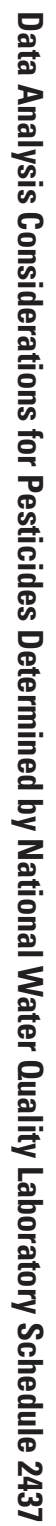

Figure 4. Graph showing distributions of recovery for pesticides in schedule 2437 by matrix. Dashed lines are at 130 percent and 70 percent. Recovery values larger than 400 percent are not shown.-Continued 
$D A$. Fipronil sulfone

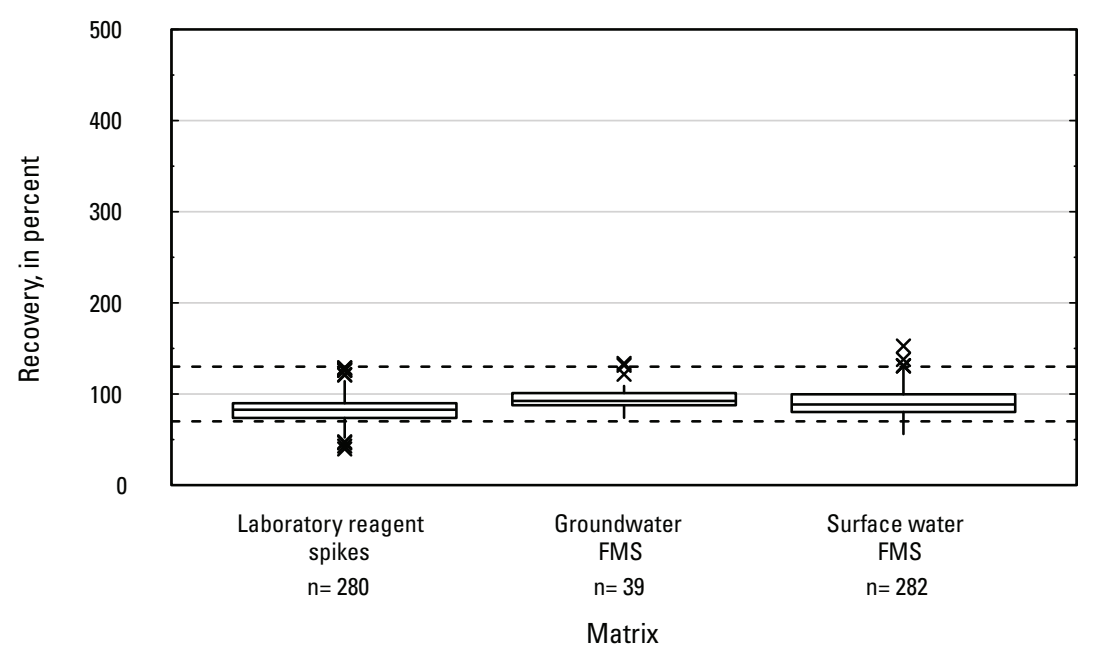

DC. Flumetsulam

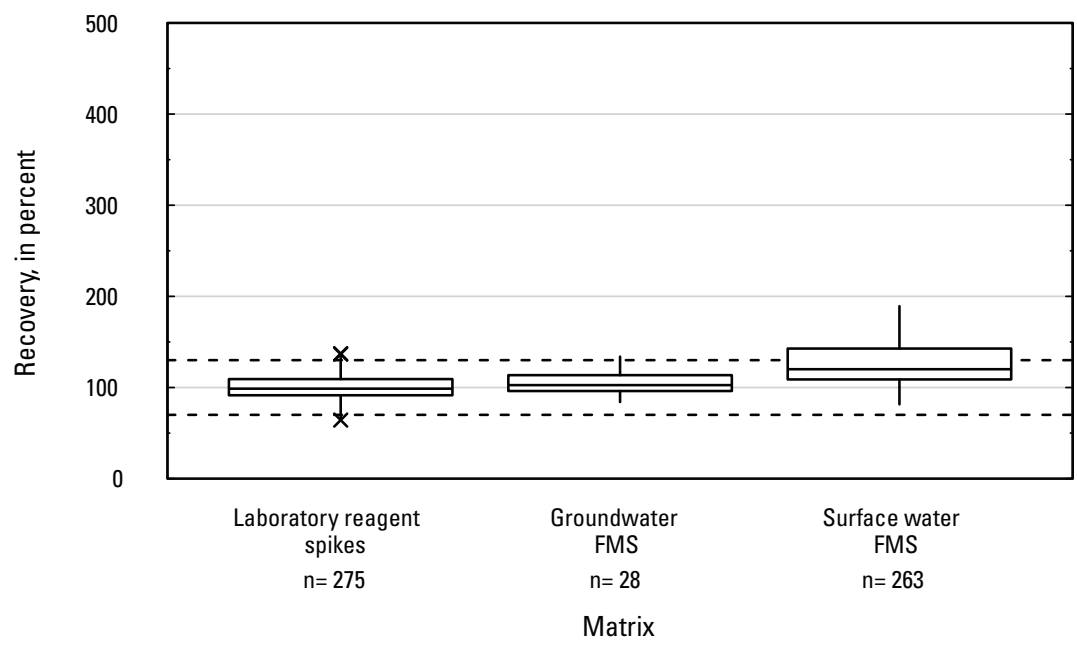

$D B$. Flubendiamide

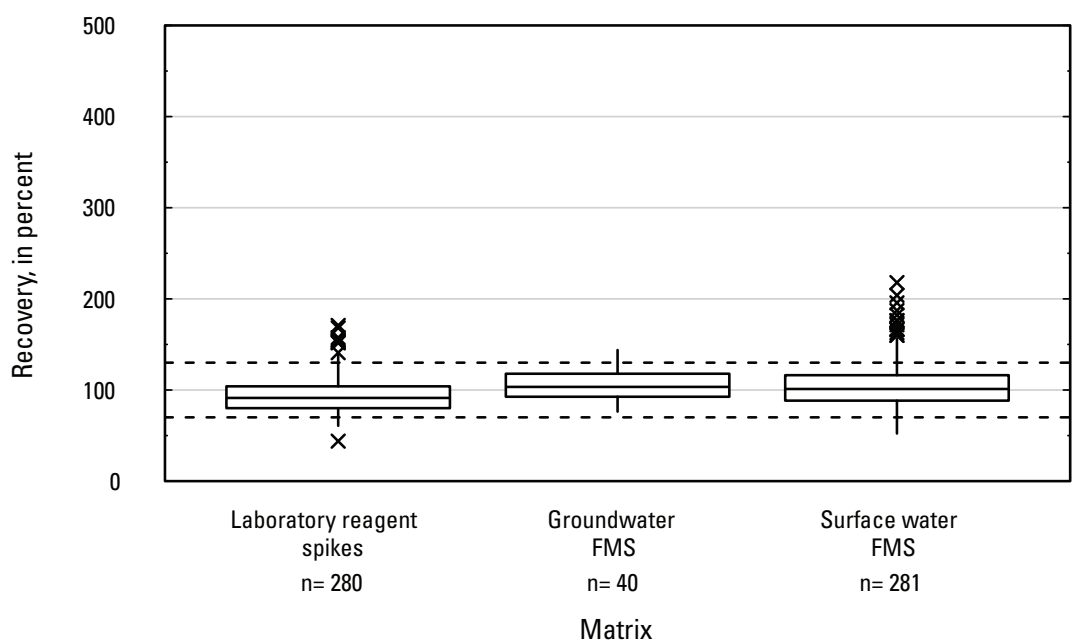

DD. Fluometuron

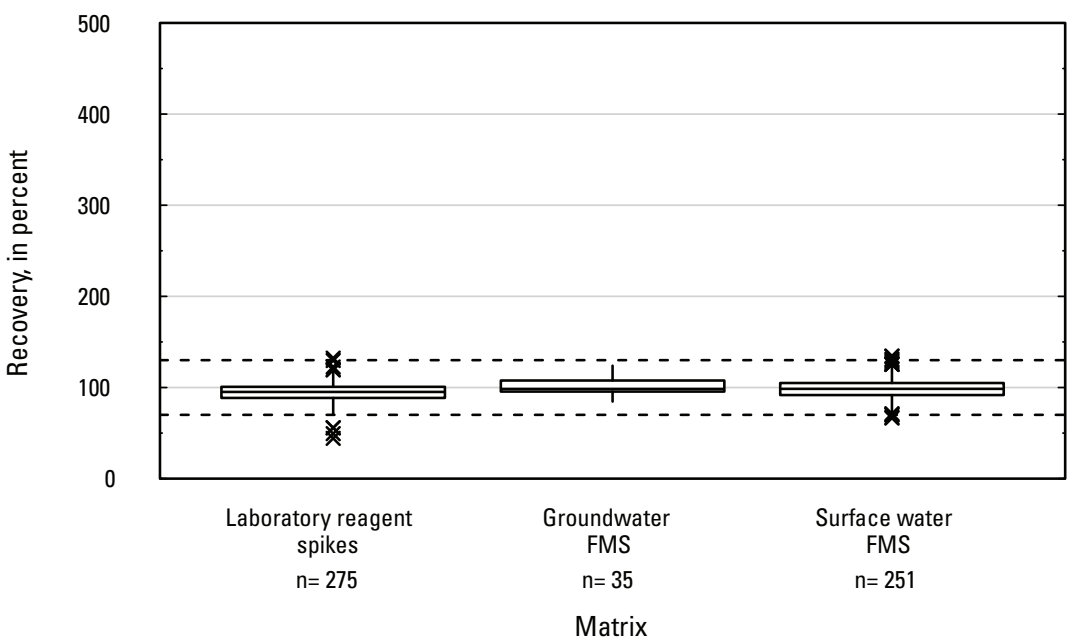

Figure 4. Graph showing distributions of recovery for pesticides in schedule 2437 by matrix. Dashed lines are at 130 percent and 70 percent. Recovery values larger than 400 percent are not shown.-Continued 
$D E$. Fonofos

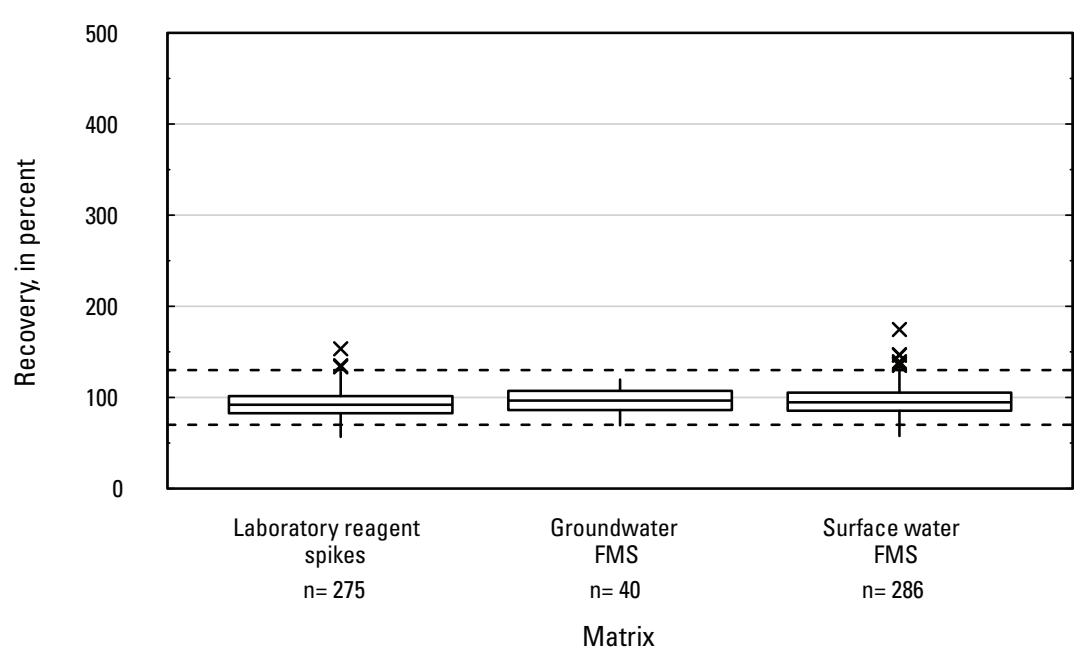

DG. Hexazinone

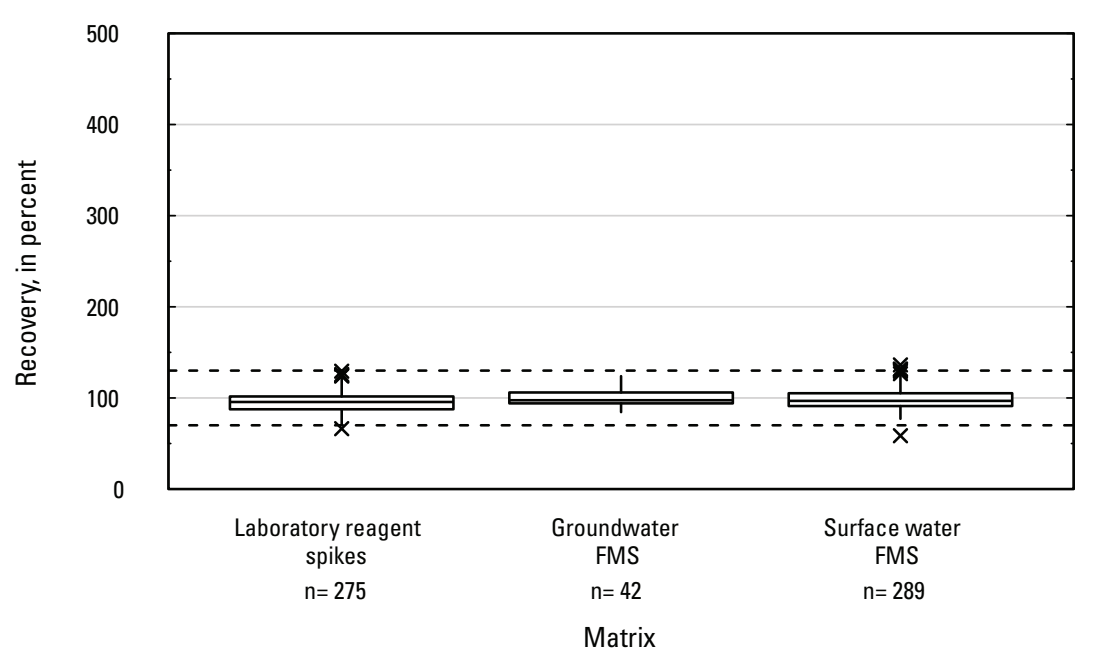

DF. Halosulfuron-methyl

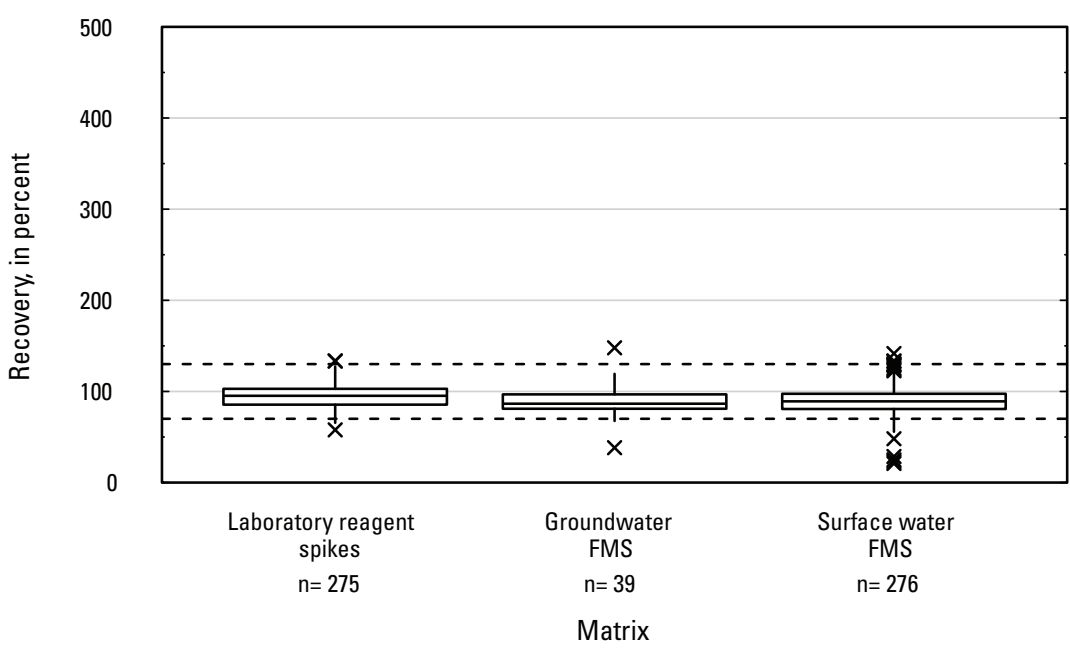

DH. Hexazinone Transformation Product C

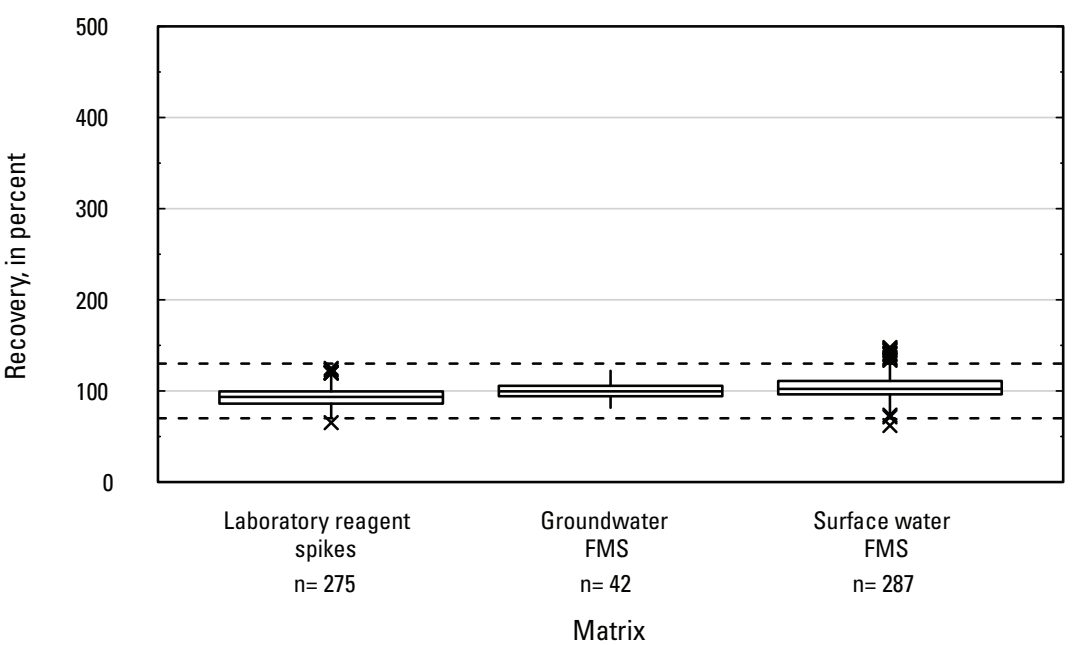

R

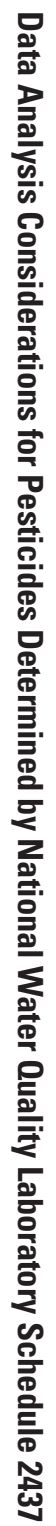

Figure 4. Graph showing distributions of recovery for pesticides in schedule 2437 by matrix. Dashed lines are at 130 percent and 70 percent. Recovery values larger than 400 percent are not shown.-Continued 
DI. Hexazinone Transformation Product D

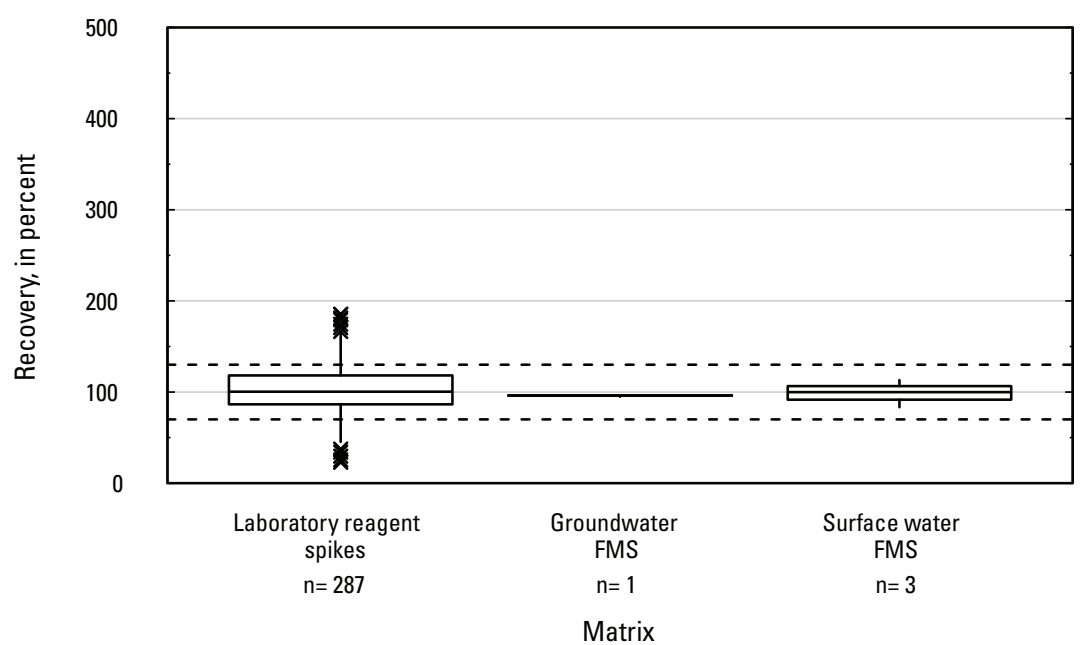

DK. Didemethyl hexazinone F

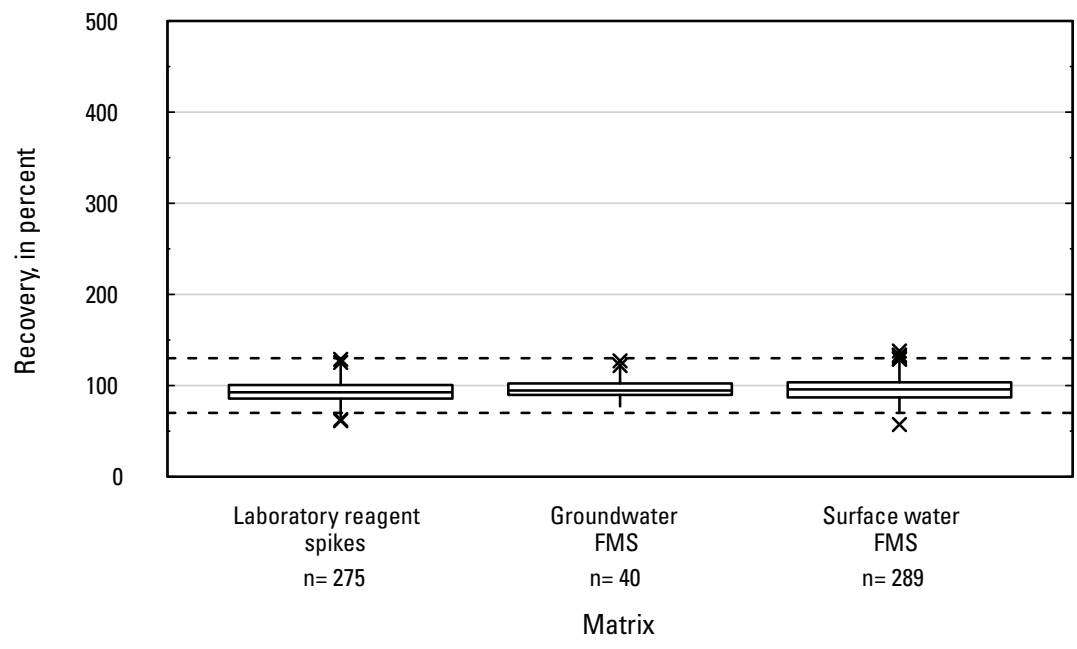

DJ. Hexazinone Transformation Product E

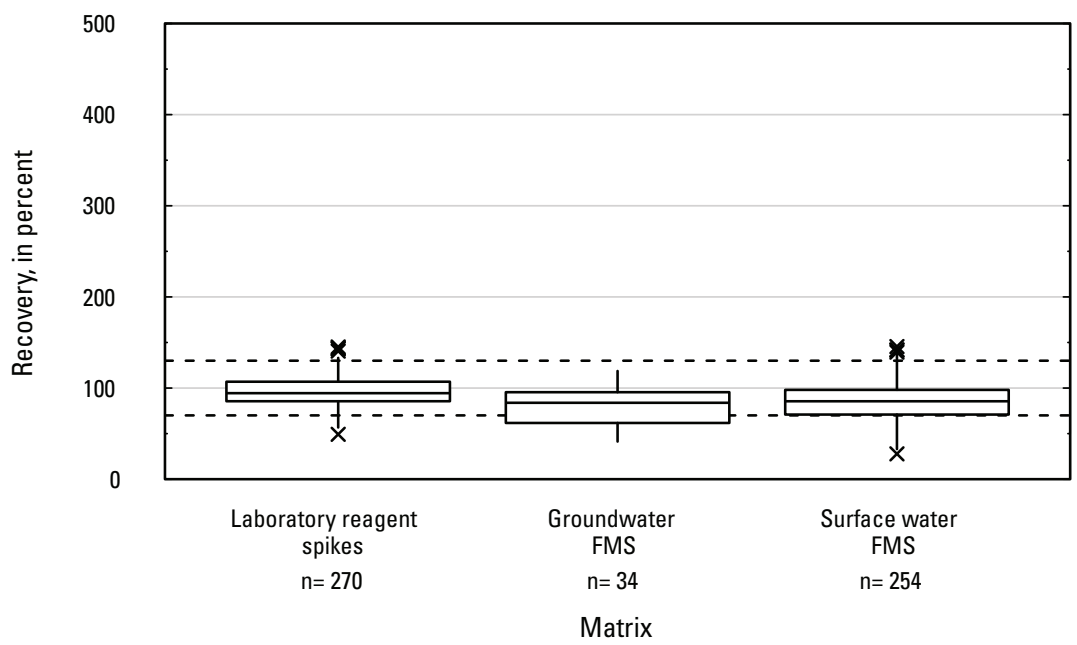

DL. Hexazinone Transformation Product G

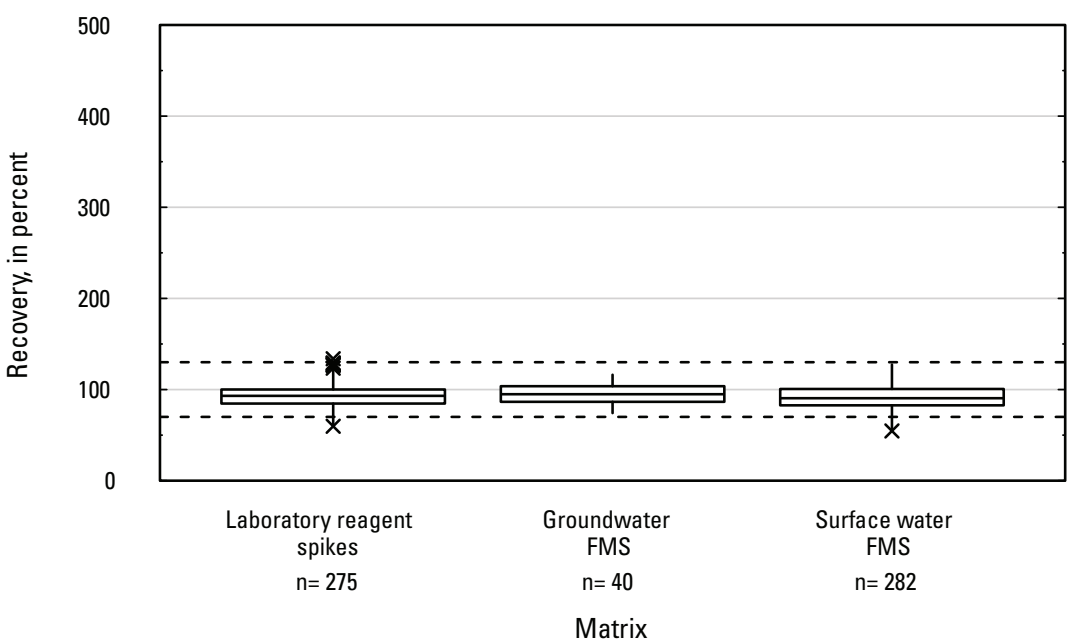

Figure 4. Graph showing distributions of recovery for pesticides in schedule 2437 by matrix. Dashed lines are at 130 percent and 70 percent. Recovery values larger than 400 percent are not shown.-Continued 
DM. Hydroxy monodemethyl fluometuron

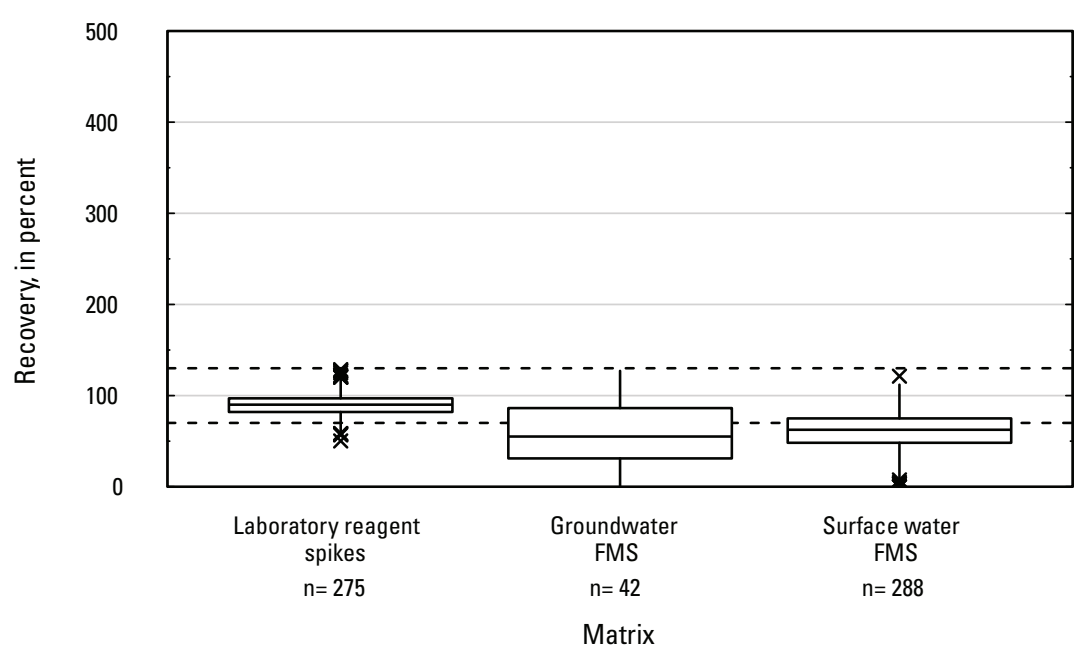

DO. Hydroxyalachlor

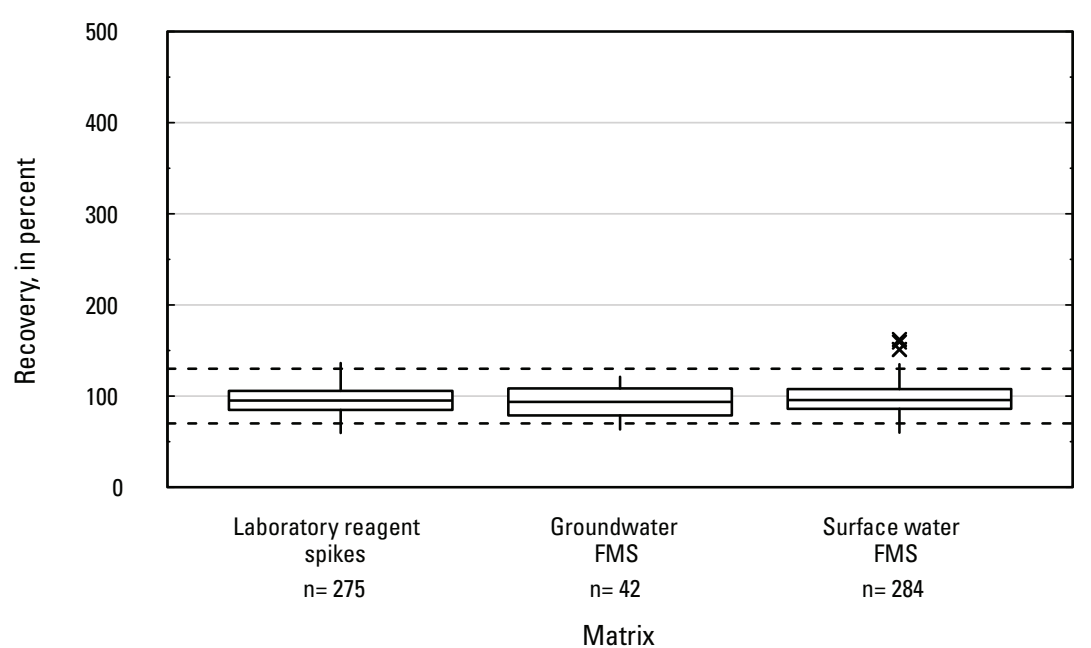

DN. Hydroxyacetochlor

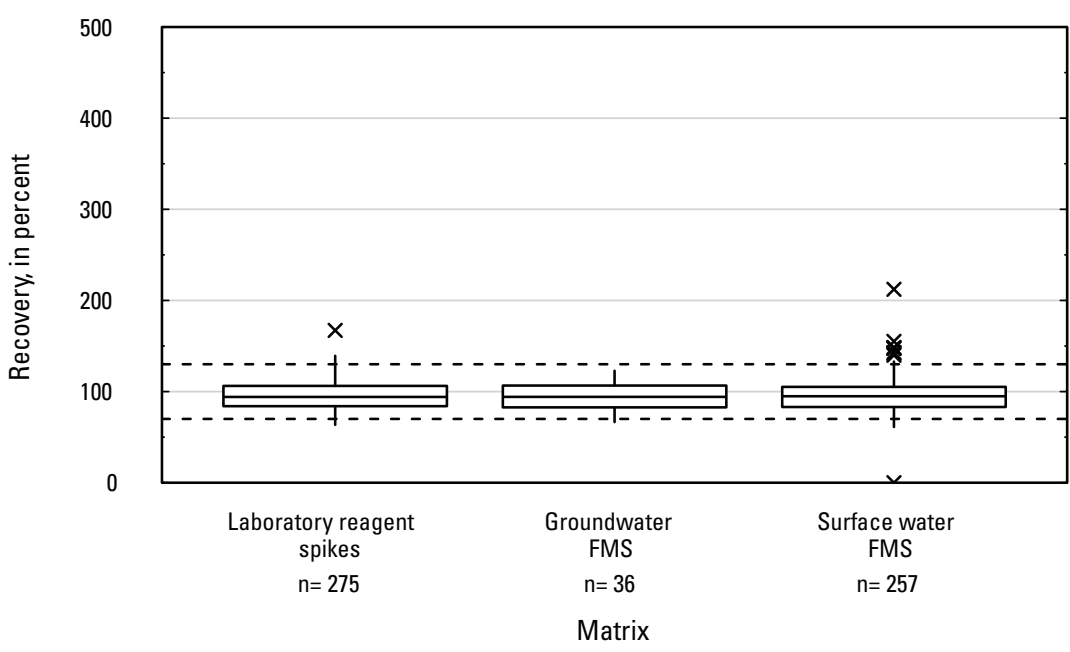

DP. Hydroxydiazinon

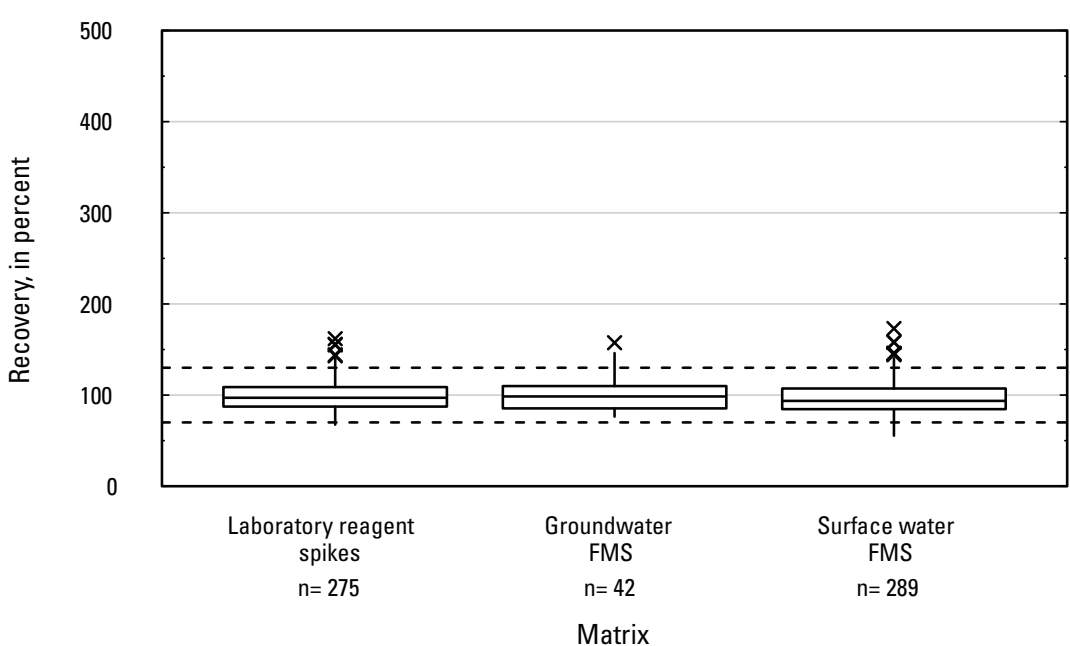

요

뭉

Figure 4. Graph showing distributions of recovery for pesticides in schedule 2437 by matrix. Dashed lines are at 130 percent and 70 percent. Recovery values larger than 400 percent are not shown.-Continued 
DQ. Hydroxyfluometuron

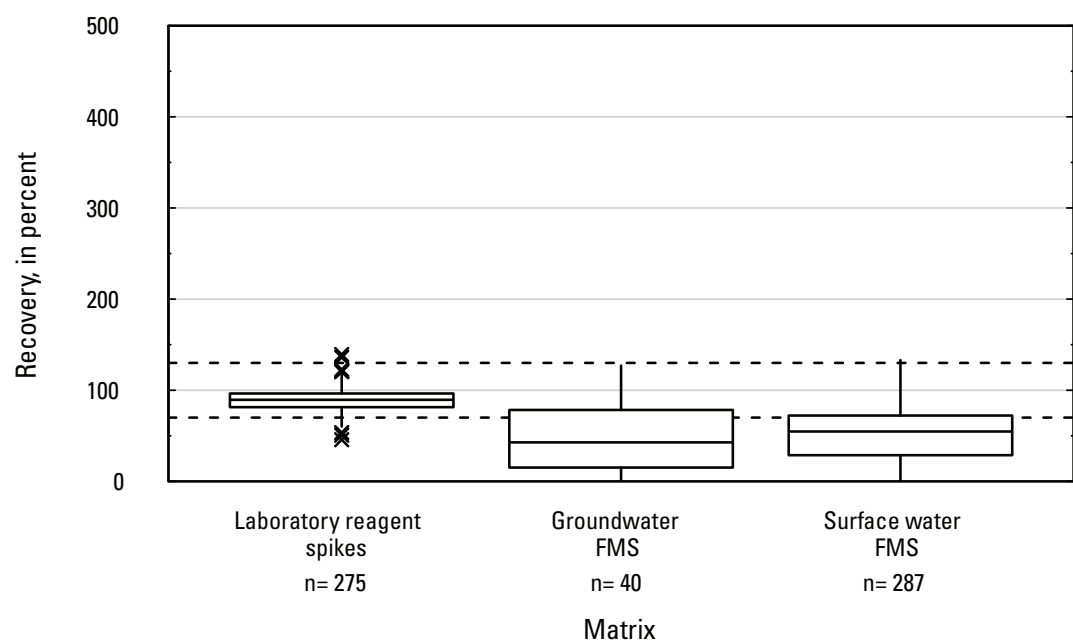

DS. Hydroxyphthalazinone

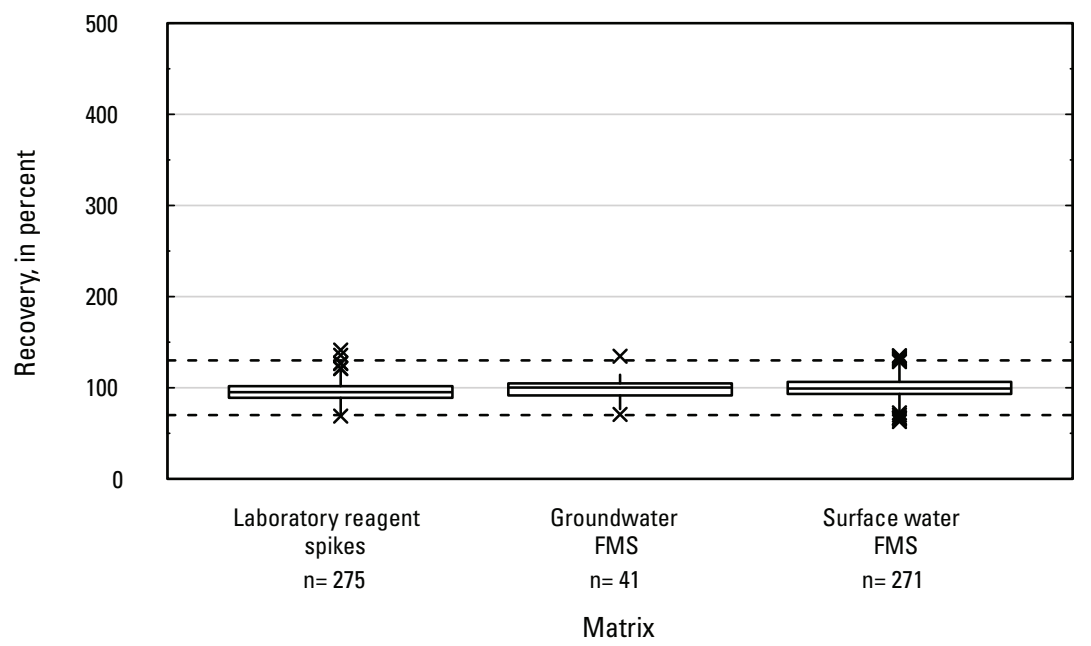

DR. Hydroxymetolachlor

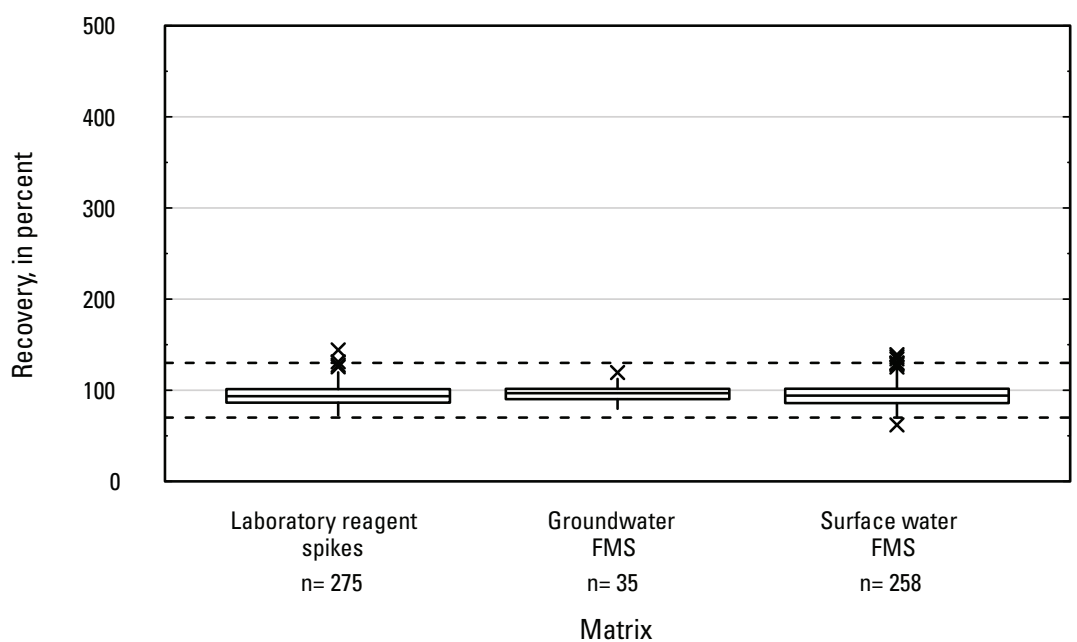

DT. Hydroxysimazine

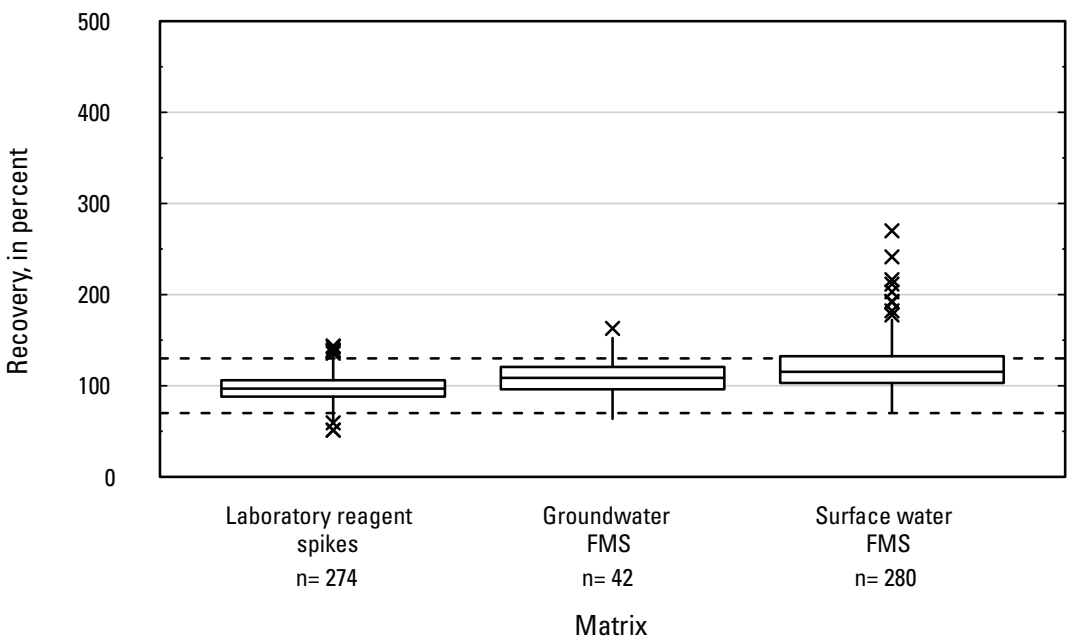

Figure 4. Graph showing distributions of recovery for pesticides in schedule 2437 by matrix. Dashed lines are at 130 percent and 70 percent. Recovery values larger 
DU. Tebuthiuron TP 109

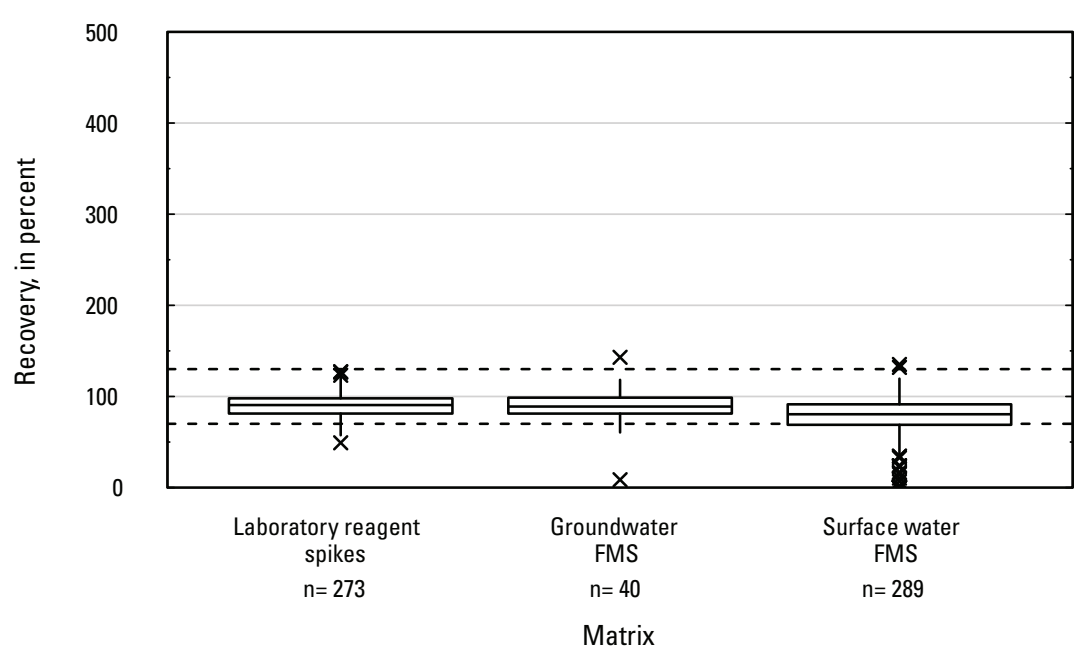

DW. Imazaquin

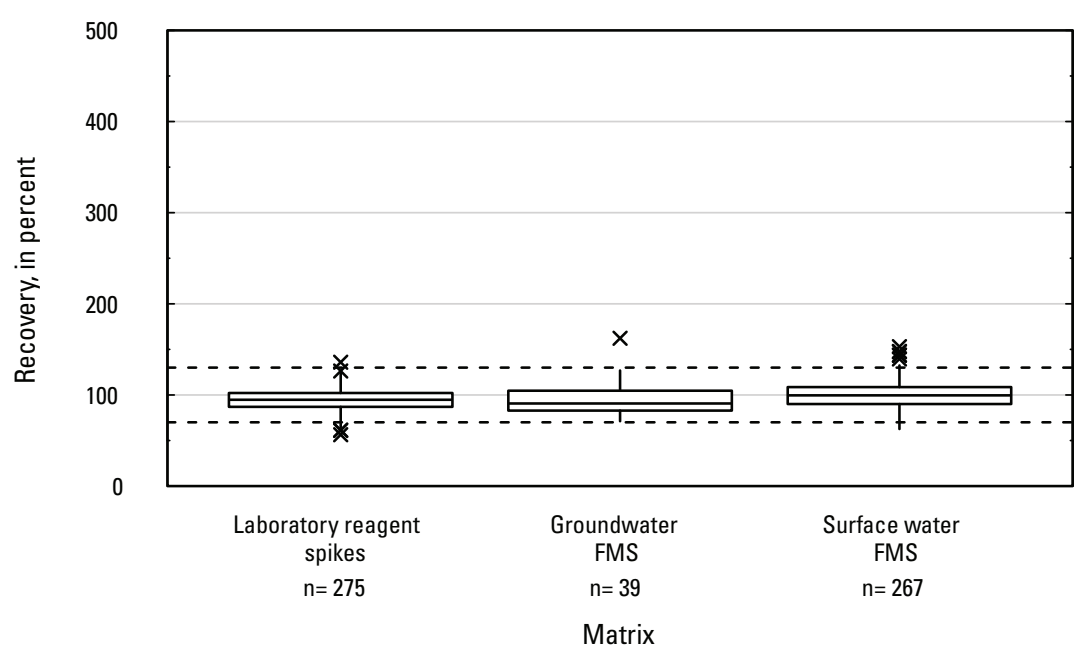

DV. Imazamox

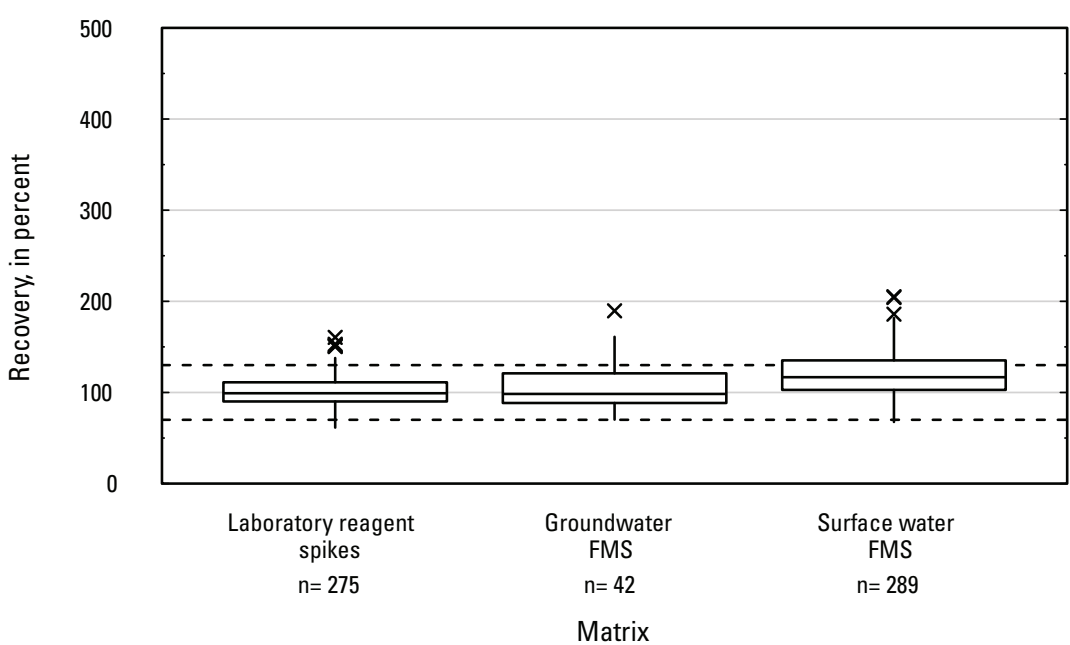

DX. Imazethapyr

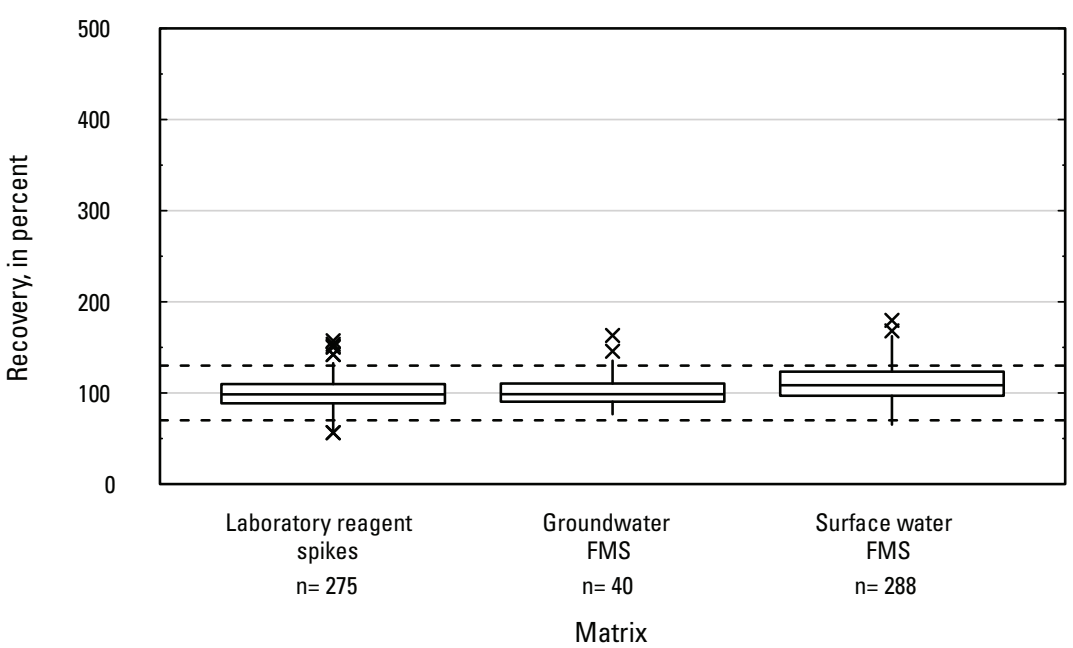

ஜ

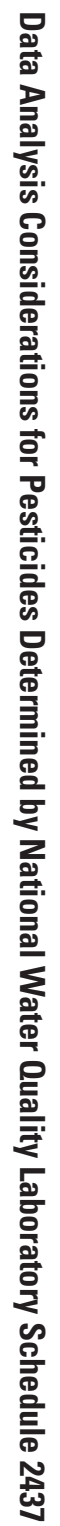

Figure 4. Graph showing distributions of recovery for pesticides in schedule 2437 by matrix. Dashed lines are at 130 percent and 70 percent. Recovery values larger than 400 percent are not shown.-Continued 
DY. Imidacloprid

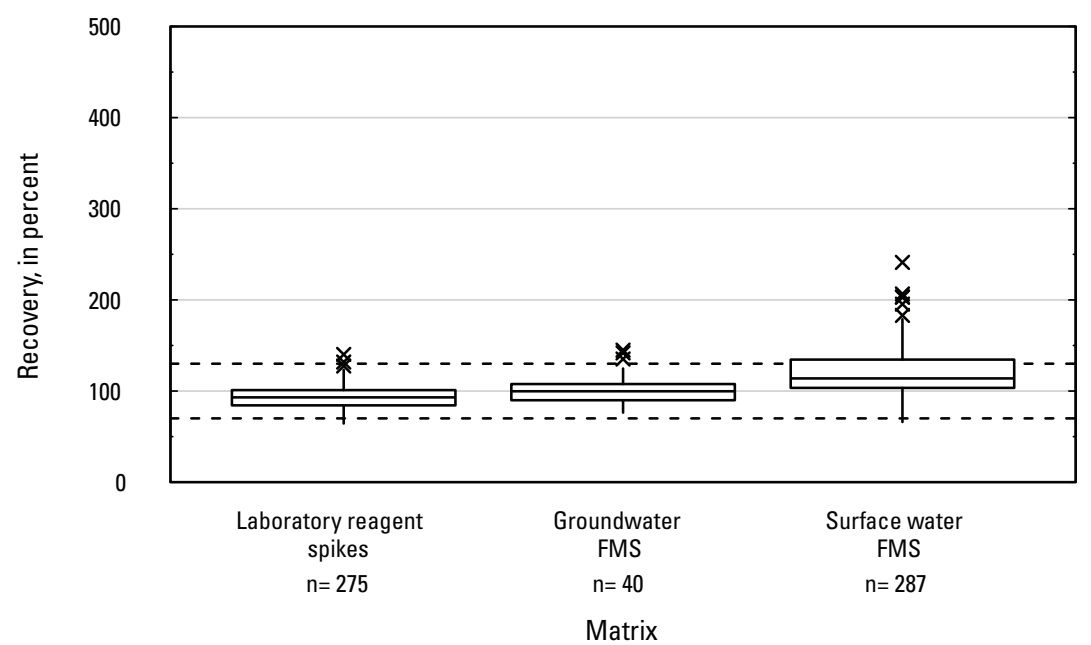

\section{$E A$. Isoxaflutole}

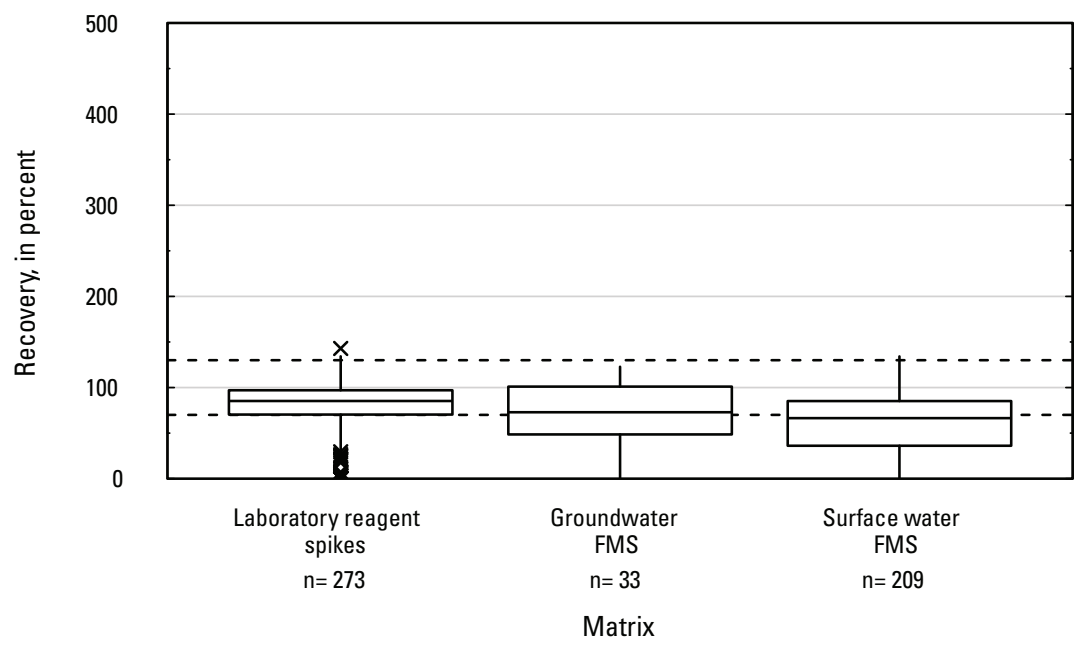

DZ. Indoxacarb

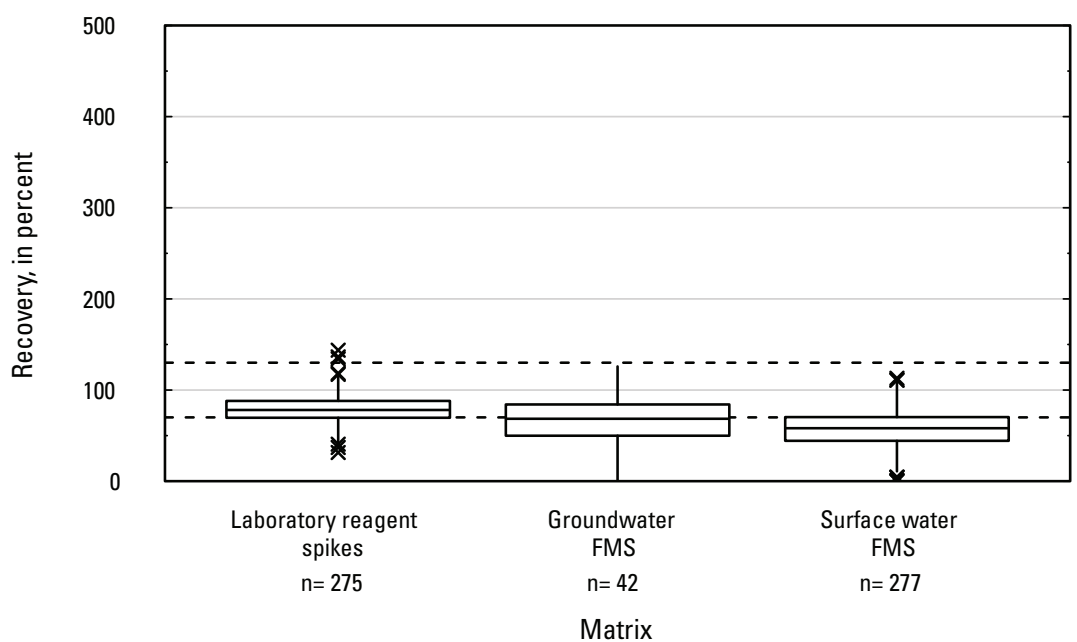

EB. Isoxaflutole acid metabolite RPA 203328

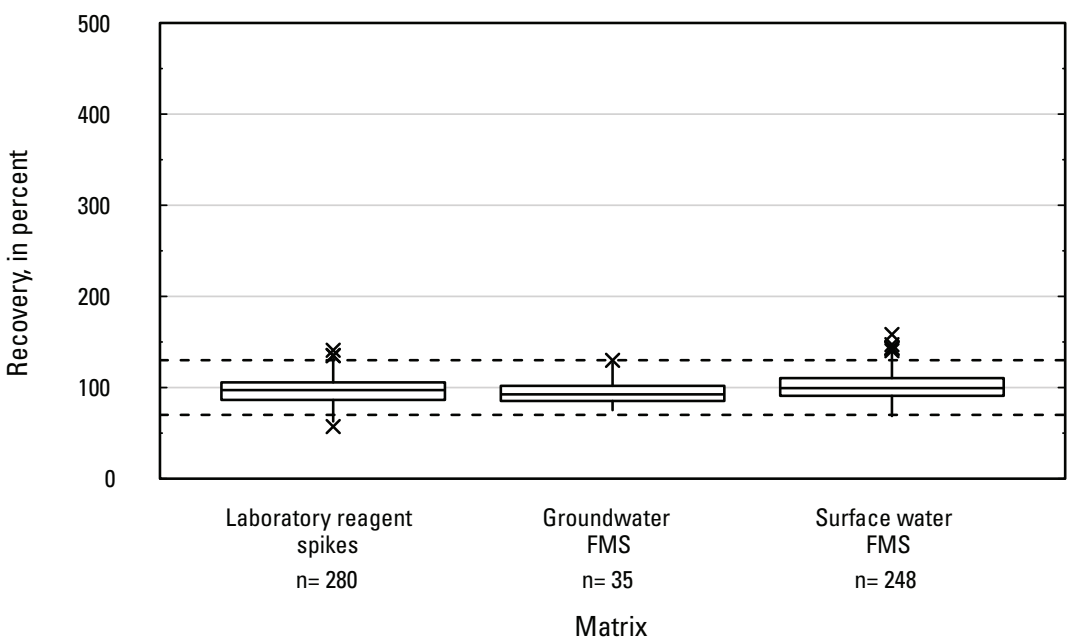

Figure 4. Graph showing distributions of recovery for pesticides in schedule 2437 by matrix. Dashed lines are at 130 percent and 70 percent. Recovery values larger than 400 percent are not shown.-Continued 
EC. Kresoxim-methyl

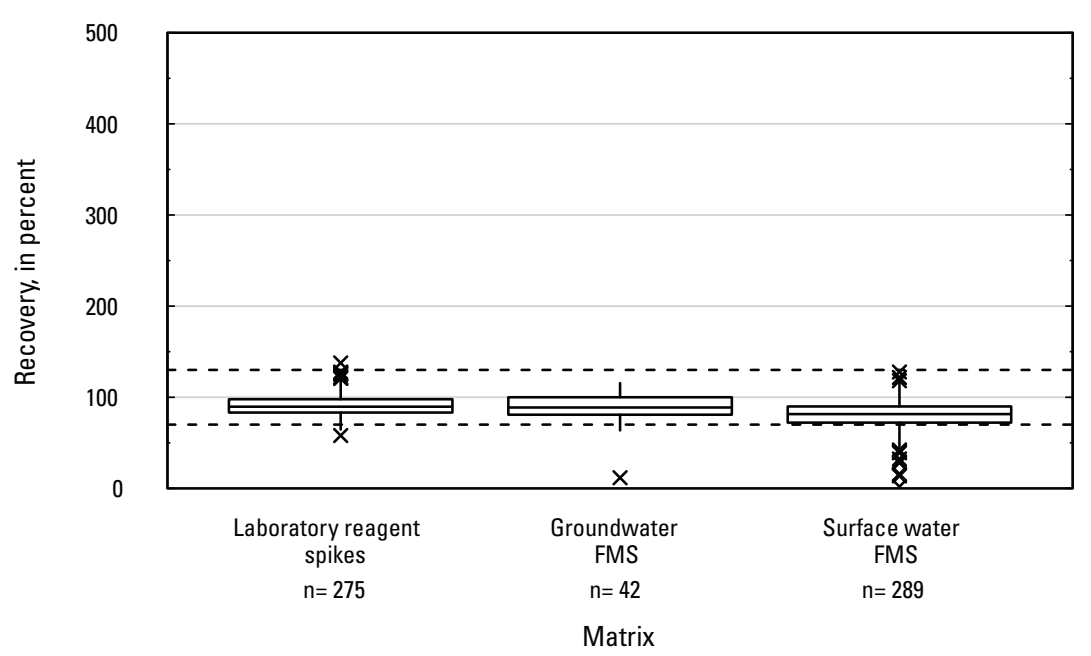

EE. Linuron

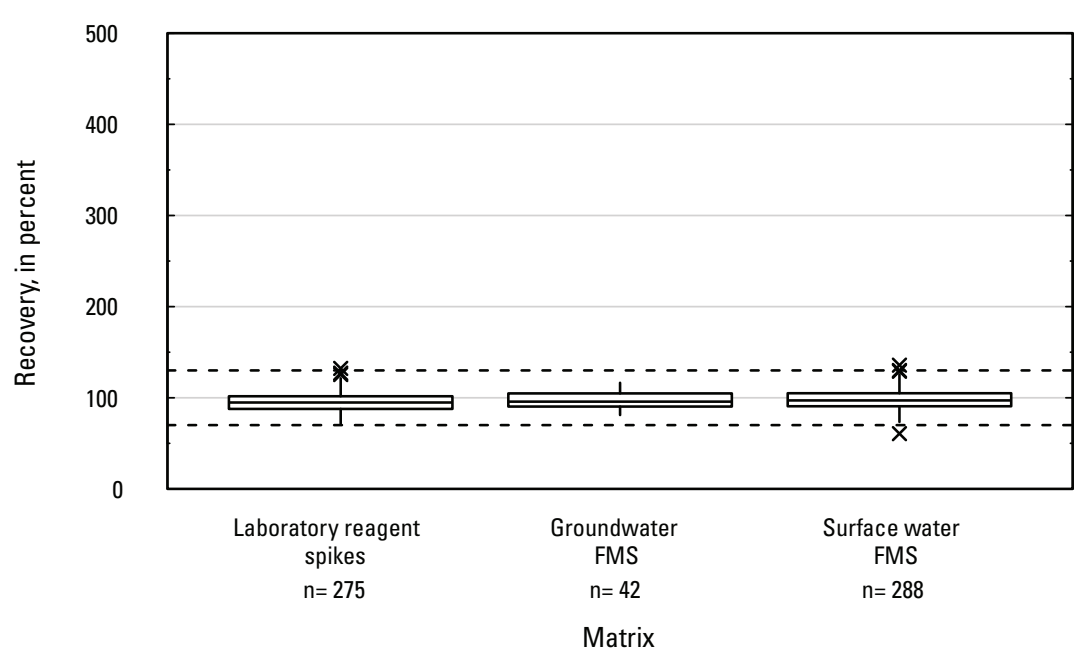

ED. Lactofen

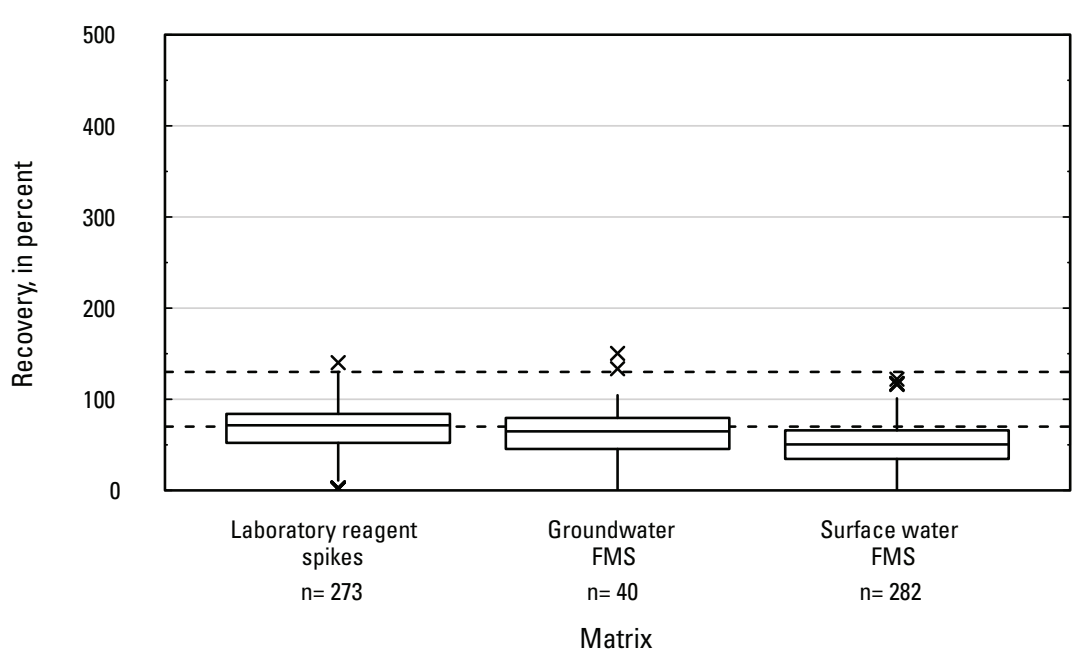

EF. Malaoxon

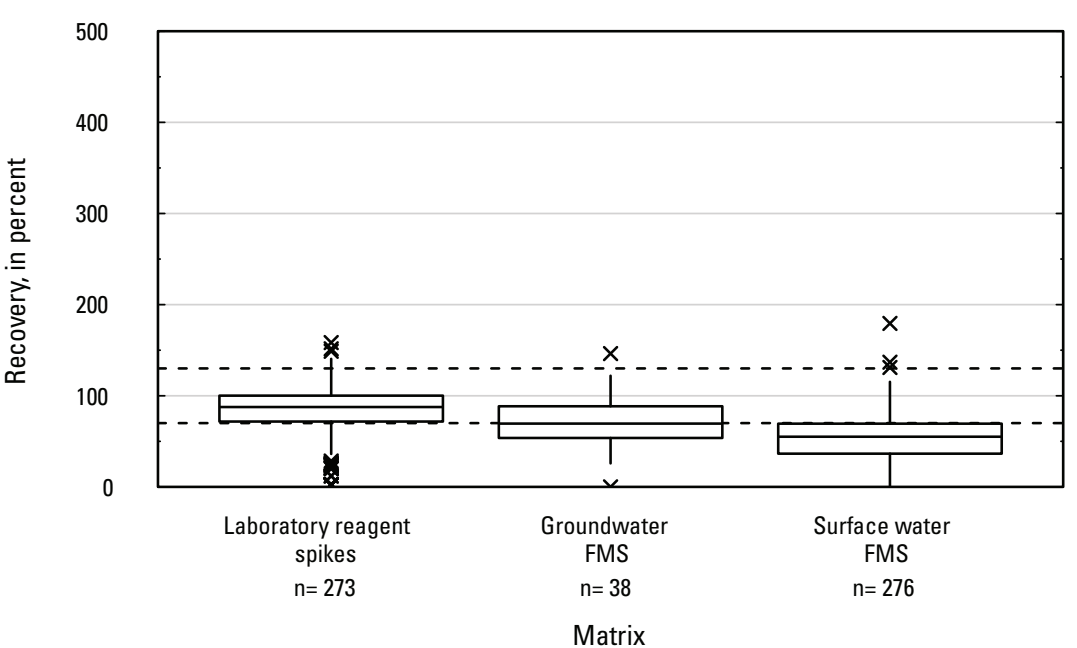

d

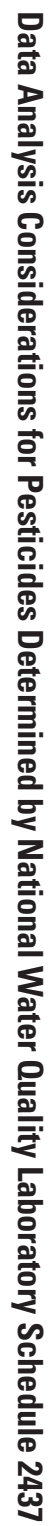

Figure 4. Graph showing distributions of recovery for pesticides in schedule 2437 by matrix. Dashed lines are at 130 percent and 70 percent. Recovery values larger than 400 percent are not shown.-Continued 
EG. Malathion

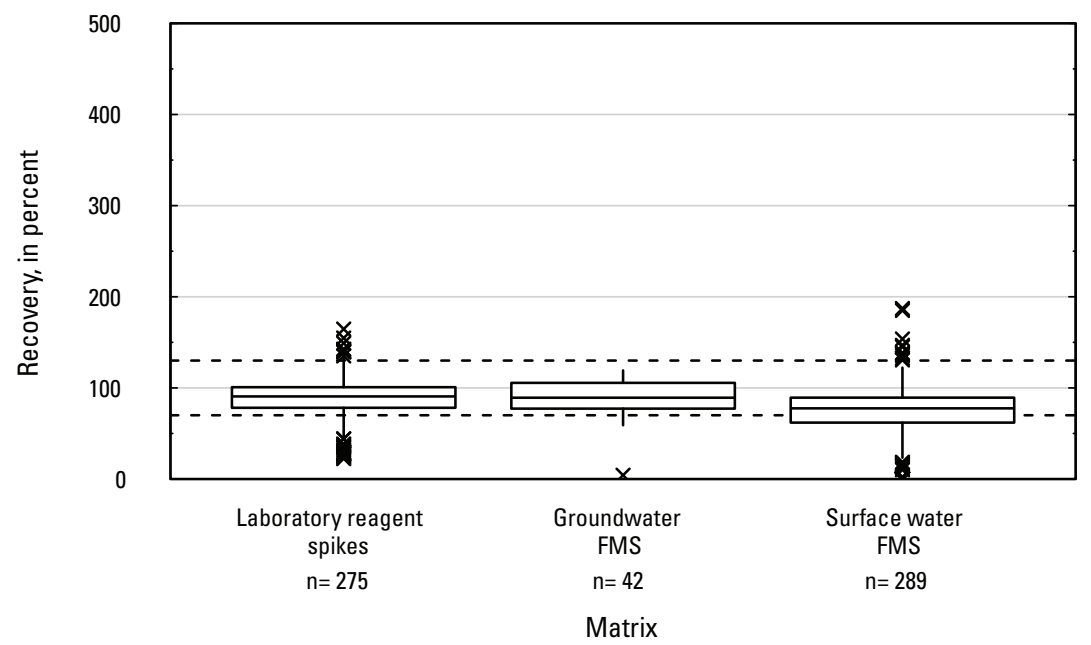

El. Metalaxyl

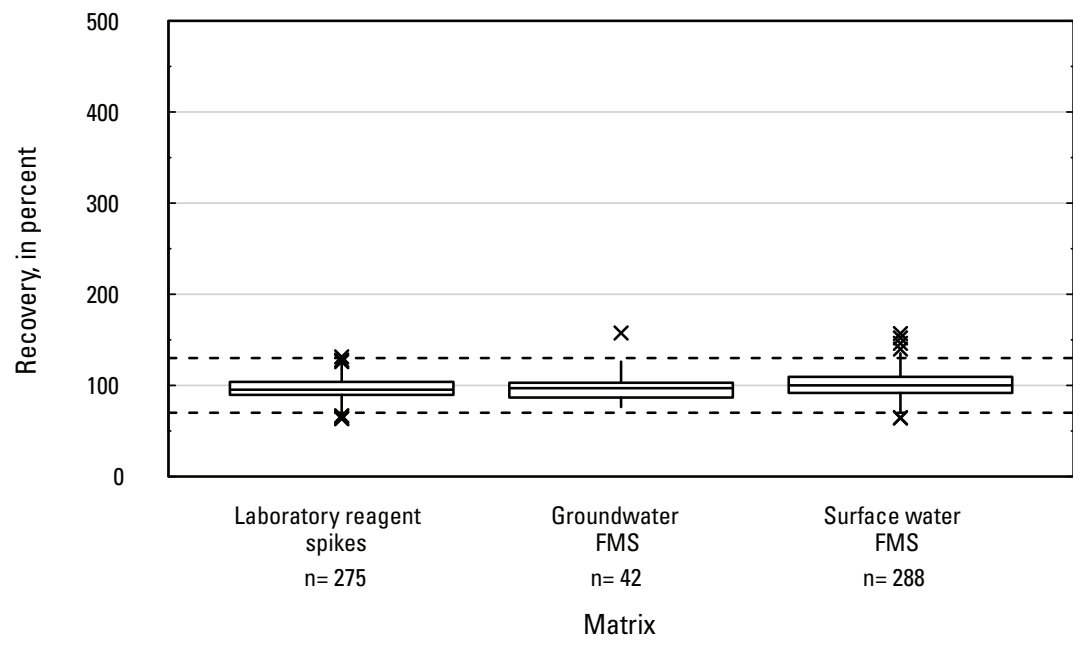

EH. МСРA

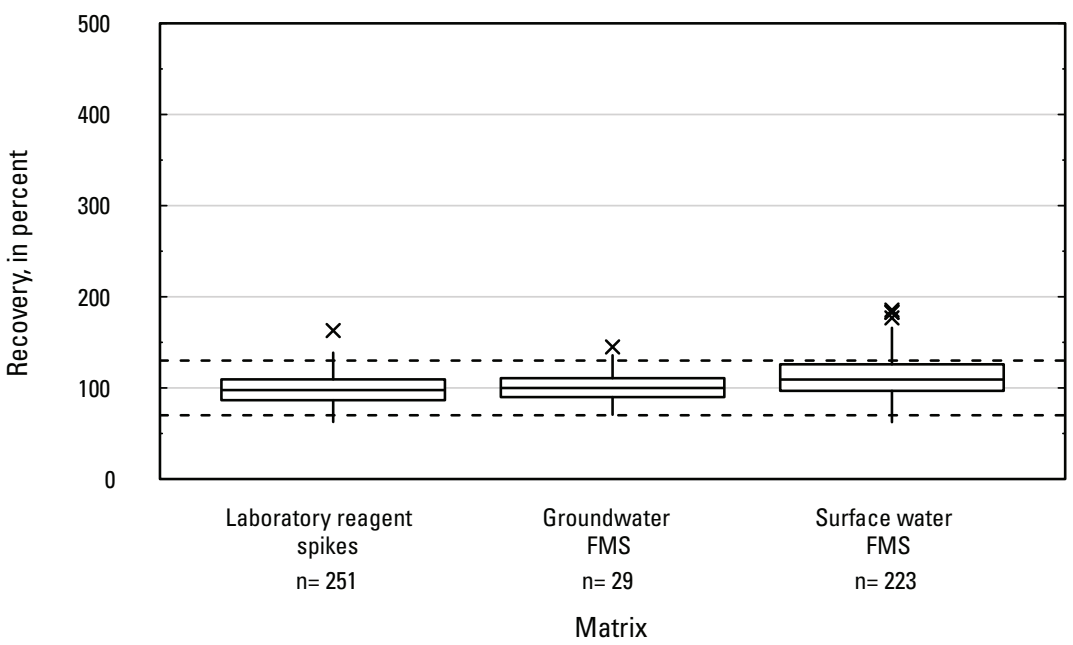

EJ. Metconazole

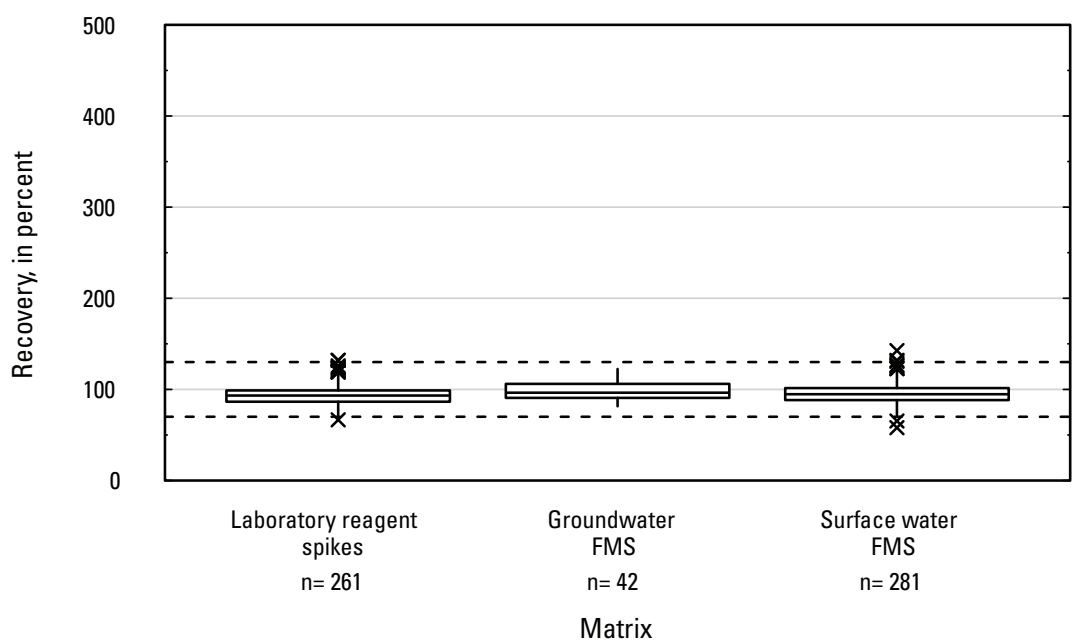

Figure 4. Graph showing distributions of recovery for pesticides in schedule 2437 by matrix. Dashed lines are at 130 percent and 70 percent. Recovery values larger than 400 percent are not shown.-Continued 
EK. Methamidophos

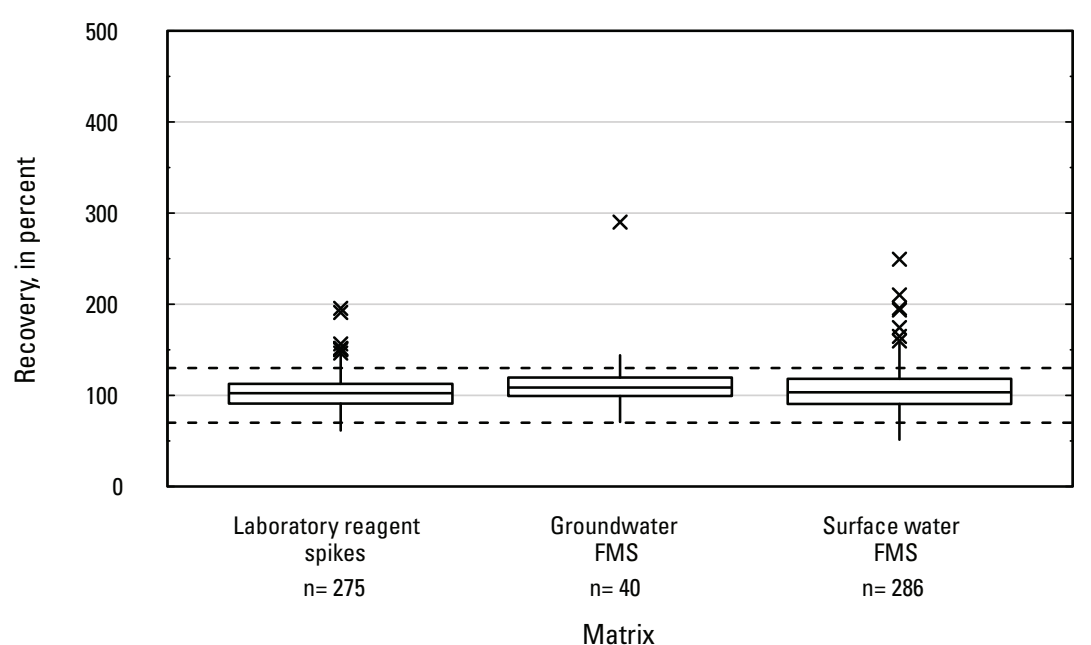

EM. Methomyl

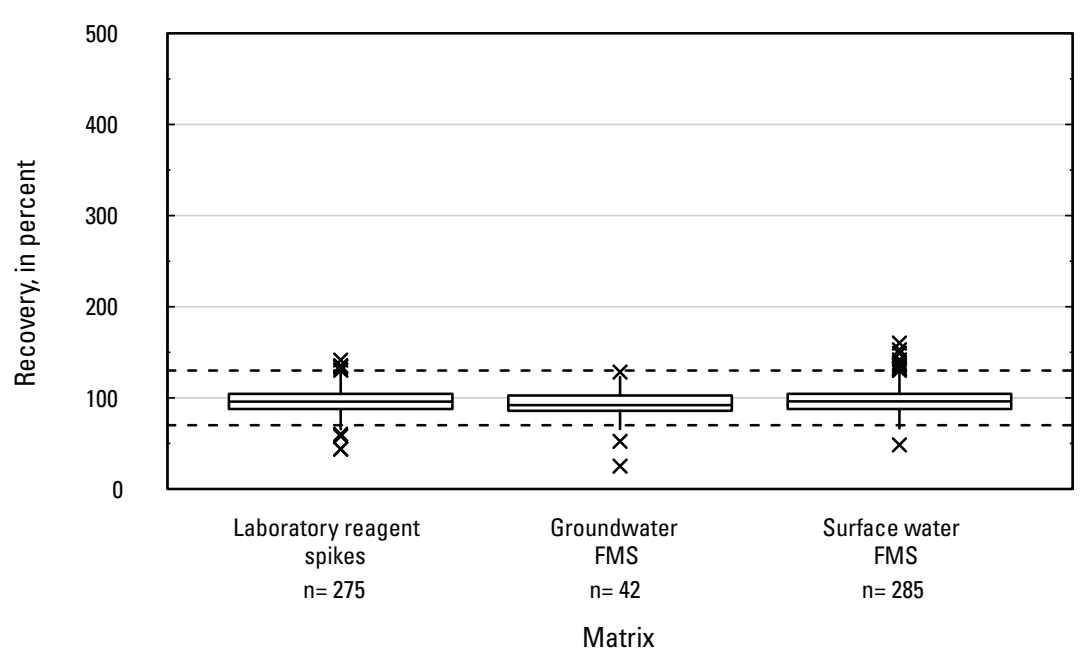

EL. Methidathion

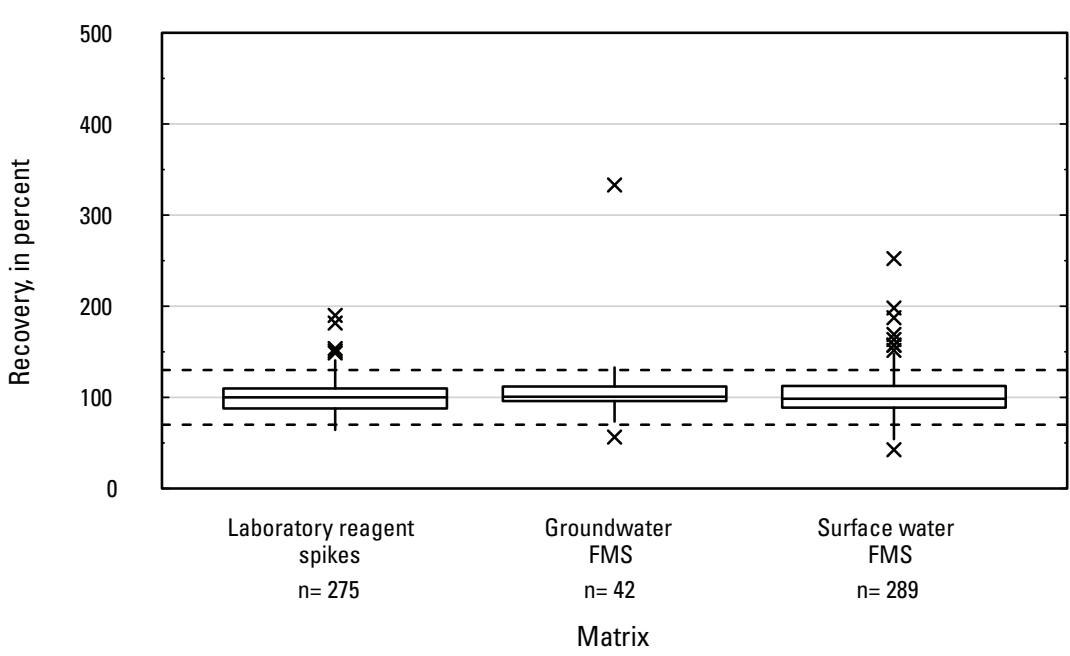

EN. Methomyl oxime

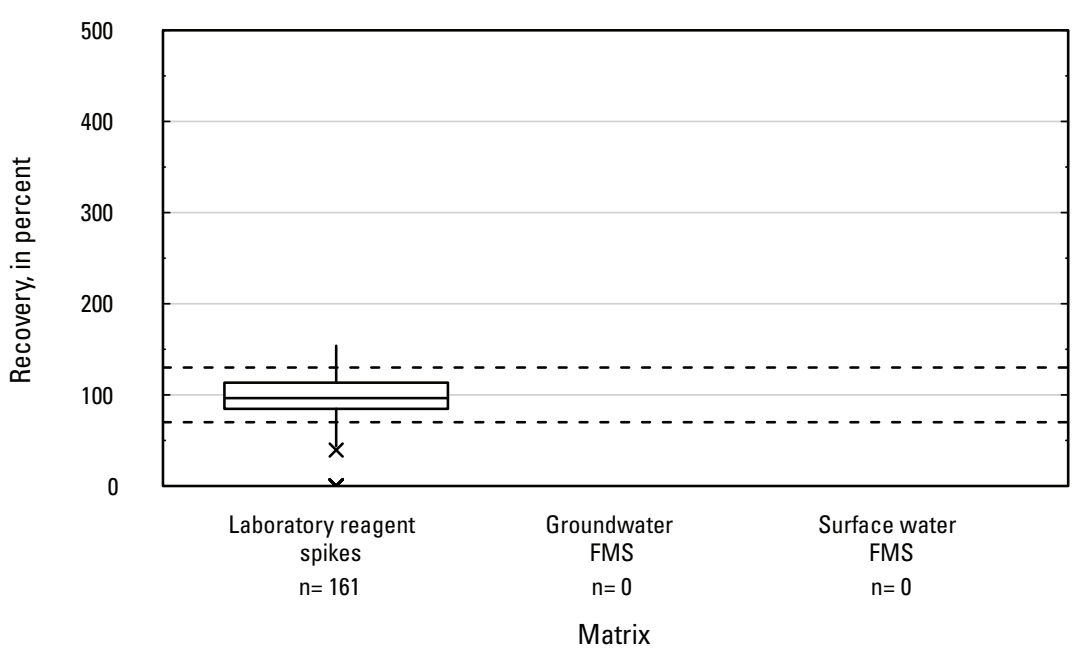

$N$

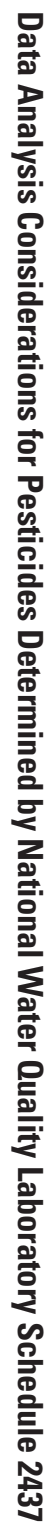

Figure 4. Graph showing distributions of recovery for pesticides in schedule 2437 by matrix. Dashed lines are at 130 percent and 70 percent. Recovery values larger than 400 percent are not shown.-Continued 
EO. Methoxyfenozide

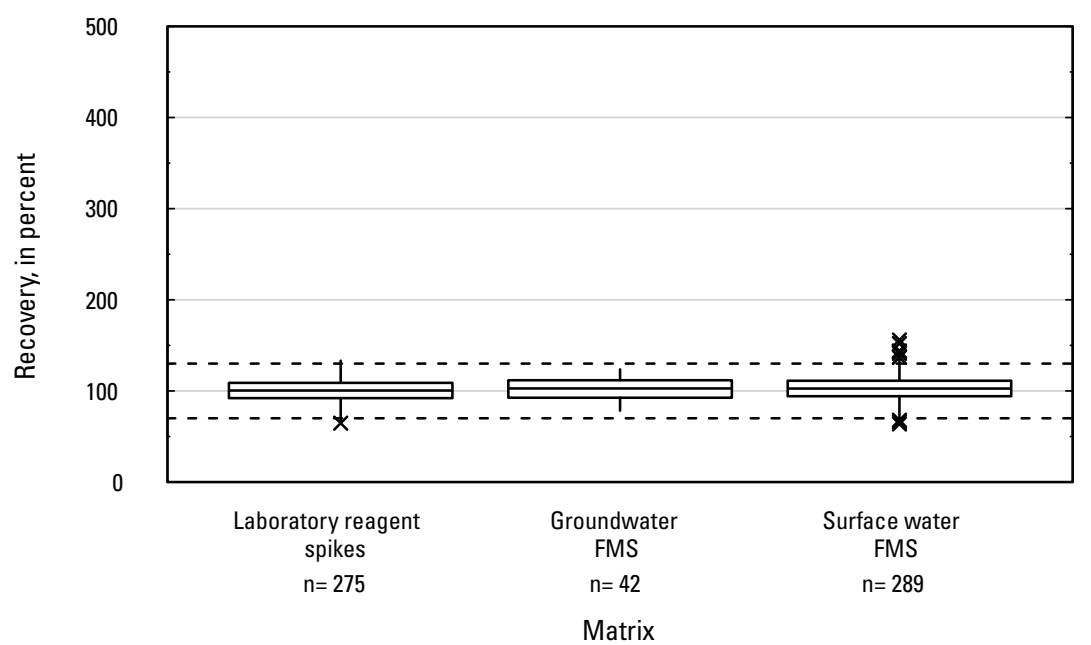

EQ. Metolachlor hydroxy morpholinone

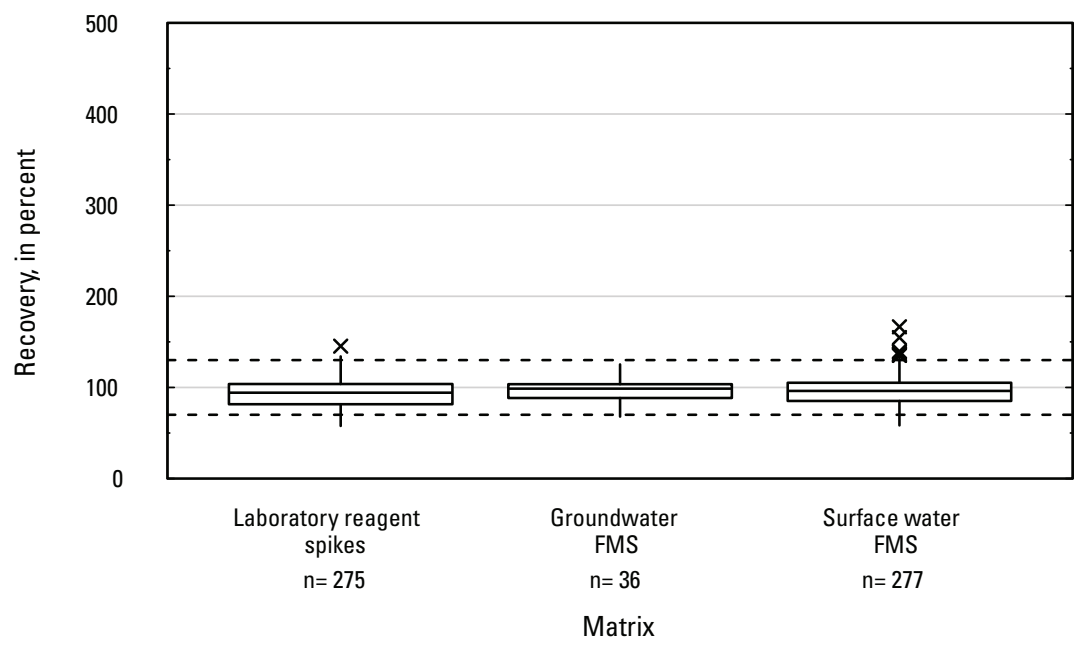

EP. Metolachlor

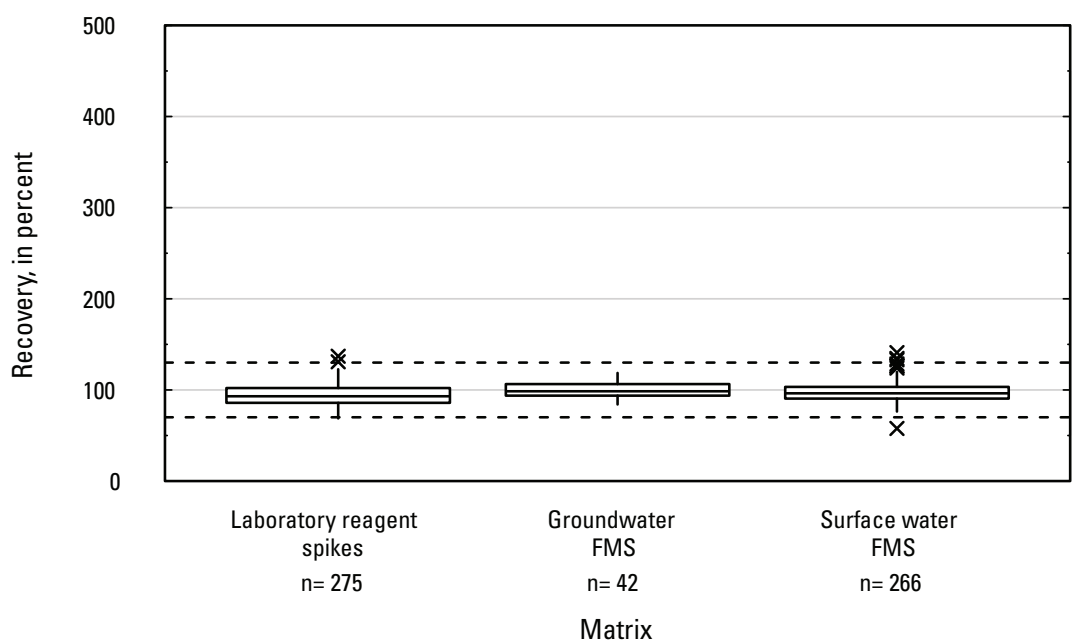

ER. Metolachlor oxanilic acid

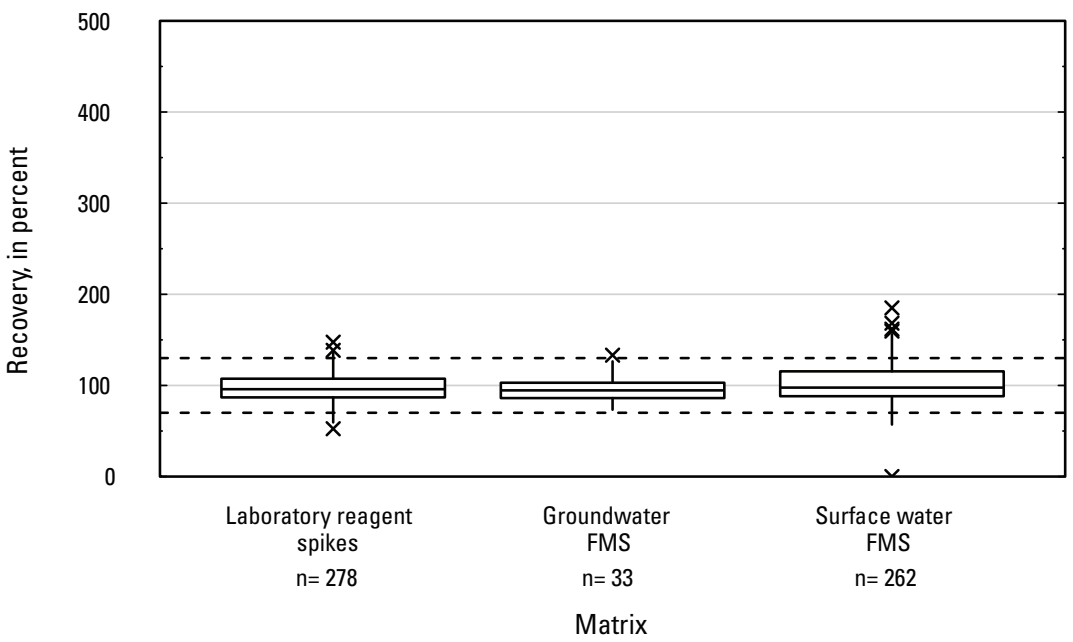

Figure 4. Graph showing distributions of recovery for pesticides in schedule 2437 by matrix. Dashed lines are at 130 percent and 70 percent. Recovery values larger than 400 percent are not shown.-Continued 
ES. Metolachlor sulfonic acid

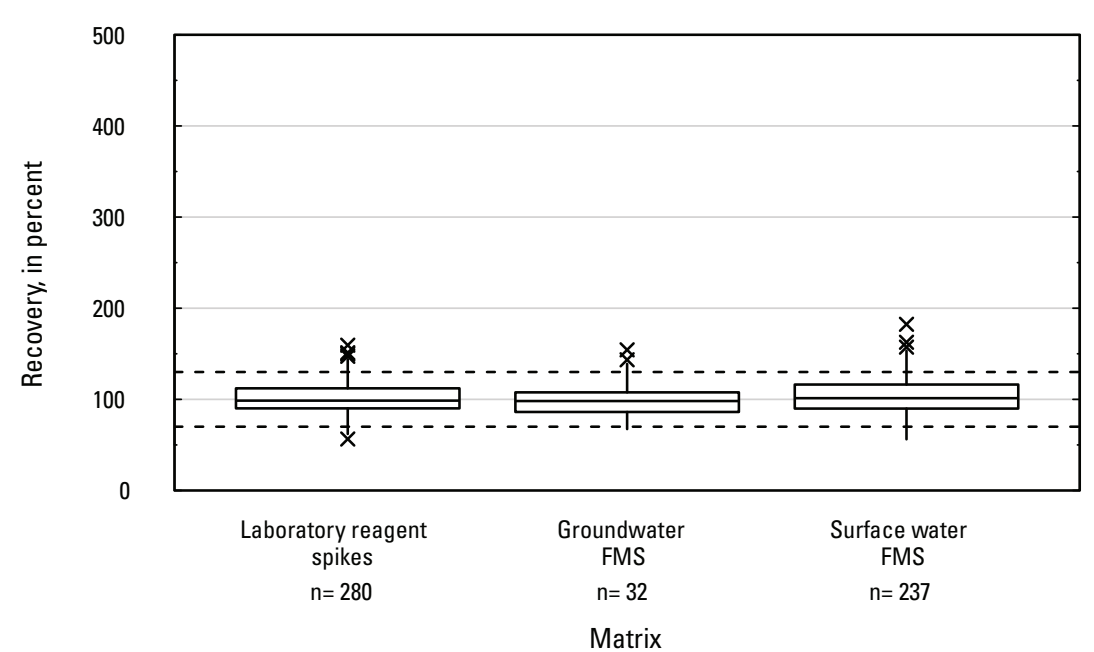

EU. Desamino metribuzin

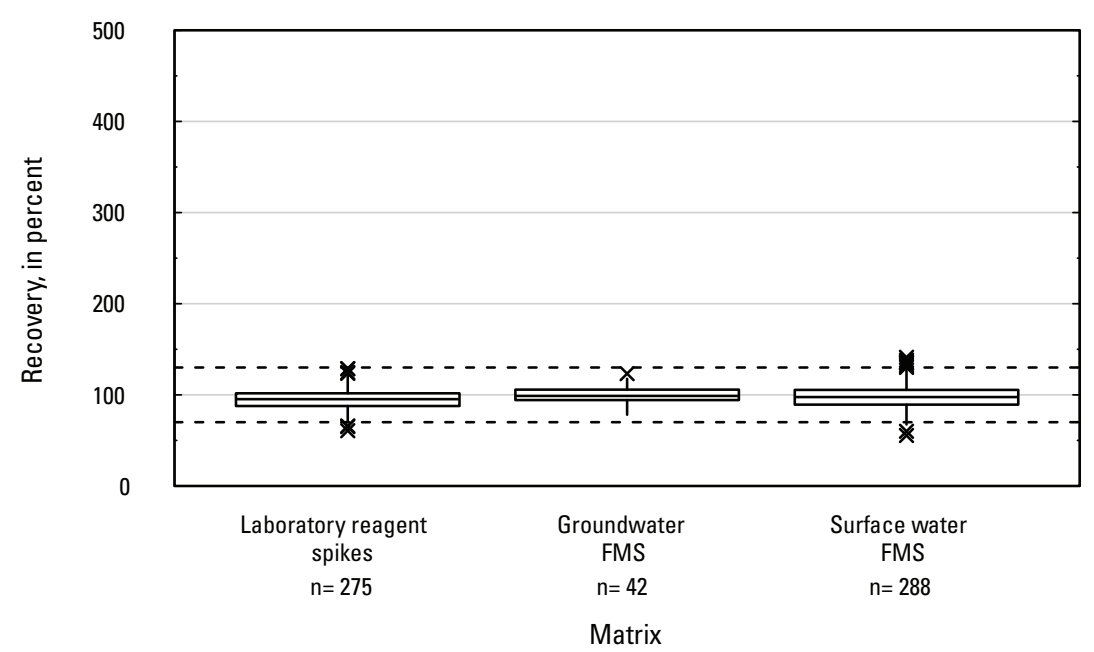

ET. Metribuzin

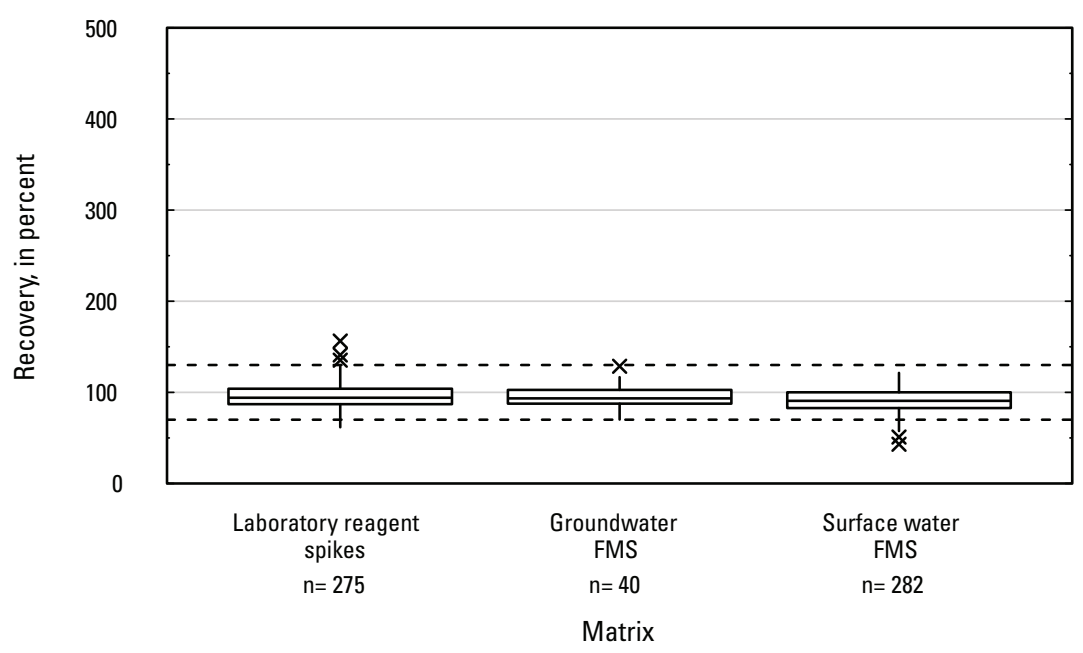

EV. Desamino-diketo metribuzin

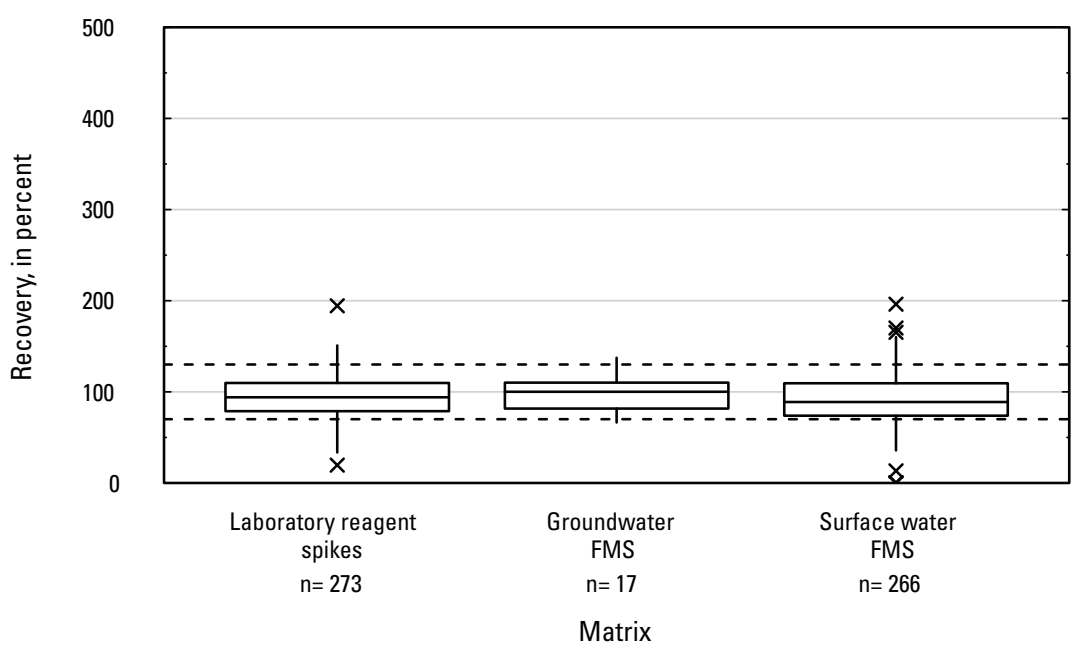

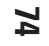

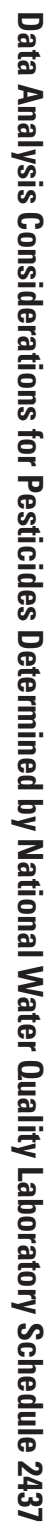

Figure 4. Graph showing distributions of recovery for pesticides in schedule 2437 by matrix. Dashed lines are at 130 percent and 70 percent. Recovery values larger than 400 percent are not shown.-Continued 
EW. Metribuzin DK

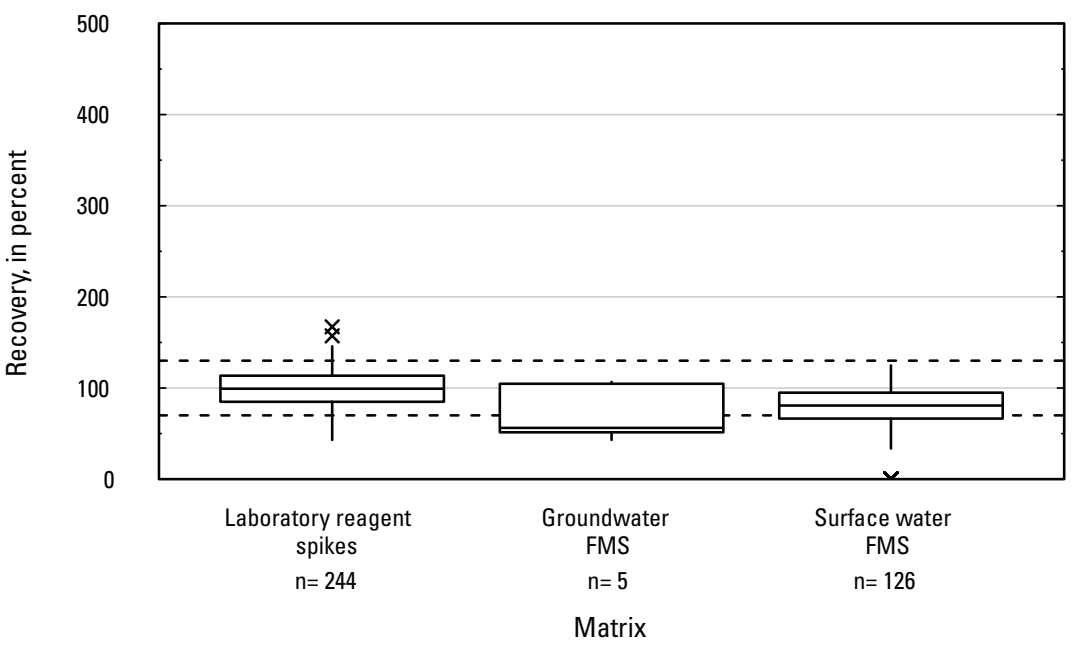

EY. Myclobutanil

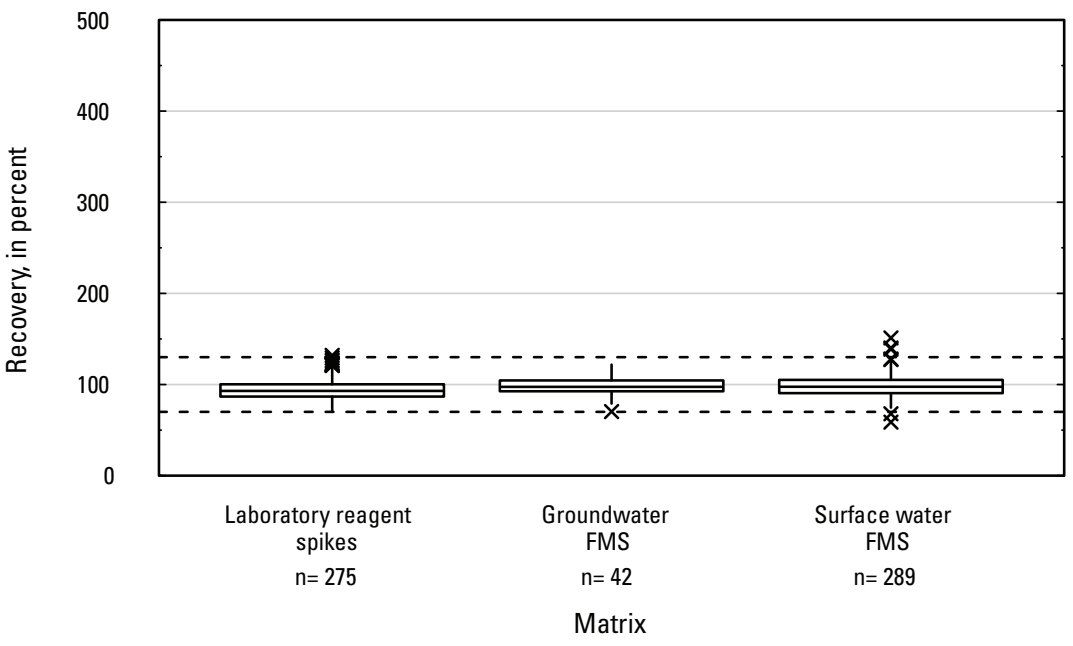

EX. Molinate

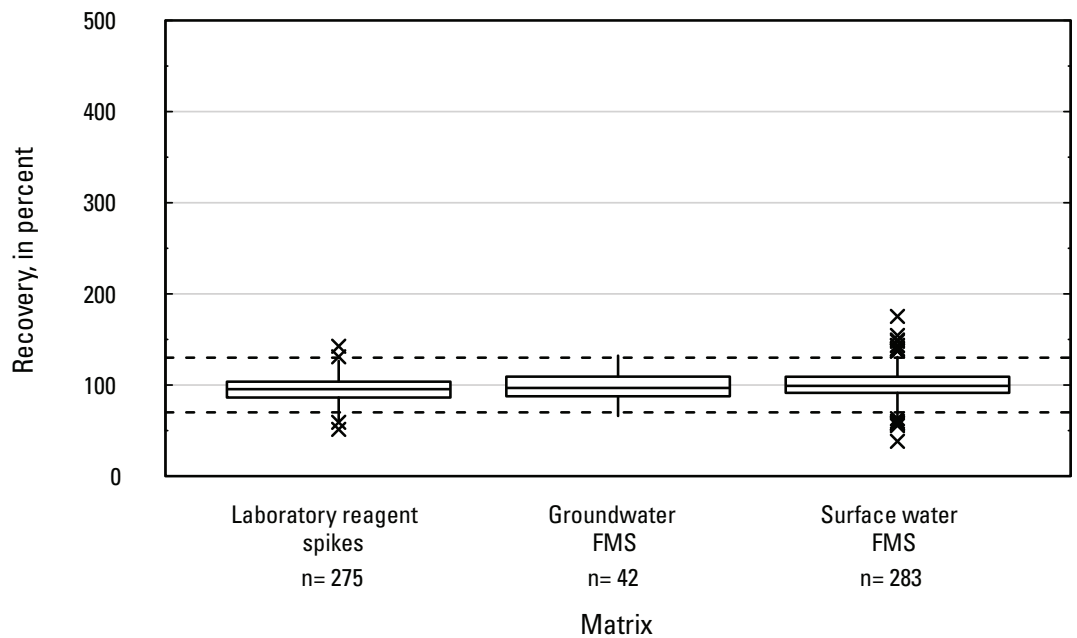

EZ. N-(3,4-Dichlorophenyl)-N'-methylurea

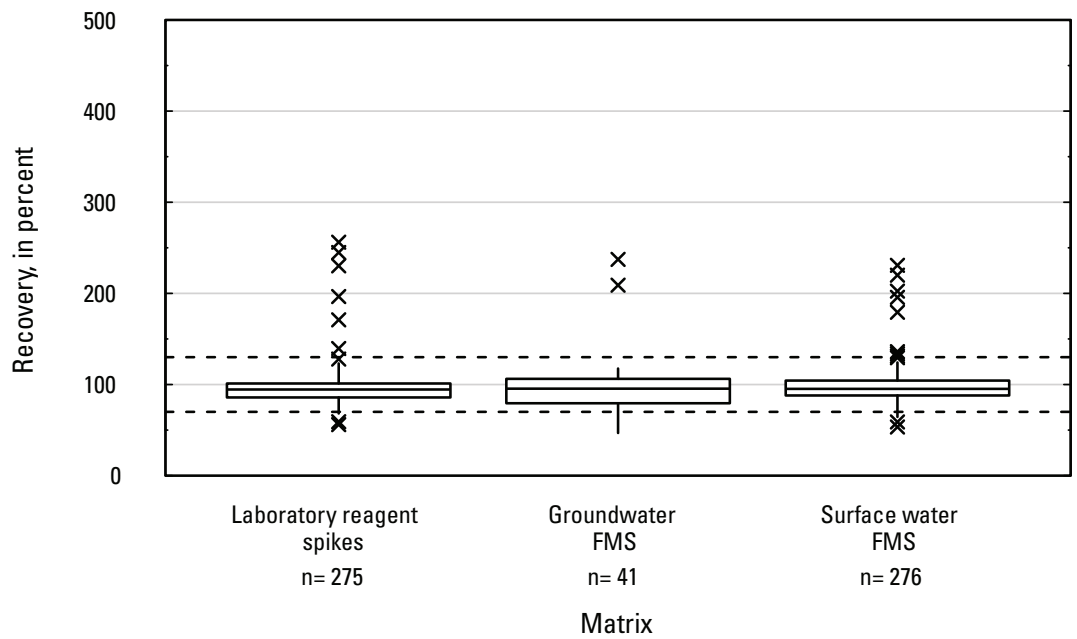

Figure 4. Graph showing distributions of recovery for pesticides in schedule 2437 by matrix. Dashed lines are at 130 percent and 70 percent. Recovery values larger 
FA. Naled

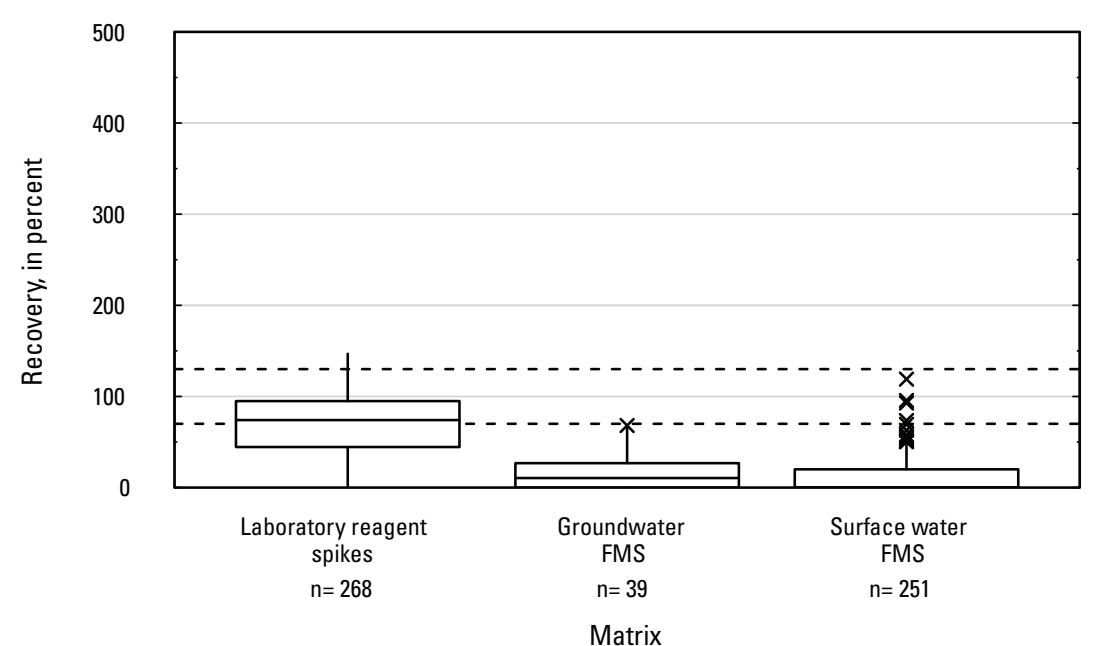

FC. Norflurazon

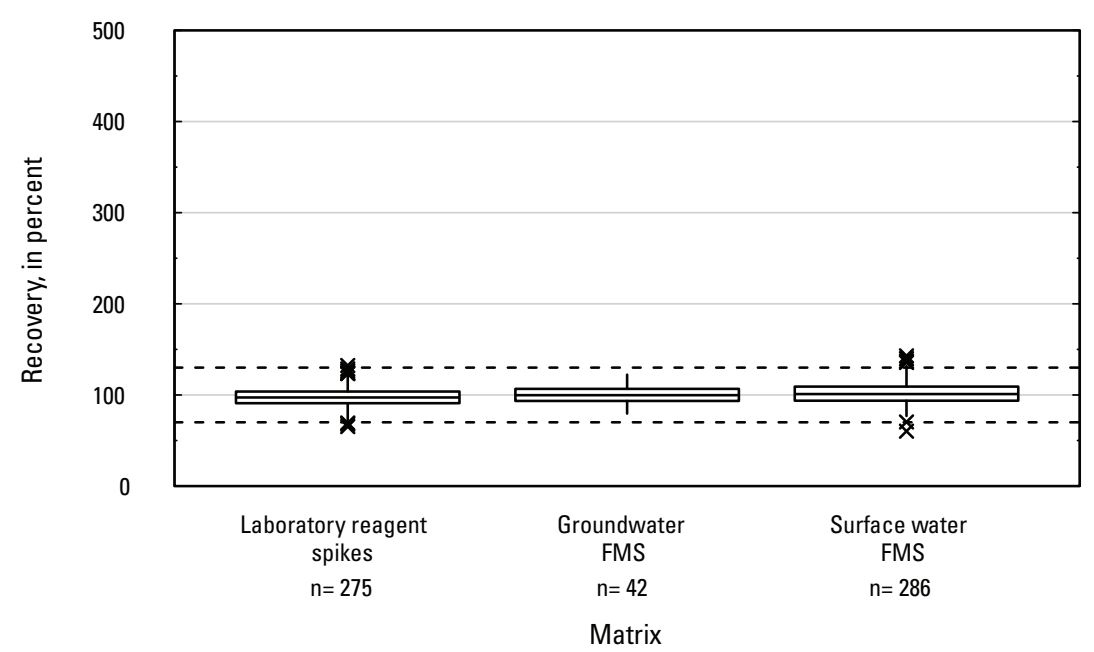

FB. Nicosulfuron

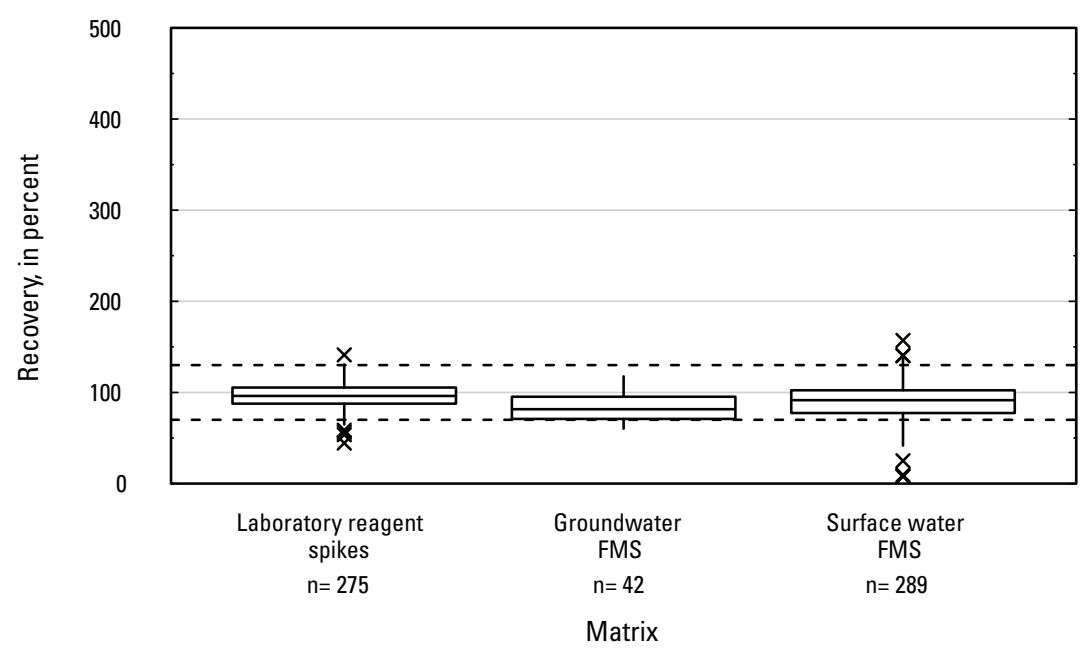

FD. Novaluron

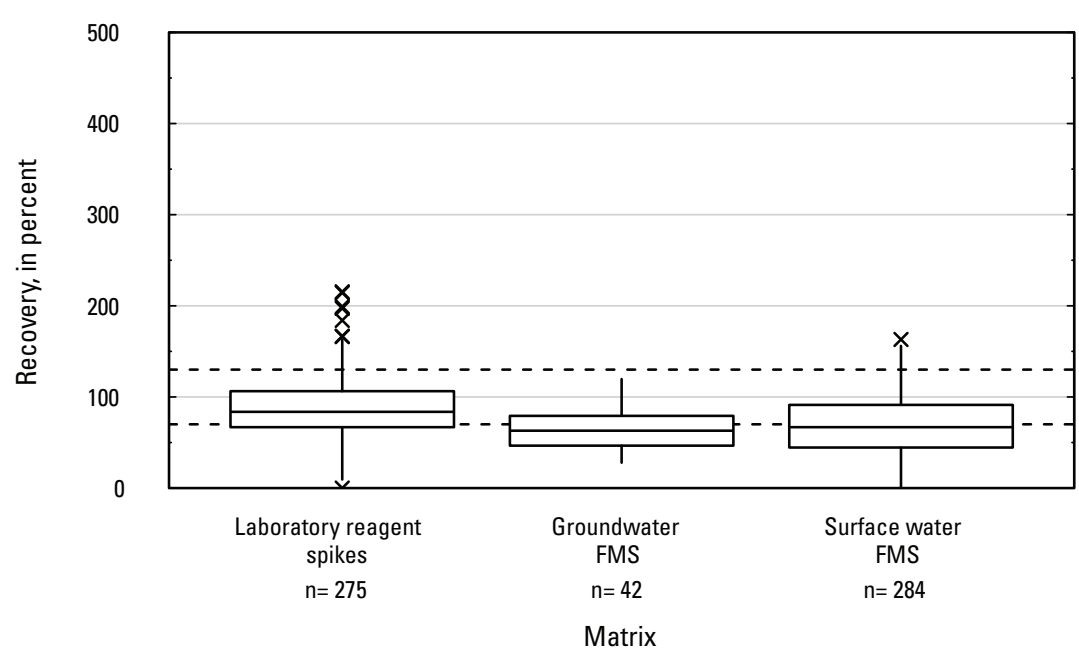

ने

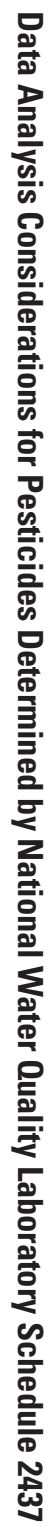

Figure 4. Graph showing distributions of recovery for pesticides in schedule 2437 by matrix. Dashed lines are at 130 percent and 70 percent. Recovery values larger than 400 percent are not shown.-Continued 
FE. 0-Ethyl-0-methyl-S-propylphosphorothioate

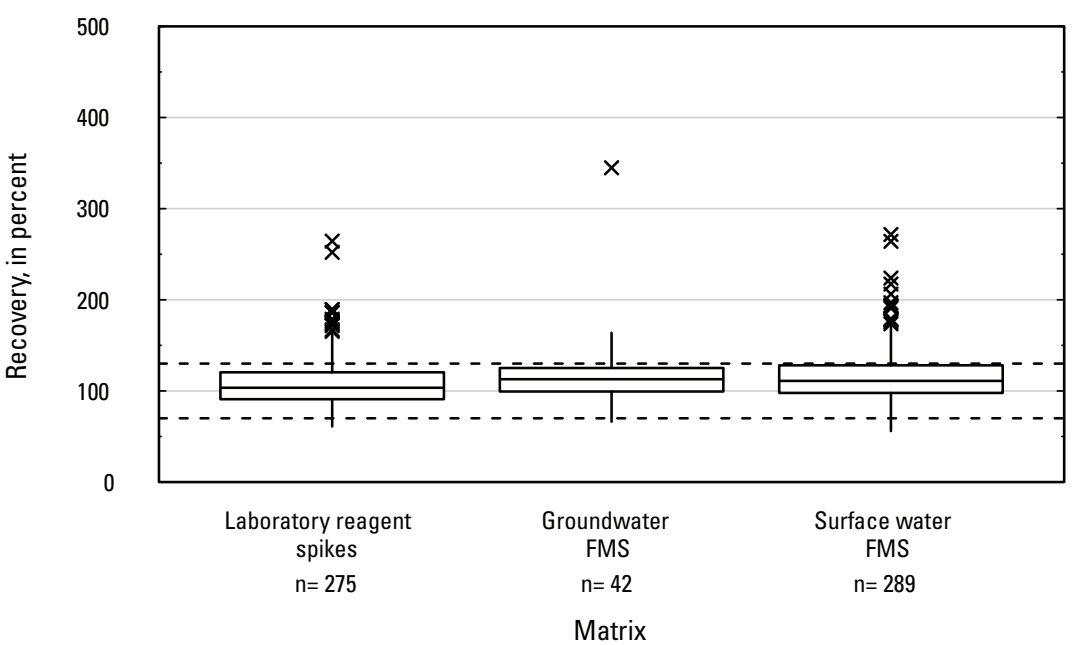

\section{FG. 0-Ethyl-S-propyl phosphorothioate}

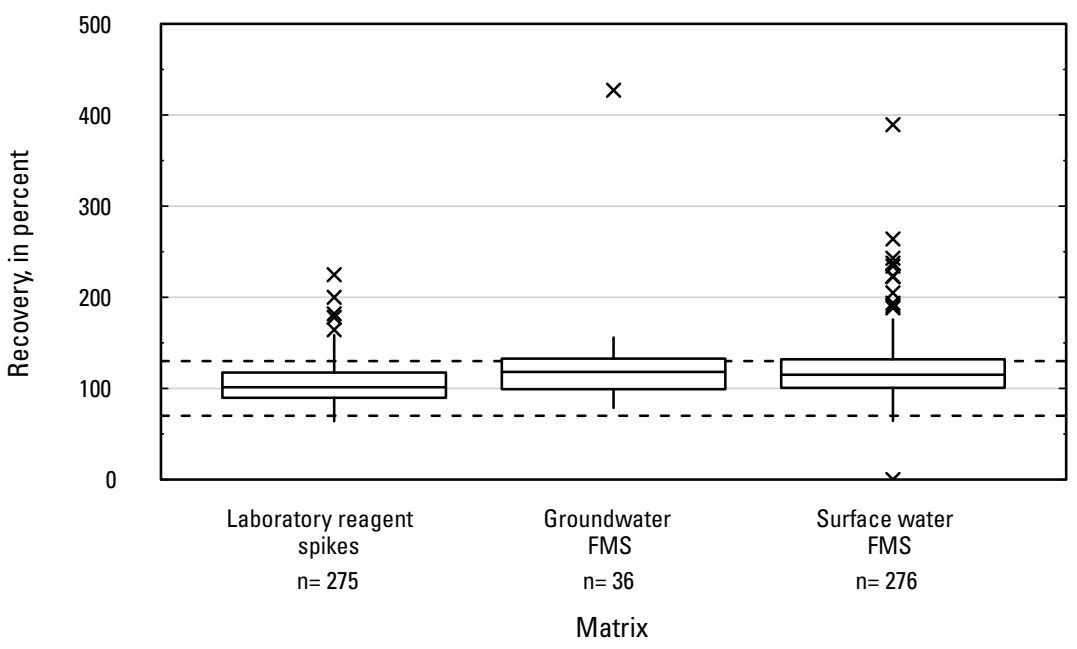

FF. 0-Ethyl-S-methyl-S-propyl phosphorodithioate

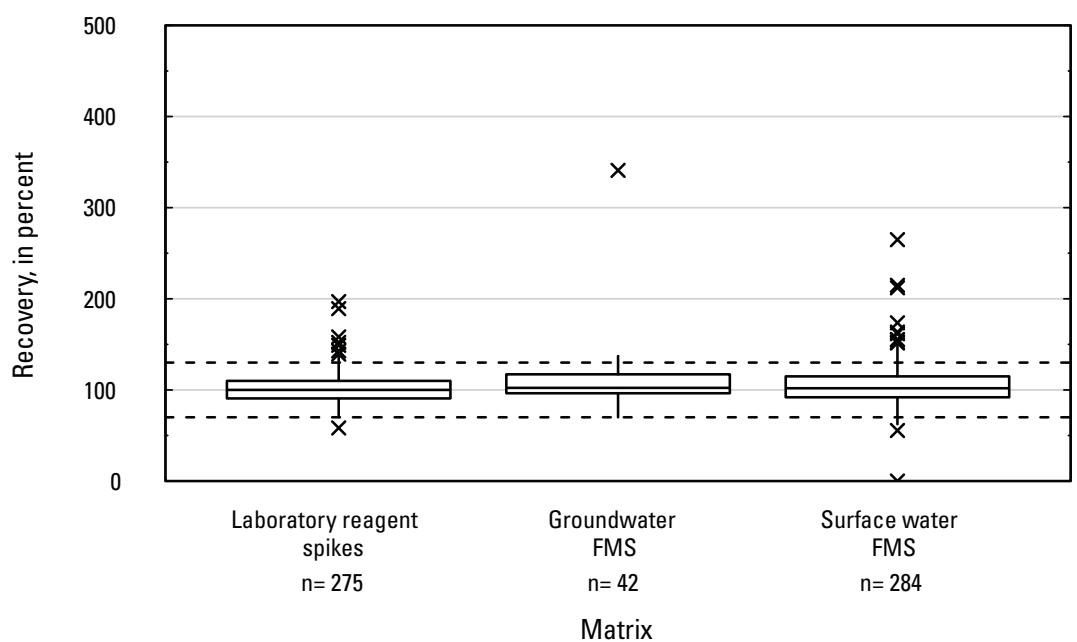

FH. Orthosulfamuron

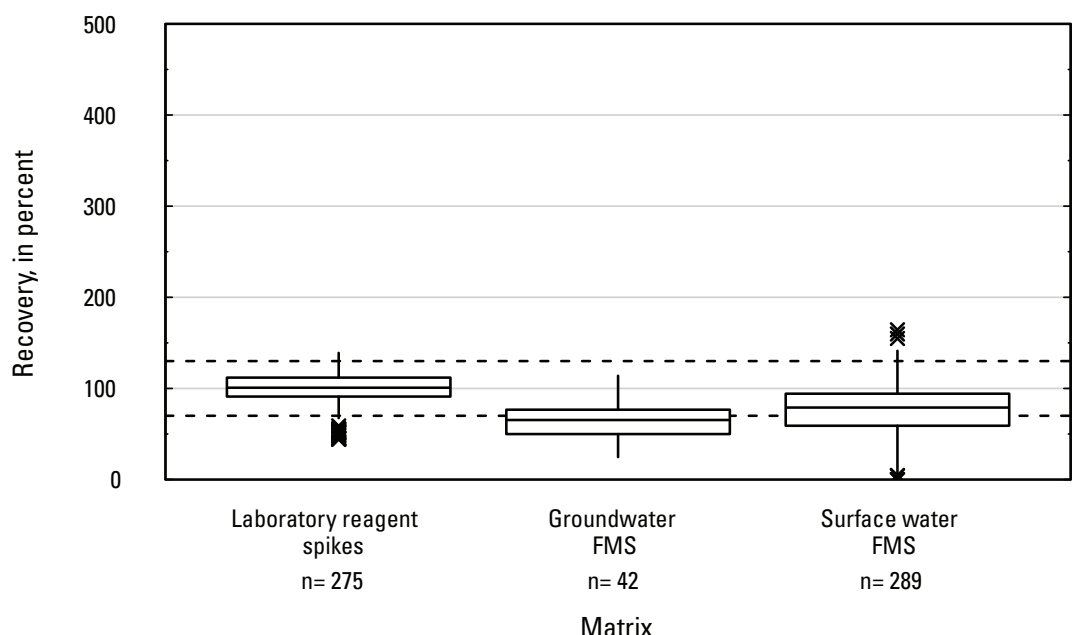

Figure 4. Graph showing distributions of recovery for pesticides in schedule 2437 by matrix. Dashed lines are at 130 percent and 70 percent. Recovery values larger 
FI. Oryzalin

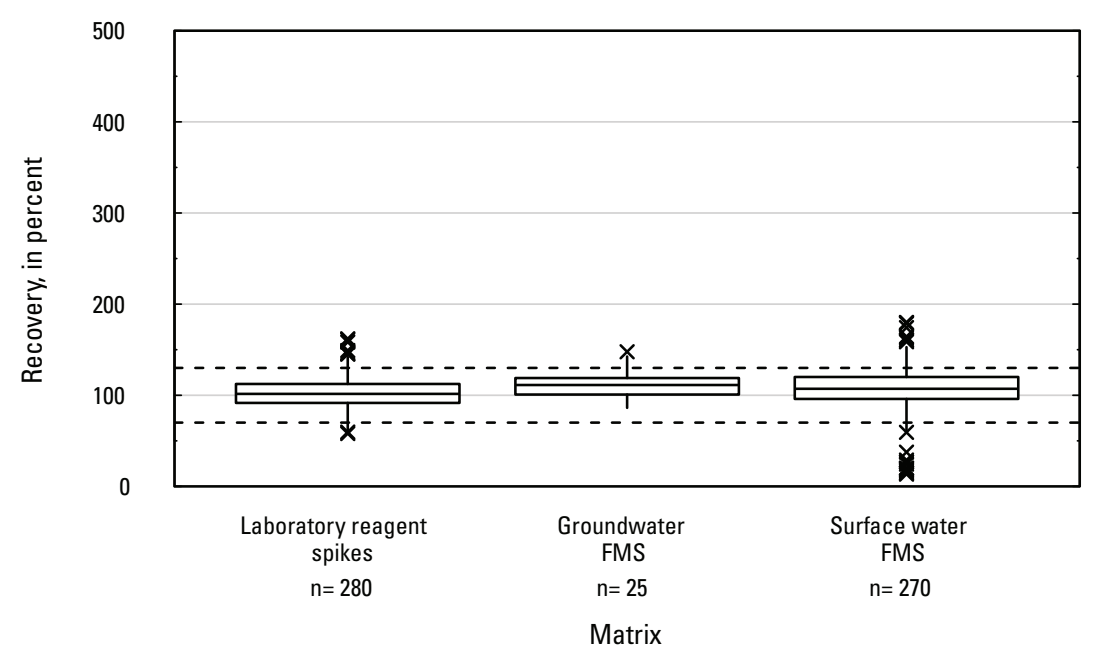

FK. Oxamyl oxime

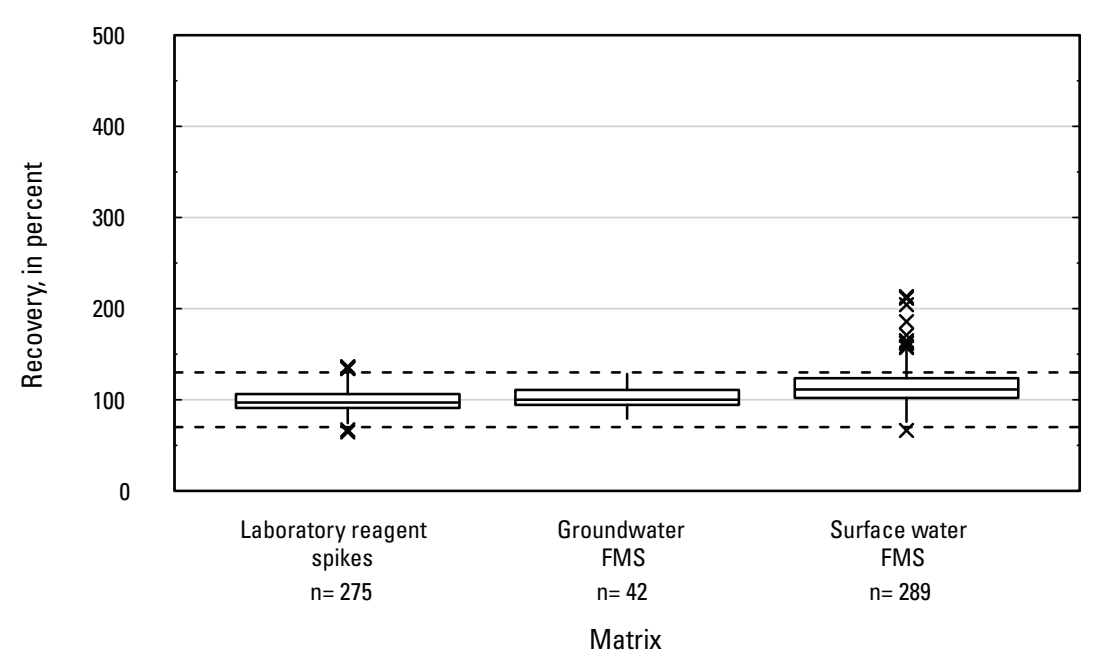

FJ. 0xamyl

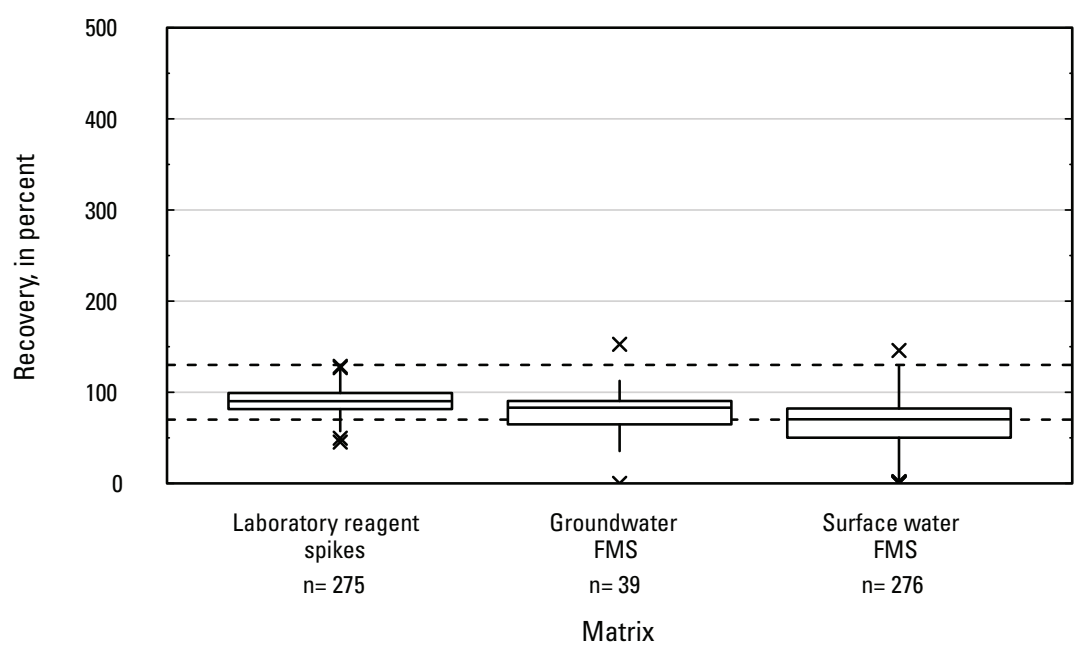

FL. Oxyfluorfen

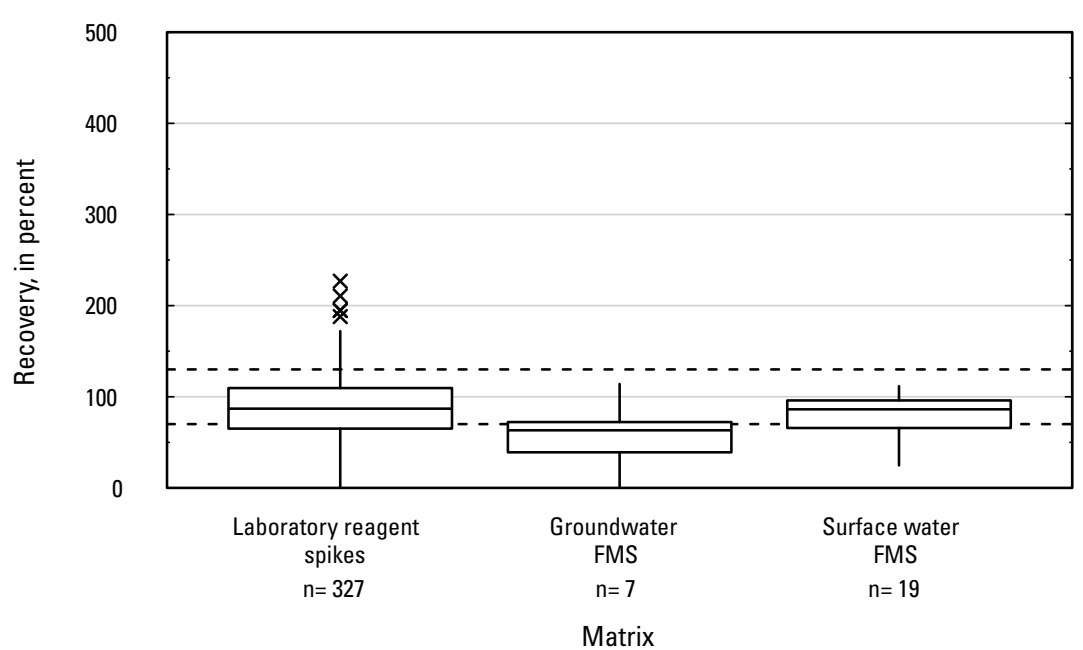

$\infty$

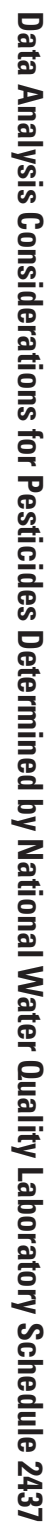

Figure 4. Graph showing distributions of recovery for pesticides in schedule 2437 by matrix. Dashed lines are at 130 percent and 70 percent. Recovery values larger than 400 percent are not shown.-Continued 
FM. Paraoxon

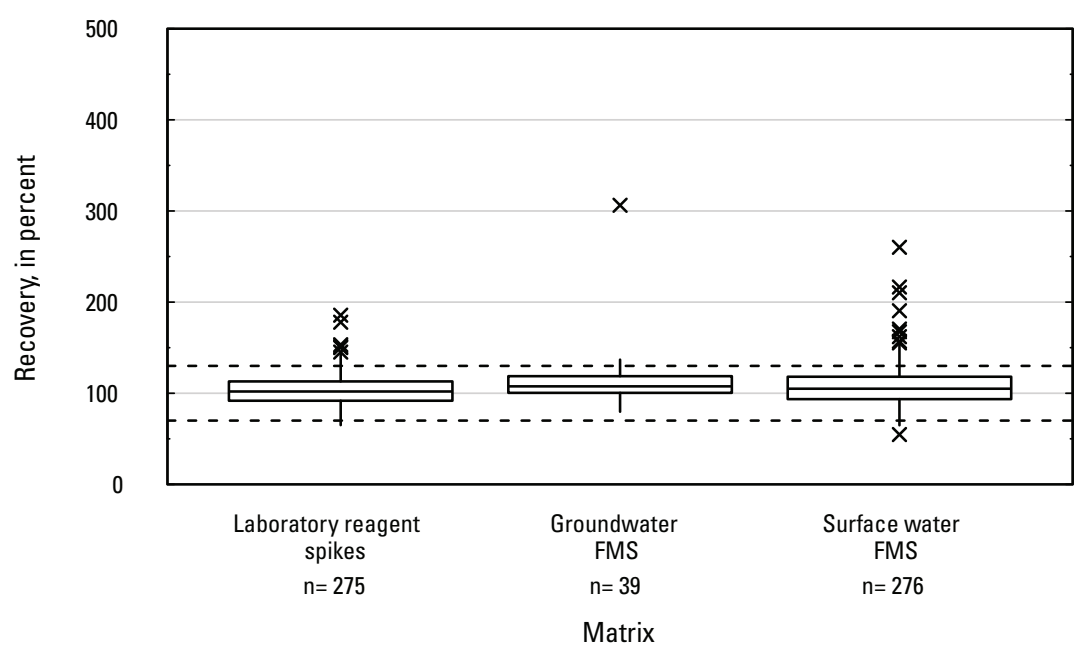

FO. Pendimethalin

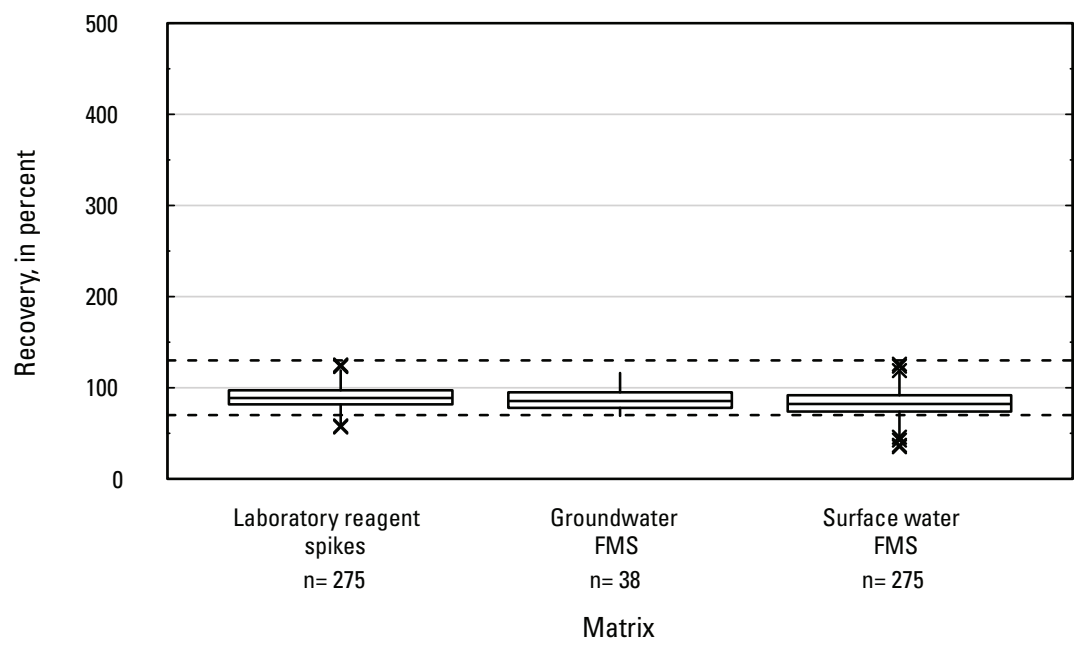

FN. Methyl paraoxon

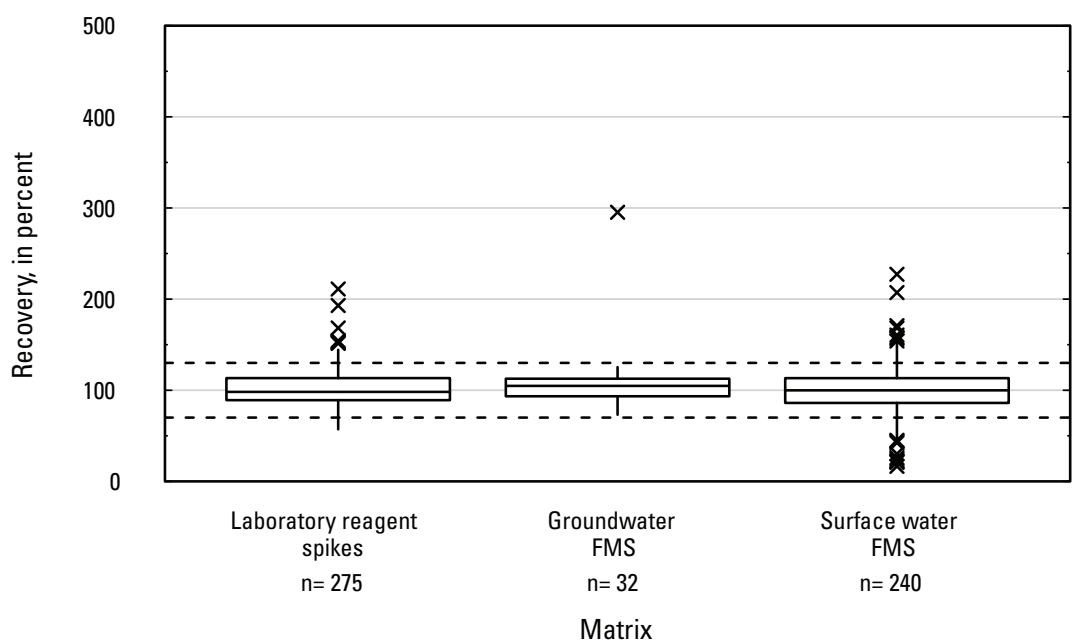

FP. Phorate

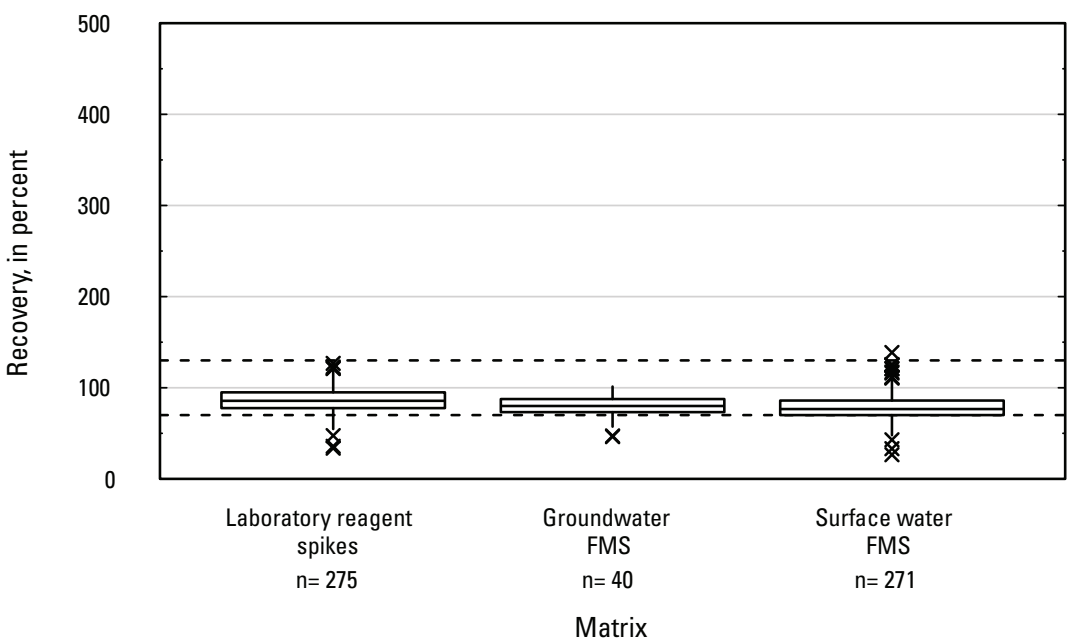

Figure 4. Graph showing distributions of recovery for pesticides in schedule 2437 by matrix. Dashed lines are at 130 percent and 70 percent. Recovery values larger 
FQ. Phorate oxon

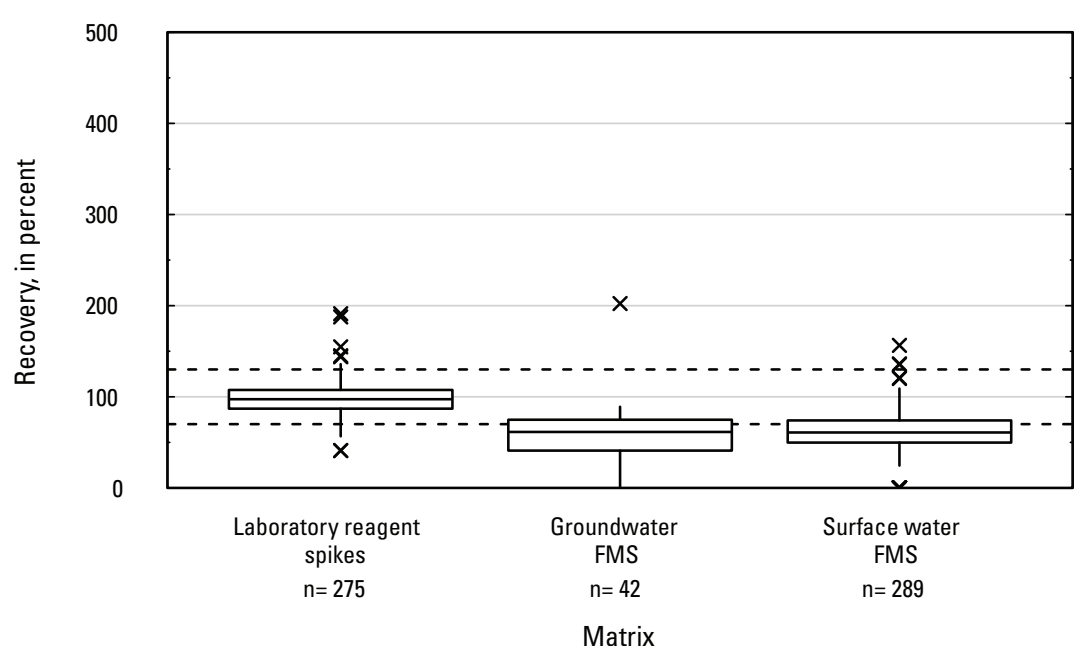

FS. Phorate oxon sulfoxide

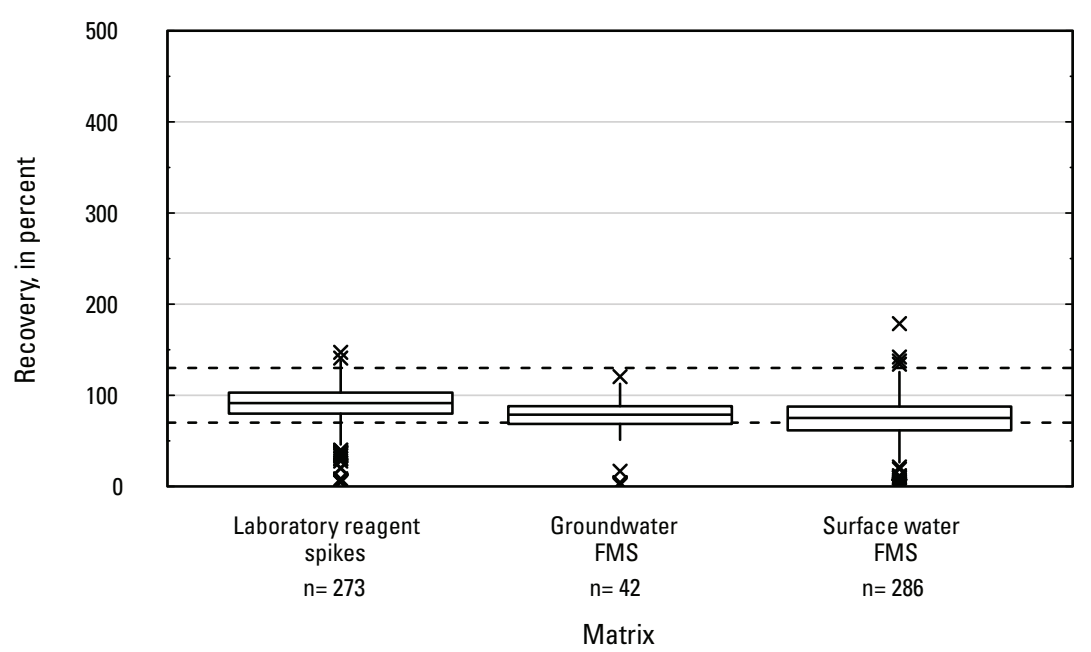

FR. Phorate oxon sulfone

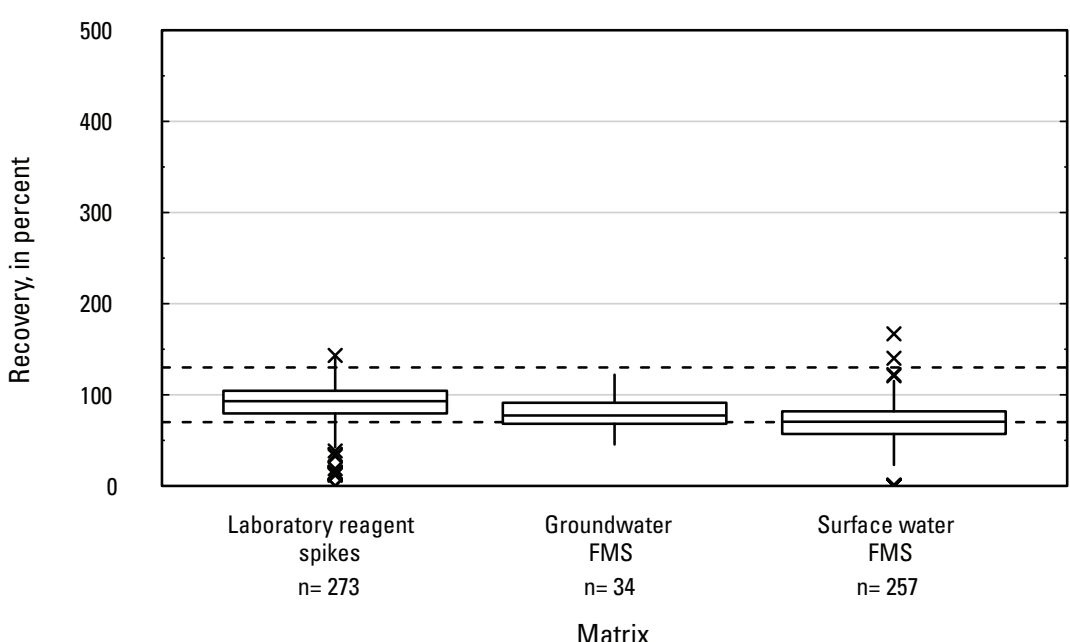

FT. Phorate sulfone

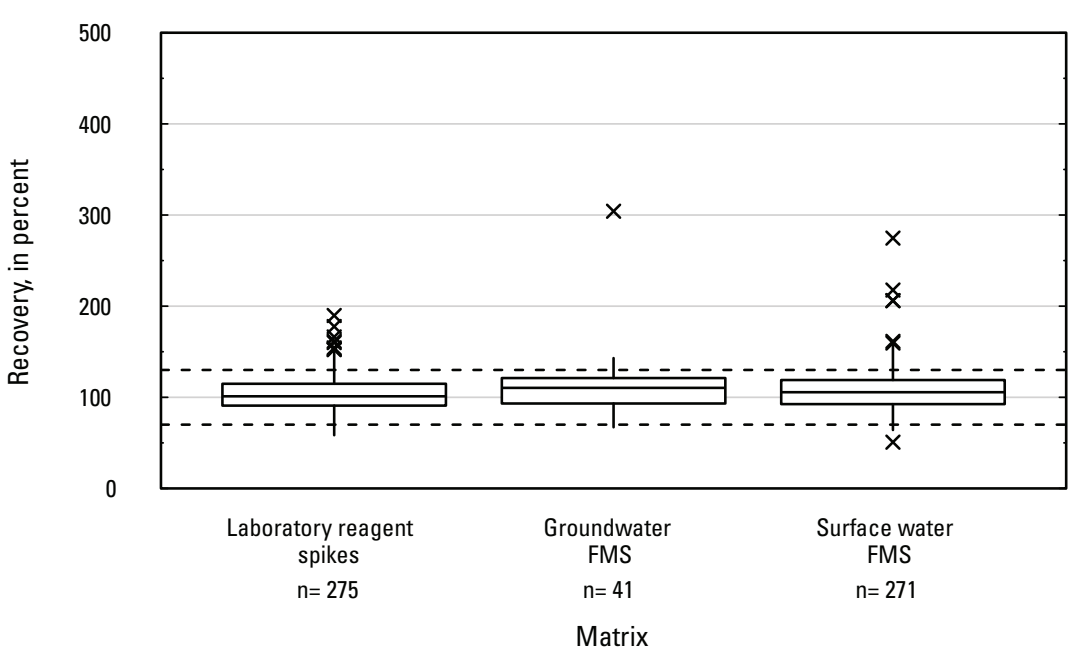

$\stackrel{9}{\circ}$

뭉

Figure 4. Graph showing distributions of recovery for pesticides in schedule 2437 by matrix. Dashed lines are at 130 percent and 70 percent. Recovery values larger than 400 percent are not shown.-Continued 
FU. Phorate sulfoxide

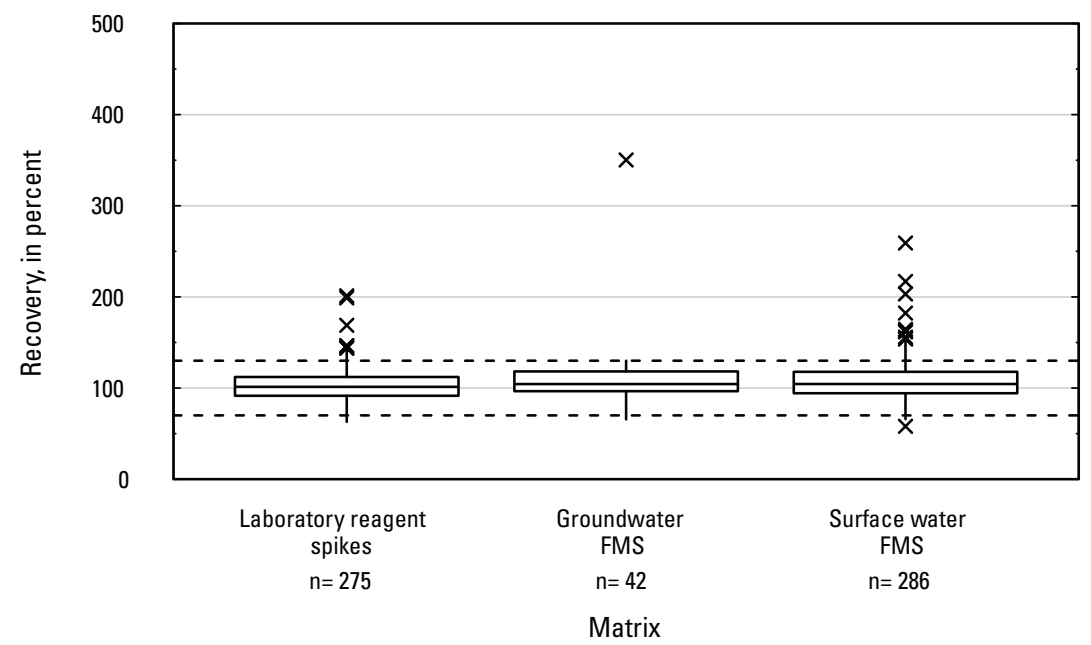

\section{FW. Piperonyl butoxide}

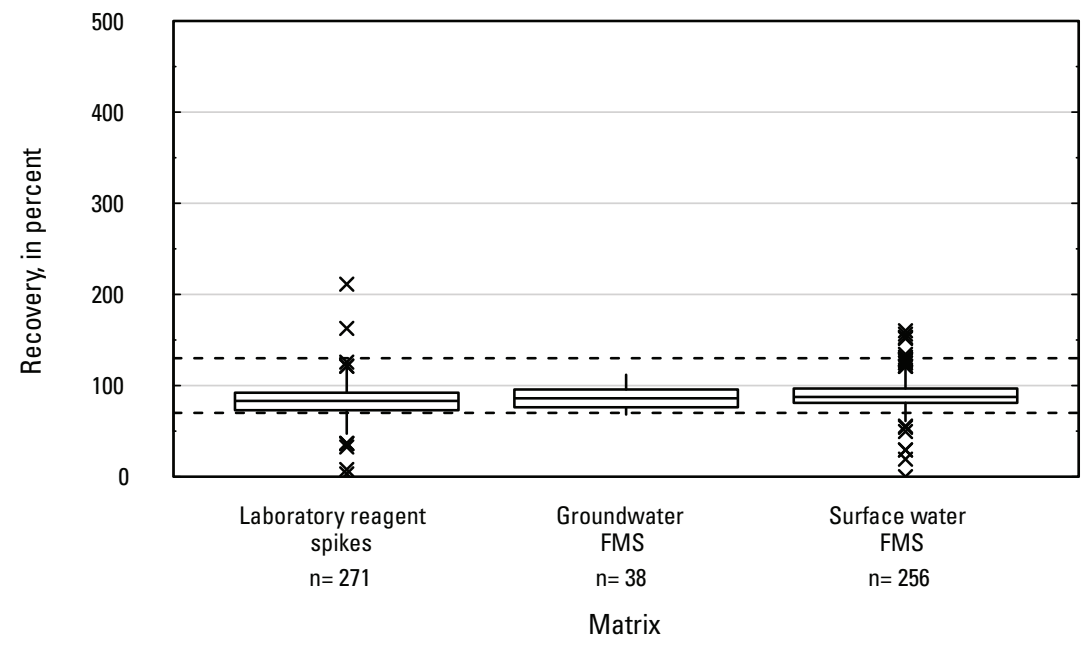

\section{FV. Phthalazinone}

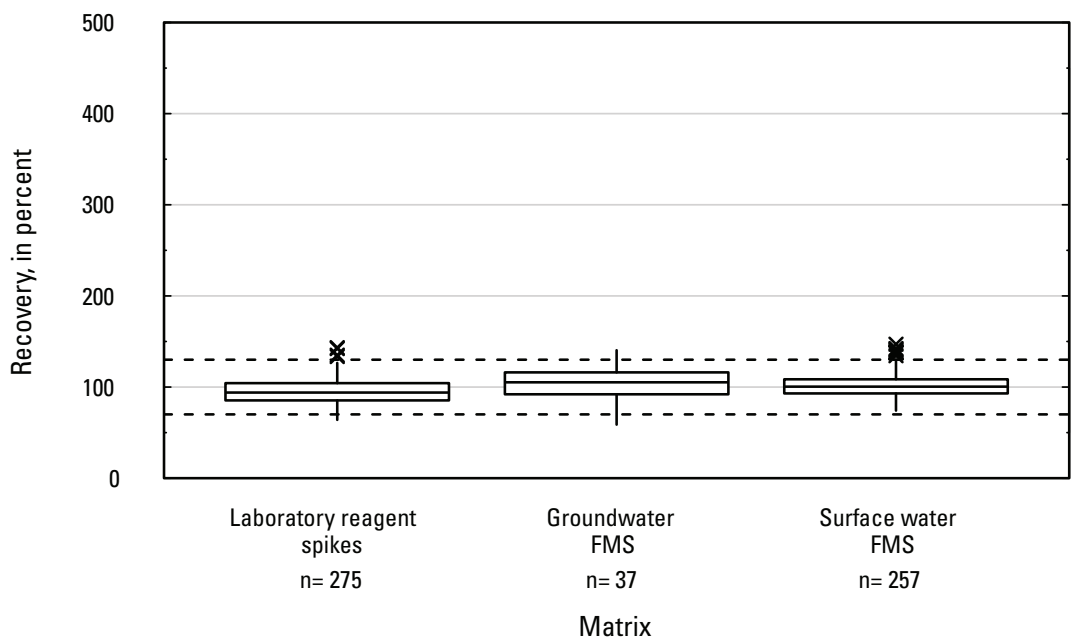

FX. Profenofos

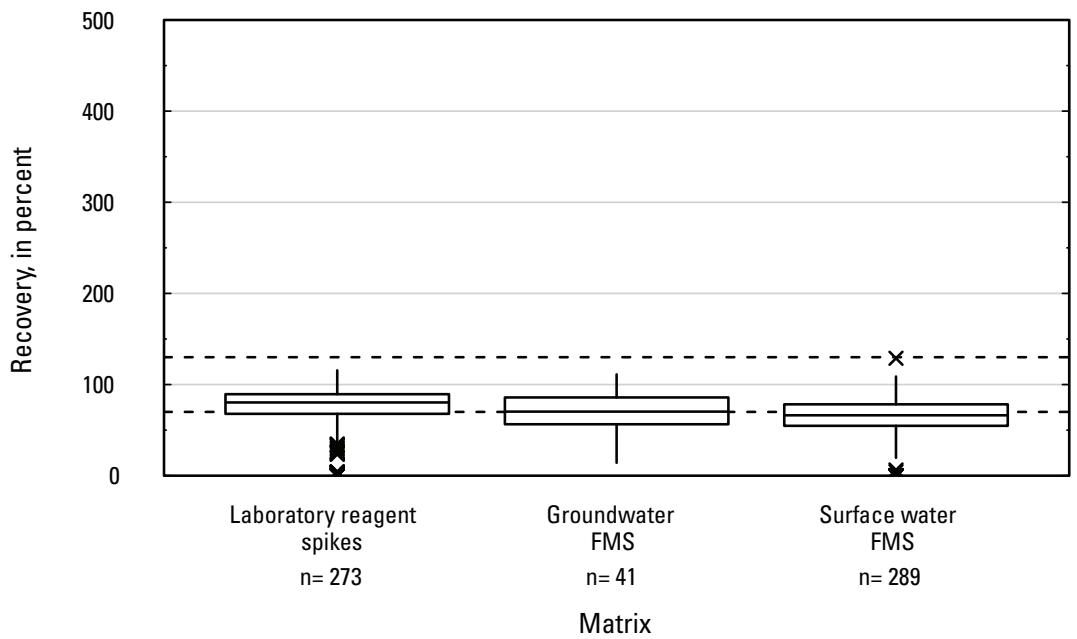

Figure 4. Graph showing distributions of recovery for pesticides in schedule 2437 by matrix. Dashed lines are at 130 percent and 70 percent. Recovery values larger 
FY. Prometon

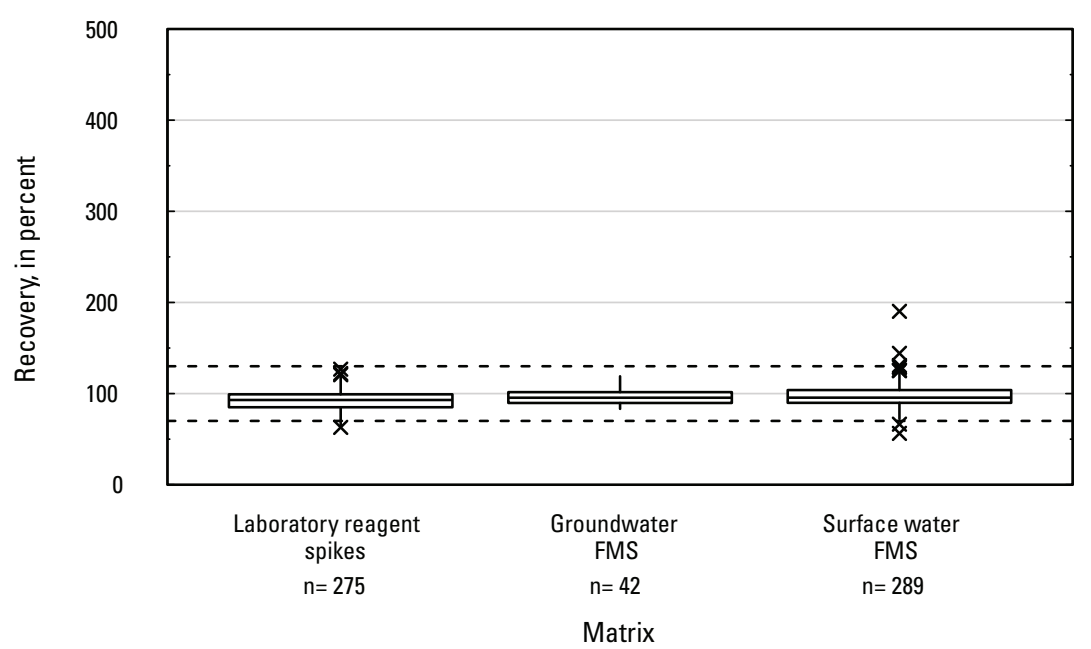

GA. Propyzamide

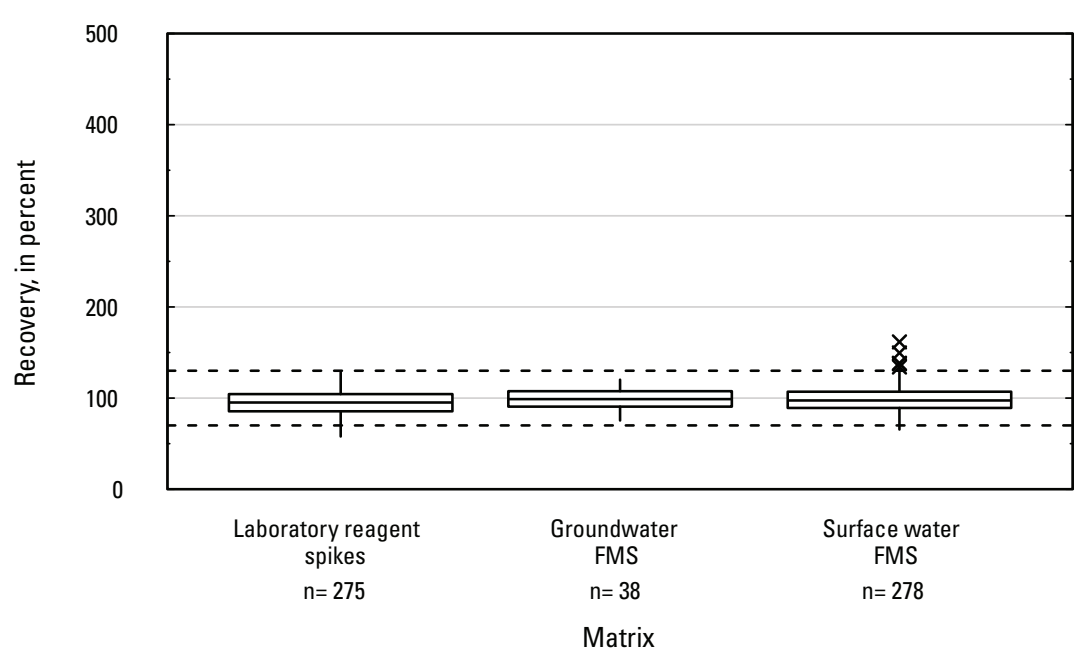

FZ. Prometryn

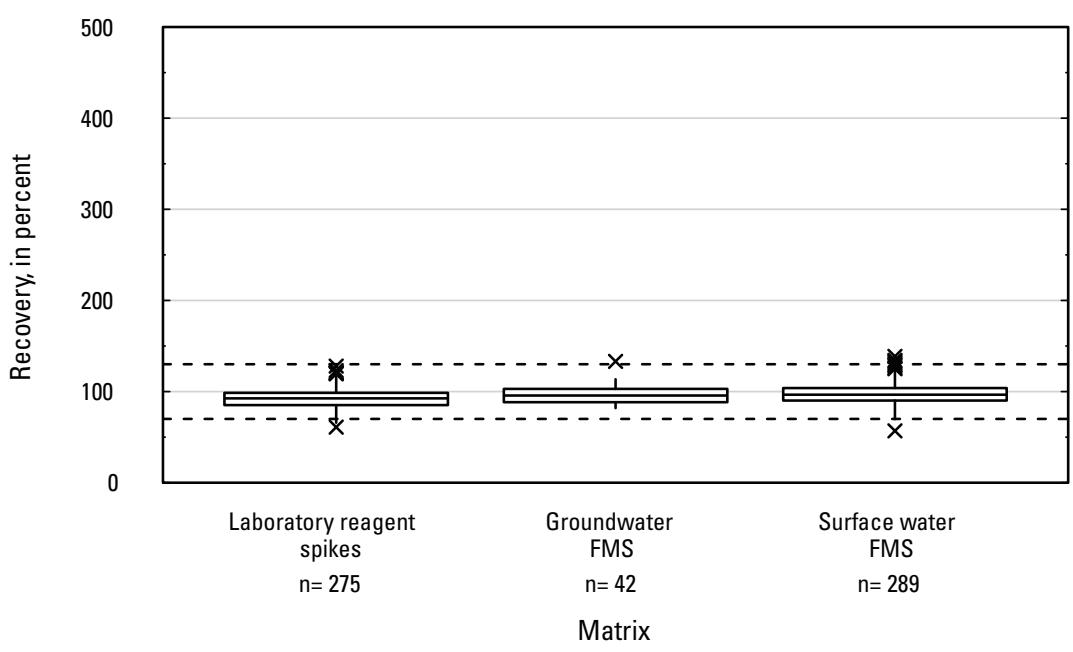

GB. Propanil

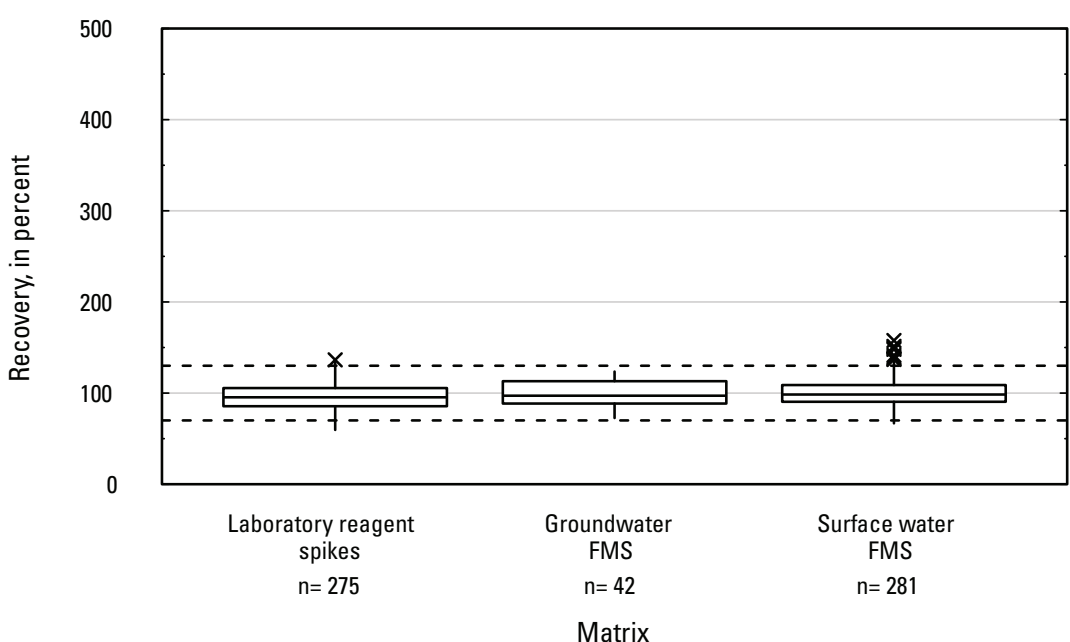

$\stackrel{\infty}{N}$

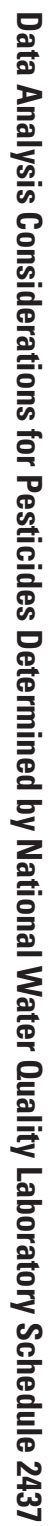

Figure 4. Graph showing distributions of recovery for pesticides in schedule 2437 by matrix. Dashed lines are at 130 percent and 70 percent. Recovery values larger than 400 percent are not shown.-Continued 
GC. Propargite

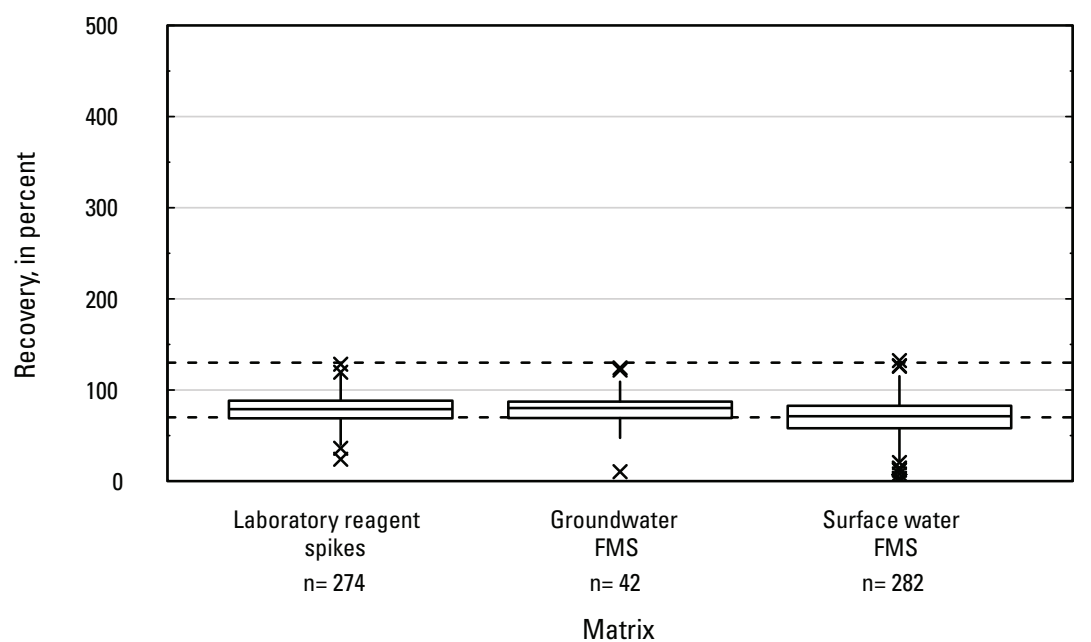

GE. Propiconazole

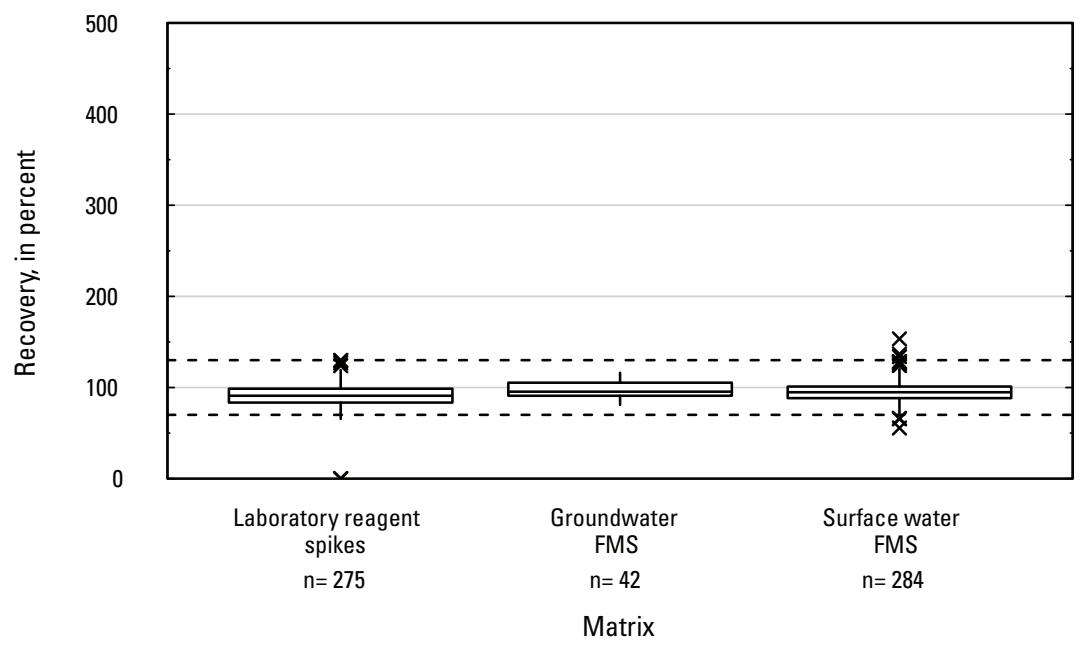

GD. Propazine

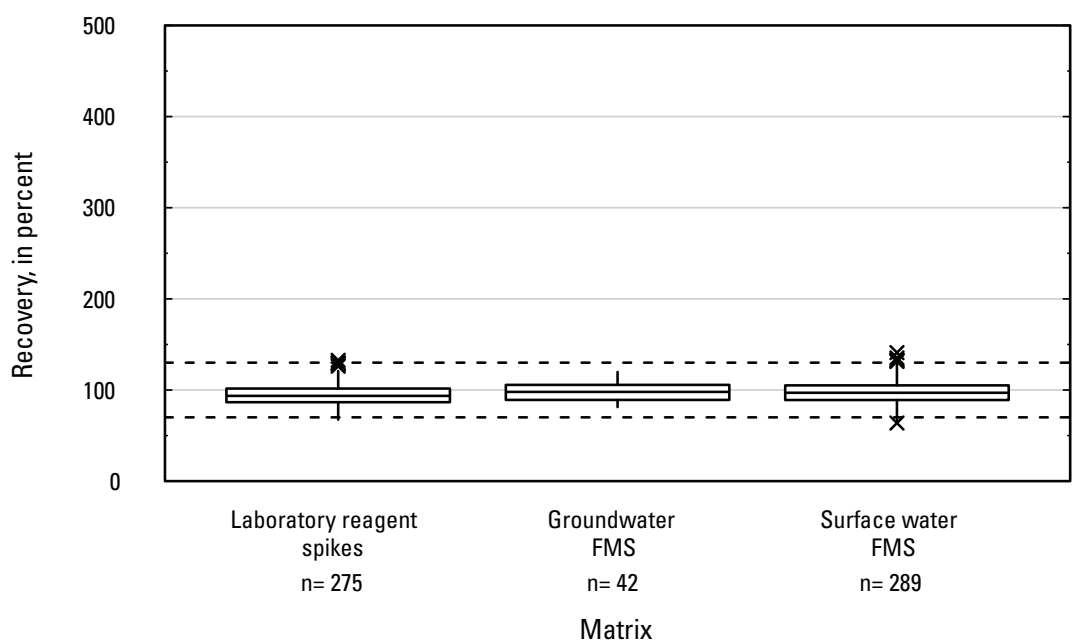

GF. Propoxur

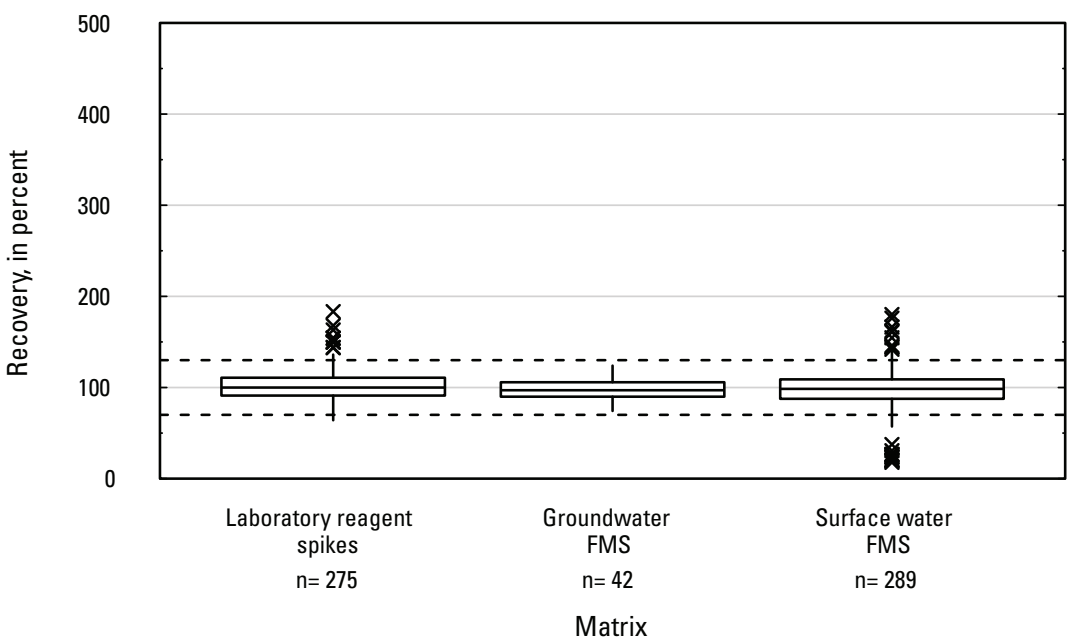

Figure 4. Graph showing distributions of recovery for pesticides in schedule 2437 by matrix. Dashed lines are at 130 percent and 70 percent. Recovery values larger 
GG. Prosulfuron

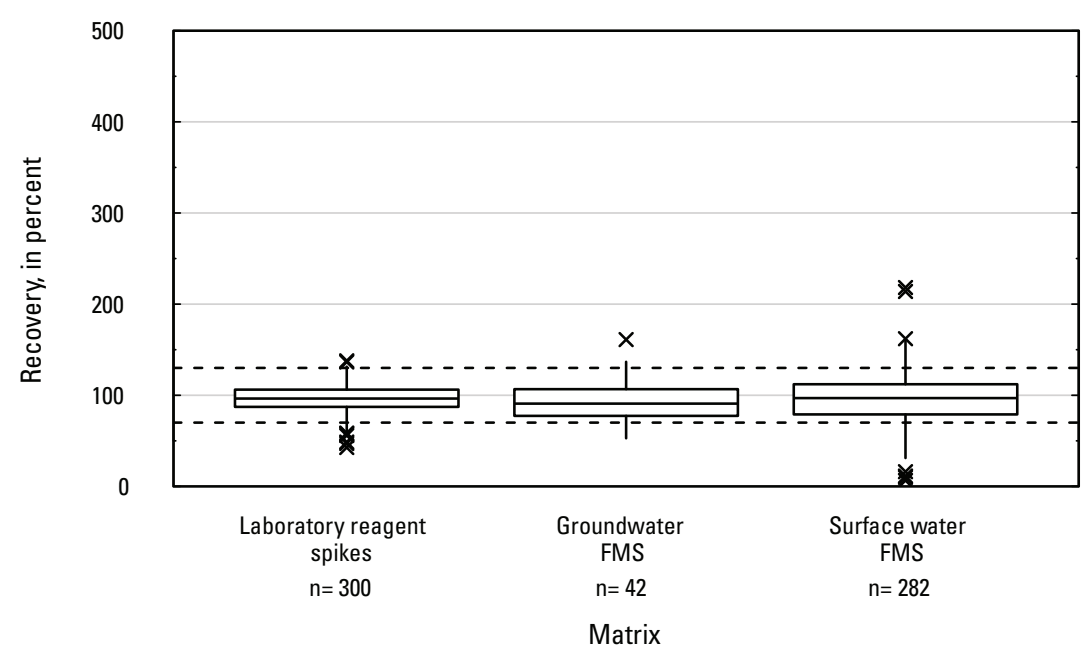

GI. Pyridaben

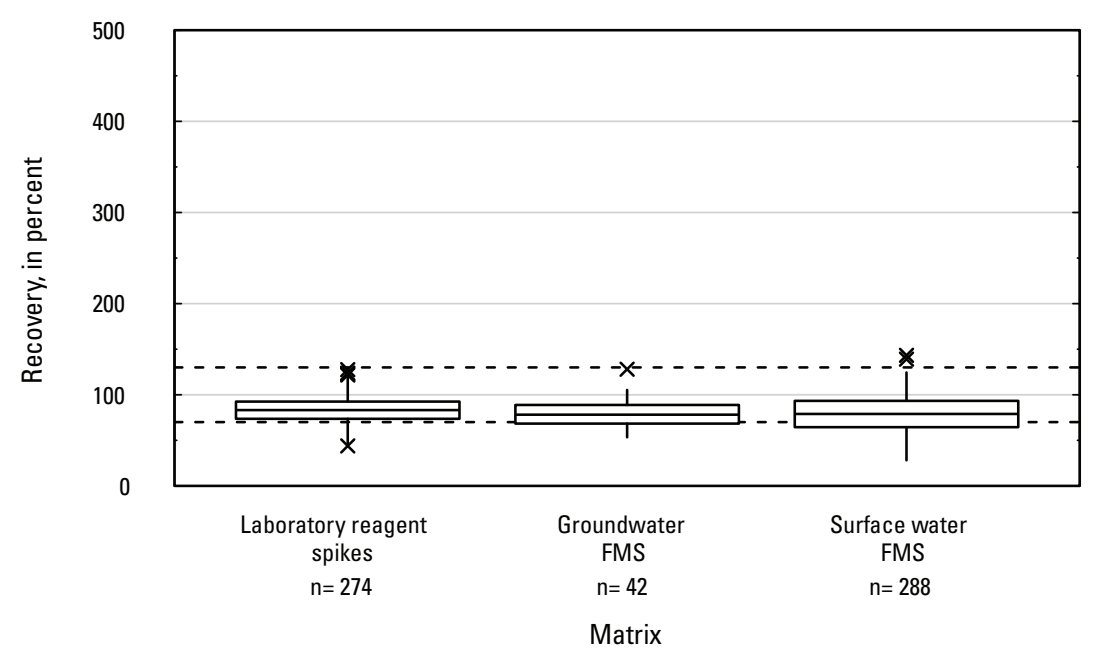

GH. Pyraclostrobin

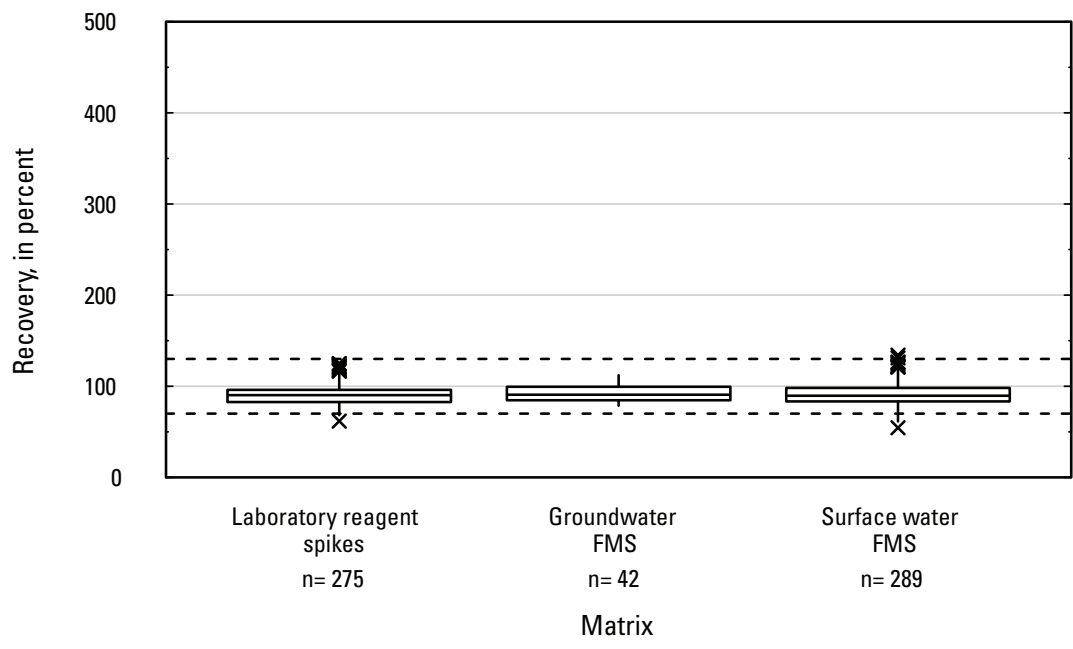

GJ. 2-Isopropyl-6-methyl-4-pyrimidinol

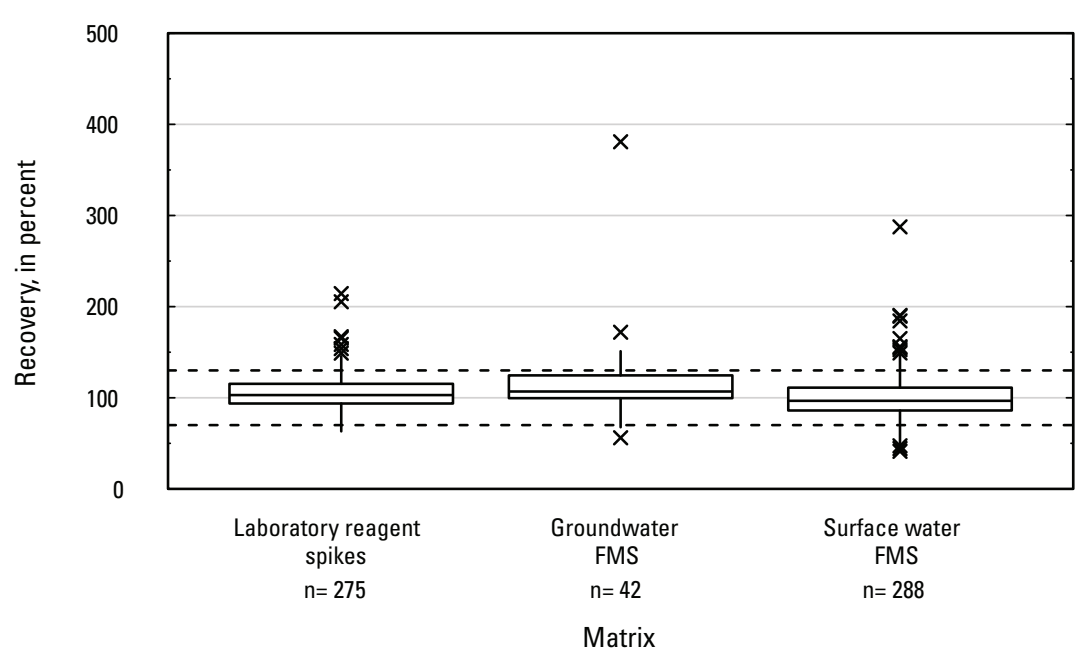

$\stackrel{\infty}{\oplus}$

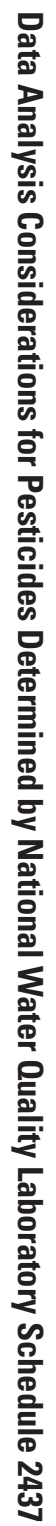

Figure 4. Graph showing distributions of recovery for pesticides in schedule 2437 by matrix. Dashed lines are at 130 percent and 70 percent. Recovery values larger than 400 percent are not shown.-Continued 
GK. Pyriproxyfen

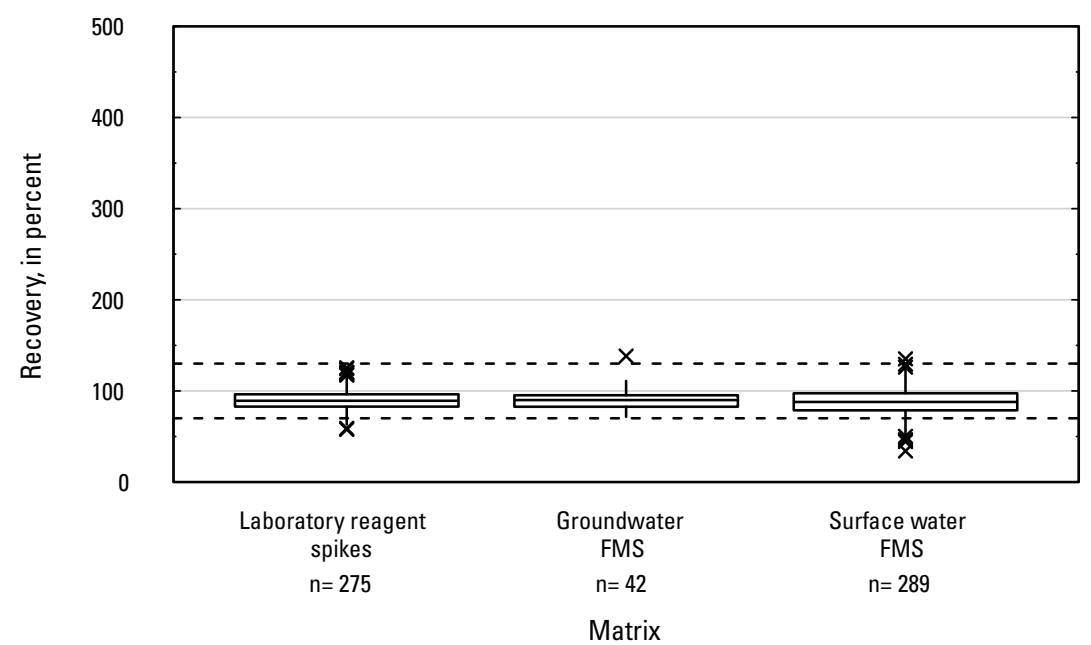

GM. sec-Alachlor oxanilic acid

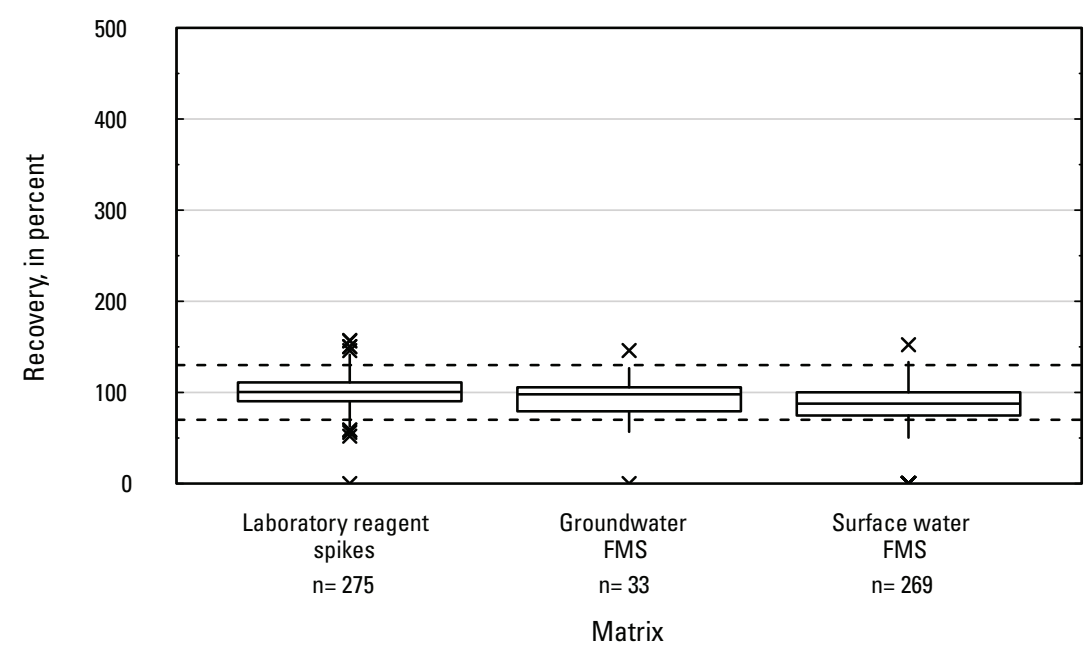

GL. sec-Acetochlor oxanilic acid

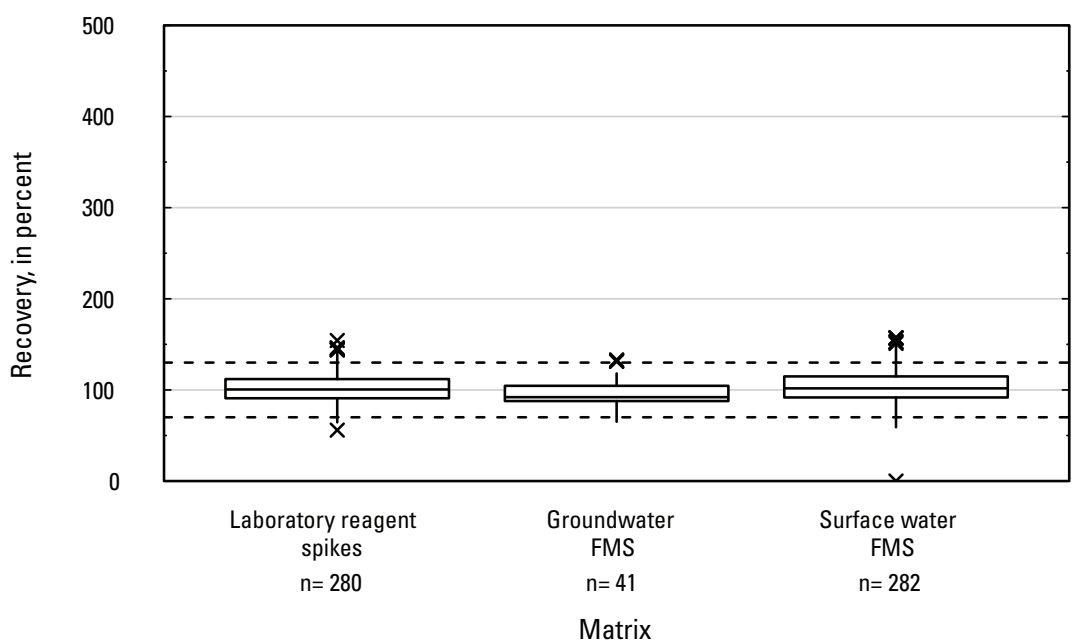

GN. Siduron

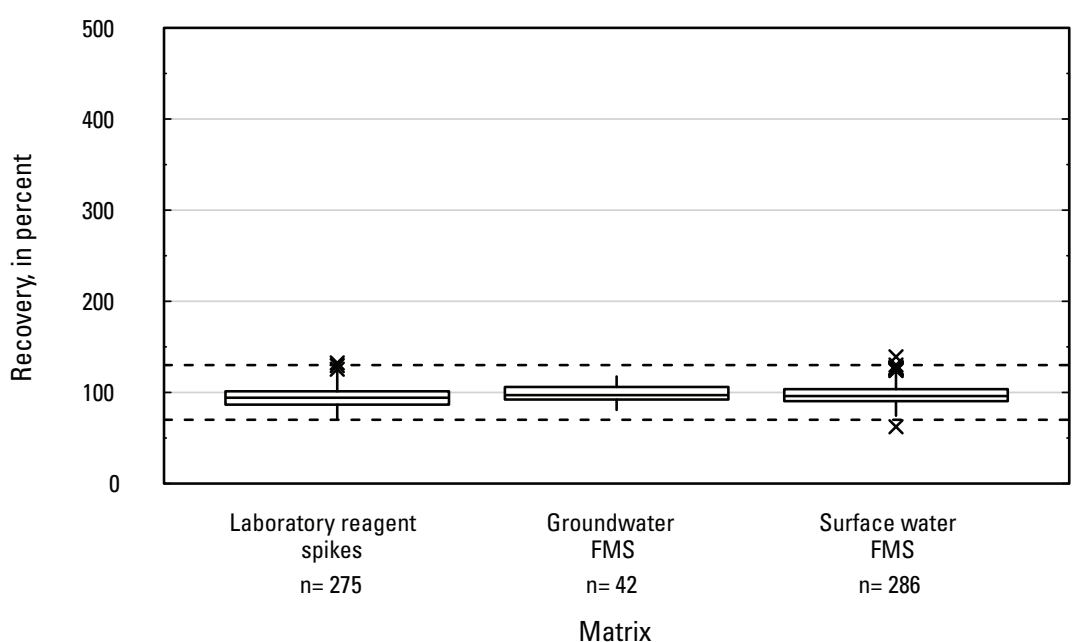

Figure 4. Graph showing distributions of recovery for pesticides in schedule 2437 by matrix. Dashed lines are at 130 percent and 70 percent. Recovery values larger 
GO. Simazine

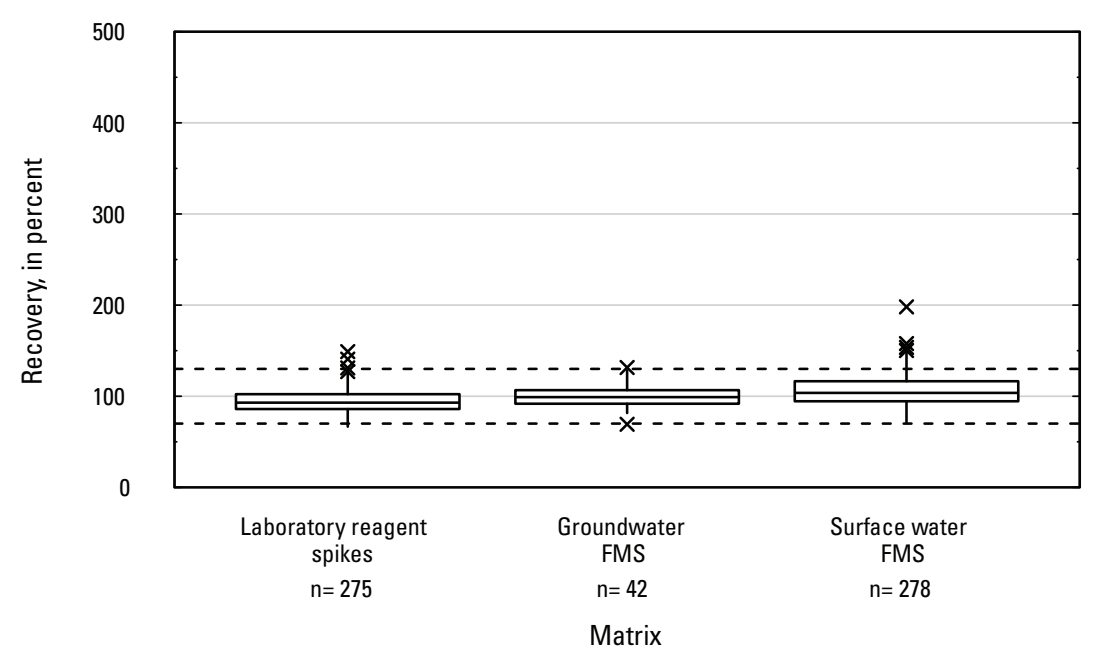

GQ. Sulfometuron-methyl

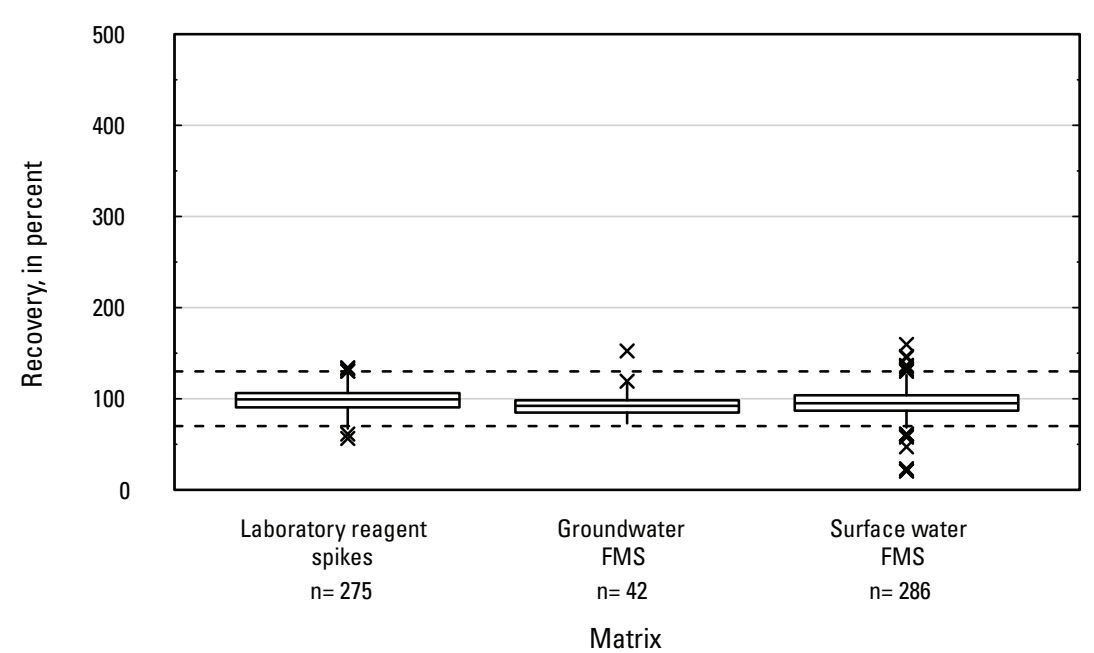

GP. Sulfentrazone

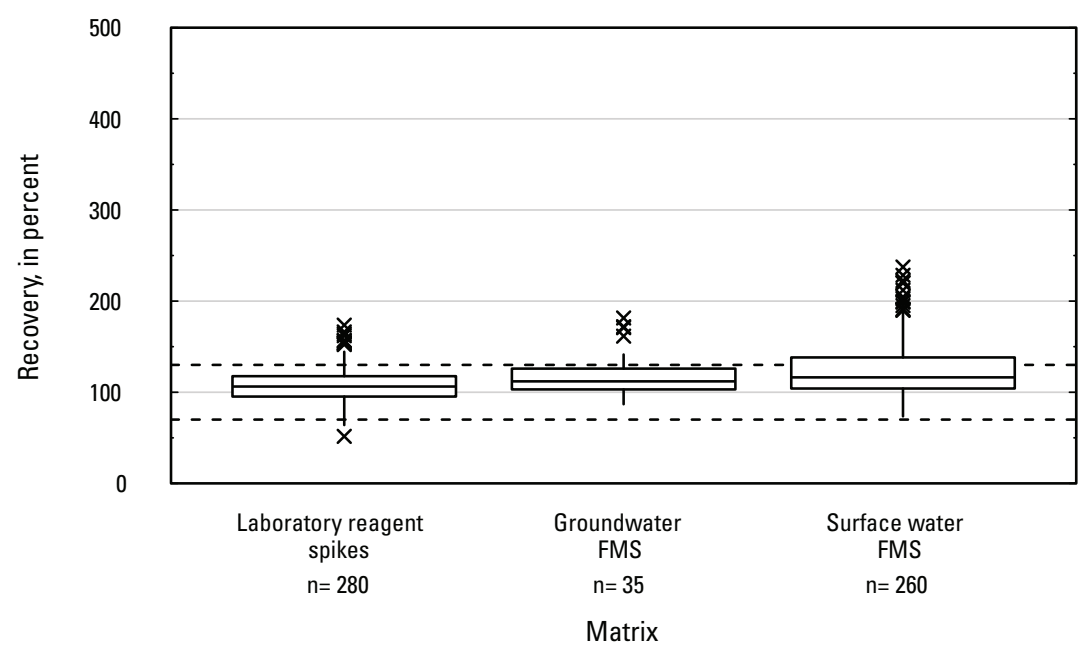

\section{GR. Sulfosulfuron}

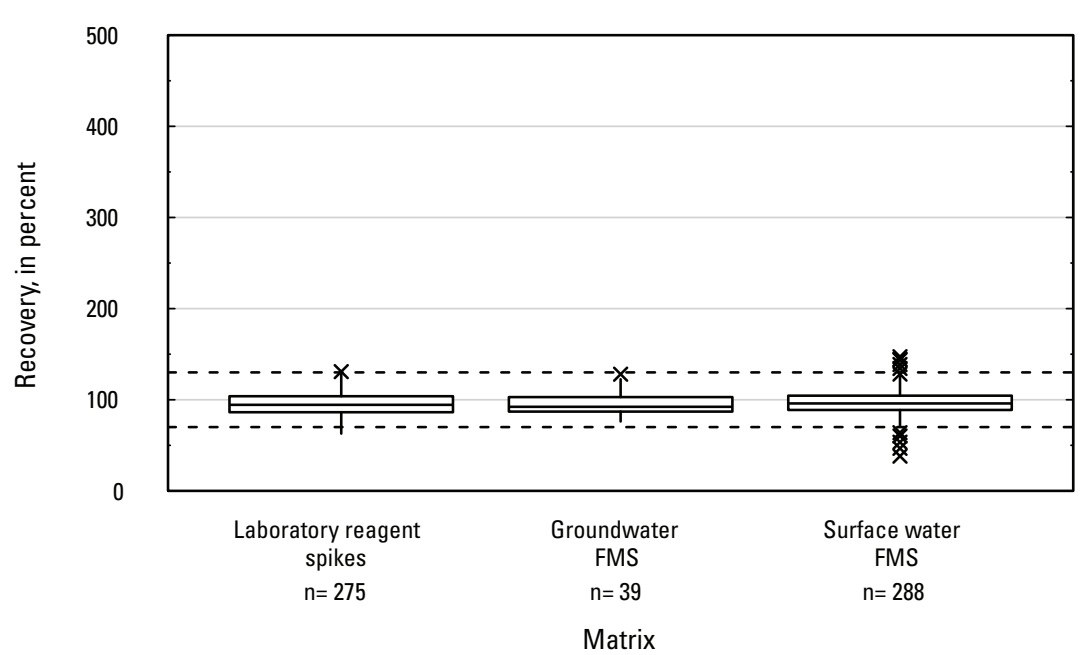

$\stackrel{\infty}{\infty}$

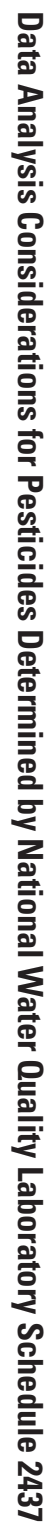

Figure 4. Graph showing distributions of recovery for pesticides in schedule 2437 by matrix. Dashed lines are at 130 percent and 70 percent. Recovery values larger than 400 percent are not shown.-Continued 
GS. Sulfosulfuron ethyl sulfone

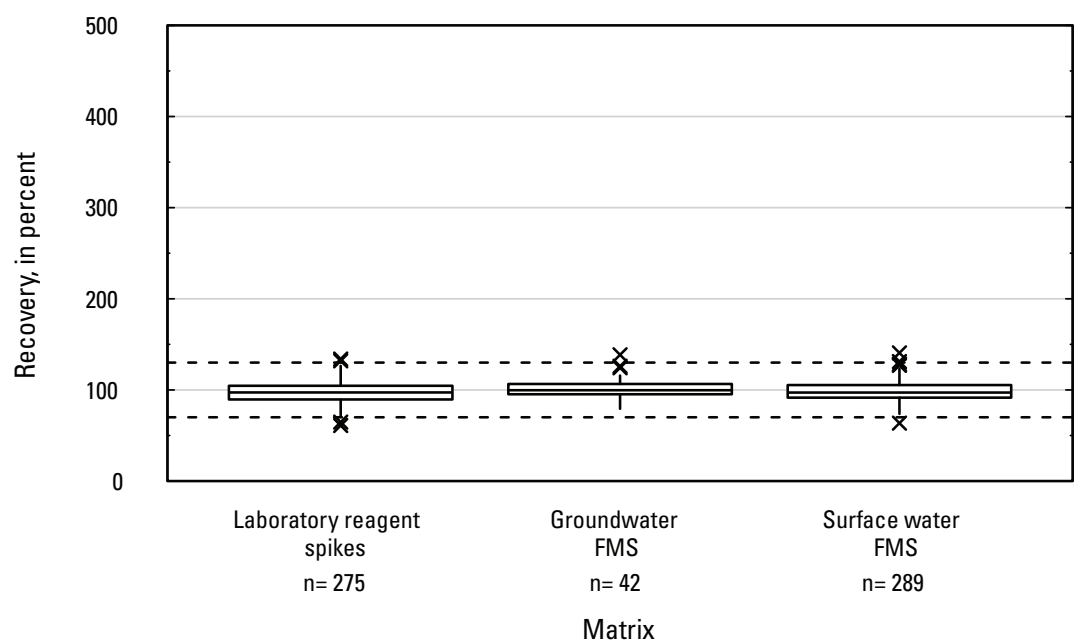

GU. Tebufenozide

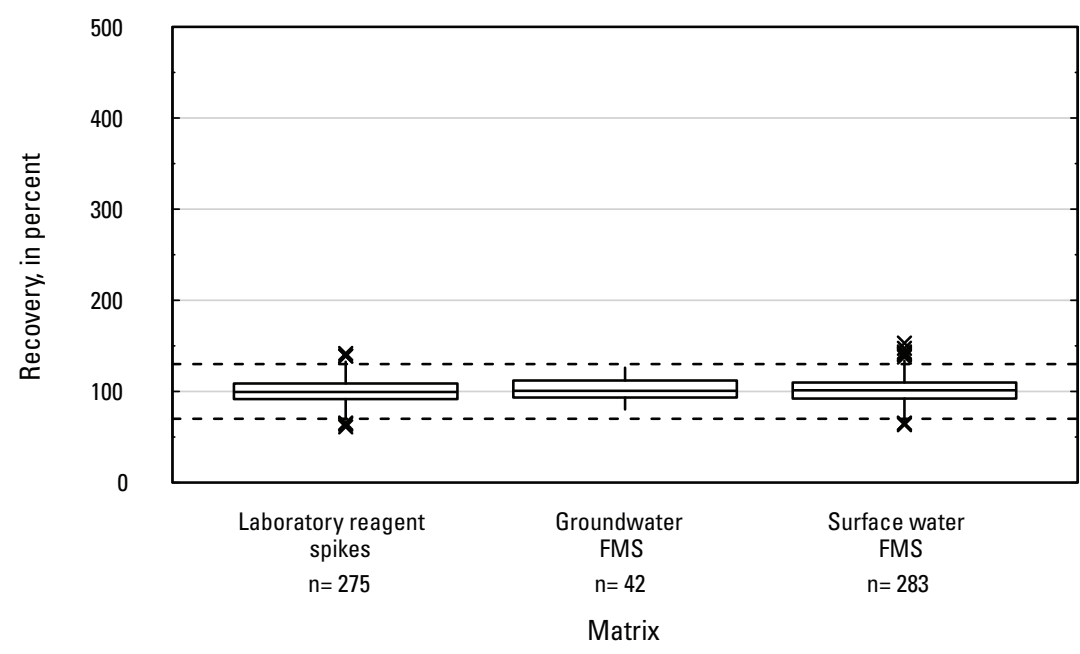

GT. Tebuconazole

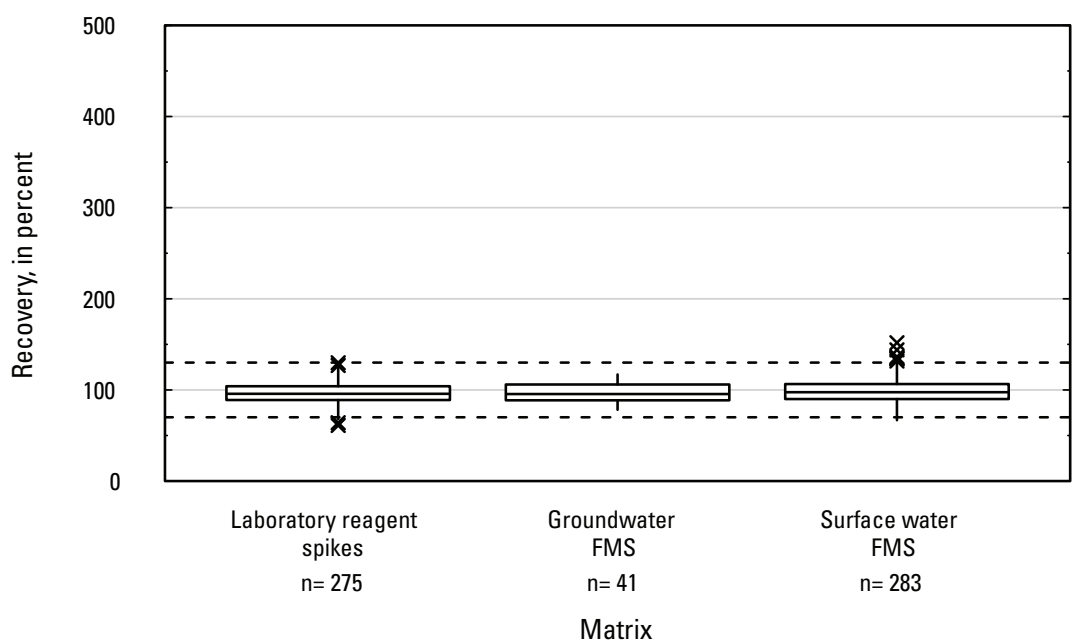

GV. Tebupirimphos

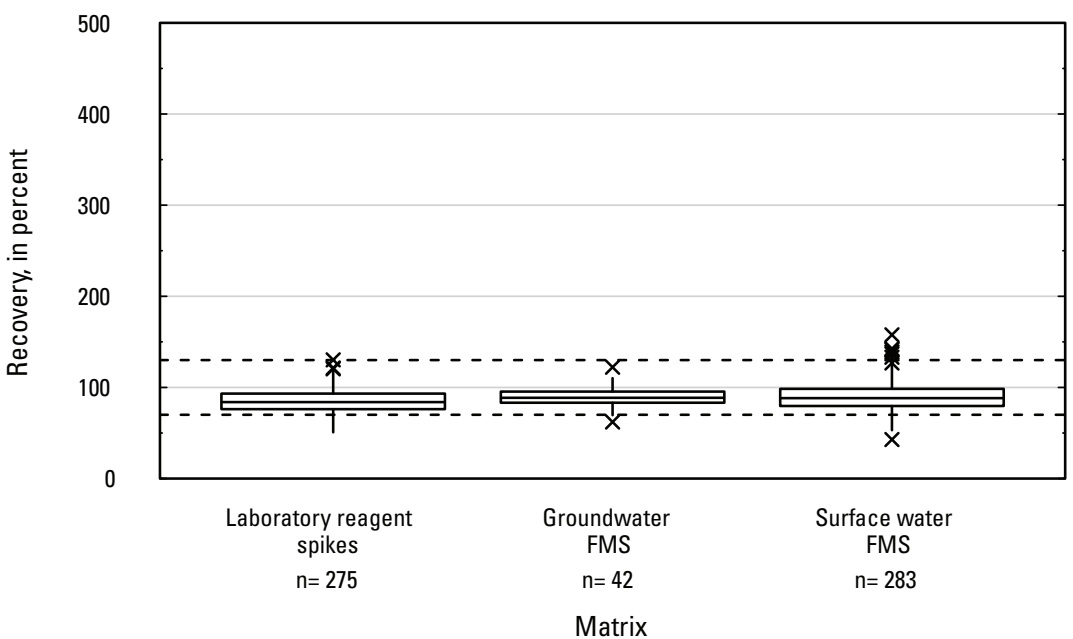

Figure 4. Graph showing distributions of recovery for pesticides in schedule 2437 by matrix. Dashed lines are at 130 percent and 70 percent. Recovery values larger than 400 percent are not shown.-Continued 
GW. Tebupirimfos oxon

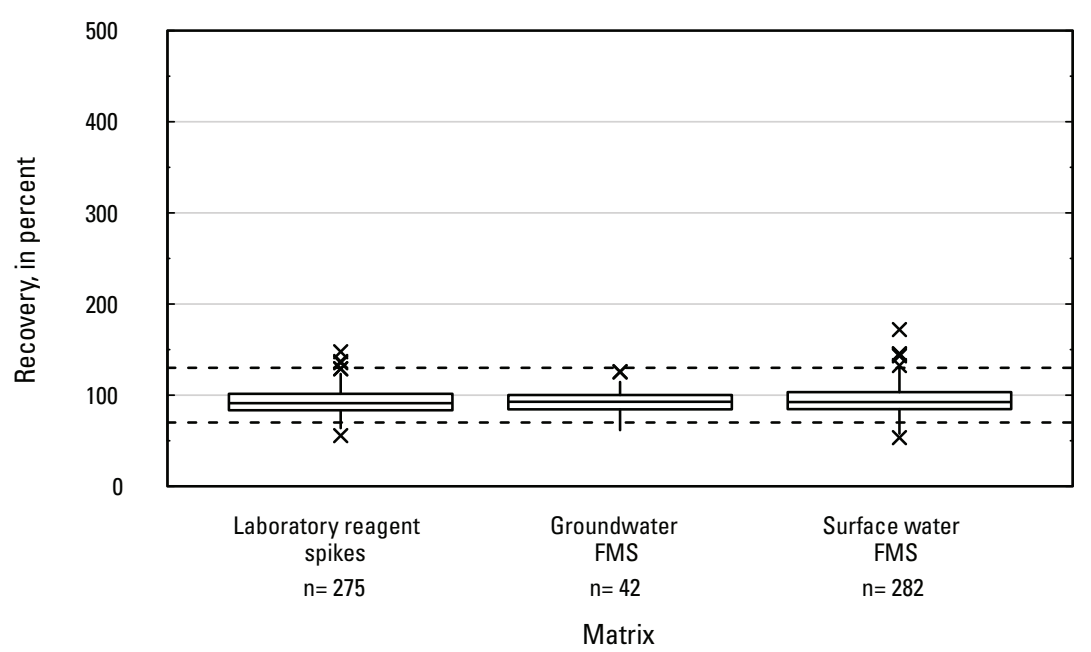

GY. Tebuthiuron TP 104

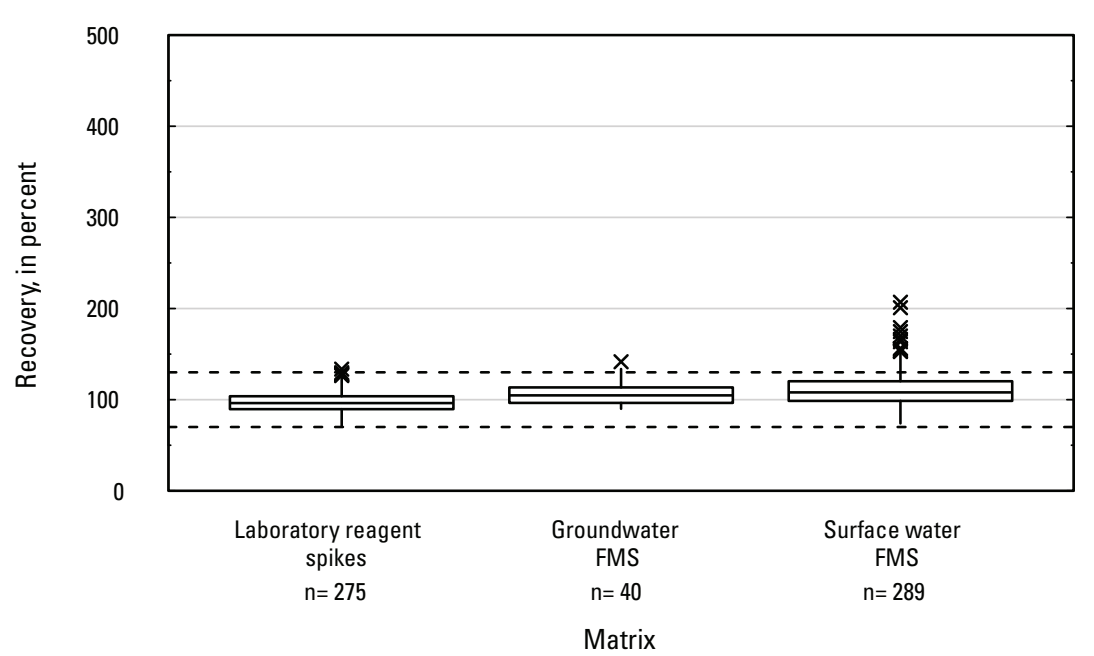

GX. Tebuthiuron

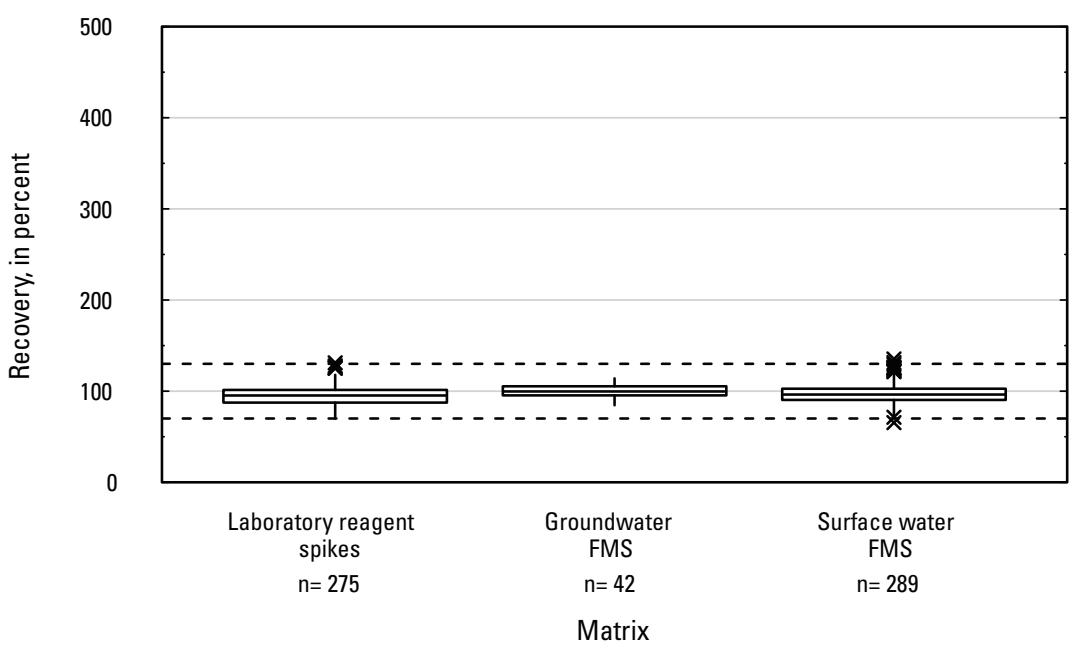

GZ. Tebuthiuron Transformation Product 106

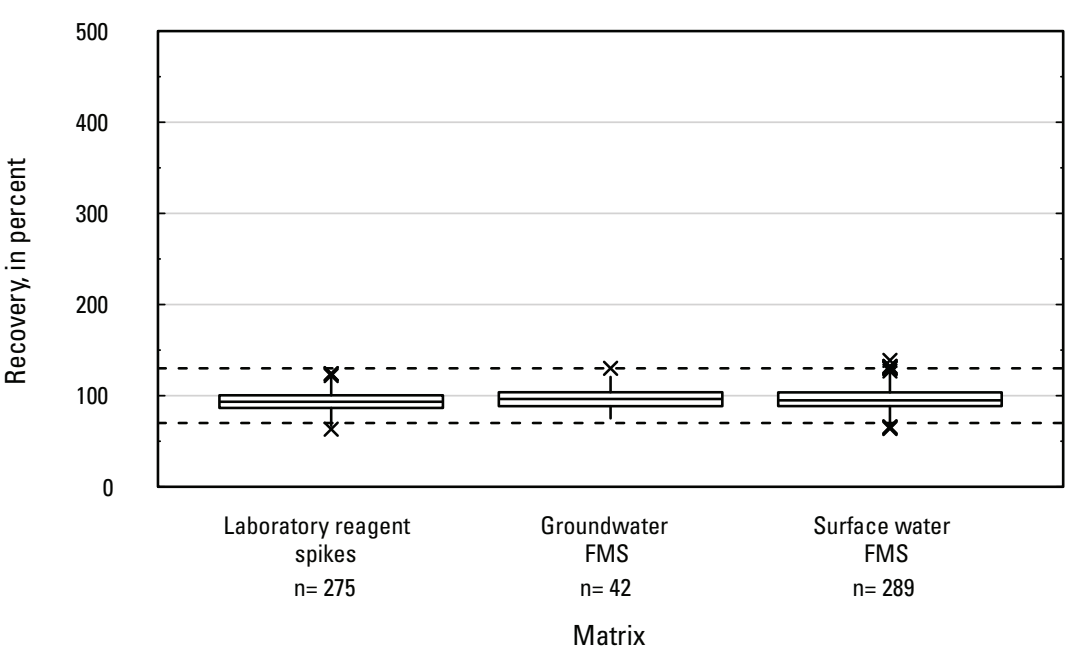

$\infty$

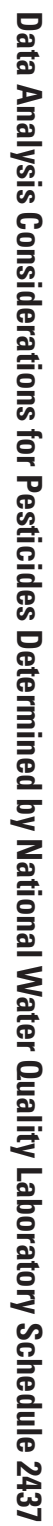

Figure 4. Graph showing distributions of recovery for pesticides in schedule 2437 by matrix. Dashed lines are at 130 percent and 70 percent. Recovery values larger than 400 percent are not shown.-Continued 
HA. Tebuthiuron TP el108

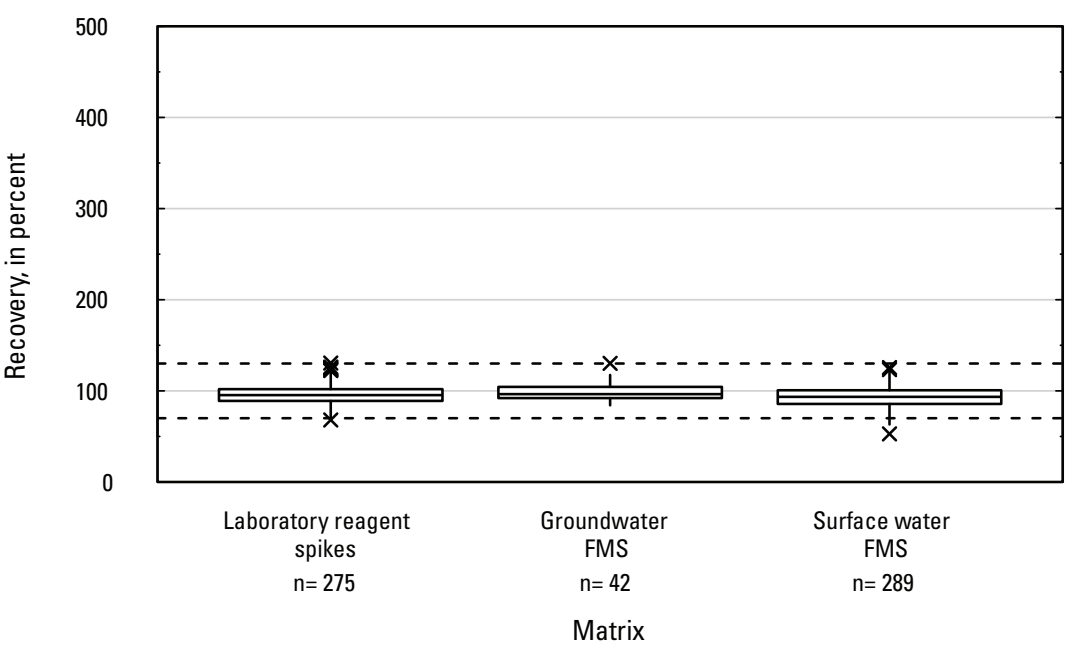

HC. Terbacil

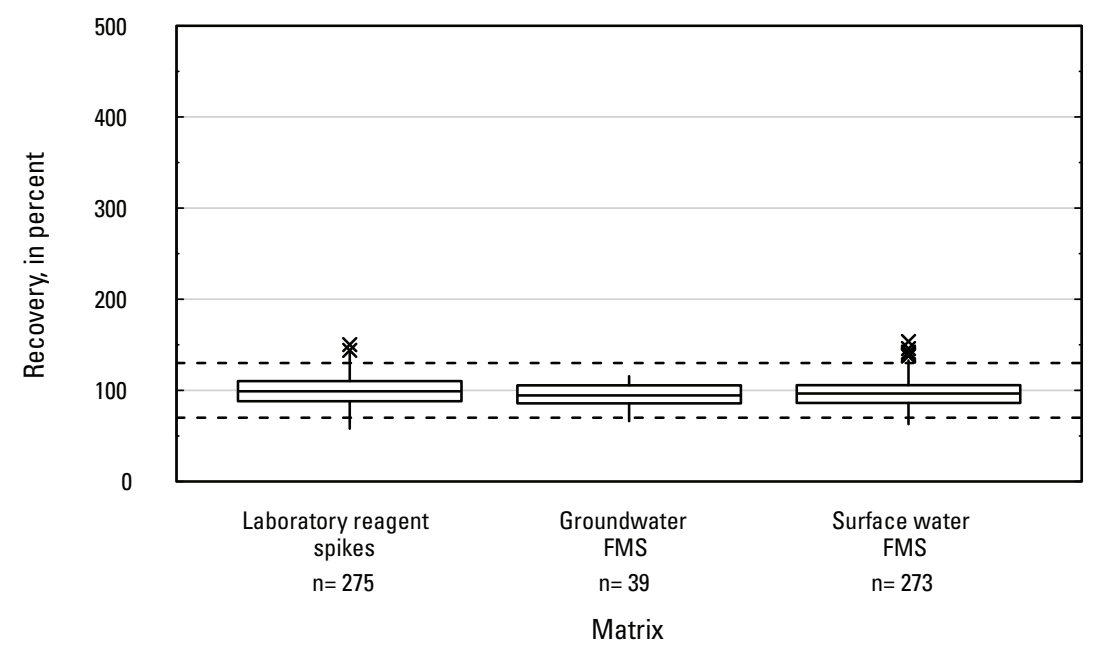

HB. Tebuthiuron TP $109(\mathrm{OH})$

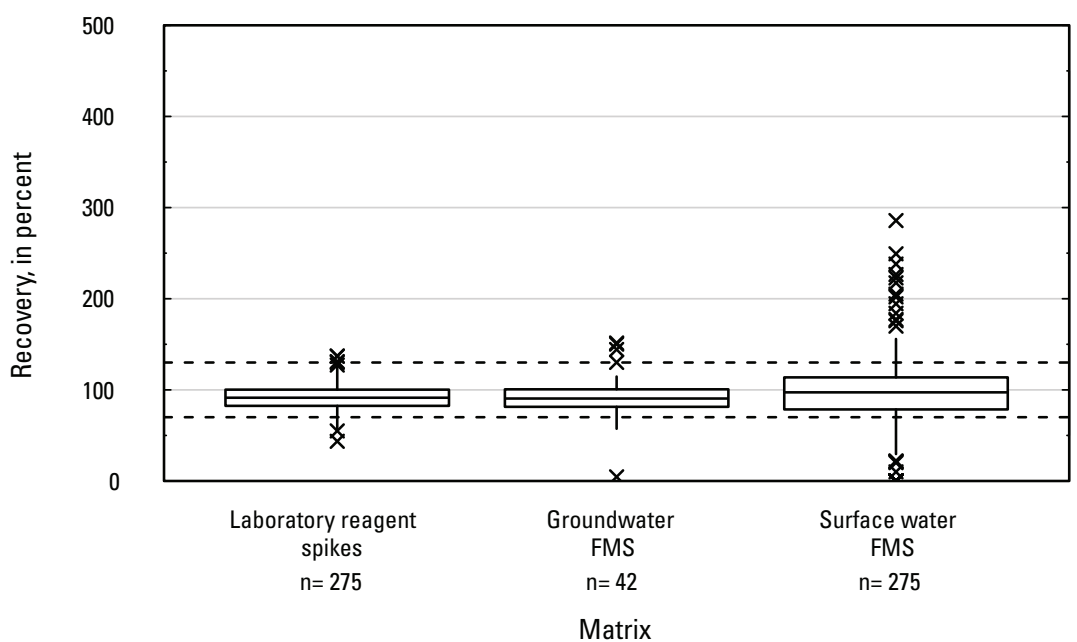

HD. Terbufos

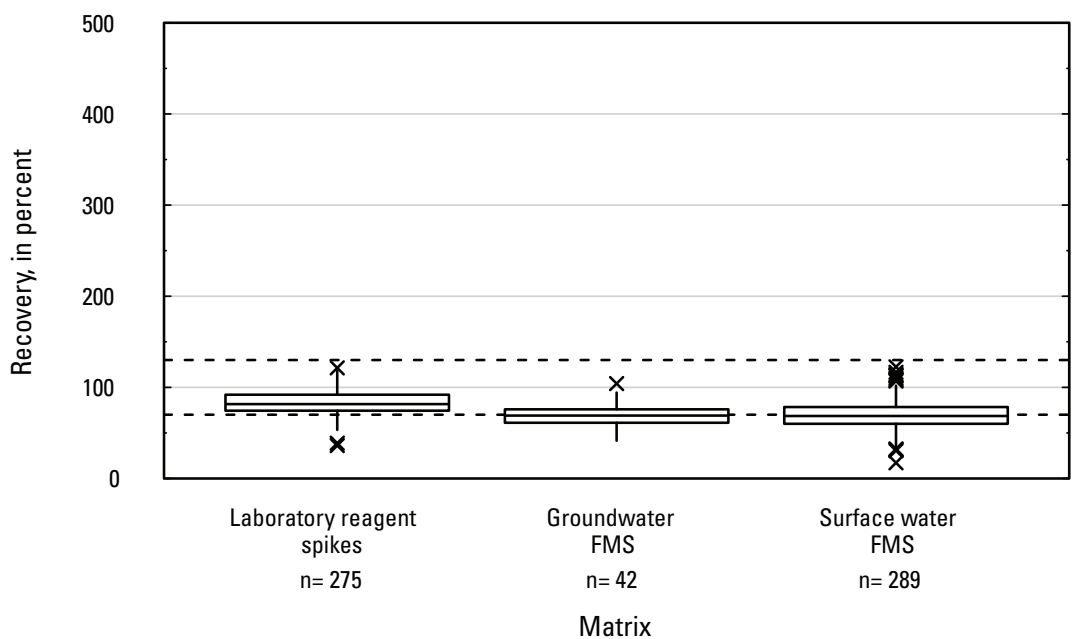

Figure 4. Graph showing distributions of recovery for pesticides in schedule 2437 by matrix. Dashed lines are at 130 percent and 70 percent. Recovery values larger 
HE. Terbufos oxon

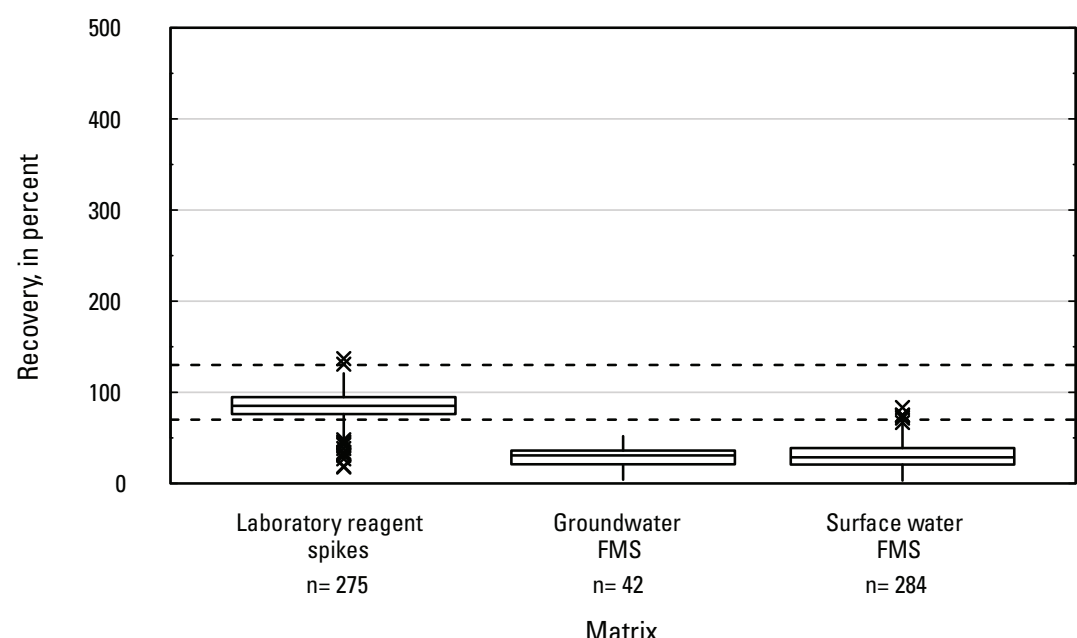

HG. Terbufos oxon sulfoxide

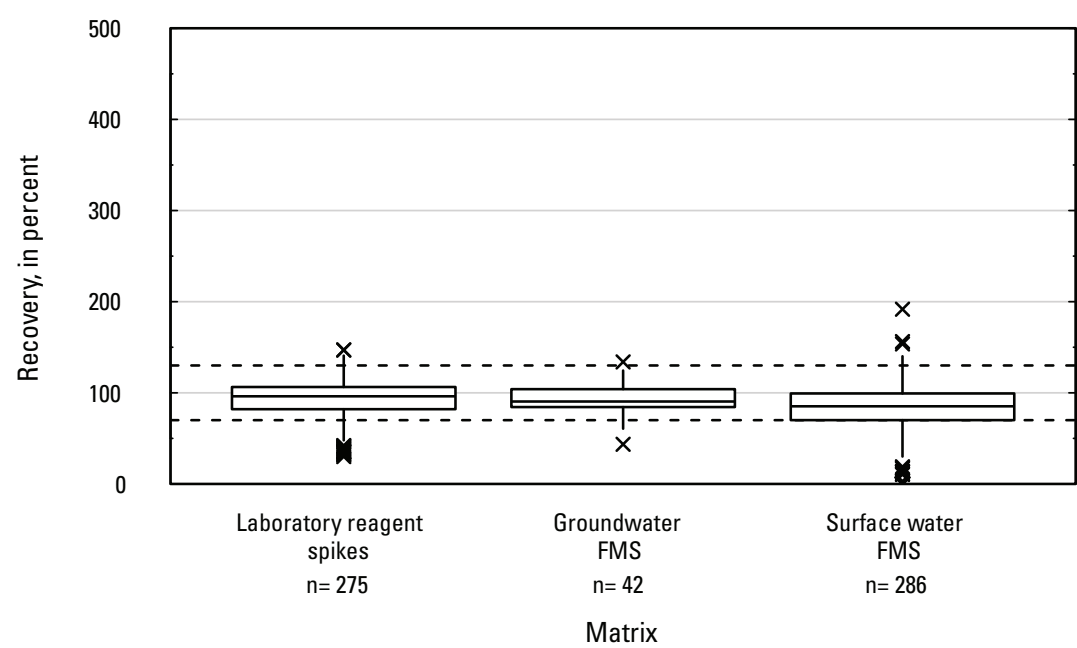

HF. Terbufos oxon sulfone

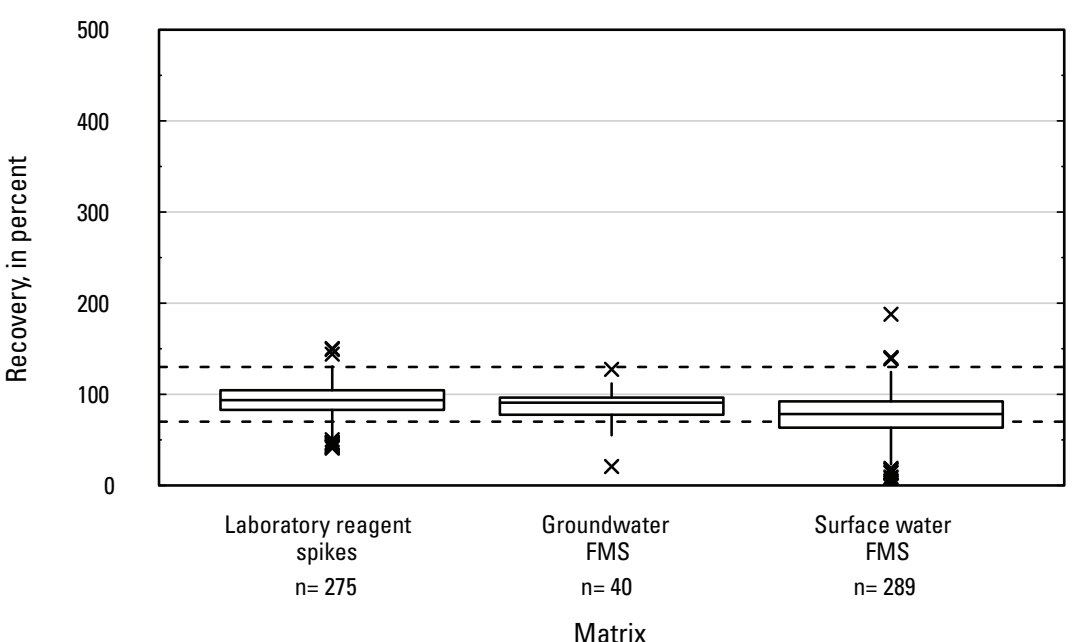

HH. Terbufos sulfone

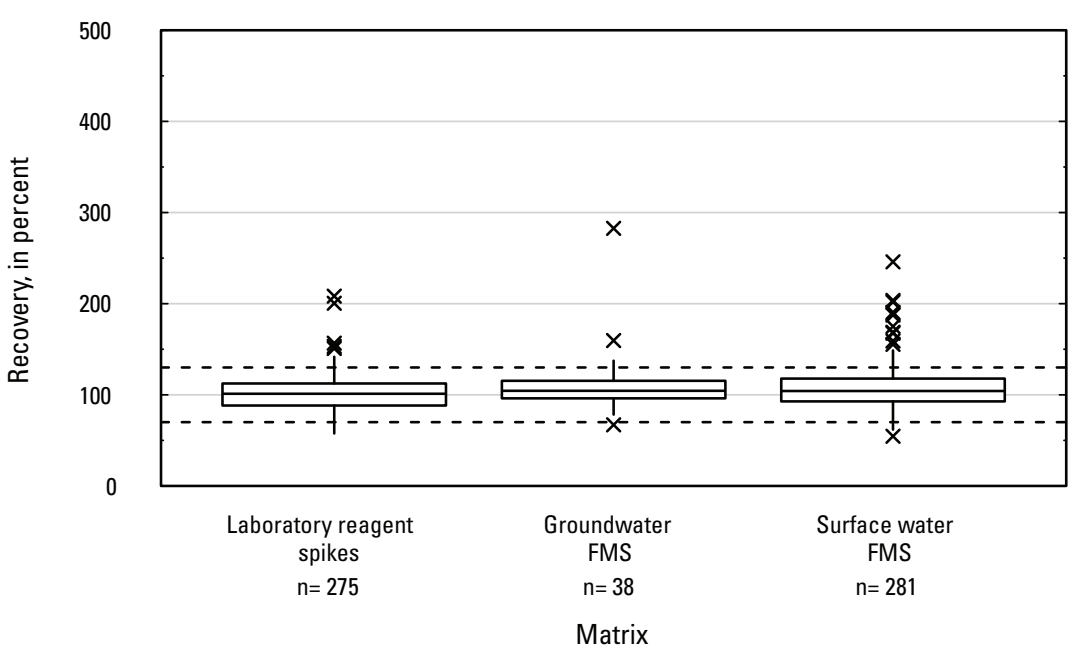

옹

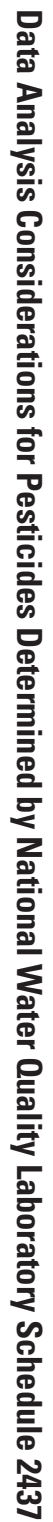

Figure 4. Graph showing distributions of recovery for pesticides in schedule 2437 by matrix. Dashed lines are at 130 percent and 70 percent. Recovery values larger than 400 percent are not shown.-Continued 
H.I Terbufos sulfoxide

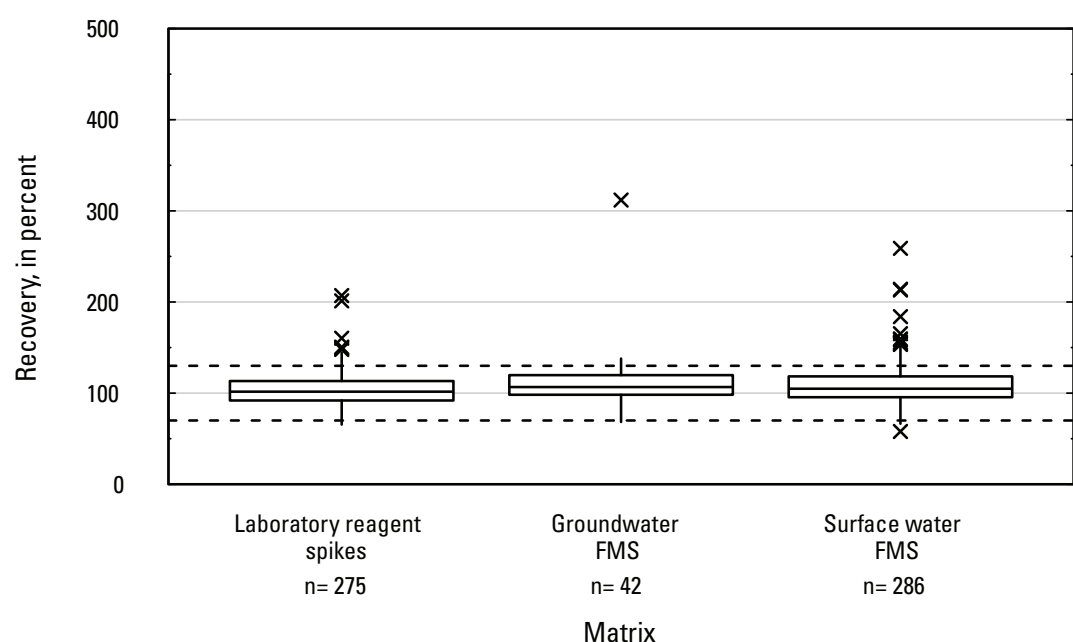

HK. Tetraconazole

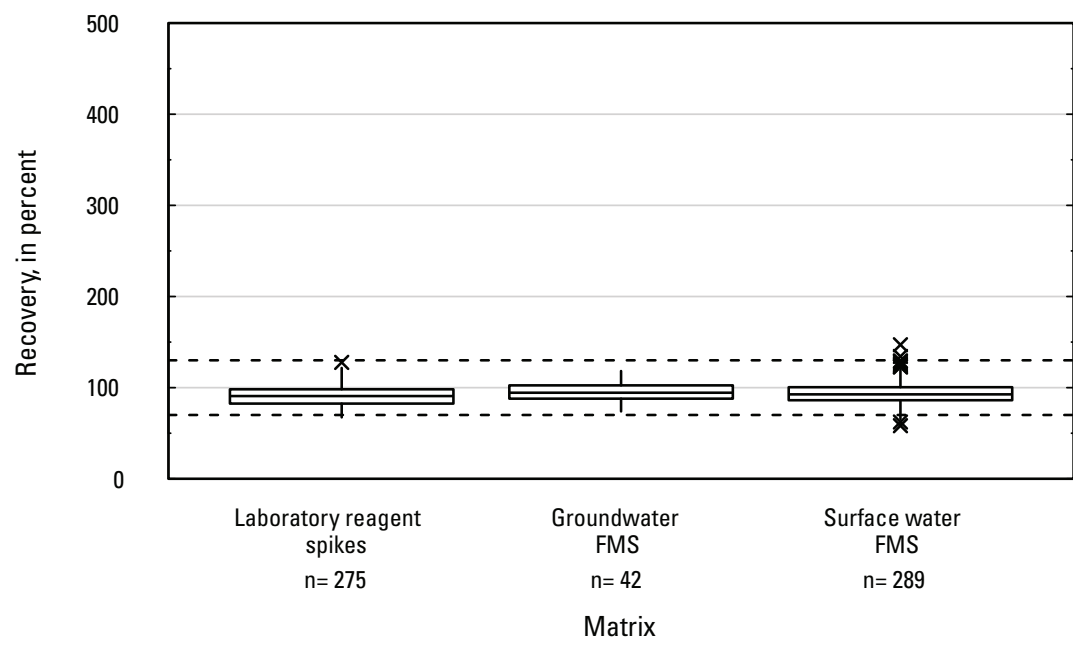

HJ. Terbuthylazine

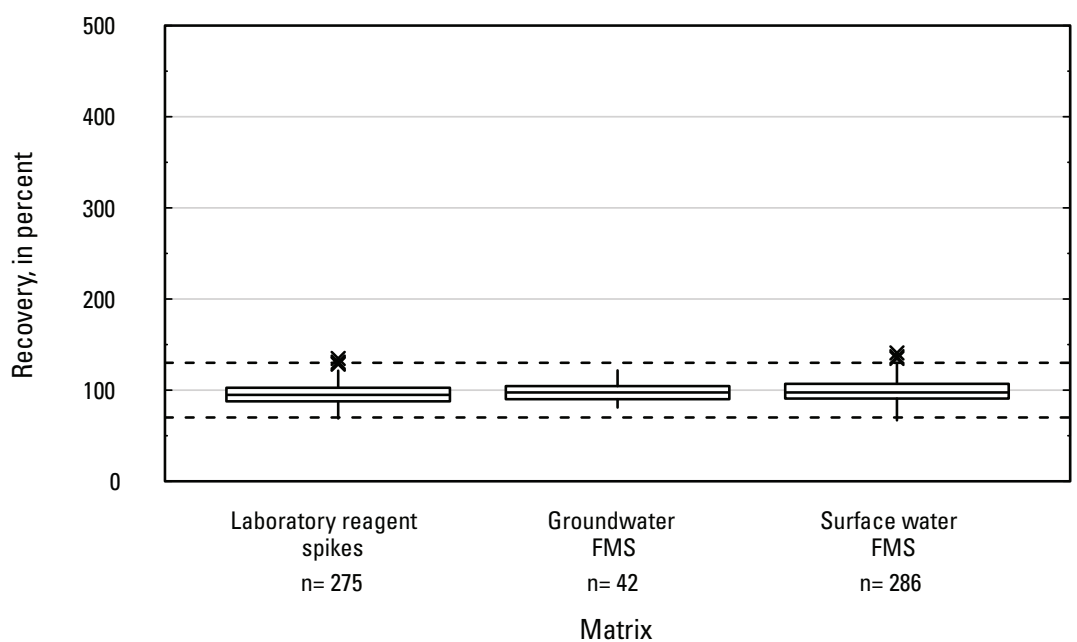

$H L$. Thiobencarb

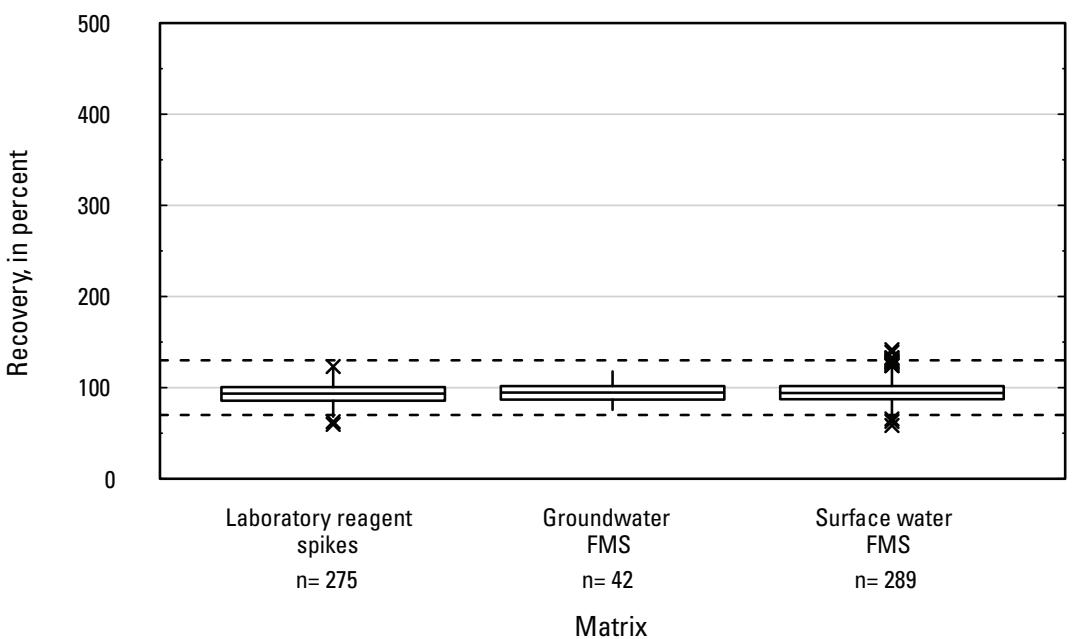

Figure 4. Graph showing distributions of recovery for pesticides in schedule 2437 by matrix. Dashed lines are at 130 percent and 70 percent. Recovery values larger 
HM. trans-Permethrin

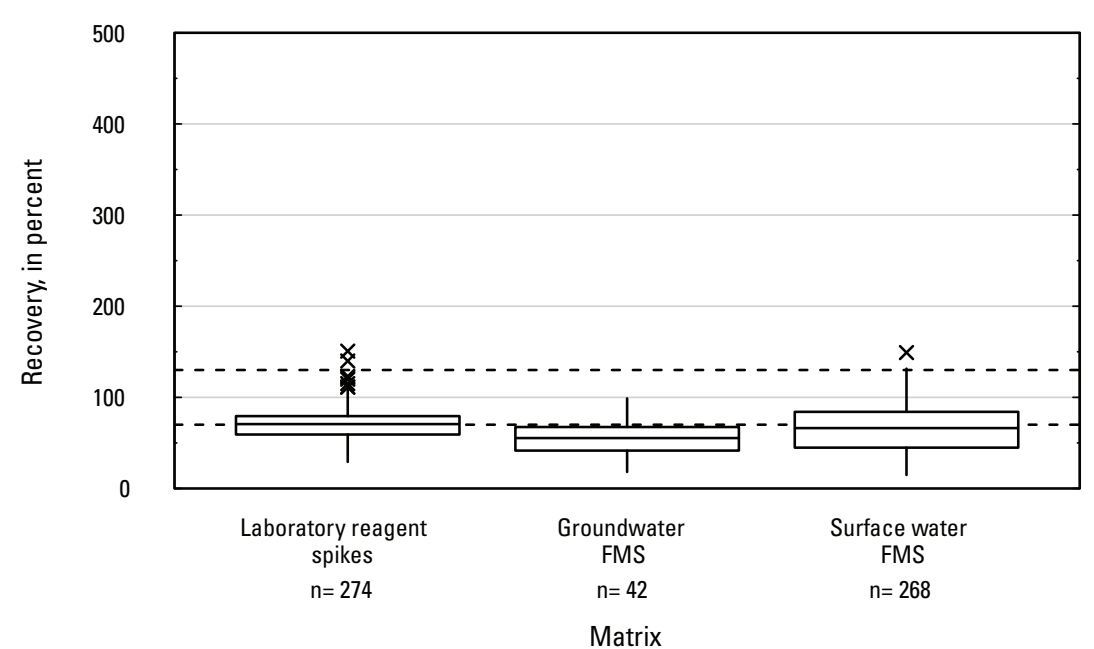

HO. Tribufos

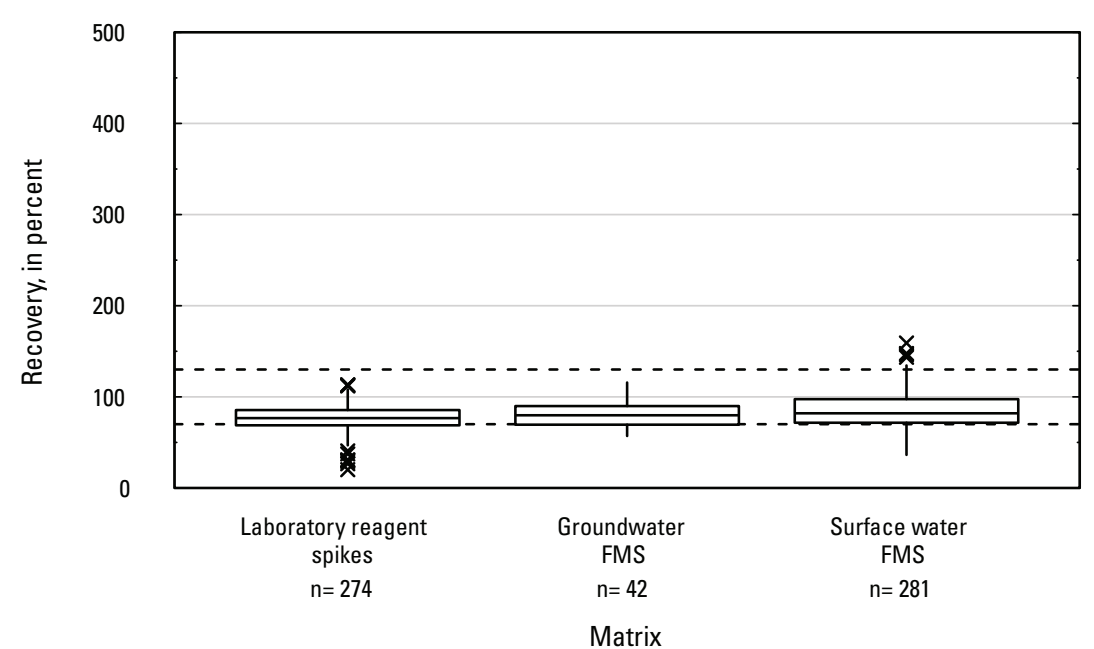

HN. Triallate

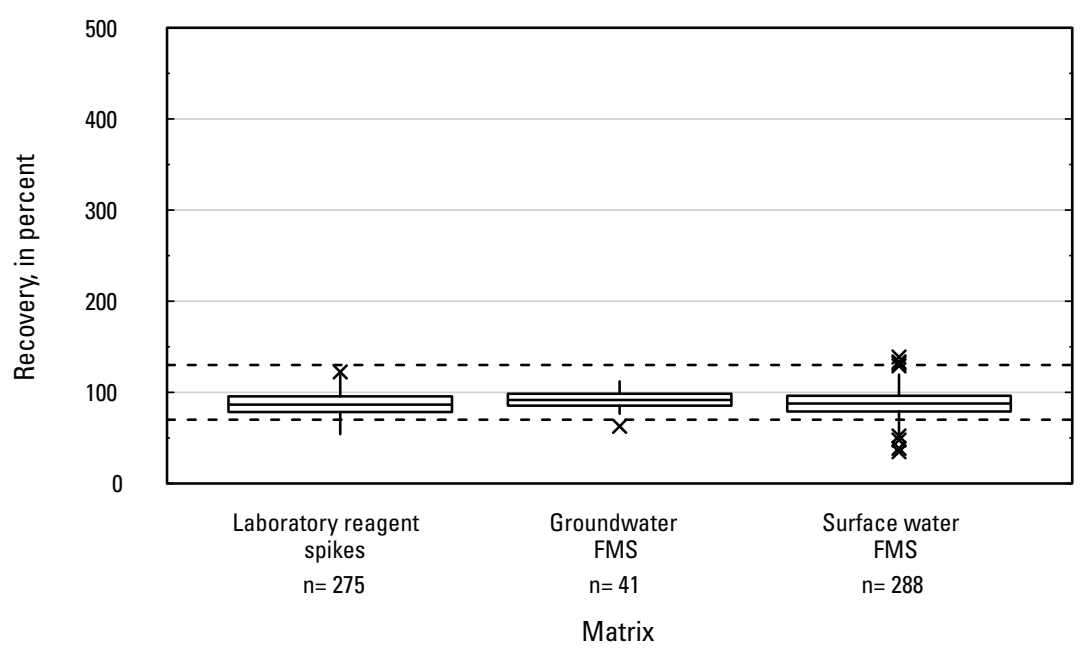

HP. Triclopyr

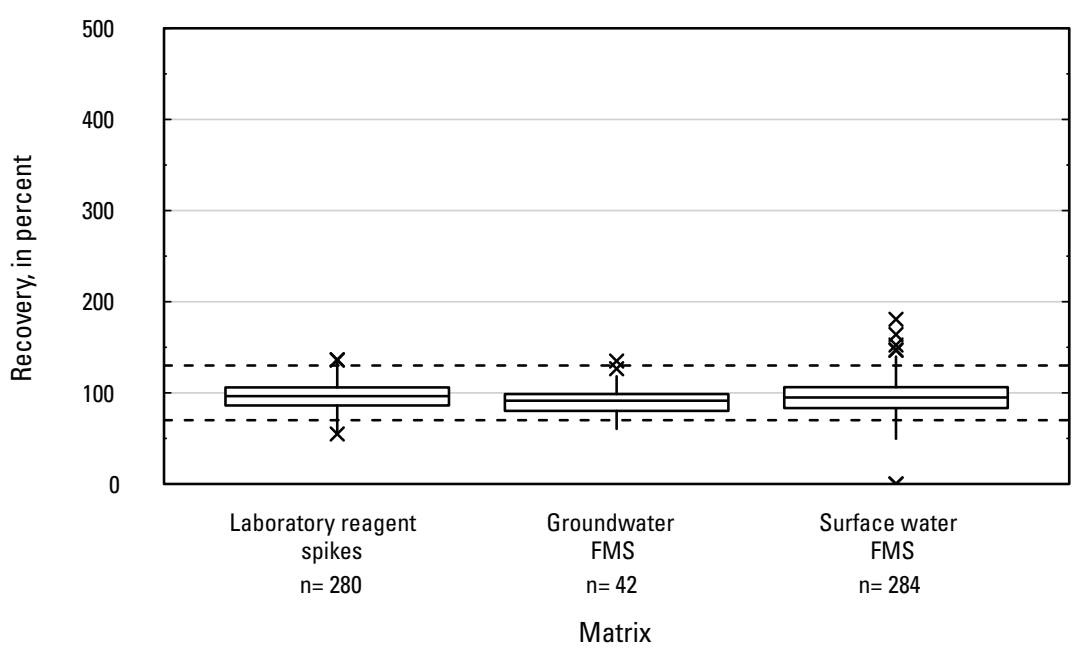

N

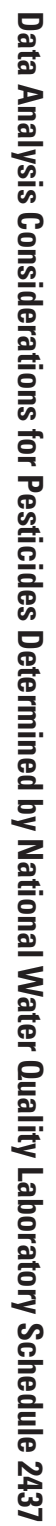

Figure 4. Graph showing distributions of recovery for pesticides in schedule 2437 by matrix. Dashed lines are at 130 percent and 70 percent. Recovery values larger than 400 percent are not shown.-Continued 
HQ. Trifloxystrobin

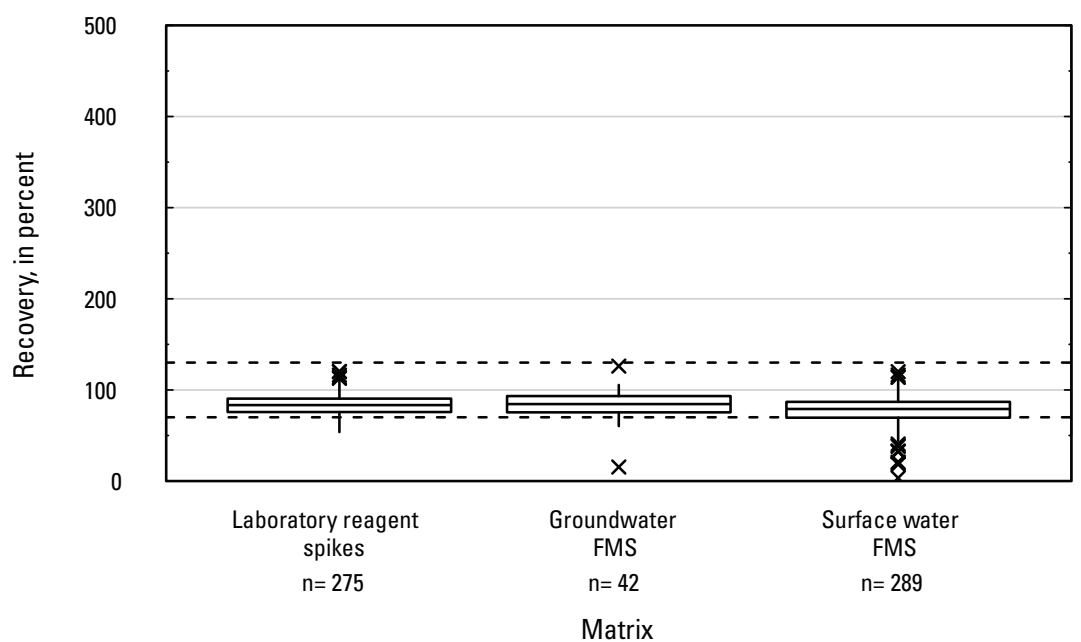

Figure 4. Graph showing distributions of recovery for pesticides in schedule 2437 by matrix. Dashed lines are at 130 percent and 70 percent. Recovery values larger than 400 percent are not shown.-Continued 


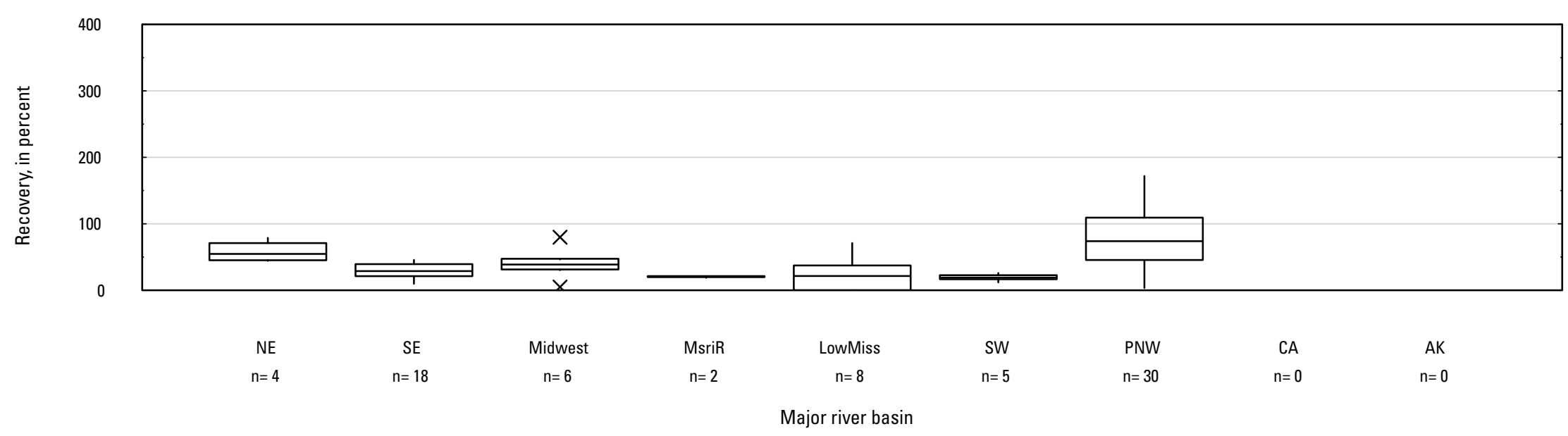

\section{B. 2-(1-Hydroxyethyl)-6-methylaniline}

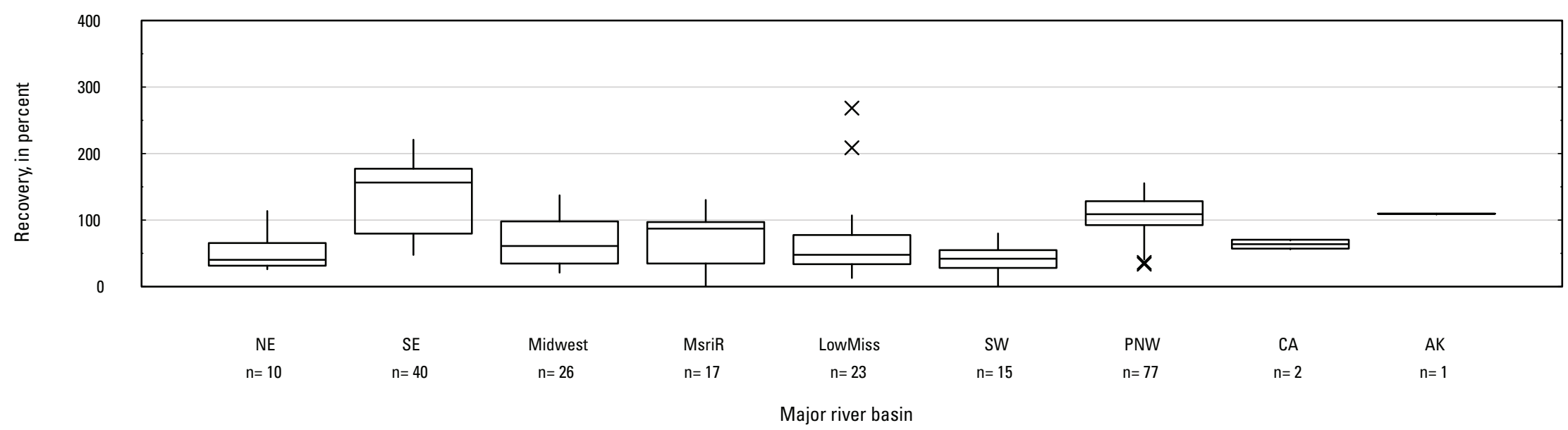

Figure 5. Graph showing distributions of recovery for pesticides in schedule 2437 in surface water by Major River Basin. Recovery values larger than 400 percent are not shown. 


\section{2-[(2-Ethyl-6-methylphenyl)amino]-1-propanol}

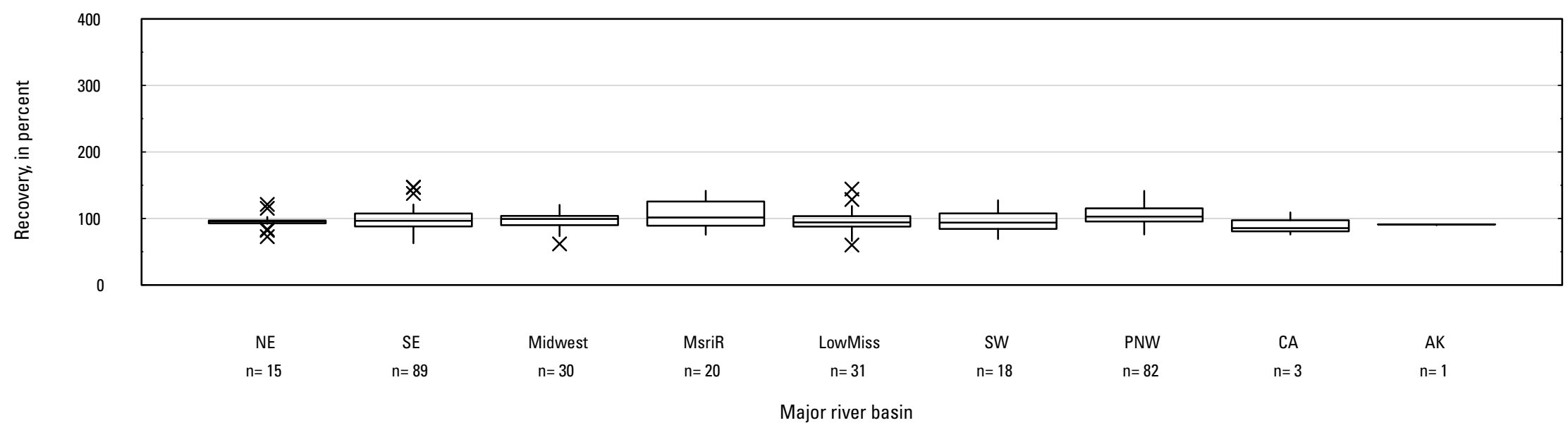

D. 2,3,3-Trichloro-2-propene-1-sulfonic acid (TCPSA)

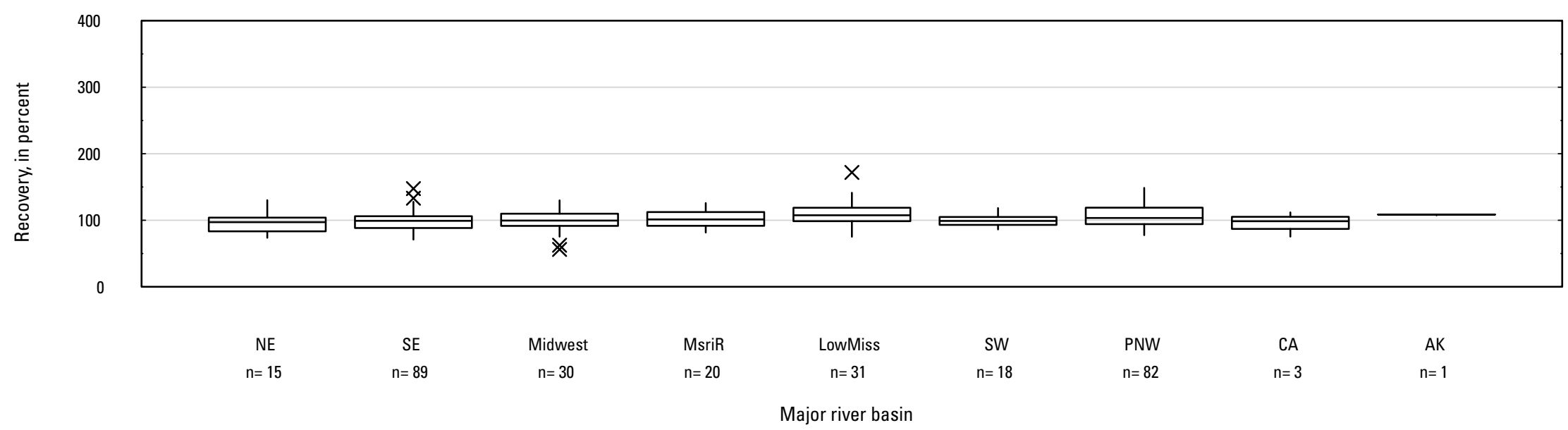

Figure 5. Graph showing distributions of recovery for pesticides in schedule 2437 in surface water by Major River Basin. Recovery values larger than 400 percent are not shown.-Continued 


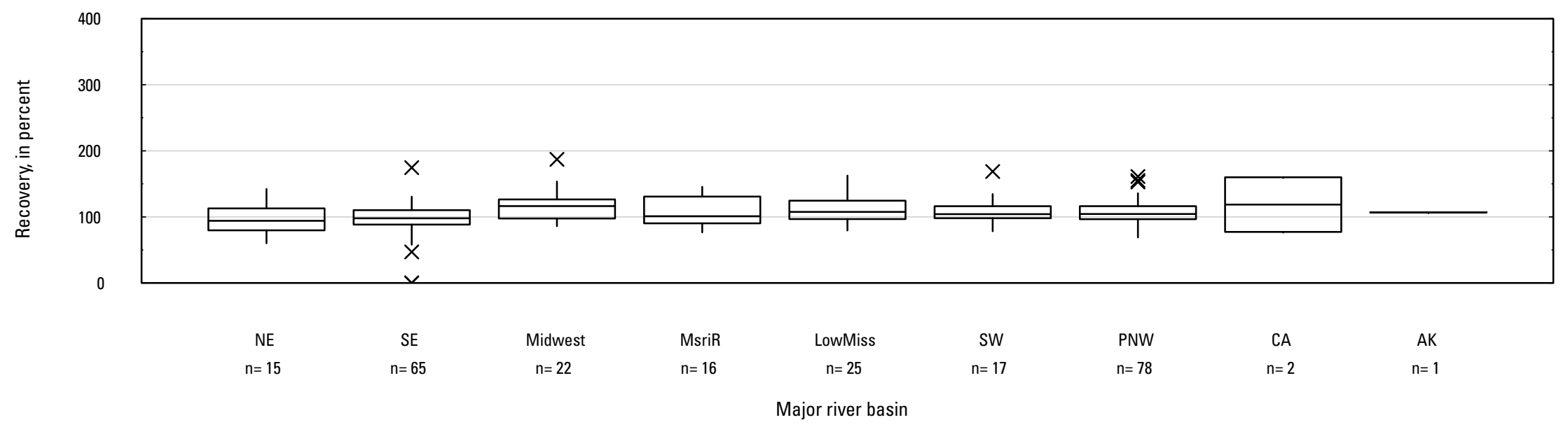

\section{F. 2-Aminobenzimidazole}

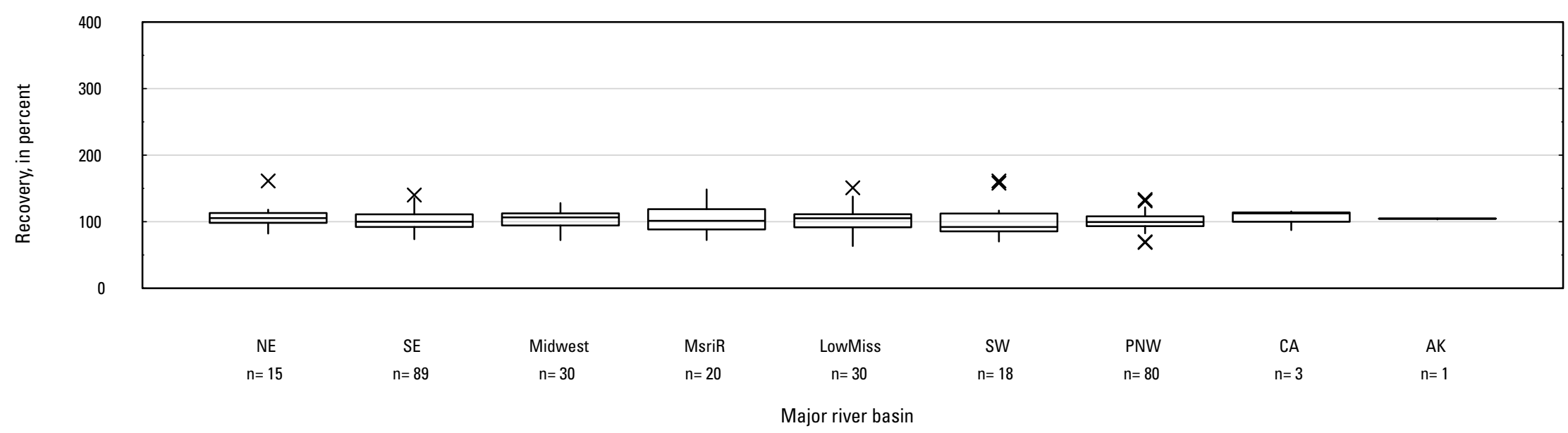

Figure 5. Graph showing distributions of recovery for pesticides in schedule 2437 in surface water by Major River Basin. Recovery values larger than 400 percent are not shown.-Continued 
G. 2-Amino-N-isopropylbenzamide

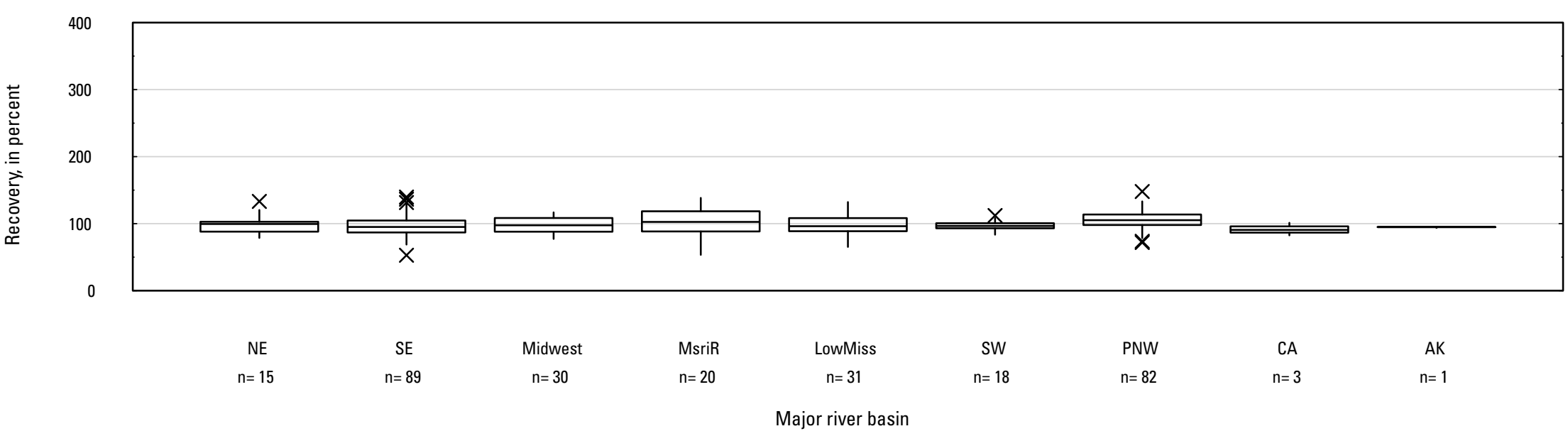

H. 2-Chloro-2',6'-diethylacetanilide

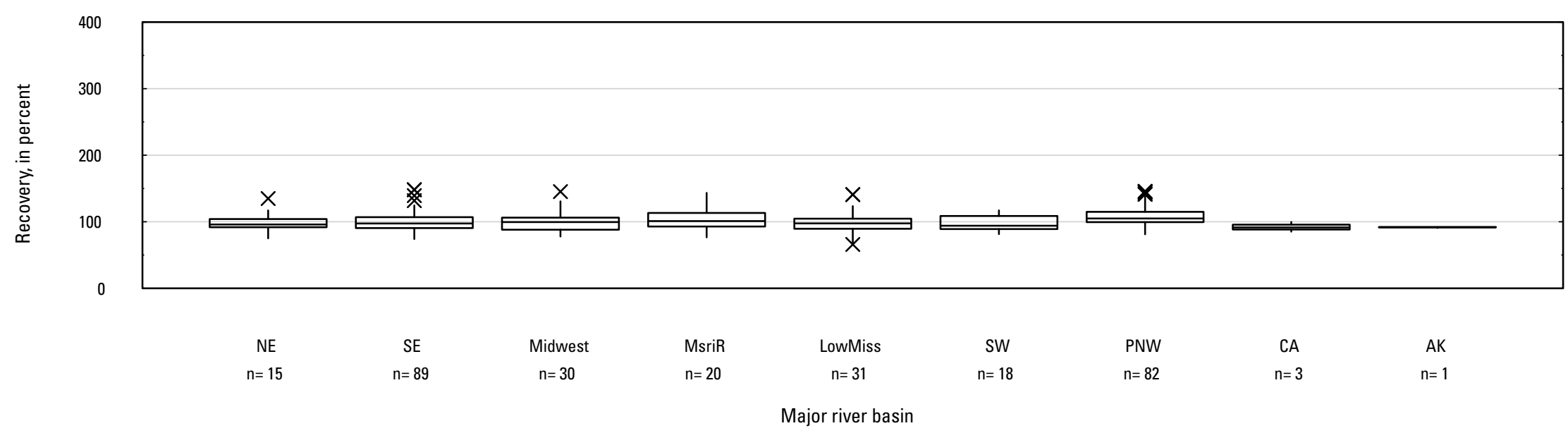

Figure 5. Graph showing distributions of recovery for pesticides in schedule 2437 in surface water by Major River Basin. Recovery values larger than 400 percent are not shown.-Continued 


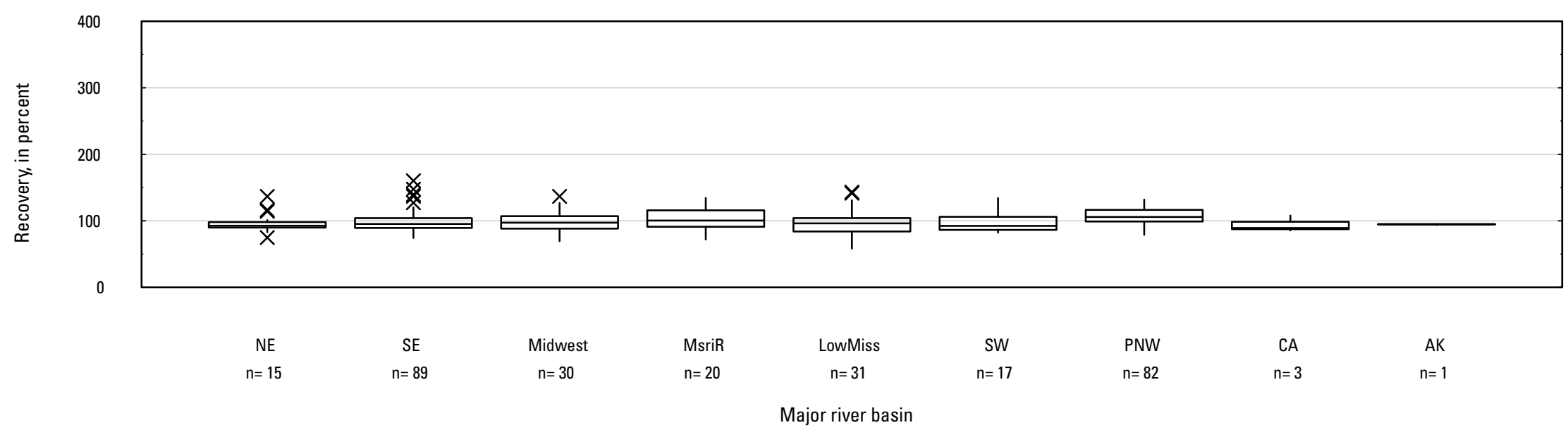

J. 2-Hydroxy-4-isopropylamino-6-amino-s-triazine

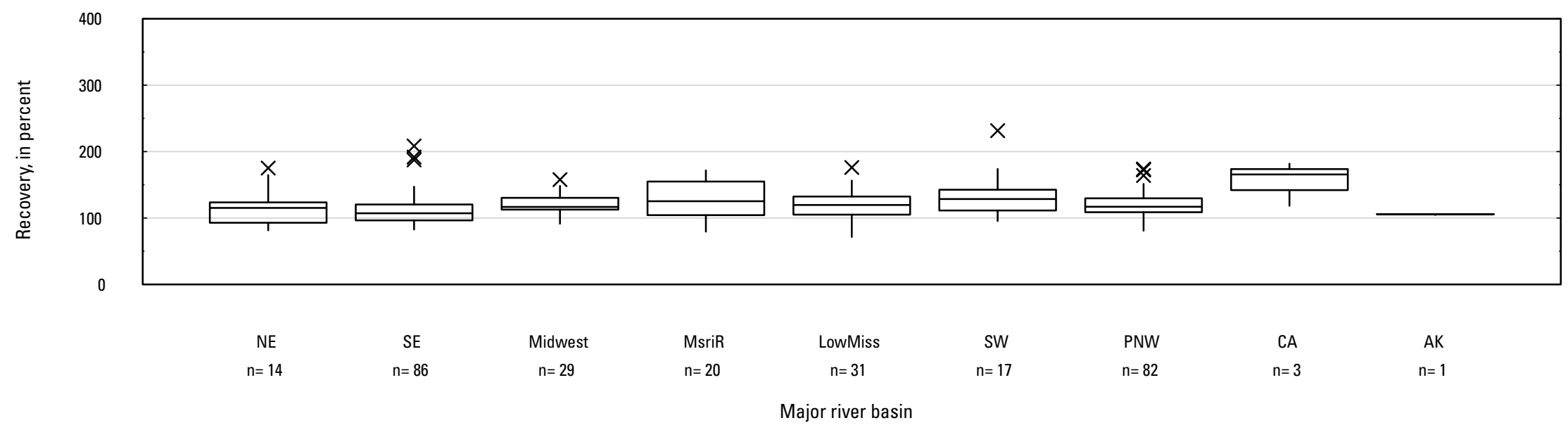

Figure 5. Graph showing distributions of recovery for pesticides in schedule 2437 in surface water by Major River Basin. Recovery values larger than 400 percent are not shown.-Continued 


\section{K. 2-Hydroxy-6-ethylamino-4-amino-s-triazine}

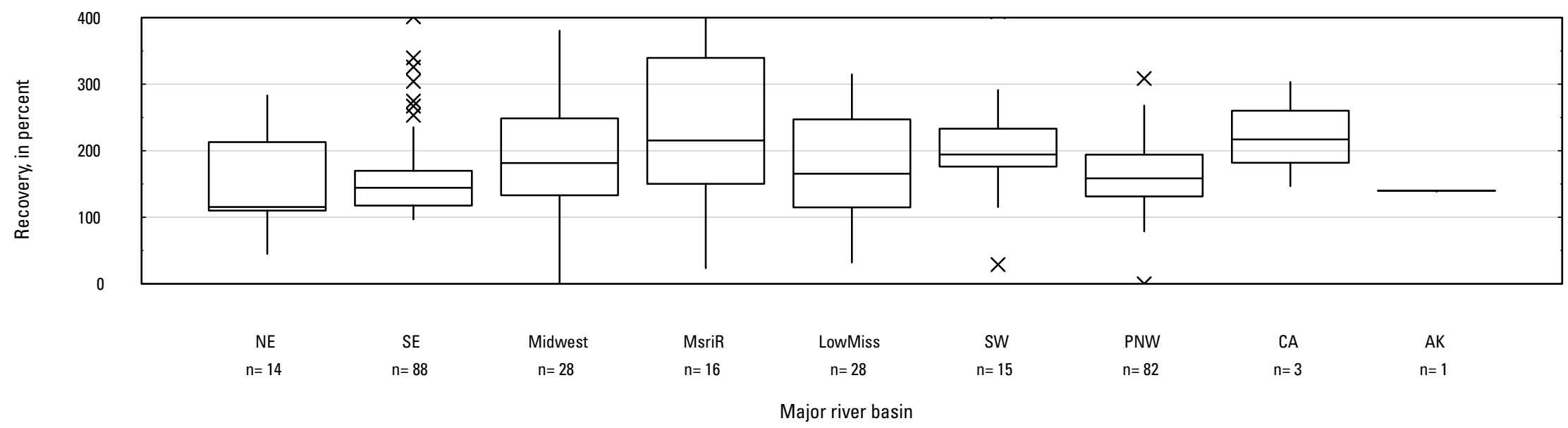

L. 2-Hydroxy-4-isopropylamino-6-ethylamino-s-triazine \{0IET\}

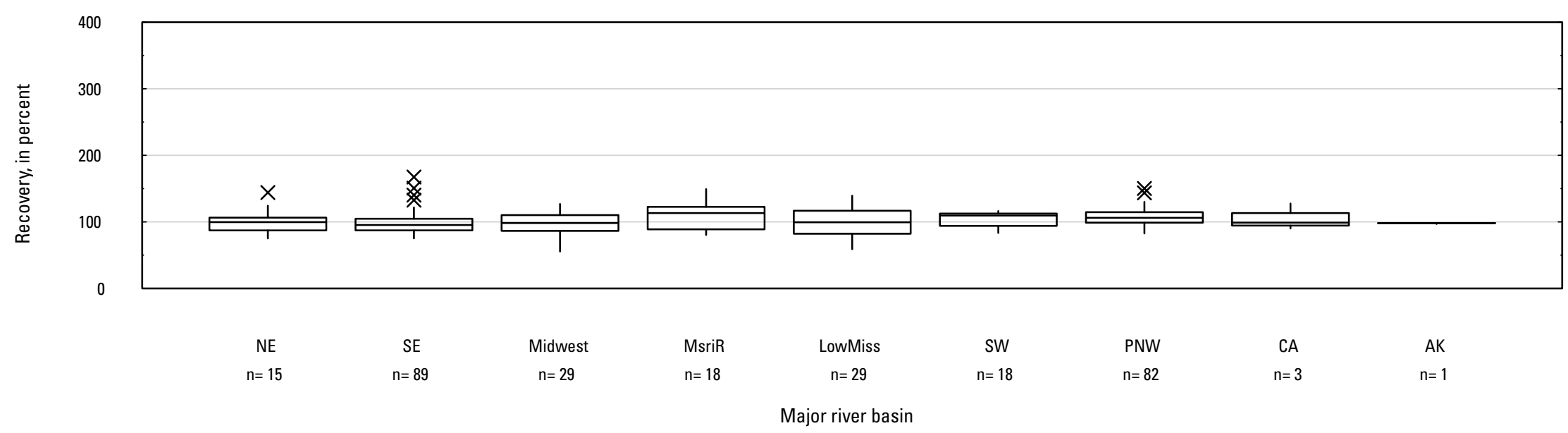

Figure 5. Graph showing distributions of recovery for pesticides in schedule 2437 in surface water by Major River Basin. Recovery values larger than 400 percent are not shown.-Continued 


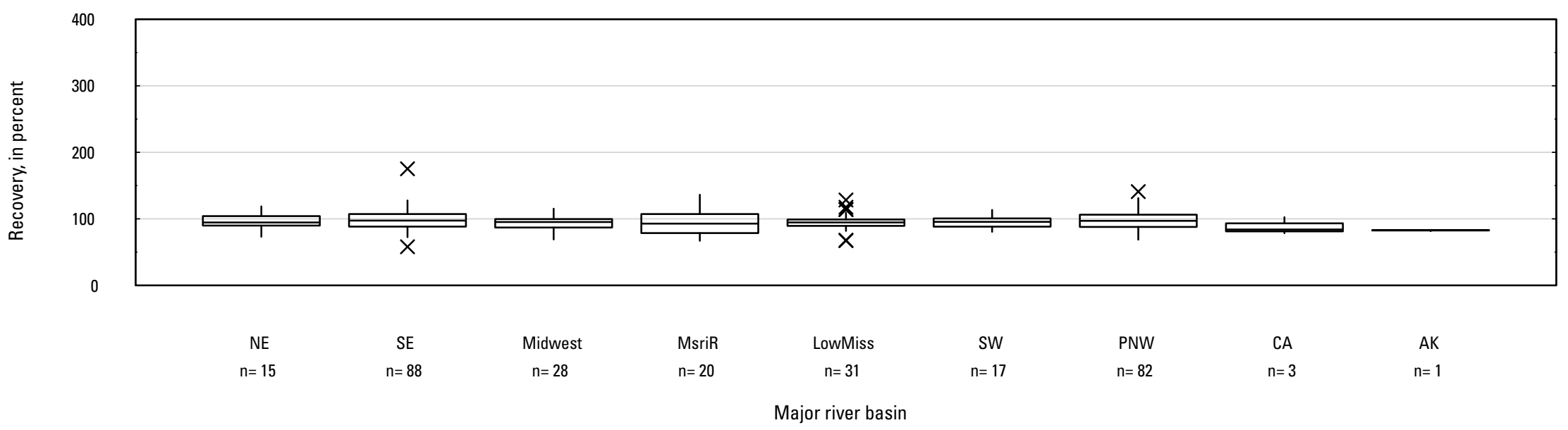

N. 3-Hydroxycarbofuran

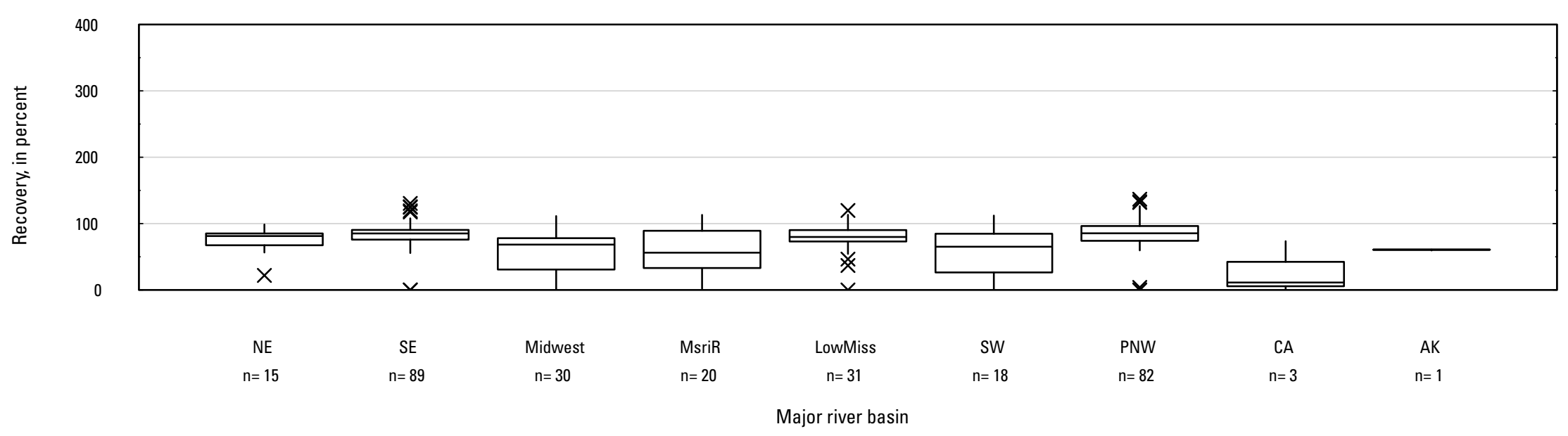

Figure 5. Graph showing distributions of recovery for pesticides in schedule 2437 in surface water by Major River Basin. Recovery values larger than 400 percent are not shown.-Continued 
0. 3-Phenoxybenzoic acid

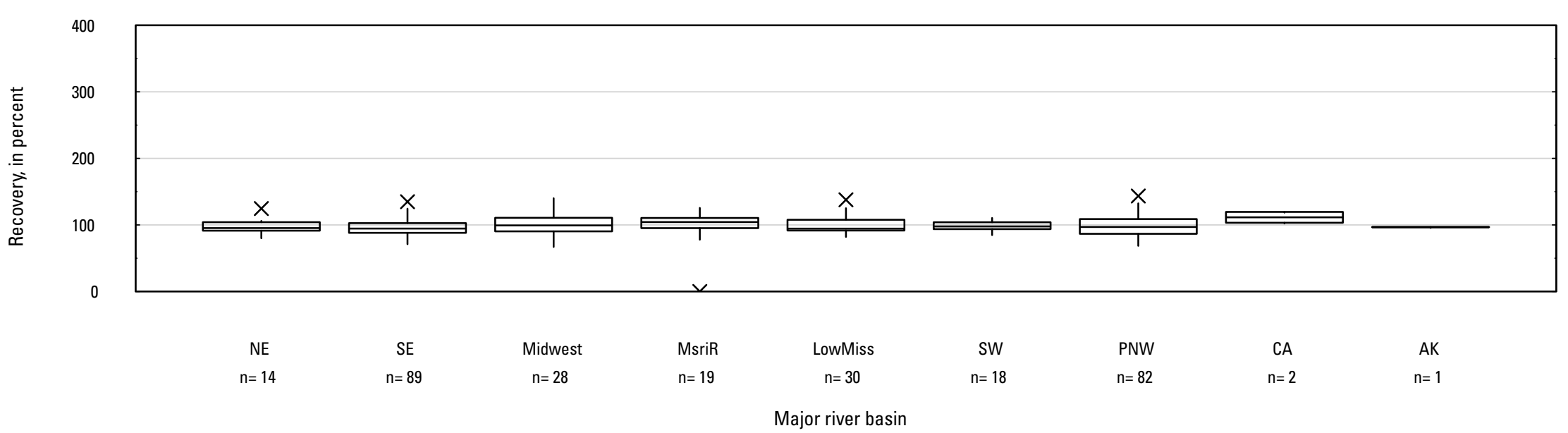

P. 4-(Hydroxymethyl)pendimethalin

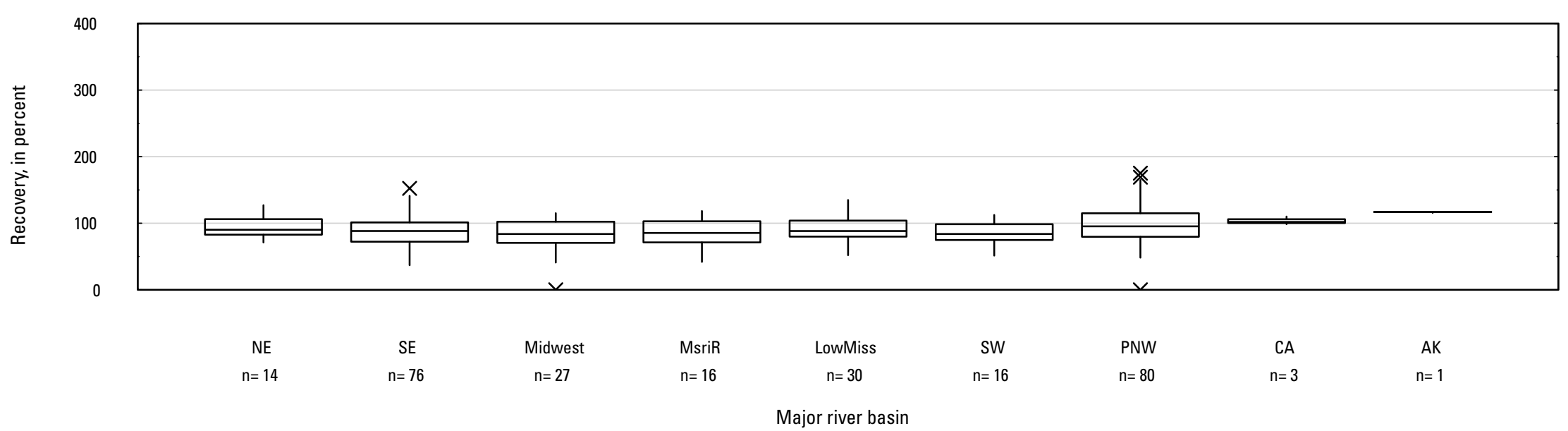

Figure 5. Graph showing distributions of recovery for pesticides in schedule 2437 in surface water by Major River Basin. Recovery values larger than 400 percent are 


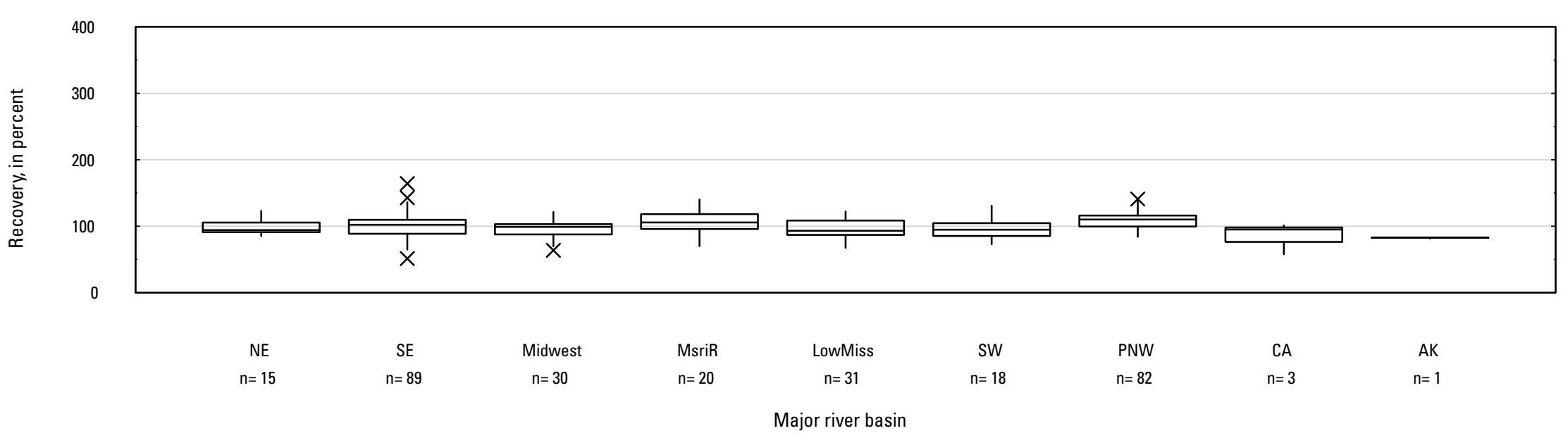

\section{R. 4-Hydroxy molinate}

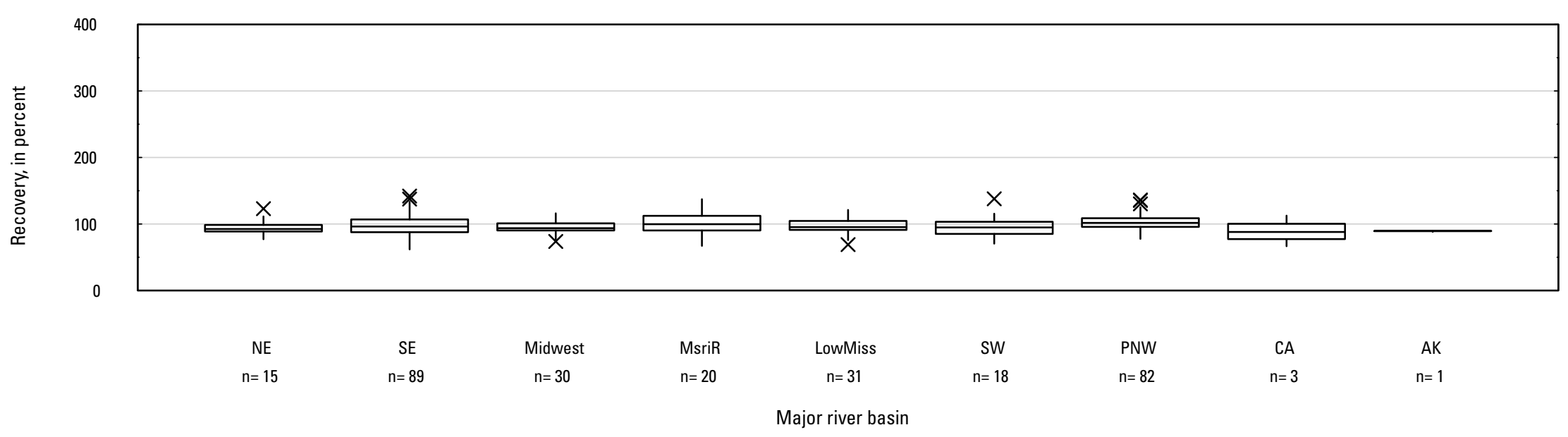

Figure 5. Graph showing distributions of recovery for pesticides in schedule 2437 in surface water by Major River Basin. Recovery values larger than 400 percent are not shown.-Continued 
S. 4-Hydroxychlorothalonil

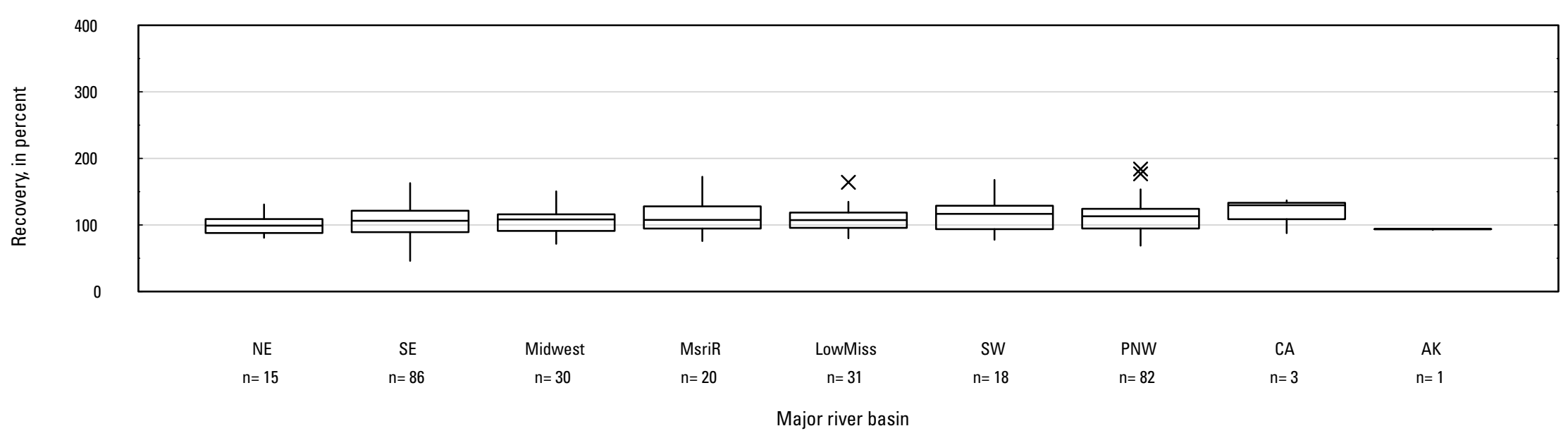

T. 4-Hydroxyhexazinone A

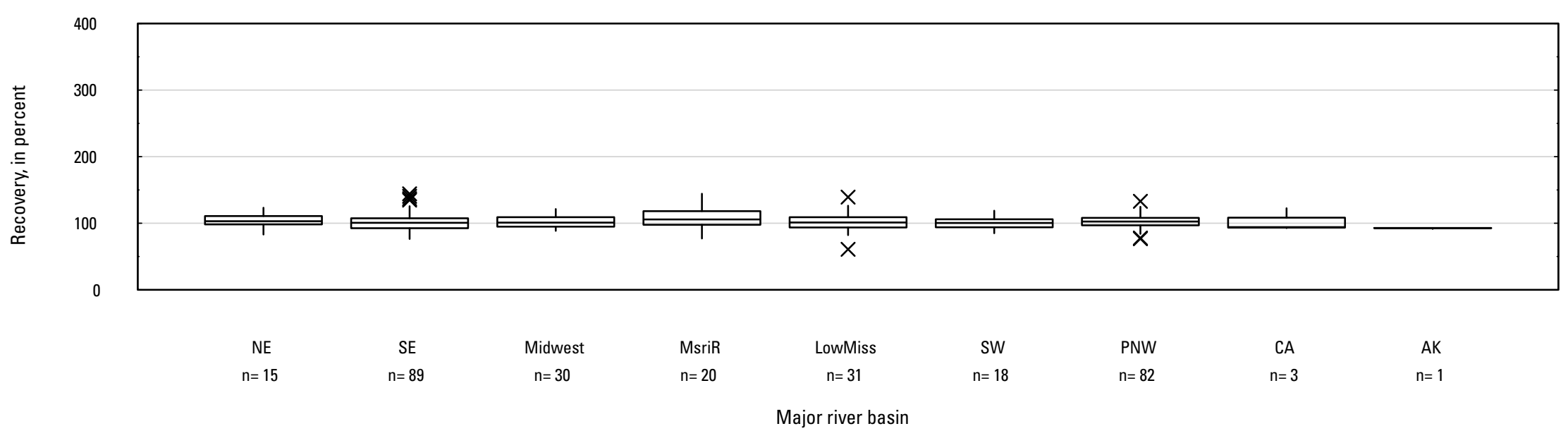

Figure 5. Graph showing distributions of recovery for pesticides in schedule 2437 in surface water by Major River Basin. Recovery values larger than 400 percent are not shown.-Continued 


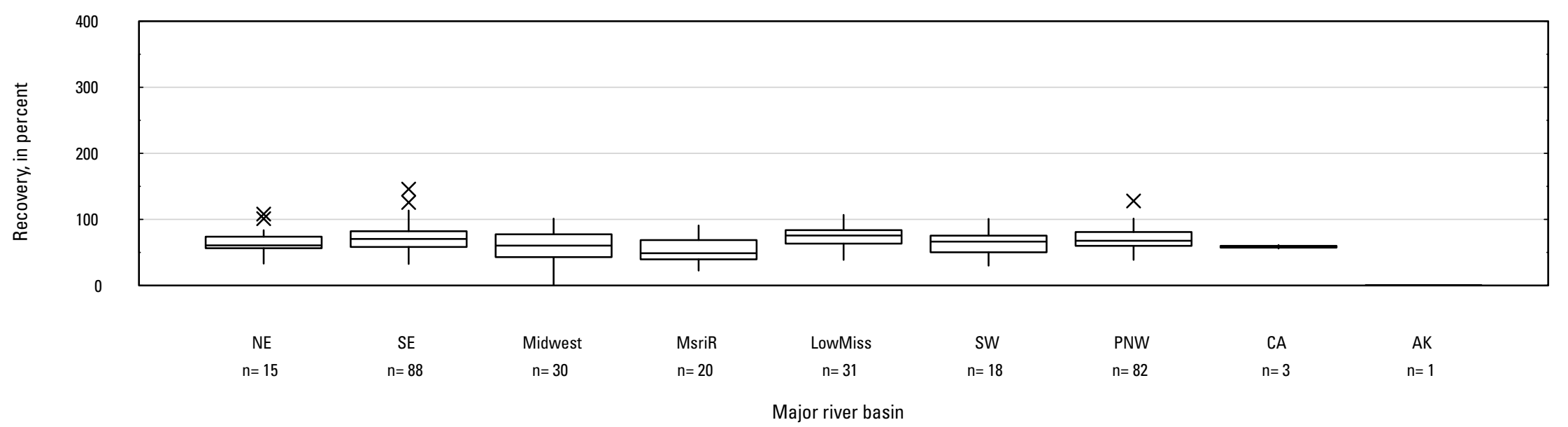

V. Acephate

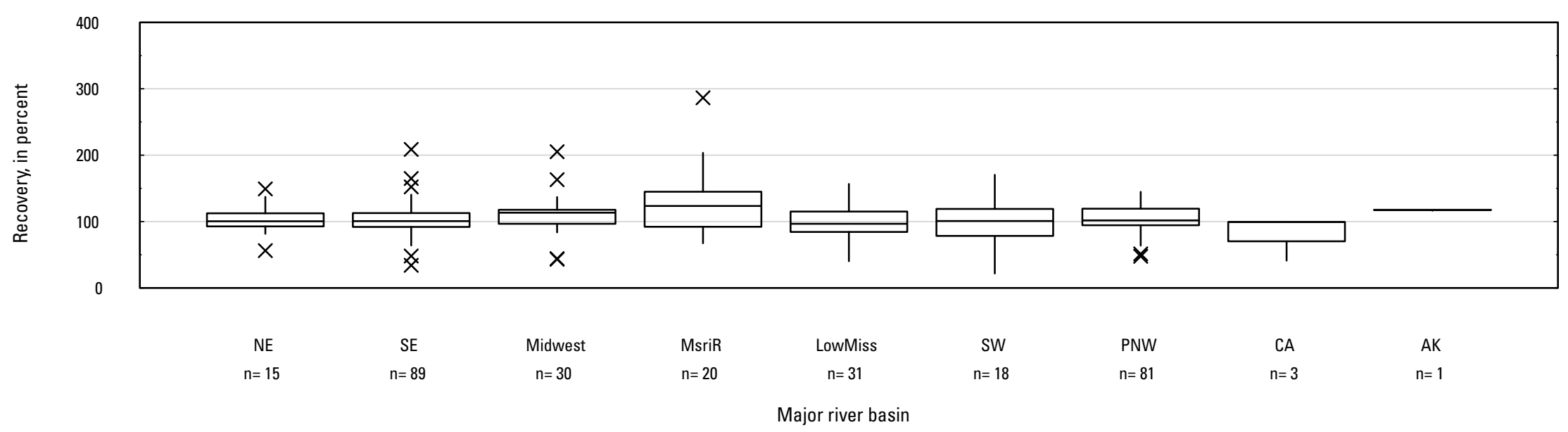

Figure 5. Graph showing distributions of recovery for pesticides in schedule 2437 in surface water by Major River Basin. Recovery values larger than 400 percent are not shown.-Continued 
W. Acetochlor

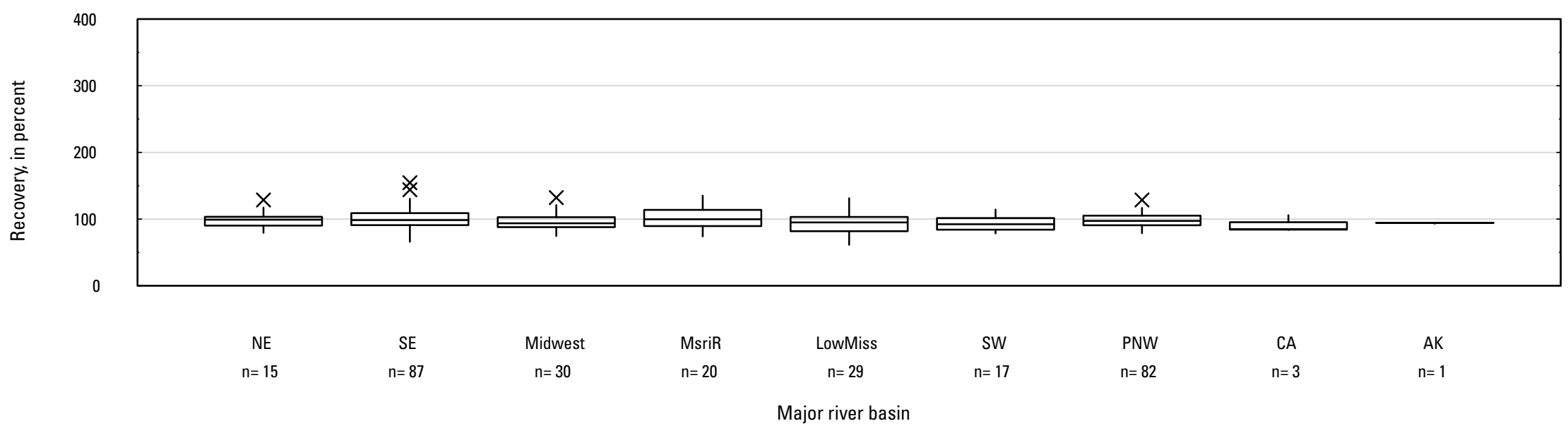

$X$. Acetochlor oxanilic acid

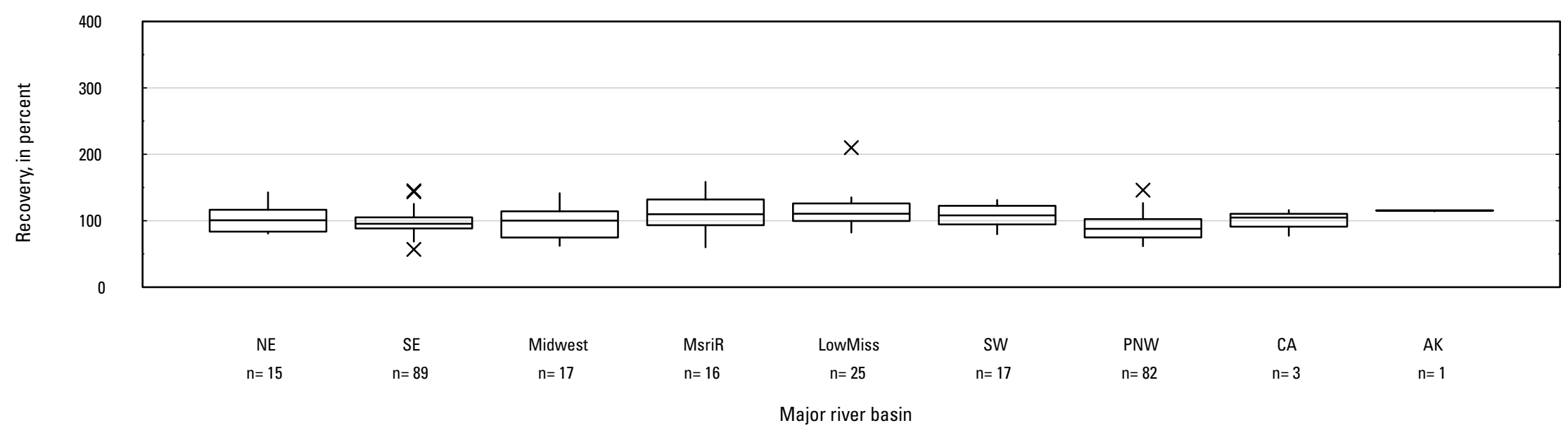

Figure 5. Graph showing distributions of recovery for pesticides in schedule 2437 in surface water by Major River Basin. Recovery values larger than 400 percent are not shown.-Continued 


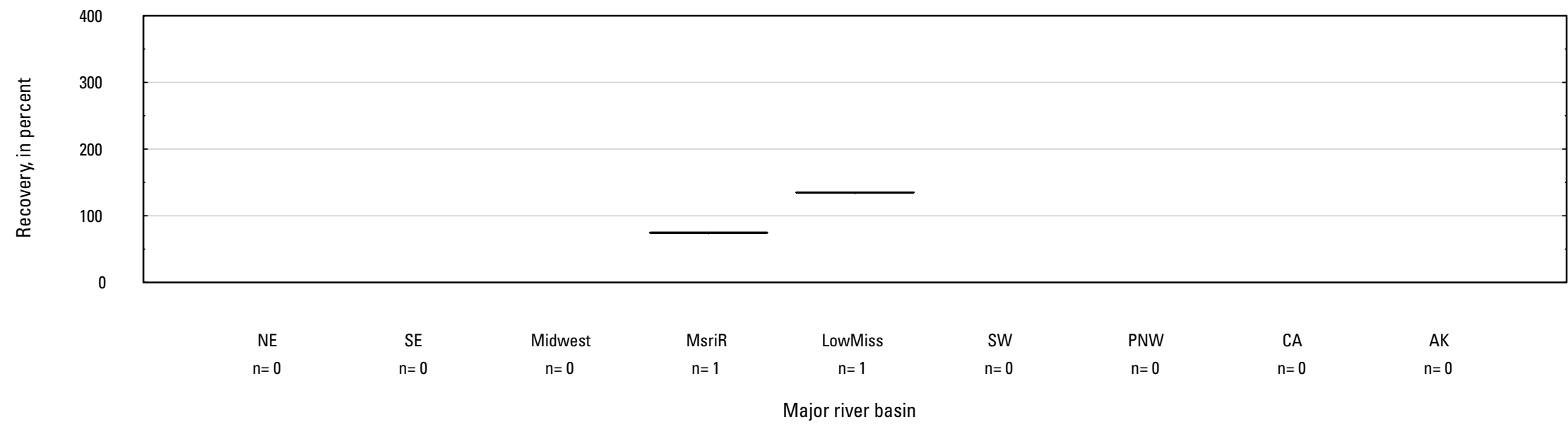

Z. Acetochlor sulfynilacetic acid

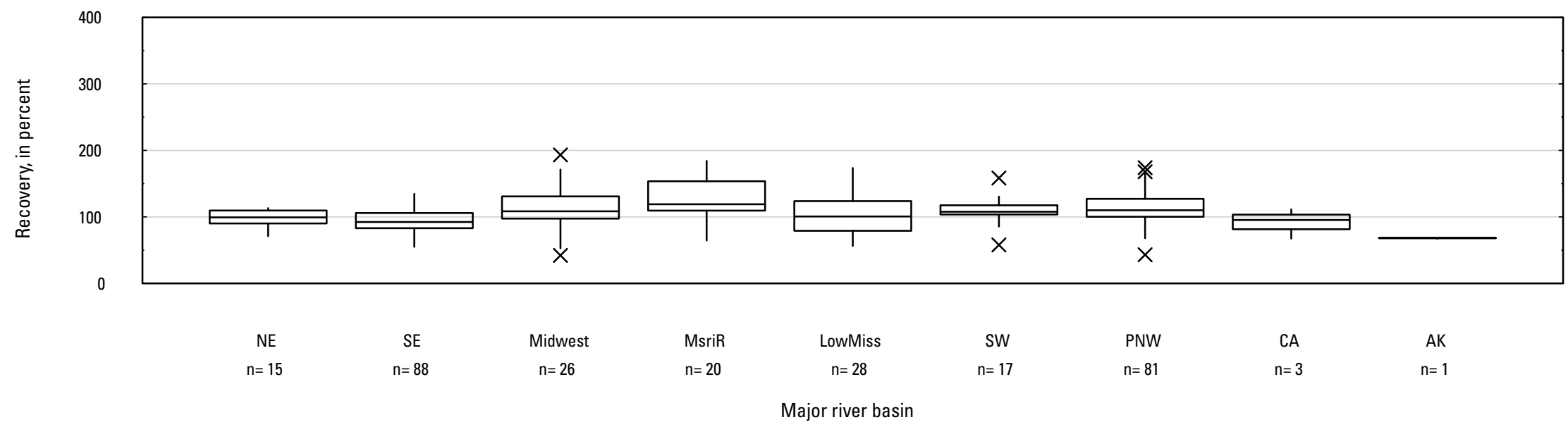

Figure 5. Graph showing distributions of recovery for pesticides in schedule 2437 in surface water by Major River Basin. Recovery values larger than 400 percent are not shown.-Continued 
AA. Alachlor

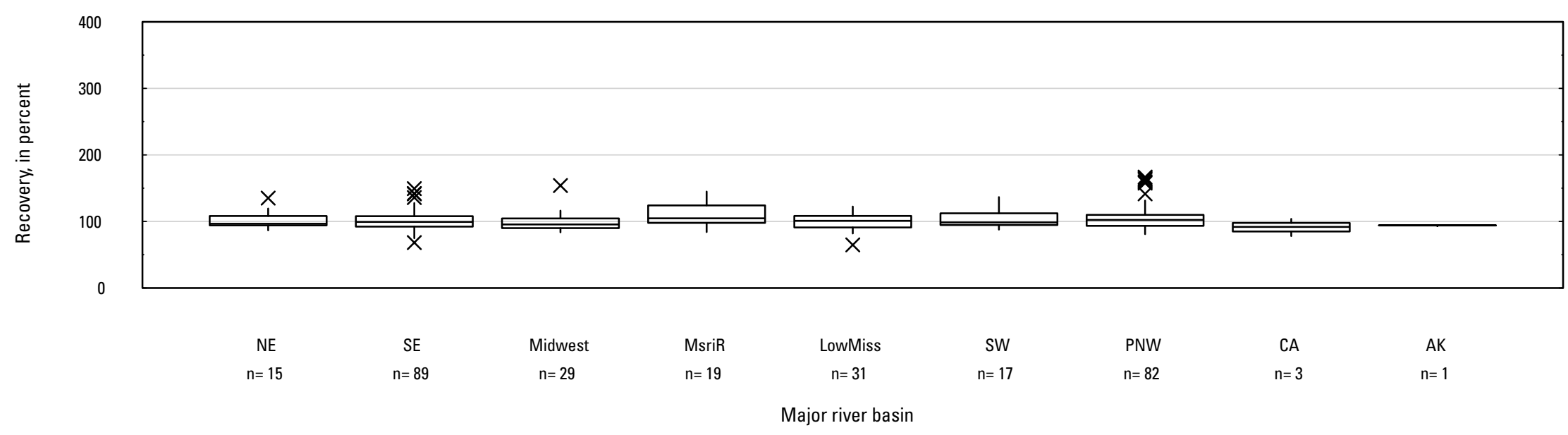

AB. Alachlor oxanilic acid

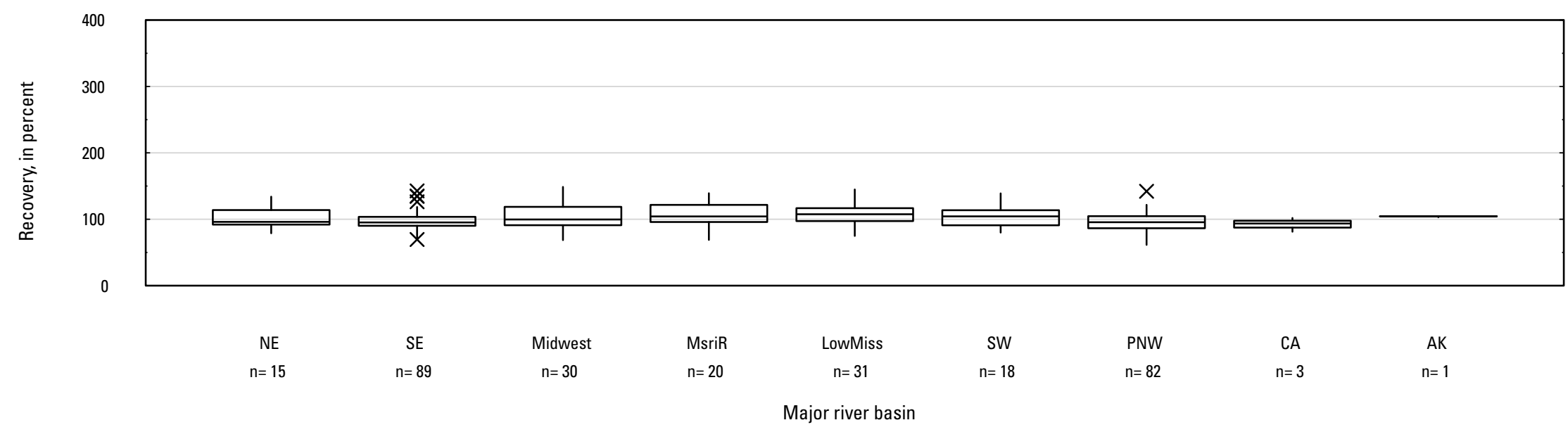

Figure 5. Graph showing distributions of recovery for pesticides in schedule 2437 in surface water by Major River Basin. Recovery values larger than 400 percent are not shown.-Continued 


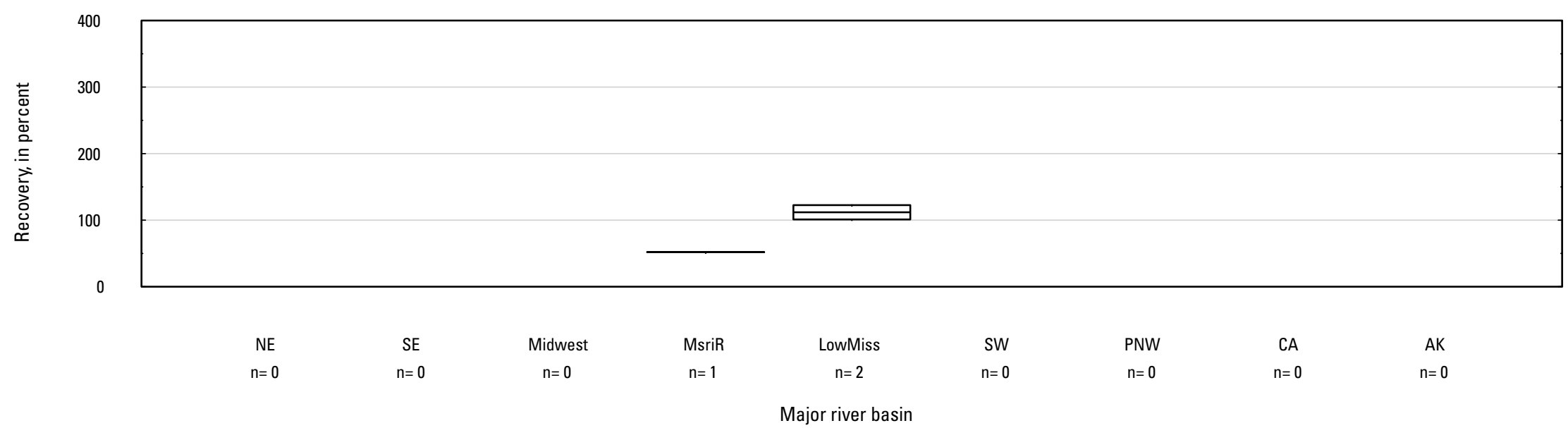

AD. Alachlor sulfynilacetic acid

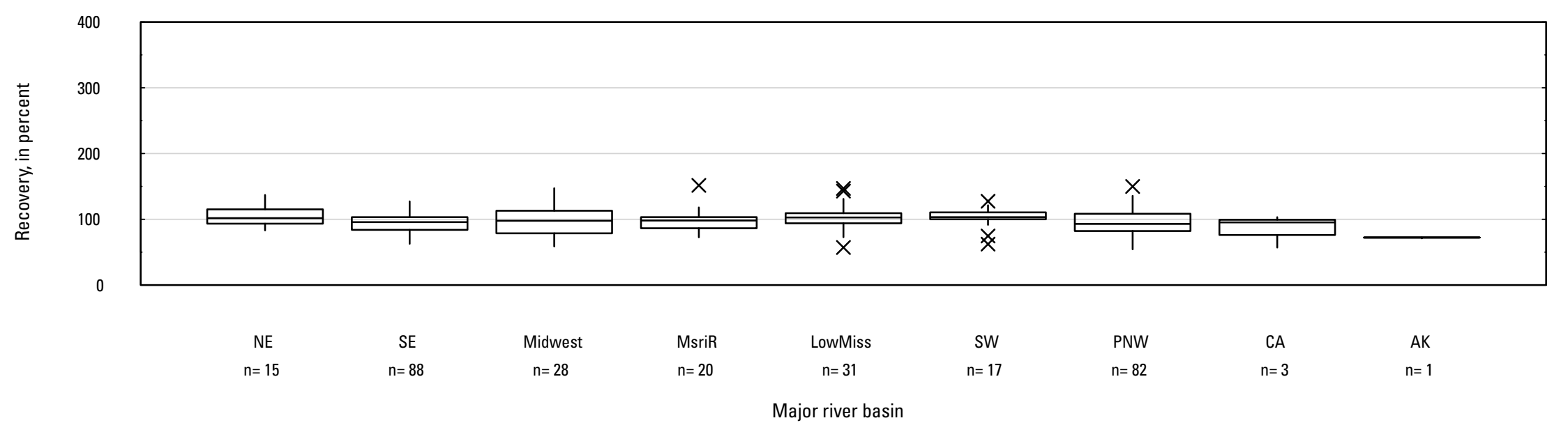

Figure 5. Graph showing distributions of recovery for pesticides in schedule 2437 in surface water by Major River Basin. Recovery values larger than 400 percent are not shown.-Continued 


\section{AE. Aldicarb}

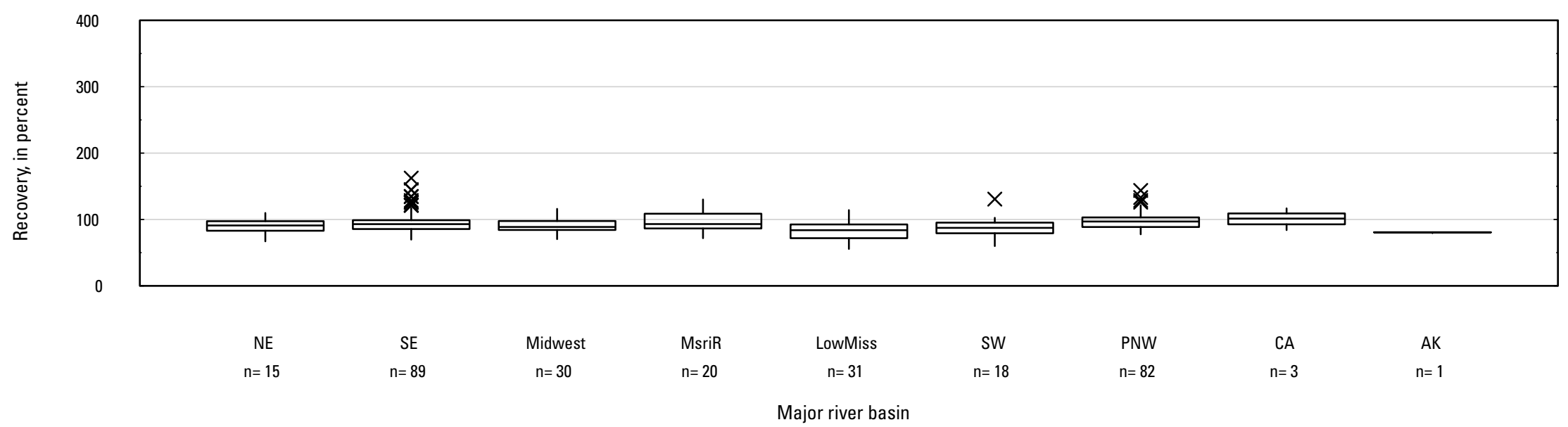

AF. Aldicarb sulfone

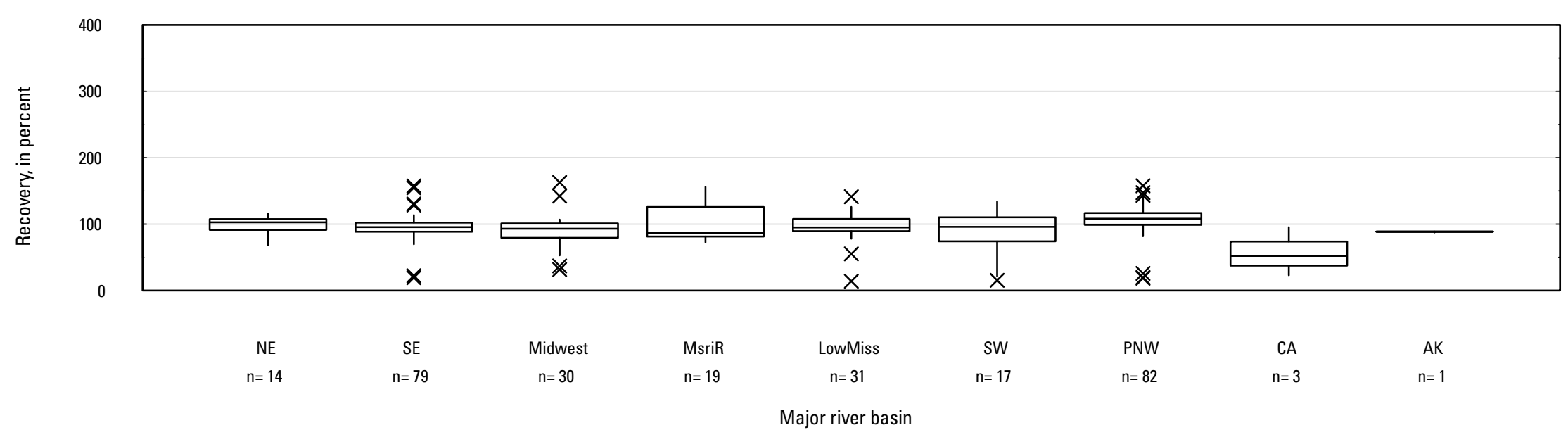

Figure 5. Graph showing distributions of recovery for pesticides in schedule 2437 in surface water by Major River Basin. Recovery values larger than 400 percent are 


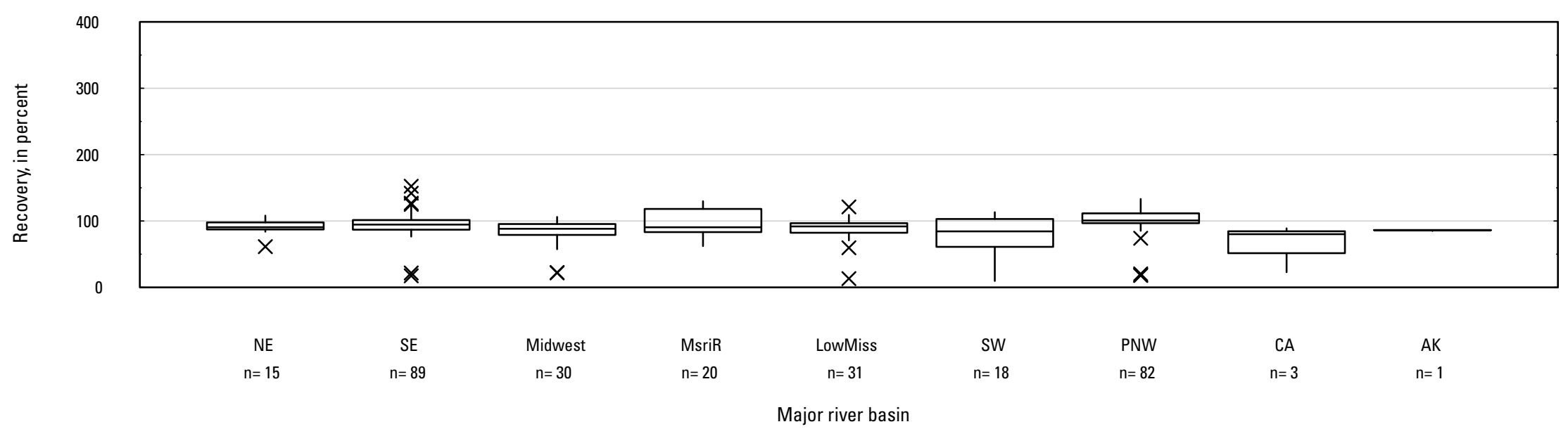

AH. Ametryn

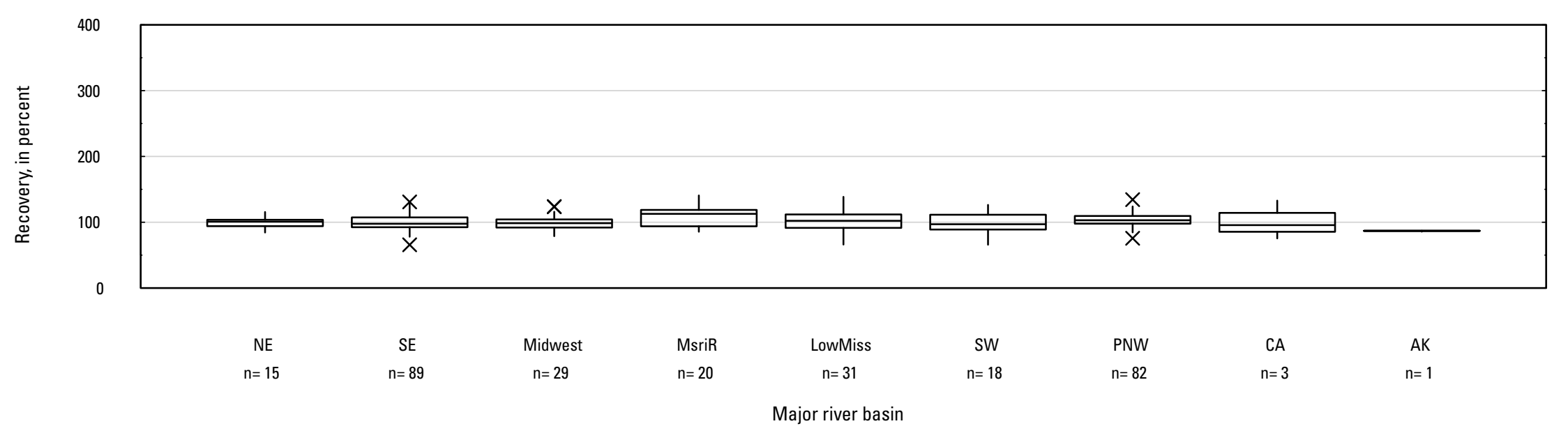

Figure 5. Graph showing distributions of recovery for pesticides in schedule 2437 in surface water by Major River Basin. Recovery values larger than 400 percent are not shown.-Continued 


\section{Al. Asulam}

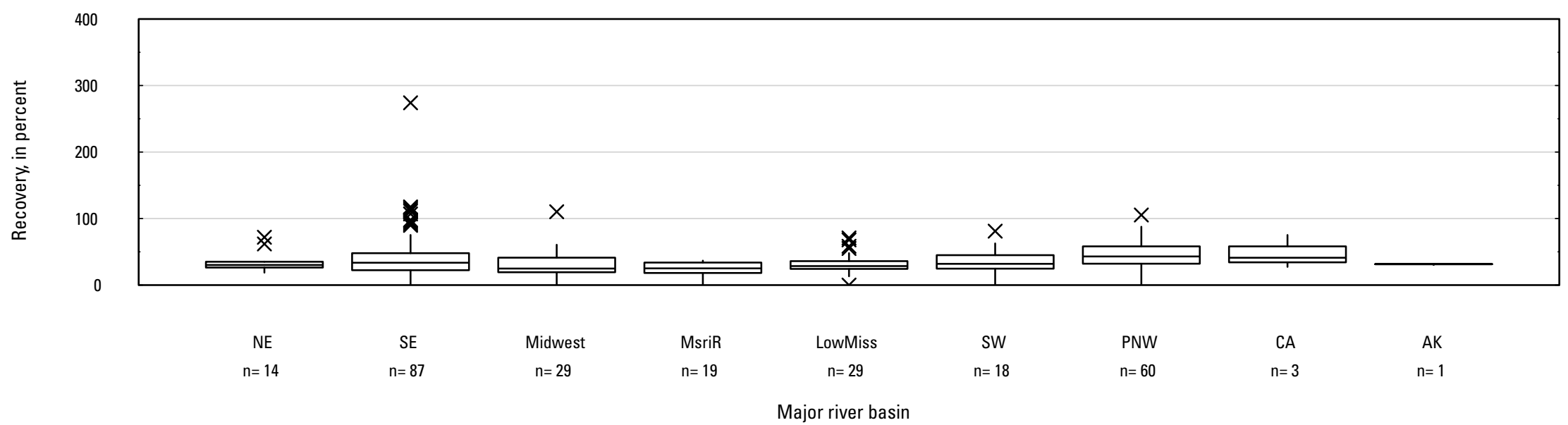

\section{AJ. Atrazine}

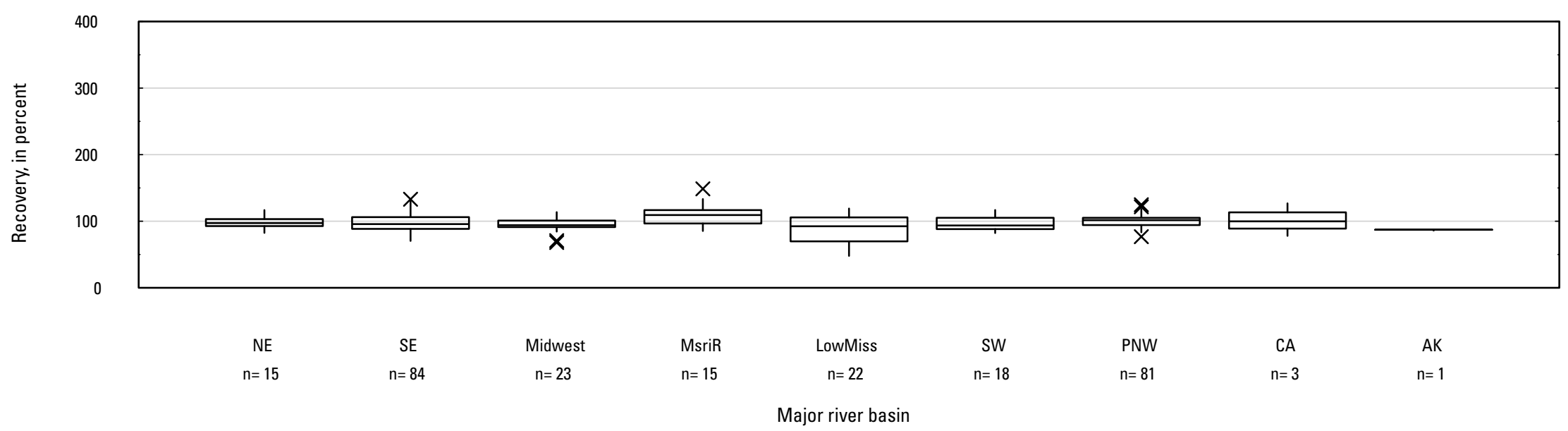

Figure 5. Graph showing distributions of recovery for pesticides in schedule 2437 in surface water by Major River Basin. Recovery values larger than 400 percent are not shown.-Continued 


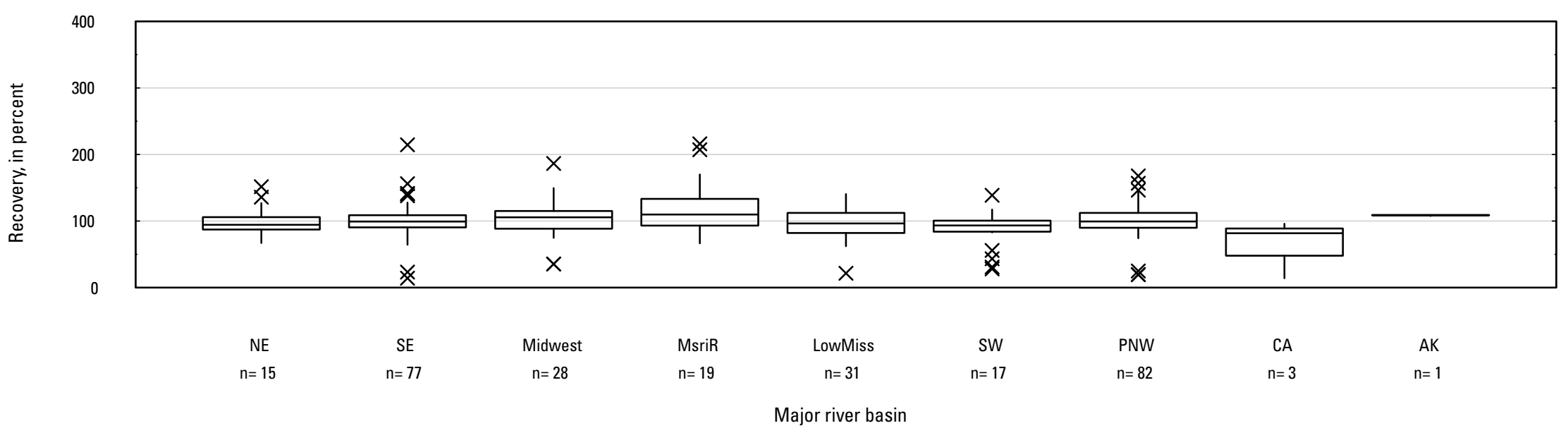

AL. Azinphos-methyl oxon

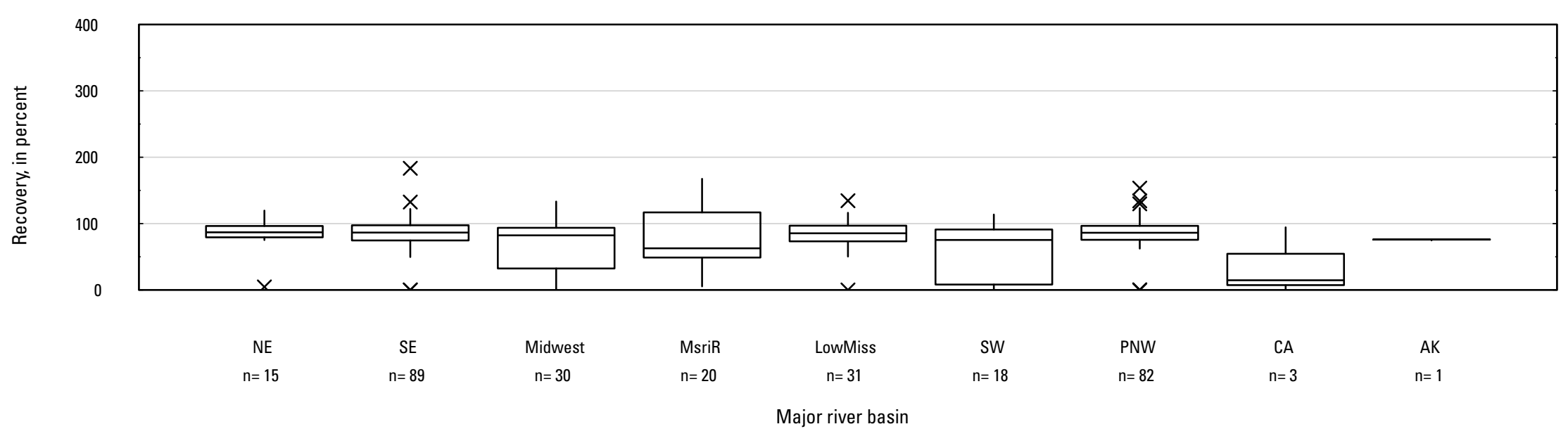

Figure 5. Graph showing distributions of recovery for pesticides in schedule 2437 in surface water by Major River Basin. Recovery values larger than 400 percent are not shown.-Continued 
AM. Azoxystrobin

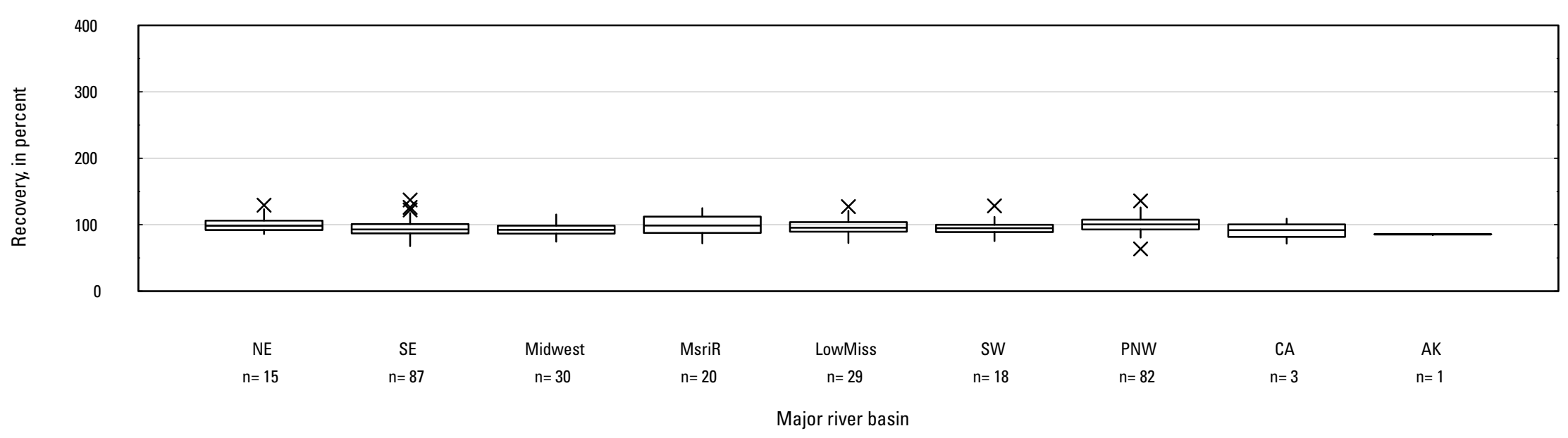

AN. Bentazone

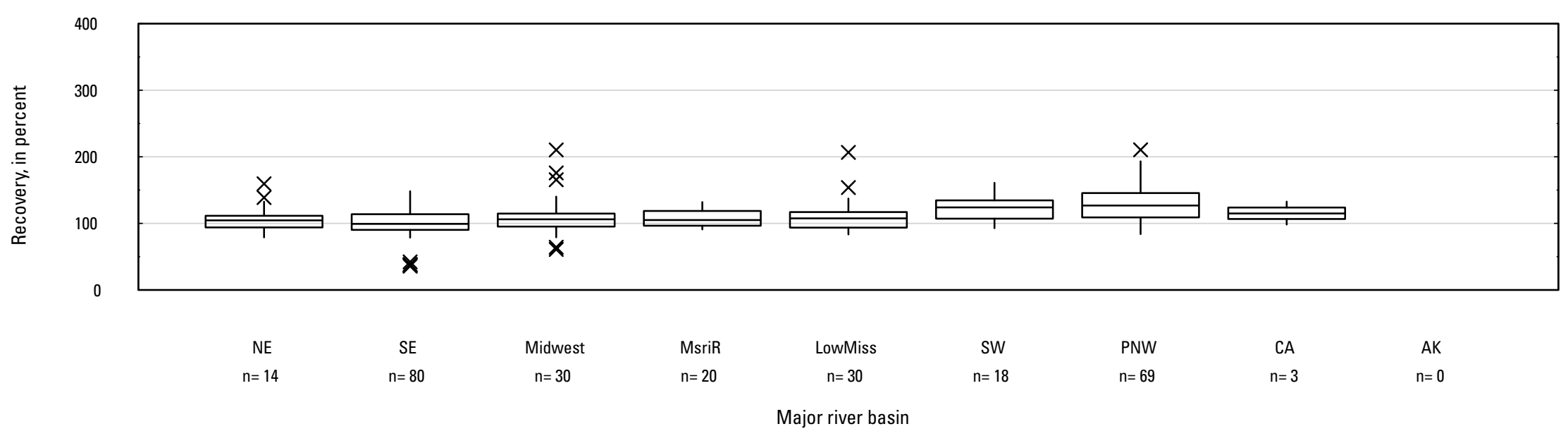

Figure 5. Graph showing distributions of recovery for pesticides in schedule 2437 in surface water by Major River Basin. Recovery values larger than 400 percent are not shown.-Continued 


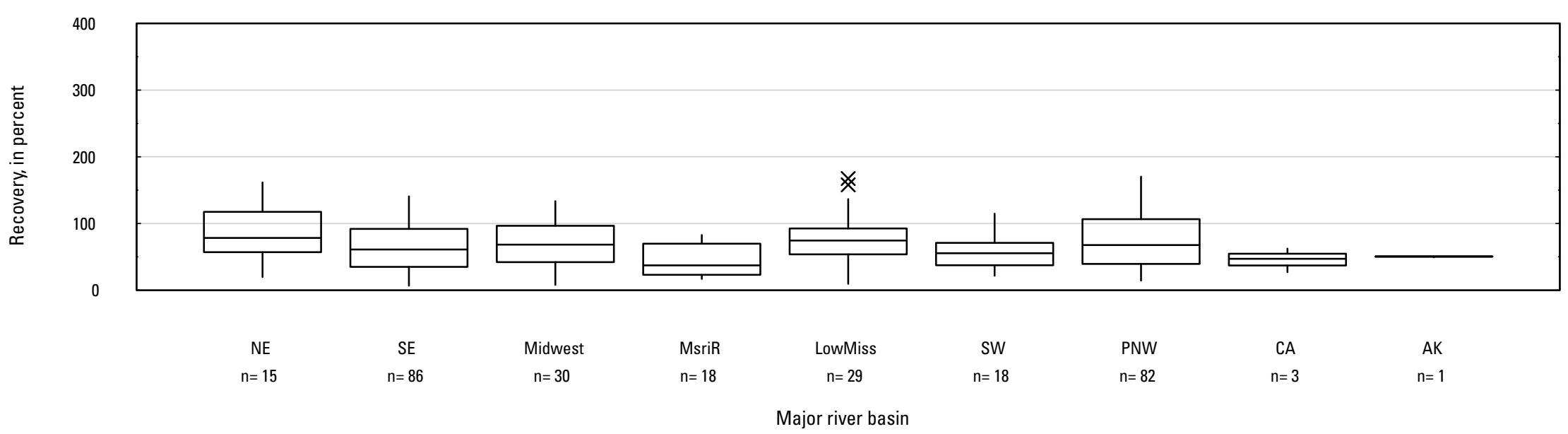

AP. Bromacil

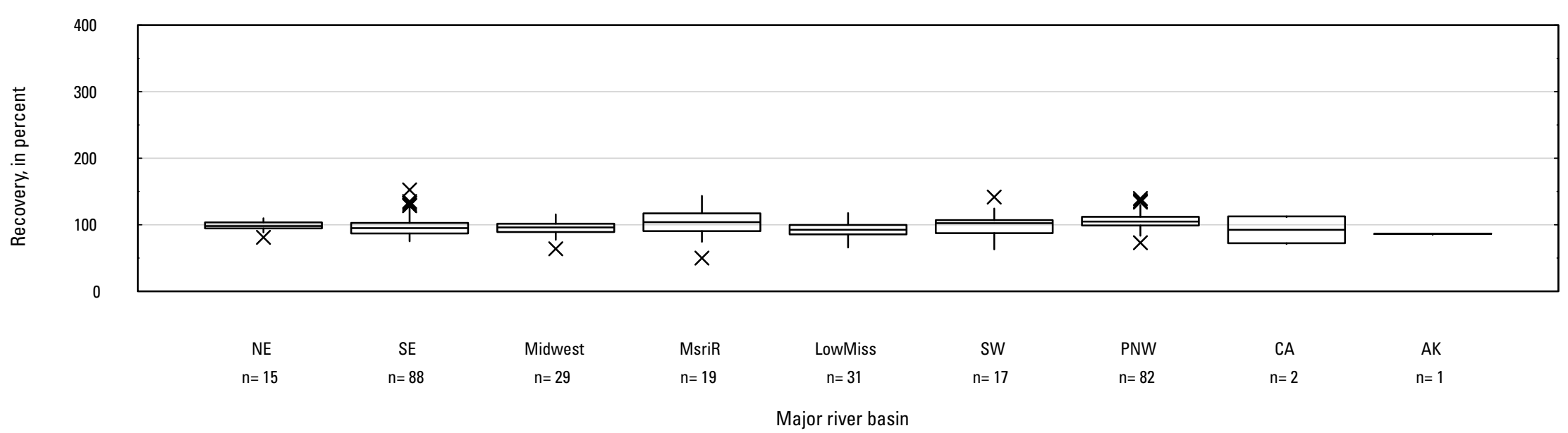

Figure 5. Graph showing distributions of recovery for pesticides in schedule 2437 in surface water by Major River Basin. Recovery values larger than 400 percent are not shown.-Continued 
AQ. Bromoxynil

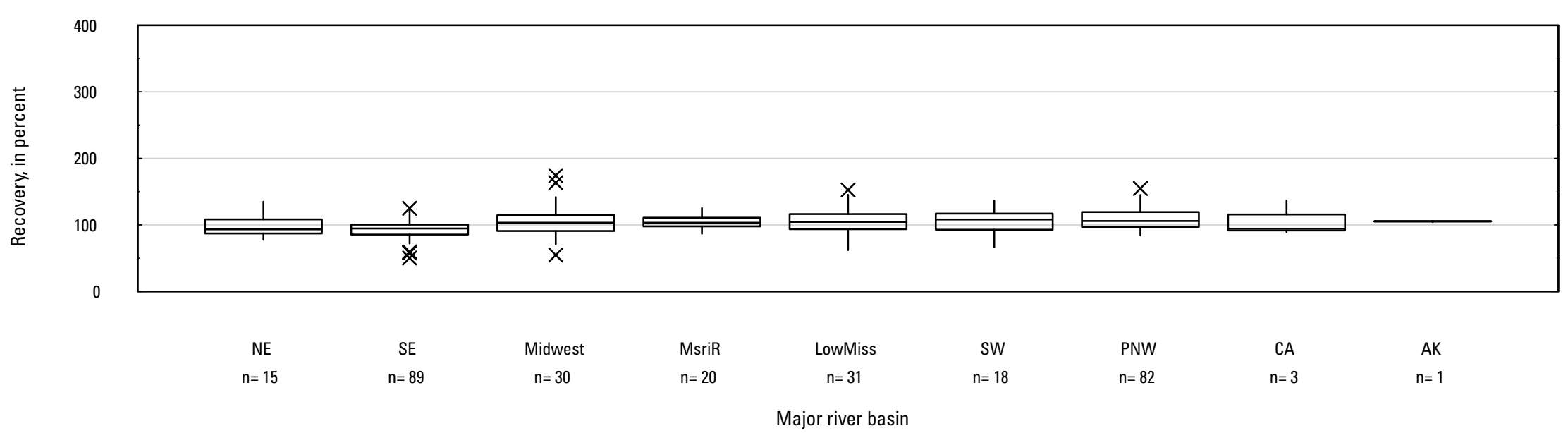

AR. Butralin

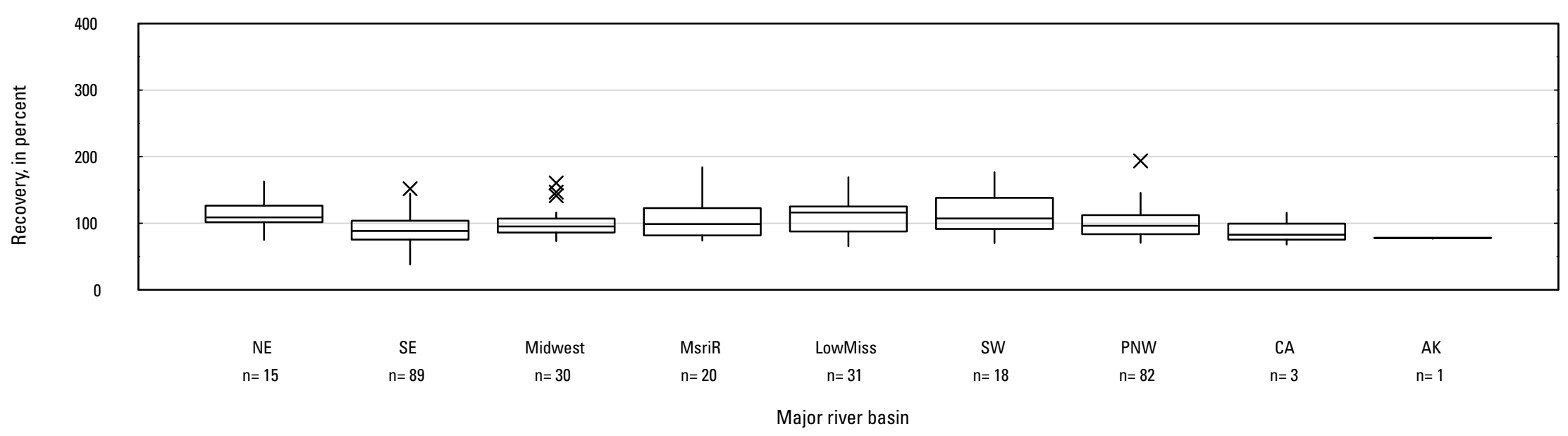

Figure 5. Graph showing distributions of recovery for pesticides in schedule 2437 in surface water by Major River Basin. Recovery values larger than 400 percent are 


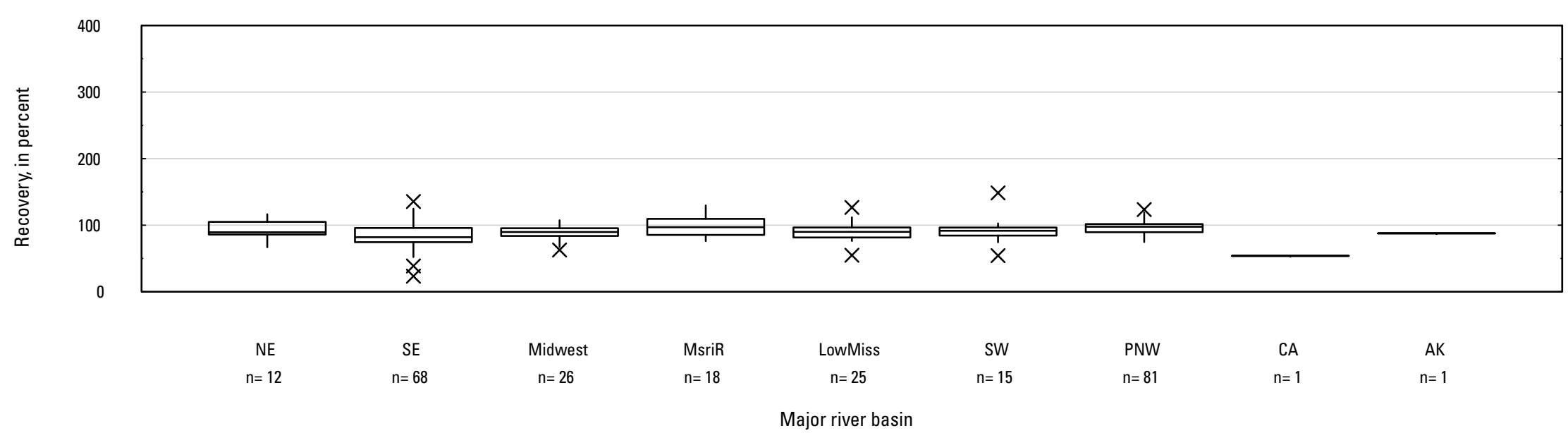

AT. Carbaryl

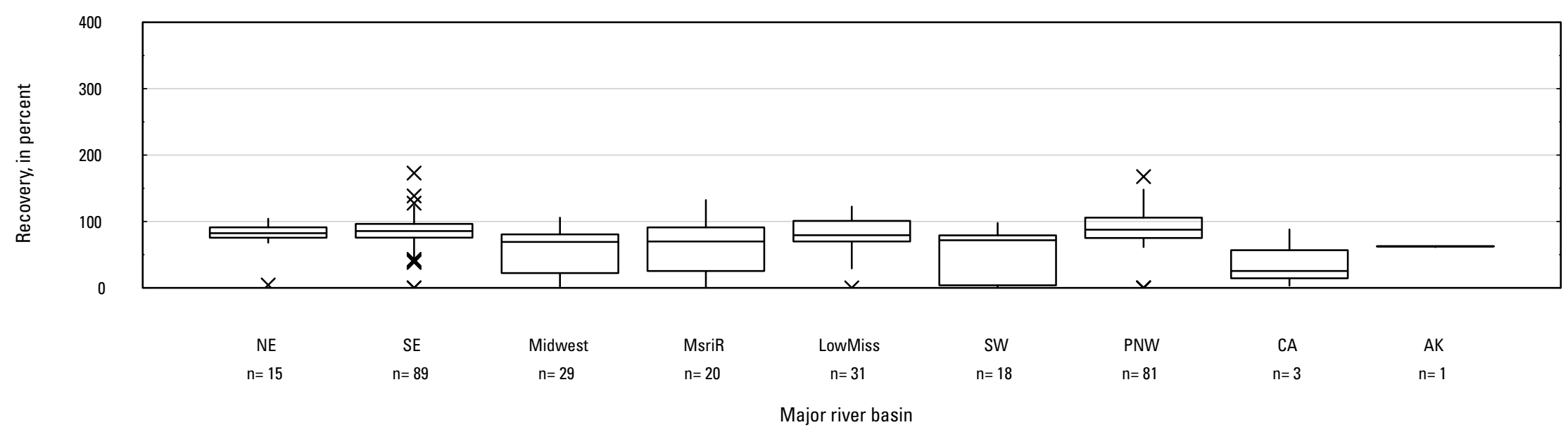

Figure 5. Graph showing distributions of recovery for pesticides in schedule 2437 in surface water by Major River Basin. Recovery values larger than 400 percent are not shown.-Continued 
AU. Carbendazim

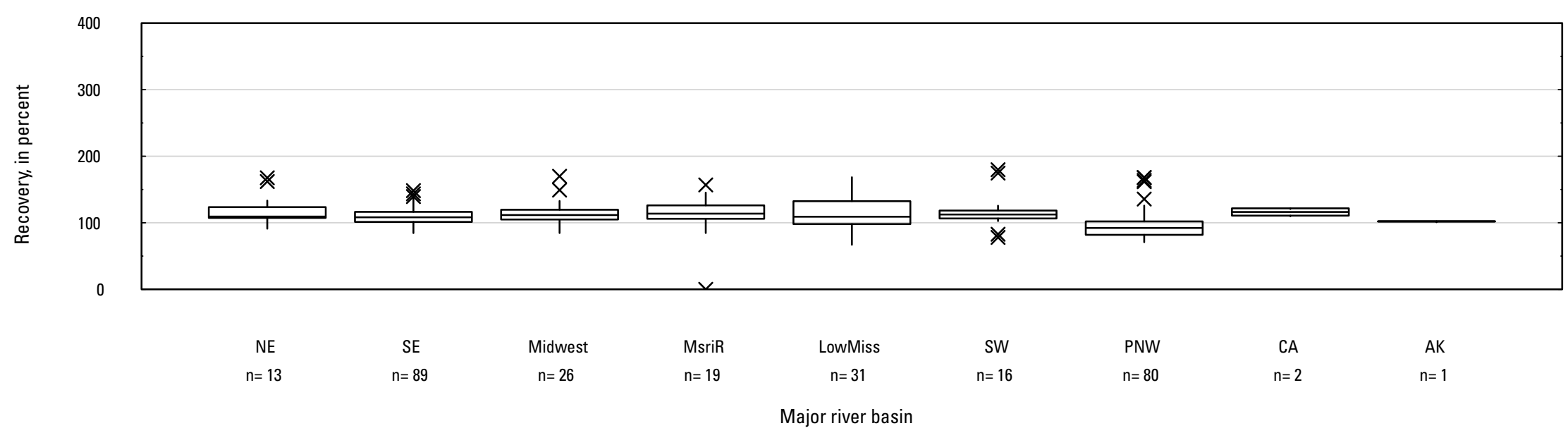

AV. Carbofuran

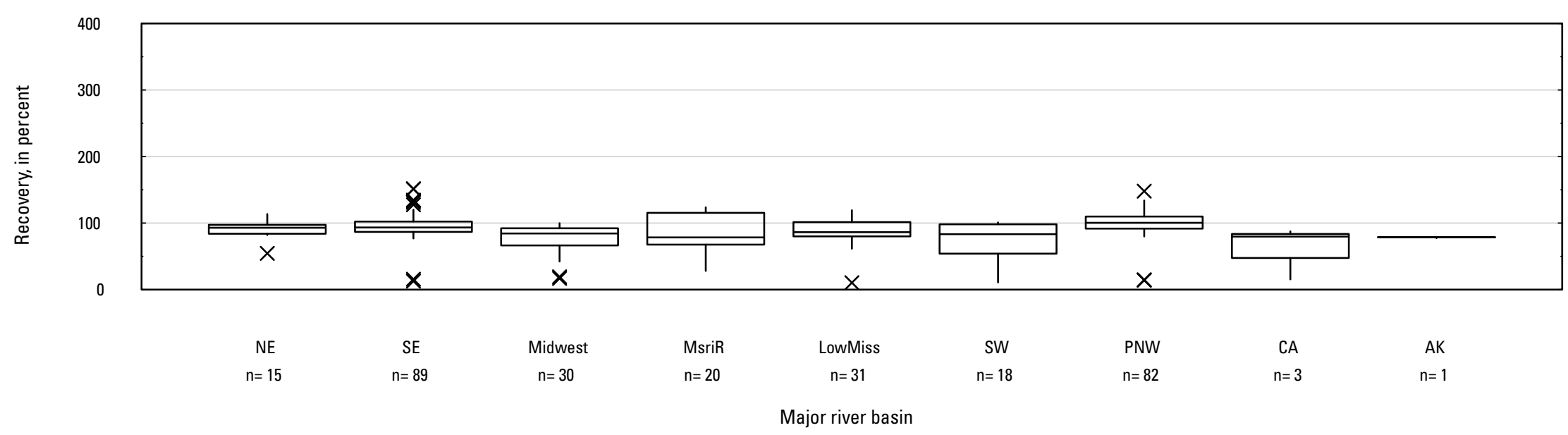

Figure 5. Graph showing distributions of recovery for pesticides in schedule 2437 in surface water by Major River Basin. Recovery values larger than 400 percent are not shown.-Continued 


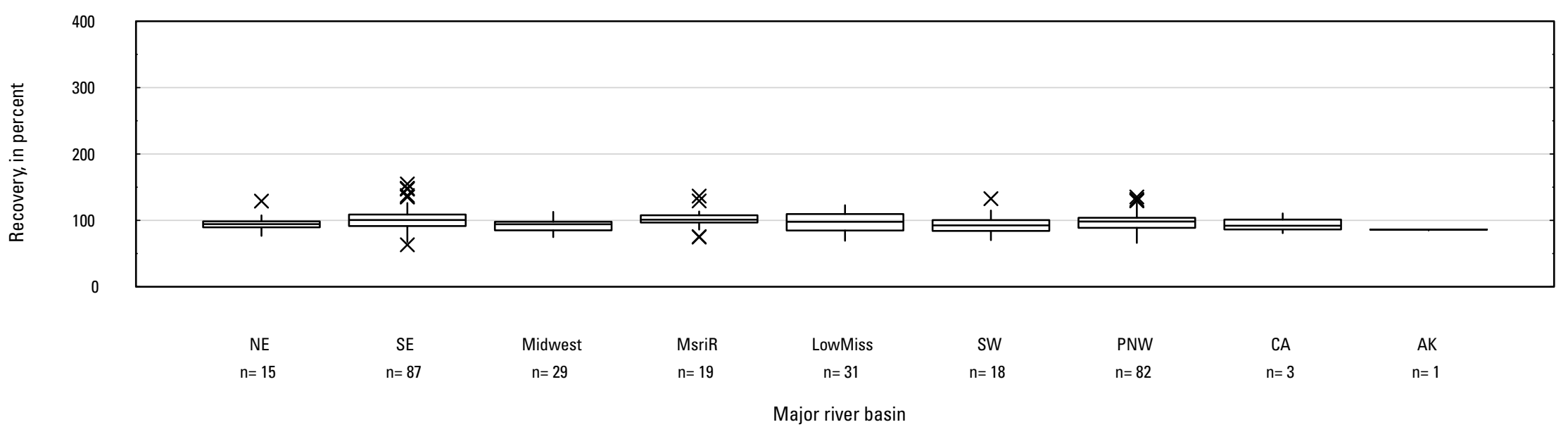

AX. Chlorimuron-ethyl

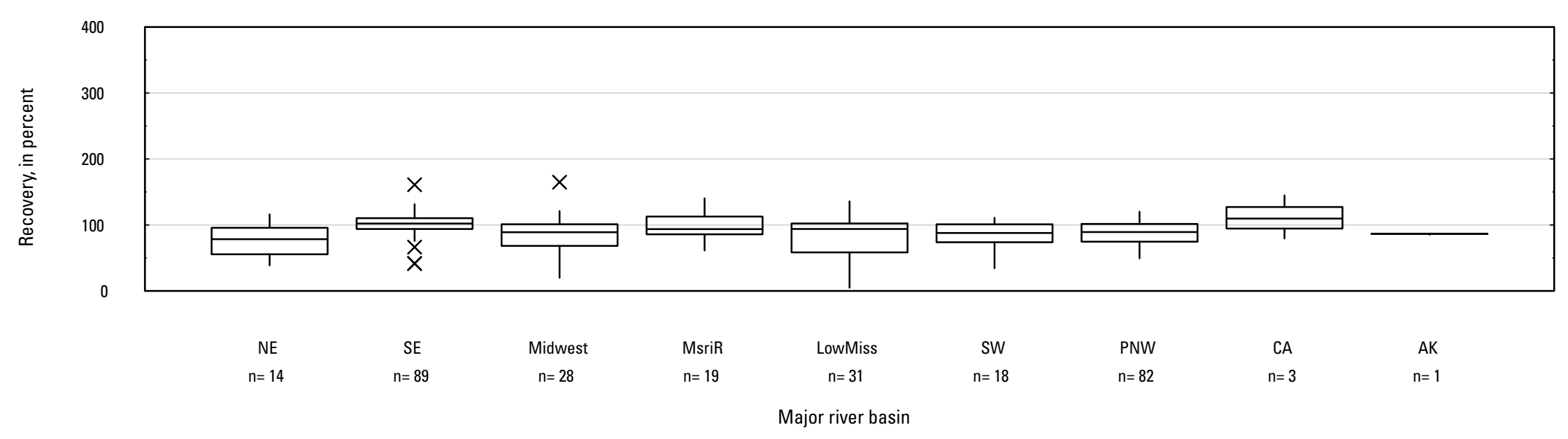

Figure 5. Graph showing distributions of recovery for pesticides in schedule 2437 in surface water by Major River Basin. Recovery values larger than 400 percent are not shown.-Continued 
AY. Chlorosulfonamide acid

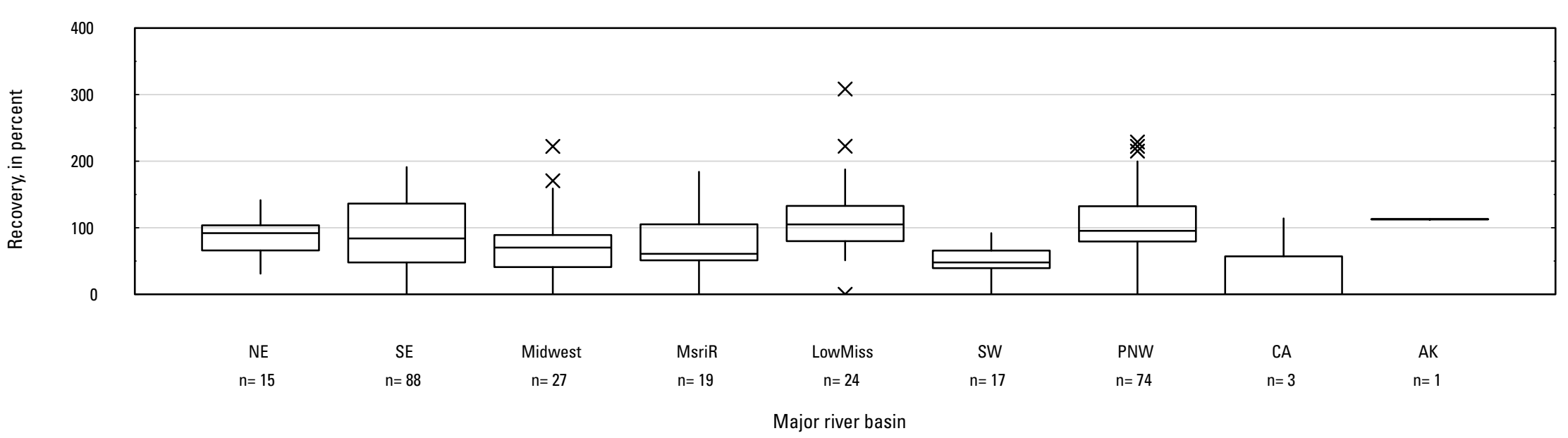

AZ. Chlorpyrifos

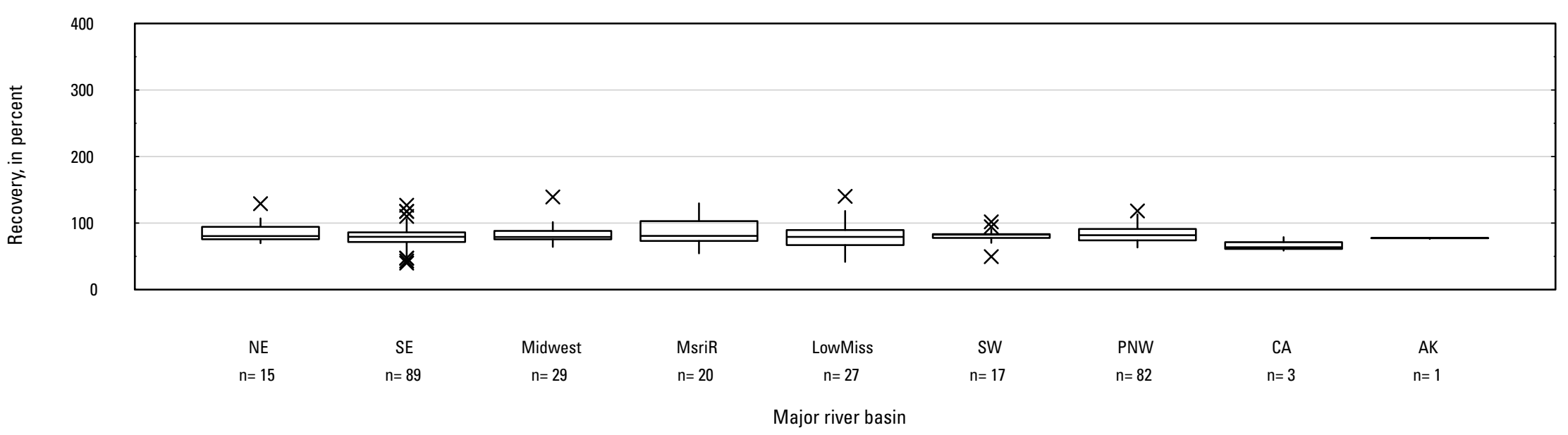

Figure 5. Graph showing distributions of recovery for pesticides in schedule 2437 in surface water by Major River Basin. Recovery values larger than 400 percent are not shown.-Continued 


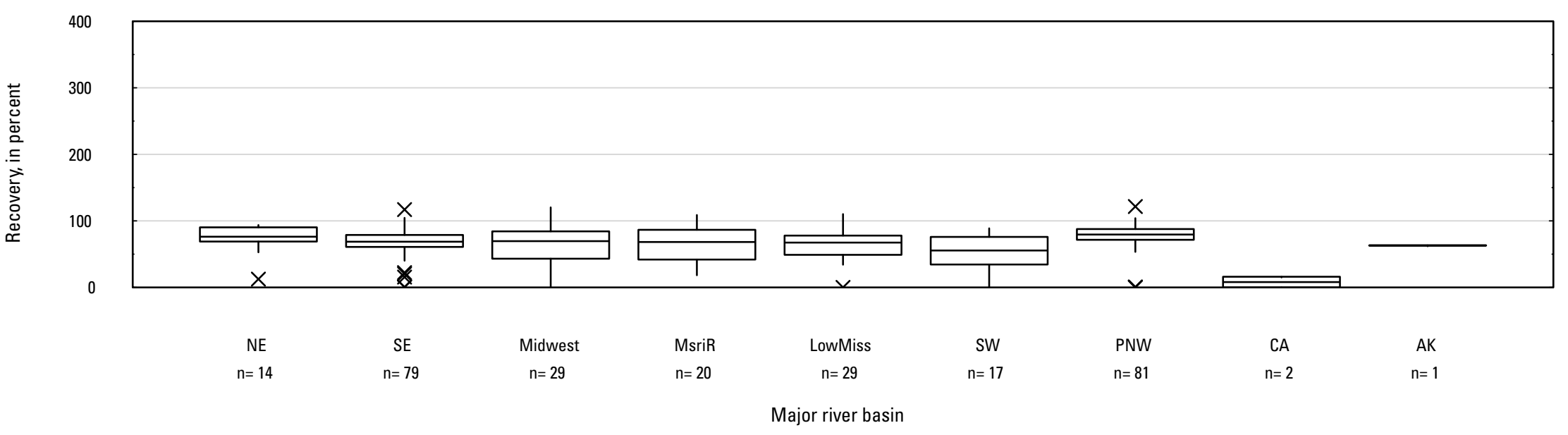

\section{BB. Chlorsulfuron}

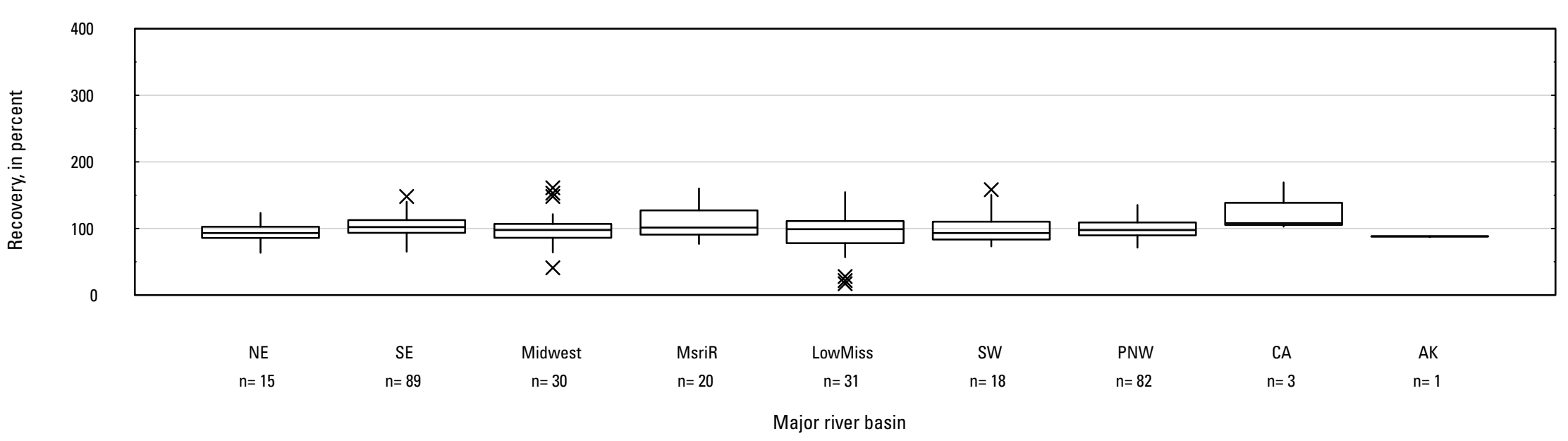

Figure 5. Graph showing distributions of recovery for pesticides in schedule 2437 in surface water by Major River Basin. Recovery values larger than 400 percent are not shown.-Continued 
BE. cis-Permethrin

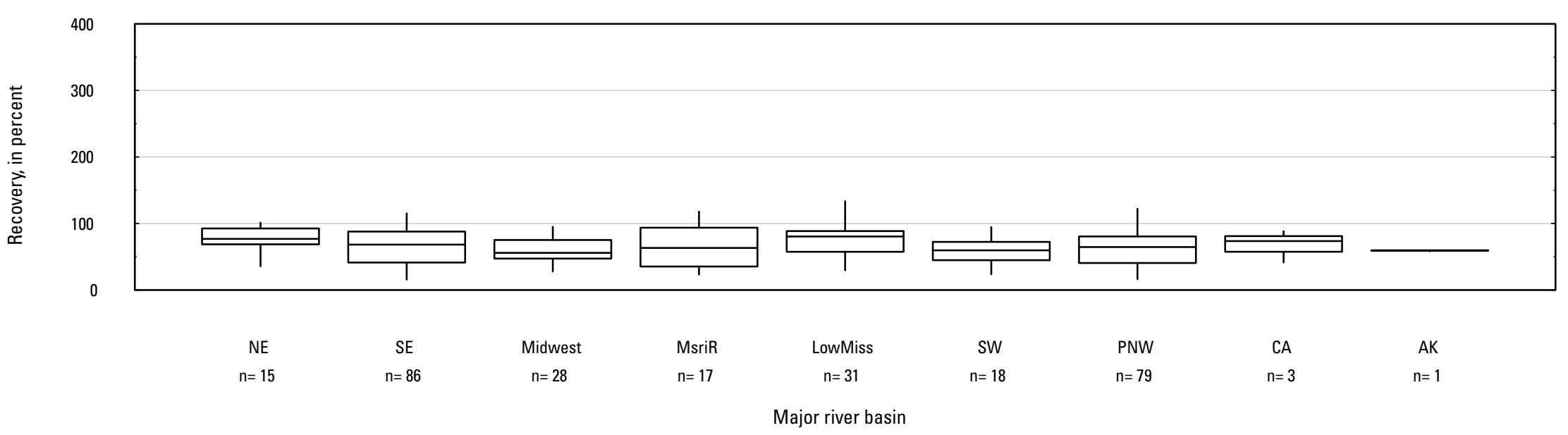

BF. Cyanazine

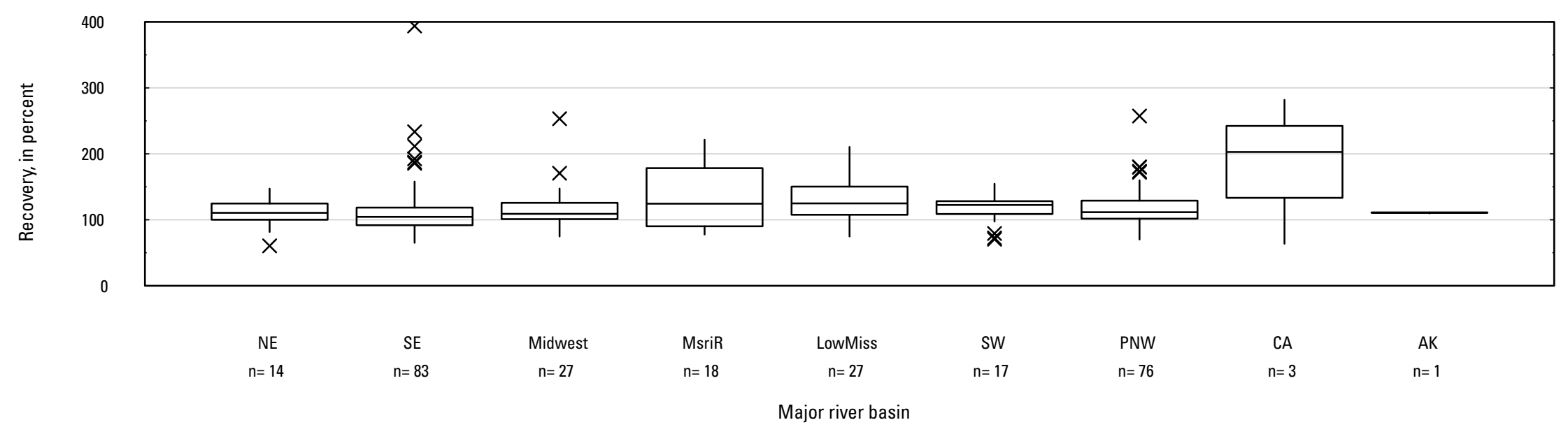

Figure 5. Graph showing distributions of recovery for pesticides in schedule 2437 in surface water by Major River Basin. Recovery values larger than 400 percent are not shown.-Continued 


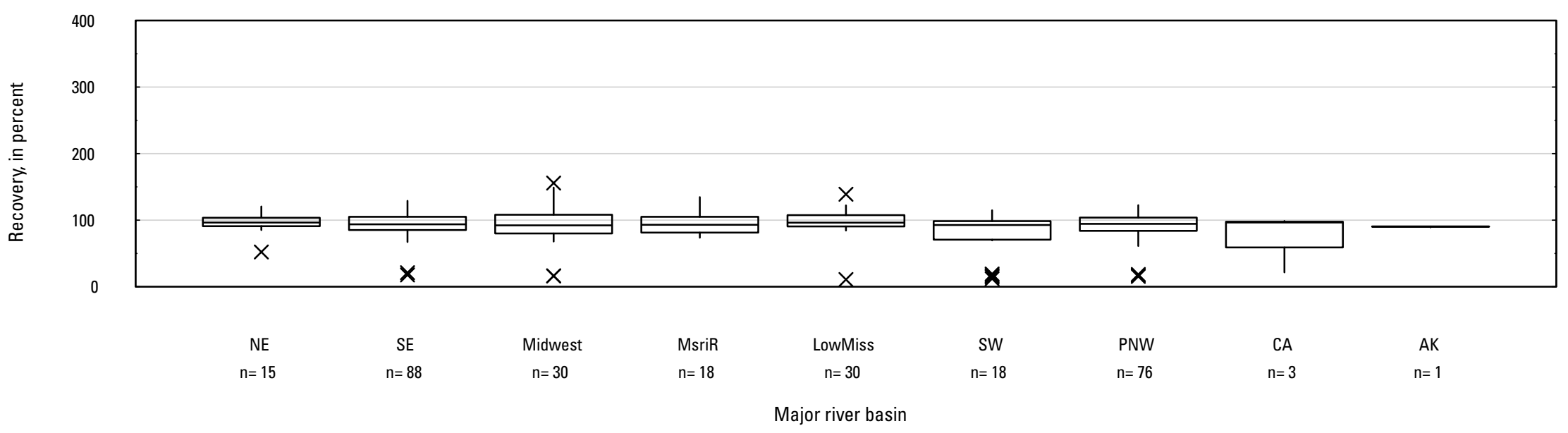

BH. Dechlorometolachlor

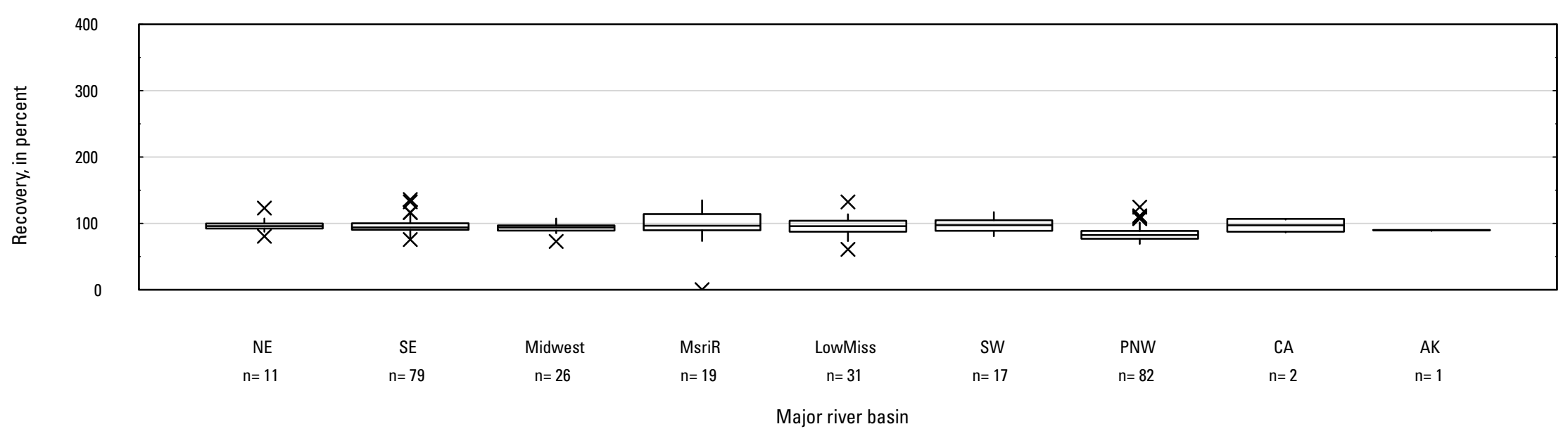

Figure 5. Graph showing distributions of recovery for pesticides in schedule 2437 in surface water by Major River Basin. Recovery values larger than 400 percent are not shown.-Continued 
BI. 2-Chloro-4-isopropylamino-6-amino-s-triazine

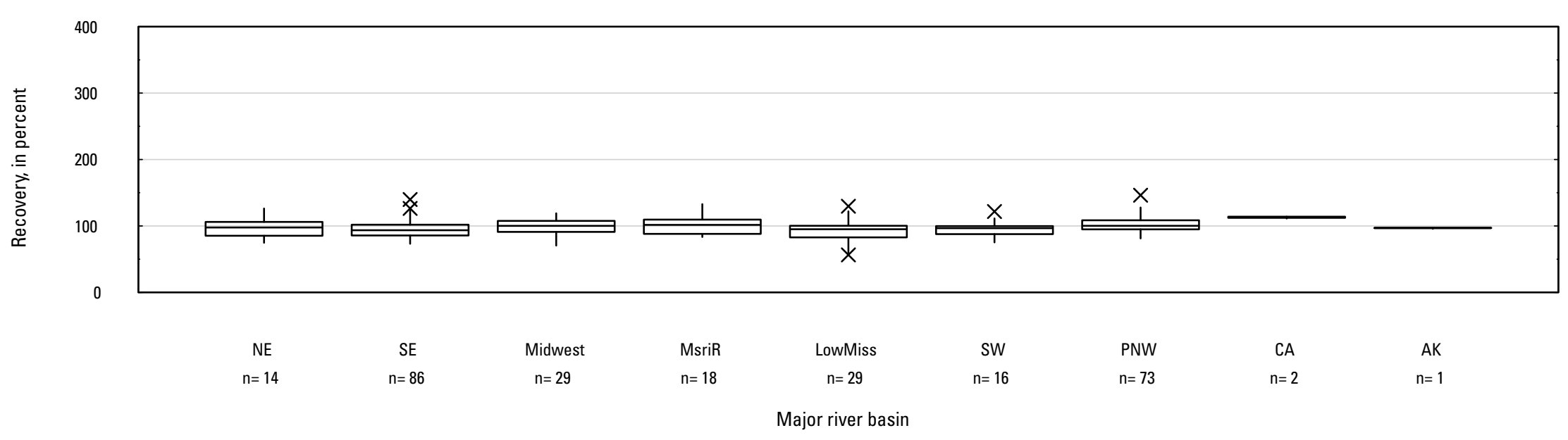

\section{BJ. Deisopropyl prometryn}

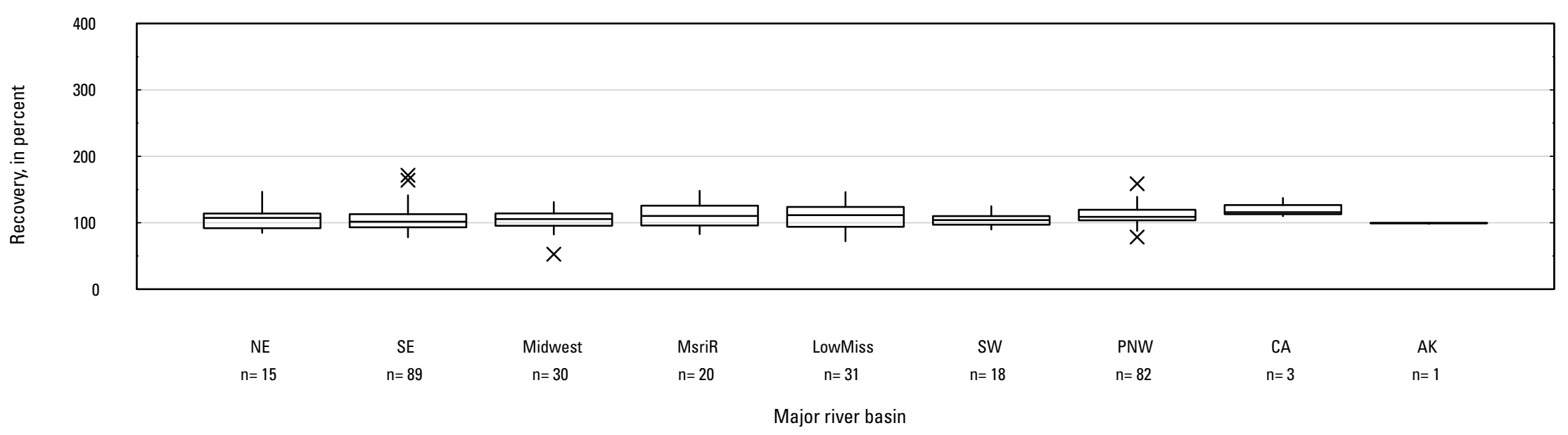

Figure 5. Graph showing distributions of recovery for pesticides in schedule 2437 in surface water by Major River Basin. Recovery values larger than 400 percent are 


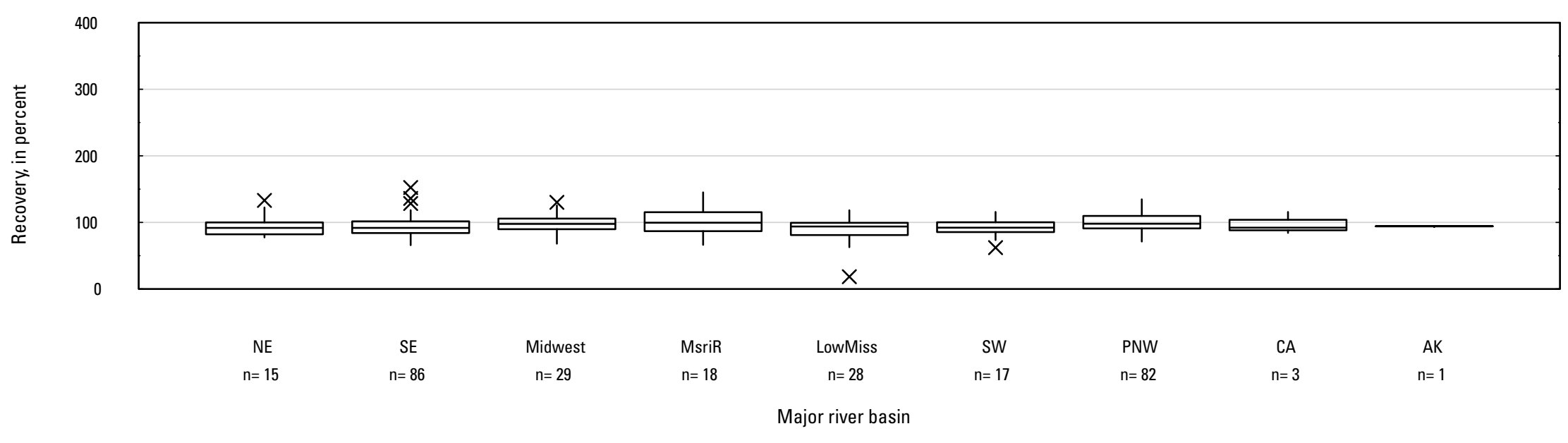

\section{$B L$. Demethyl fluometuron}

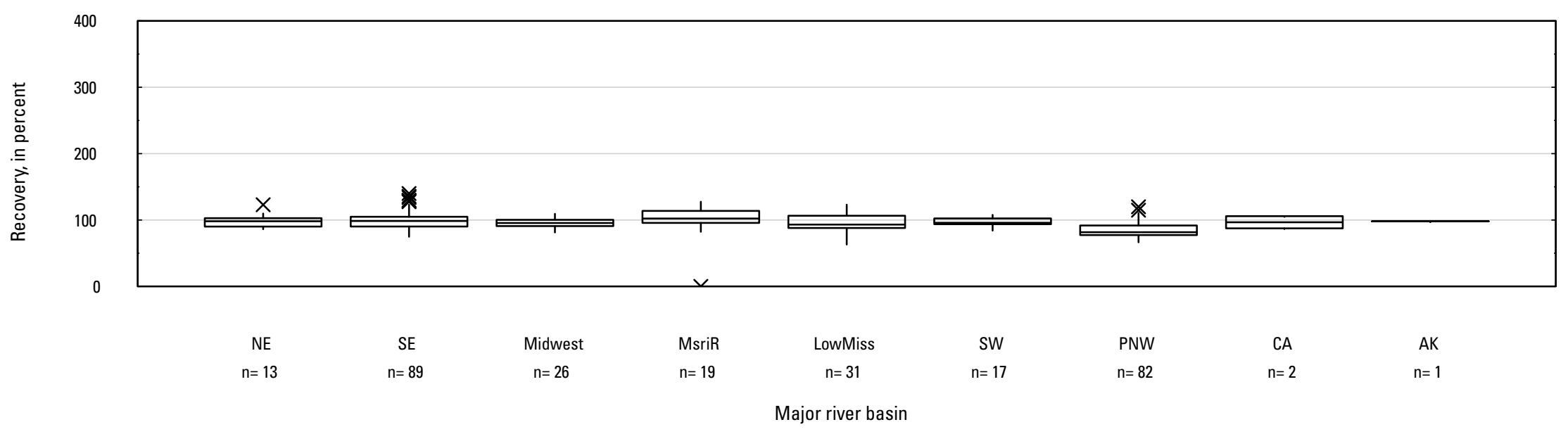

Figure 5. Graph showing distributions of recovery for pesticides in schedule 2437 in surface water by Major River Basin. Recovery values larger than 400 percent are not shown.-Continued 
BM. Demethyl hexazinone B

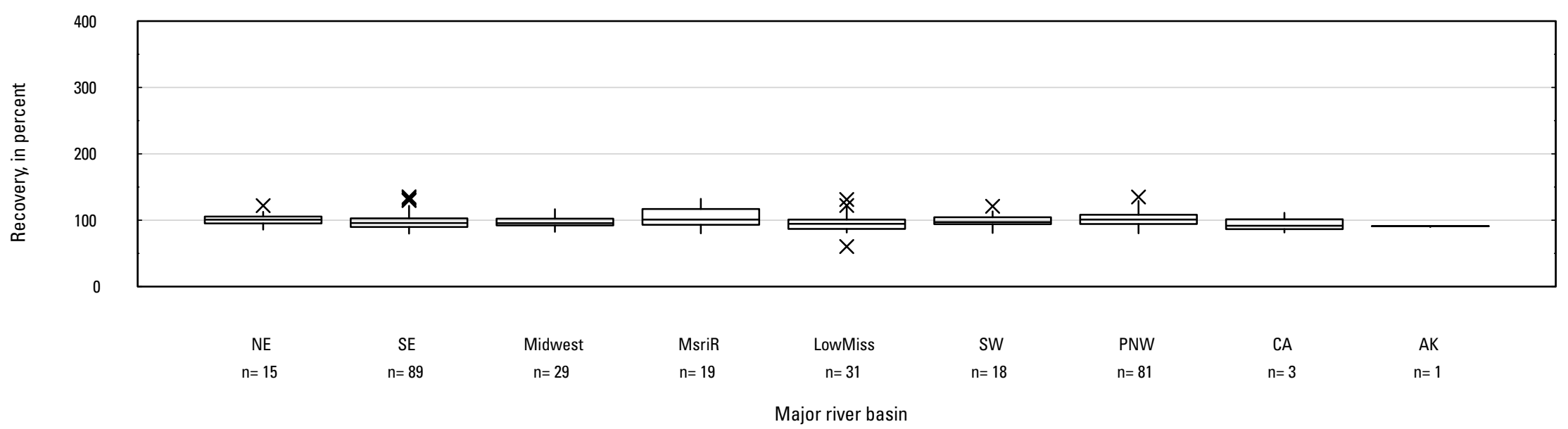

$B N$. Demethyl norflurazon

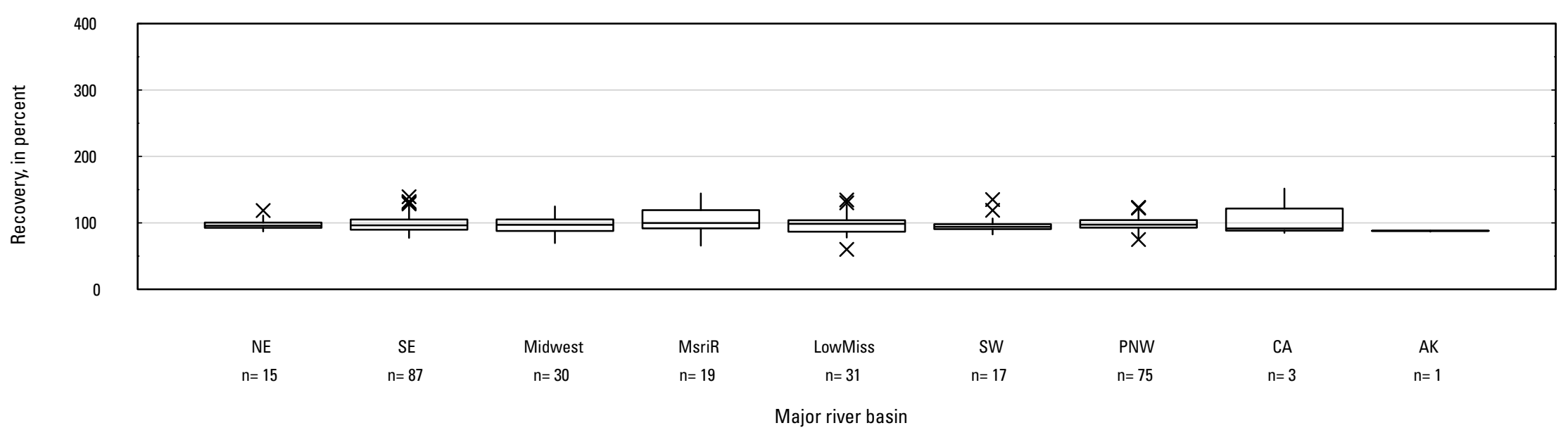

Figure 5. Graph showing distributions of recovery for pesticides in schedule 2437 in surface water by Major River Basin. Recovery values larger than 400 percent are 


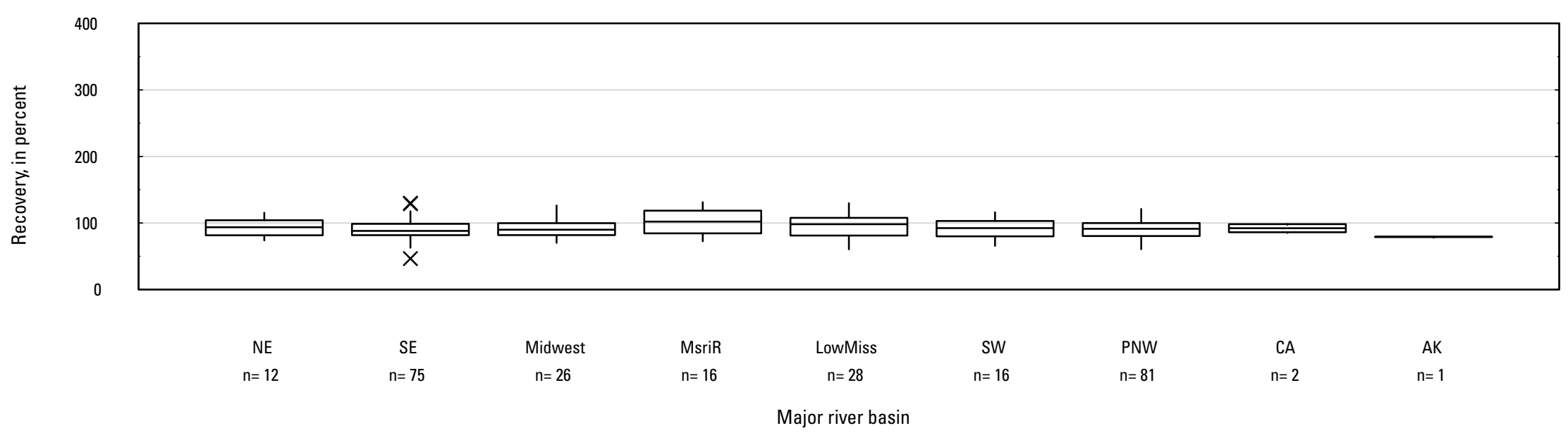

\section{$B P$. Desulfinylfipronil}

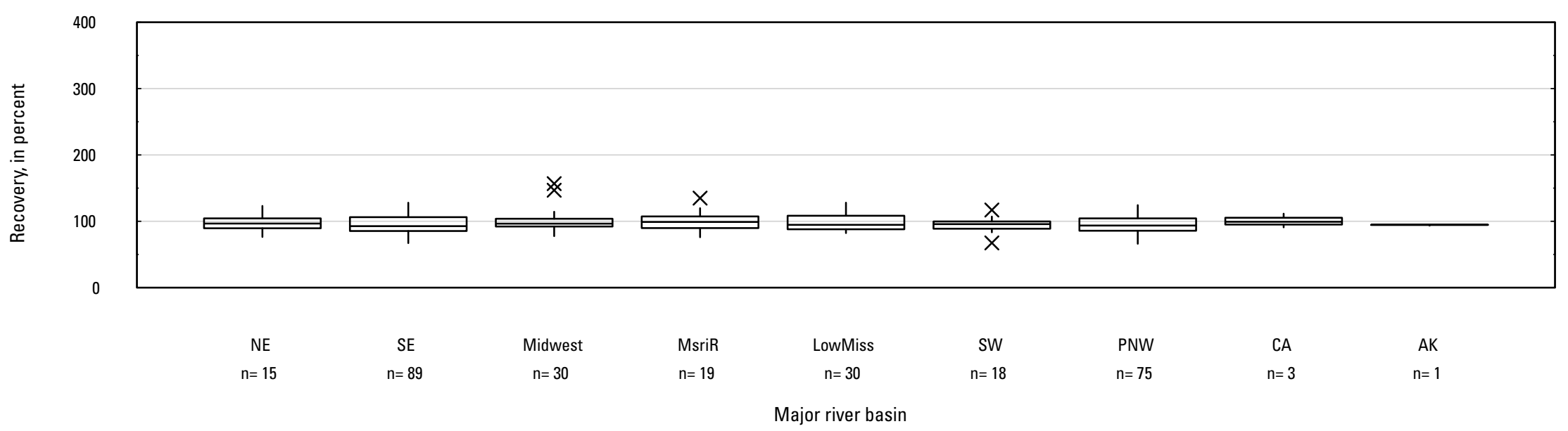

Figure 5. Graph showing distributions of recovery for pesticides in schedule 2437 in surface water by Major River Basin. Recovery values larger than 400 percent are not shown.-Continued 
BO. Desulfinylfipronil amide

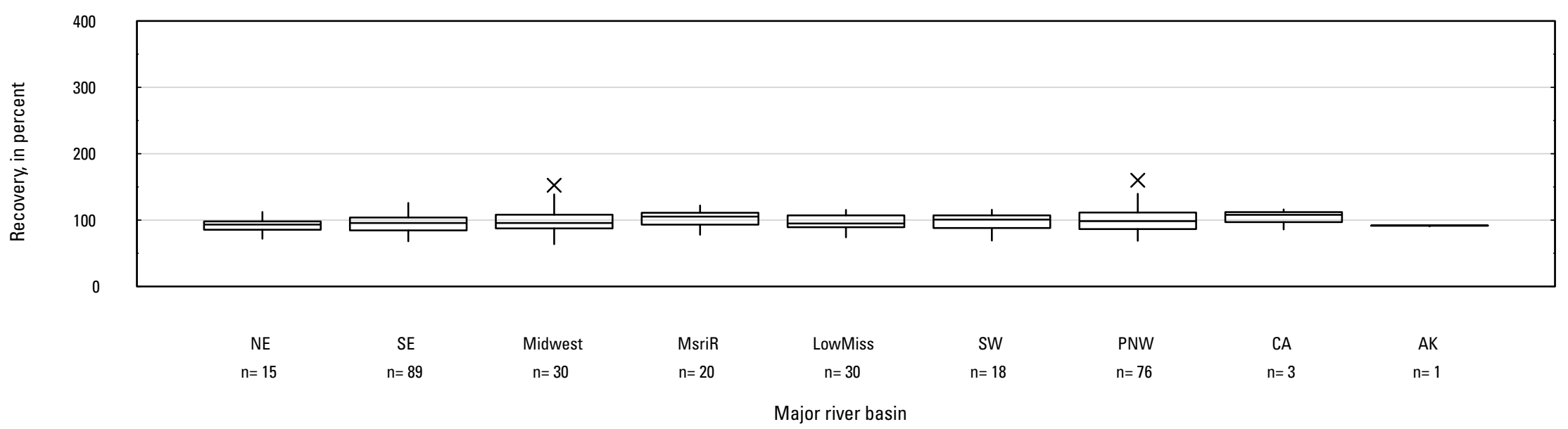

BR. Diazinon

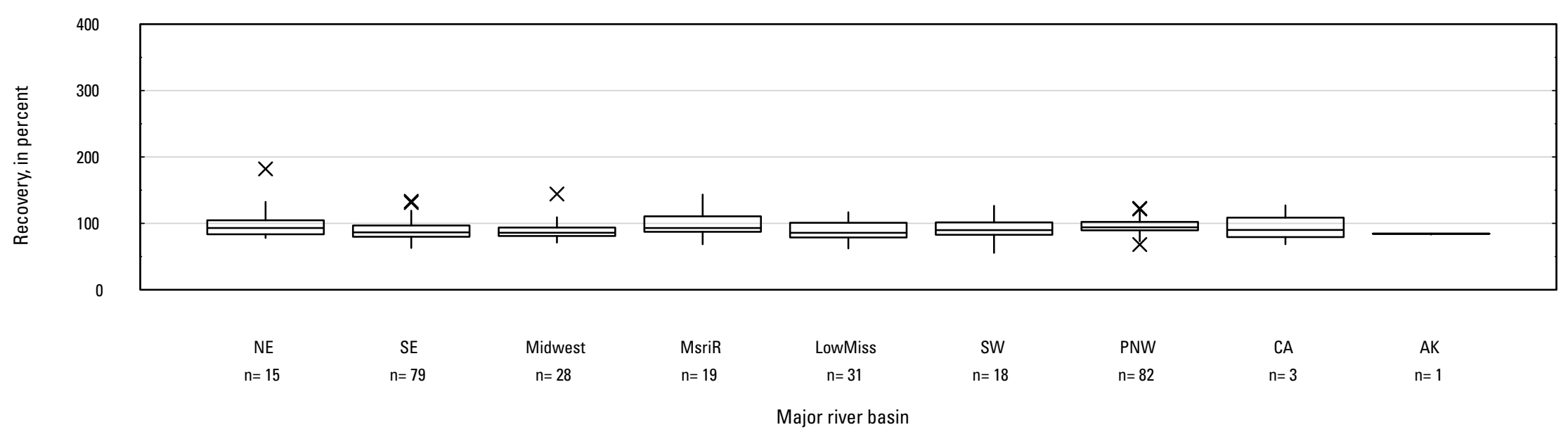

Figure 5. Graph showing distributions of recovery for pesticides in schedule 2437 in surface water by Major River Basin. Recovery values larger than 400 percent are not shown.-Continued 


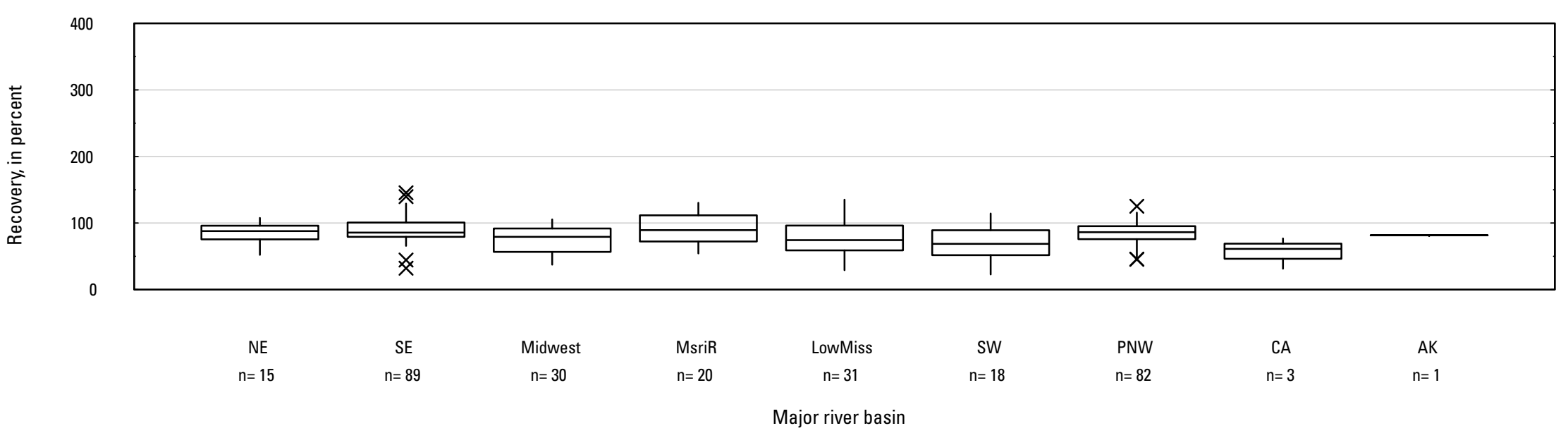

BU. Dichlorvos

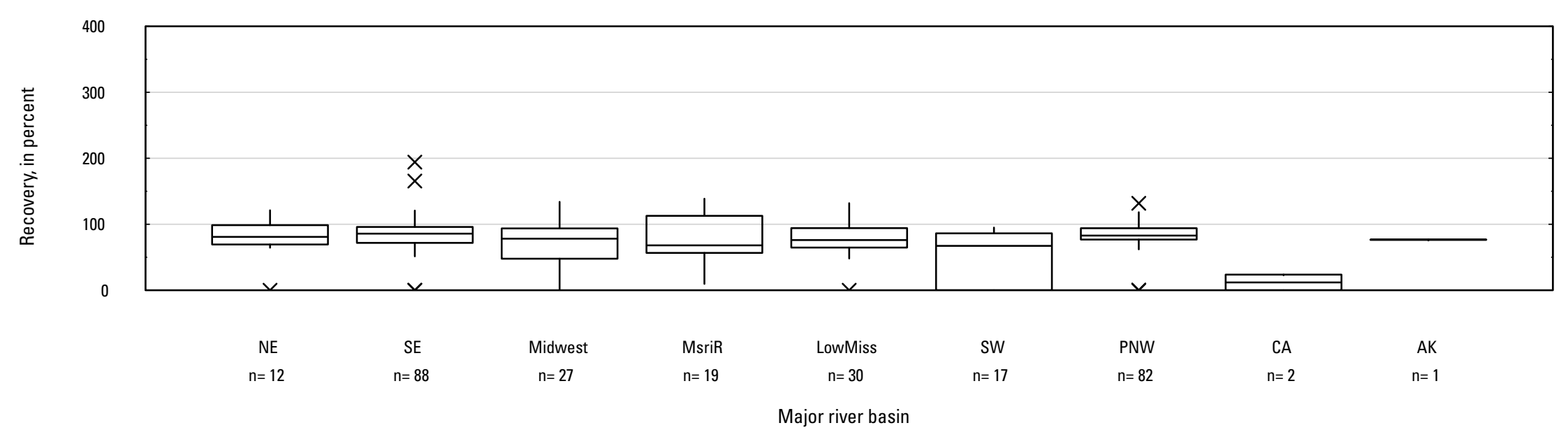

Figure 5. Graph showing distributions of recovery for pesticides in schedule 2437 in surface water by Major River Basin. Recovery values larger than 400 percent are not shown.-Continued 


\section{BV. Dicrotophos}

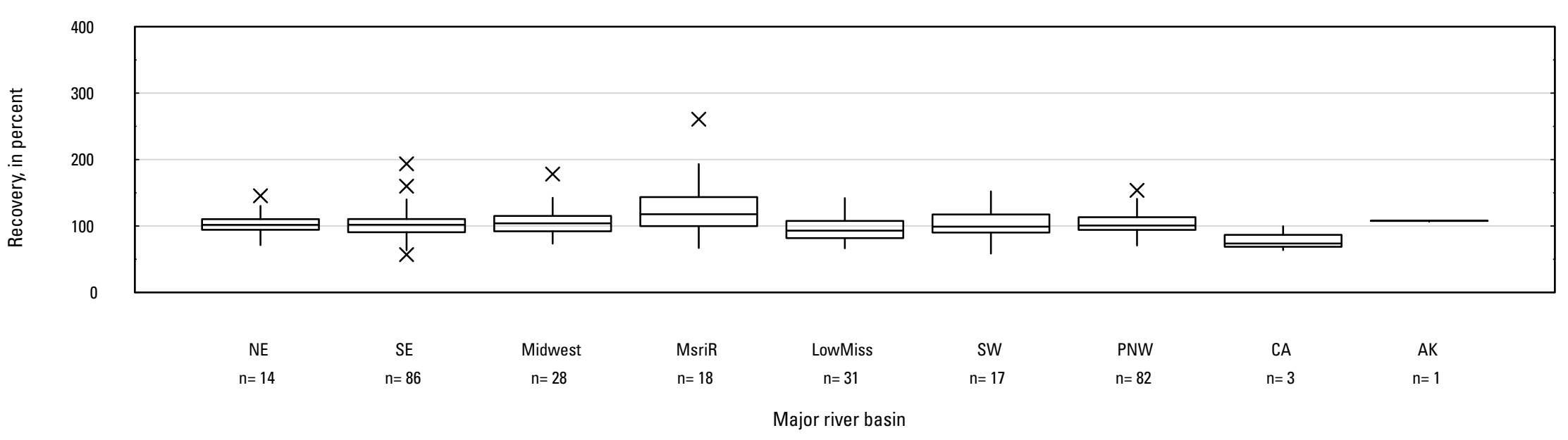

BW. 2-Chloro-4,6-diamino-s-triazine \{CAAT\} (Didealkylatrazine)

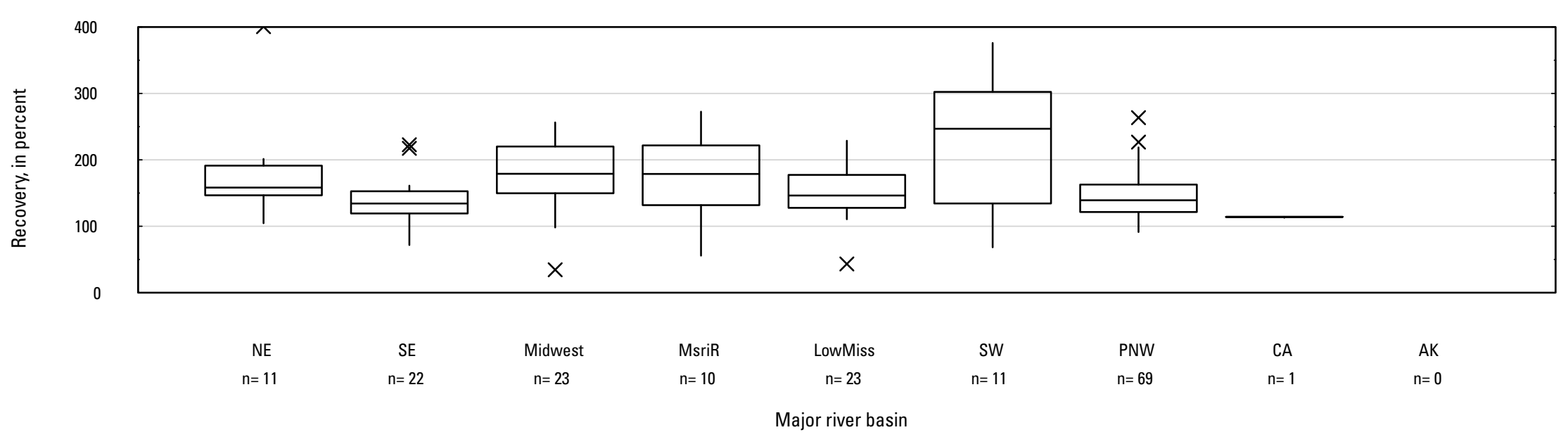

Figure 5. Graph showing distributions of recovery for pesticides in schedule 2437 in surface water by Major River Basin. Recovery values larger than 400 percent are not shown.-Continued 


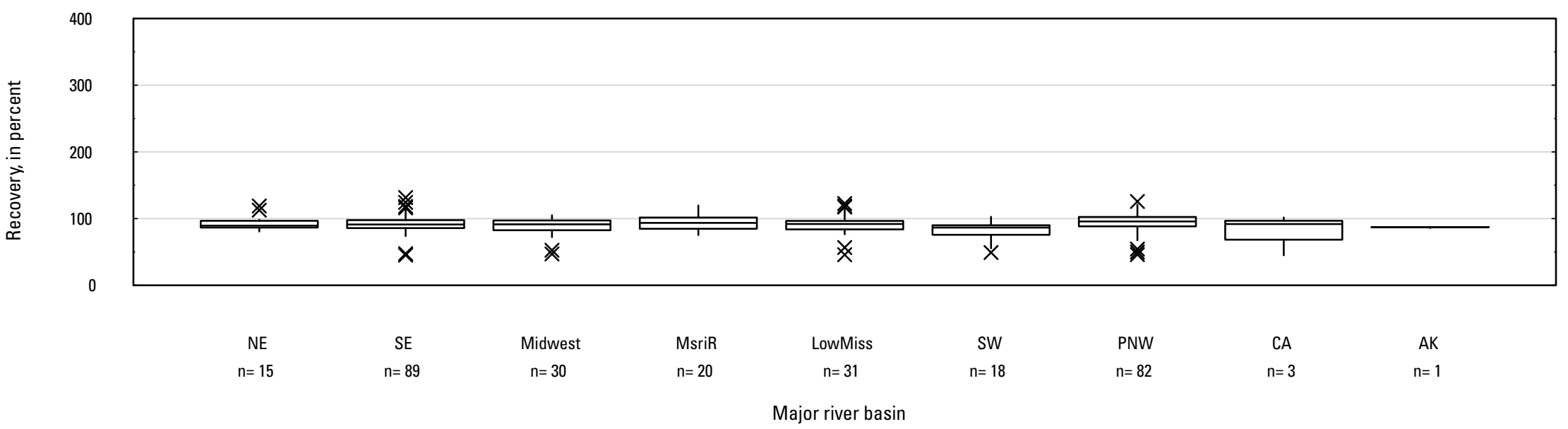

BY. Diflufenzopyr

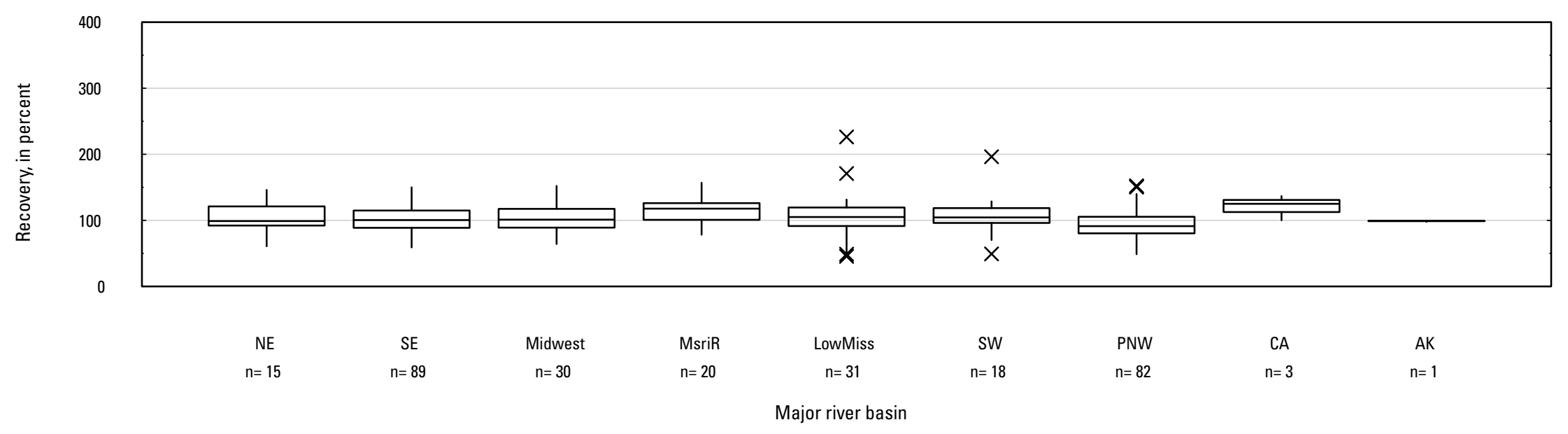

Figure 5. Graph showing distributions of recovery for pesticides in schedule 2437 in surface water by Major River Basin. Recovery values larger than 400 percent are not shown.-Continued 
BZ. Diketonitrile-isoxaflutole

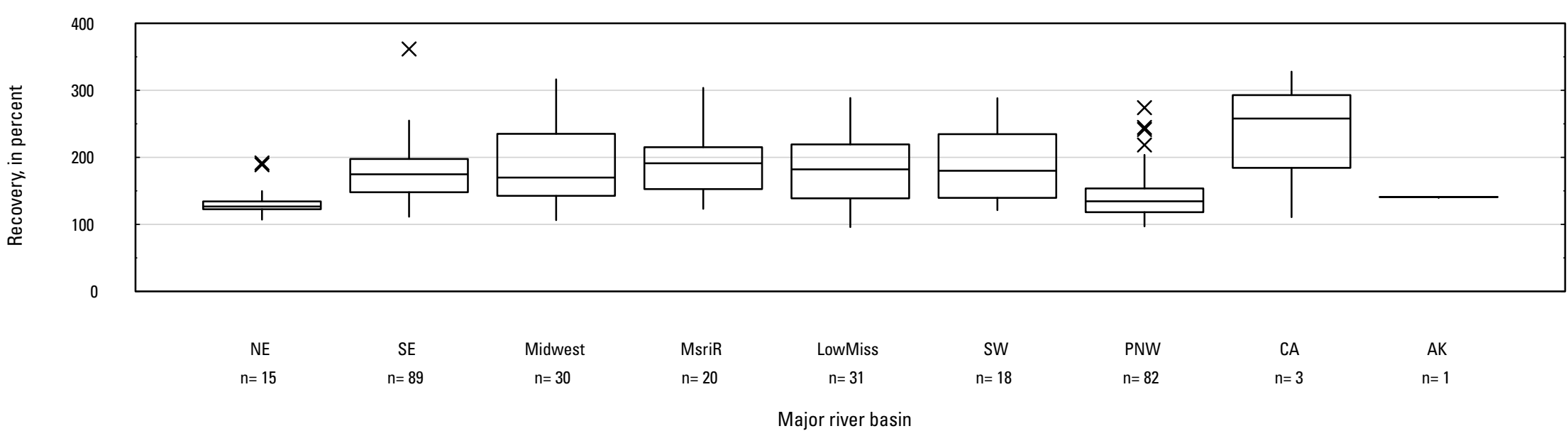

CA. Dimethenamid

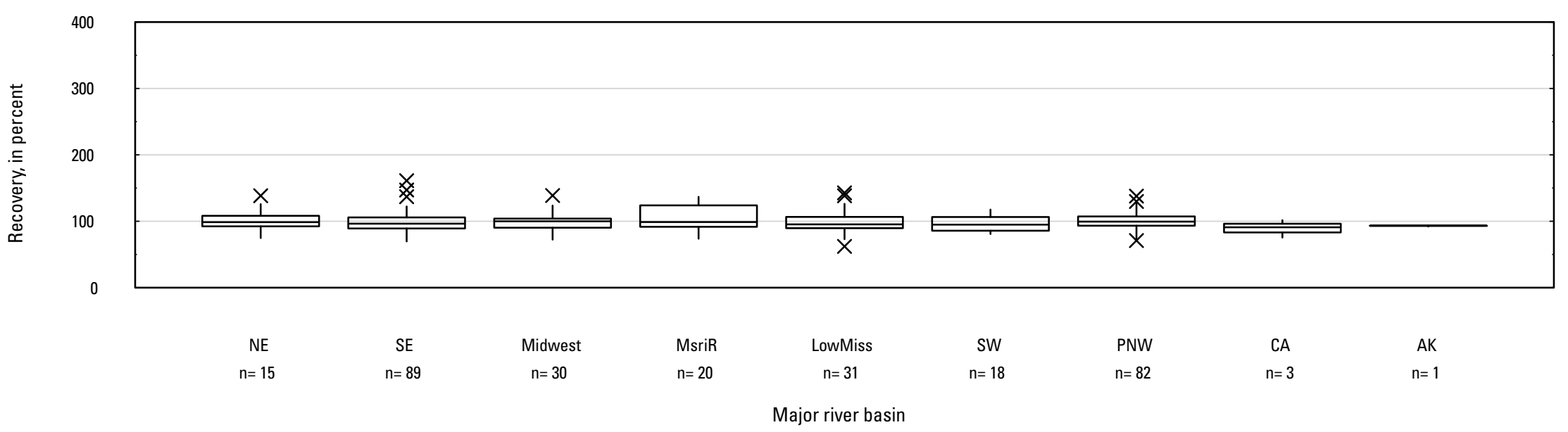

Figure 5. Graph showing distributions of recovery for pesticides in schedule 2437 in surface water by Major River Basin. Recovery values larger than 400 percent are not shown.-Continued 


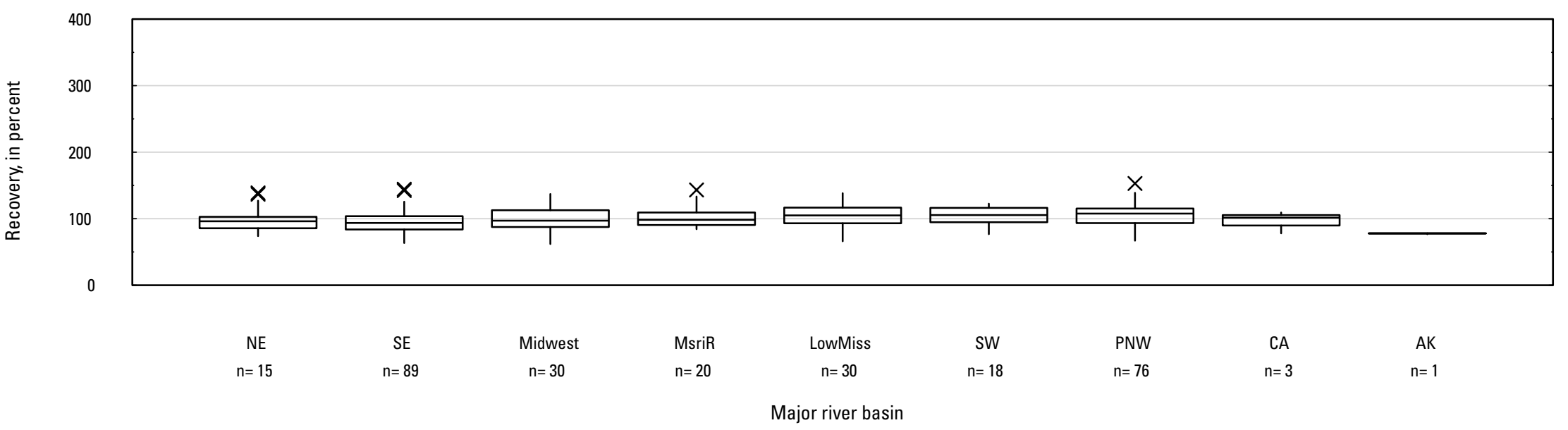

CC. Dimethenamid sulfonic acid

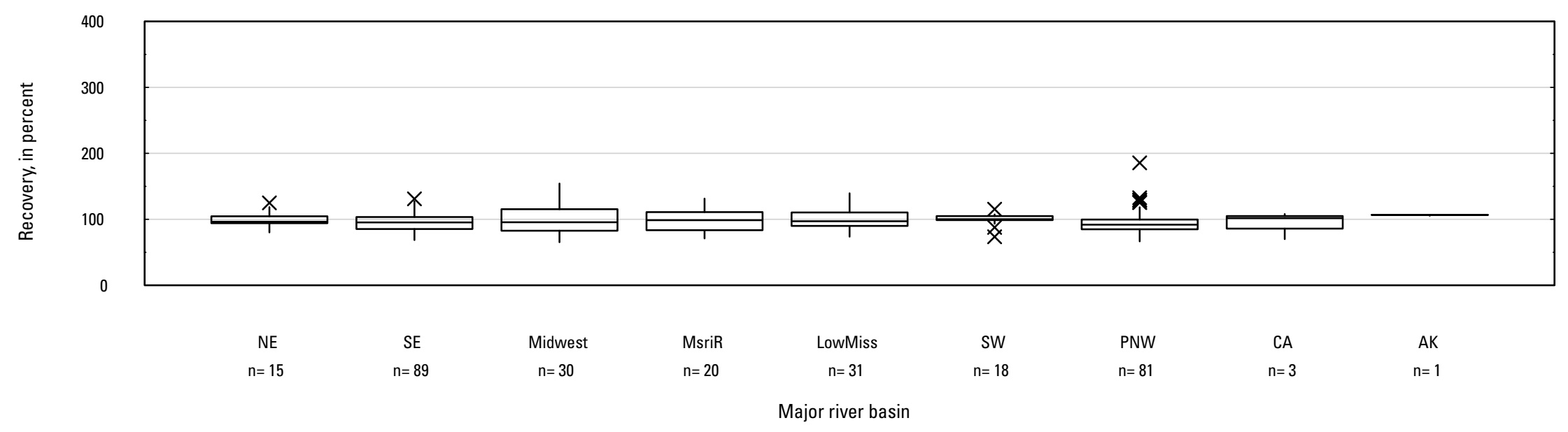

Figure 5. Graph showing distributions of recovery for pesticides in schedule 2437 in surface water by Major River Basin. Recovery values larger than 400 percent are not shown.-Continued 
CD. Dimethenamid SAA

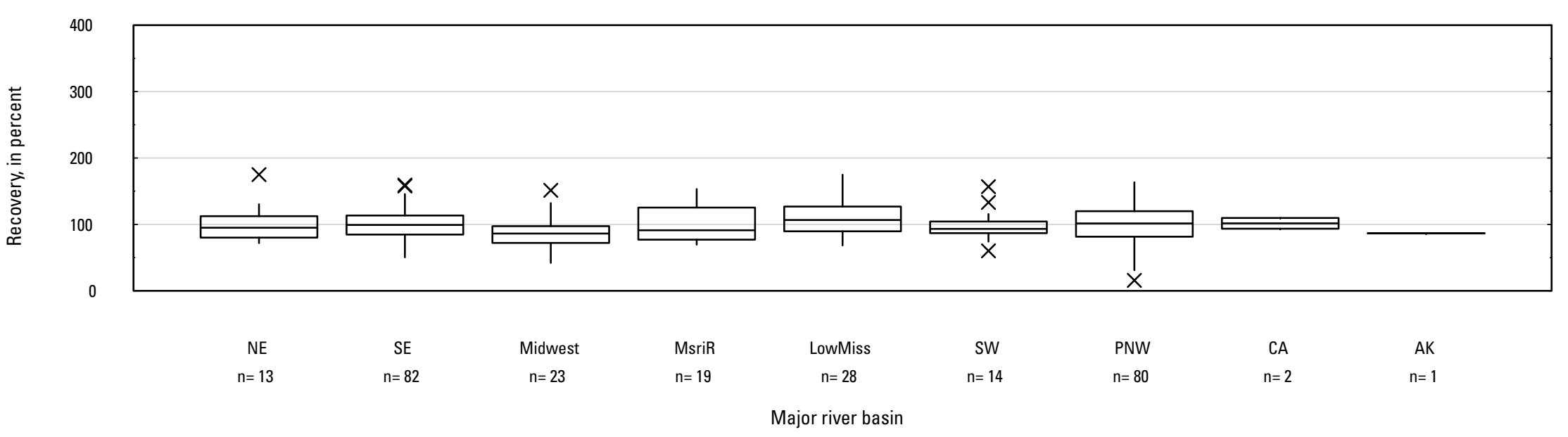

CE. Dimethoate

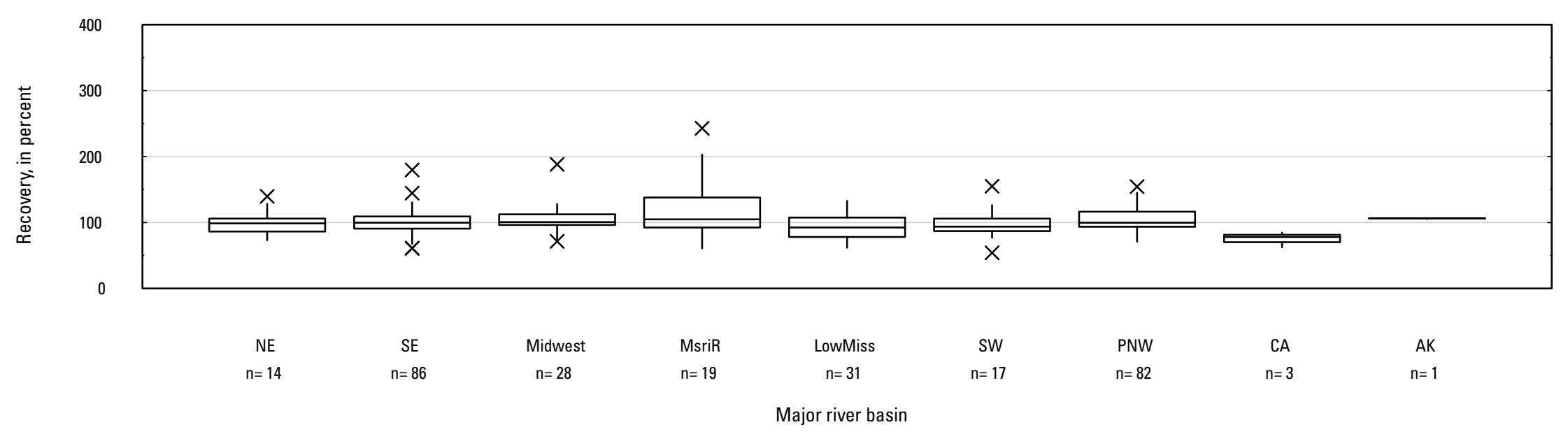

Figure 5. Graph showing distributions of recovery for pesticides in schedule 2437 in surface water by Major River Basin. Recovery values larger than 400 percent are not shown.-Continued 


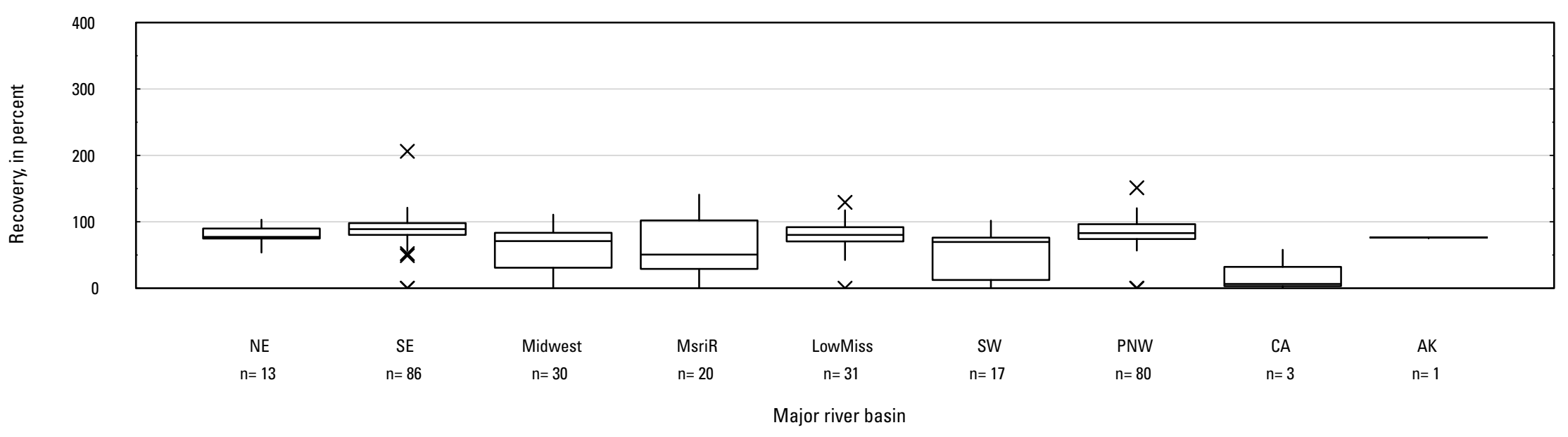

CG. Disulfoton

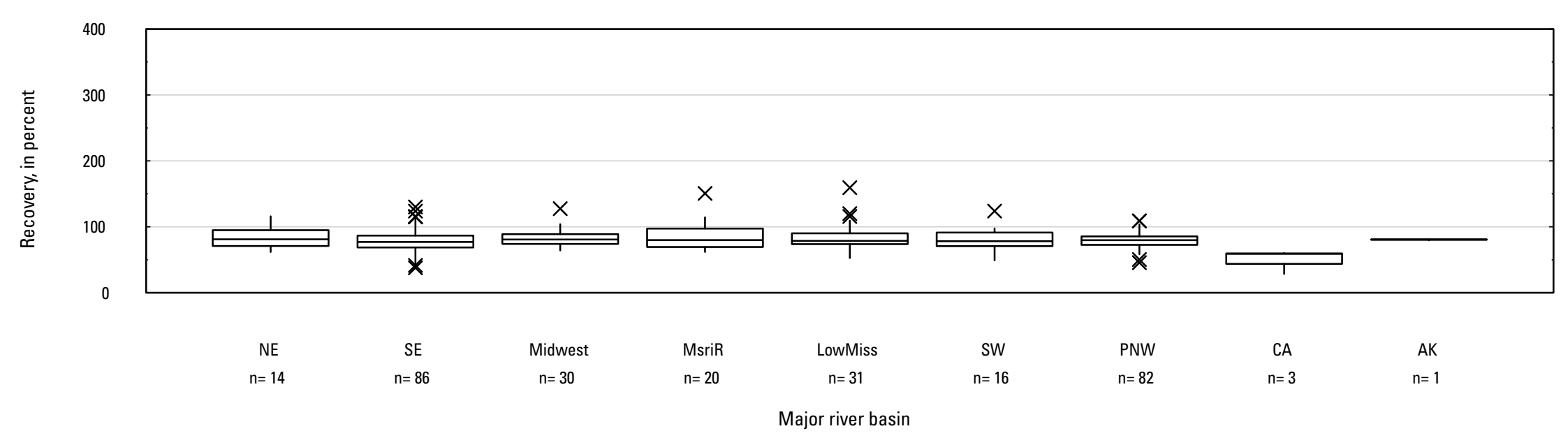

Figure 5. Graph showing distributions of recovery for pesticides in schedule 2437 in surface water by Major River Basin. Recovery values larger than 400 percent are not shown.-Continued 
CH. Disulfoton oxon

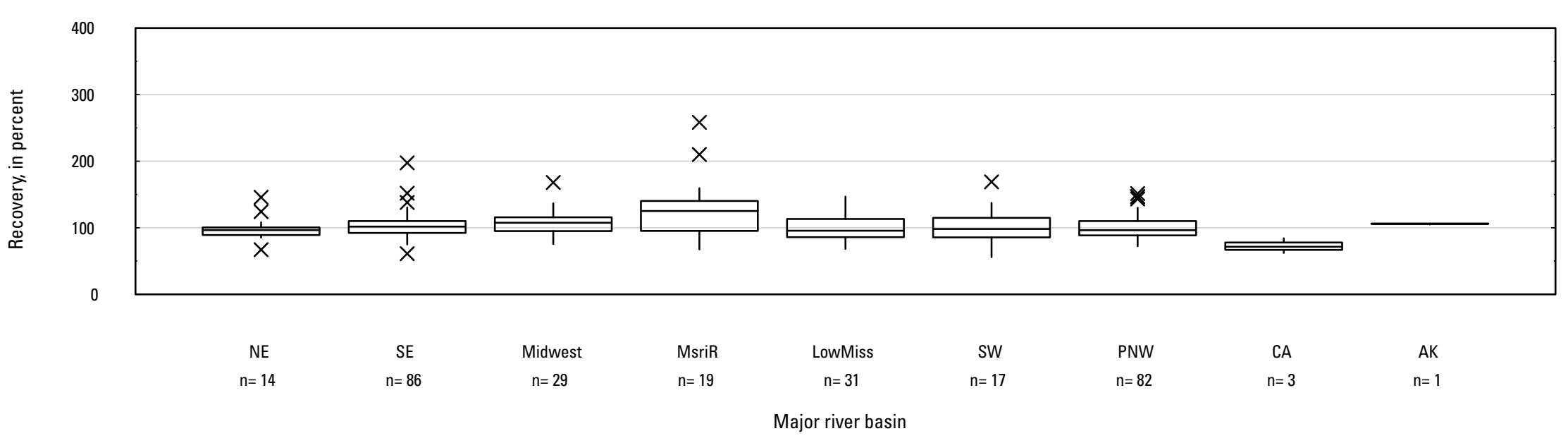

Cl. Disulfoton oxon sulfone

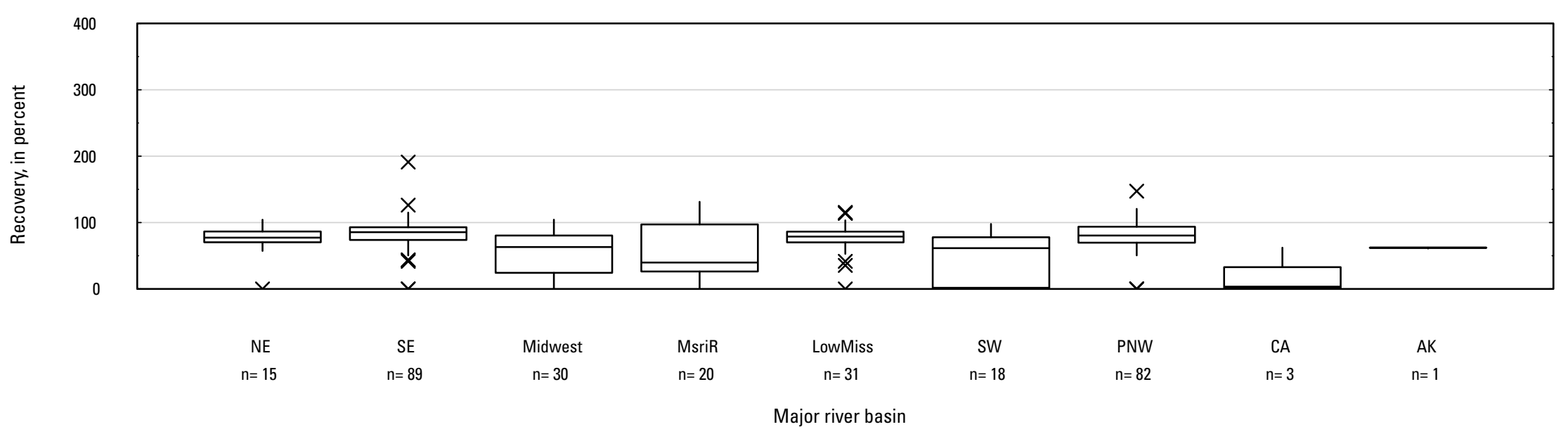

Figure 5. Graph showing distributions of recovery for pesticides in schedule 2437 in surface water by Major River Basin. Recovery values larger than 400 percent are not shown.-Continued 


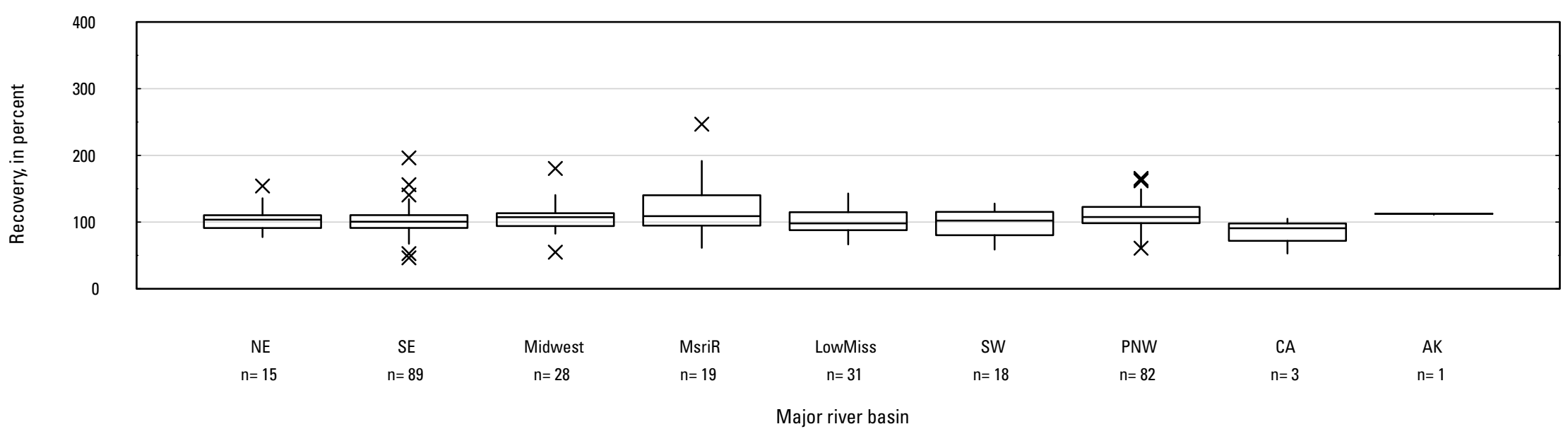

CK. Disulfoton sulfone

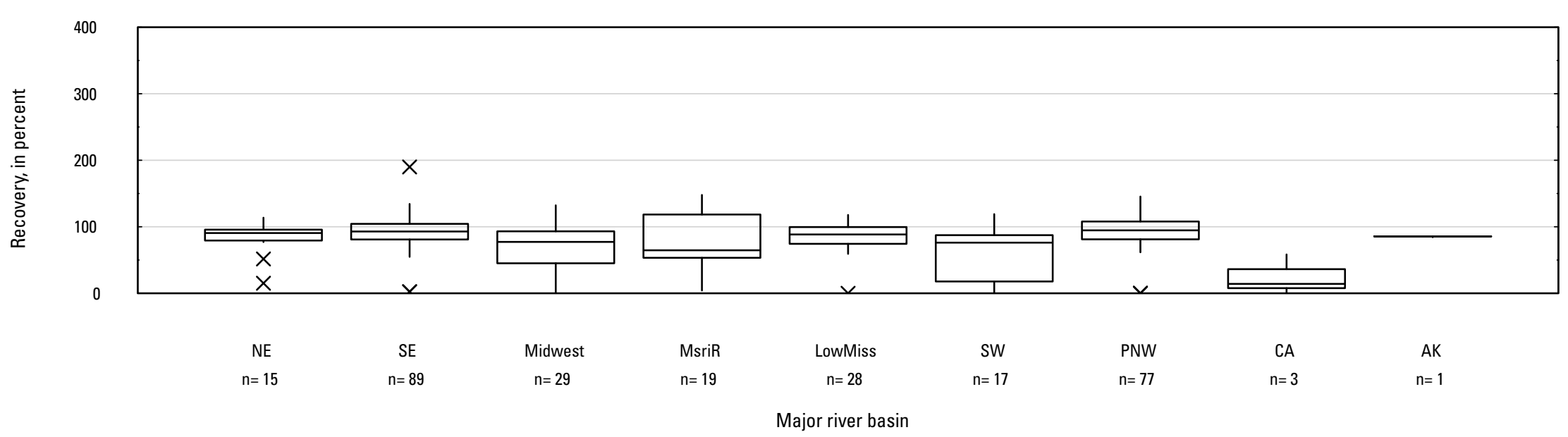

Figure 5. Graph showing distributions of recovery for pesticides in schedule 2437 in surface water by Major River Basin. Recovery values larger than 400 percent are not shown.-Continued 
CL. Disulfoton sulfoxide

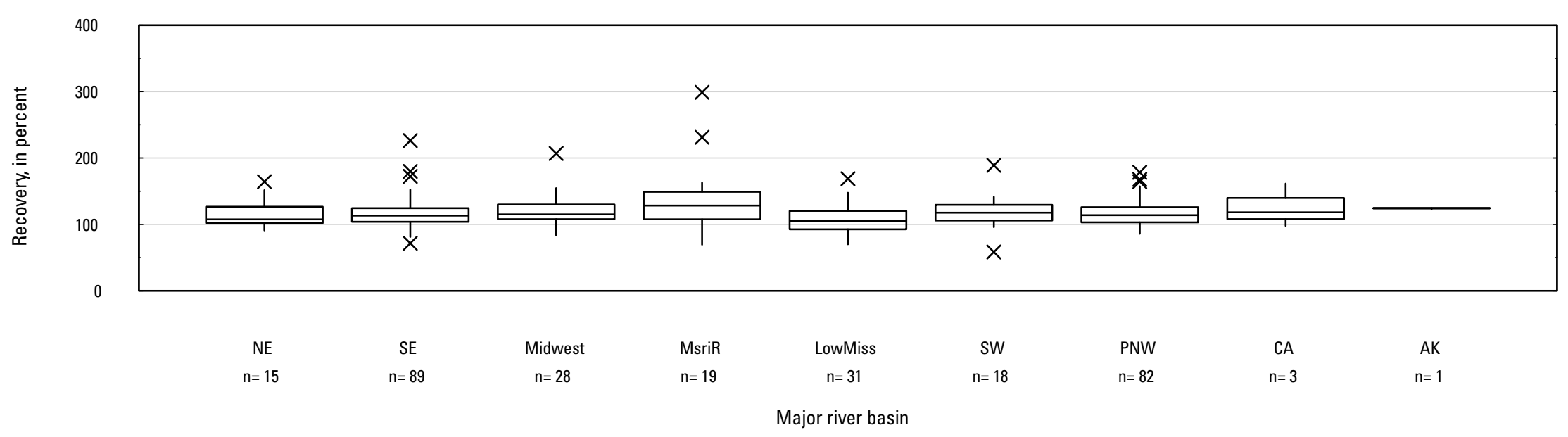

CM. Diuron

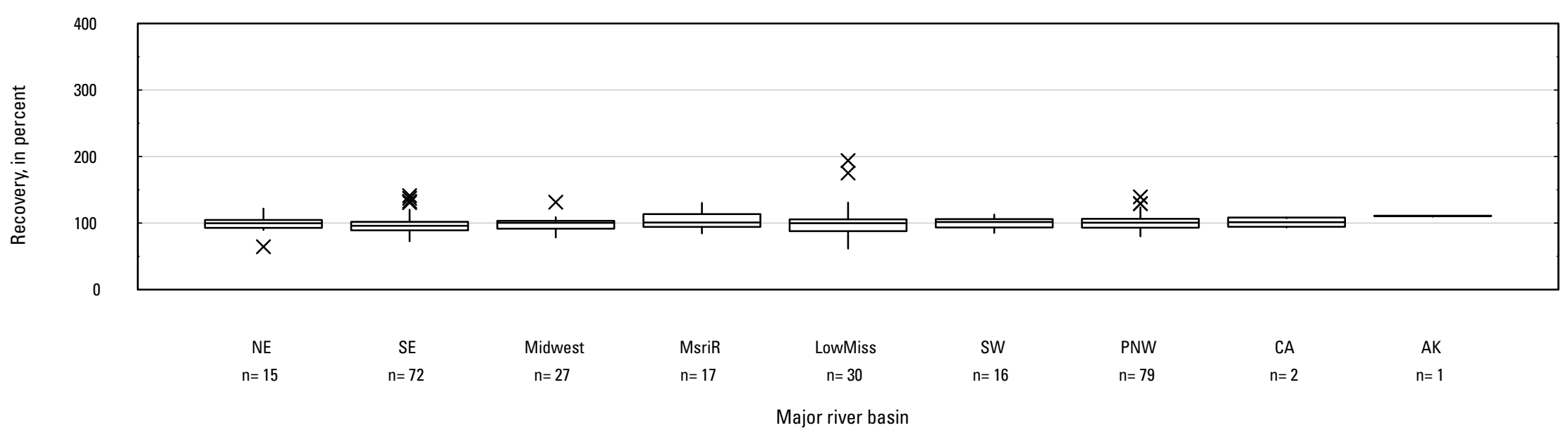

Figure 5. Graph showing distributions of recovery for pesticides in schedule 2437 in surface water by Major River Basin. Recovery values larger than 400 percent are not shown.-Continued 


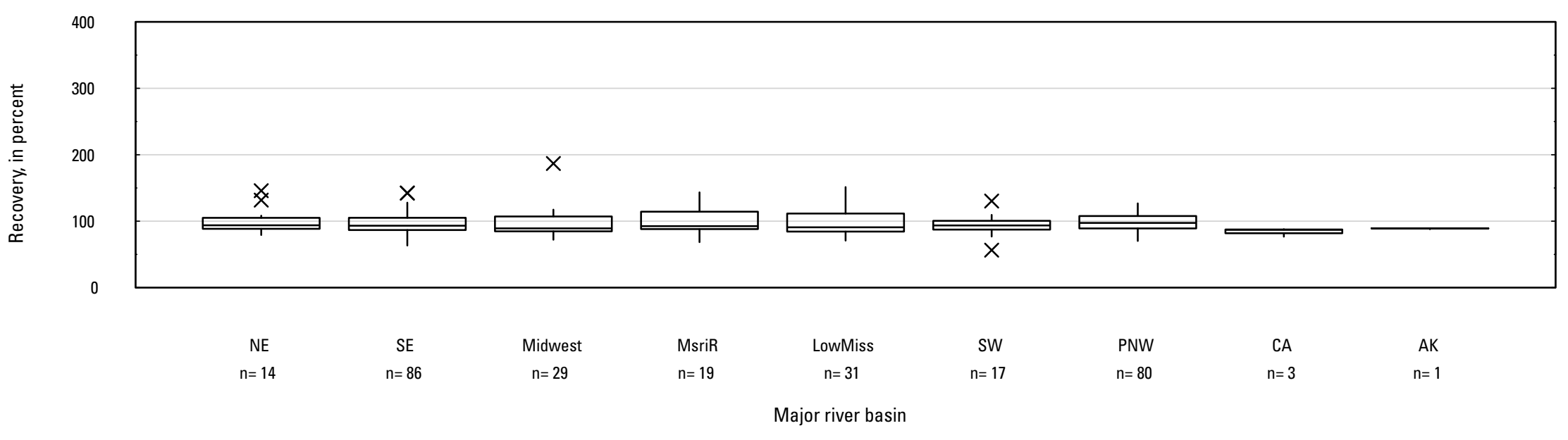

CS. Fenamiphos sulfone

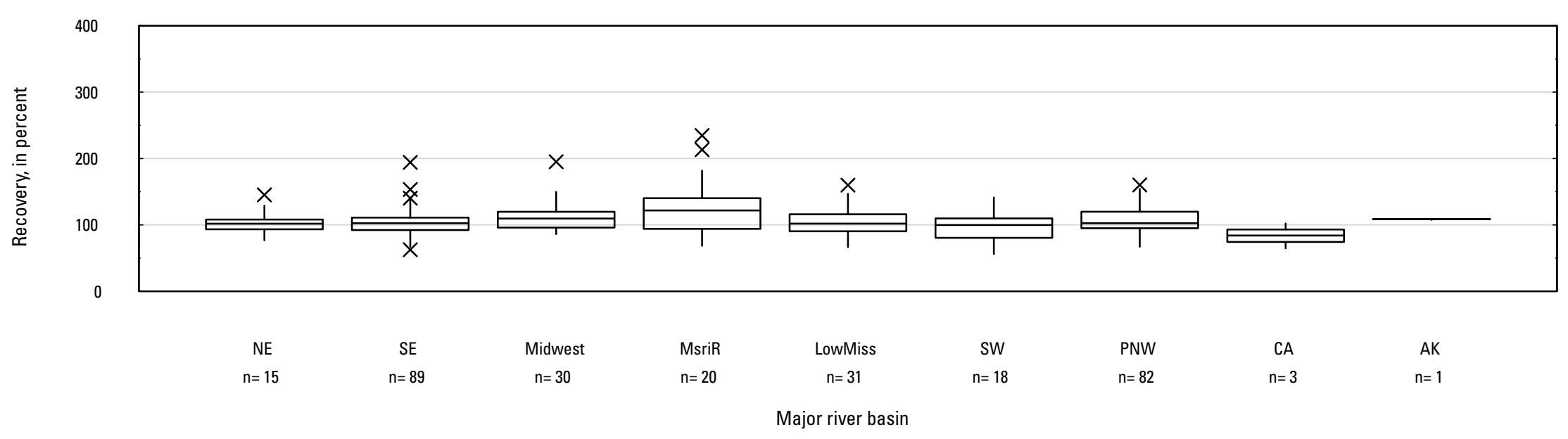

Figure 5. Graph showing distributions of recovery for pesticides in schedule 2437 in surface water by Major River Basin. Recovery values larger than 400 percent are not shown.-Continued 
CT. Fenamiphos sulfoxide

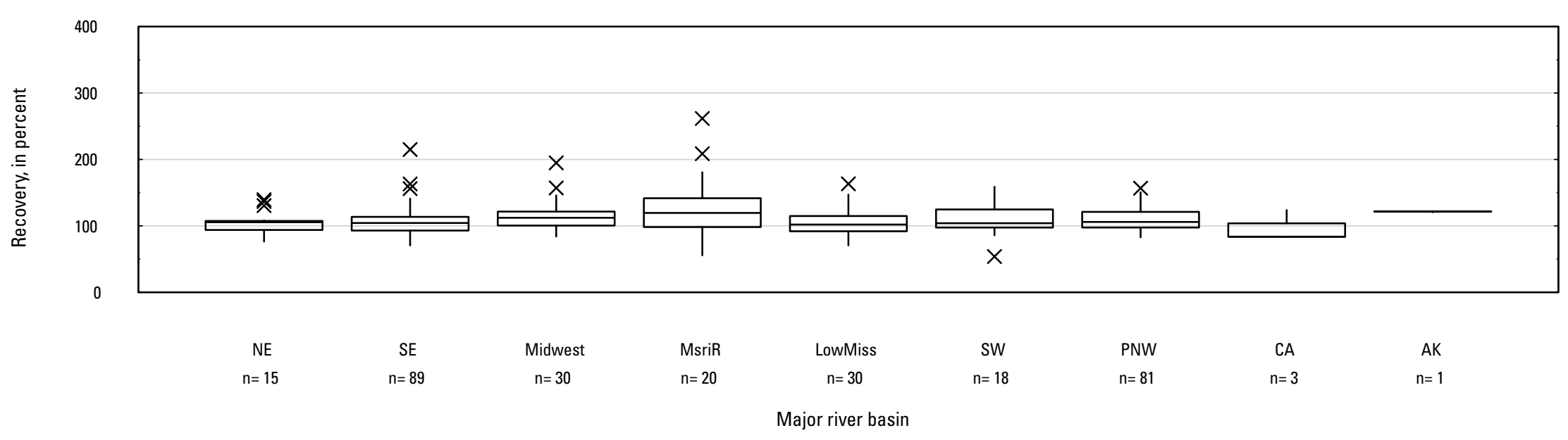

CU. Fenbutatin oxide

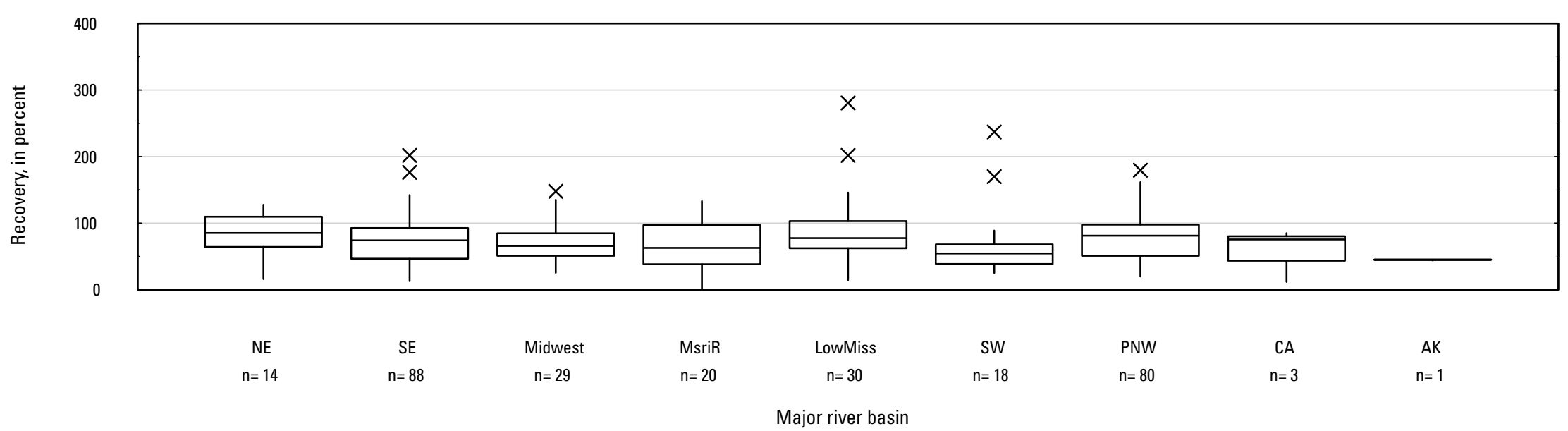

Figure 5. Graph showing distributions of recovery for pesticides in schedule 2437 in surface water by Major River Basin. Recovery values larger than 400 percent are not shown.-Continued 


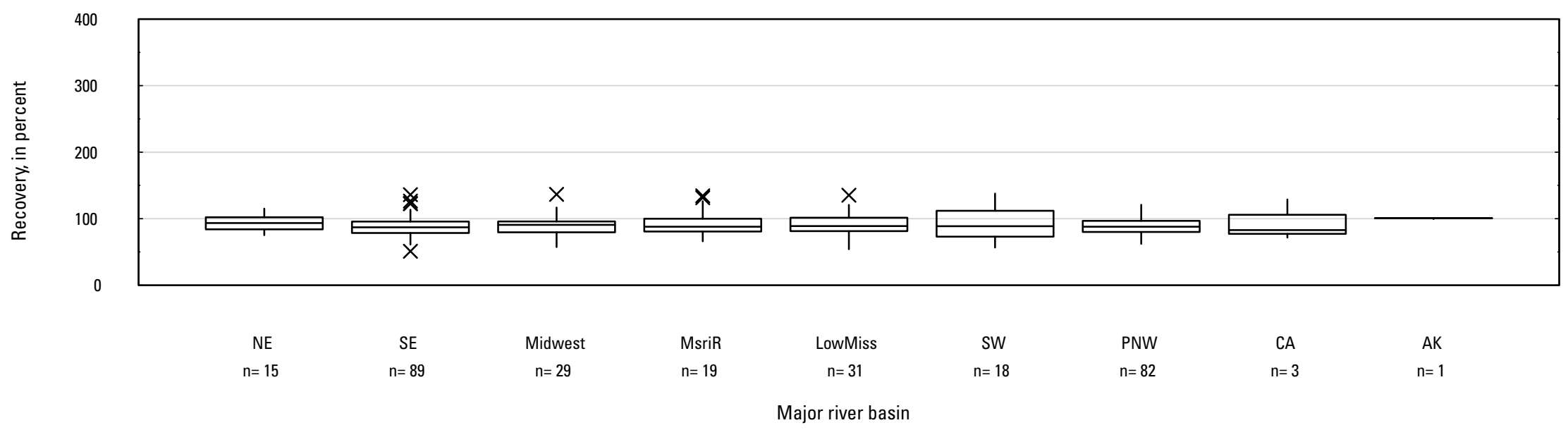

\section{CW. Fipronil}

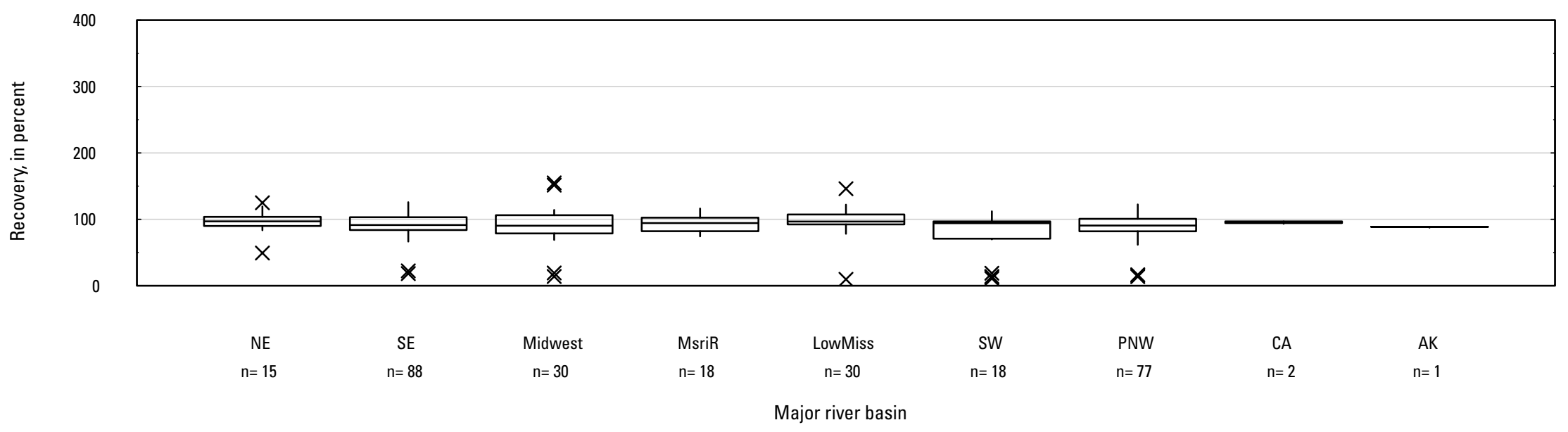

Figure 5. Graph showing distributions of recovery for pesticides in schedule 2437 in surface water by Major River Basin. Recovery values larger than 400 percent are not shown.-Continued 
CX. Fipronil amide

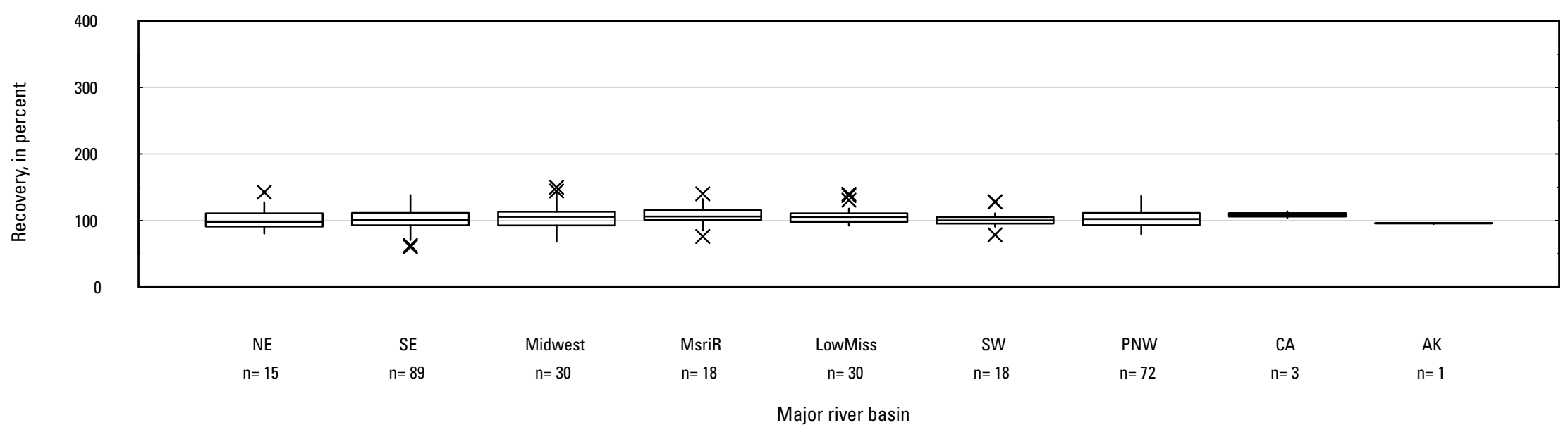

CY. Fipronil sulfide

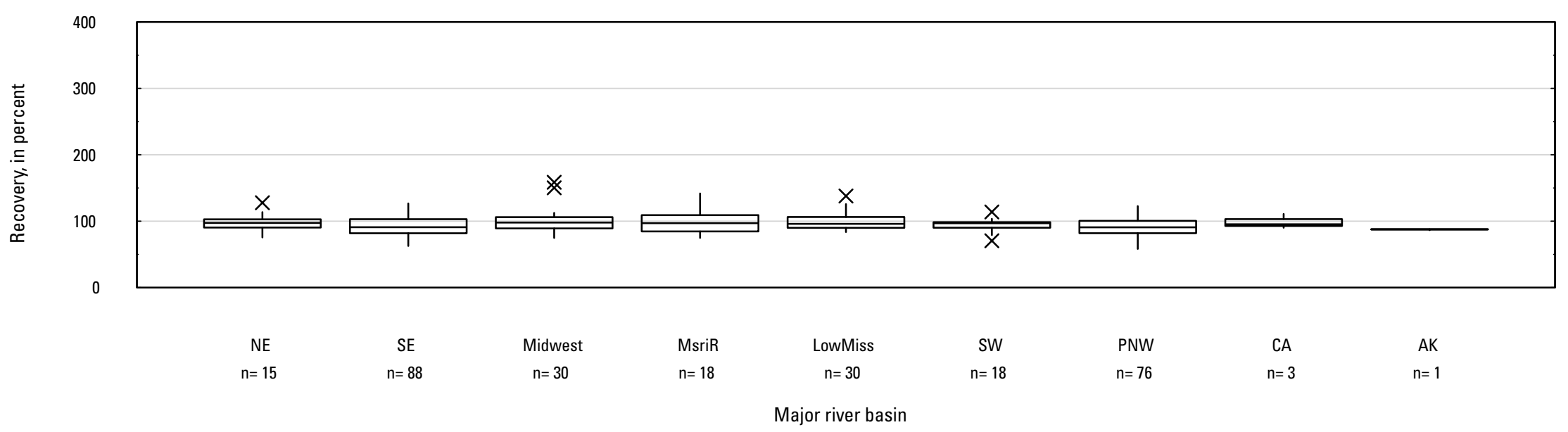

Figure 5. Graph showing distributions of recovery for pesticides in schedule 2437 in surface water by Major River Basin. Recovery values larger than 400 percent are not shown.-Continued 


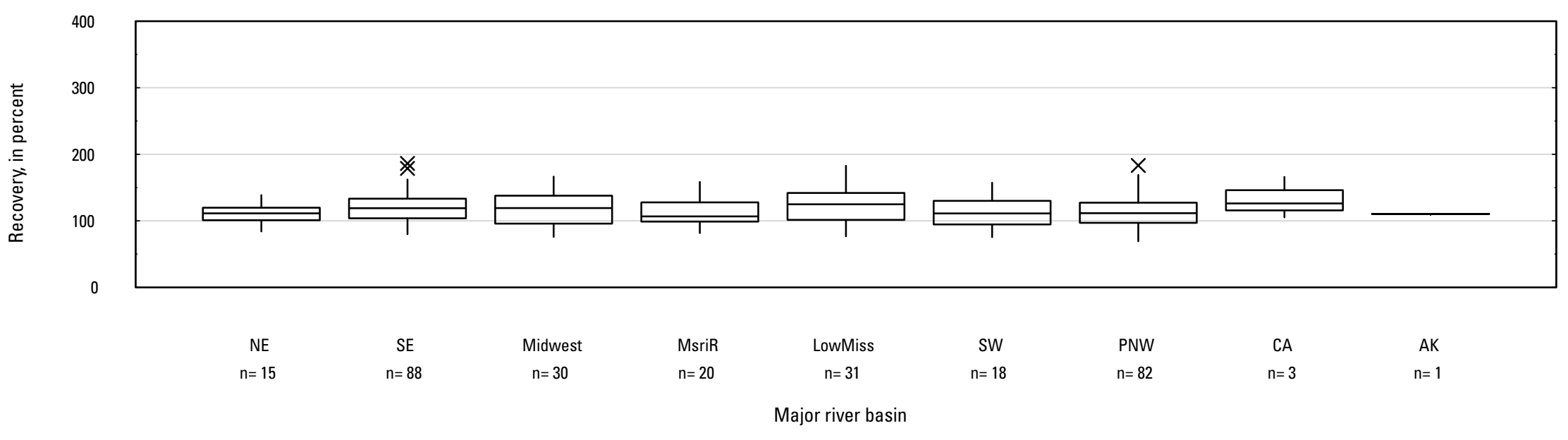

\section{DA. Fipronil sulfone}

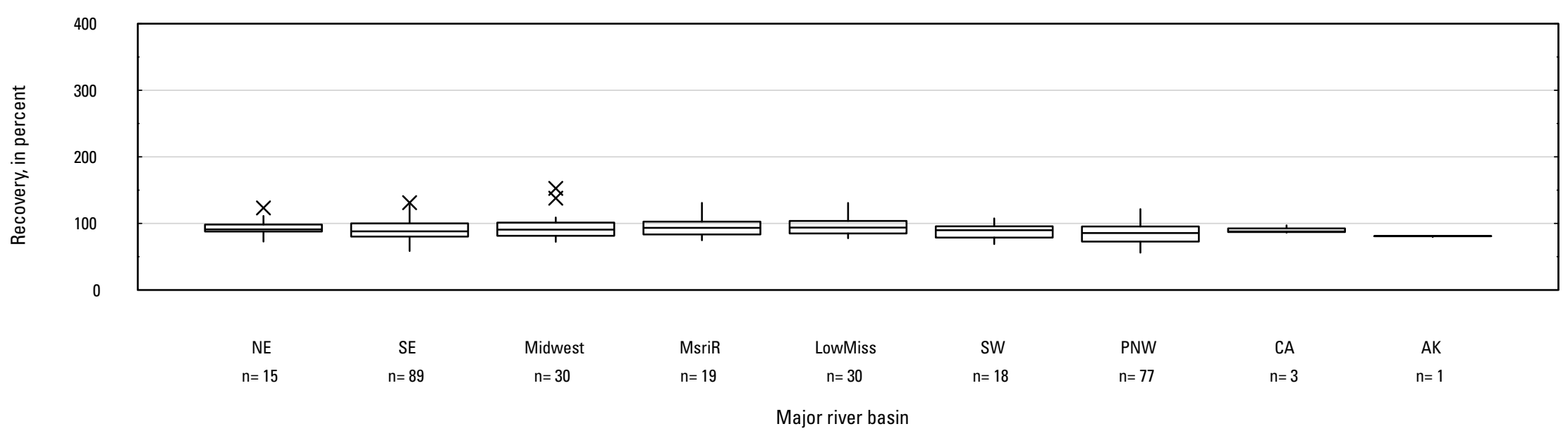

Figure 5. Graph showing distributions of recovery for pesticides in schedule 2437 in surface water by Major River Basin. Recovery values larger than 400 percent are not shown.-Continued 
DB. Flubendiamide

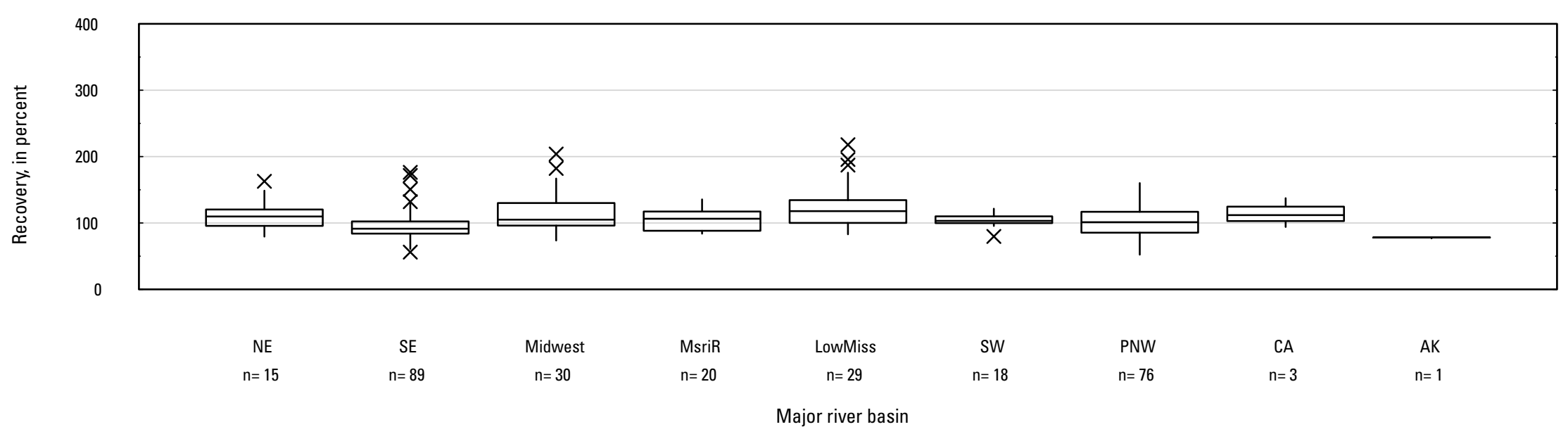

DC. Flumetsulam

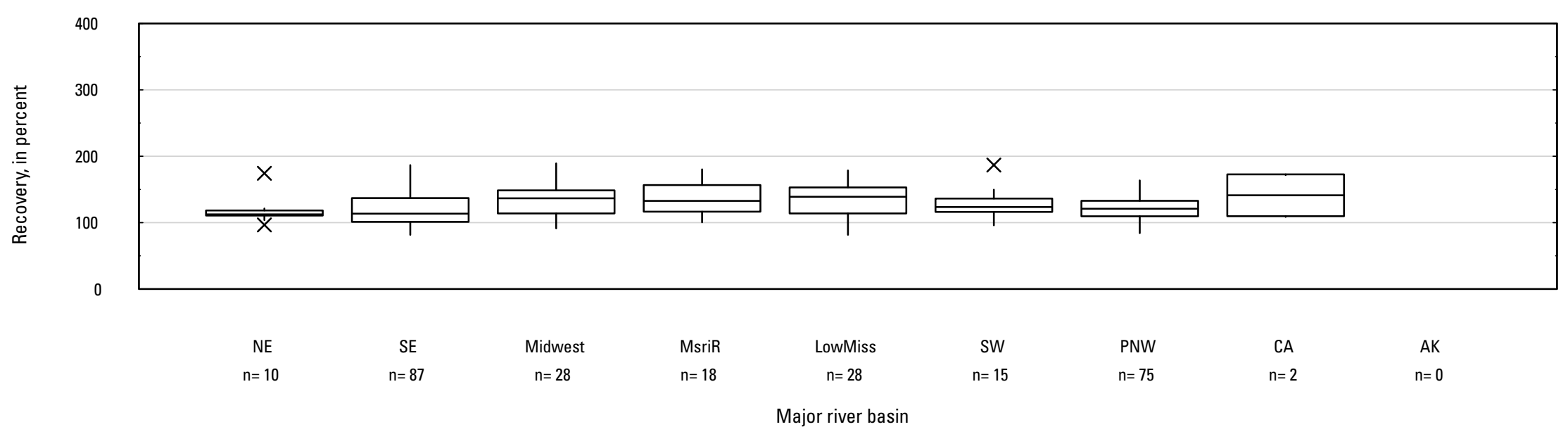

Figure 5. Graph showing distributions of recovery for pesticides in schedule 2437 in surface water by Major River Basin. Recovery values larger than 400 percent are not shown.-Continued 


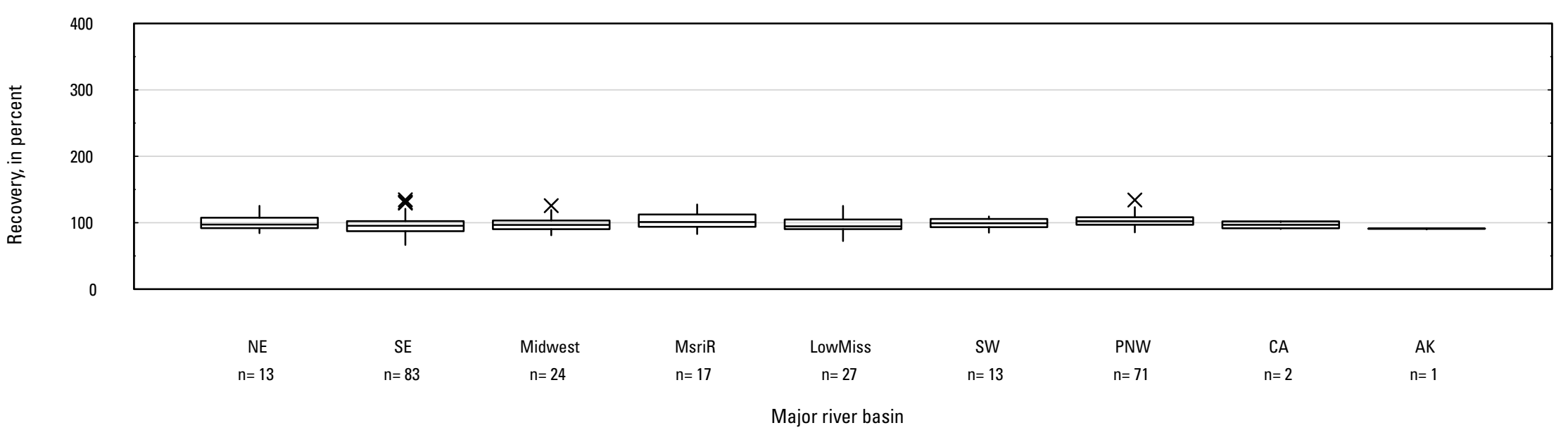

DE. Fonofos

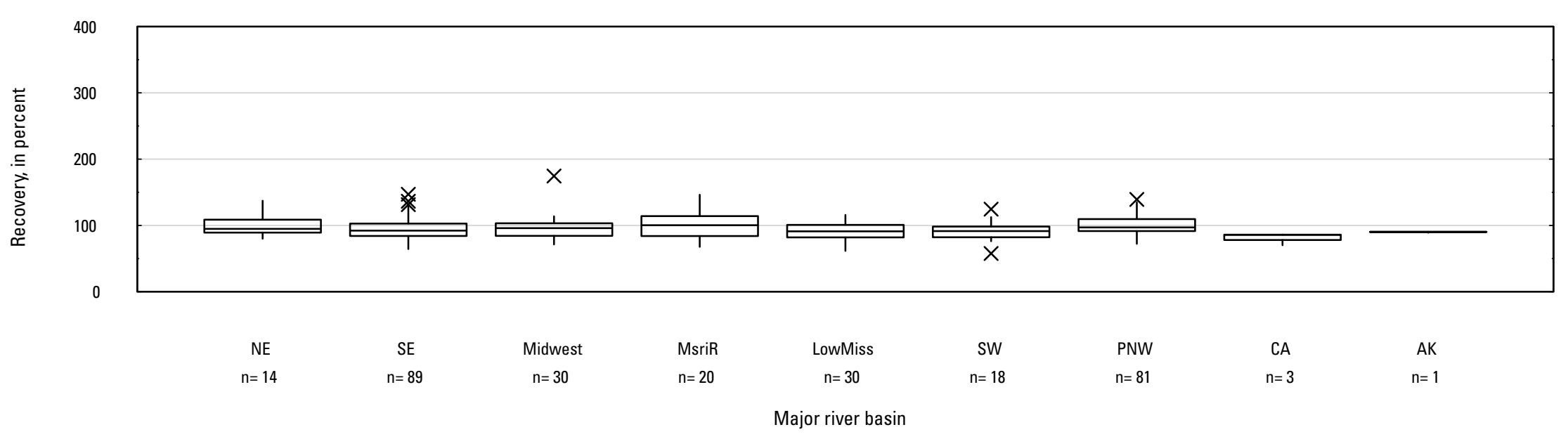

Figure 5. Graph showing distributions of recovery for pesticides in schedule 2437 in surface water by Major River Basin. Recovery values larger than 400 percent are not shown.-Continued 
DF. Halosulfuron-methyl

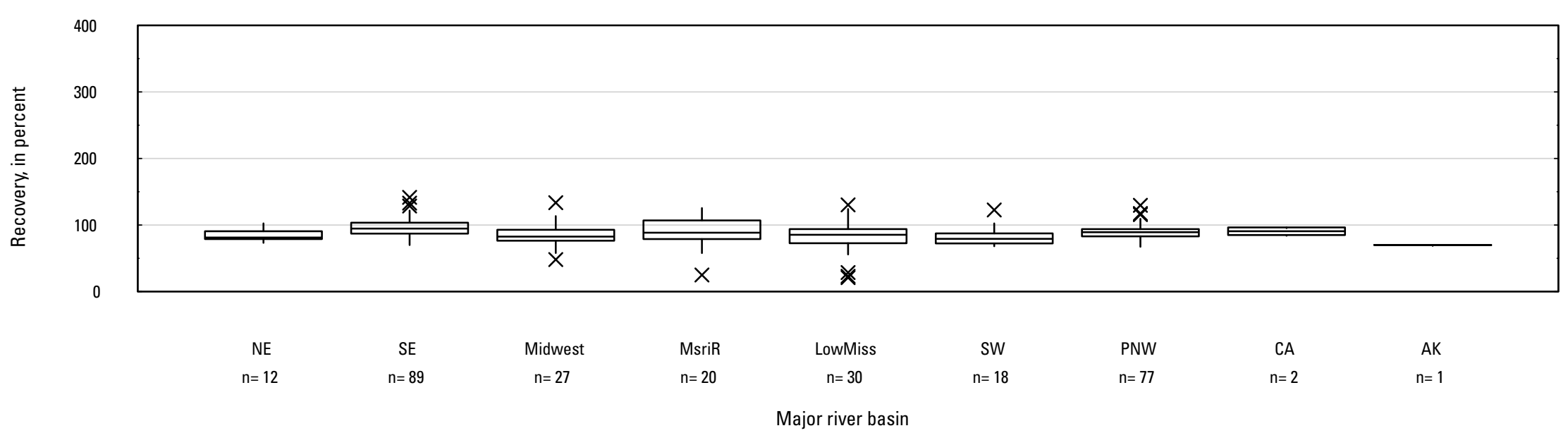

DG. Hexazinone

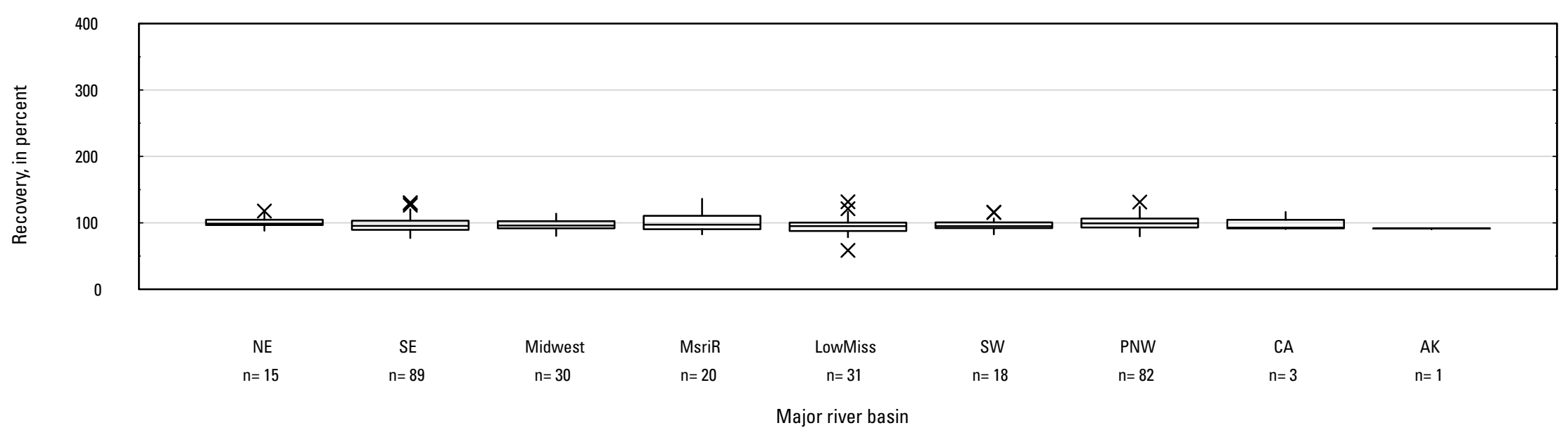

Figure 5. Graph showing distributions of recovery for pesticides in schedule 2437 in surface water by Major River Basin. Recovery values larger than 400 percent are not shown.-Continued 


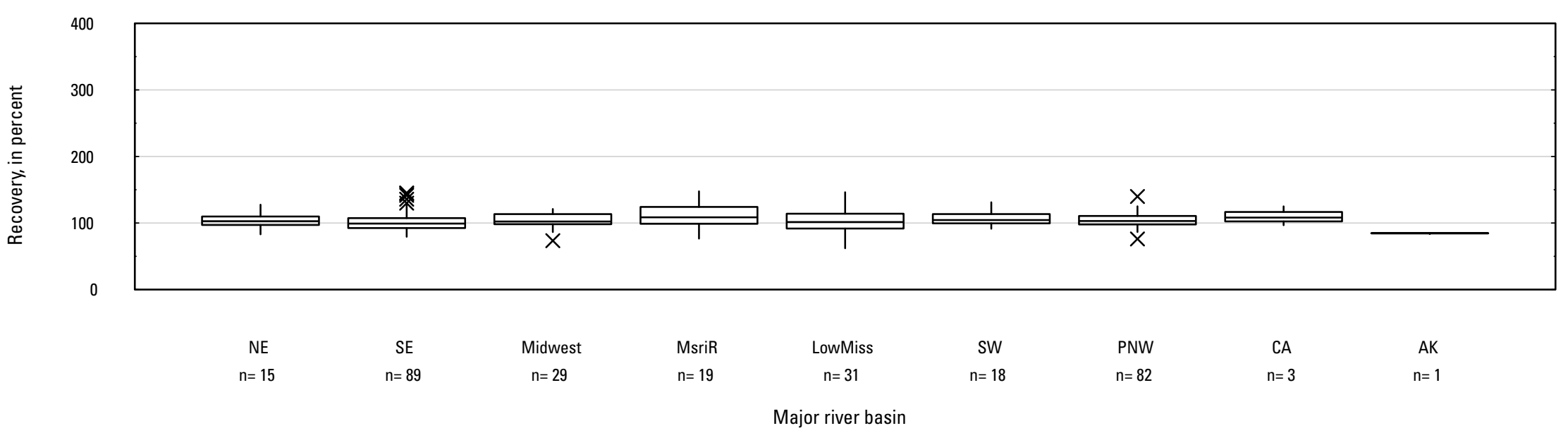

DI. Hexazinone Transformation Product D

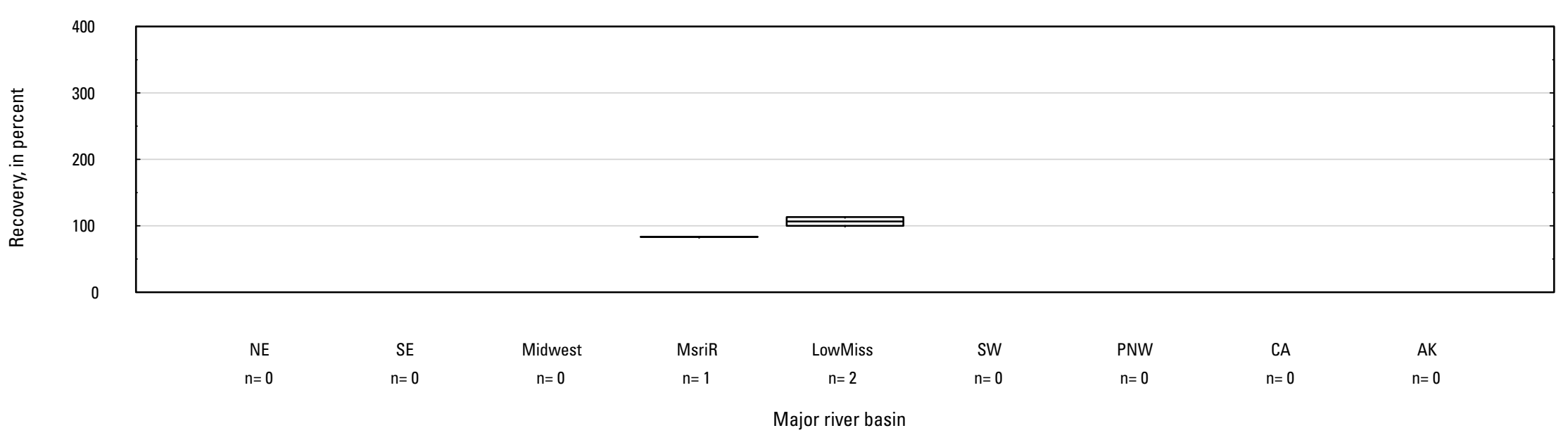

Figure 5. Graph showing distributions of recovery for pesticides in schedule 2437 in surface water by Major River Basin. Recovery values larger than 400 percent are not shown.-Continued 
DJ. Hexazinone Transformation Product E

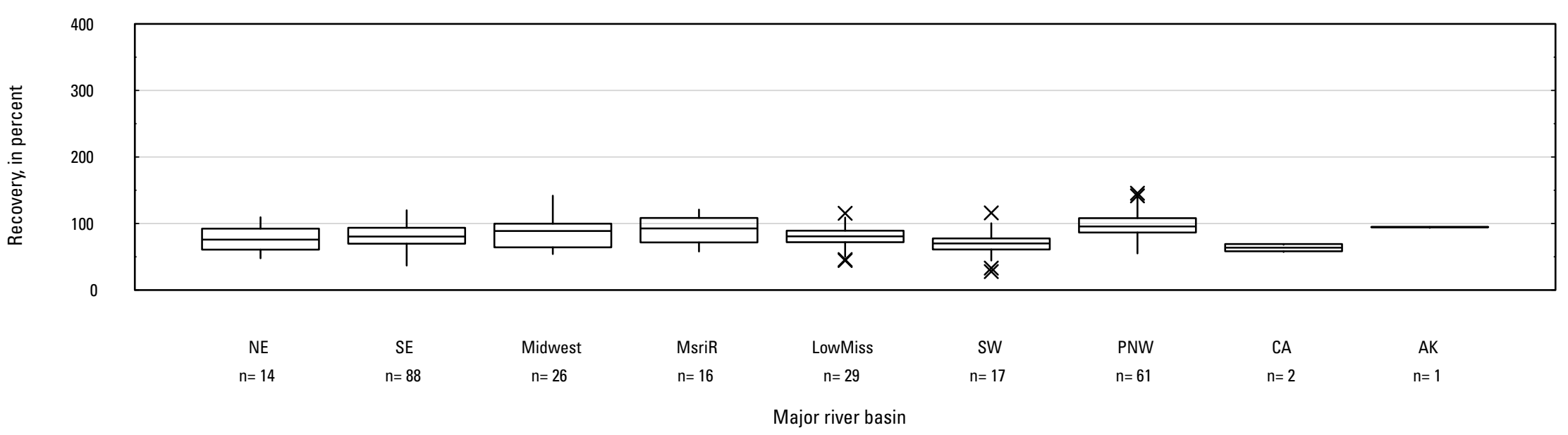

DK. Didemethyl hexazinone F

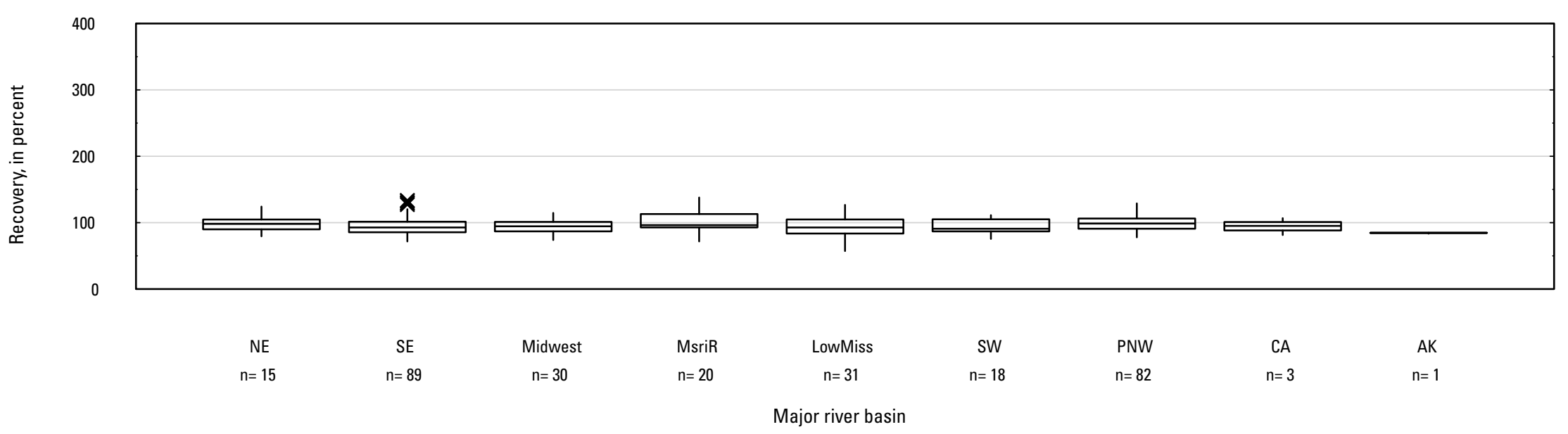

Figure 5. Graph showing distributions of recovery for pesticides in schedule 2437 in surface water by Major River Basin. Recovery values larger than 400 percent are not shown.-Continued 


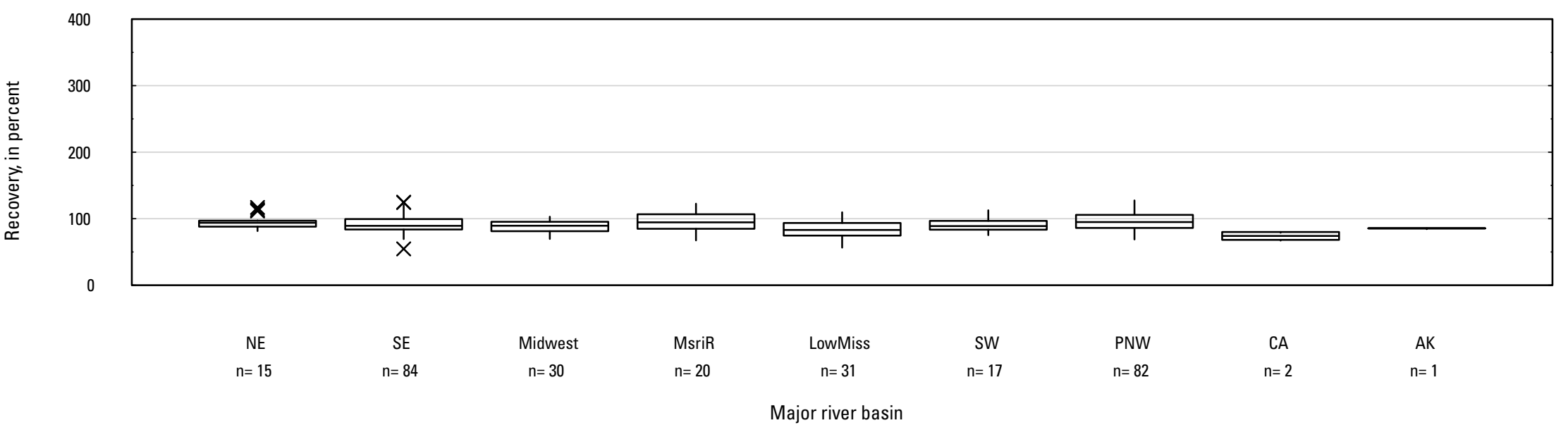

DM. Hydroxy monodemethyl fluometuron

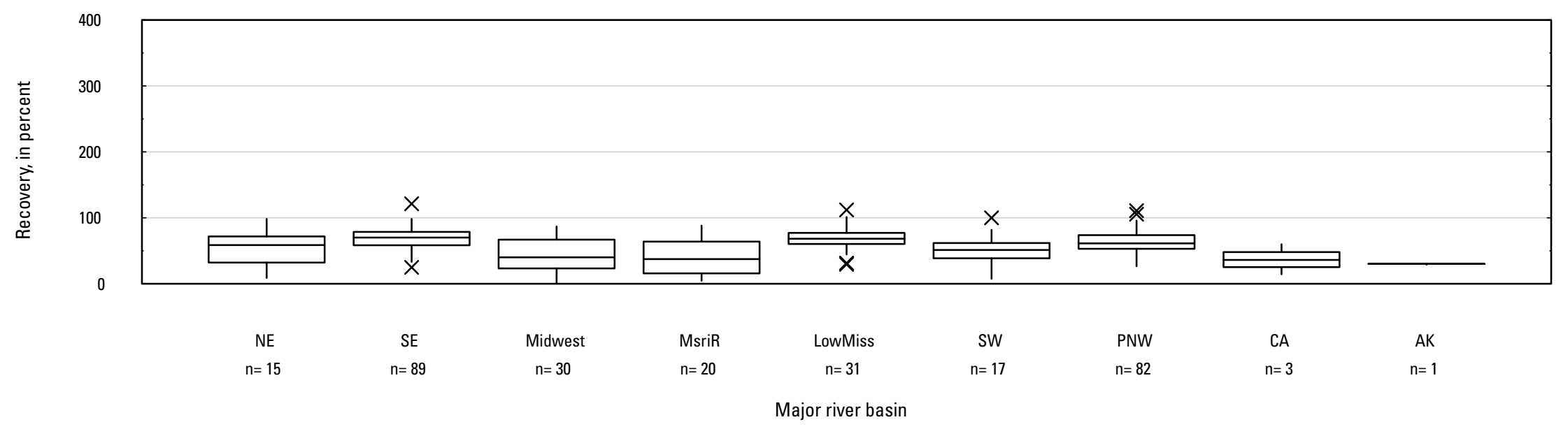

Figure 5. Graph showing distributions of recovery for pesticides in schedule 2437 in surface water by Major River Basin. Recovery values larger than 400 percent are not shown.-Continued 
DN. Hydroxyacetochlor

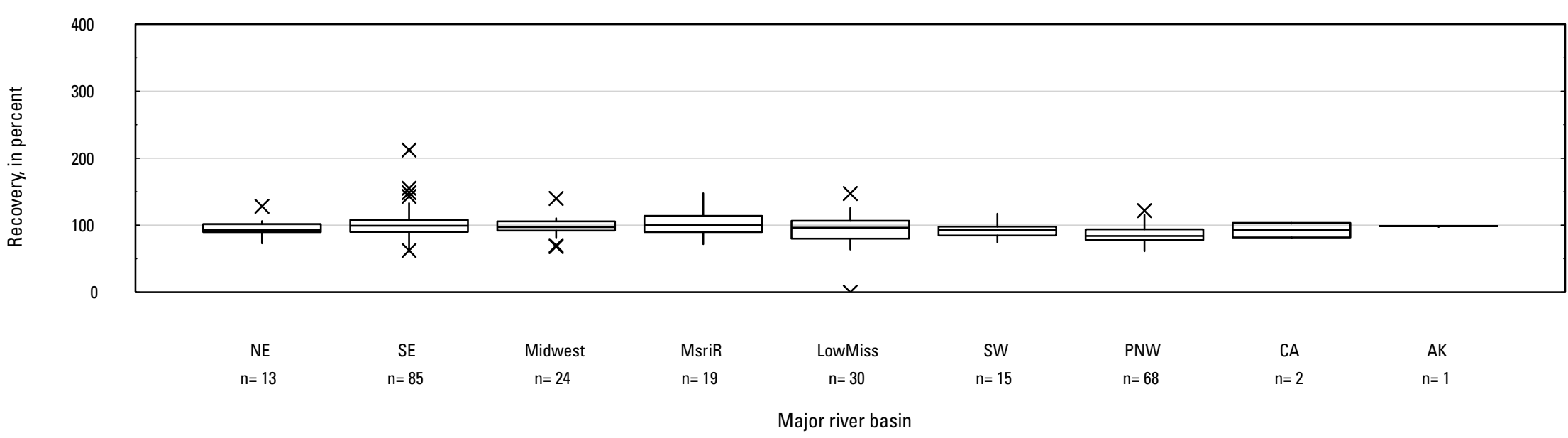

\section{DO. Hydroxyalachlor}

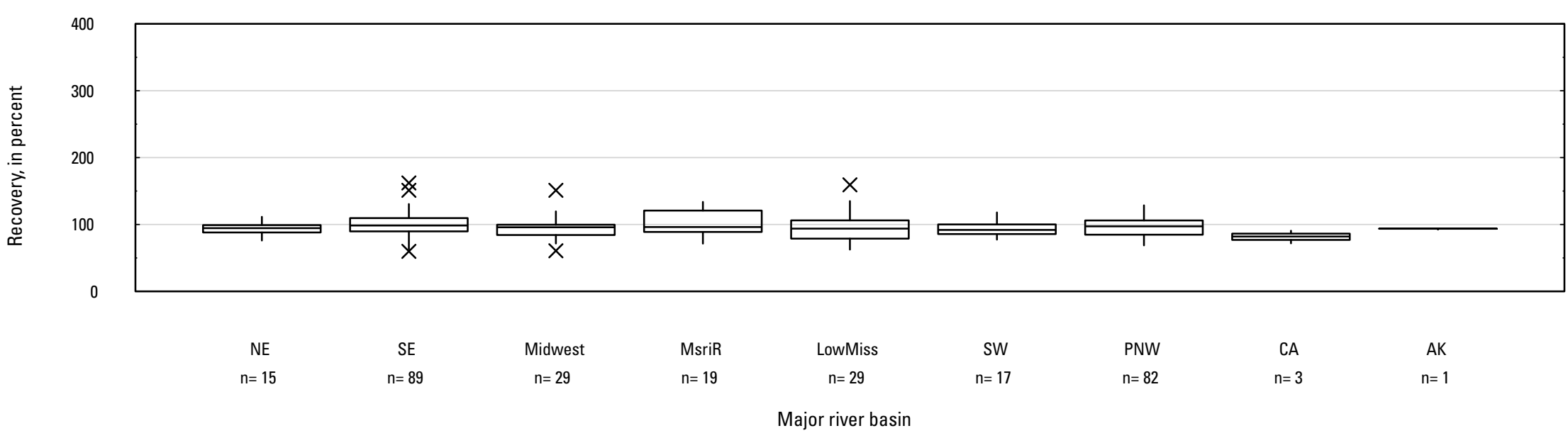

Figure 5. Graph showing distributions of recovery for pesticides in schedule 2437 in surface water by Major River Basin. Recovery values larger than 400 percent are not shown.-Continued 


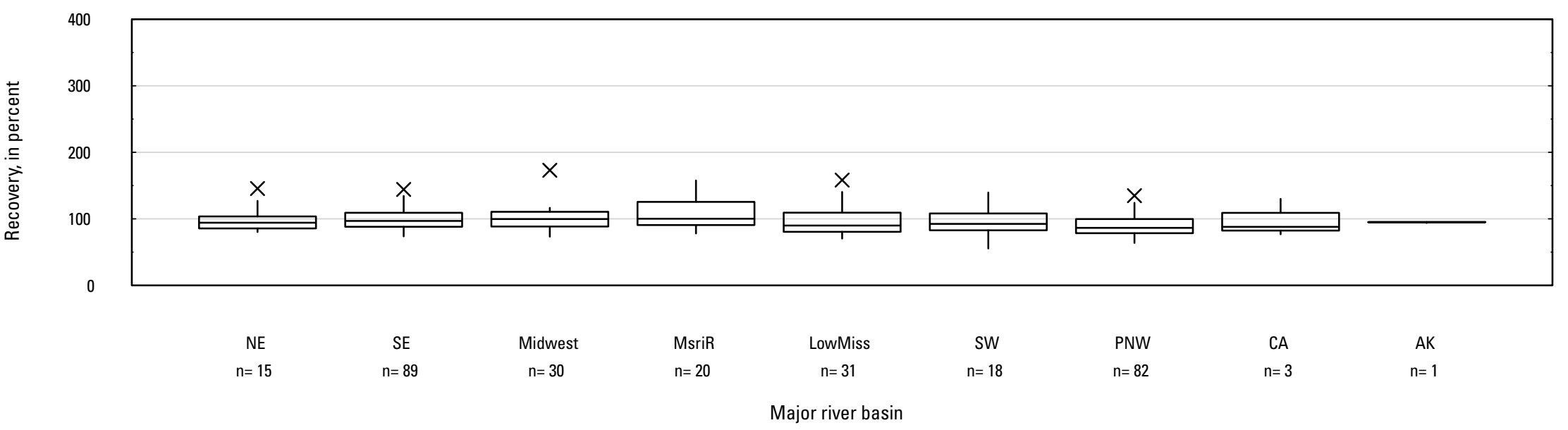

DQ. Hydroxyfluometuron

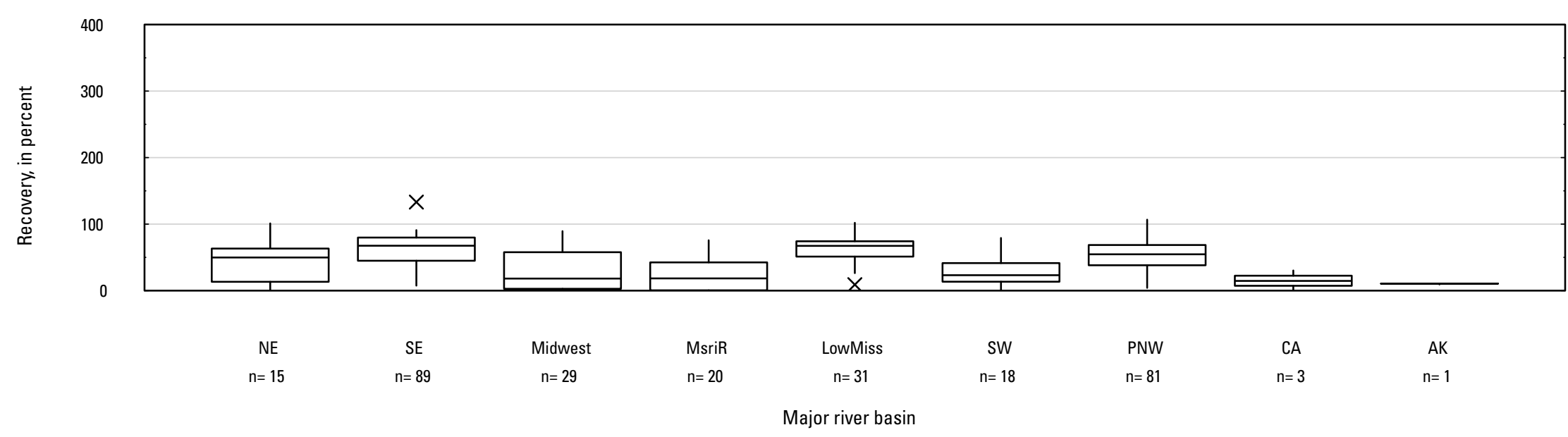

Figure 5. Graph showing distributions of recovery for pesticides in schedule 2437 in surface water by Major River Basin. Recovery values larger than 400 percent are not shown.-Continued 
DR. Hydroxymetolachlor

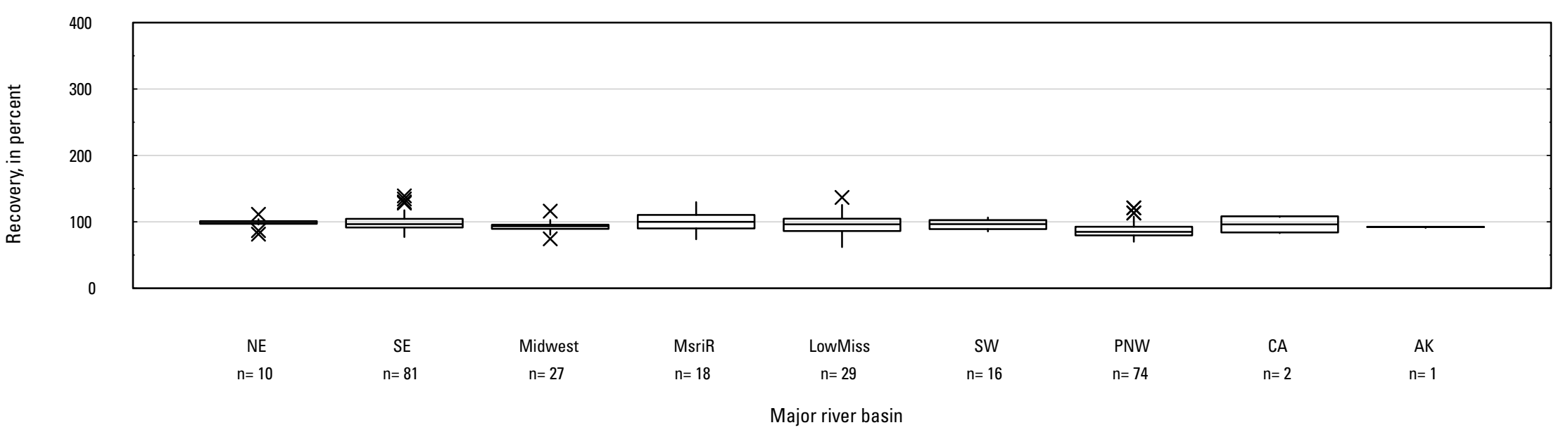

DS. Hydroxyphthalazinone

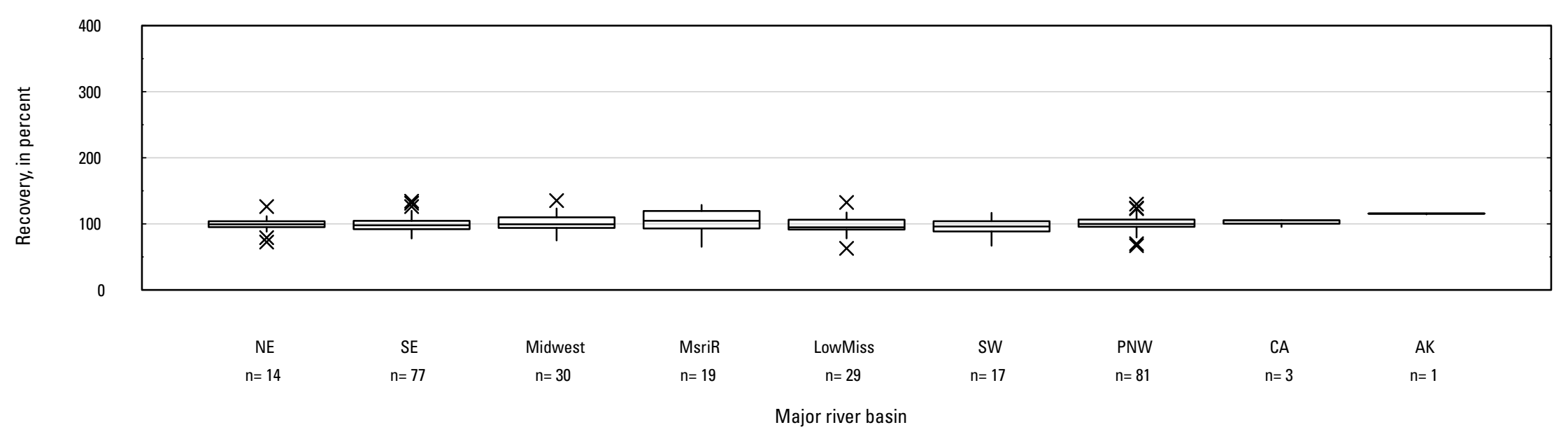

Figure 5. Graph showing distributions of recovery for pesticides in schedule 2437 in surface water by Major River Basin. Recovery values larger than 400 percent are not shown.-Continued 


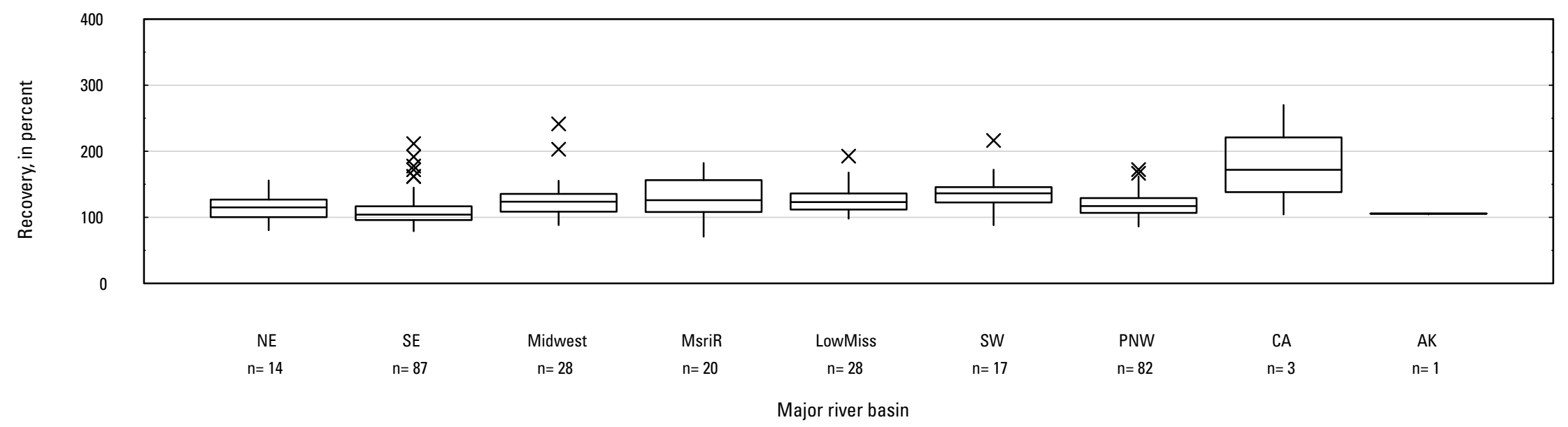

\section{DU. Tebuthiuron TP 109}

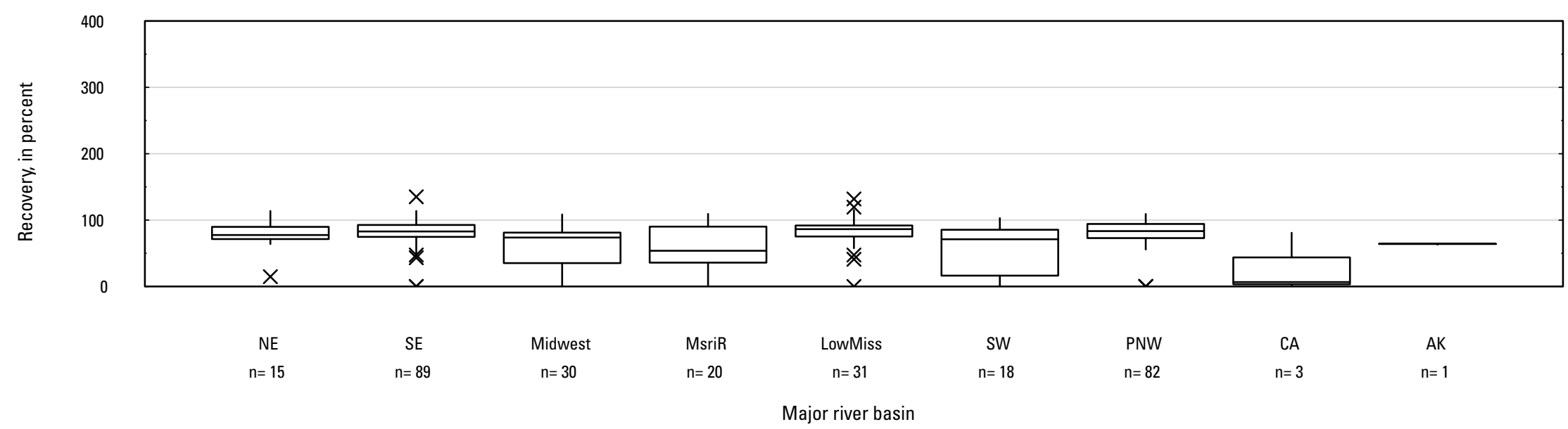

Figure 5. Graph showing distributions of recovery for pesticides in schedule 2437 in surface water by Major River Basin. Recovery values larger than 400 percent are not shown.-Continued 
DV. Imazamox

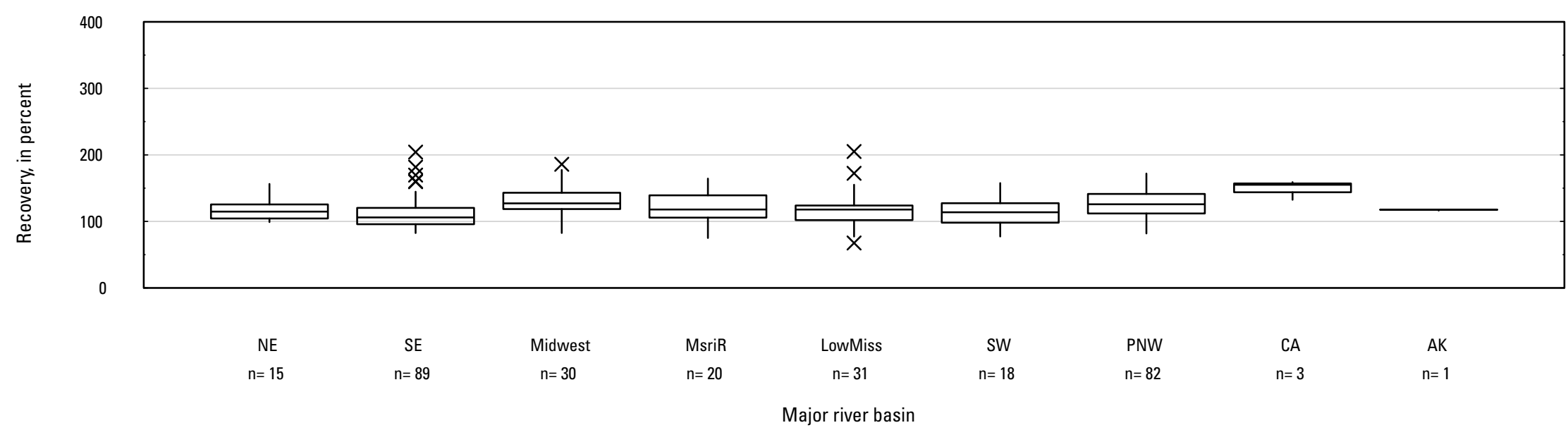

DW. Imazaquin

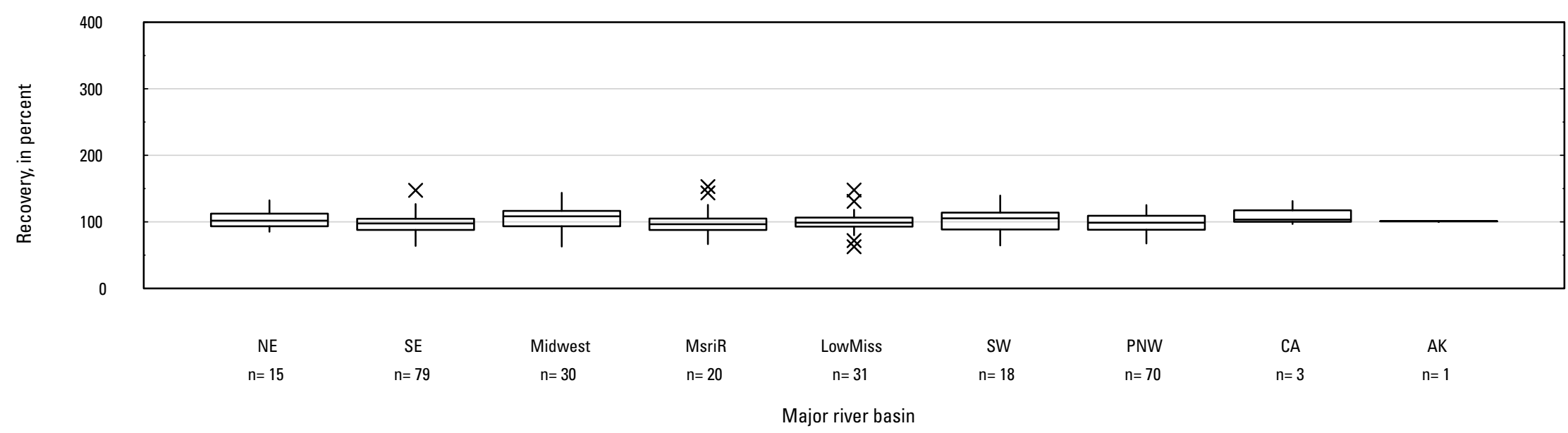

Figure 5. Graph showing distributions of recovery for pesticides in schedule 2437 in surface water by Major River Basin. Recovery values larger than 400 percent are 


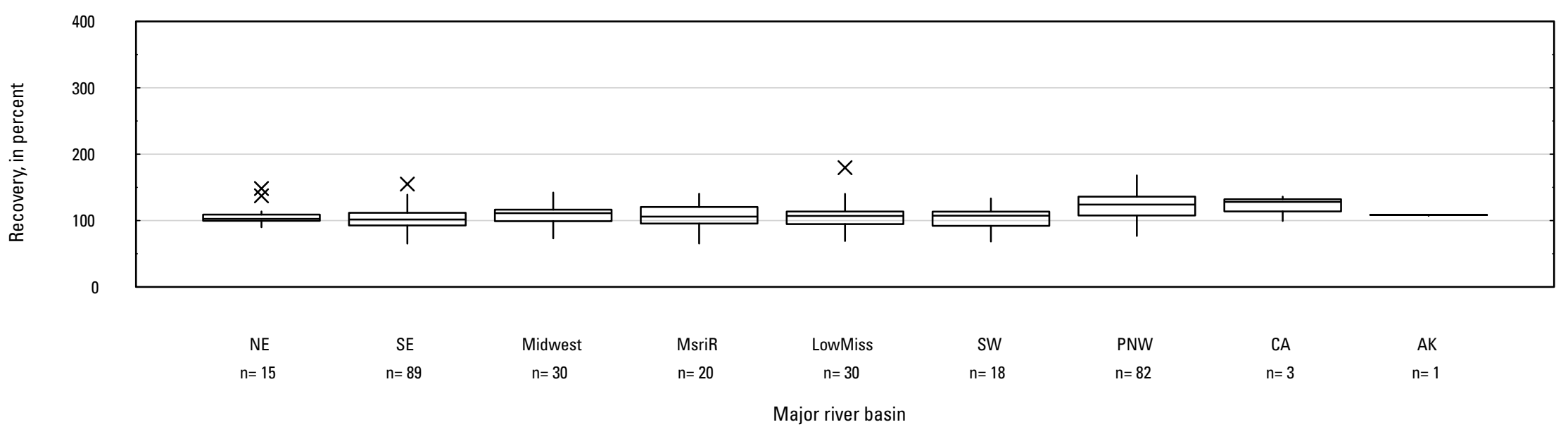

DY. Imidacloprid

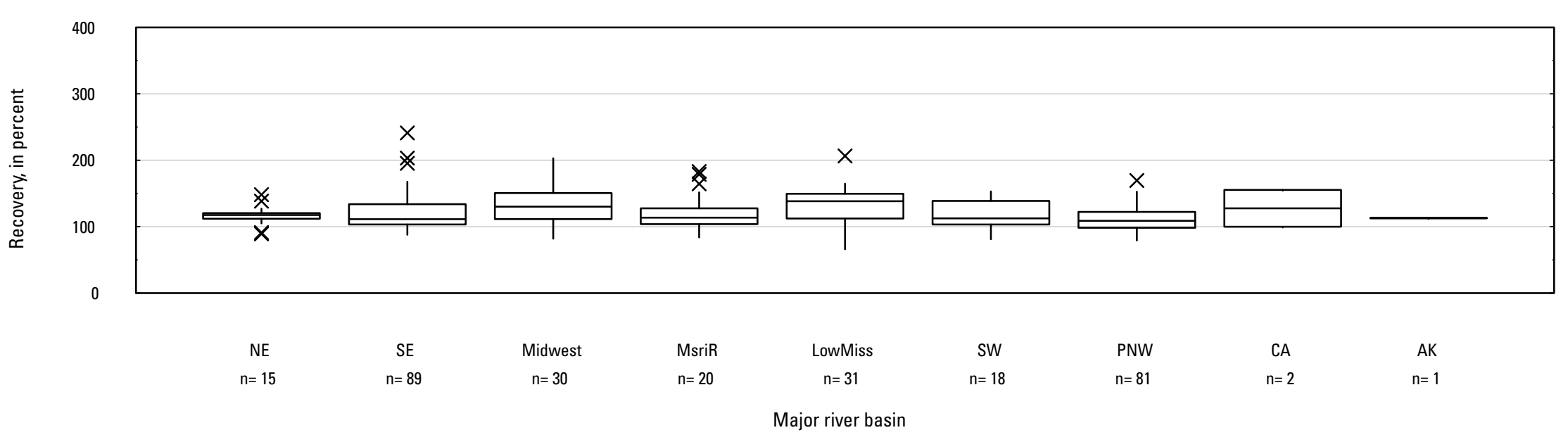

Figure 5. Graph showing distributions of recovery for pesticides in schedule 2437 in surface water by Major River Basin. Recovery values larger than 400 percent are not shown.-Continued 
DZ. Indoxacarb

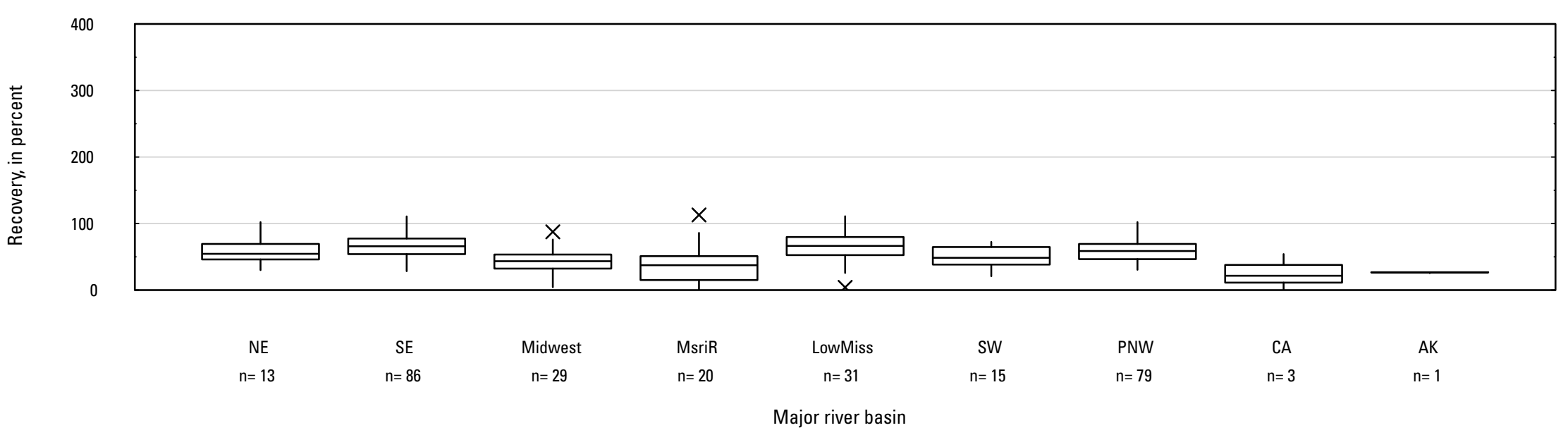

EA. Isoxaflutole

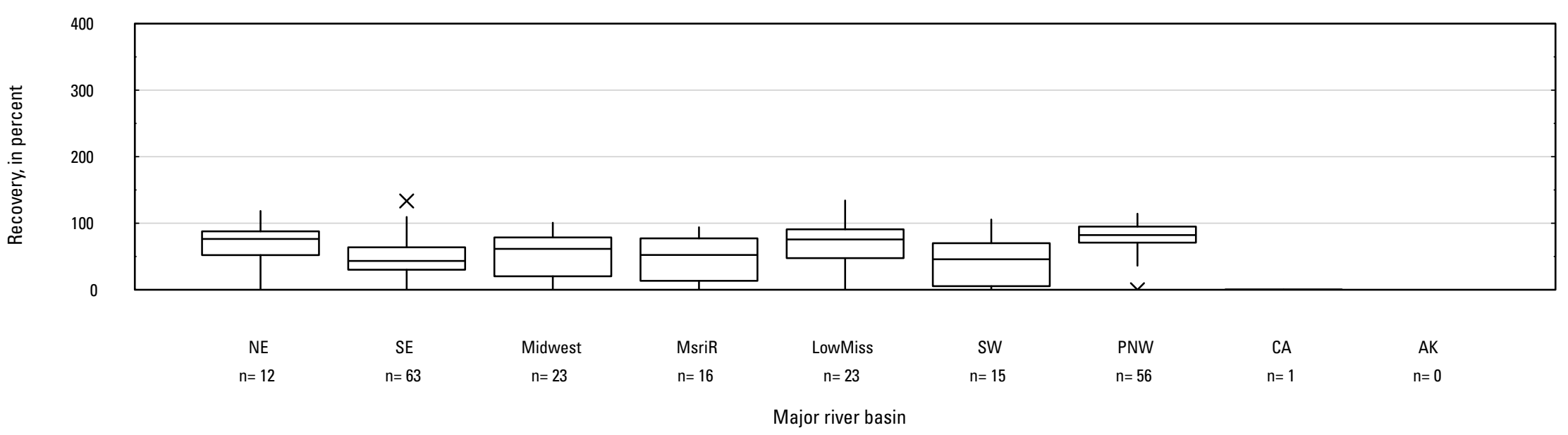

Figure 5. Graph showing distributions of recovery for pesticides in schedule 2437 in surface water by Major River Basin. Recovery values larger than 400 percent are not shown.-Continued 


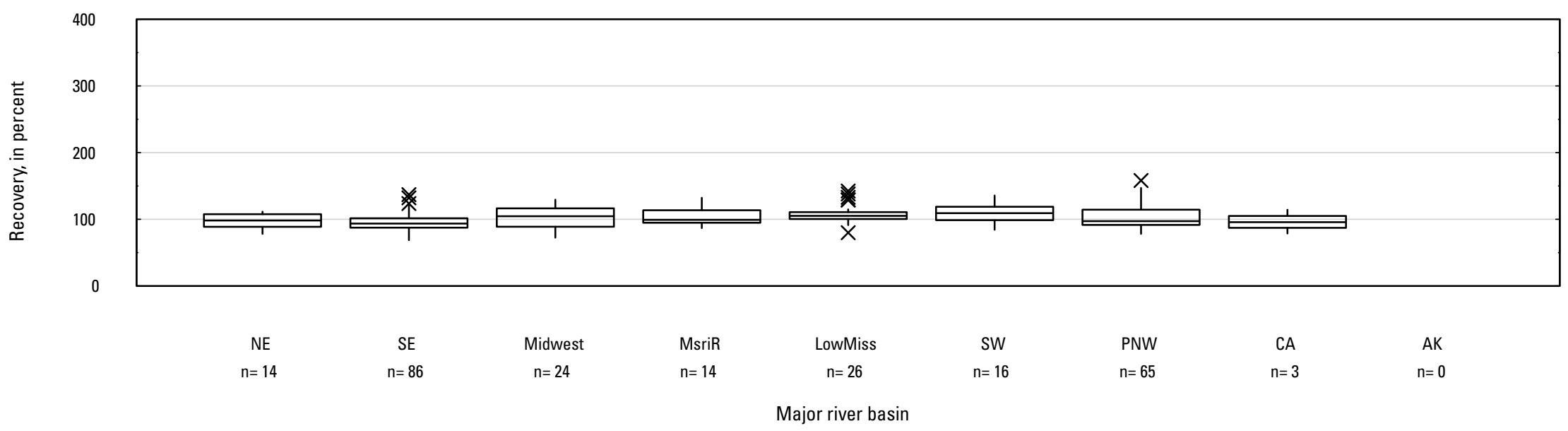

EC. Kresoxim-methyl

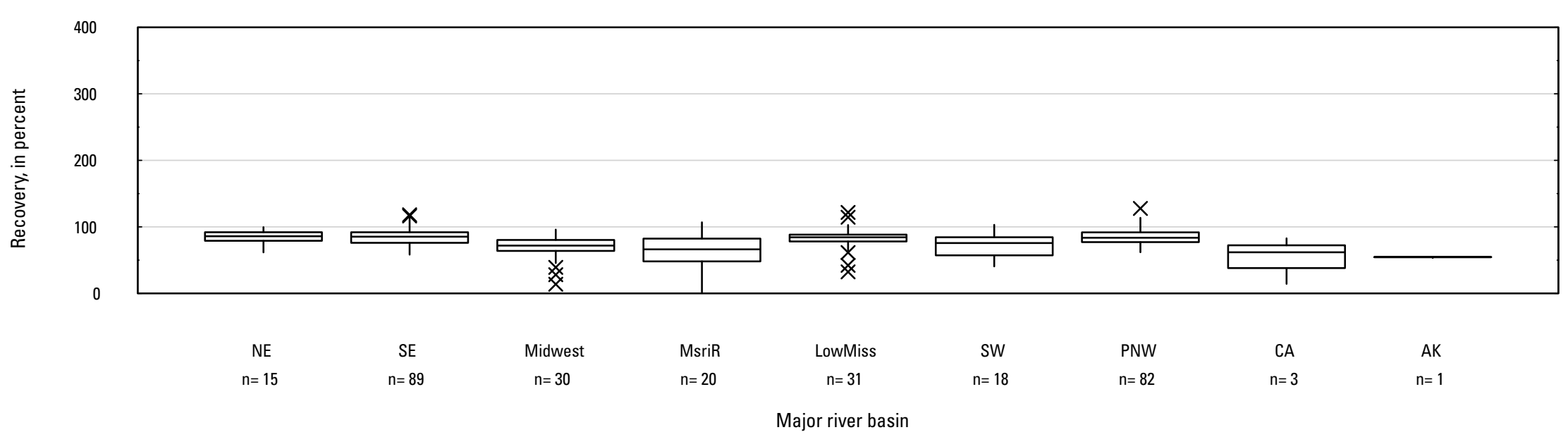

Figure 5. Graph showing distributions of recovery for pesticides in schedule 2437 in surface water by Major River Basin. Recovery values larger than 400 percent are not shown.-Continued 
ED. Lactofen

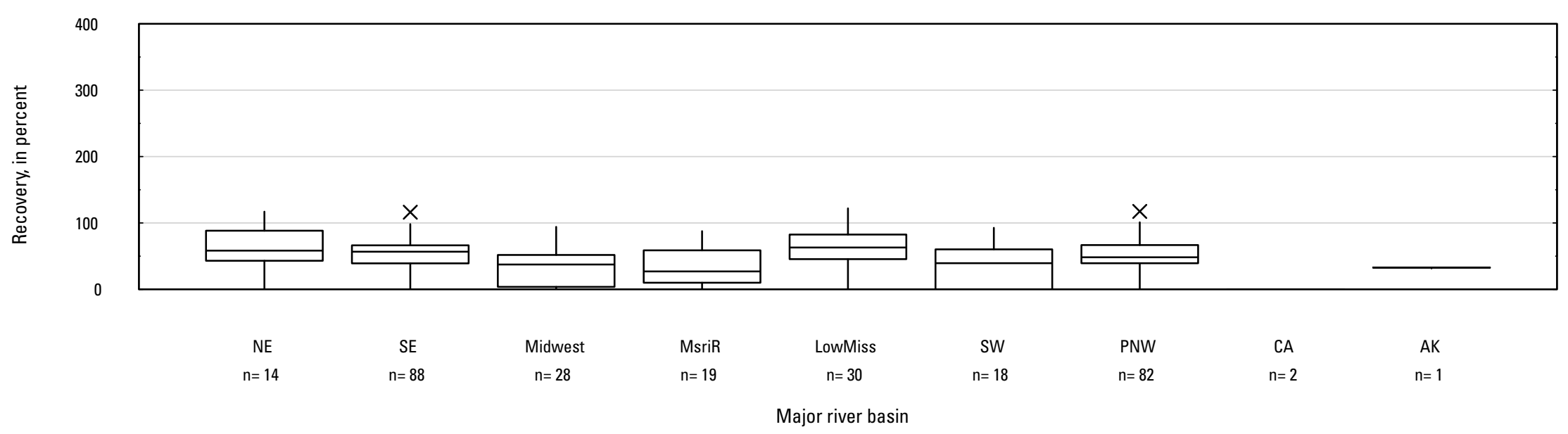

EE. Linuron

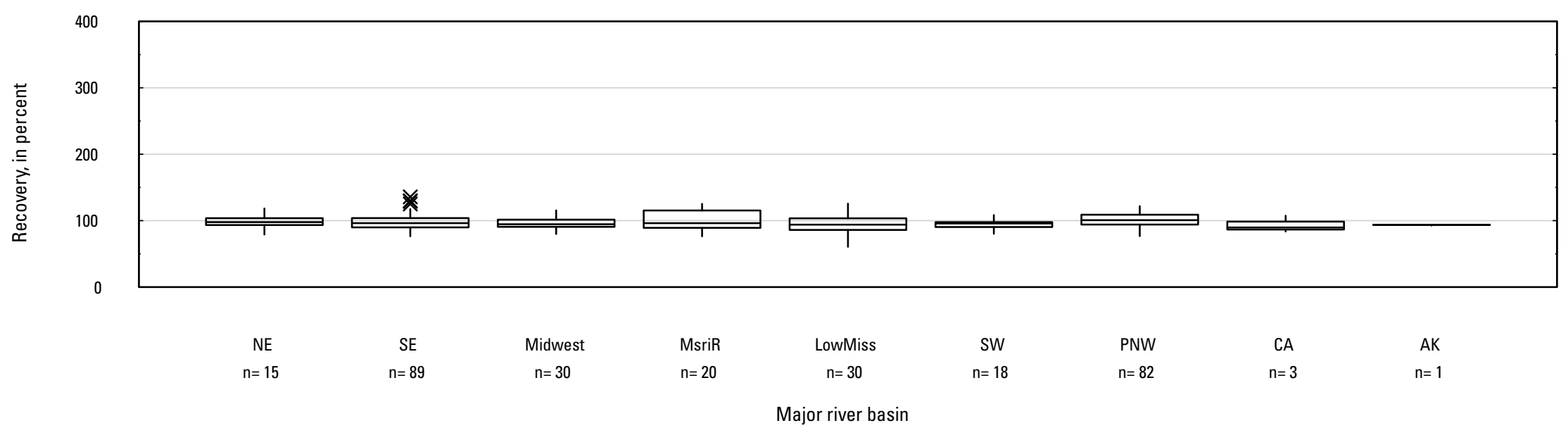

Figure 5. Graph showing distributions of recovery for pesticides in schedule 2437 in surface water by Major River Basin. Recovery values larger than 400 percent are not shown.-Continued 


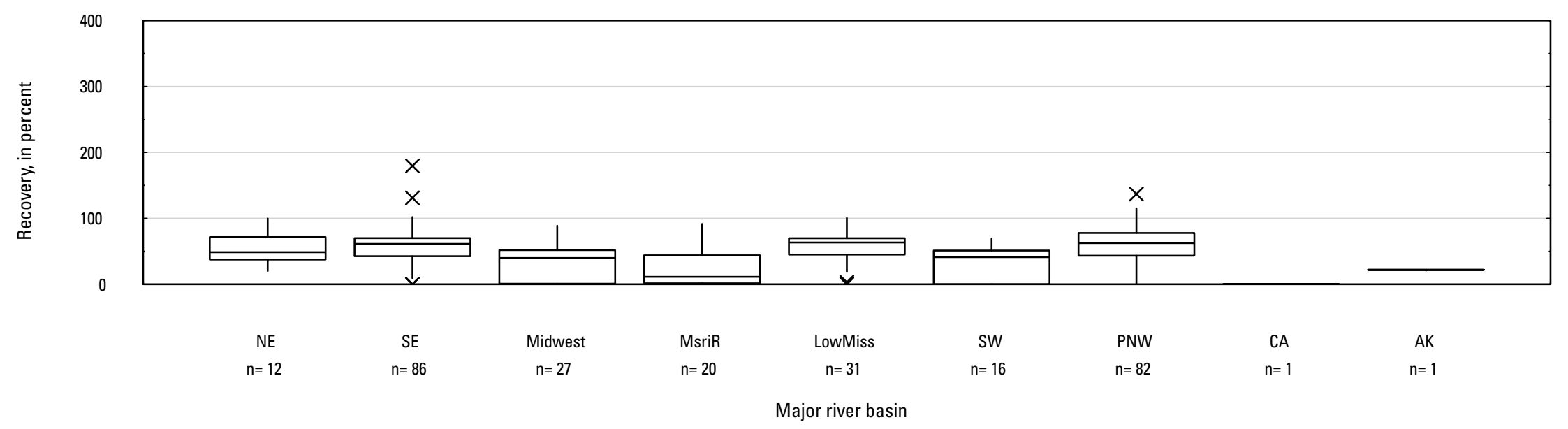

EG. Malathion

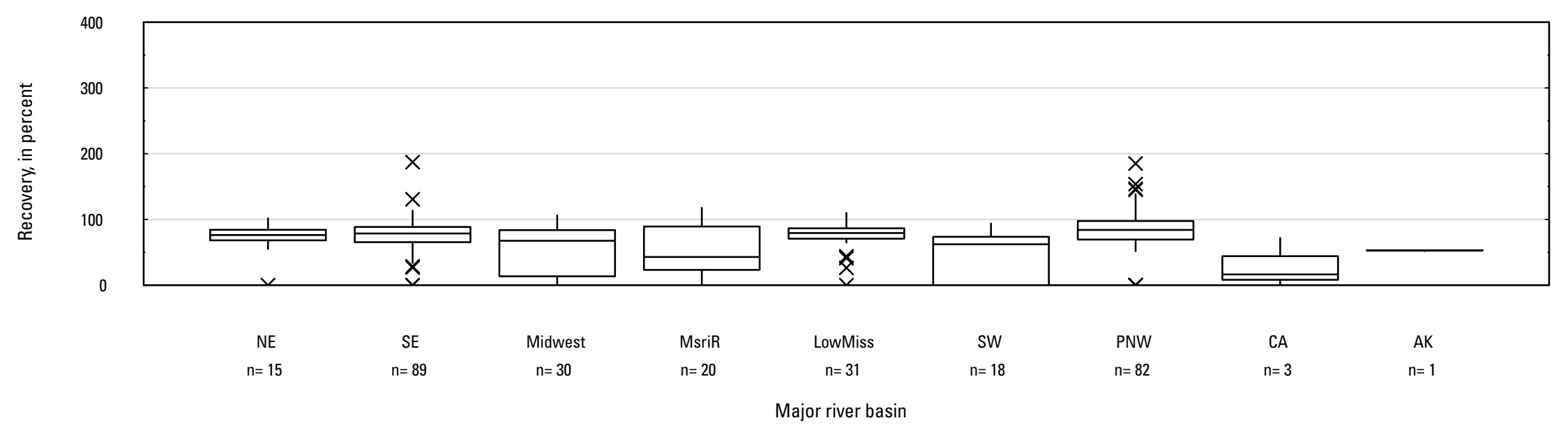

Figure 5. Graph showing distributions of recovery for pesticides in schedule 2437 in surface water by Major River Basin. Recovery values larger than 400 percent are not shown.-Continued 
EH. МСРA

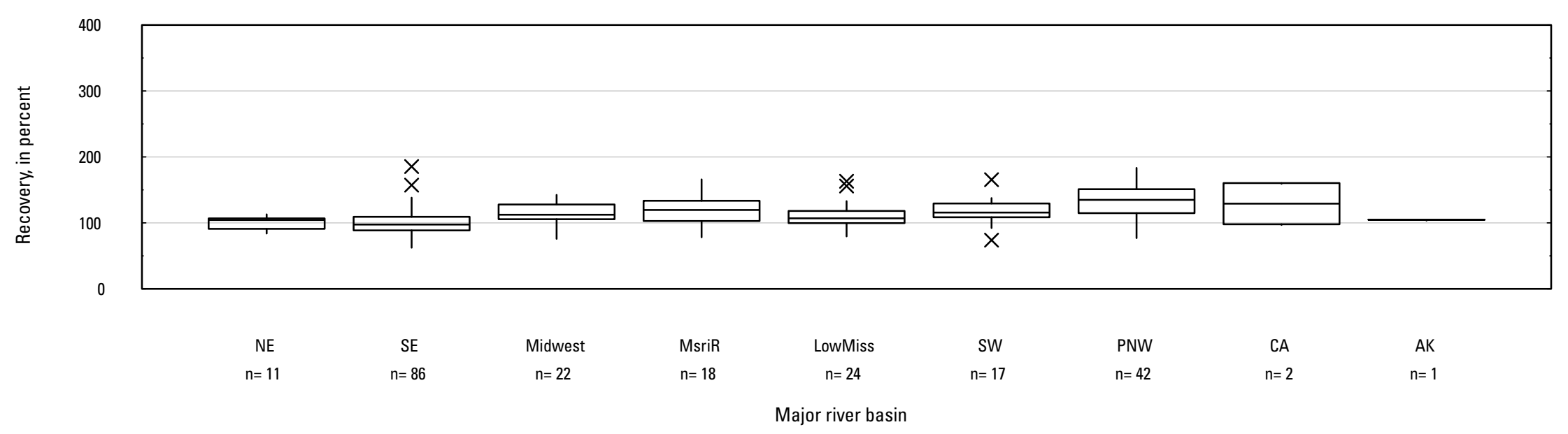

El. Metalaxyl

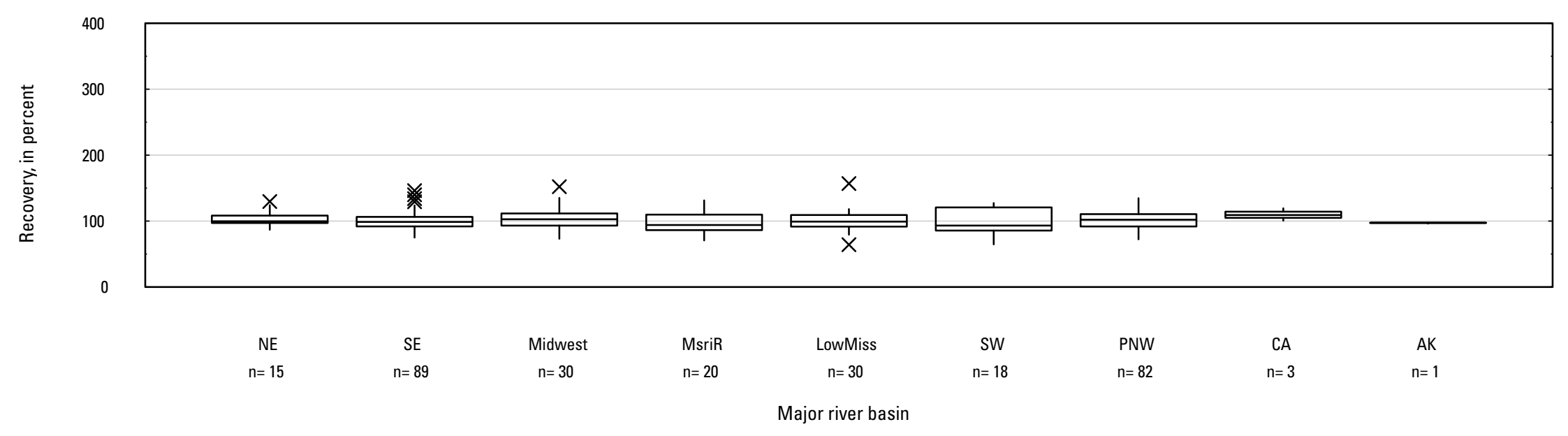

Figure 5. Graph showing distributions of recovery for pesticides in schedule 2437 in surface water by Major River Basin. Recovery values larger than 400 percent are not shown.-Continued 


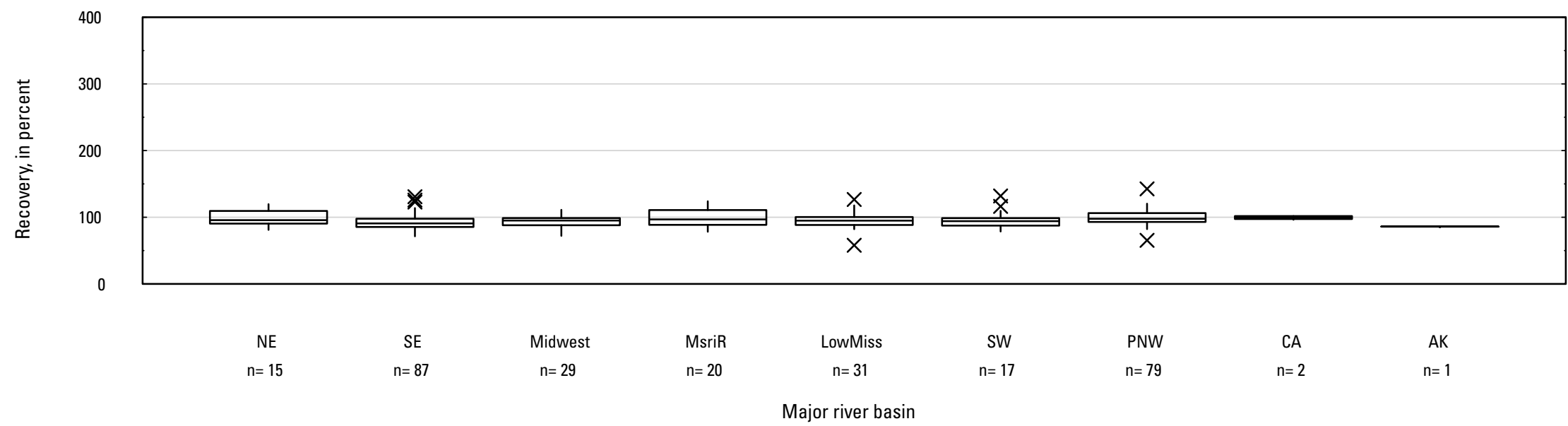

EK. Methamidophos

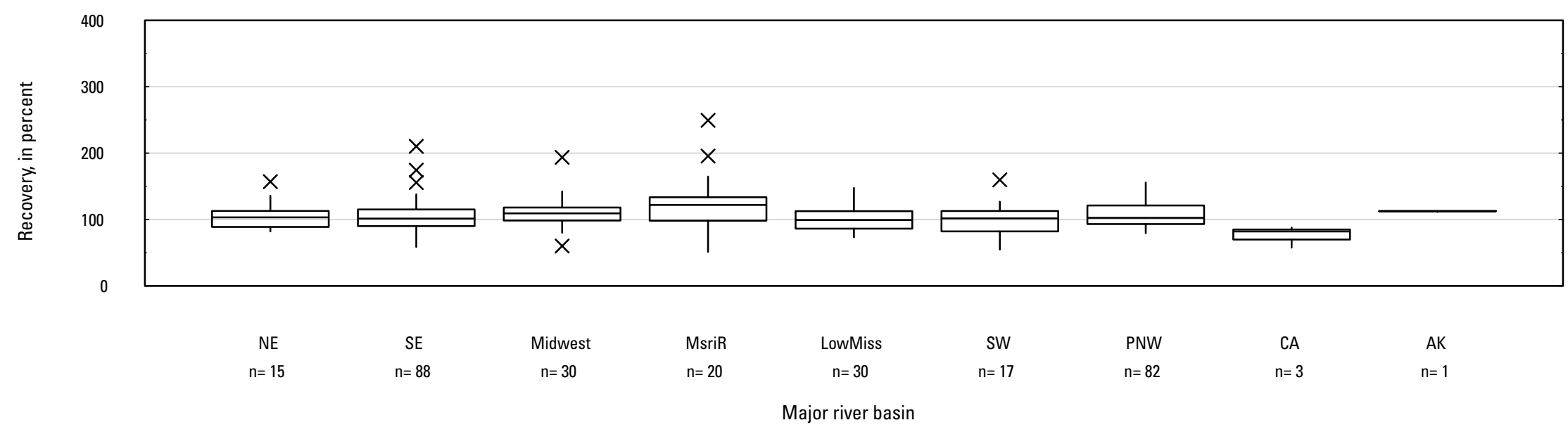

Figure 5. Graph showing distributions of recovery for pesticides in schedule 2437 in surface water by Major River Basin. Recovery values larger than 400 percent are not shown.-Continued 
EL. Methidathion

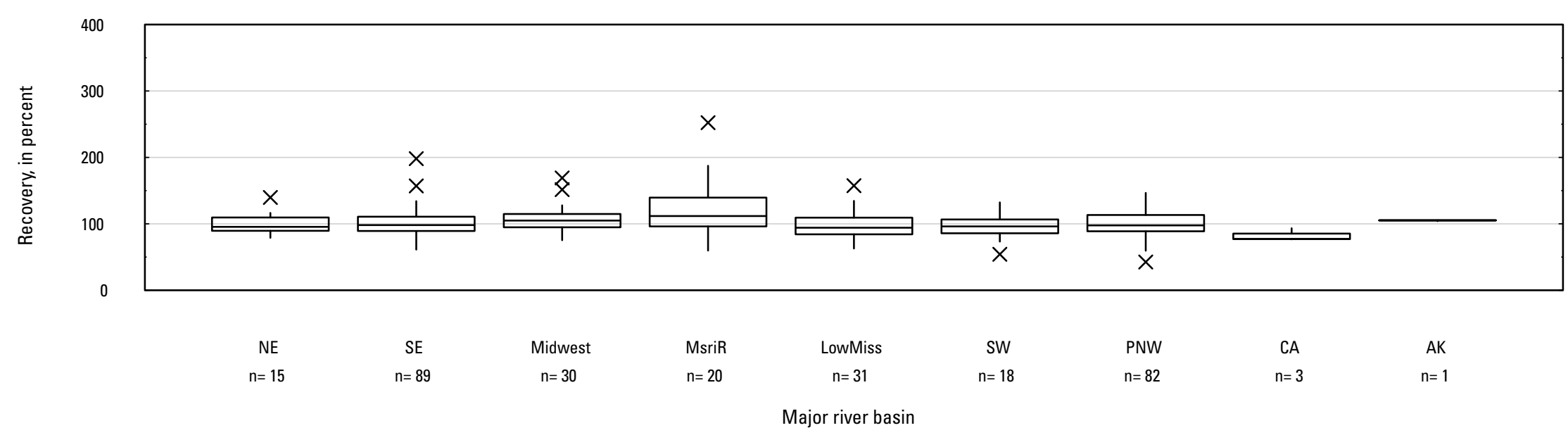

EM. Methomyl

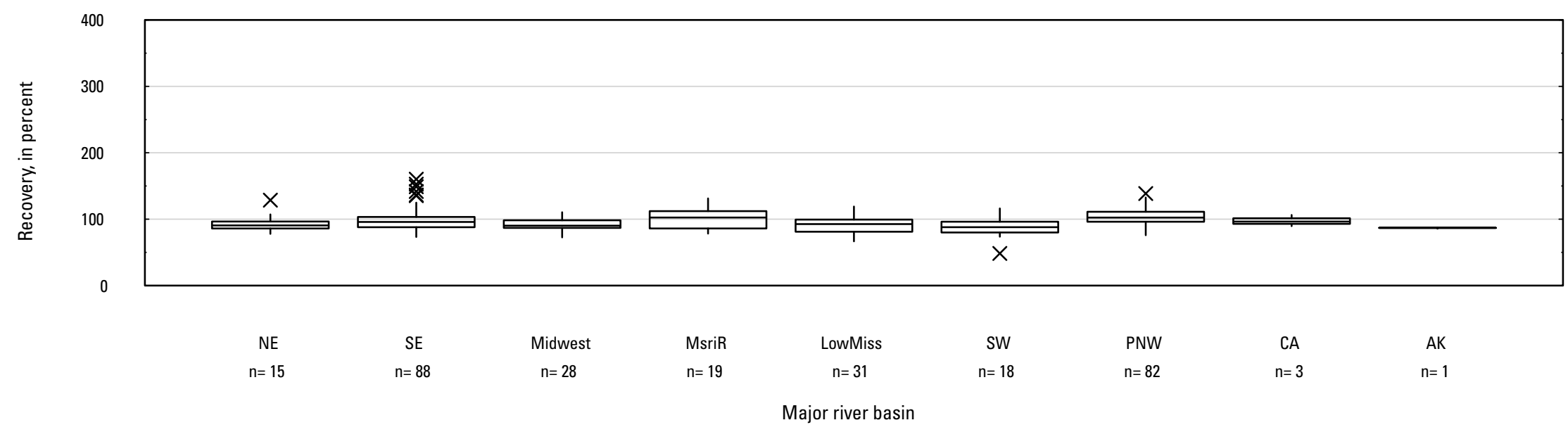

Figure 5. Graph showing distributions of recovery for pesticides in schedule 2437 in surface water by Major River Basin. Recovery values larger than 400 percent are 


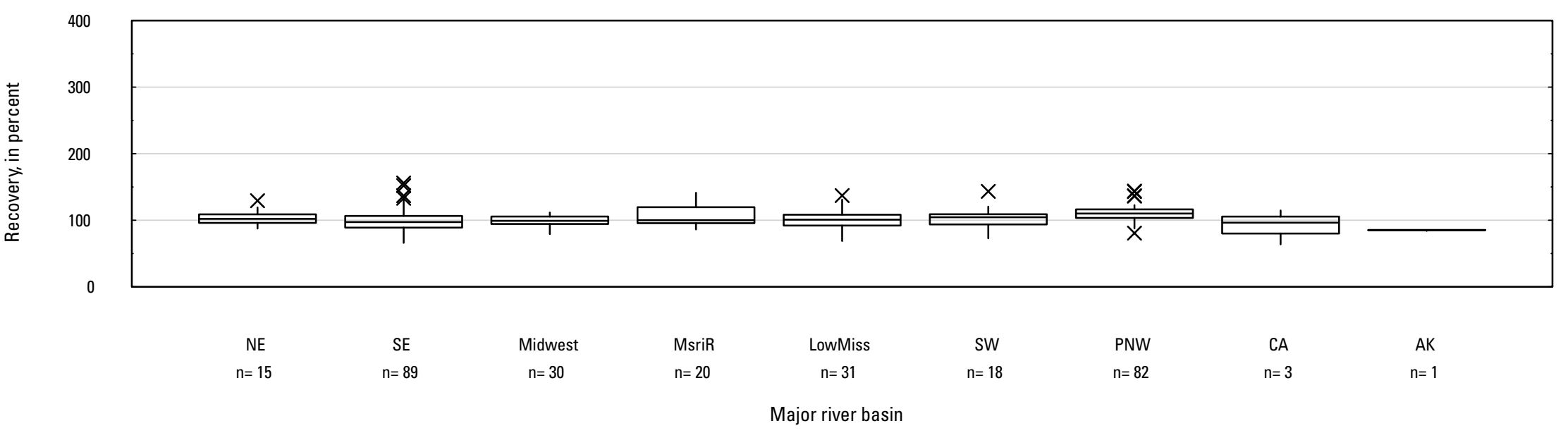

EP. Metolachlor

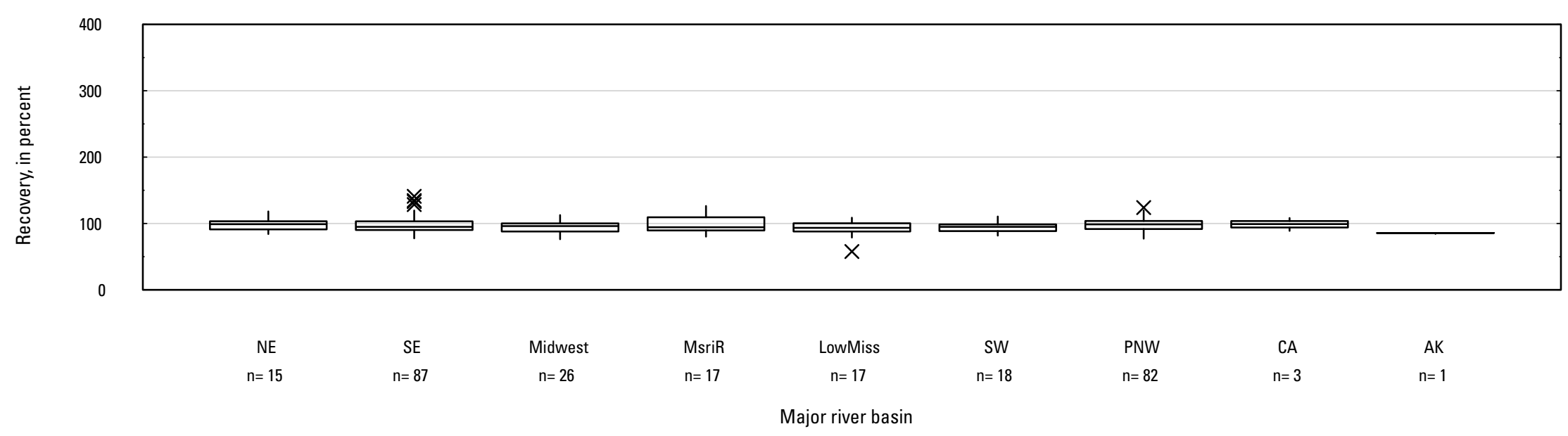

Figure 5. Graph showing distributions of recovery for pesticides in schedule 2437 in surface water by Major River Basin. Recovery values larger than 400 percent are not shown.-Continued 
EQ. Metolachlor hydroxy morpholinone

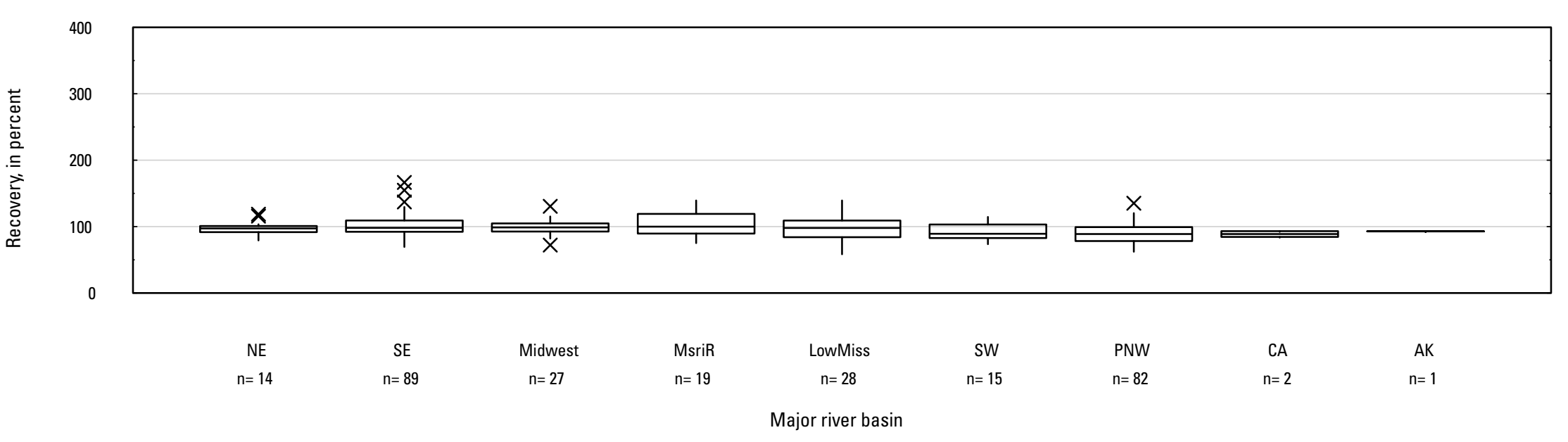

ER. Metolachlor oxanilic acid

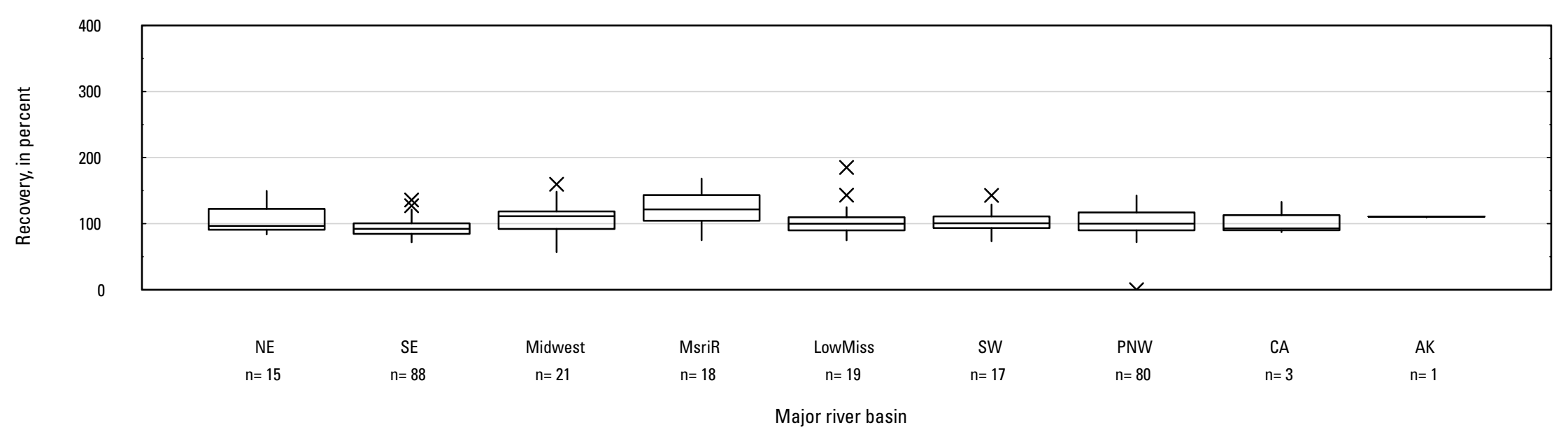

Figure 5. Graph showing distributions of recovery for pesticides in schedule 2437 in surface water by Major River Basin. Recovery values larger than 400 percent are not shown.-Continued 


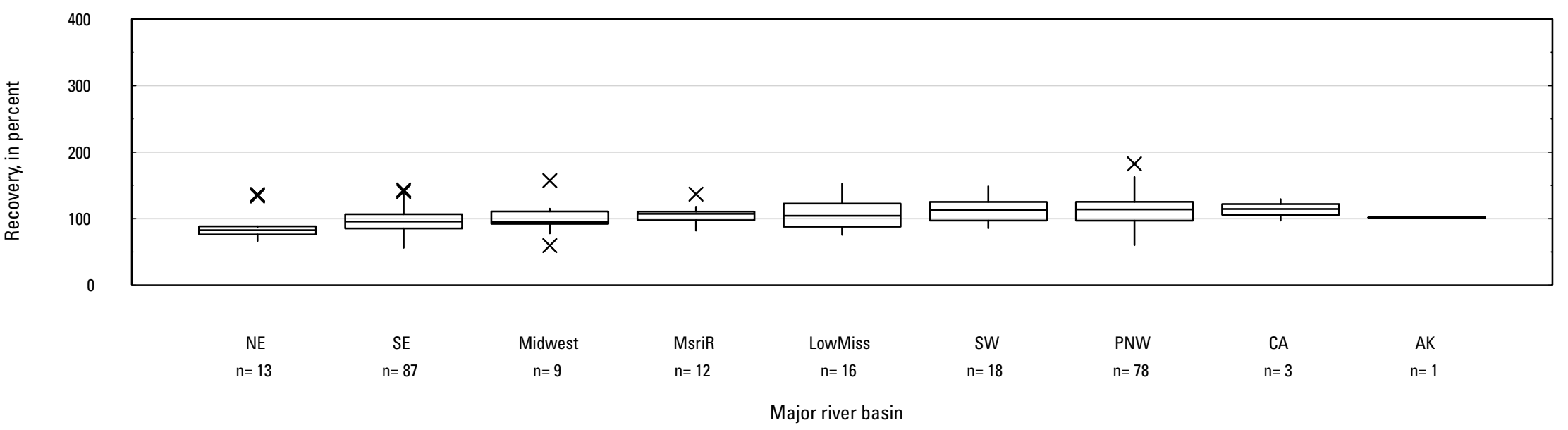

ET. Metribuzin

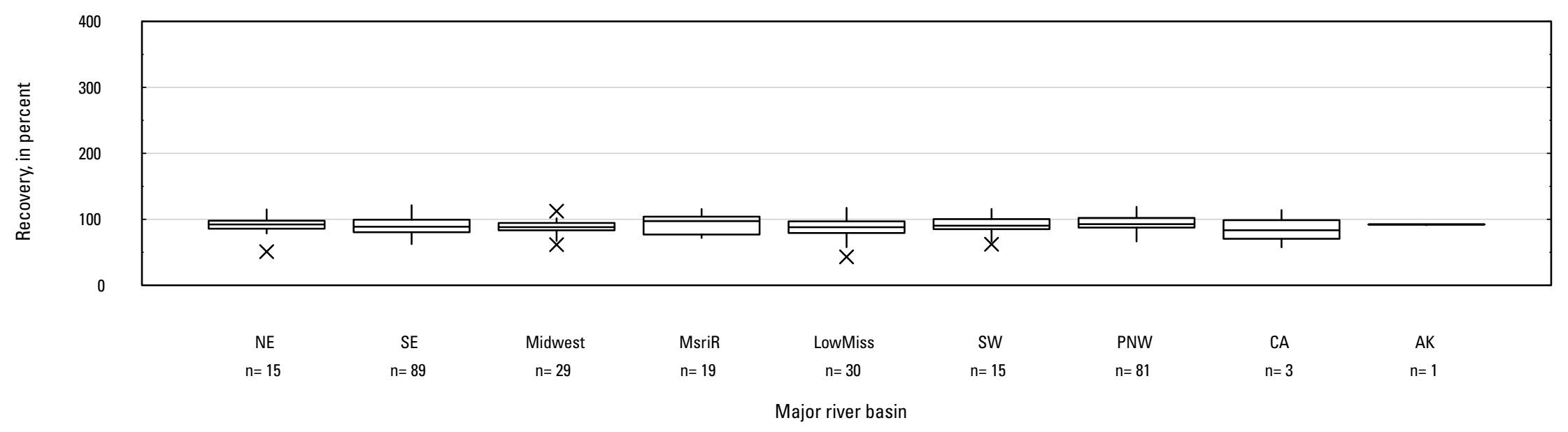

Figure 5. Graph showing distributions of recovery for pesticides in schedule 2437 in surface water by Major River Basin. Recovery values larger than 400 percent are not shown.-Continued 
EU. Desamino metribuzin

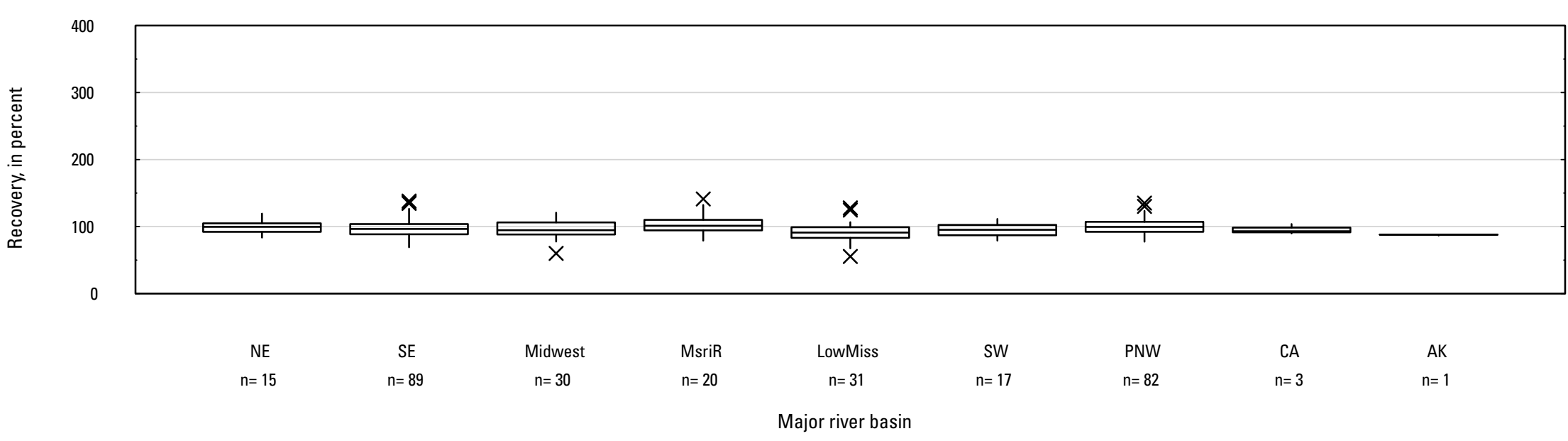

EV. Desamino-diketo metribuzin

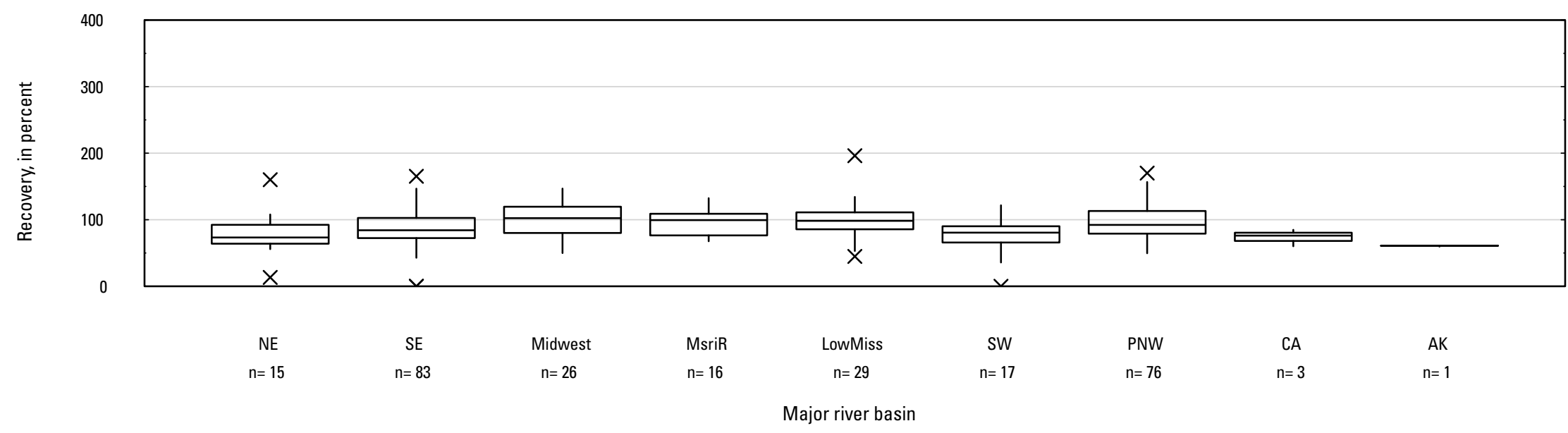

Figure 5. Graph showing distributions of recovery for pesticides in schedule 2437 in surface water by Major River Basin. Recovery values larger than 400 percent are 


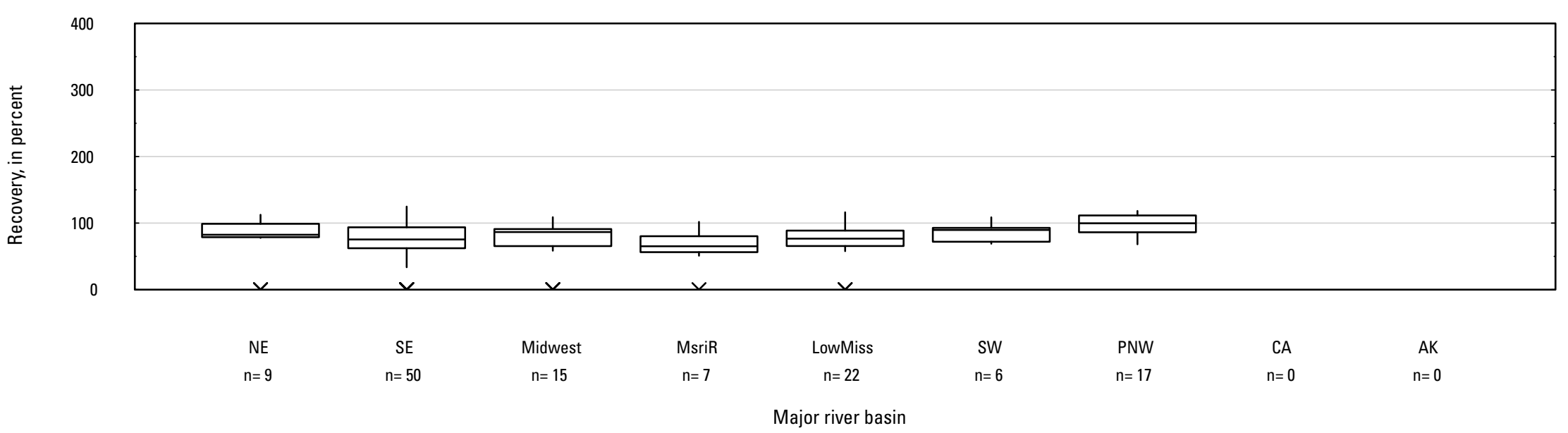

EX. Molinate

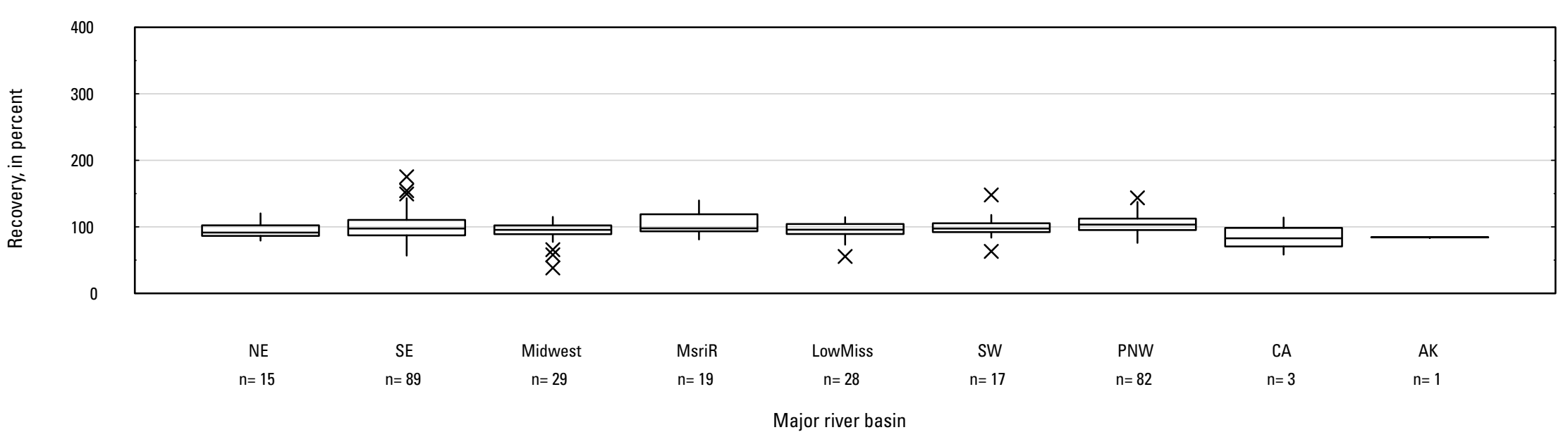

Figure 5. Graph showing distributions of recovery for pesticides in schedule 2437 in surface water by Major River Basin. Recovery values larger than 400 percent are not shown.-Continued 
EY. Myclobutanil

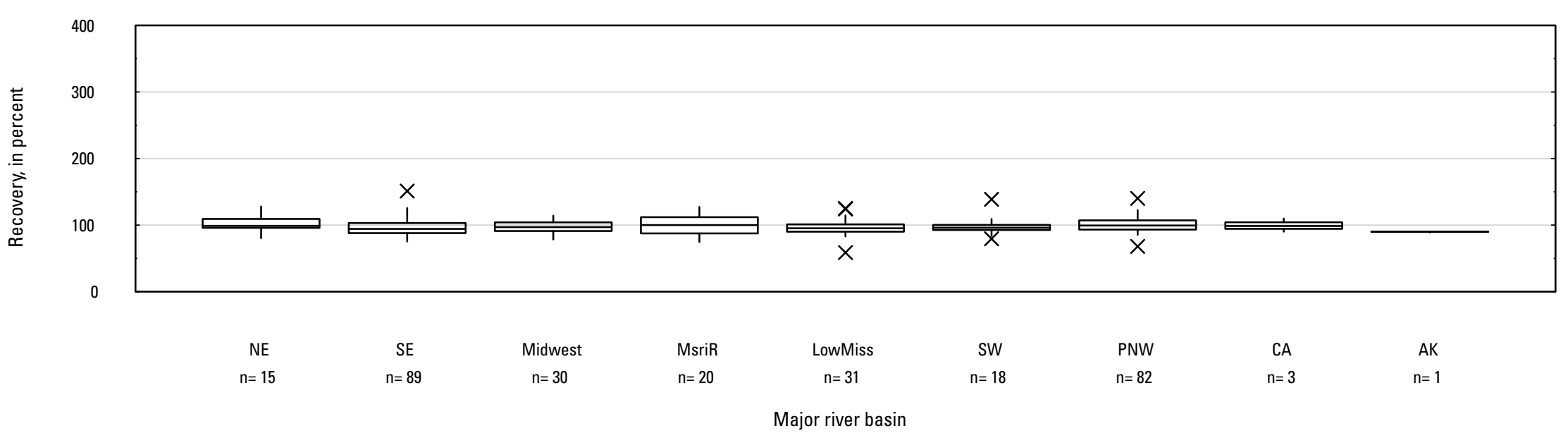

EZ. N-(3,4-Dichlorophenyl)-N'-methylurea

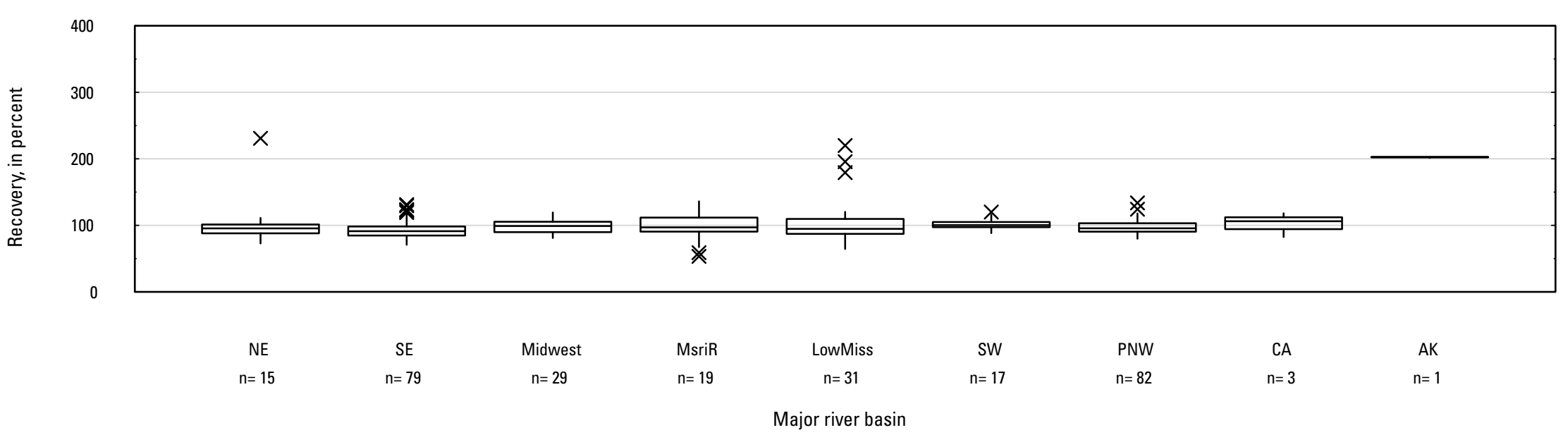

Figure 5. Graph showing distributions of recovery for pesticides in schedule 2437 in surface water by Major River Basin. Recovery values larger than 400 percent are not shown.-Continued 


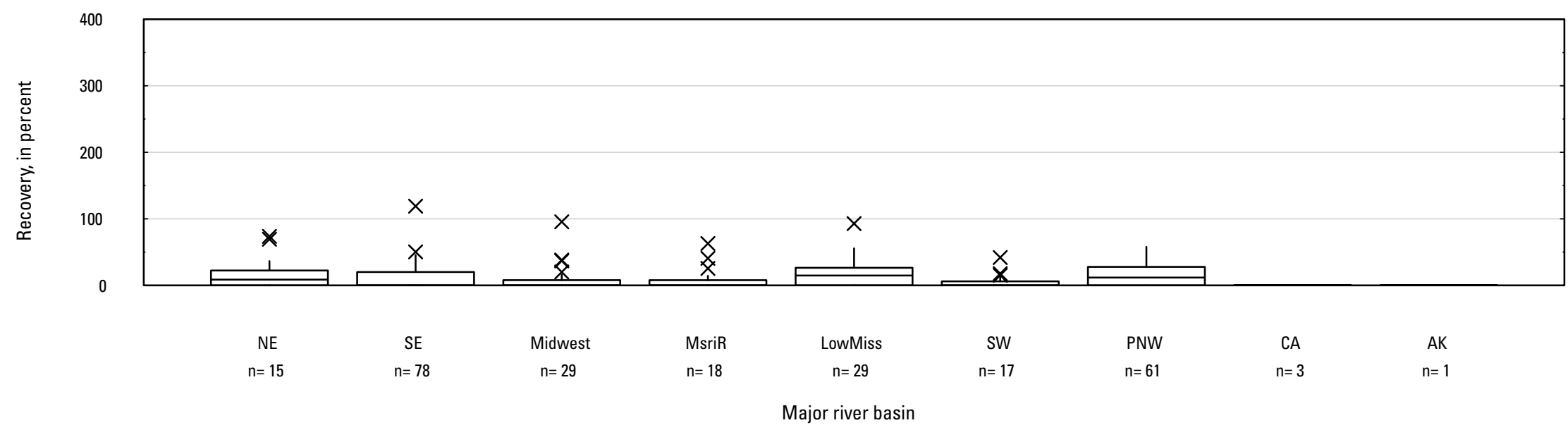

FB. Nicosulfuron

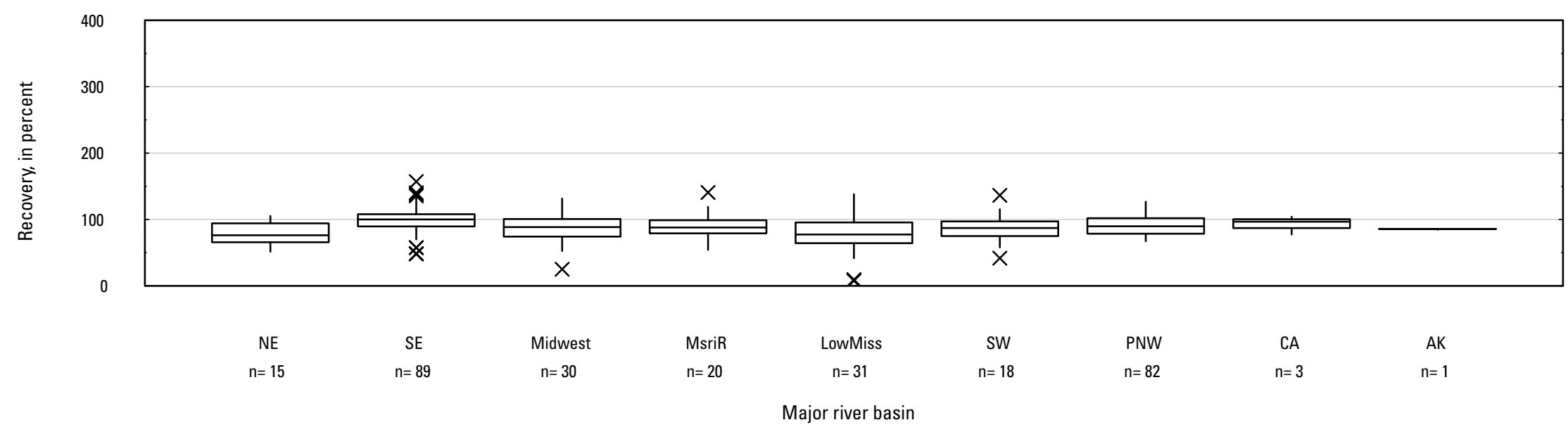

Figure 5. Graph showing distributions of recovery for pesticides in schedule 2437 in surface water by Major River Basin. Recovery values larger than 400 percent are not shown.-Continued 
FC. Norflurazon

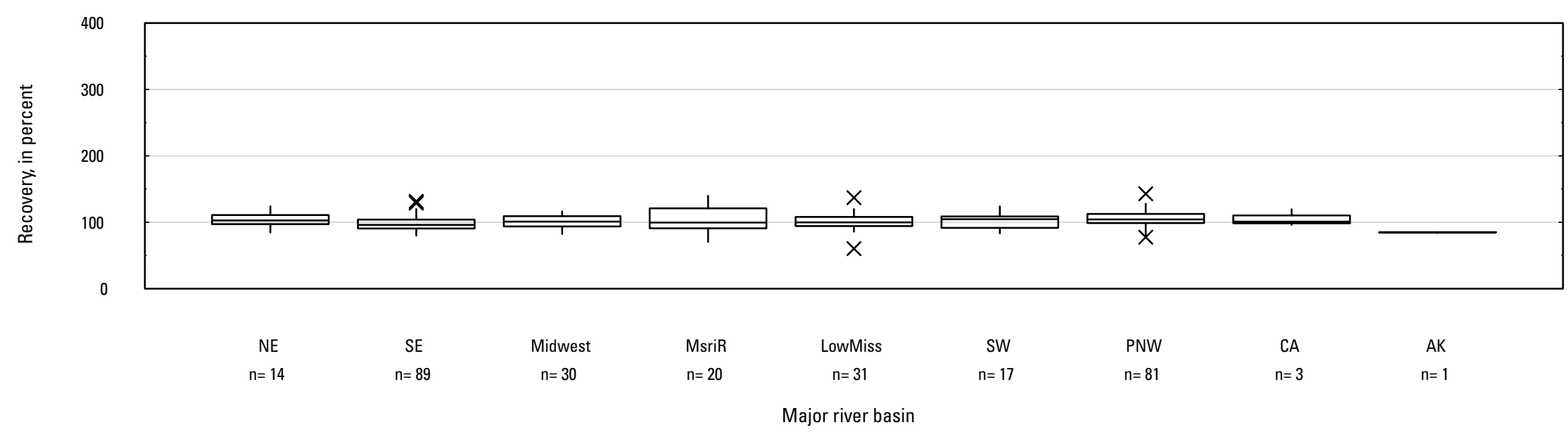

FD. Novaluron

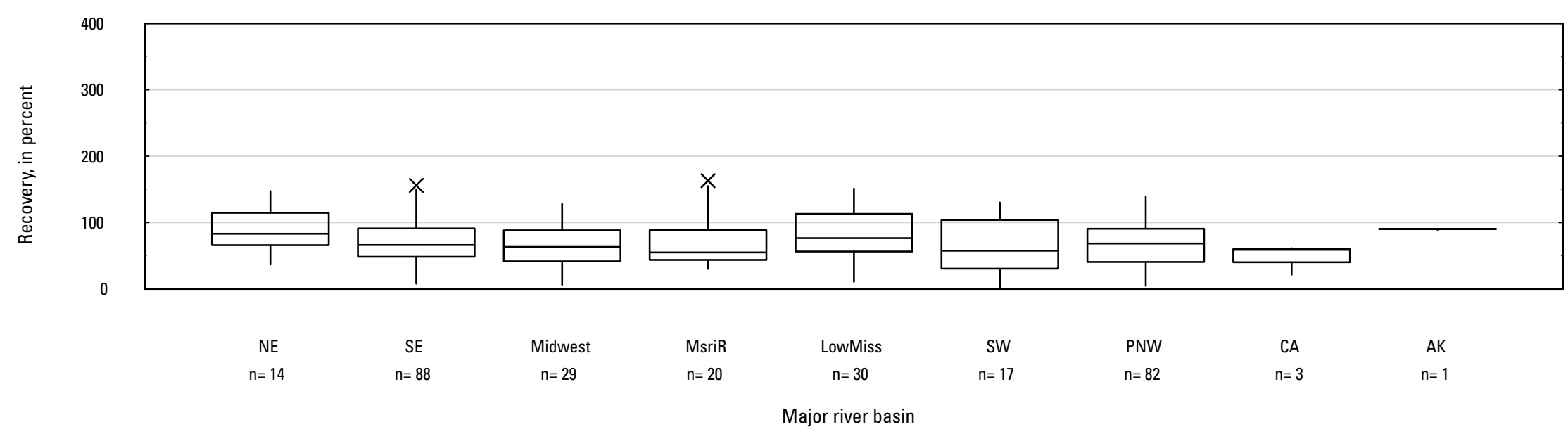

Figure 5. Graph showing distributions of recovery for pesticides in schedule 2437 in surface water by Major River Basin. Recovery values larger than 400 percent are 


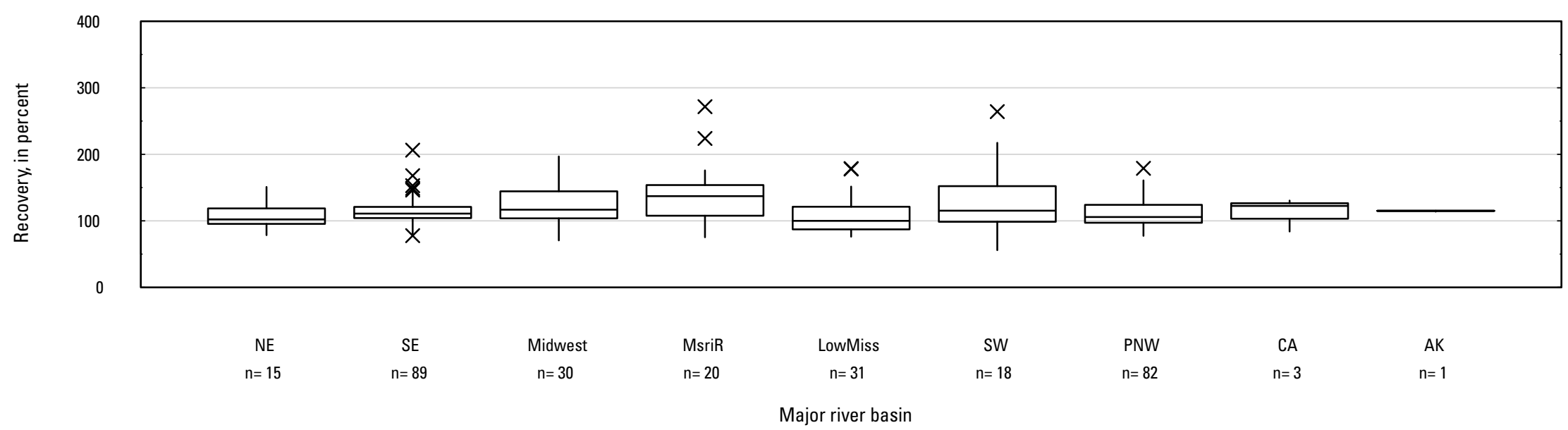

FF. 0-Ethyl-S-methyl-S-propyl phosphorodithioate

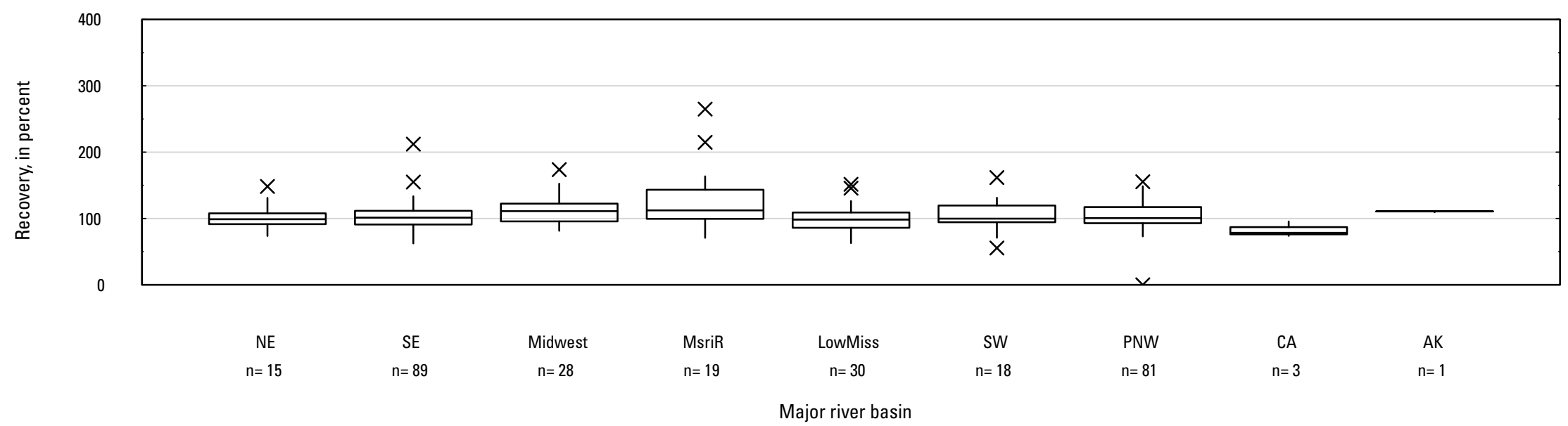

Figure 5. Graph showing distributions of recovery for pesticides in schedule 2437 in surface water by Major River Basin. Recovery values larger than 400 percent are not shown.-Continued 
FG. 0-Ethyl-S-propyl phosphorothioate

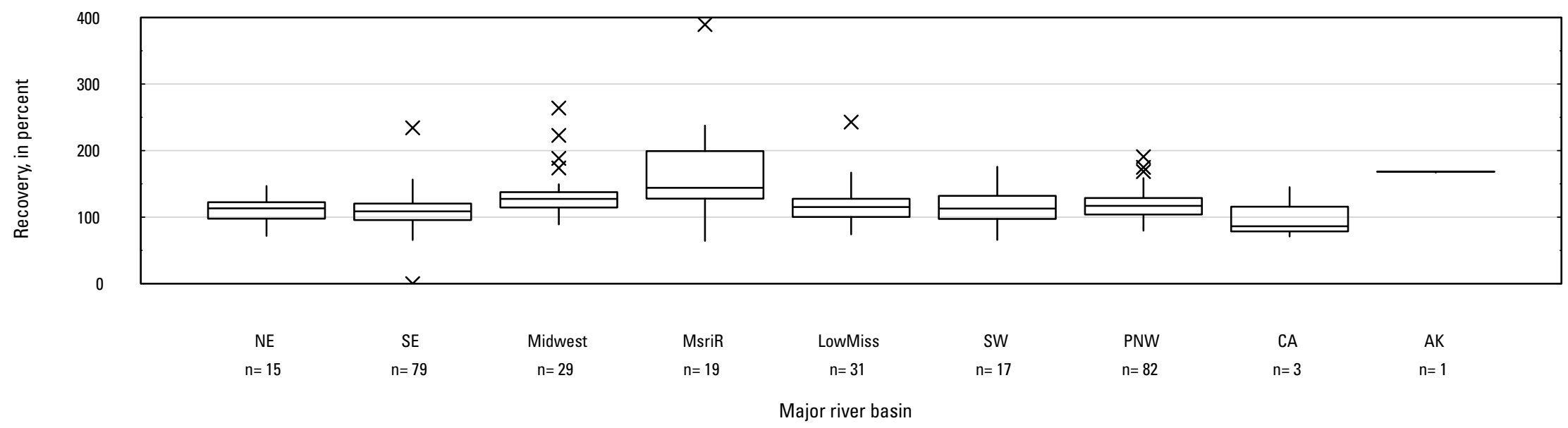

FH. Orthosulfamuron

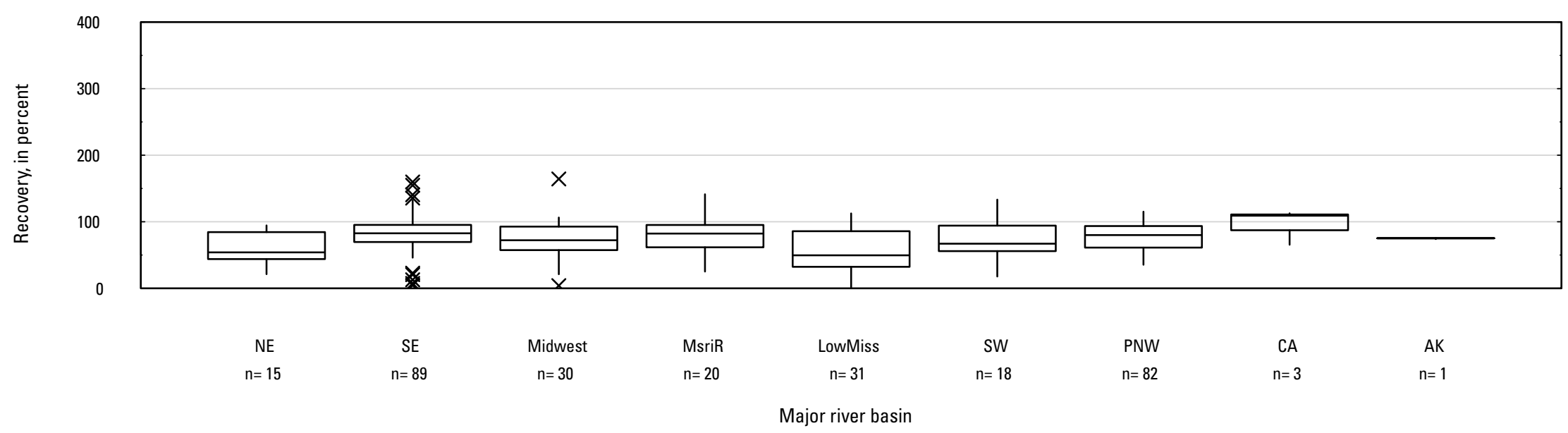

Figure 5. Graph showing distributions of recovery for pesticides in schedule 2437 in surface water by Major River Basin. Recovery values larger than 400 percent are not shown.-Continued 


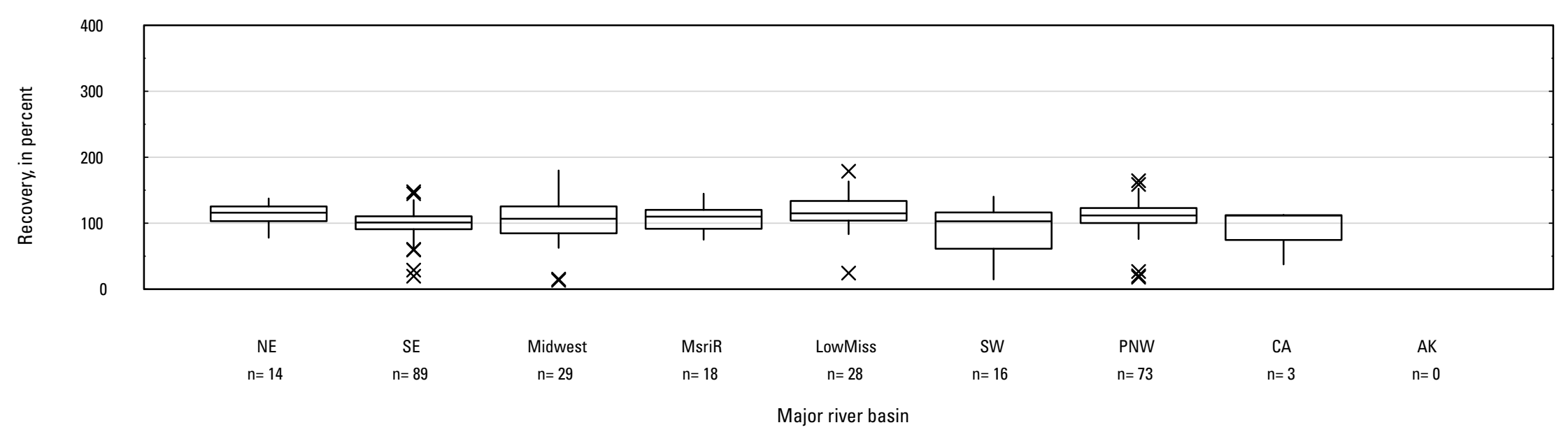

FJ. Oxamyl

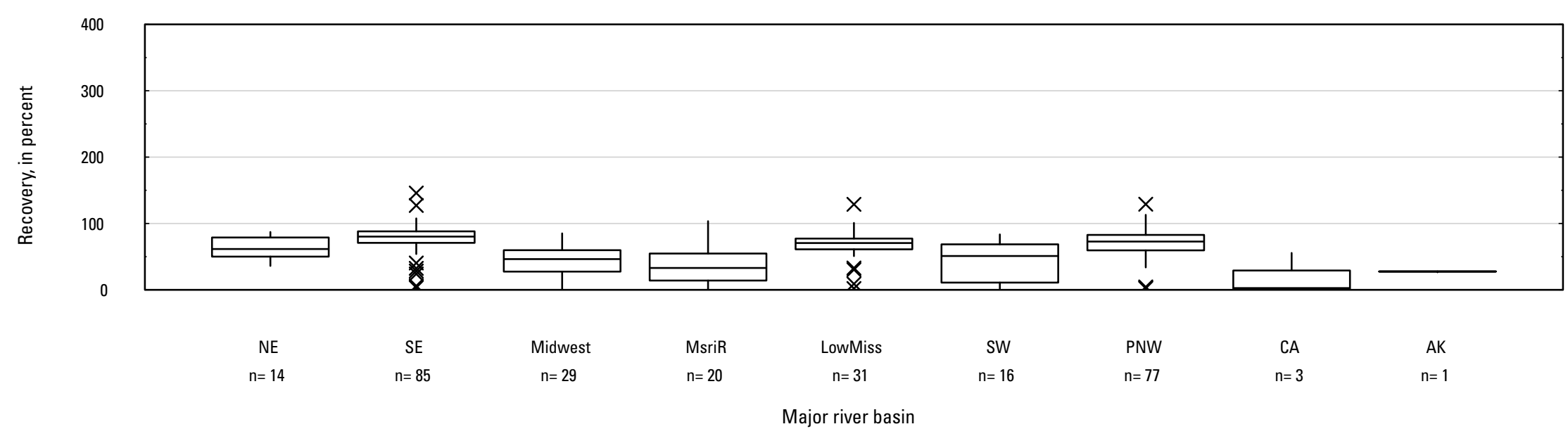

Figure 5. Graph showing distributions of recovery for pesticides in schedule 2437 in surface water by Major River Basin. Recovery values larger than 400 percent are not shown.-Continued 
FK. Oxamyl oxime

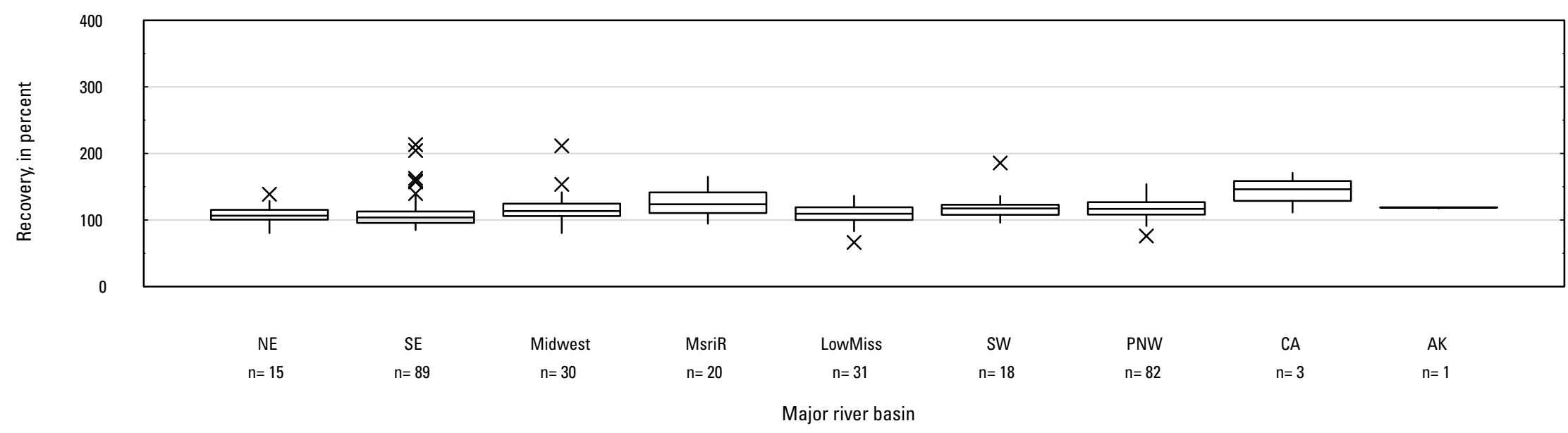

FL. Oxyfluorfen

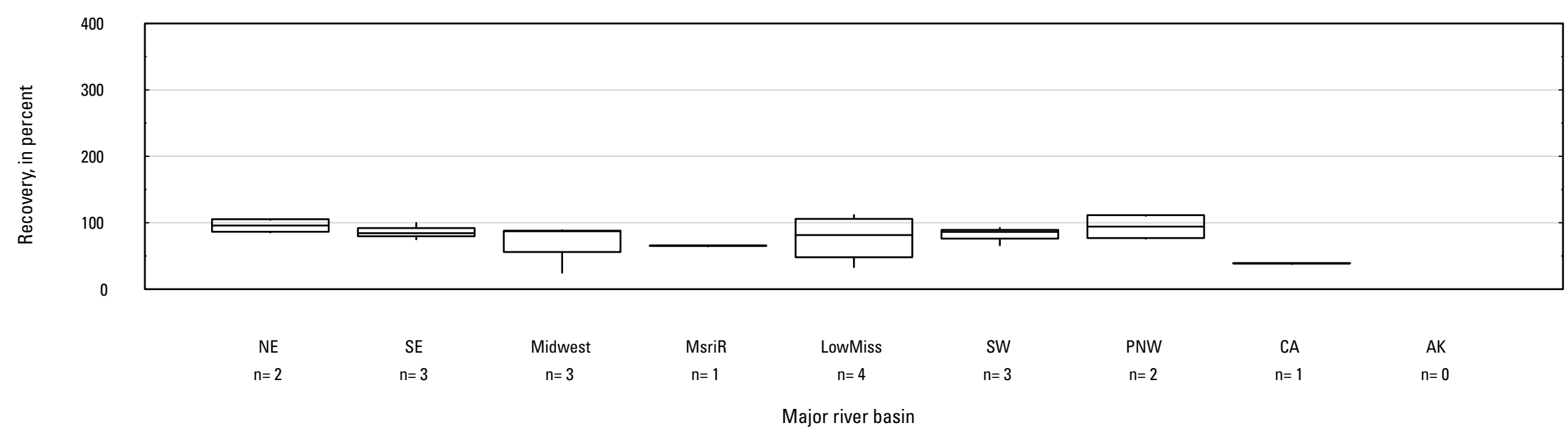

Figure 5. Graph showing distributions of recovery for pesticides in schedule 2437 in surface water by Major River Basin. Recovery values larger than 400 percent are not shown.-Continued 


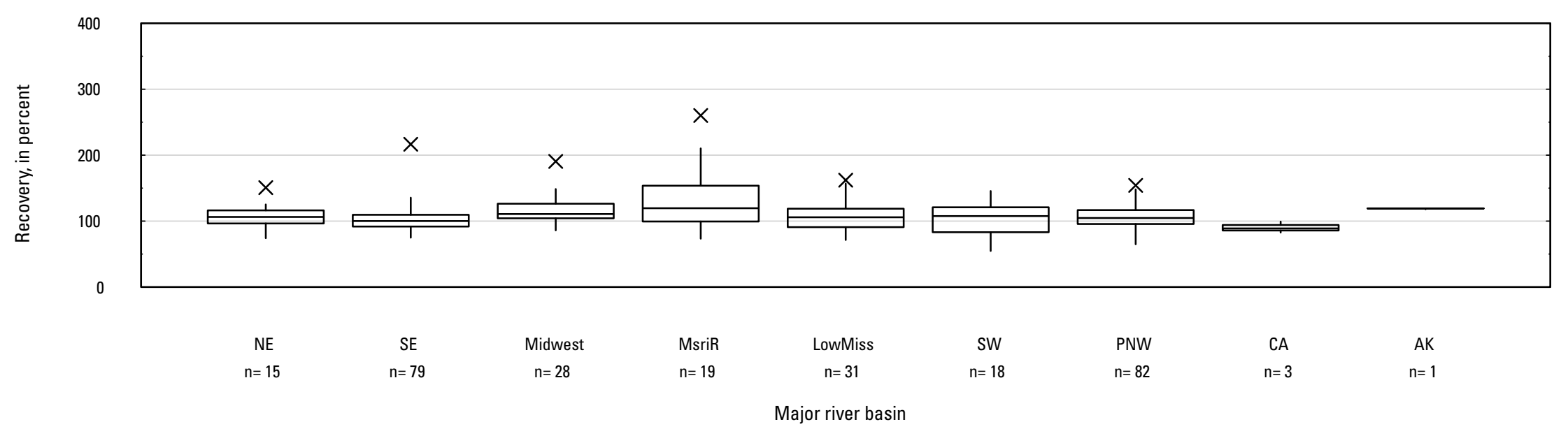

FN. Methyl paraoxon

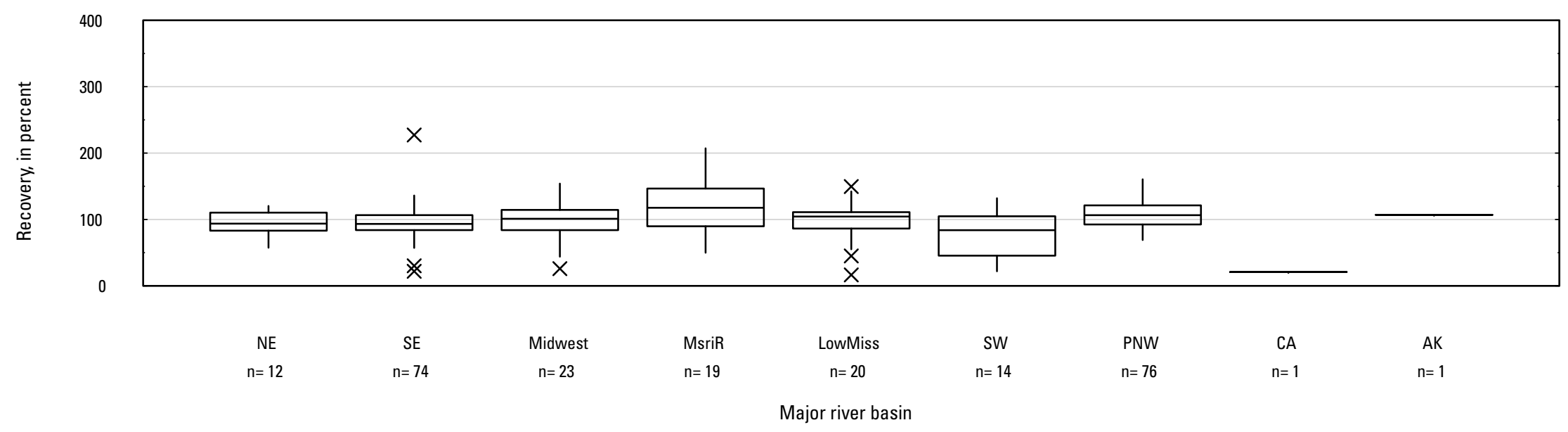

Figure 5. Graph showing distributions of recovery for pesticides in schedule 2437 in surface water by Major River Basin. Recovery values larger than 400 percent are not shown.-Continued 
FO. Pendimethalin

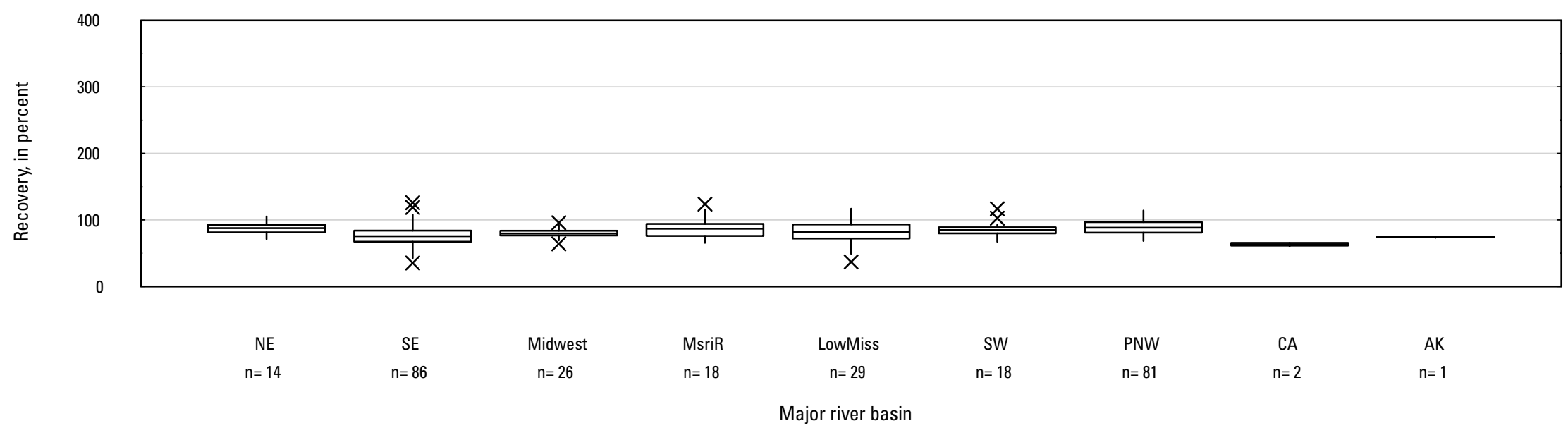

FP. Phorate

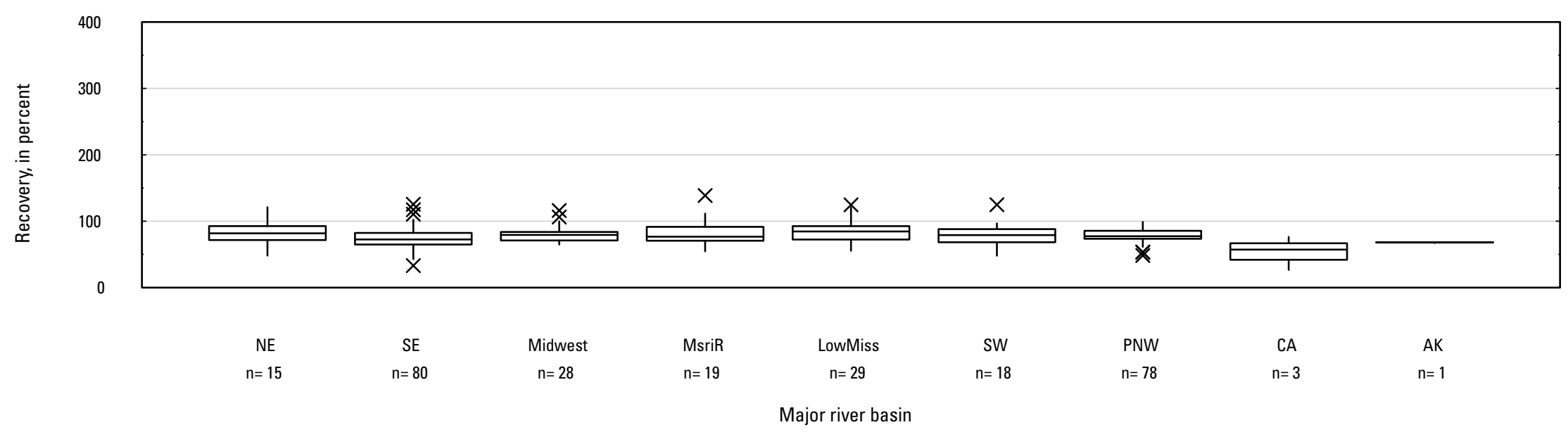

Figure 5. Graph showing distributions of recovery for pesticides in schedule 2437 in surface water by Major River Basin. Recovery values larger than 400 percent are not shown.-Continued 


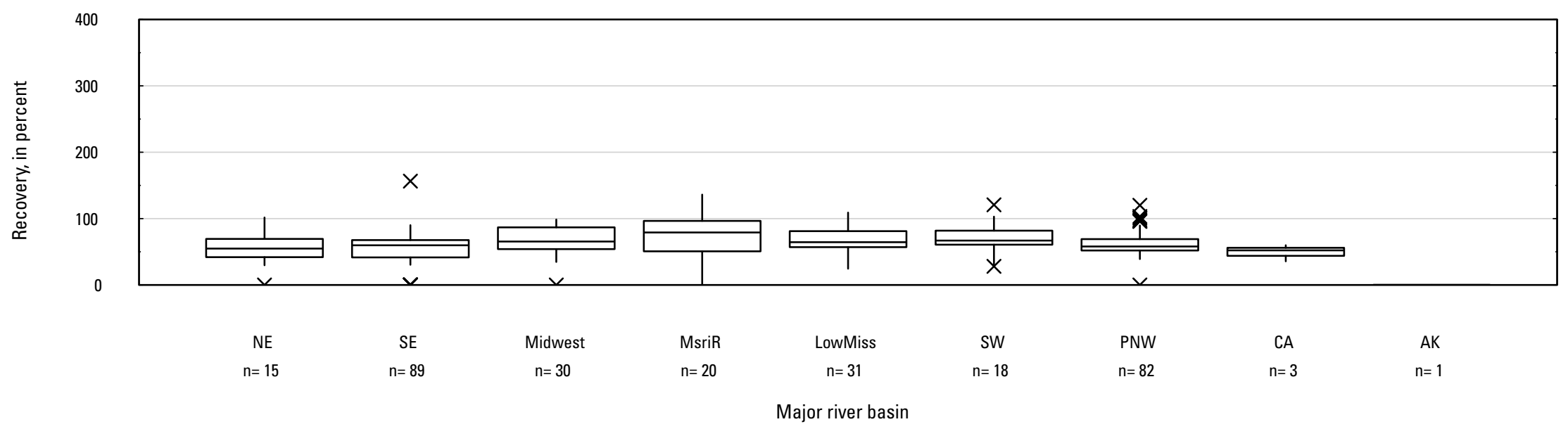

FR. Phorate oxon sulfone

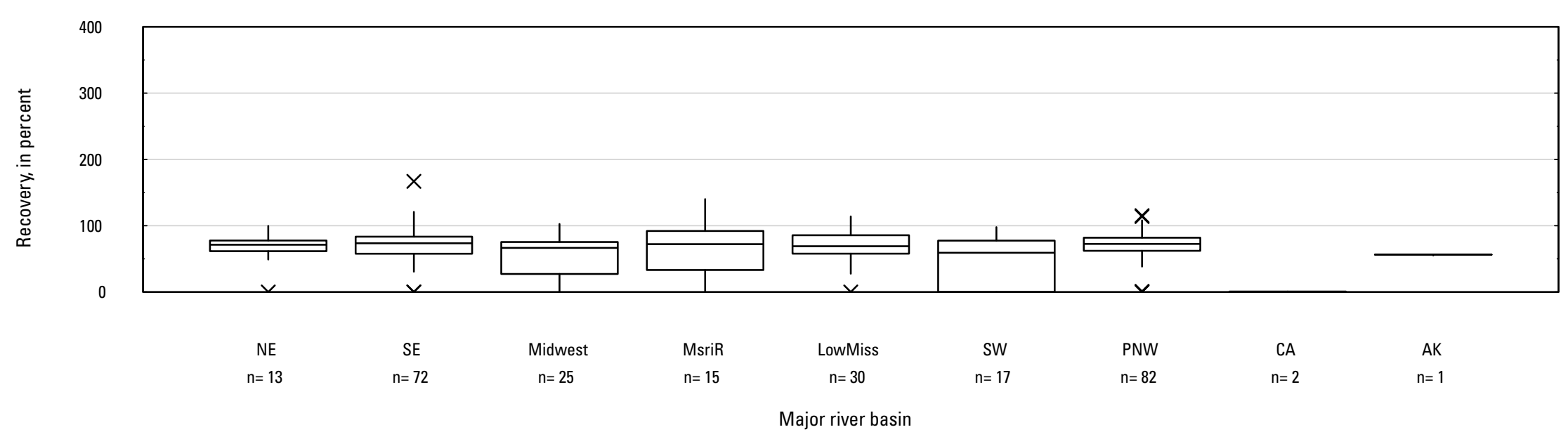

Figure 5. Graph showing distributions of recovery for pesticides in schedule 2437 in surface water by Major River Basin. Recovery values larger than 400 percent are not shown.-Continued 
FS. Phorate oxon sulfoxide

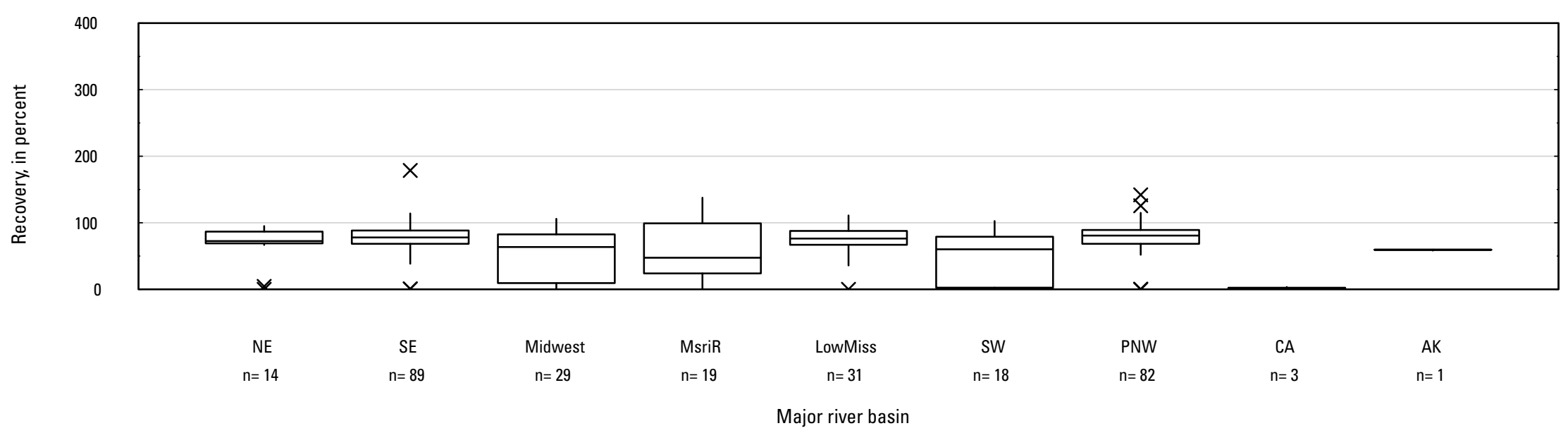

FT. Phorate sulfone

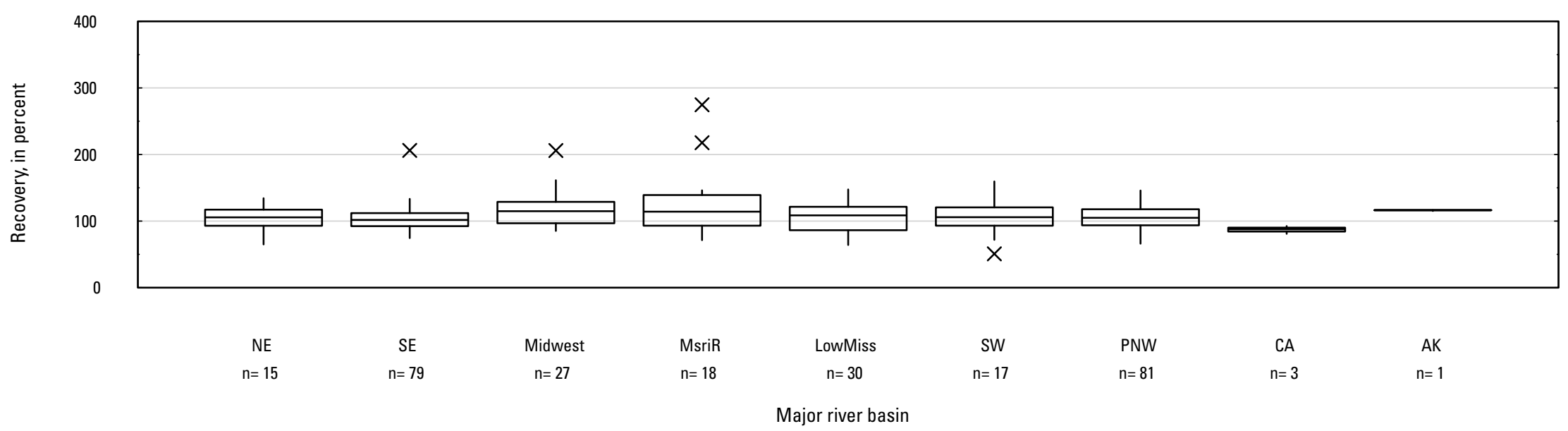

Figure 5. Graph showing distributions of recovery for pesticides in schedule 2437 in surface water by Major River Basin. Recovery values larger than 400 percent are 


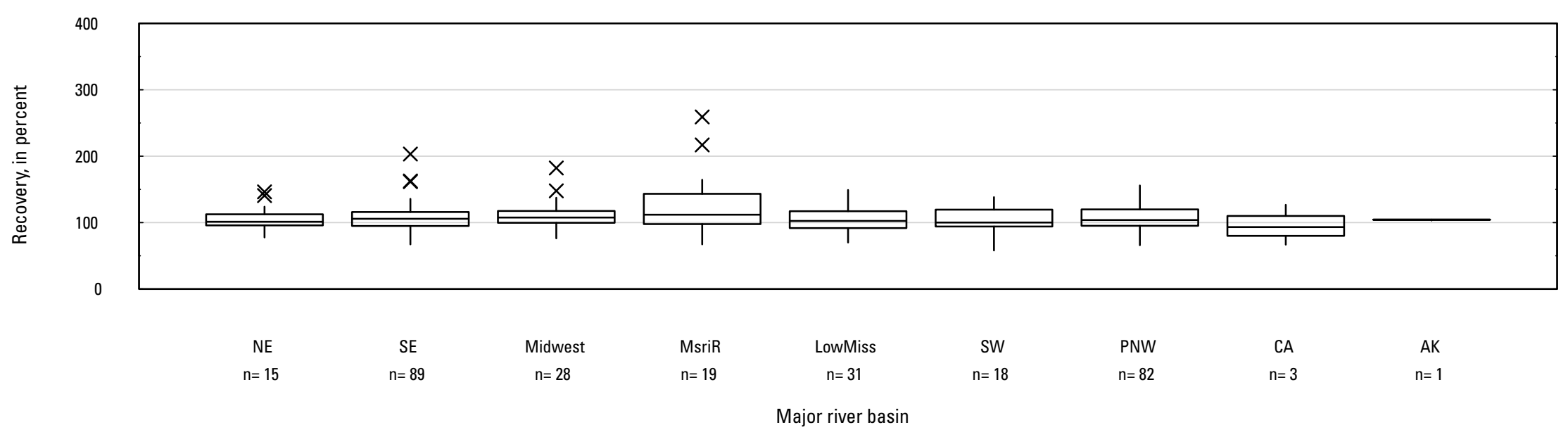

\section{FV. Phthalazinone}

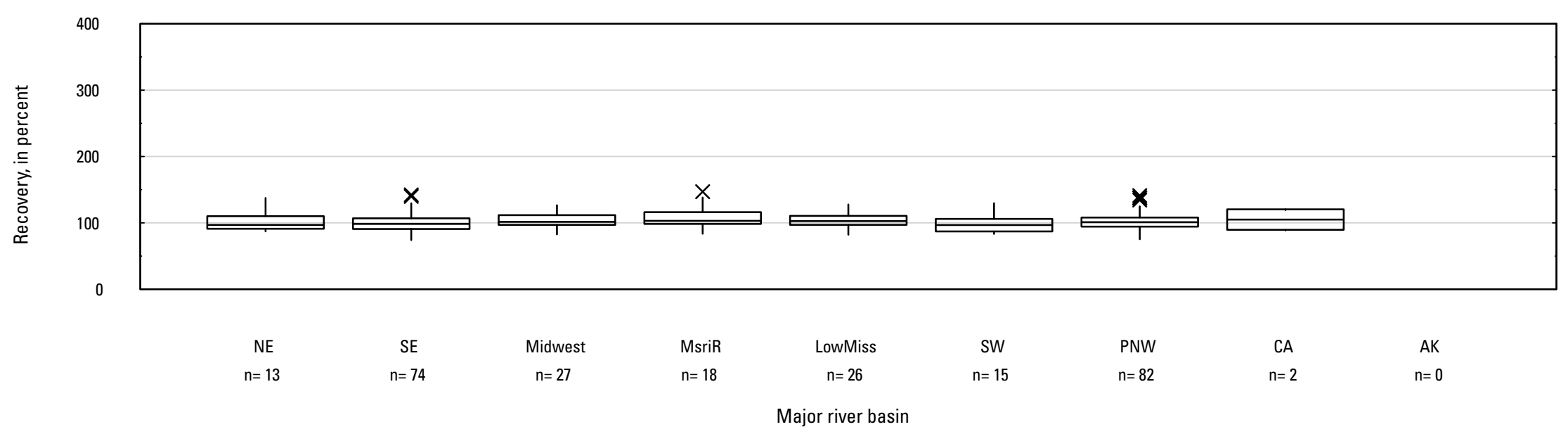

Figure 5. Graph showing distributions of recovery for pesticides in schedule 2437 in surface water by Major River Basin. Recovery values larger than 400 percent are not shown.-Continued 
FW. Piperonyl butoxide

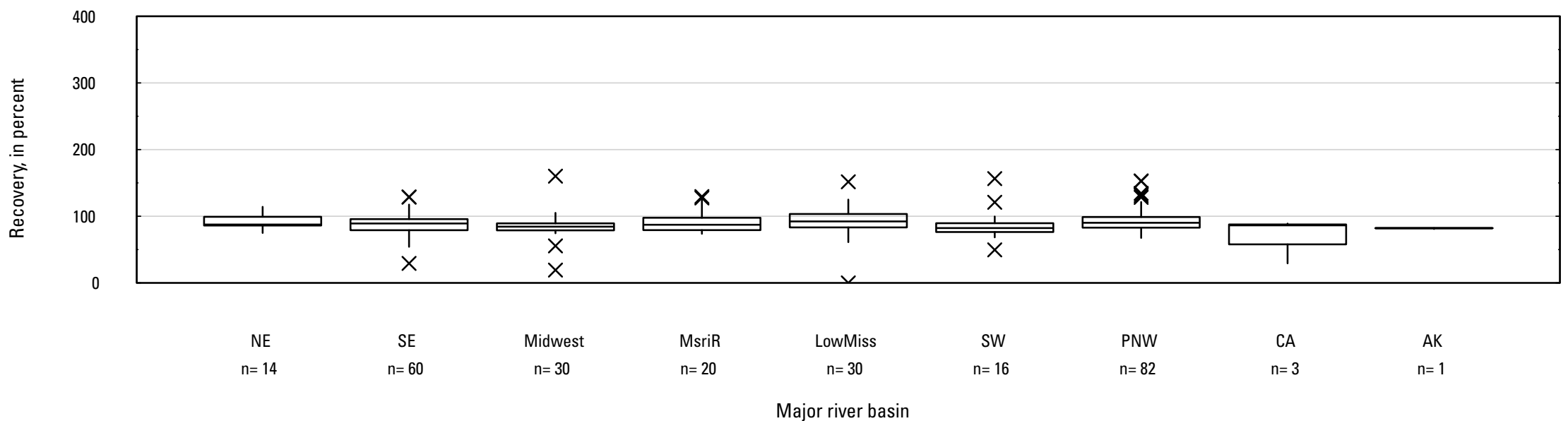

FX. Profenofos

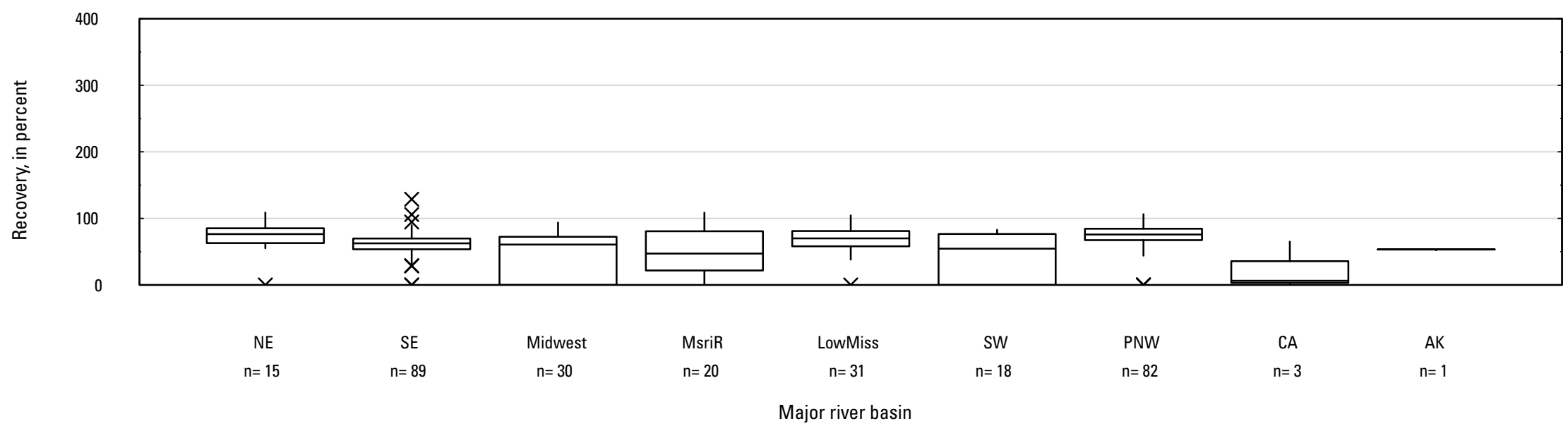

Figure 5. Graph showing distributions of recovery for pesticides in schedule 2437 in surface water by Major River Basin. Recovery values larger than 400 percent are not shown.-Continued 


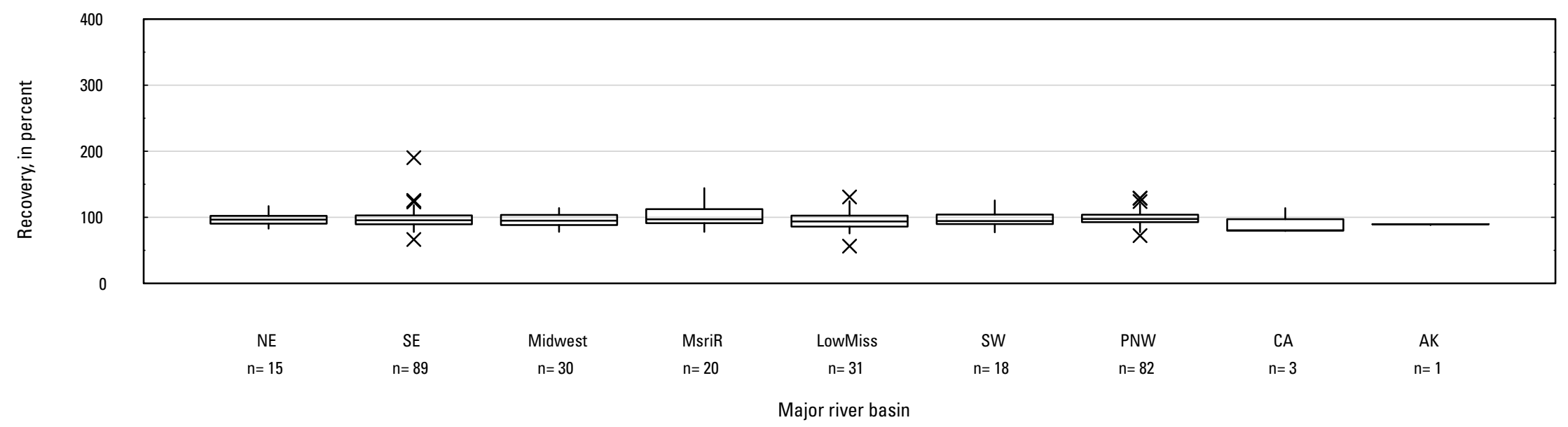

FZ. Prometryn

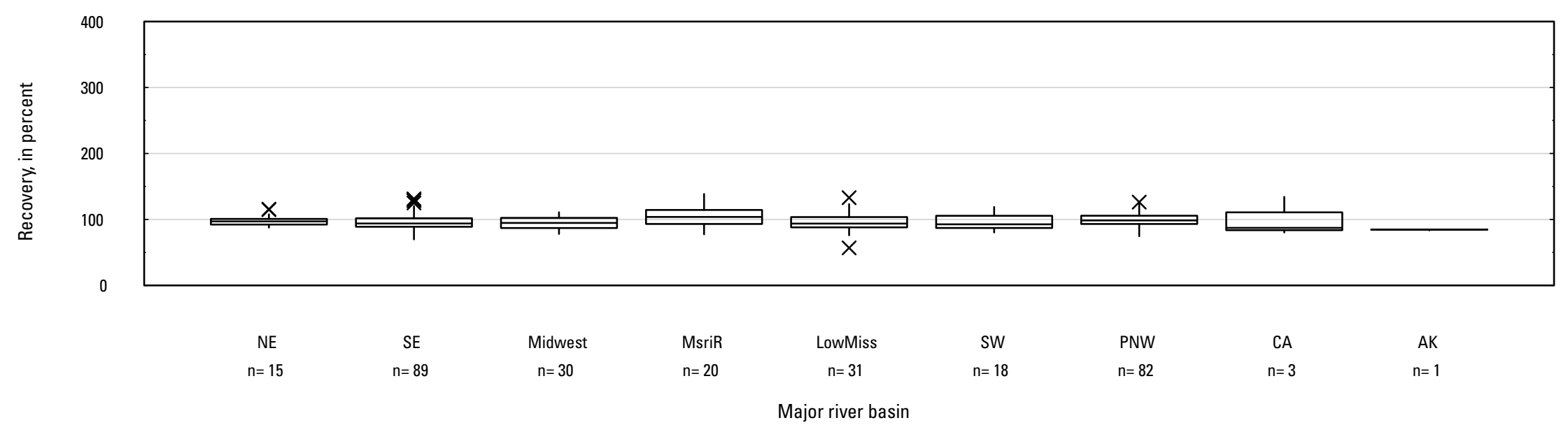

Figure 5. Graph showing distributions of recovery for pesticides in schedule 2437 in surface water by Major River Basin. Recovery values larger than 400 percent are not shown.-Continued 
GA. Propyzamide

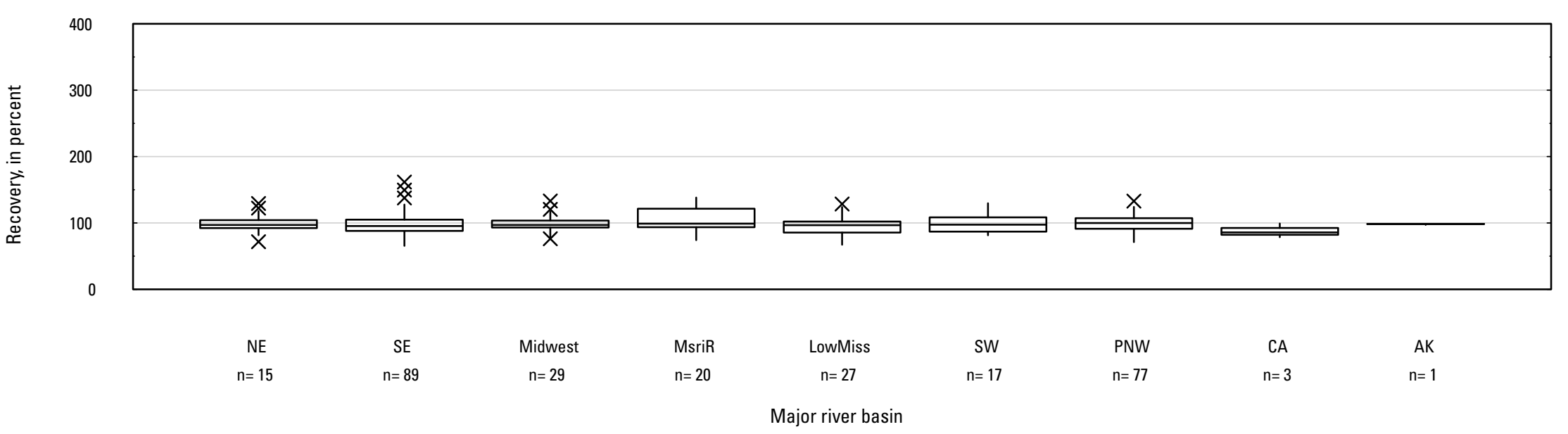

GB. Propanil

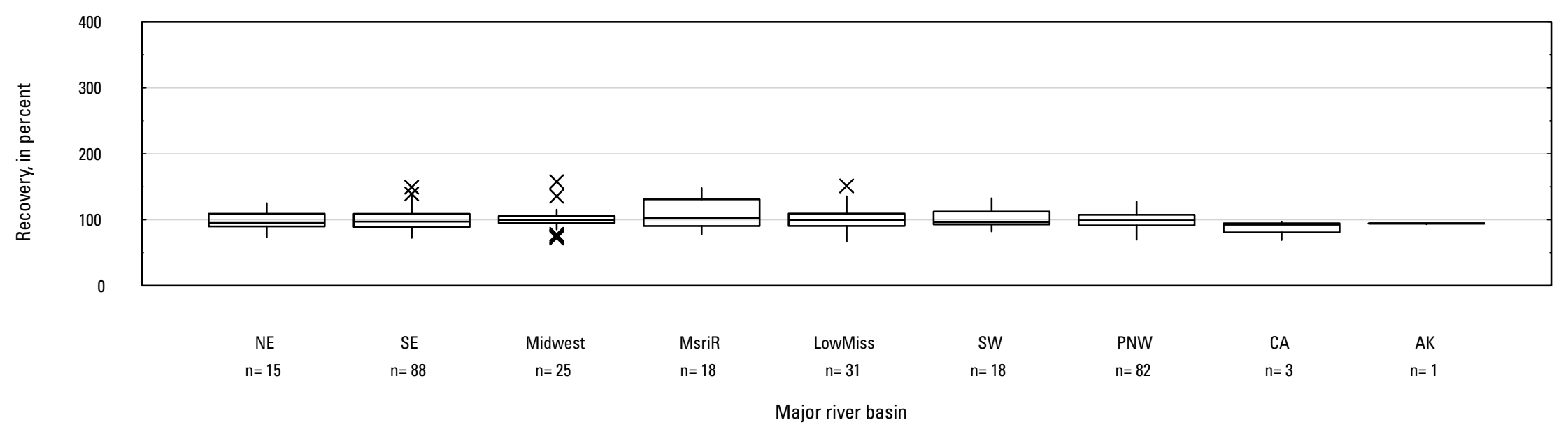

Figure 5. Graph showing distributions of recovery for pesticides in schedule 2437 in surface water by Major River Basin. Recovery values larger than 400 percent are not shown.-Continued 


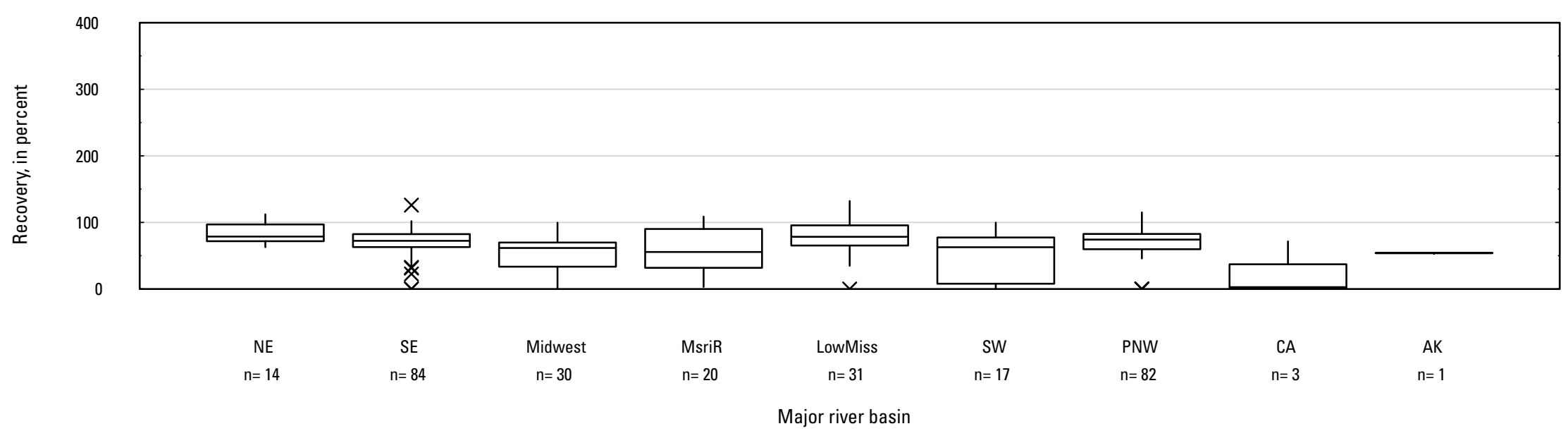

GD. Propazine

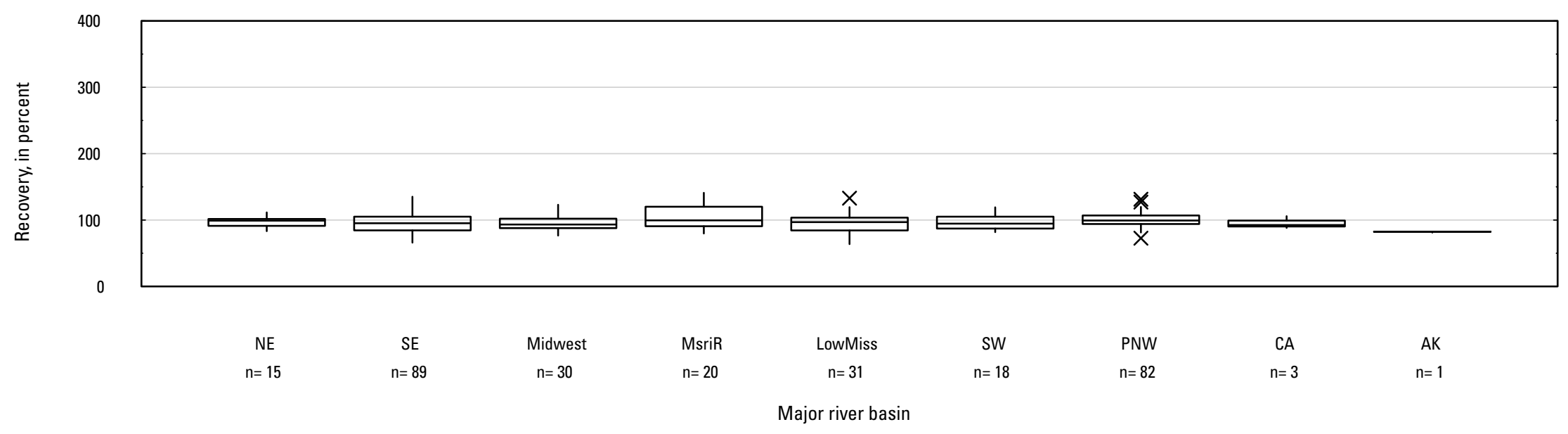

Figure 5. Graph showing distributions of recovery for pesticides in schedule 2437 in surface water by Major River Basin. Recovery values larger than 400 percent are not shown.-Continued 
GE. Propiconazole

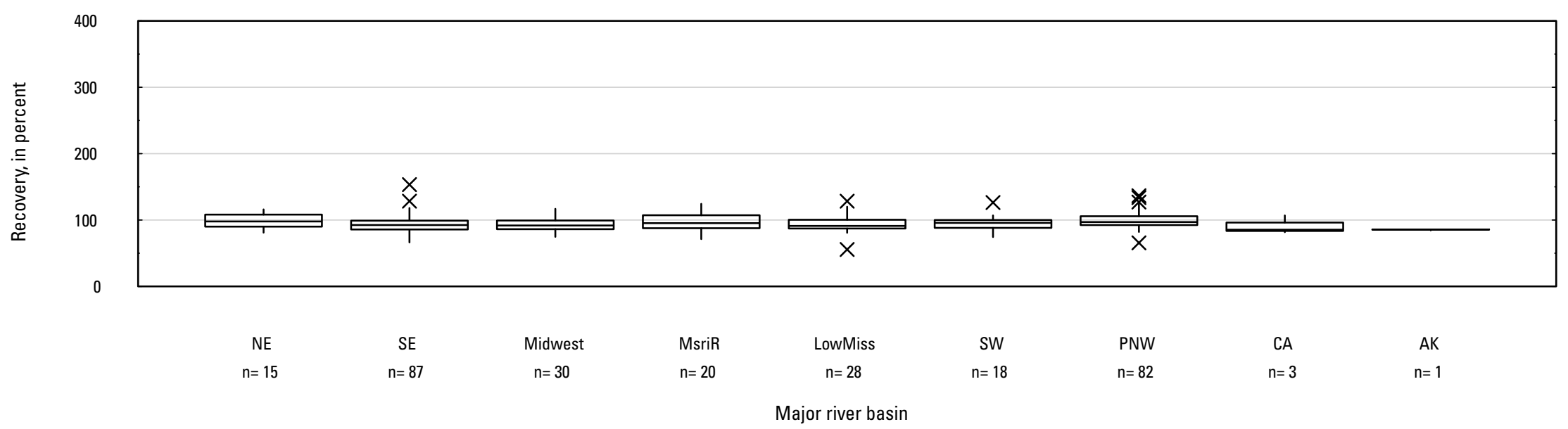

GF Propoxur

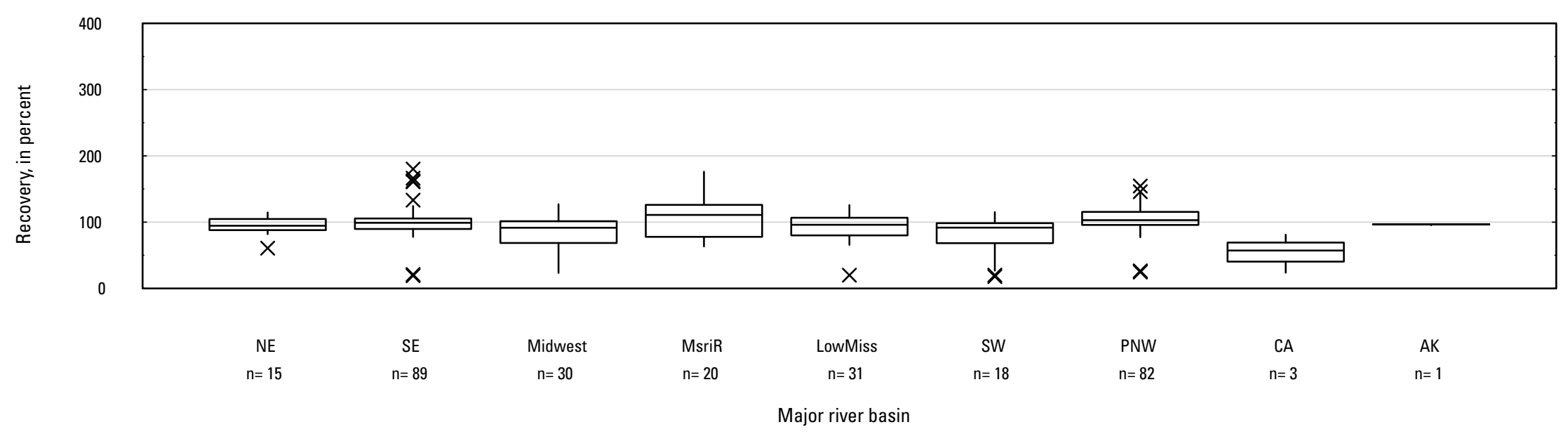

Figure 5. Graph showing distributions of recovery for pesticides in schedule 2437 in surface water by Major River Basin. Recovery values larger than 400 percent are 


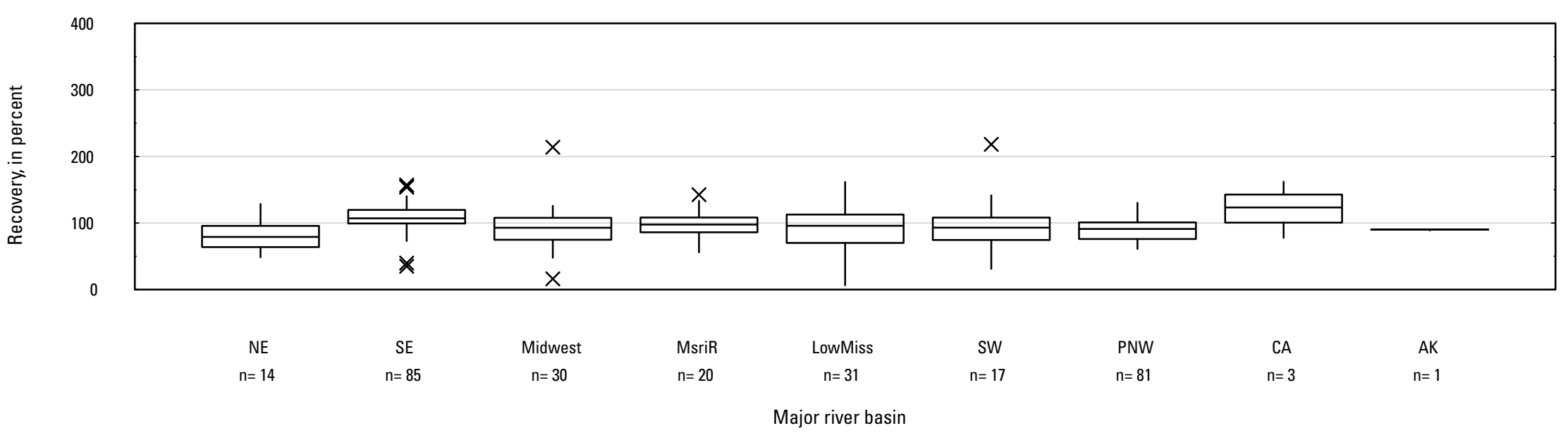

GH. Pyraclostrobin

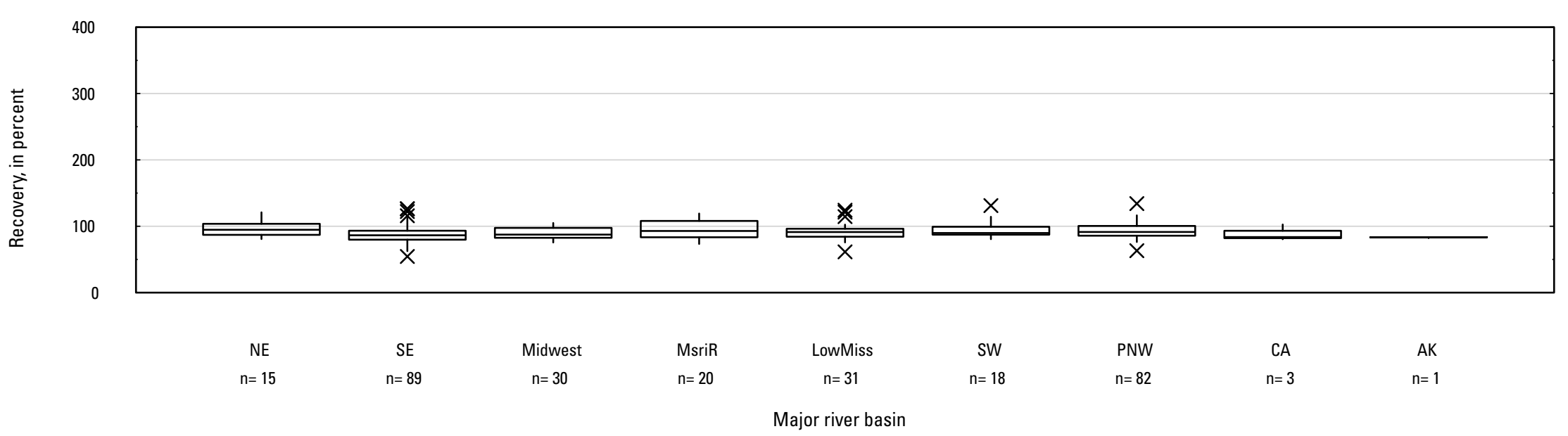

Figure 5. Graph showing distributions of recovery for pesticides in schedule 2437 in surface water by Major River Basin. Recovery values larger than 400 percent are not shown.-Continued 
GI. Pyridaben

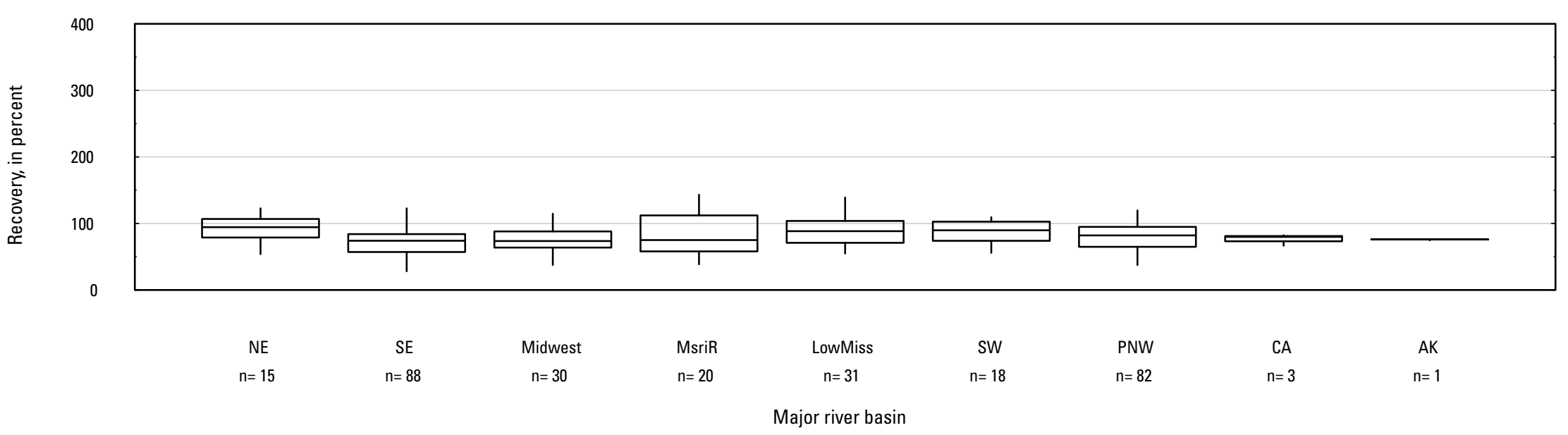

GJ. 2-Isopropyl-6-methyl-4-pyrimidinol

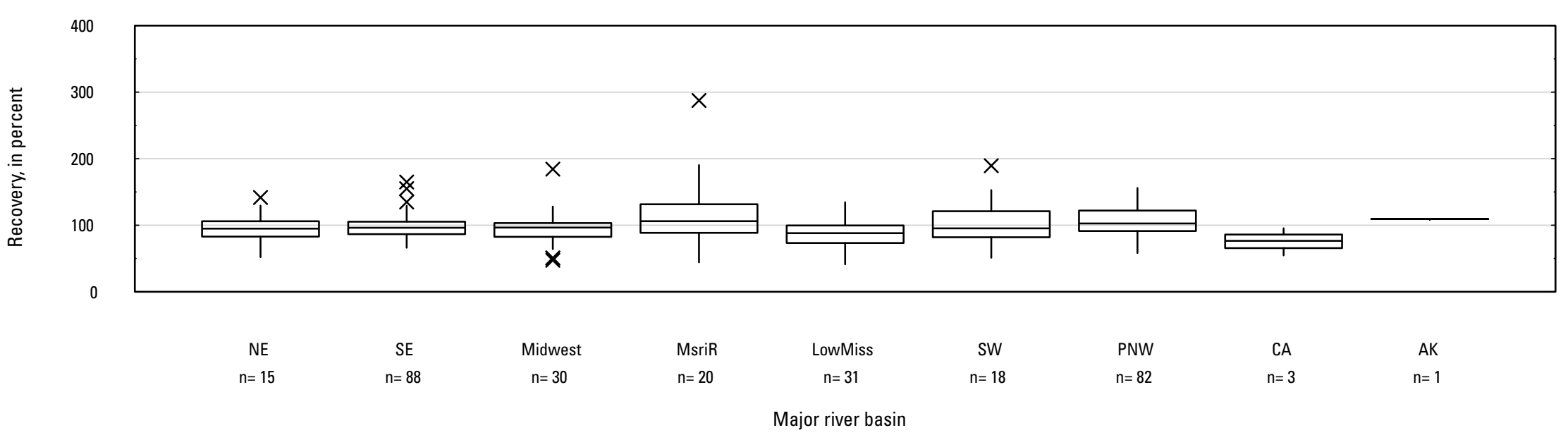

Figure 5. Graph showing distributions of recovery for pesticides in schedule 2437 in surface water by Major River Basin. Recovery values larger than 400 percent are 


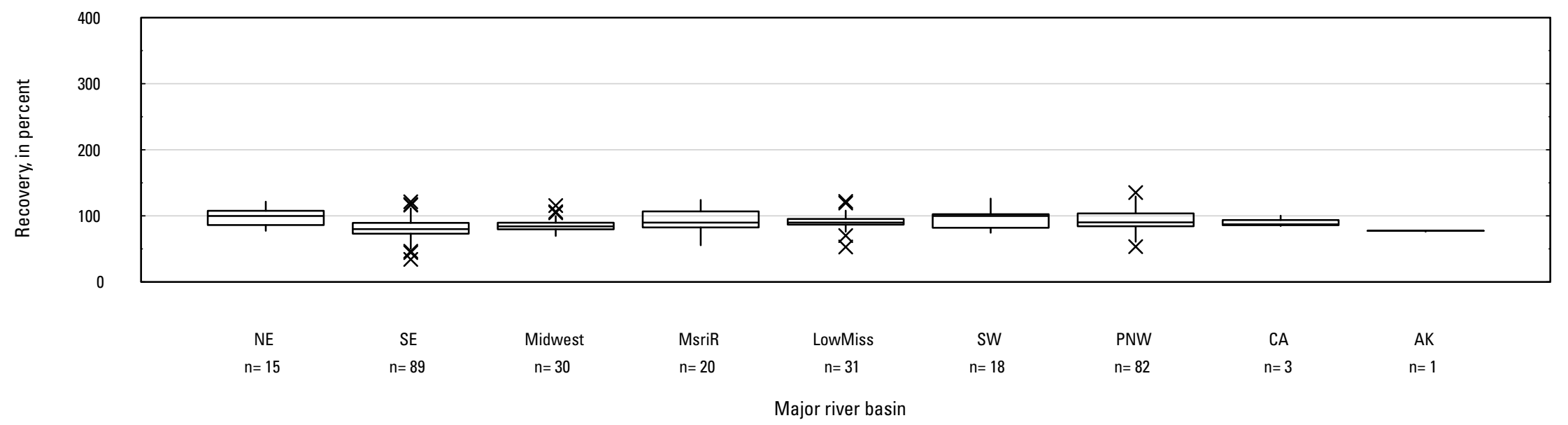

GL. sec-Acetochlor oxanilic acid

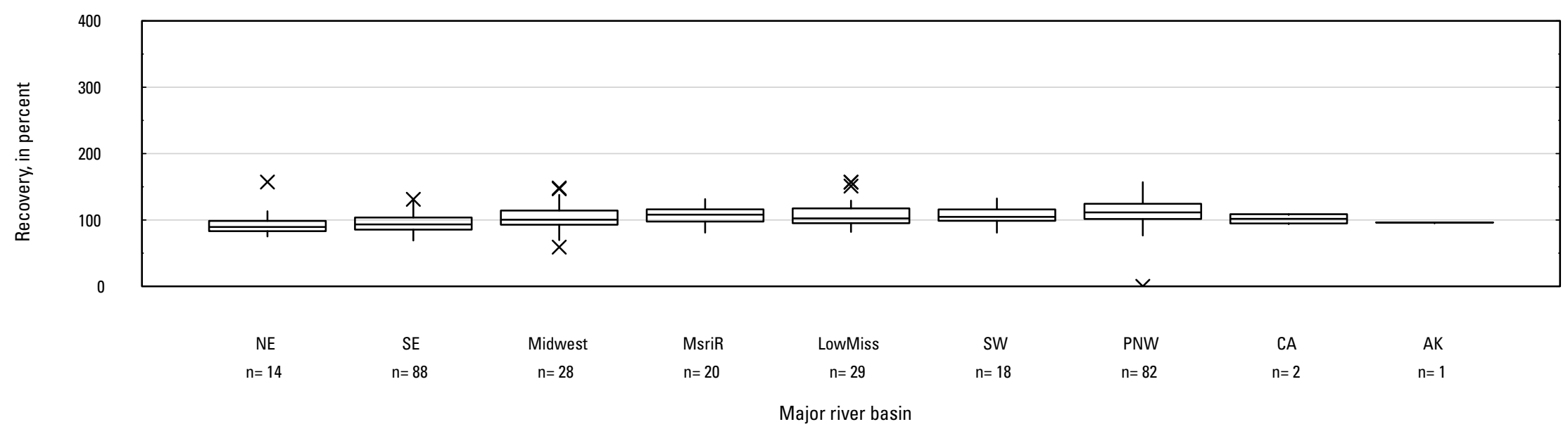

Figure 5. Graph showing distributions of recovery for pesticides in schedule 2437 in surface water by Major River Basin. Recovery values larger than 400 percent are not shown.-Continued 
GM. sec-Alachlor oxanilic acid

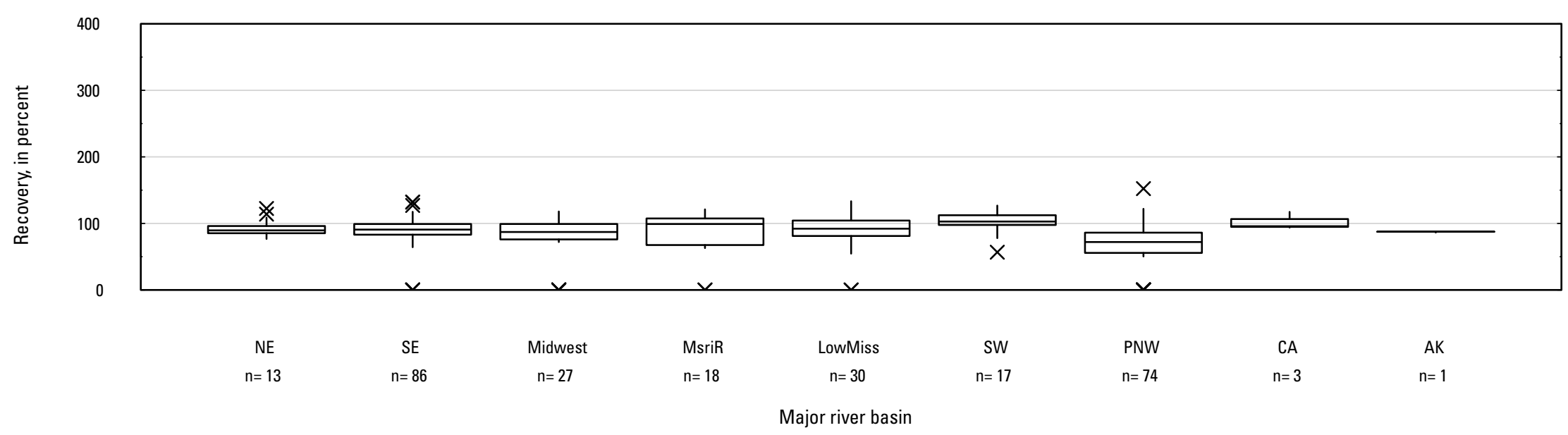

GN. Siduron

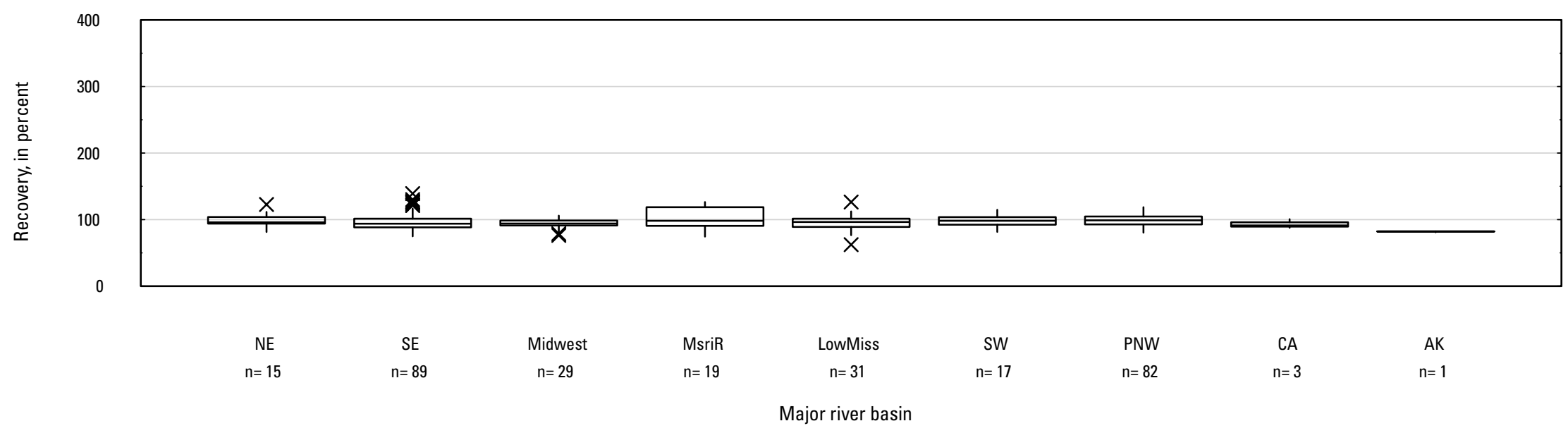

Figure 5. Graph showing distributions of recovery for pesticides in schedule 2437 in surface water by Major River Basin. Recovery values larger than 400 percent are not shown.-Continued 


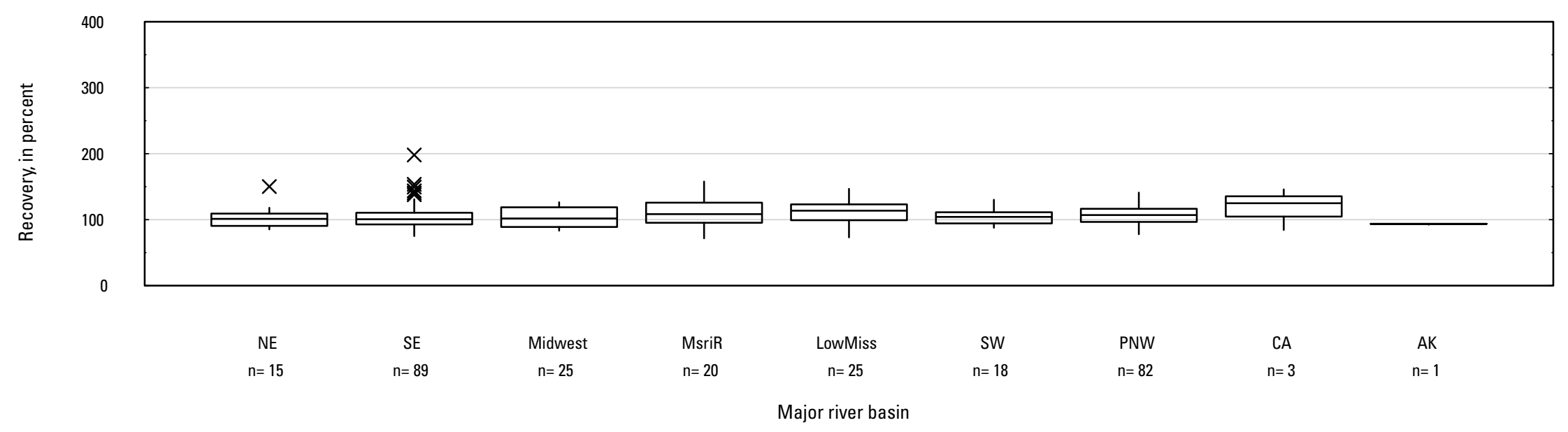

GP. Sulfentrazone

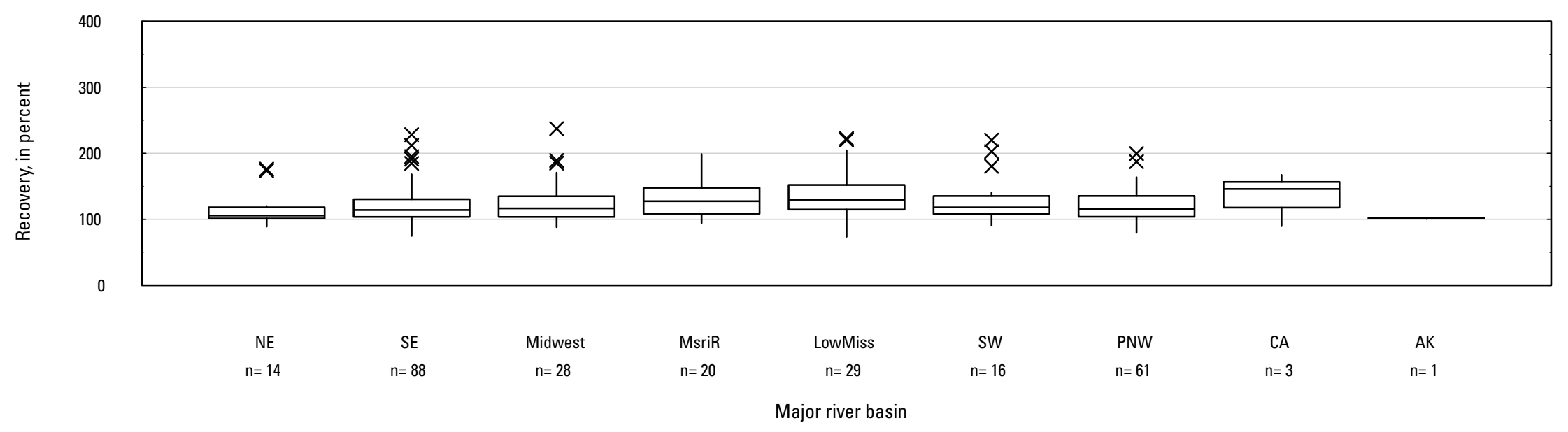

Figure 5. Graph showing distributions of recovery for pesticides in schedule 2437 in surface water by Major River Basin. Recovery values larger than 400 percent are not shown.-Continued 
GQ. Sulfometuron-methyl

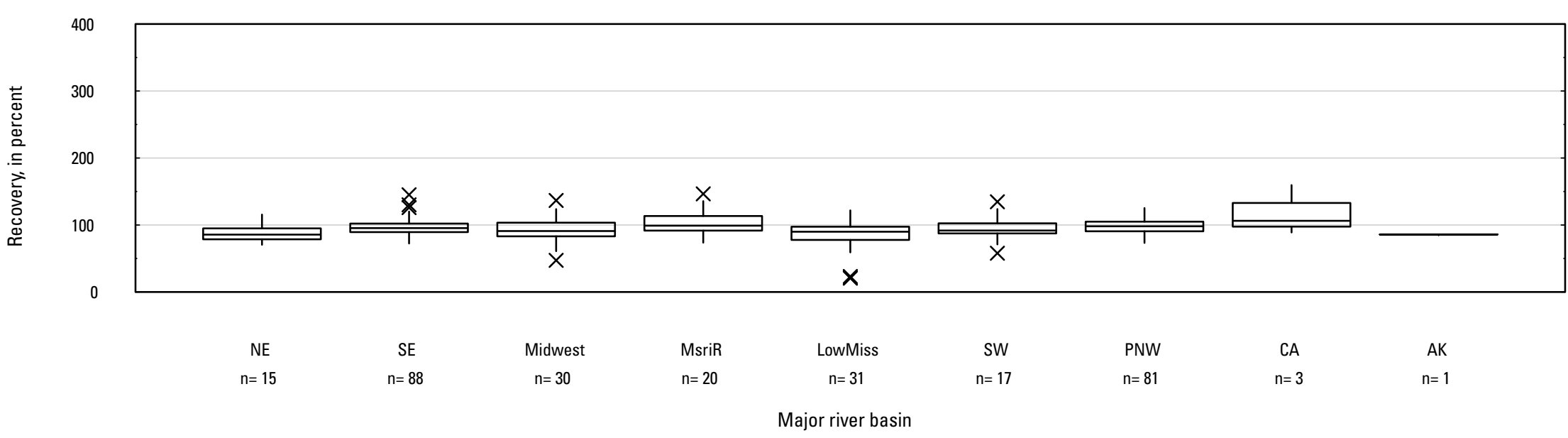

GR. Sulfosulfuron

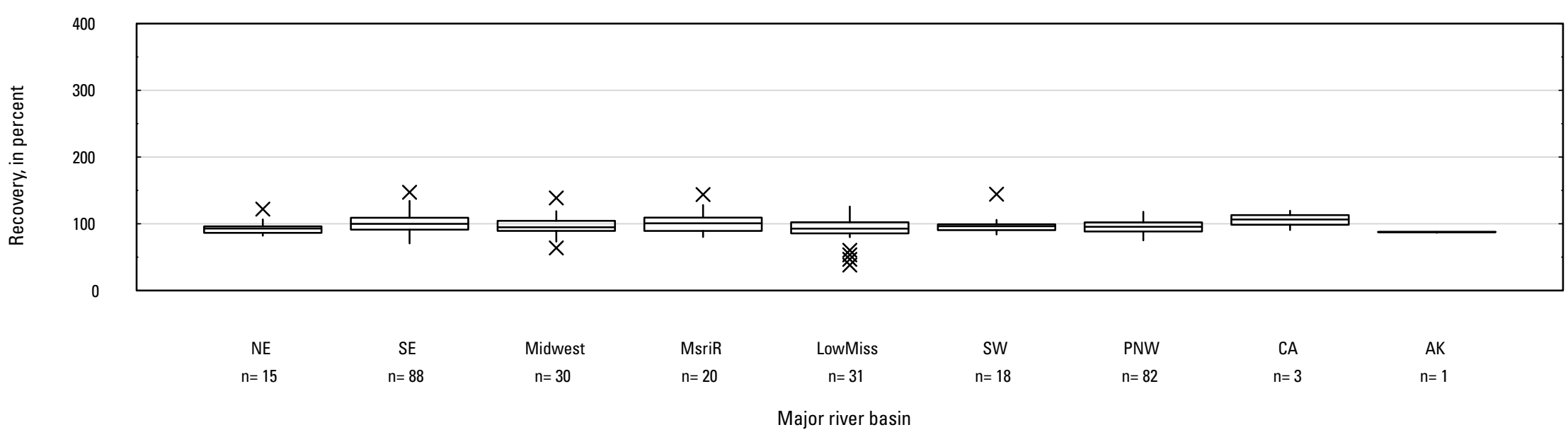

Figure 5. Graph showing distributions of recovery for pesticides in schedule 2437 in surface water by Major River Basin. Recovery values larger than 400 percent are not shown.-Continued 


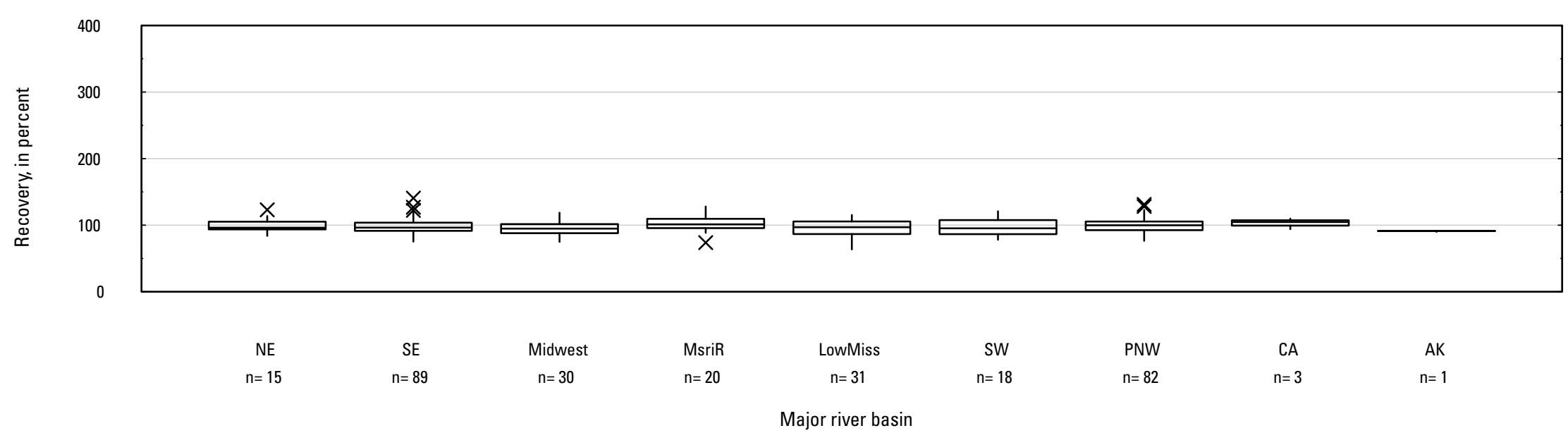

GT. Tebuconazole

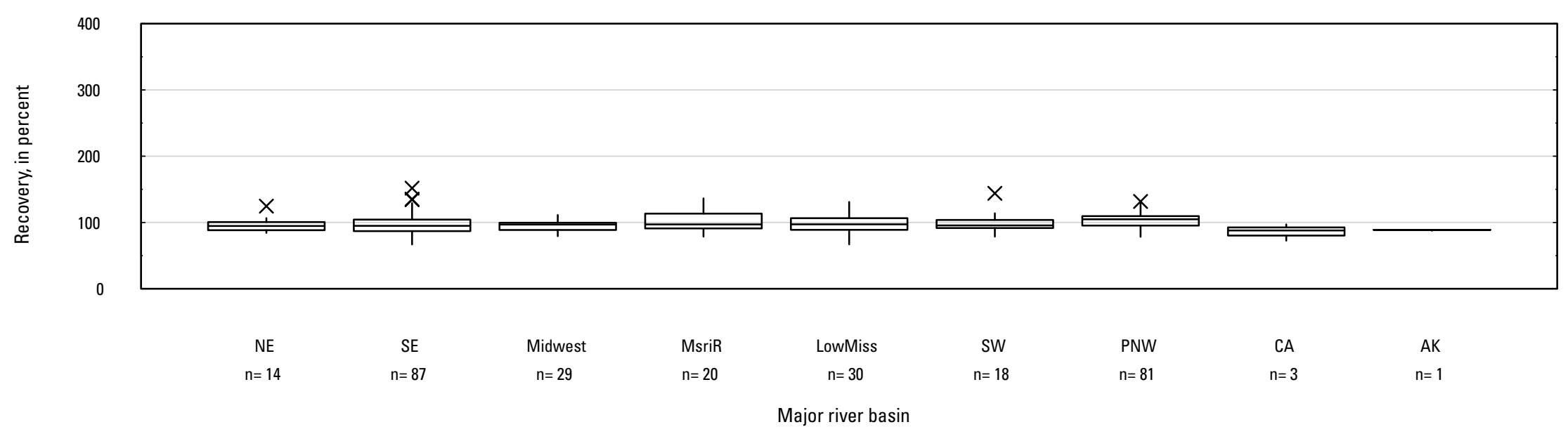

Figure 5. Graph showing distributions of recovery for pesticides in schedule 2437 in surface water by Major River Basin. Recovery values larger than 400 percent are not shown.-Continued 
GU. Tebufenozide

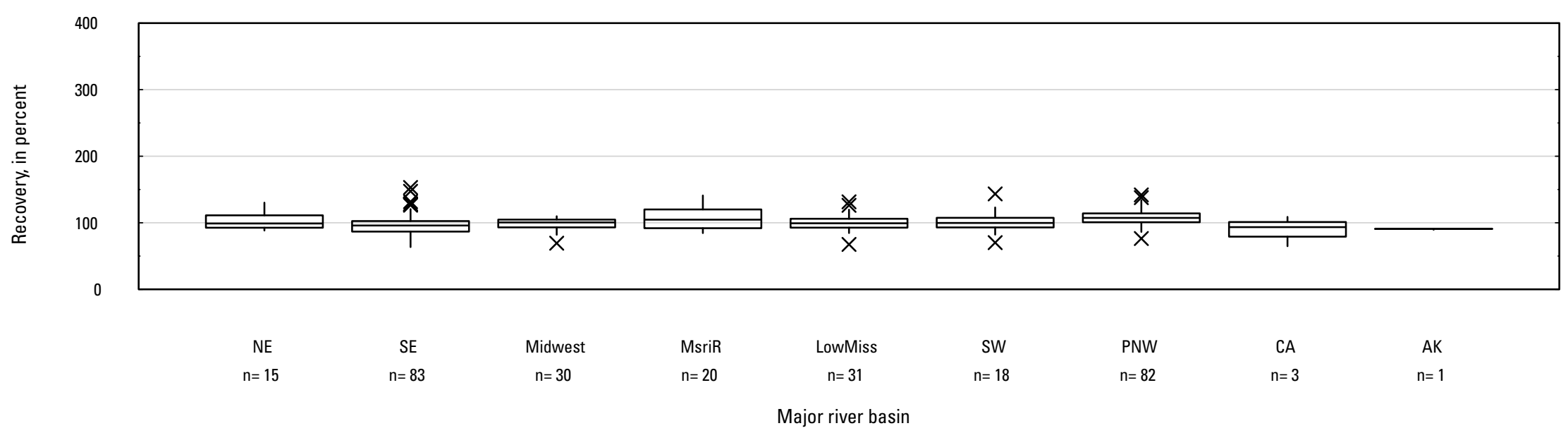

GV. Tebupirimphos

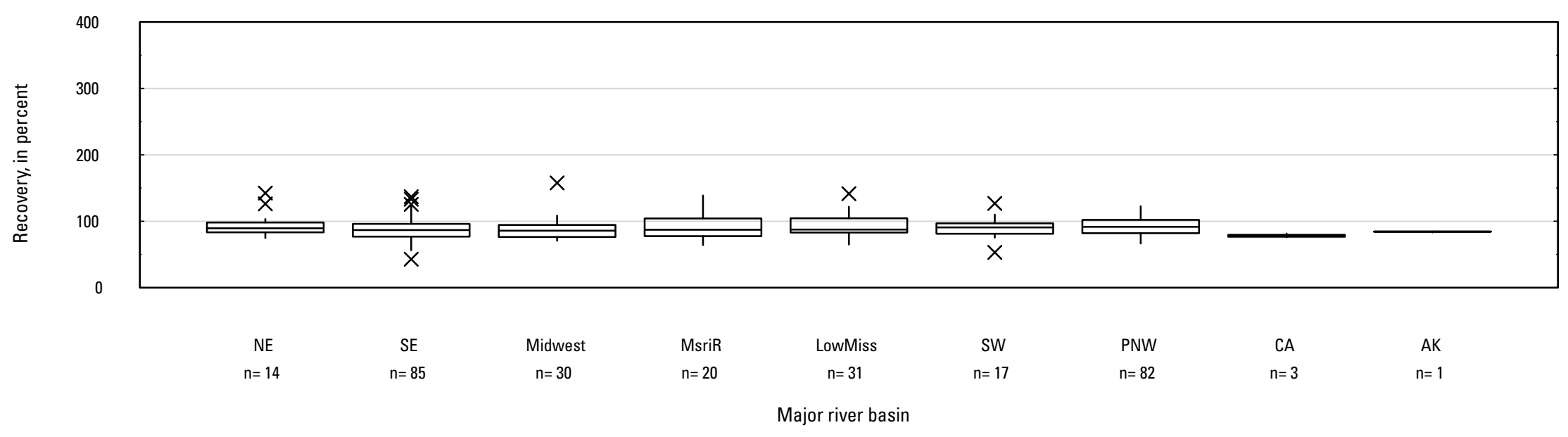

Figure 5. Graph showing distributions of recovery for pesticides in schedule 2437 in surface water by Major River Basin. Recovery values larger than 400 percent are 


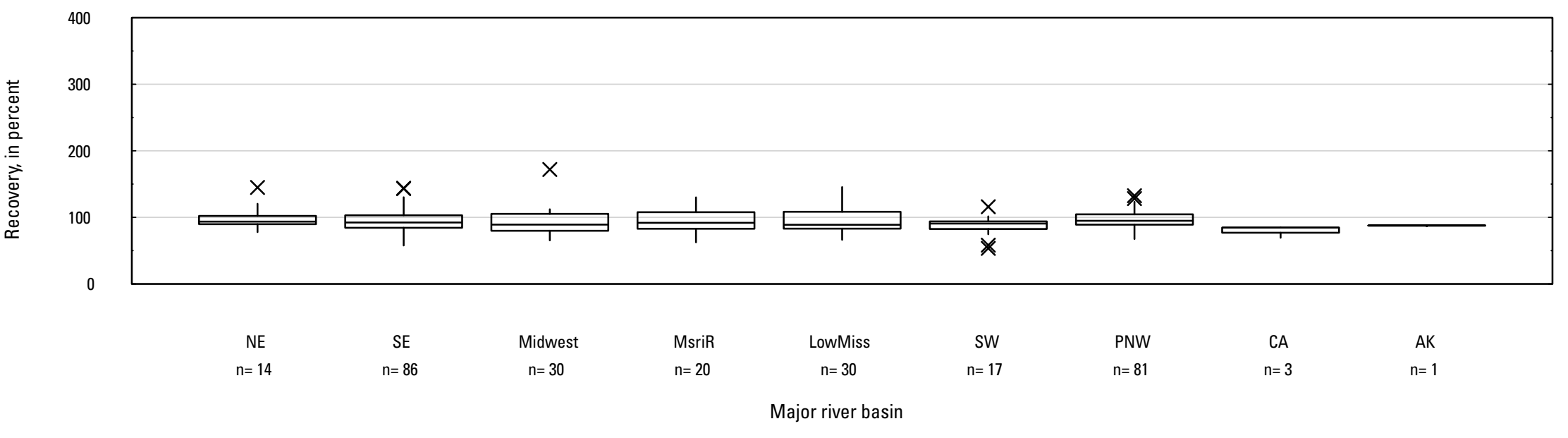

GX. Tebuthiuron

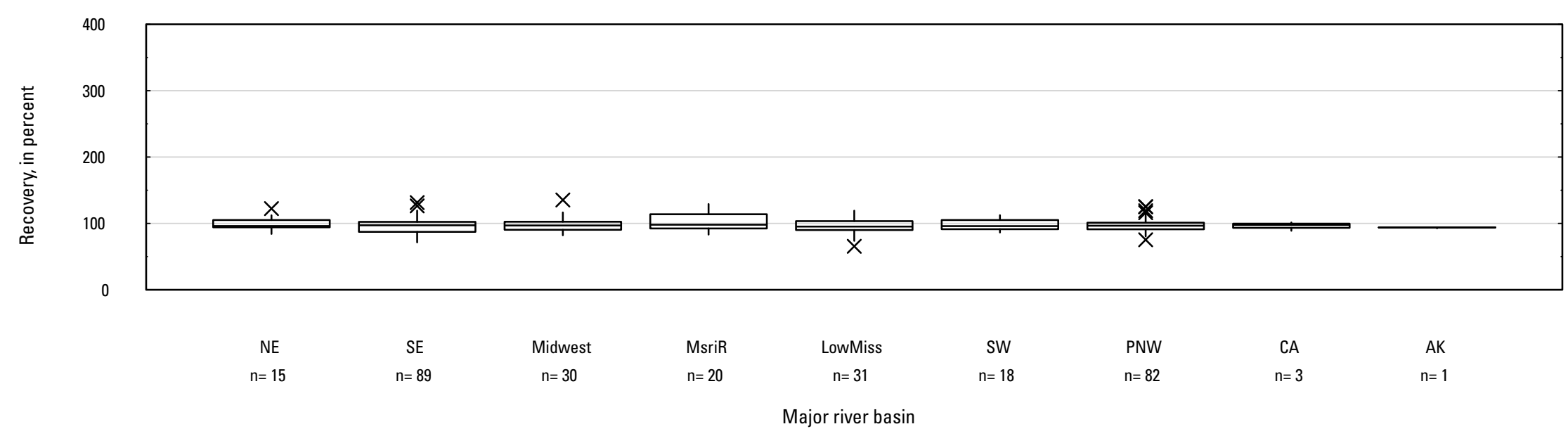

Figure 5. Graph showing distributions of recovery for pesticides in schedule 2437 in surface water by Major River Basin. Recovery values larger than 400 percent are not shown.-Continued 
GY. Tebuthiuron TP 104

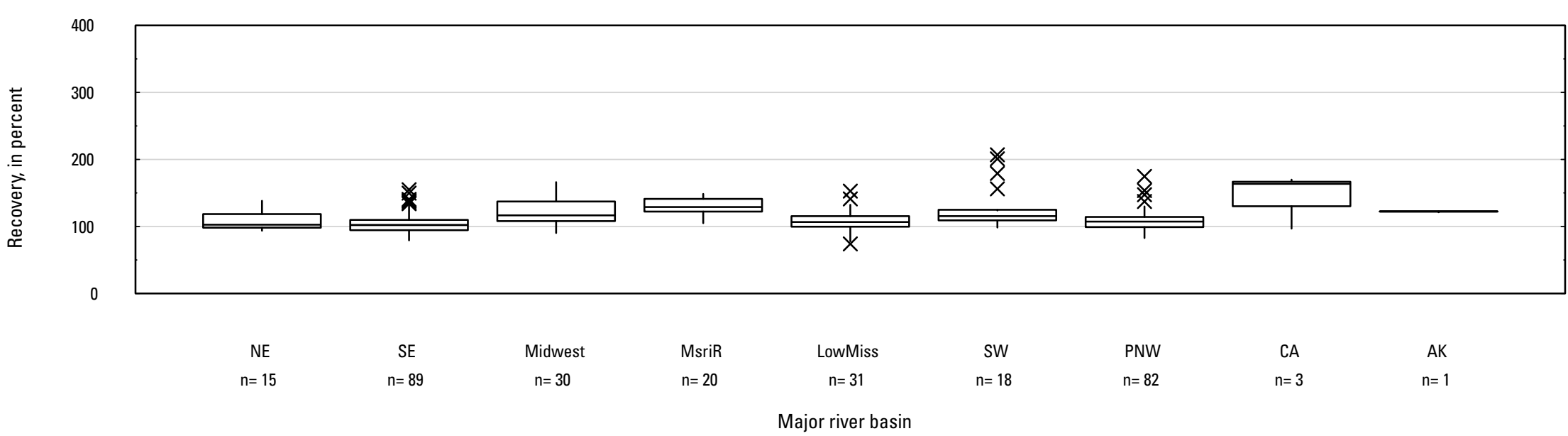

GZ. Tebuthiuron Transformation Product 106

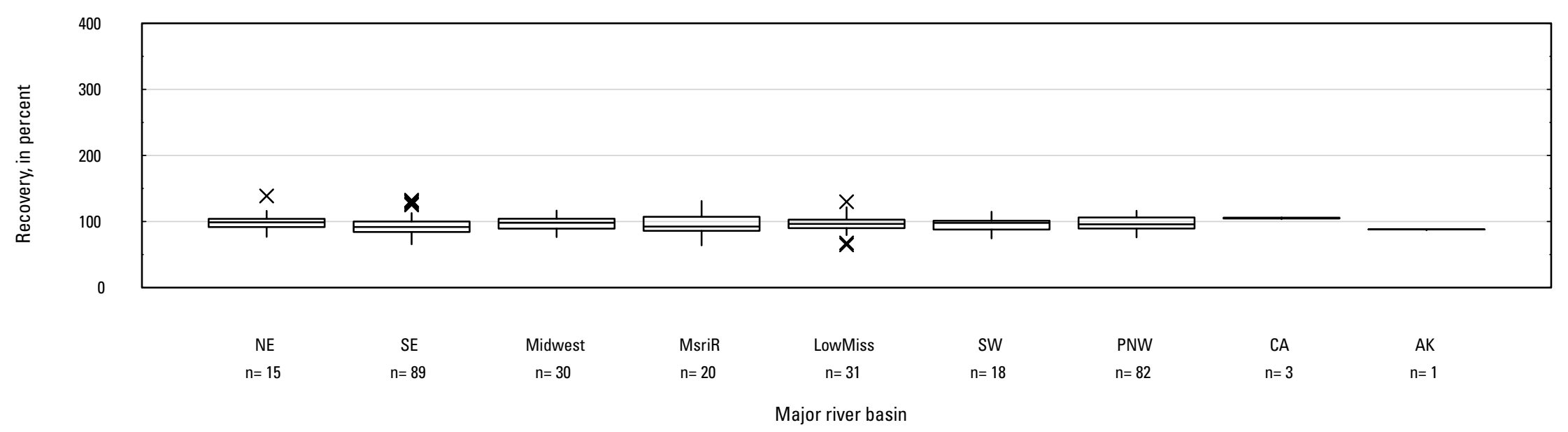

Figure 5. Graph showing distributions of recovery for pesticides in schedule 2437 in surface water by Major River Basin. Recovery values larger than 400 percent are not shown.-Continued 


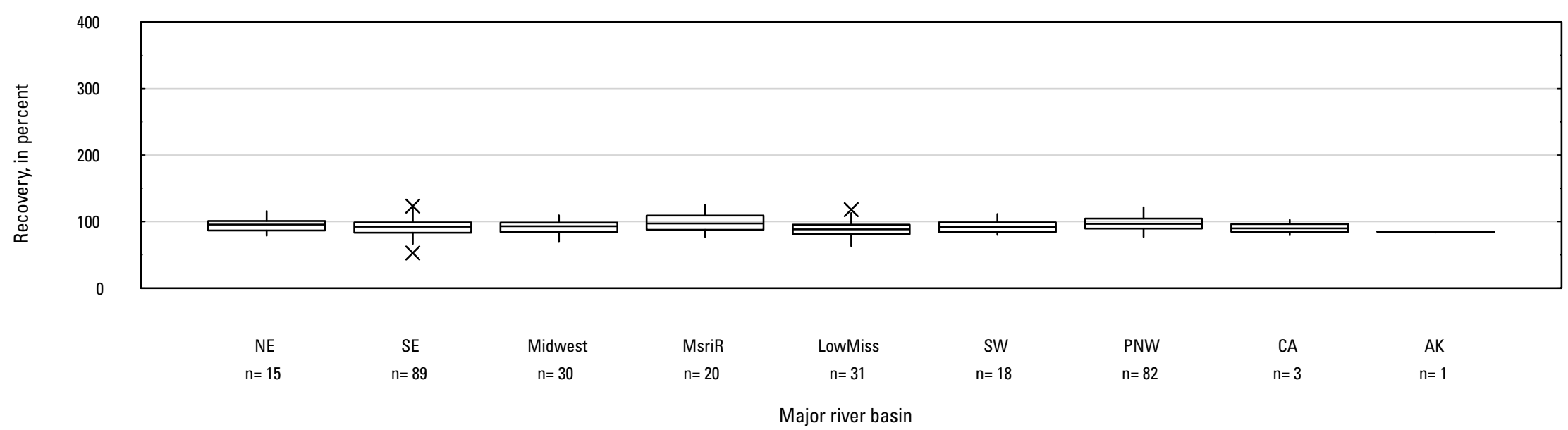

HB. Tebuthiuron TP 109 (OH)

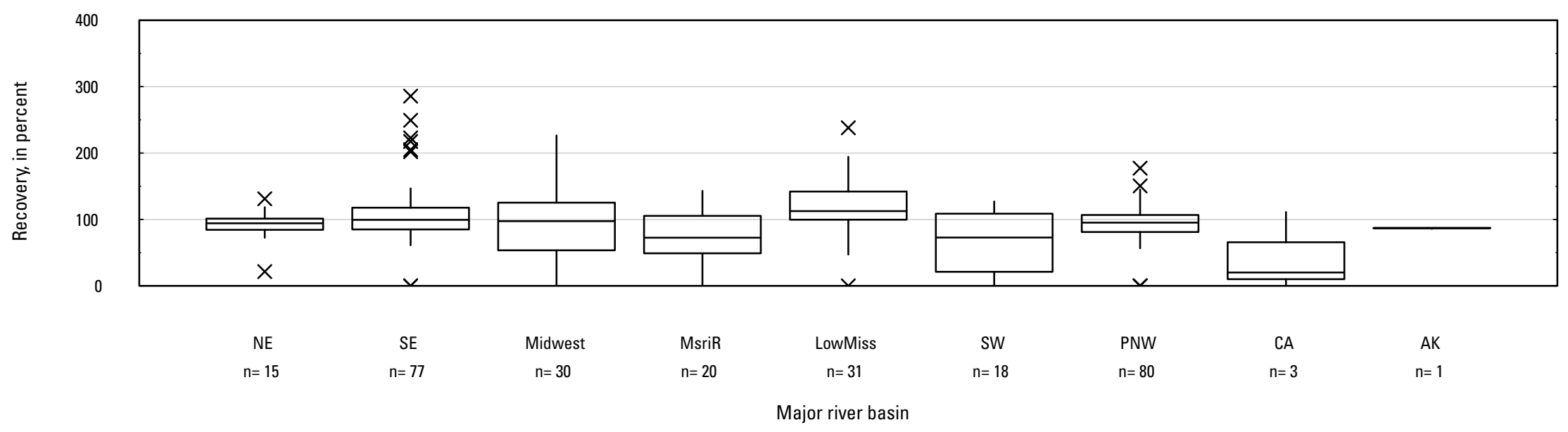

Figure 5. Graph showing distributions of recovery for pesticides in schedule 2437 in surface water by Major River Basin. Recovery values larger than 400 percent are not shown.-Continued 
HC. Terbacil

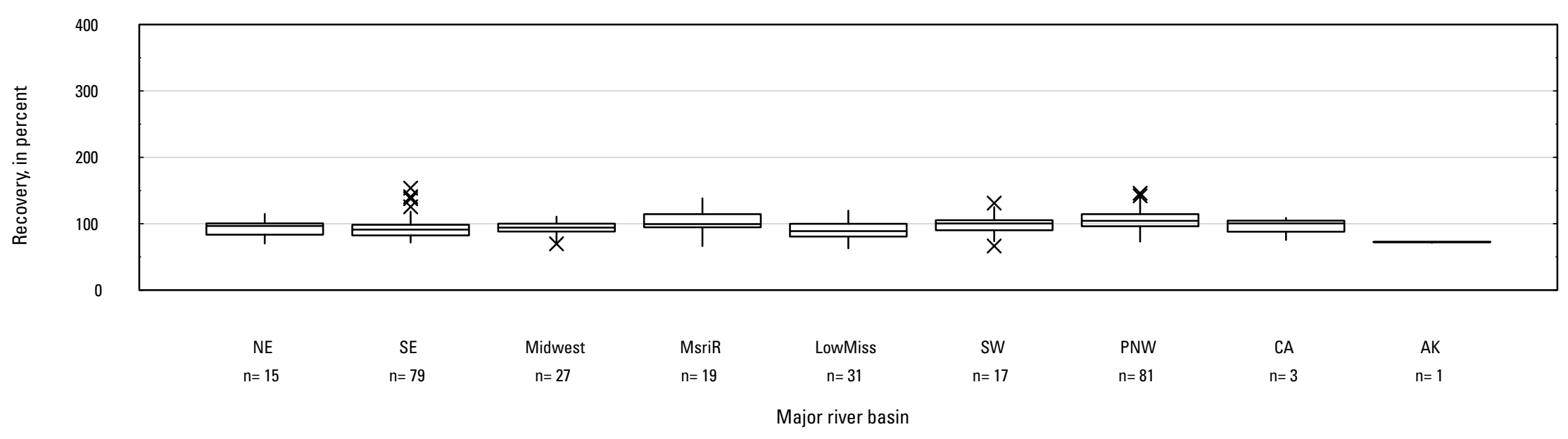

HD. Terbufos

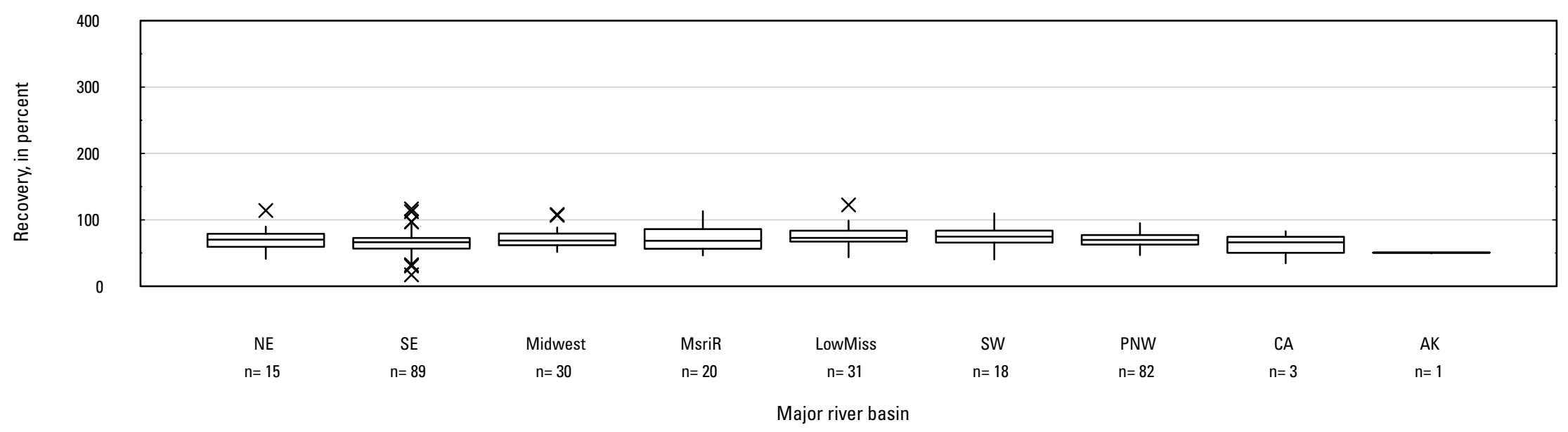

Figure 5. Graph showing distributions of recovery for pesticides in schedule 2437 in surface water by Major River Basin. Recovery values larger than 400 percent are not shown.-Continued 


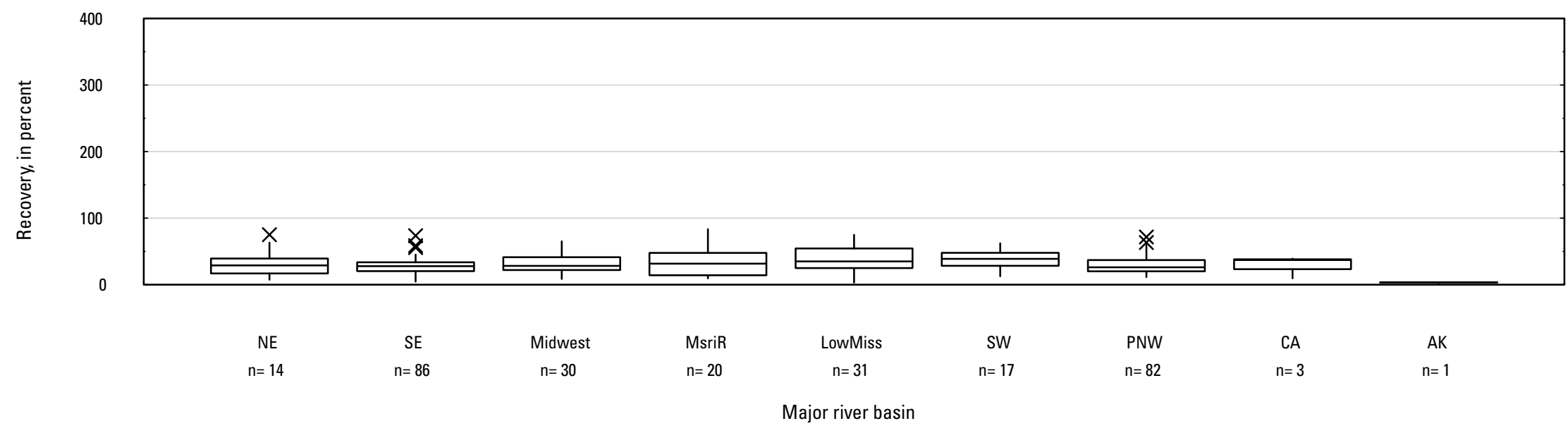

HF. Terbufos oxon sulfone

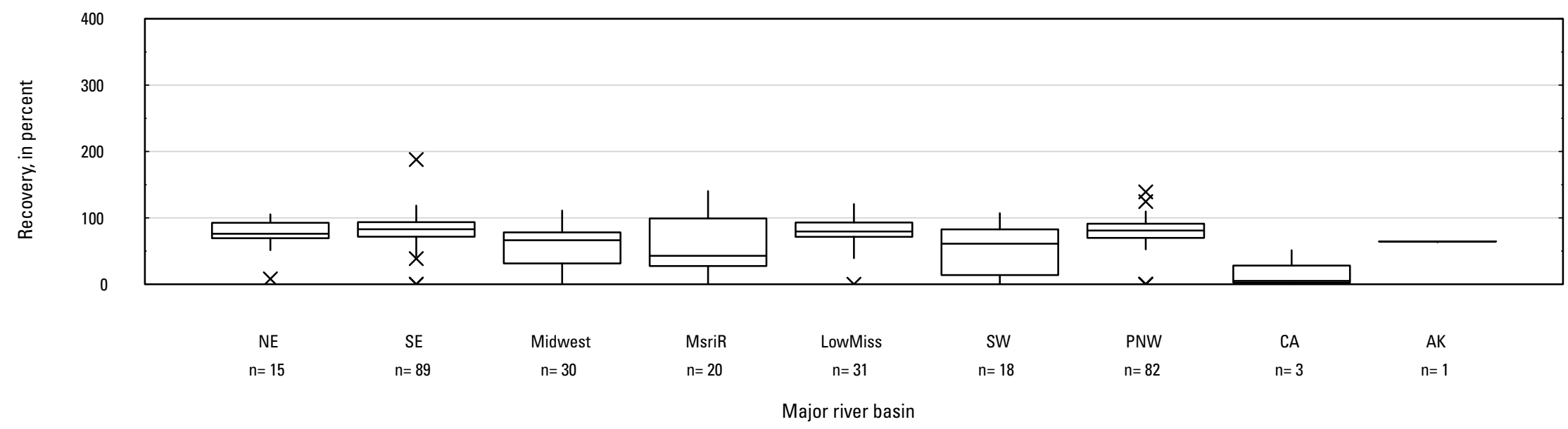

Figure 5. Graph showing distributions of recovery for pesticides in schedule 2437 in surface water by Major River Basin. Recovery values larger than 400 percent are not shown.-Continued 
HG. Terbufos oxon sulfoxide

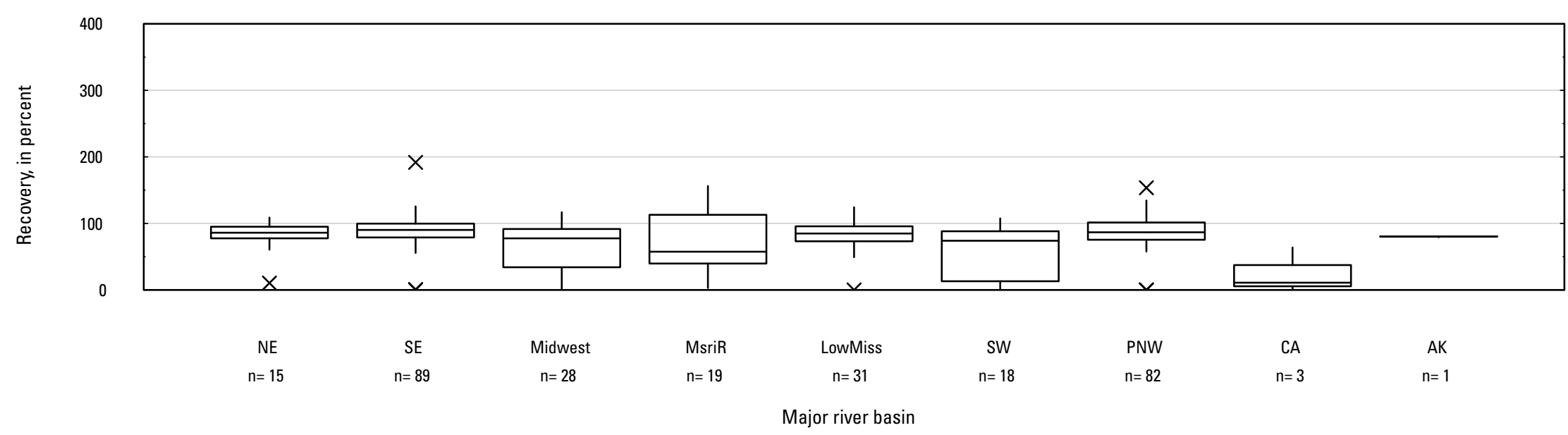

HH. Terbufos sulfone

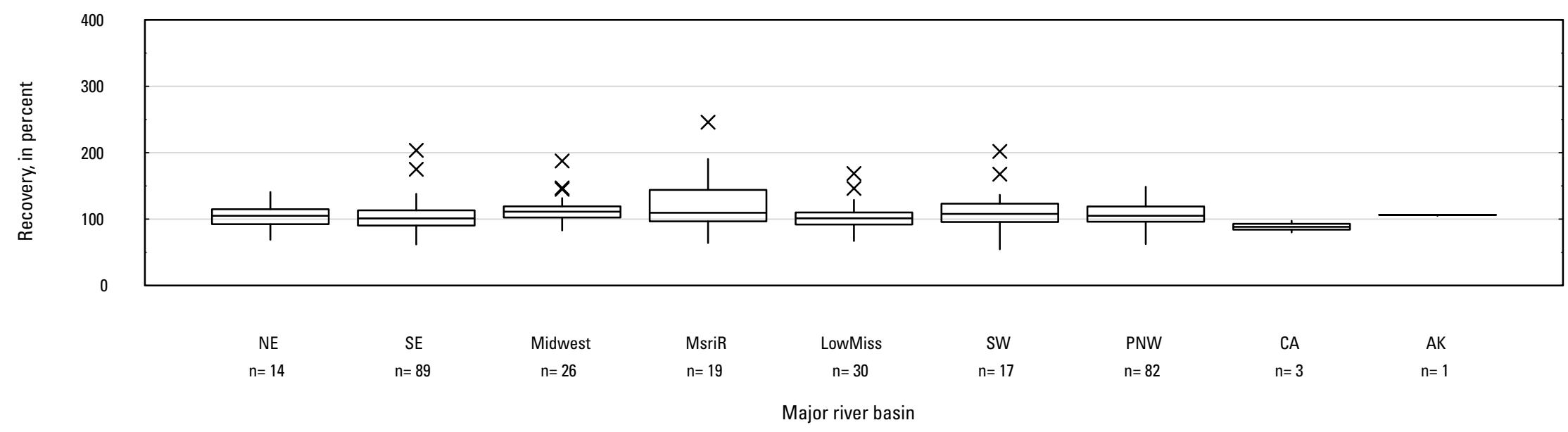

Figure 5. Graph showing distributions of recovery for pesticides in schedule 2437 in surface water by Major River Basin. Recovery values larger than 400 percent are 


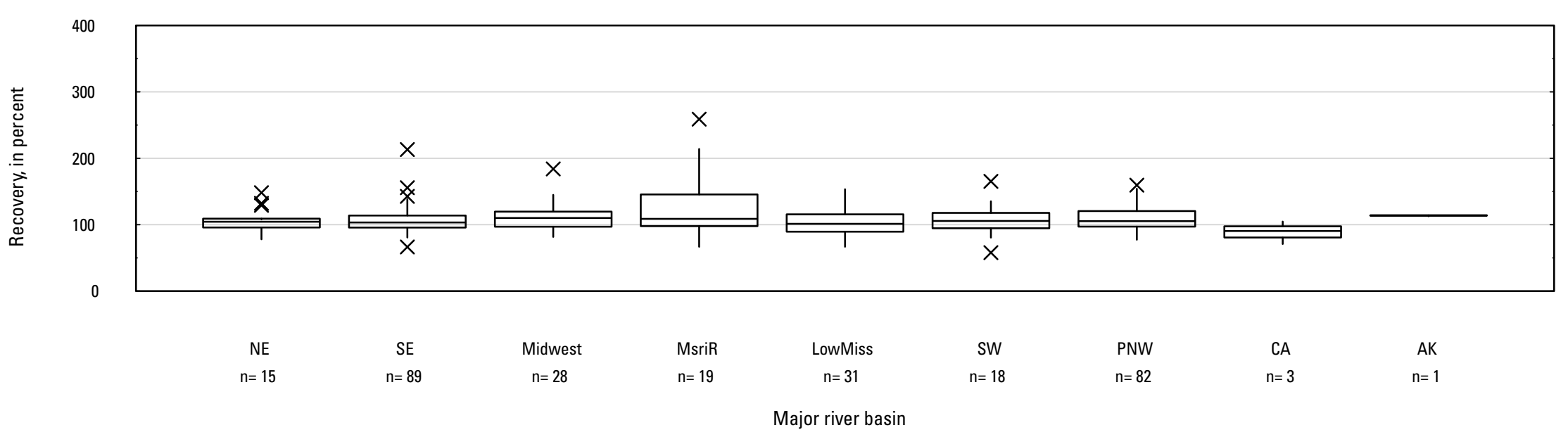

HJ. Terbuthylazine

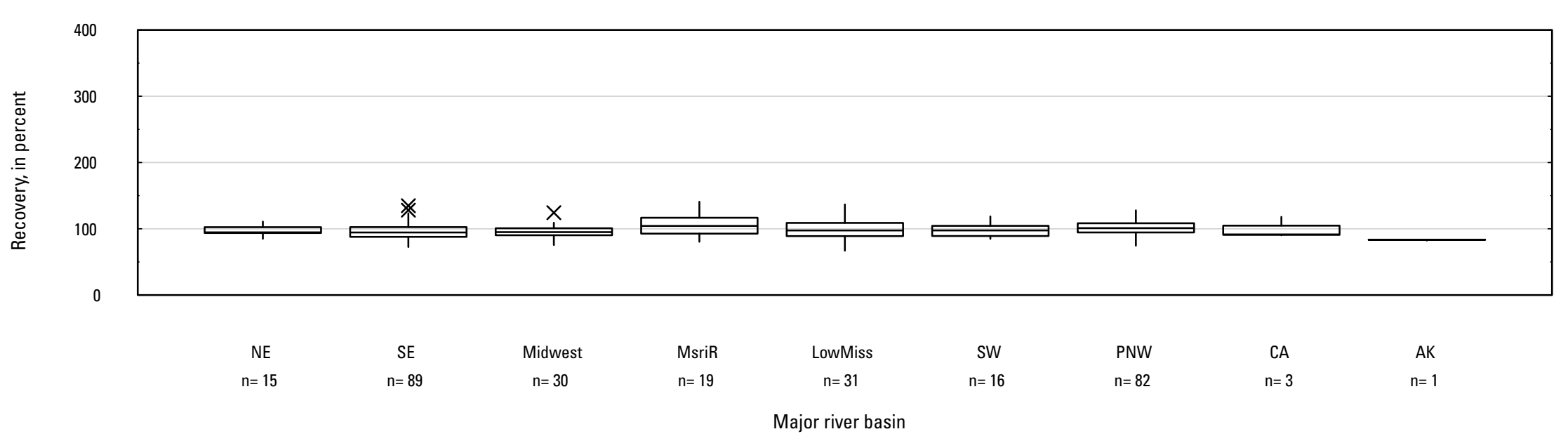

Figure 5. Graph showing distributions of recovery for pesticides in schedule 2437 in surface water by Major River Basin. Recovery values larger than 400 percent are not shown.-Continued 
HK. Tetraconazole

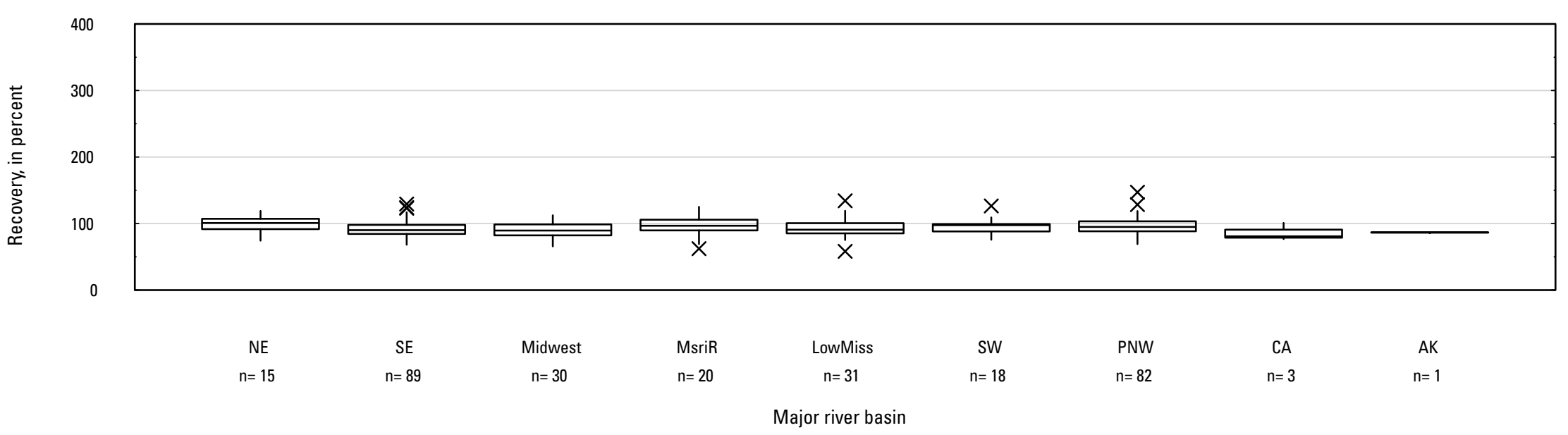

HL. Thiobencarb

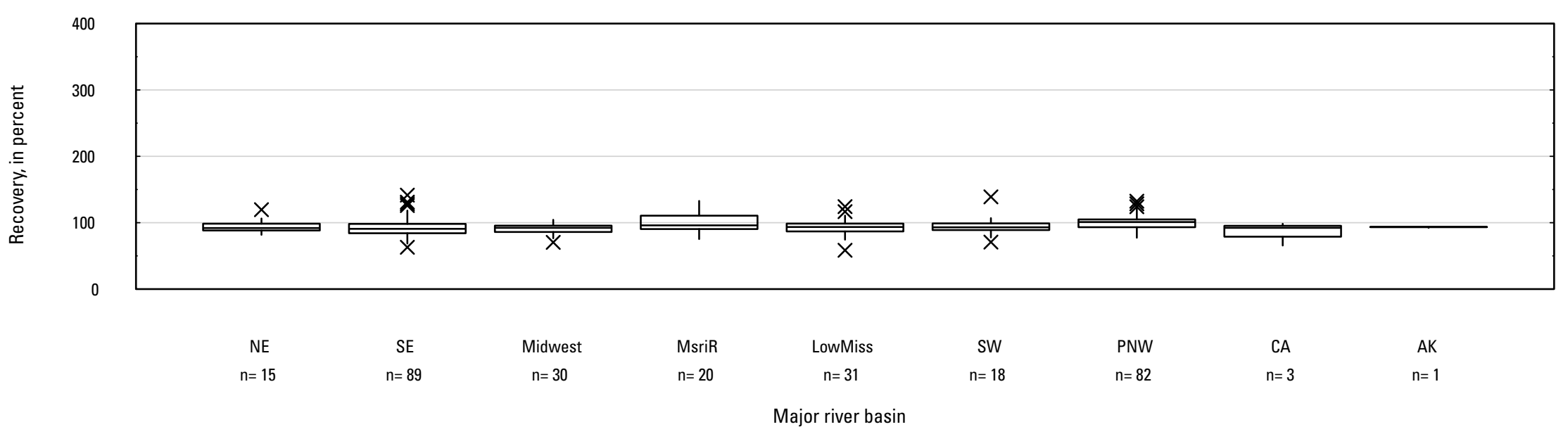

Figure 5. Graph showing distributions of recovery for pesticides in schedule 2437 in surface water by Major River Basin. Recovery values larger than 400 percent are not shown.-Continued 


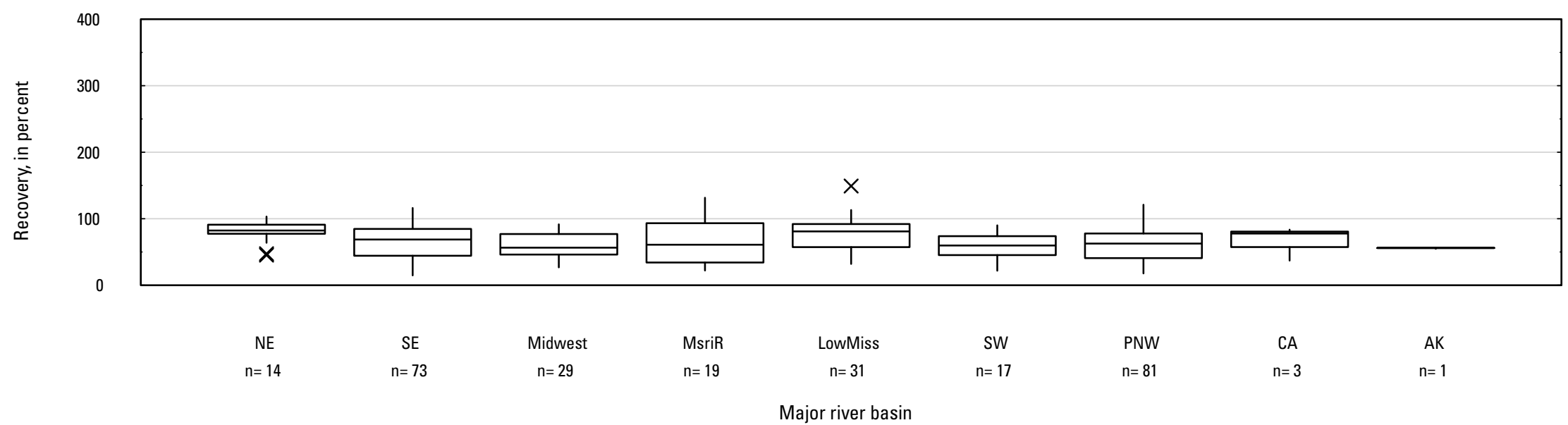

HN. Triallate

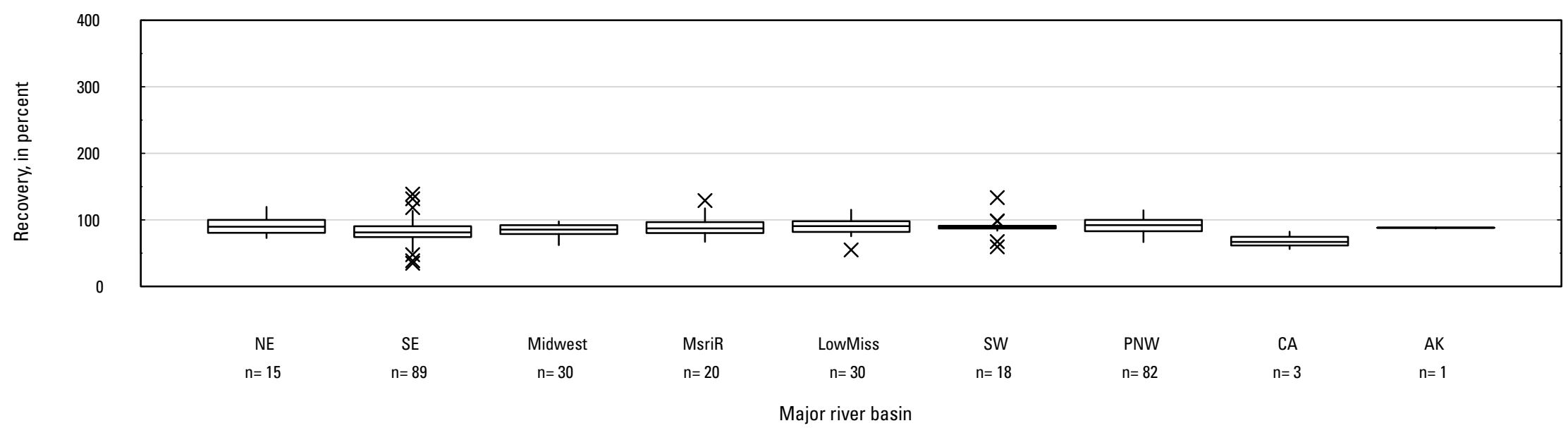

Figure 5. Graph showing distributions of recovery for pesticides in schedule 2437 in surface water by Major River Basin. Recovery values larger than 400 percent are not shown.-Continued 
HO. Tribufos

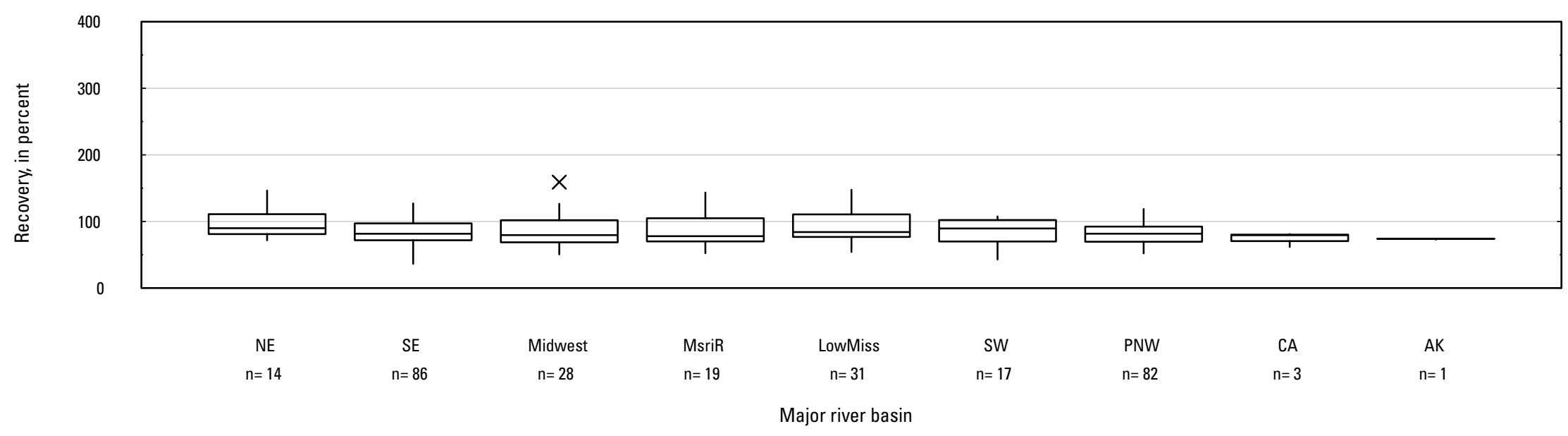

HP. Triclopyr

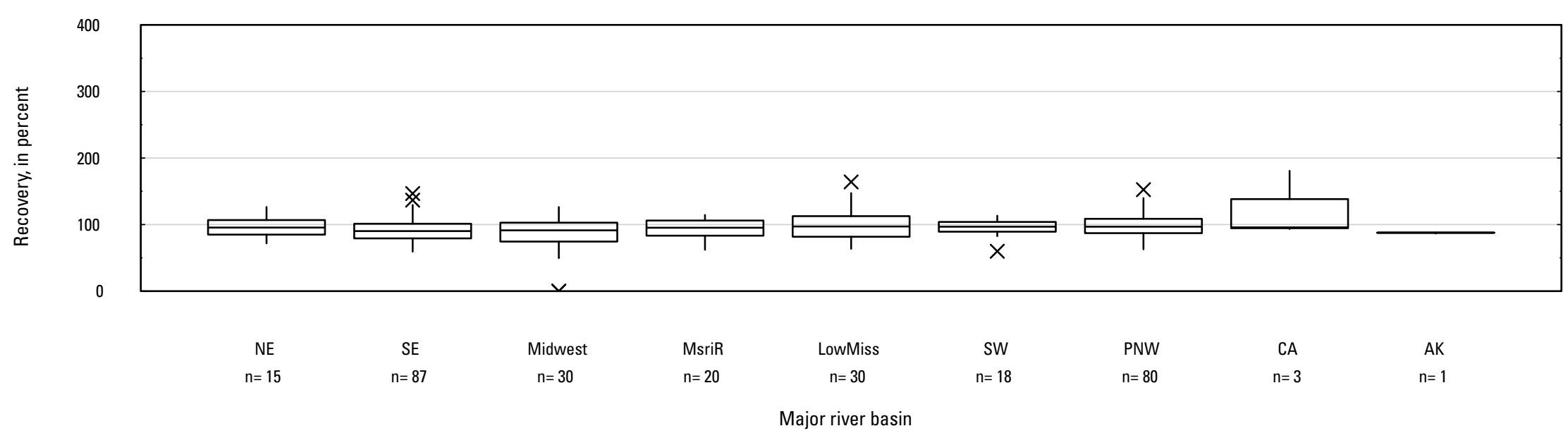

Figure 5. Graph showing distributions of recovery for pesticides in schedule 2437 in surface water by Major River Basin. Recovery values larger than 400 percent are 


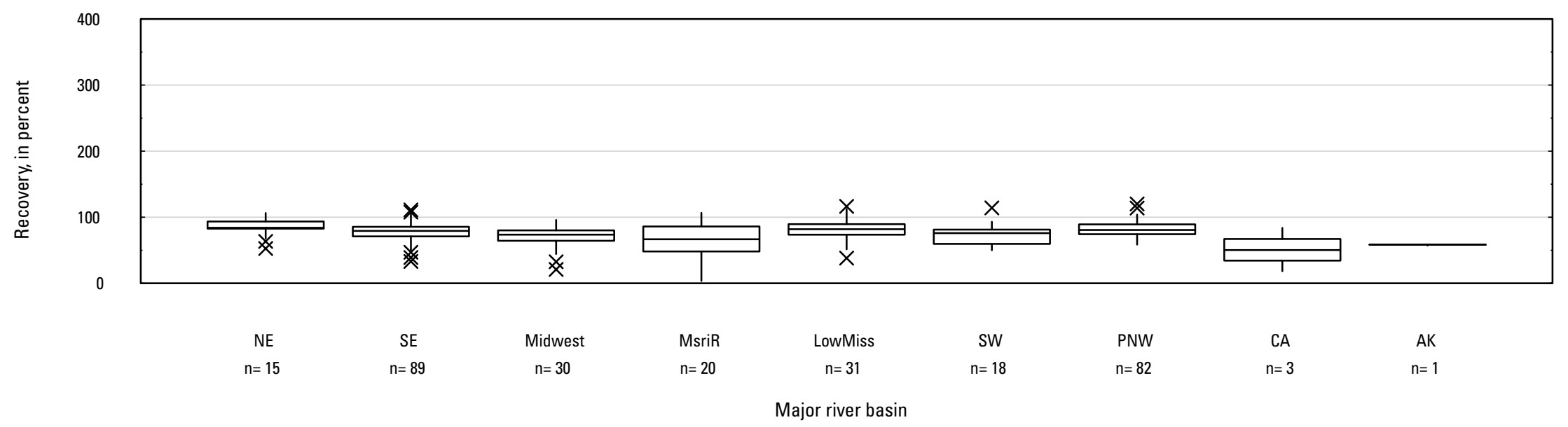

Figure 5. Graph showing distributions of recovery for pesticides in schedule 2437 in surface water by Major River Basin. Recovery values larger than 400 percent are not shown.-Continued 
Table 4. Summary statistics for the recovery of schedule 2437 pesticides in lab reagent spikes, and groundwater and surface-water field matrix spike samples.

[ _ , values that do not meet method validation data-quality objectives; ___ , recovery values in which the first quartile is greater than or third quartile is less than 100 percent]

\begin{tabular}{|c|c|c|c|c|c|c|c|c|c|}
\hline \multirow[b]{2}{*}{ Pcode } & \multirow[b]{2}{*}{ Parameter name } & \multicolumn{8}{|c|}{ Lab reagent spikes } \\
\hline & & Count & Minimum & $\begin{array}{c}\text { First } \\
\text { quartile }\end{array}$ & Mean & Median & $\begin{array}{c}\text { Third } \\
\text { quartile }\end{array}$ & Maximum & $\begin{array}{c}\text { Relative } \\
\text { standard } \\
\text { deviation }\end{array}$ \\
\hline 61678 & Chlorsulfuron & 275 & 57.16 & 86.53 & 96.41 & 95.16 & 106.36 & 154.84 & 15.24 \\
\hline 61679 & Flumetsulam & 275 & 64.25 & 91.39 & 99.93 & 98.73 & 109.31 & 137.17 & 13.69 \\
\hline 61680 & Halosulfuron-methyl & 275 & 57.81 & 85.50 & 94.87 & 95.23 & 102.91 & 133.55 & 12.90 \\
\hline 61682 & Imazaquin & 275 & 56.36 & 86.73 & 94.44 & 94.74 & 102.17 & 135.90 & 13.47 \\
\hline 61683 & Imazethapyr & 275 & 56.37 & 88.51 & 99.24 & 98.45 & 109.76 & 157.23 & 17.28 \\
\hline 61685 & Nicosulfuron & 275 & 44.43 & 87.67 & 96.49 & 96.19 & 105.43 & 141.21 & 14.39 \\
\hline 61687 & Prosulfuron & 300 & 42.69 & 87.25 & 95.93 & 96.39 & 106.19 & 137.82 & 16.45 \\
\hline 65064 & Alachlor & 275 & 64.51 & 84.59 & 94.46 & 94.06 & 102.77 & 129.91 & 12.83 \\
\hline 65065 & Atrazine & 275 & 70.00 & 87.83 & 95.89 & 94.74 & 103.45 & 147.44 & 12.99 \\
\hline 65066 & Azinphos-methyl & 275 & 67.54 & 88.55 & 101.11 & 99.31 & 109.78 & 193.00 & 17.75 \\
\hline 65067 & Bifenthrin & 274 & 14.17 & 59.17 & 80.23 & 74.25 & 90.52 & 312.34 & 48.88 \\
\hline 65068 & Butylate & 275 & 35.99 & 78.78 & 86.98 & 87.13 & 95.70 & 116.19 & 15.89 \\
\hline 65069 & Carbaryl & 275 & 50.92 & 84.65 & 95.71 & 93.77 & 103.94 & 165.49 & 18.13 \\
\hline 65070 & Carbofuran & 275 & 68.12 & 87.38 & 97.03 & 96.42 & 104.64 & 141.47 & 13.03 \\
\hline 65072 & Chlorpyrifos & 275 & 45.41 & 79.53 & 87.84 & 87.42 & 96.30 & 137.12 & 14.92 \\
\hline 65078 & Diazinon & 275 & 56.75 & 81.13 & 89.75 & 89.34 & 97.79 & 127.63 & 13.88 \\
\hline 65080 & EPTC & 275 & 3.66 & 86.37 & 98.29 & 97.67 & 111.45 & 171.28 & 22.56 \\
\hline 65084 & Fonofos & 275 & 56.75 & 82.53 & 93.24 & 91.98 & 101.66 & 153.35 & 15.50 \\
\hline 65085 & Hexazinone & 275 & 66.28 & 87.43 & 95.17 & 95.56 & 101.64 & 129.33 & 11.23 \\
\hline 65087 & Malathion & 275 & 22.38 & 78.10 & 89.07 & 90.64 & 100.92 & 164.55 & 26.25 \\
\hline 65088 & Methidathion & 275 & 64.30 & 87.81 & 100.88 & 100.03 & 109.88 & 189.94 & 17.13 \\
\hline 65090 & Metolachlor & 275 & 68.75 & 85.83 & 94.20 & 93.06 & 102.35 & 136.97 & 12.03 \\
\hline 65091 & Molinate & 275 & 51.32 & 86.12 & 95.37 & 95.45 & 103.72 & 142.53 & 14.46 \\
\hline 65093 & Oxyfluorfen & 327 & 0 & 64.61 & 88.43 & 86.96 & 109.54 & 226.87 & 39.86 \\
\hline 65098 & Pendimethalin & 275 & 56.95 & 81.75 & 89.76 & 88.73 & 97.25 & 124.99 & 13.75 \\
\hline 65102 & Piperonyl butoxide & 271 & 3.09 & 72.81 & 83.47 & 83.14 & 92.37 & 211.23 & 22.66 \\
\hline 65103 & Prometryn & 275 & 60.97 & 85.12 & 92.35 & 92.55 & 98.62 & 127.96 & 11.45 \\
\hline 65105 & Simazine & 275 & 66.63 & 85.91 & 94.41 & 92.97 & 102.36 & 148.93 & 13.64 \\
\hline
\end{tabular}


Table 4. Summary statistics for the recovery of schedule 2437 pesticides in lab reagent spikes, and groundwater and surface-water field matrix spike samples.-Continued

$[\quad$ [ values that do not meet method validation data-quality objectives; _ـ , recovery values in which the first quartile is greater than or third quartile is less than 100 percent]

\begin{tabular}{|c|c|c|c|c|c|c|c|c|c|}
\hline \multirow[b]{2}{*}{ Pcode } & \multirow[b]{2}{*}{ Parameter name } & \multicolumn{8}{|c|}{ Groundwater } \\
\hline & & Count & Minimum & $\begin{array}{c}\text { First } \\
\text { quartile }\end{array}$ & Mean & Median & $\begin{array}{c}\text { Third } \\
\text { quartile }\end{array}$ & Maximum & $\begin{array}{l}\text { Relative } \\
\text { standard } \\
\text { deviation }\end{array}$ \\
\hline 61678 & Chlorsulfuron & 42 & 57.27 & 84.78 & 100.73 & 99.33 & 109.28 & 189.16 & 22.72 \\
\hline 61679 & Flumetsulam & 28 & 84.05 & 96.14 & 105.35 & 102.63 & 113.54 & 133.87 & 12.51 \\
\hline 61680 & Halosulfuron-methyl & 39 & 38.26 & 80.45 & 90.07 & 86.55 & 98.38 & 147.88 & 19.66 \\
\hline 61682 & Imazaquin & 39 & 71.20 & 82.70 & 94.98 & 90.64 & 105.31 & 162.20 & 17.72 \\
\hline 61683 & Imazethapyr & 40 & 76.65 & 90.42 & 101.92 & 98.67 & 110.33 & 163.08 & 17.87 \\
\hline 61685 & Nicosulfuron & 42 & 60.26 & 70.96 & 83.46 & 81.61 & 95.31 & 117.69 & 18.52 \\
\hline 61687 & Prosulfuron & 42 & 52.80 & 77.38 & 94.31 & 90.84 & 106.70 & 161.09 & 25.95 \\
\hline 65064 & Alachlor & 40 & 85.76 & 95.40 & 103.39 & 100.94 & 111.76 & 123.27 & 10.08 \\
\hline 65065 & Atrazine & 40 & 80.26 & 91.39 & 101.10 & 98.82 & 111.39 & 144.23 & 12.78 \\
\hline 65066 & Azinphos-methyl & 42 & 69.94 & 97.20 & 110.23 & 103.65 & 116.98 & 308.17 & 31.24 \\
\hline 65067 & Bifenthrin & 36 & 7.91 & 18.87 & 35.34 & 26.72 & 46.27 & 136.37 & 77.85 \\
\hline 65068 & Butylate & 34 & 67.57 & 82.65 & 93.68 & 96.76 & 100.06 & 112.13 & 12.57 \\
\hline 65069 & Carbaryl & 42 & 8.79 & 84.71 & 98.18 & 96.41 & 123.95 & 135.24 & 25.85 \\
\hline 65070 & Carbofuran & 42 & 49.36 & 91.06 & 96.53 & 95.33 & 103.03 & 120.57 & 13.13 \\
\hline 65072 & Chlorpyrifos & 40 & 58.26 & 76.85 & 84.78 & 85.61 & 91.48 & 115.25 & 13.26 \\
\hline 65078 & Diazinon & 41 & 67.40 & 83.25 & 95.40 & 92.10 & 104.07 & 143.67 & 18.47 \\
\hline 65080 & EPTC & 16 & 61.79 & 92.96 & 108.60 & 110.48 & 123.27 & 151.38 & 21.78 \\
\hline 65084 & Fonofos & 40 & 69.45 & 86.17 & 96.47 & 96.56 & 107.23 & 119.59 & 12.70 \\
\hline 65085 & Hexazinone & 42 & 84.45 & 94.06 & 100.08 & 97.48 & 106.02 & 123.92 & 9.28 \\
\hline 65087 & Malathion & 42 & 4.21 & 77.27 & 88.18 & 89.23 & 105.56 & 119.20 & 23.87 \\
\hline 65088 & Methidathion & 42 & 56.43 & 95.94 & 106.76 & 100.72 & 111.92 & 333.02 & 36.68 \\
\hline 65090 & Metolachlor & 42 & 84.07 & 93.73 & 99.60 & 98.54 & 106.44 & 118.66 & 9.03 \\
\hline 65091 & Molinate & 42 & 66.04 & 87.63 & 98.82 & 96.80 & 109.22 & 132.20 & 15.07 \\
\hline 65093 & Oxyfluorfen & 7 & 0 & 29.60 & 57.14 & 63.23 & 77.56 & 114.10 & 63.47 \\
\hline 65098 & Pendimethalin & 38 & 69.18 & 77.91 & 86.61 & 85.42 & 94.99 & 116.19 & 13.04 \\
\hline 65102 & Piperonyl butoxide & 38 & 68.20 & 76.16 & 86.00 & 85.99 & 95.69 & 111.83 & 14.23 \\
\hline 65103 & Prometryn & 42 & 81.84 & 88.37 & 96.77 & 95.69 & 102.97 & 133.15 & 10.61 \\
\hline 65105 & Simazine & 42 & 69.23 & 91.78 & 100.69 & 99.03 & 106.70 & 131.54 & 13.81 \\
\hline
\end{tabular}


Table 4. Summary statistics for the recovery of schedule 2437 pesticides in lab reagent spikes, and groundwater and surface-water field matrix spike samples.-Continued [ _ , values that do not meet method validation data-quality objectives; _ـ , recovery values in which the first quartile is greater than or third quartile is less than 100 percent]

\begin{tabular}{|c|c|c|c|c|c|c|c|c|c|}
\hline \multirow[b]{2}{*}{ Pcode } & \multirow[b]{2}{*}{ Parameter name } & \multicolumn{8}{|c|}{ Surface water } \\
\hline & & Count & Minimum & $\begin{array}{c}\text { First } \\
\text { quartile }\end{array}$ & Mean & Median & $\begin{array}{c}\text { Third } \\
\text { quartile }\end{array}$ & Maximum & $\begin{array}{l}\text { Relative } \\
\text { standard } \\
\text { deviation }\end{array}$ \\
\hline 61678 & Chlorsulfuron & 289 & 17.11 & 89.18 & 100.43 & 99.23 & 110.22 & 169.13 & 20.84 \\
\hline 61679 & Flumetsulam & 263 & 81.56 & 108.80 & 125.58 & 120.05 & 142.89 & 189.23 & 18.37 \\
\hline 61680 & Halosulfuron-methyl & 276 & 20.97 & 80.77 & 89.54 & 89.11 & 97.44 & 141.58 & 18.14 \\
\hline 61682 & Imazaquin & 267 & 62.61 & 89.96 & 100.34 & 99.59 & 108.75 & 153.00 & 15.28 \\
\hline 61683 & Imazethapyr & 288 & 65.26 & 96.94 & 110.39 & 108.48 & 123.39 & 179.65 & 17.88 \\
\hline 61685 & Nicosulfuron & 289 & 7.82 & 77.45 & 90.17 & 91.53 & 102.40 & 157.07 & 22.95 \\
\hline 61687 & Prosulfuron & 282 & 6.49 & 79.00 & 96.83 & 96.88 & 112.09 & 218.19 & 27.60 \\
\hline 65064 & Alachlor & 286 & 64.74 & 92.31 & 102.59 & 99.90 & 109.03 & 166.67 & 15.42 \\
\hline 65065 & Atrazine & 262 & 47.94 & 90.94 & 98.45 & 98.08 & 105.75 & 148.65 & 13.64 \\
\hline 65066 & Azinphos-methyl & 273 & 14.09 & 89.01 & 100.90 & 98.47 & 112.76 & 216.00 & 27.25 \\
\hline 65067 & Bifenthrin & 282 & 6.86 & 39.49 & 68.54 & 64.02 & 95.63 & 170.40 & 52.64 \\
\hline 65068 & Butylate & 247 & 23.32 & 82.45 & 91.25 & 92.07 & 99.15 & 148.46 & 17.24 \\
\hline 65069 & Carbaryl & 287 & 0.00 & 69.69 & 78.72 & 82.39 & 96.37 & 172.94 & 39.22 \\
\hline 65070 & Carbofuran & 289 & 10.38 & 83.21 & 91.07 & 93.38 & 102.23 & 151.63 & 25.43 \\
\hline 65072 & Chlorpyrifos & 283 & 39.83 & 72.90 & 81.70 & 80.28 & 89.93 & 140.14 & 18.62 \\
\hline 65078 & Diazinon & 276 & 55.55 & 82.02 & 92.46 & 90.74 & 99.98 & 182.02 & 16.63 \\
\hline 65080 & ЕРTC & 271 & 0 & 84.65 & 98.42 & 98.11 & 113.96 & 165.89 & 26.60 \\
\hline 65084 & Fonofos & 286 & 57.70 & 85.44 & 96.58 & 94.67 & 105.29 & 174.53 & 17.22 \\
\hline 65085 & Hexazinone & 289 & 58.55 & 91.12 & 98.29 & 96.80 & 105.18 & 136.22 & 11.18 \\
\hline 65087 & Malathion & 289 & 0 & 61.92 & 72.38 & 77.60 & 89.31 & 187.11 & 44.69 \\
\hline 65088 & Methidathion & 289 & 42.53 & 88.76 & 102.15 & 98.48 & 112.53 & 252.28 & 21.73 \\
\hline 65090 & Metolachlor & 266 & 57.73 & 90.50 & 97.55 & 96.30 & 103.34 & 140.93 & 11.16 \\
\hline 65091 & Molinate & 283 & 38.22 & 91.38 & 100.12 & 99.09 & 109.14 & 175.28 & 16.88 \\
\hline 65093 & Oxyfluorfen & 19 & 24.71 & 65.49 & 78.76 & 86.25 & 99.69 & 111.64 & 32.00 \\
\hline 65098 & Pendimethalin & 275 & 35.29 & 73.79 & 82.71 & 82.18 & 91.80 & 125.82 & 17.44 \\
\hline 65102 & Piperonyl butoxide & 256 & 0 & 81.01 & 90.08 & 87.51 & 96.73 & 160.12 & 20.86 \\
\hline 65103 & Prometryn & 289 & 56.90 & 90.16 & 97.96 & 96.60 & 103.89 & 138.64 & 12.43 \\
\hline 65105 & Simazine & 278 & 71.73 & 94.51 & 106.54 & 103.67 & 116.49 & 198.07 & 16.27 \\
\hline
\end{tabular}


Table 4. Summary statistics for the recovery of schedule 2437 pesticides in lab reagent spikes, and groundwater and surface-water field matrix spike samples.-Continued

$[\quad$, values that do not meet method validation data-quality objectives; ___ , recovery values in which the first quartile is greater than or third quartile is less than 100 percent $]$

\begin{tabular}{|c|c|c|c|c|c|c|c|c|c|}
\hline \multirow[b]{2}{*}{ Pcode } & \multirow[b]{2}{*}{ Parameter name } & \multicolumn{8}{|c|}{ Lab reagent spike } \\
\hline & & Count & Minimum & $\begin{array}{c}\text { First } \\
\text { quartile }\end{array}$ & Mean & Median & $\begin{array}{c}\text { Third } \\
\text { quartile }\end{array}$ & Maximum & $\begin{array}{l}\text { Relative } \\
\text { standard } \\
\text { deviation }\end{array}$ \\
\hline 65107 & Thiobencarb & 275 & 59.73 & 85.64 & 93.56 & 93.48 & 100.60 & 123.17 & 11.94 \\
\hline 66589 & Azoxystrobin & 275 & 66.97 & 86.75 & 94.08 & 92.73 & 100.05 & 132.53 & 11.77 \\
\hline 66592 & Cyanazine & 275 & 15.32 & 83.57 & 94.38 & 94.97 & 106.00 & 148.67 & 19.04 \\
\hline 66596 & Dimethoate & 275 & 67.73 & 92.24 & 103.68 & 101.98 & 113.08 & 191.86 & 16.62 \\
\hline 66598 & Diuron & 275 & 68.09 & 87.79 & 95.66 & 95.00 & 101.67 & 211.52 & 13.67 \\
\hline 66604 & Fipronil & 280 & 50.98 & 80.55 & 89.49 & 88.79 & 97.80 & 141.39 & 16.13 \\
\hline 66607 & Desulfinylfipronil & 280 & 57.38 & 84.72 & 93.13 & 91.48 & 100.75 & 142.00 & 14.68 \\
\hline 66610 & Fipronil sulfide & 280 & 50.50 & 78.96 & 87.90 & 87.55 & 95.04 & 136.75 & 16.22 \\
\hline 66613 & Fipronil sulfone & 280 & 39.76 & 73.75 & 82.85 & 82.75 & 89.90 & 128.85 & 17.39 \\
\hline 66620 & Metconazole & 261 & 66.61 & 86.51 & 93.63 & 93.28 & 98.87 & 131.93 & 12.21 \\
\hline 66632 & Myclobutanil & 275 & 70.01 & 86.69 & 93.78 & 92.99 & 100.46 & 131.98 & 12.33 \\
\hline 66641 & Propanil & 275 & 59.55 & 85.50 & 96.12 & 95.37 & 105.53 & 136.44 & 14.59 \\
\hline 66643 & Propiconazole & 275 & 0.00 & 83.38 & 91.39 & 90.95 & 98.81 & 129.68 & 15.47 \\
\hline 66646 & Pyraclostrobin & 275 & 61.77 & 82.66 & 90.45 & 90.27 & 96.24 & 124.94 & 12.09 \\
\hline 66649 & Tebuconazole & 275 & 61.05 & 88.81 & 96.40 & 95.75 & 104.09 & 129.80 & 12.73 \\
\hline 66651 & Terbuthylazine & 275 & 68.89 & 87.70 & 95.45 & 94.82 & 102.64 & 134.77 & 11.72 \\
\hline 66654 & Tetraconazole & 275 & 67.42 & 82.46 & 91.24 & 90.77 & 98.25 & 127.70 & 12.63 \\
\hline 66660 & Trifloxystrobin & 275 & 53.75 & 75.76 & 83.27 & 83.38 & 90.47 & 120.26 & 14.16 \\
\hline 67595 & Disulfoton & 275 & 39.13 & 79.10 & 88.25 & 86.59 & 97.51 & 145.02 & 17.55 \\
\hline 67670 & Kresoxim-methyl & 275 & 58.00 & 83.14 & 91.08 & 89.67 & 97.97 & 137.65 & 13.58 \\
\hline 67685 & Norflurazon & 275 & 65.28 & 90.86 & 97.31 & 97.26 & 103.83 & 132.30 & 11.57 \\
\hline 67702 & Prometon & 275 & 62.91 & 85.00 & 92.83 & 92.98 & 99.29 & 126.76 & 11.34 \\
\hline 67706 & Propyzamide & 275 & 57.87 & 85.45 & 95.32 & 95.13 & 104.48 & 128.20 & 13.82 \\
\hline 68211 & Azinphos-methyl oxon & 275 & 27.97 & 83.20 & 93.31 & 96.68 & 105.49 & 158.09 & 24.44 \\
\hline 68216 & Chlorpyrifos oxon & 275 & 56.26 & 83.19 & 93.32 & 93.82 & 103.25 & 138.65 & 16.04 \\
\hline 68226 & 3,4-Dichlorophenylurea & 275 & 54.25 & 85.08 & 95.55 & 94.43 & 105.00 & 167.88 & 15.90 \\
\hline 68231 & N-(3,4-Dichlorophenyl)-N'-methylurea & 275 & 55.99 & 85.79 & 96.18 & 94.52 & 101.18 & 256.14 & 21.80 \\
\hline 68236 & Diazinon oxon & 275 & 44.70 & 82.41 & 92.21 & 93.40 & 103.41 & 135.99 & 17.83 \\
\hline 68240 & Malaoxon & 273 & 1.92 & 71.72 & 82.63 & 87.63 & 100.12 & 158.36 & 36.47 \\
\hline
\end{tabular}


Table 4. Summary statistics for the recovery of schedule 2437 pesticides in lab reagent spikes, and groundwater and surface-water field matrix spike samples.-Continued

[ _ , values that do not meet method validation data-quality objectives; _ , recovery values in which the first quartile is greater than or third quartile is less than 100 percent]

\begin{tabular}{|c|c|c|c|c|c|c|c|c|c|}
\hline \multirow[b]{2}{*}{ Pcode } & \multirow[b]{2}{*}{ Parameter name } & \multicolumn{8}{|c|}{ Groundwater } \\
\hline & & Count & Minimum & $\begin{array}{c}\text { First } \\
\text { quartile }\end{array}$ & Mean & Median & $\begin{array}{c}\text { Third } \\
\text { quartile }\end{array}$ & Maximum & $\begin{array}{l}\text { Relative } \\
\text { standard } \\
\text { deviation }\end{array}$ \\
\hline 65107 & Thiobencarb & 42 & 75.65 & 86.89 & 94.53 & 94.60 & 101.68 & 117.83 & 10.09 \\
\hline 66589 & Azoxystrobin & 42 & 84.36 & 91.71 & 97.85 & 96.90 & 104.72 & 127.26 & 9.33 \\
\hline 66592 & Cyanazine & 34 & 72.16 & 84.84 & 102.55 & 97.05 & 114.87 & 219.51 & 27.40 \\
\hline 66596 & Dimethoate & 42 & 67.81 & 93.33 & 108.66 & 104.28 & 113.49 & 303.17 & 31.35 \\
\hline 66598 & Diuron & 35 & 83.60 & 94.90 & 102.28 & 100.86 & 109.26 & 128.41 & 10.17 \\
\hline 66604 & Fipronil & 39 & 79.41 & 90.35 & 100.78 & 99.95 & 107.80 & 144.49 & 14.25 \\
\hline 66607 & Desulfinylfipronil & 40 & 78.46 & 93.61 & 101.57 & 100.19 & 108.23 & 140.49 & 13.62 \\
\hline 66610 & Fipronil sulfide & 39 & 77.74 & 88.50 & 99.31 & 99.64 & 106.83 & 143.45 & 15.36 \\
\hline 66613 & Fipronil sulfone & 39 & 73.89 & 87.12 & 95.01 & 92.41 & 101.77 & 133.50 & 14.27 \\
\hline 66620 & Metconazole & 42 & 81.48 & 90.69 & 97.18 & 96.42 & 106.10 & 122.43 & 10.12 \\
\hline 66632 & Myclobutanil & 42 & 70.32 & 92.61 & 98.51 & 97.44 & 104.48 & 121.82 & 10.14 \\
\hline 66641 & Propanil & 42 & 72.65 & 88.52 & 98.73 & 97.12 & 113.05 & 123.62 & 14.36 \\
\hline 66643 & Propiconazole & 42 & 80.95 & 90.94 & 96.94 & 95.40 & 105.28 & 116.12 & 9.69 \\
\hline 66646 & Pyraclostrobin & 42 & 78.65 & 84.69 & 92.21 & 90.72 & 99.46 & 112.10 & 9.78 \\
\hline 66649 & Tebuconazole & 41 & 78.23 & 88.64 & 97.56 & 95.48 & 105.98 & 116.99 & 10.80 \\
\hline 66651 & Terbuthylazine & 42 & 80.91 & 90.09 & 98.36 & 97.45 & 104.43 & 121.65 & 10.16 \\
\hline 66654 & Tetraconazole & 42 & 73.94 & 87.91 & 95.25 & 94.42 & 102.50 & 118.22 & 11.11 \\
\hline 66660 & Trifloxystrobin & 42 & 15.42 & 75.40 & 83.60 & 84.49 & 93.30 & 126.11 & 20.25 \\
\hline 67595 & Disulfoton & 40 & 48.51 & 74.09 & 82.96 & 83.42 & 93.63 & 109.88 & 16.95 \\
\hline 67670 & Kresoxim-methyl & 42 & 11.69 & 80.88 & 87.23 & 88.77 & 100.01 & 115.51 & 19.80 \\
\hline 67685 & Norflurazon & 42 & 79.58 & 93.43 & 100.42 & 99.75 & 106.70 & 122.27 & 9.29 \\
\hline 67702 & Prometon & 42 & 83.22 & 89.69 & 96.23 & 95.44 & 101.60 & 118.96 & 8.80 \\
\hline 67706 & Propyzamide & 38 & 75.40 & 90.62 & 99.52 & 98.88 & 107.56 & 120.20 & 11.12 \\
\hline 68211 & Azinphos-methyl oxon & 40 & 64.54 & 86.77 & 96.39 & 95.74 & 106.32 & 127.79 & 15.30 \\
\hline 68216 & Chlorpyrifos oxon & 40 & 32.37 & 55.60 & 67.83 & 65.45 & 79.28 & 103.86 & 25.01 \\
\hline 68226 & 3,4-Dichlorophenylurea & 37 & 71.95 & 89.25 & 98.61 & 98.48 & 109.59 & 123.49 & 12.23 \\
\hline 68231 & $\mathrm{~N}$-(3,4-Dichlorophenyl)-N'-methylurea & 41 & 46.81 & 79.44 & 96.75 & 95.47 & 106.22 & 237.30 & 35.77 \\
\hline 68236 & Diazinon oxon & 42 & 45.61 & 71.10 & 82.80 & 81.35 & 95.16 & 138.71 & 25.03 \\
\hline 68240 & Malaoxon & 38 & 0 & 53.70 & 69.04 & 69.46 & 88.49 & 146.29 & 42.47 \\
\hline
\end{tabular}


Table 4. Summary statistics for the recovery of schedule 2437 pesticides in lab reagent spikes, and groundwater and surface-water field matrix spike samples.-Continued

$[\quad$ [ values that do not meet method validation data-quality objectives; ___ , recovery values in which the first quartile is greater than or third quartile is less than 100 percent $]$

\begin{tabular}{|c|c|c|c|c|c|c|c|c|c|}
\hline \multirow[b]{2}{*}{ Pcode } & \multirow[b]{2}{*}{ Parameter name } & \multicolumn{8}{|c|}{ Surface water } \\
\hline & & Count & Minimum & $\begin{array}{c}\text { First } \\
\text { quartile }\end{array}$ & Mean & Median & $\begin{array}{c}\text { Third } \\
\text { quartile }\end{array}$ & Maximum & $\begin{array}{l}\text { Relative } \\
\text { standard } \\
\text { deviation }\end{array}$ \\
\hline 65107 & Thiobencarb & 289 & 58.47 & 87.29 & 95.18 & 94.13 & 101.73 & 141.55 & 13.41 \\
\hline 66589 & Azoxystrobin & 285 & 63.56 & 89.50 & 96.97 & 94.98 & 103.85 & 137.18 & 12.31 \\
\hline 66592 & Cyanazine & 266 & 60.47 & 96.50 & 119.36 & 111.01 & 128.21 & 393.83 & 31.68 \\
\hline 66596 & Dimethoate & 281 & 54.11 & 88.94 & 102.50 & 99.21 & 112.26 & 242.89 & 21.71 \\
\hline 66598 & Diuron & 259 & 61.58 & 91.64 & 100.07 & 99.13 & 105.28 & 193.92 & 14.77 \\
\hline 66604 & Fipronil & 279 & 9.65 & 83.69 & 91.14 & 92.23 & 102.75 & 154.85 & 23.79 \\
\hline 66607 & Desulfinylfipronil & 280 & 66.13 & 86.65 & 96.30 & 94.65 & 104.92 & 156.74 & 14.29 \\
\hline 66610 & Fipronil sulfide & 279 & 58.28 & 84.17 & 94.36 & 93.70 & 103.34 & 158.58 & 15.77 \\
\hline 66613 & Fipronil sulfone & 282 & 56.09 & 80.24 & 90.40 & 88.51 & 99.67 & 152.60 & 16.83 \\
\hline 66620 & Metconazole & 281 & 57.98 & 88.31 & 95.96 & 94.72 & 101.46 & 142.58 & 12.39 \\
\hline 66632 & Myclobutanil & 289 & 58.73 & 90.52 & 98.42 & 97.56 & 105.08 & 151.11 & 12.28 \\
\hline 66641 & Propanil & 281 & 66.79 & 90.48 & 100.18 & 98.42 & 108.81 & 157.66 & 15.48 \\
\hline 66643 & Propiconazole & 284 & 55.58 & 88.34 & 95.74 & 94.80 & 101.07 & 153.34 & 12.60 \\
\hline 66646 & Pyraclostrobin & 289 & 54.46 & 83.38 & 91.50 & 89.63 & 98.21 & 134.12 & 13.15 \\
\hline 66649 & Tebuconazole & 283 & 66.77 & 89.96 & 98.72 & 97.50 & 106.50 & 151.75 & 13.45 \\
\hline 66651 & Terbuthylazine & 286 & 66.88 & 90.75 & 98.64 & 97.46 & 106.91 & 140.81 & 12.71 \\
\hline 66654 & Tetraconazole & 289 & 58.23 & 86.19 & 93.93 & 92.65 & 100.48 & 147.05 & 13.23 \\
\hline 66660 & Trifloxystrobin & 289 & 3.42 & 69.53 & 77.45 & 79.12 & 86.91 & 120.05 & 21.44 \\
\hline 67595 & Disulfoton & 283 & 28.60 & 70.52 & 80.47 & 78.65 & 88.31 & 159.33 & 20.70 \\
\hline 67670 & Kresoxim-methyl & 289 & 1.93 & 72.19 & 80.30 & 81.55 & 89.93 & 127.71 & 21.54 \\
\hline 67685 & Norflurazon & 286 & 60.26 & 93.72 & 101.72 & 100.96 & 109.18 & 142.90 & 12.12 \\
\hline 67702 & Prometon & 289 & 56.34 & 89.86 & 97.67 & 95.55 & 103.86 & 190.26 & 13.18 \\
\hline 67706 & Propyzamide & 278 & 65.50 & 89.18 & 98.66 & 97.38 & 106.99 & 161.63 & 14.64 \\
\hline 68211 & Azinphos-methyl oxon & 289 & 0 & 70.66 & 81.21 & 84.64 & 96.62 & 183.34 & 36.84 \\
\hline 68216 & Chlorpyrifos oxon & 272 & 0.00 & 60.32 & 68.29 & 72.07 & 84.51 & 121.55 & 35.10 \\
\hline 68226 & 3,4-Dichlorophenylurea & 285 & 58.02 & 87.56 & 96.57 & 96.05 & 104.90 & 175.23 & 14.48 \\
\hline 68231 & N-(3,4-Dichlorophenyl)-N'-methylurea & 276 & 53.40 & 87.99 & 98.12 & 95.22 & 104.31 & 230.78 & 19.77 \\
\hline 68236 & Diazinon oxon & 289 & 22.70 & 74.26 & 83.96 & 83.77 & 98.35 & 145.39 & 24.57 \\
\hline 68240 & Malaoxon & 276 & 0 & 36.48 & 52.14 & 55.05 & 69.40 & 179.39 & 54.39 \\
\hline
\end{tabular}


Table 4. Summary statistics for the recovery of schedule 2437 pesticides in lab reagent spikes, and groundwater and surface-water field matrix spike samples.-Continued [ _ , values that do not meet method validation data-quality objectives; ___ , recovery values in which the first quartile is greater than or third quartile is less than 100 percent]

\begin{tabular}{|c|c|c|c|c|c|c|c|c|c|}
\hline \multirow[b]{2}{*}{ Pcode } & \multirow[b]{2}{*}{ Parameter name } & \multicolumn{8}{|c|}{ Lab reagent spikes } \\
\hline & & Count & Minimum & $\begin{array}{c}\text { First } \\
\text { quartile }\end{array}$ & Mean & Median & $\begin{array}{c}\text { Third } \\
\text { quartile }\end{array}$ & Maximum & $\begin{array}{l}\text { Relative } \\
\text { standard } \\
\text { deviation }\end{array}$ \\
\hline 68336 & 4-Hydroxychlorothalonil & 280 & 52.54 & 87.59 & 100.82 & 98.84 & 111.94 & 176.37 & 20.06 \\
\hline 68426 & Imidacloprid & 275 & 64.38 & 84.27 & 93.23 & 93.05 & 101.12 & 140.14 & 13.66 \\
\hline 68437 & Metalaxyl & 275 & 63.41 & 89.60 & 95.83 & 95.32 & 103.97 & 131.29 & 12.78 \\
\hline 68498 & 1H-1,2,4-Triazole & 275 & 35.24 & 62.52 & 81.94 & 78.99 & 99.46 & 155.43 & 31.21 \\
\hline 68500 & $2,4-\mathrm{D}$ & 280 & 45.78 & 84.56 & 95.87 & 95.95 & 107.44 & 148.71 & 18.07 \\
\hline 68502 & 2-Aminobenzimidazole & 275 & 61.86 & 86.30 & 94.29 & 92.96 & 102.21 & 154.77 & 13.91 \\
\hline 68503 & 2-Amino-N-isopropylbenzamide & 275 & 62.69 & 86.73 & 95.10 & 94.67 & 102.35 & 135.97 & 12.91 \\
\hline 68505 & 2-Isopropyl-6-methyl-4-pyrimidinol & 275 & 63.14 & 93.57 & 105.53 & 102.98 & 115.35 & 214.31 & 18.43 \\
\hline 68508 & 3-Hydroxycarbofuran & 275 & 60.93 & 85.44 & 93.78 & 92.28 & 101.83 & 139.10 & 13.98 \\
\hline 68511 & 4-(Hydroxymethyl)pendimethalin & 280 & 32.76 & 81.83 & 96.02 & 95.25 & 107.69 & 173.93 & 22.96 \\
\hline 68514 & 4-Chlorobenzylmethyl sulfoxide & 275 & 62.94 & 91.00 & 99.18 & 99.11 & 106.97 & 132.14 & 12.12 \\
\hline 68515 & 4-Hydroxy molinate & 275 & 62.89 & 88.81 & 97.21 & 96.73 & 105.15 & 130.70 & 12.47 \\
\hline 68517 & 4-Hydroxyhexazinone A & 275 & 63.94 & 87.42 & 94.36 & 94.79 & 101.72 & 126.28 & 11.15 \\
\hline 68519 & Acephate & 275 & 64.18 & 90.51 & 102.02 & 99.69 & 111.46 & 179.37 & 17.15 \\
\hline 68520 & Acetochlor & 275 & 60.75 & 83.74 & 93.94 & 93.65 & 102.96 & 127.42 & 14.30 \\
\hline 68521 & 2-Chloro-N-(2-ethyl-6-methylphenyl)acetamide & 275 & 63.35 & 89.34 & 98.19 & 97.57 & 106.64 & 137.46 & 13.33 \\
\hline 68522 & Acetochlor oxanilic acid & 280 & 49.32 & 82.91 & 93.86 & 94.52 & 104.97 & 146.51 & 18.26 \\
\hline 68523 & Acetochlor sulfonic acid & 280 & 12.09 & 81.28 & 102.63 & 98.90 & 122.23 & 239.06 & 32.18 \\
\hline 68524 & Acetochlor sulfynilacetic acid & 280 & 37.79 & 85.71 & 100.43 & 99.62 & 114.68 & 171.97 & 20.55 \\
\hline 68525 & 2-Chloro-2',6'-diethylacetanilide & 275 & 65.92 & 88.86 & 98.89 & 98.75 & 107.29 & 133.71 & 13.01 \\
\hline 68526 & Alachlor oxanilic acid & 280 & 56.72 & 85.67 & 95.64 & 95.27 & 105.34 & 158.66 & 16.67 \\
\hline 68527 & Alachlor sulfynilacetic acid & 280 & 52.77 & 83.06 & 96.46 & 94.78 & 109.31 & 151.98 & 19.72 \\
\hline 68528 & Aldicarb & 275 & 63.53 & 86.34 & 95.41 & 94.49 & 103.04 & 145.48 & 14.72 \\
\hline 68529 & Aldicarb sulfone & 275 & 70.60 & 89.23 & 99.31 & 97.31 & 106.95 & 150.86 & 14.36 \\
\hline 68530 & Aldicarb sulfoxide & 275 & 64.74 & 88.34 & 96.75 & 95.62 & 103.66 & 143.41 & 12.80 \\
\hline 68533 & Ametryn & 275 & 60.42 & 88.15 & 95.09 & 95.07 & 102.76 & 133.28 & 11.91 \\
\hline 68536 & Asulam & 273 & 33.50 & 87.17 & 98.09 & 97.14 & 108.00 & 161.19 & 18.05 \\
\hline 68538 & Bentazone & 280 & 58.23 & 93.44 & 106.17 & 103.50 & 116.24 & 172.63 & 17.55 \\
\hline 68542 & Bromacil & 275 & 71.12 & 91.55 & 101.28 & 99.95 & 109.96 & 149.90 & 13.95 \\
\hline 68543 & Bromoxynil & 280 & 56.91 & 87.07 & 99.06 & 97.34 & 109.24 & 170.34 & 17.46 \\
\hline
\end{tabular}




\begin{tabular}{|c|c|c|c|c|c|c|c|c|c|}
\hline \multirow[b]{2}{*}{ Pcode } & \multirow[b]{2}{*}{ Parameter name } & \multicolumn{8}{|c|}{ Groundwater } \\
\hline & & Count & Minimum & $\begin{array}{c}\text { First } \\
\text { quartile }\end{array}$ & Mean & Median & $\begin{array}{c}\text { Third } \\
\text { quartile }\end{array}$ & Maximum & $\begin{array}{l}\text { Relative } \\
\text { standard } \\
\text { deviation }\end{array}$ \\
\hline 68336 & 4-Hydroxychlorothalonil & 41 & 61.11 & 89.62 & 101.75 & 97.94 & 114.95 & 148.91 & 19.39 \\
\hline 68426 & Imidacloprid & 40 & 76.27 & 89.93 & 100.87 & 99.74 & 107.67 & 144.82 & 16.70 \\
\hline 68437 & Metalaxyl & 42 & 76.25 & 86.69 & 97.19 & 96.98 & 102.91 & 157.50 & 15.53 \\
\hline 68498 & 1H-1,2,4-Triazole & 21 & 11.22 & 22.22 & 30.17 & 26.15 & 40.23 & 64.73 & 40.53 \\
\hline 68500 & $2,4-\mathrm{D}$ & 38 & 0 & 66.82 & 87.32 & 94.83 & 108.95 & 147.66 & 40.81 \\
\hline 68502 & 2-Aminobenzimidazole & 40 & 77.81 & 87.52 & 100.80 & 96.99 & 106.88 & 179.90 & 20.41 \\
\hline 68503 & 2-Amino-N-isopropylbenzamide & 42 & 73.88 & 89.23 & 95.45 & 95.32 & 100.22 & 118.46 & 11.01 \\
\hline 68505 & 2-Isopropyl-6-methyl-4-pyrimidinol & 42 & 56.10 & 99.56 & 114.26 & 106.89 & 124.56 & 380.88 & 42.28 \\
\hline 68508 & 3-Hydroxycarbofuran & 42 & 7.70 & 80.24 & 86.47 & 89.75 & 97.57 & 120.98 & 22.79 \\
\hline 68511 & 4-(Hydroxymethyl)pendimethalin & 14 & 0 & 68.82 & 90.72 & 91.57 & 111.63 & 144.76 & 40.79 \\
\hline 68514 & 4-Chlorobenzylmethyl sulfoxide & 40 & 78.46 & 95.48 & 106.40 & 104.33 & 118.90 & 134.58 & 13.47 \\
\hline 68515 & 4-Hydroxy molinate & 40 & 80.66 & 89.96 & 98.94 & 98.53 & 106.25 & 117.85 & 9.94 \\
\hline 68517 & 4-Hydroxyhexazinone A & 42 & 81.31 & 92.52 & 98.99 & 97.29 & 105.14 & 123.06 & 9.13 \\
\hline 68519 & Acephate & 42 & 73.00 & 98.97 & 117.46 & 114.38 & 129.65 & 328.80 & 32.36 \\
\hline 68520 & Acetochlor & 42 & 72.41 & 86.44 & 97.19 & 93.55 & 107.48 & 128.13 & 14.60 \\
\hline 68521 & 2-Chloro-N-(2-ethyl-6-methylphenyl)acetamide & 42 & 78.93 & 89.14 & 98.70 & 97.84 & 107.32 & 122.61 & 12.59 \\
\hline 68522 & Acetochlor oxanilic acid & 38 & 58.35 & 81.98 & 97.06 & 94.81 & 107.24 & 135.96 & 19.32 \\
\hline 68523 & Acetochlor sulfonic acid & 1 & 93.89 & 93.89 & 93.89 & 93.89 & 93.89 & 93.89 & \\
\hline 68524 & Acetochlor sulfynilacetic acid & 27 & 72.05 & 91.61 & 103.85 & 102.69 & 116.61 & 141.65 & 15.80 \\
\hline 68525 & 2-Chloro-2',6'-diethylacetanilide & 42 & 77.12 & 91.93 & 100.74 & 98.94 & 110.92 & 124.45 & 12.21 \\
\hline 68526 & Alachlor oxanilic acid & 41 & 70.56 & 90.48 & 98.03 & 96.57 & 105.02 & 133.58 & 15.04 \\
\hline 68527 & Alachlor sulfynilacetic acid & 28 & 59.39 & 86.82 & 97.93 & 97.69 & 113.24 & 136.09 & 18.82 \\
\hline 68528 & Aldicarb & 42 & 60.23 & 82.24 & 90.63 & 91.69 & 97.36 & 111.24 & 12.74 \\
\hline 68529 & Aldicarb sulfone & 40 & 60.82 & 87.86 & 97.03 & 98.86 & 104.77 & 132.66 & 16.30 \\
\hline 68530 & Aldicarb sulfoxide & 42 & 79.09 & 88.34 & 95.97 & 95.62 & 103.63 & 124.16 & 10.90 \\
\hline 68533 & Ametryn & 42 & 84.81 & 93.41 & 101.58 & 101.57 & 107.35 & 129.60 & 10.97 \\
\hline 68536 & Asulam & 38 & 0 & 17.57 & 33.39 & 23.23 & 39.54 & 139.84 & 94.78 \\
\hline 68538 & Bentazone & 39 & 54.07 & 93.11 & 104.36 & 102.93 & 117.39 & 137.97 & 16.74 \\
\hline 68542 & Bromacil & 41 & 49.54 & 88.38 & 95.97 & 98.27 & 105.64 & 120.40 & 15.37 \\
\hline 68543 & Bromoxynil & 42 & 66.71 & 84.17 & 96.95 & 94.82 & 107.37 & 149.19 & 18.20 \\
\hline
\end{tabular}


Table 4. Summary statistics for the recovery of schedule 2437 pesticides in lab reagent spikes, and groundwater and surface-water field matrix spike samples.-Continued

[ _ , values that do not meet method validation data-quality objectives; ___ , recovery values in which the first quartile is greater than or third quartile is less than 100 percent]

\begin{tabular}{|c|c|c|c|c|c|c|c|c|c|}
\hline \multirow[b]{2}{*}{ Pcode } & \multirow[b]{2}{*}{ Parameter name } & \multicolumn{8}{|c|}{ Surface water } \\
\hline & & Count & Minimum & $\begin{array}{c}\text { First } \\
\text { quartile }\end{array}$ & Mean & Median & $\begin{array}{c}\text { Third } \\
\text { quartile }\end{array}$ & Maximum & $\begin{array}{l}\text { Relative } \\
\text { standard } \\
\text { deviation }\end{array}$ \\
\hline 68336 & 4-Hydroxychlorothalonil & 286 & 46.02 & 92.35 & 108.50 & 108.65 & 121.98 & 183.98 & 20.37 \\
\hline 68426 & Imidacloprid & 287 & 65.98 & 103.35 & 119.94 & 113.87 & 134.51 & 241.12 & 20.91 \\
\hline 68437 & Metalaxyl & 288 & 64.16 & 91.77 & 100.91 & 100.08 & 109.39 & 156.87 & 14.09 \\
\hline 68498 & 1H-1,2,4-Triazole & 73 & 0 & 22.56 & 49.62 & 39.34 & 71.01 & 171.89 & 76.52 \\
\hline 68500 & $2,4-\mathrm{D}$ & 241 & 0 & 93.15 & 105.14 & 102.86 & 117.56 & 187.13 & 21.67 \\
\hline 68502 & 2-Aminobenzimidazole & 286 & 63.42 & 92.10 & 102.53 & 100.57 & 111.12 & 161.21 & 15.29 \\
\hline 68503 & 2-Amino-N-isopropylbenzamide & 289 & 52.86 & 90.68 & 99.82 & 99.36 & 108.32 & 147.92 & 14.81 \\
\hline 68505 & 2-Isopropyl-6-methyl-4-pyrimidinol & 288 & 41.23 & 86.03 & 99.65 & 96.66 & 111.11 & 287.50 & 25.94 \\
\hline 68508 & 3-Hydroxycarbofuran & 289 & 0 & 68.74 & 76.89 & 80.79 & 91.03 & 136.73 & 35.36 \\
\hline 68511 & 4-(Hydroxymethyl)pendimethalin & 263 & 0 & 78.01 & 91.08 & 89.55 & 104.79 & 175.20 & 26.86 \\
\hline 68514 & 4-Chlorobenzylmethyl sulfoxide & 289 & 51.34 & 91.63 & 101.62 & 102.04 & 112.89 & 164.18 & 15.62 \\
\hline 68515 & 4-Hydroxy molinate & 289 & 61.73 & 89.47 & 98.41 & 96.63 & 106.24 & 141.96 & 13.86 \\
\hline 68517 & 4-Hydroxyhexazinone A & 289 & 60.82 & 94.58 & 102.70 & 101.26 & 108.60 & 144.28 & 11.95 \\
\hline 68519 & Acephate & 288 & 21.81 & 90.92 & 105.22 & 101.71 & 117.49 & 286.40 & 26.64 \\
\hline 68520 & Acetochlor & 284 & 61.42 & 89.90 & 97.76 & 96.51 & 105.57 & 154.53 & 13.38 \\
\hline 68521 & 2-Chloro-N-(2-ethyl-6-methylphenyl)acetamide & 288 & 58.23 & 90.90 & 101.05 & 98.64 & 109.77 & 160.24 & 15.28 \\
\hline 68522 & Acetochlor oxanilic acid & 265 & 56.89 & 83.27 & 98.38 & 97.38 & 109.67 & 209.99 & 20.64 \\
\hline 68523 & Acetochlor sulfonic acid & 2 & 74.73 & 74.73 & 104.87 & 104.87 & 135.00 & 135.00 & 40.64 \\
\hline 68524 & Acetochlor sulfynilacetic acid & 279 & 42.08 & 90.10 & 106.02 & 105.03 & 118.61 & 193.22 & 24.16 \\
\hline 68525 & 2-Chloro-2',6'-diethylacetanilide & 289 & 65.90 & 91.88 & 102.00 & 99.90 & 109.53 & 148.22 & 15.01 \\
\hline 68526 & Alachlor oxanilic acid & 289 & 61.40 & 89.27 & 99.58 & 97.93 & 108.49 & 148.67 & 15.57 \\
\hline 68527 & Alachlor sulfynilacetic acid & 285 & 54.35 & 85.10 & 96.84 & 96.94 & 107.07 & 151.59 & 18.33 \\
\hline 68528 & Aldicarb & 289 & 55.64 & 84.02 & 93.92 & 92.52 & 99.77 & 162.34 & 16.43 \\
\hline 68529 & Aldicarb sulfone & 276 & 14.00 & 88.78 & 97.70 & 98.38 & 109.43 & 162.76 & 24.49 \\
\hline 68530 & Aldicarb sulfoxide & 289 & 9.65 & 86.25 & 93.37 & 95.45 & 103.14 & 152.35 & 22.84 \\
\hline 68533 & Ametryn & 288 & 65.70 & 93.18 & 101.27 & 100.85 & 108.45 & 140.69 & 13.09 \\
\hline 68536 & Asulam & 260 & 0 & 24.07 & 37.99 & 32.50 & 46.28 & 274.04 & 73.16 \\
\hline 68538 & Bentazone & 264 & 35.73 & 95.54 & 112.68 & 108.35 & 126.86 & 210.48 & 23.39 \\
\hline 68542 & Bromacil & 284 & 49.91 & 91.51 & 99.49 & 98.61 & 107.53 & 152.41 & 14.53 \\
\hline 68543 & Bromoxynil & 289 & 50.33 & 89.97 & 101.43 & 99.66 & 110.84 & 174.13 & 17.60 \\
\hline
\end{tabular}




\begin{tabular}{|c|c|c|c|c|c|c|c|c|c|}
\hline \multirow[b]{2}{*}{ Pcode } & \multirow[b]{2}{*}{ Parameter name } & \multicolumn{8}{|c|}{ Lab reagent spikes } \\
\hline & & Count & Minimum & $\begin{array}{c}\text { First } \\
\text { quartile }\end{array}$ & Mean & Median & $\begin{array}{c}\text { Third } \\
\text { quartile }\end{array}$ & Maximum & $\begin{array}{l}\text { Relative } \\
\text { standard } \\
\text { deviation }\end{array}$ \\
\hline 68545 & Butralin & 274 & 53.52 & 79.57 & 87.84 & 87.25 & 96.19 & 125.08 & 13.82 \\
\hline 68547 & $\begin{array}{l}\text { 2-Chloro-4,6-diamino-s-triazine }\{\text { CAAT }\} \\
\text { (Didealkylatrazine) }\end{array}$ & 275 & 61.64 & 86.55 & 95.15 & 94.10 & 103.52 & 148.57 & 14.54 \\
\hline 68548 & Carbendazim & 275 & 67.78 & 90.79 & 101.40 & 97.59 & 107.31 & 185.33 & 19.34 \\
\hline 68549 & Carboxy molinate & 275 & 62.04 & 87.42 & 96.66 & 96.17 & 105.43 & 129.76 & 13.21 \\
\hline 68550 & 2-Chloro-6-ethylamino-4-amino-s-triazine $\{\mathrm{CEAT}\}$ & 275 & 57.66 & 86.05 & 95.28 & 93.76 & 104.77 & 135.92 & 14.30 \\
\hline 68551 & Chlorosulfonamide acid & 282 & 46.03 & 85.20 & 95.59 & 95.27 & 105.26 & 151.95 & 16.59 \\
\hline 68552 & 2-Chloro-4-isopropylamino-6-amino-s-triazine & 275 & 63.23 & 87.80 & 95.17 & 94.90 & 102.89 & 129.54 & 12.37 \\
\hline 68561 & Dechlorofipronil & 280 & 52.40 & 80.00 & 89.64 & 87.91 & 97.72 & 141.19 & 16.10 \\
\hline 68562 & Dechlorometolachlor & 275 & 68.32 & 85.84 & 94.11 & 92.62 & 100.78 & 144.02 & 12.24 \\
\hline 68563 & Deiodo flubendiamide & 275 & 45.47 & 75.48 & 85.23 & 85.75 & 94.01 & 128.06 & 16.45 \\
\hline 68564 & Deisopropyl prometryn & 275 & 65.76 & 87.08 & 94.58 & 93.35 & 102.43 & 135.99 & 12.38 \\
\hline 68566 & Demethyl hexazinone B & 275 & 64.68 & 88.74 & 96.26 & 97.04 & 102.55 & 129.68 & 10.95 \\
\hline 68567 & Demethyl norflurazon & 275 & 62.31 & 84.15 & 92.39 & 92.25 & 100.04 & 131.56 & 12.58 \\
\hline 68568 & Desamino metribuzin & 275 & 60.66 & 87.74 & 94.99 & 95.25 & 101.90 & 128.75 & 11.73 \\
\hline 68569 & Desamino-diketo metribuzin & 273 & 19.59 & 78.79 & 94.71 & 94.06 & 109.74 & 194.43 & 25.20 \\
\hline 68570 & Desulfinylfipronil amide & 280 & 58.41 & 87.52 & 97.96 & 96.70 & 106.77 & 146.51 & 15.98 \\
\hline 68572 & Dichlorvos & 275 & 37.66 & 84.00 & 97.17 & 97.39 & 110.82 & 190.81 & 24.15 \\
\hline 68573 & Dicrotophos & 275 & 66.76 & 91.09 & 102.77 & 101.46 & 112.05 & 187.46 & 16.55 \\
\hline 68574 & Didemethyl hexazinone F & 275 & 61.41 & 85.70 & 93.28 & 92.60 & 100.69 & 128.73 & 12.37 \\
\hline 68575 & Tebuthiuron TP 104 & 275 & 70.35 & 89.56 & 97.04 & 96.26 & 103.79 & 133.47 & 11.84 \\
\hline 68576 & Diflubenzuron & 275 & 62.74 & 83.93 & 91.11 & 91.02 & 97.29 & 133.79 & 12.08 \\
\hline 68577 & Diflufenzopyr & 280 & 53.81 & 93.41 & 107.35 & 107.45 & 119.14 & 220.98 & 21.36 \\
\hline 68578 & Diketonitrile-isoxaflutole & 280 & 64.02 & 95.54 & 125.26 & 112.83 & 140.19 & 285.88 & 33.19 \\
\hline 68580 & Dimethenamid & 275 & 64.62 & 86.43 & 96.21 & 96.61 & 104.93 & 135.83 & 13.51 \\
\hline 68581 & Dimethenamid oxanilic acid & 280 & 55.59 & 87.89 & 98.07 & 97.53 & 107.62 & 145.46 & 15.66 \\
\hline 68582 & Dimethenamid sulfonic acid & 280 & 47.90 & 84.60 & 97.08 & 96.10 & 108.76 & 157.43 & 18.80 \\
\hline 68583 & Dimethenamid SAA & 280 & 37.86 & 88.06 & 107.32 & 104.56 & 122.12 & 220.07 & 25.41 \\
\hline 68586 & Disulfoton oxon & 275 & 64.04 & 90.46 & 103.30 & 102.70 & 112.26 & 206.68 & 17.74 \\
\hline 68587 & Disulfoton oxon sulfoxide & 275 & 61.19 & 90.73 & 101.88 & 100.82 & 110.75 & 186.23 & 17.10 \\
\hline 68588 & Disulfoton oxon sulfone & 275 & 50.36 & 82.96 & 95.03 & 95.20 & 105.61 & 147.35 & 18.20 \\
\hline
\end{tabular}


Table 4. Summary statistics for the recovery of schedule 2437 pesticides in lab reagent spikes, and groundwater and surface-water field matrix spike samples. - Continued

[ _ , values that do not meet method validation data-quality objectives; ___ , recovery values in which the first quartile is greater than or third quartile is less than 100 percent]

\begin{tabular}{|c|c|c|c|c|c|c|c|c|c|}
\hline \multirow[b]{2}{*}{ Pcode } & \multirow[b]{2}{*}{ Parameter name } & \multicolumn{8}{|c|}{ Groundwater } \\
\hline & & Count & Minimum & $\begin{array}{c}\text { First } \\
\text { quartile }\end{array}$ & Mean & Median & $\begin{array}{c}\text { Third } \\
\text { quartile }\end{array}$ & Maximum & $\begin{array}{l}\text { Relative } \\
\text { standard } \\
\text { deviation }\end{array}$ \\
\hline 68545 & Butralin & 42 & 70.44 & 84.64 & 97.05 & 95.56 & 100.81 & 155.07 & 21.17 \\
\hline 68547 & $\begin{array}{l}\text { 2-Chloro-4,6-diamino-s-triazine }\{\text { CAAT }\} \\
\text { (Didealkylatrazine) }\end{array}$ & 14 & 82.34 & 115.09 & 140.14 & 126.53 & 151.17 & 249.97 & 35.91 \\
\hline 68548 & Carbendazim & 38 & 84.09 & 99.01 & 108.32 & 104.64 & 118.26 & 156.91 & 14.56 \\
\hline 68549 & Carboxy molinate & 40 & 79.94 & 87.80 & 98.38 & 97.22 & 107.01 & 122.92 & 11.72 \\
\hline 68550 & 2-Chloro-6-ethylamino-4-amino-s-triazine $\{\mathrm{CEAT}\}$ & 39 & 78.01 & 87.90 & 98.62 & 97.08 & 106.05 & 131.77 & 13.63 \\
\hline 68551 & Chlorosulfonamide acid & 40 & 0 & 23.77 & 56.35 & 49.85 & 86.31 & 154.00 & 72.59 \\
\hline 68552 & 2-Chloro-4-isopropylamino-6-amino-s-triazine & 35 & 69.87 & 93.56 & 98.39 & 98.18 & 106.51 & 120.76 & 10.86 \\
\hline 68561 & Dechlorofipronil & 39 & 80.22 & 89.07 & 100.66 & 97.69 & 106.56 & 134.75 & 13.56 \\
\hline 68562 & Dechlorometolachlor & 37 & 82.35 & 90.06 & 97.58 & 97.50 & 103.93 & 117.82 & 9.52 \\
\hline 68563 & Deiodo flubendiamide & 38 & 64.65 & 82.41 & 94.06 & 95.25 & 106.22 & 122.27 & 17.15 \\
\hline 68564 & Deisopropyl prometryn & 42 & 79.28 & 90.57 & 100.04 & 99.49 & 110.50 & 124.16 & 11.21 \\
\hline 68566 & Demethyl hexazinone B & 42 & 87.45 & 96.63 & 102.01 & 100.91 & 107.23 & 122.29 & 8.13 \\
\hline 68567 & Demethyl norflurazon & 42 & 77.20 & 90.86 & 99.76 & 95.75 & 105.88 & 163.69 & 14.98 \\
\hline 68568 & Desamino metribuzin & 42 & 78.21 & 94.28 & 99.78 & 98.83 & 105.74 & 123.25 & 9.48 \\
\hline 68569 & Desamino-diketo metribuzin & 17 & 66.39 & 81.67 & 99.17 & 100.10 & 110.13 & 137.41 & 21.48 \\
\hline 68570 & Desulfinylfipronil amide & 36 & 76.28 & 84.88 & 94.63 & 92.15 & 102.21 & 133.08 & 13.13 \\
\hline 68572 & Dichlorvos & 35 & 0 & 68.95 & 78.52 & 79.78 & 93.53 & 117.45 & 27.32 \\
\hline 68573 & Dicrotophos & 42 & 64.32 & 95.76 & 111.01 & 105.88 & 116.92 & 339.73 & 35.41 \\
\hline 68574 & Didemethyl hexazinone F & 40 & 77.20 & 89.72 & 96.63 & 94.57 & 102.31 & 127.06 & 11.43 \\
\hline 68575 & Tebuthiuron TP 104 & 40 & 89.98 & 96.44 & 105.96 & 104.72 & 113.42 & 141.58 & 11.17 \\
\hline 68576 & Diflubenzuron & 42 & 78.65 & 87.76 & 94.35 & 93.28 & 99.51 & 111.01 & 9.49 \\
\hline 68577 & Diflufenzopyr & 42 & 65.56 & 83.38 & 100.70 & 93.69 & 110.72 & 167.17 & 24.01 \\
\hline 68578 & Diketonitrile-isoxaflutole & 42 & 85.18 & 117.05 & 143.63 & 138.97 & 169.54 & 245.77 & 25.45 \\
\hline 68580 & Dimethenamid & 42 & 77.12 & 89.52 & 98.83 & 96.70 & 109.23 & 130.08 & 12.88 \\
\hline 68581 & Dimethenamid oxanilic acid & 37 & 71.96 & 86.13 & 98.02 & 94.76 & 104.22 & 141.39 & 18.29 \\
\hline 68582 & Dimethenamid sulfonic acid & 42 & 63.32 & 83.96 & 98.25 & 98.26 & 109.53 & 136.32 & 19.97 \\
\hline 68583 & Dimethenamid SAA & 17 & 0 & 70.42 & 84.96 & 89.71 & 105.16 & 136.42 & 37.19 \\
\hline 68586 & Disulfoton oxon & 42 & 65.89 & 93.20 & 106.41 & 101.73 & 112.03 & 314.82 & 33.61 \\
\hline 68587 & Disulfoton oxon sulfoxide & 42 & 67.95 & 95.97 & 110.82 & 103.86 & 119.61 & 303.32 & 30.73 \\
\hline 68588 & Disulfoton oxon sulfone & 42 & 13.13 & 79.78 & 89.69 & 89.57 & 101.51 & 129.71 & 22.59 \\
\hline
\end{tabular}




\begin{tabular}{|c|c|c|c|c|c|c|c|c|c|}
\hline \multirow[b]{2}{*}{ Pcode } & \multirow[b]{2}{*}{ Parameter name } & \multicolumn{8}{|c|}{ Surface water } \\
\hline & & Count & Minimum & $\begin{array}{c}\text { First } \\
\text { quartile }\end{array}$ & Mean & Median & $\begin{array}{c}\text { Third } \\
\text { quartile }\end{array}$ & Maximum & $\begin{array}{l}\text { Relative } \\
\text { standard } \\
\text { deviation }\end{array}$ \\
\hline 68545 & Butralin & 289 & 37.93 & 82.84 & 99.86 & 95.62 & 114.34 & 193.66 & 24.63 \\
\hline 68547 & $\begin{array}{l}\text { 2-Chloro-4,6-diamino-s-triazine }\{\mathrm{CAAT}\} \\
\text { (Didealkylatrazine) }\end{array}$ & 170 & 34.49 & 126.58 & 160.32 & 148.26 & 183.08 & 421.09 & 36.11 \\
\hline 68548 & Carbendazim & 277 & 0 & 95.02 & 107.54 & 106.67 & 116.01 & 179.68 & 20.04 \\
\hline 68549 & Carboxy molinate & 285 & 63.22 & 88.00 & 98.06 & 97.68 & 105.74 & 154.86 & 15.71 \\
\hline 68550 & 2-Chloro-6-ethylamino-4-amino-s-triazine $\{\mathrm{CEAT}\}$ & 279 & 18.42 & 86.42 & 96.31 & 95.01 & 105.00 & 152.41 & 16.09 \\
\hline 68551 & Chlorosulfonamide acid & 268 & 0 & 50.88 & 90.43 & 87.29 & 120.40 & 308.40 & 57.00 \\
\hline 68552 & 2-Chloro-4-isopropylamino-6-amino-s-triazine & 268 & 56.44 & 88.43 & 97.88 & 97.24 & 106.17 & 146.25 & 13.99 \\
\hline 68561 & Dechlorofipronil & 279 & 10.77 & 85.02 & 92.16 & 94.42 & 104.39 & 155.75 & 24.12 \\
\hline 68562 & Dechlorometolachlor & 268 & 0.20 & 83.33 & 92.27 & 92.08 & 98.93 & 135.91 & 14.78 \\
\hline 68563 & Deiodo flubendiamide & 257 & 46.54 & 81.22 & 92.46 & 91.01 & 101.74 & 131.33 & 16.27 \\
\hline 68564 & Deisopropyl prometryn & 289 & 52.66 & 96.67 & 107.76 & 106.80 & 117.14 & 171.48 & 14.68 \\
\hline 68566 & Demethyl hexazinone B & 286 & 60.34 & 91.48 & 99.35 & 97.83 & 104.98 & 135.25 & 11.84 \\
\hline 68567 & Demethyl norflurazon & 278 & 60.08 & 90.17 & 99.10 & 96.74 & 104.83 & 151.57 & 13.73 \\
\hline 68568 & Desamino metribuzin & 288 & 55.40 & 89.28 & 98.05 & 97.60 & 105.45 & 141.22 & 13.54 \\
\hline 68569 & Desamino-diketo metribuzin & 266 & 0 & 73.84 & 91.69 & 88.87 & 109.44 & 196.18 & 30.28 \\
\hline 68570 & Desulfinylfipronil amide & 282 & 63.71 & 86.83 & 97.32 & 96.33 & 107.08 & 160.05 & 14.98 \\
\hline 68572 & Dichlorvos & 278 & 0 & 68.37 & 79.33 & 81.90 & 94.82 & 194.14 & 36.91 \\
\hline 68573 & Dicrotophos & 280 & 56.91 & 90.67 & 103.76 & 101.33 & 113.36 & 260.71 & 21.53 \\
\hline 68574 & Didemethyl hexazinone F & 289 & 57.23 & 86.99 & 96.43 & 95.80 & 103.58 & 137.88 & 13.60 \\
\hline 68575 & Tebuthiuron TP 104 & 289 & 73.94 & 98.65 & 111.78 & 107.97 & 120.21 & 206.93 & 17.49 \\
\hline 68576 & Diflubenzuron & 289 & 44.96 & 85.99 & 91.81 & 91.79 & 98.77 & 131.53 & 15.28 \\
\hline 68577 & Diflufenzopyr & 289 & 45.49 & 87.42 & 101.58 & 99.11 & 115.59 & 226.24 & 24.18 \\
\hline 68578 & Diketonitrile-isoxaflutole & 289 & 95.90 & 131.90 & 168.37 & 158.02 & 193.77 & 361.68 & 28.68 \\
\hline 68580 & Dimethenamid & 289 & 62.10 & 90.15 & 99.18 & 97.19 & 107.02 & 161.10 & 14.73 \\
\hline 68581 & Dimethenamid oxanilic acid & 282 & 61.90 & 88.95 & 100.14 & 98.54 & 110.94 & 152.93 & 16.81 \\
\hline 68582 & Dimethenamid sulfonic acid & 288 & 65.30 & 86.36 & 97.09 & 95.89 & 104.96 & 185.58 & 16.55 \\
\hline 68583 & Dimethenamid SAA & 262 & 15.74 & 82.90 & 100.18 & 98.20 & 115.35 & 174.99 & 25.55 \\
\hline 68586 & Disulfoton oxon & 282 & 55.83 & 89.07 & 103.26 & 99.20 & 112.92 & 258.37 & 21.99 \\
\hline 68587 & Disulfoton oxon sulfoxide & 286 & 46.39 & 91.20 & 105.90 & 103.51 & 118.75 & 246.75 & 22.33 \\
\hline 68588 & Disulfoton oxon sulfone & 289 & 0 & 63.85 & 74.09 & 78.71 & 91.55 & 191.14 & 40.02 \\
\hline
\end{tabular}


Table 4. Summary statistics for the recovery of schedule 2437 pesticides in lab reagent spikes, and groundwater and surface-water field matrix spike samples.-Continued

[ _ , values that do not meet method validation data-quality objectives; __ , recovery values in which the first quartile is greater than or third quartile is less than 100 percent]

\begin{tabular}{|c|c|c|c|c|c|c|c|c|c|}
\hline \multirow[b]{2}{*}{ Pcode } & \multirow[b]{2}{*}{ Parameter name } & \multicolumn{8}{|c|}{ Lab reagent spikes } \\
\hline & & Count & Minimum & $\begin{array}{c}\text { First } \\
\text { quartile }\end{array}$ & Mean & Median & $\begin{array}{c}\text { Third } \\
\text { quartile }\end{array}$ & Maximum & $\begin{array}{l}\text { Relative } \\
\text { standard } \\
\text { deviation }\end{array}$ \\
\hline 68589 & Disulfoton sulfone & 275 & 57.06 & 85.83 & 98.25 & 97.78 & 107.58 & 153.67 & 17.36 \\
\hline 68590 & Disulfoton sulfoxide & 275 & 64.90 & 93.14 & 105.44 & 103.53 & 116.21 & 194.81 & 17.29 \\
\hline 68591 & Demethyl fluometuron & 275 & 0.00 & 87.50 & 94.82 & 95.08 & 101.94 & 133.16 & 12.75 \\
\hline 68594 & EPTC degradate R248722 & 275 & 63.58 & 88.17 & 97.02 & 96.72 & 106.14 & 128.92 & 12.57 \\
\hline 68595 & 2-[(2-Ethyl-6-methylphenyl)amino]-1-propanol & 275 & 60.78 & 86.99 & 96.30 & 96.08 & 105.18 & 135.11 & 13.49 \\
\hline 68596 & Ethoprophos & 275 & 61.24 & 86.71 & 95.87 & 95.21 & 104.55 & 147.64 & 14.47 \\
\hline 68597 & O-Ethyl-O-methyl-S-propylphosphorothioate & 275 & 61.03 & 90.86 & 109.99 & 103.49 & 120.50 & 264.38 & 25.19 \\
\hline 68598 & Etoxazole & 274 & 37.21 & 71.03 & 79.96 & 80.29 & 88.97 & 121.14 & 17.68 \\
\hline 68599 & Fenamiphos & 275 & 59.79 & 84.04 & 93.64 & 92.60 & 101.99 & 148.24 & 14.15 \\
\hline 68600 & Fenamiphos sulfone & 275 & 61.37 & 87.87 & 100.35 & 98.85 & 109.28 & 200.15 & 17.76 \\
\hline 68601 & Fenamiphos sulfoxide & 275 & 61.44 & 89.83 & 101.26 & 98.85 & 109.64 & 181.96 & 16.89 \\
\hline 68602 & Fenbutatin oxide & 275 & 14.22 & 68.10 & 104.81 & 92.98 & 125.93 & 357.55 & 51.74 \\
\hline 68603 & Fentin & 275 & 57.48 & 83.56 & 93.54 & 92.72 & 101.99 & 158.84 & 15.51 \\
\hline 68604 & Fipronil amide & 280 & 58.00 & 87.27 & 99.05 & 97.74 & 109.16 & 154.48 & 16.68 \\
\hline 68605 & Fipronil sulfonate & 278 & 58.52 & 86.94 & 98.71 & 97.18 & 109.26 & 154.63 & 17.73 \\
\hline 68606 & Flubendiamide & 280 & 43.85 & 80.11 & 93.75 & 91.30 & 104.03 & 170.84 & 20.50 \\
\hline 68608 & Fluometuron & 275 & 44.22 & 88.37 & 94.57 & 95.16 & 100.78 & 132.20 & 11.67 \\
\hline 68611 & 2-(1-Hydroxyethyl)-6-methylaniline & 274 & 14.15 & 82.21 & 109.37 & 98.51 & 129.57 & 305.99 & 41.56 \\
\hline 68612 & Hexazinone Transformation Product C & 275 & 65.21 & 86.05 & 93.66 & 93.44 & 99.56 & 124.76 & 11.01 \\
\hline 68613 & Hexazinone Transformation Product D & 287 & 22.94 & 86.06 & 102.19 & 100.40 & 118.48 & 185.25 & 27.06 \\
\hline 68614 & Hexazinone Transformation Product E & 270 & 49.25 & 85.53 & 96.32 & 94.39 & 106.95 & 145.06 & 17.39 \\
\hline 68615 & Hydroxyacetochlor & 275 & 63.49 & 83.95 & 95.37 & 94.12 & 106.49 & 167.16 & 16.17 \\
\hline 68616 & Hydroxyalachlor & 275 & 59.50 & 84.77 & 95.45 & 95.10 & 105.71 & 136.24 & 15.09 \\
\hline 68617 & Hydroxy monodemethyl fluometuron & 275 & 50.21 & 81.94 & 89.49 & 90.02 & 96.98 & 128.47 & 14.48 \\
\hline 68618 & Hydroxydiazinon & 275 & 67.54 & 87.31 & 98.13 & 97.05 & 108.95 & 161.85 & 16.24 \\
\hline 68619 & Hydroxy didemethyl fluometuron & 275 & 56.87 & 82.39 & 92.71 & 92.41 & 100.44 & 151.29 & 16.05 \\
\hline 68620 & Hydroxyfluometuron & 275 & 45.69 & 81.41 & 89.14 & 89.52 & 96.46 & 139.17 & 14.40 \\
\hline 68621 & Tebuthiuron TP 109 & 273 & 49.16 & 81.23 & 90.18 & 90.58 & 97.91 & 127.21 & 13.73 \\
\hline 68622 & Hydroxymetolachlor & 275 & 72.49 & 86.08 & 94.66 & 93.49 & 101.49 & 144.22 & 12.04 \\
\hline 68623 & Hydroxyphthalazinone & 275 & 68.92 & 88.95 & 95.64 & 95.14 & 101.78 & 141.34 & 11.66 \\
\hline 68624 & Hydroxysimazine & 274 & 51.20 & 88.04 & 97.53 & 96.88 & 106.05 & 143.67 & 16.57 \\
\hline
\end{tabular}




\begin{tabular}{|c|c|c|c|c|c|c|c|c|c|}
\hline \multirow[b]{2}{*}{ Pcode } & \multirow[b]{2}{*}{ Parameter name } & \multicolumn{8}{|c|}{ Groundwater } \\
\hline & & Count & Minimum & $\begin{array}{c}\text { First } \\
\text { quartile }\end{array}$ & Mean & Median & $\begin{array}{c}\text { Third } \\
\text { quartile }\end{array}$ & Maximum & $\begin{array}{l}\text { Relative } \\
\text { standard } \\
\text { deviation }\end{array}$ \\
\hline 68589 & Disulfoton sulfone & 42 & 56.23 & 87.89 & 94.93 & 96.75 & 107.99 & 146.06 & 21.48 \\
\hline 68590 & Disulfoton sulfoxide & 42 & 71.57 & 104.17 & 120.76 & 111.16 & 125.17 & 427.38 & 42.19 \\
\hline 68591 & Demethyl fluometuron & 38 & 82.23 & 93.81 & 101.10 & 100.13 & 109.12 & 118.15 & 9.58 \\
\hline 68594 & EPTC degradate R248722 & 42 & 69.91 & 90.82 & 99.47 & 99.42 & 107.27 & 120.09 & 11.71 \\
\hline 68595 & 2-[(2-Ethyl-6-methylphenyl)amino]-1-propanol & 42 & 71.24 & 92.58 & 100.89 & 100.17 & 109.46 & 144.89 & 13.59 \\
\hline 68596 & Ethoprophos & 42 & 65.34 & 91.87 & 98.87 & 98.30 & 105.74 & 132.37 & 12.73 \\
\hline 68597 & O-Ethyl-O-methyl-S-propylphosphorothioate & 42 & 66.27 & 99.36 & 117.66 & 112.87 & 125.16 & 344.83 & 34.45 \\
\hline 68598 & Etoxazole & 42 & 60.01 & 73.03 & 81.94 & 81.33 & 89.96 & 115.93 & 15.15 \\
\hline 68599 & Fenamiphos & 42 & 68.15 & 87.13 & 95.96 & 95.32 & 103.90 & 126.51 & 13.40 \\
\hline 68600 & Fenamiphos sulfone & 41 & 63.90 & 95.09 & 109.36 & 102.62 & 114.72 & 334.19 & 35.77 \\
\hline 68601 & Fenamiphos sulfoxide & 42 & 71.87 & 97.27 & 111.78 & 106.54 & 115.19 & 361.06 & 37.88 \\
\hline 68602 & Fenbutatin oxide & 35 & 0 & 24.55 & 45.92 & 36.42 & 64.57 & 148.94 & 75.61 \\
\hline 68603 & Fentin & 42 & 64.41 & 79.71 & 89.19 & 87.82 & 97.99 & 125.77 & 16.72 \\
\hline 68604 & Fipronil amide & 39 & 77.73 & 87.42 & 100.41 & 99.79 & 110.14 & 143.55 & 14.32 \\
\hline 68605 & Fipronil sulfonate & 42 & 69.49 & 89.10 & 108.87 & 105.79 & 124.85 & 175.18 & 22.70 \\
\hline 68606 & Flubendiamide & 40 & 76.29 & 92.59 & 105.08 & 103.42 & 117.85 & 143.86 & 15.93 \\
\hline 68608 & Fluometuron & 35 & 84.55 & 93.91 & 101.26 & 98.47 & 108.00 & 123.94 & 8.82 \\
\hline 68611 & 2-(1-Hydroxyethyl)-6-methylaniline & 31 & 16.18 & 42.18 & 86.70 & 71.30 & 106.00 & 239.20 & 72.42 \\
\hline 68612 & Hexazinone Transformation Product C & 42 & 81.53 & 94.18 & 100.07 & 99.56 & 105.64 & 122.08 & 9.62 \\
\hline 68613 & Hexazinone Transformation Product D & 1 & 96.23 & 96.23 & 96.23 & 96.23 & 96.23 & 96.23 & \\
\hline 68614 & Hexazinone Transformation Product E & 34 & 41.29 & 61.71 & 81.02 & 83.84 & 95.45 & 118.71 & 25.89 \\
\hline 68615 & Hydroxyacetochlor & 36 & 66.51 & 82.69 & 95.32 & 94.20 & 106.61 & 122.85 & 16.20 \\
\hline 68616 & Hydroxyalachlor & 42 & 63.38 & 78.77 & 93.14 & 93.61 & 108.39 & 121.15 & 17.34 \\
\hline 68617 & Hydroxy monodemethyl fluometuron & 42 & 0 & 31.07 & 57.90 & 55.05 & 86.29 & 127.11 & 56.05 \\
\hline 68618 & Hydroxydiazinon & 42 & 76.32 & 85.38 & 101.80 & 98.51 & 109.90 & 157.45 & 19.18 \\
\hline 68619 & Hydroxy didemethyl fluometuron & 42 & 0 & 53.55 & 70.95 & 69.85 & 94.43 & 112.05 & 35.27 \\
\hline 68620 & Hydroxyfluometuron & 40 & 0 & 15.19 & 48.35 & 42.82 & 78.40 & 127.07 & 77.53 \\
\hline 68621 & Tebuthiuron TP 109 & 40 & 8.62 & 81.21 & 88.41 & 88.92 & 98.69 & 142.95 & 22.22 \\
\hline 68622 & Hydroxymetolachlor & 35 & 79.66 & 89.55 & 96.44 & 96.78 & 101.68 & 119.41 & 9.50 \\
\hline 68623 & Hydroxyphthalazinone & 41 & 70.74 & 91.58 & 98.76 & 100.22 & 104.78 & 134.57 & 11.64 \\
\hline 68624 & Hydroxysimazine & 42 & 63.73 & 96.11 & 109.32 & 108.63 & 120.63 & 162.92 & 18.47 \\
\hline
\end{tabular}


Table 4. Summary statistics for the recovery of schedule 2437 pesticides in lab reagent spikes, and groundwater and surface-water field matrix spike samples.-Continued [ _ , values that do not meet method validation data-quality objectives; ___ , recovery values in which the first quartile is greater than or third quartile is less than 100 percent]

\begin{tabular}{|c|c|c|c|c|c|c|c|c|c|}
\hline \multirow[b]{2}{*}{ Pcode } & \multirow[b]{2}{*}{ Parameter name } & \multicolumn{8}{|c|}{ Surface water } \\
\hline & & Count & Minimum & $\begin{array}{c}\text { First } \\
\text { quartile }\end{array}$ & Mean & Median & $\begin{array}{c}\text { Third } \\
\text { quartile }\end{array}$ & Maximum & $\begin{array}{l}\text { Relative } \\
\text { standard } \\
\text { deviation }\end{array}$ \\
\hline 68589 & Disulfoton sulfone & 278 & 0 & 76.44 & 85.34 & 90.82 & 104.32 & 189.92 & 35.32 \\
\hline 68590 & Disulfoton sulfoxide & 286 & 58.46 & 103.08 & 117.67 & 113.88 & 127.29 & 298.86 & 21.76 \\
\hline 68591 & Demethyl fluometuron & 280 & 0.00 & 84.69 & 94.29 & 94.12 & 102.79 & 139.92 & 15.59 \\
\hline 68594 & EPTC degradate R248722 & 269 & 70.30 & 90.12 & 99.21 & 98.33 & 107.47 & 148.95 & 13.60 \\
\hline 68595 & 2-[(2-Ethyl-6-methylphenyl)amino]-1-propanol & 289 & 60.10 & 89.94 & 99.79 & 98.34 & 108.84 & 147.17 & 15.97 \\
\hline 68596 & Ethoprophos & 288 & 53.62 & 89.40 & 100.63 & 96.93 & 110.97 & 187.50 & 17.13 \\
\hline 68597 & O-Ethyl-O-methyl-S-propylphosphorothioate & 289 & 55.92 & 97.80 & 116.26 & 110.99 & 128.10 & 271.61 & 24.76 \\
\hline 68598 & Etoxazole & 288 & 31.46 & 69.83 & 81.43 & 80.37 & 92.34 & 130.97 & 21.63 \\
\hline 68599 & Fenamiphos & 280 & 56.40 & 86.76 & 97.40 & 94.32 & 105.57 & 186.83 & 16.80 \\
\hline 68600 & Fenamiphos sulfone & 289 & 56.17 & 92.79 & 106.56 & 102.84 & 115.93 & 234.67 & 21.12 \\
\hline 68601 & Fenamiphos sulfoxide & 287 & 53.87 & 96.04 & 109.36 & 105.33 & 118.82 & 261.62 & 21.35 \\
\hline 68602 & Fenbutatin oxide & 283 & 0 & 48.93 & 74.95 & 72.97 & 95.44 & 280.52 & 50.13 \\
\hline 68603 & Fentin & 287 & 51.02 & 79.20 & 89.73 & 88.07 & 97.41 & 137.75 & 17.13 \\
\hline 68604 & Fipronil amide & 276 & 60.34 & 93.56 & 103.47 & 102.32 & 111.49 & 149.96 & 14.30 \\
\hline 68605 & Fipronil sulfonate & 288 & 69.28 & 100.21 & 116.84 & 113.42 & 132.86 & 186.23 & 19.84 \\
\hline 68606 & Flubendiamide & 281 & 52.25 & 88.33 & 104.93 & 101.14 & 116.26 & 217.87 & 23.68 \\
\hline 68608 & Fluometuron & 251 & 66.52 & 91.66 & 98.90 & 98.54 & 104.98 & 134.52 & 12.15 \\
\hline 68611 & 2-(1-Hydroxyethyl)-6-methylaniline & 211 & 0 & 48.47 & 92.72 & 95.45 & 124.78 & 268.35 & 53.43 \\
\hline 68612 & Hexazinone Transformation Product C & 287 & 62.04 & 96.21 & 104.14 & 102.23 & 111.05 & 147.63 & 13.01 \\
\hline 68613 & Hexazinone Transformation Product D & 3 & 83.34 & 83.34 & 98.81 & 99.92 & 113.15 & 113.15 & 15.11 \\
\hline 68614 & Hexazinone Transformation Product E & 254 & 27.79 & 70.93 & 85.55 & 85.52 & 98.00 & 145.53 & 24.19 \\
\hline 68615 & Hydroxyacetochlor & 257 & 0 & 83.06 & 95.46 & 94.89 & 105.15 & 212.23 & 20.48 \\
\hline 68616 & Hydroxyalachlor & 284 & 59.84 & 86.01 & 97.26 & 95.66 & 107.69 & 162.10 & 17.13 \\
\hline 68617 & Hydroxy monodemethyl fluometuron & 288 & 0 & 48.20 & 59.91 & 62.49 & 74.94 & 121.28 & 36.84 \\
\hline 68618 & Hydroxydiazinon & 289 & 55.42 & 84.57 & 97.03 & 93.64 & 107.23 & 173.03 & 18.59 \\
\hline 68619 & Hydroxy didemethyl fluometuron & 288 & 0 & 56.57 & 68.43 & 67.26 & 80.75 & 145.86 & 28.49 \\
\hline 68620 & Hydroxyfluometuron & 287 & 0 & 28.69 & 50.55 & 54.72 & 72.49 & 133.11 & 54.90 \\
\hline 68621 & Tebuthiuron TP 109 & 289 & 0 & 68.89 & 75.45 & 80.49 & 91.36 & 135.10 & 34.38 \\
\hline 68622 & Hydroxymetolachlor & 258 & 61.97 & 85.90 & 94.64 & 94.13 & 101.67 & 139.02 & 13.42 \\
\hline 68623 & Hydroxyphthalazinone & 271 & 62.92 & 93.19 & 99.94 & 99.15 & 106.51 & 135.20 & 12.30 \\
\hline 68624 & Hydroxysimazine & 280 & 70.77 & 103.04 & 120.31 & 115.31 & 132.42 & 270.00 & 21.85 \\
\hline
\end{tabular}


Table 4. Summary statistics for the recovery of schedule 2437 pesticides in lab reagent spikes, and groundwater and surface-water field matrix spike samples.-Continued

[ _ , values that do not meet method validation data-quality objectives; ___ , recovery values in which the first quartile is greater than or third quartile is less than 100 percent]

\begin{tabular}{|c|c|c|c|c|c|c|c|c|c|}
\hline \multirow[b]{2}{*}{ Pcode } & \multirow[b]{2}{*}{ Parameter name } & \multicolumn{8}{|c|}{ Lab reagent spikes } \\
\hline & & Count & Minimum & $\begin{array}{c}\text { First } \\
\text { quartile }\end{array}$ & Mean & Median & $\begin{array}{c}\text { Third } \\
\text { quartile }\end{array}$ & Maximum & $\begin{array}{l}\text { Relative } \\
\text { standard } \\
\text { deviation }\end{array}$ \\
\hline 68625 & Imazamox & 275 & 61.44 & 90.04 & 101.37 & 99.16 & 111.23 & 160.43 & 16.69 \\
\hline 68627 & Indoxacarb & 275 & 31.44 & 69.53 & 78.53 & 78.14 & 88.15 & 143.73 & 20.88 \\
\hline 68632 & Isoxaflutole & 273 & 0 & 70.34 & 76.78 & 85.24 & 97.03 & 142.89 & 42.49 \\
\hline 68633 & Isoxaflutole acid metabolite RPA 203328 & 280 & 57.17 & 86.42 & 96.49 & 97.19 & 105.65 & 140.90 & 14.89 \\
\hline 68638 & Lactofen & 273 & 1.26 & 52.18 & 66.76 & 71.45 & 83.95 & 140.19 & 40.56 \\
\hline 68639 & Linuron & 275 & 71.46 & 87.74 & 95.04 & 94.80 & 101.66 & 132.20 & 11.58 \\
\hline 68641 & MCPA & 251 & 62.67 & 86.47 & 98.65 & 97.61 & 109.41 & 162.92 & 16.38 \\
\hline 68644 & Methamidophos & 275 & 61.42 & 90.53 & 103.79 & 102.40 & 112.90 & 195.44 & 17.88 \\
\hline 68645 & Methomyl & 275 & 43.72 & 87.66 & 96.48 & 95.89 & 104.45 & 141.49 & 14.84 \\
\hline 68647 & Methoxyfenozide & 275 & 64.64 & 92.10 & 100.41 & 100.44 & 108.95 & 133.20 & 12.65 \\
\hline 68648 & Methyl paraoxon & 275 & 57.14 & 89.06 & 102.47 & 98.19 & 113.48 & 211.10 & 20.19 \\
\hline 68649 & Metolachlor hydroxy morpholinone & 275 & 57.73 & 81.59 & 93.16 & 94.22 & 103.76 & 145.30 & 16.37 \\
\hline 68650 & Metolachlor oxanilic acid & 278 & 52.49 & 86.89 & 97.63 & 95.88 & 107.39 & 147.47 & 16.23 \\
\hline 68651 & Metolachlor sulfonic acid & 280 & 56.49 & 90.10 & 101.17 & 98.62 & 112.11 & 159.41 & 17.27 \\
\hline 68652 & Metribuzin & 275 & 61.68 & 87.01 & 95.81 & 94.06 & 104.06 & 156.30 & 14.04 \\
\hline 68653 & Metribuzin DK & 244 & 43.03 & 84.90 & 98.59 & 99.25 & 113.55 & 167.10 & 20.89 \\
\hline 68654 & Naled & 268 & 0 & 44.51 & 66.94 & 74.09 & 94.93 & 146.80 & 57.01 \\
\hline 68655 & Novaluron & 275 & 0 & 66.70 & 86.58 & 83.67 & 106.81 & 215.38 & 38.93 \\
\hline 68656 & 2-Hydroxy-6-ethylamino-4-amino-s-triazine & 274 & 43.94 & 85.44 & 95.00 & 94.28 & 103.24 & 169.40 & 16.10 \\
\hline 68657 & O-Ethyl-S-methyl-S-propyl phosphorodithioate & 275 & 58.29 & 90.47 & 101.91 & 100.02 & 110.05 & 196.92 & 17.01 \\
\hline 68658 & O-Ethyl-S-propyl phosphorothioate & 275 & 64.08 & 89.71 & 104.85 & 101.32 & 117.49 & 224.74 & 21.82 \\
\hline 68659 & 2-Hydroxy-4-isopropylamino-6-amino-s-triazine & 275 & 65.00 & 85.34 & 93.91 & 93.22 & 102.67 & 137.19 & 13.27 \\
\hline 68660 & $\begin{array}{l}\text { 2-Hydroxy-4-isopropylamino-6-ethylamino-s- } \\
\text { triazine }\{\text { OIET }\}\end{array}$ & 275 & 65.49 & 84.39 & 93.05 & 92.73 & 100.88 & 135.05 & 13.42 \\
\hline 68661 & Omethoate (Dimethoate oxon) & 275 & 40.57 & 83.65 & 94.21 & 94.84 & 104.20 & 150.38 & 20.24 \\
\hline 68662 & Orthosulfamuron & 275 & 44.13 & 90.75 & 99.47 & 100.80 & 111.95 & 138.98 & 19.35 \\
\hline 68663 & Oryzalin & 280 & 57.99 & 91.62 & 102.50 & 101.53 & 112.42 & 161.97 & 17.29 \\
\hline 68664 & Oxamyl & 275 & 45.35 & 81.43 & 90.51 & 90.22 & 99.31 & 128.45 & 15.51 \\
\hline 68665 & Oxamyl oxime & 275 & 64.67 & 90.96 & 98.77 & 96.99 & 106.52 & 136.20 & 13.18 \\
\hline 68666 & Paraoxon & 275 & 65.01 & 91.76 & 103.34 & 101.93 & 113.00 & 185.65 & 17.13 \\
\hline
\end{tabular}


Table 4. Summary statistics for the recovery of schedule 2437 pesticides in lab reagent spikes, and groundwater and surface-water field matrix spike samples.-Continued [ _ values that do not meet method validation data-quality objectives; _ _ , recovery values in which the first quartile is greater than or third quartile is less than 100 percent]

\begin{tabular}{|c|c|c|c|c|c|c|c|c|c|}
\hline \multirow[b]{2}{*}{ Pcode } & \multirow[b]{2}{*}{ Parameter name } & \multicolumn{8}{|c|}{ Groundwater } \\
\hline & & Count & Minimum & $\begin{array}{c}\text { First } \\
\text { quartile }\end{array}$ & Mean & Median & $\begin{array}{l}\text { Third } \\
\text { quartile }\end{array}$ & Maximum & $\begin{array}{l}\text { Relative } \\
\text { standard } \\
\text { deviation }\end{array}$ \\
\hline 68625 & Imazamox & 42 & 70.19 & 88.43 & 105.32 & 98.38 & 120.97 & 189.55 & 22.85 \\
\hline 68627 & Indoxacarb & 42 & 0.73 & 49.89 & 67.20 & 68.48 & 84.18 & 125.90 & 36.45 \\
\hline 68632 & Isoxaflutole & 33 & 0.71 & 48.56 & 70.46 & 72.88 & 101.01 & 122.82 & 48.84 \\
\hline 68633 & Isoxaflutole acid metabolite RPA 203328 & 35 & 75.18 & 84.42 & 95.72 & 92.51 & 103.11 & 129.86 & 14.95 \\
\hline 68638 & Lactofen & 40 & 1.25 & 45.46 & 65.22 & 64.78 & 79.53 & 150.33 & 43.25 \\
\hline 68639 & Linuron & 42 & 81.04 & 90.35 & 96.27 & 95.76 & 104.78 & 116.45 & 9.21 \\
\hline 68641 & MCPA & 29 & 70.56 & 89.91 & 103.09 & 99.93 & 110.71 & 144.96 & 17.68 \\
\hline 68644 & Methamidophos & 40 & 70.85 & 99.33 & 113.09 & 108.60 & 119.59 & 290.09 & 29.03 \\
\hline 68645 & Methomyl & 42 & 25.09 & 85.80 & 92.27 & 92.01 & 102.62 & 128.31 & 20.31 \\
\hline 68647 & Methoxyfenozide & 42 & 78.37 & 92.56 & 101.87 & 102.71 & 111.72 & 123.63 & 11.37 \\
\hline 68648 & Methyl paraoxon & 32 & 73.09 & 93.40 & 109.03 & 104.78 & 112.68 & 295.27 & 33.33 \\
\hline 68649 & Metolachlor hydroxy morpholinone & 36 & 68.00 & 88.40 & 97.48 & 98.70 & 103.56 & 125.24 & 13.26 \\
\hline 68650 & Metolachlor oxanilic acid & 33 & 73.36 & 86.10 & 97.14 & 94.57 & 102.97 & 133.22 & 15.11 \\
\hline 68651 & Metolachlor sulfonic acid & 32 & 67.37 & 86.15 & 99.40 & 98.15 & 107.66 & 154.32 & 20.75 \\
\hline 68652 & Metribuzin & 40 & 70.17 & 87.59 & 95.30 & 93.38 & 102.70 & 128.62 & 12.39 \\
\hline 68653 & Metribuzin DK & 5 & 43.06 & 51.43 & 72.42 & 56.25 & 104.68 & 106.69 & 42.44 \\
\hline 68654 & Naled & 39 & 0 & 0 & 16.80 & 10.50 & 28.92 & 68.14 & 125.98 \\
\hline 68655 & Novaluron & 42 & 27.88 & 46.55 & 65.25 & 62.98 & 79.24 & 119.61 & 32.88 \\
\hline 68656 & 2-Hydroxy-6-ethylamino-4-amino-s-triazine & 42 & 22.42 & 156.73 & 216.94 & 217.48 & 258.43 & 518.22 & 44.91 \\
\hline 68657 & O-Ethyl-S-methyl-S-propyl phosphorodithioate & 42 & 69.96 & 96.36 & 110.17 & 102.27 & 117.12 & 340.91 & 35.83 \\
\hline 68658 & O-Ethyl-S-propyl phosphorothioate & 36 & 78.62 & 99.18 & 122.95 & 118.13 & 132.70 & 427.20 & 45.40 \\
\hline 68659 & 2-Hydroxy-4-isopropylamino-6-amino-s-triazine & 42 & 75.65 & 93.54 & 106.81 & 102.71 & 116.52 & 169.30 & 19.17 \\
\hline 68660 & $\begin{array}{l}\text { 2-Hydroxy-4-isopropylamino-6-ethylamino-s- } \\
\text { triazine }\{\text { OIET }\}\end{array}$ & 42 & 73.95 & 93.62 & 101.17 & 101.39 & 109.56 & 124.88 & 11.42 \\
\hline 68661 & Omethoate (Dimethoate oxon) & 41 & 24.59 & 80.63 & 88.61 & 85.87 & 101.80 & 125.47 & 21.29 \\
\hline 68662 & Orthosulfamuron & 42 & 24.68 & 49.93 & 64.20 & 65.37 & 76.68 & 113.88 & 30.33 \\
\hline 68663 & Oryzalin & 25 & 86.19 & 100.78 & 112.11 & 111.27 & 118.90 & 147.74 & 14.44 \\
\hline 68664 & Oxamyl & 39 & 0 & 64.03 & 77.53 & 83.12 & 90.41 & 152.62 & 35.08 \\
\hline 68665 & Oxamyl oxime & 42 & 79.27 & 94.37 & 101.97 & 100.06 & 110.79 & 128.33 & 12.50 \\
\hline 68666 & Paraoxon & 39 & 79.72 & 99.92 & 112.61 & 107.56 & 120.28 & 306.26 & 30.71 \\
\hline
\end{tabular}


Table 4. Summary statistics for the recovery of schedule 2437 pesticides in lab reagent spikes, and groundwater and surface-water field matrix spike samples.—Continued

[ _ _ values that do not meet method validation data-quality objectives; ___ , recovery values in which the first quartile is greater than or third quartile is less than 100 percent]

\begin{tabular}{|c|c|c|c|c|c|c|c|c|c|}
\hline \multirow[b]{2}{*}{ Pcode } & \multirow[b]{2}{*}{ Parameter name } & \multicolumn{8}{|c|}{ Surface water } \\
\hline & & Count & Minimum & $\begin{array}{c}\text { First } \\
\text { quartile }\end{array}$ & Mean & Median & $\begin{array}{l}\text { Third } \\
\text { quartile }\end{array}$ & Maximum & $\begin{array}{l}\text { Relative } \\
\text { standard } \\
\text { deviation }\end{array}$ \\
\hline 68625 & Imazamox & 289 & 67.60 & 102.80 & 120.06 & 116.76 & 135.18 & 205.06 & 19.67 \\
\hline 68627 & Indoxacarb & 277 & 0 & 44.27 & 57.50 & 58.13 & 70.32 & 112.95 & 37.16 \\
\hline 68632 & Isoxaflutole & 209 & 0 & 36.13 & 60.17 & 66.42 & 85.18 & 134.17 & 54.25 \\
\hline 68633 & Isoxaflutole acid metabolite RPA 203328 & 248 & 68.73 & 90.93 & 100.89 & 99.37 & 110.34 & 158.19 & 15.26 \\
\hline 68638 & Lactofen & 282 & 0 & 34.54 & 50.06 & 50.45 & 65.90 & 122.01 & 53.34 \\
\hline 68639 & Linuron & 288 & 60.62 & 90.70 & 98.33 & 97.10 & 104.98 & 135.76 & 11.32 \\
\hline 68641 & MCPA & 223 & 62.41 & 96.75 & 112.58 & 109.19 & 125.91 & 185.38 & 21.06 \\
\hline 68644 & Methamidophos & 286 & 51.32 & 90.58 & 105.97 & 103.38 & 118.18 & 249.37 & 22.31 \\
\hline 68645 & Methomyl & 285 & 48.39 & 87.80 & 98.18 & 96.14 & 104.44 & 160.19 & 15.46 \\
\hline 68647 & Methoxyfenozide & 289 & 63.71 & 94.19 & 103.27 & 102.62 & 111.20 & 155.89 & 14.00 \\
\hline 68648 & Methyl paraoxon & 240 & 16.53 & 86.09 & 100.01 & 99.93 & 113.28 & 227.22 & 28.51 \\
\hline 68649 & Metolachlor hydroxy morpholinone & 277 & 58.35 & 85.18 & 96.72 & 96.18 & 105.11 & 166.49 & 16.65 \\
\hline 68650 & Metolachlor oxanilic acid & 262 & 0 & 88.19 & 101.86 & 97.62 & 115.46 & 185.07 & 20.48 \\
\hline 68651 & Metolachlor sulfonic acid & 237 & 56.16 & 89.90 & 103.93 & 101.28 & 116.25 & 182.35 & 20.15 \\
\hline 68652 & Metribuzin & 282 & 43.07 & 82.76 & 90.88 & 90.71 & 100.07 & 121.34 & 14.94 \\
\hline 68653 & Metribuzin DK & 126 & 0 & 66.47 & 75.08 & 80.78 & 94.93 & 124.84 & 41.96 \\
\hline 68654 & Naled & 251 & 0 & 0 & 12.74 & 0 & 20.07 & 119.00 & 146.48 \\
\hline 68655 & Novaluron & 284 & 0.98 & 44.52 & 70.68 & 66.84 & 91.32 & 163.08 & 47.17 \\
\hline 68656 & 2-Hydroxy-6-ethylamino-4-amino-s-triazine & 275 & 0 & 127.34 & 173.86 & 154.74 & 209.70 & 508.73 & 42.00 \\
\hline 68657 & O-Ethyl-S-methyl-S-propyl phosphorodithioate & 284 & 0 & 91.93 & 105.13 & 101.85 & 114.88 & 264.86 & 22.96 \\
\hline 68658 & O-Ethyl-S-propyl phosphorothioate & 276 & 0 & 100.63 & 120.26 & 115.04 & 131.93 & 389.36 & 29.11 \\
\hline 68659 & 2-Hydroxy-4-isopropylamino-6-amino-s-triazine & 283 & 71.43 & 103.61 & 118.94 & 114.32 & 130.13 & 231.54 & 19.63 \\
\hline 68660 & $\begin{array}{l}\text { 2-Hydroxy-4-isopropylamino-6-ethylamino-s- } \\
\text { triazine }\{\text { OIET }\}\end{array}$ & 284 & 55.44 & 90.63 & 102.11 & 100.56 & 112.77 & 167.41 & 16.26 \\
\hline 68661 & Omethoate (Dimethoate oxon) & 281 & 0 & 70.36 & 78.23 & 82.31 & 96.27 & 206.14 & 37.34 \\
\hline 68662 & Orthosulfamuron & 289 & 0 & 59.07 & 75.67 & 79.02 & 94.23 & 164.31 & 37.27 \\
\hline 68663 & Oryzalin & 270 & 13.46 & 95.97 & 105.59 & 107.13 & 120.05 & 179.90 & 26.51 \\
\hline 68664 & Oxamyl & 276 & 0 & 50.14 & 64.10 & 70.35 & 82.18 & 145.87 & 43.04 \\
\hline 68665 & Oxamyl oxime & 289 & 66.34 & 102.01 & 114.48 & 111.29 & 123.64 & 213.21 & 17.12 \\
\hline 68666 & Paraoxon & 276 & 54.70 & 93.53 & 107.72 & 104.99 & 118.11 & 260.16 & 21.86 \\
\hline
\end{tabular}


Table 4. Summary statistics for the recovery of schedule 2437 pesticides in lab reagent spikes, and groundwater and surface-water field matrix spike samples.-Continued [ _ values that do not meet method validation data-quality objectives; ___ , recovery values in which the first quartile is greater than or third quartile is less than 100 percent]

\begin{tabular}{|c|c|c|c|c|c|c|c|c|c|}
\hline \multirow[b]{2}{*}{ Pcode } & \multirow[b]{2}{*}{ Parameter name } & \multicolumn{8}{|c|}{ Lab reagent spikes } \\
\hline & & Count & Minimum & $\begin{array}{c}\text { First } \\
\text { quartile }\end{array}$ & Mean & Median & $\begin{array}{c}\text { Third } \\
\text { quartile }\end{array}$ & Maximum & $\begin{array}{l}\text { Relative } \\
\text { standard } \\
\text { deviation }\end{array}$ \\
\hline 68668 & Phorate & 275 & 33.68 & 77.67 & 86.29 & 85.67 & 95.17 & 126.73 & 16.17 \\
\hline 68669 & Phorate oxon & 275 & 41.02 & 86.96 & 97.57 & 97.36 & 107.60 & 191.15 & 19.20 \\
\hline 68670 & Phorate oxon sulfone & 273 & 0 & 79.58 & 88.62 & 93.00 & 104.41 & 143.21 & 28.62 \\
\hline 68671 & Phorate oxon sulfoxide & 273 & 4.48 & 79.91 & 87.83 & 91.45 & 102.94 & 147.15 & 30.95 \\
\hline 68672 & Phorate sulfone & 275 & 58.38 & 90.87 & 103.65 & 101.09 & 115.00 & 189.73 & 19.18 \\
\hline 68673 & Phorate sulfoxide & 275 & 62.87 & 91.51 & 103.74 & 101.33 & 112.34 & 201.20 & 17.54 \\
\hline 68675 & Phthalazinone & 275 & 63.97 & 85.25 & 94.92 & 93.98 & 104.39 & 143.21 & 14.65 \\
\hline 68676 & Profenofos & 273 & 0 & 67.86 & 72.33 & 80.35 & 89.45 & 115.57 & 39.49 \\
\hline 68677 & Propargite & 274 & 24.02 & 68.96 & 78.17 & 79.01 & 88.30 & 128.28 & 20.27 \\
\hline 68678 & Propazine & 275 & 67.43 & 86.61 & 94.79 & 93.57 & 101.65 & 132.54 & 12.17 \\
\hline 68679 & Propoxur & 275 & 64.05 & 90.92 & 101.83 & 99.95 & 110.77 & 183.26 & 17.52 \\
\hline 68682 & Pyridaben & 274 & 43.89 & 73.61 & 83.33 & 83.19 & 92.51 & 127.58 & 18.29 \\
\hline 68683 & Pyriproxyfen & 275 & 57.76 & 82.69 & 89.86 & 89.20 & 96.35 & 125.42 & 12.72 \\
\hline 68684 & sec-Acetochlor oxanilic acid & 280 & 55.90 & 90.87 & 102.21 & 100.51 & 111.92 & 154.22 & 16.72 \\
\hline 68685 & sec-Alachlor oxanilic acid & 275 & 0 & 90.26 & 101.10 & 100.44 & 111.14 & 157.06 & 18.59 \\
\hline 68686 & Siduron & 275 & 70.77 & 86.57 & 94.77 & 94.18 & 101.55 & 132.37 & 11.75 \\
\hline 68687 & Sulfentrazone & 280 & 51.59 & 95.27 & 106.87 & 106.28 & 117.63 & 173.62 & 18.08 \\
\hline 68688 & Sulfometuron-methyl & 275 & 56.39 & 90.48 & 99.14 & 99.30 & 106.33 & 134.04 & 12.37 \\
\hline 68689 & Sulfosulfuron & 275 & 62.83 & 86.27 & 95.15 & 94.43 & 103.95 & 130.86 & 13.62 \\
\hline 68690 & Sulfosulfuron ethyl sulfone & 275 & 60.98 & 89.54 & 97.12 & 97.23 & 104.59 & 133.89 & 11.60 \\
\hline 68691 & $\begin{array}{l}\text { 2,3,3-Trichloro-2-propene-1-sulfonic acid } \\
\text { (TCPSA) }\end{array}$ & 280 & 59.83 & 88.94 & 99.29 & 98.45 & 107.25 & 174.03 & 15.94 \\
\hline 68692 & Tebufenozide & 275 & 61.13 & 91.58 & 100.06 & 99.43 & 108.72 & 141.62 & 13.55 \\
\hline 68693 & Tebupirimphos & 275 & 50.78 & 75.98 & 84.86 & 83.90 & 93.38 & 130.22 & 15.13 \\
\hline 68694 & Tebupirimfos oxon & 275 & 55.61 & 83.44 & 92.53 & 91.23 & 101.55 & 147.66 & 15.03 \\
\hline 68695 & Tebuthiuron & 275 & 69.88 & 87.36 & 95.08 & 95.27 & 101.46 & 131.09 & 11.16 \\
\hline 68696 & Tebuthiuron TP el108 & 275 & 68.08 & 88.89 & 95.42 & 95.35 & 101.89 & 130.49 & 11.52 \\
\hline 68697 & Tebuthiuron TP $109(\mathrm{OH})$ & 275 & 43.56 & 82.46 & 91.67 & 91.37 & 100.26 & 137.20 & 16.18 \\
\hline 68698 & Terbacil & 275 & 57.98 & 87.90 & 99.73 & 98.98 & 110.26 & 150.17 & 15.55 \\
\hline
\end{tabular}


Table 4. Summary statistics for the recovery of schedule 2437 pesticides in lab reagent spikes, and groundwater and surface-water field matrix spike samples.-Continued

[ _ v values that do not meet method validation data-quality objectives; ___ , recovery values in which the first quartile is greater than or third quartile is less than 100 percent]

\begin{tabular}{|c|c|c|c|c|c|c|c|c|c|}
\hline \multirow[b]{2}{*}{ Pcode } & \multirow[b]{2}{*}{ Parameter name } & \multicolumn{8}{|c|}{ Groundwater } \\
\hline & & Count & Minimum & $\begin{array}{c}\text { First } \\
\text { quartile }\end{array}$ & Mean & Median & $\begin{array}{c}\text { Third } \\
\text { quartile }\end{array}$ & Maximum & $\begin{array}{l}\text { Relative } \\
\text { standard } \\
\text { deviation }\end{array}$ \\
\hline 68668 & Phorate & 40 & 46.36 & 73.23 & 79.00 & 80.00 & 87.60 & 101.32 & 15.19 \\
\hline 68669 & Phorate oxon & 42 & 0 & 40.98 & 57.15 & 61.45 & 74.83 & 202.32 & 61.55 \\
\hline 68670 & Phorate oxon sulfone & 34 & 45.52 & 68.22 & 78.67 & 77.33 & 91.24 & 122.06 & 21.52 \\
\hline 68671 & Phorate oxon sulfoxide & 42 & 1.61 & 68.61 & 73.38 & 78.76 & 88.07 & 120.47 & 39.40 \\
\hline 68672 & Phorate sulfone & 41 & 67.12 & 93.20 & 111.93 & 110.36 & 121.14 & 304.16 & 31.78 \\
\hline 68673 & Phorate sulfoxide & 42 & 65.58 & 96.57 & 111.04 & 104.36 & 118.25 & 350.24 & 36.42 \\
\hline 68675 & Phthalazinone & 37 & 58.78 & 92.05 & 102.99 & 105.21 & 116.12 & 140.41 & 16.49 \\
\hline 68676 & Profenofos & 41 & 14.16 & 56.45 & 70.22 & 70.32 & 85.87 & 111.18 & 26.79 \\
\hline 68677 & Propargite & 42 & 10.36 & 69.26 & 78.50 & 80.20 & 87.26 & 124.25 & 25.97 \\
\hline 68678 & Propazine & 42 & 81.23 & 89.13 & 97.72 & 97.94 & 105.61 & 119.85 & 10.43 \\
\hline 68679 & Propoxur & 42 & 74.28 & 89.92 & 97.80 & 97.00 & 105.76 & 123.97 & 12.28 \\
\hline 68682 & Pyridaben & 42 & 53.33 & 68.36 & 78.80 & 78.16 & 88.84 & 128.03 & 19.46 \\
\hline 68683 & Pyriproxyfen & 42 & 71.51 & 82.67 & 90.54 & 89.96 & 95.28 & 138.25 & 13.20 \\
\hline 68684 & sec-Acetochlor oxanilic acid & 41 & 65.07 & 87.81 & 96.17 & 92.06 & 104.53 & 132.97 & 17.74 \\
\hline 68685 & sec-Alachlor oxanilic acid & 33 & 0 & 79.29 & 93.38 & 97.91 & 105.62 & 145.96 & 27.13 \\
\hline 68686 & Siduron & 42 & 80.80 & 92.22 & 98.37 & 97.05 & 106.02 & 117.52 & 8.98 \\
\hline 68687 & Sulfentrazone & 35 & 86.84 & 103.10 & 116.82 & 111.95 & 128.11 & 181.48 & 20.68 \\
\hline 68688 & Sulfometuron-methyl & 42 & 73.04 & 84.82 & 94.24 & 92.21 & 98.30 & 152.41 & 15.40 \\
\hline 68689 & Sulfosulfuron & 39 & 76.12 & 86.79 & 96.20 & 92.24 & 103.94 & 128.01 & 12.28 \\
\hline 68690 & Sulfosulfuron ethyl sulfone & 42 & 79.32 & 95.28 & 101.77 & 99.70 & 106.54 & 138.53 & 11.25 \\
\hline 68691 & $\begin{array}{l}\text { 2,3,3-Trichloro-2-propene-1-sulfonic acid } \\
\text { (TCPSA) }\end{array}$ & 40 & 75.16 & 85.41 & 96.97 & 95.72 & 103.15 & 163.47 & 16.49 \\
\hline 68692 & Tebufenozide & 42 & 80.24 & 93.32 & 101.94 & 100.69 & 111.99 & 126.04 & 11.60 \\
\hline 68693 & Tebupirimphos & 42 & 62.09 & 83.22 & 89.06 & 88.65 & 95.44 & 122.22 & 13.11 \\
\hline 68694 & Tebupirimfos oxon & 42 & 61.63 & 84.46 & 91.78 & 92.74 & 100.16 & 125.86 & 15.46 \\
\hline 68695 & Tebuthiuron & 42 & 84.50 & 95.38 & 100.38 & 99.70 & 105.37 & 114.07 & 7.57 \\
\hline 68696 & Tebuthiuron TP el108 & 42 & 84.12 & 92.05 & 98.84 & 96.42 & 104.37 & 130.20 & 9.55 \\
\hline 68697 & Tebuthiuron TP $109(\mathrm{OH})$ & 42 & 4.56 & 81.41 & 91.31 & 90.49 & 100.59 & 151.40 & 27.62 \\
\hline 68698 & Terbacil & 39 & 66.23 & 85.35 & 94.41 & 94.47 & 105.85 & 115.52 & 13.81 \\
\hline
\end{tabular}


Table 4. Summary statistics for the recovery of schedule 2437 pesticides in lab reagent spikes, and groundwater and surface-water field matrix spike samples.-Continued [ _ _ values that do not meet method validation data-quality objectives; ___ , recovery values in which the first quartile is greater than or third quartile is less than 100 percent]

\begin{tabular}{|c|c|c|c|c|c|c|c|c|c|}
\hline \multirow[b]{2}{*}{ Pcode } & \multirow[b]{2}{*}{ Parameter name } & \multicolumn{8}{|c|}{ Surface water } \\
\hline & & Count & Minimum & $\begin{array}{c}\text { First } \\
\text { quartile }\end{array}$ & Mean & Median & $\begin{array}{c}\text { Third } \\
\text { quartile }\end{array}$ & Maximum & $\begin{array}{l}\text { Relative } \\
\text { standard } \\
\text { deviation }\end{array}$ \\
\hline 68668 & Phorate & 271 & 26.52 & 69.96 & 78.29 & 76.61 & 86.09 & 138.67 & 19.73 \\
\hline 68669 & Phorate oxon & 289 & 0 & 49.78 & 61.51 & 60.86 & 74.06 & 156.45 & 38.69 \\
\hline 68670 & Phorate oxon sulfone & 257 & 0 & 57.01 & 66.41 & 70.46 & 81.87 & 166.90 & 42.46 \\
\hline 68671 & Phorate oxon sulfoxide & 286 & 0 & 61.54 & 70.34 & 75.13 & 87.64 & 178.62 & 43.09 \\
\hline 68672 & Phorate sulfone & 271 & 50.78 & 92.51 & 107.22 & 105.59 & 118.99 & 274.67 & 22.47 \\
\hline 68673 & Phorate sulfoxide & 286 & 57.99 & 94.32 & 108.21 & 104.45 & 117.80 & 259.13 & 21.36 \\
\hline 68675 & Phthalazinone & 257 & 74.14 & 93.02 & 102.05 & 100.46 & 108.52 & 146.99 & 13.37 \\
\hline 68676 & Profenofos & 289 & 0 & 54.71 & 62.70 & 66.28 & 78.30 & 128.82 & 40.45 \\
\hline 68677 & Propargite & 282 & 0 & 58.05 & 67.60 & 71.23 & 82.69 & 132.27 & 37.49 \\
\hline 68678 & Propazine & 289 & 63.74 & 89.01 & 97.13 & 96.97 & 105.09 & 141.01 & 13.33 \\
\hline 68679 & Propoxur & 289 & 17.90 & 87.60 & 97.15 & 98.50 & 108.95 & 180.12 & 25.23 \\
\hline 68682 & Pyridaben & 288 & 28.11 & 64.43 & 78.82 & 78.94 & 93.33 & 143.27 & 27.20 \\
\hline 68683 & Pyriproxyfen & 289 & 34.10 & 78.72 & 88.47 & 87.91 & 97.44 & 135.21 & 17.46 \\
\hline 68684 & sec-Acetochlor oxanilic acid & 282 & 0 & 91.77 & 103.63 & 101.73 & 114.84 & 157.17 & 17.95 \\
\hline 68685 & sec-Alachlor oxanilic acid & 269 & 0 & 74.69 & 82.34 & 87.73 & 100.14 & 152.32 & 37.67 \\
\hline 68686 & Siduron & 286 & 62.34 & 90.43 & 97.54 & 96.02 & 103.60 & 139.06 & 11.42 \\
\hline 68687 & Sulfentrazone & 260 & 73.53 & 104.09 & 124.83 & 116.33 & 138.18 & 237.37 & 24.49 \\
\hline 68688 & Sulfometuron-methyl & 286 & 20.67 & 86.92 & 95.55 & 95.09 & 103.83 & 159.56 & 17.50 \\
\hline 68689 & Sulfosulfuron & 288 & 38.11 & 88.79 & 97.24 & 95.96 & 104.50 & 147.20 & 14.12 \\
\hline 68690 & Sulfosulfuron ethyl sulfone & 289 & 63.58 & 91.54 & 98.47 & 97.15 & 105.38 & 140.62 & 11.26 \\
\hline 68691 & $\begin{array}{l}\text { 2,3,3-Trichloro-2-propene-1-sulfonic acid } \\
\text { (TCPSA) }\end{array}$ & 289 & 56.15 & 91.91 & 102.54 & 100.81 & 111.75 & 171.84 & 15.47 \\
\hline 68692 & Tebufenozide & 283 & 63.50 & 92.17 & 101.75 & 101.24 & 109.84 & 153.02 & 14.42 \\
\hline 68693 & Tebupirimphos & 283 & 42.84 & 79.64 & 90.62 & 88.27 & 98.49 & 157.72 & 17.75 \\
\hline 68694 & Tebupirimfos oxon & 282 & 53.41 & 84.69 & 94.80 & 92.46 & 103.35 & 171.94 & 17.02 \\
\hline 68695 & Tebuthiuron & 289 & 65.48 & 90.39 & 97.34 & 96.30 & 102.69 & 135.30 & 10.98 \\
\hline 68696 & Tebuthiuron TP el108 & 289 & 52.78 & 85.65 & 93.74 & 93.43 & 100.66 & 125.56 & 12.53 \\
\hline 68697 & Tebuthiuron TP $109(\mathrm{OH})$ & 275 & 0 & 78.47 & 96.28 & 97.29 & 113.74 & 285.74 & 44.03 \\
\hline 68698 & Terbacil & 273 & 62.92 & 86.18 & 97.50 & 96.57 & 105.71 & 153.59 & 16.82 \\
\hline
\end{tabular}


Table 4. Summary statistics for the recovery of schedule 2437 pesticides in lab reagent spikes, and groundwater and surface-water field matrix spike samples.-Continued

[_ values that do not meet method validation data-quality objectives; ___ , recovery values in which the first quartile is greater than or third quartile is less than 100 percent]

\begin{tabular}{|c|c|c|c|c|c|c|c|c|c|}
\hline \multirow[b]{2}{*}{ Pcode } & \multirow[b]{2}{*}{ Parameter name } & \multicolumn{8}{|c|}{ Lab reagent spikes } \\
\hline & & Count & Minimum & $\begin{array}{c}\text { First } \\
\text { quartile }\end{array}$ & Mean & Median & $\begin{array}{c}\text { Third } \\
\text { quartile }\end{array}$ & Maximum & $\begin{array}{l}\text { Relative } \\
\text { standard } \\
\text { deviation }\end{array}$ \\
\hline 68699 & Terbufos & 275 & 36.13 & 74.38 & 82.70 & 81.64 & 91.99 & 121.37 & 15.46 \\
\hline 68700 & Terbufos oxon & 275 & 17.99 & 76.14 & 84.57 & 85.17 & 94.91 & 136.95 & 20.02 \\
\hline 68701 & Terbufos oxon sulfone & 275 & 40.88 & 82.85 & 92.63 & 93.61 & 104.64 & 150.22 & 20.79 \\
\hline 68702 & Terbufos oxon sulfoxide & 275 & 30.03 & 82.04 & 93.68 & 96.16 & 106.66 & 147.08 & 22.83 \\
\hline 68703 & Terbufos sulfone & 275 & 57.57 & 88.15 & 101.99 & 101.19 & 112.50 & 208.25 & 18.63 \\
\hline 68704 & Terbufos sulfoxide & 275 & 65.53 & 91.93 & 103.48 & 101.63 & 113.30 & 207.02 & 17.39 \\
\hline 68708 & trans-Permethrin & 274 & 29.19 & 59.24 & 70.98 & 70.65 & 79.43 & 150.68 & 24.37 \\
\hline 68710 & Triallate & 275 & 54.03 & 78.37 & 86.98 & 86.54 & 95.67 & 122.38 & 13.94 \\
\hline 68711 & Tribufos & 274 & 20.08 & 68.77 & 76.44 & 76.66 & 85.53 & 113.19 & 20.00 \\
\hline 68712 & Triclopyr & 280 & 54.87 & 86.17 & 96.19 & 96.42 & 105.97 & 136.42 & 15.59 \\
\hline 68713 & Hexazinone Transformation Product G & 275 & 59.70 & 84.62 & 92.90 & 93.09 & 100.16 & 133.76 & 14.01 \\
\hline 68714 & Tebuthiuron Transformation Product 106 & 275 & 63.24 & 86.51 & 94.25 & 93.30 & 100.52 & 124.17 & 11.73 \\
\hline 68769 & cis-Permethrin & 274 & 29.94 & 59.56 & 71.79 & 70.81 & 82.41 & 144.35 & 23.76 \\
\hline 68871 & Alachlor sulfonic acid & 300 & 0 & 69.60 & 101.86 & 99.98 & 129.21 & 299.06 & 45.83 \\
\hline 68872 & Chlorimuron-ethyl & 275 & 40.95 & 88.43 & 97.01 & 97.06 & 107.81 & 129.62 & 16.03 \\
\hline 68873 & 3-Phenoxybenzoic acid & 280 & 60.27 & 85.78 & 95.25 & 94.12 & 103.15 & 146.00 & 14.57 \\
\hline 68553 & $\begin{array}{l}\text { cis-Bifenthrin acid/cis-Cyhalothrin acid/cis-Teflu- } \\
\text { thrin acid }\end{array}$ & 259 & 0 & 84.48 & 94.15 & 92.88 & 103.98 & 168.65 & 21.46 \\
\hline 68560 & Dacthal monoacid & 323 & 0 & 70.57 & 106.61 & 101.01 & 131.38 & 407.65 & 53.07 \\
\hline 68571 & Dicamba & 302 & 0 & 78.22 & 98.48 & 98.73 & 117.12 & 200.73 & 32.49 \\
\hline 68646 & Methonyl oxime & 161 & 0 & 84.68 & 92.92 & 96.45 & 113.39 & 153.90 & 33.43 \\
\hline
\end{tabular}


Table 4. Summary statistics for the recovery of schedule 2437 pesticides in lab reagent spikes, and groundwater and surface-water field matrix spike samples.-Continued [ _ values that do not meet method validation data-quality objectives; ___ , recovery values in which the first quartile is greater than or third quartile is less than 100 percent]

\begin{tabular}{|c|c|c|c|c|c|c|c|c|c|}
\hline \multirow[b]{2}{*}{ Pcode } & \multirow[b]{2}{*}{ Parameter name } & \multicolumn{8}{|c|}{ Groundwater } \\
\hline & & Count & Minimum & $\begin{array}{c}\text { First } \\
\text { quartile }\end{array}$ & Mean & Median & $\begin{array}{l}\text { Third } \\
\text { quartile }\end{array}$ & Maximum & $\begin{array}{l}\text { Relative } \\
\text { standard } \\
\text { deviation }\end{array}$ \\
\hline 68699 & Terbufos & 42 & 41.43 & 61.17 & 68.31 & 69.03 & 75.89 & 104.12 & 18.94 \\
\hline 68700 & Terbufos oxon & 42 & 4.09 & 21.04 & 28.57 & 30.78 & 36.11 & 51.91 & 45.90 \\
\hline 68701 & Terbufos oxon sulfone & 40 & 20.78 & 77.57 & 86.90 & 90.84 & 96.39 & 127.38 & 21.21 \\
\hline 68702 & Terbufos oxon sulfoxide & 42 & 43.52 & 84.37 & 92.19 & 90.47 & 104.03 & 133.88 & 17.95 \\
\hline 68703 & Terbufos sulfone & 38 & 67.06 & 96.14 & 110.39 & 104.41 & 115.37 & 282.66 & 30.27 \\
\hline 68704 & Terbufos sulfoxide & 42 & 68.38 & 98.23 & 112.58 & 106.70 & 119.75 & 311.84 & 30.58 \\
\hline 68708 & trans-Permethrin & 42 & 18.14 & 41.57 & 55.13 & 55.32 & 67.45 & 98.89 & 35.80 \\
\hline 68710 & Triallate & 41 & 62.73 & 85.40 & 91.64 & 91.65 & 98.48 & 112.06 & 10.90 \\
\hline 68711 & Tribufos & 42 & 57.07 & 69.46 & 80.60 & 79.81 & 89.91 & 115.73 & 18.26 \\
\hline 68712 & Triclopyr & 42 & 60.41 & 80.22 & 91.35 & 91.40 & 98.64 & 134.89 & 16.30 \\
\hline 68713 & Hexazinone Transformation Product G & 40 & 74.16 & 86.52 & 94.91 & 94.87 & 103.68 & 116.26 & 11.59 \\
\hline 68714 & Tebuthiuron Transformation Product 106 & 42 & 75.13 & 88.49 & 97.24 & 96.37 & 103.74 & 130.02 & 12.23 \\
\hline 68769 & cis-Permethrin & 42 & 19.66 & 39.52 & 52.09 & 48.98 & 63.87 & 89.36 & 35.87 \\
\hline 68871 & Alachlor sulfonic acid & 1 & 76.31 & 76.31 & 76.31 & 76.31 & 76.31 & 76.31 & \\
\hline 68872 & Chlorimuron-ethyl & 41 & 54.60 & 70.00 & 86.41 & 85.41 & 100.99 & 136.10 & 24.19 \\
\hline 68873 & 3-Phenoxybenzoic acid & 41 & 73.67 & 85.19 & 97.25 & 96.26 & 107.07 & 124.11 & 14.47 \\
\hline 68553 & $\begin{array}{l}\text { cis-Bifenthrin acid/cis-Cyhalothrin acid/cis-Teflu- } \\
\text { thrin acid }\end{array}$ & & & & & & & & \\
\hline 68560 & Dacthal monoacid & & & & & & & & \\
\hline 68571 & Dicamba & & & & & & & & \\
\hline 68646 & Methonyl oxime & & & & & & & & \\
\hline
\end{tabular}


Table 4. Summary statistics for the recovery of schedule 2437 pesticides in lab reagent spikes, and groundwater and surface-water field matrix spike samples.-Continued

[ _ values that do not meet method validation data-quality objectives; ___ , recovery values in which the first quartile is greater than or third quartile is less than 100 percent]

\begin{tabular}{|c|c|c|c|c|c|c|c|c|c|}
\hline \multirow[b]{2}{*}{ Pcode } & \multirow[b]{2}{*}{ Parameter name } & \multicolumn{8}{|c|}{ Surface water } \\
\hline & & Count & Minimum & $\begin{array}{c}\text { First } \\
\text { quartile }\end{array}$ & Mean & Median & $\begin{array}{l}\text { Third } \\
\text { quartile }\end{array}$ & Maximum & $\begin{array}{l}\text { Relative } \\
\text { standard } \\
\text { deviation }\end{array}$ \\
\hline 68699 & Terbufos & 289 & 17.25 & 60.03 & 69.34 & 68.58 & 78.43 & 122.51 & 22.30 \\
\hline 68700 & Terbufos oxon & 284 & 3.14 & 20.74 & 30.84 & 28.66 & 38.85 & 83.31 & 49.65 \\
\hline 68701 & Terbufos oxon sulfone & 289 & 0 & 63.42 & 74.17 & 78.35 & 92.25 & 187.88 & 38.89 \\
\hline 68702 & Terbufos oxon sulfoxide & 286 & 0 & 70.06 & 81.29 & 85.22 & 99.29 & 191.74 & 36.77 \\
\hline 68703 & Terbufos sulfone & 281 & 54.52 & 92.78 & 107.32 & 104.18 & 117.81 & 245.91 & 22.08 \\
\hline 68704 & Terbufos sulfoxide & 286 & 57.92 & 95.51 & 108.58 & 104.85 & 118.39 & 258.90 & 20.71 \\
\hline 68708 & trans-Permethrin & 268 & 14.82 & 44.81 & 65.31 & 66.26 & 84.12 & 149.14 & 38.91 \\
\hline 68710 & Triallate & 288 & 34.80 & 78.99 & 87.71 & 87.76 & 96.24 & 138.74 & 16.43 \\
\hline 68711 & Tribufos & 281 & 36.33 & 71.61 & 85.17 & 81.95 & 97.46 & 159.06 & 23.07 \\
\hline 68712 & Triclopyr & 284 & 0 & 83.30 & 94.78 & 94.92 & 106.25 & 180.70 & 20.87 \\
\hline 68713 & Hexazinone Transformation Product G & 282 & 54.59 & 82.70 & 91.80 & 90.49 & 100.64 & 127.43 & 14.43 \\
\hline 68714 & Tebuthiuron Transformation Product 106 & 289 & 63.95 & 88.56 & 95.98 & 94.86 & 103.61 & 138.71 & 13.04 \\
\hline 68769 & cis-Permethrin & 278 & 15.76 & 43.29 & 65.54 & 66.78 & 85.14 & 133.42 & 38.44 \\
\hline 68871 & Alachlor sulfonic acid & 3 & 51.90 & 51.90 & 91.85 & 101.08 & 122.59 & 122.59 & 39.45 \\
\hline 68872 & Chlorimuron-ethyl & 285 & 4.89 & 81.01 & 91.28 & 94.46 & 104.70 & 164.78 & 25.05 \\
\hline 68873 & 3-Phenoxybenzoic acid & 283 & 0 & 89.22 & 97.48 & 96.50 & 106.01 & 143.43 & 14.78 \\
\hline 68553 & $\begin{array}{l}\text { cis-Bifenthrin acid/cis-Cyhalothrin acid/cis-Teflu- } \\
\text { thrin acid }\end{array}$ & & & & & & & & \\
\hline 68560 & Dacthal monoacid & & & & & & & & \\
\hline 68571 & Dicamba & & & & & & & & \\
\hline 68646 & Methonyl oxime & & & & & & & & \\
\hline
\end{tabular}




\section{Appendix 1. Supporting Tables and Figures}

\section{Figures}

1-1. Distributions of recovery for individual pesticides in schedule 2437 by matrix, spike lot, and spike lot age. Recovery values larger than 400 percent are not shown

1-2. Distributions of recovery for schedule 2437 pesticides by spike lot, and spike lot age, pooled by matrix. Recovery values larger than 400 percent are not shown

1-3. Distributions of recovery for pesticides in schedule 2437 in surface water by analytical method group and Major River Basin. Recovery values larger than 400 percent are not shown

\section{Tables}

1-1. Number of pesticides in each analytical method group

1-2. Pesticides that have at least one result reported with a VQC of " $\mathrm{m}$ ".

Table 1-1. Number of pesticides in each analytical method group.

\begin{tabular}{lc}
\hline \multicolumn{1}{c}{ Analytical method group } & Number of pesticides \\
\hline Acetanilide and amide & 28 \\
Acid & 8 \\
Carbamate and thiocarbamate & 22 \\
Fungicide & 15 \\
Miscellaneous & 28 \\
Organophosphate & 50 \\
Pyrethroid, organochlorine and phenylpyrazine & 14 \\
Sulfonylurea and urea & 32 \\
Triazine & 28 \\
\hline
\end{tabular}




\section{A. 2-(1-Hydroxyethyl)-6-methylaniline: laboratory reagent spikes}

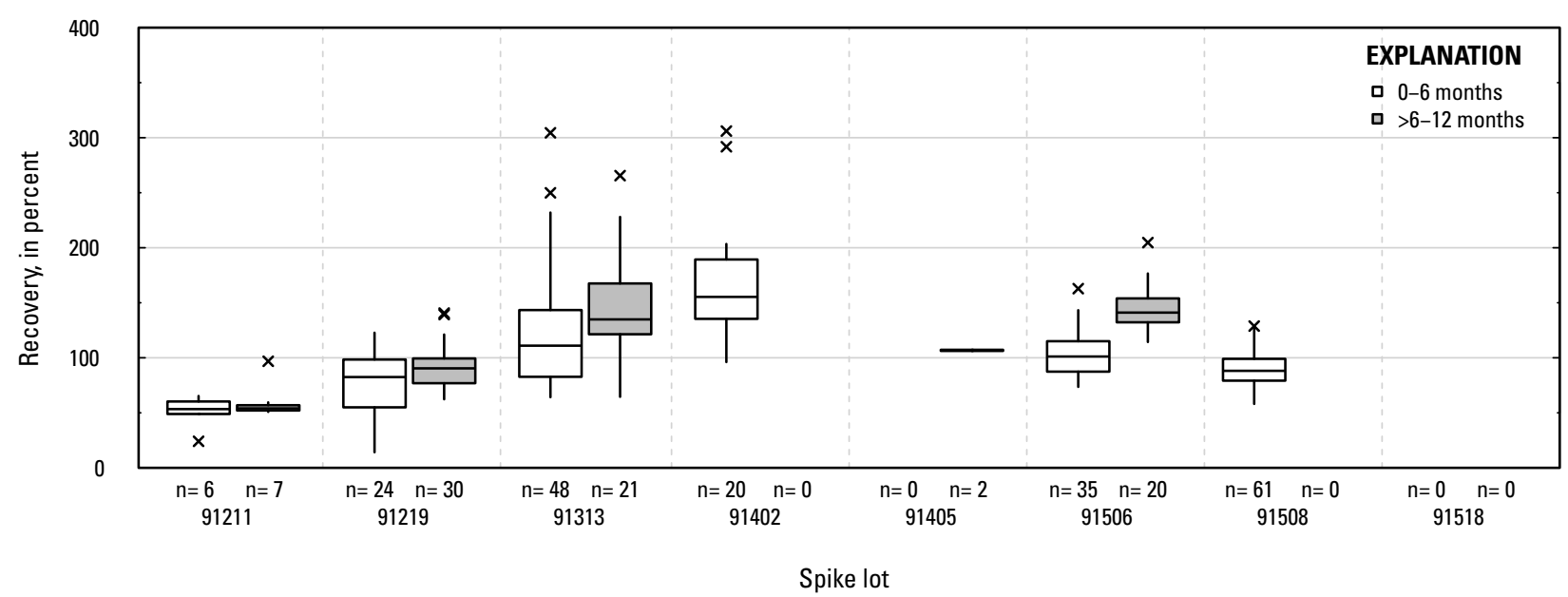

\section{B. 2-(1-Hydroxyethyl)-6-methylaniline: groundwater field matrix spikes}

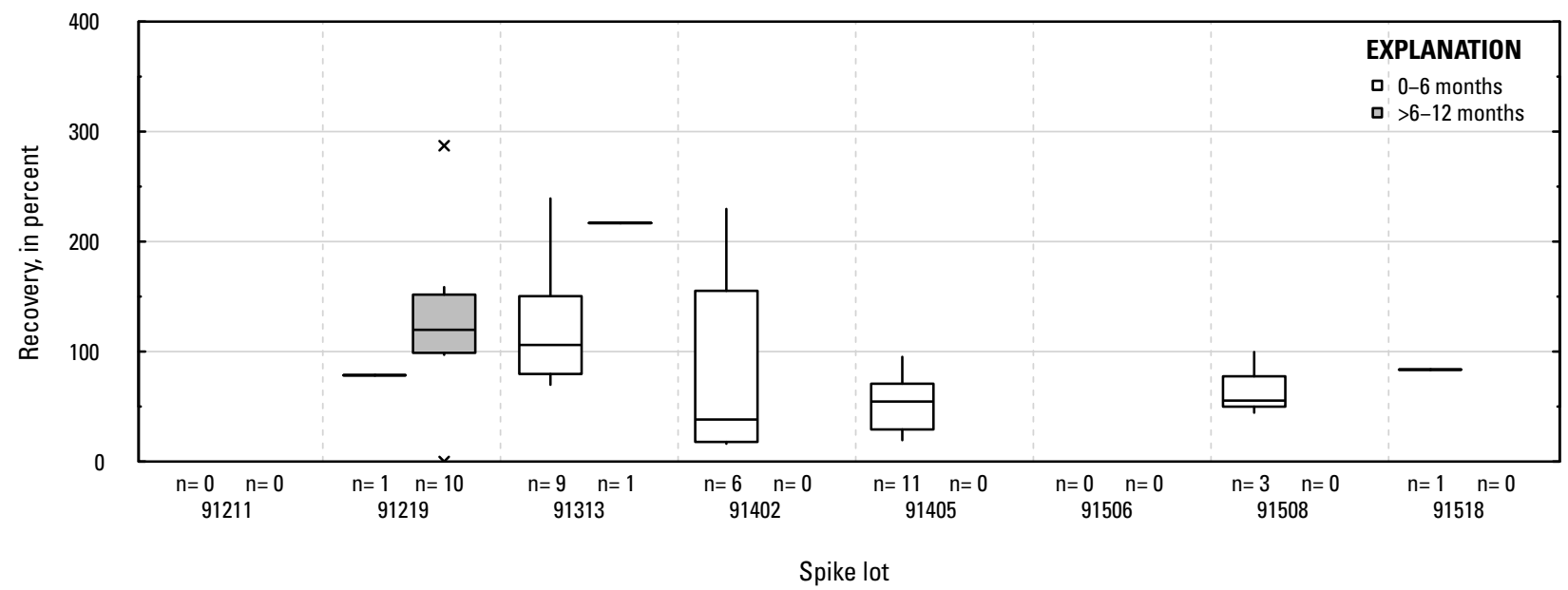

\section{2-(1-Hydroxyethyl)-6-methylaniline: surface water field matrix spikes}

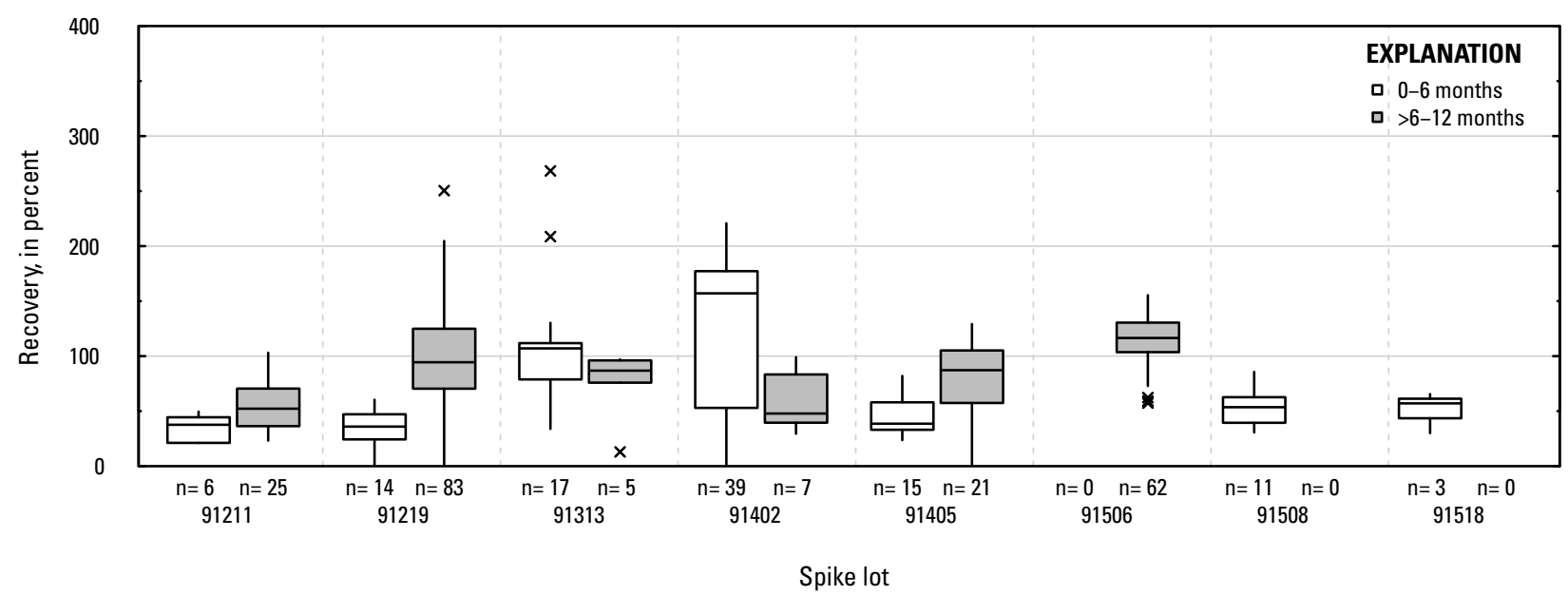

Figure 1-1. Distributions of recovery for individual pesticides in schedule 2437 by matrix, spike lot, and spike lot age. Recovery values larger than 400 percent are not shown. 
D. 2-[(2-Ethyl-6-methylphenyl)amino]-1-propanol: laboratory reagent spikes

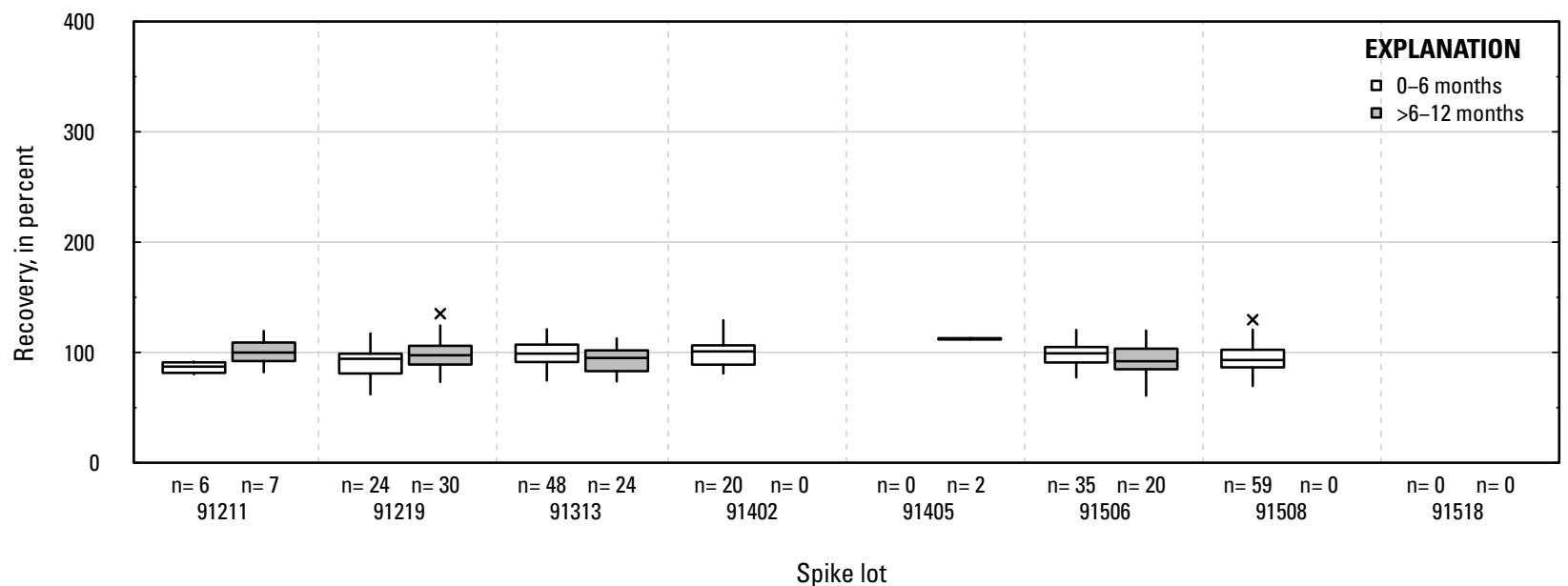

\section{E. 2-[(2-Ethyl-6-methylphenyl)amino]-1-propanol: groundwater field matrix spikes}

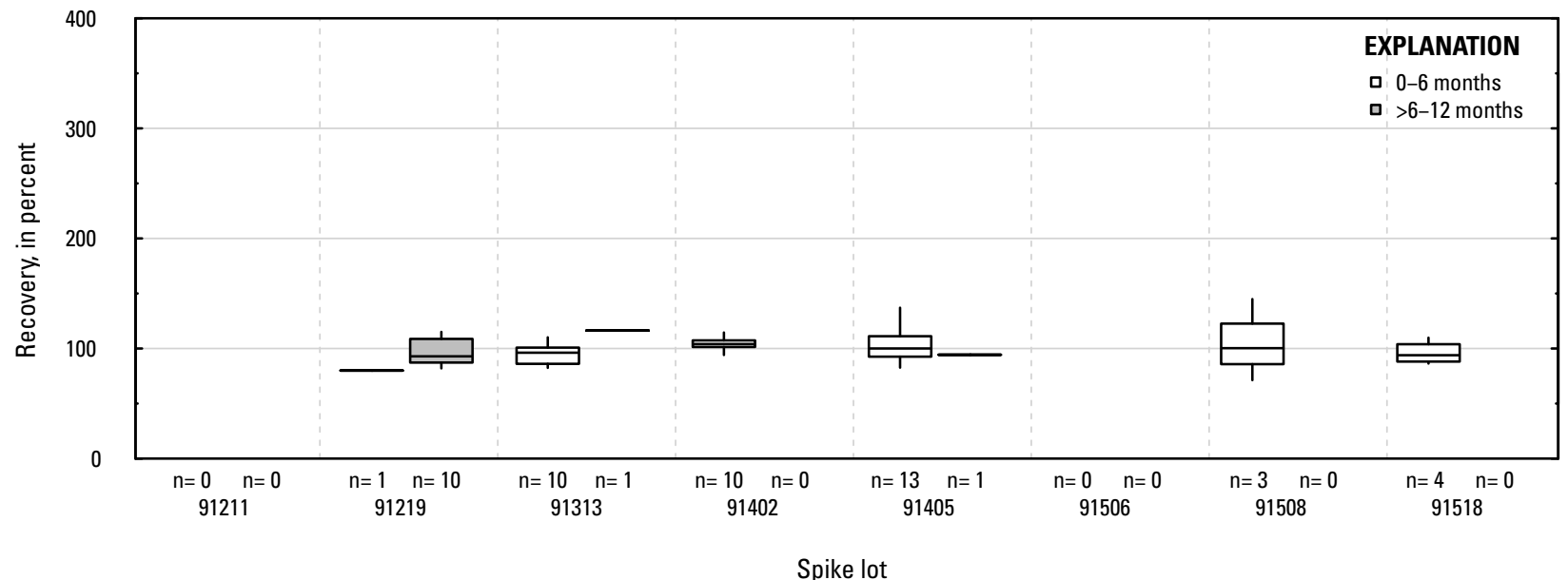

\section{F. 2-[(2-Ethyl-6-methylphenyl)amino]-1-propanol: surface water field matrix spikes}

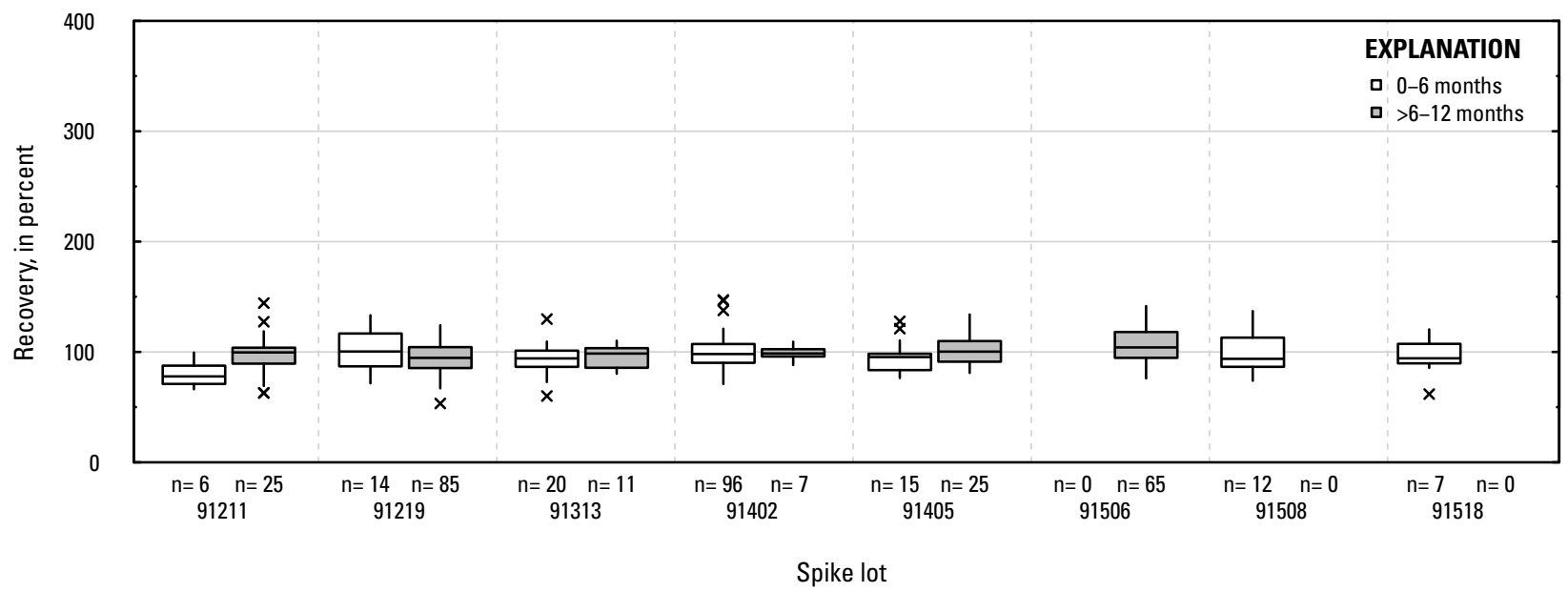

Figure 1-1. Distributions of recovery for individual pesticides in schedule 2437 by matrix, spike lot, and spike lot age. Recovery values larger than 400 percent are not shown.-Continued 


\section{G. 2,3,3-Trichloro-2-propene-1-sulfonic acid (TCPSA): laboratory reagent spikes}

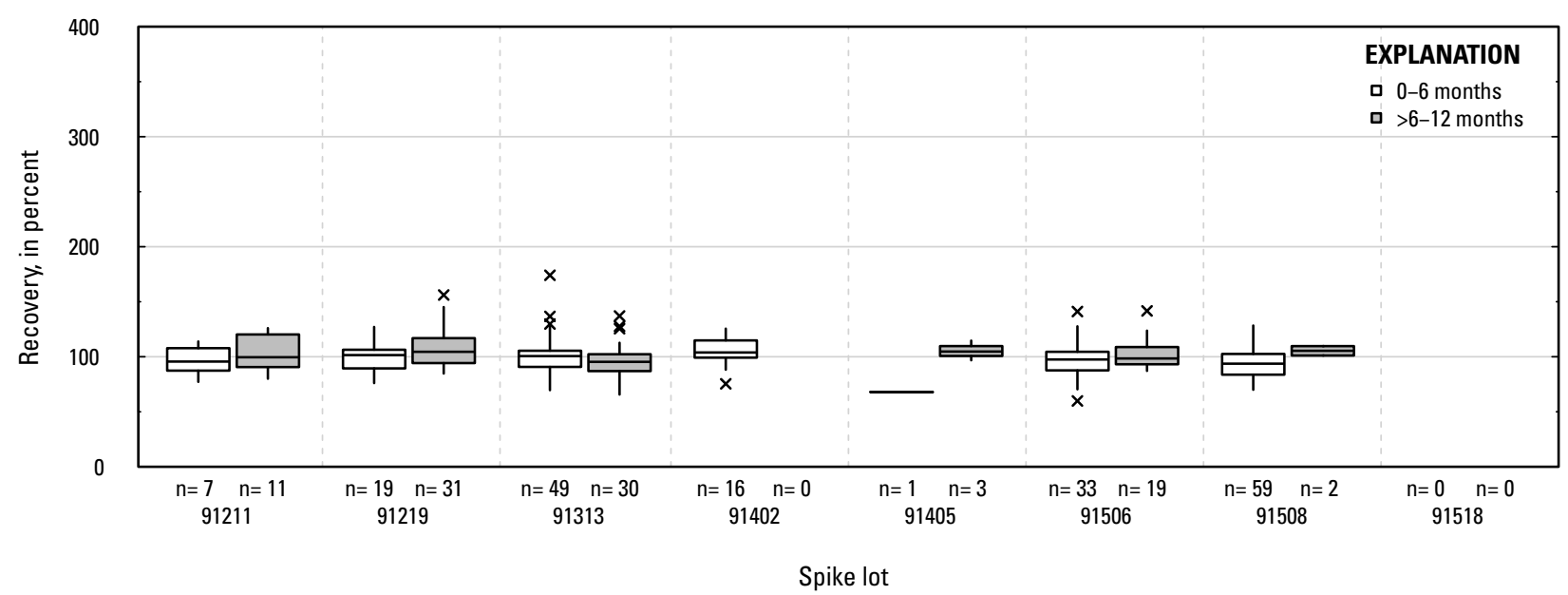

H. 2,3,3-Trichloro-2-propene-1-sulfonic acid (TCPSA): groundwater field matrix spikes

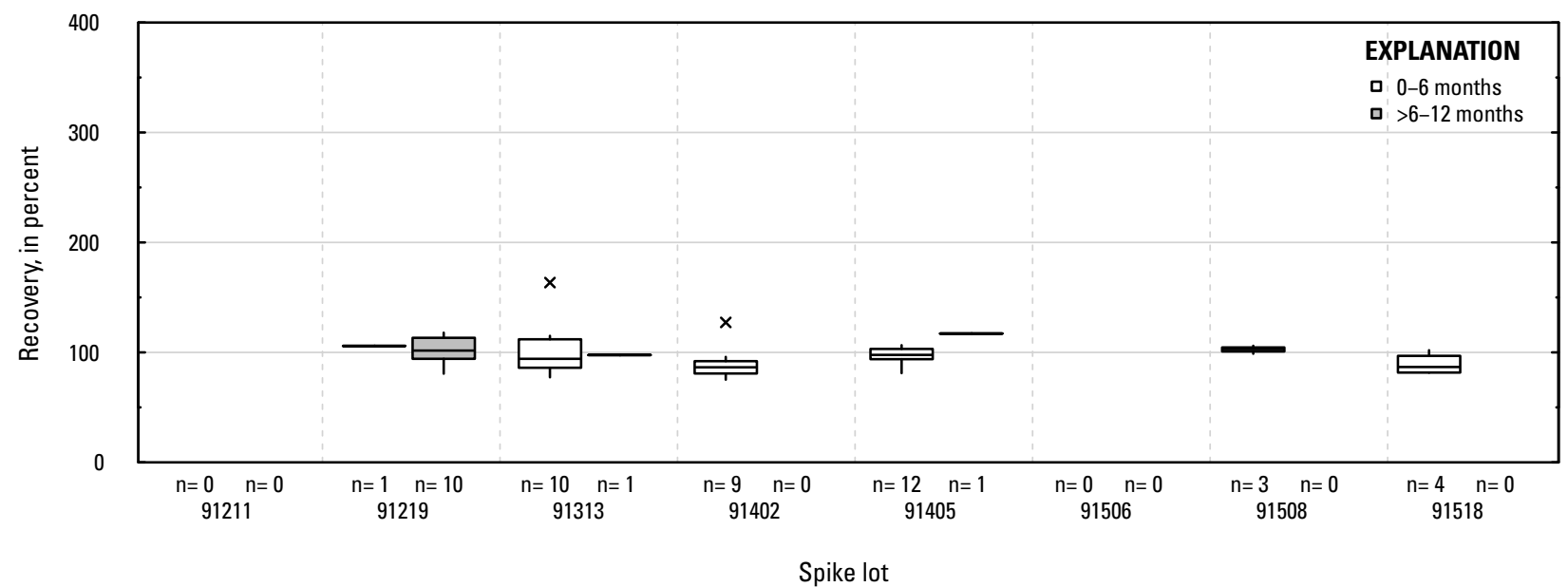

I. 2,3,3-Trichloro-2-propene-1-sulfonic acid (TCPSA): surface water field matrix spikes

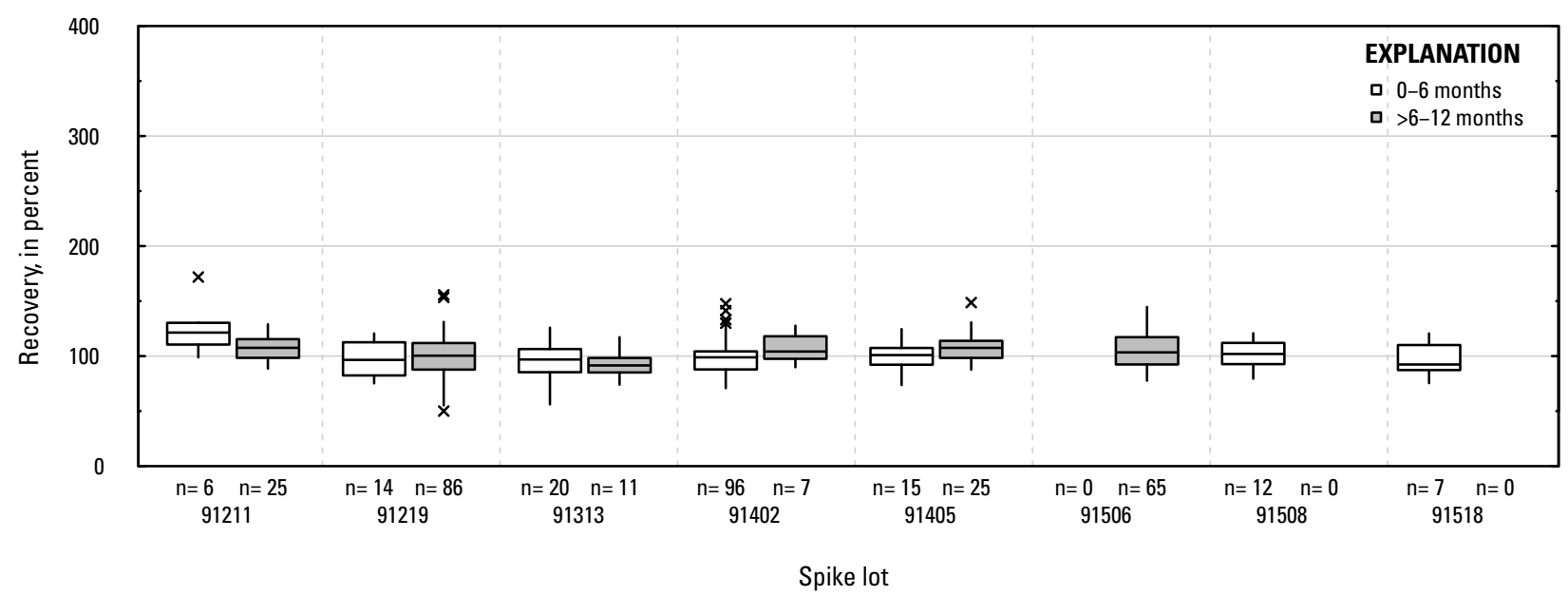

Figure 1-1. Distributions of recovery for individual pesticides in schedule 2437 by matrix, spike lot, and spike lot age. Recovery values larger than 400 percent are not shown.-Continued 


\section{J. 2,4-D: laboratory reagent spikes}

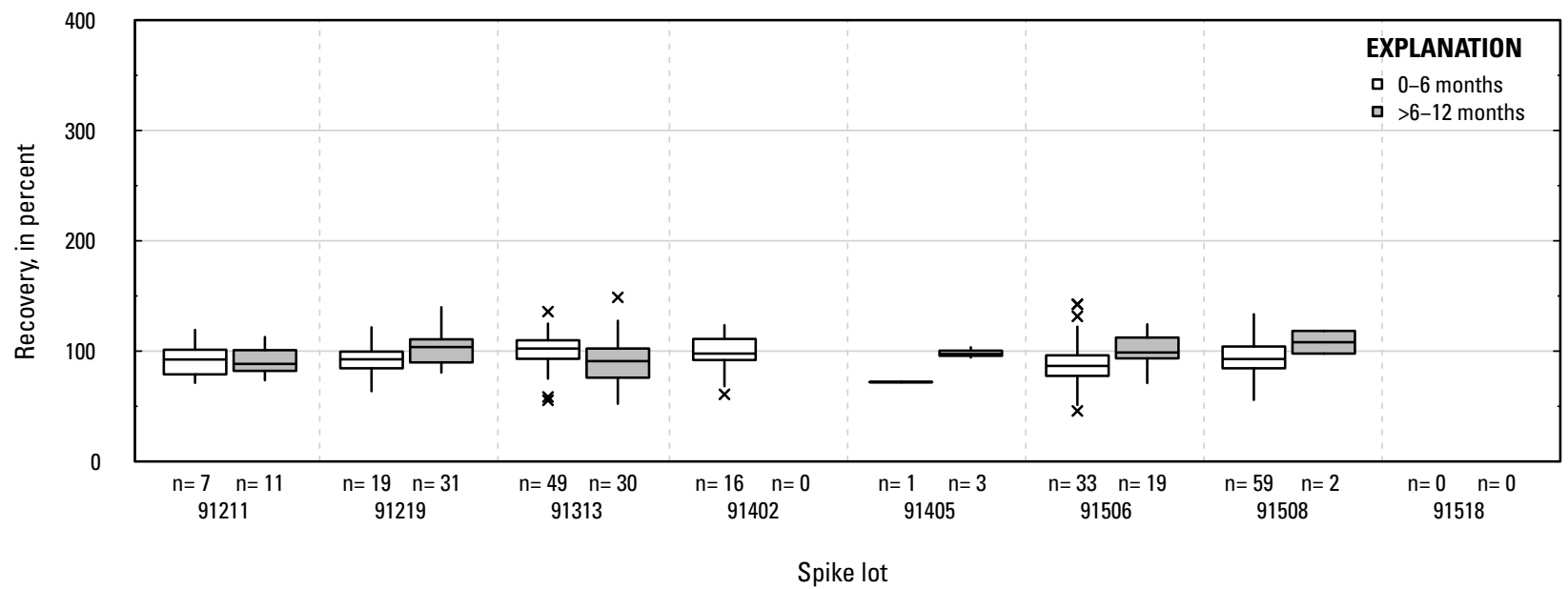

\section{K. 2,4-D: groundwater field matrix spikes}

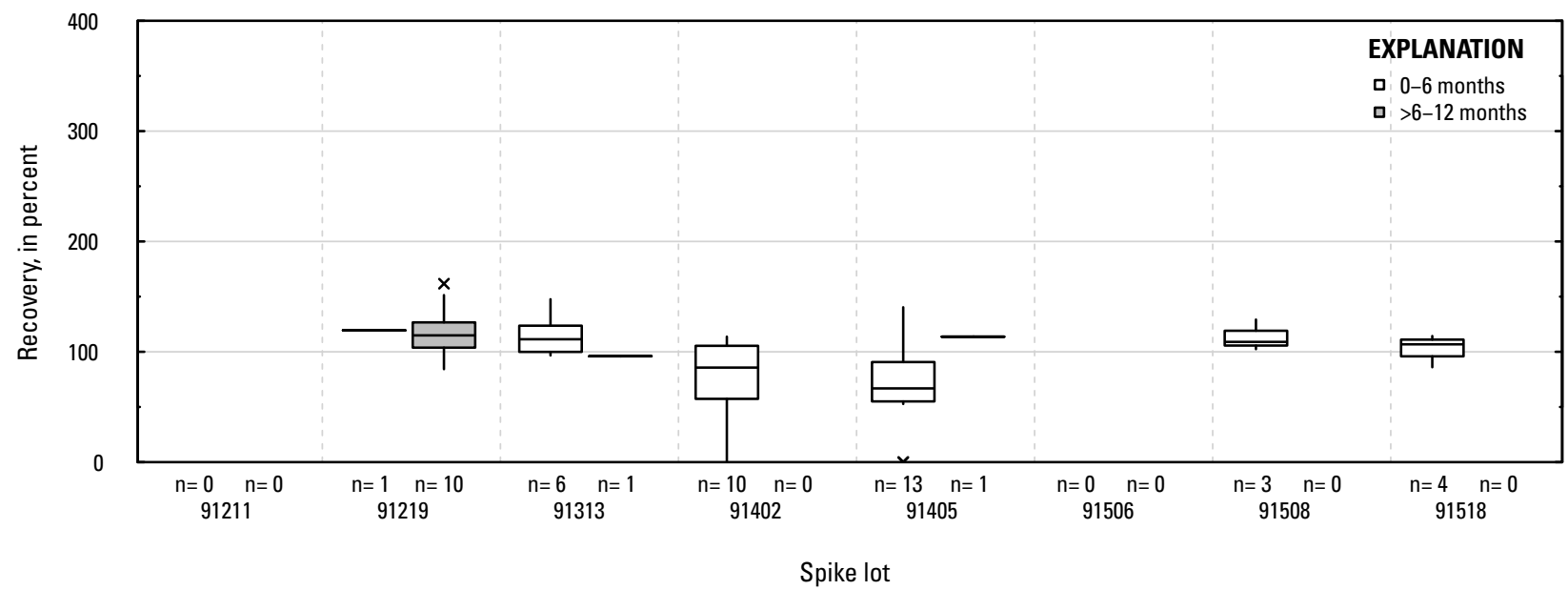

\section{2,4-D: surface water field matrix spikes}

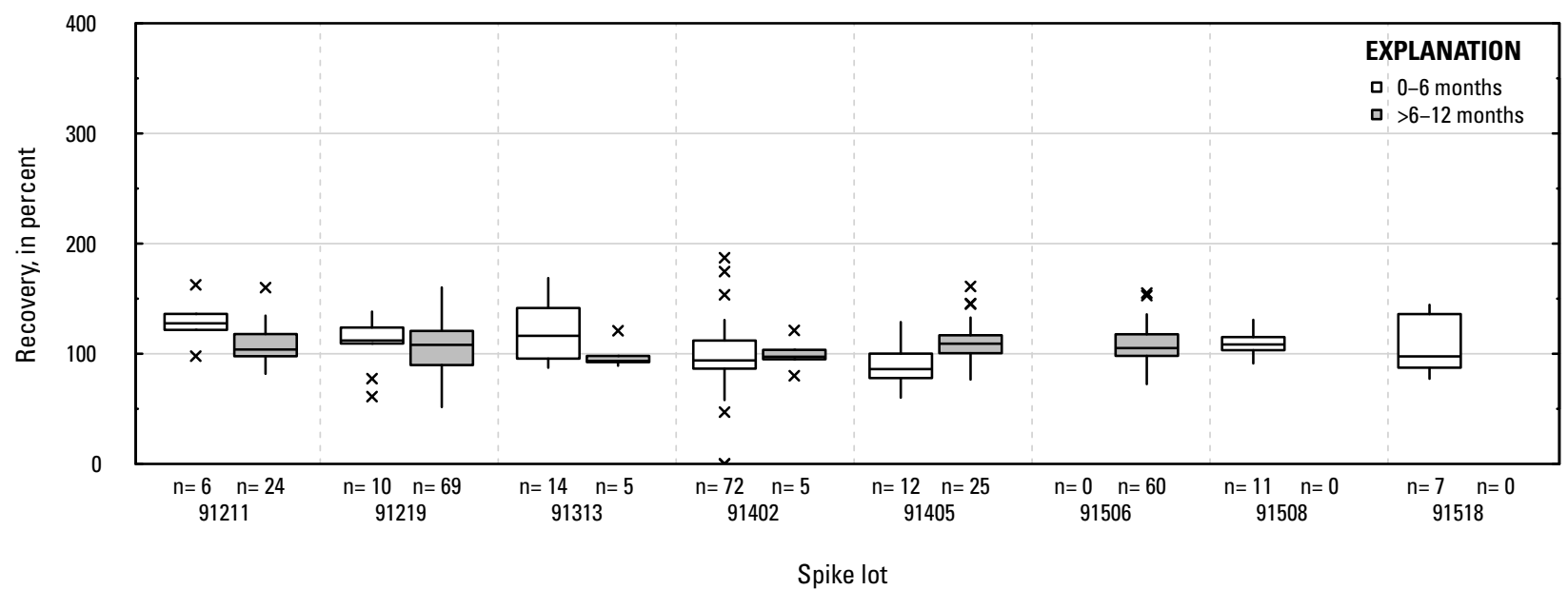

Figure 1-1. Distributions of recovery for individual pesticides in schedule 2437 by matrix, spike lot, and spike lot age. Recovery values larger than 400 percent are not shown.-Continued 


\section{2-Aminobenzimidazole: laboratory reagent spikes}

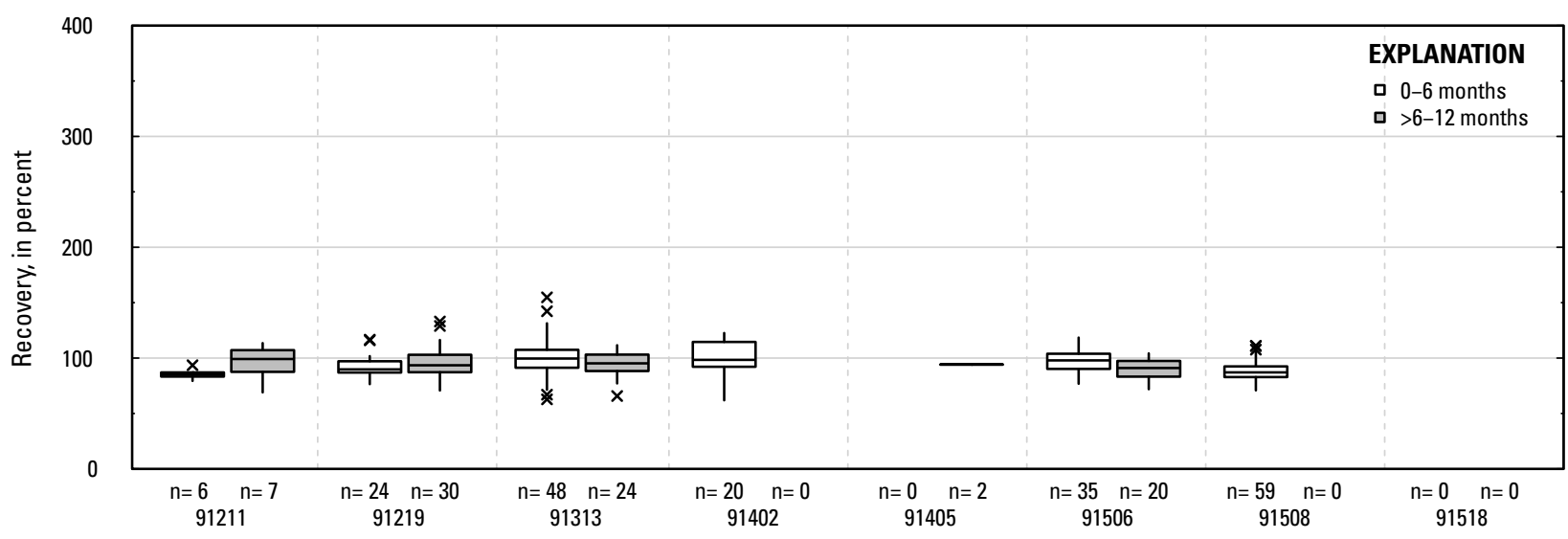

Spike lot

\section{N. 2-Aminobenzimidazole: groundwater field matrix spikes}

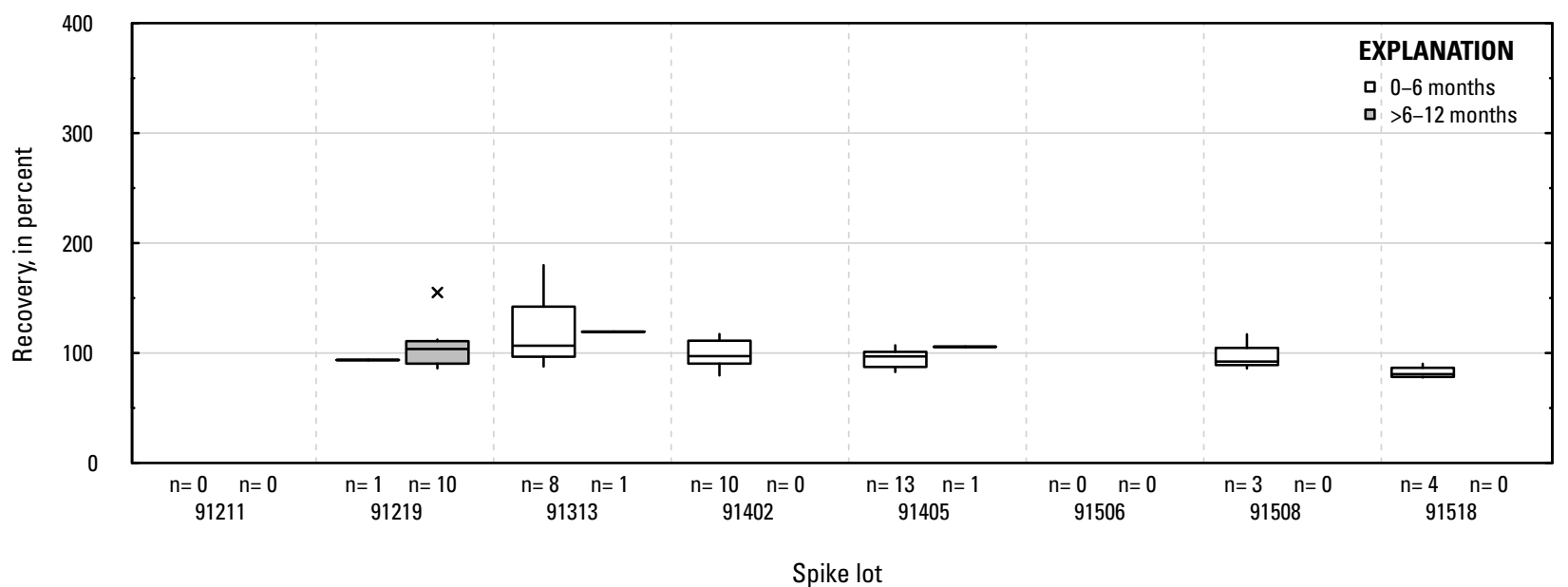

0. 2-Aminobenzimidazole: surface water field matrix spikes

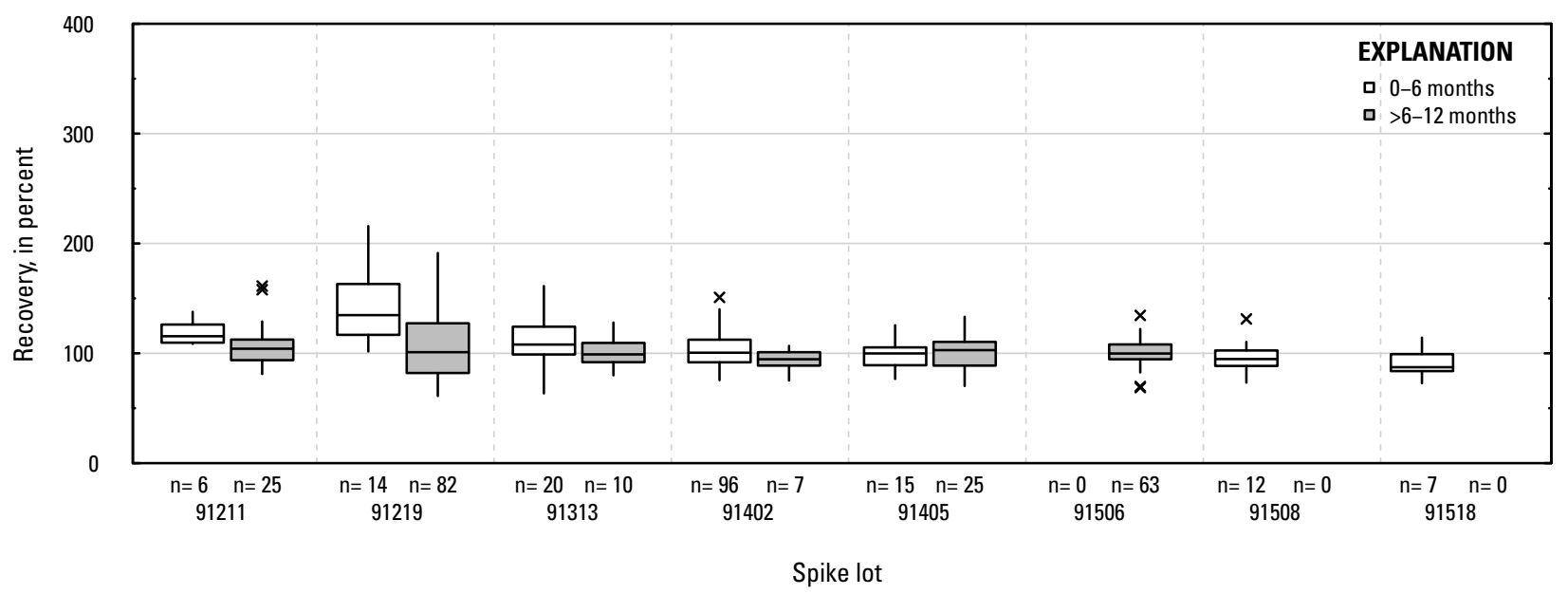

Figure 1-1. Distributions of recovery for individual pesticides in schedule 2437 by matrix, spike lot, and spike lot age. Recovery values larger than 400 percent are not shown.-Continued 


\section{P. 2-Amino-N-isopropylbenzamide: laboratory reagent spikes}

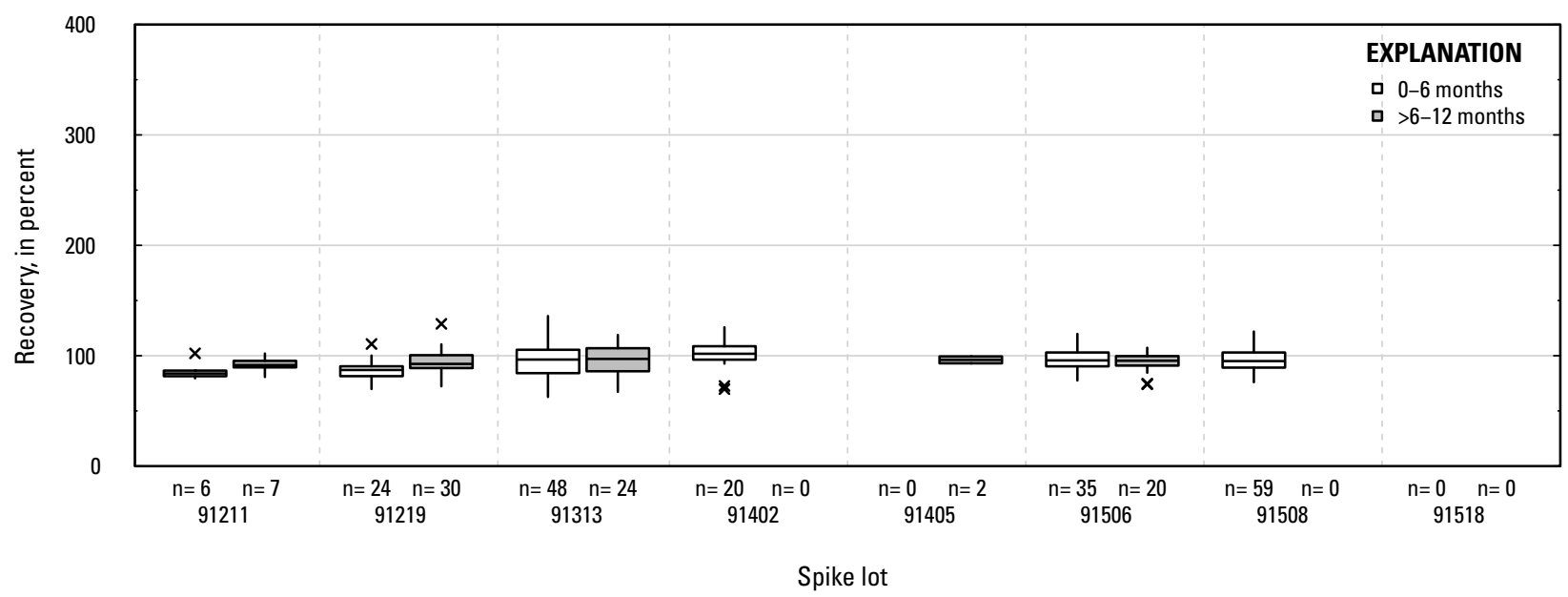

Q. 2-Amino- $\mathrm{N}$-isopropylbenzamide: groundwater field matrix spikes

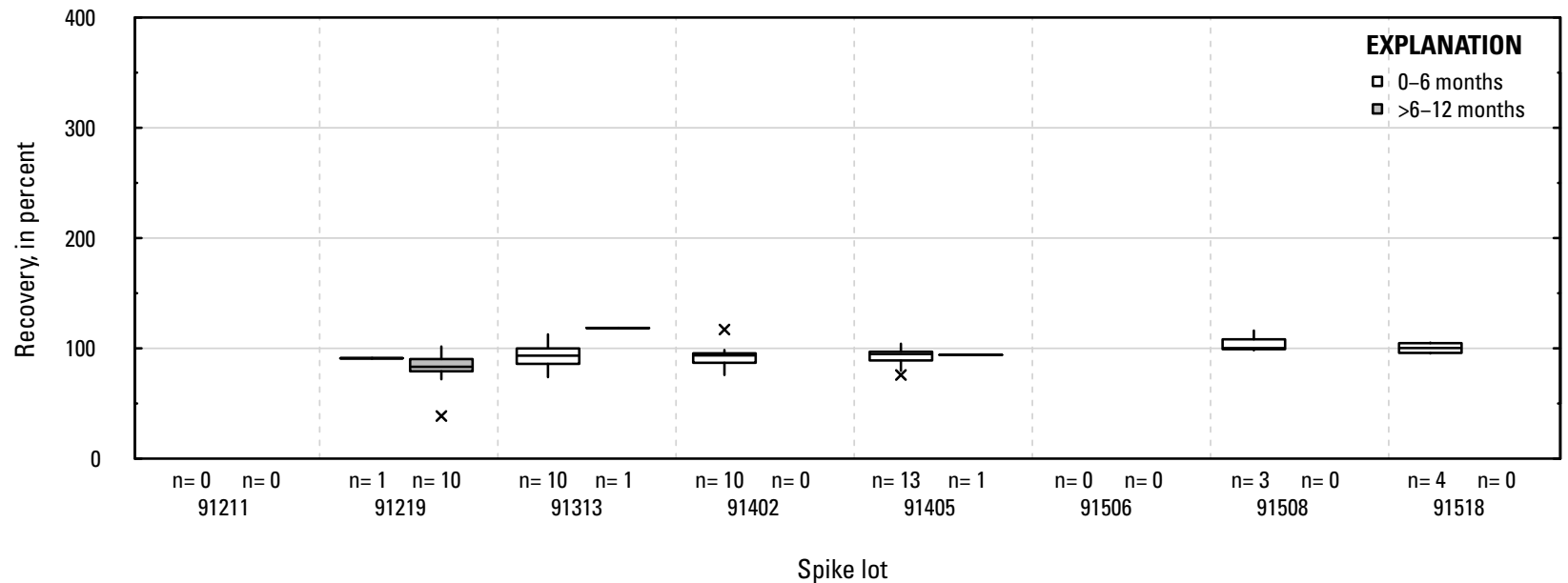

\section{R. 2-Amino-N-isopropylbenzamide: surface water field matrix spikes}

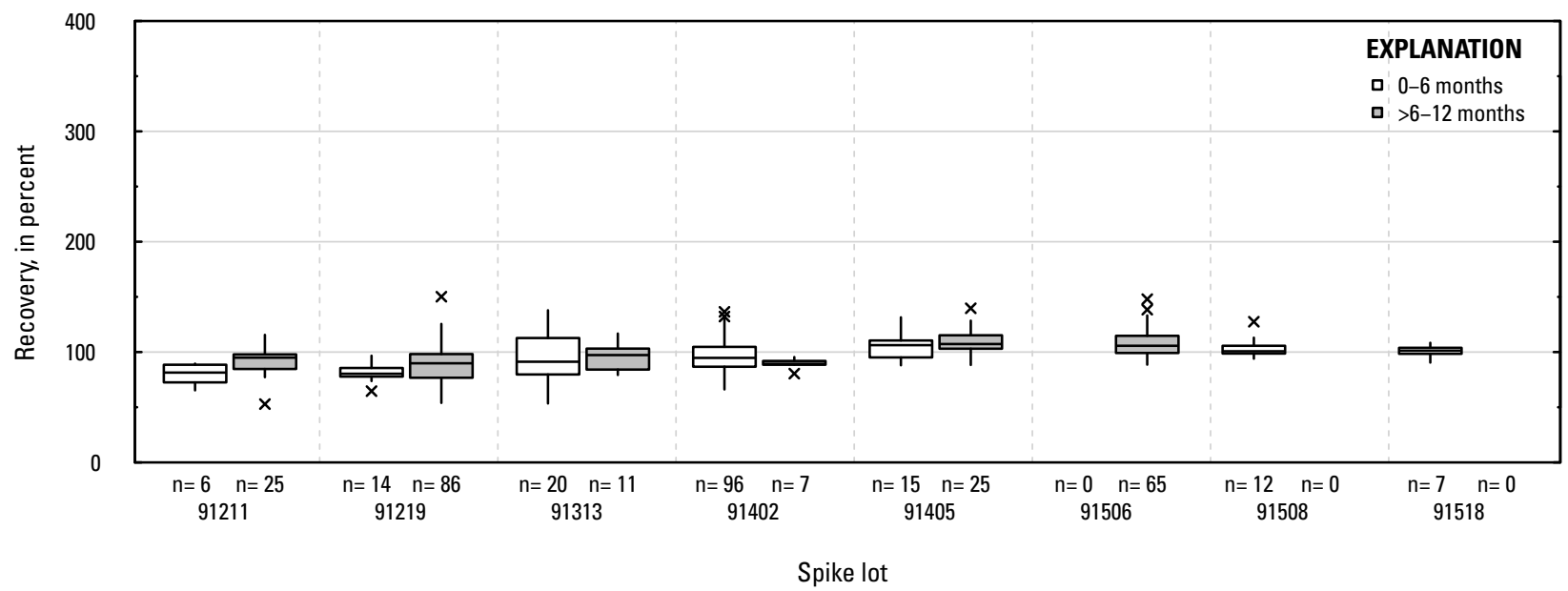

Figure 1-1. Distributions of recovery for individual pesticides in schedule 2437 by matrix, spike lot, and spike lot age. Recovery values larger than 400 percent are not shown.-Continued 


\section{S. 2-Chloro-2',6'-diethylacetanilide: laboratory reagent spikes}

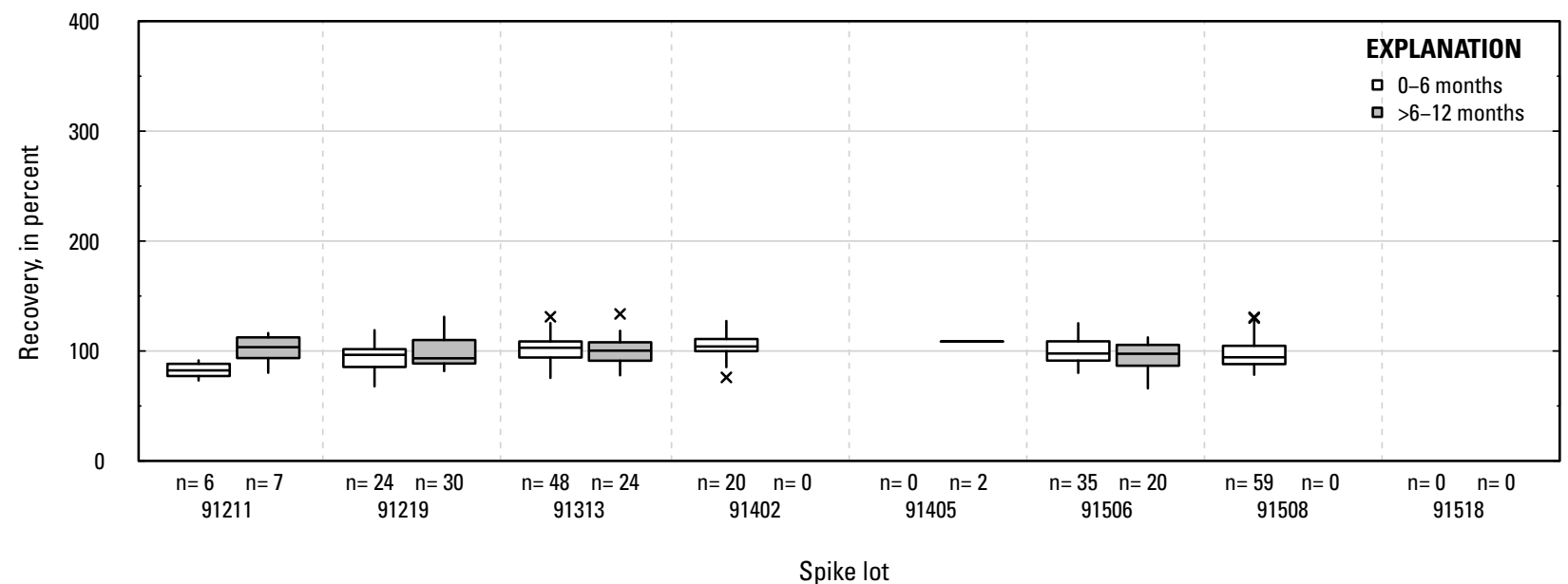

\section{T. 2-Chloro-2',6'-diethylacetanilide: groundwater field matrix spikes}

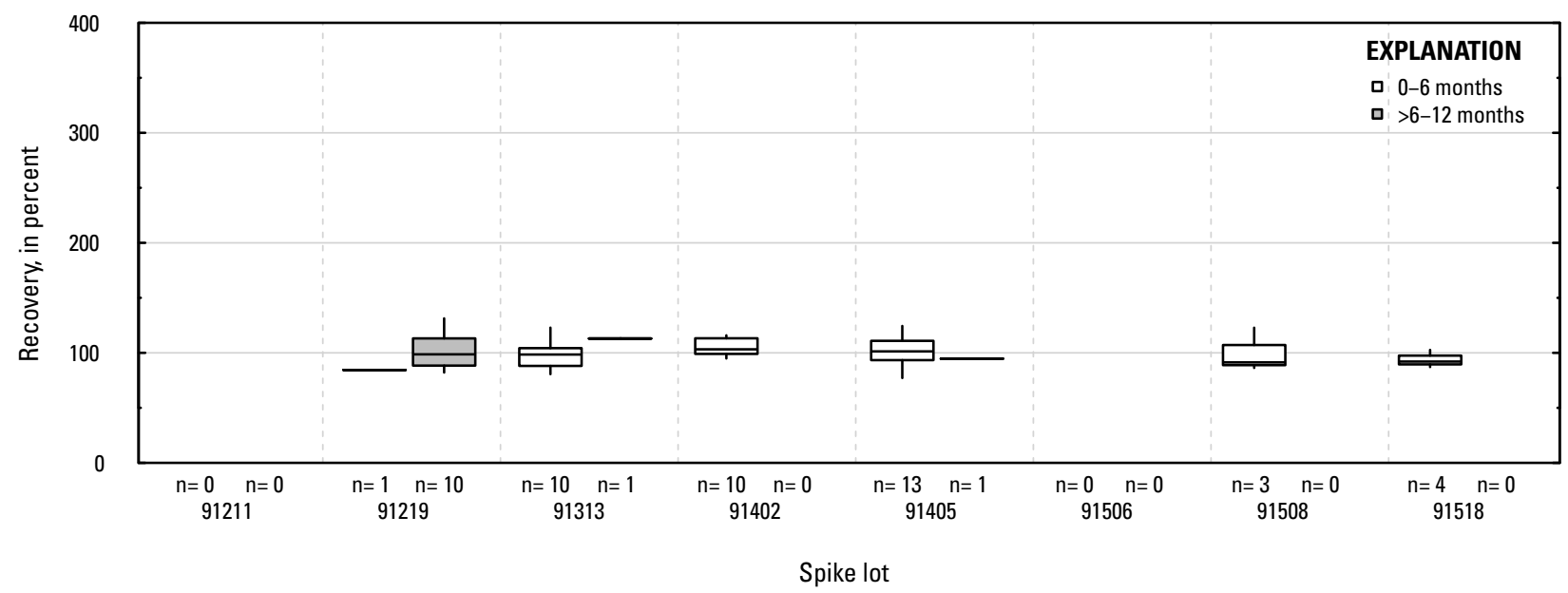

U. 2-Chloro-2',6'-diethylacetanilide: surface water field matrix spikes

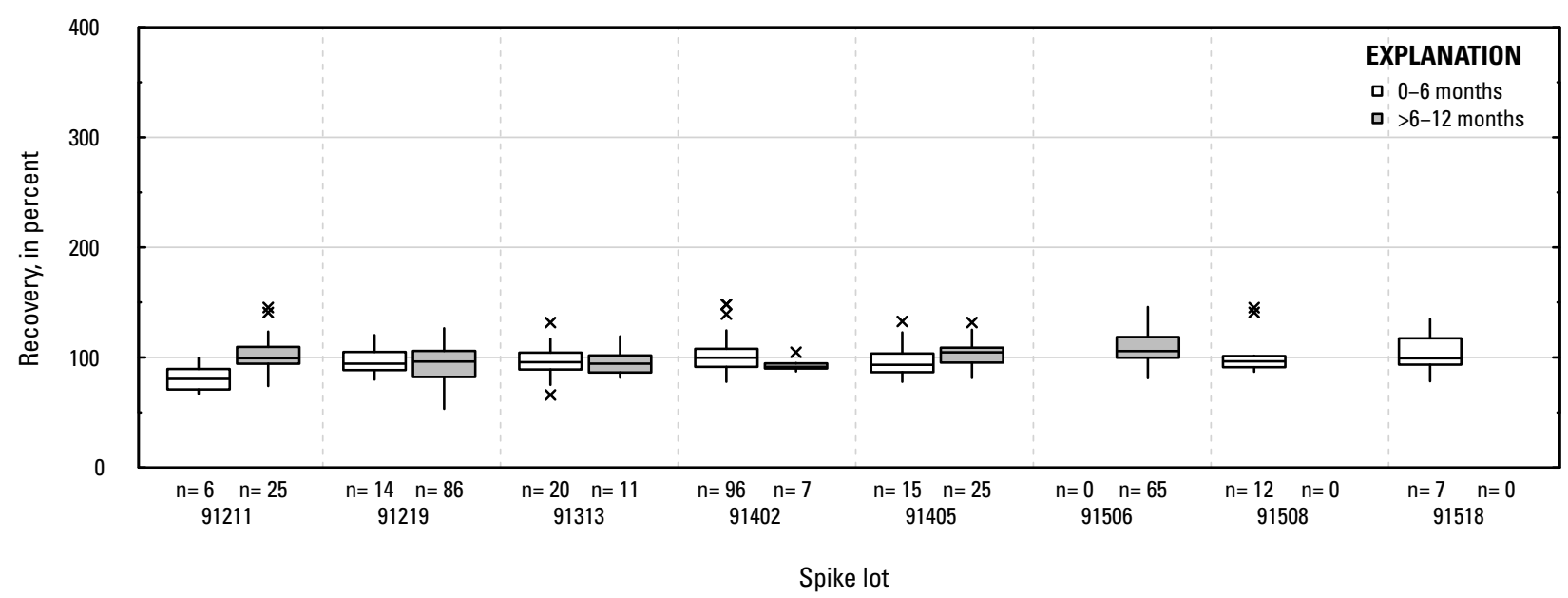

Figure 1-1. Distributions of recovery for individual pesticides in schedule 2437 by matrix, spike lot, and spike lot age. Recovery values larger than 400 percent are not shown.-Continued 
V. 2-Chloro-N-(2-ethyl-6-methylphenyl)acetamide: laboratory reagent spikes

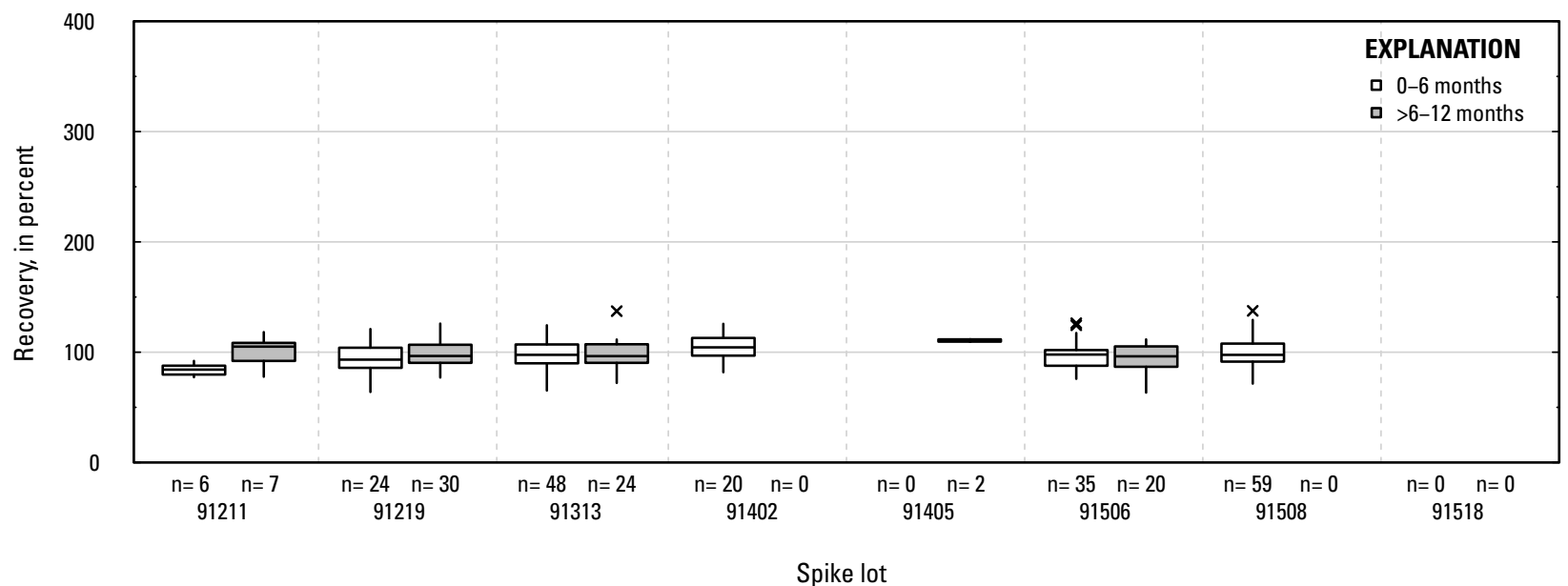

W. 2-Chloro-N-(2-ethyl-6-methylphenyl)acetamide: groundwater field matrix spikes

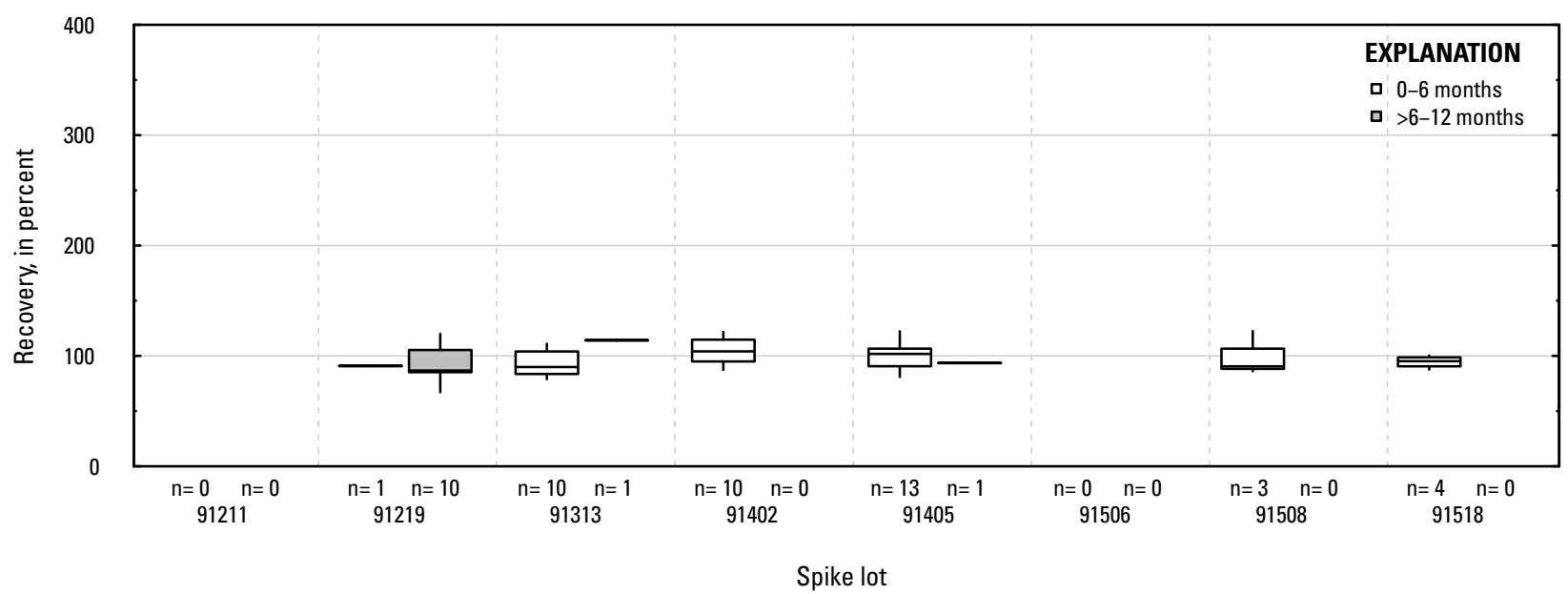

X. 2-Chloro-N-(2-ethyl-6-methylphenyl)acetamide: surface water field matrix spikes

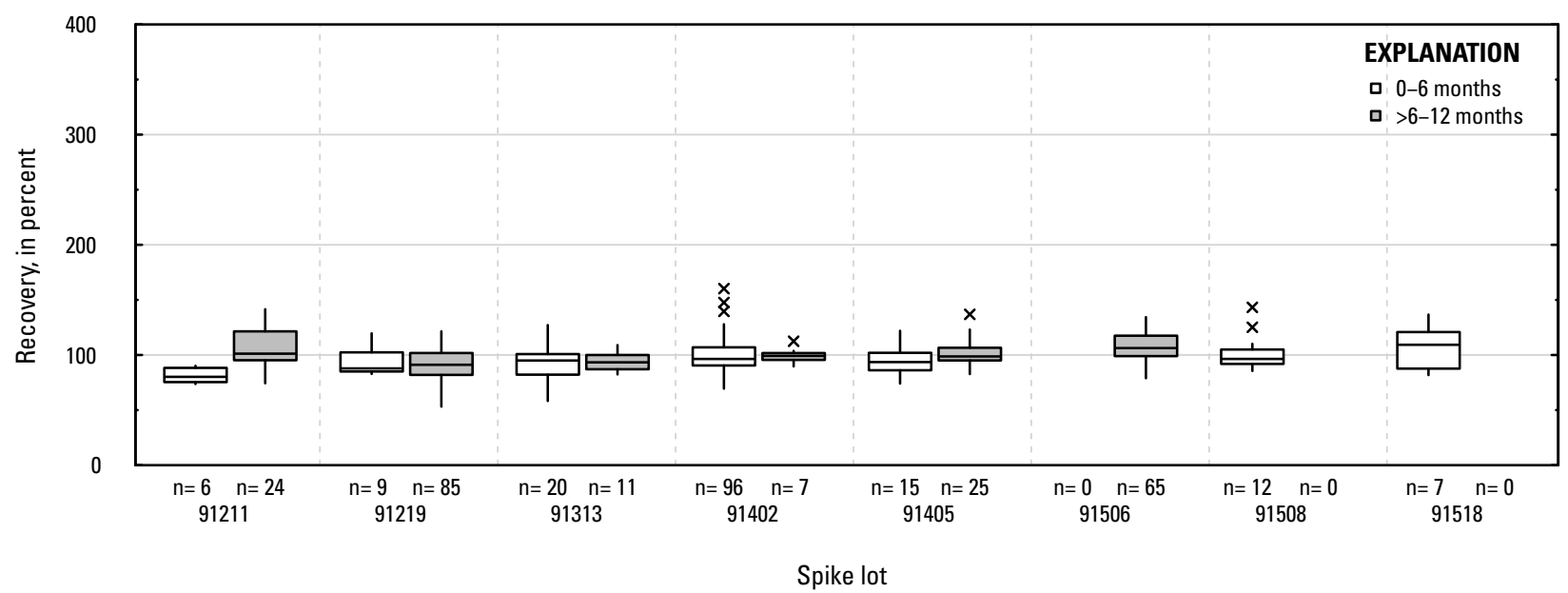

Figure 1-1. Distributions of recovery for individual pesticides in schedule 2437 by matrix, spike lot, and spike lot age. Recovery values larger than 400 percent are not shown.-Continued 
Y. 2-Hydroxy-4-isopropylamino-6-amino-s-triazine: laboratory reagent spikes

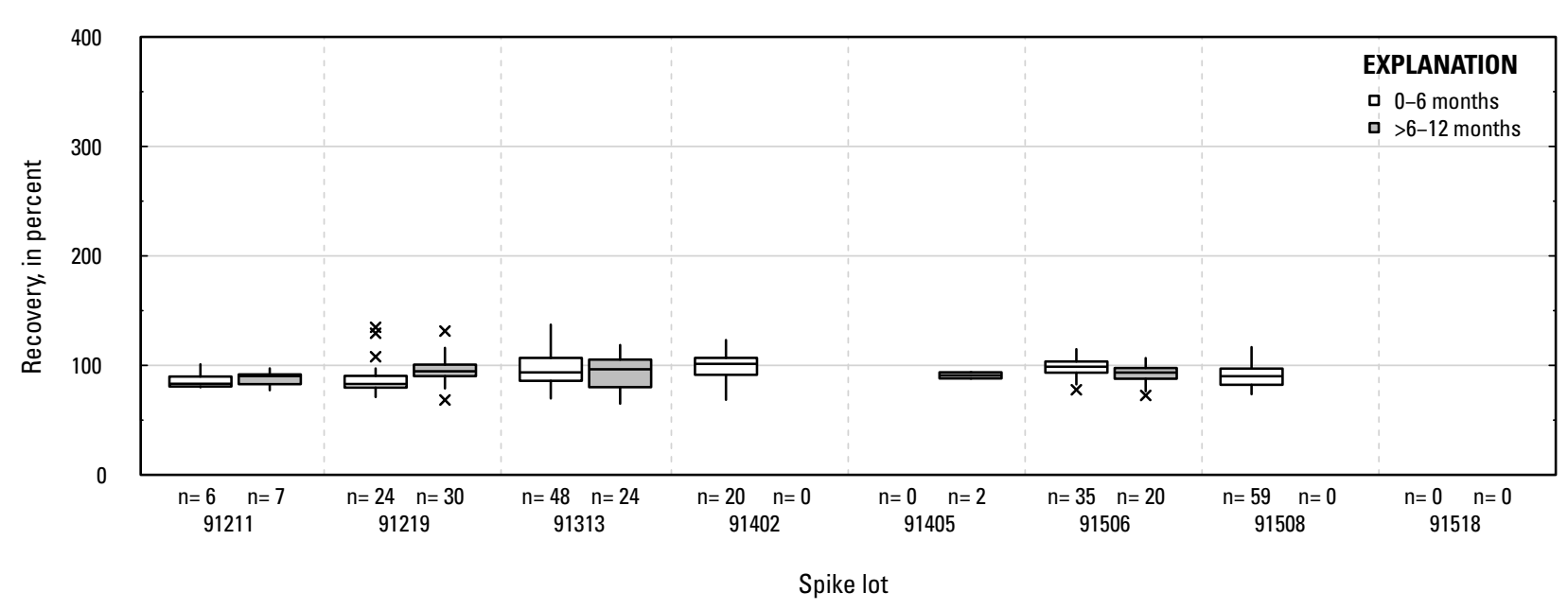

Z. 2-Hydroxy-4-isopropylamino-6-amino-s-triazine: groundwater field matrix spikes

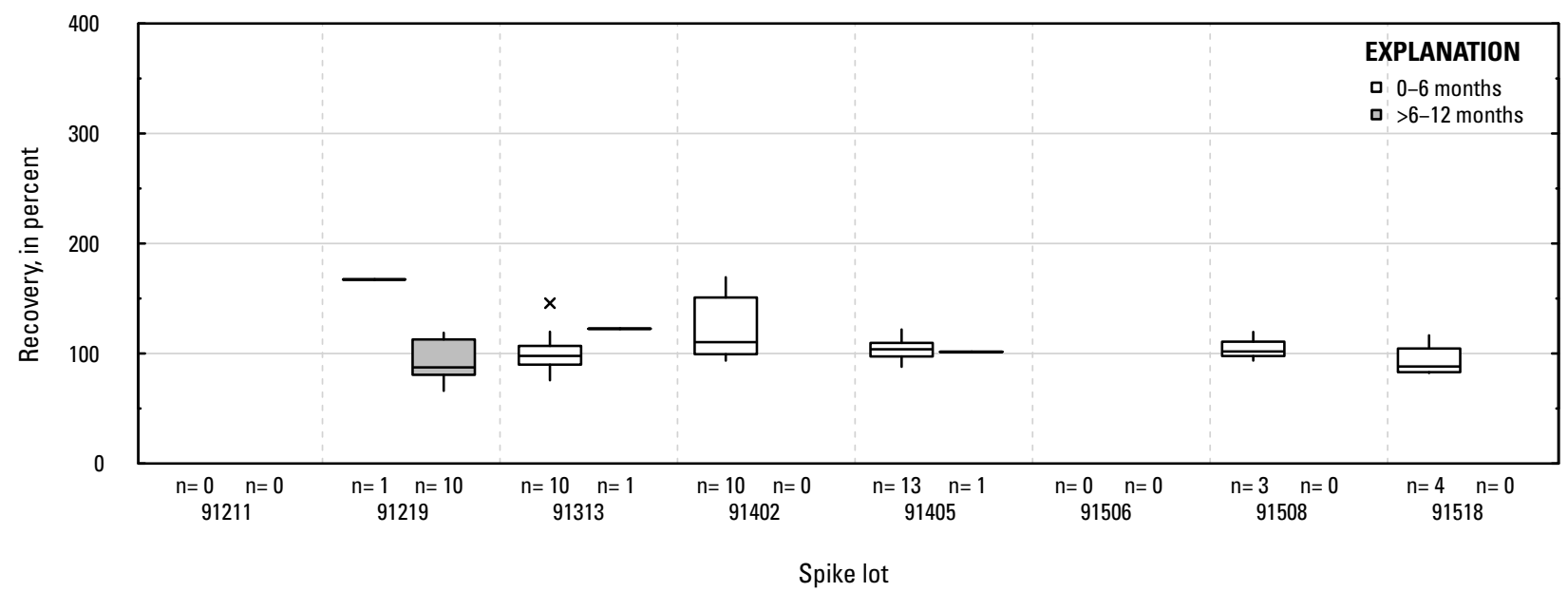

AA. 2-Hydroxy-4-isopropylamino-6-amino-s-triazine: surface water field matrix spikes

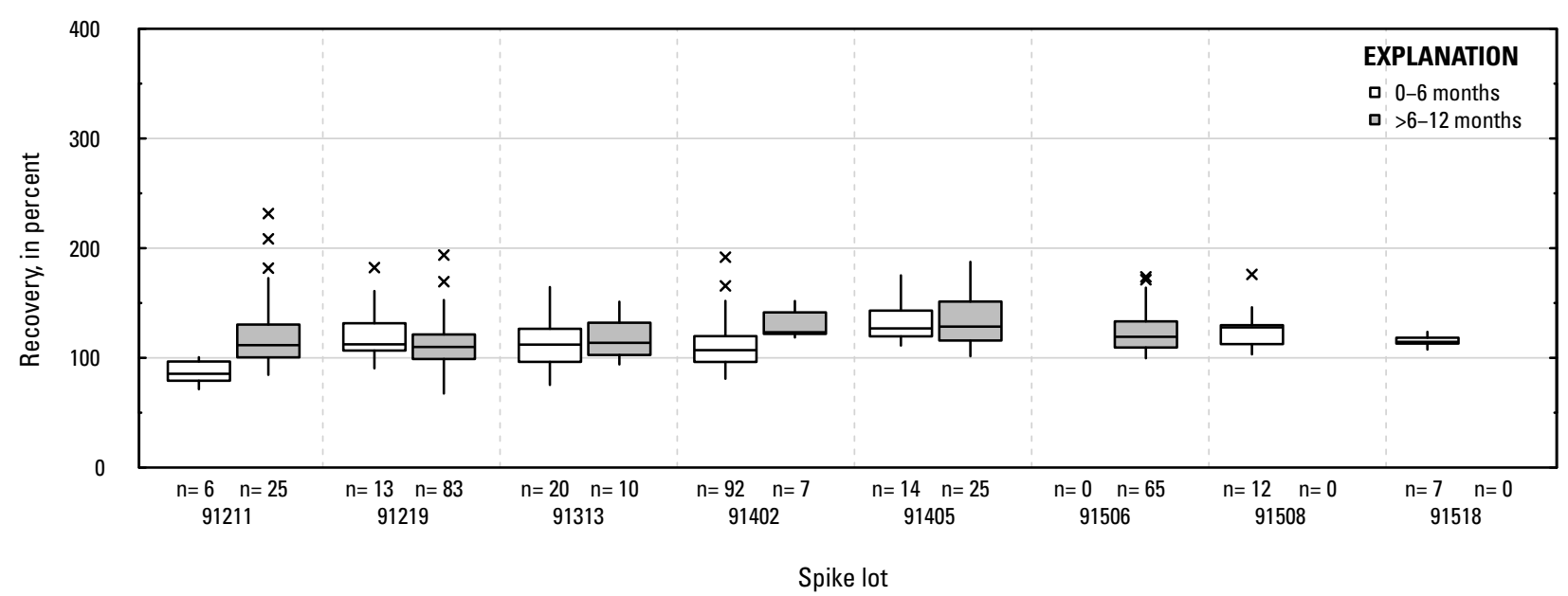

Figure 1-1. Distributions of recovery for individual pesticides in schedule 2437 by matrix, spike lot, and spike lot age. Recovery values larger than 400 percent are not shown.-Continued 
AB. 2-Hydroxy-6-ethylamino-4-amino-s-triazine: laboratory reagent spikes

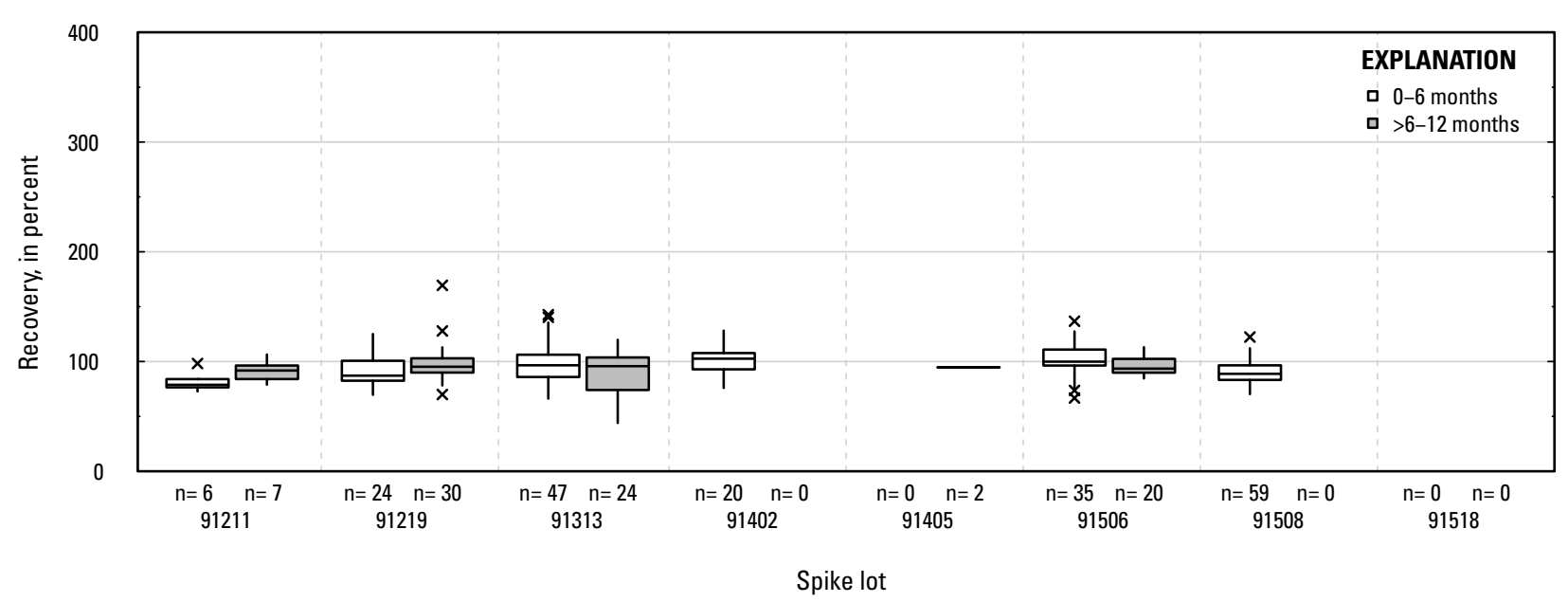

AC. 2-Hydroxy-6-ethylamino-4-amino-s-triazine: groundwater field matrix spikes

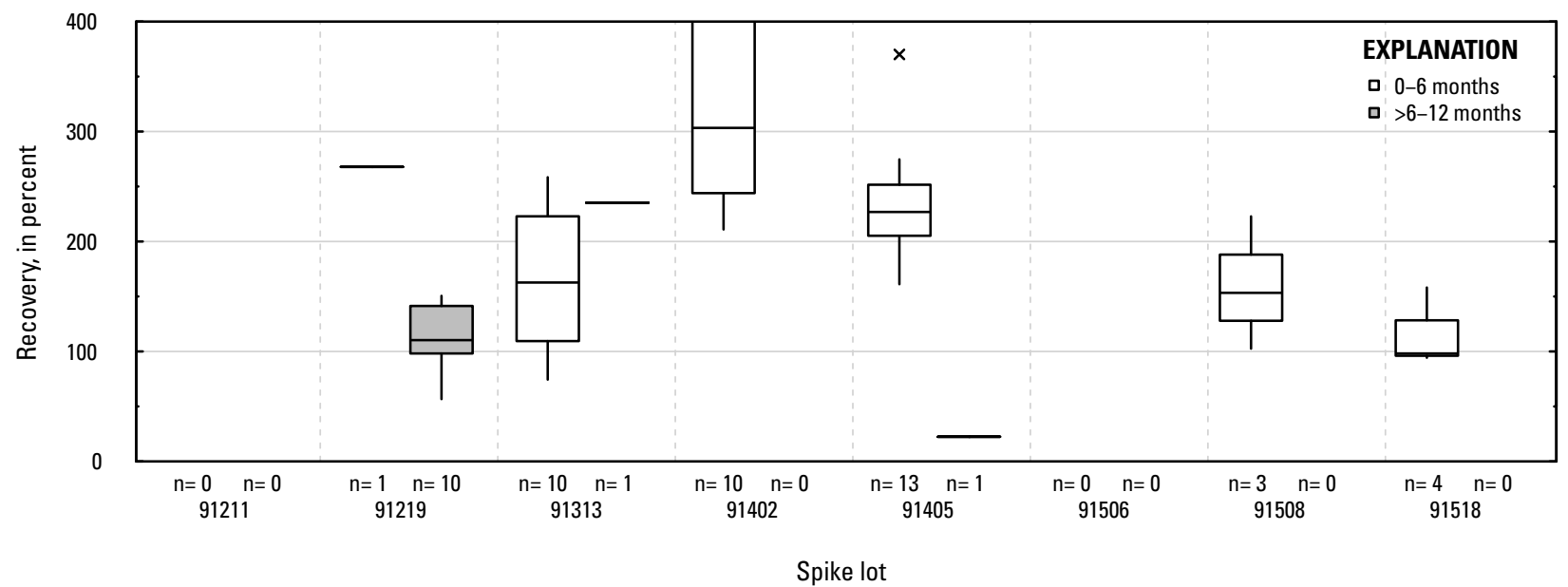

$A D$. 2-Hydroxy-6-ethylamino-4-amino-s-triazine: surface water field matrix spikes

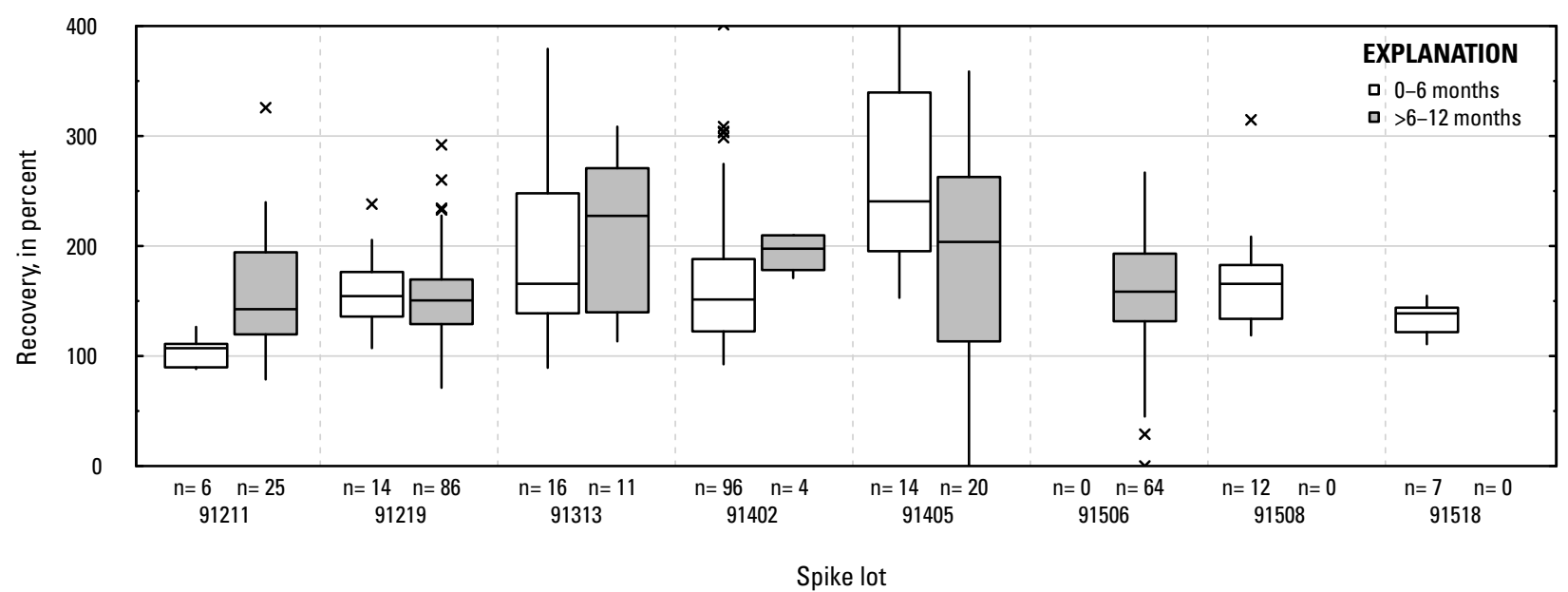

Figure 1-1. Distributions of recovery for individual pesticides in schedule 2437 by matrix, spike lot, and spike lot age. Recovery values larger than 400 percent are not shown.-Continued 


\section{AE. 2-Hydroxy-4-isopropylamino-6-ethylamino-s-triazine \{0IET\}: laboratory reagent spikes}

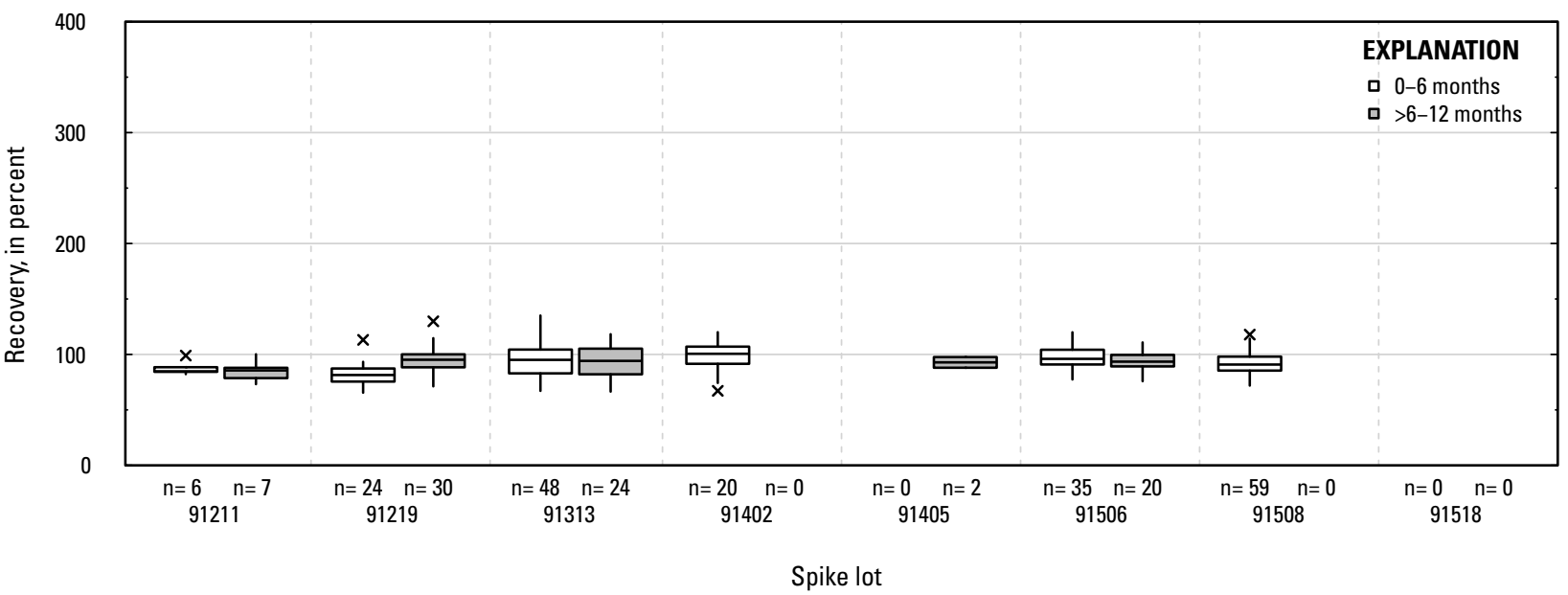

AF. 2-Hydroxy-4-isopropylamino-6-ethylamino-s-triazine \{0IET\}: groundwater field matrix spikes

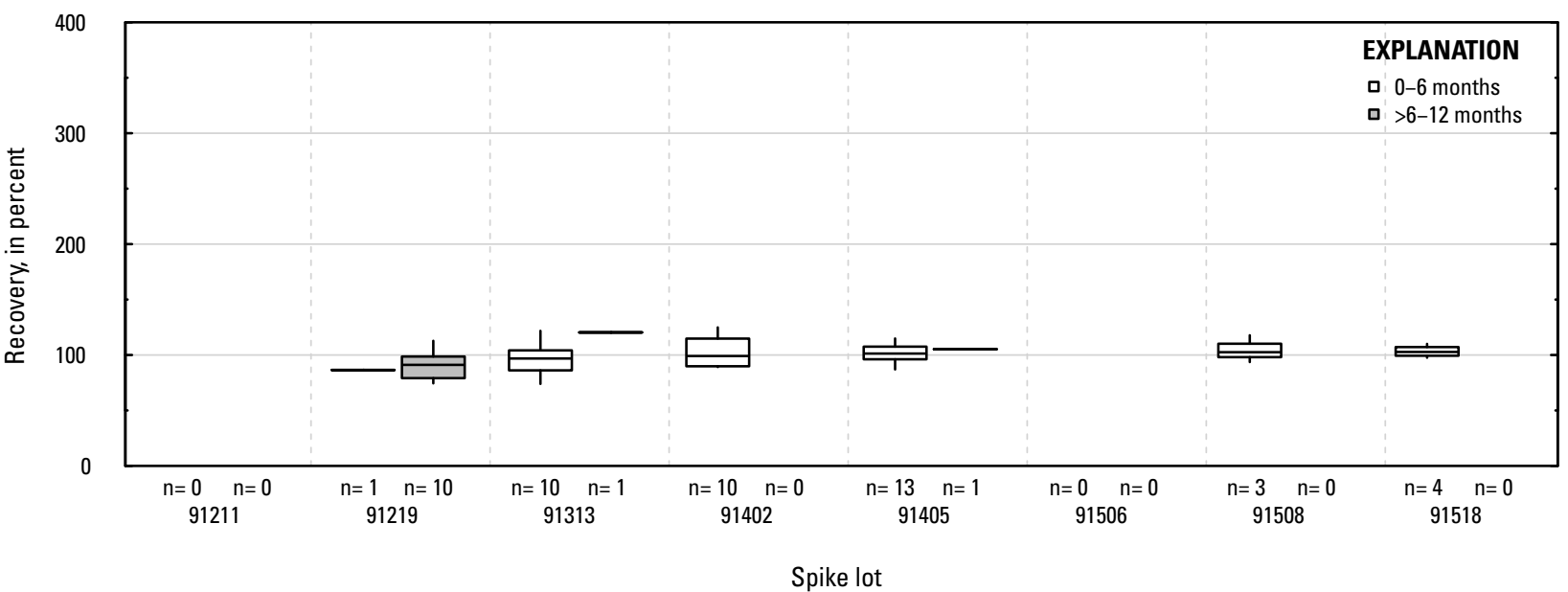

AG. 2-Hydroxy-4-isopropylamino-6-ethylamino-s-triazine \{OIET\}: surface water field matrix spikes

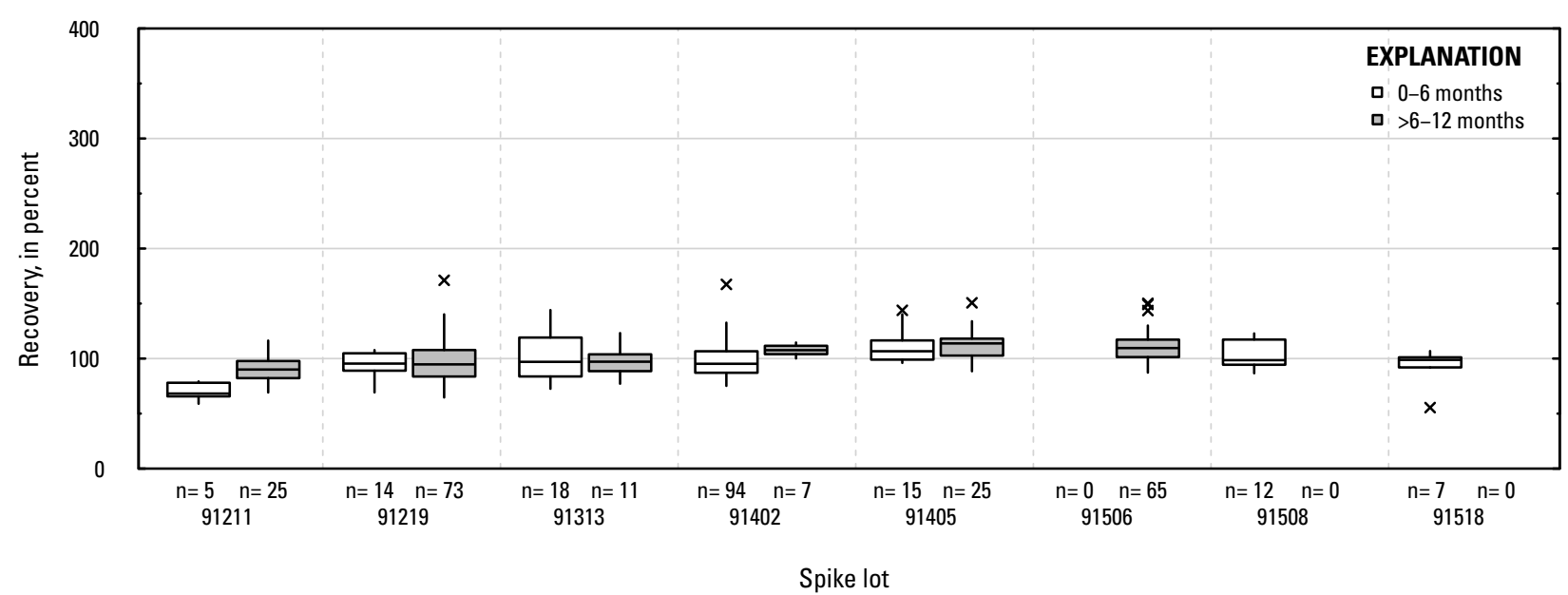

Figure 1-1. Distributions of recovery for individual pesticides in schedule 2437 by matrix, spike lot, and spike lot age. Recovery values larger than 400 percent are not shown.-Continued 
AH. 3,4-Dichlorophenylurea: laboratory reagent spikes

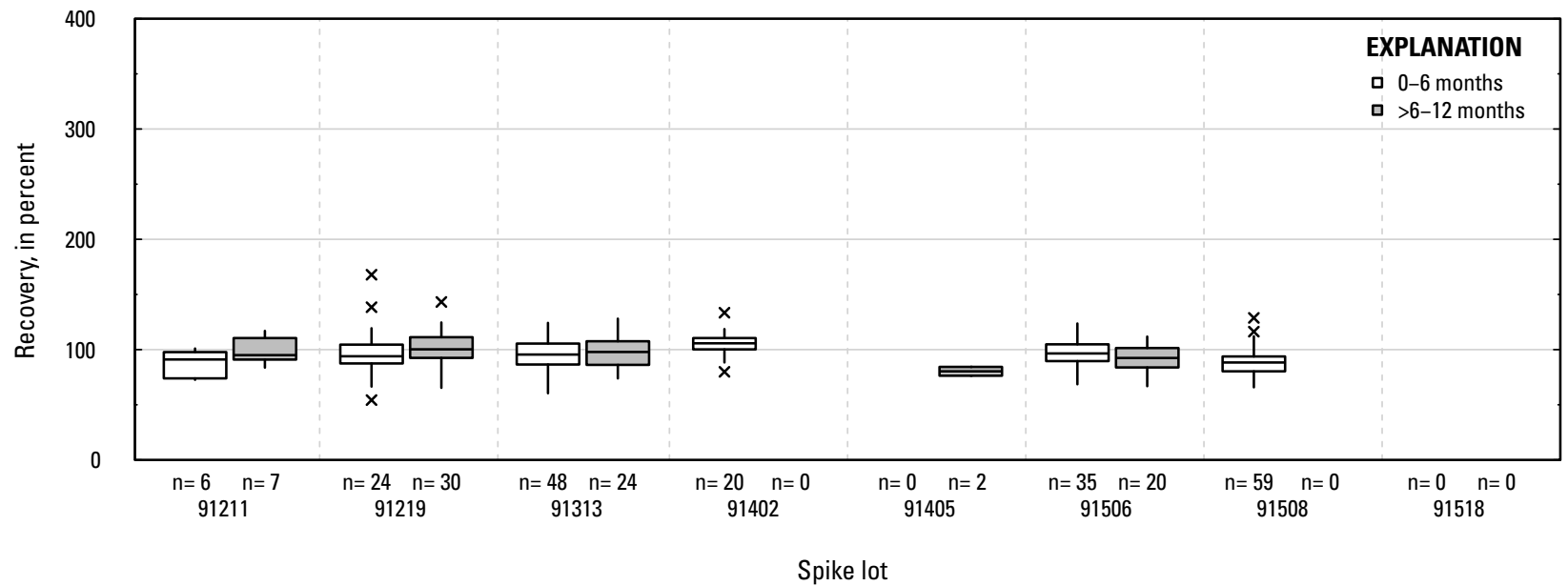

Al. 3,4-Dichlorophenylurea: groundwater field matrix spikes

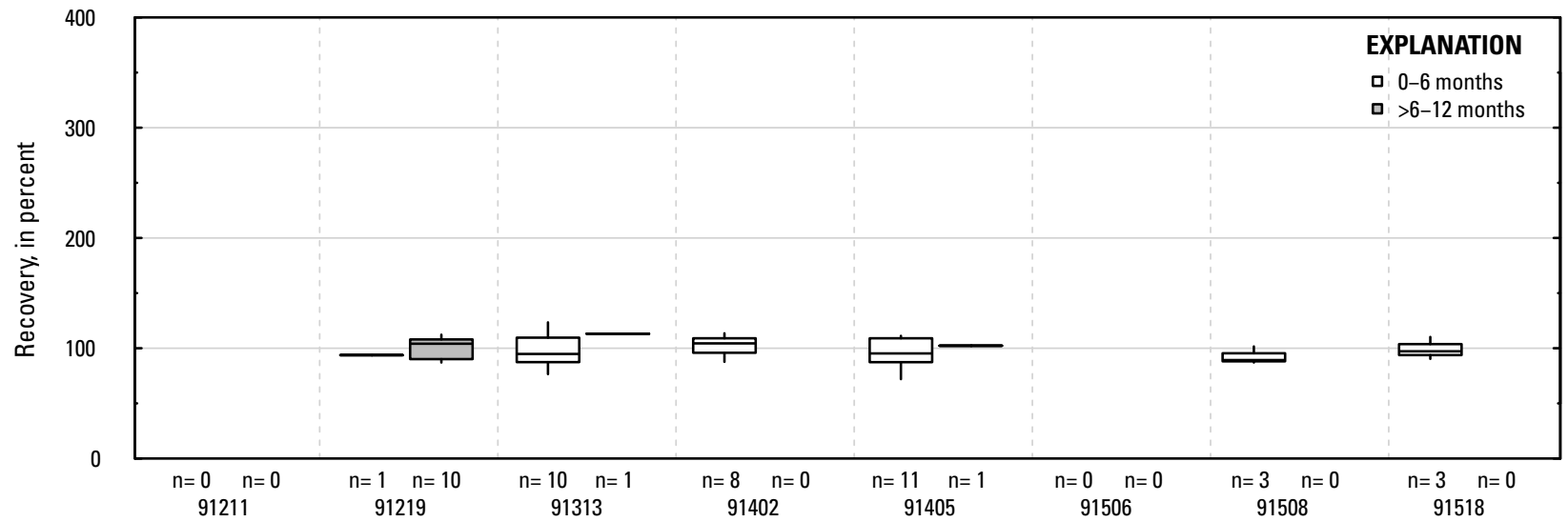

Spike lot

\section{AJ. 3,4-Dichlorophenylurea: surface water field matrix spikes}

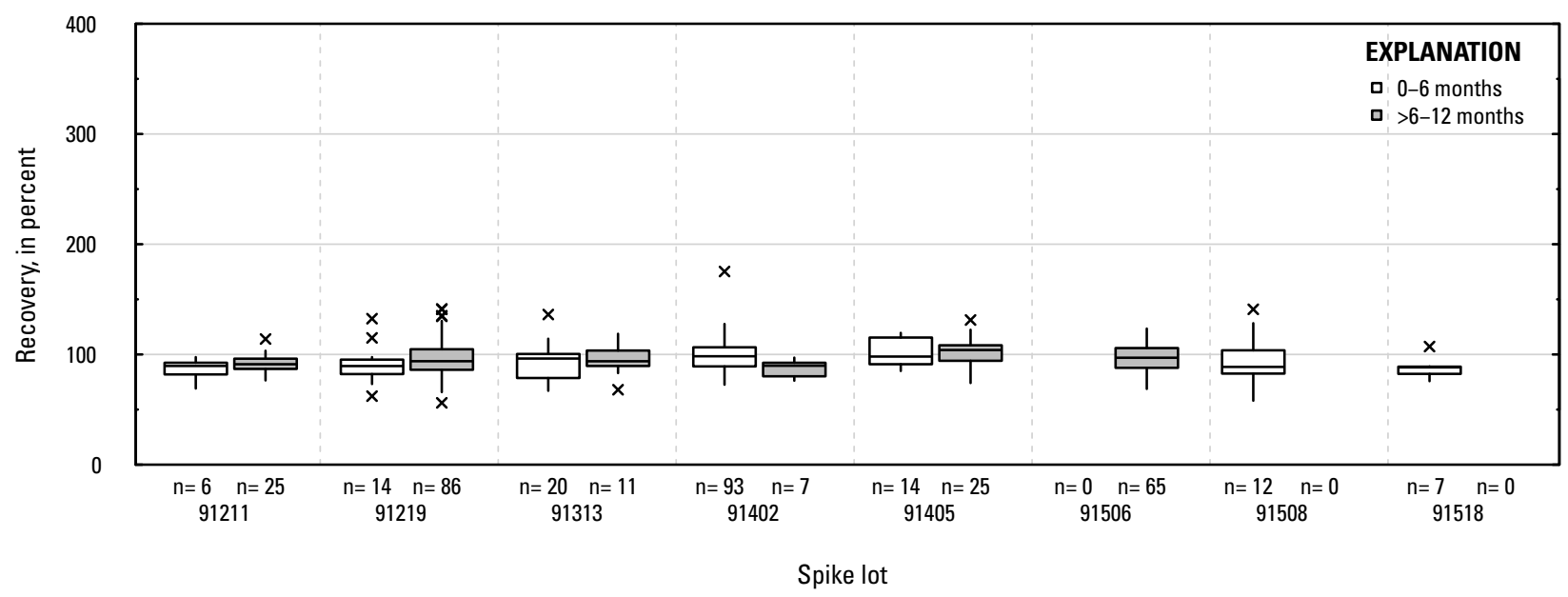

Figure 1-1. Distributions of recovery for individual pesticides in schedule 2437 by matrix, spike lot, and spike lot age. Recovery values larger than 400 percent are not shown.-Continued 
AK. 3-Hydroxycarbofuran: laboratory reagent spikes

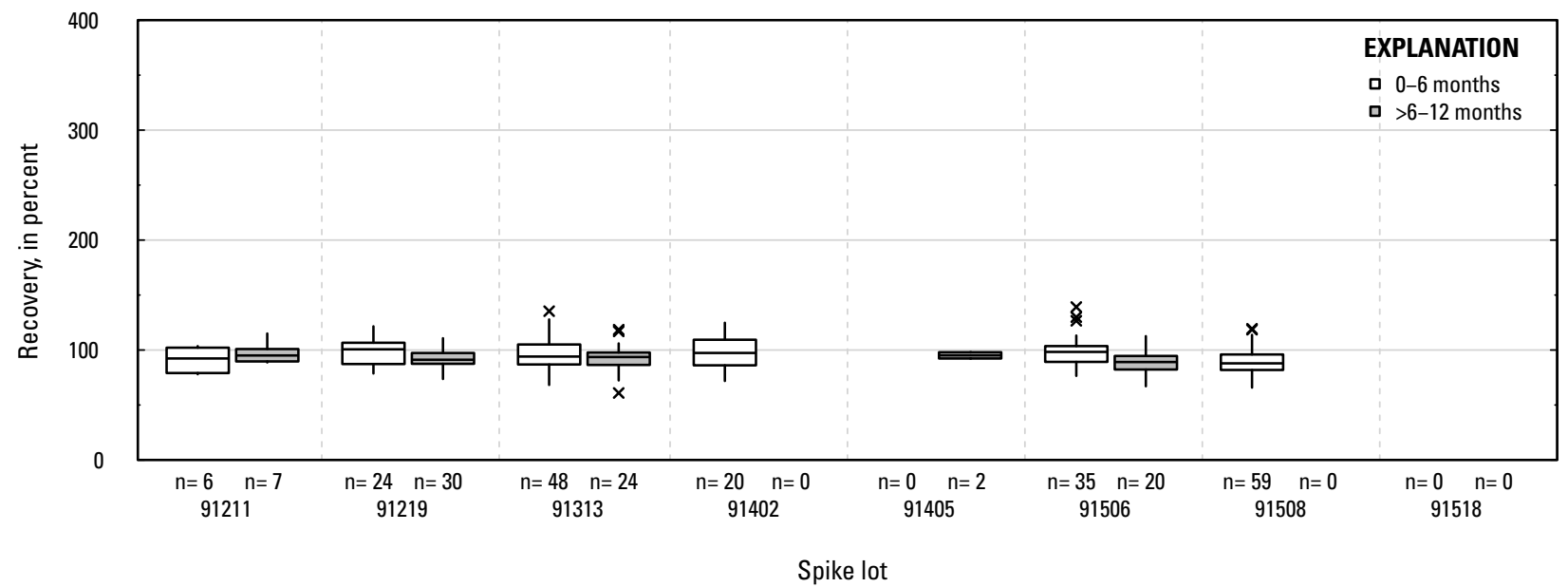

AL. 3-Hydroxycarbofuran: groundwater field matrix spikes

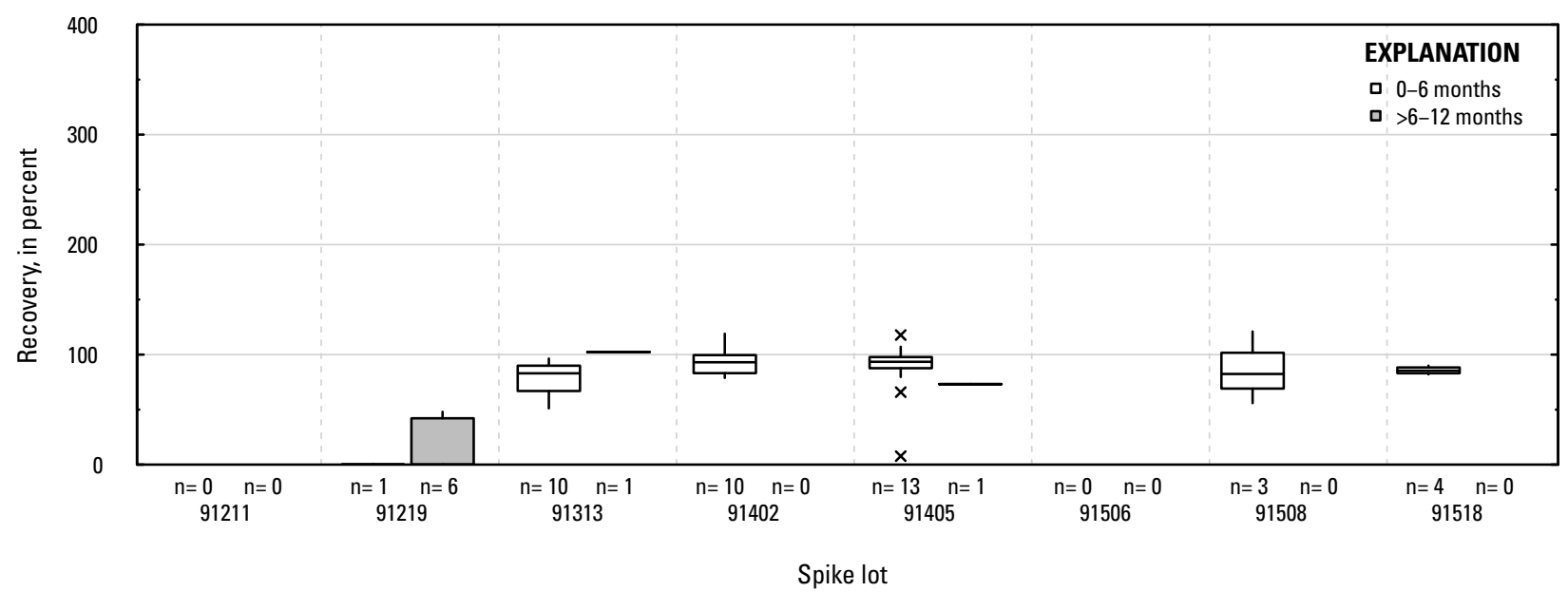

AM. 3-Hydroxycarbofuran: surface water field matrix spikes

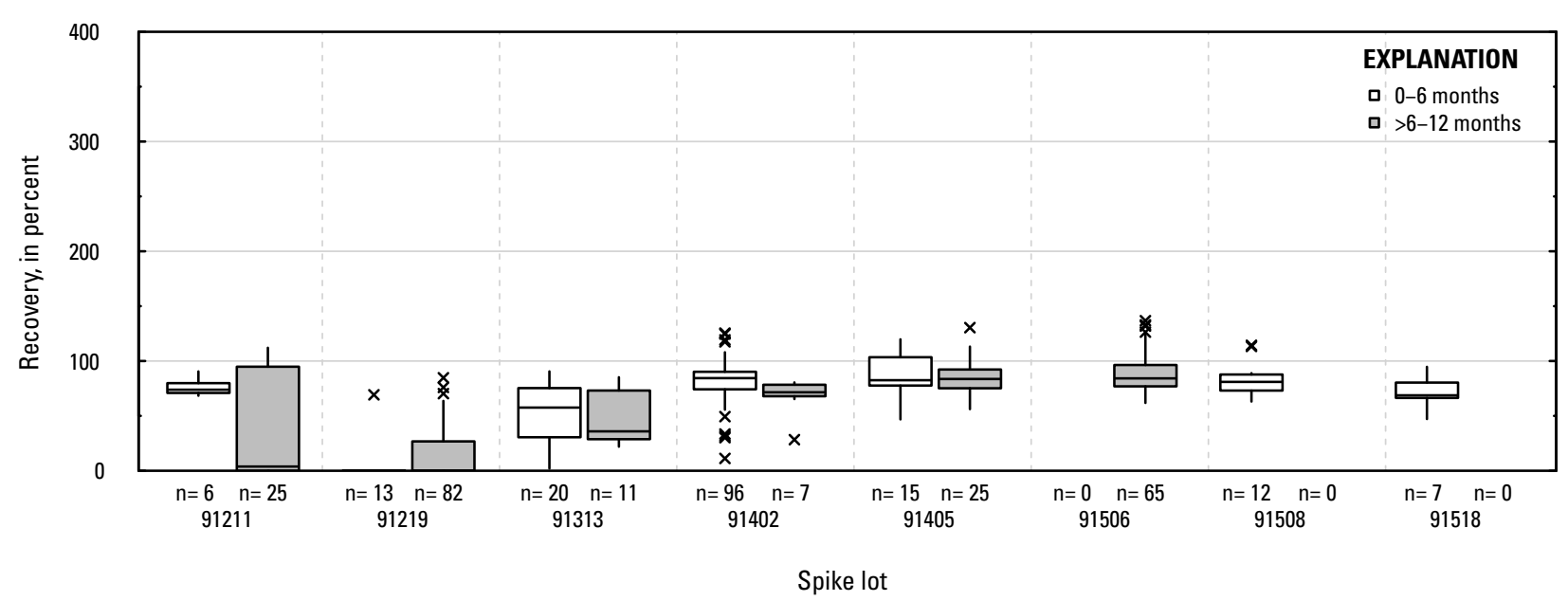

Figure 1-1. Distributions of recovery for individual pesticides in schedule 2437 by matrix, spike lot, and spike lot age. Recovery values larger than 400 percent are not shown.-Continued 
AN. 3-Phenoxybenzoic acid: laboratory reagent spikes

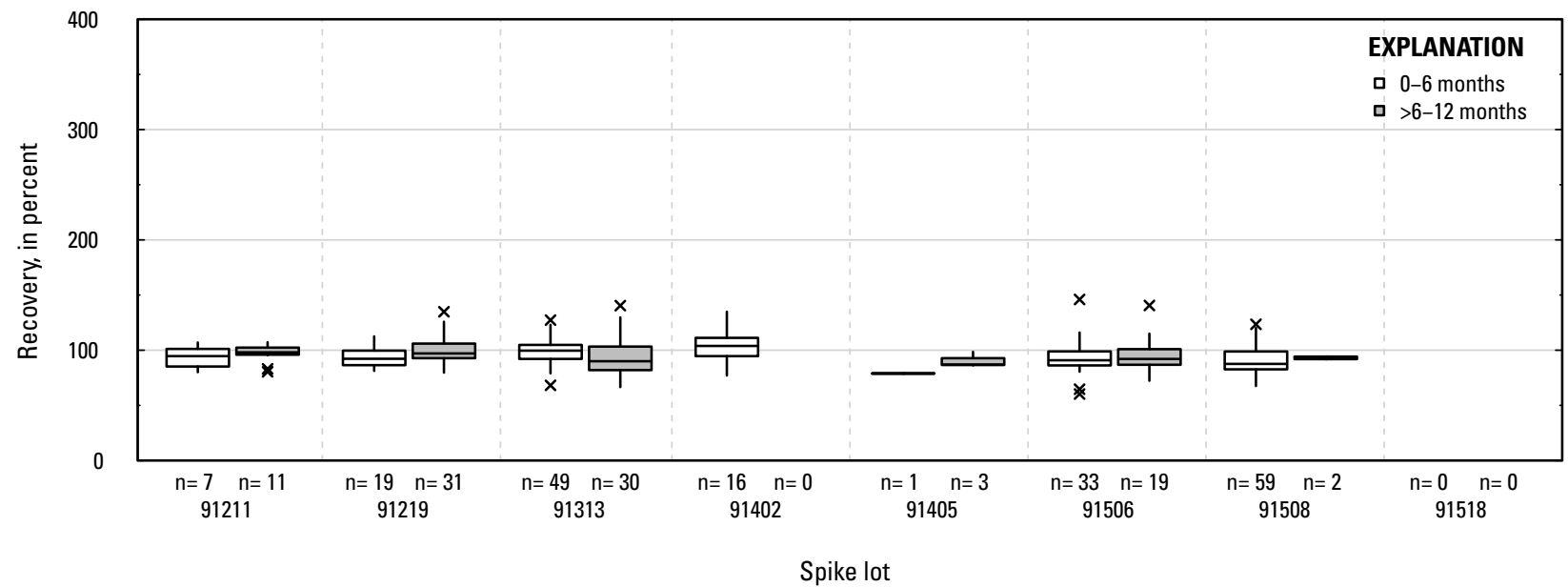

A0. 3-Phenoxybenzoic acid: groundwater field matrix spikes

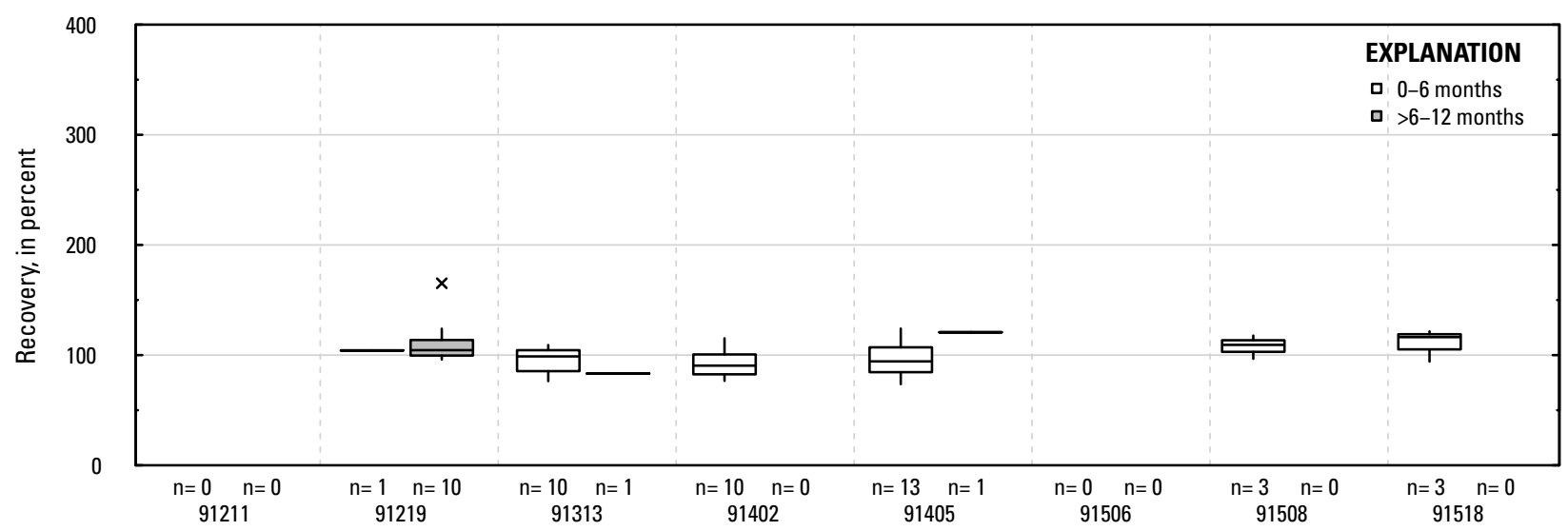

Spike lot

AP. 3-Phenoxybenzoic acid: surface water field matrix spikes

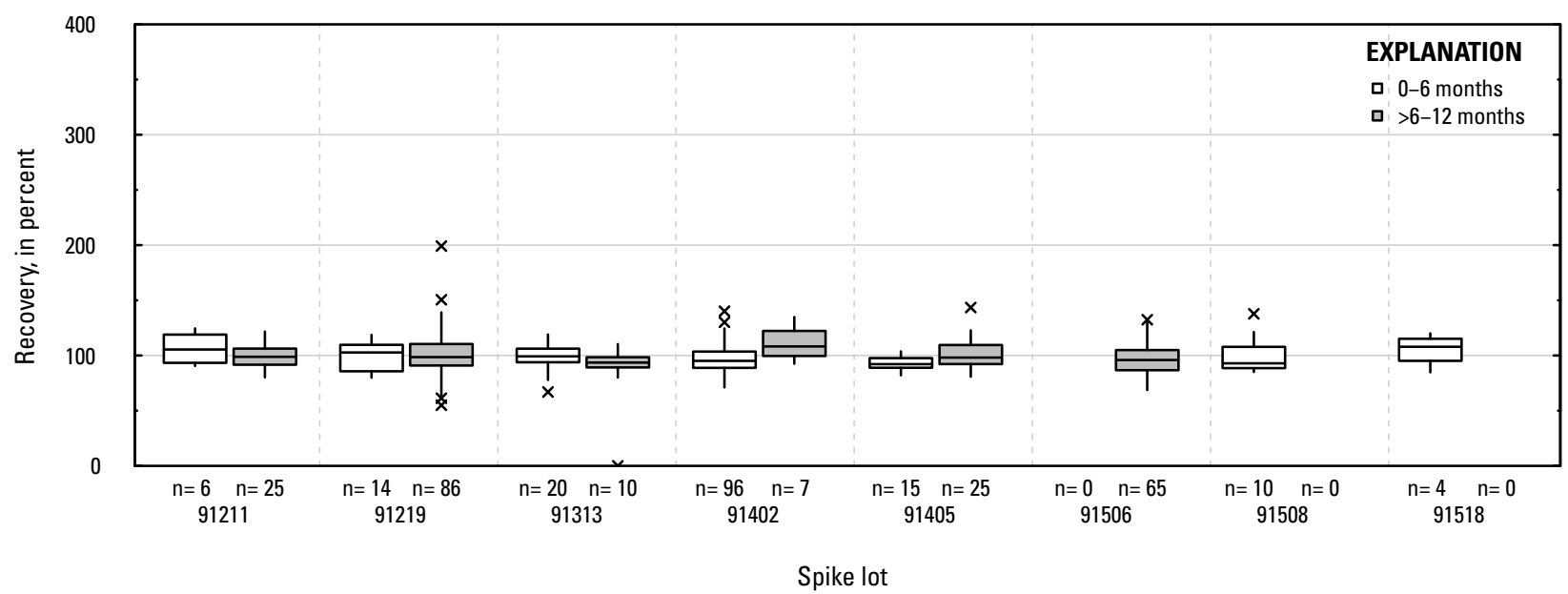

Figure 1-1. Distributions of recovery for individual pesticides in schedule 2437 by matrix, spike lot, and spike lot age. Recovery values larger than 400 percent are not shown.-Continued 
AQ. 4-(Hydroxymethyl)pendimethalin: laboratory reagent spikes

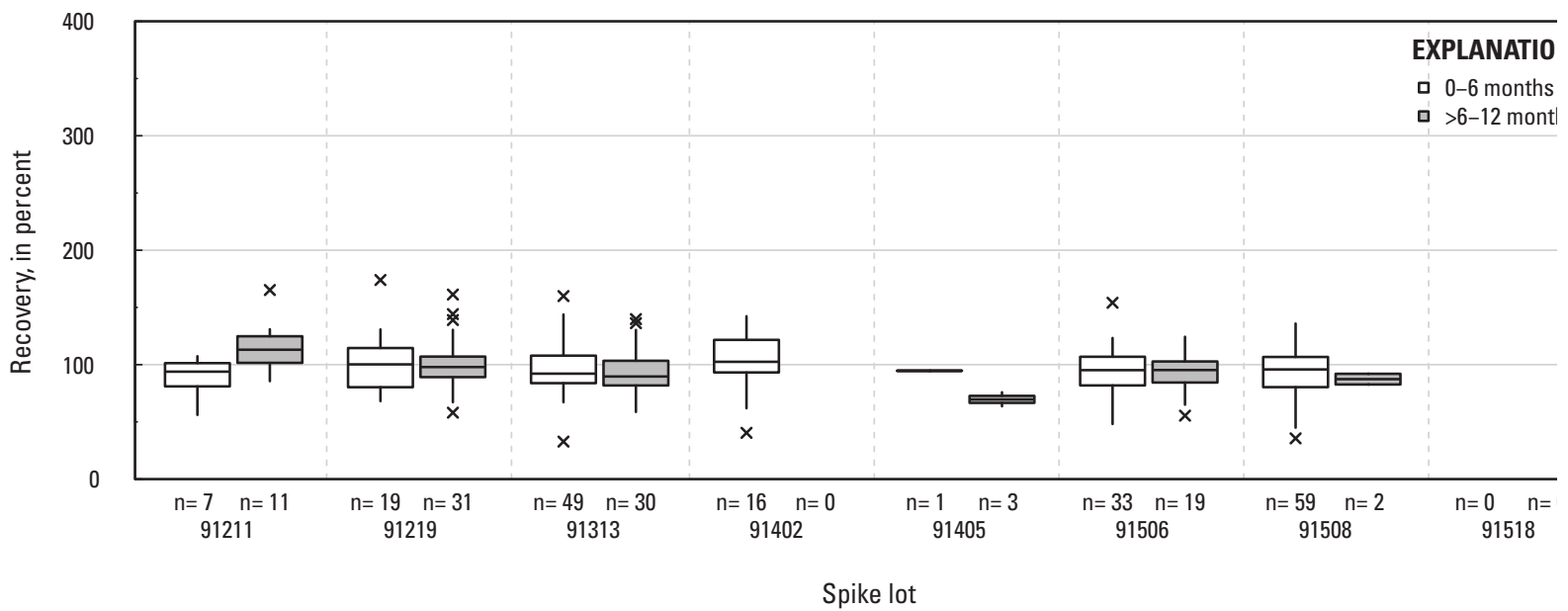

AR. 4-(Hydroxymethyl)pendimethalin: groundwater field matrix spikes

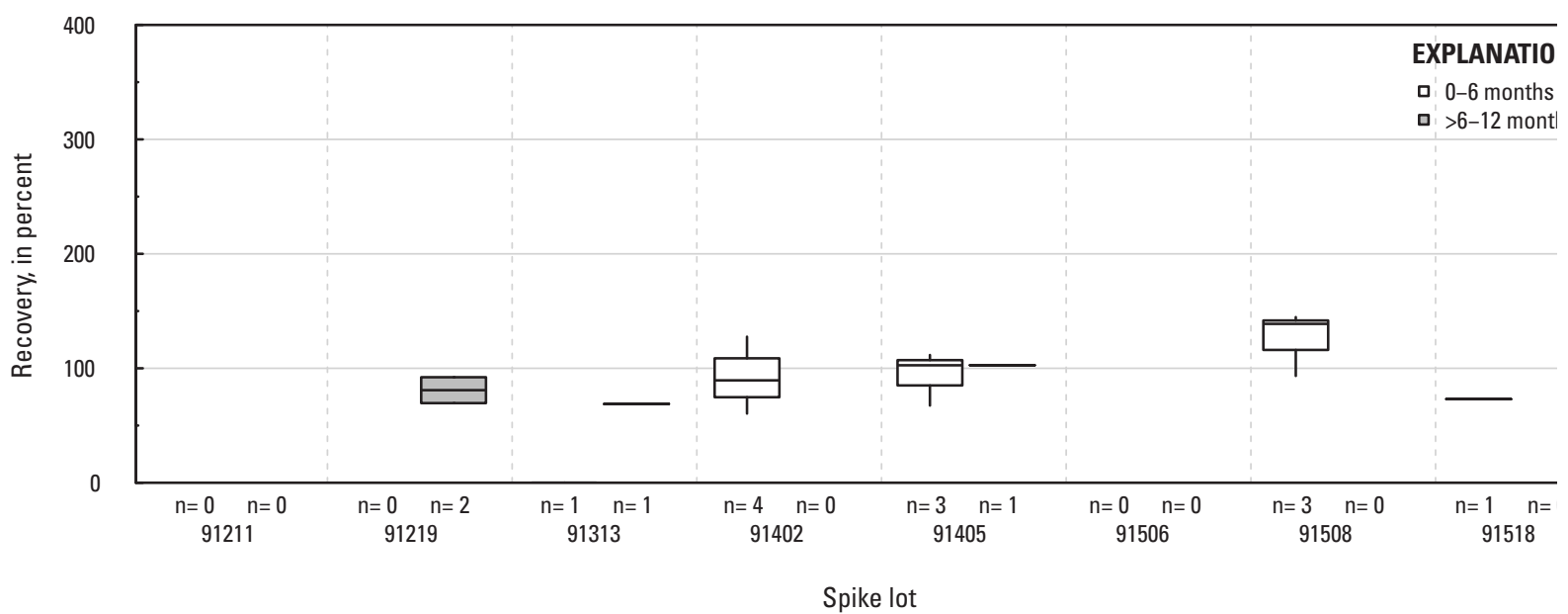

AS. 4-(Hydroxymethyl)pendimethalin: surface water field matrix spikes

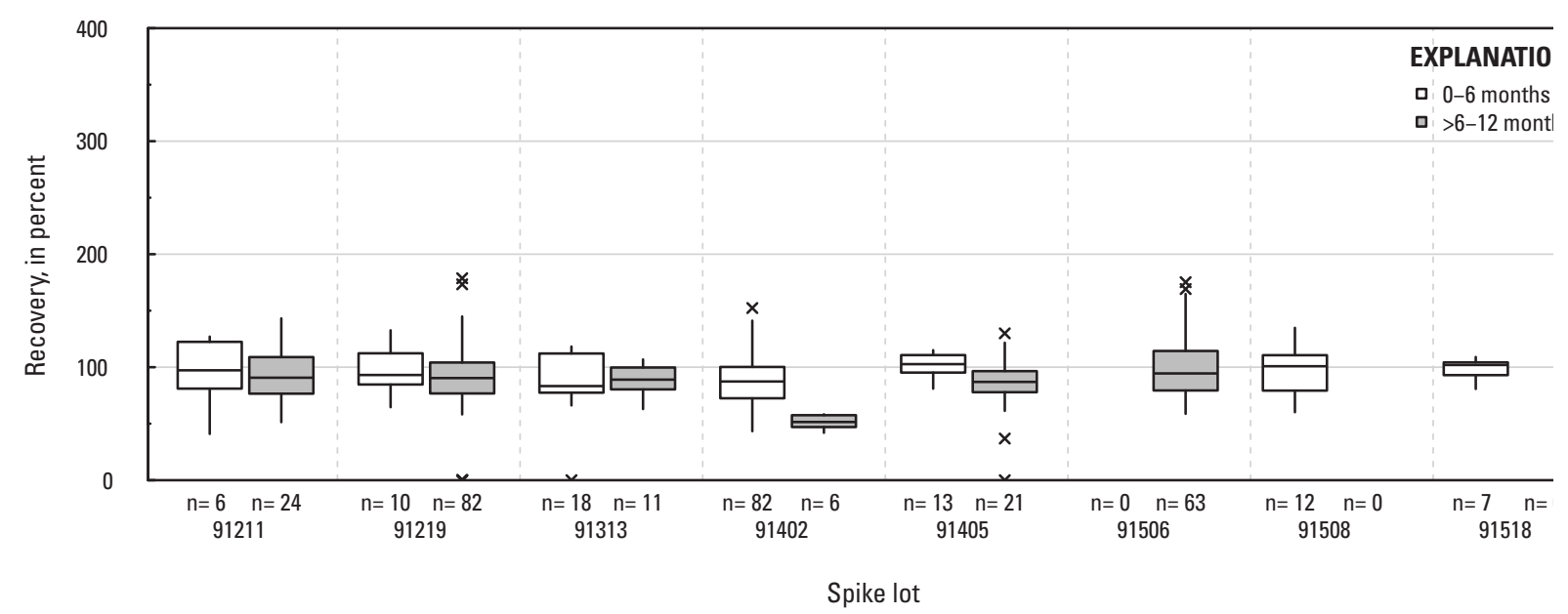

Figure 1-1. Distributions of recovery for individual pesticides in schedule 2437 by matrix, spike lot, and spike lot age. Recovery values larger than 400 percent are not shown.-Continued 
AT. 4-Chlorobenzylmethyl sulfoxide: laboratory reagent spikes

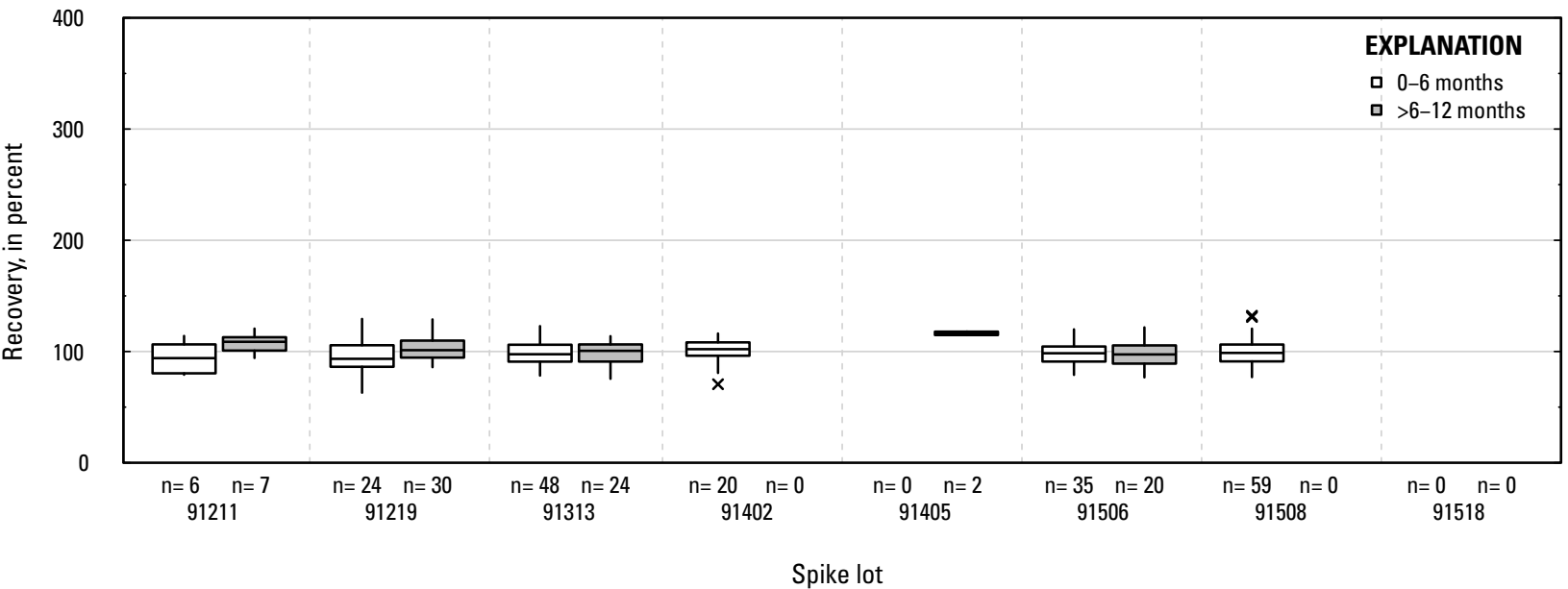

$A U$. 4-Chlorobenzylmethyl sulfoxide: groundwater field matrix spikes

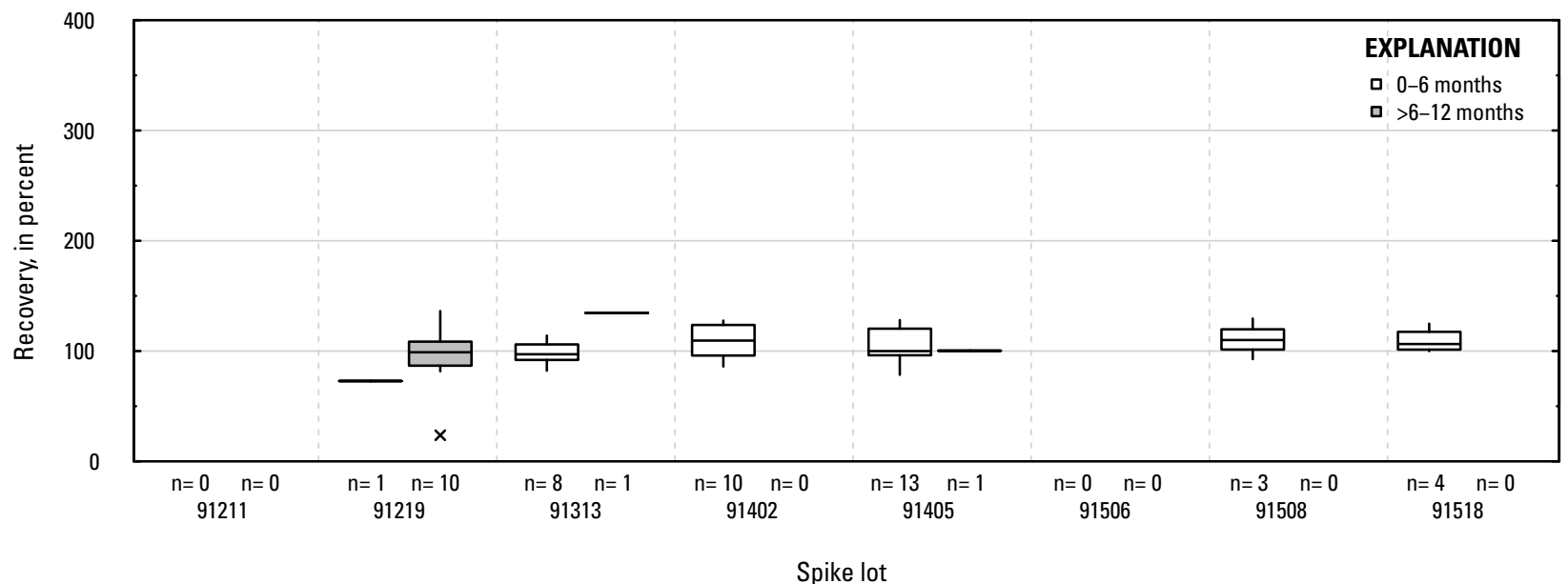

AV. 4-Chlorobenzylmethyl sulfoxide: surface water field matrix spikes

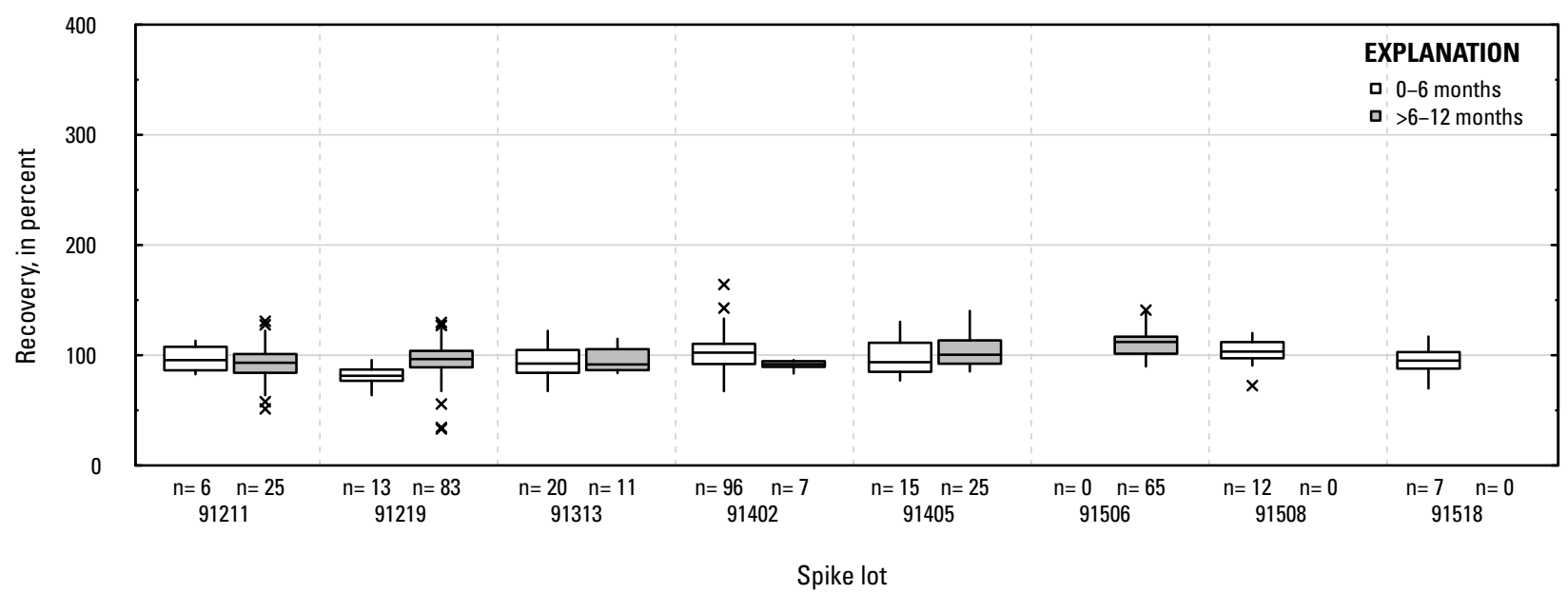

Figure 1-1. Distributions of recovery for individual pesticides in schedule 2437 by matrix, spike lot, and spike lot age. Recovery values larger than 400 percent are not shown.-Continued 
AW. 4-Hydroxy molinate: laboratory reagent spikes

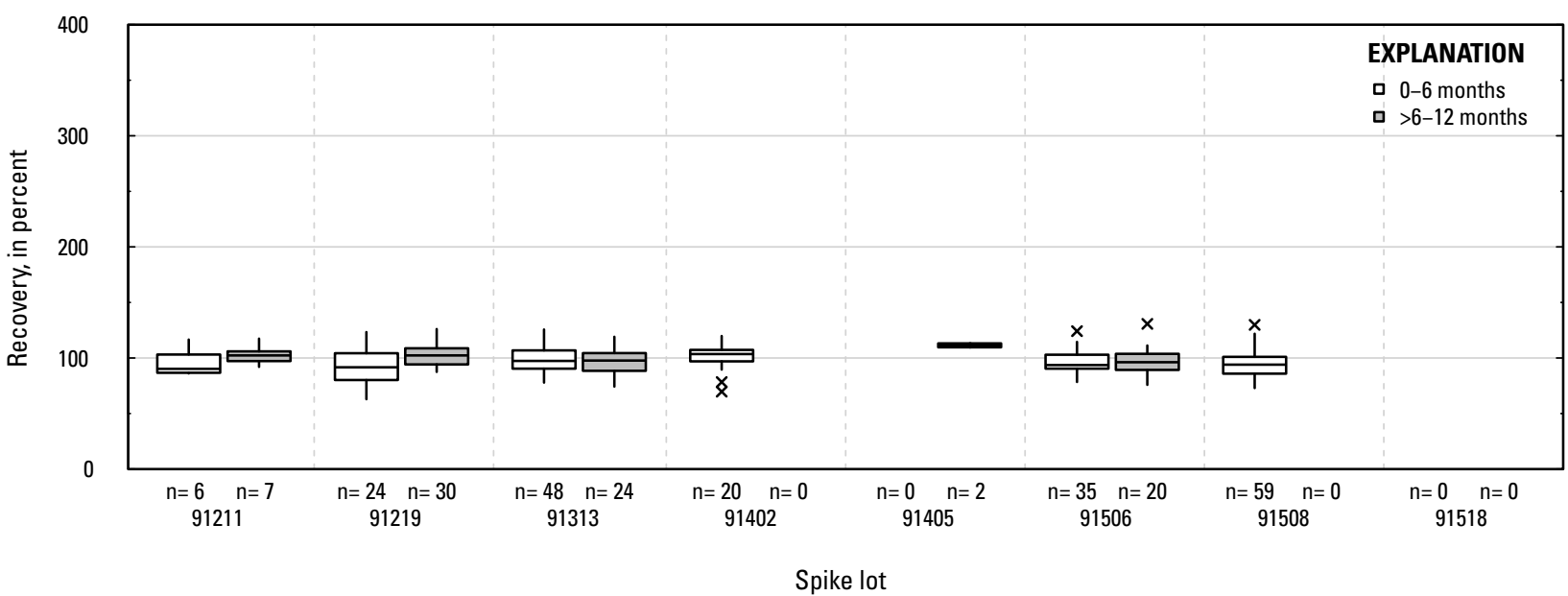

AX. 4-Hydroxy molinate: groundwater field matrix spikes

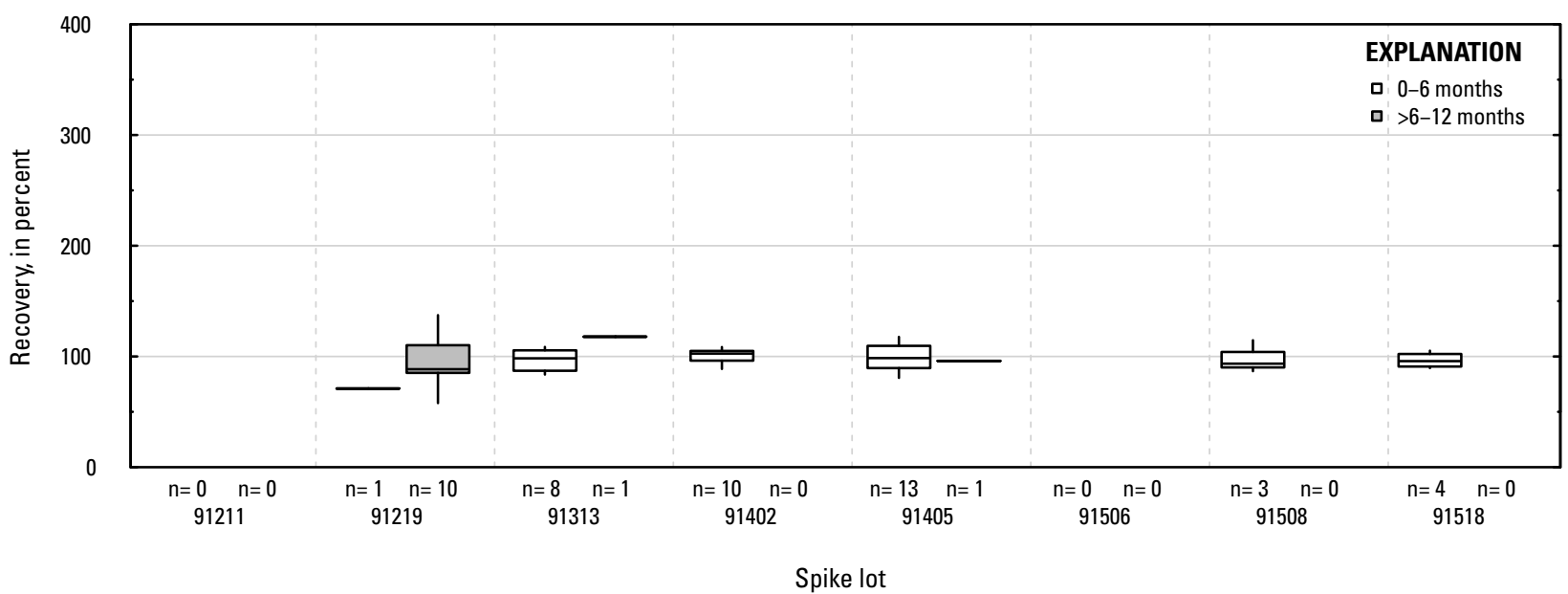

AY. 4-Hydroxy molinate: surface water field matrix spikes

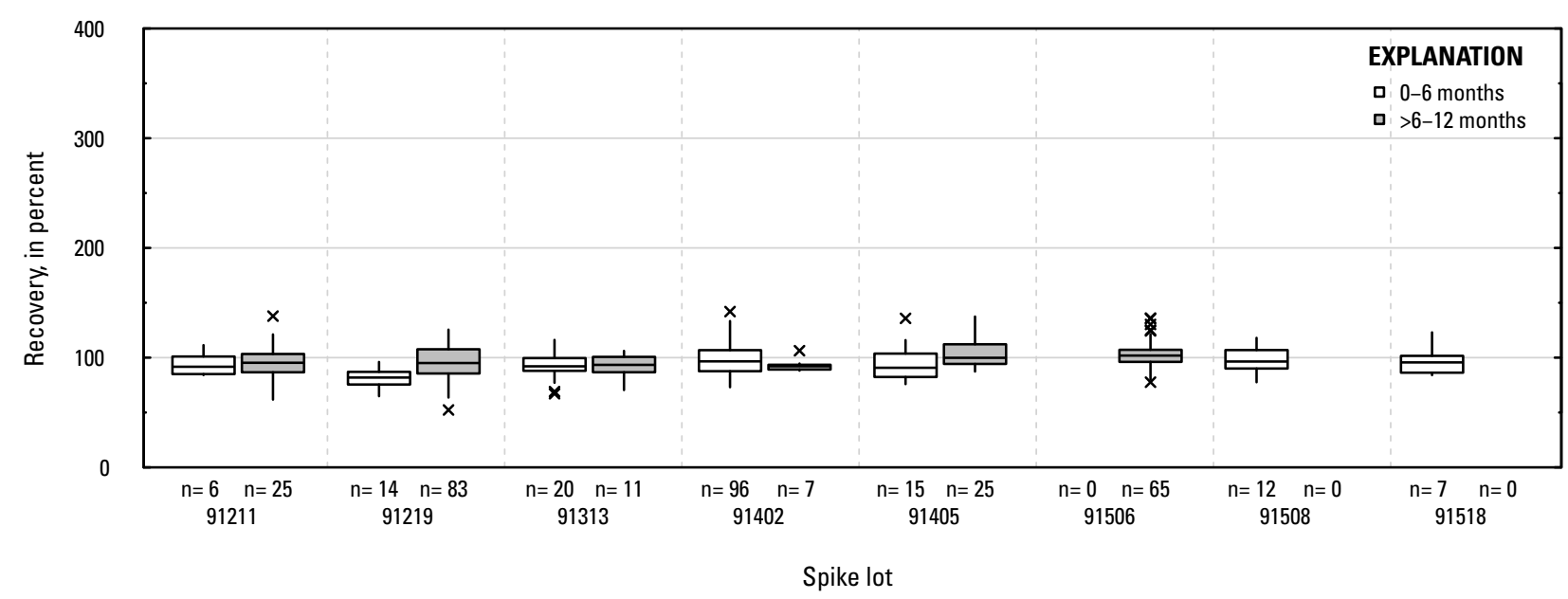

Figure 1-1. Distributions of recovery for individual pesticides in schedule 2437 by matrix, spike lot, and spike lot age. Recovery values larger than 400 percent are not shown.-Continued 
AZ. 4-Hydroxychlorothalonil: laboratory reagent spikes

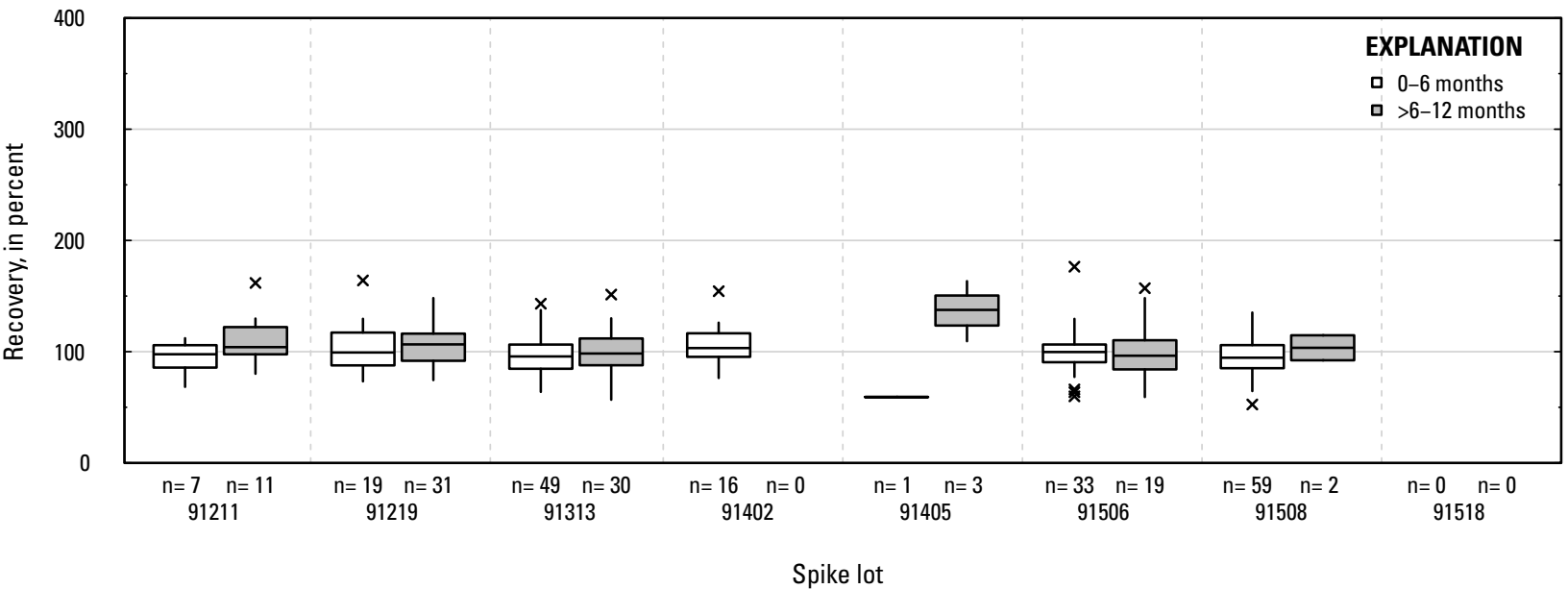

BA. 4-Hydroxychlorothalonil: groundwater field matrix spikes

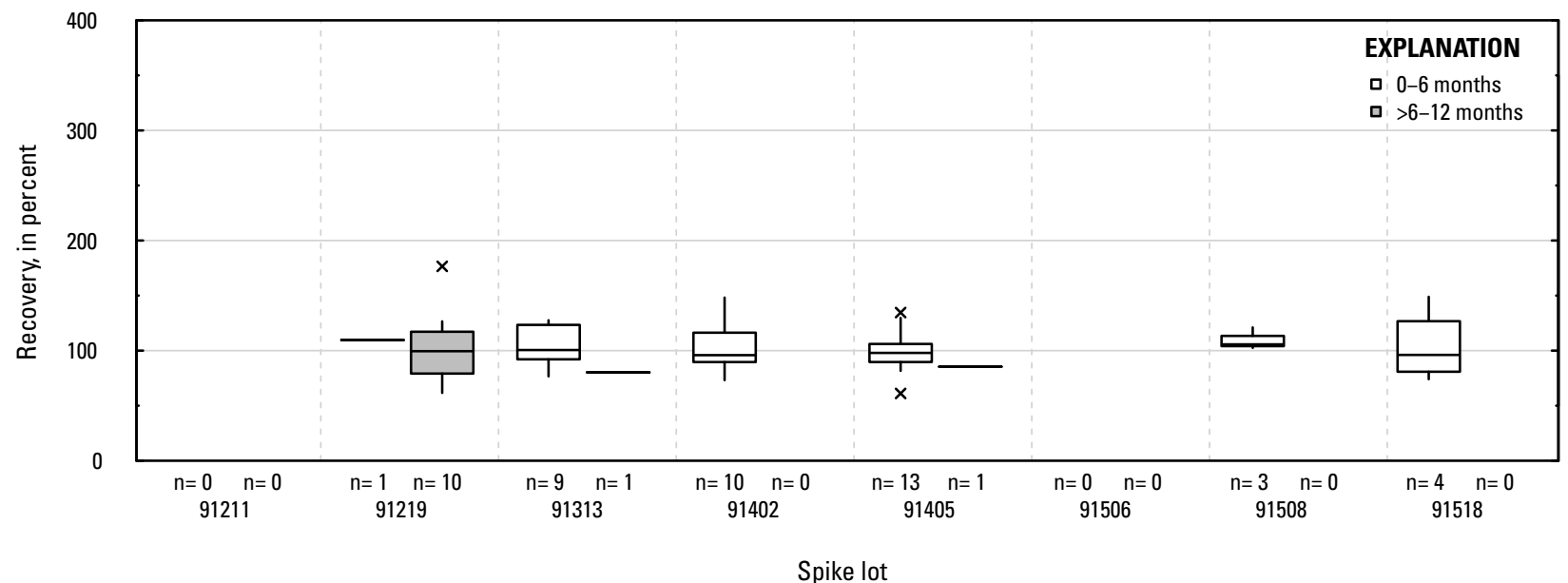

BB. 4-Hydroxychlorothalonil: surface water field matrix spikes

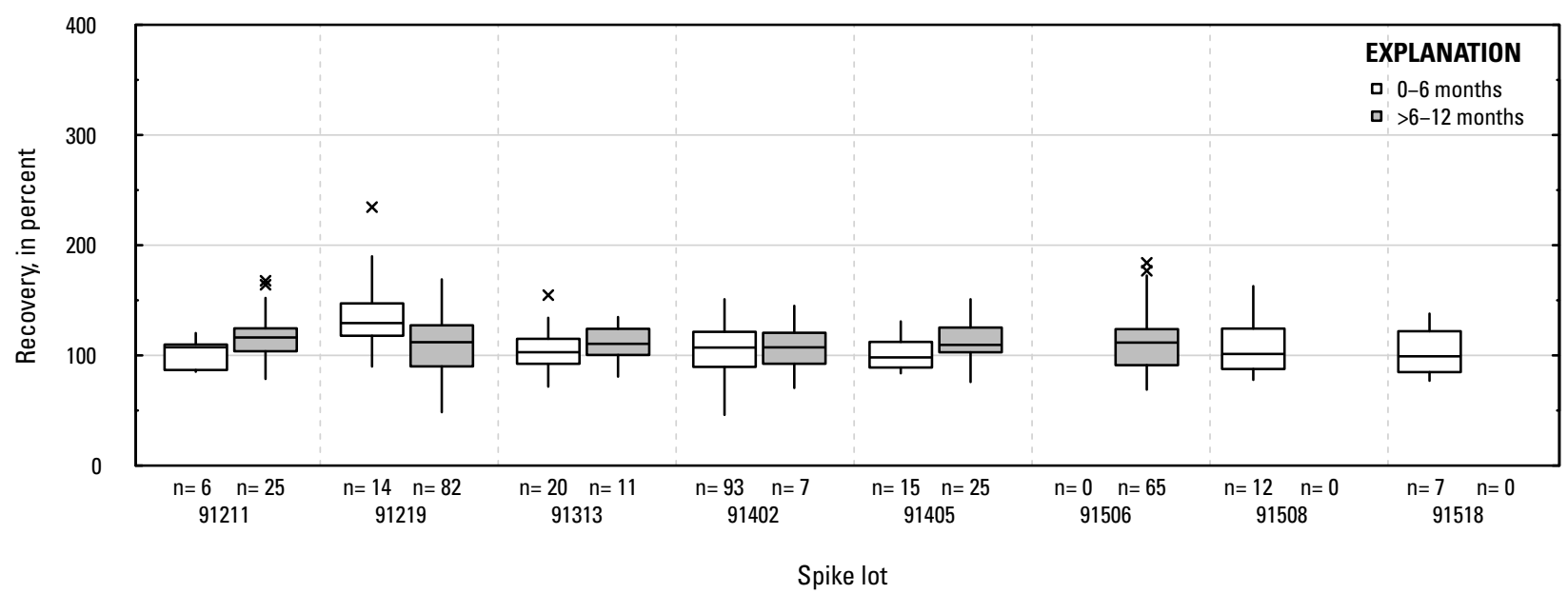

Figure 1-1. Distributions of recovery for individual pesticides in schedule 2437 by matrix, spike lot, and spike lot age. Recovery values larger than 400 percent are not shown.-Continued 


\section{BC. 4-Hydroxyhexazinone A: laboratory reagent spikes}

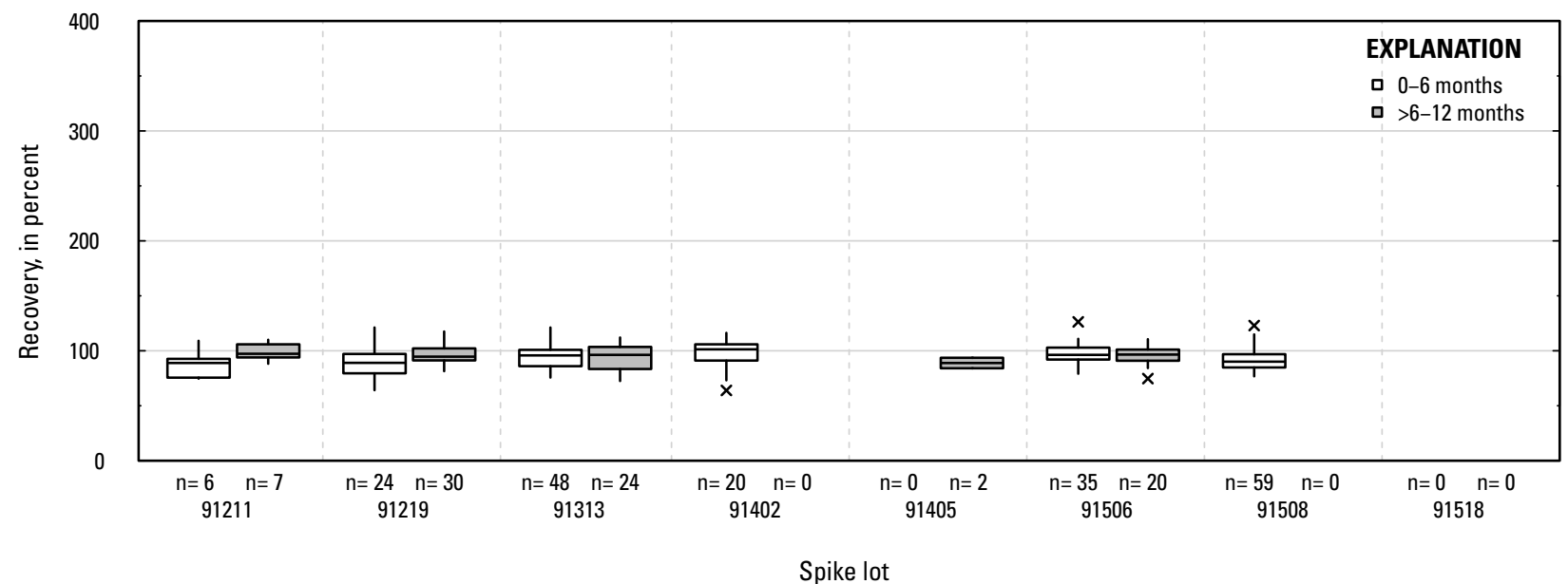

BD. 4-Hydroxyhexazinone A: groundwater field matrix spikes

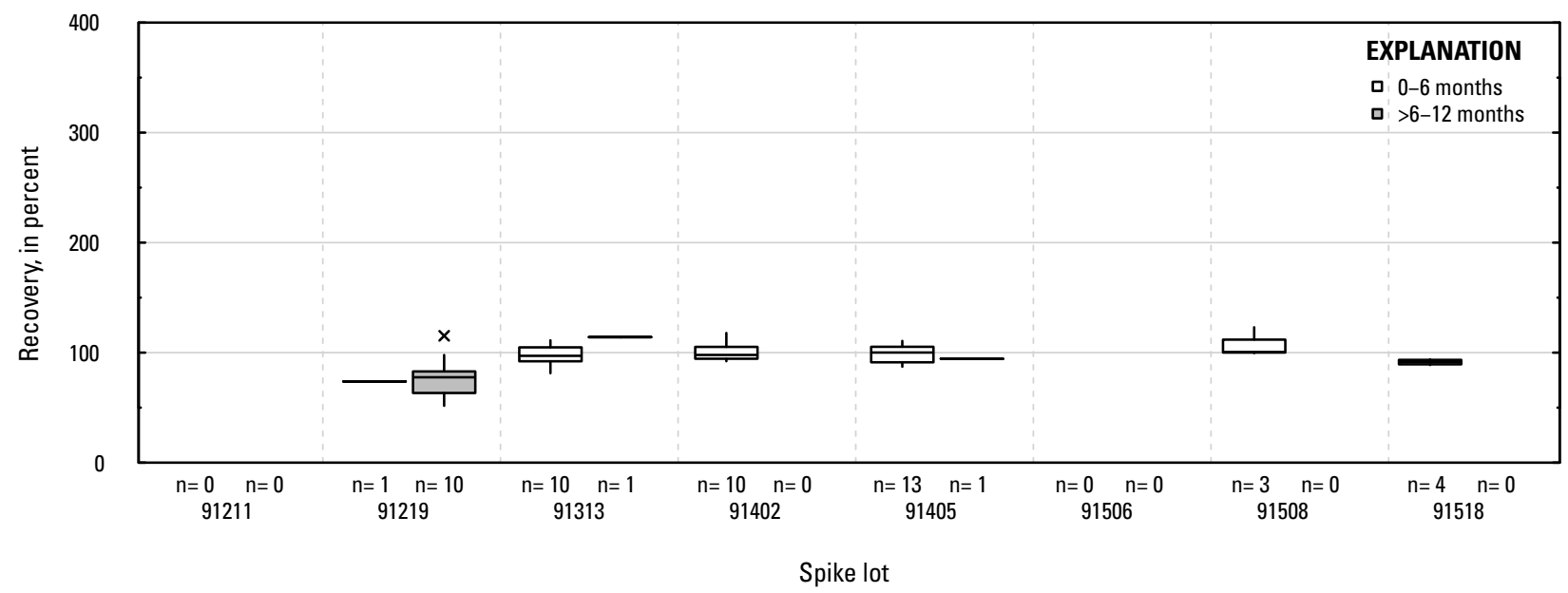

BE. 4-Hydroxyhexazinone A: surface water field matrix spikes

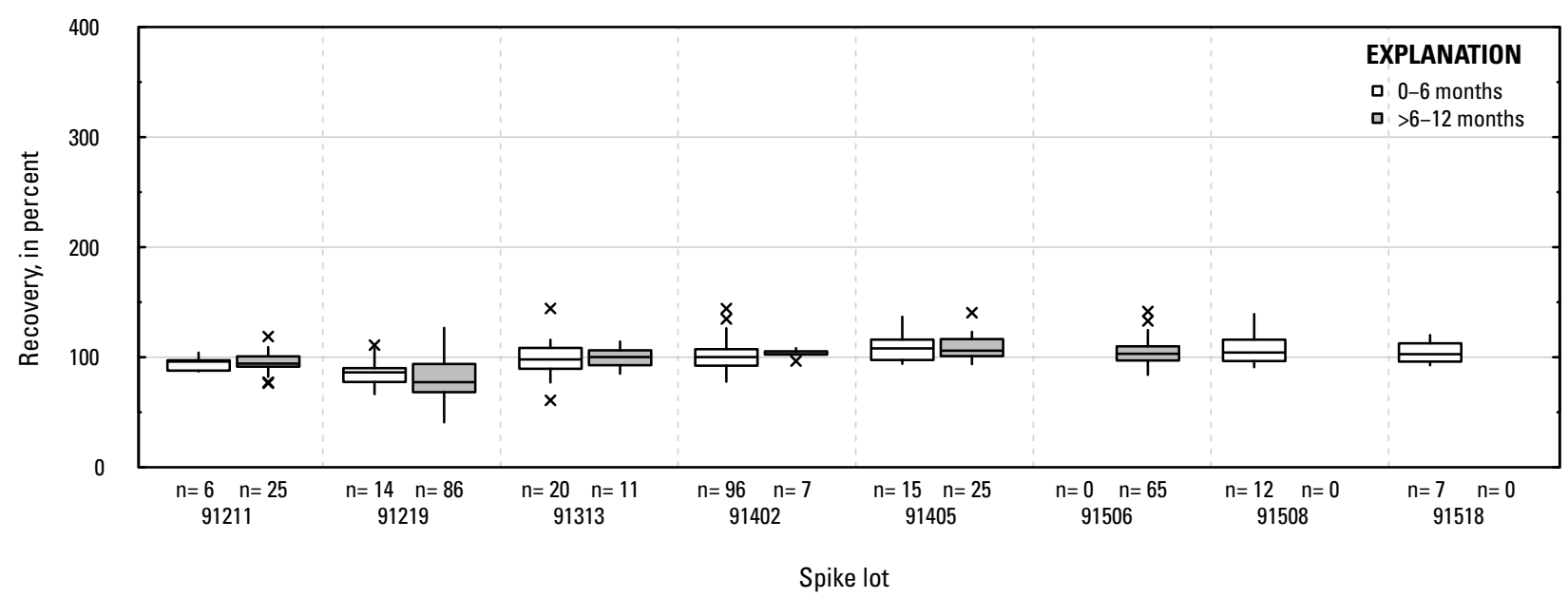

Figure 1-1. Distributions of recovery for individual pesticides in schedule 2437 by matrix, spike lot, and spike lot age. Recovery values larger than 400 percent are not shown.-Continued 
BF. Hydroxy didemethyl fluometuron: laboratory reagent spikes

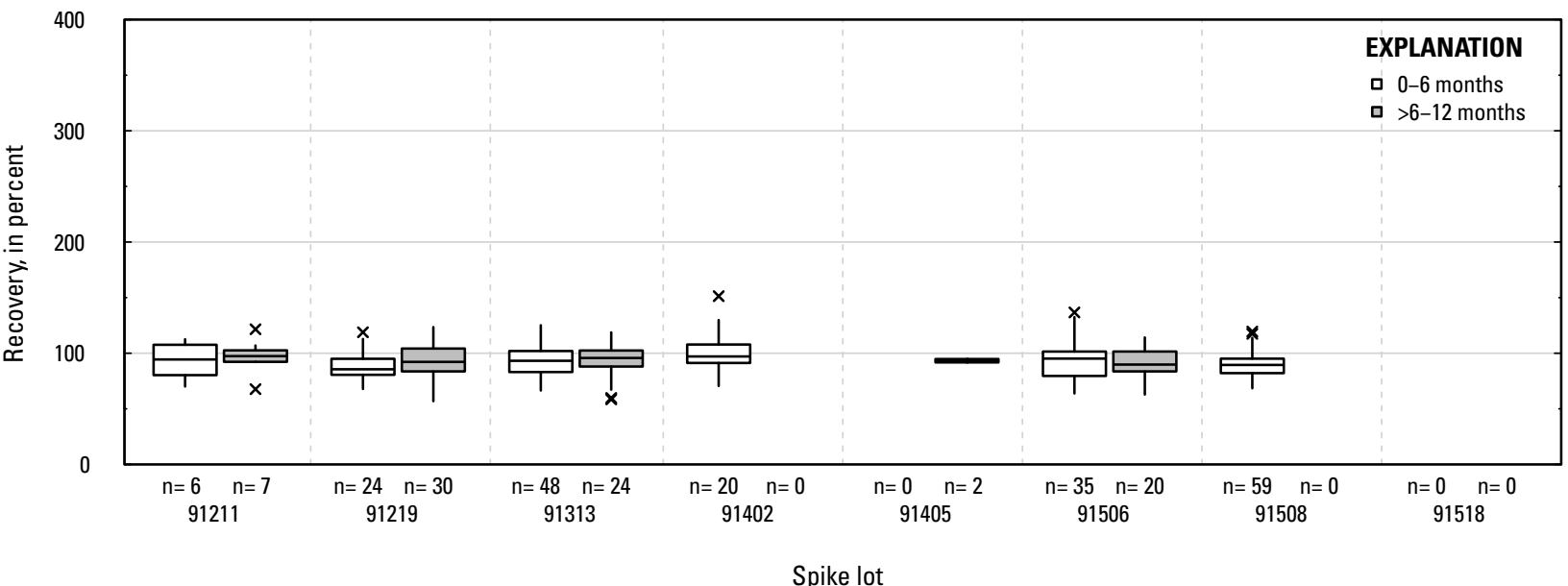

BG. Hydroxy didemethyl fluometuron: groundwater field matrix spikes

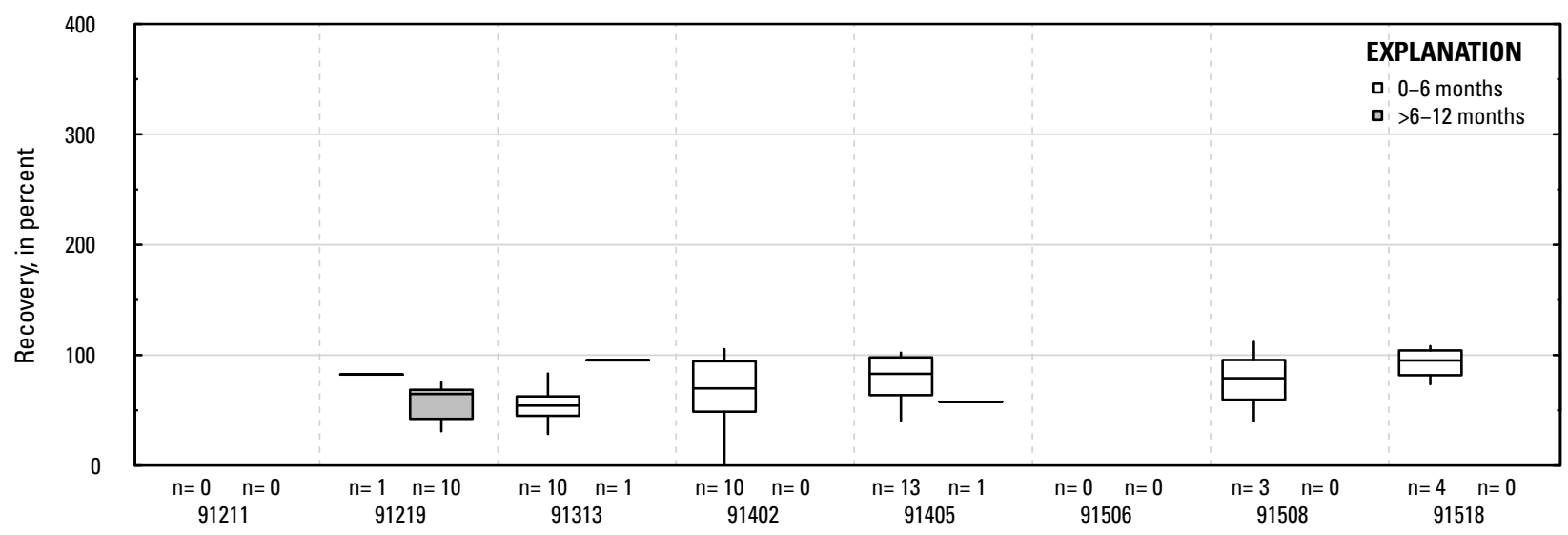

Spike lot

\section{BH. Hydroxy didemethyl fluometuron: surface water field matrix spikes}

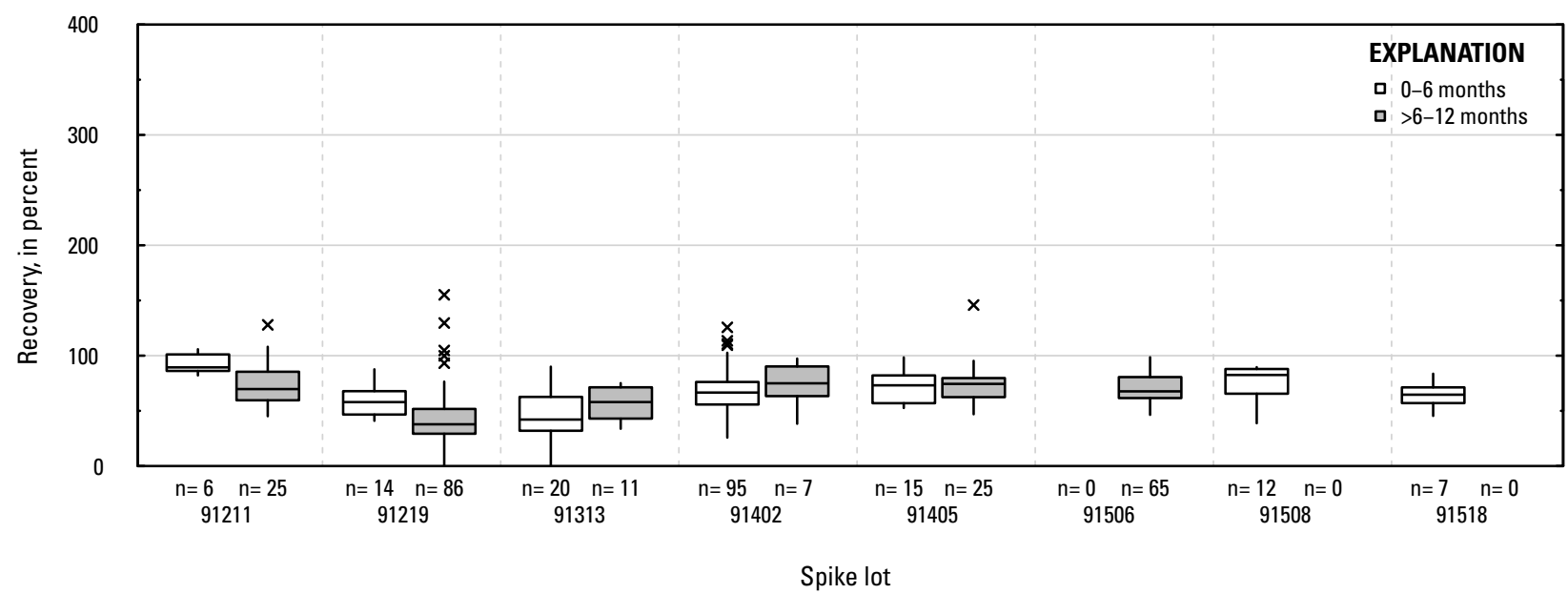

Figure 1-1. Distributions of recovery for individual pesticides in schedule 2437 by matrix, spike lot, and spike lot age. Recovery values larger than 400 percent are not shown.-Continued 


\section{BI. Acephate: laboratory reagent spikes}

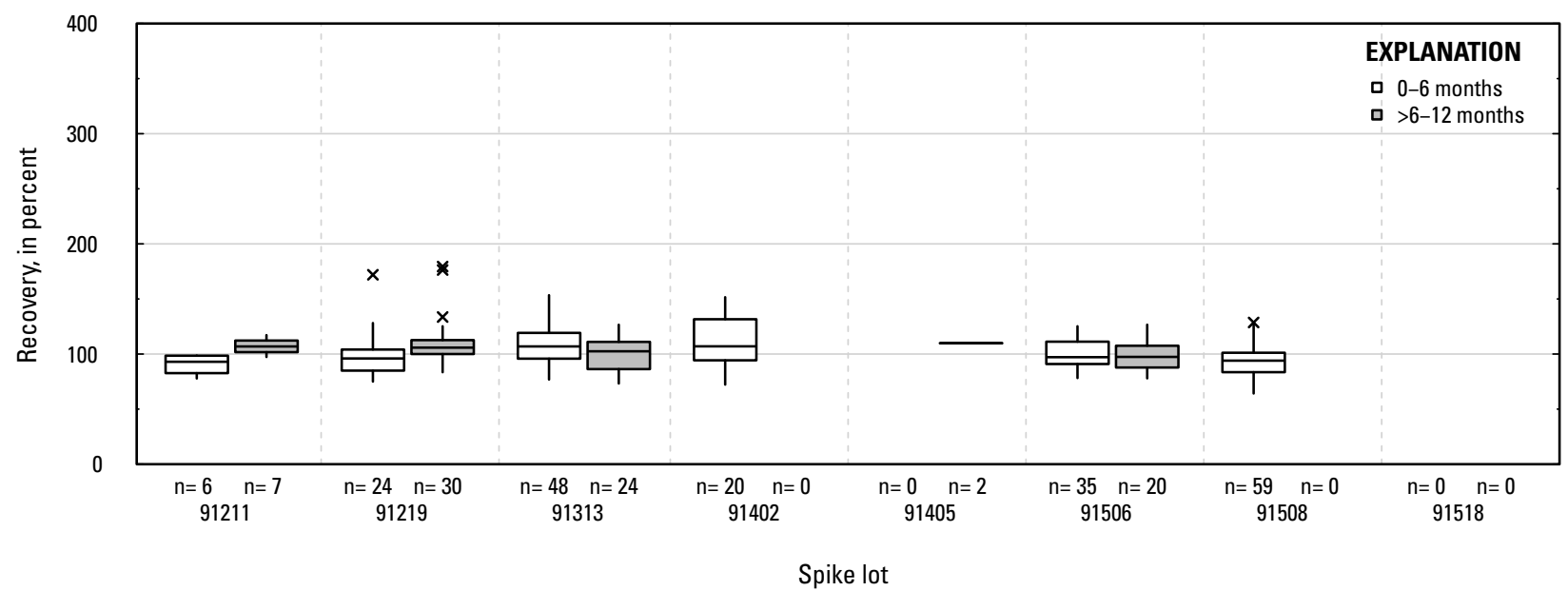

\section{BJ. Acephate: groundwater field matrix spikes}

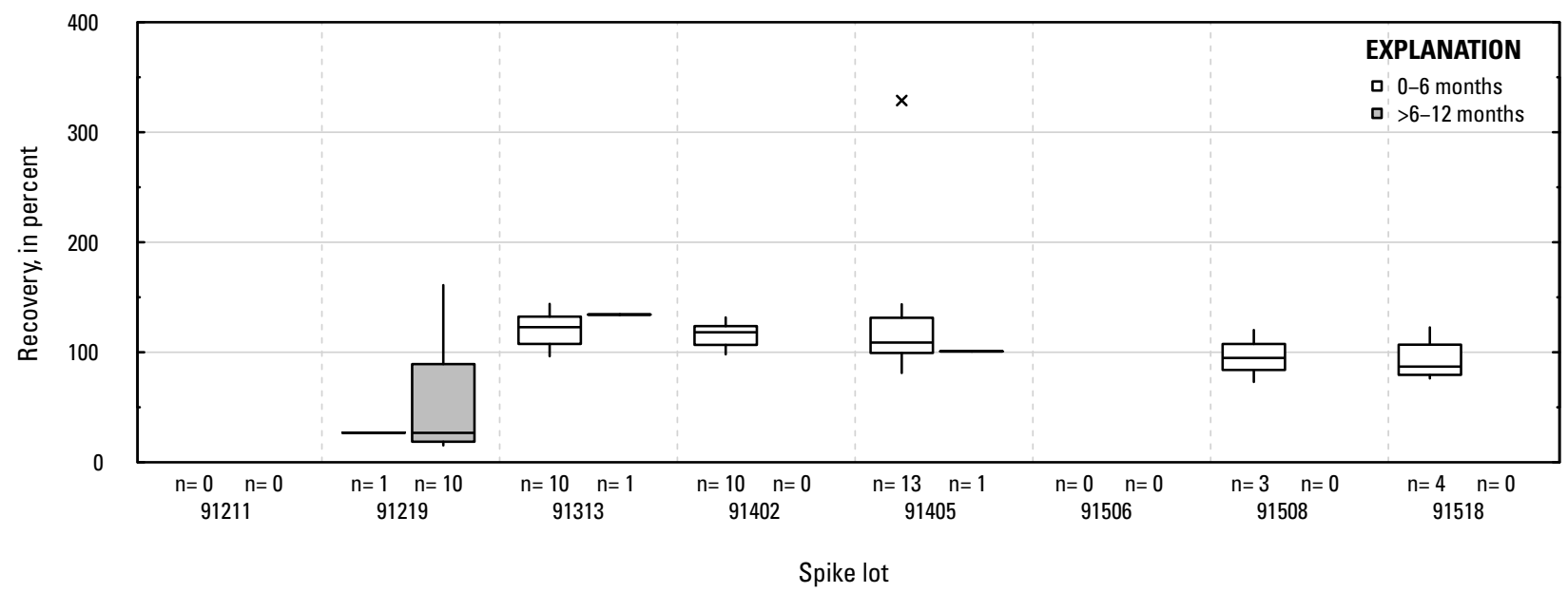

BK. Acephate: surface water field matrix spikes

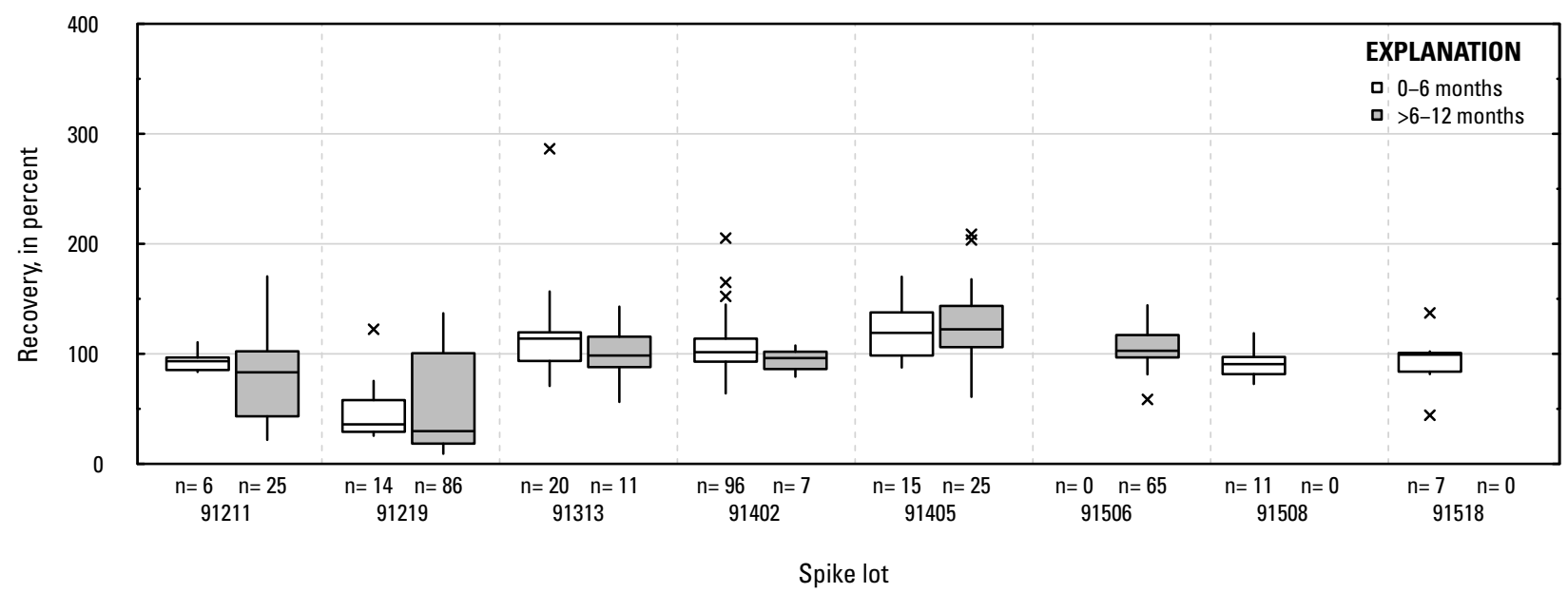

Figure 1-1. Distributions of recovery for individual pesticides in schedule 2437 by matrix, spike lot, and spike lot age. Recovery values larger than 400 percent are not shown.-Continued 


\section{BL. Acetochlor: laboratory reagent spikes}

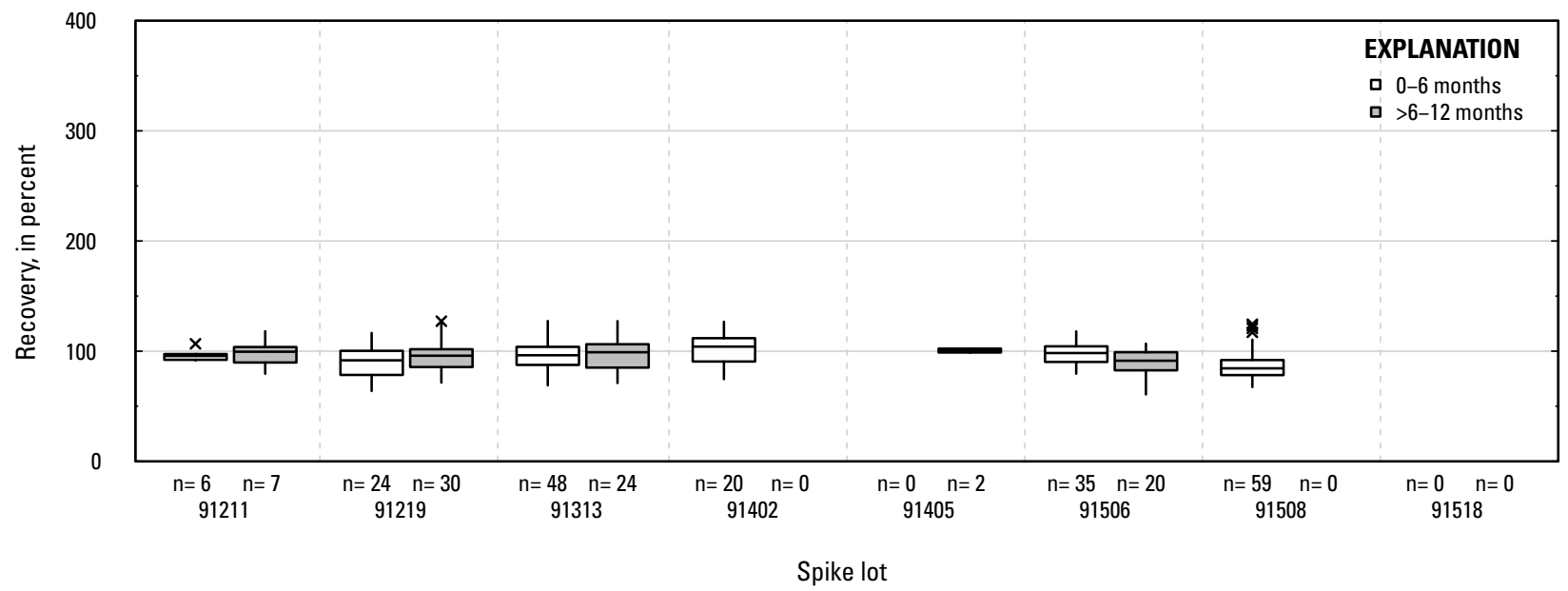

BM. Acetochlor: groundwater field matrix spikes

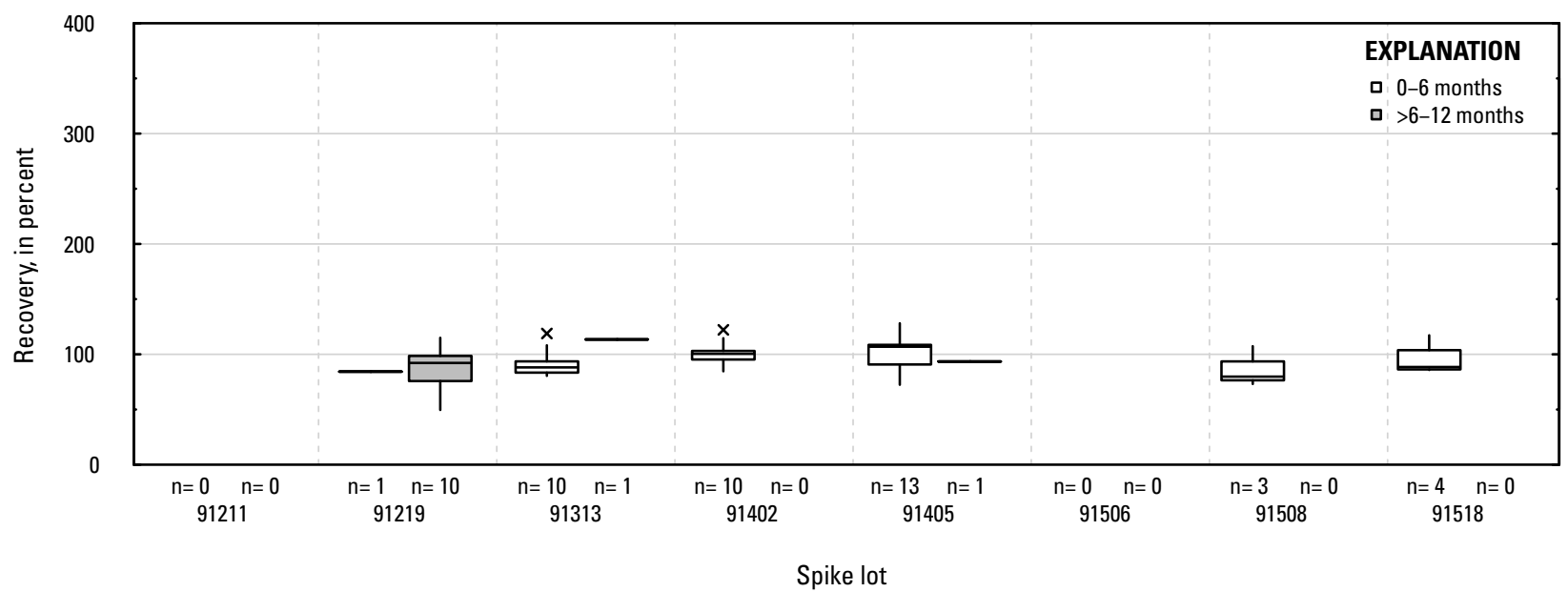

$B N$. Acetochlor: surface water field matrix spikes

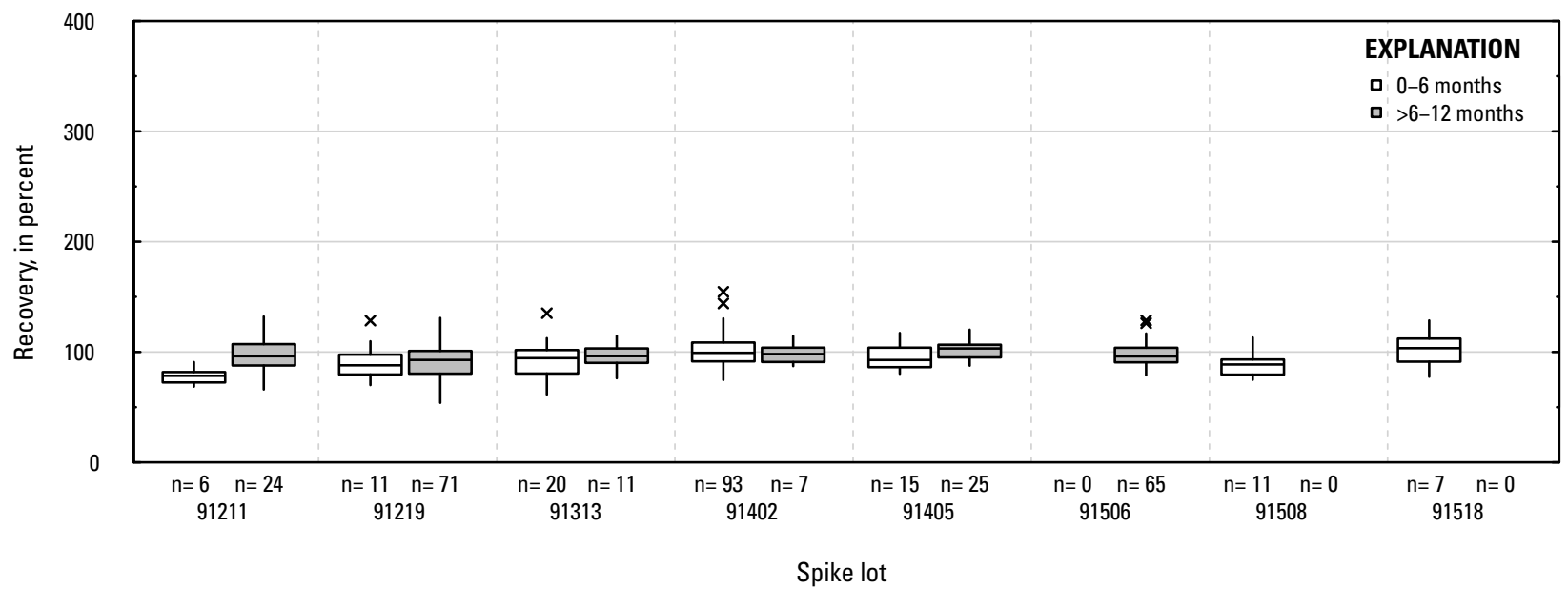

Figure 1-1. Distributions of recovery for individual pesticides in schedule 2437 by matrix, spike lot, and spike lot age. Recovery values larger than 400 percent are not shown.-Continued 
BO. Acetochlor oxanilic acid: laboratory reagent spikes

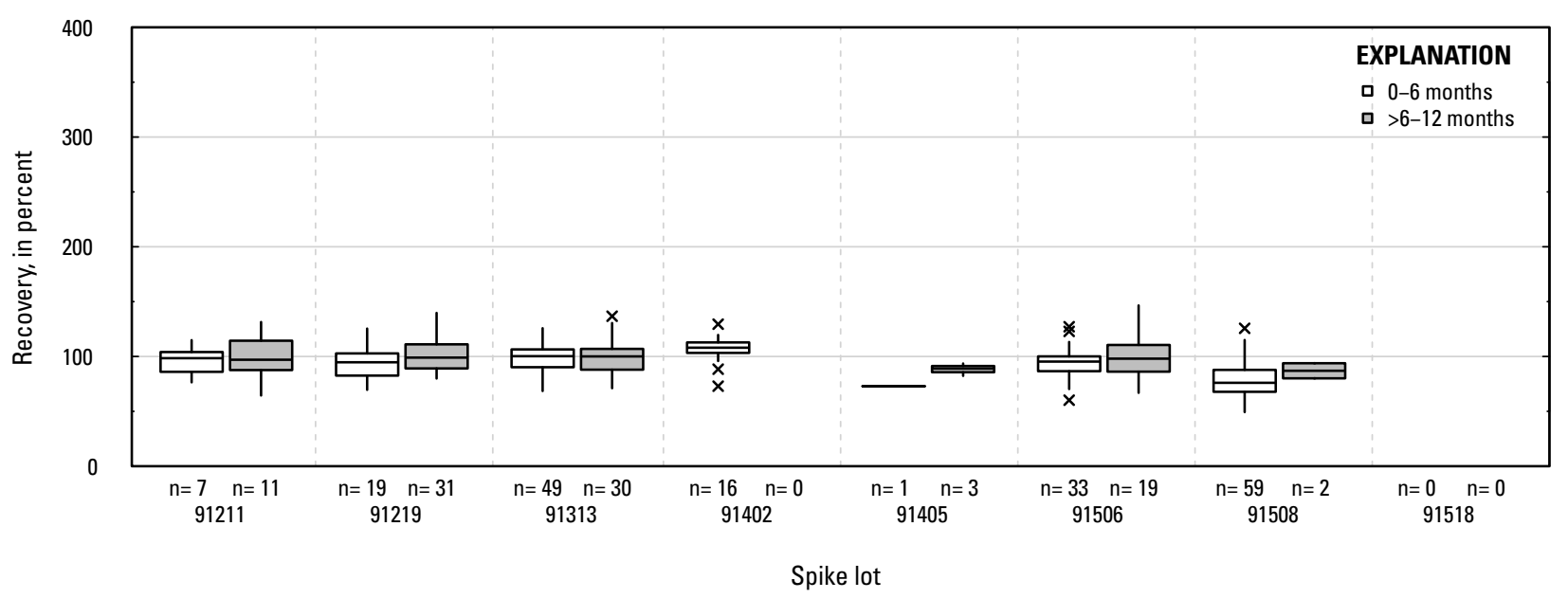

$B P$. Acetochlor oxanilic acid: groundwater field matrix spikes

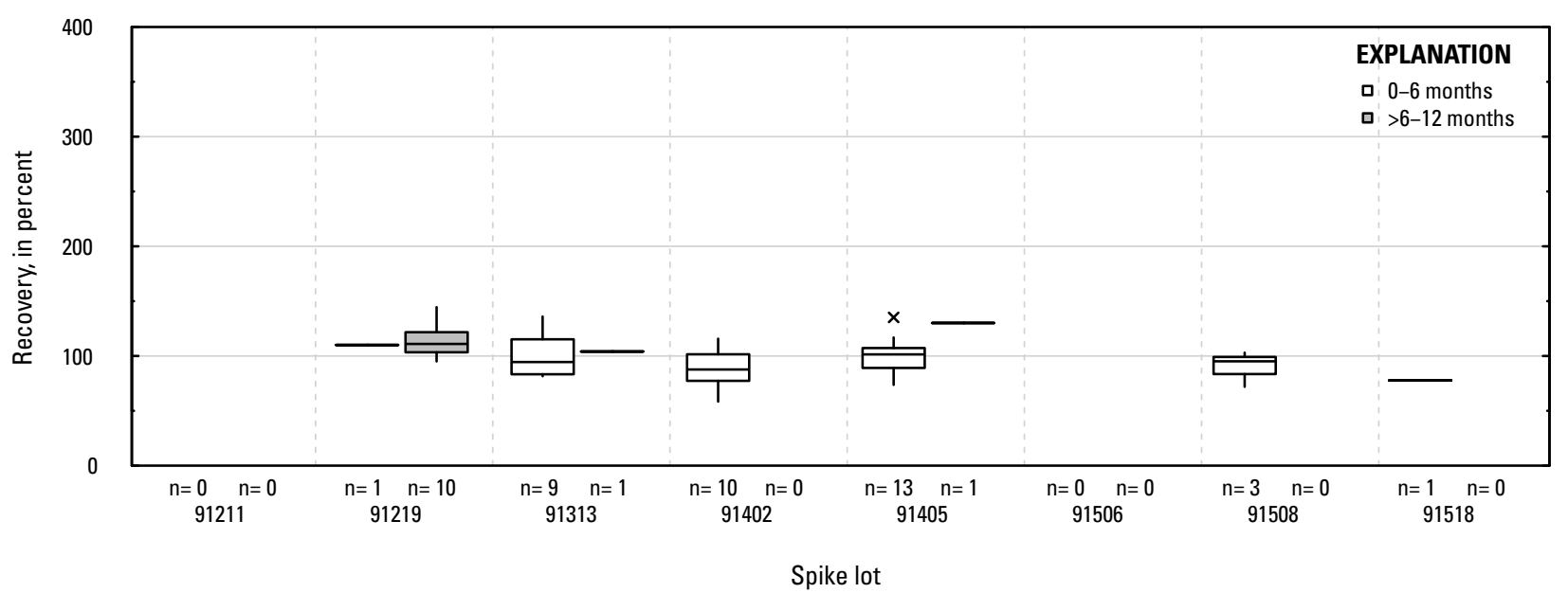

BO. Acetochlor oxanilic acid: surface water field matrix spikes

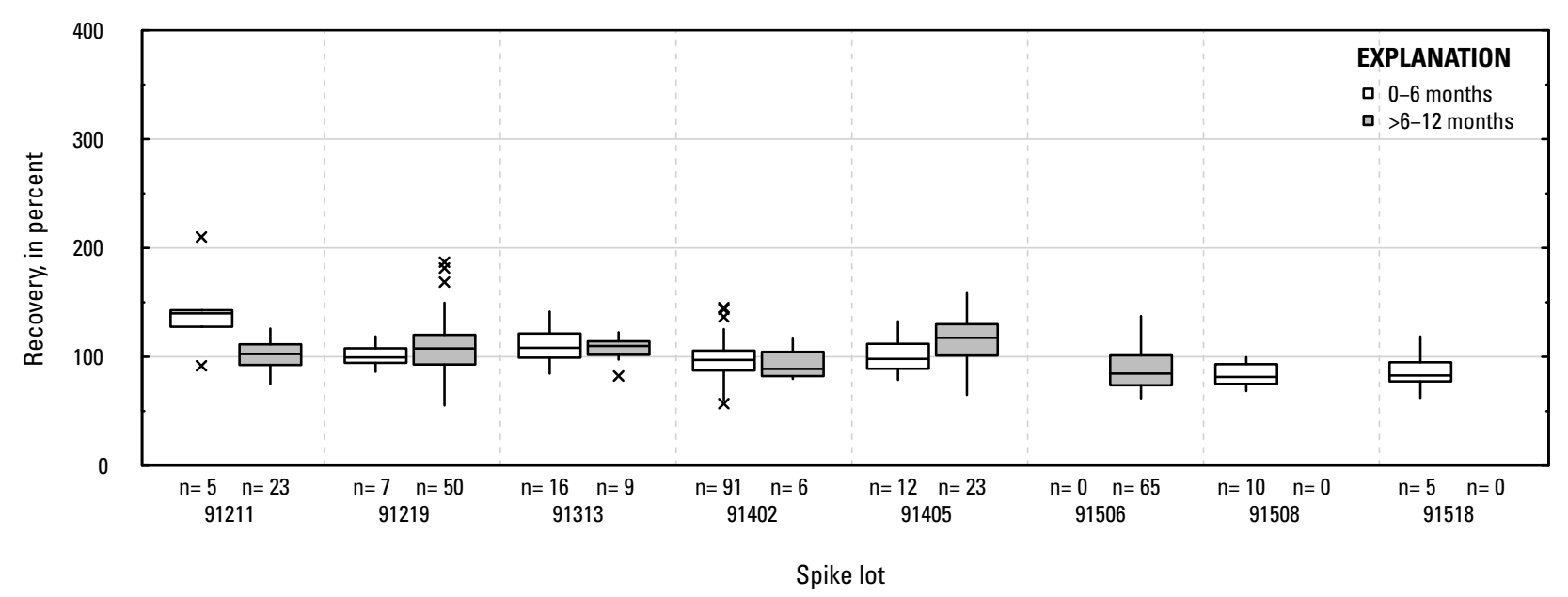

Figure 1-1. Distributions of recovery for individual pesticides in schedule 2437 by matrix, spike lot, and spike lot age. Recovery values larger than 400 percent are not shown.-Continued 
$B R$. Acetochlor sulfonic acid: laboratory reagent spikes

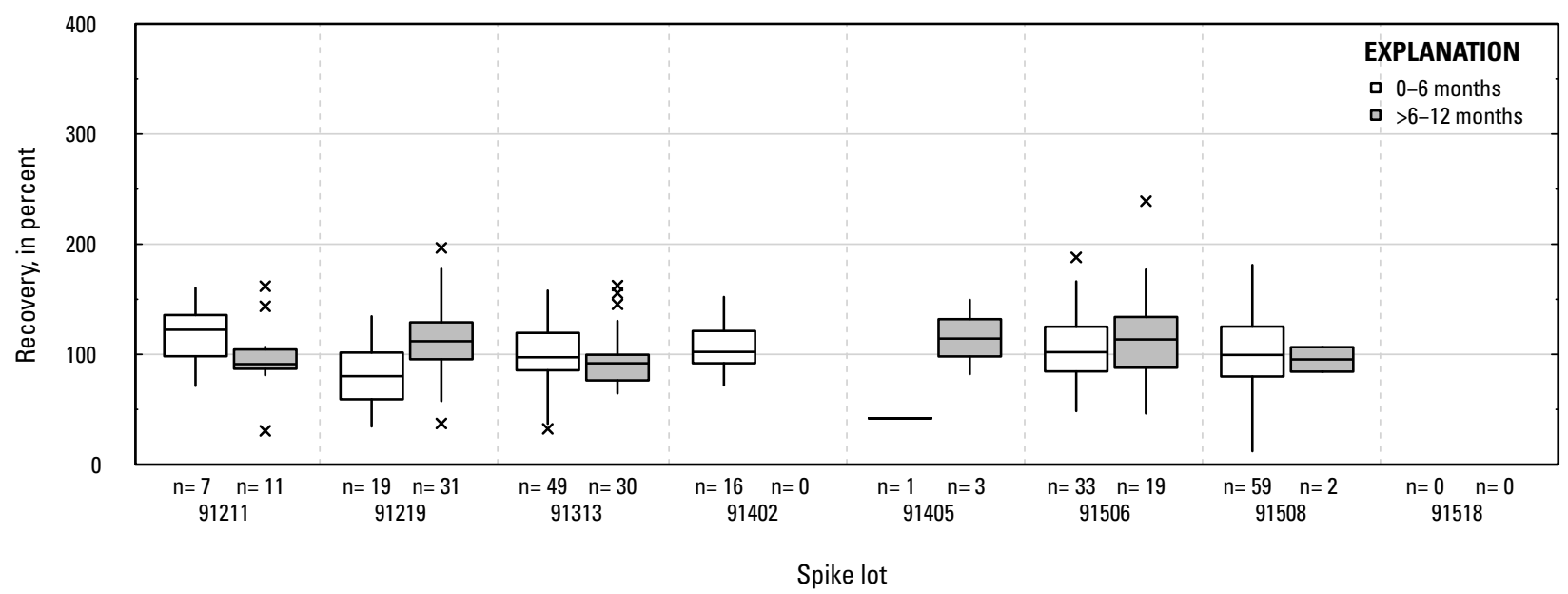

BS. Acetochlor sulfonic acid: groundwater field matrix spikes

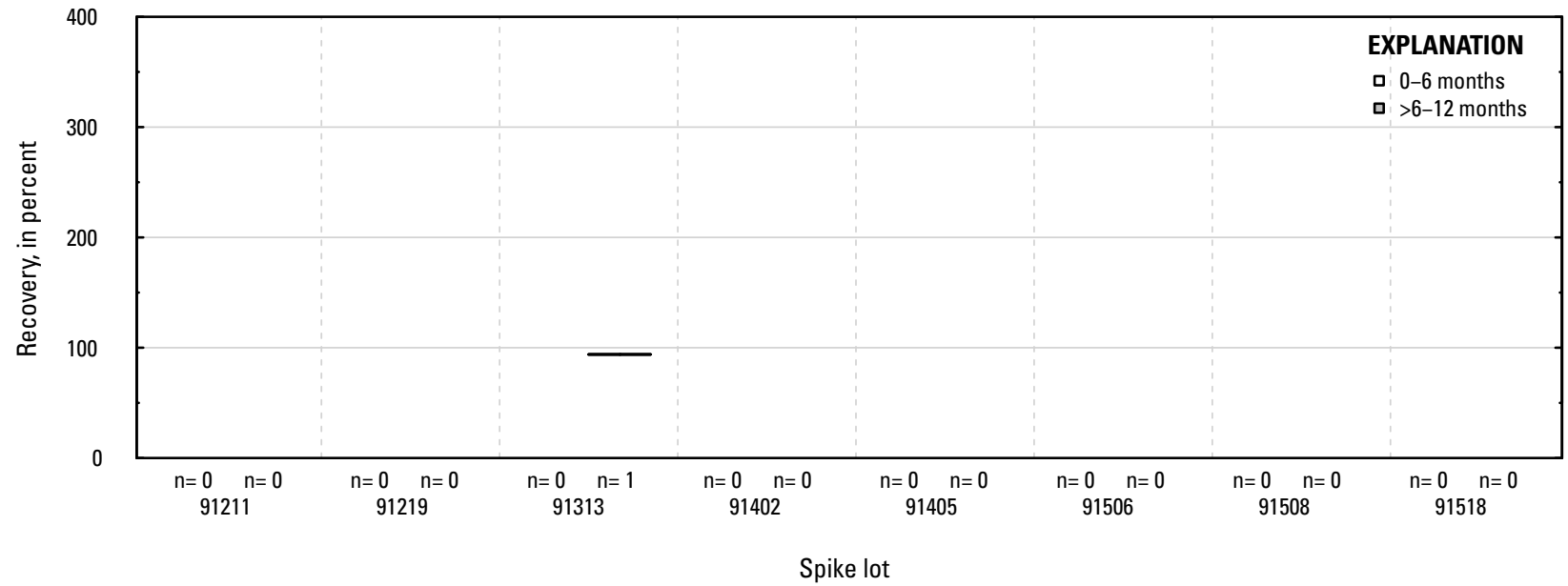

BT. Acetochlor sulfonic acid: surface water field matrix spikes

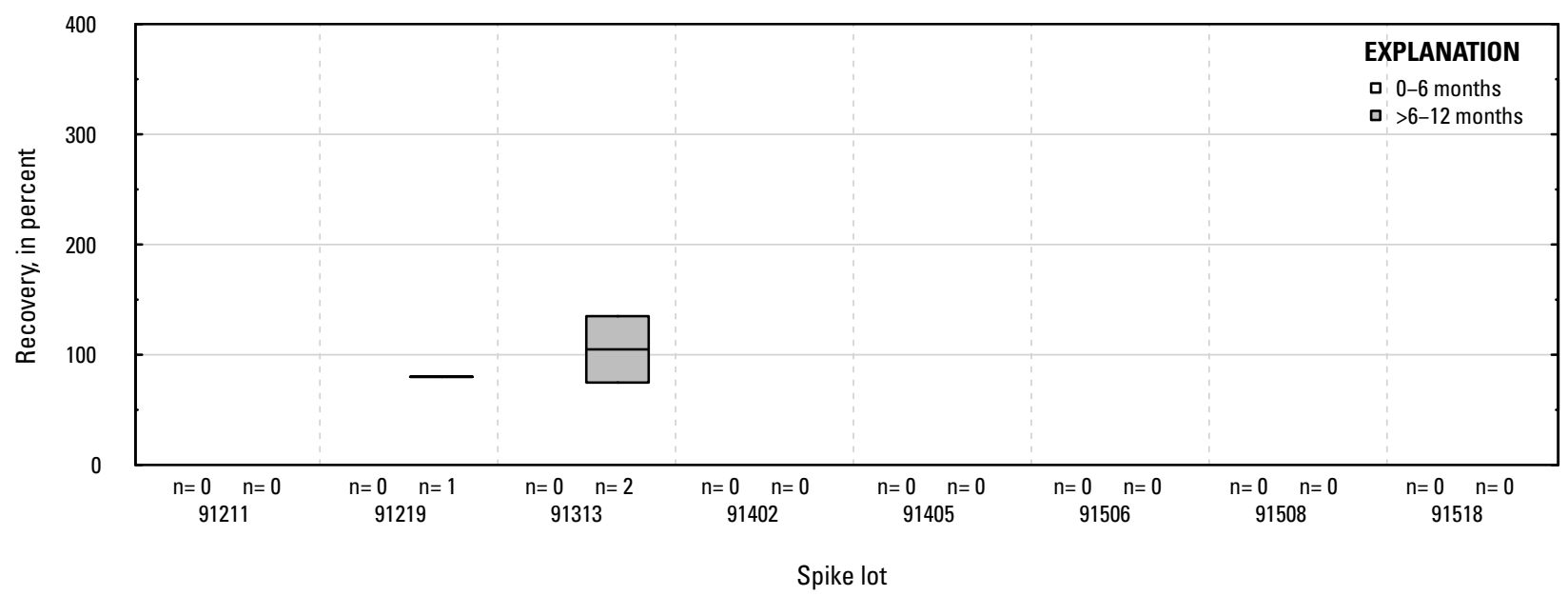

Figure 1-1. Distributions of recovery for individual pesticides in schedule 2437 by matrix, spike lot, and spike lot age. Recovery values larger than 400 percent are not shown.-Continued 
$B U$. Acetochlor sulfynilacetic acid: laboratory reagent spikes

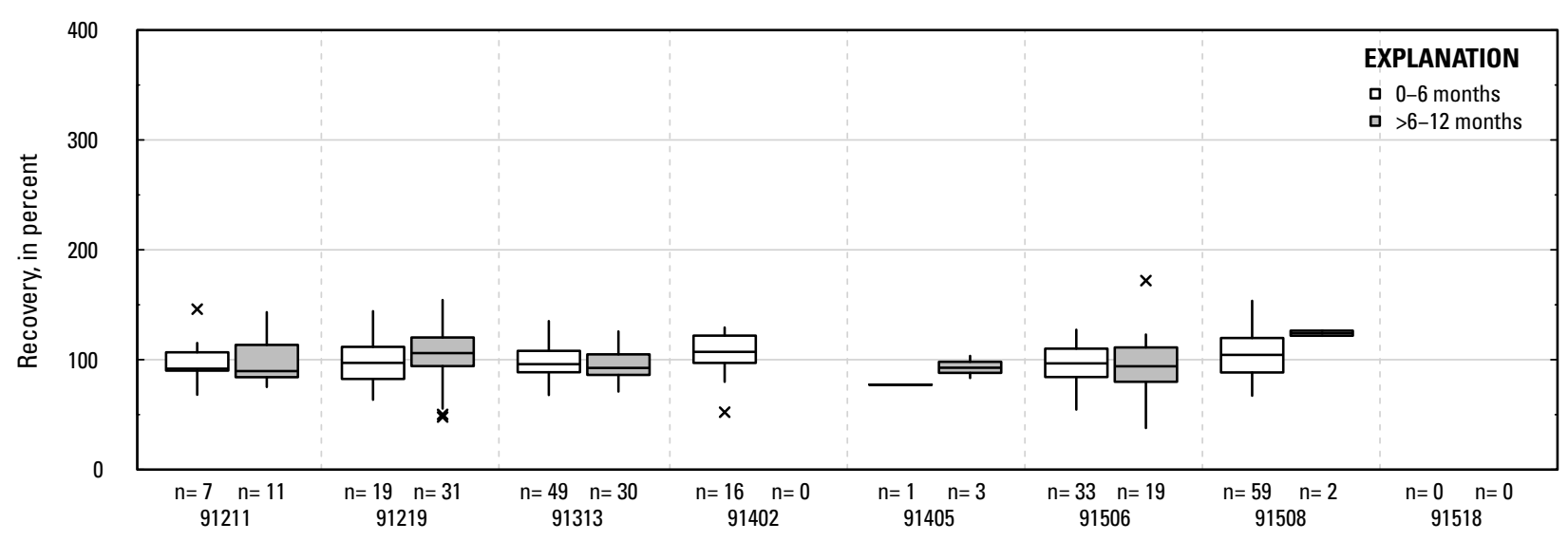

Spike lot

BV. Acetochlor sulfynilacetic acid: groundwater field matrix spikes

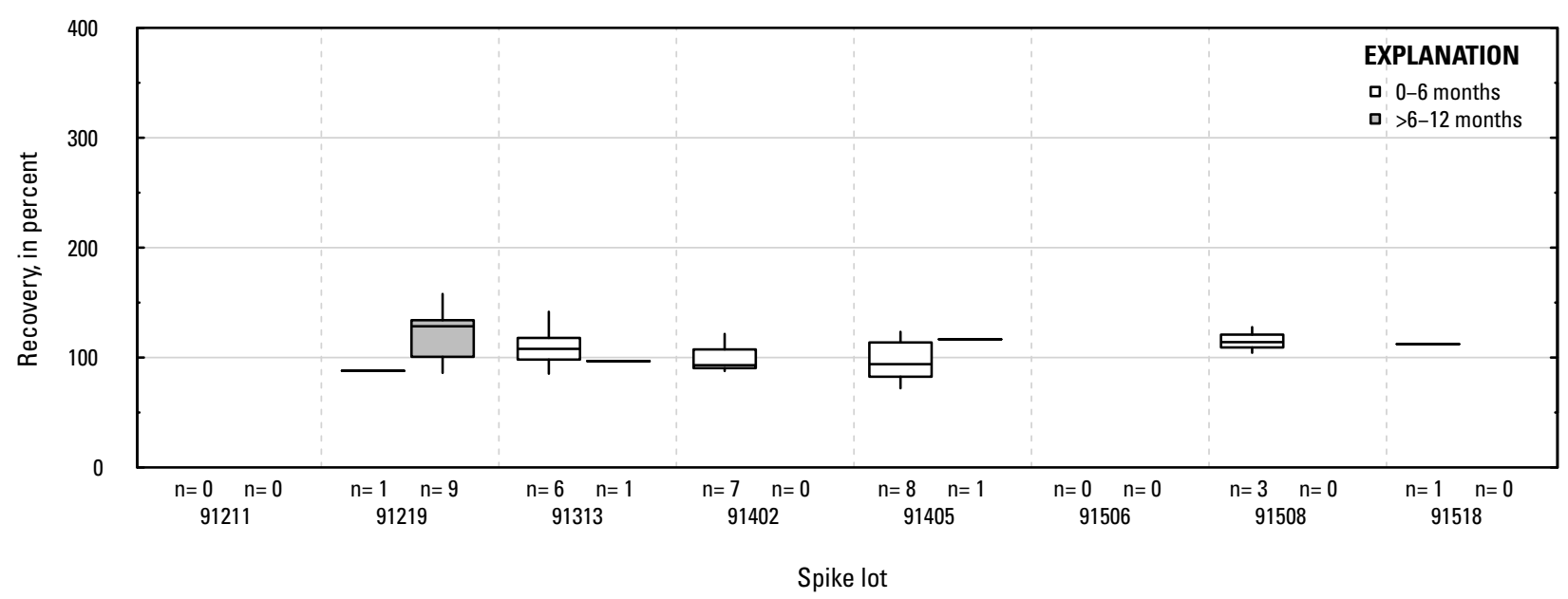

BW. Acetochlor sulfynilacetic acid: surface water field matrix spikes

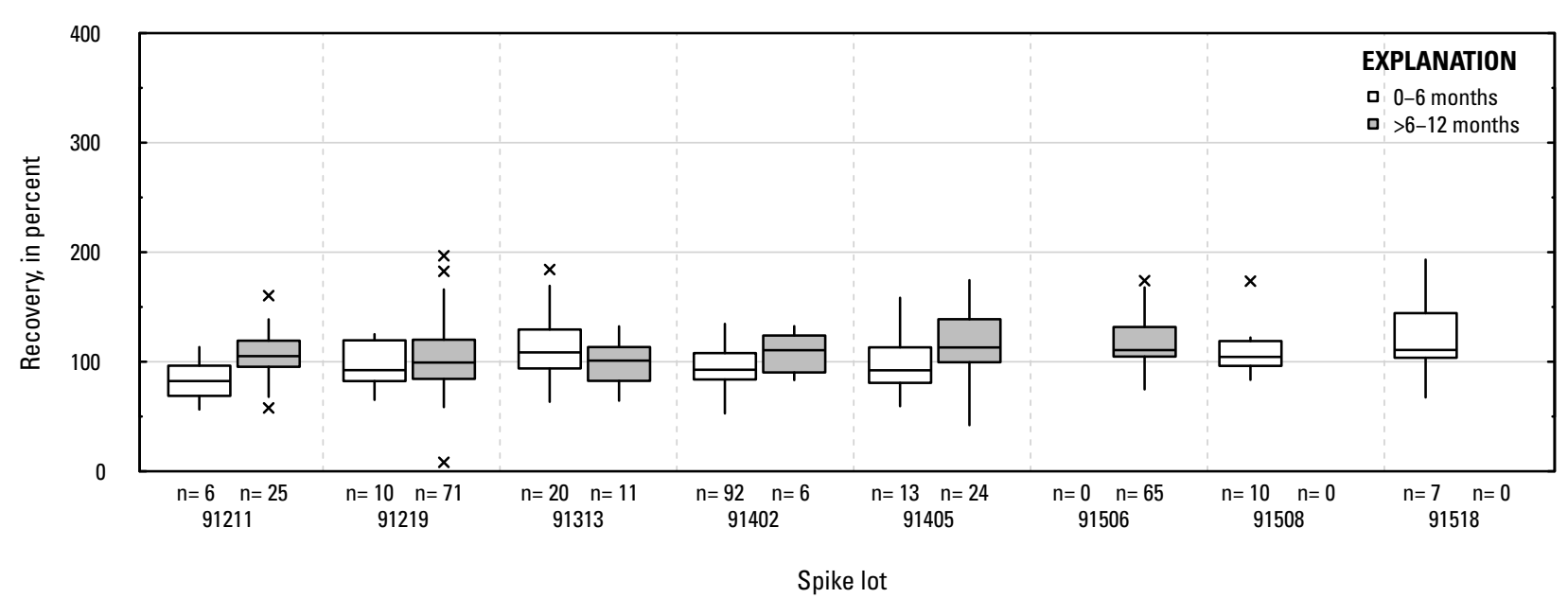

Figure 1-1. Distributions of recovery for individual pesticides in schedule 2437 by matrix, spike lot, and spike lot age. Recovery values larger than 400 percent are not shown.-Continued 
$B X$. Alachlor: laboratory reagent spikes

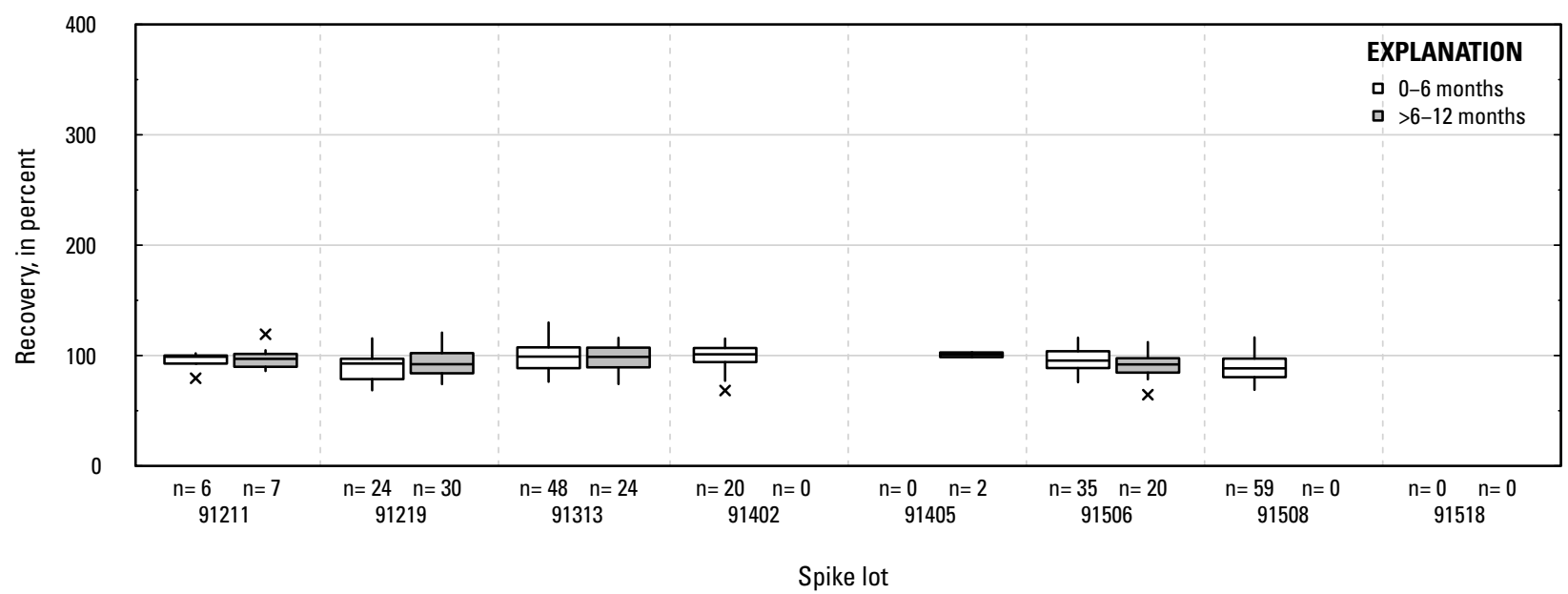

BY. Alachlor: groundwater field matrix spikes

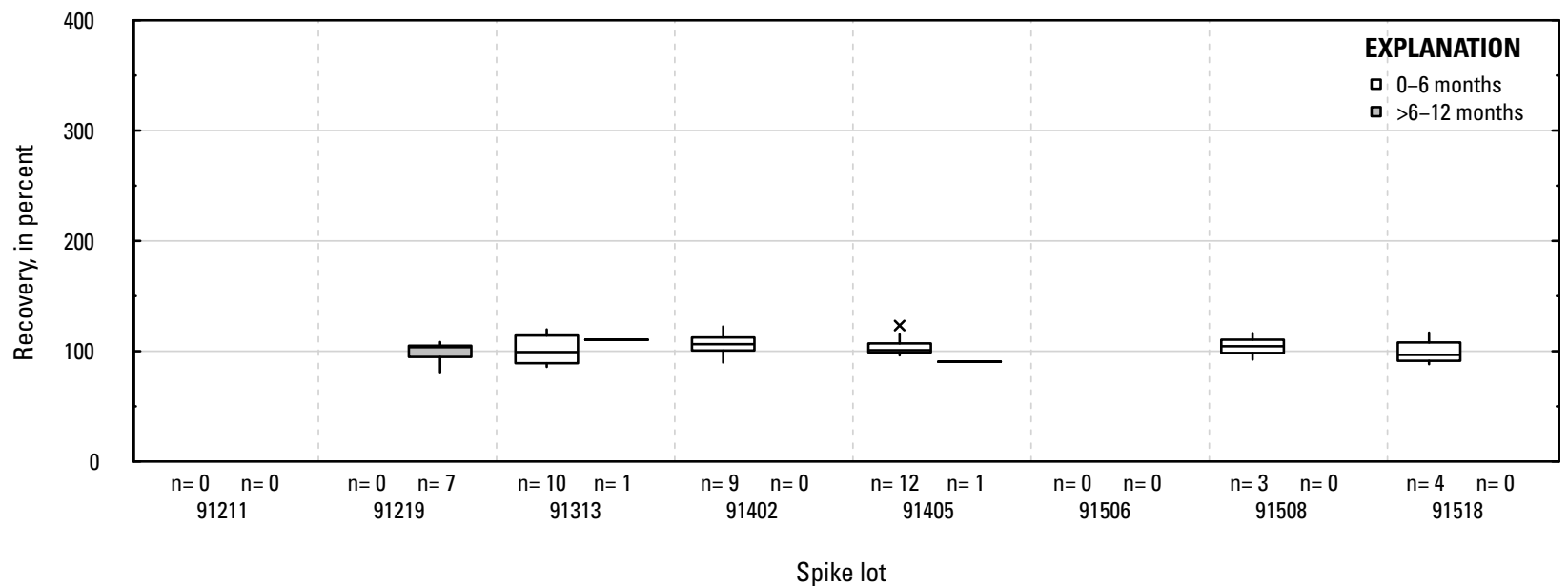

BZ. Alachlor: surface water field matrix spikes

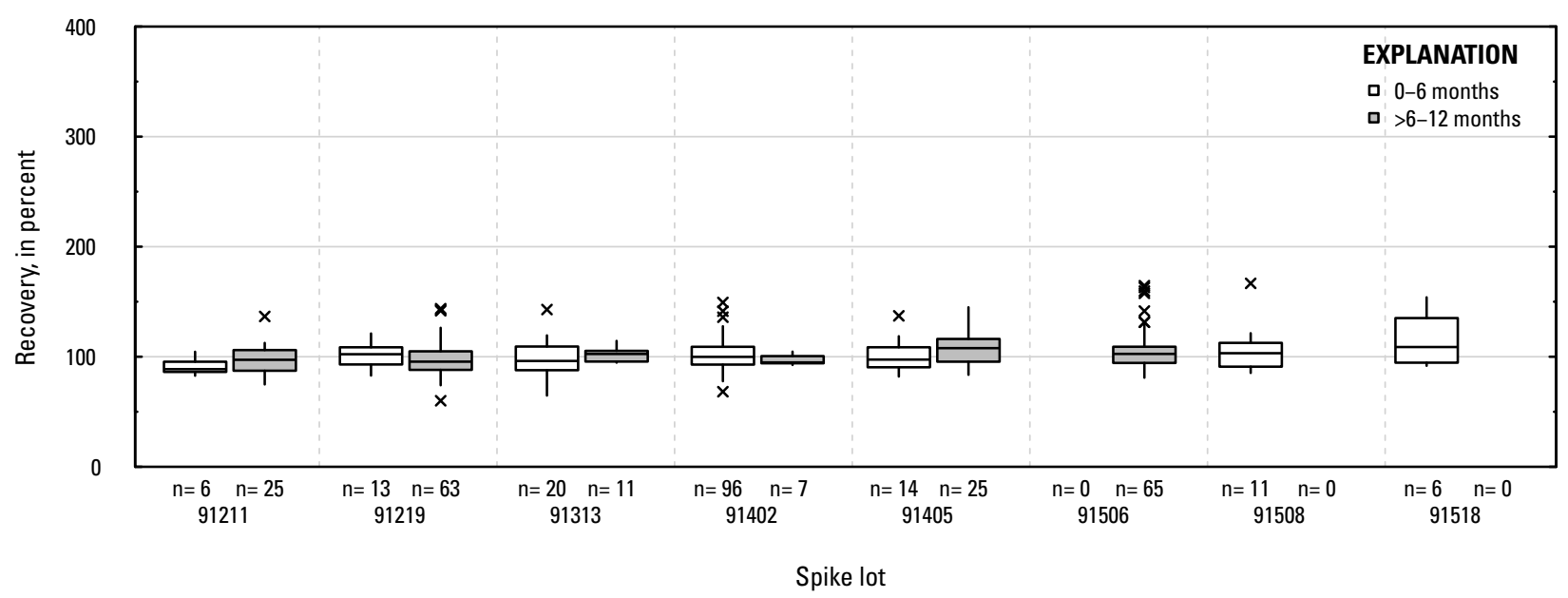

Figure 1-1. Distributions of recovery for individual pesticides in schedule 2437 by matrix, spike lot, and spike lot age. Recovery values larger than 400 percent are not shown.-Continued 
CA. Alachlor oxanilic acid: laboratory reagent spikes

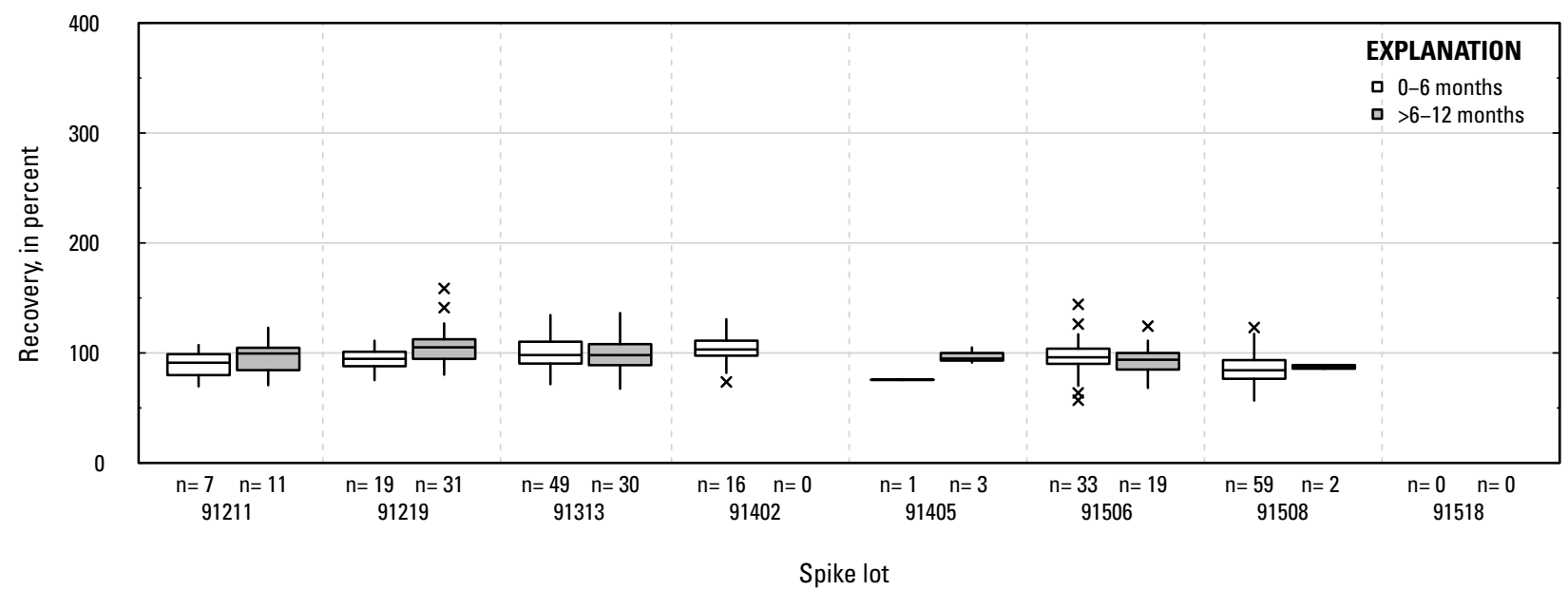

CB. Alachlor oxanilic acid: groundwater field matrix spikes

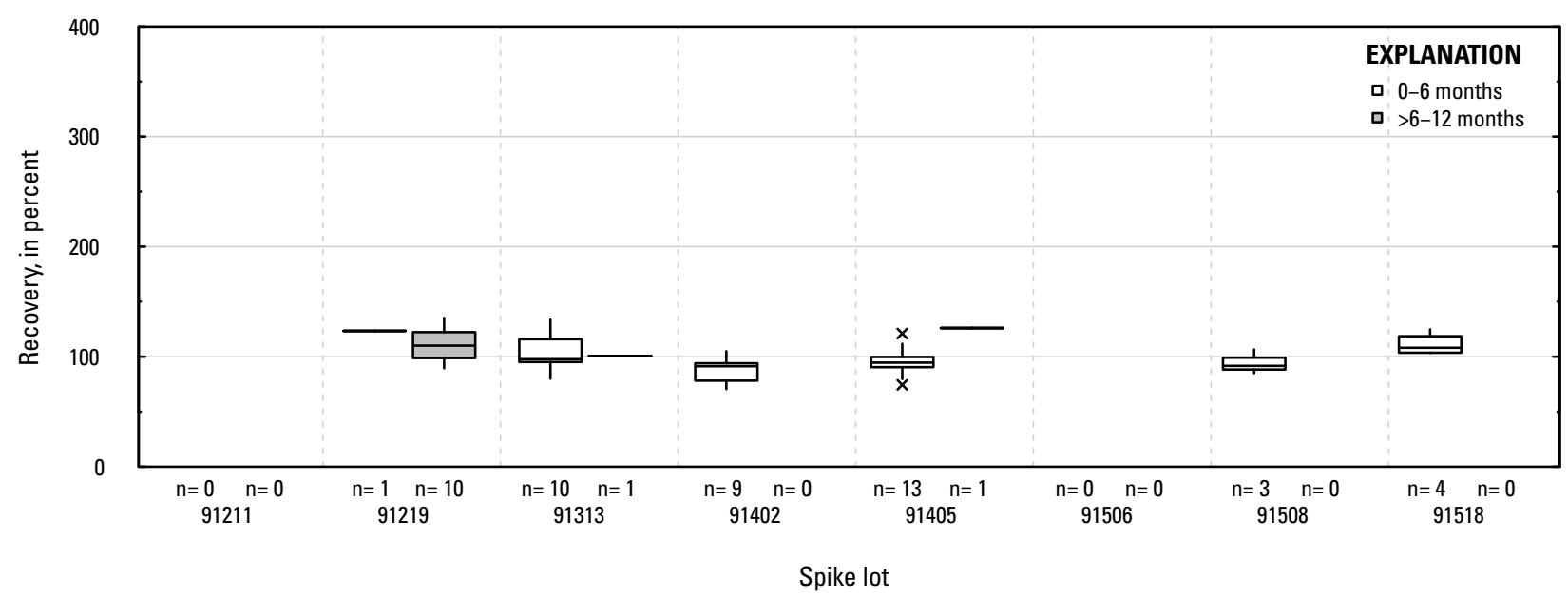

CC. Alachlor oxanilic acid: surface water field matrix spikes

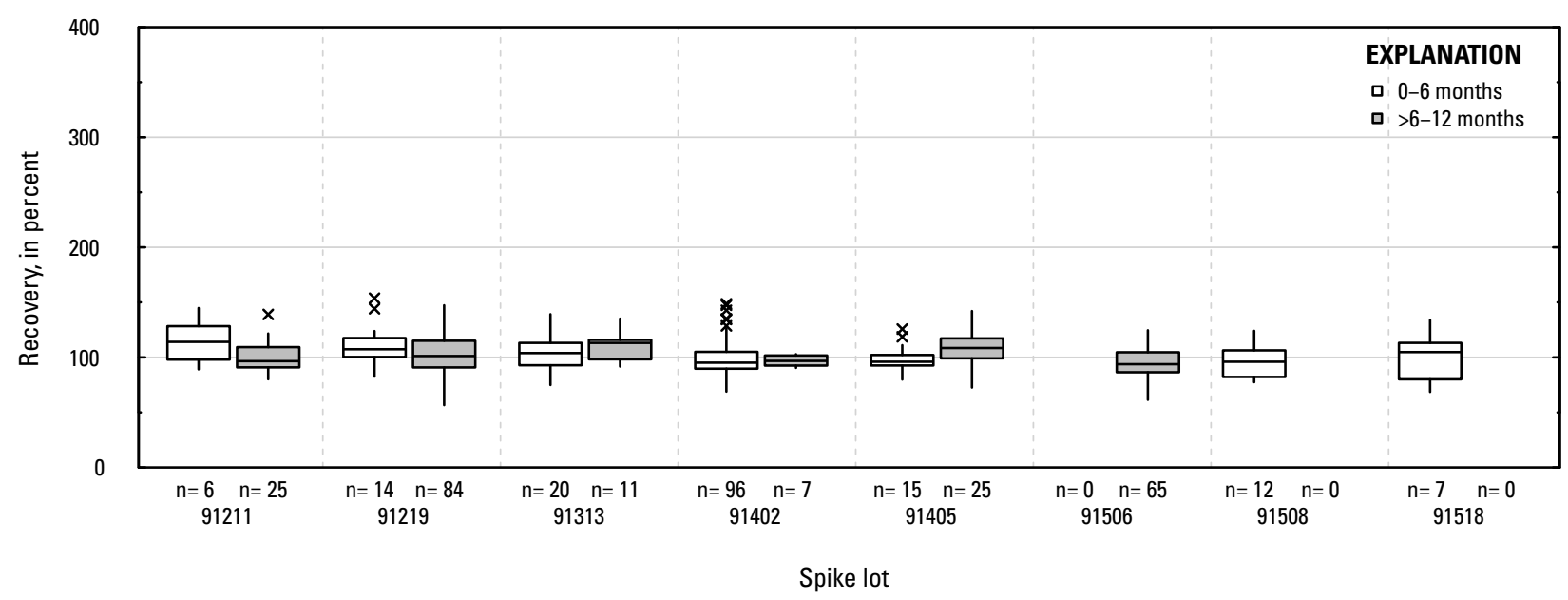

Figure 1-1. Distributions of recovery for individual pesticides in schedule 2437 by matrix, spike lot, and spike lot age. Recovery values larger than 400 percent are not shown.-Continued 
CD. Alachlor sulfonic acid: laboratory reagent spikes

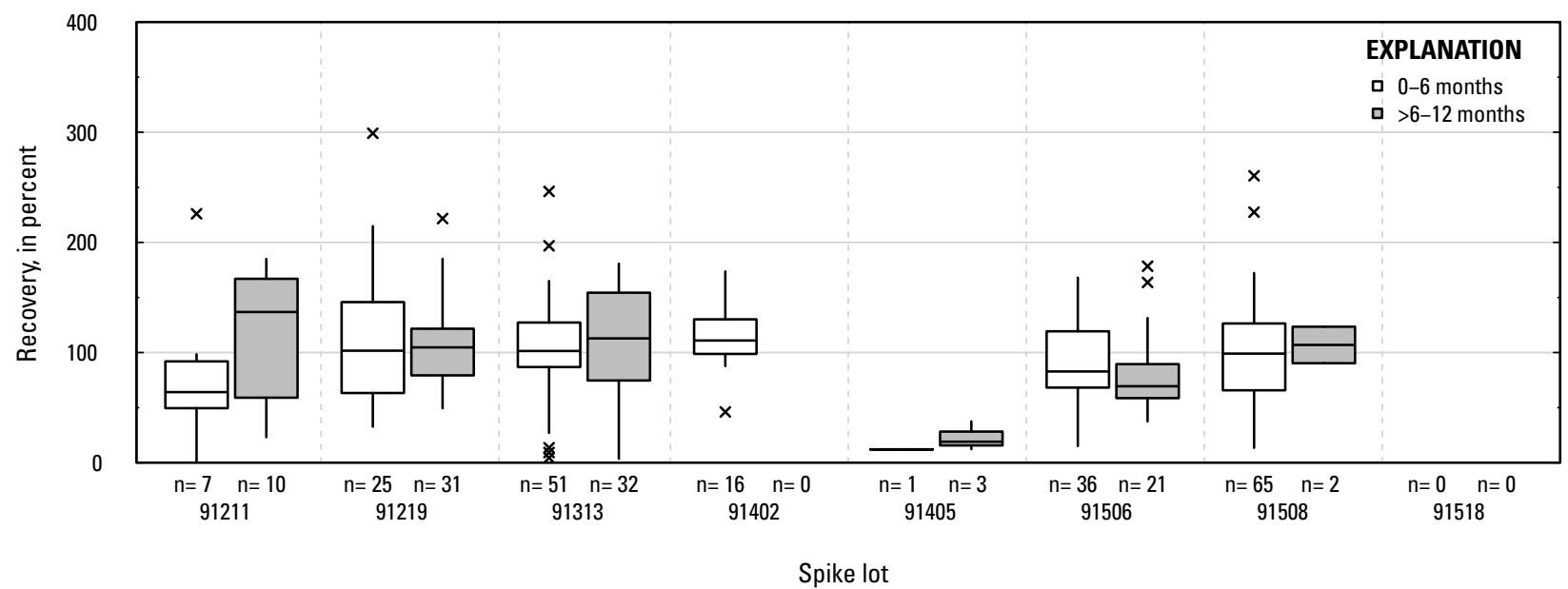

CE. Alachlor sulfonic acid: groundwater field matrix spikes

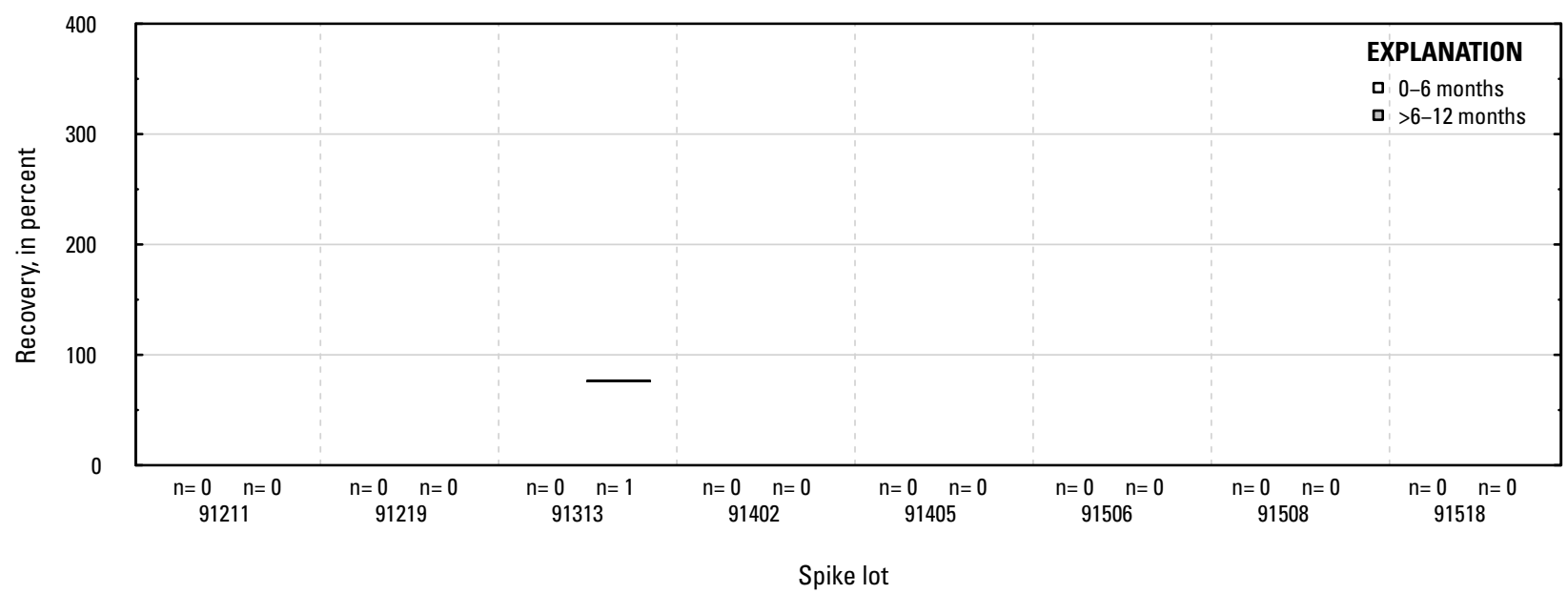

CF. Alachlor sulfonic acid: surface water field matrix spikes

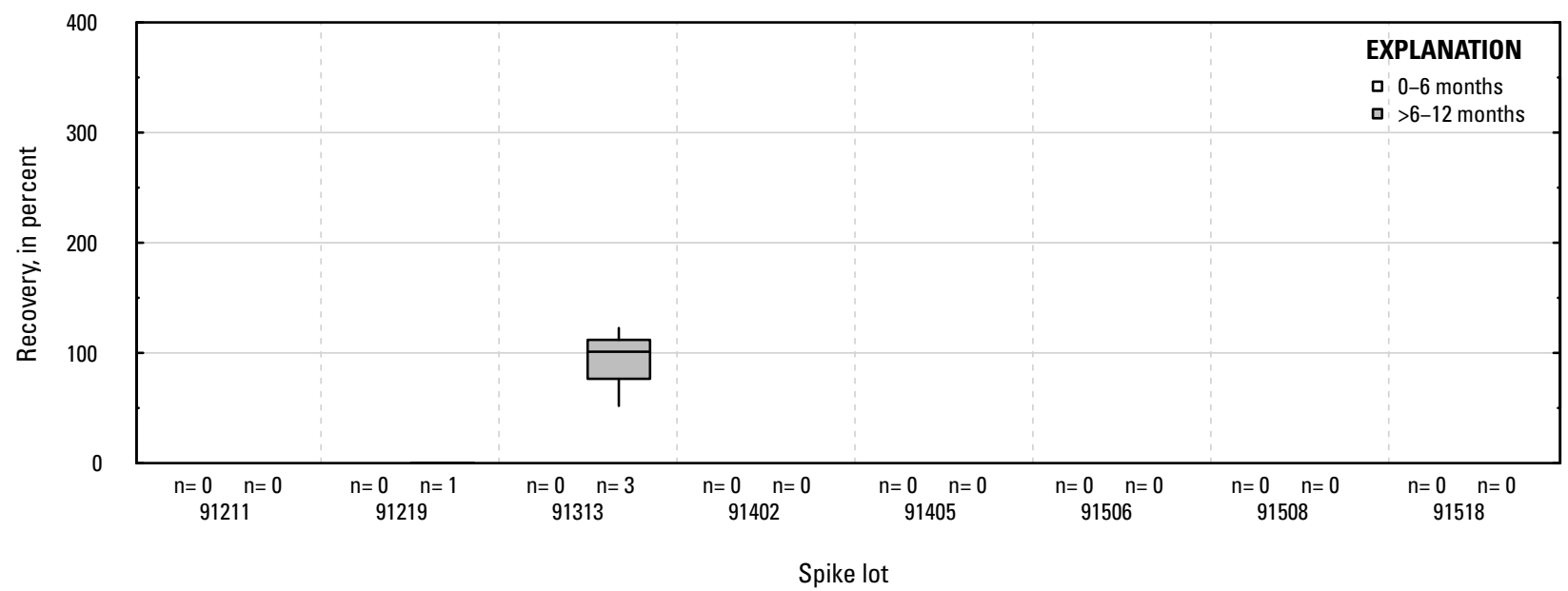

Figure 1-1. Distributions of recovery for individual pesticides in schedule 2437 by matrix, spike lot, and spike lot age. Recovery values larger than 400 percent are not shown.-Continued 


\section{CG. Alachlor sulfynilacetic acid: laboratory reagent spikes}

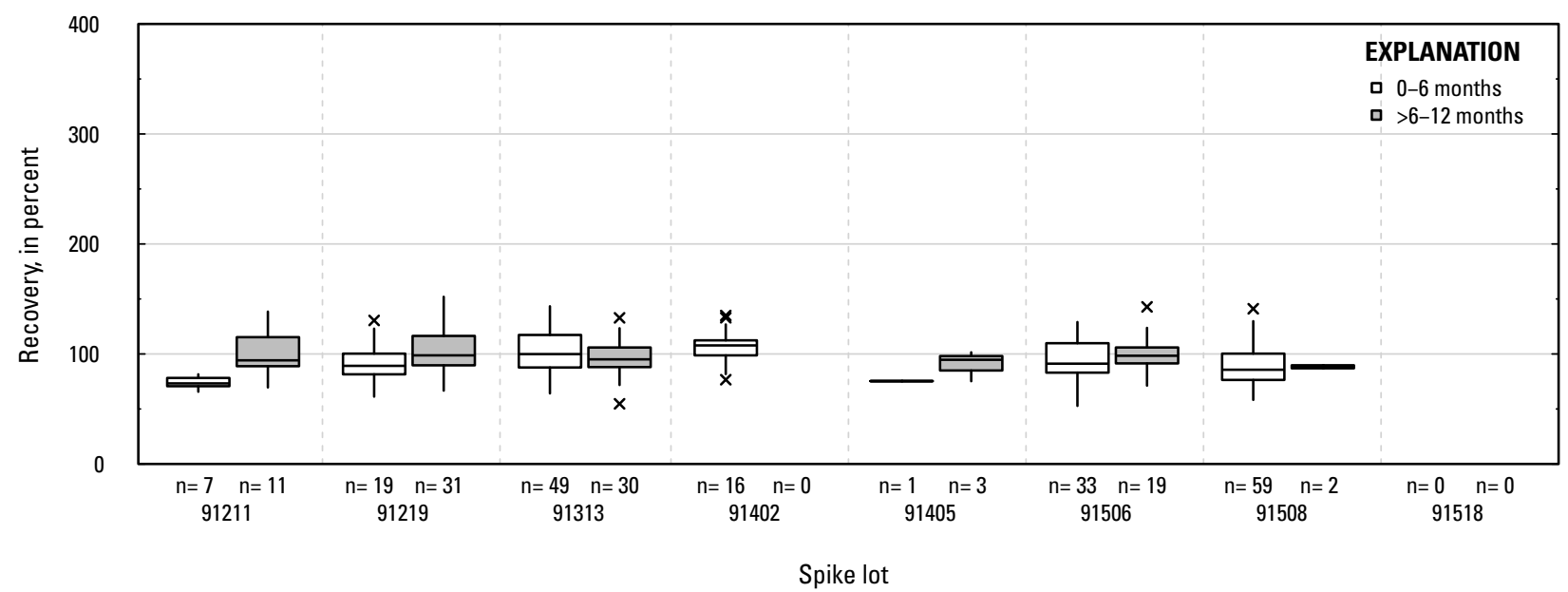

CH. Alachlor sulfynilacetic acid: groundwater field matrix spikes

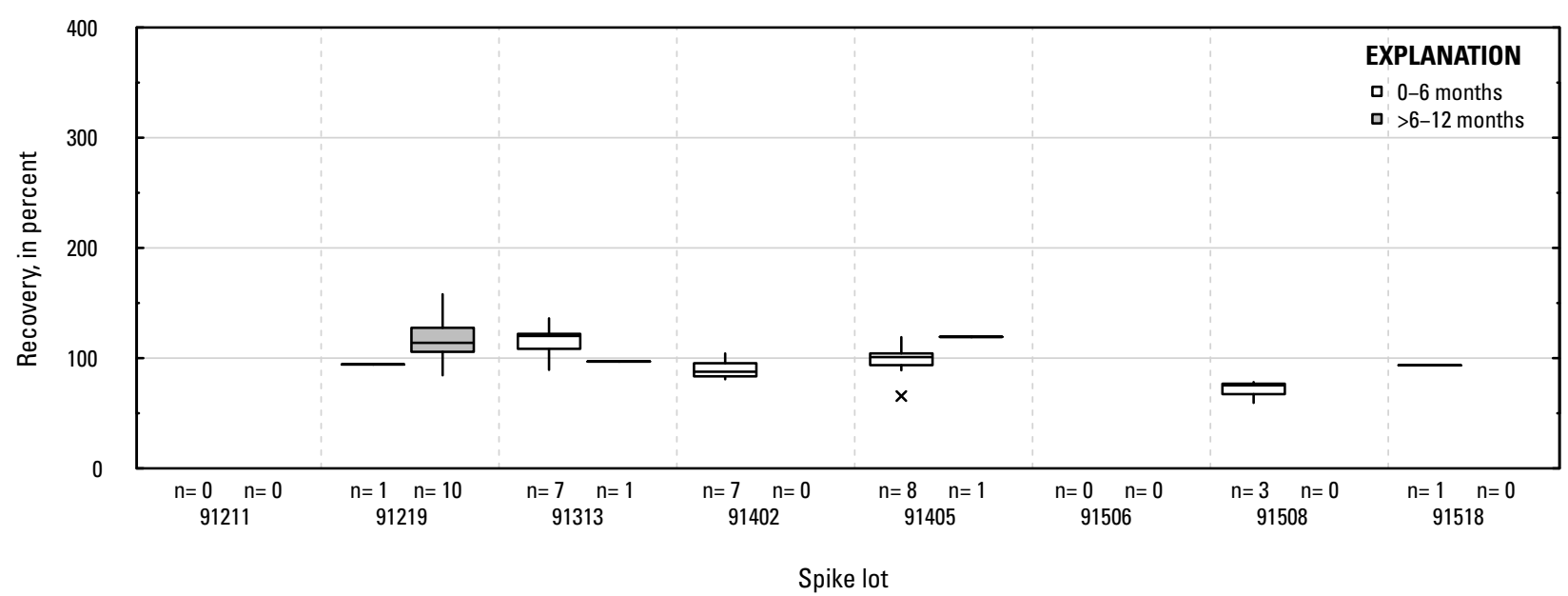

CI. Alachlor sulfynilacetic acid: surface water field matrix spikes

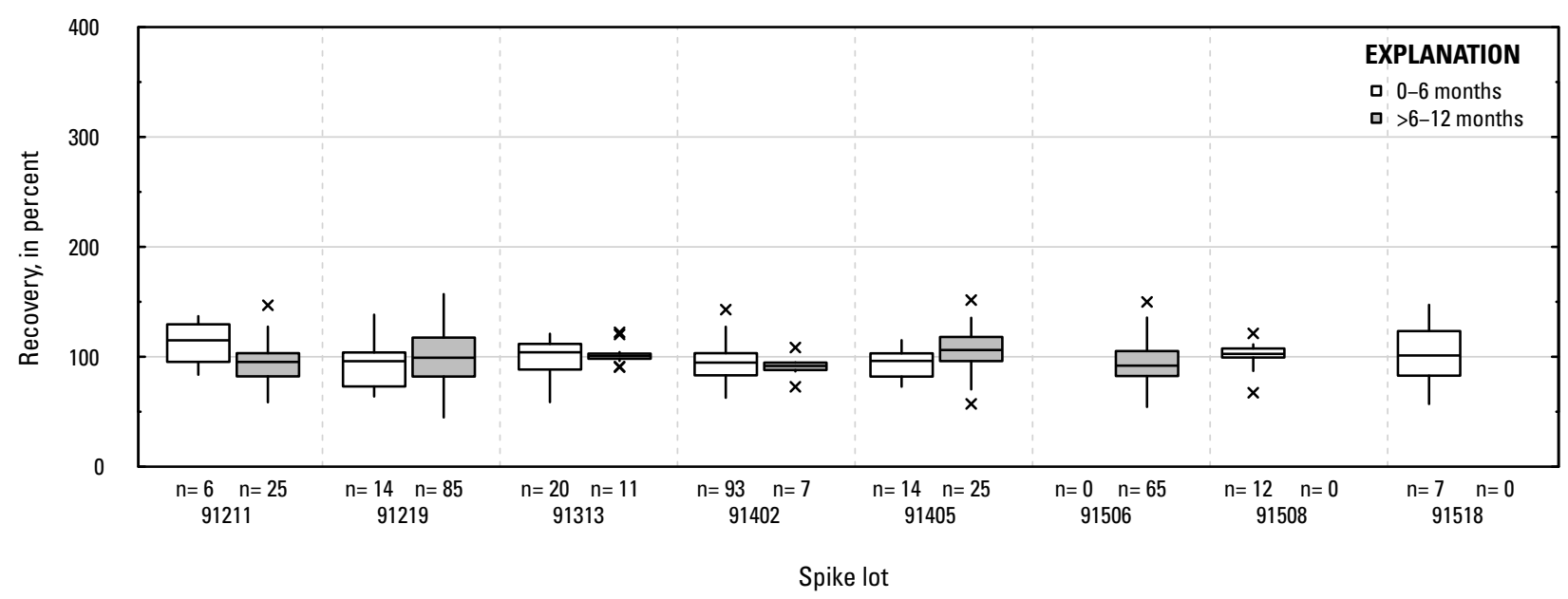

Figure 1-1. Distributions of recovery for individual pesticides in schedule 2437 by matrix, spike lot, and spike lot age. Recovery values larger than 400 percent are not shown.-Continued 


\section{CJ. Aldicarb: laboratory reagent spikes}

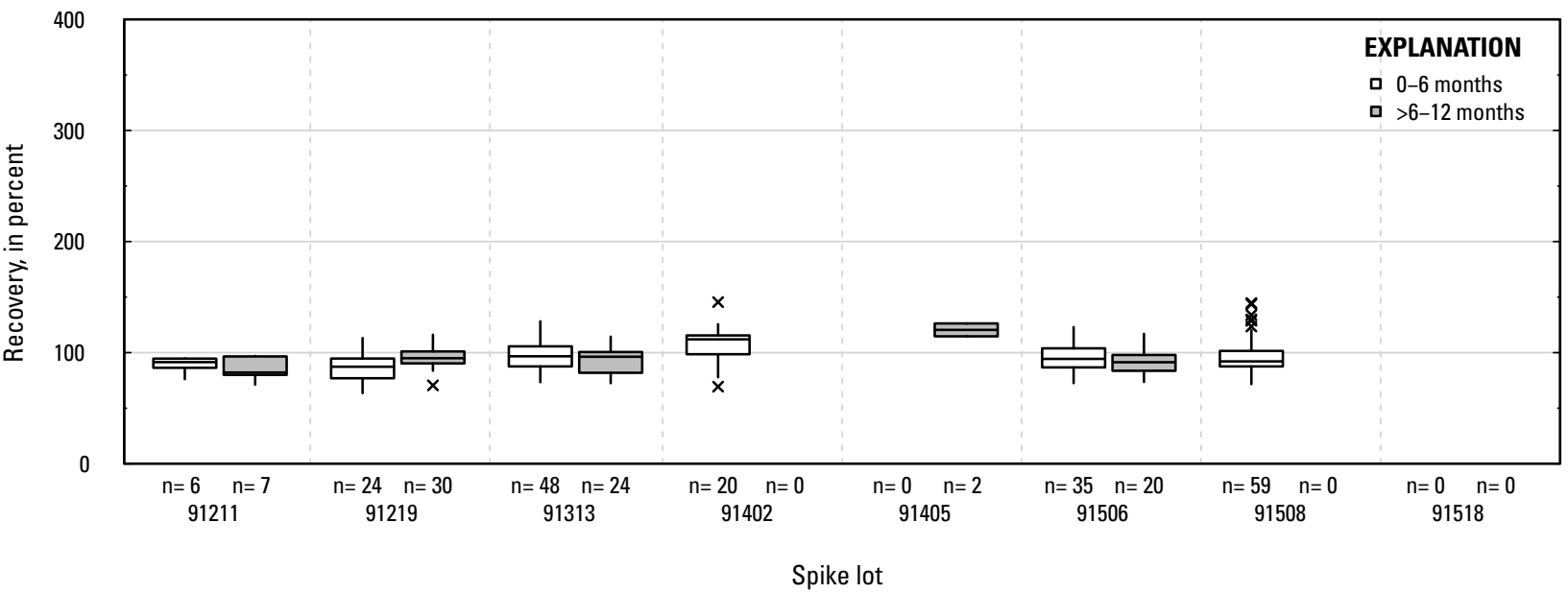

\section{CK. Aldicarb: groundwater field matrix spikes}

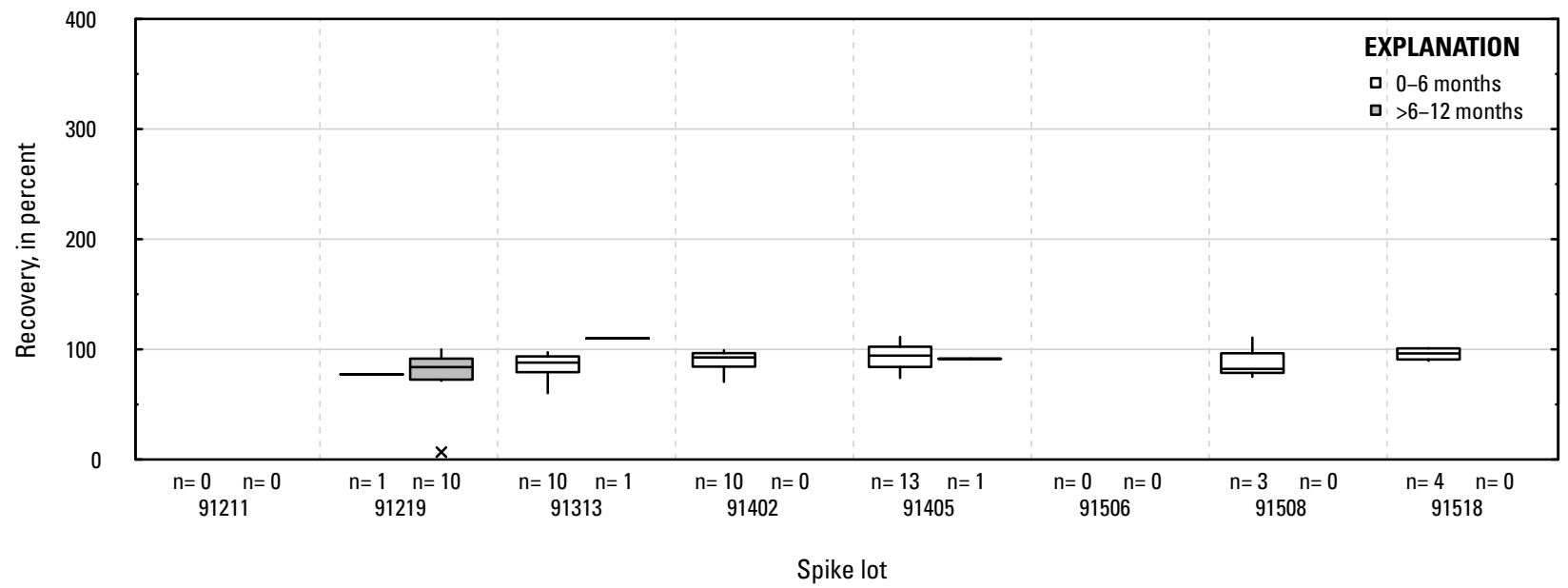

\section{Aldicarb: surface water field matrix spikes}

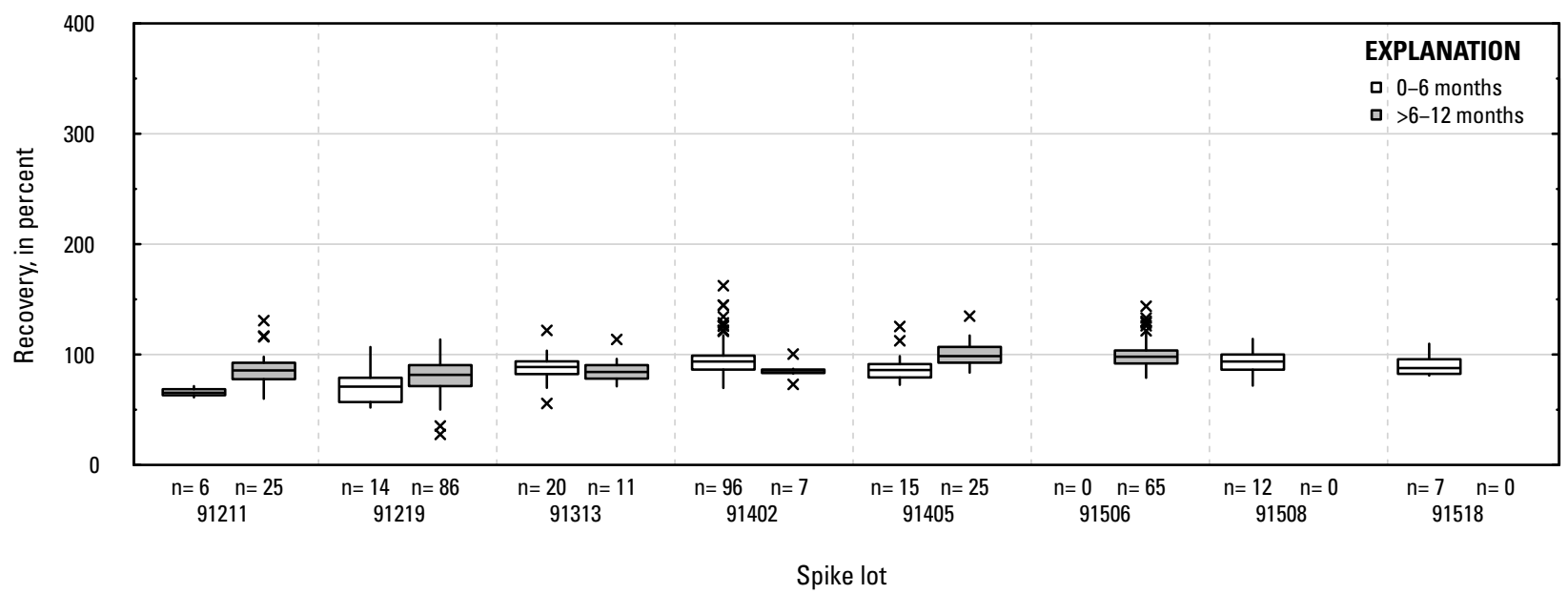

Figure 1-1. Distributions of recovery for individual pesticides in schedule 2437 by matrix, spike lot, and spike lot age. Recovery values larger than 400 percent are not shown.-Continued 
CM. Aldicarb sulfone: laboratory reagent spikes

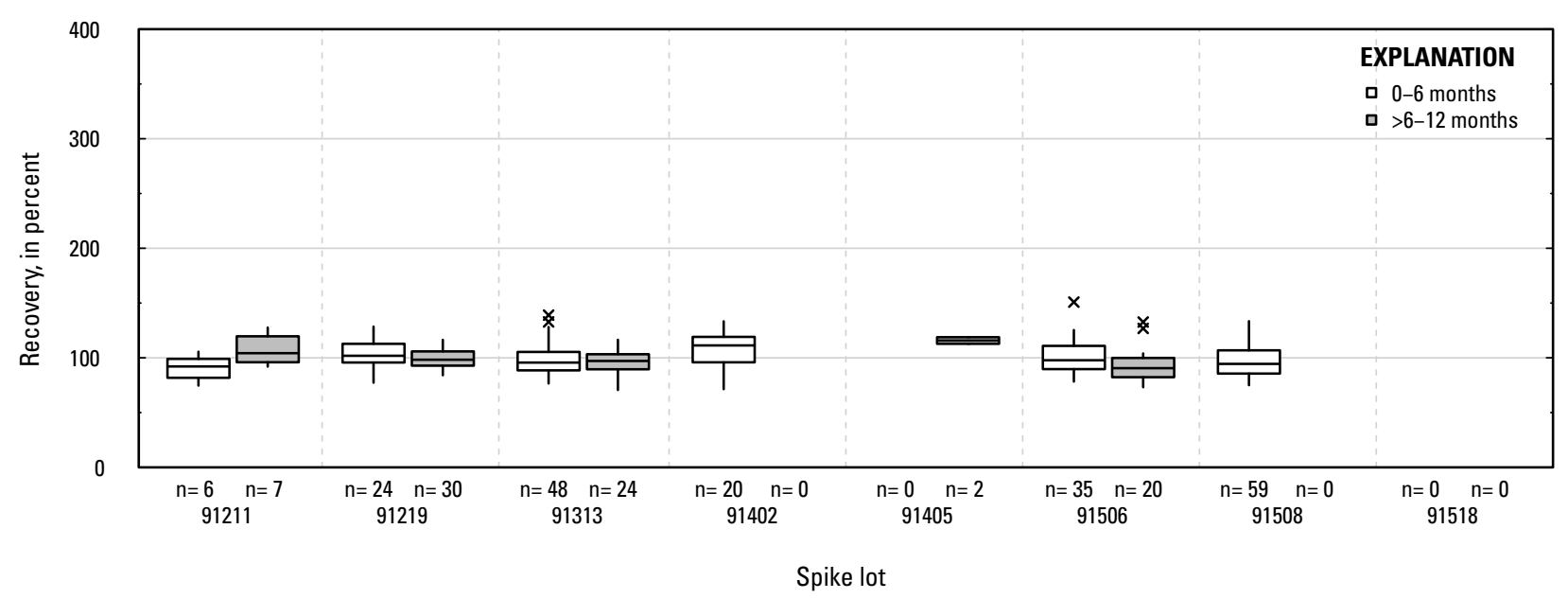

CN. Aldicarb sulfone: groundwater field matrix spikes

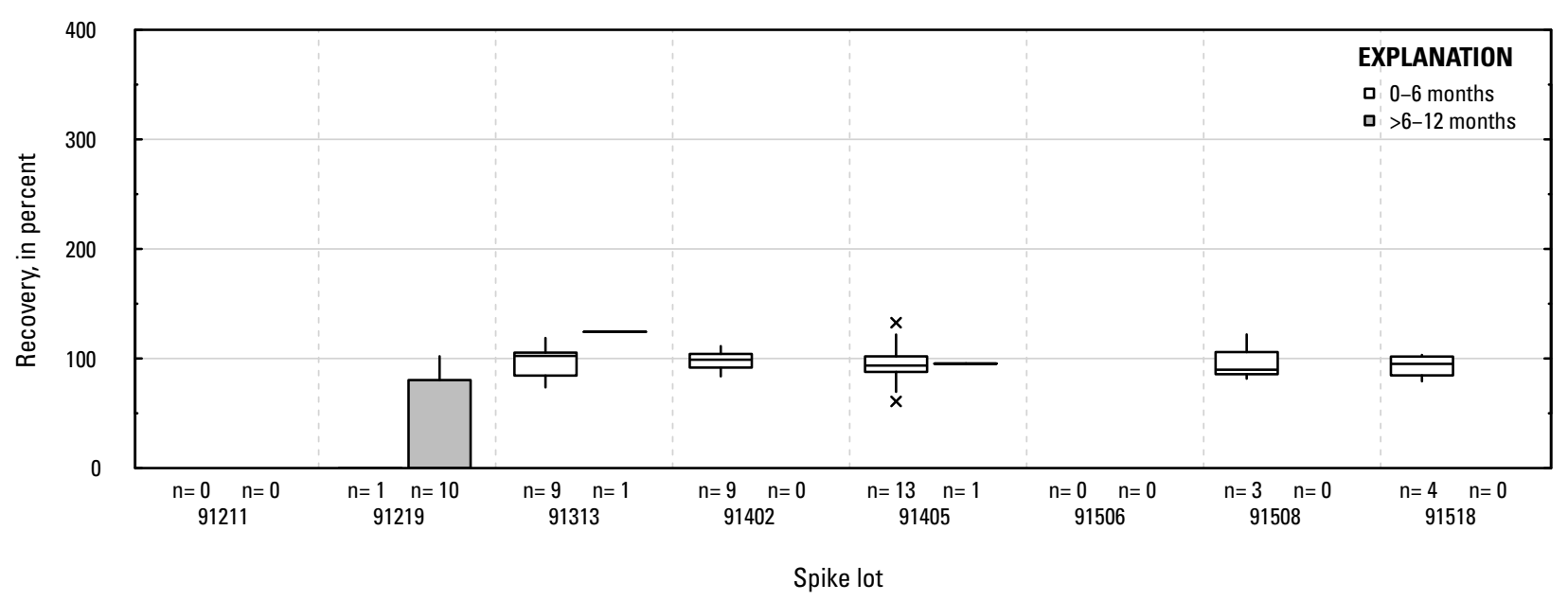

CO. Aldicarb sulfone: surface water field matrix spikes

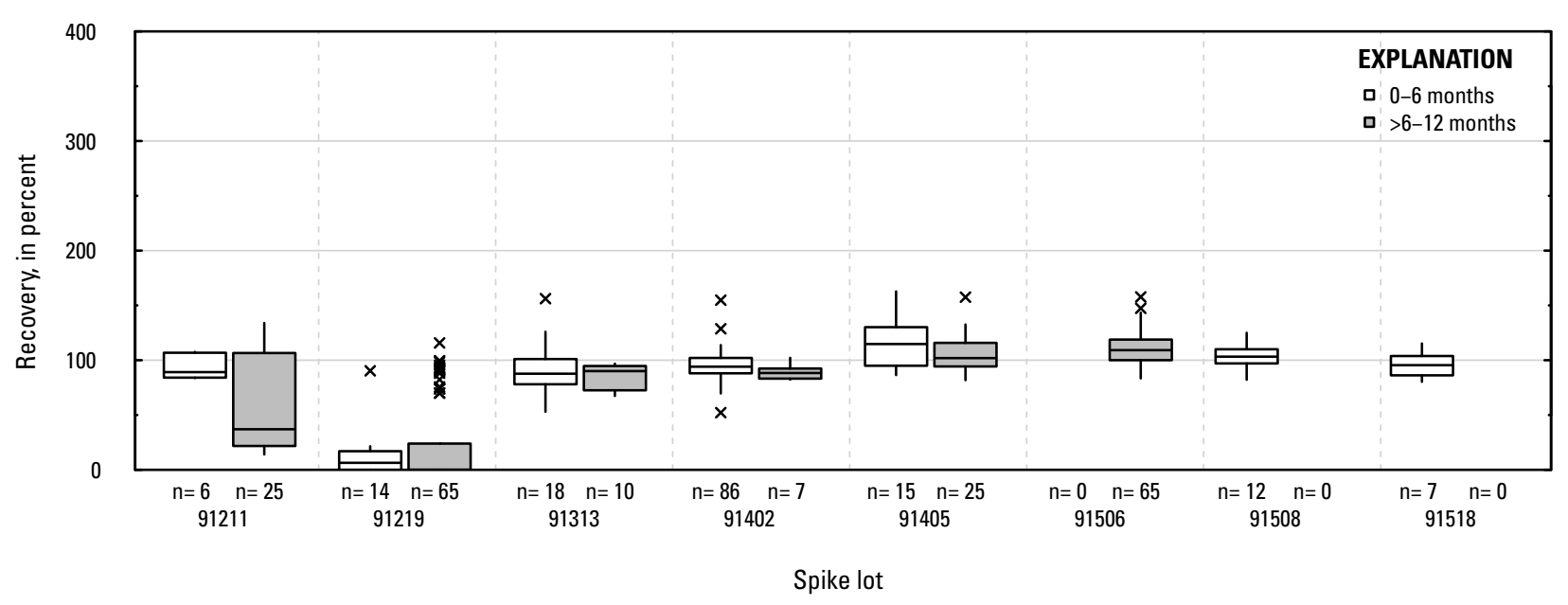

Figure 1-1. Distributions of recovery for individual pesticides in schedule 2437 by matrix, spike lot, and spike lot age. Recovery values larger than 400 percent are not shown.-Continued 
CP. Aldicarb sulfoxide: laboratory reagent spikes

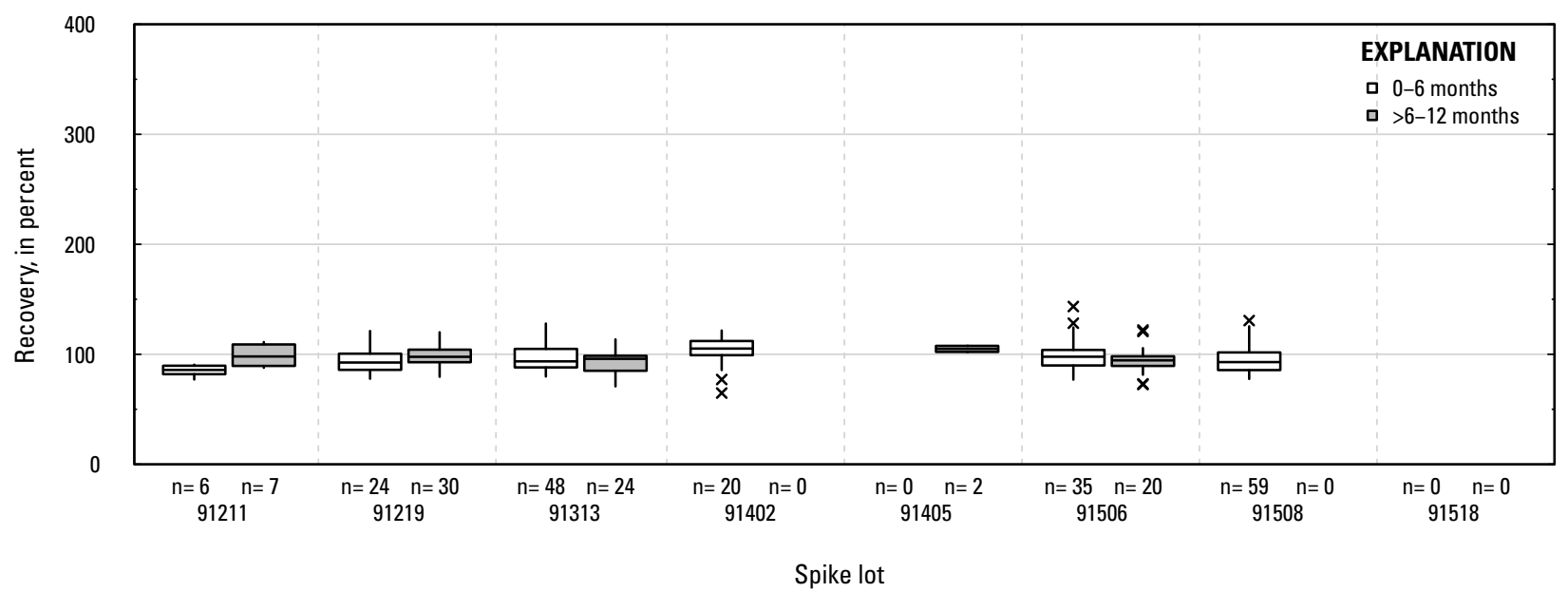

CQ. Aldicarb sulfoxide: groundwater field matrix spikes

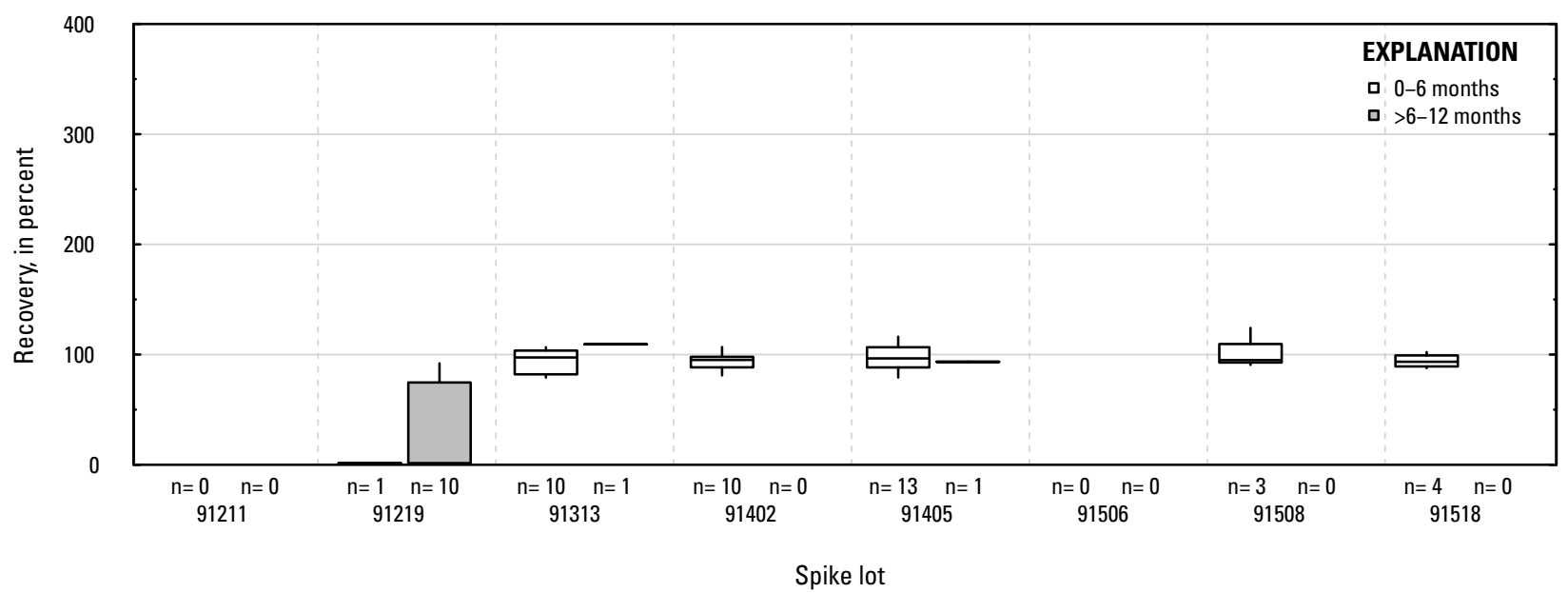

CR. Aldicarb sulfoxide: surface water field matrix spikes

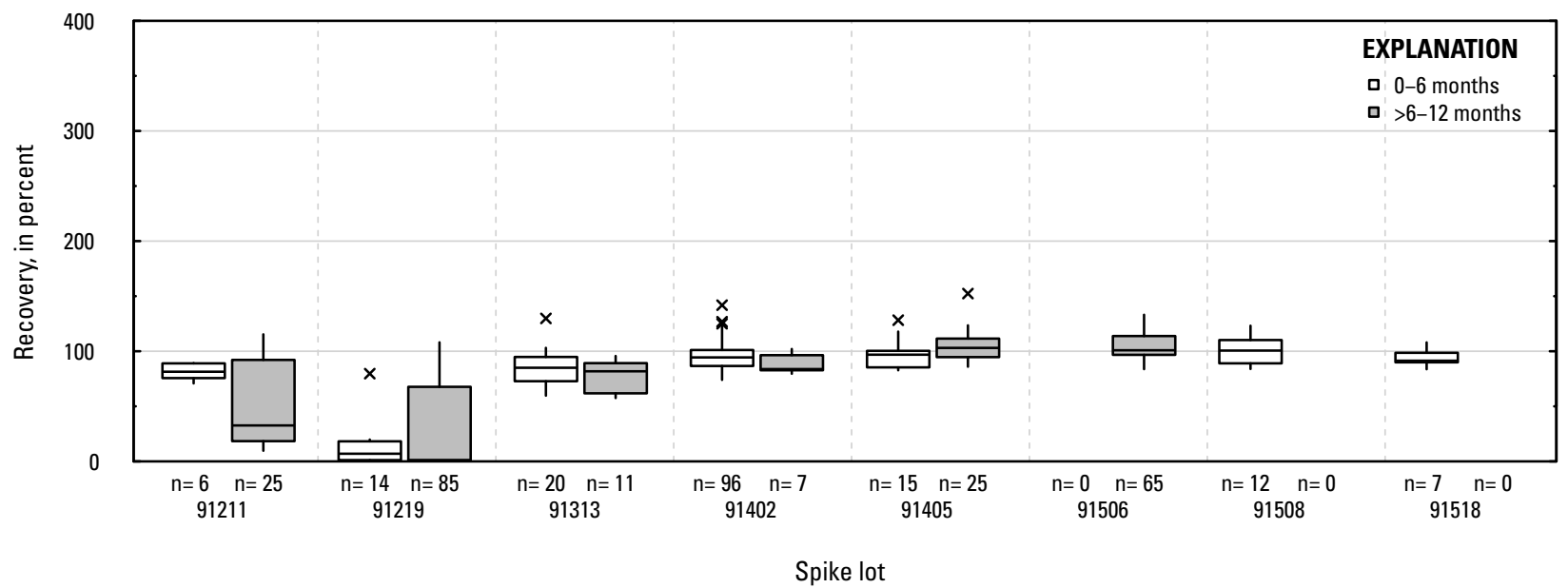

Figure 1-1. Distributions of recovery for individual pesticides in schedule 2437 by matrix, spike lot, and spike lot age. Recovery values larger than 400 percent are not shown.-Continued 


\section{CS. Ametryn: laboratory reagent spikes}

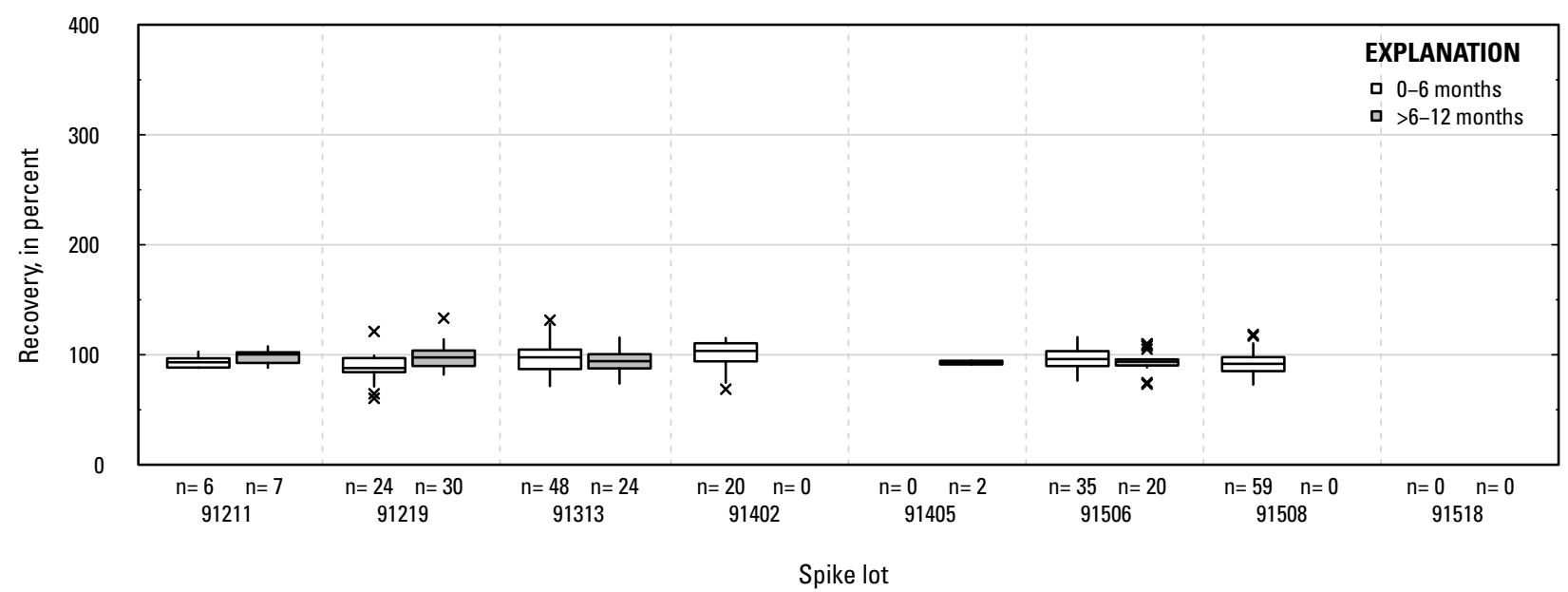

CT. Ametryn: groundwater field matrix spikes

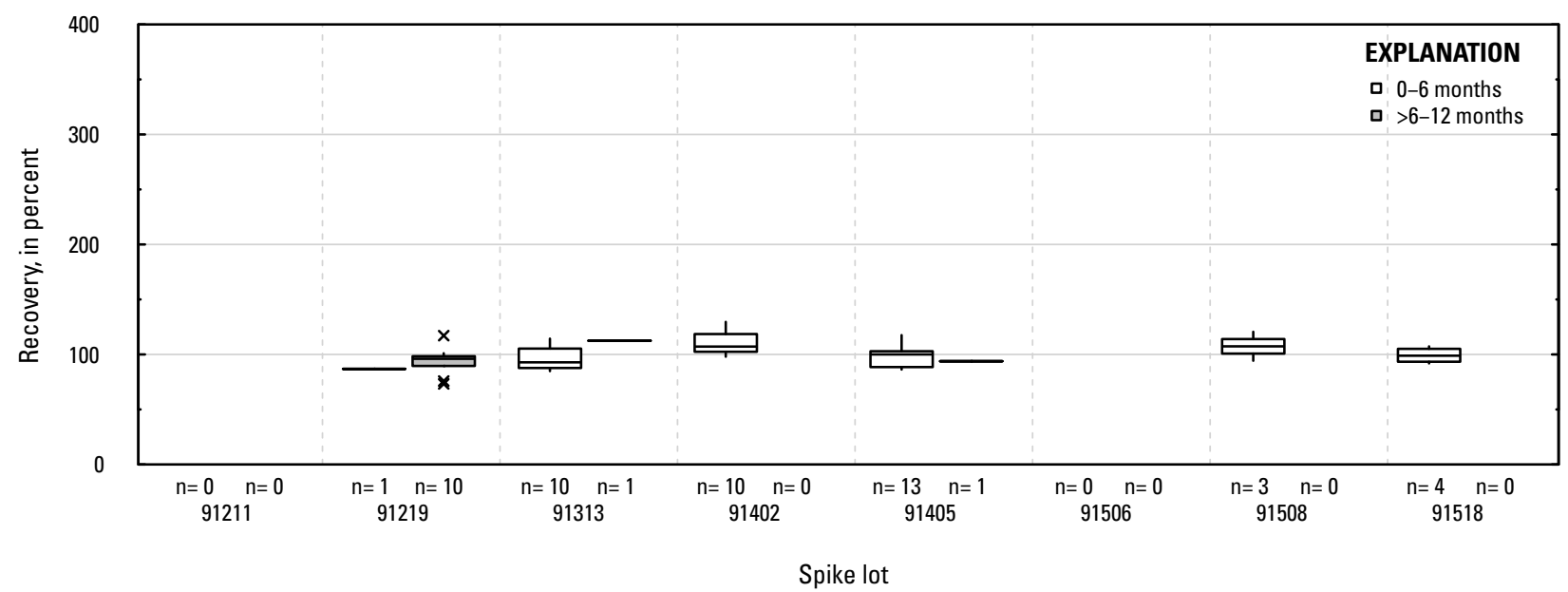

CU. Ametryn: surface water field matrix spikes

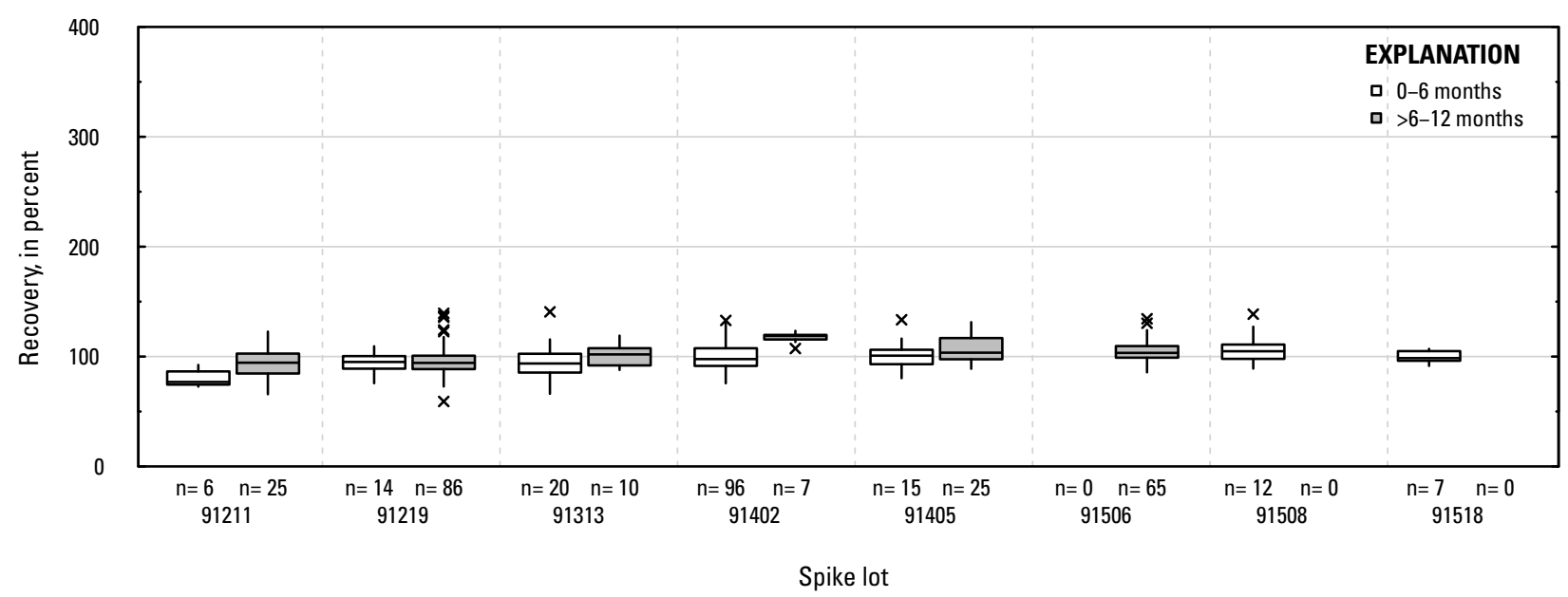

Figure 1-1. Distributions of recovery for individual pesticides in schedule 2437 by matrix, spike lot, and spike lot age. Recovery values larger than 400 percent are not shown.-Continued 
CV. Asulam: laboratory reagent spikes

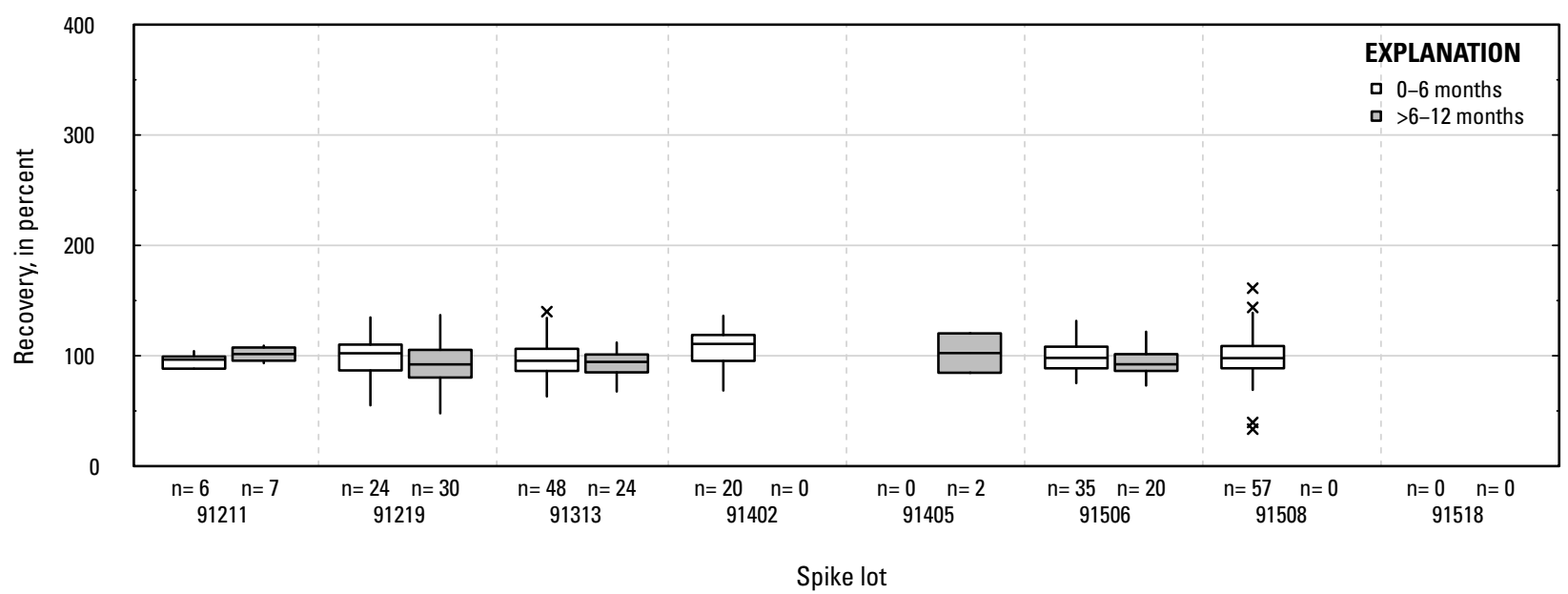

CW. Asulam: groundwater field matrix spikes

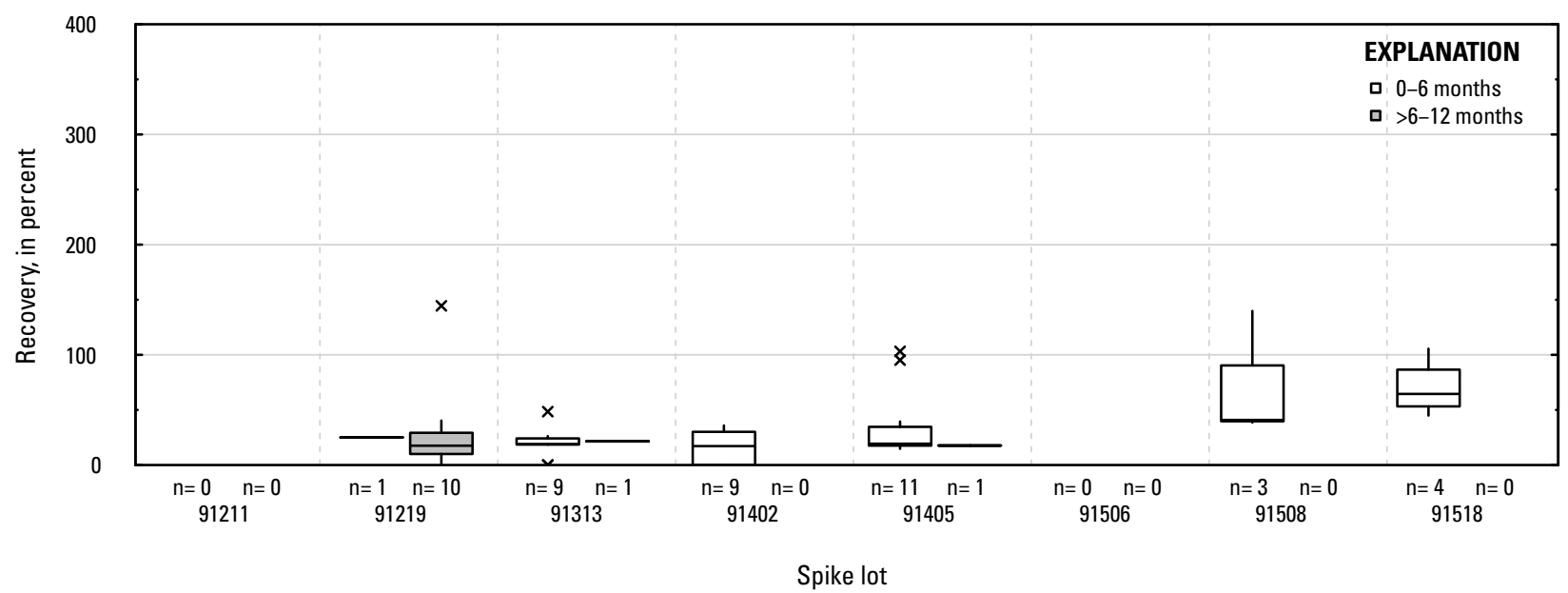

\section{Asulam: surface water field matrix spikes}

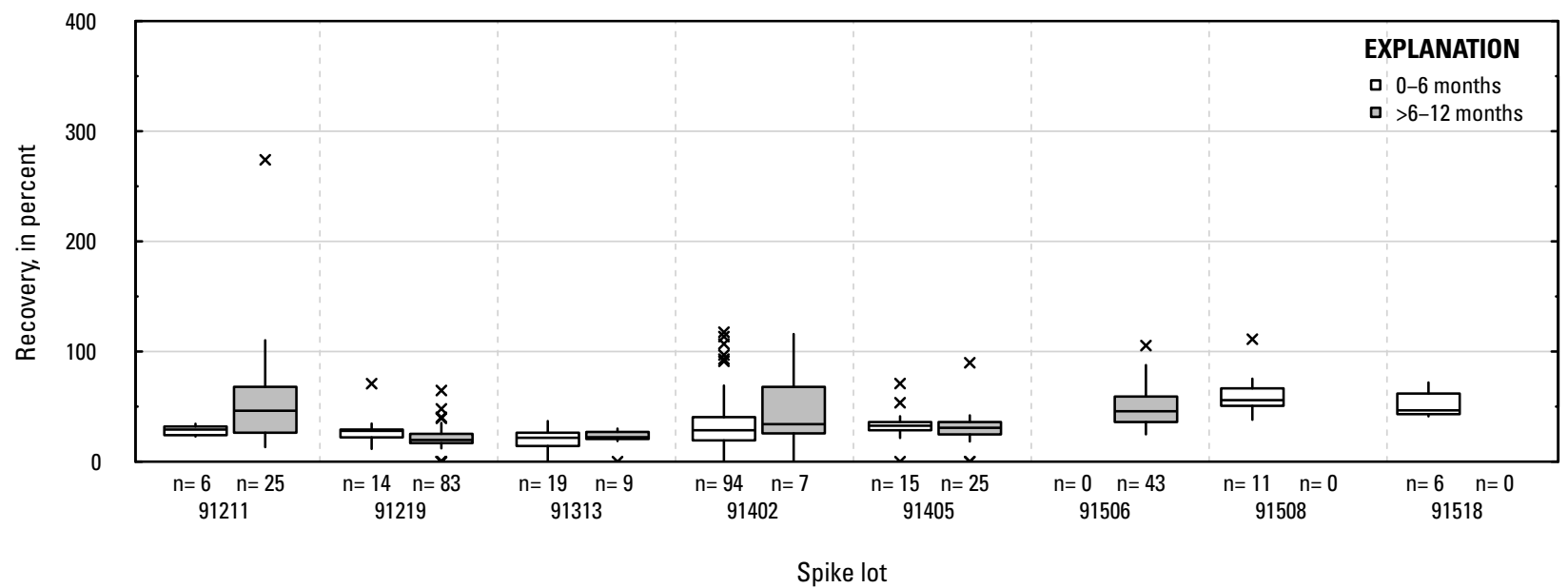

Figure 1-1. Distributions of recovery for individual pesticides in schedule 2437 by matrix, spike lot, and spike lot age. Recovery values larger than 400 percent are not shown.-Continued 
CY. Atrazine: laboratory reagent spikes

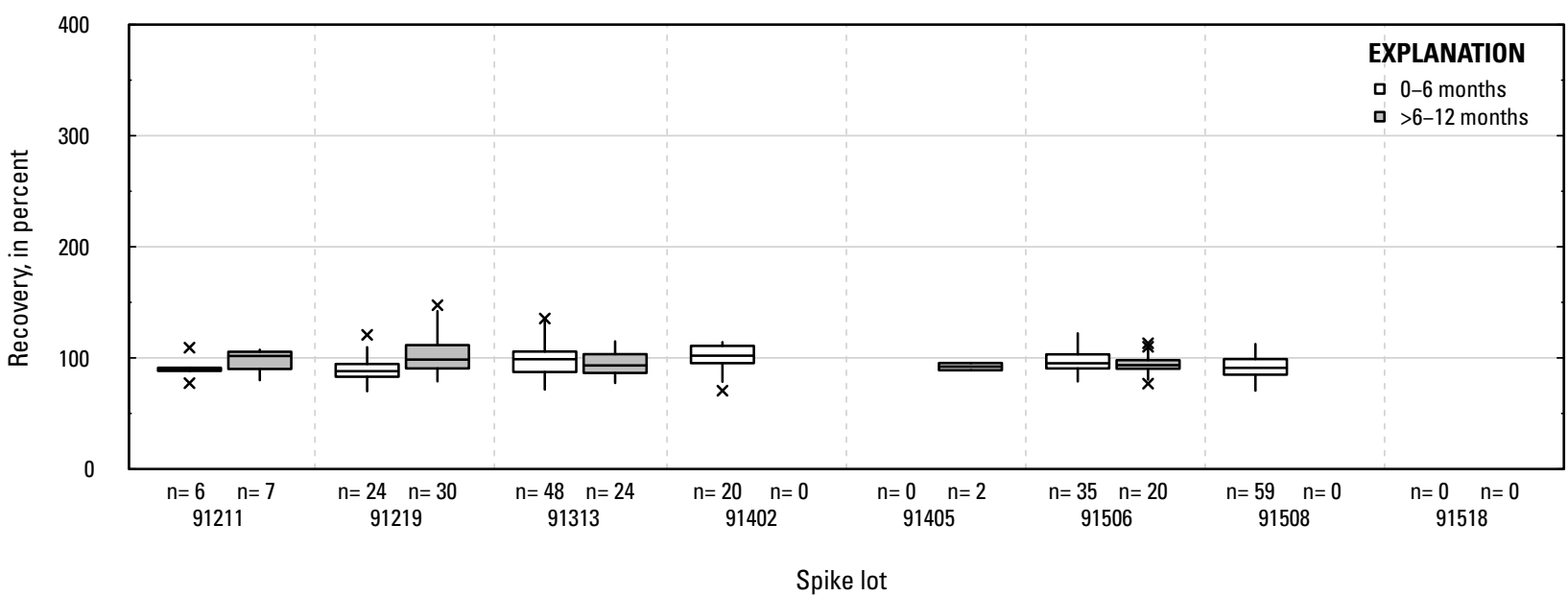

CZ. Atrazine: groundwater field matrix spikes

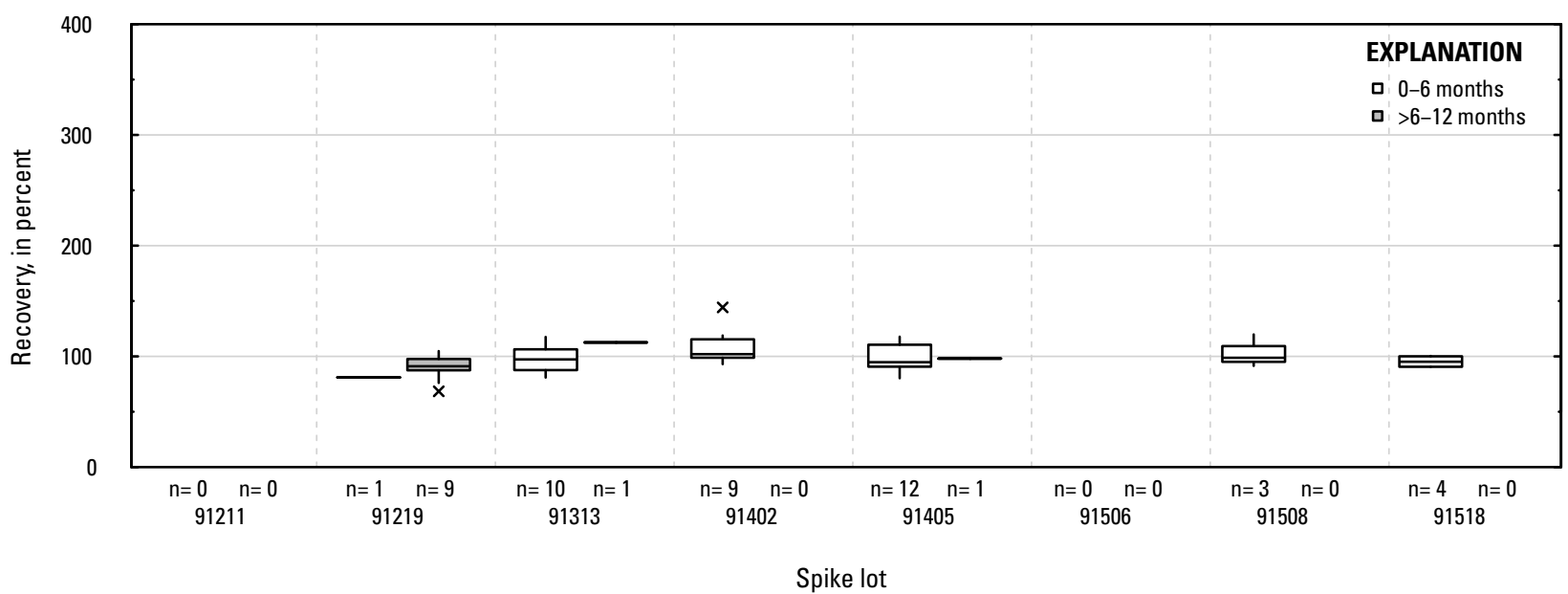

DA. Atrazine: surface water field matrix spikes

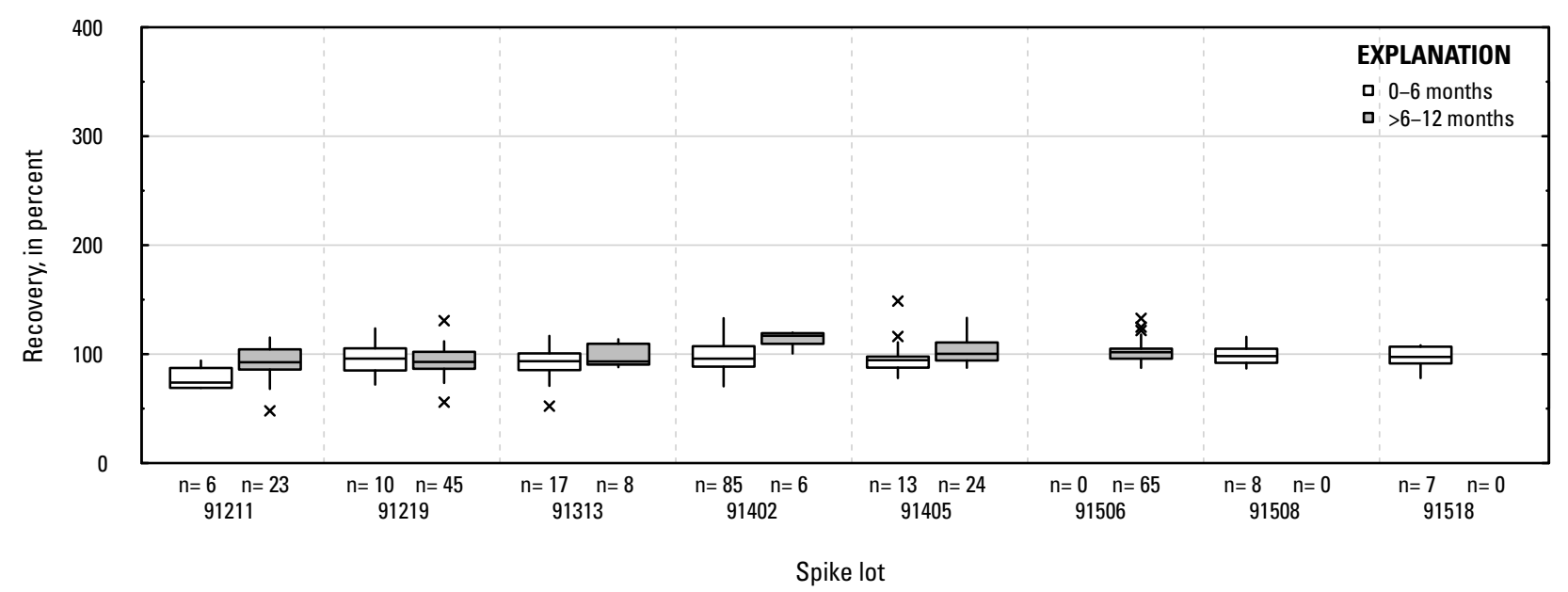

Figure 1-1. Distributions of recovery for individual pesticides in schedule 2437 by matrix, spike lot, and spike lot age. Recovery values larger than 400 percent are not shown.-Continued 
DB. Azinphos-methyl: laboratory reagent spikes

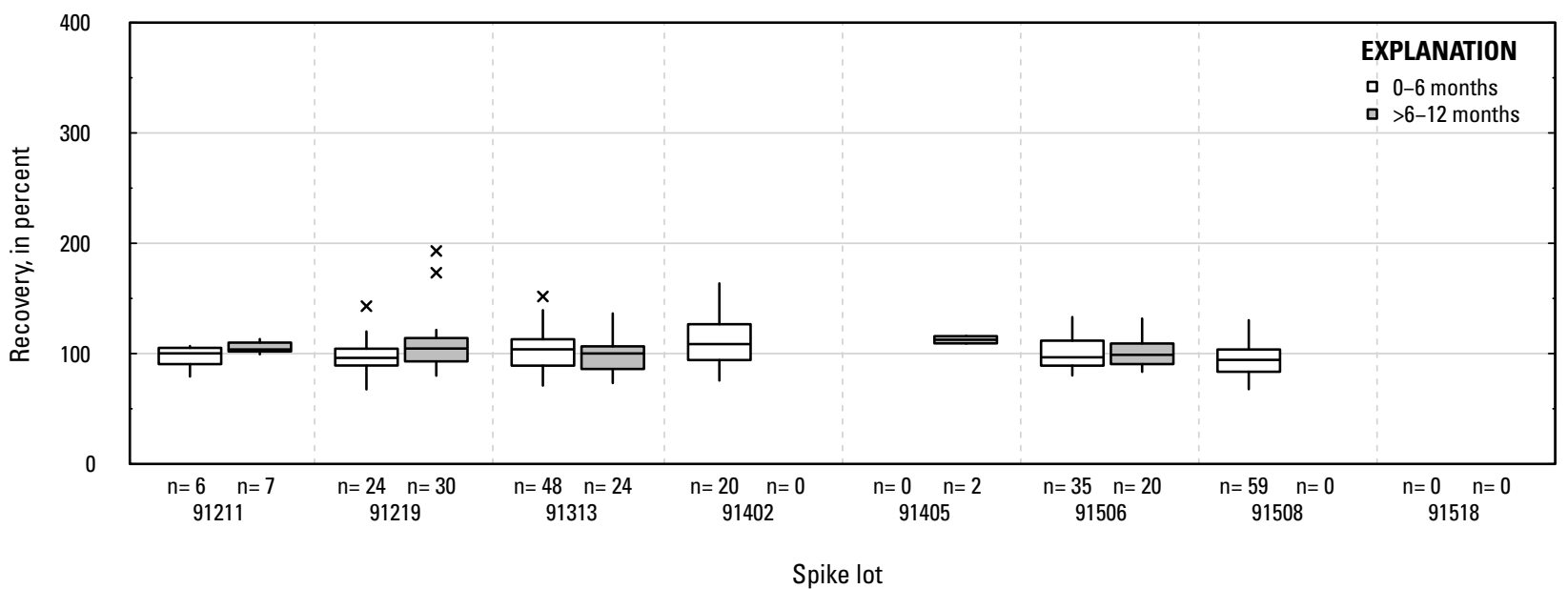

DC. Azinphos-methyl: groundwater field matrix spikes

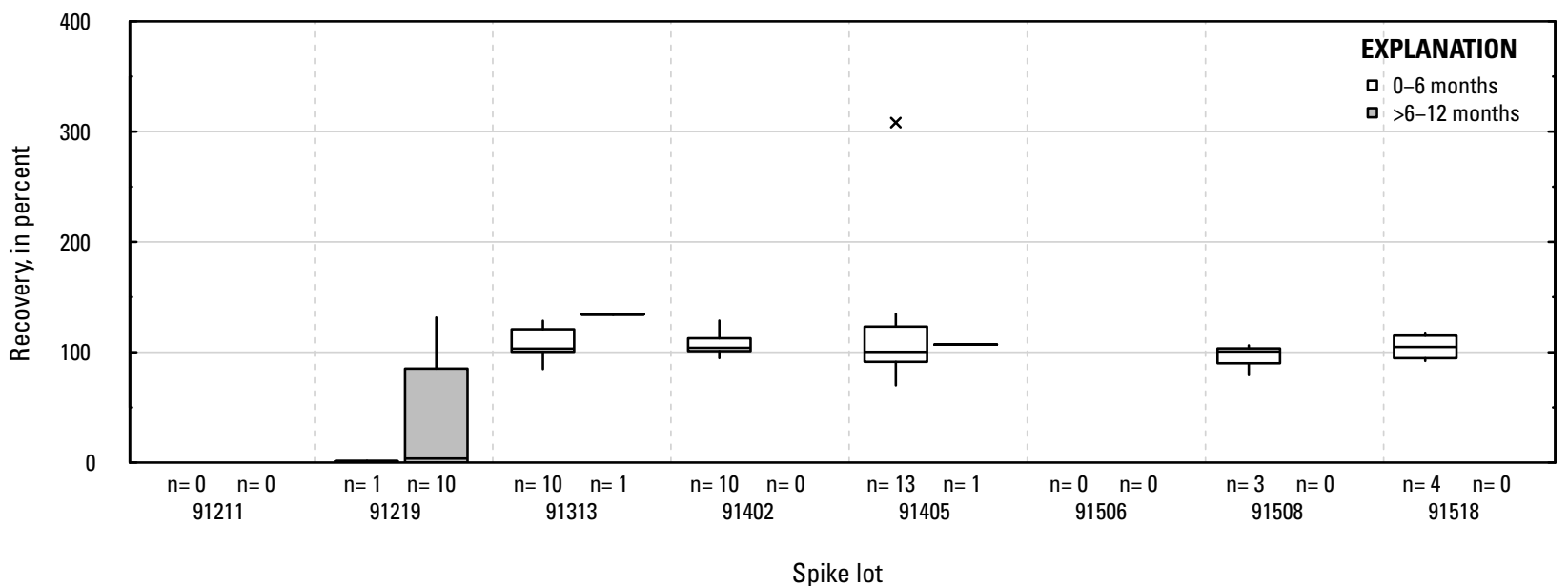

DD. Azinphos-methyl: surface water field matrix spikes

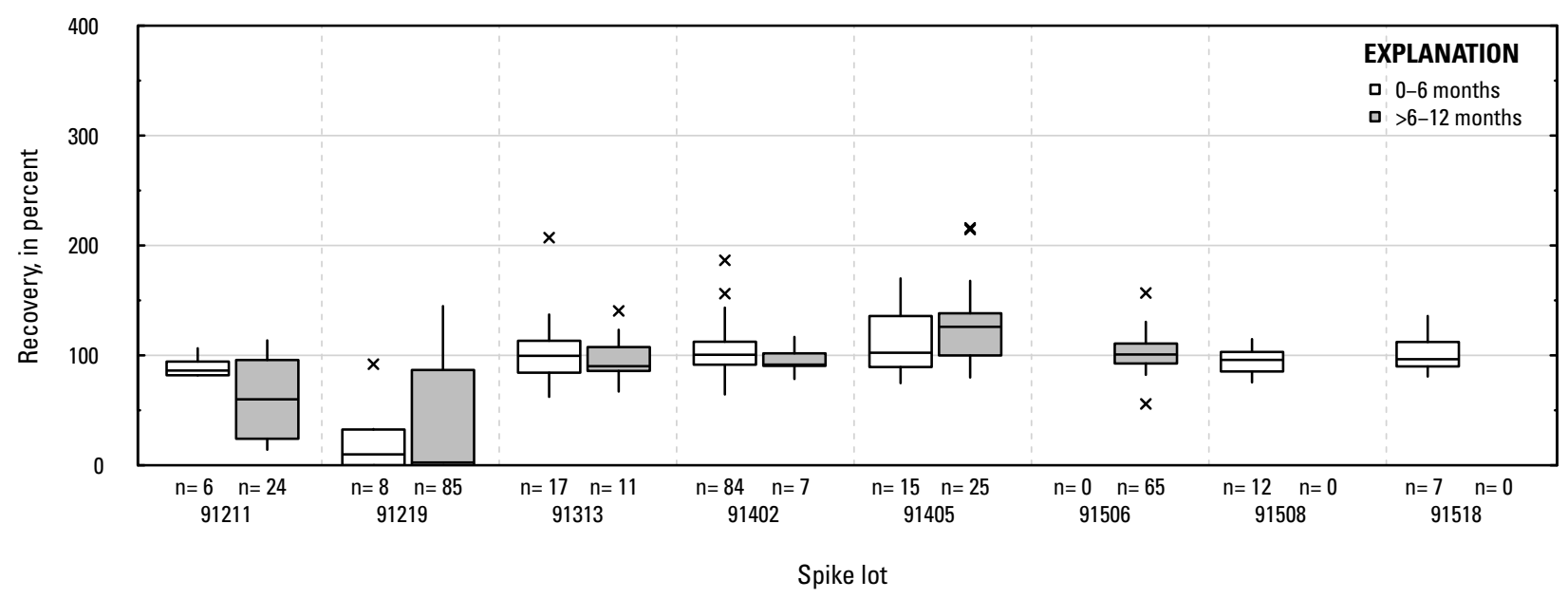

Figure 1-1. Distributions of recovery for individual pesticides in schedule 2437 by matrix, spike lot, and spike lot age. Recovery values larger than 400 percent are not shown.-Continued 
DE. Azinphos-methyl oxon: laboratory reagent spikes

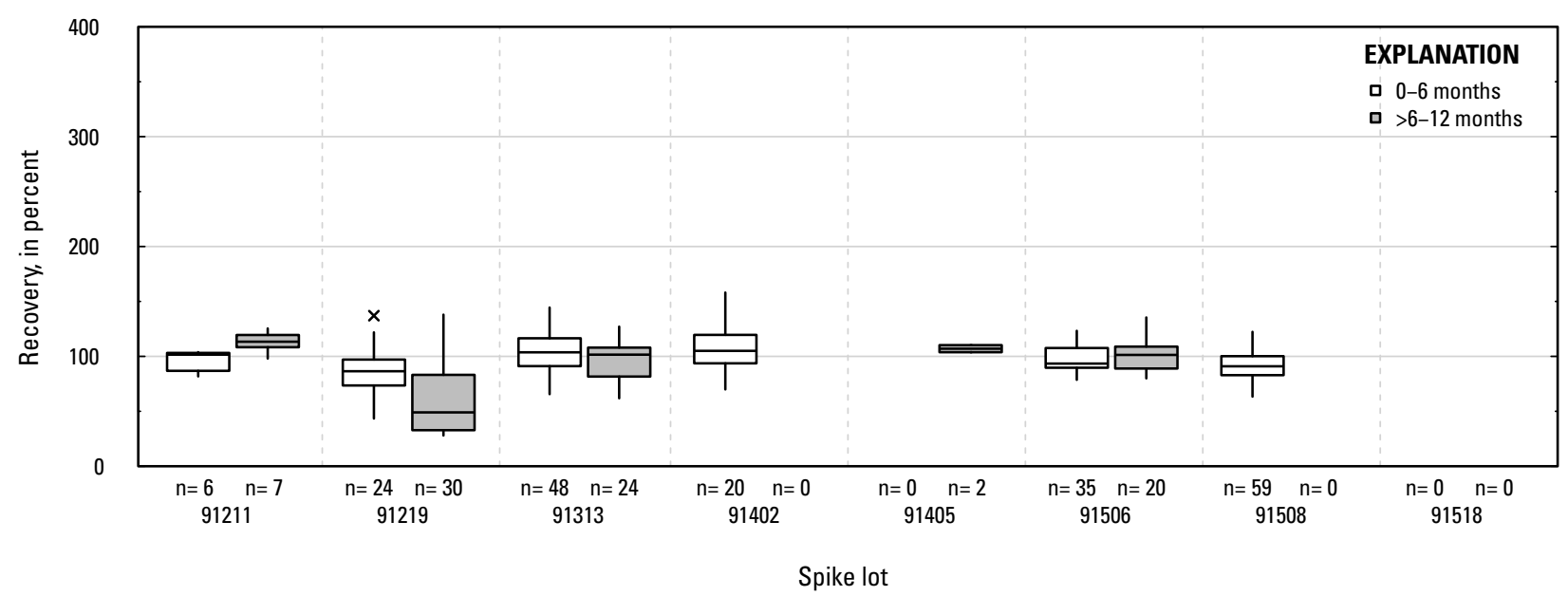

DF. Azinphos-methyl oxon: groundwater field matrix spikes

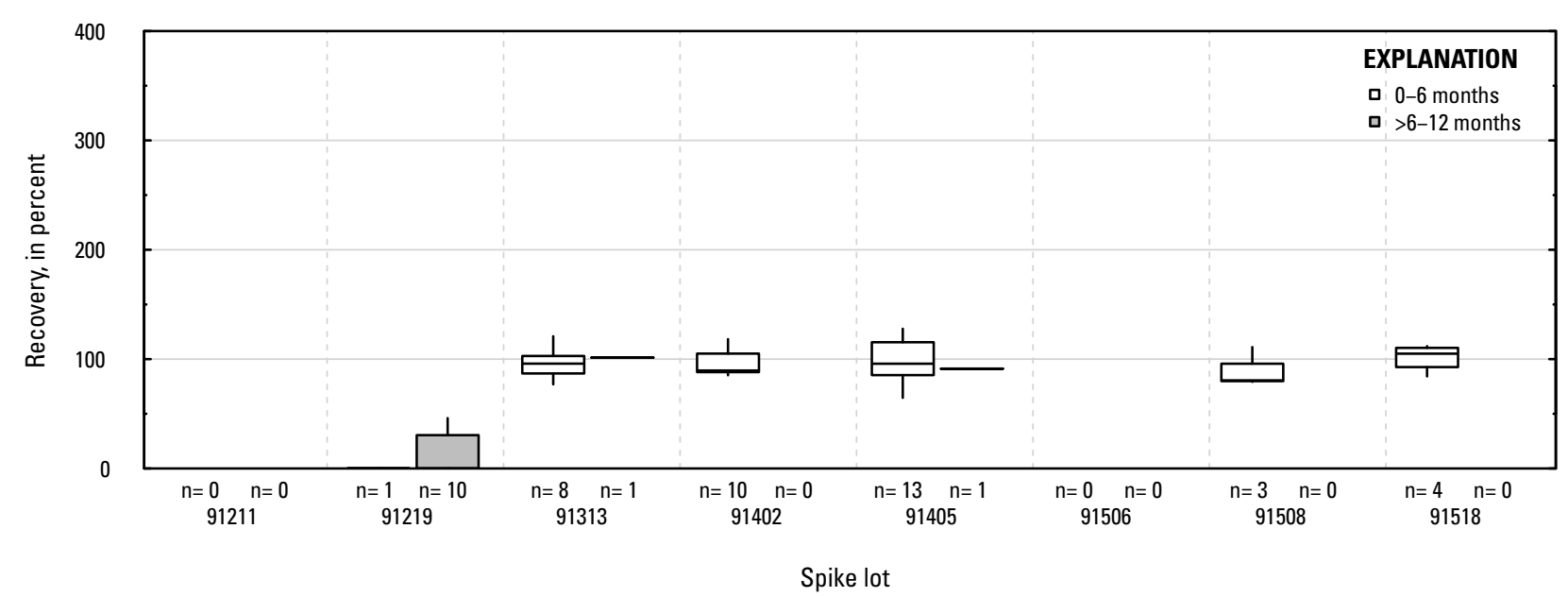

DG. Azinphos-methyl oxon: surface water field matrix spikes

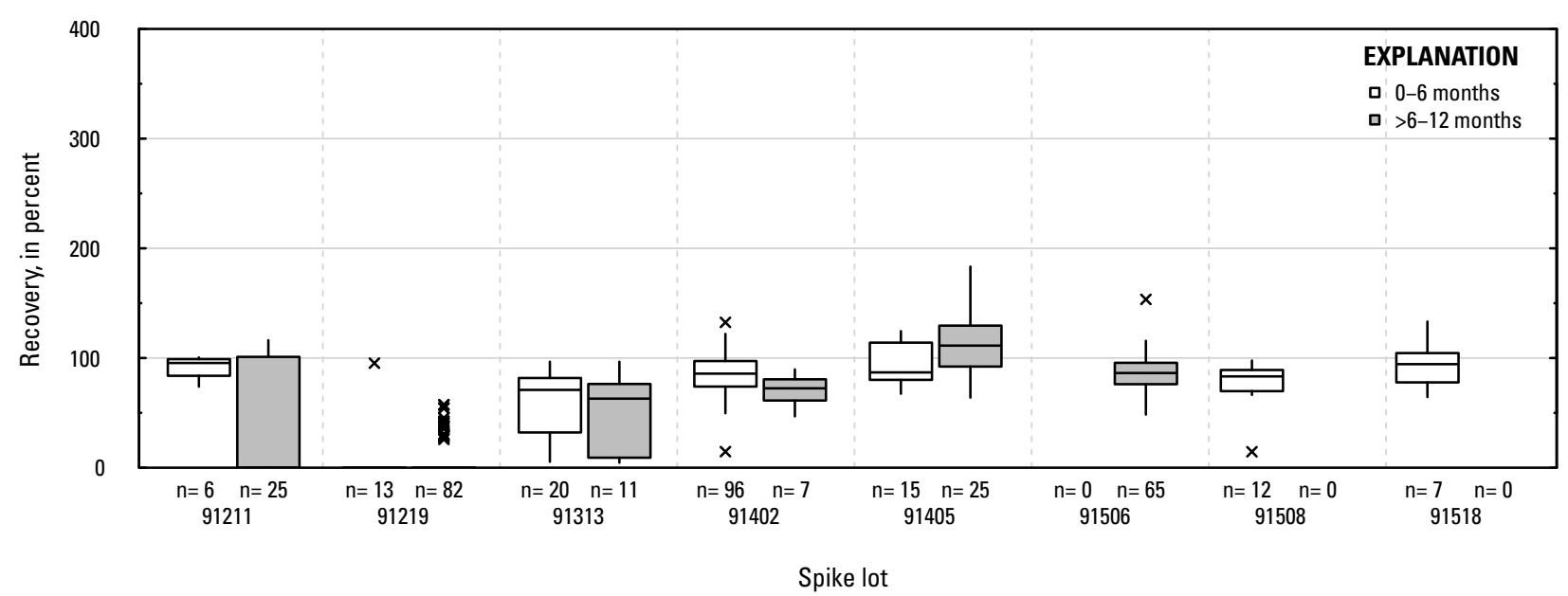

Figure 1-1. Distributions of recovery for individual pesticides in schedule 2437 by matrix, spike lot, and spike lot age. Recovery values larger than 400 percent are not shown.-Continued 


\section{DH. Azoxystrobin: laboratory reagent spikes}

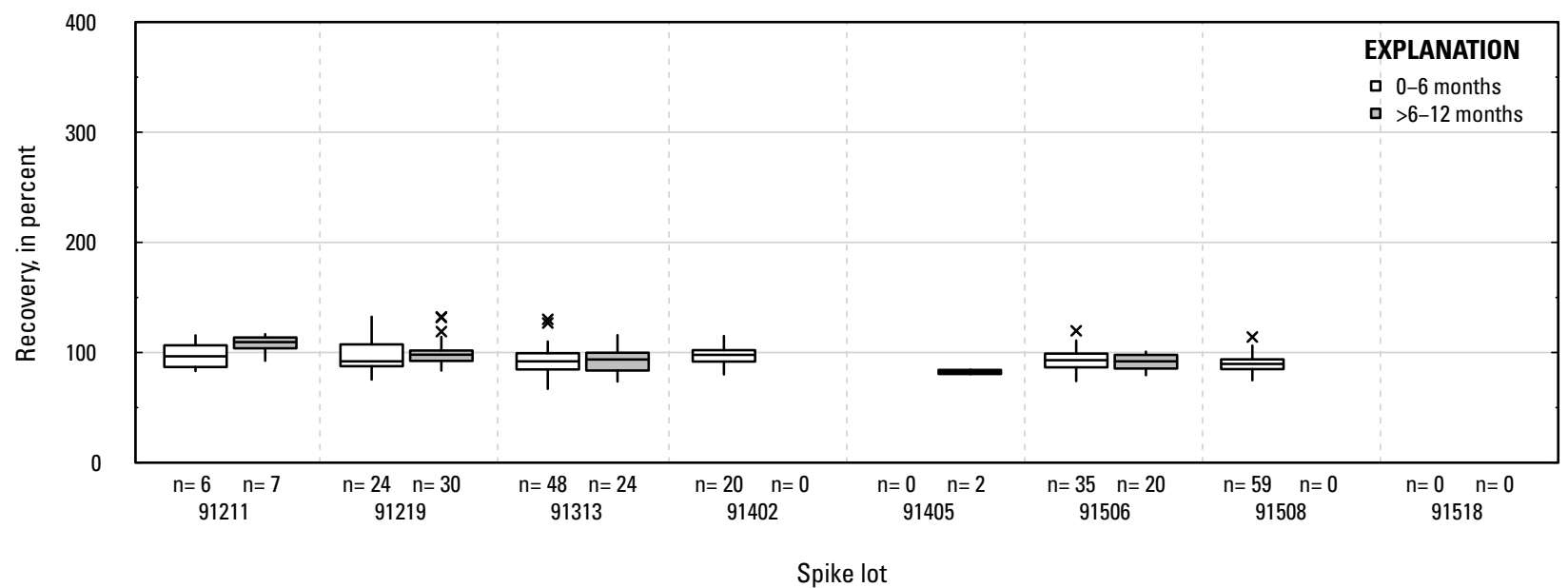

\section{Azoxystrobin: groundwater field matrix spikes}

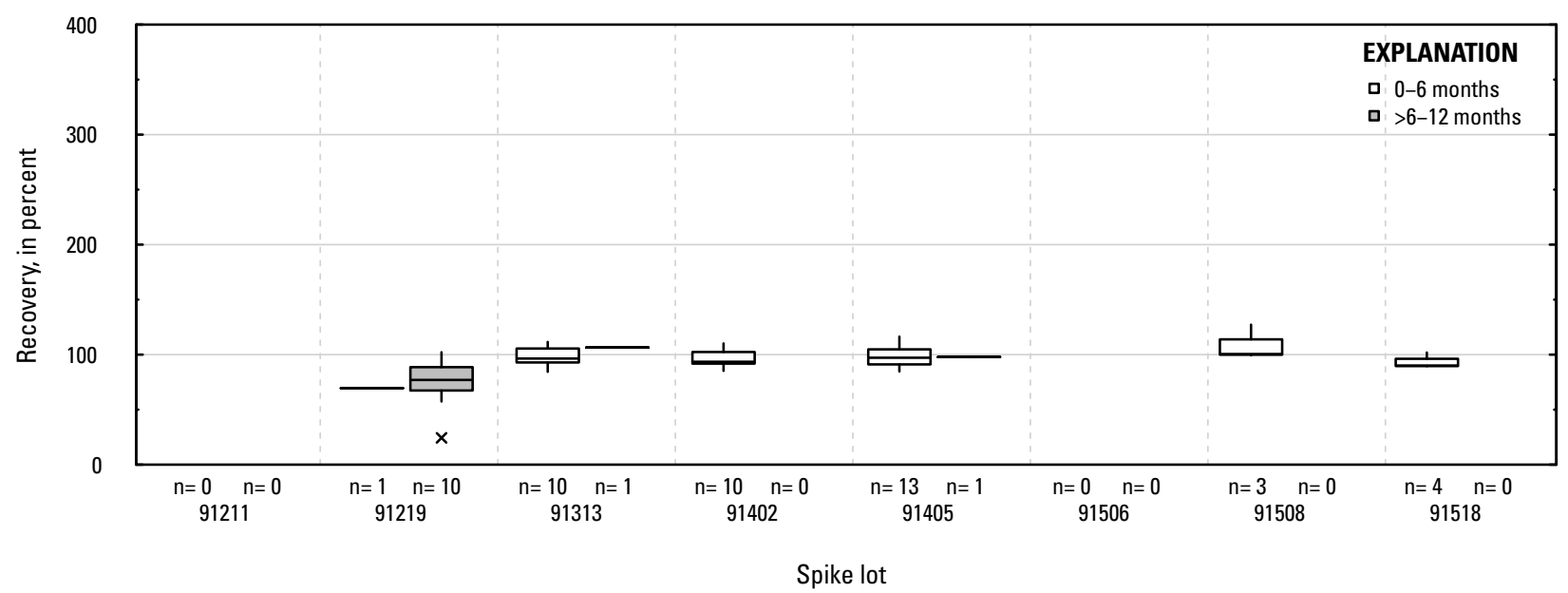

\section{DJ. Azoxystrobin: surface water field matrix spikes}

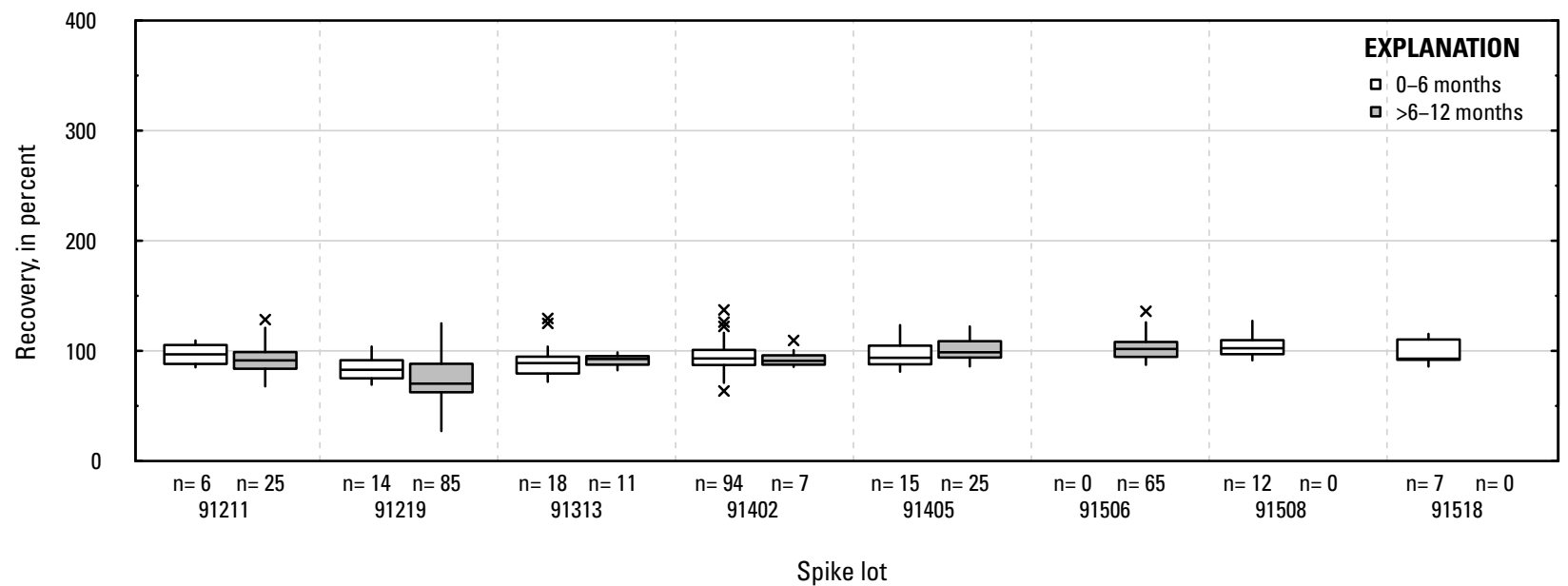

Figure 1-1. Distributions of recovery for individual pesticides in schedule 2437 by matrix, spike lot, and spike lot age. Recovery values larger than 400 percent are not shown.-Continued 
DK. Bentazone: laboratory reagent spikes

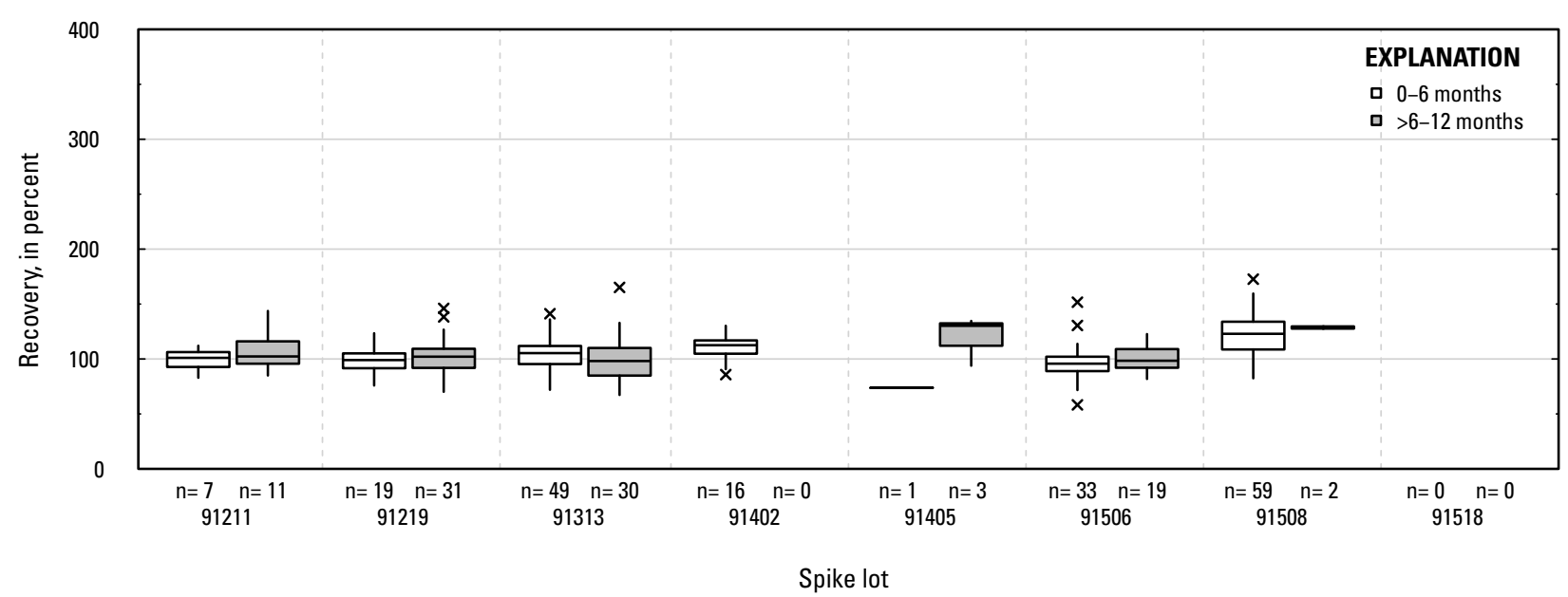

DL. Bentazone: groundwater field matrix spikes

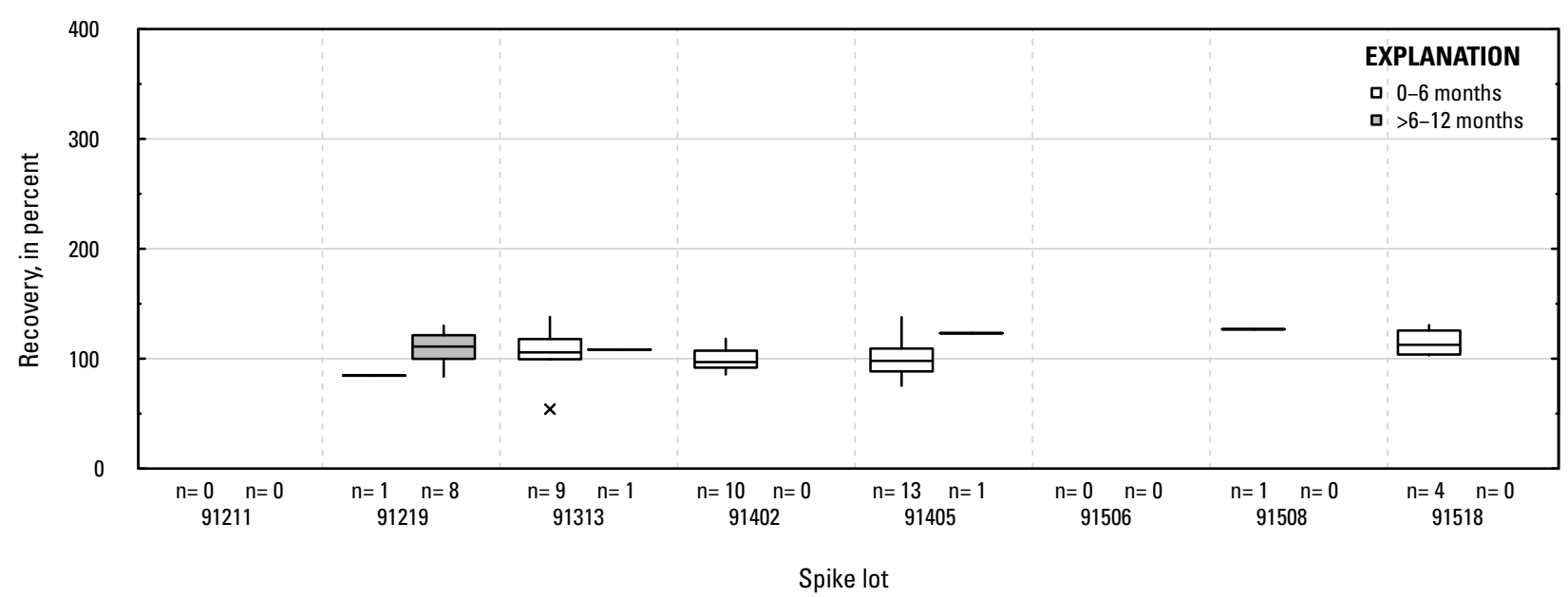

DM. Bentazone: surface water field matrix spikes

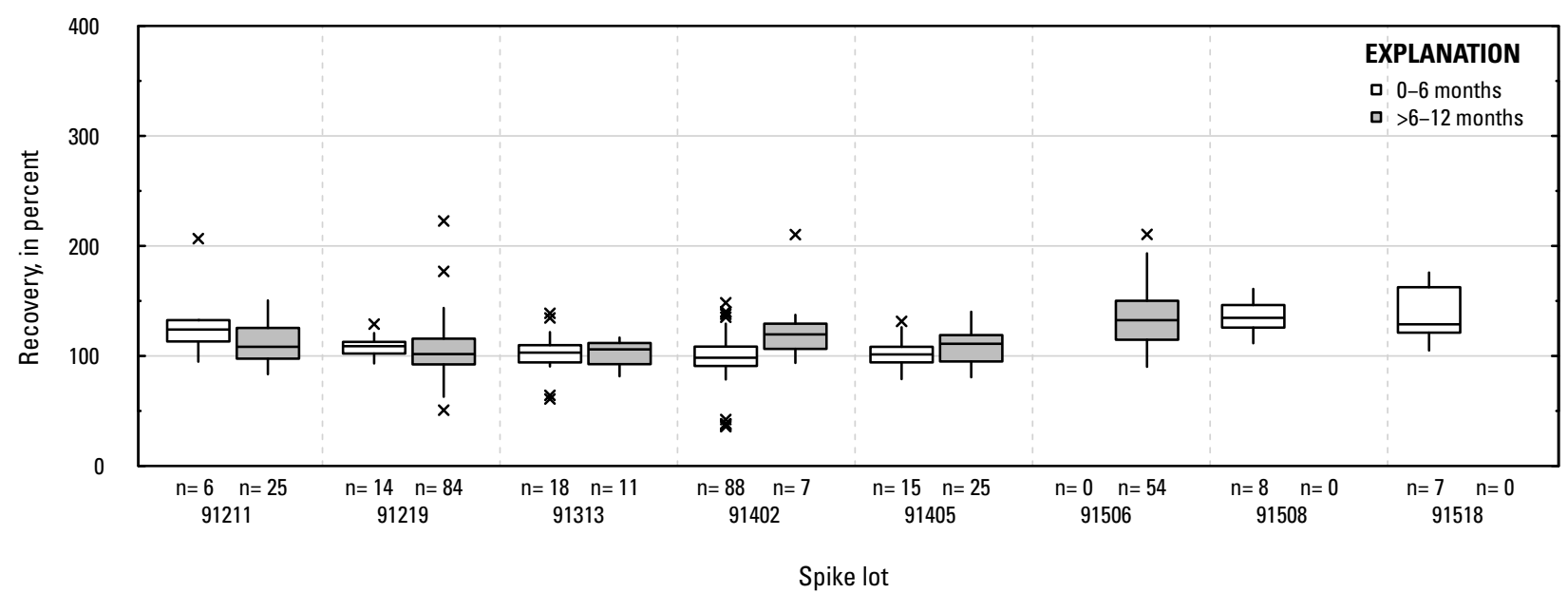

Figure 1-1. Distributions of recovery for individual pesticides in schedule 2437 by matrix, spike lot, and spike lot age. Recovery values larger than 400 percent are not shown.-Continued 
DN. Bifenthrin: laboratory reagent spikes

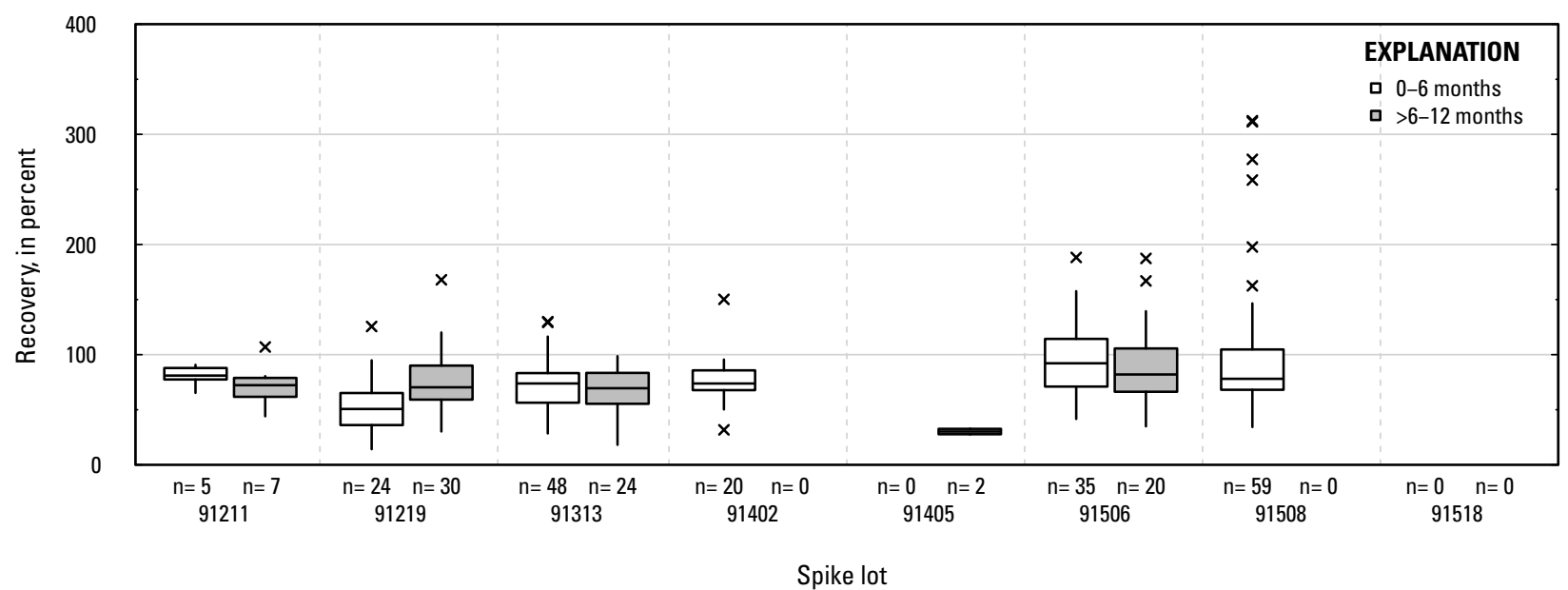

DO. Bifenthrin: groundwater field matrix spikes

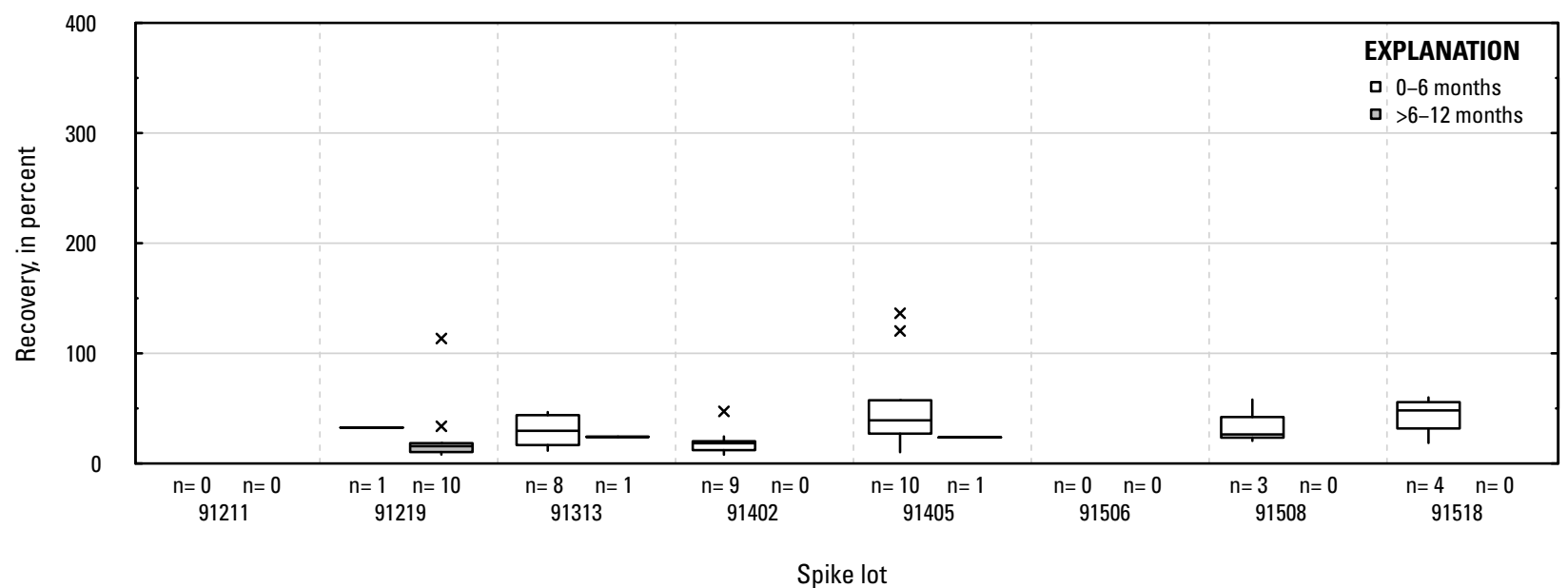

DP. Bifenthrin: surface water field matrix spikes

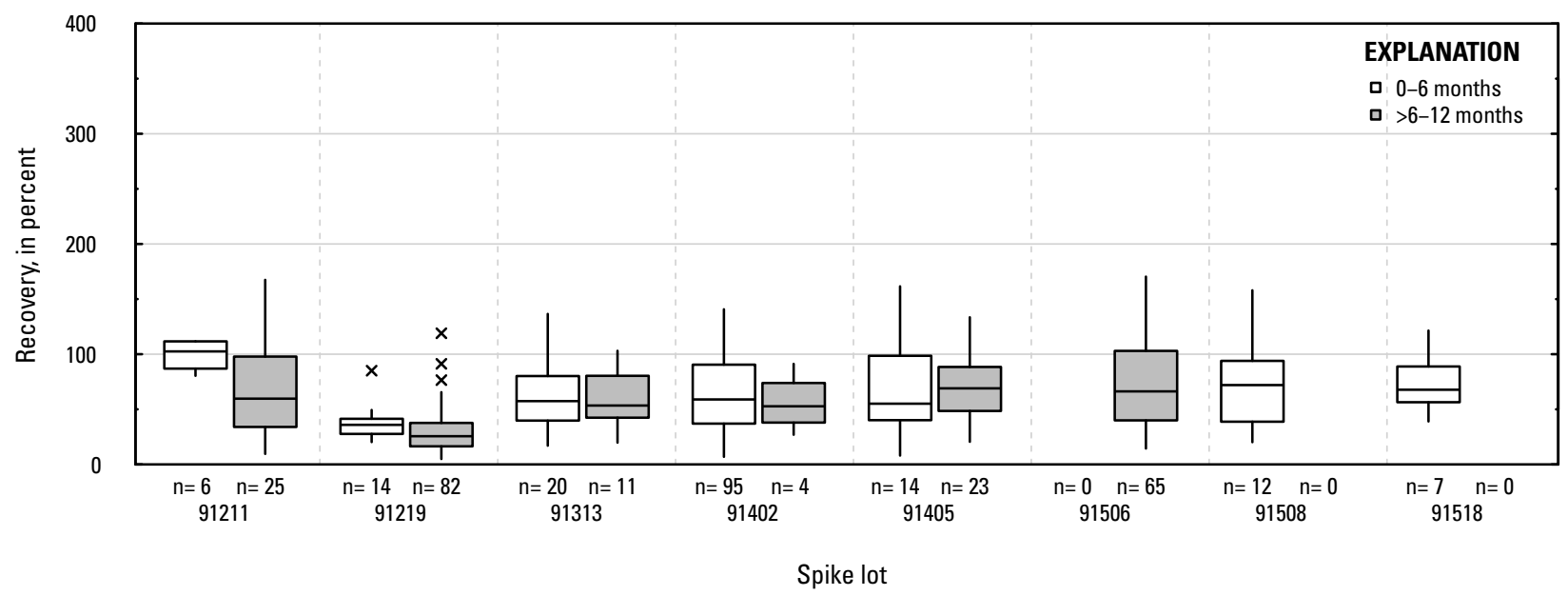

Figure 1-1. Distributions of recovery for individual pesticides in schedule 2437 by matrix, spike lot, and spike lot age. Recovery values larger than 400 percent are not shown.-Continued 


\section{DQ. Bromacil: laboratory reagent spikes}

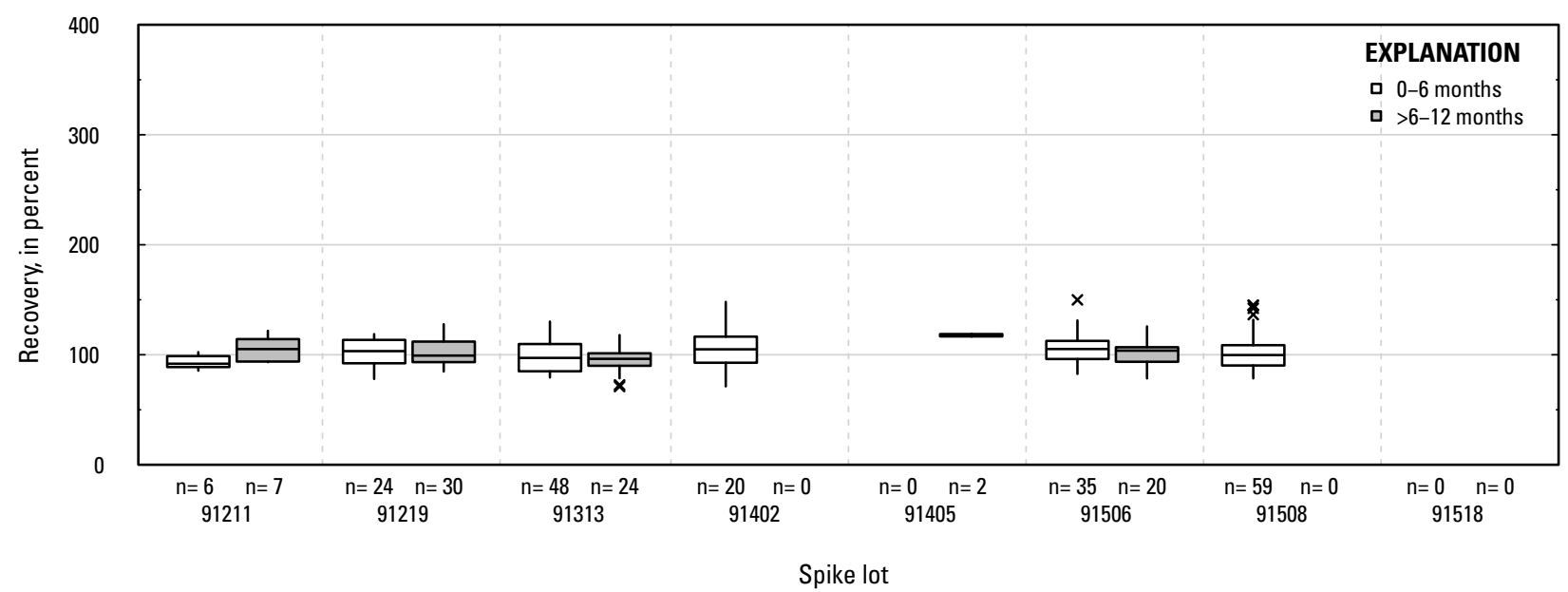

DR. Bromacil: groundwater field matrix spikes

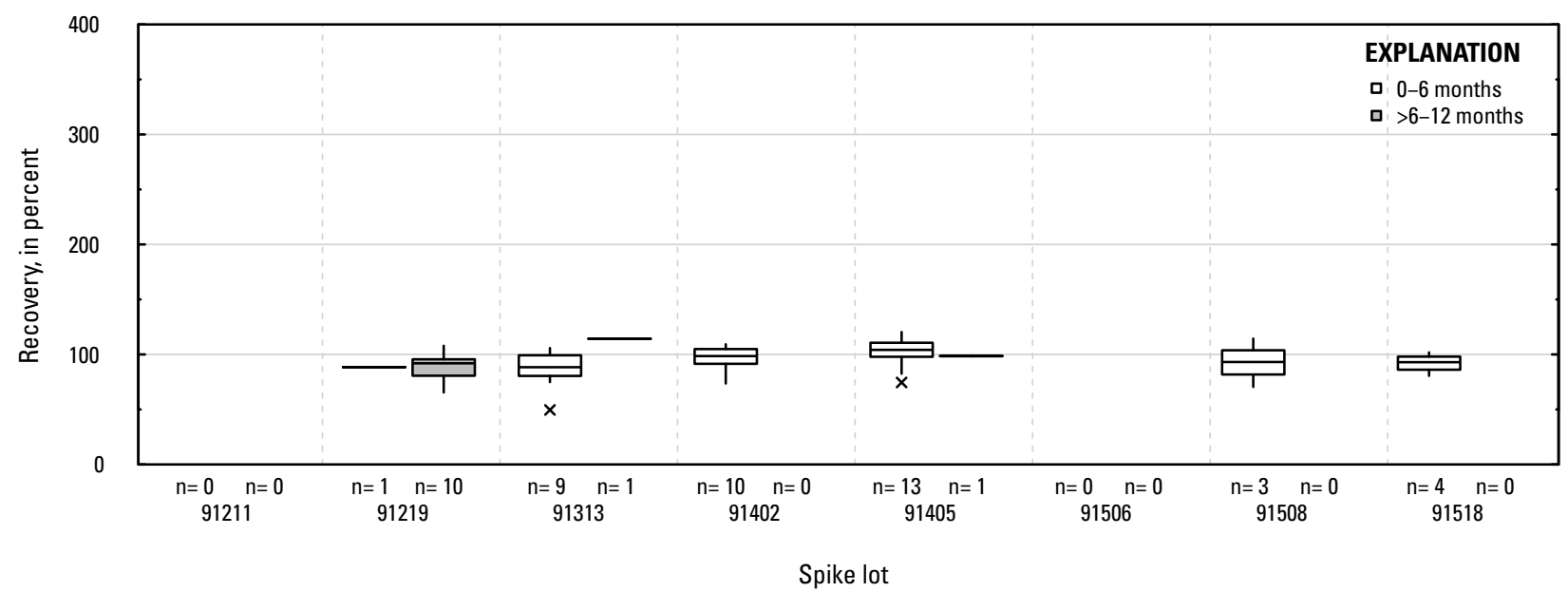

DS. Bromacil: surface water field matrix spikes

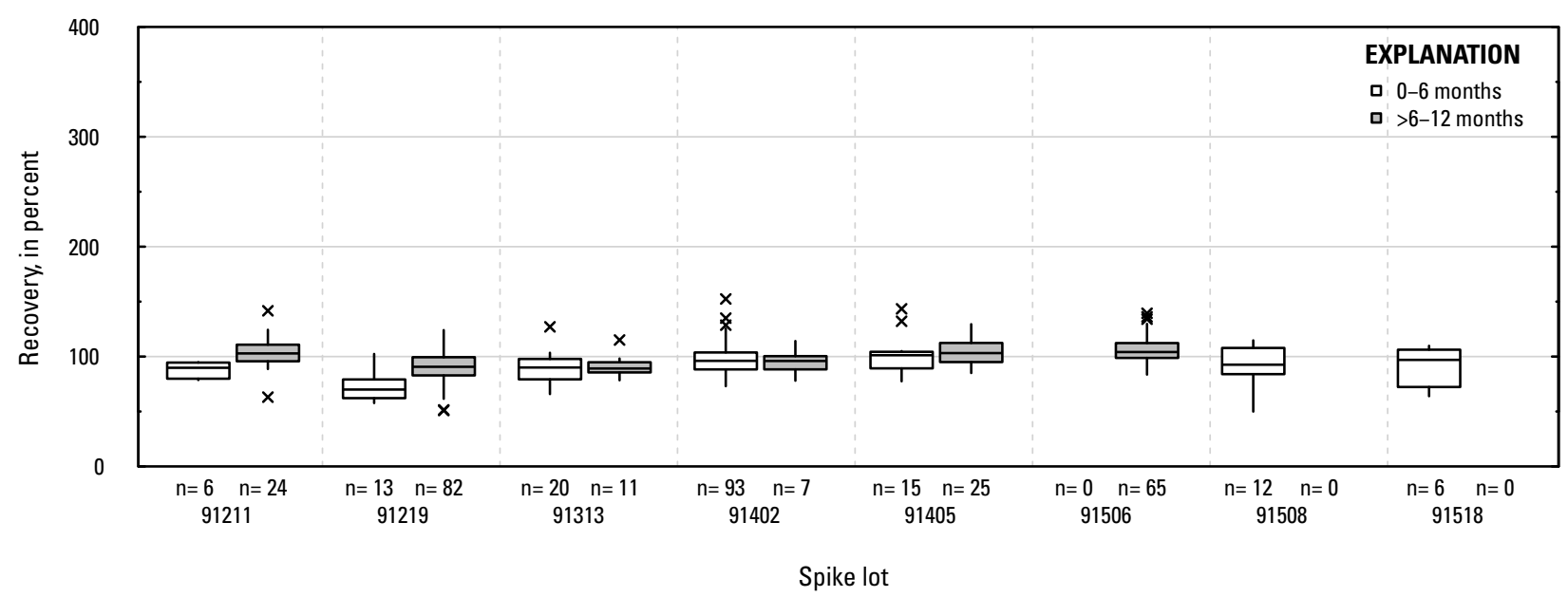

Figure 1-1. Distributions of recovery for individual pesticides in schedule 2437 by matrix, spike lot, and spike lot age. Recovery values larger than 400 percent are not shown.-Continued 
DT. Bromoxynil: laboratory reagent spikes

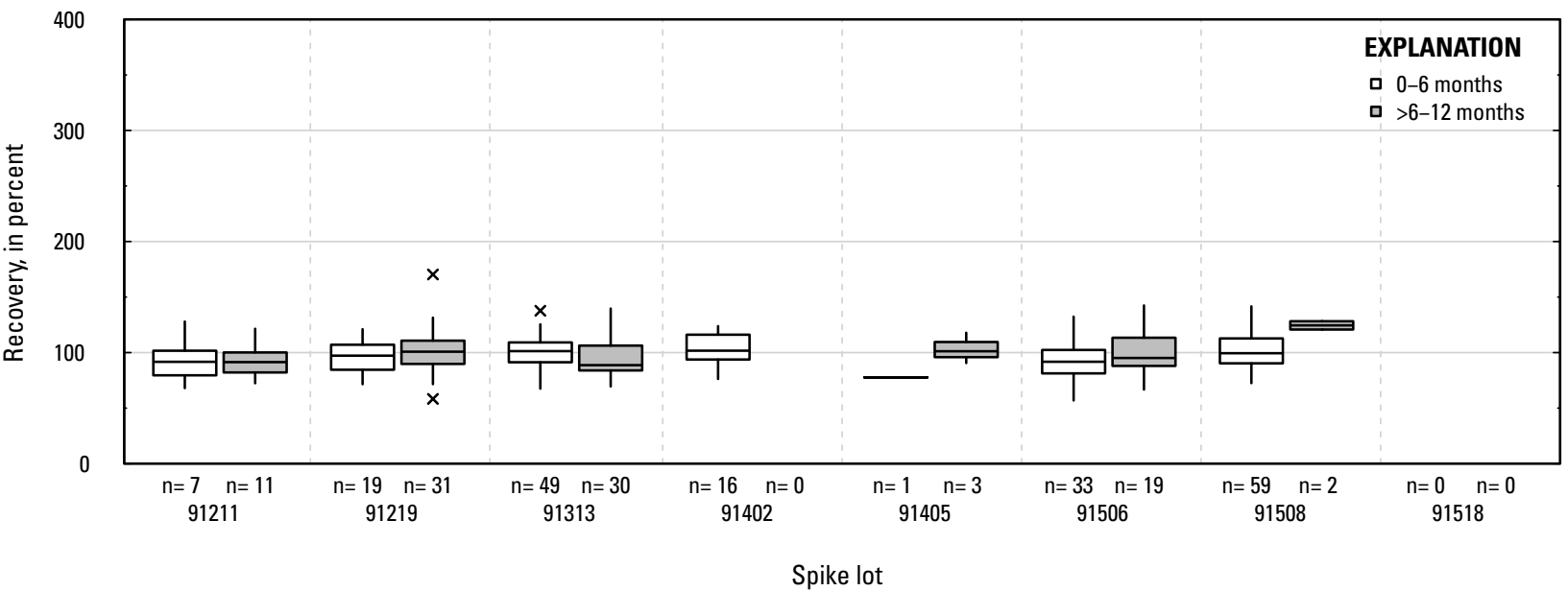

DU. Bromoxynil: groundwater field matrix spikes

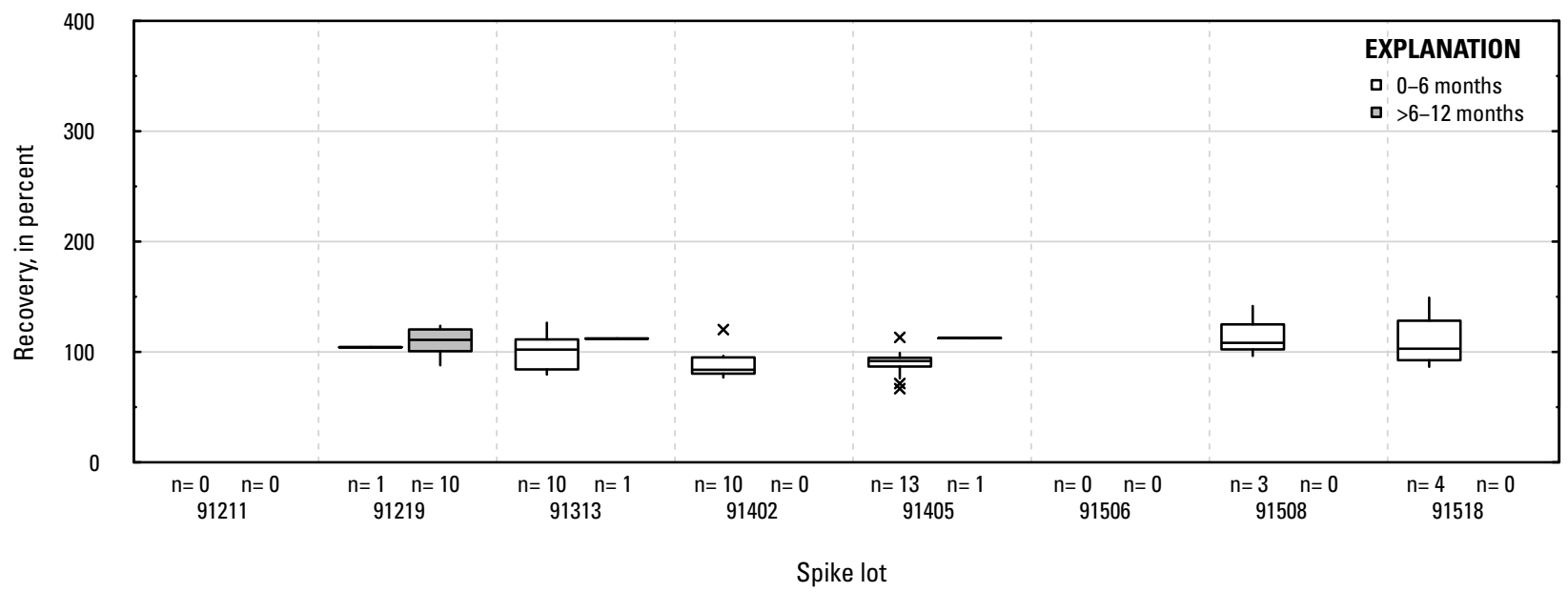

DV. Bromoxynil: surface water field matrix spikes

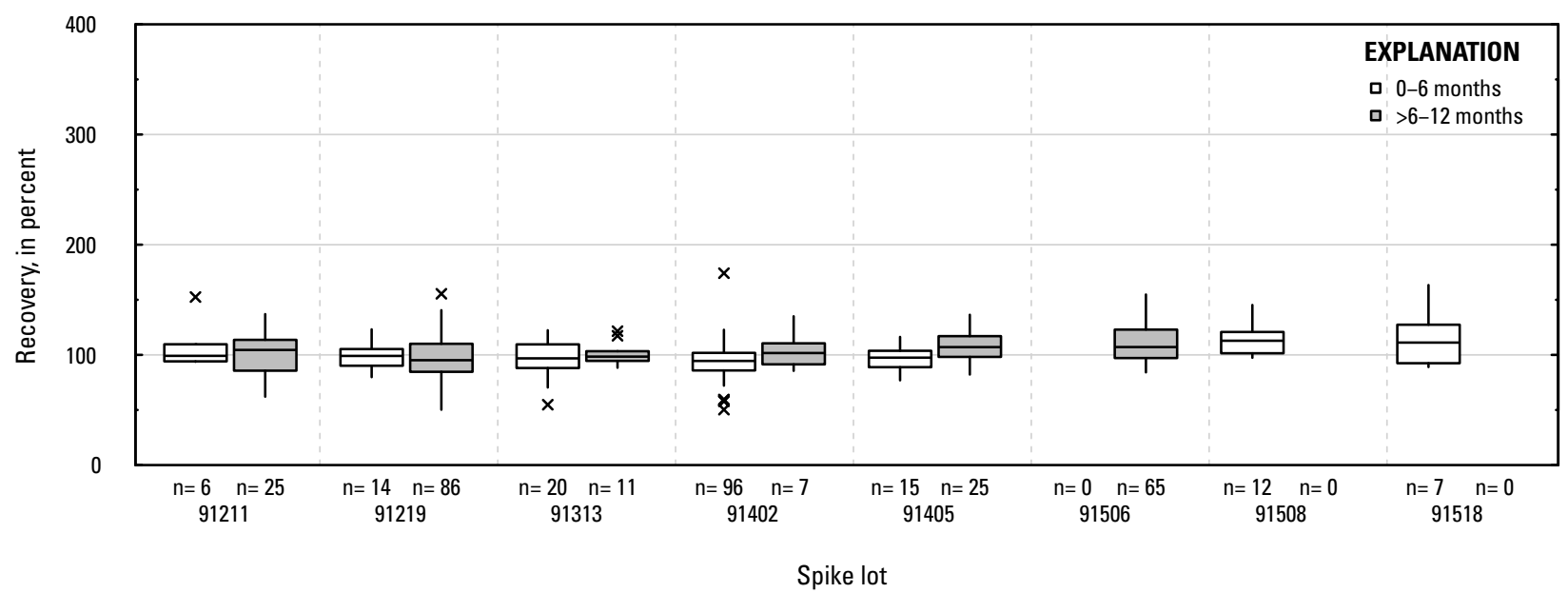

Figure 1-1. Distributions of recovery for individual pesticides in schedule 2437 by matrix, spike lot, and spike lot age. Recovery values larger than 400 percent are not shown.-Continued 
DW. Butralin: laboratory reagent spikes

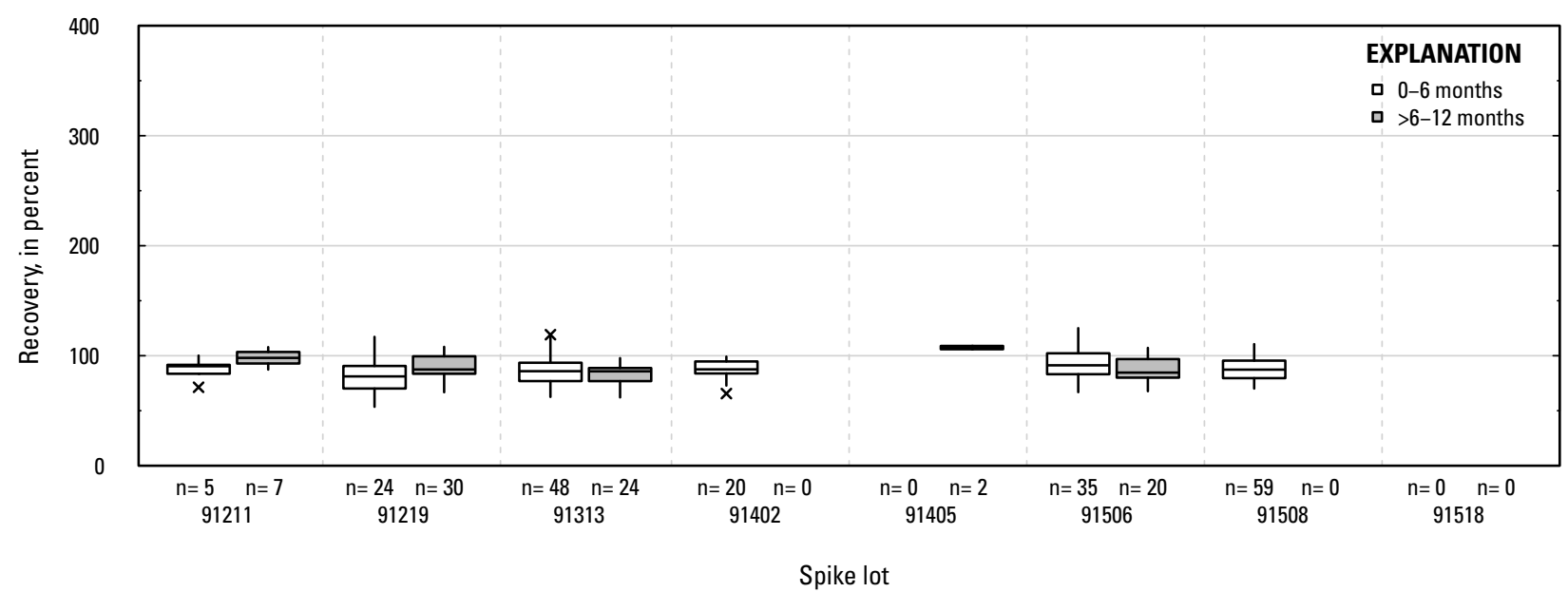

DX. Butralin: groundwater field matrix spikes

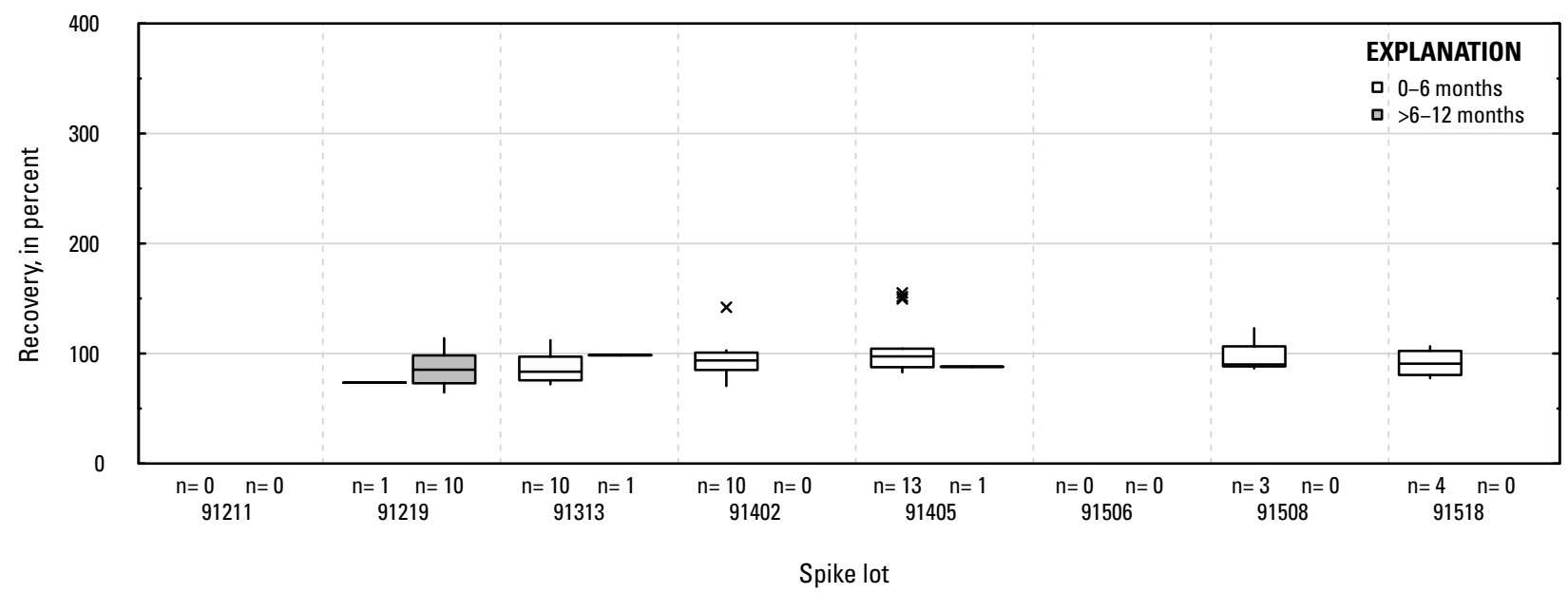

DY. Butralin: surface water field matrix spikes

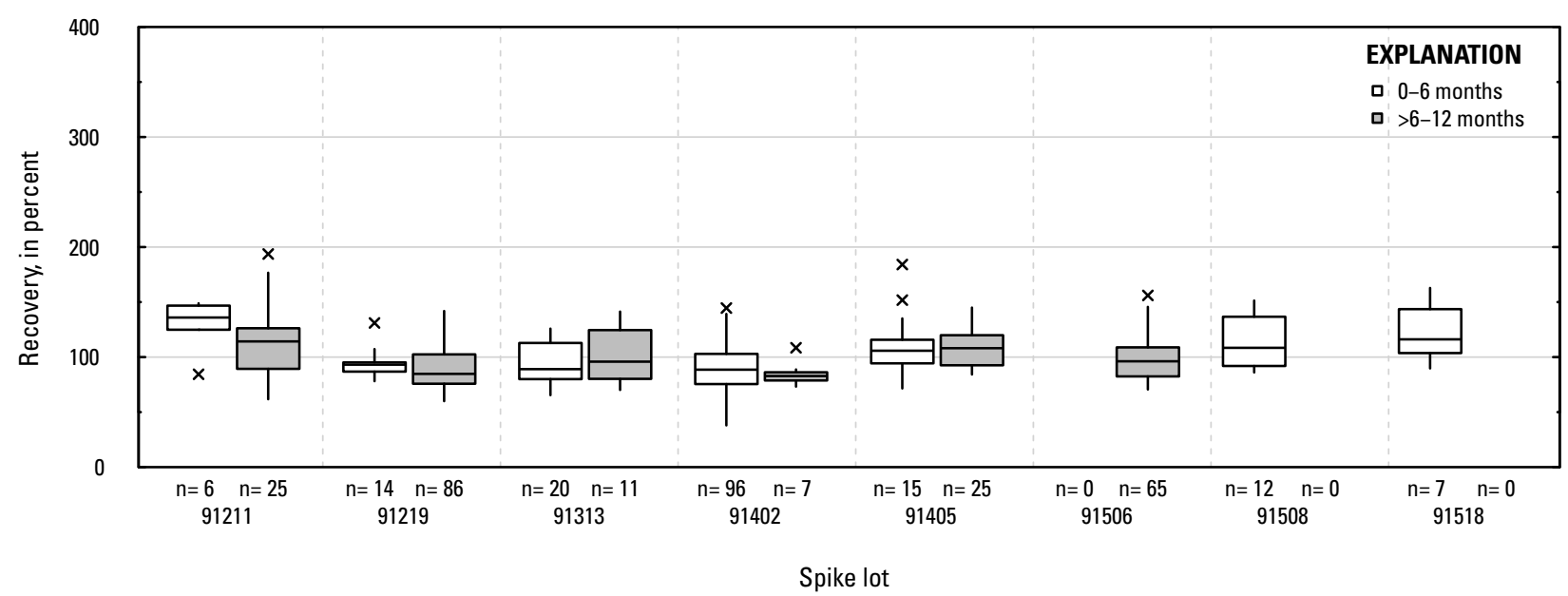

Figure 1-1. Distributions of recovery for individual pesticides in schedule 2437 by matrix, spike lot, and spike lot age. Recovery values larger than 400 percent are not shown.-Continued 


\section{DZ. Butylate: laboratory reagent spikes}

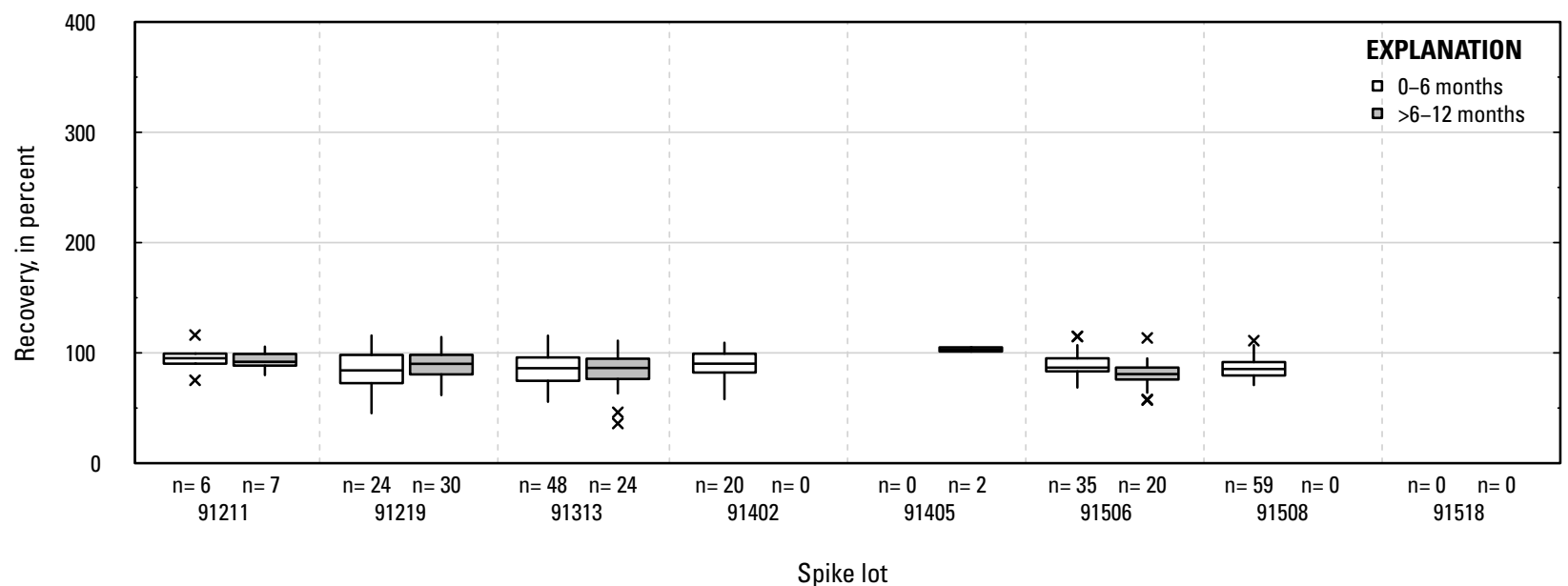

EA. Butylate: groundwater field matrix spikes

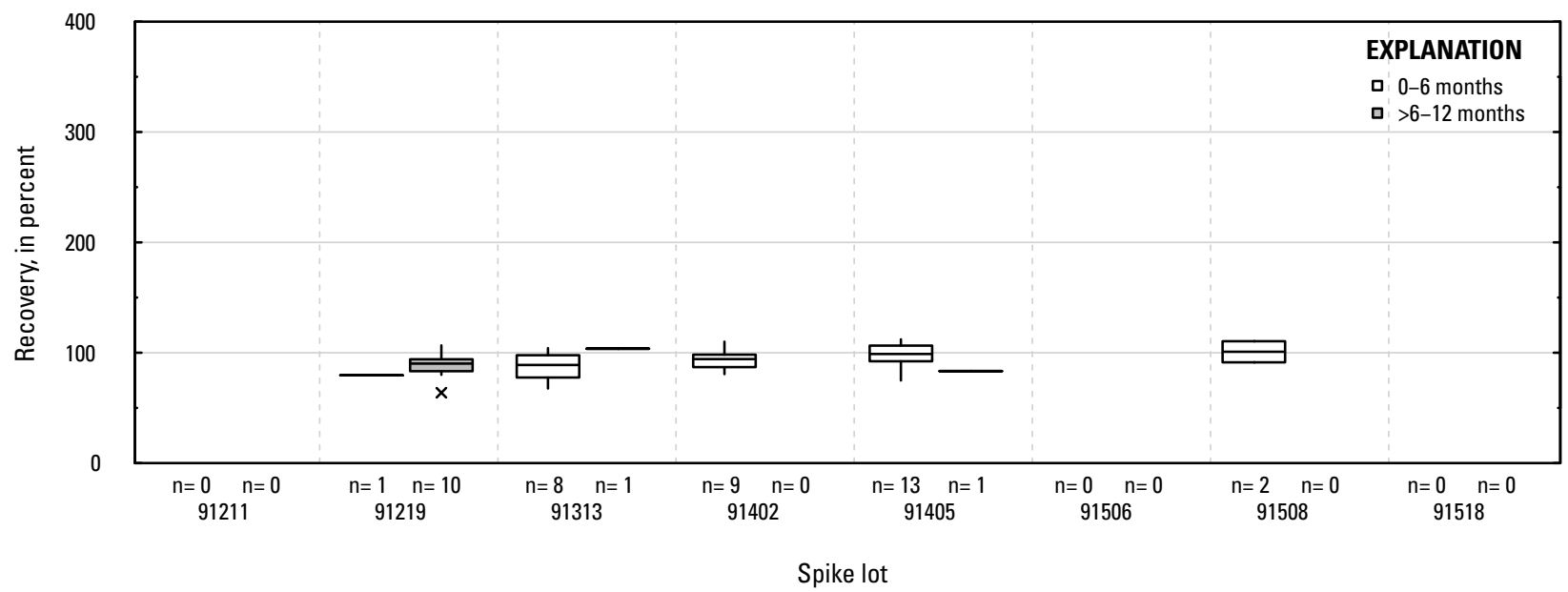

EB. Butylate: surface water field matrix spikes

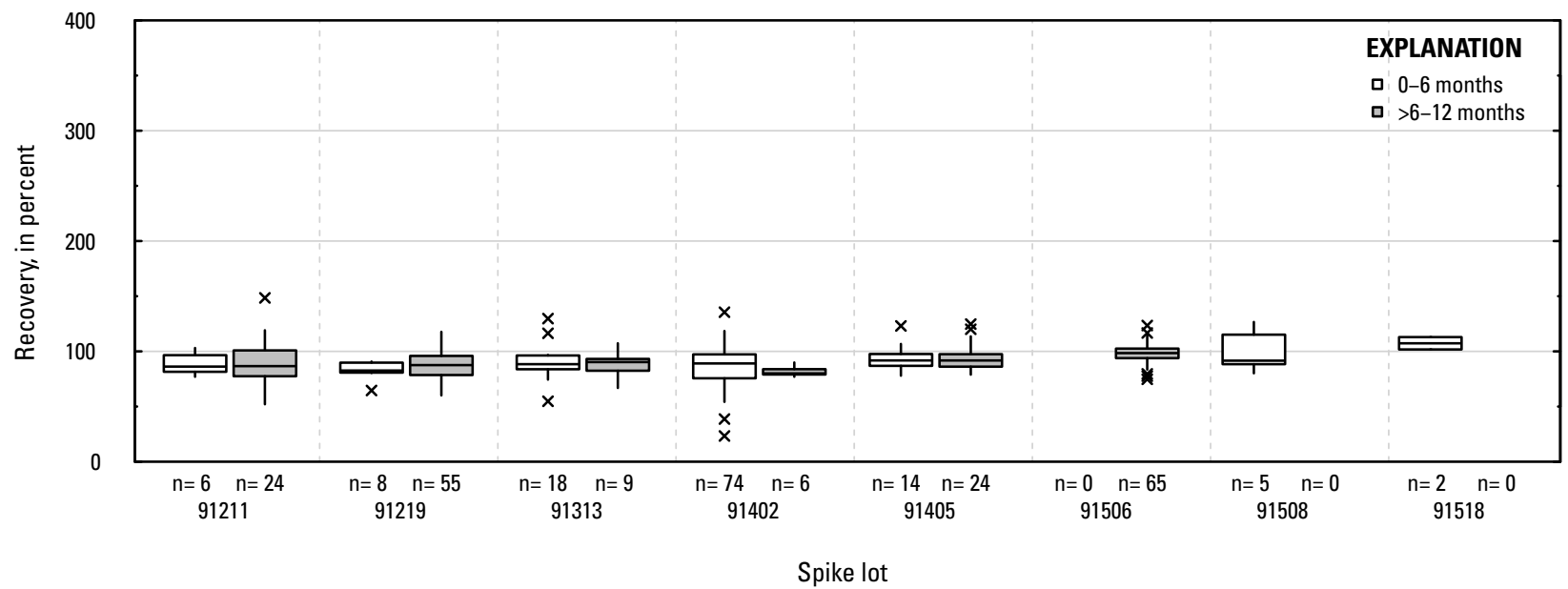

Figure 1-1. Distributions of recovery for individual pesticides in schedule 2437 by matrix, spike lot, and spike lot age. Recovery values larger than 400 percent are not shown.-Continued 


\section{EC. Carbaryl: laboratory reagent spikes}

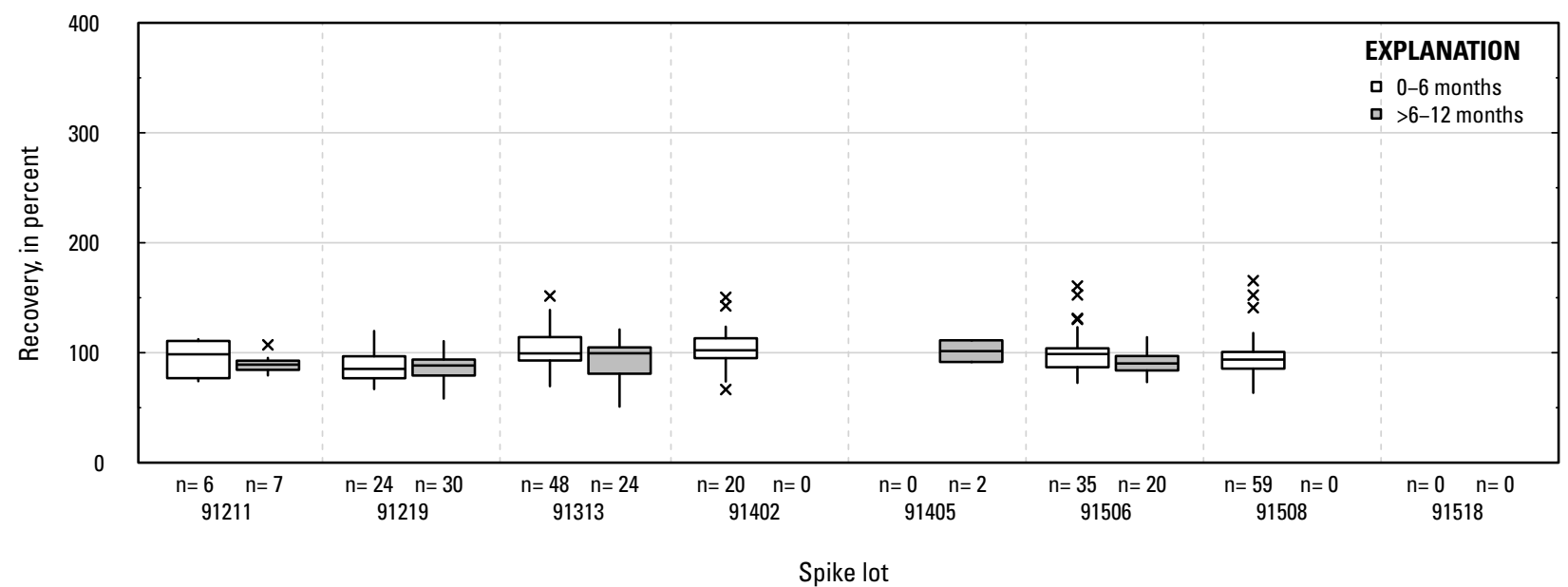

ED. Carbaryl: groundwater field matrix spikes

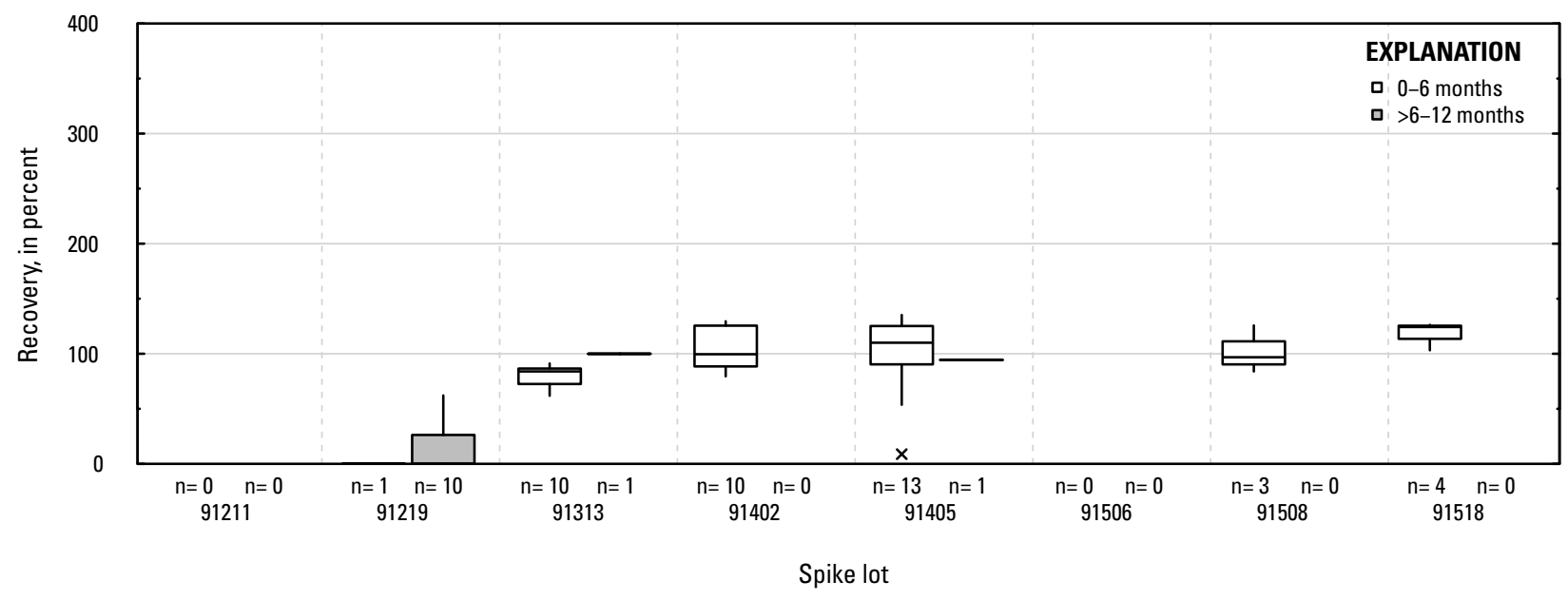

EE. Carbaryl: surface water field matrix spikes

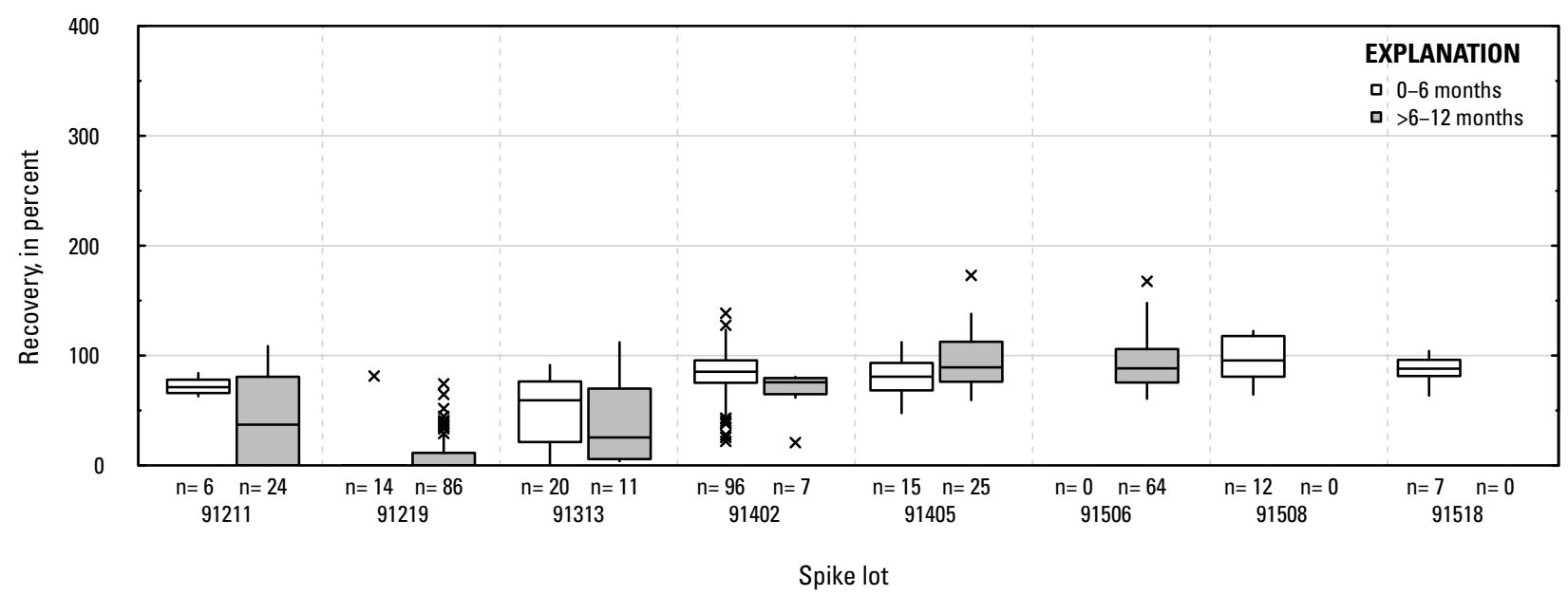

Figure 1-1. Distributions of recovery for individual pesticides in schedule 2437 by matrix, spike lot, and spike lot age. Recovery values larger than 400 percent are not shown.-Continued 


\section{EF. Carbendazim: laboratory reagent spikes}

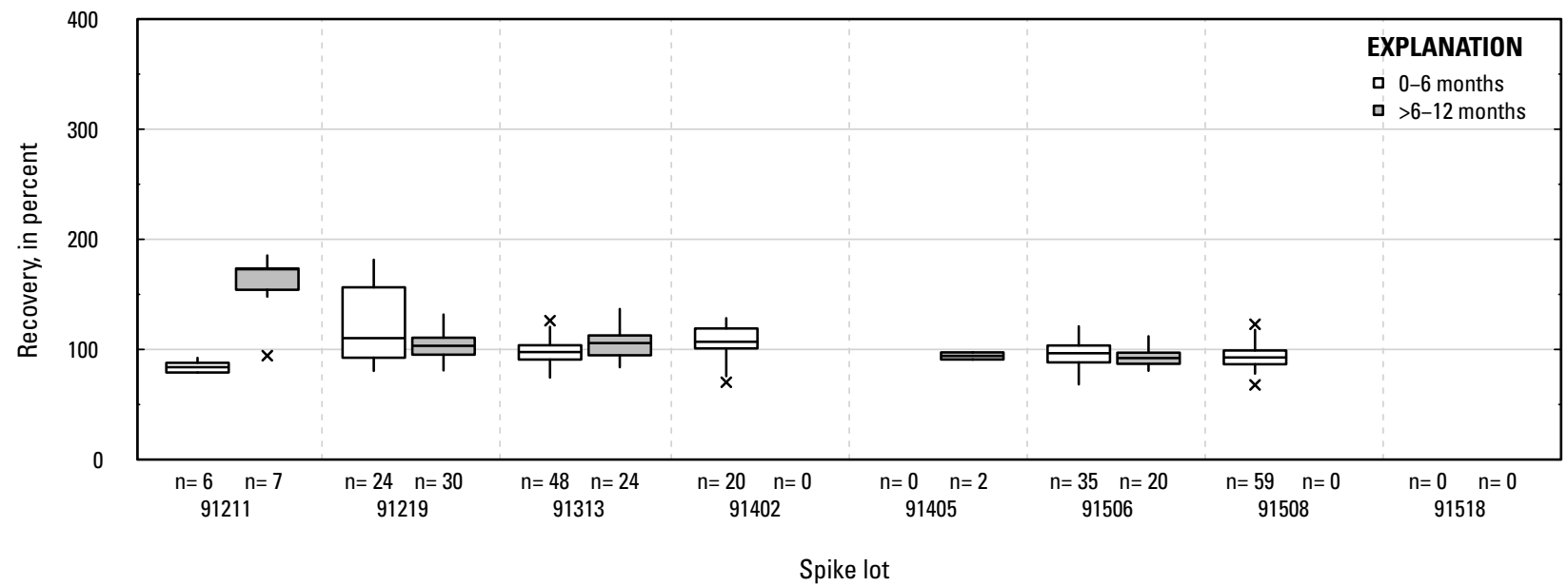

EG. Carbendazim: groundwater field matrix spikes

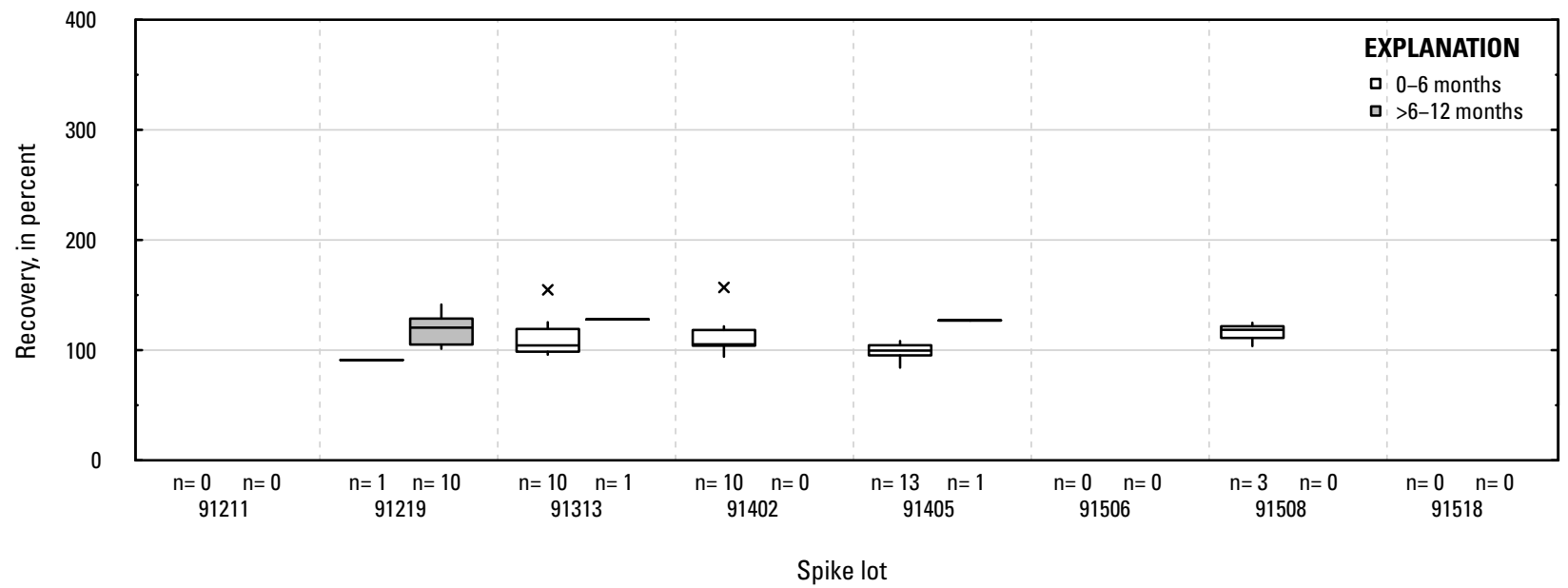

EH. Carbendazim: surface water field matrix spikes

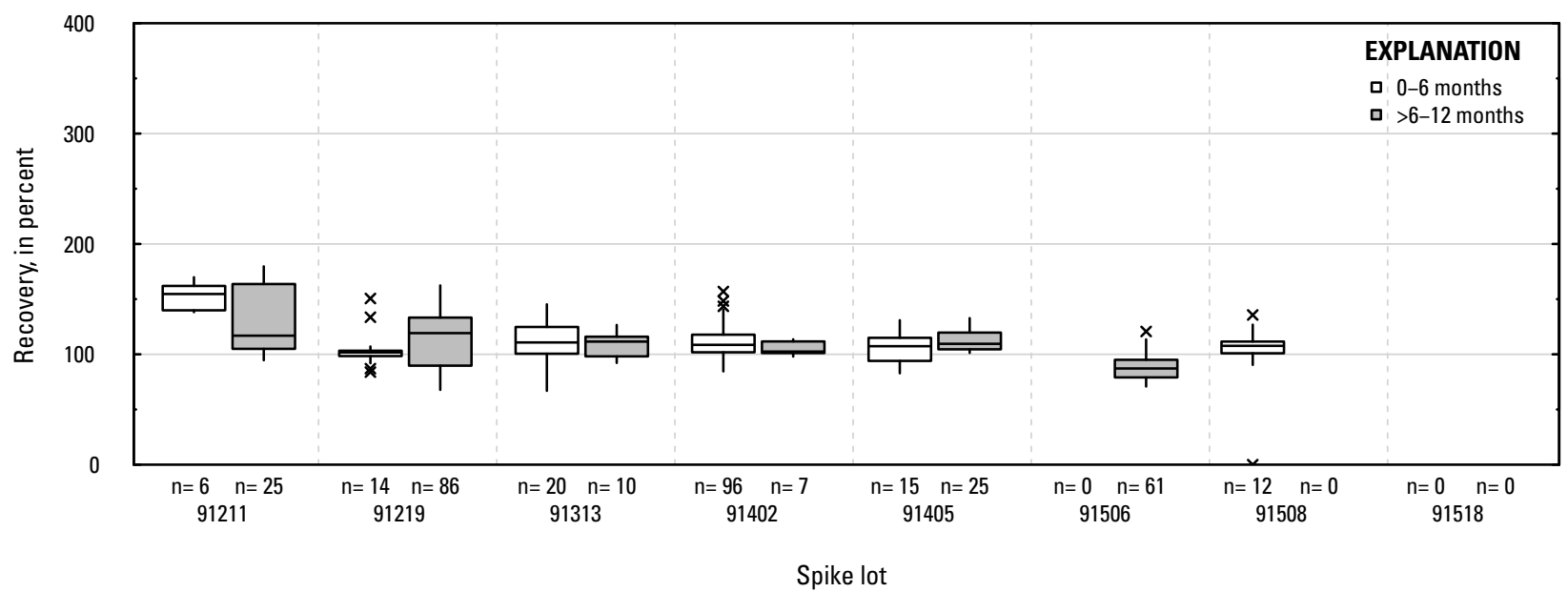

Figure 1-1. Distributions of recovery for individual pesticides in schedule 2437 by matrix, spike lot, and spike lot age. Recovery values larger than 400 percent are not shown.-Continued 


\section{El. Carbofuran: laboratory reagent spikes}

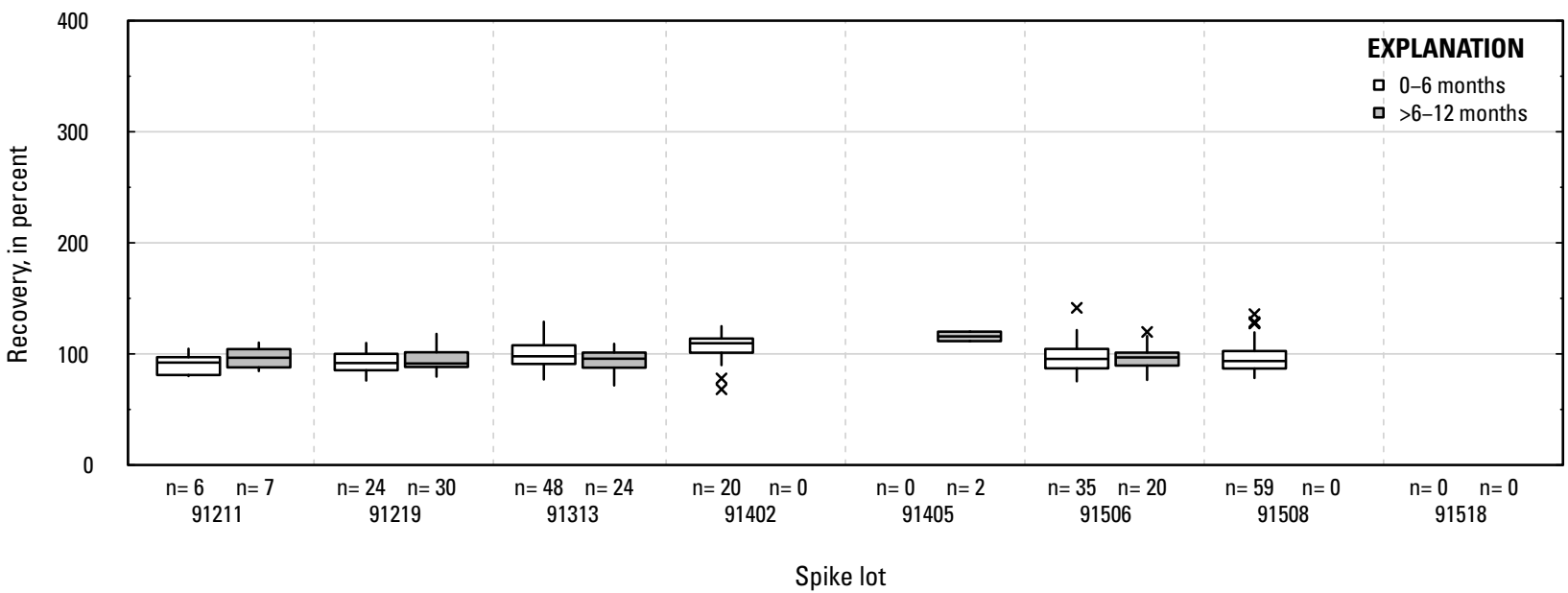

\section{EJ. Carbofuran: groundwater field matrix spikes}

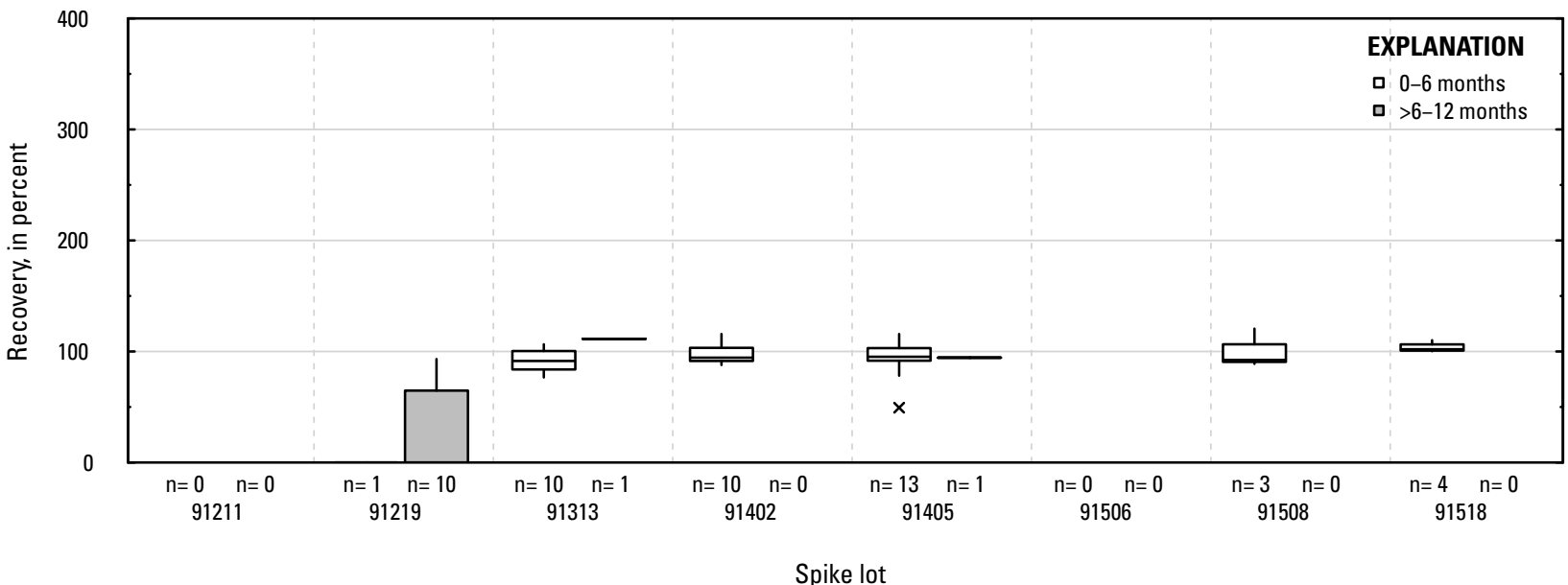

\section{EK. Carbofuran: surface water field matrix spikes}

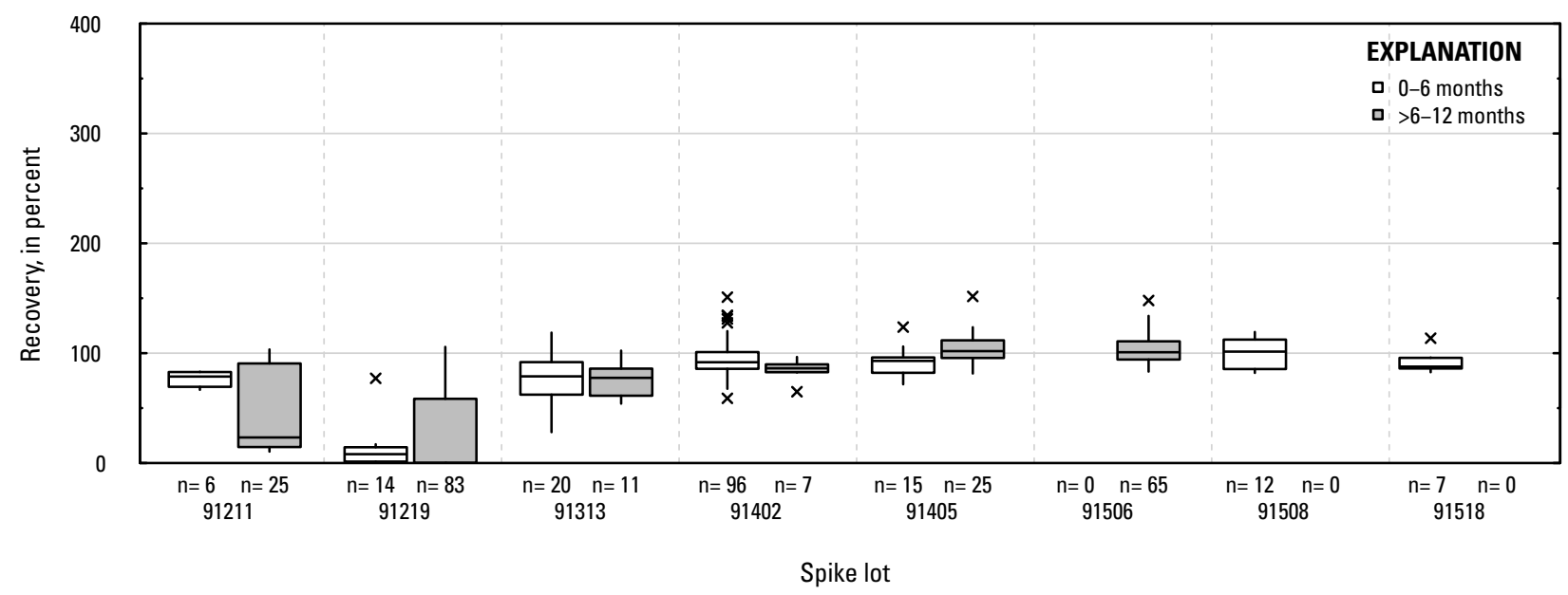

Figure 1-1. Distributions of recovery for individual pesticides in schedule 2437 by matrix, spike lot, and spike lot age. Recovery values larger than 400 percent are not shown.-Continued 
EL. Carboxy molinate: laboratory reagent spikes

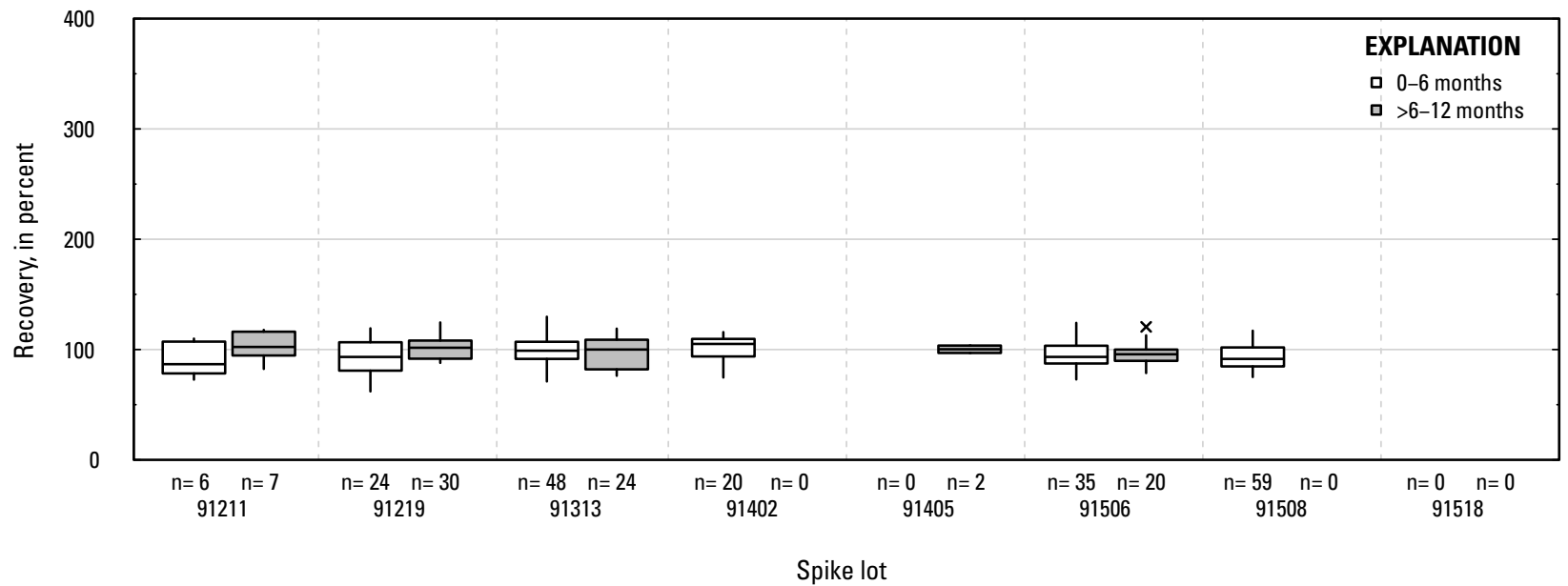

EM. Carboxy molinate: groundwater field matrix spikes

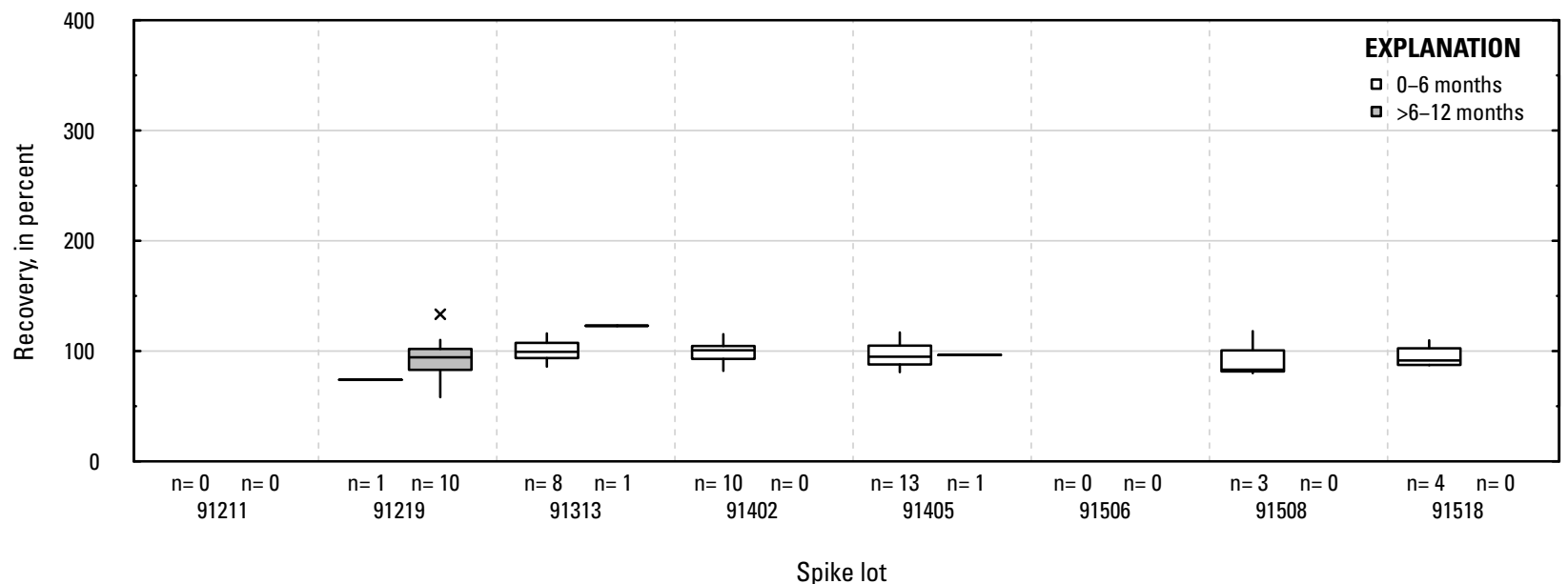

EN. Carboxy molinate: surface water field matrix spikes

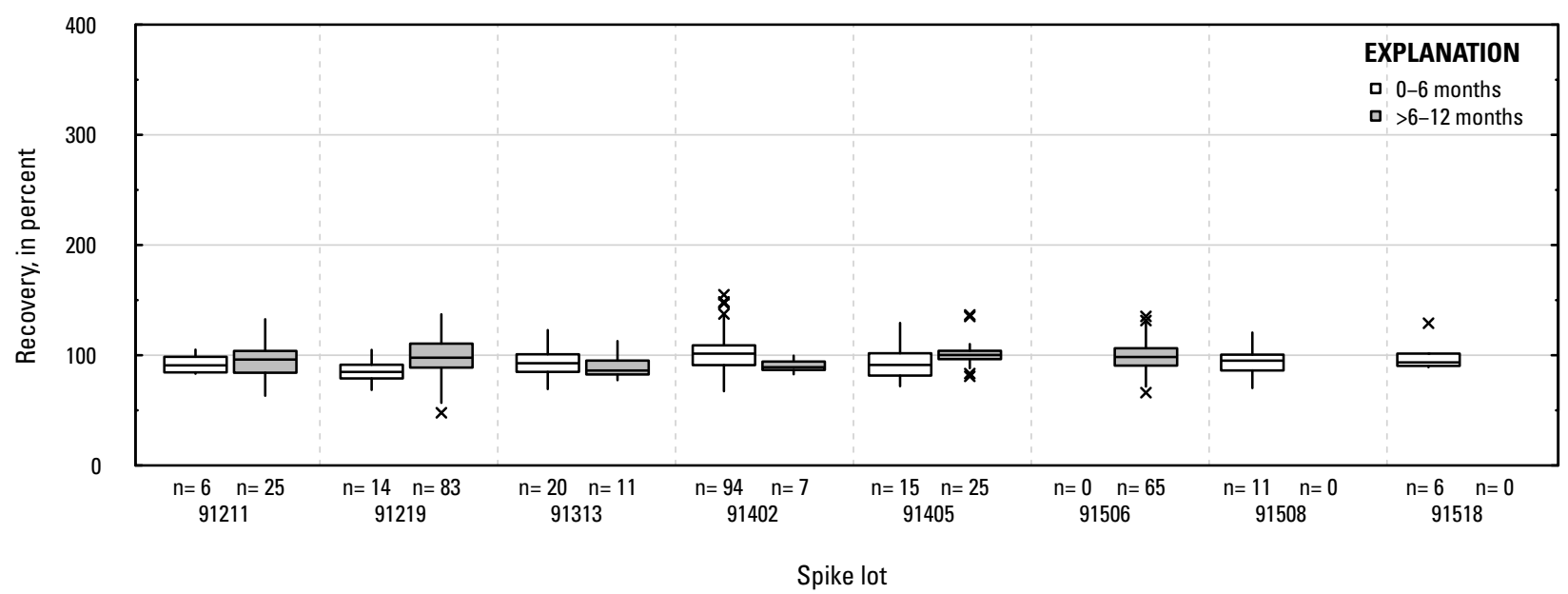

Figure 1-1. Distributions of recovery for individual pesticides in schedule 2437 by matrix, spike lot, and spike lot age. Recovery values larger than 400 percent are not shown.-Continued 
EO. Chlorimuron-ethyl: laboratory reagent spikes

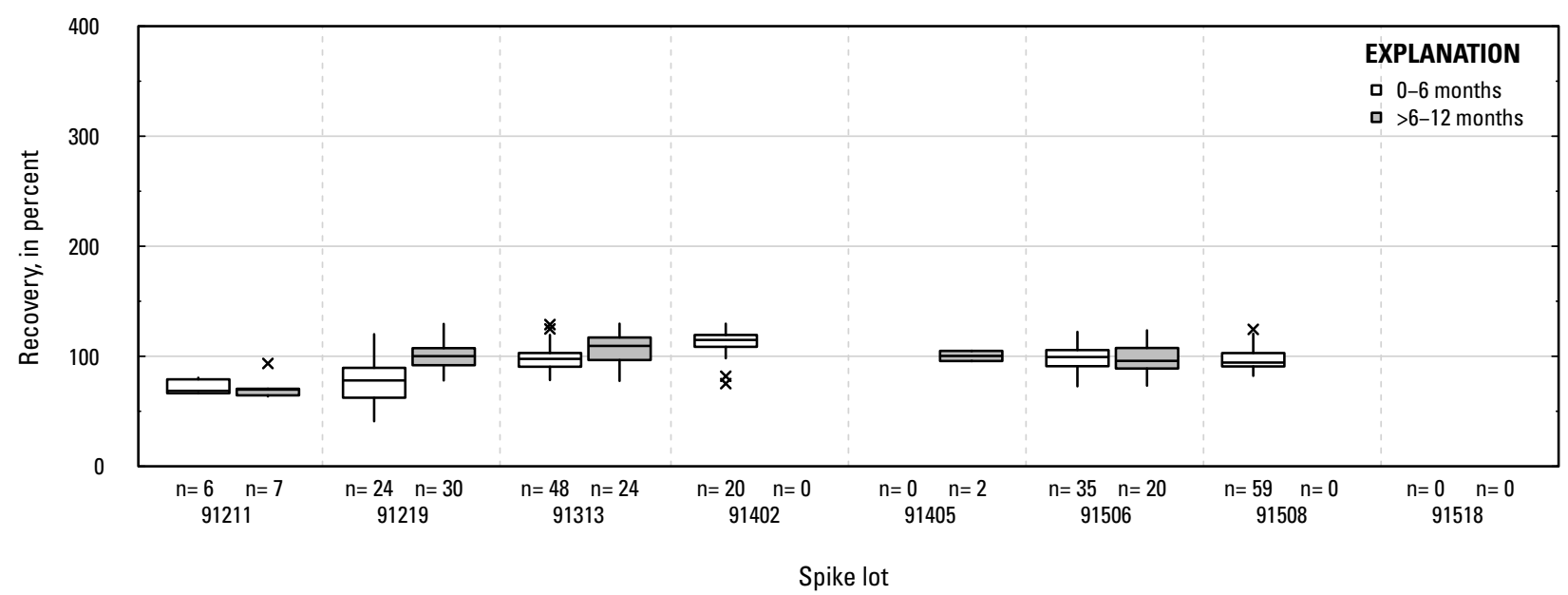

EP. Chlorimuron-ethyl: groundwater field matrix spikes

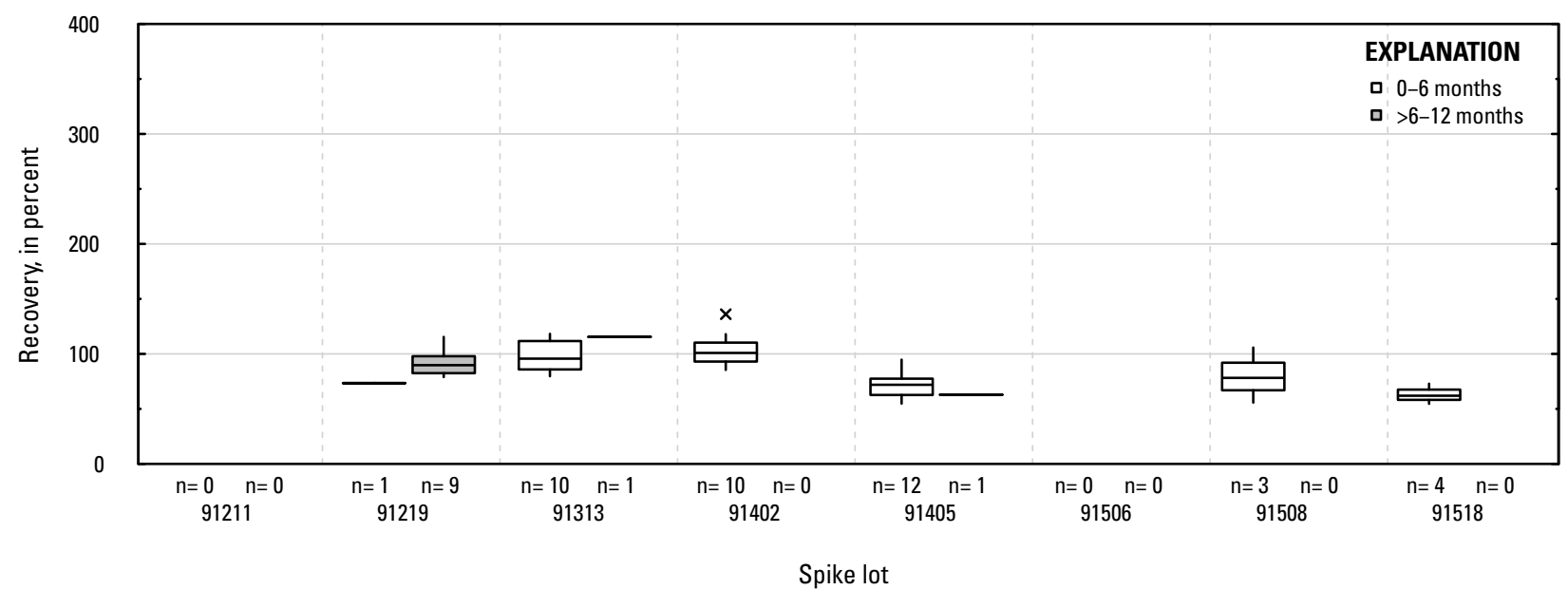

EQ. Chlorimuron-ethyl: surface water field matrix spikes

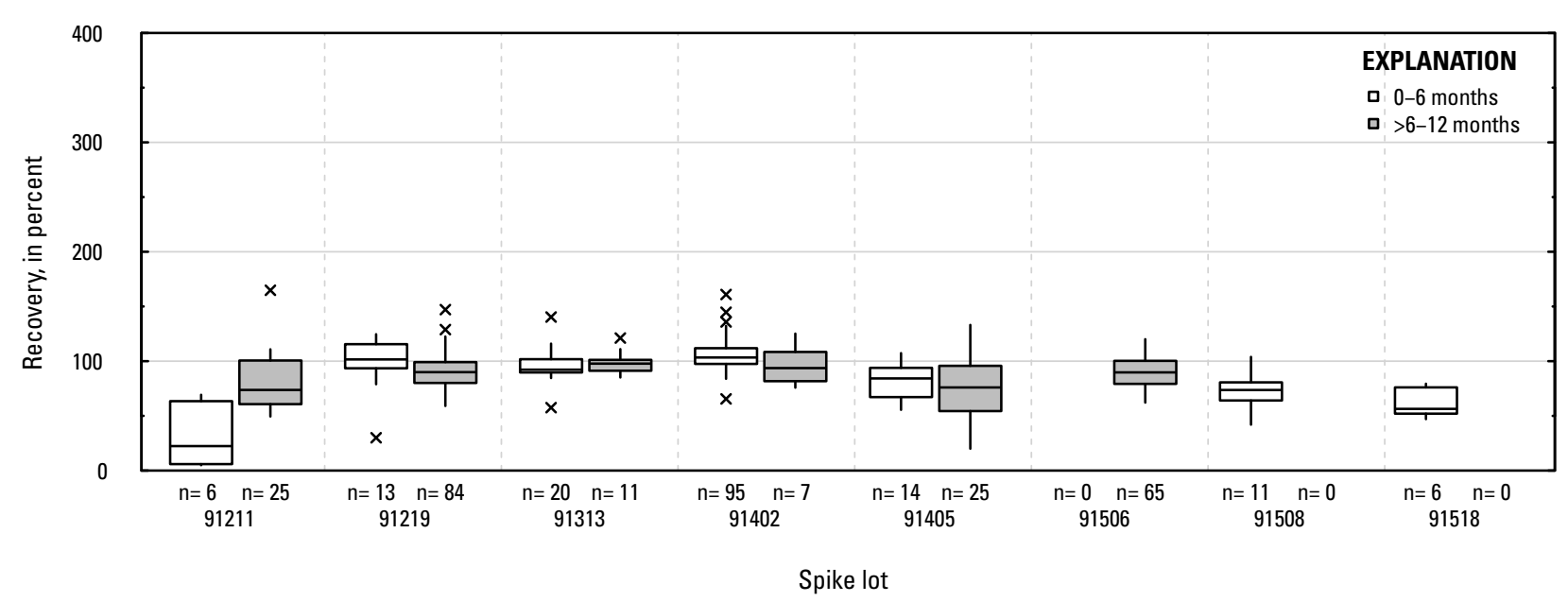

Figure 1-1. Distributions of recovery for individual pesticides in schedule 2437 by matrix, spike lot, and spike lot age. Recovery values larger than 400 percent are not shown.-Continued 
ER. Chlorosulfonamide acid: laboratory reagent spikes

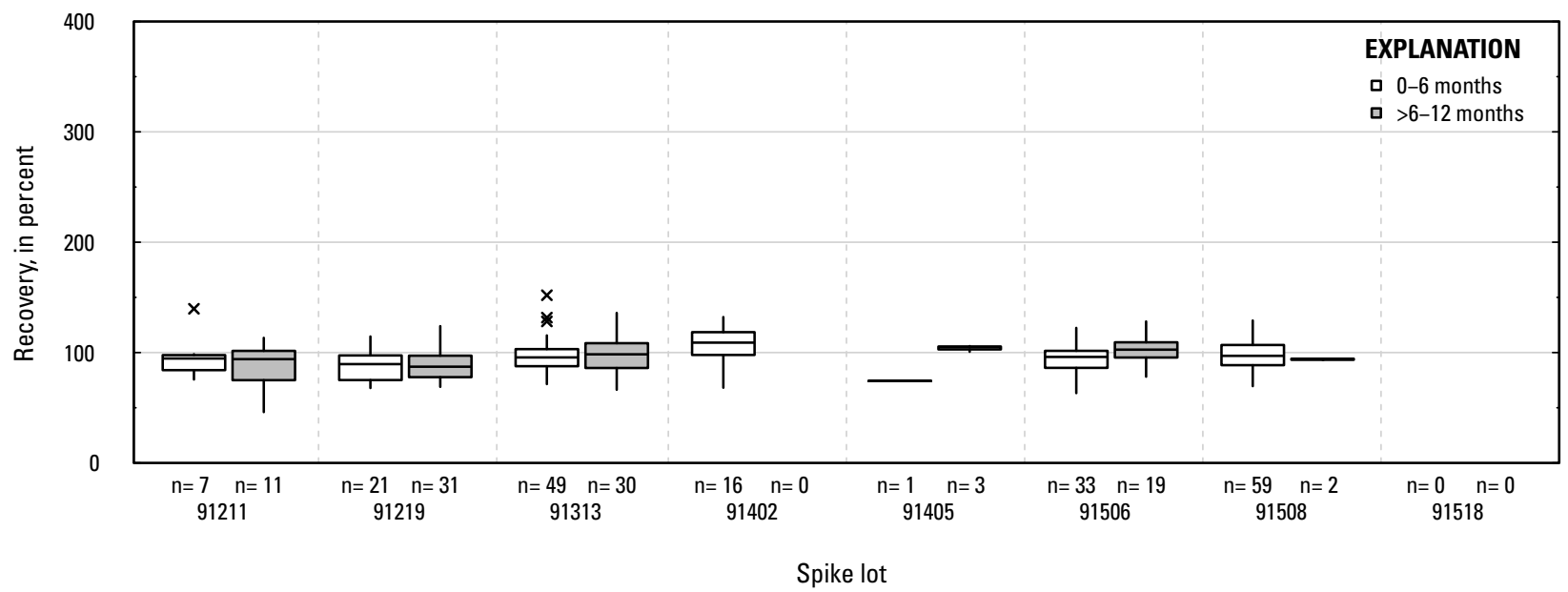

ES. Chlorosulfonamide acid: groundwater field matrix spikes

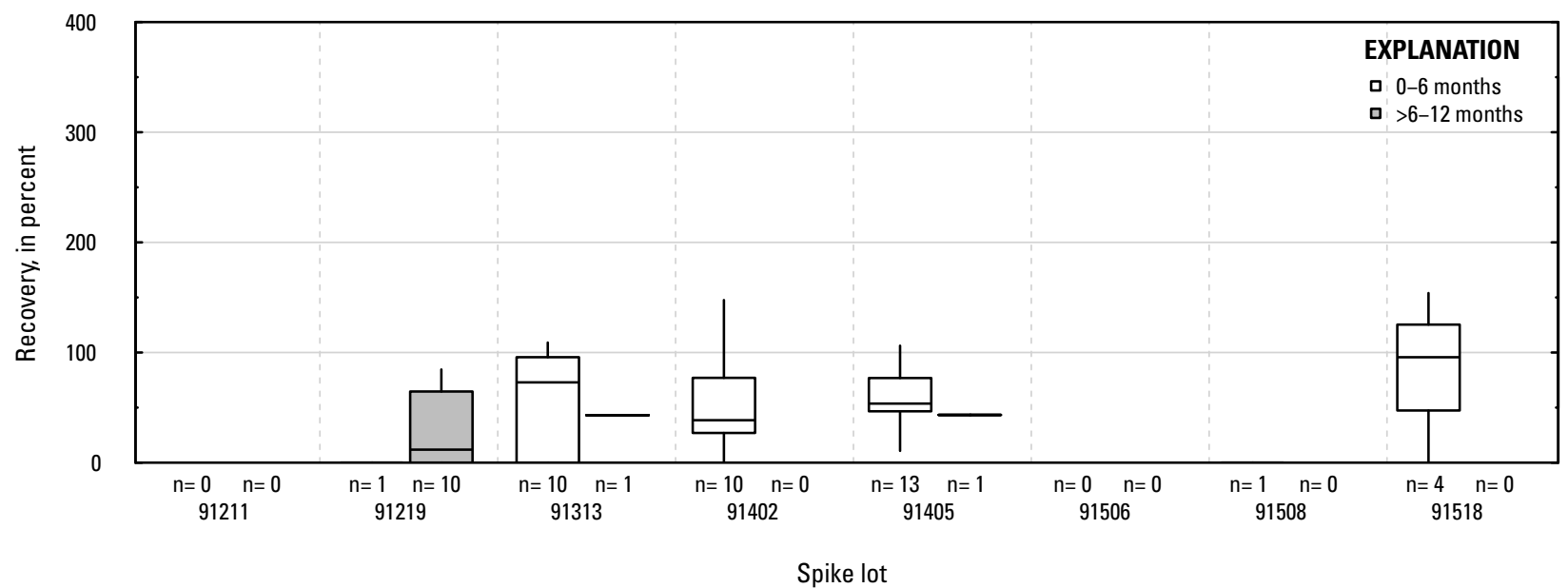

ET. Chlorosulfonamide acid: surface water field matrix spikes

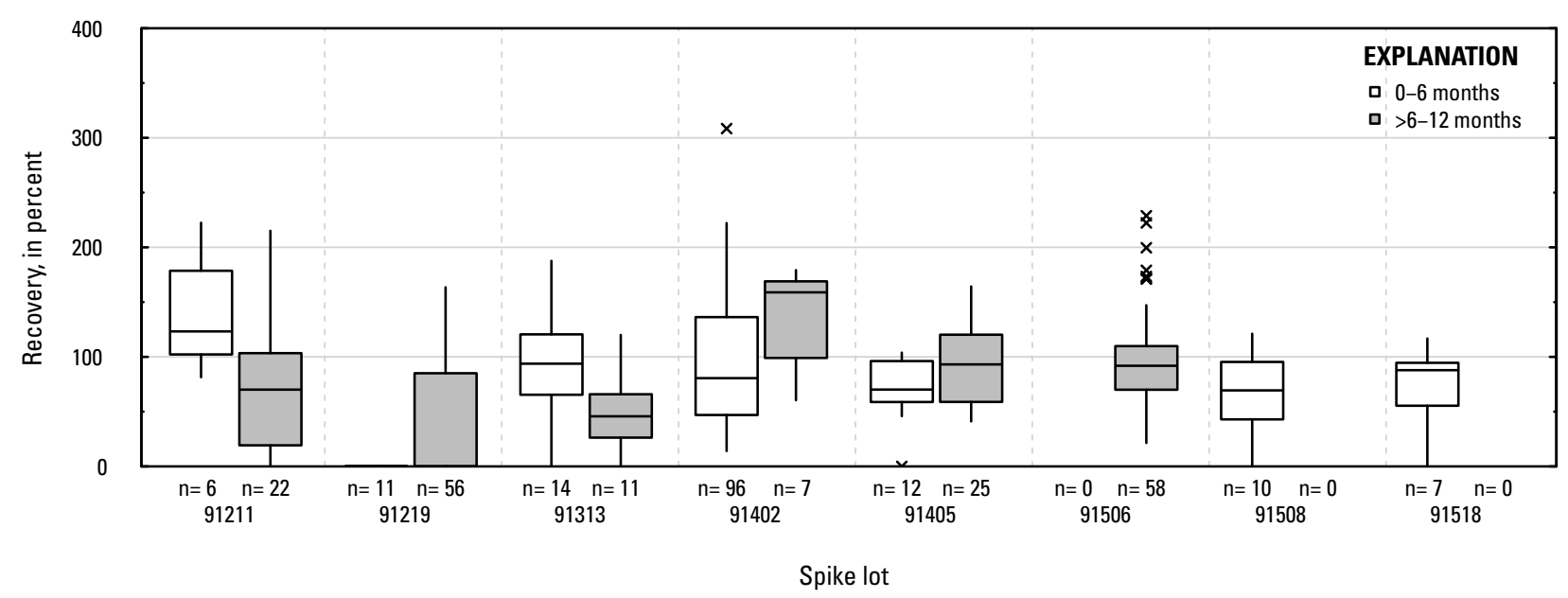

Figure 1-1. Distributions of recovery for individual pesticides in schedule 2437 by matrix, spike lot, and spike lot age. Recovery values larger than 400 percent are not shown.-Continued 
EU. Chlorpyrifos: laboratory reagent spikes

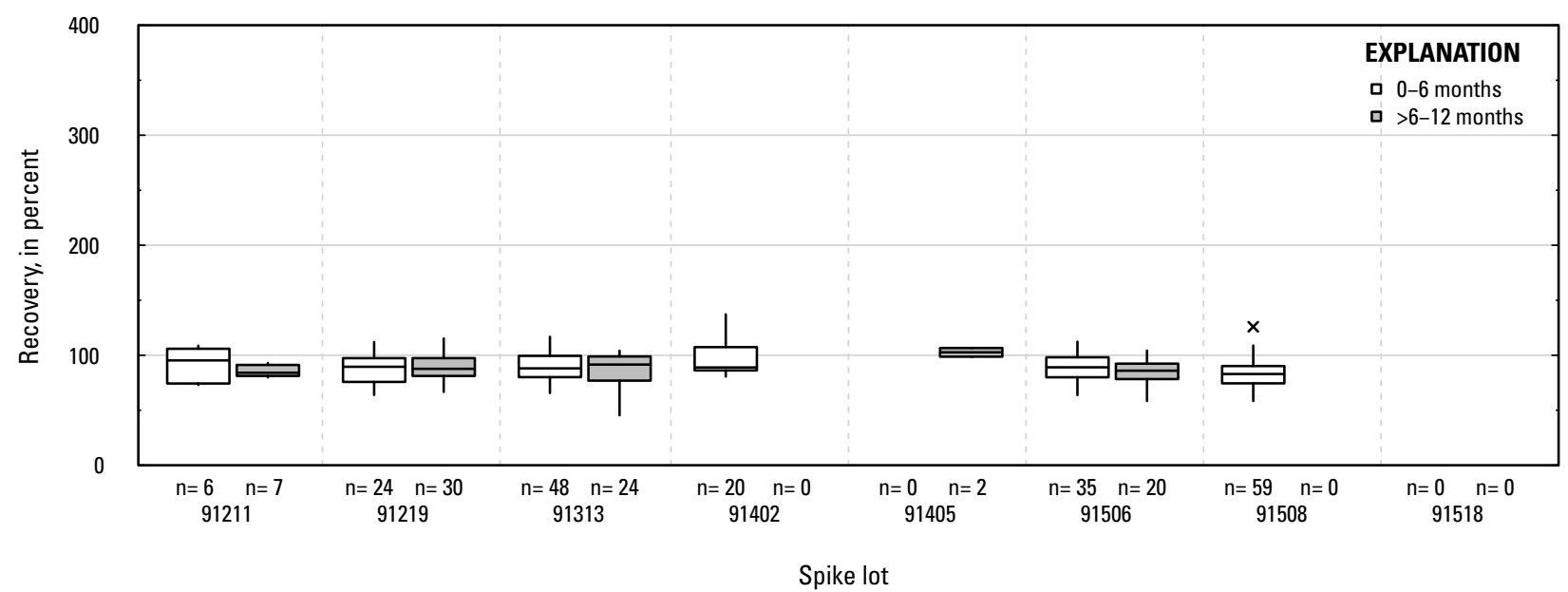

EV. Chlorpyrifos: groundwater field matrix spikes

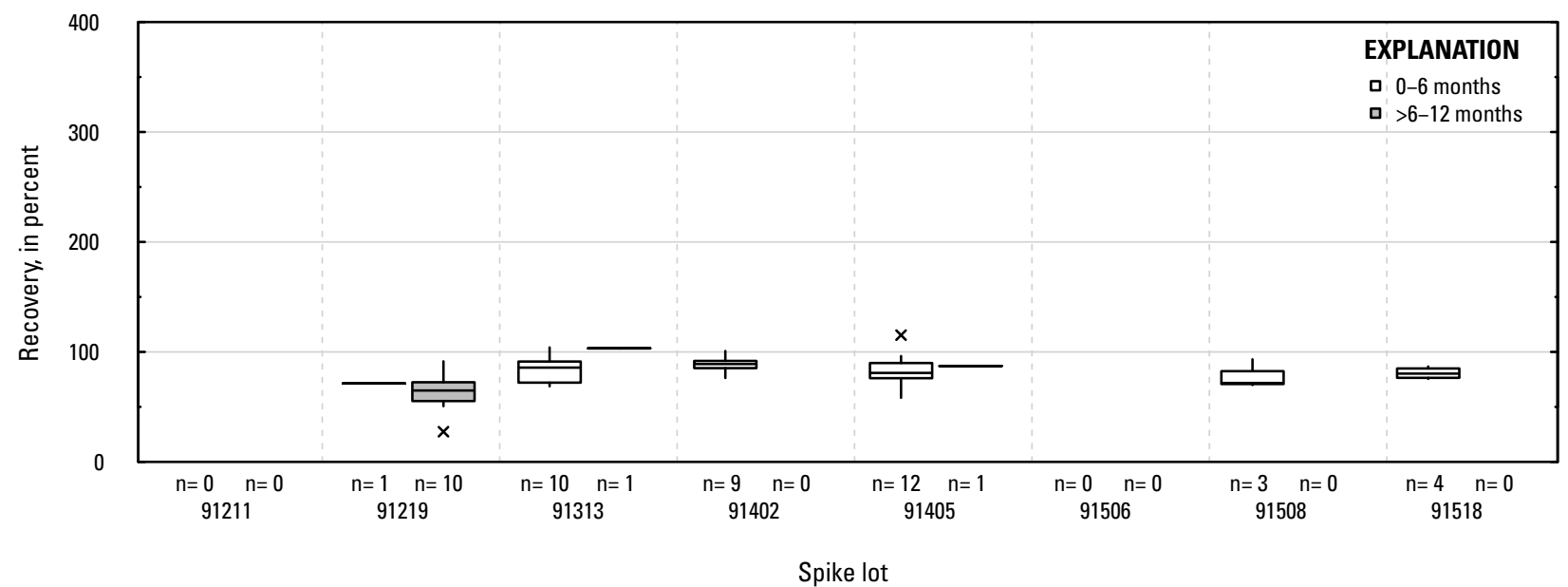

EW. Chlorpyrifos: surface water field matrix spikes

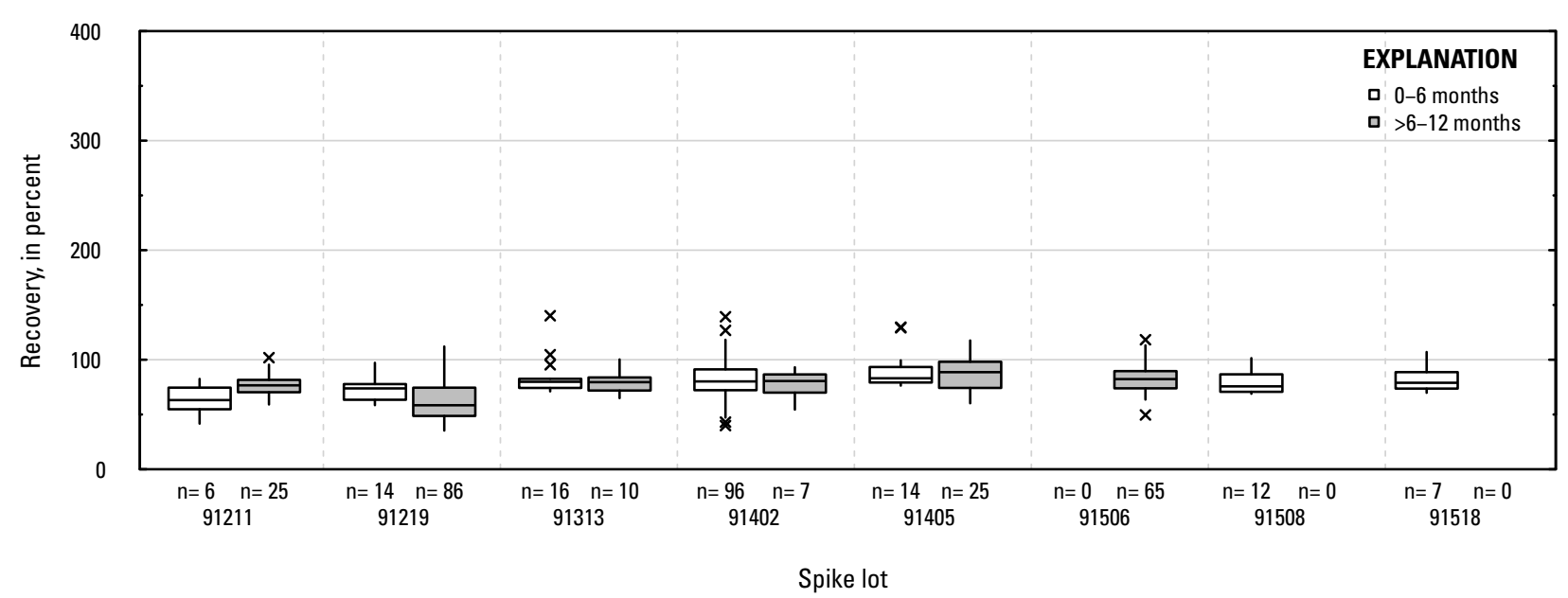

Figure 1-1. Distributions of recovery for individual pesticides in schedule 2437 by matrix, spike lot, and spike lot age. Recovery values larger than 400 percent are not shown.-Continued 


\section{EX. Chlorpyrifos oxon: laboratory reagent spikes}

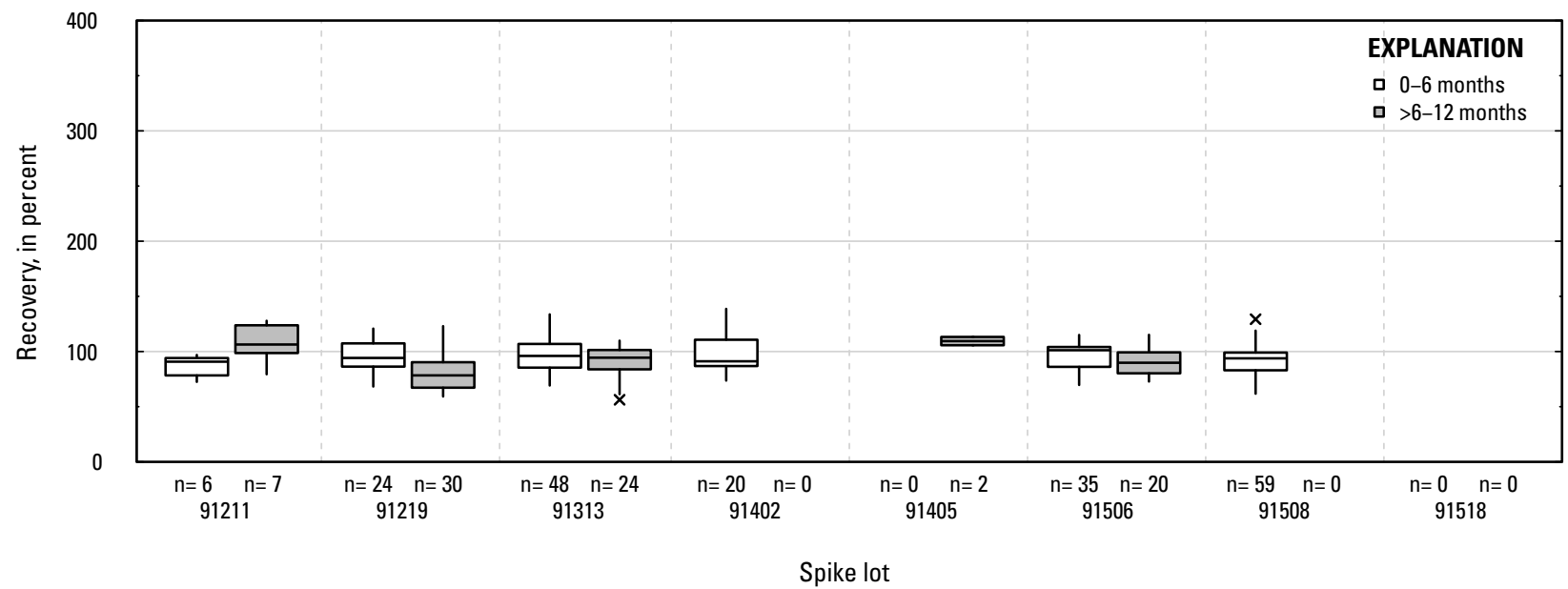

\section{EY. Chlorpyrifos oxon: groundwater field matrix spikes}

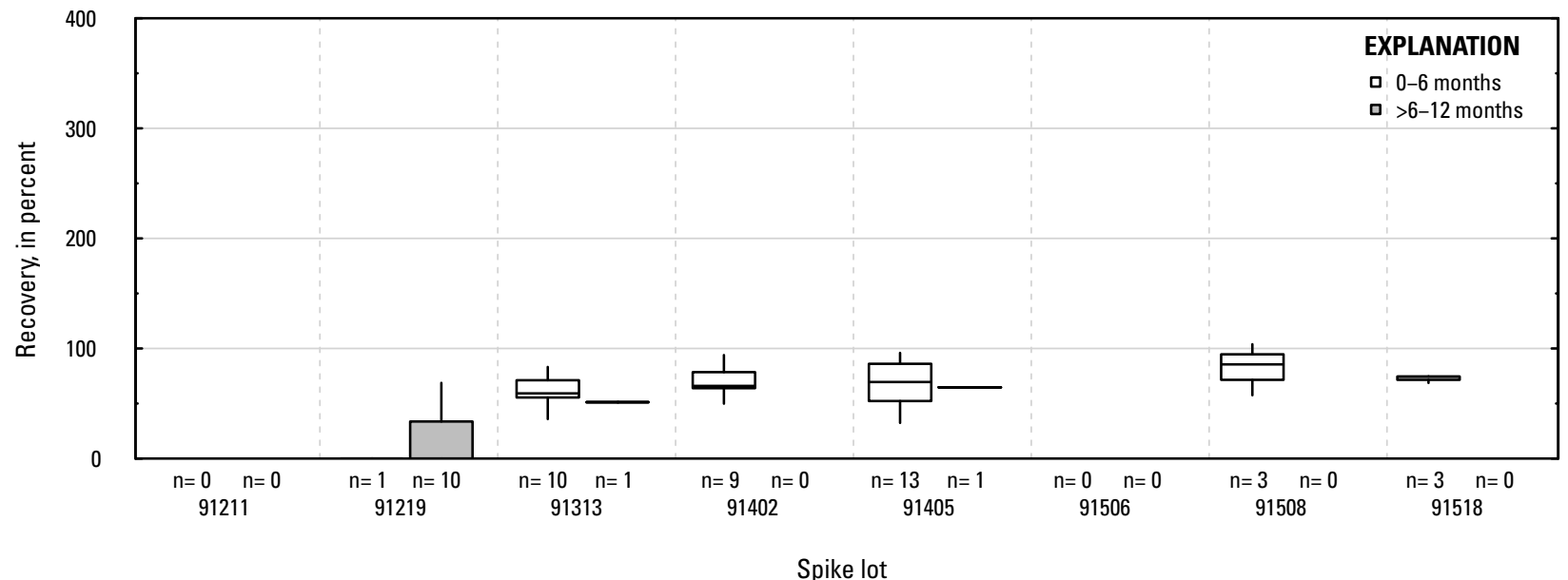

\section{EZ. Chlorpyrifos oxon: surface water field matrix spikes}

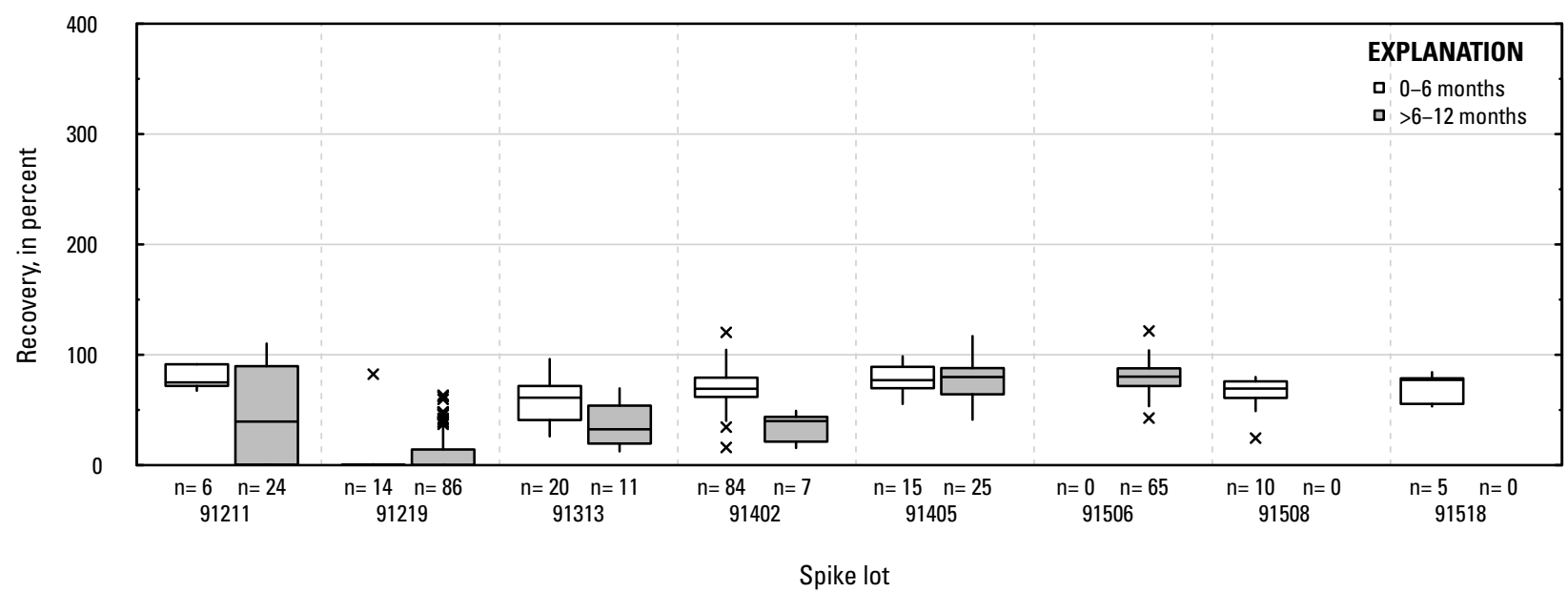

Figure 1-1. Distributions of recovery for individual pesticides in schedule 2437 by matrix, spike lot, and spike lot age. Recovery values larger than 400 percent are not shown.-Continued 


\section{FA. Chlorsulfuron: laboratory reagent spikes}

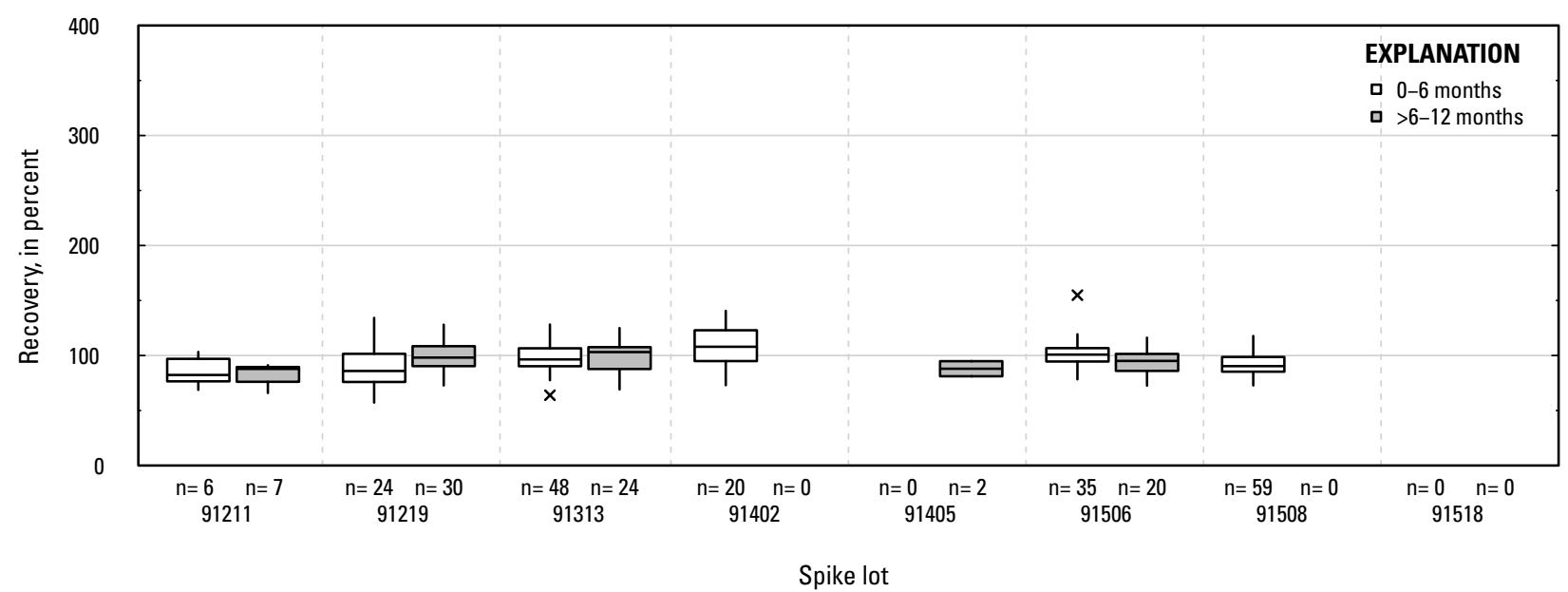

FB. Chlorsulfuron: groundwater field matrix spikes

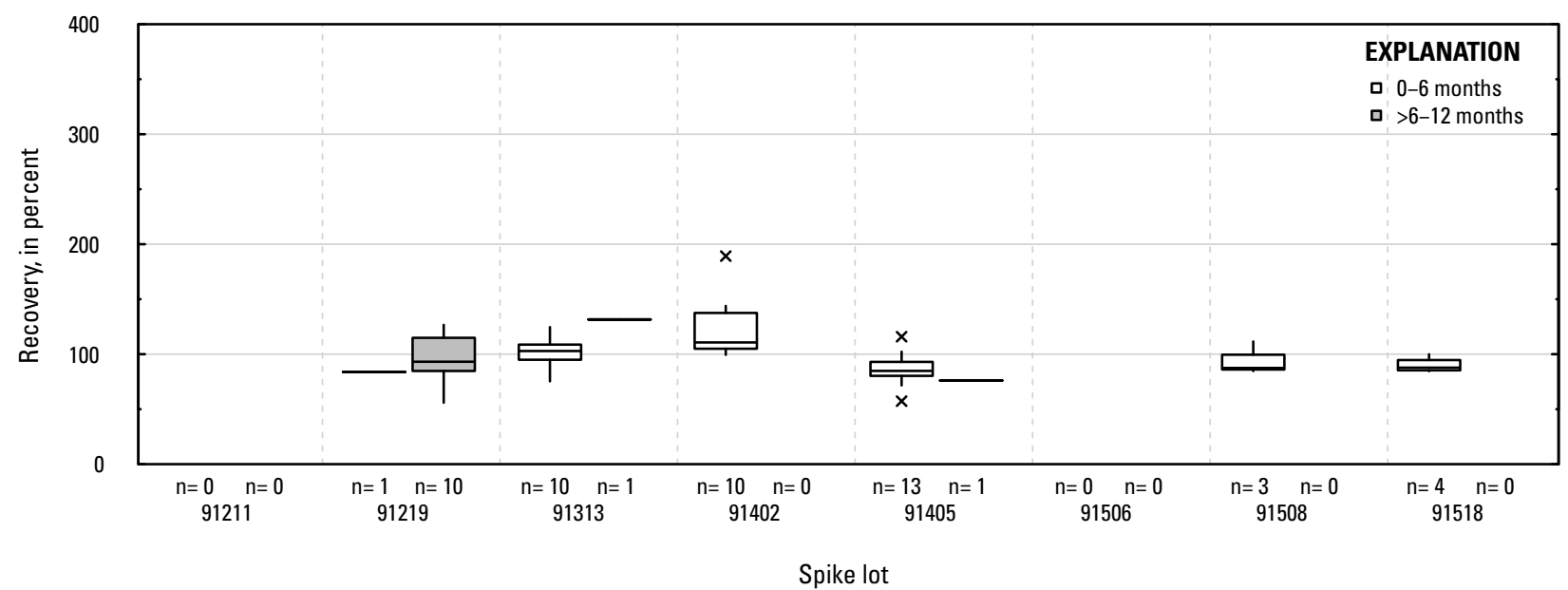

FC. Chlorsulfuron: surface water field matrix spikes

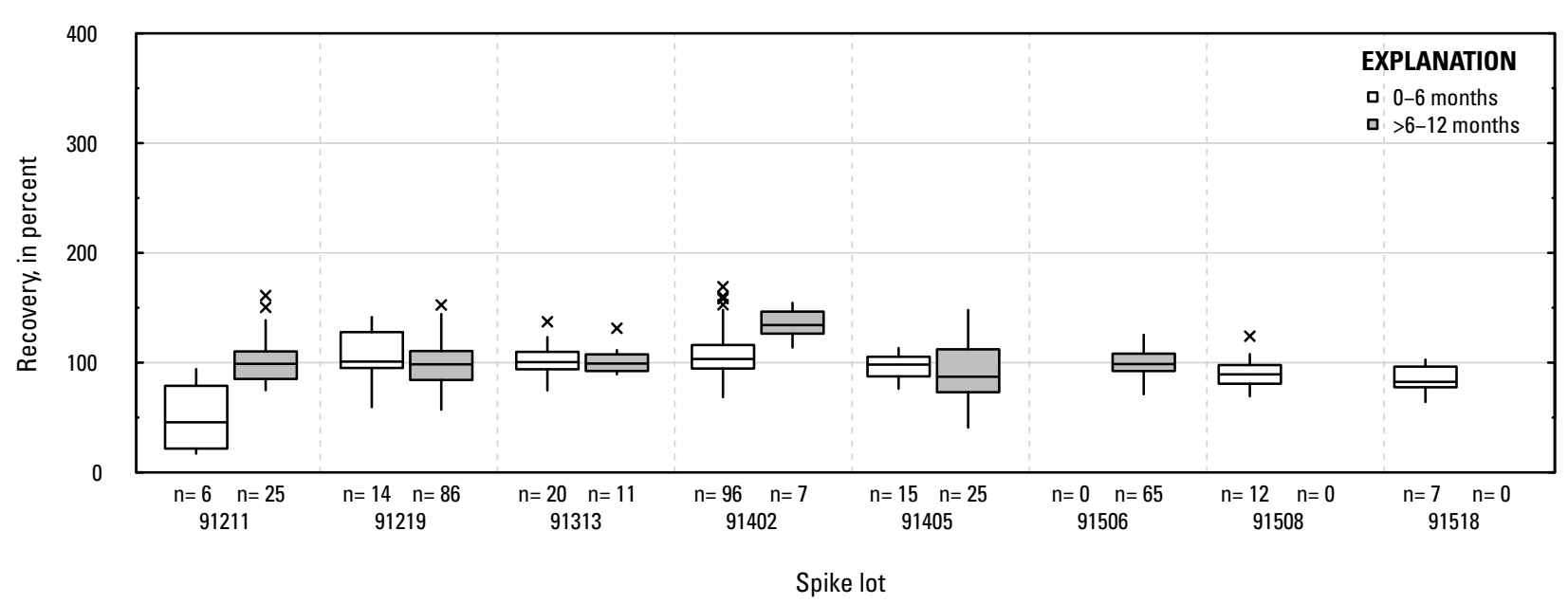

Figure 1-1. Distributions of recovery for individual pesticides in schedule 2437 by matrix, spike lot, and spike lot age. Recovery values larger than 400 percent are not shown.-Continued 
FD. Dacthal monoacid: laboratory reagent spikes

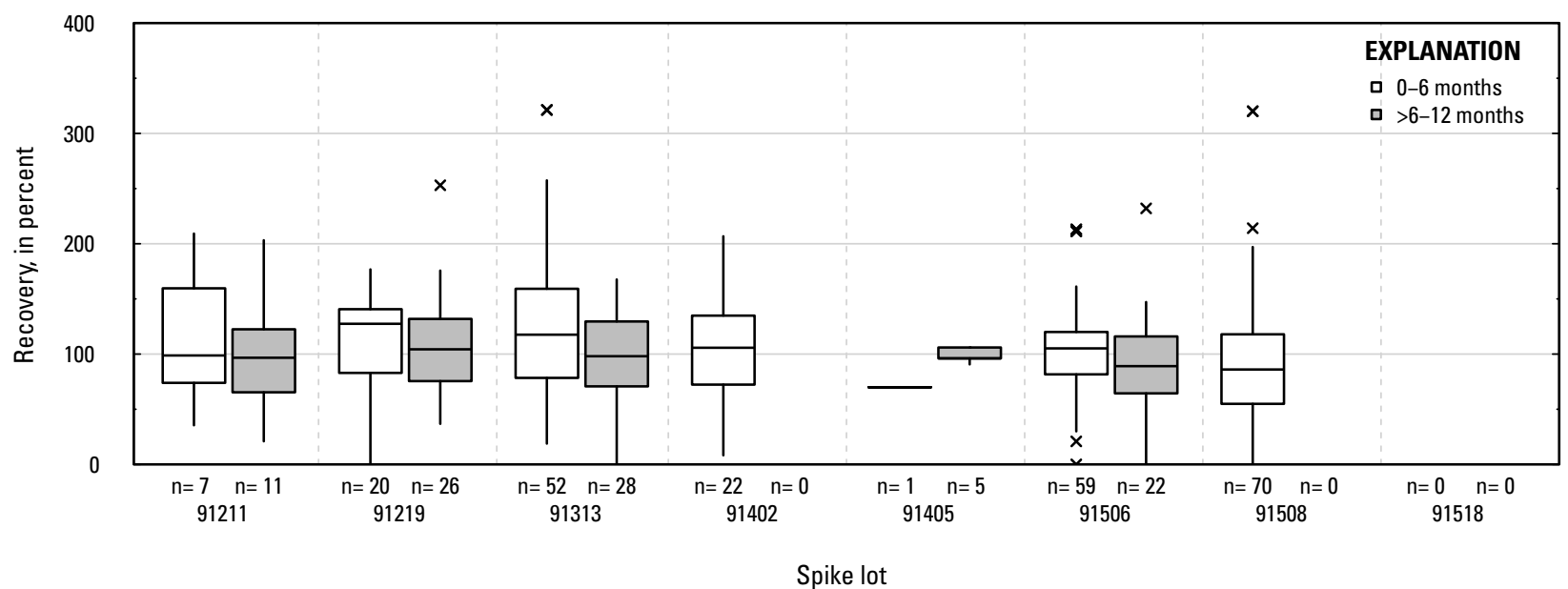

FE. Dacthal monoacid: groundwater field matrix spikes

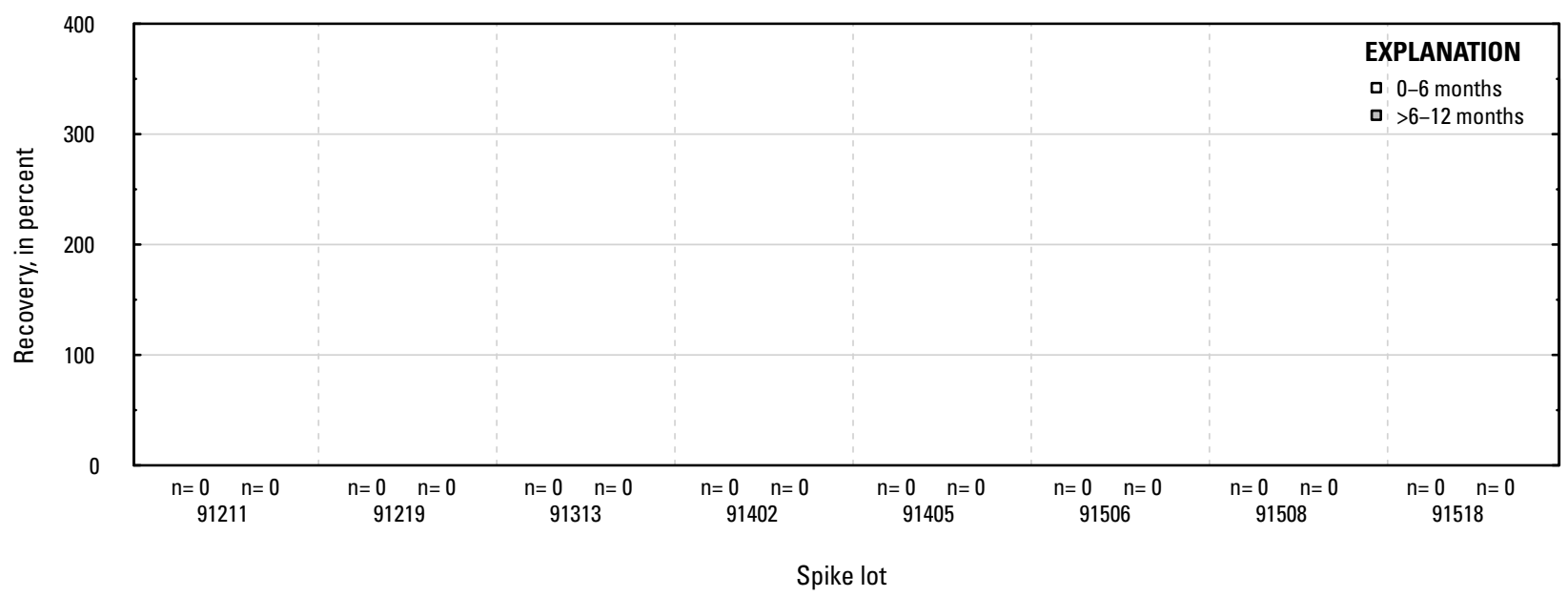

FF. Dacthal monoacid: surface water field matrix spikes

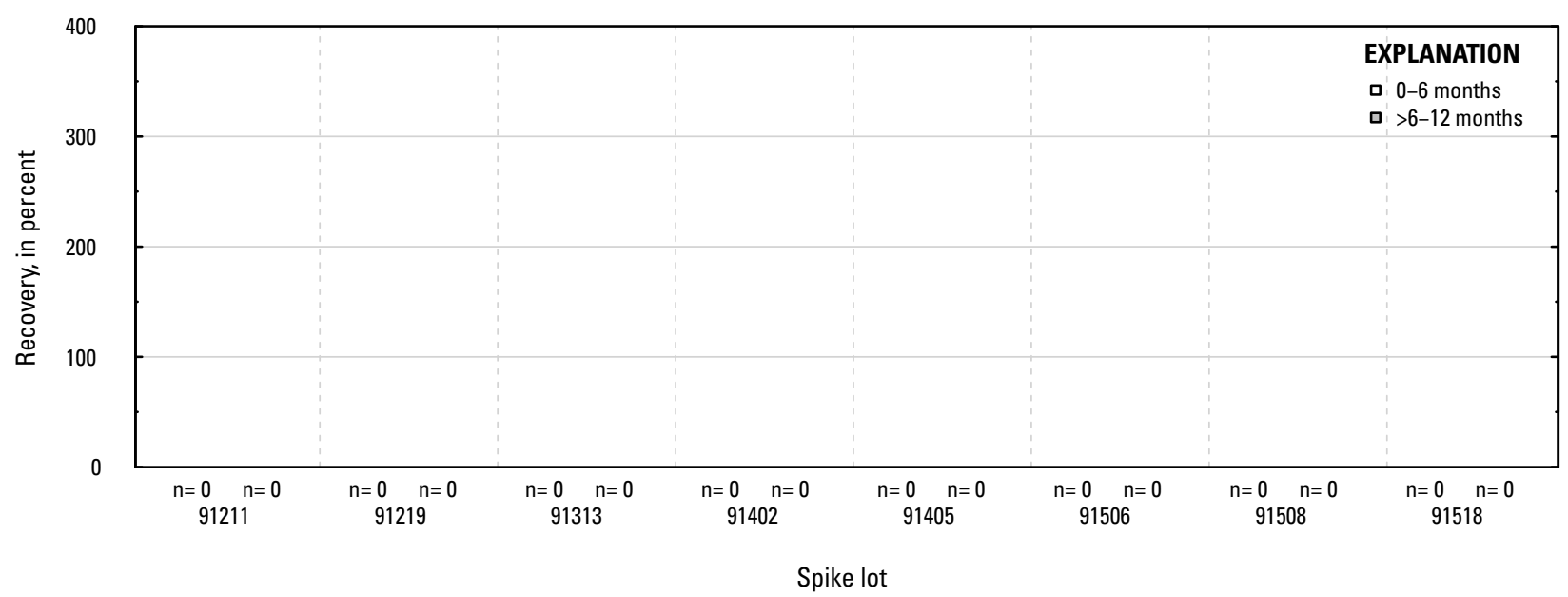

Figure 1-1. Distributions of recovery for individual pesticides in schedule 2437 by matrix, spike lot, and spike lot age. Recovery values larger than 400 percent are not shown.-Continued 
FG. cis-Bifenthrin acid/cis-Cyhalothrin acid/cis-Tefluthrin acid: laboratory reagent spikes

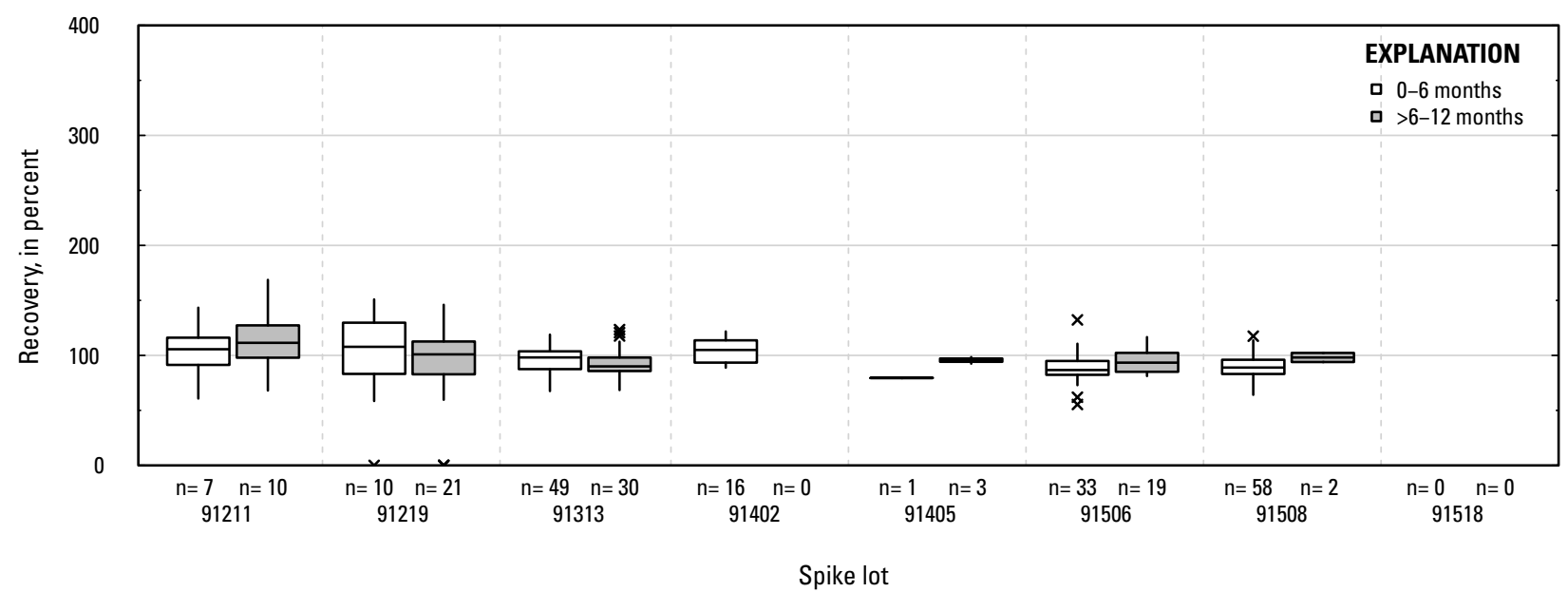

FH. cis-Bifenthrin acid/cis-Cyhalothrin acid/cis-Tefluthrin acid: groundwater field matrix spikes

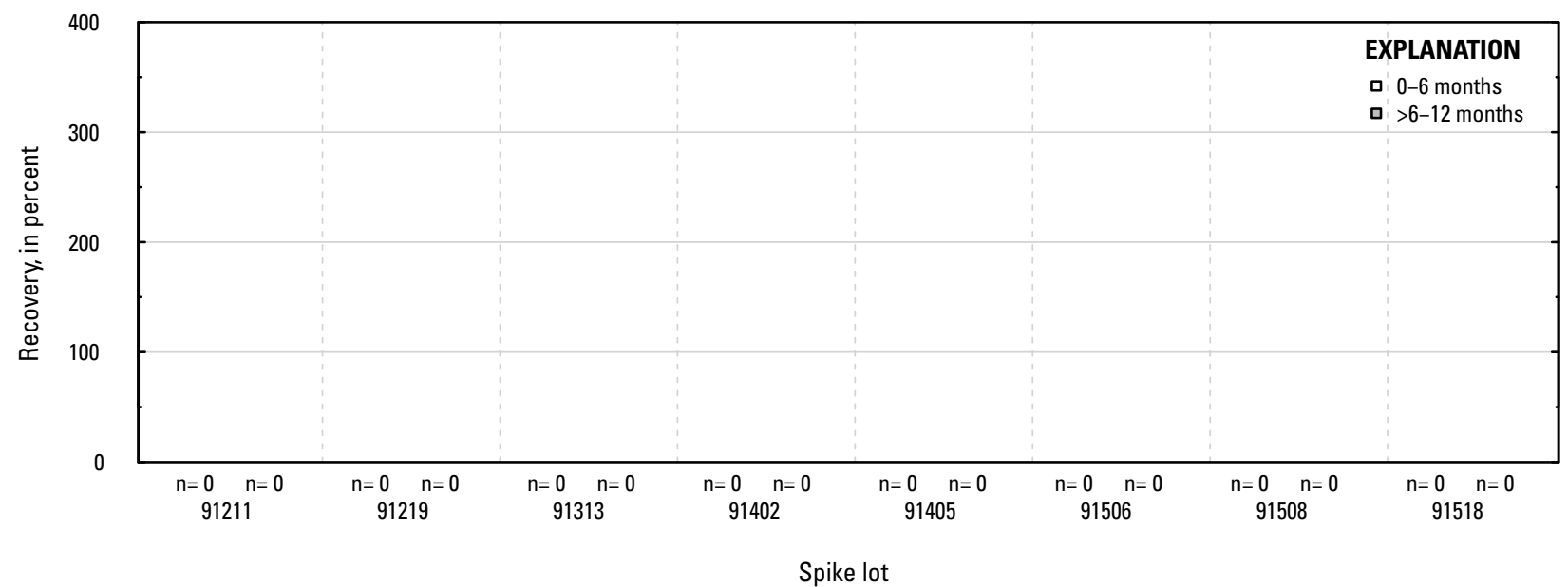

FI. cis-Bifenthrin acid/cis-Cyhalothrin acid/cis-Tefluthrin acid: surface water field matrix spikes

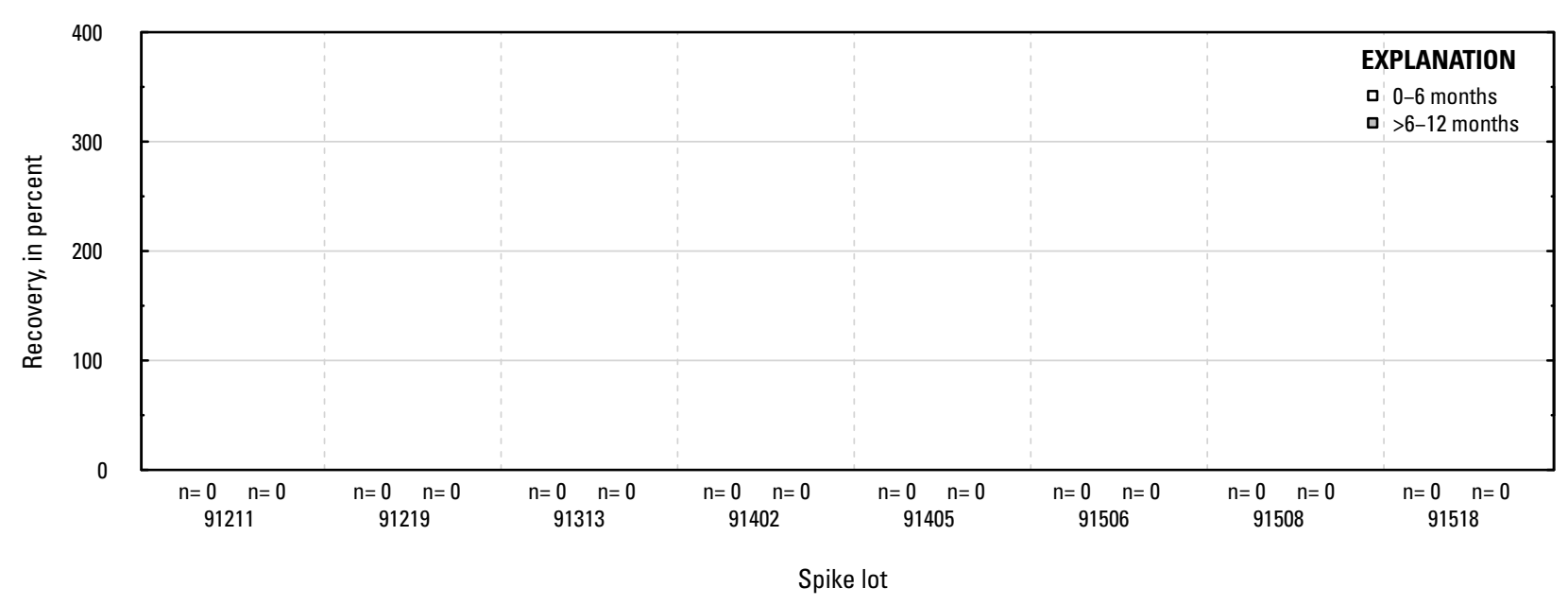

Figure 1-1. Distributions of recovery for individual pesticides in schedule 2437 by matrix, spike lot, and spike lot age. Recovery values larger than 400 percent are not shown.-Continued 
FJ. cis-Permethrin: laboratory reagent spikes

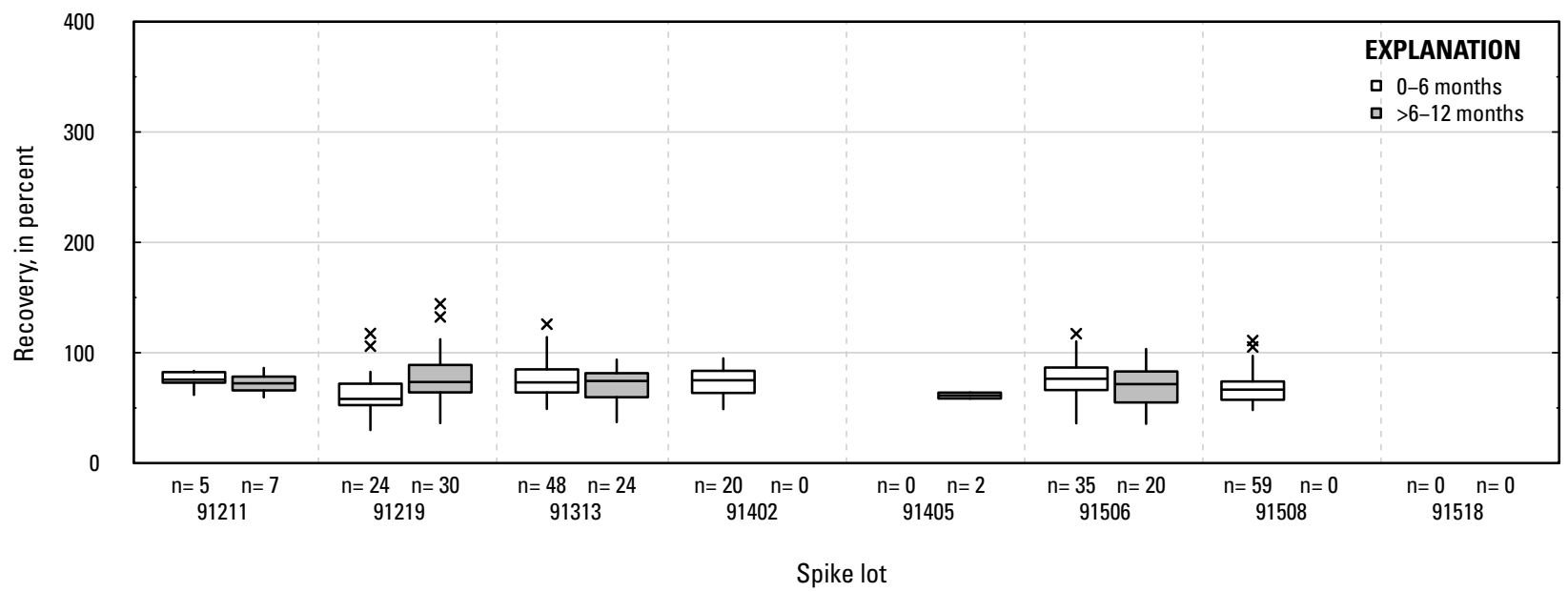

FK. cis-Permethrin: groundwater field matrix spikes

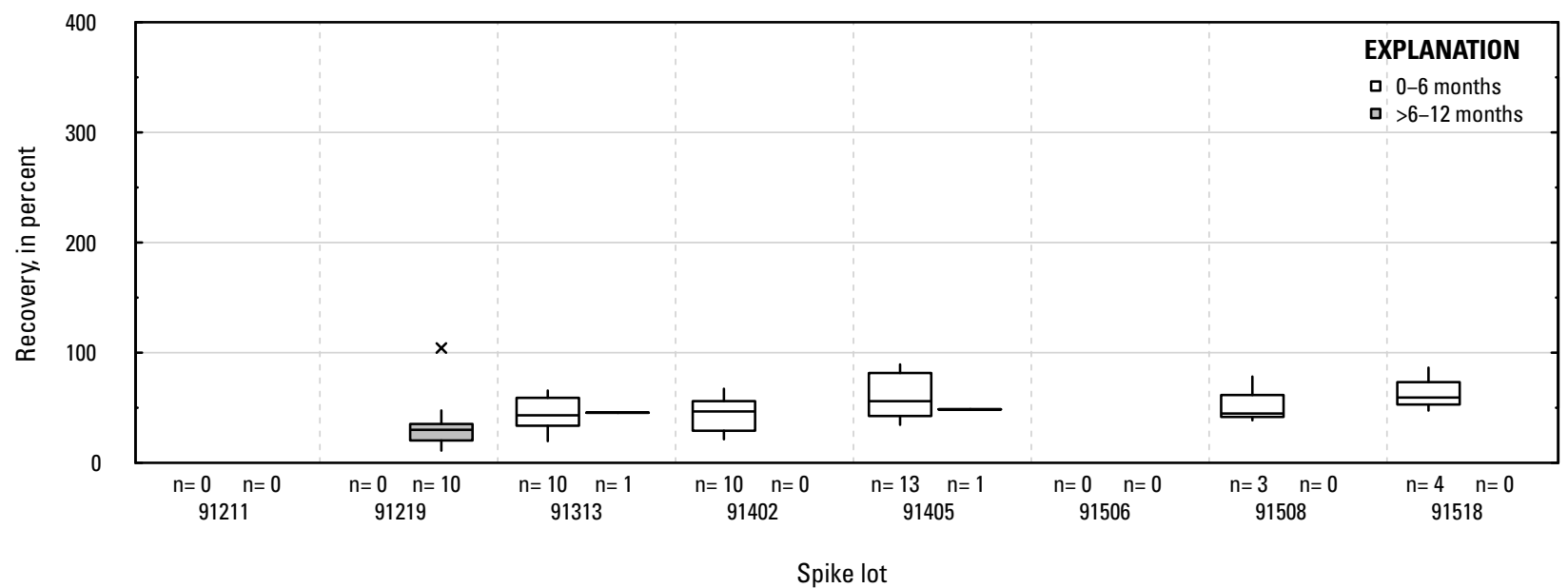

FL. cis-Permethrin: surface water field matrix spikes

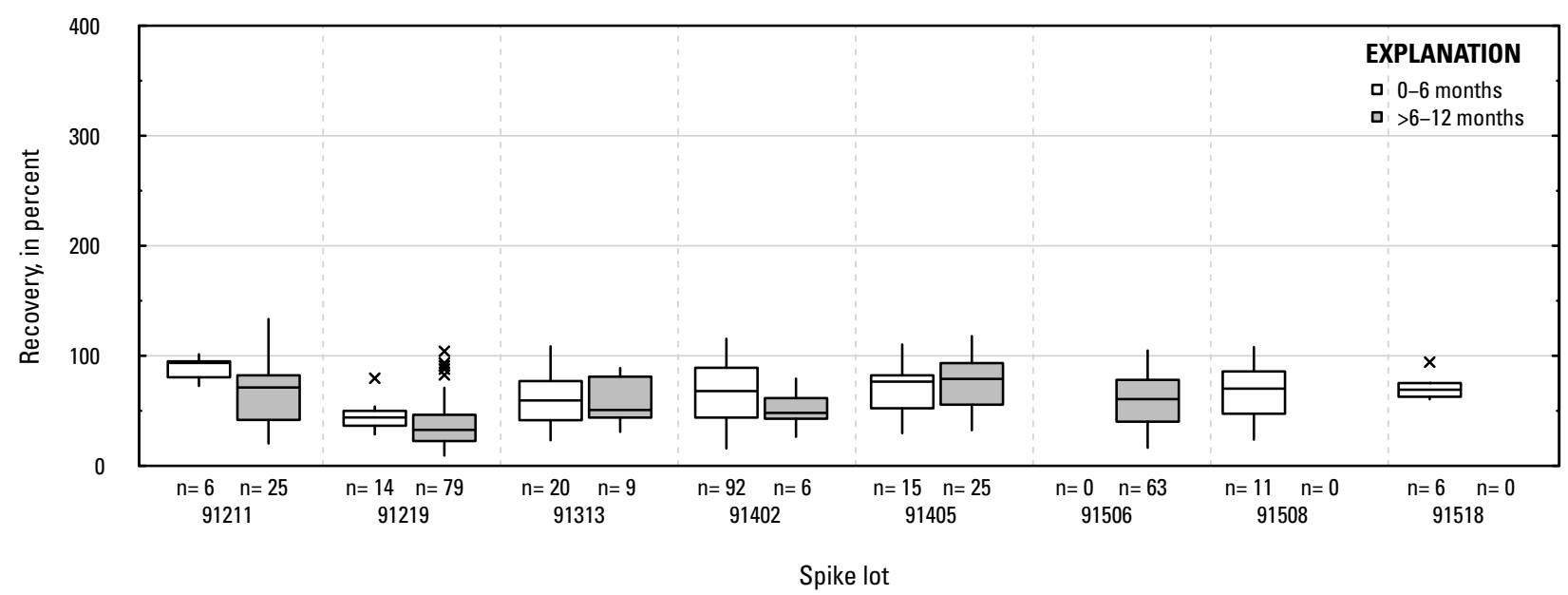

Figure 1-1. Distributions of recovery for individual pesticides in schedule 2437 by matrix, spike lot, and spike lot age. Recovery values larger than 400 percent are not shown.-Continued 
FM. Cyanazine: laboratory reagent spikes

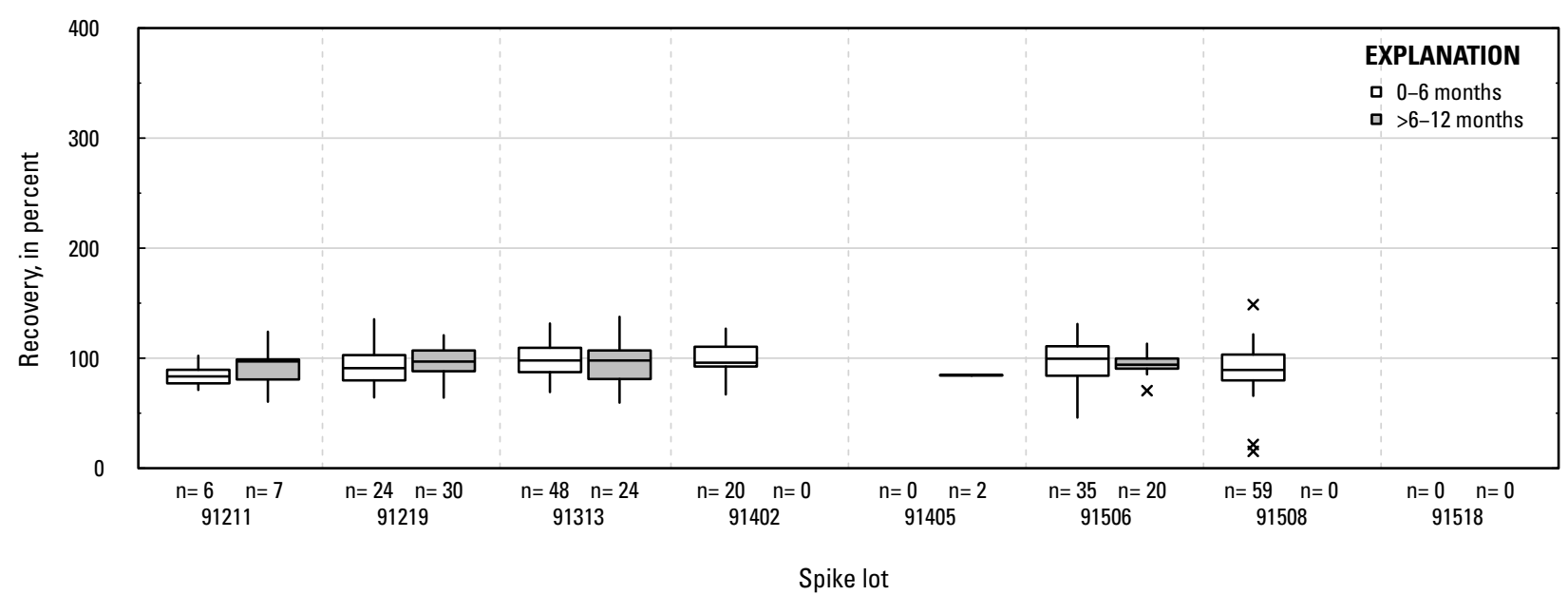

FN. Cyanazine: groundwater field matrix spikes

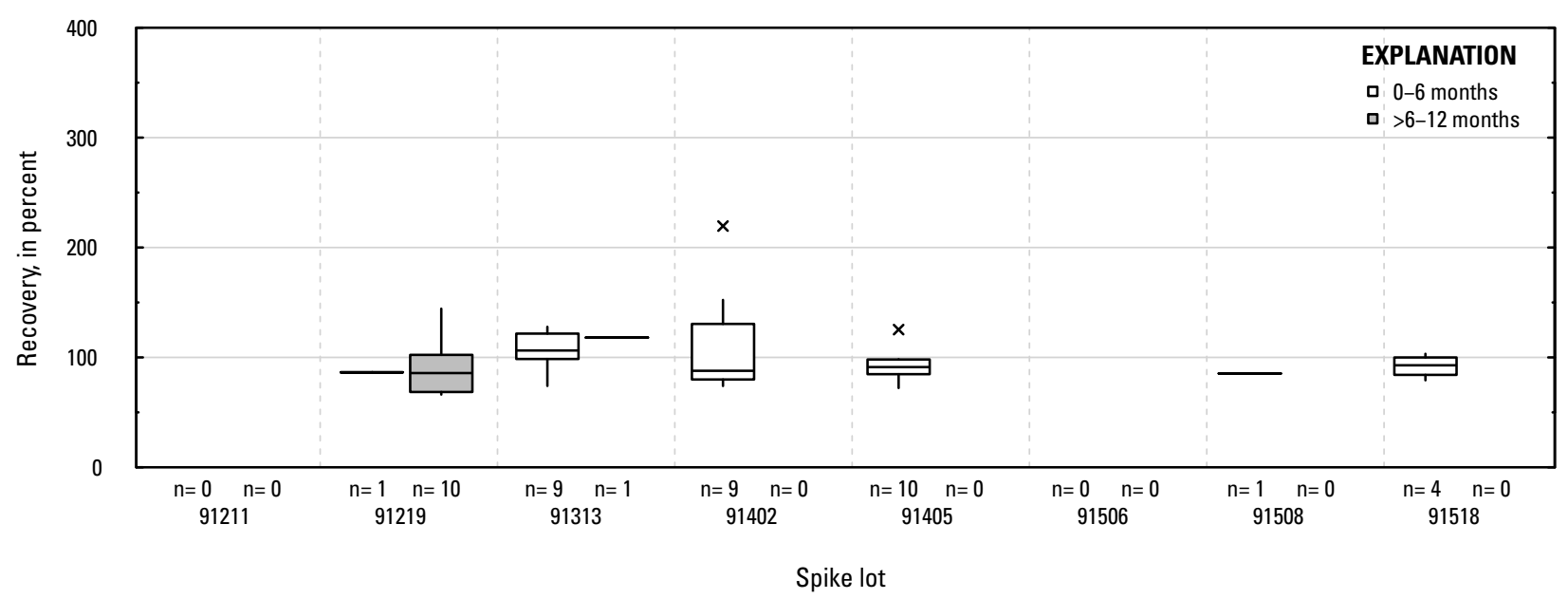

FO. Cyanazine: surface water field matrix spikes

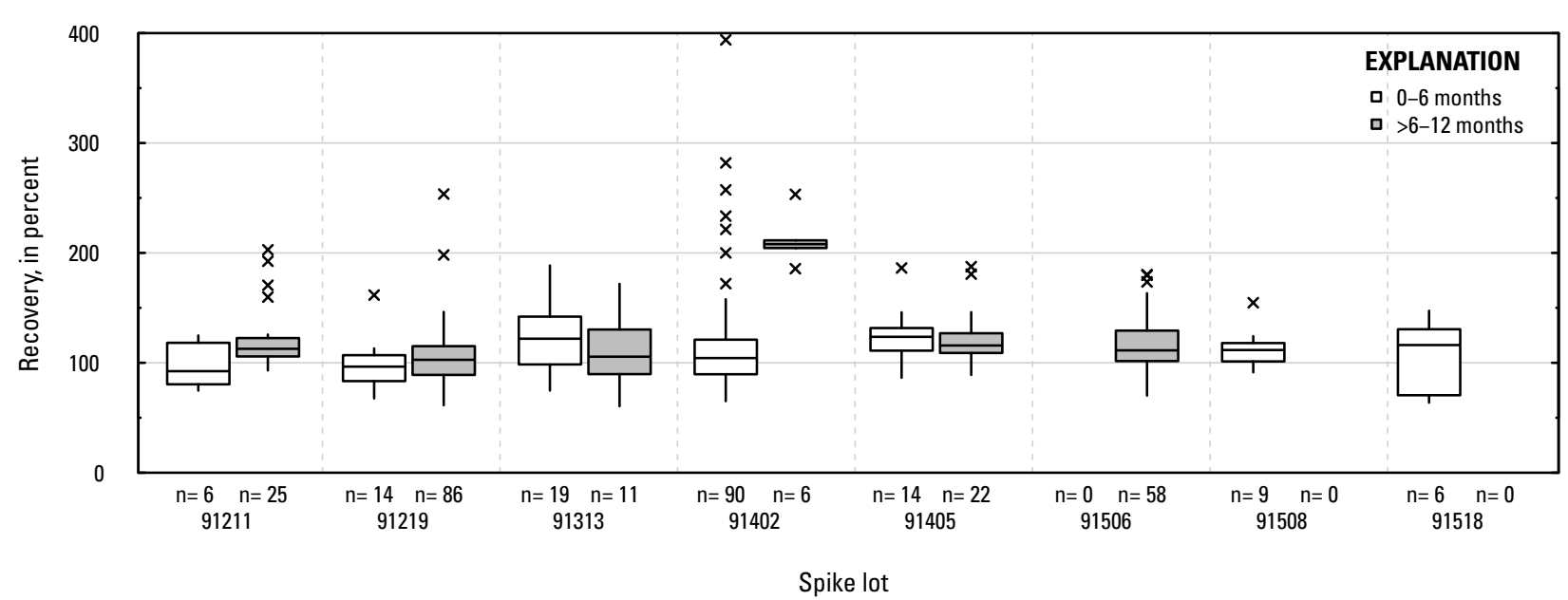

Figure 1-1. Distributions of recovery for individual pesticides in schedule 2437 by matrix, spike lot, and spike lot age. Recovery values larger than 400 percent are not shown.-Continued 
FP. Dechlorofipronil: laboratory reagent spikes

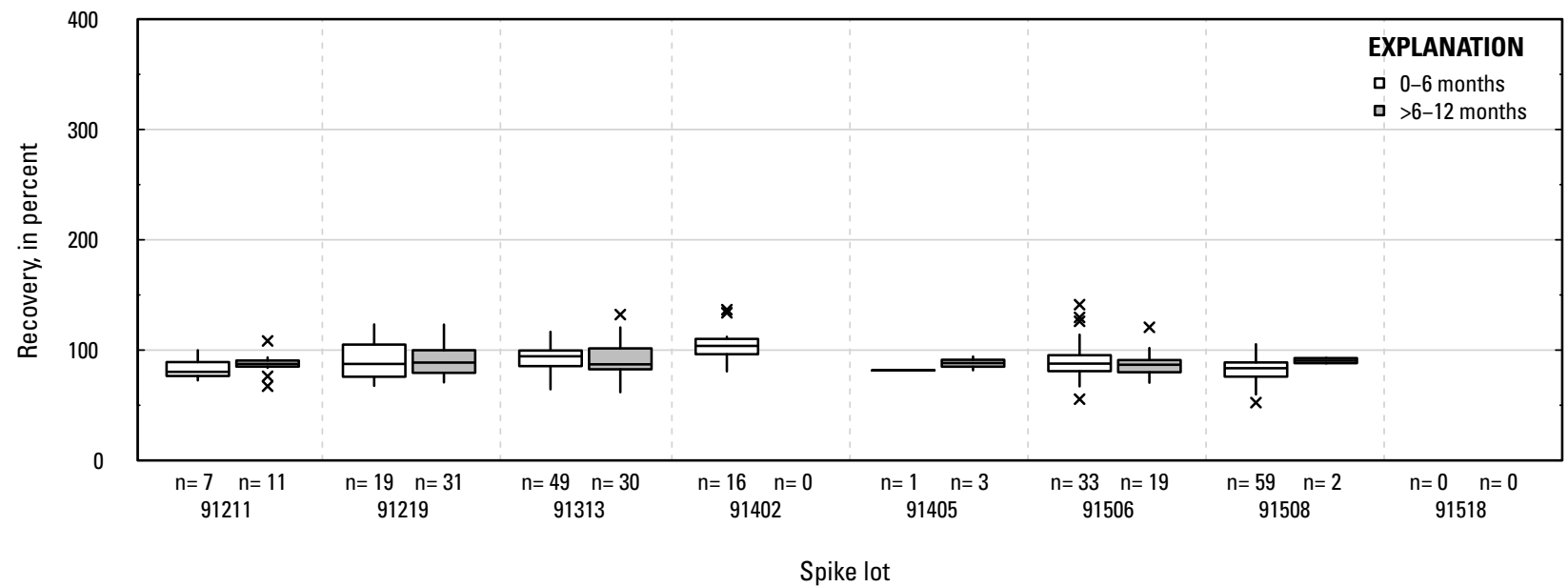

FQ. Dechlorofipronil: groundwater field matrix spikes

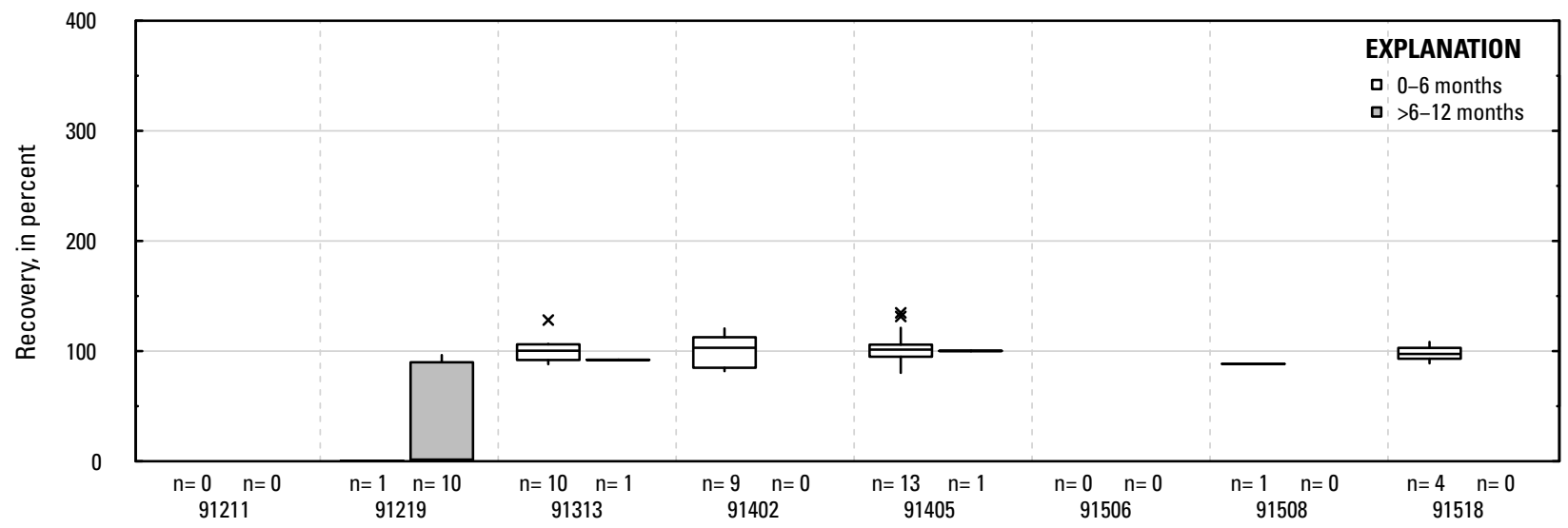

Spike lot

\section{FR. Dechlorofipronil: surface water field matrix spikes}

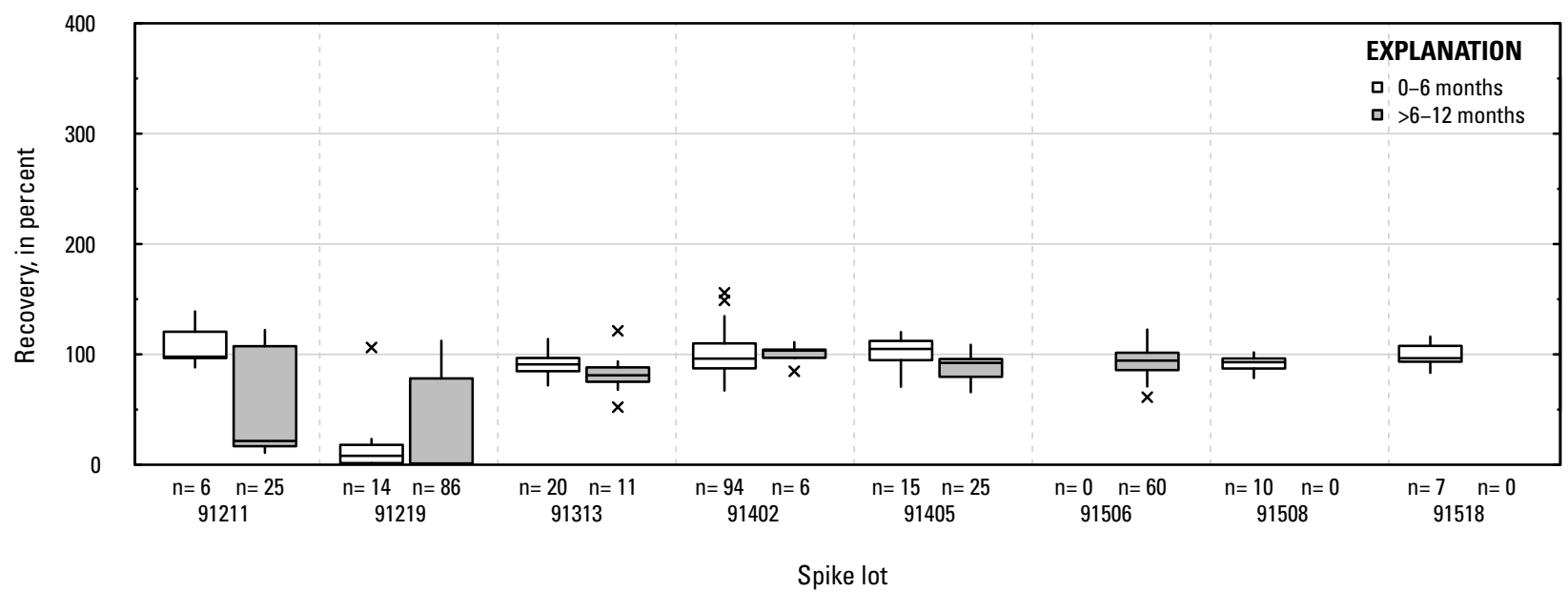

Figure 1-1. Distributions of recovery for individual pesticides in schedule 2437 by matrix, spike lot, and spike lot age. Recovery values larger than 400 percent are not shown.-Continued 


\section{FS. Dechlorometolachlor: laboratory reagent spikes}

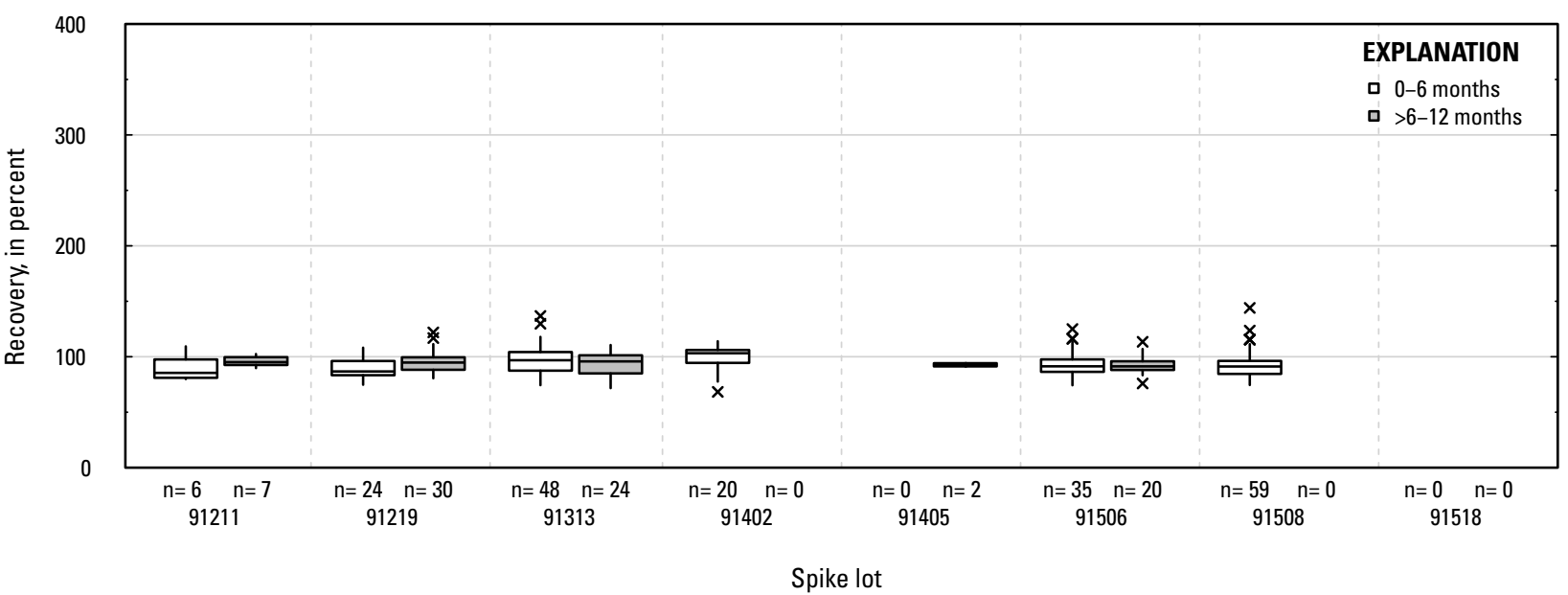

\section{FT. Dechlorometolachlor: groundwater field matrix spikes}

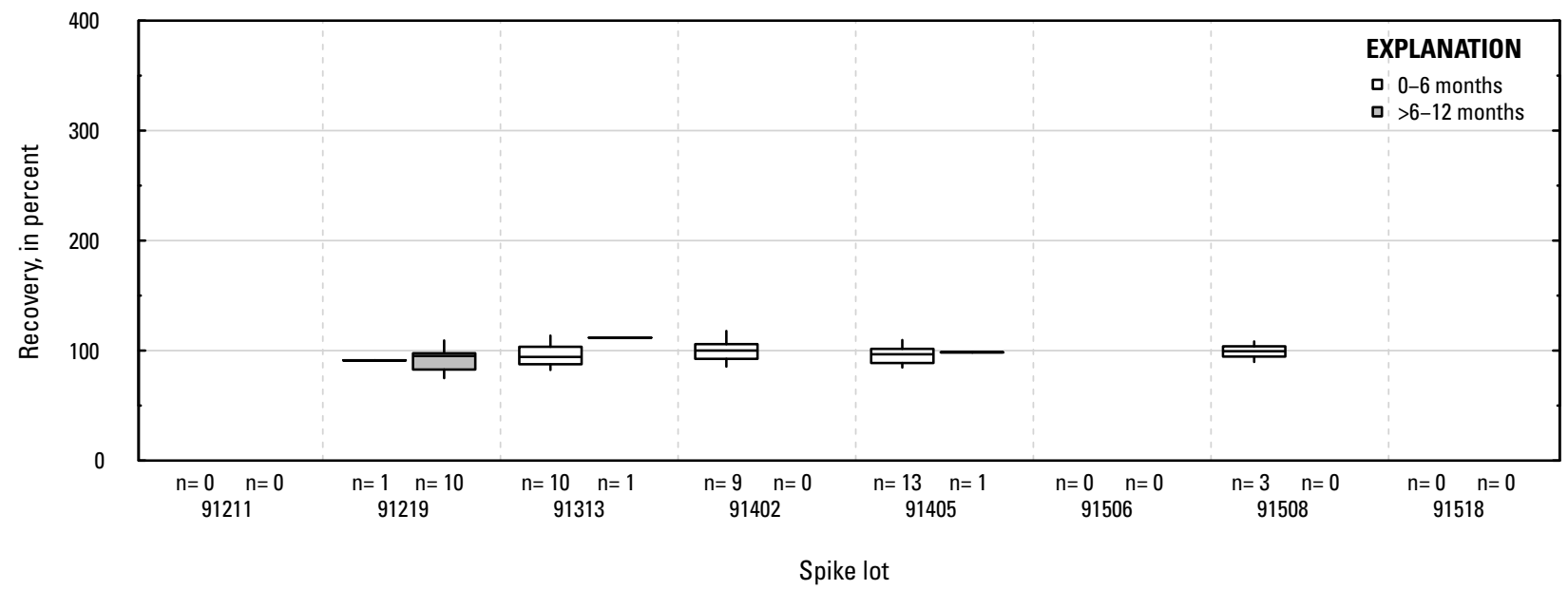

FU. Dechlorometolachlor: surface water field matrix spikes

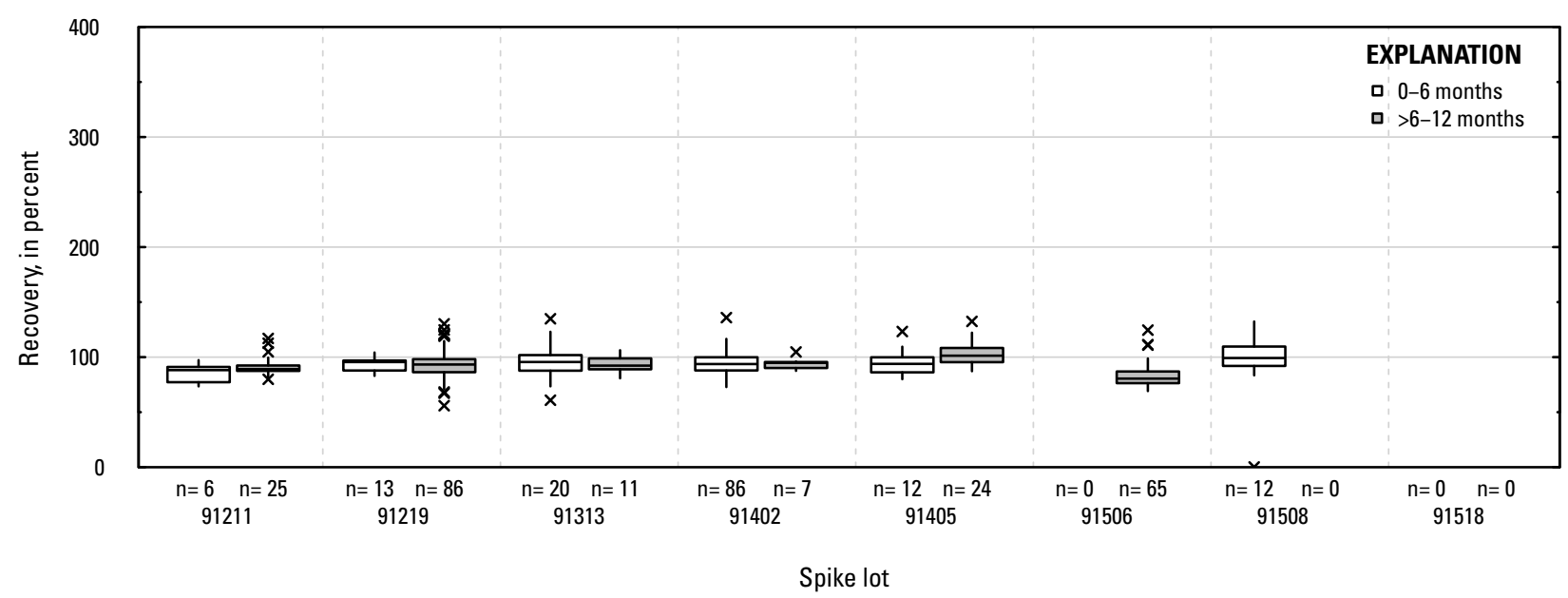

Figure 1-1. Distributions of recovery for individual pesticides in schedule 2437 by matrix, spike lot, and spike lot age. Recovery values larger than 400 percent are not shown.-Continued 
FV. 2-Chloro-4-isopropylamino-6-amino-s-triazine: laboratory reagent spikes

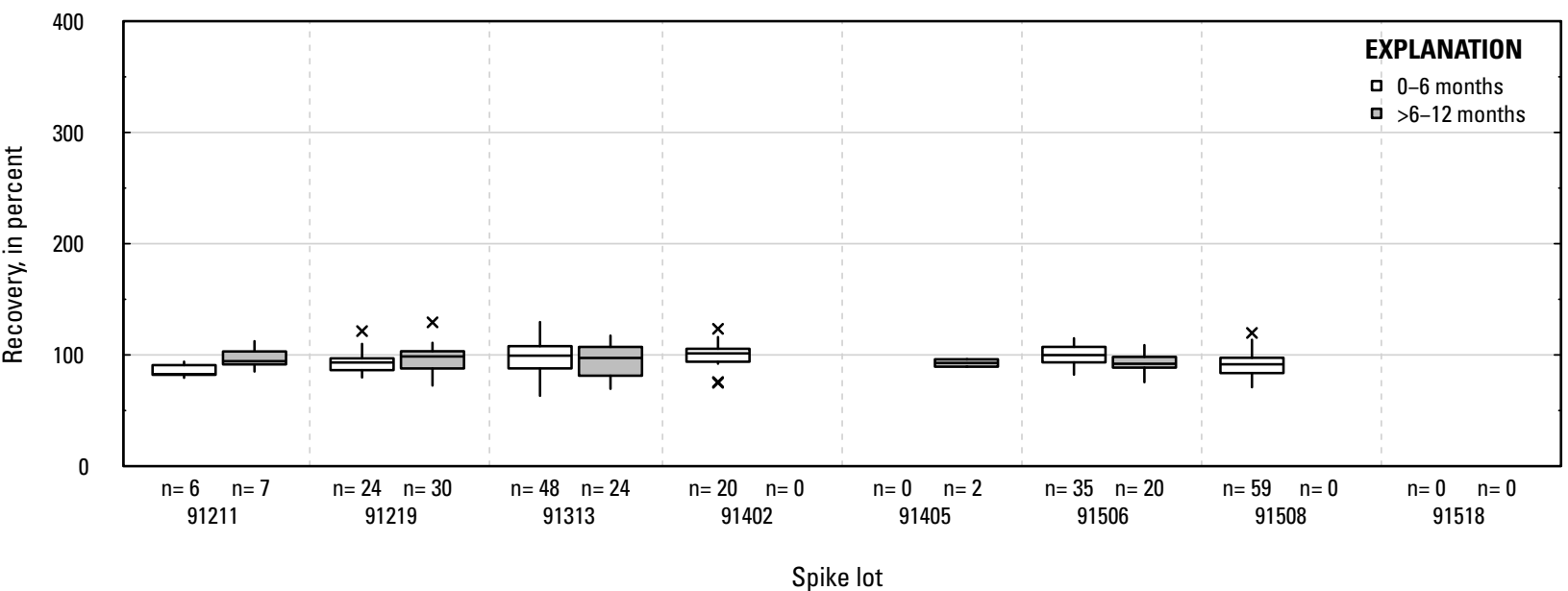

FW. 2-Chloro-4-isopropylamino-6-amino-s-triazine: groundwater field matrix spikes

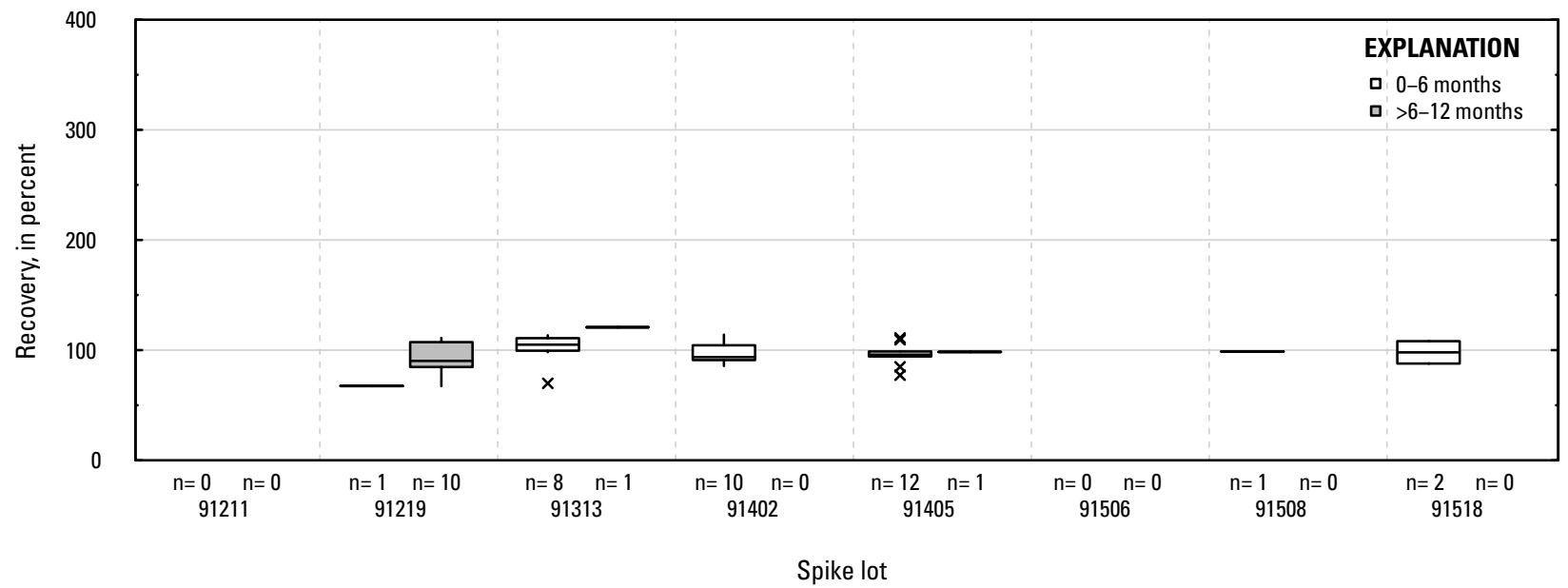

FX. 2-Chloro-4-isopropylamino-6-amino-s-triazine: surface water field matrix spikes

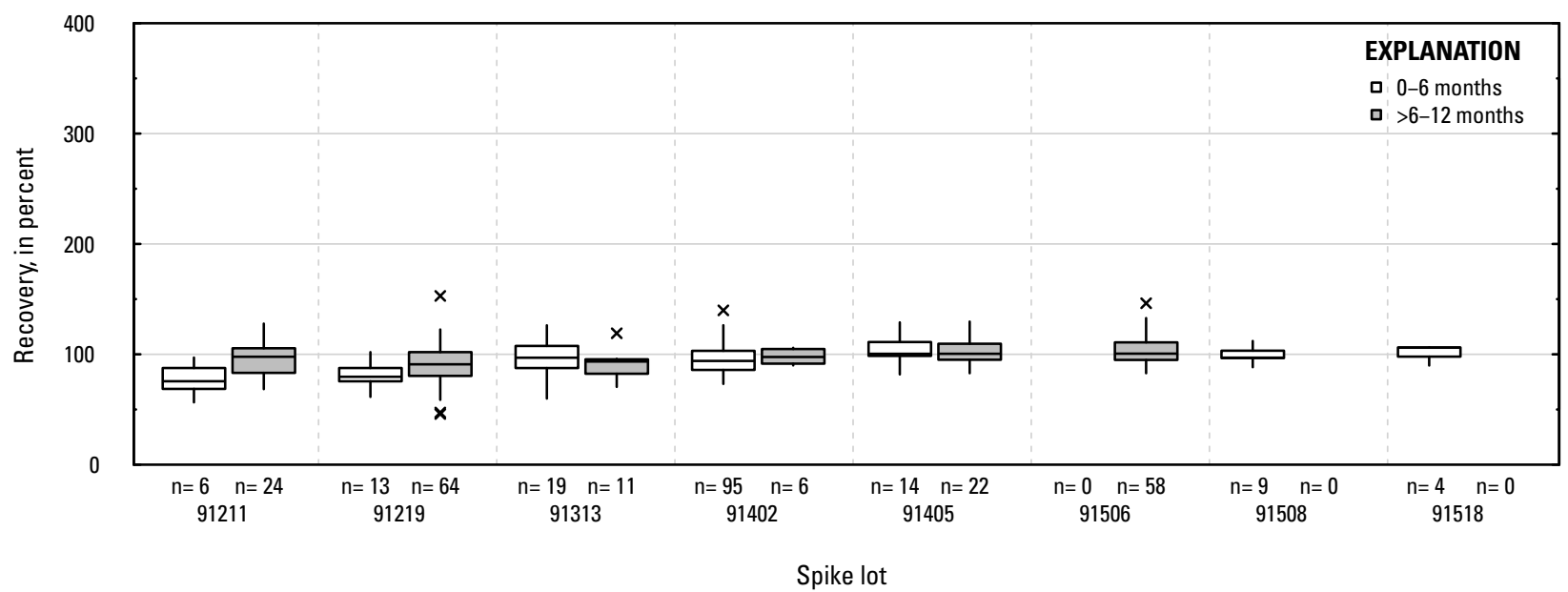

Figure 1-1. Distributions of recovery for individual pesticides in schedule 2437 by matrix, spike lot, and spike lot age. Recovery values larger than 400 percent are not shown.-Continued 


\section{FY. Deisopropyl prometryn: laboratory reagent spikes}

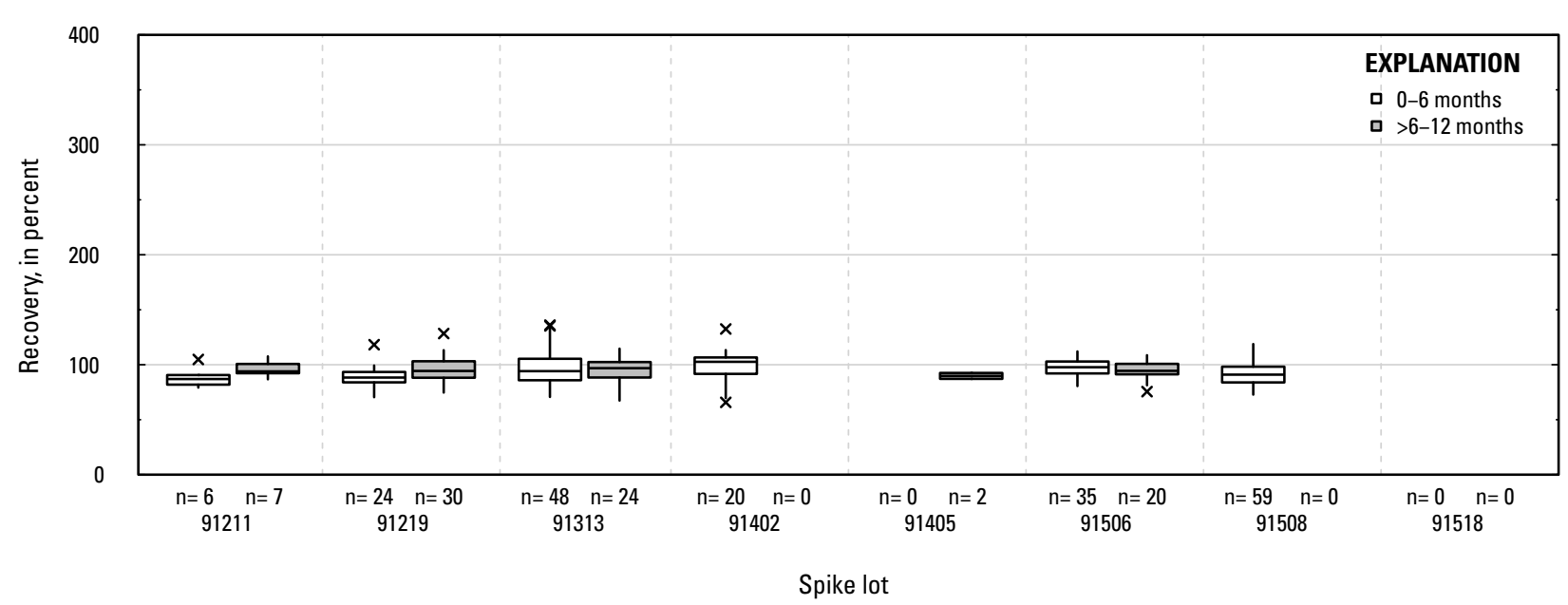

FZ. Deisopropyl prometryn: groundwater field matrix spikes

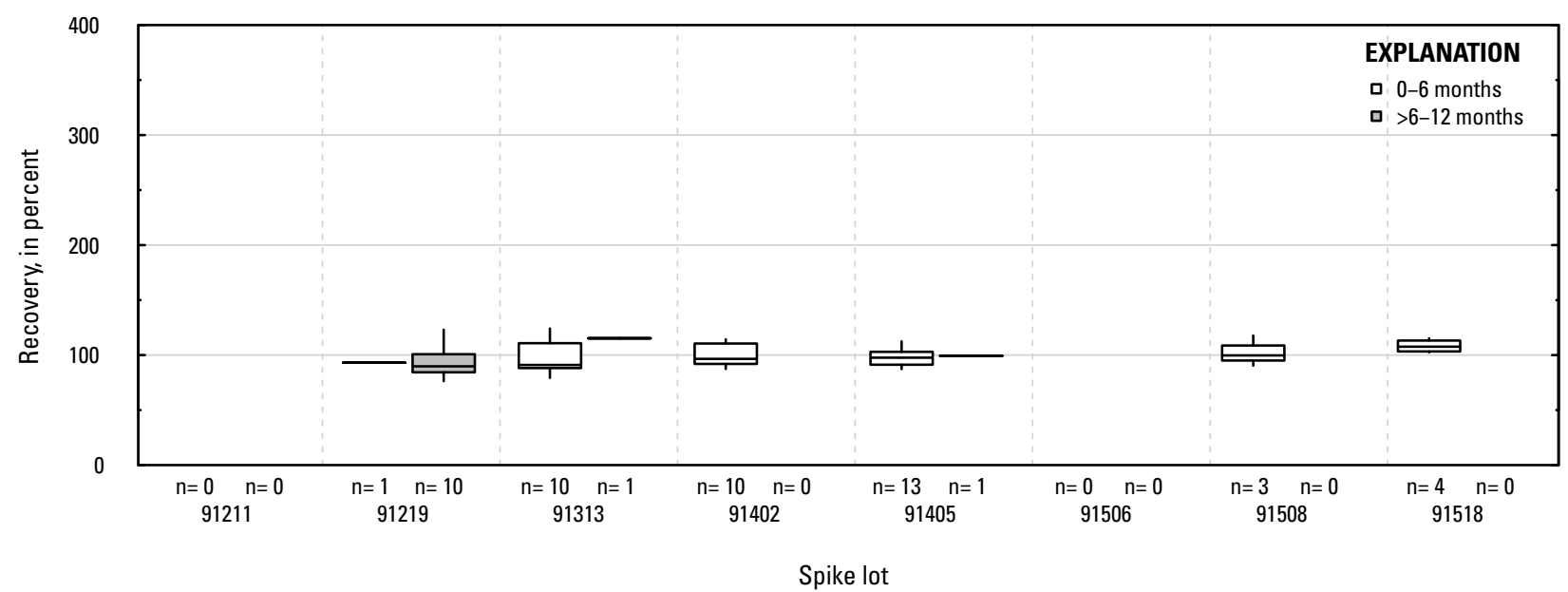

GA. Deisopropyl prometryn: surface water field matrix spikes

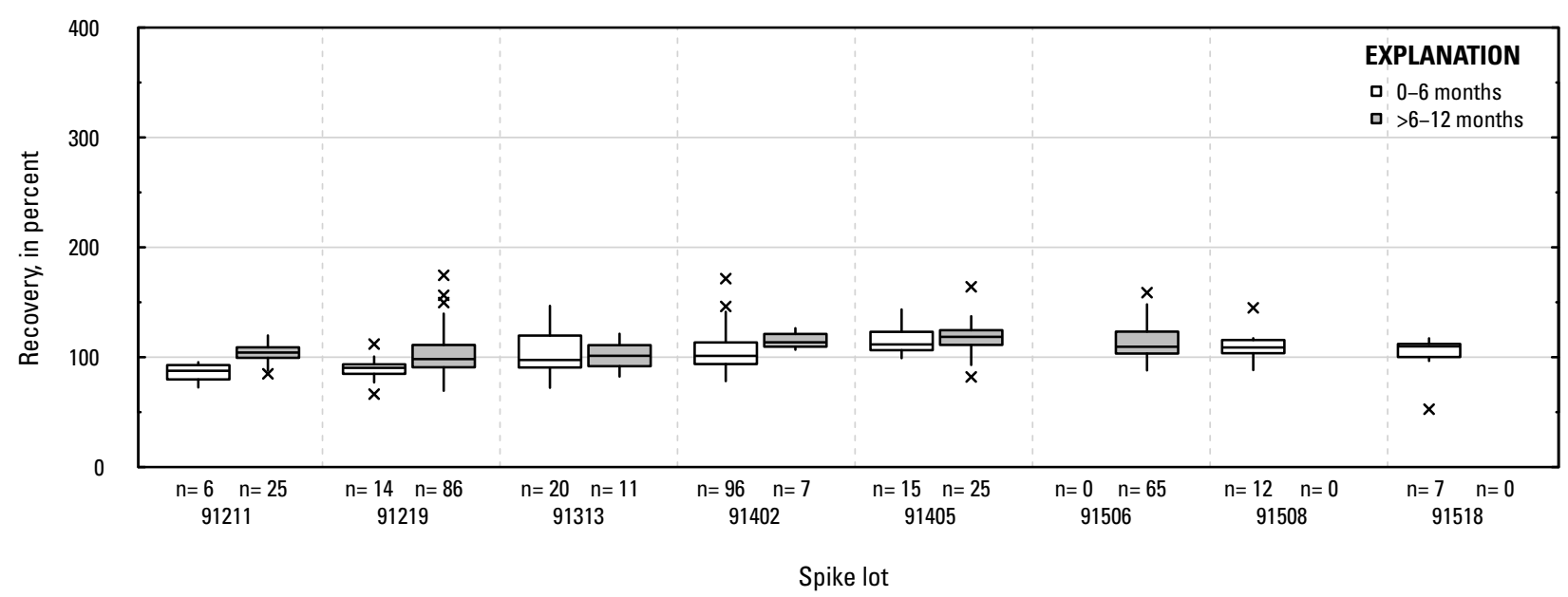

Figure 1-1. Distributions of recovery for individual pesticides in schedule 2437 by matrix, spike lot, and spike lot age. Recovery values larger than 400 percent are not shown.-Continued 
GB. 2-Chloro-6-ethylamino-4-amino-s-triazine \{CEAT\}: laboratory reagent spikes

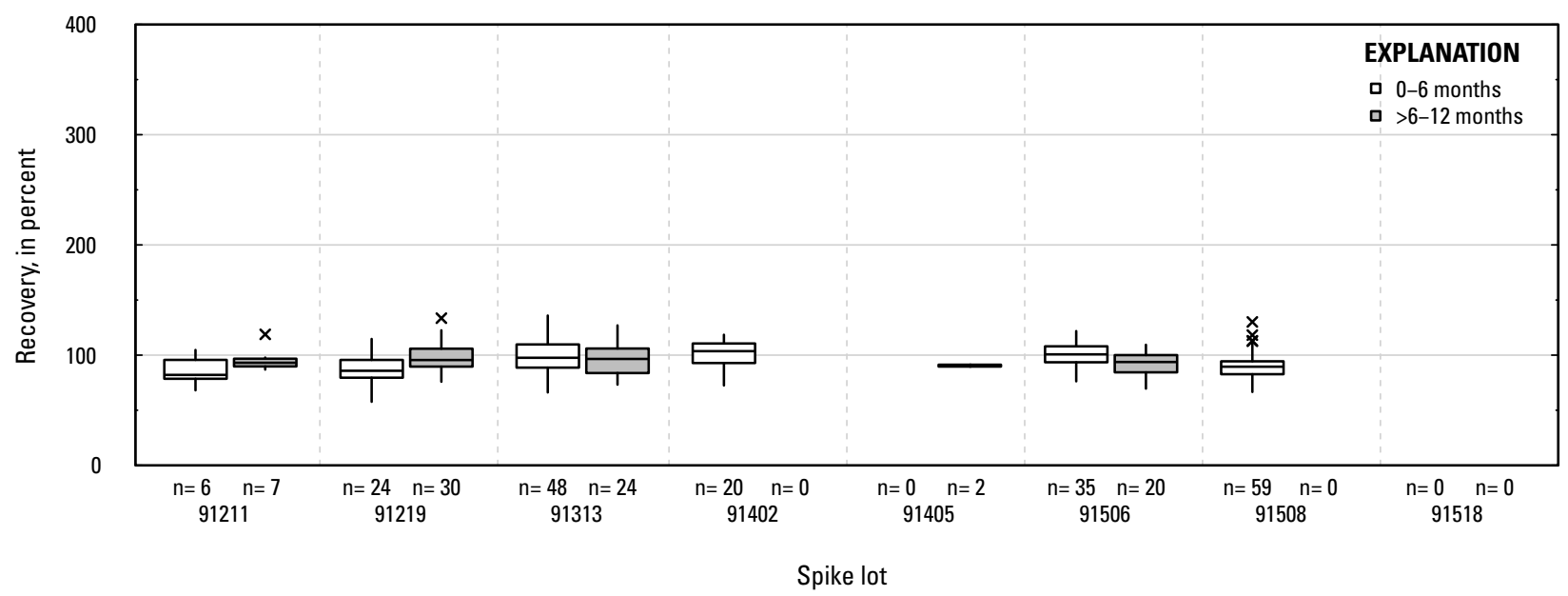

GC. 2-Chloro-6-ethylamino-4-amino-s-triazine \{CEAT\}: groundwater field matrix spikes

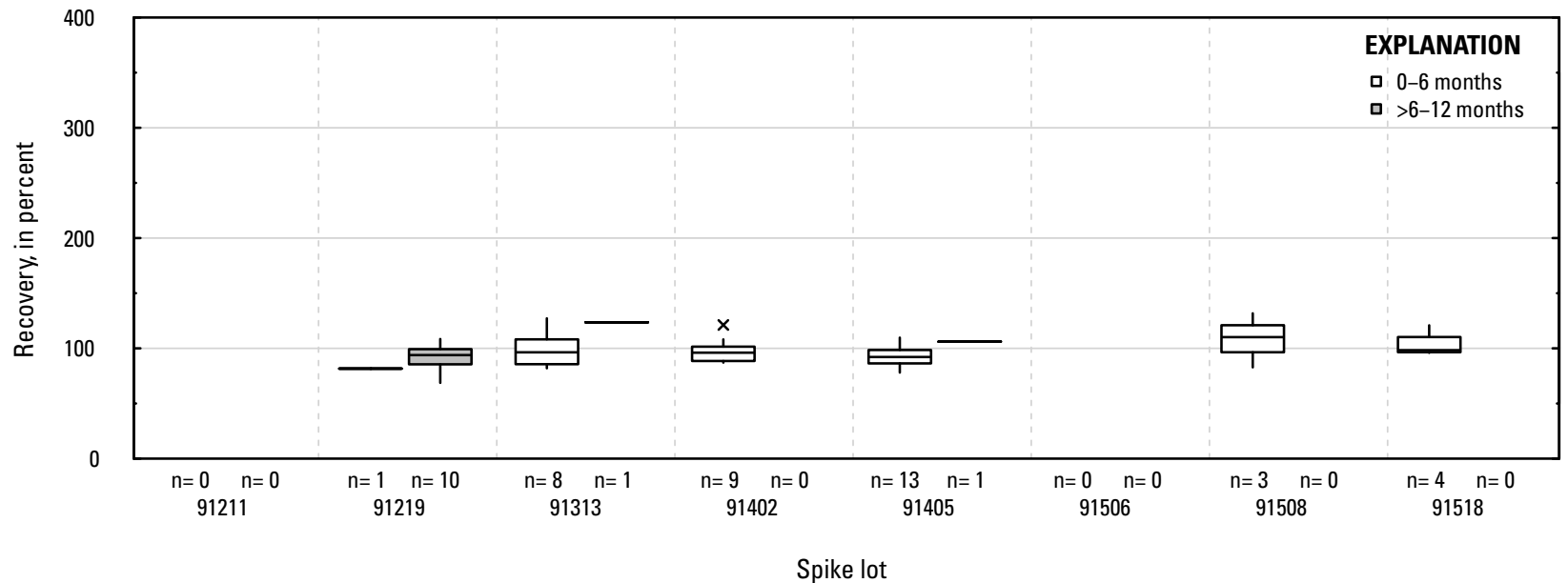

GD. 2-Chloro-6-ethylamino-4-amino-s-triazine \{CEAT\}: surface water field matrix spikes

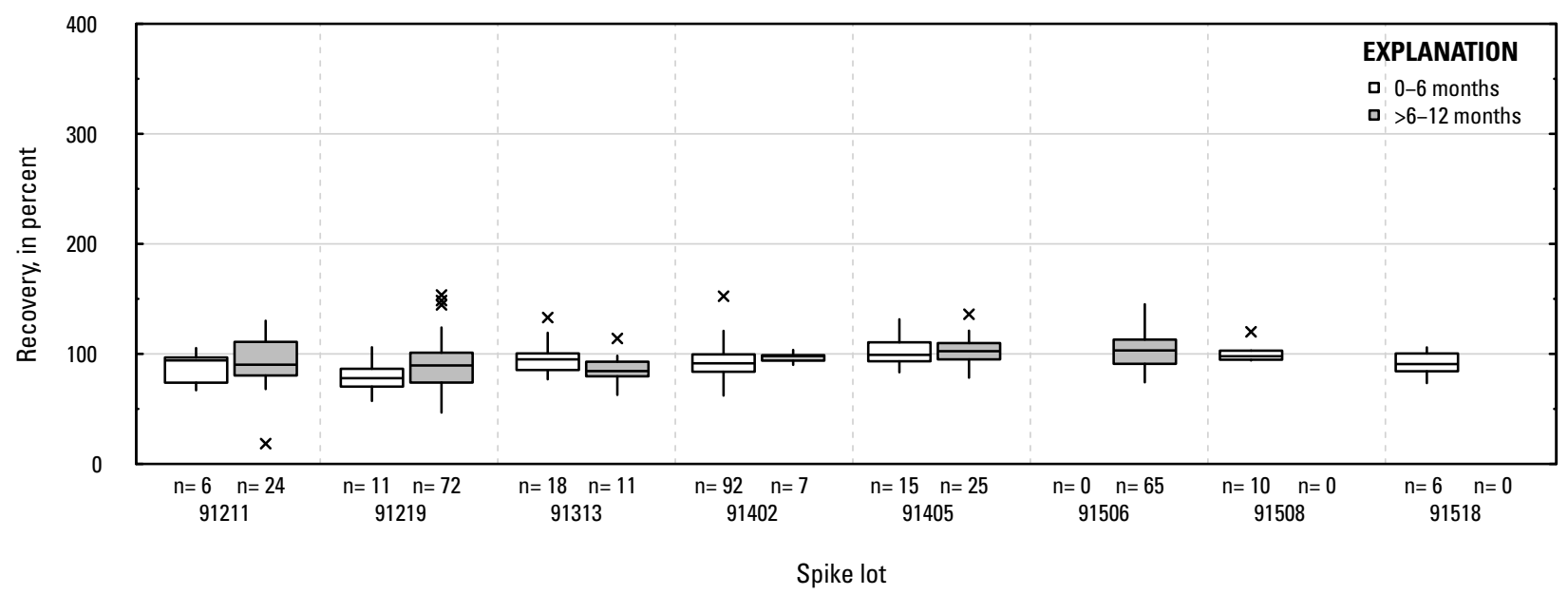

Figure 1-1. Distributions of recovery for individual pesticides in schedule 2437 by matrix, spike lot, and spike lot age. Recovery values larger than 400 percent are not shown.-Continued 
GE. Demethyl fluometuron: laboratory reagent spikes

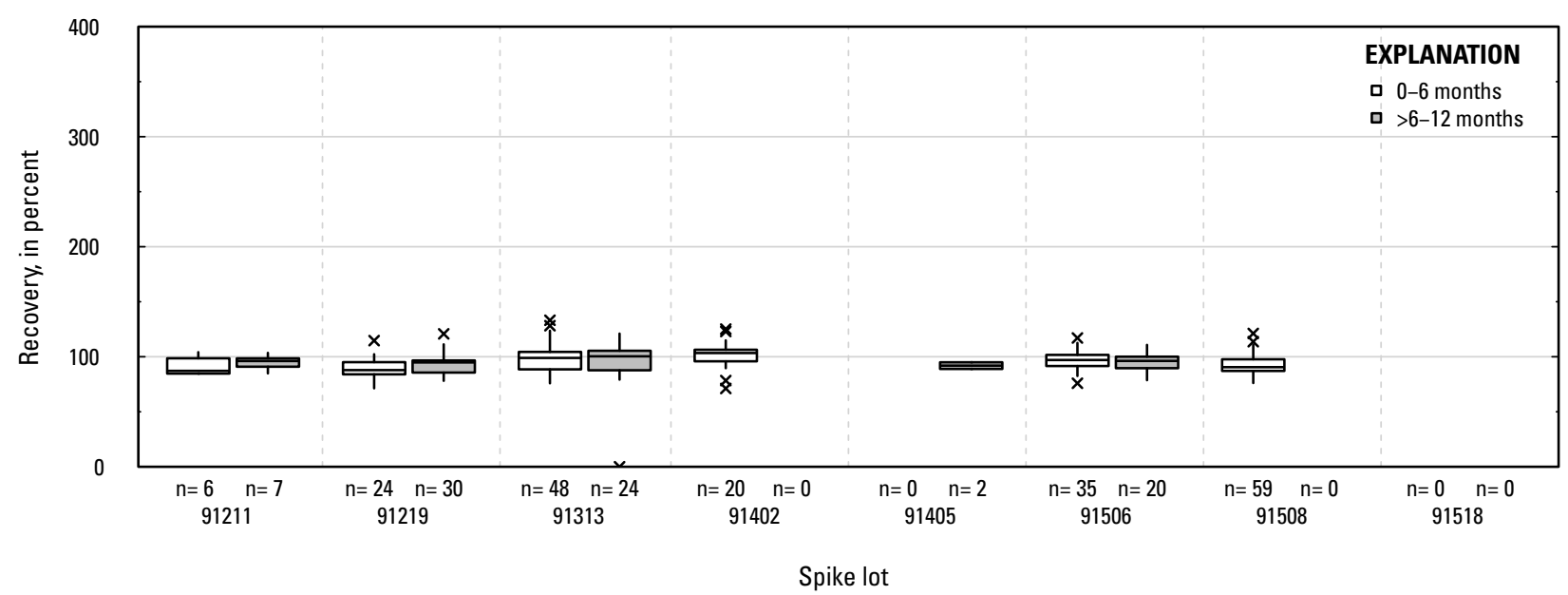

GF. Demethyl fluometuron: groundwater field matrix spikes

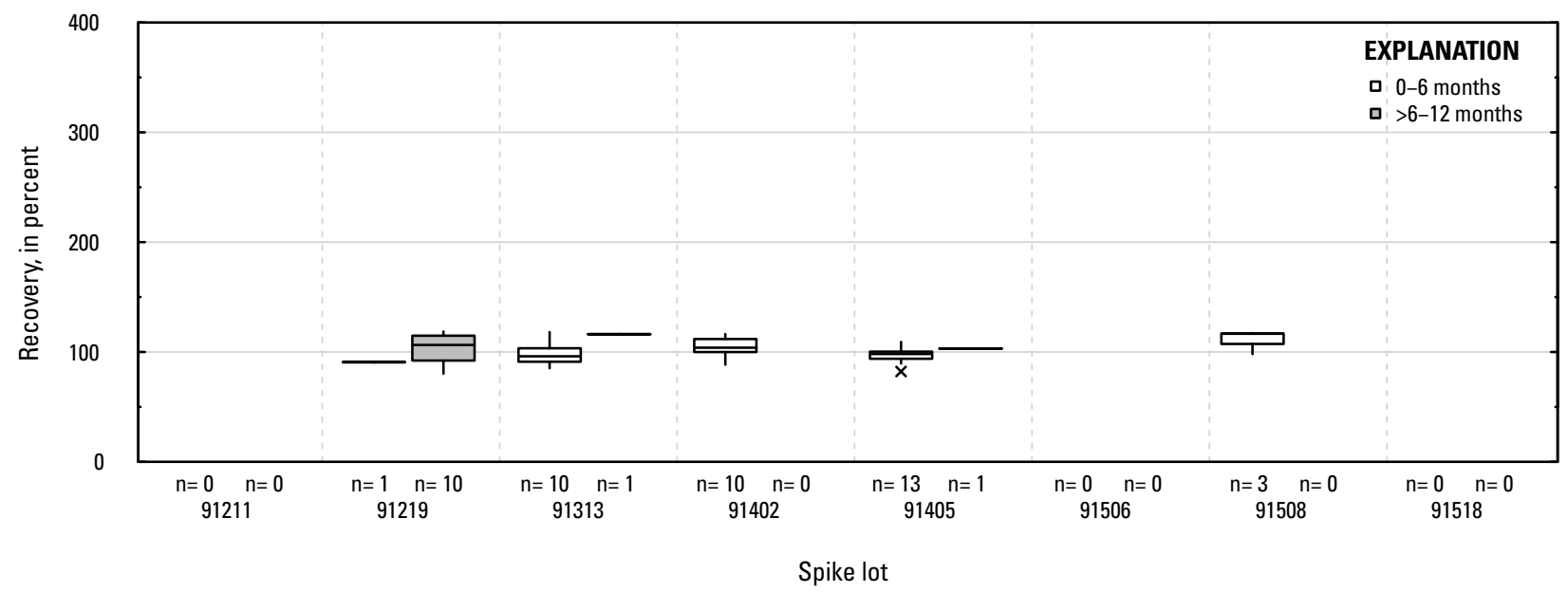

GG. Demethyl fluometuron: surface water field matrix spikes

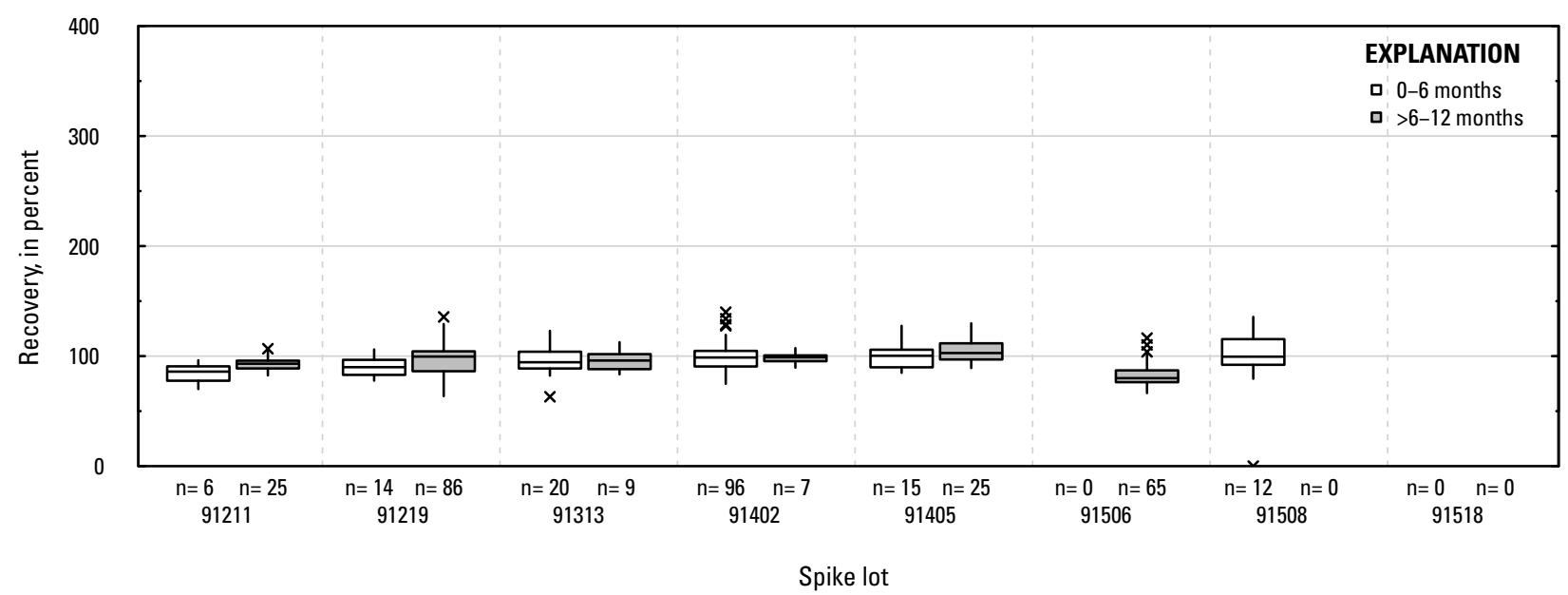

Figure 1-1. Distributions of recovery for individual pesticides in schedule 2437 by matrix, spike lot, and spike lot age. Recovery values larger than 400 percent are not shown.-Continued 
GH. Demethyl hexazinone B: laboratory reagent spikes

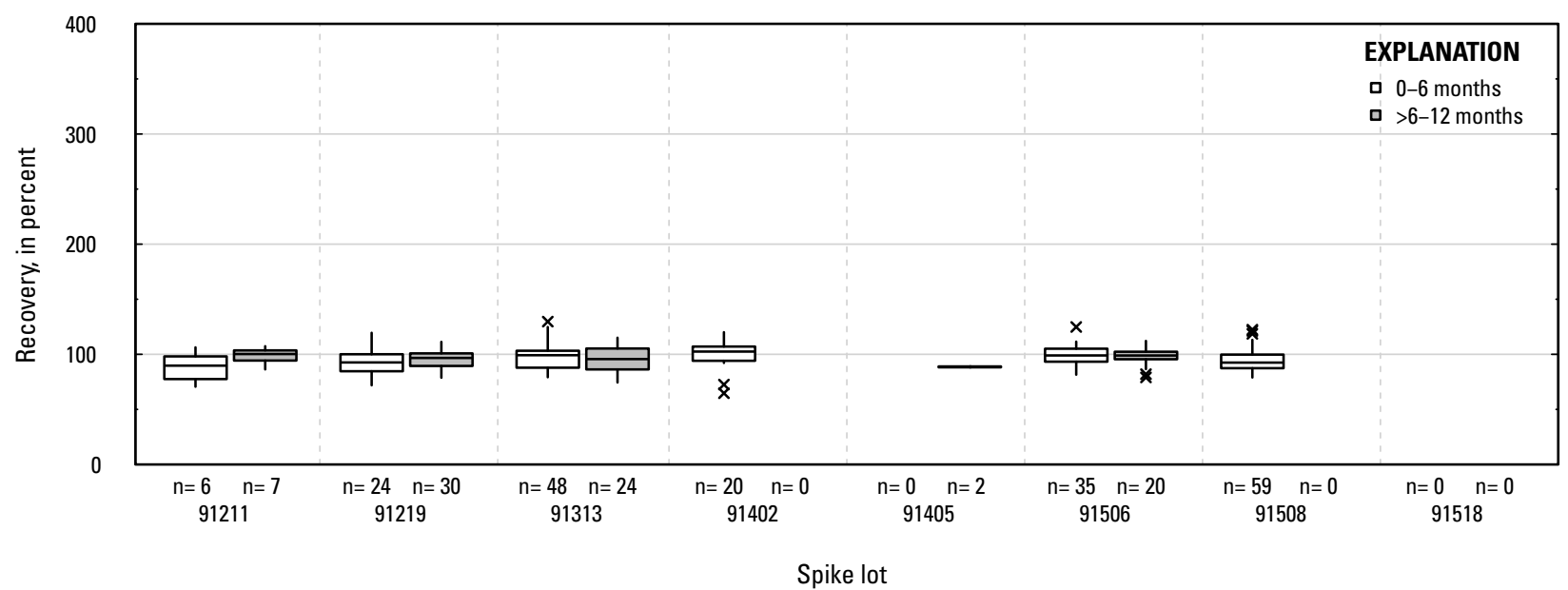

\section{GI. Demethyl hexazinone B: groundwater field matrix spikes}

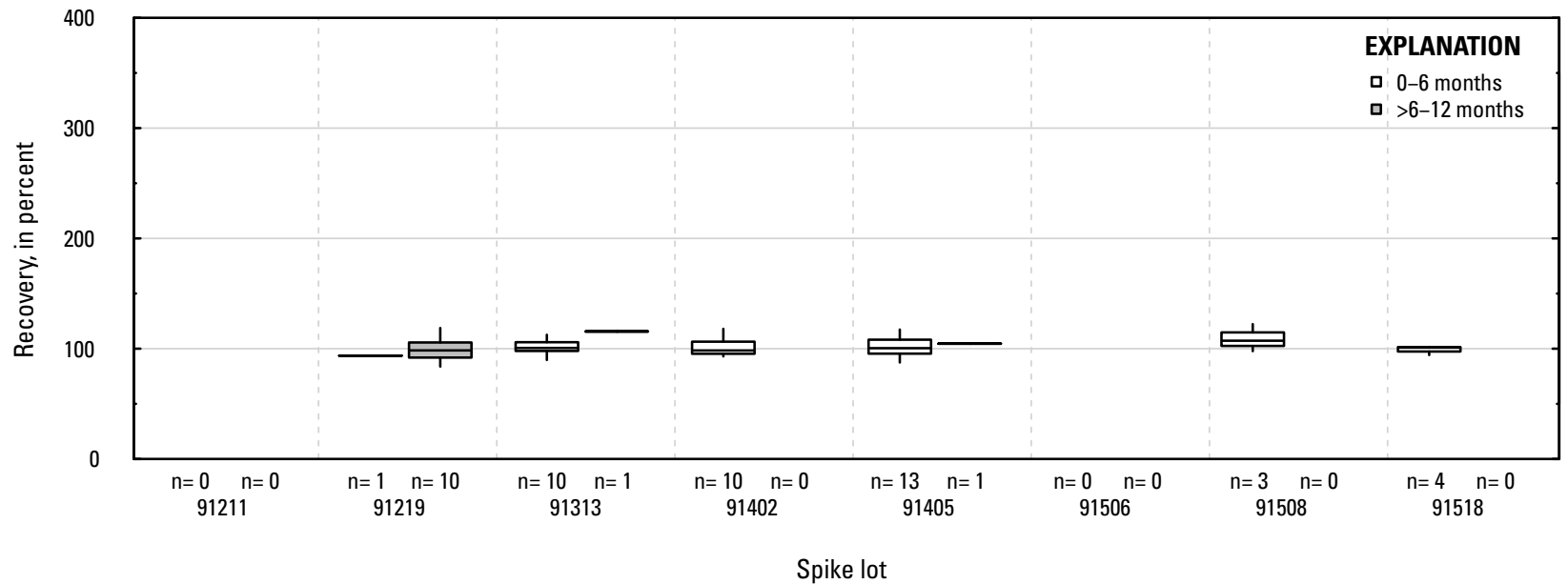

\section{GJ. Demethyl hexazinone B: surface water field matrix spikes}

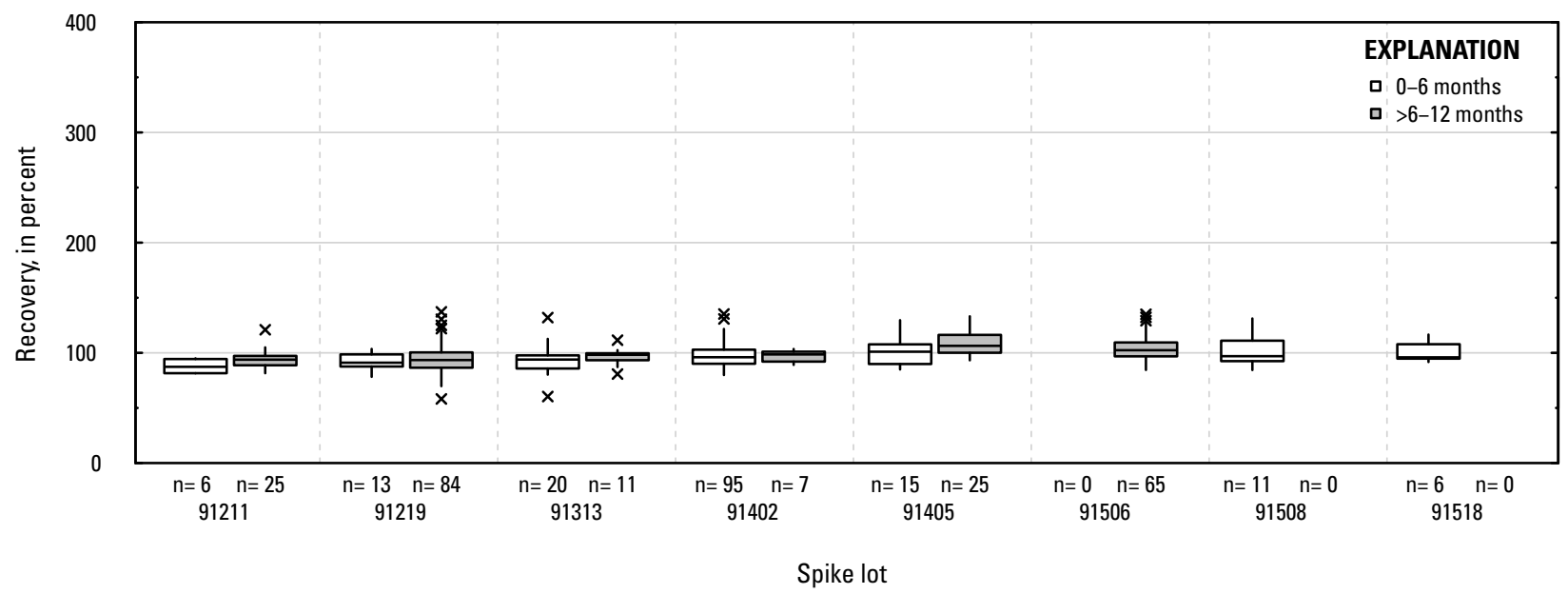

Figure 1-1. Distributions of recovery for individual pesticides in schedule 2437 by matrix, spike lot, and spike lot age. Recovery values larger than 400 percent are not shown.-Continued 
GK. Demethyl norflurazon: laboratory reagent spikes

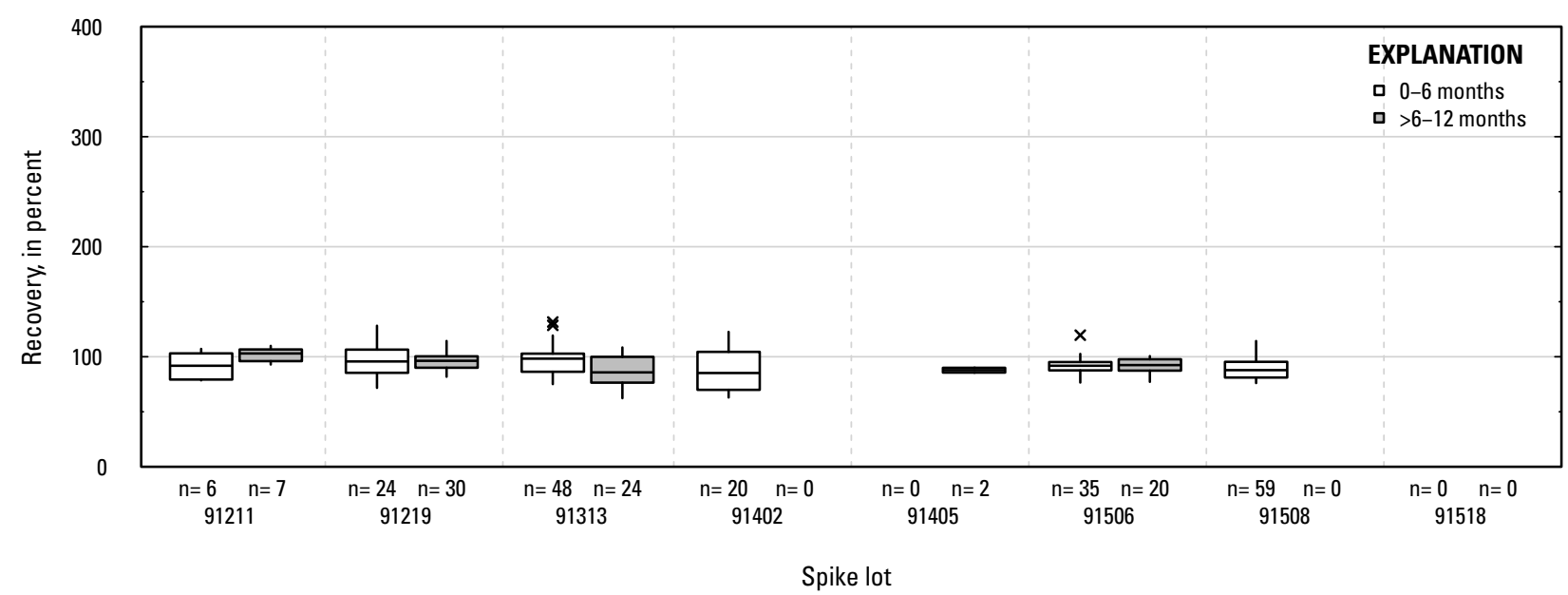

GL. Demethyl norflurazon: groundwater field matrix spikes

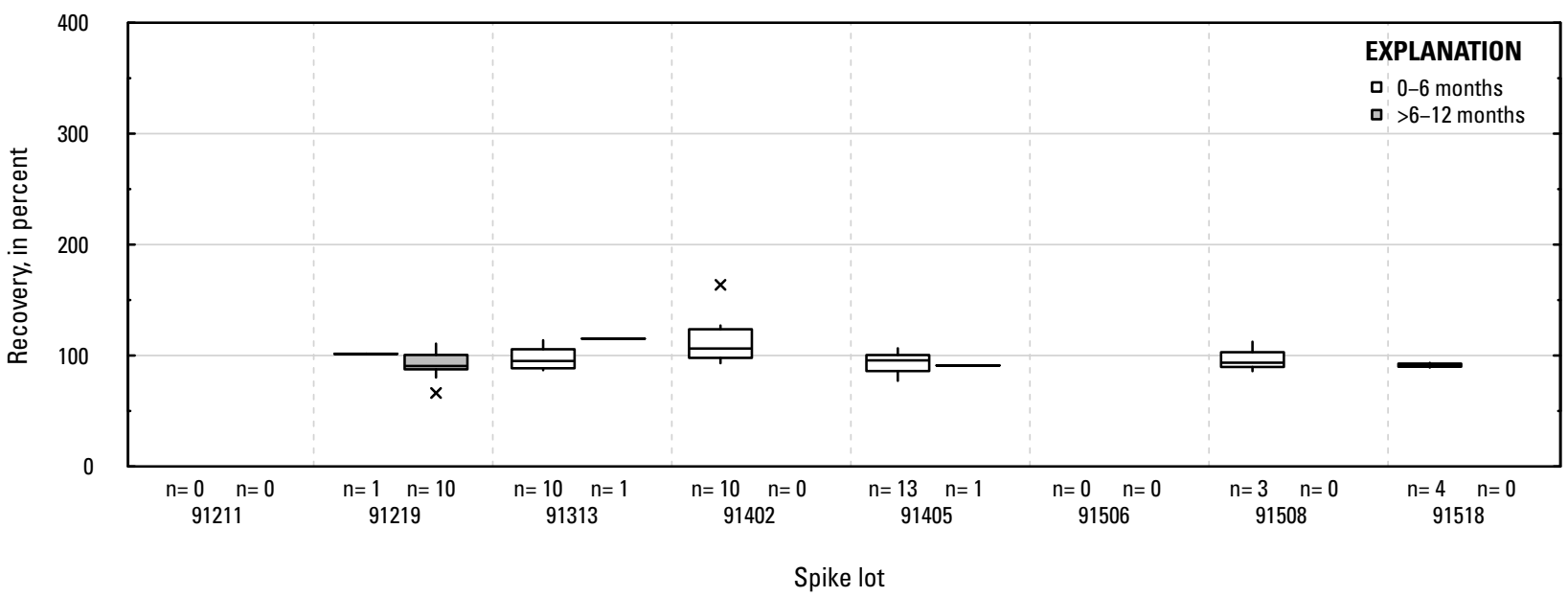

GM. Demethyl norflurazon: surface water field matrix spikes

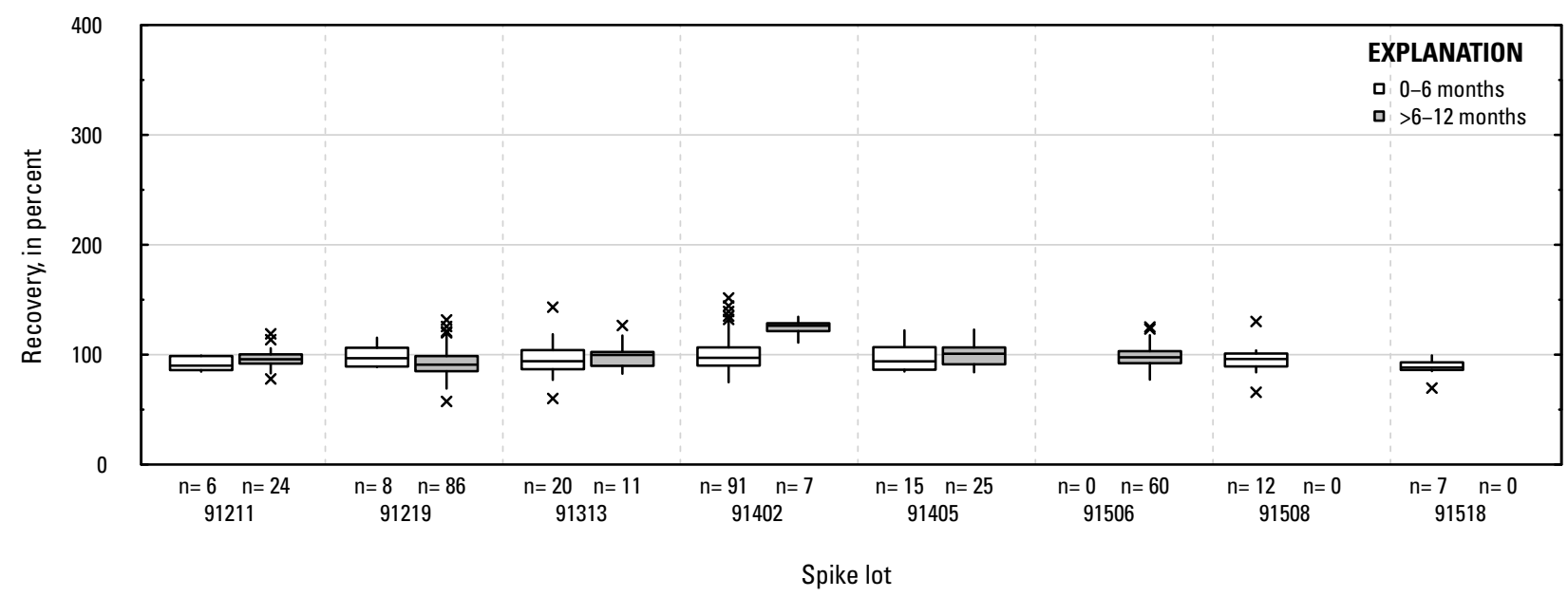

Figure 1-1. Distributions of recovery for individual pesticides in schedule 2437 by matrix, spike lot, and spike lot age. Recovery values larger than 400 percent are not shown.-Continued 
GN. Deiodo flubendiamide: laboratory reagent spikes

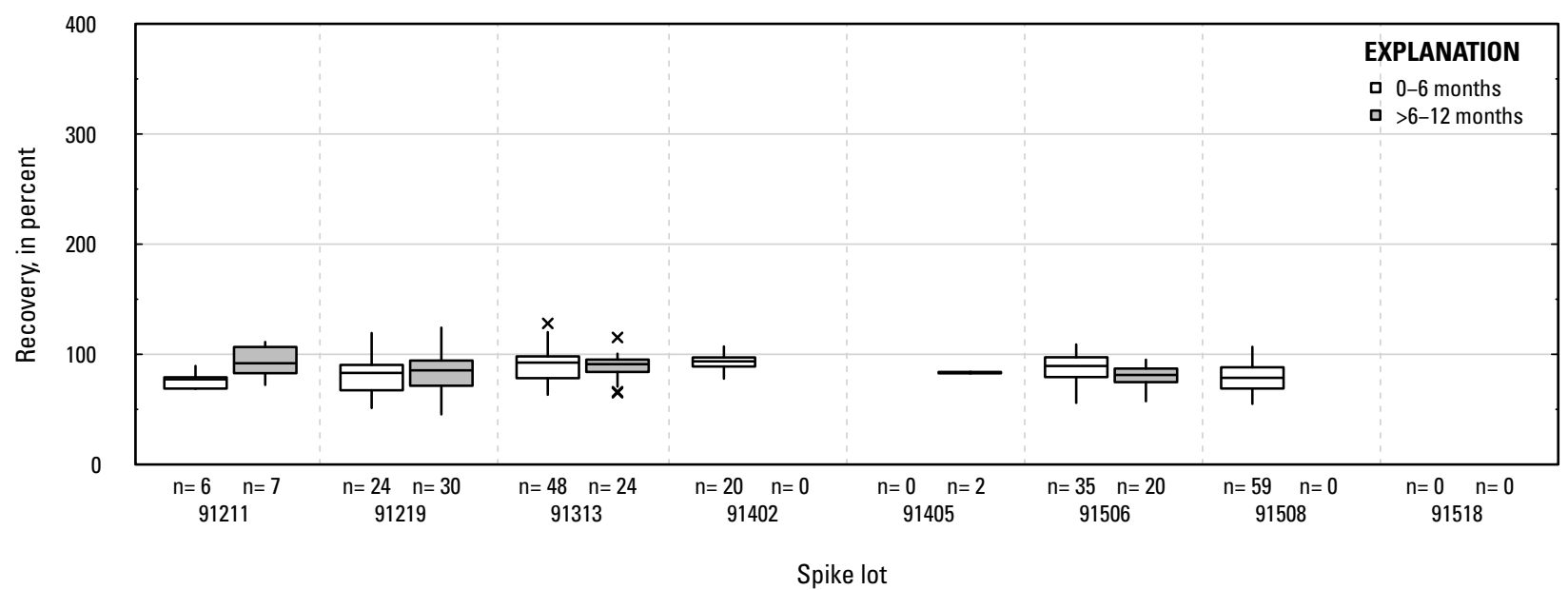

GO. Deiodo flubendiamide: groundwater field matrix spikes

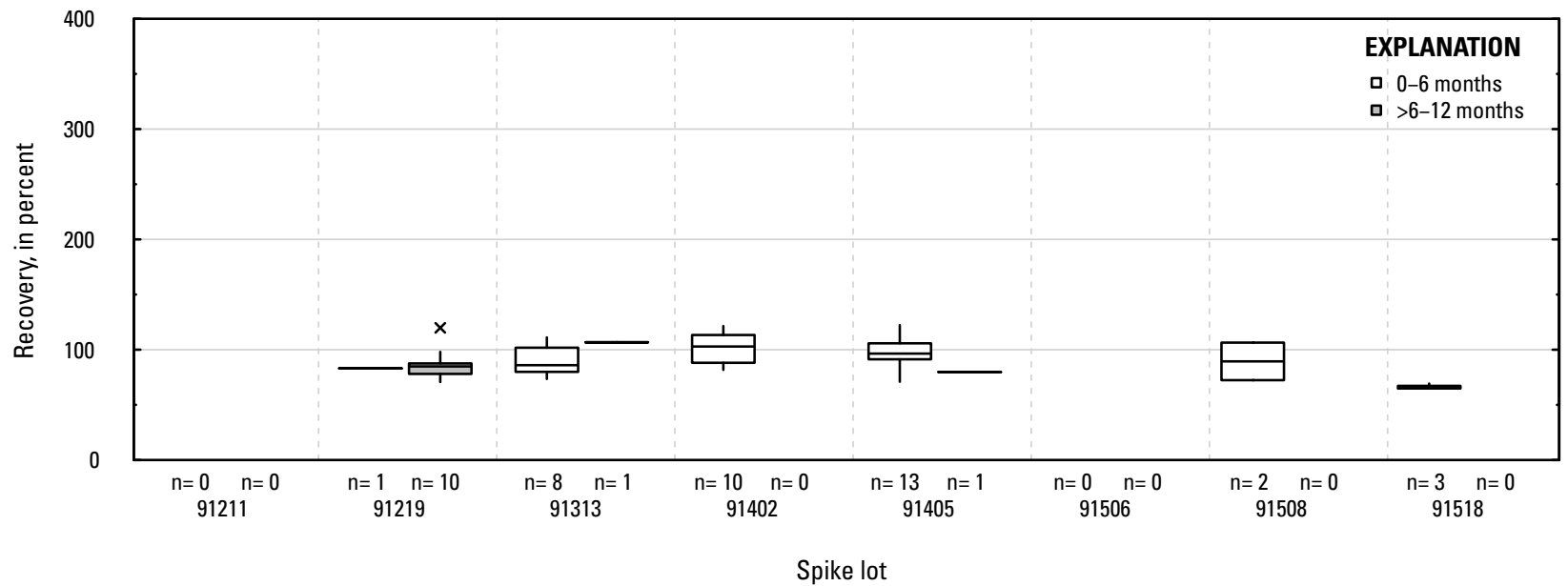

GP. Deiodo flubendiamide: surface water field matrix spikes

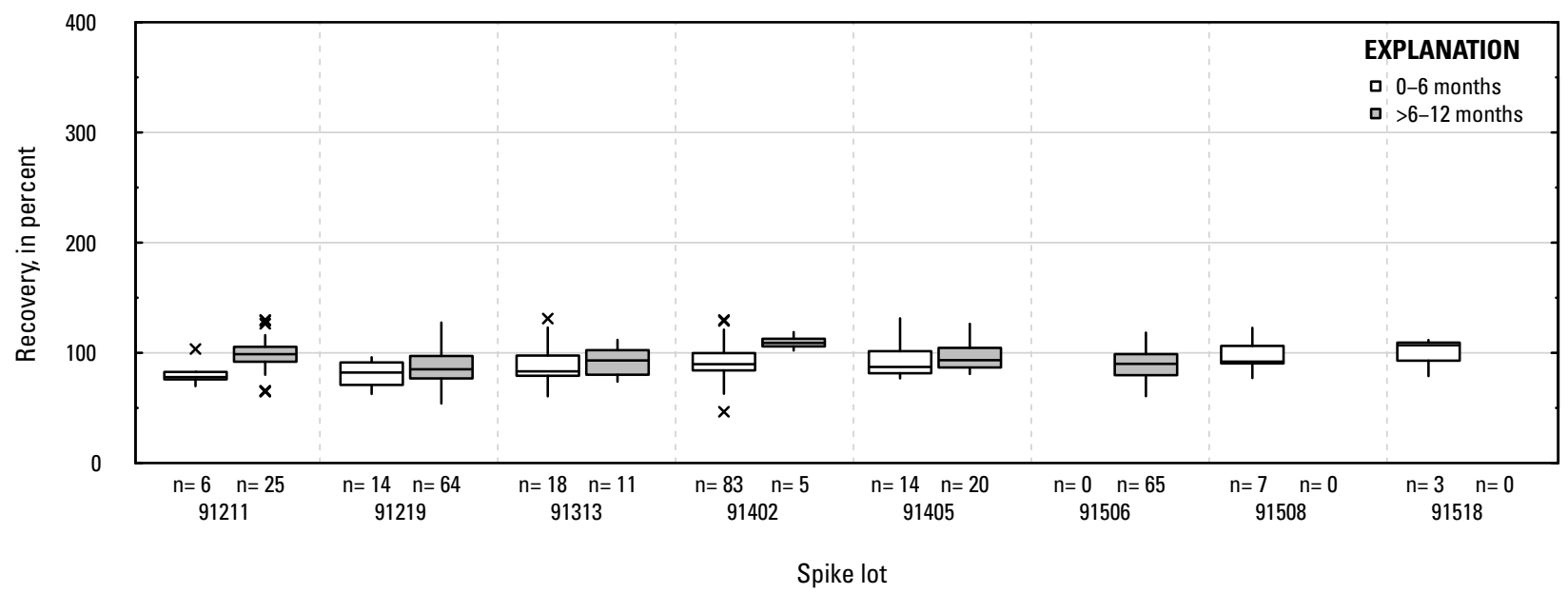

Figure 1-1. Distributions of recovery for individual pesticides in schedule 2437 by matrix, spike lot, and spike lot age. Recovery values larger than 400 percent are not shown.-Continued 
GO. Desulfinylfipronil: laboratory reagent spikes

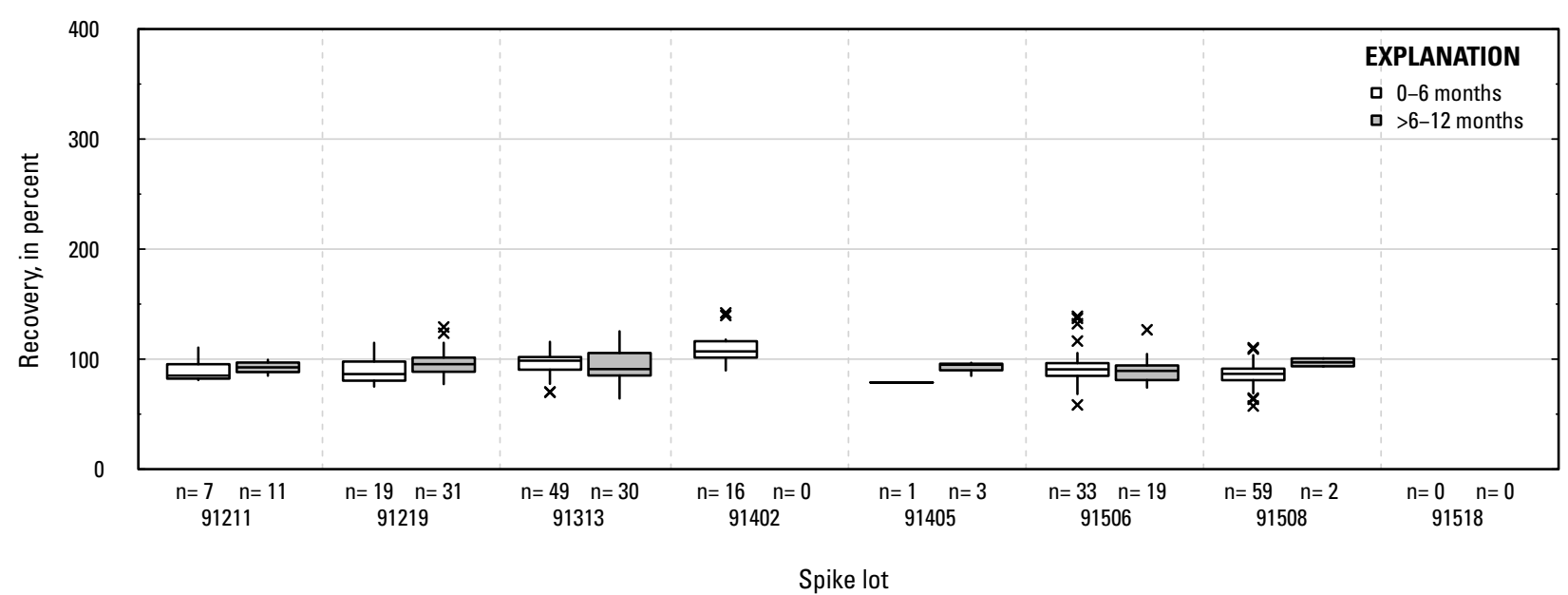

GR. Desulfinylfipronil: groundwater field matrix spikes

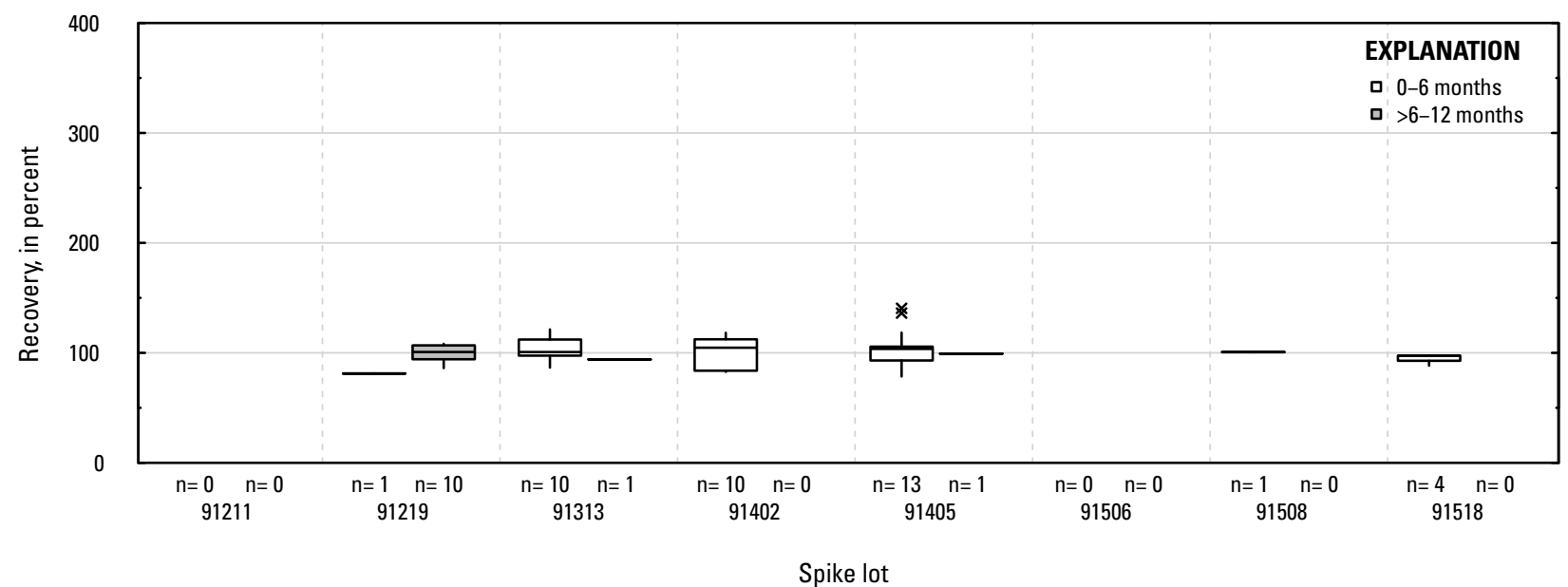

GS. Desulfinylfipronil: surface water field matrix spikes

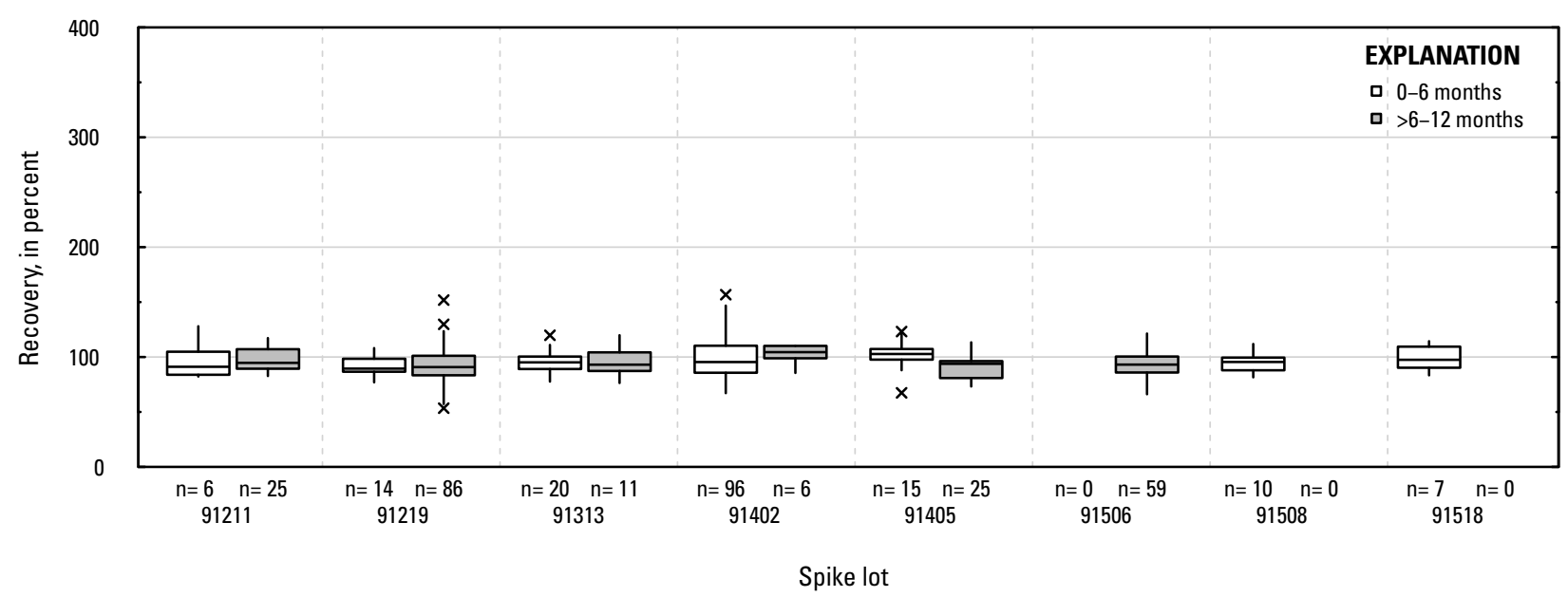

Figure 1-1. Distributions of recovery for individual pesticides in schedule 2437 by matrix, spike lot, and spike lot age. Recovery values larger than 400 percent are not shown.-Continued 


\section{GT. Desulfinylfipronil amide: laboratory reagent spikes}

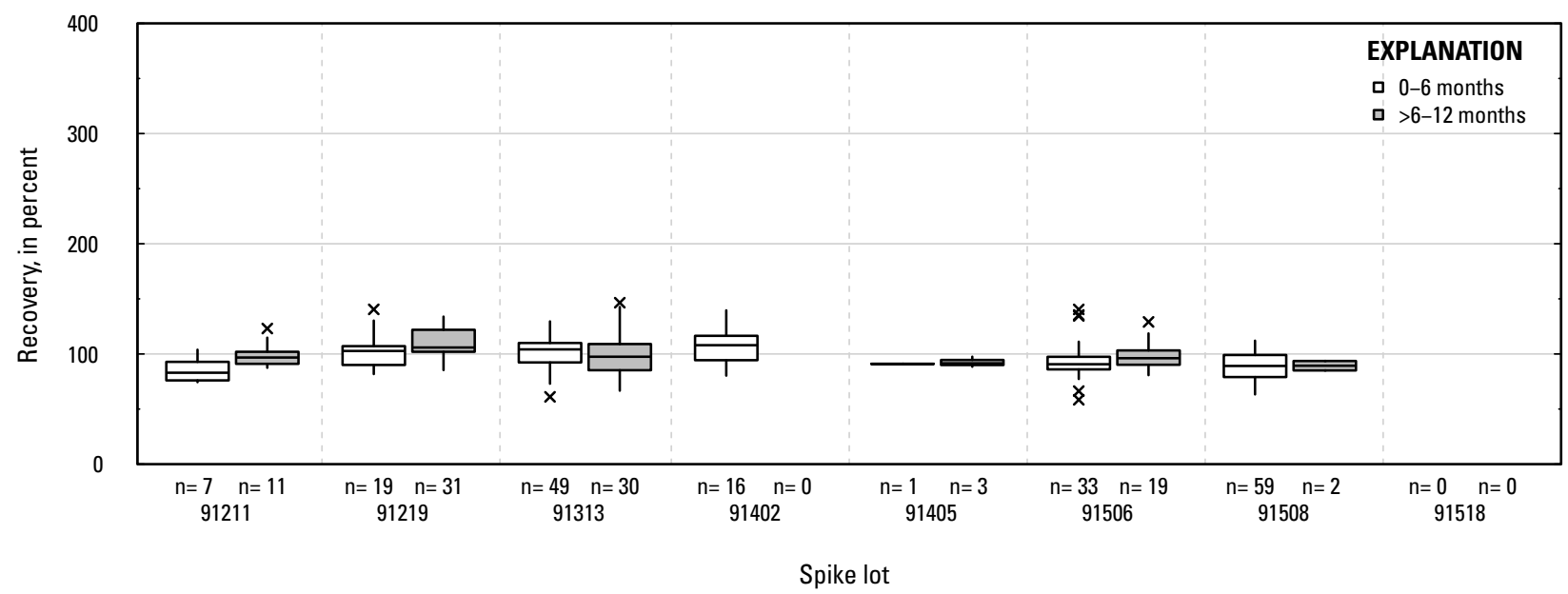

GU. Desulfinylfipronil amide: groundwater field matrix spikes

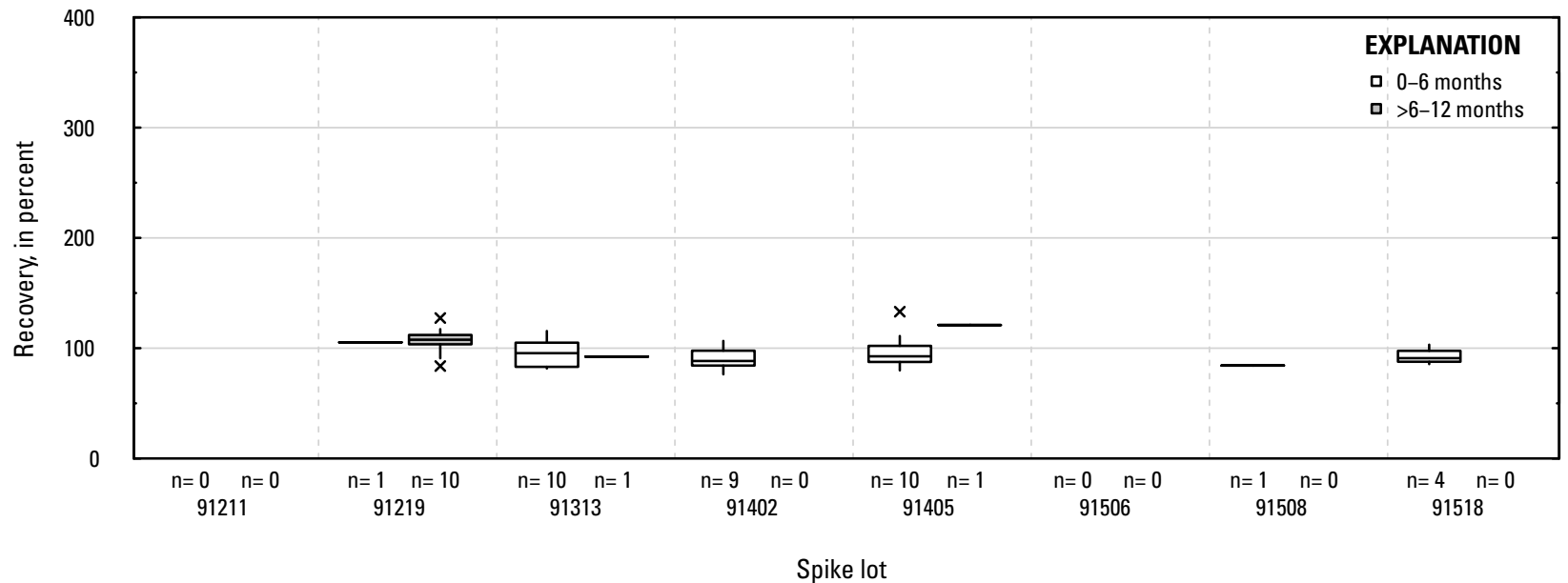

GV. Desulfinylfipronil amide: surface water field matrix spikes

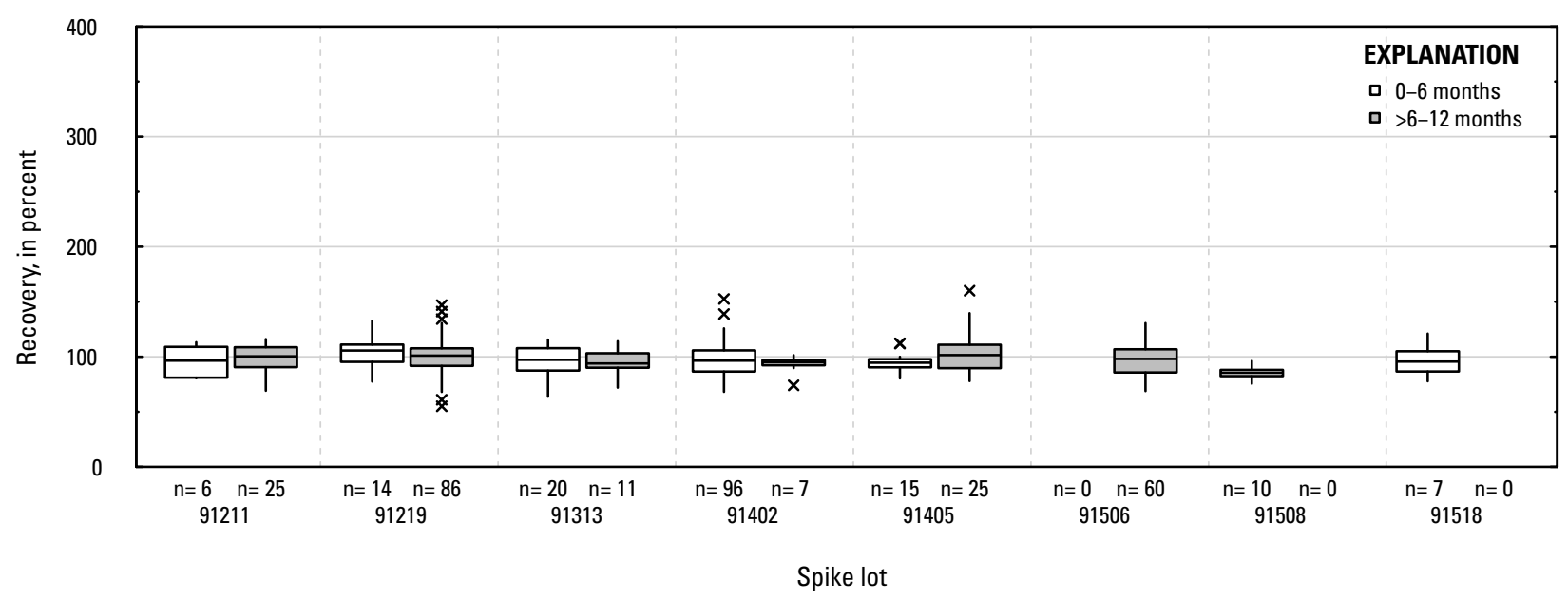

Figure 1-1. Distributions of recovery for individual pesticides in schedule 2437 by matrix, spike lot, and spike lot age. Recovery values larger than 400 percent are not shown.-Continued 
GW. Diazinon: laboratory reagent spikes

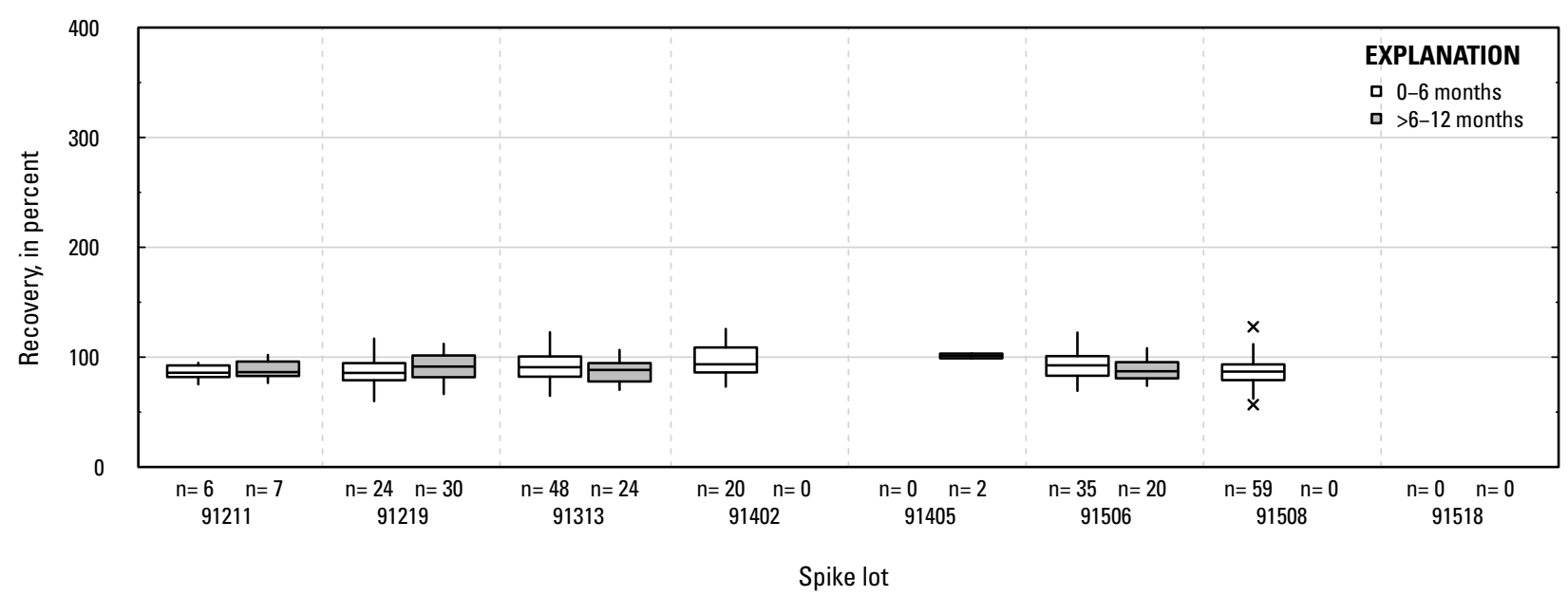

GX. Diazinon: groundwater field matrix spikes

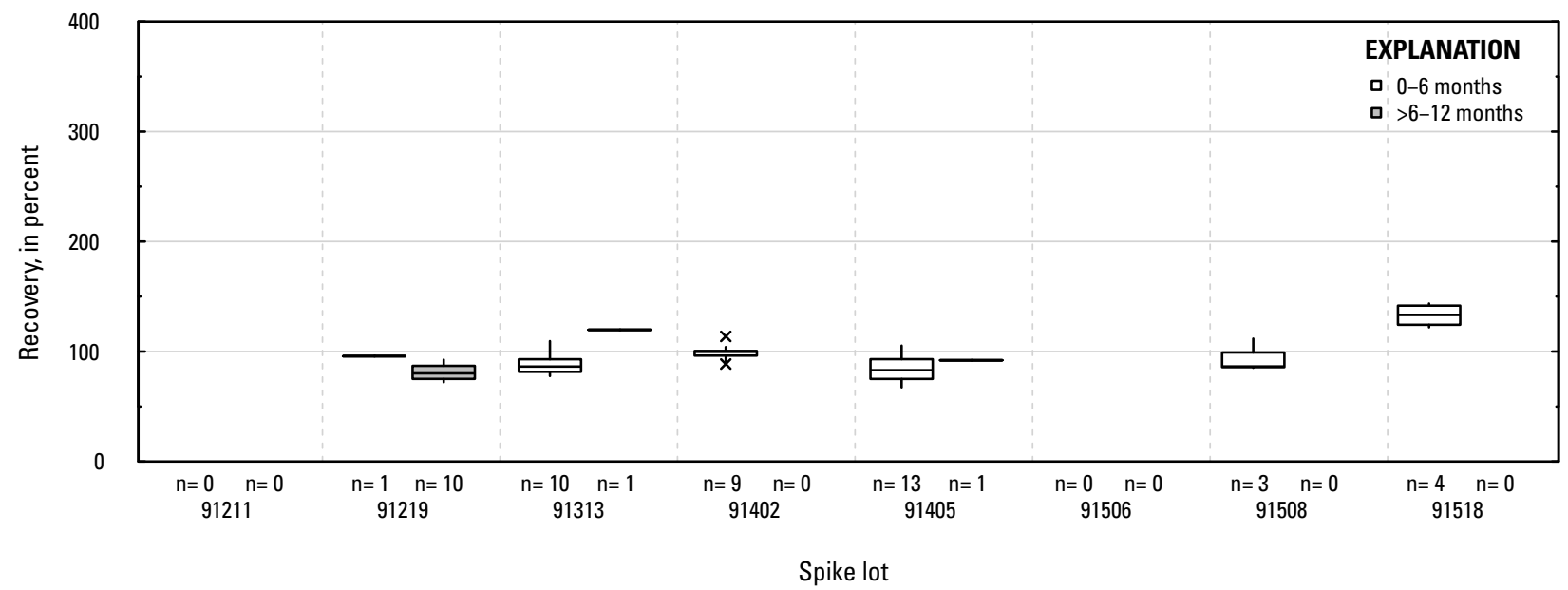

GY. Diazinon: surface water field matrix spikes

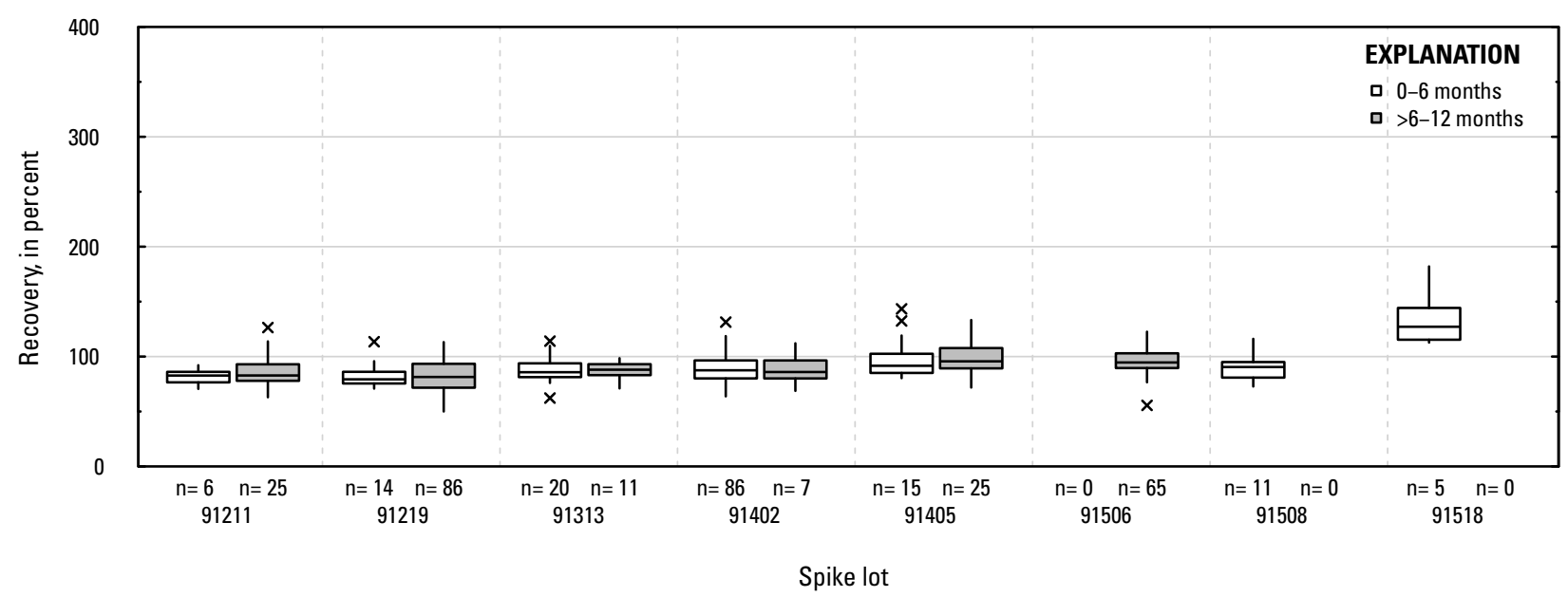

Figure 1-1. Distributions of recovery for individual pesticides in schedule 2437 by matrix, spike lot, and spike lot age. Recovery values larger than 400 percent are not shown.-Continued 


\section{GZ. Diazinon oxon: laboratory reagent spikes}

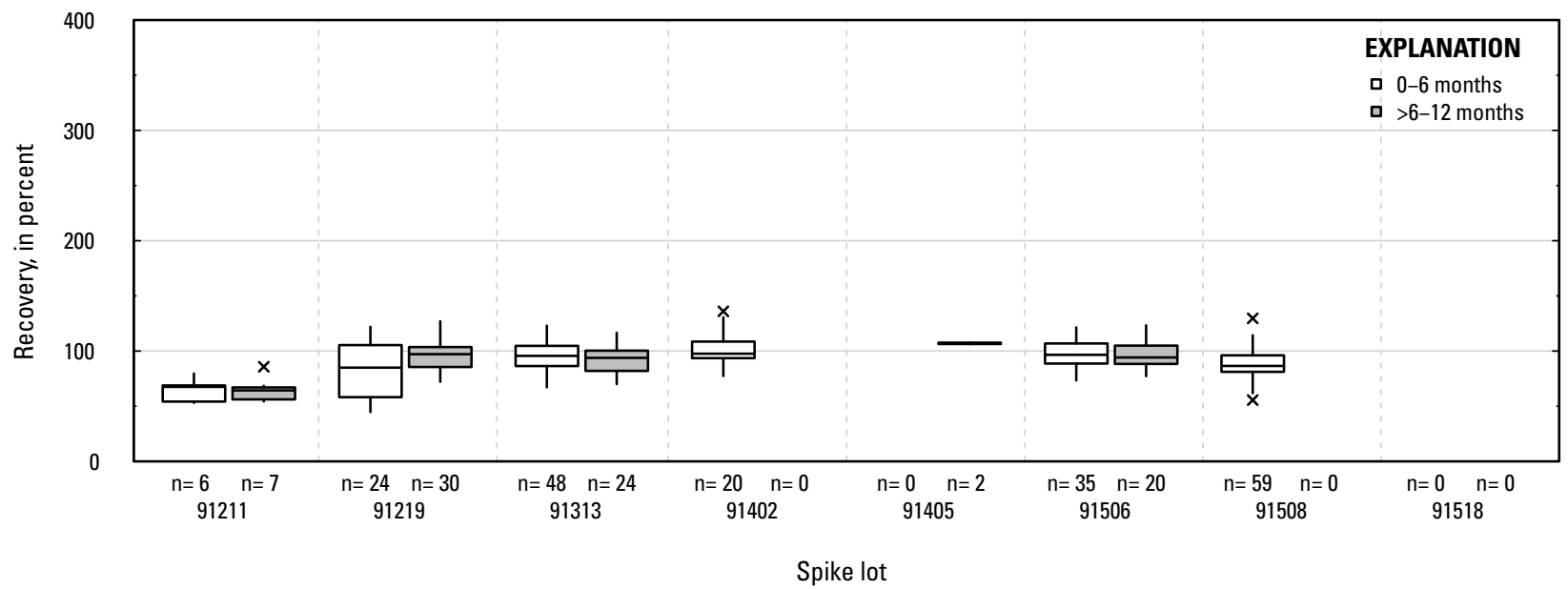

\section{HA. Diazinon oxon: groundwater field matrix spikes}

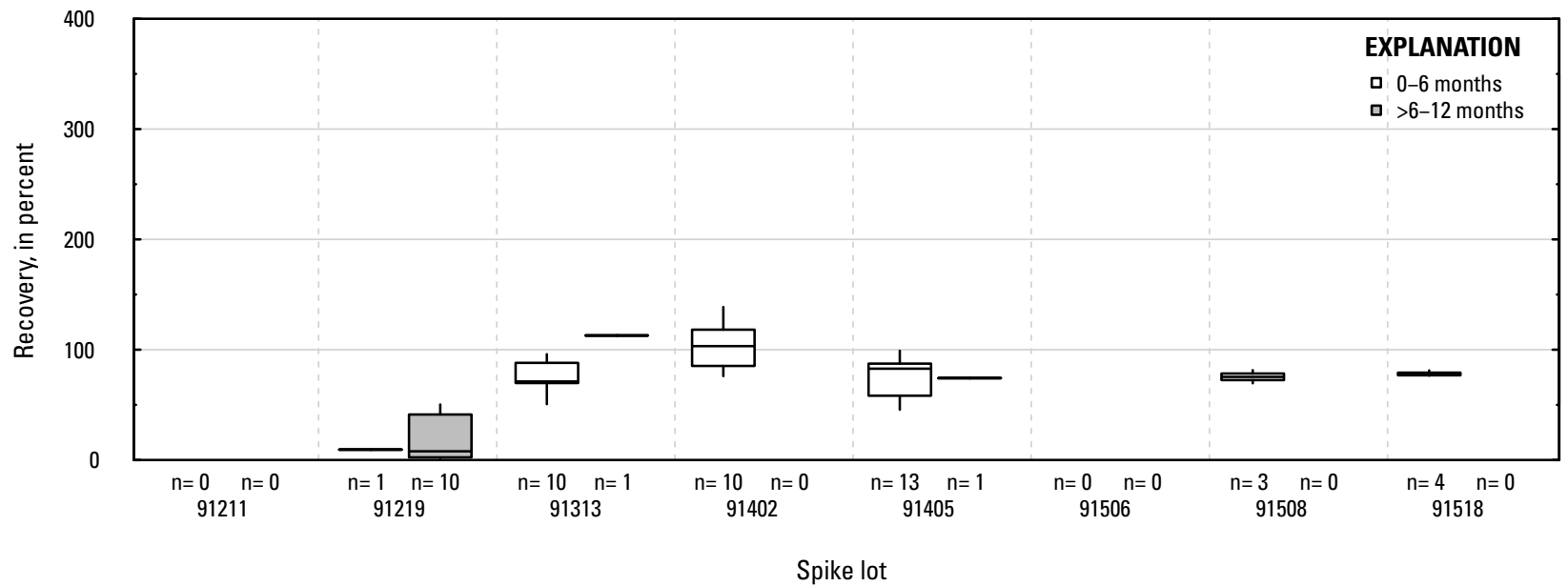

HB. Diazinon oxon: surface water field matrix spikes

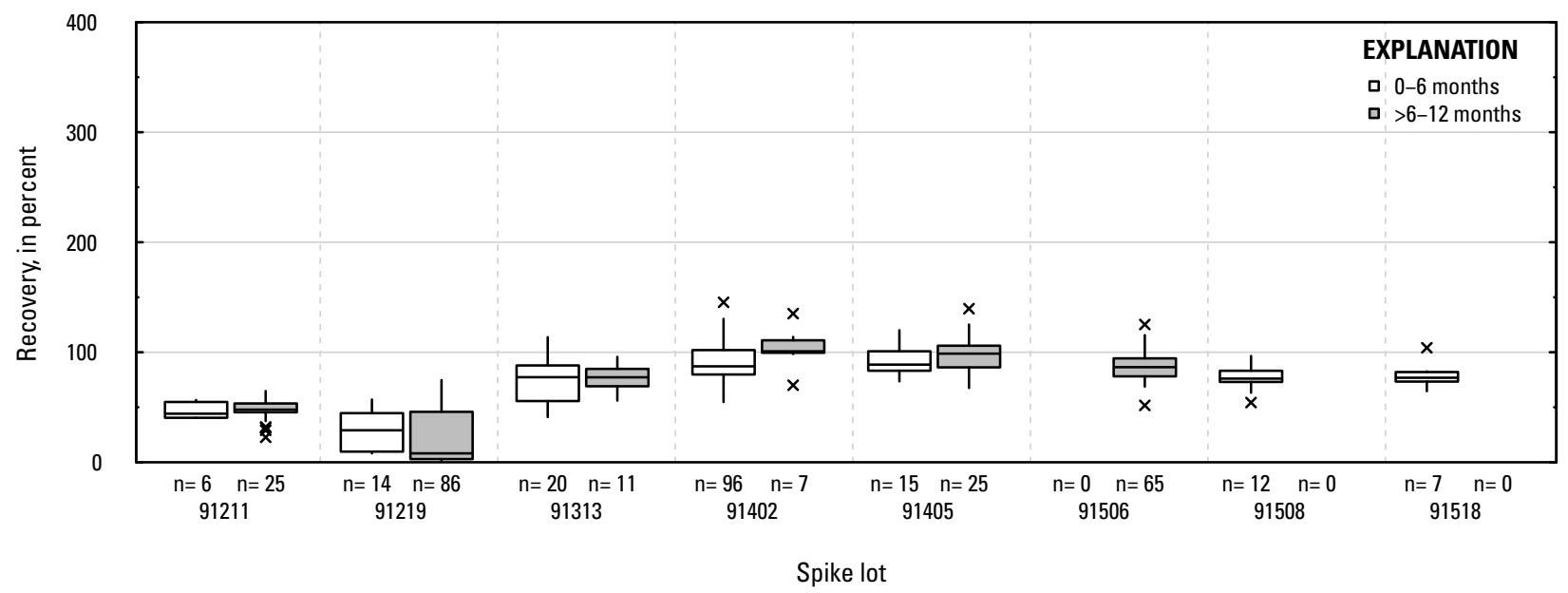

Figure 1-1. Distributions of recovery for individual pesticides in schedule 2437 by matrix, spike lot, and spike lot age. Recovery values larger than 400 percent are not shown.-Continued 
HC. Dicamba: laboratory reagent spikes

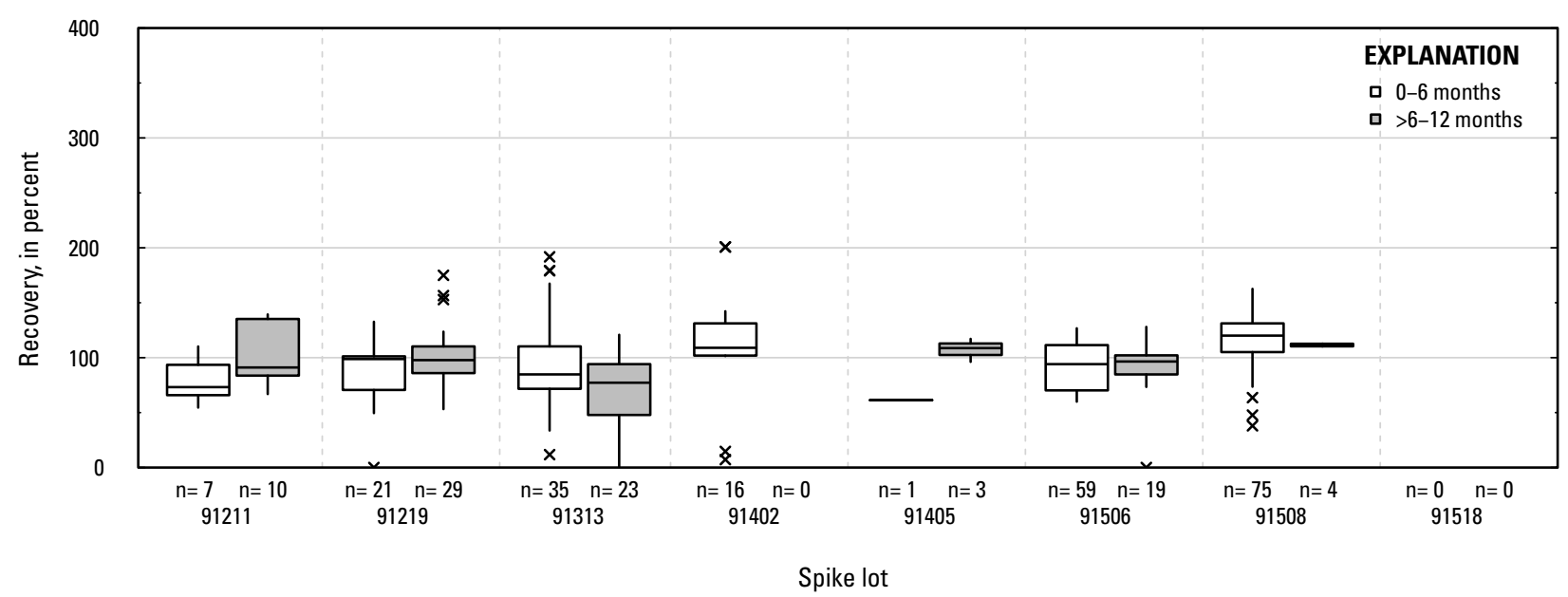

HD. Dicamba: groundwater field matrix spikes

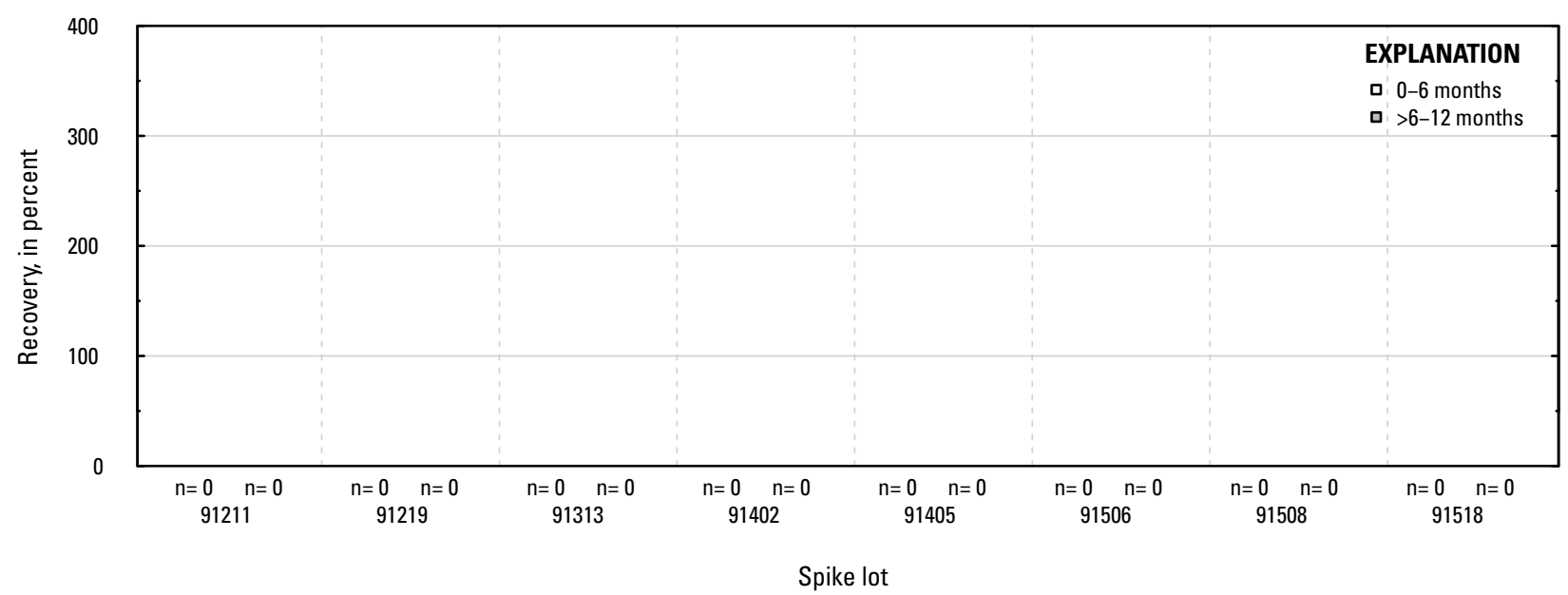

HE. Dicamba: surface water field matrix spikes

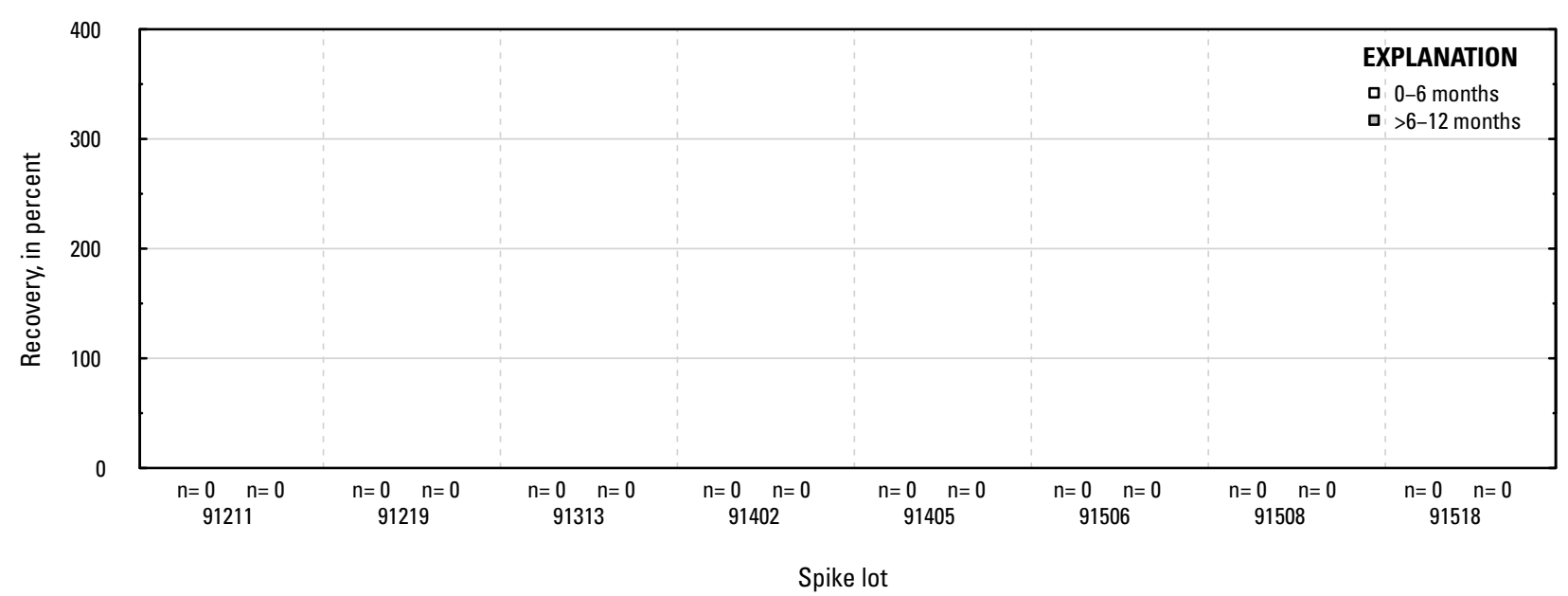

Figure 1-1. Distributions of recovery for individual pesticides in schedule 2437 by matrix, spike lot, and spike lot age. Recovery values larger than 400 percent are not shown.-Continued 
HF. Dichlorvos: laboratory reagent spikes

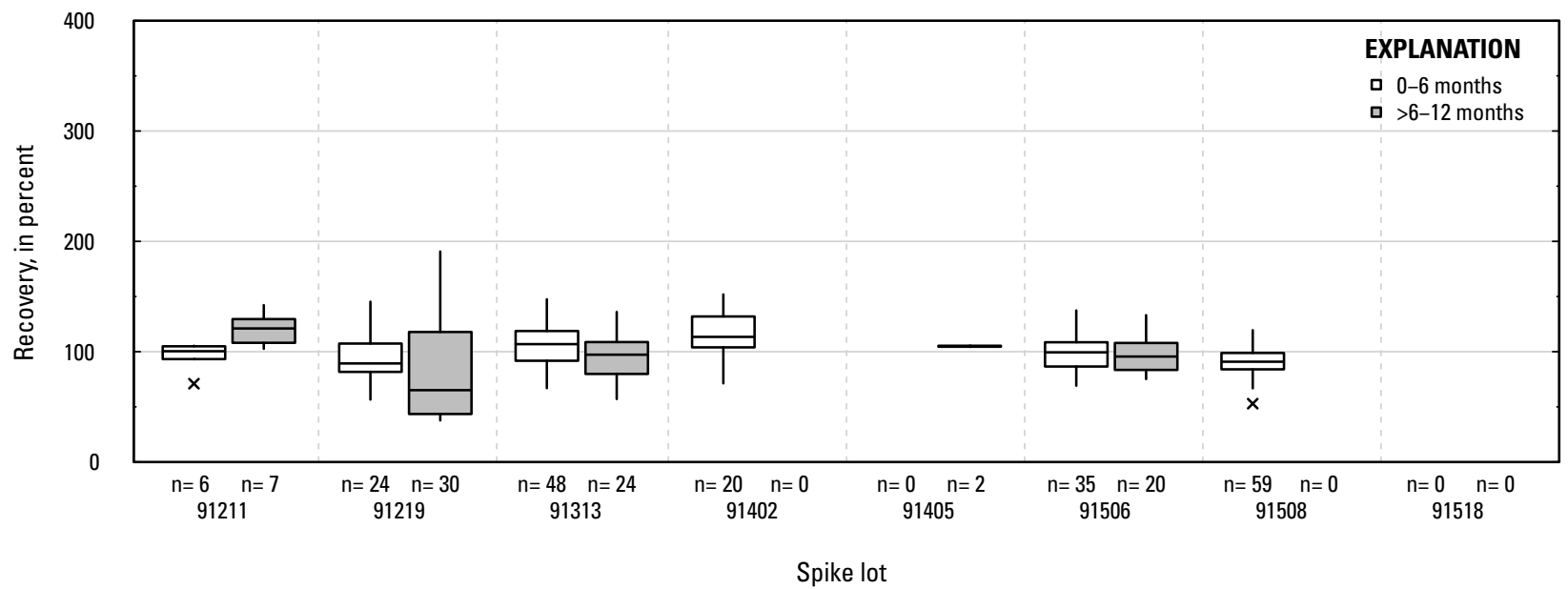

HG. Dichlorvos: groundwater field matrix spikes

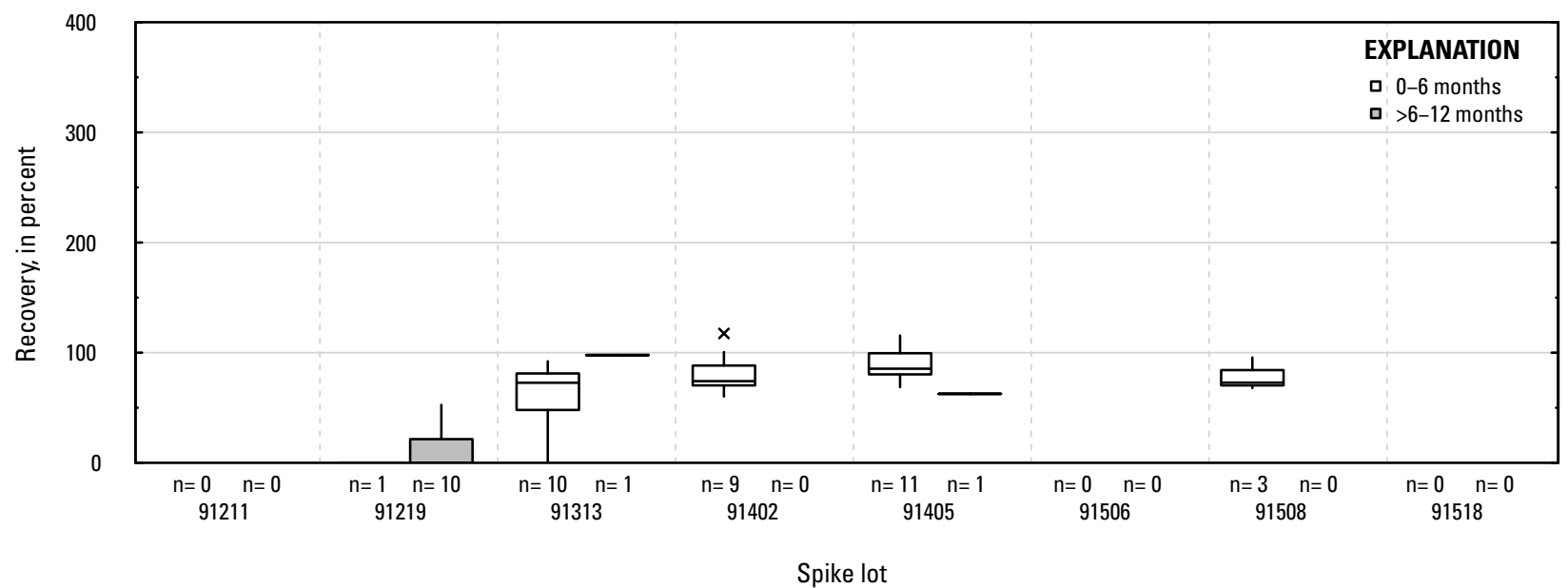

HH. Dichlorvos: surface water field matrix spikes

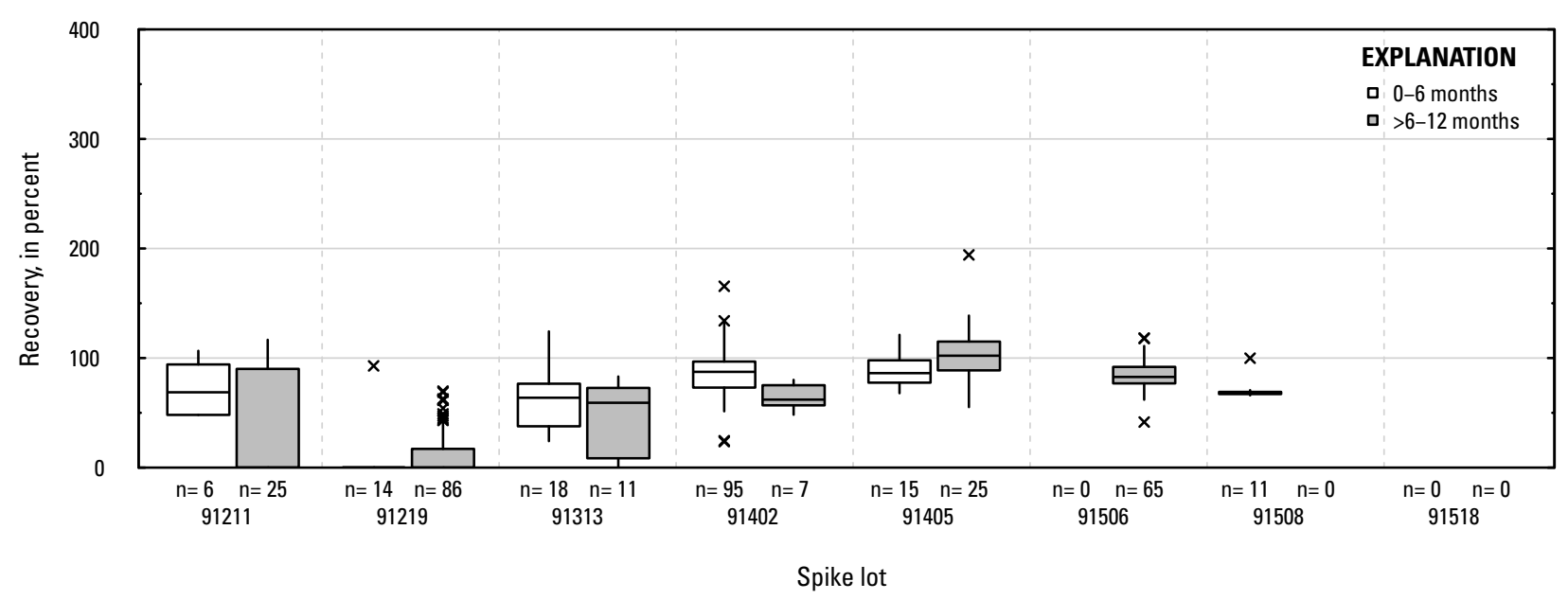

Figure 1-1. Distributions of recovery for individual pesticides in schedule 2437 by matrix, spike lot, and spike lot age. Recovery values larger than 400 percent are not shown.-Continued 


\section{HI. Dicrotophos: laboratory reagent spikes}

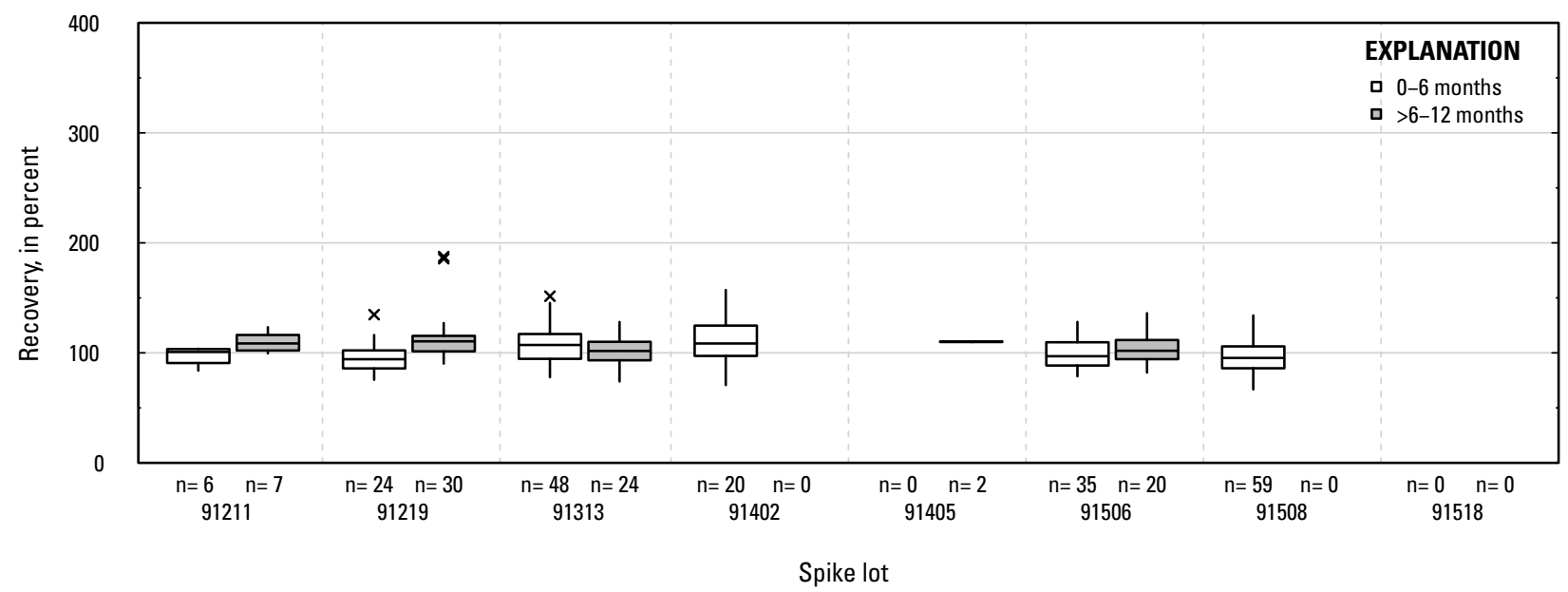

\section{HJ. Dicrotophos: groundwater field matrix spikes}

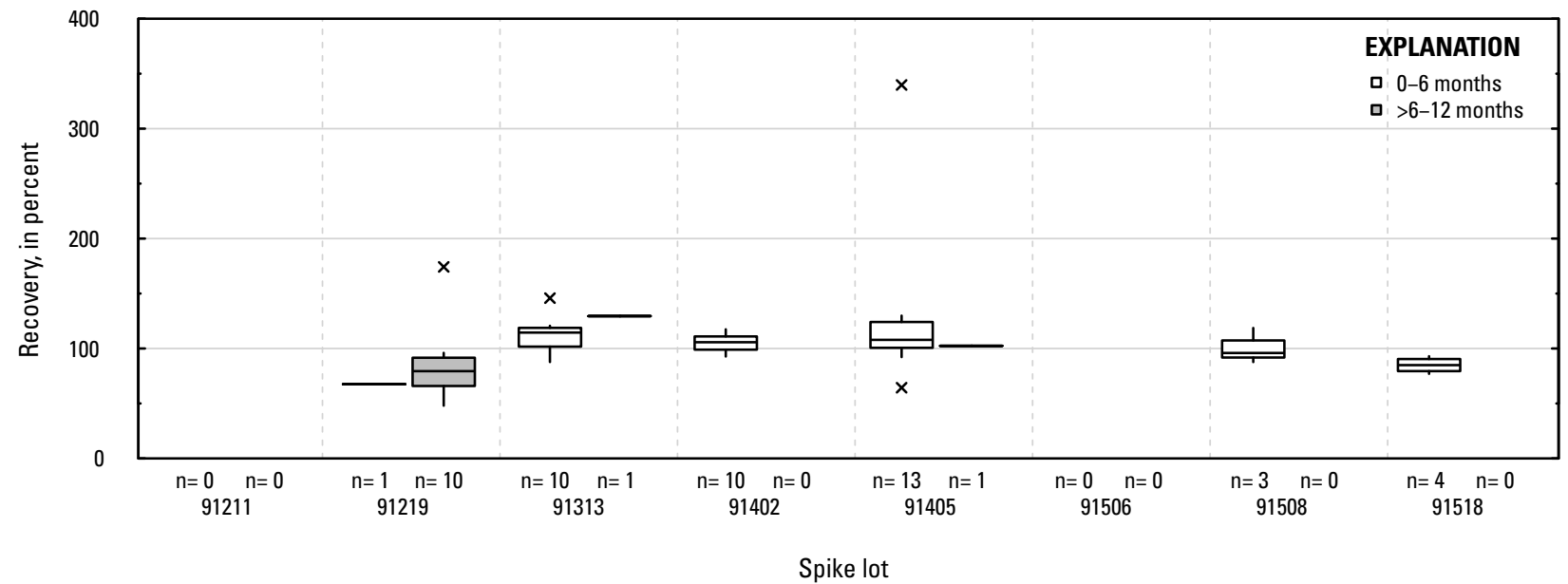

HK. Dicrotophos: surface water field matrix spikes

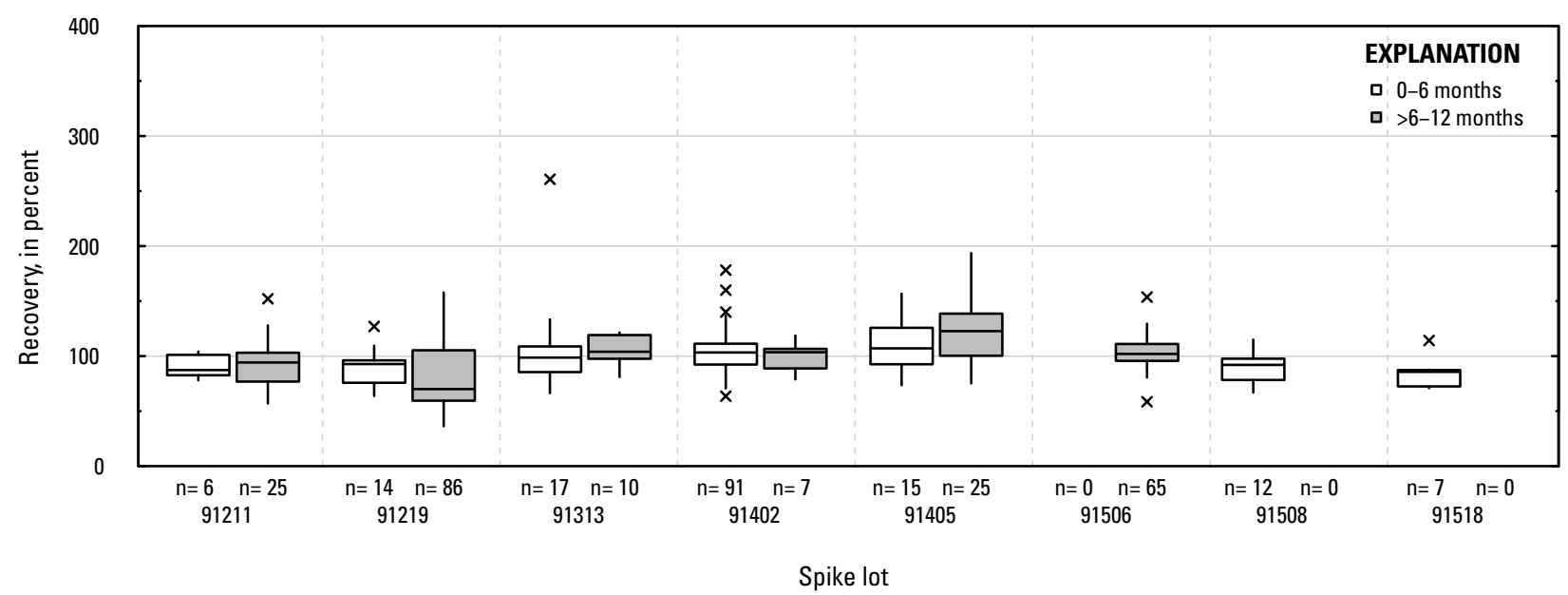

Figure 1-1. Distributions of recovery for individual pesticides in schedule 2437 by matrix, spike lot, and spike lot age. Recovery values larger than 400 percent are not shown.-Continued 
HL. 2-Chloro-4,6-diamino-s-triazine \{CAAT\} (Didealkylatrazine): laboratory reagent spikes

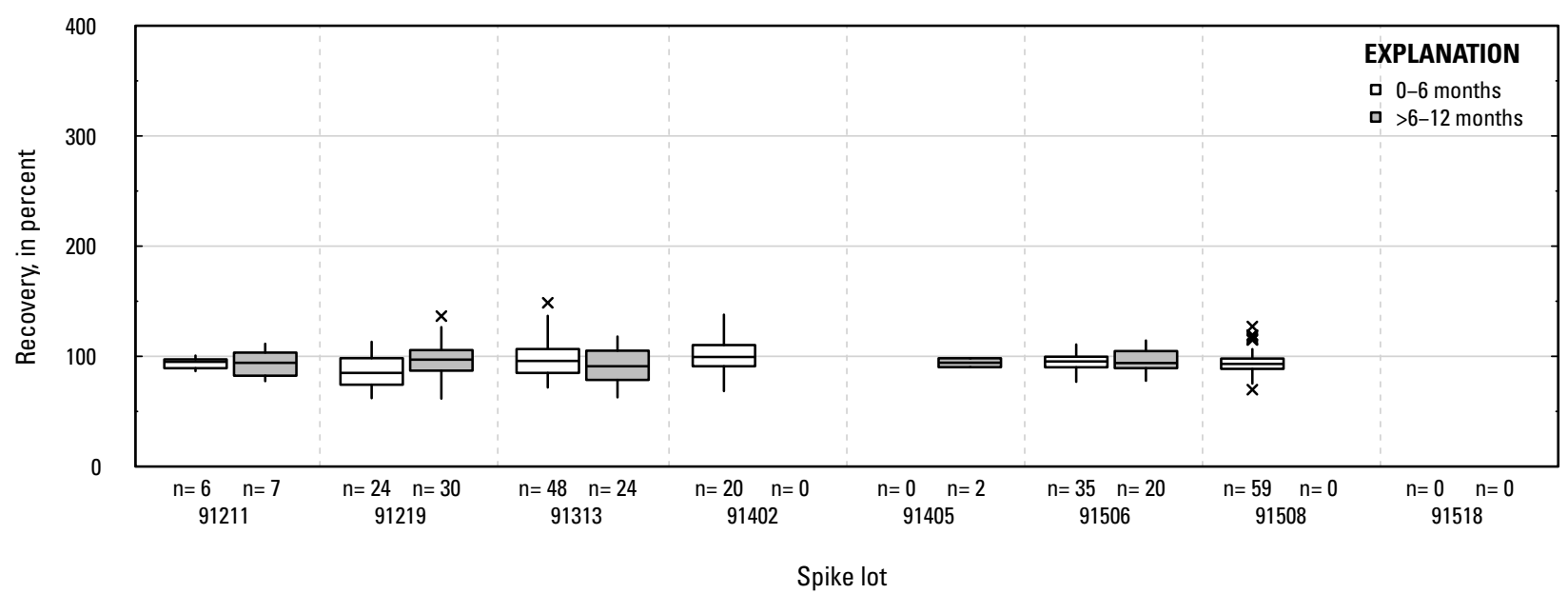

HM. 2-Chloro-4,6-diamino-s-triazine \{CAAT\} (Didealkylatrazine): groundwater field matrix spikes

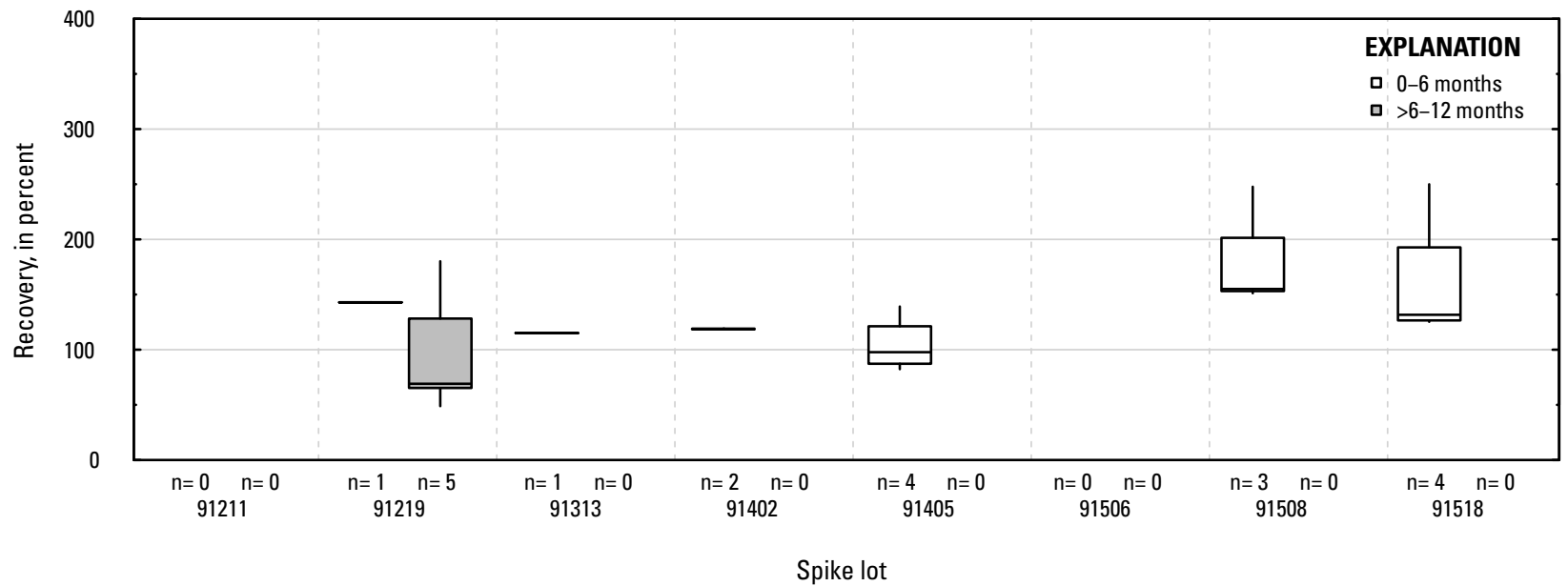

HN. 2-Chloro-4,6-diamino-s-triazine \{CAAT\} (Didealkylatrazine): surface water field matrix spikes

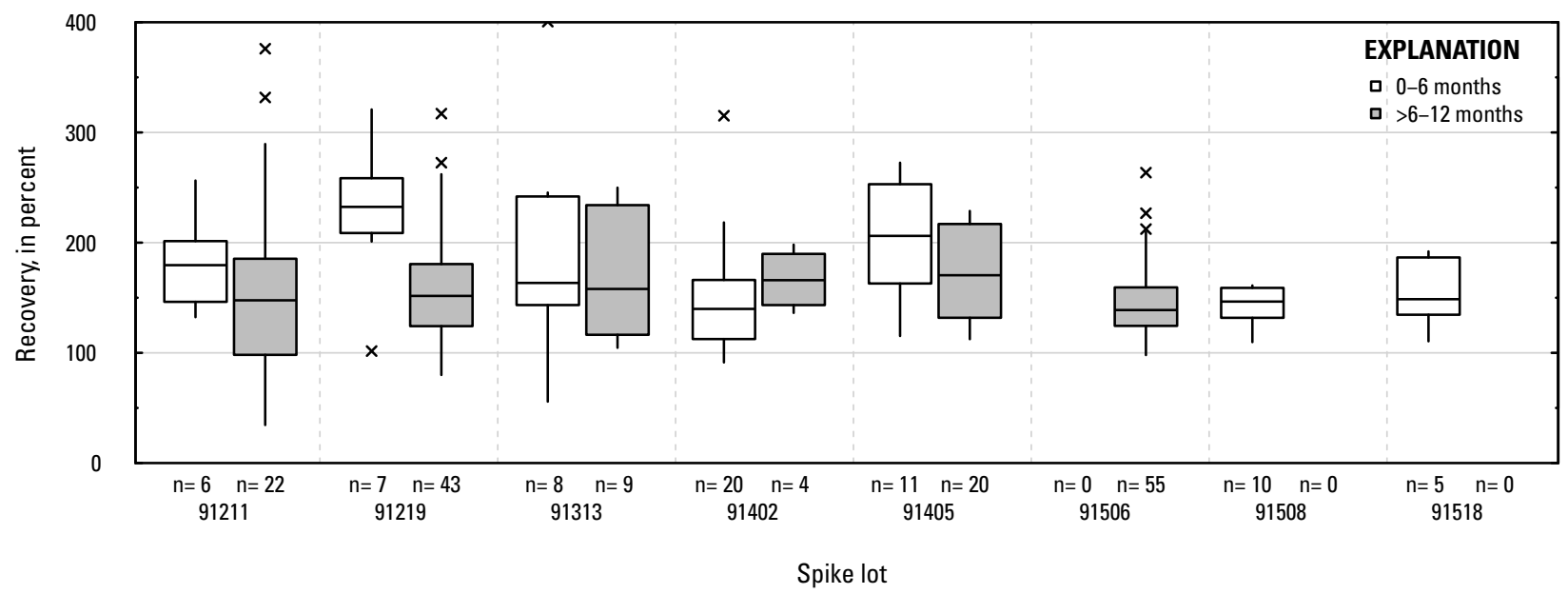

Figure 1-1. Distributions of recovery for individual pesticides in schedule 2437 by matrix, spike lot, and spike lot age. Recovery values larger than 400 percent are not shown.-Continued 
HO. Diflubenzuron: laboratory reagent spikes

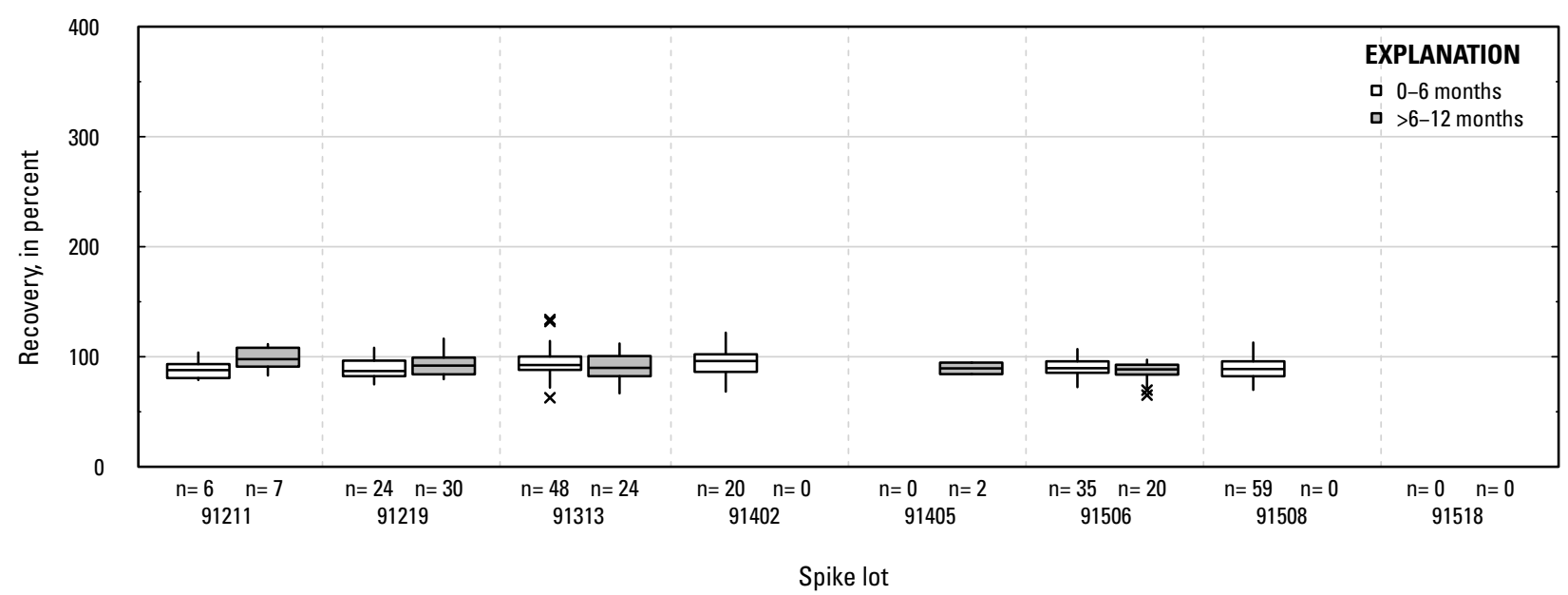

HP. Diflubenzuron: groundwater field matrix spikes

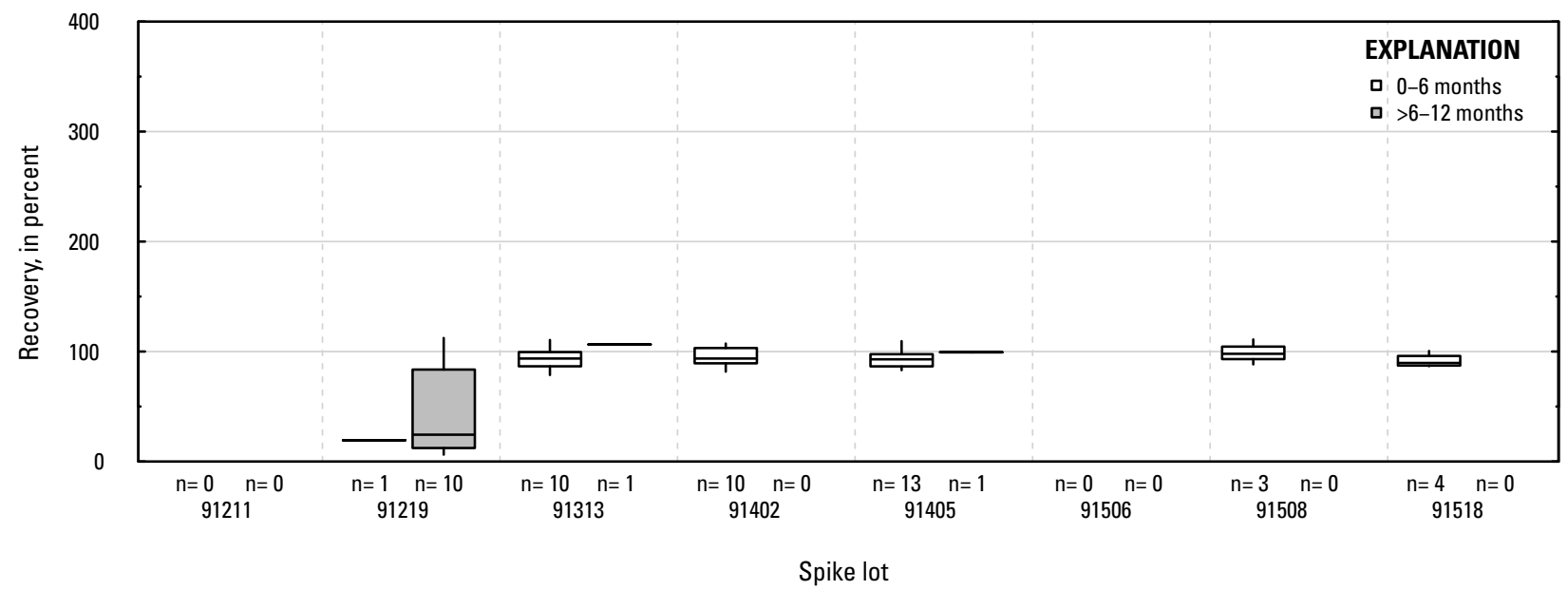

HQ. Diflubenzuron: surface water field matrix spikes

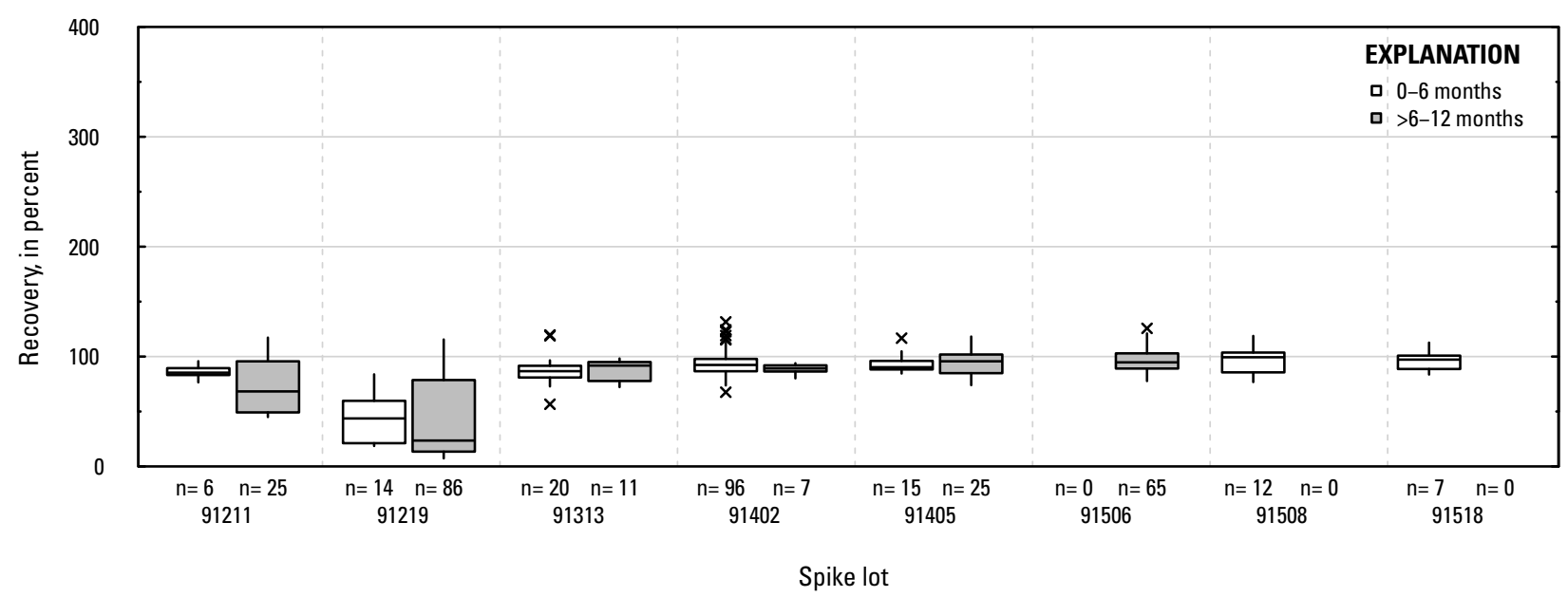

Figure 1-1. Distributions of recovery for individual pesticides in schedule 2437 by matrix, spike lot, and spike lot age. Recovery values larger than 400 percent are not shown.-Continued 
HR. Diflufenzopyr: laboratory reagent spikes

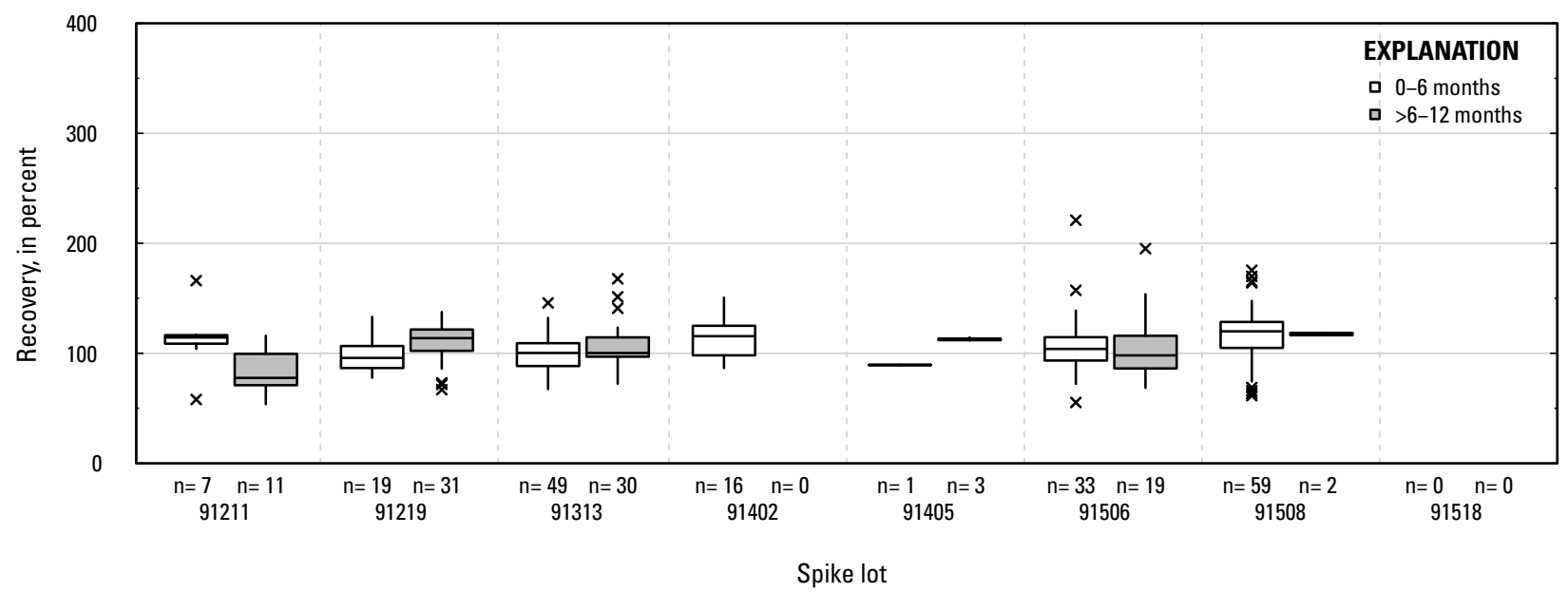

HS. Diflufenzopyr: groundwater field matrix spikes

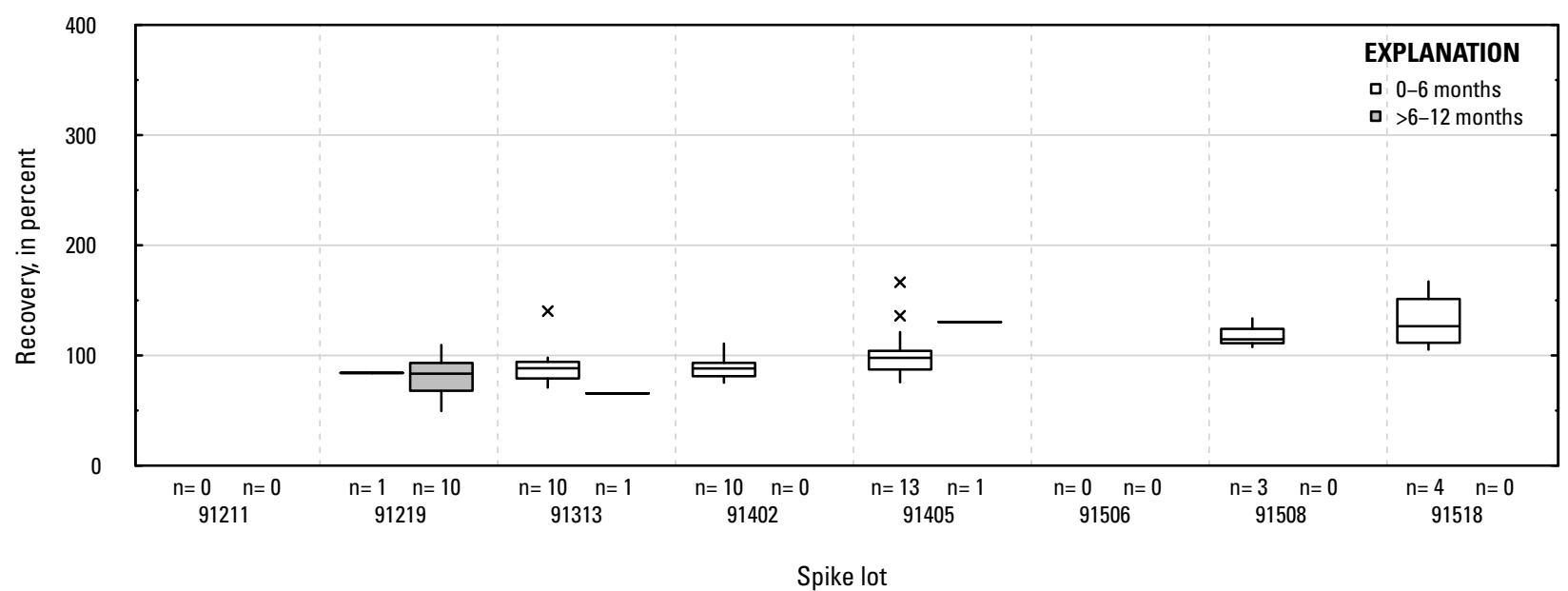

\section{HT. Diflufenzopyr: surface water field matrix spikes}

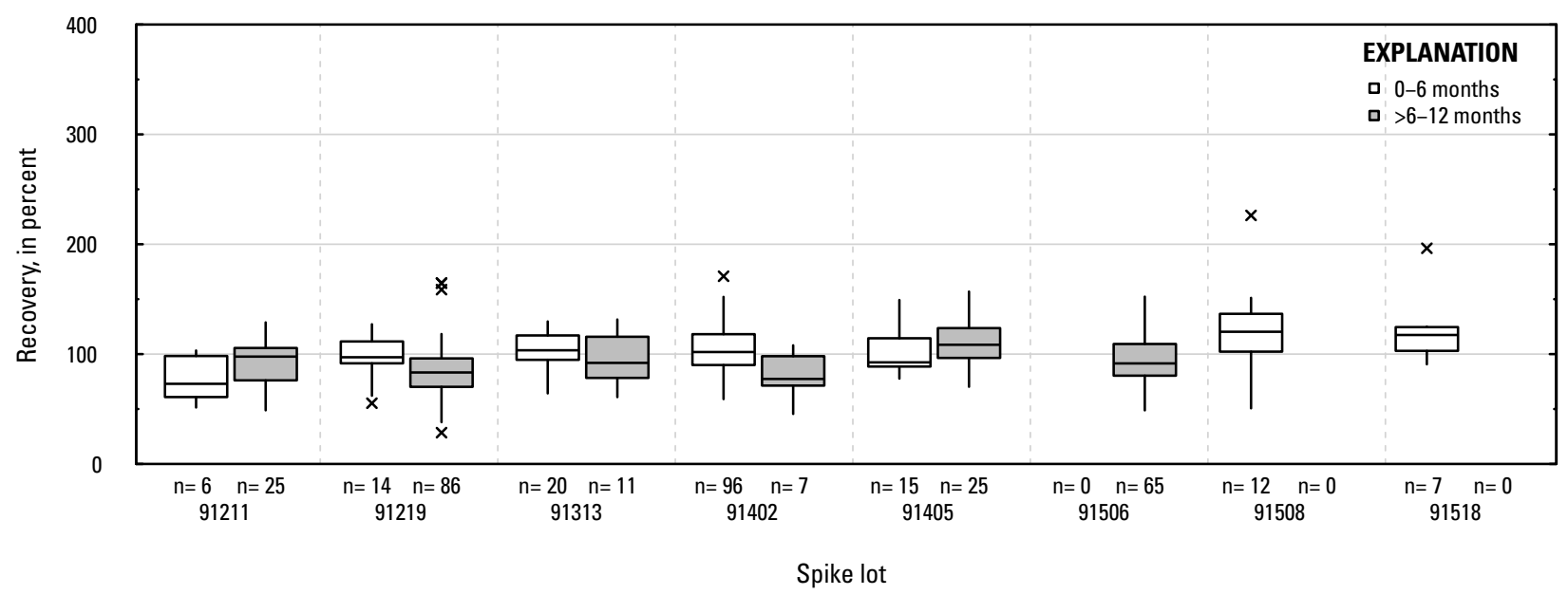

Figure 1-1. Distributions of recovery for individual pesticides in schedule 2437 by matrix, spike lot, and spike lot age. Recovery values larger than 400 percent are not shown.-Continued 
HU. Diketonitrile-isoxaflutole: laboratory reagent spikes

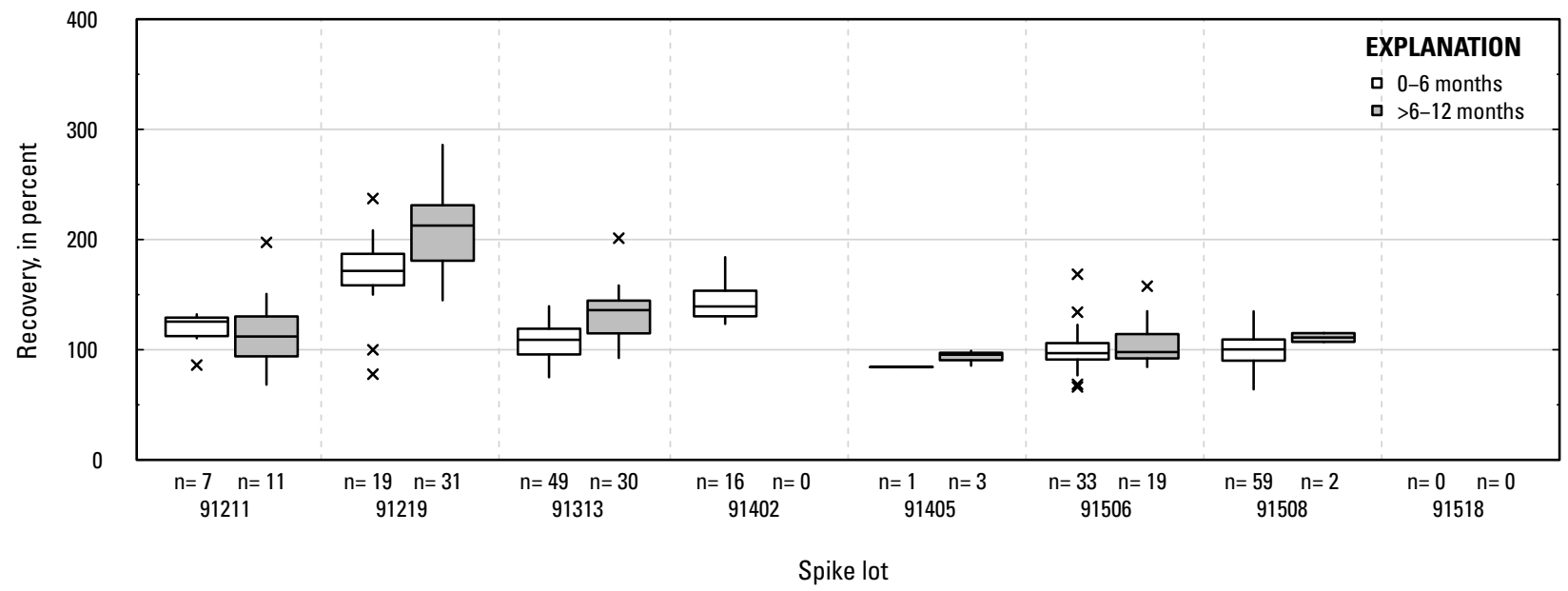

HV. Diketonitrile-isoxaflutole: groundwater field matrix spikes

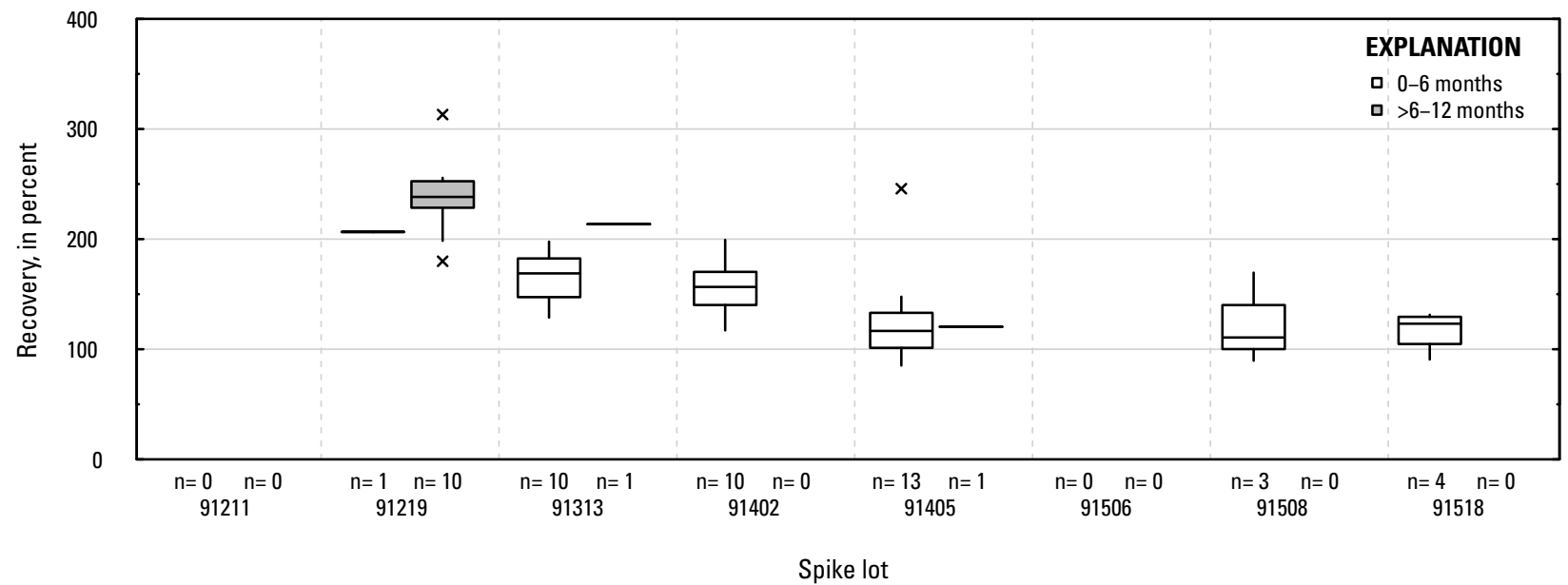

HW. Diketonitrile-isoxaflutole: surface water field matrix spikes

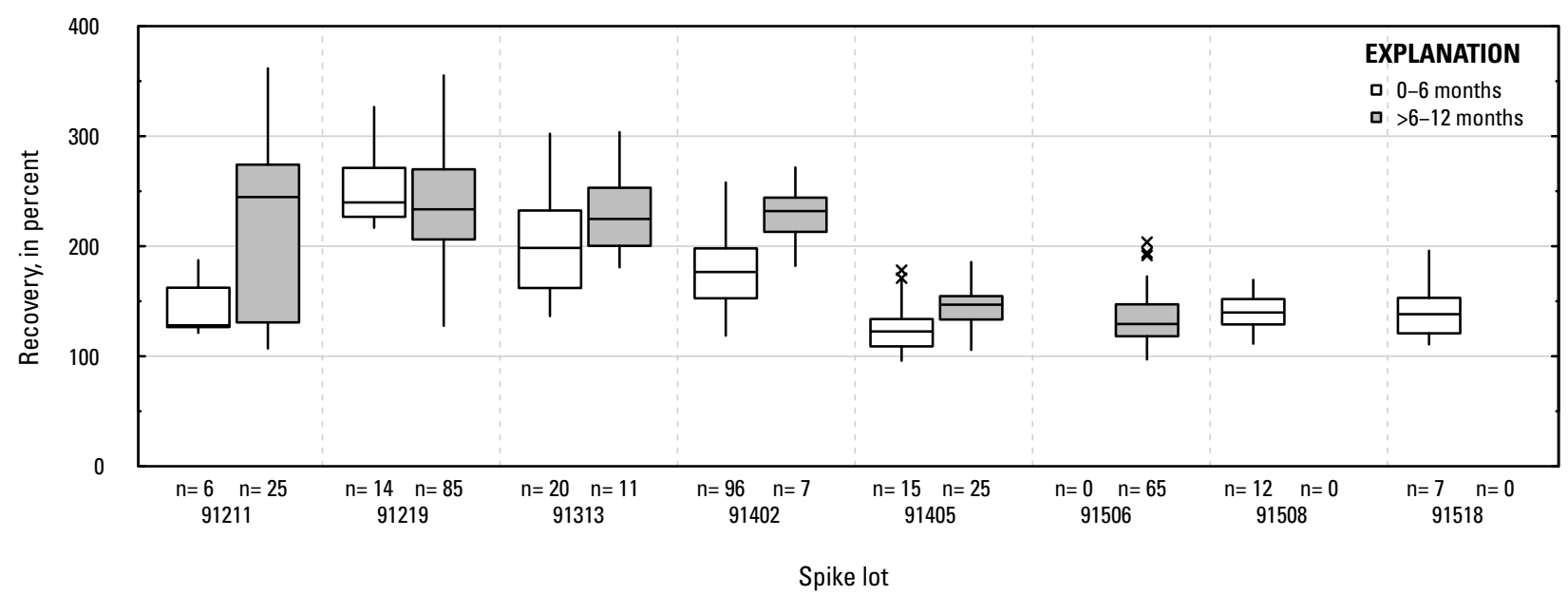

Figure 1-1. Distributions of recovery for individual pesticides in schedule 2437 by matrix, spike lot, and spike lot age. Recovery values larger than 400 percent are not shown.-Continued 
$H X$. Dimethenamid: laboratory reagent spikes

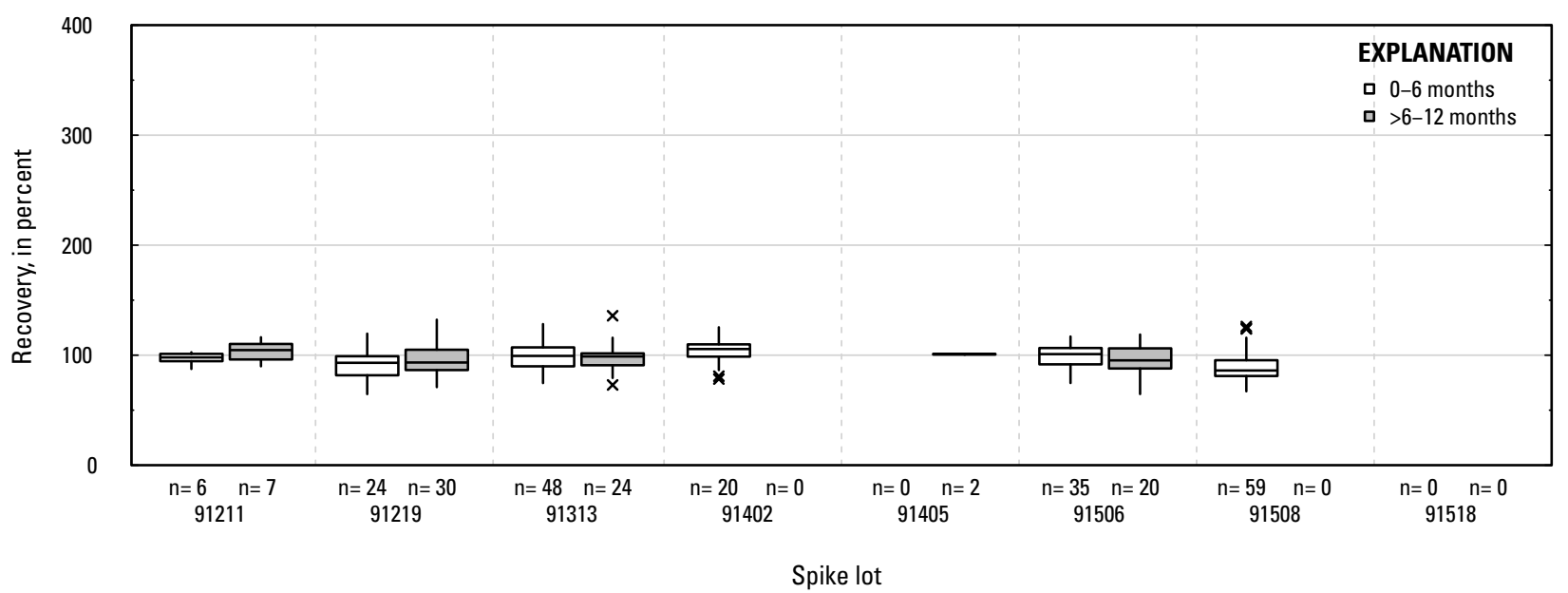

HY. Dimethenamid: groundwater field matrix spikes

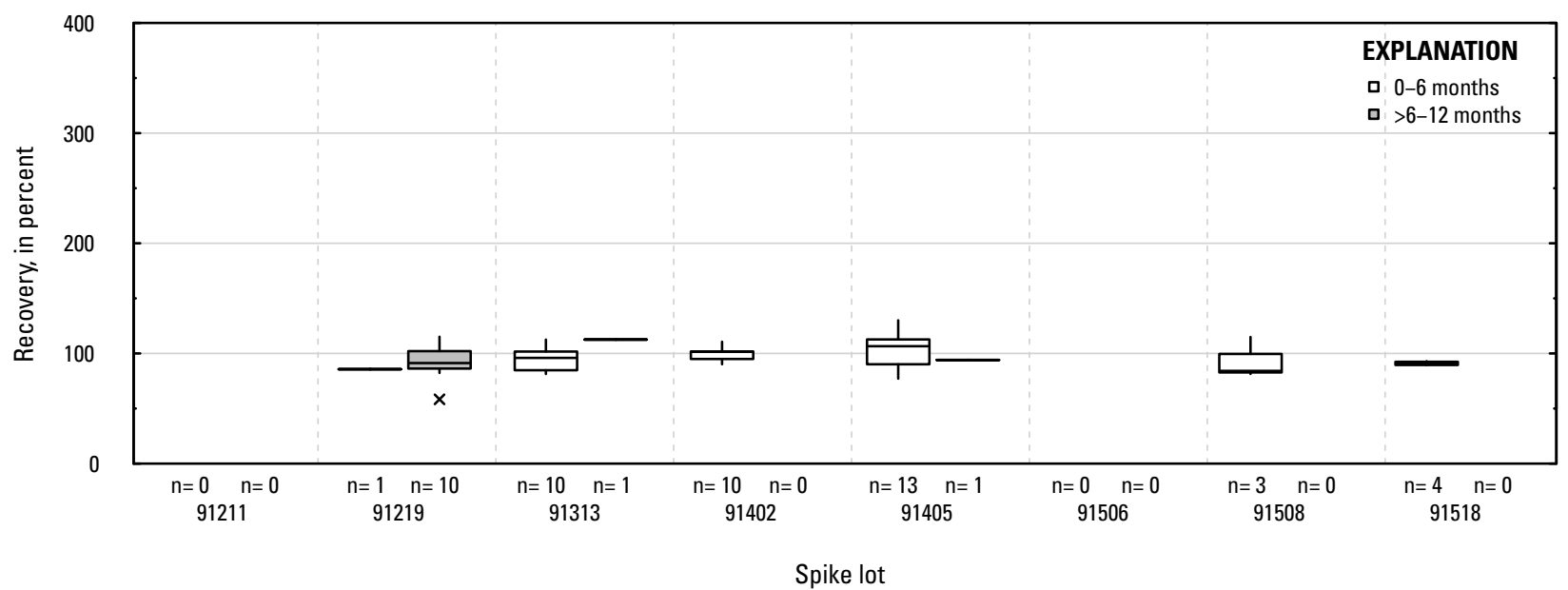

HZ. Dimethenamid: surface water field matrix spikes

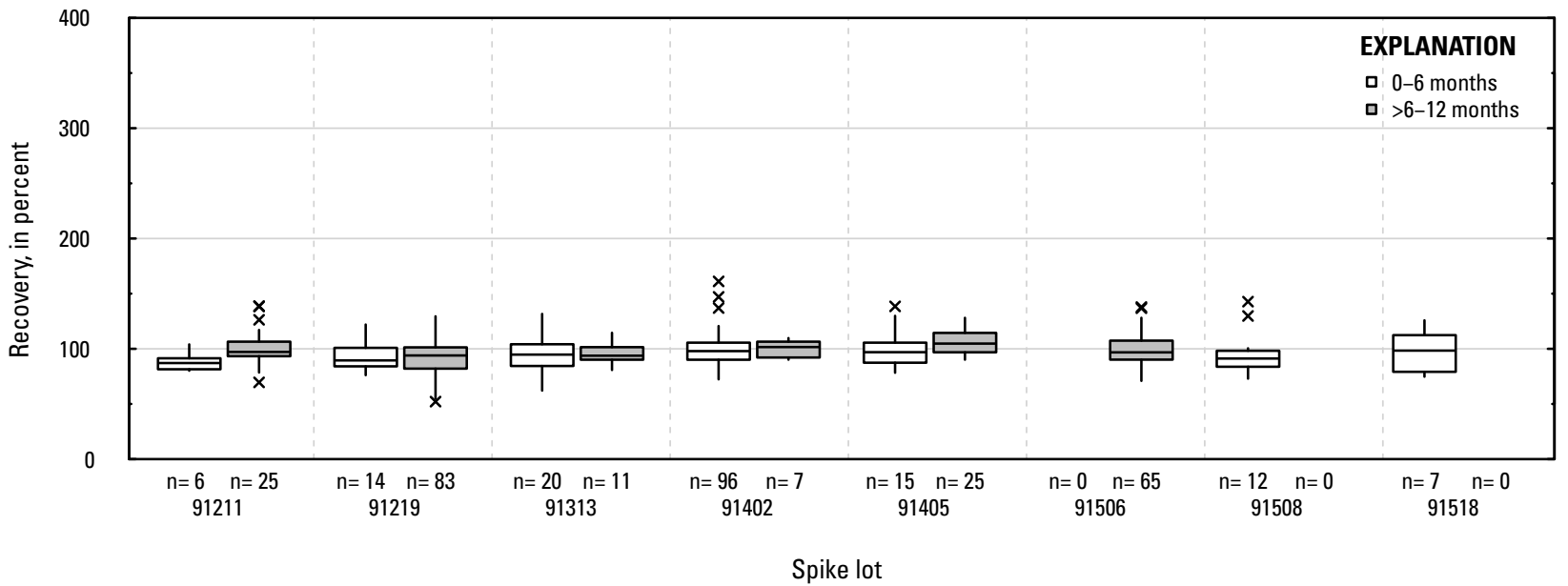

Figure 1-1. Distributions of recovery for individual pesticides in schedule 2437 by matrix, spike lot, and spike lot age. Recovery values larger than 400 percent are not shown.-Continued 


\section{IA. Dimethenamid oxanilic acid: laboratory reagent spikes}

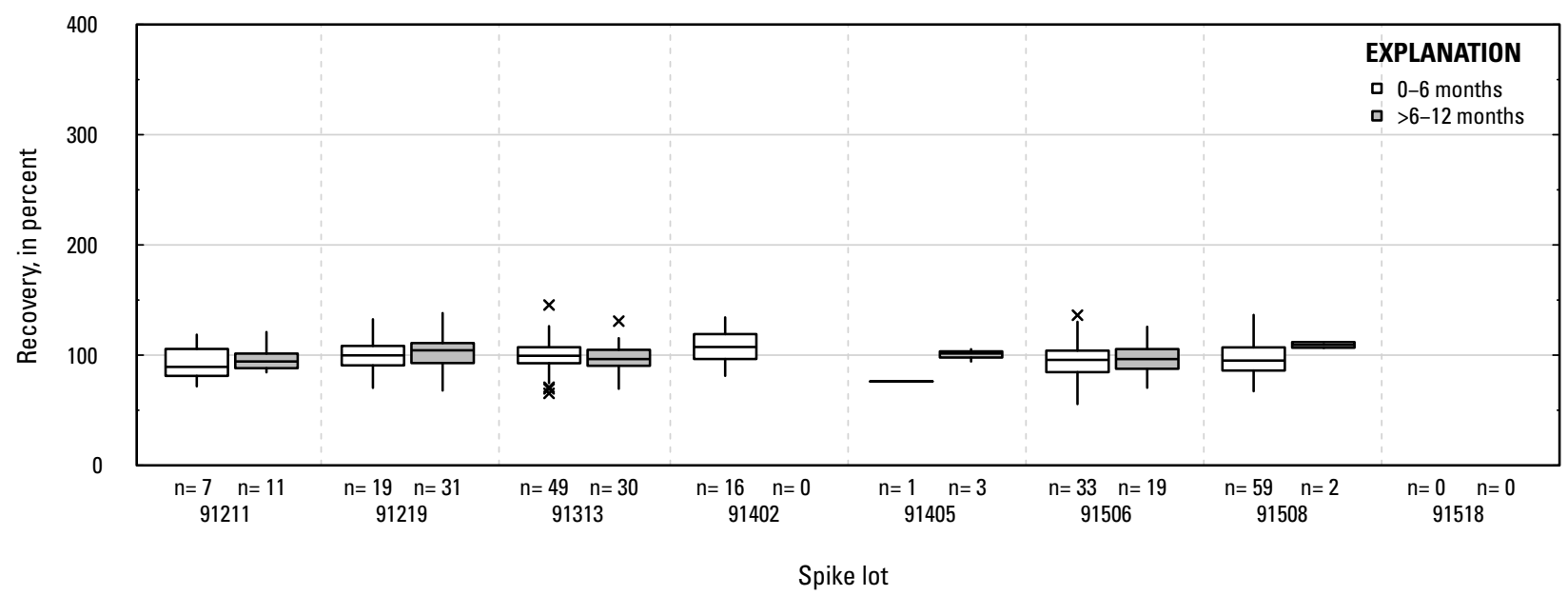

IB. Dimethenamid oxanilic acid: groundwater field matrix spikes

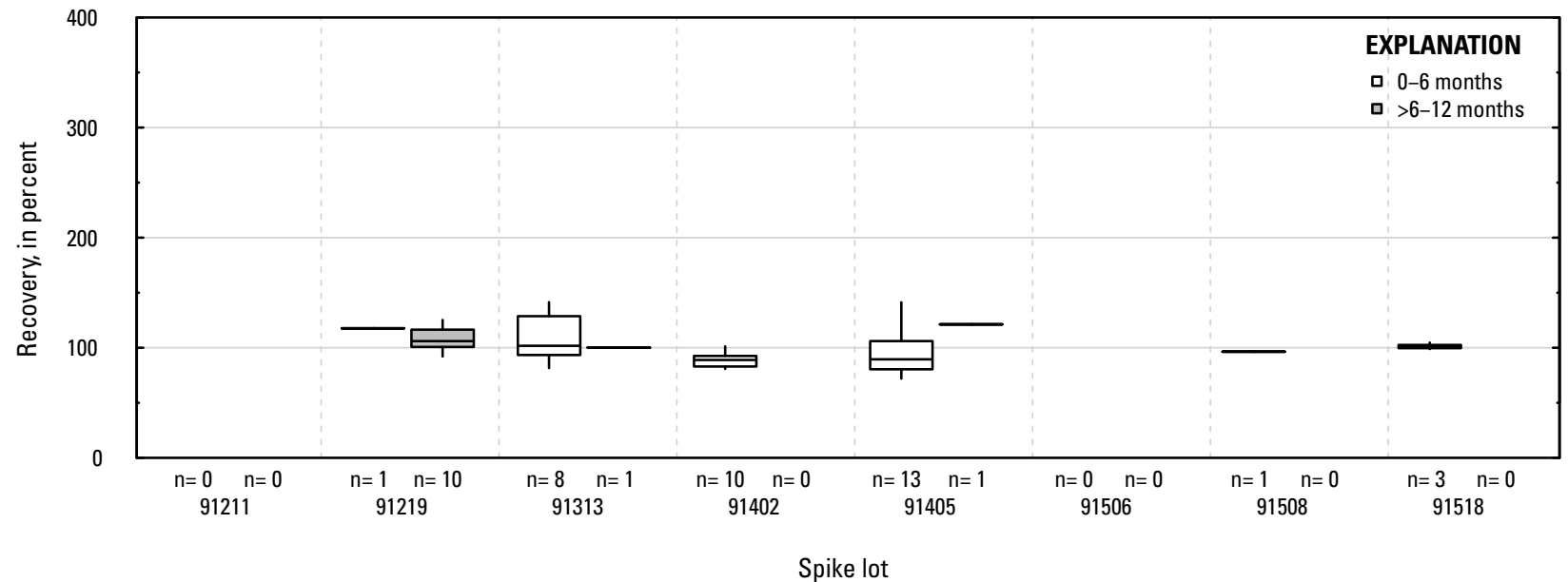

IC. Dimethenamid oxanilic acid: surface water field matrix spikes

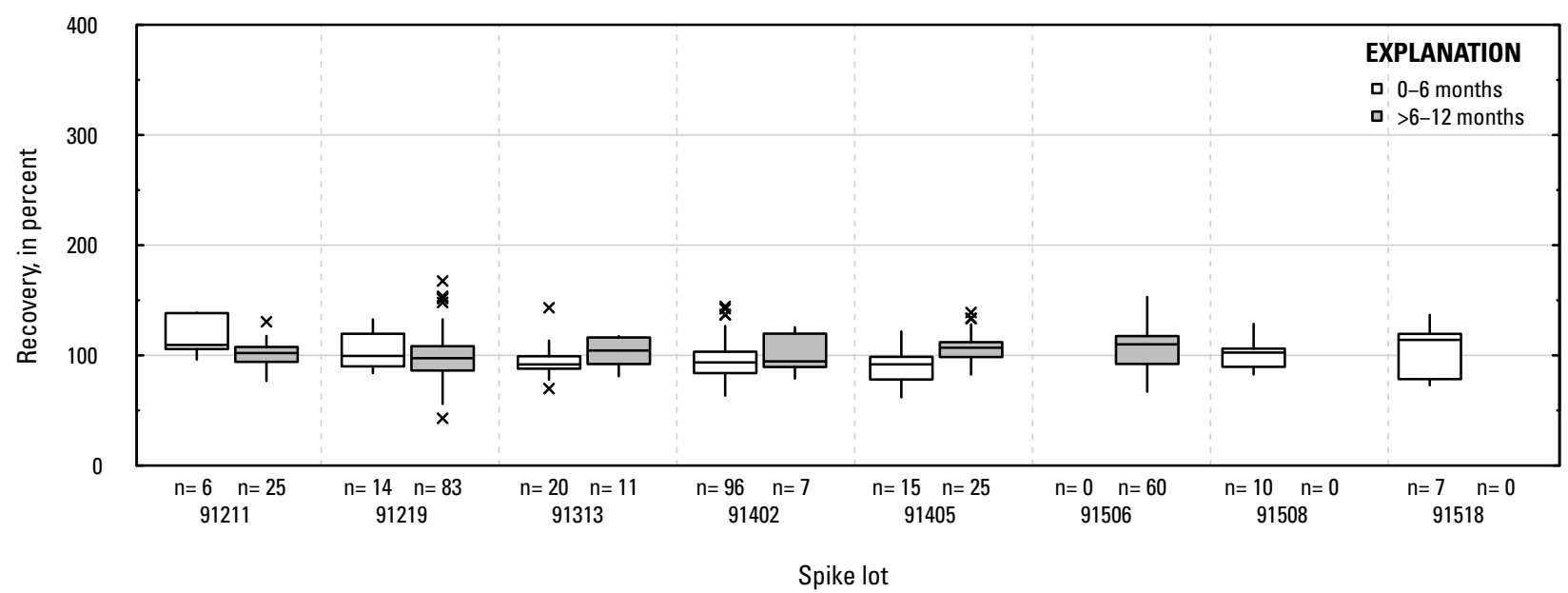

Figure 1-1. Distributions of recovery for individual pesticides in schedule 2437 by matrix, spike lot, and spike lot age. Recovery values larger than 400 percent are not shown.-Continued 


\section{ID. Dimethenamid sulfonic acid: laboratory reagent spikes}

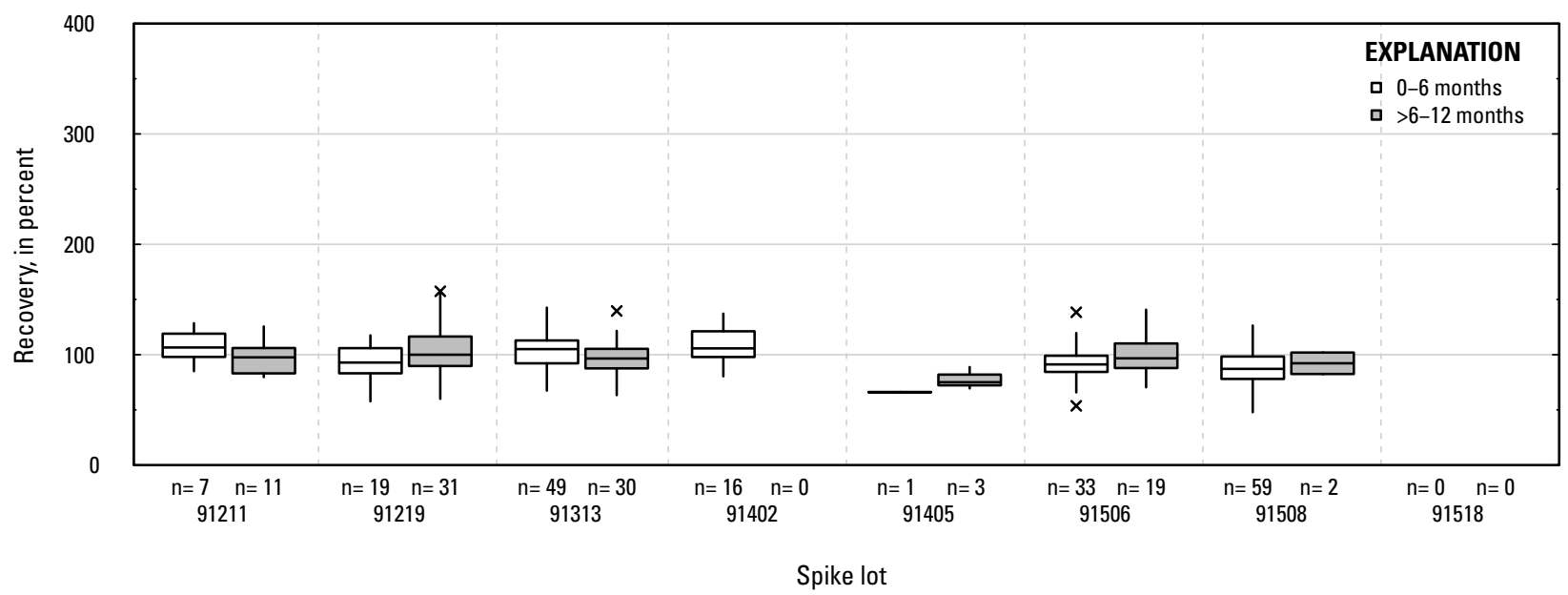

IE. Dimethenamid sulfonic acid: groundwater field matrix spikes

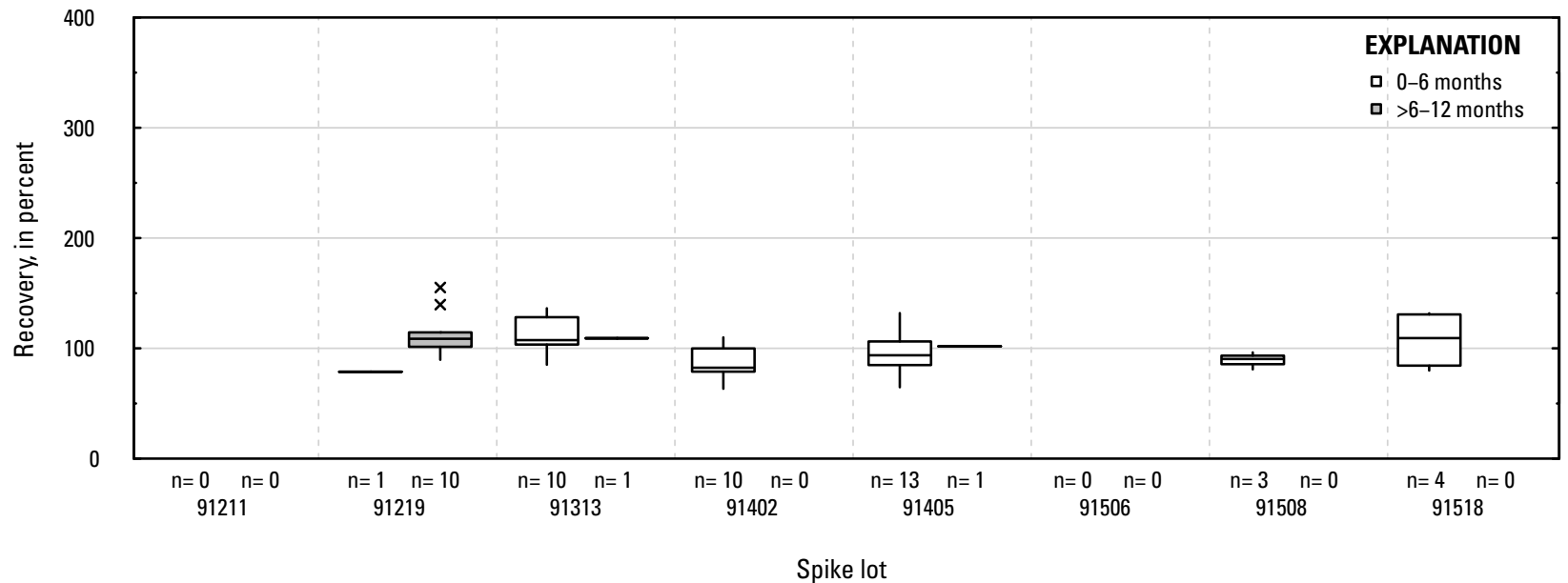

\section{IF. Dimethenamid sulfonic acid: surface water field matrix spikes}

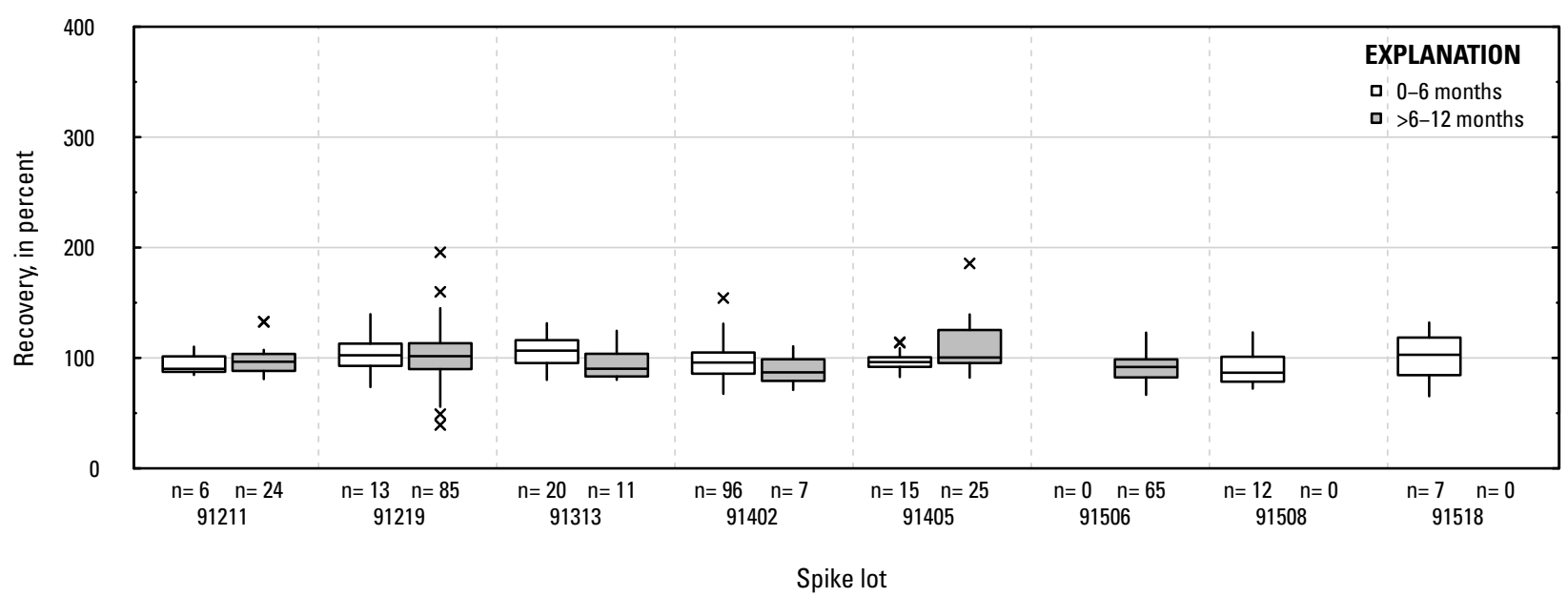

Figure 1-1. Distributions of recovery for individual pesticides in schedule 2437 by matrix, spike lot, and spike lot age. Recovery values larger than 400 percent are not shown.-Continued 


\section{IG. Dimethenamid SAA: laboratory reagent spikes}

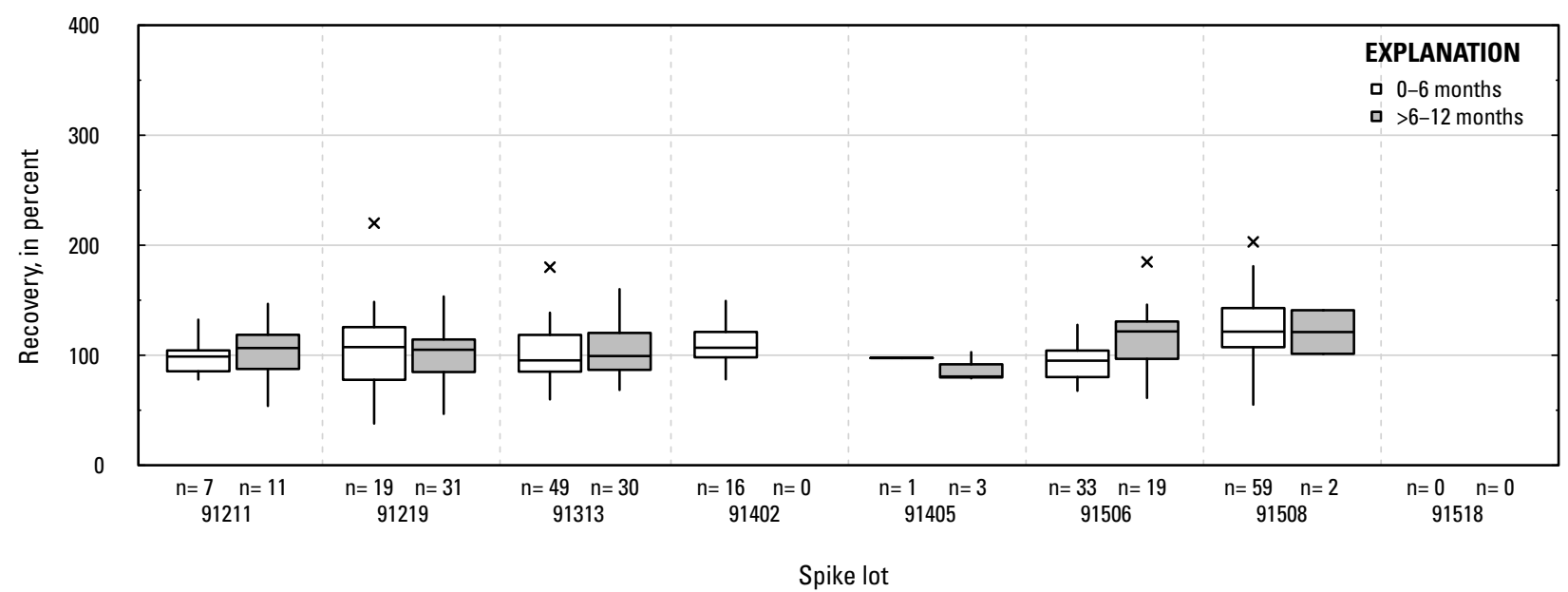

\section{IH. Dimethenamid SAA: groundwater field matrix spikes}

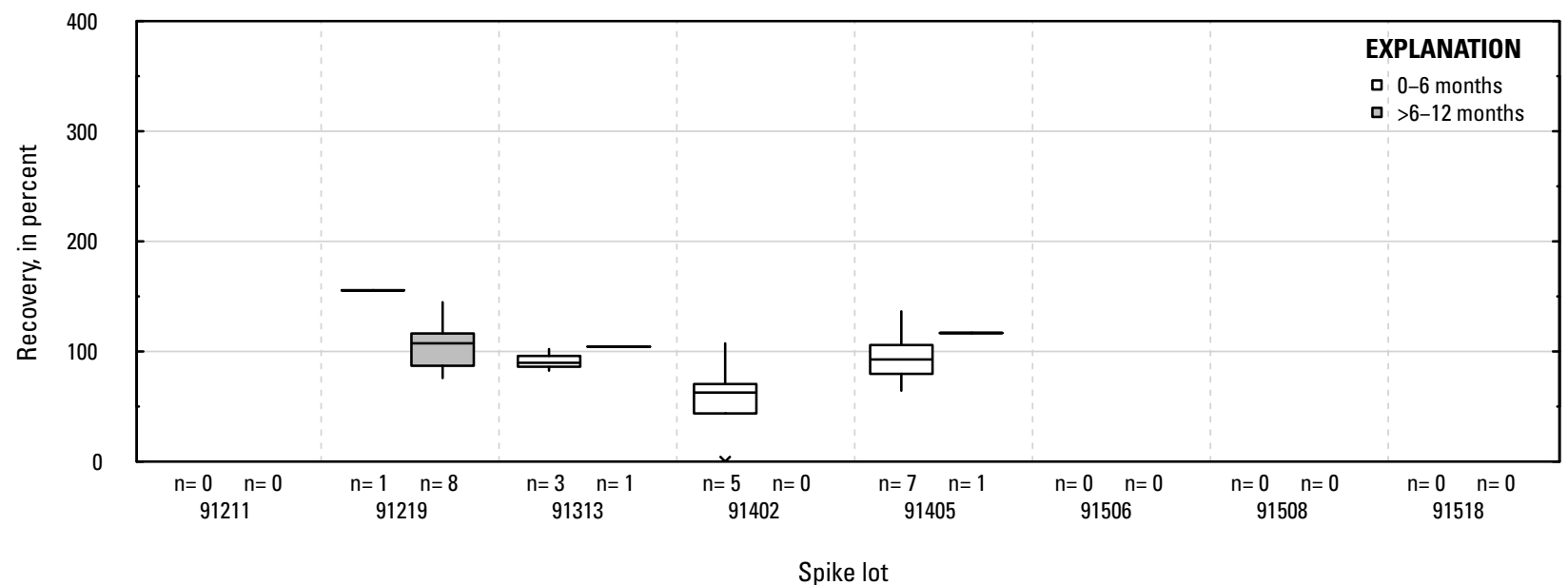

\section{Dimethenamid SAA: surface water field matrix spikes}

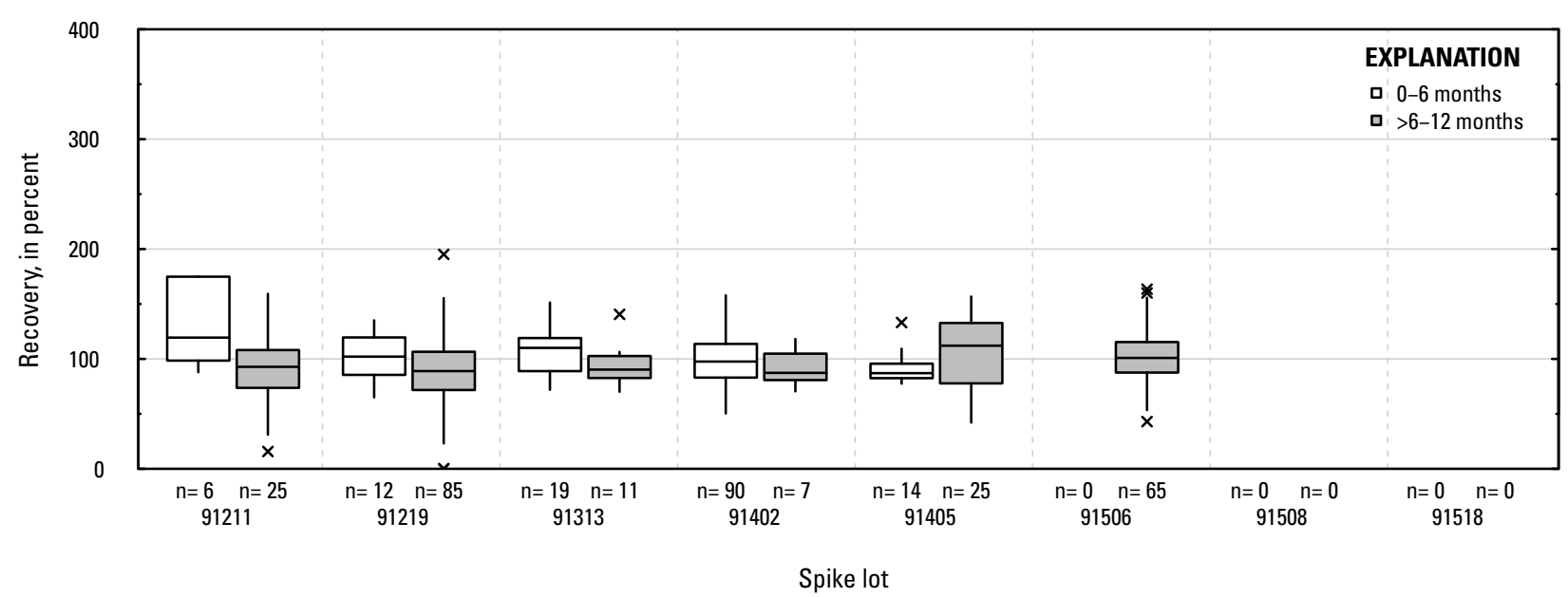

Figure 1-1. Distributions of recovery for individual pesticides in schedule 2437 by matrix, spike lot, and spike lot age. Recovery values larger than 400 percent are not shown.-Continued 
IJ. Dimethoate: laboratory reagent spikes

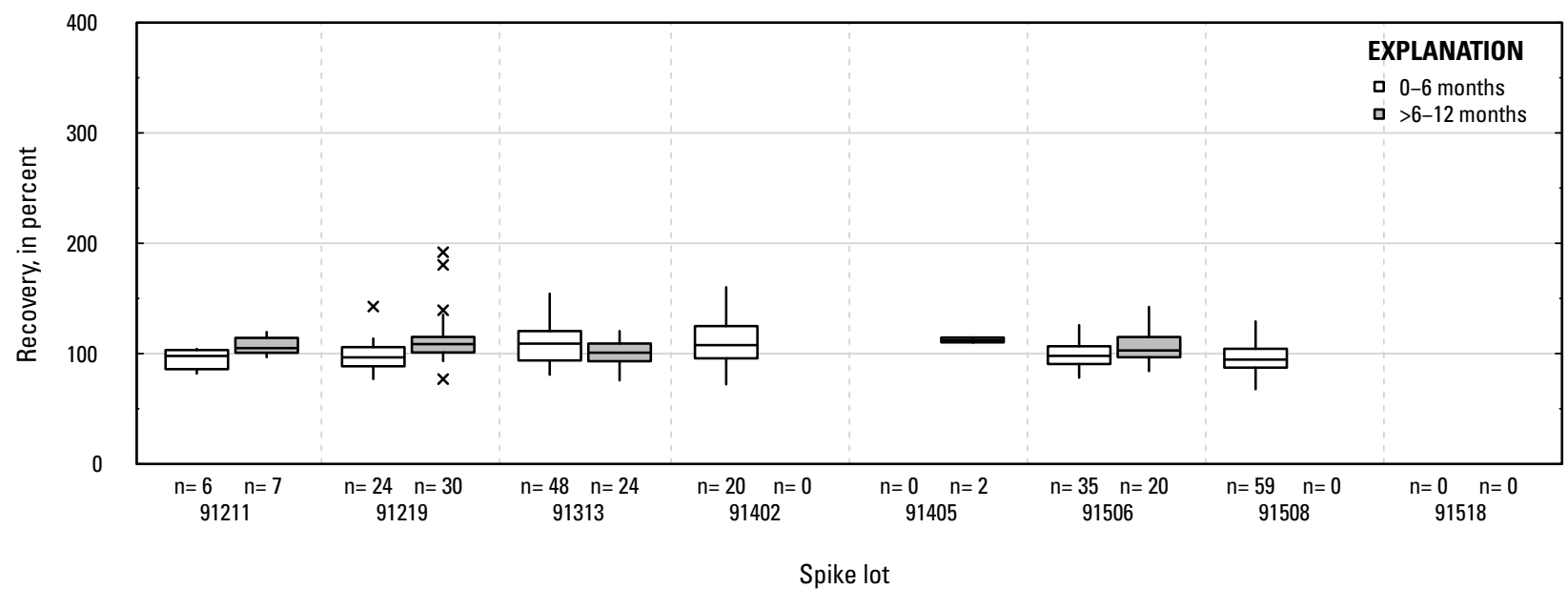

\section{IK. Dimethoate: groundwater field matrix spikes}

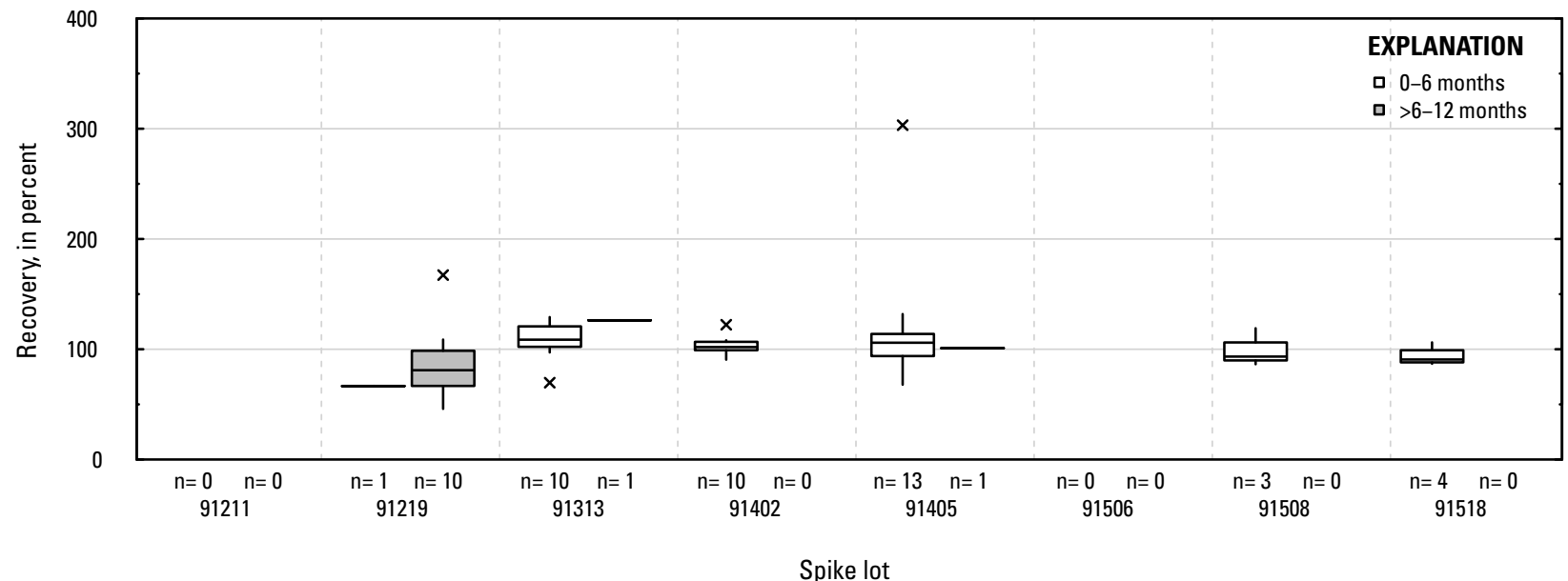

\section{IL. Dimethoate: surface water field matrix spikes}

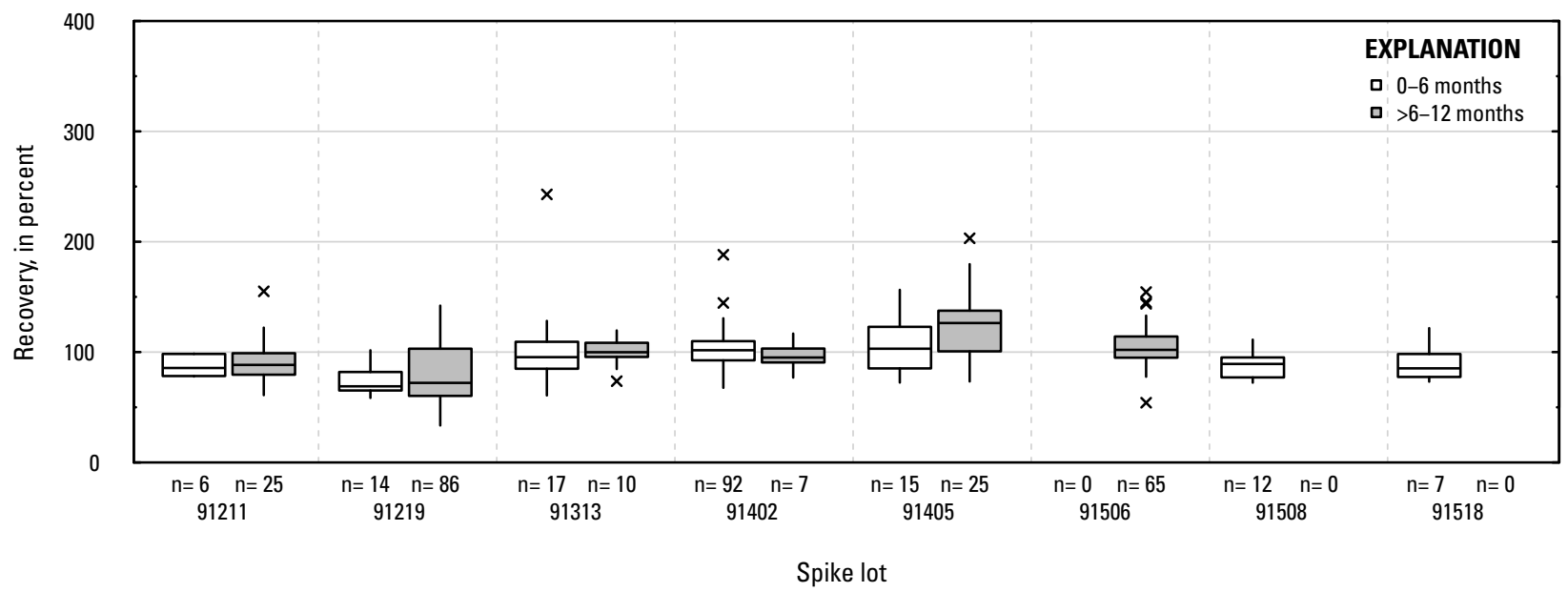

Figure 1-1. Distributions of recovery for individual pesticides in schedule 2437 by matrix, spike lot, and spike lot age. Recovery values larger than 400 percent are not shown.-Continued 
IM. Omethoate (Dimethoate oxon): laboratory reagent spikes

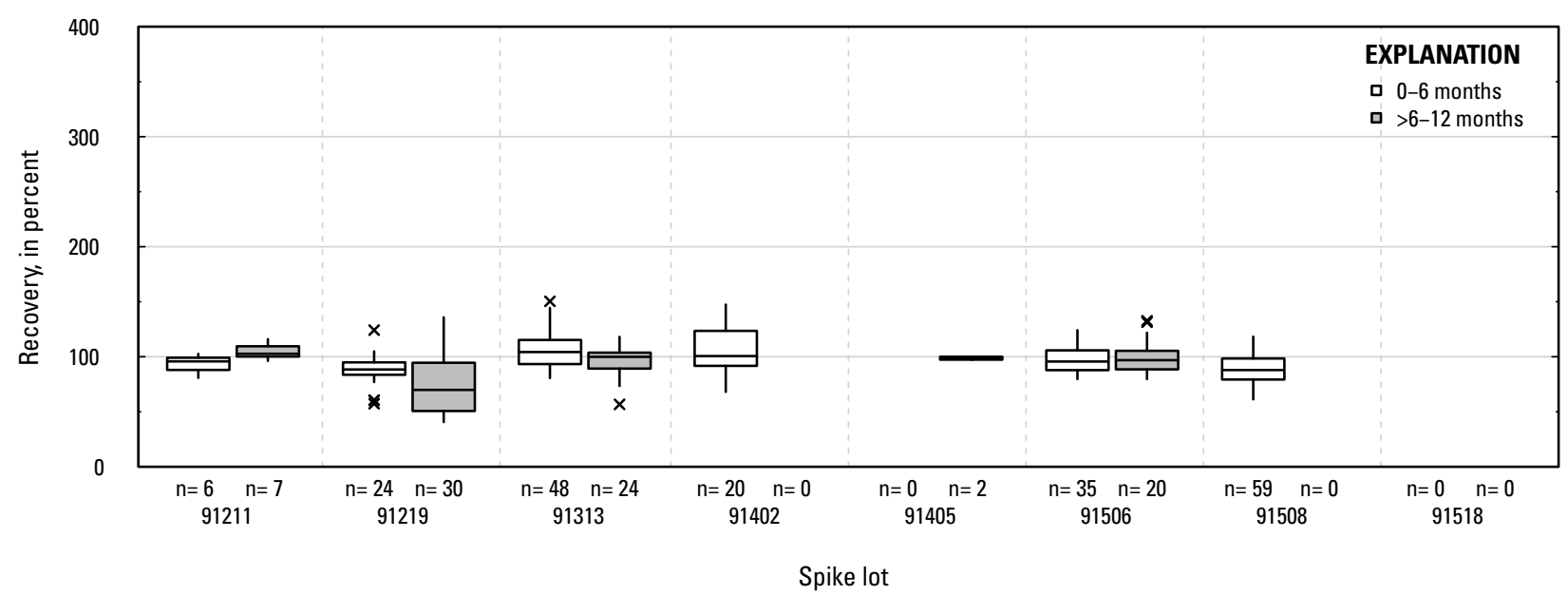

IN. Omethoate (Dimethoate oxon): groundwater field matrix spikes

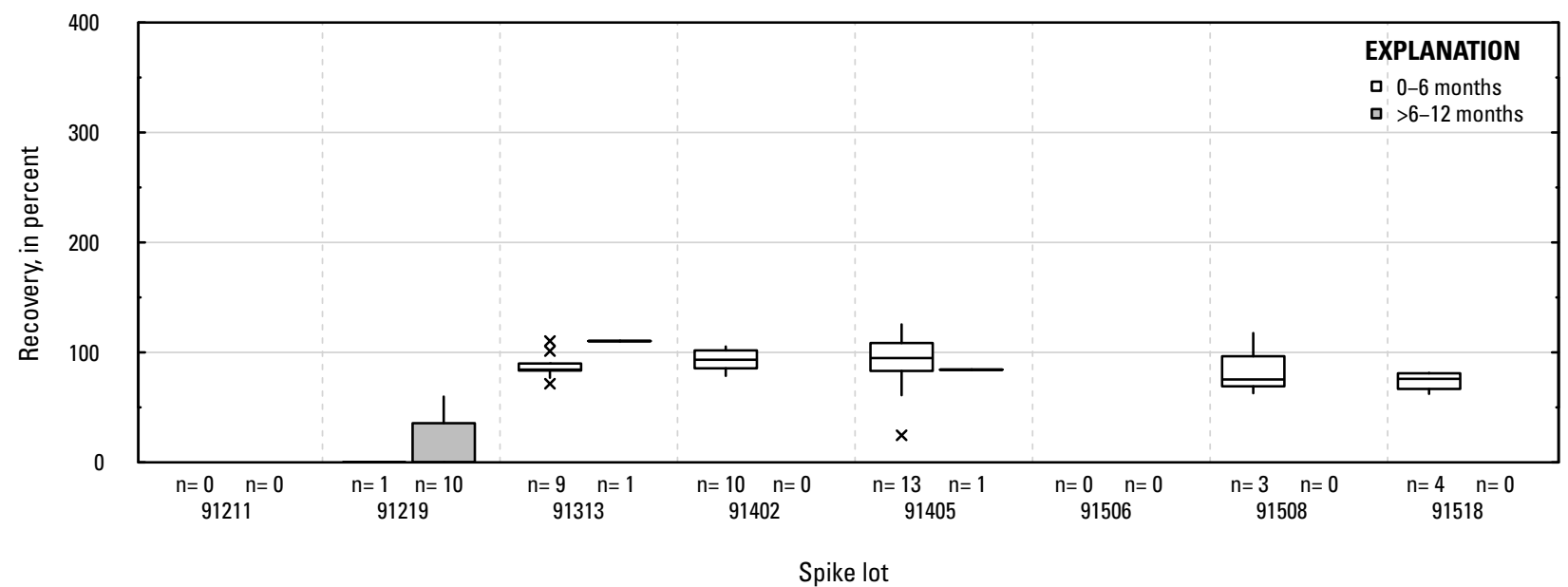

10. Omethoate (Dimethoate oxon): surface water field matrix spikes

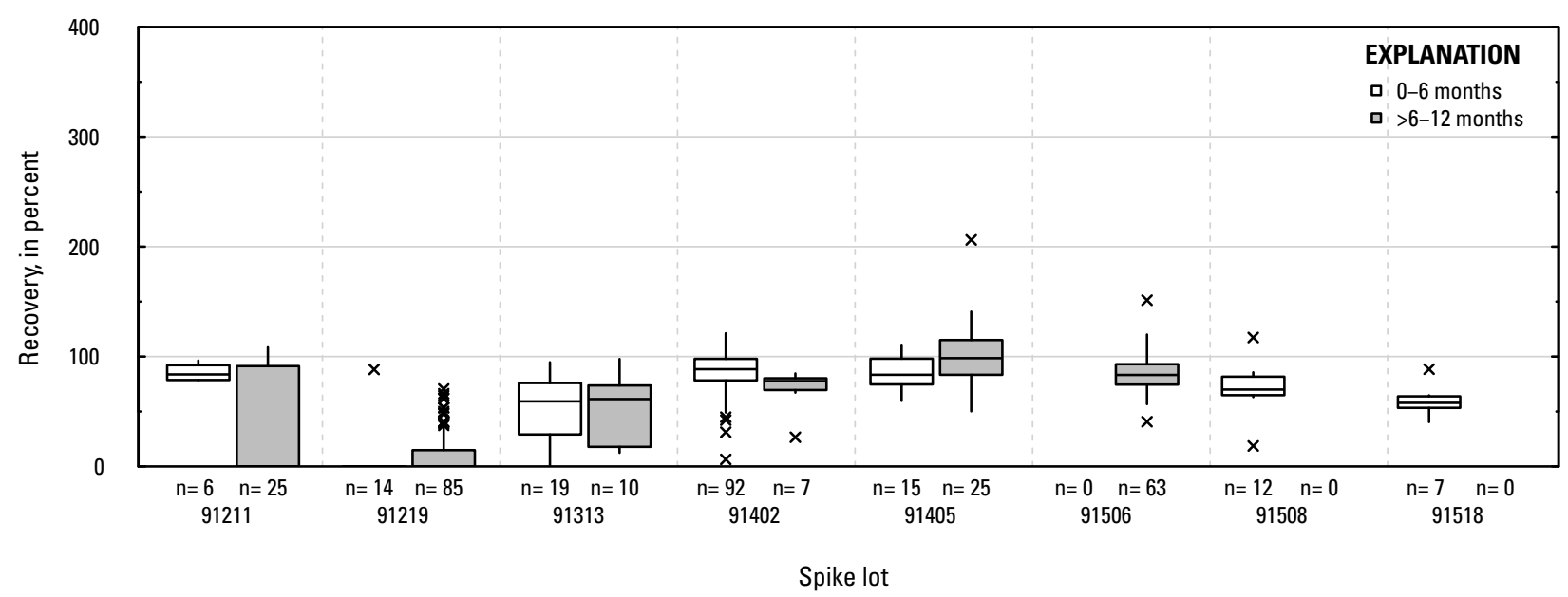

Figure 1-1. Distributions of recovery for individual pesticides in schedule 2437 by matrix, spike lot, and spike lot age. Recovery values larger than 400 percent are not shown.-Continued 
IP. Disulfoton: laboratory reagent spikes

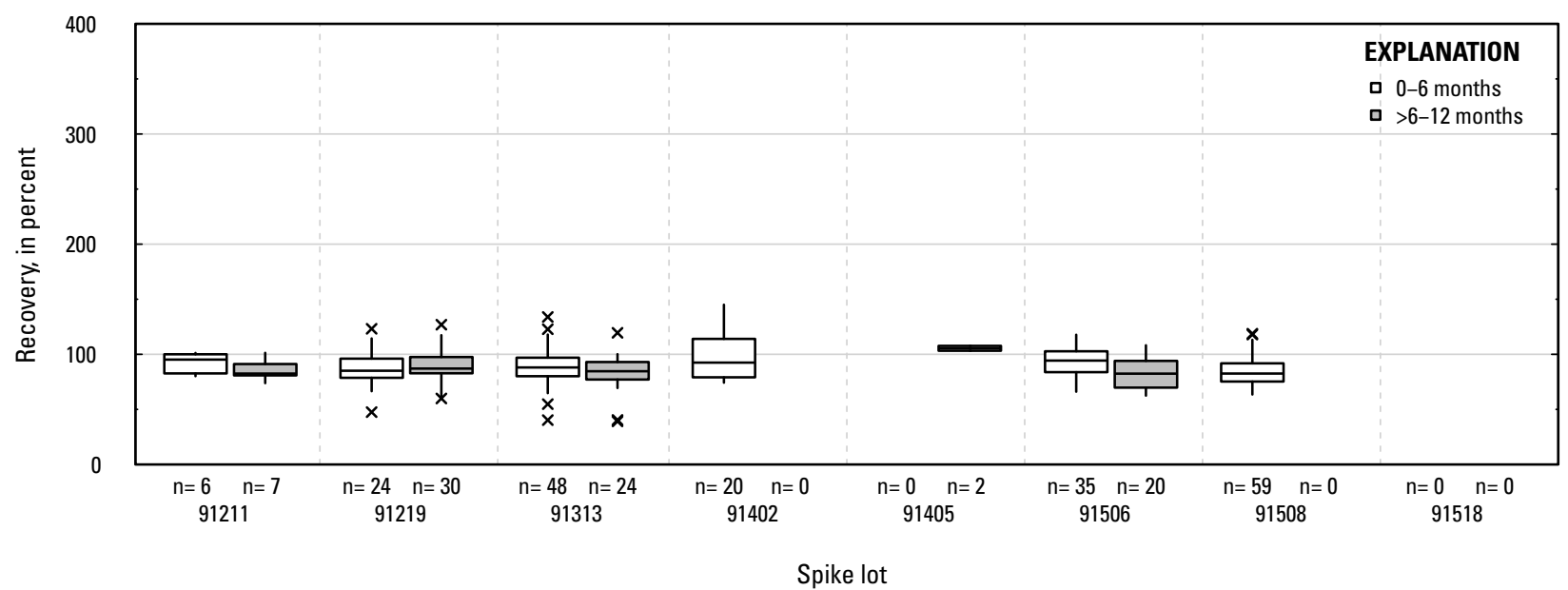

\section{Disulfoton: groundwater field matrix spikes}

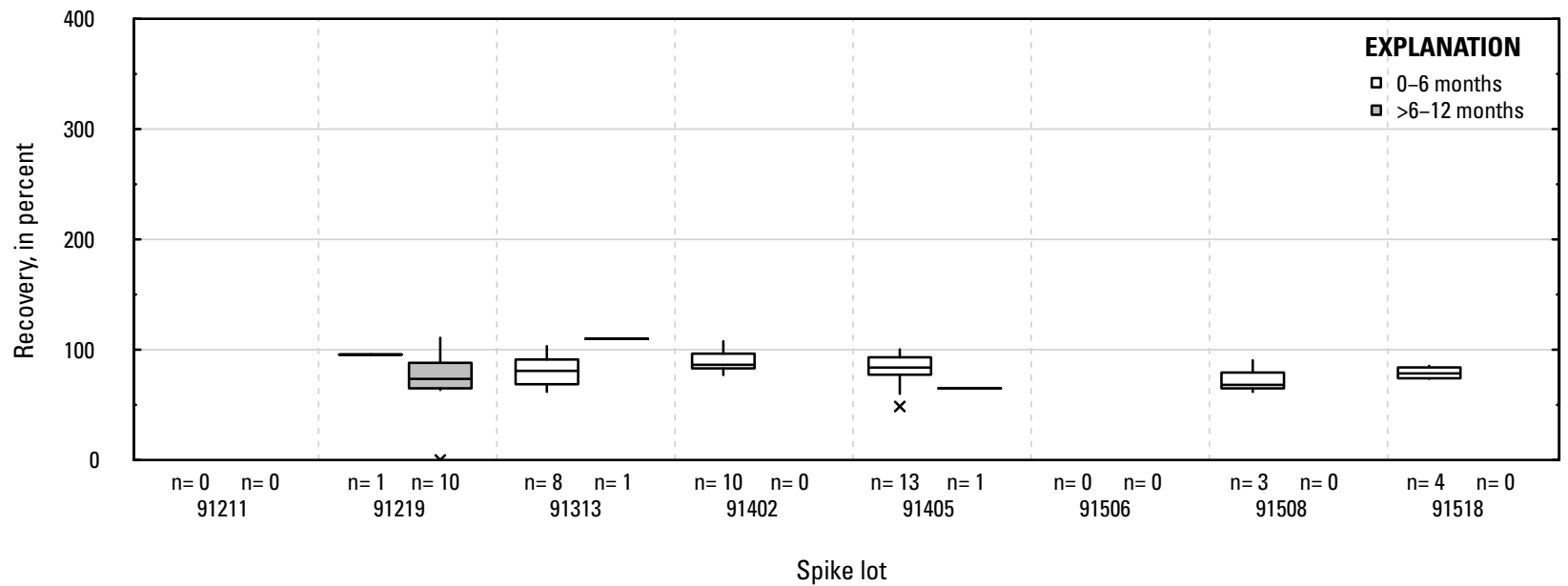

\section{IR. Disulfoton: surface water field matrix spikes}

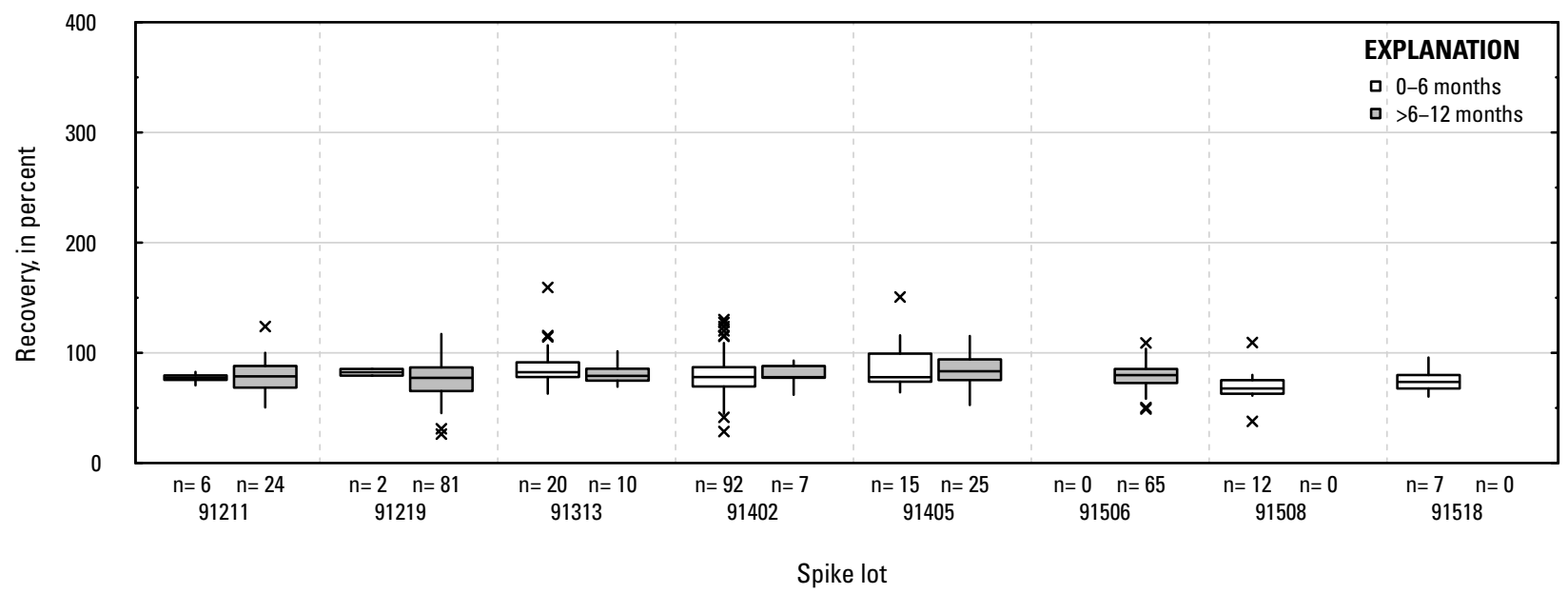

Figure 1-1. Distributions of recovery for individual pesticides in schedule 2437 by matrix, spike lot, and spike lot age. Recovery values larger than 400 percent are not shown.-Continued 


\section{IS. Disulfoton oxon: laboratory reagent spikes}

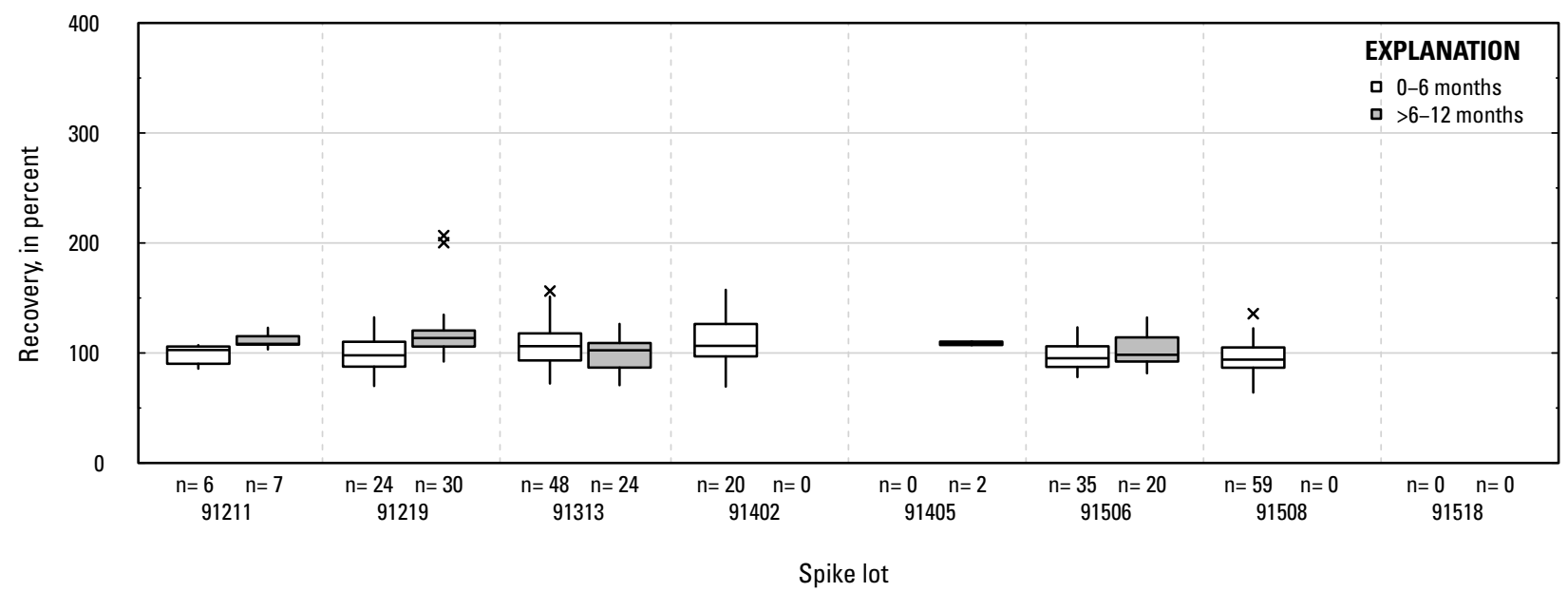

\section{IT. Disulfoton oxon: groundwater field matrix spikes}

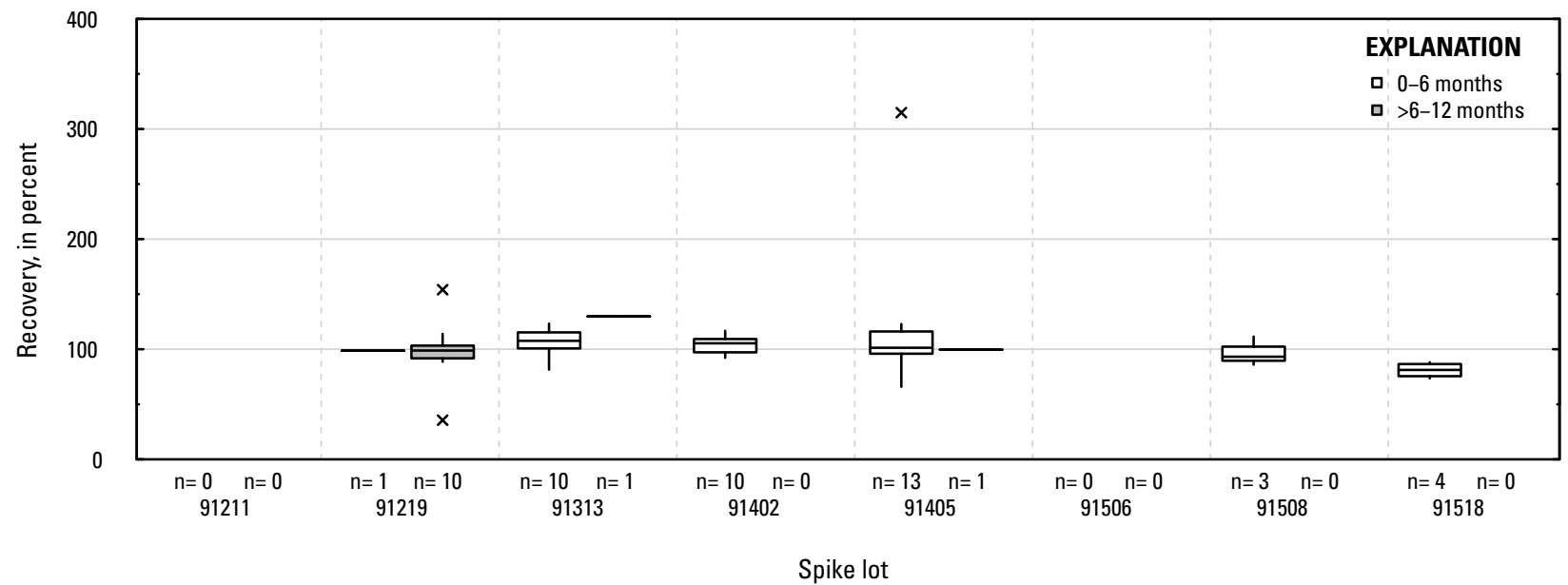

IU. Disulfoton oxon: surface water field matrix spikes

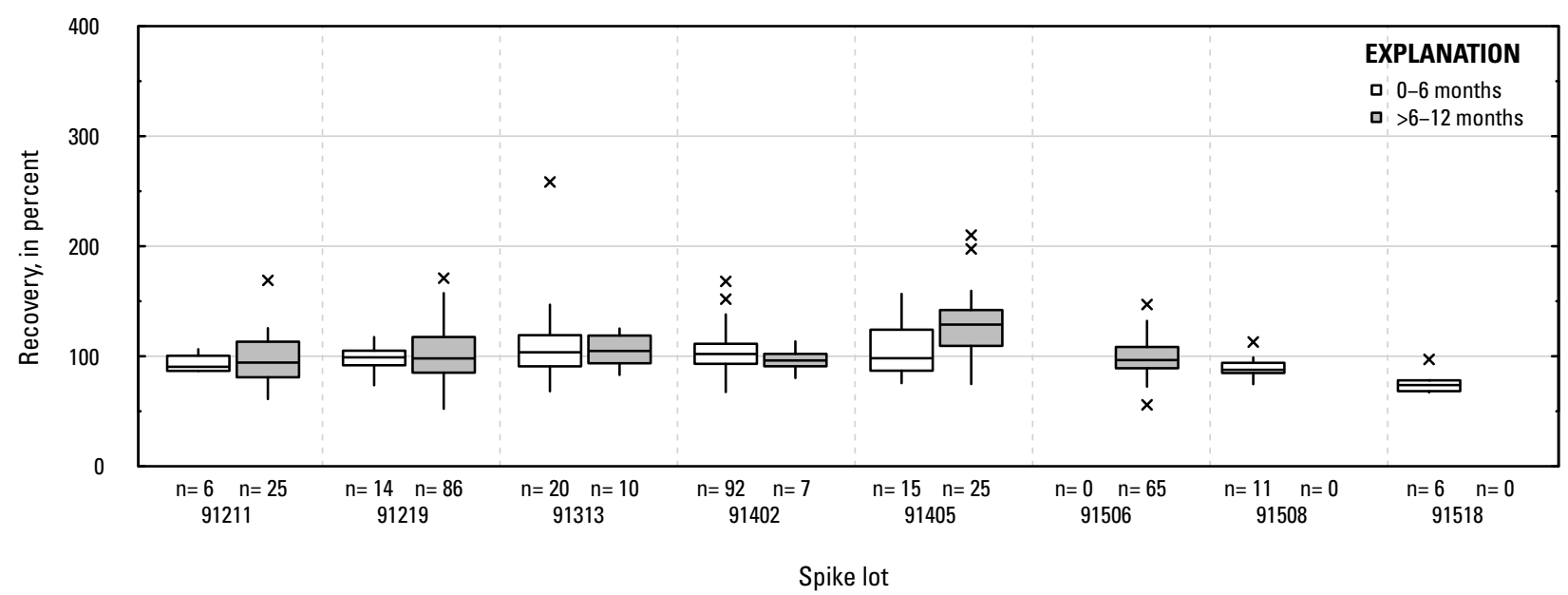

Figure 1-1. Distributions of recovery for individual pesticides in schedule 2437 by matrix, spike lot, and spike lot age. Recovery values larger than 400 percent are not shown.-Continued 
IV. Disulfoton oxon sulfone: laboratory reagent spikes

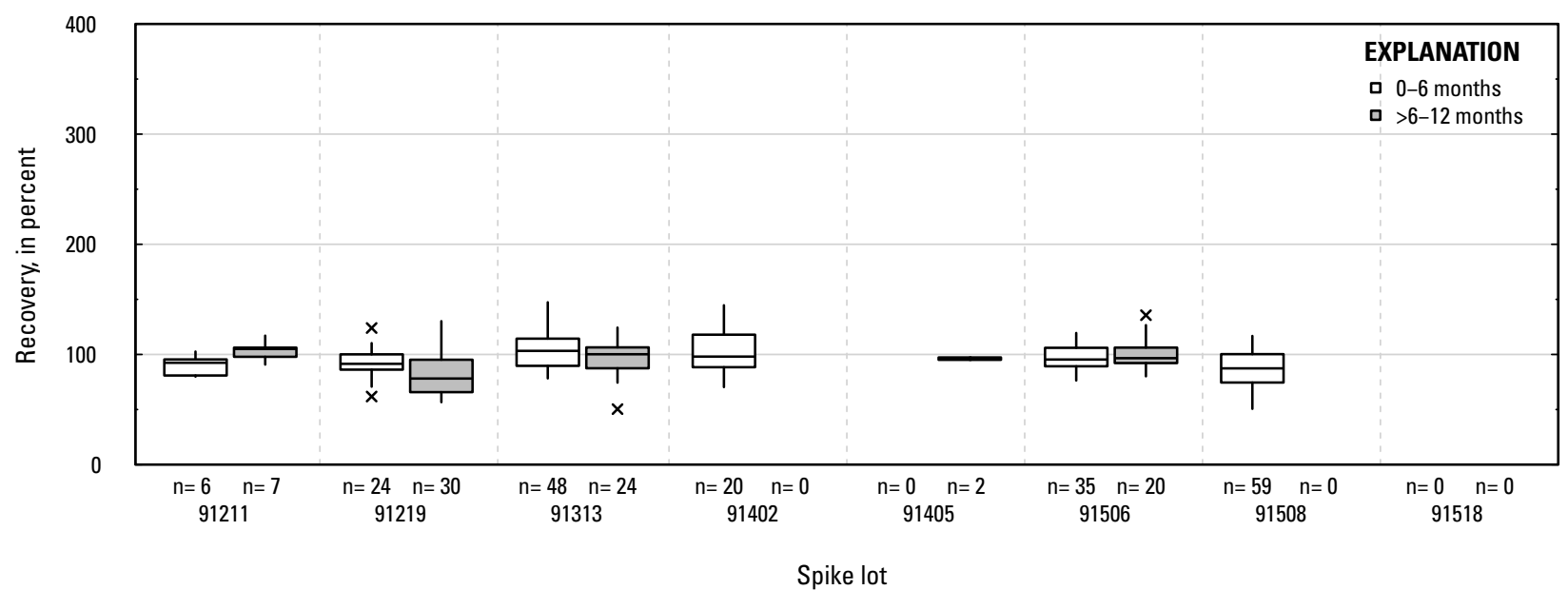

IW. Disulfoton oxon sulfone: groundwater field matrix spikes

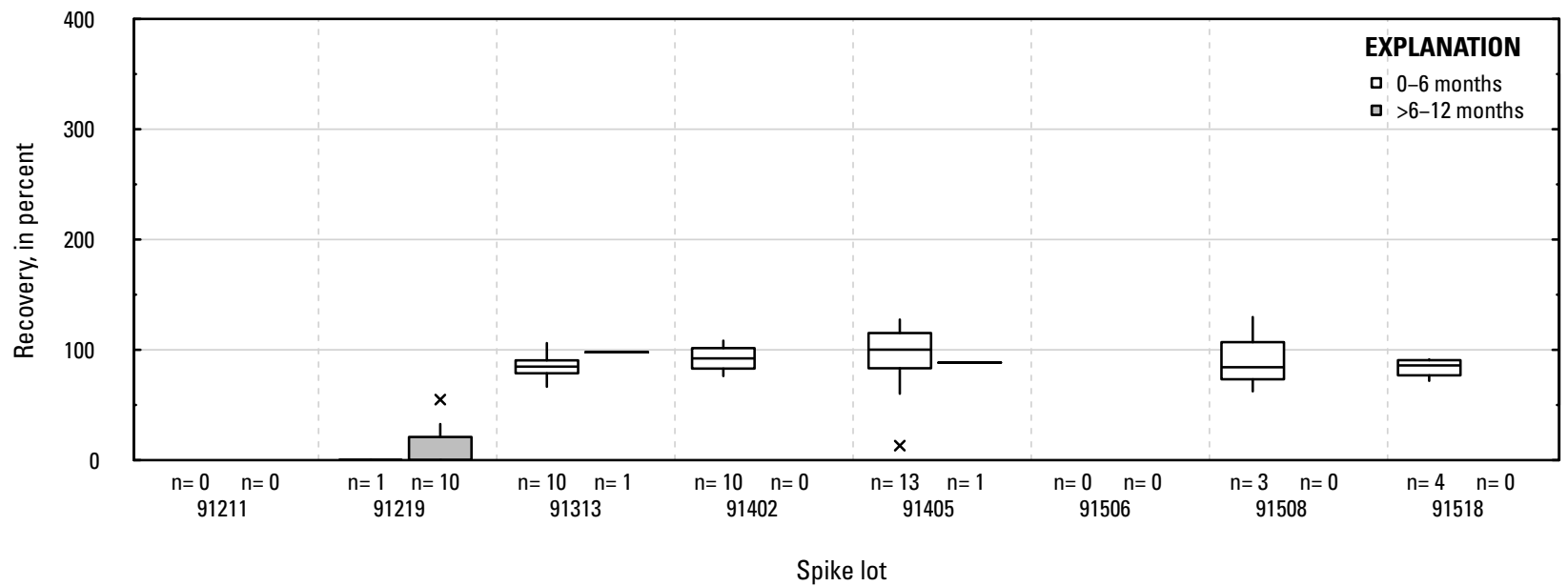

IX. Disulfoton oxon sulfone: surface water field matrix spikes

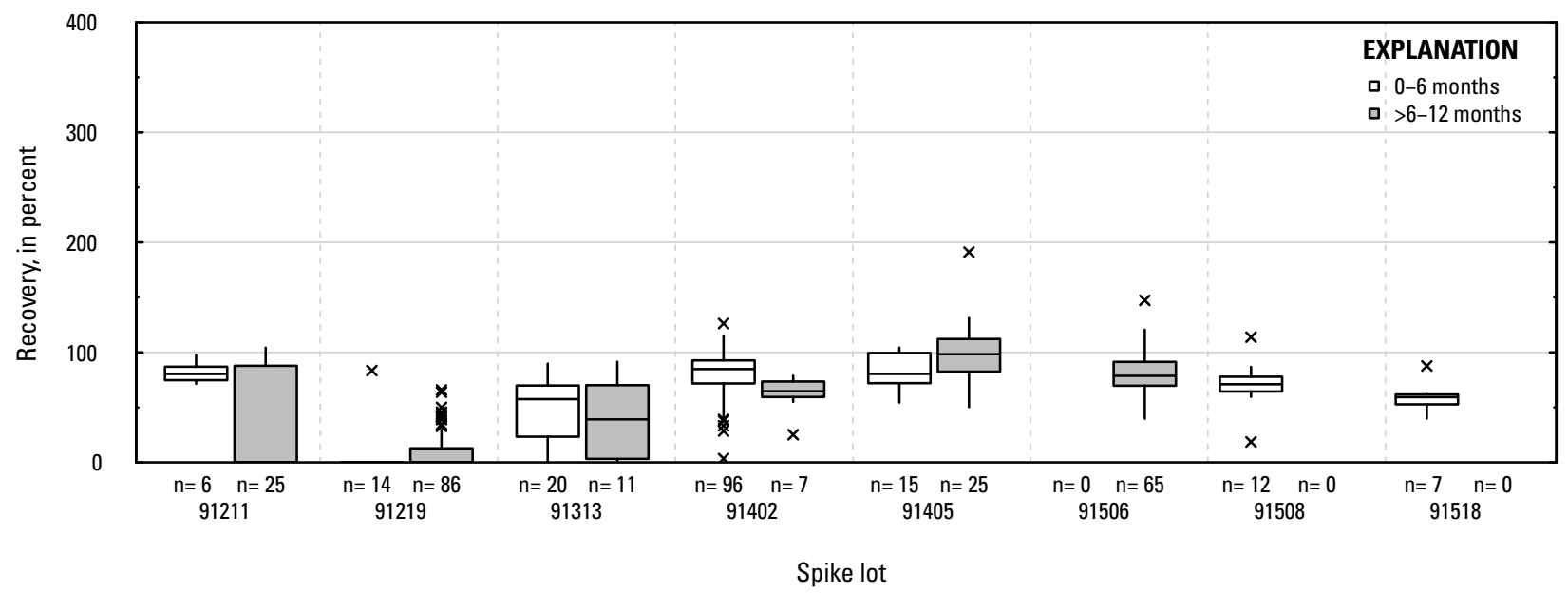

Figure 1-1. Distributions of recovery for individual pesticides in schedule 2437 by matrix, spike lot, and spike lot age. Recovery values larger than 400 percent are not shown.-Continued 
IY. Disulfoton oxon sulfoxide: laboratory reagent spikes

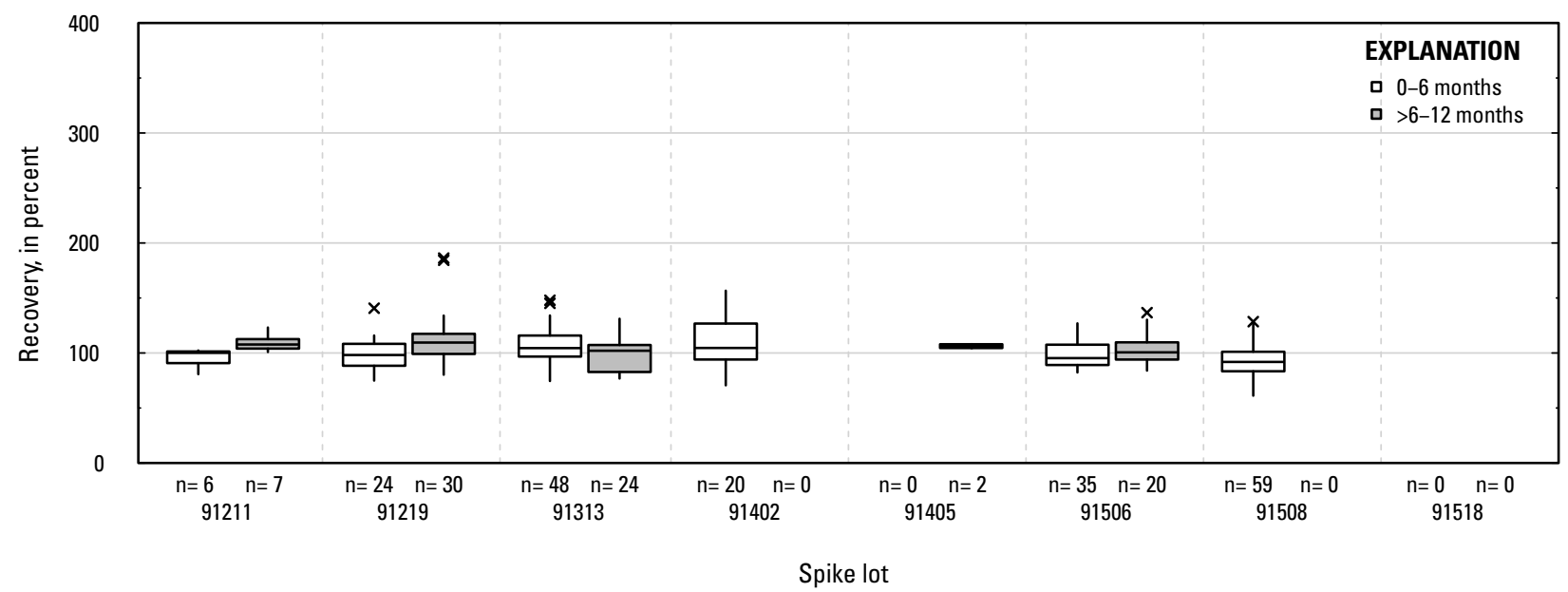

\section{IZ. Disulfoton oxon sulfoxide: groundwater field matrix spikes}

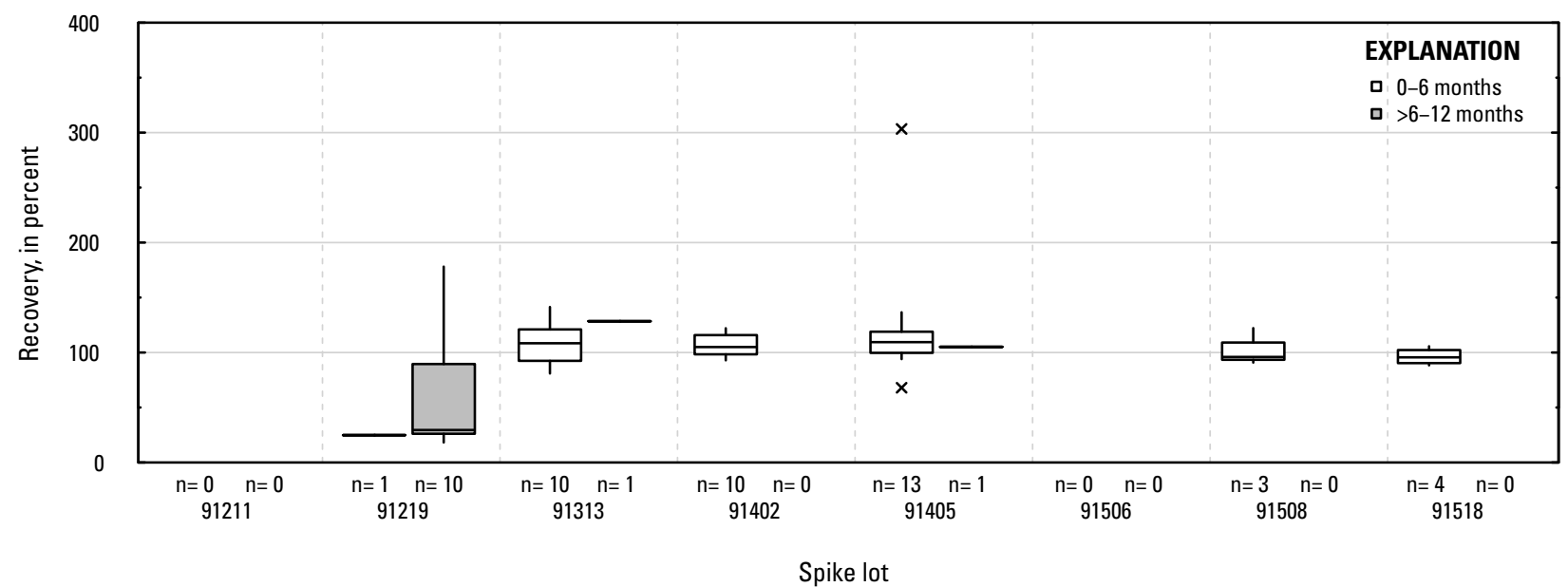

JA. Disulfoton oxon sulfoxide: surface water field matrix spikes

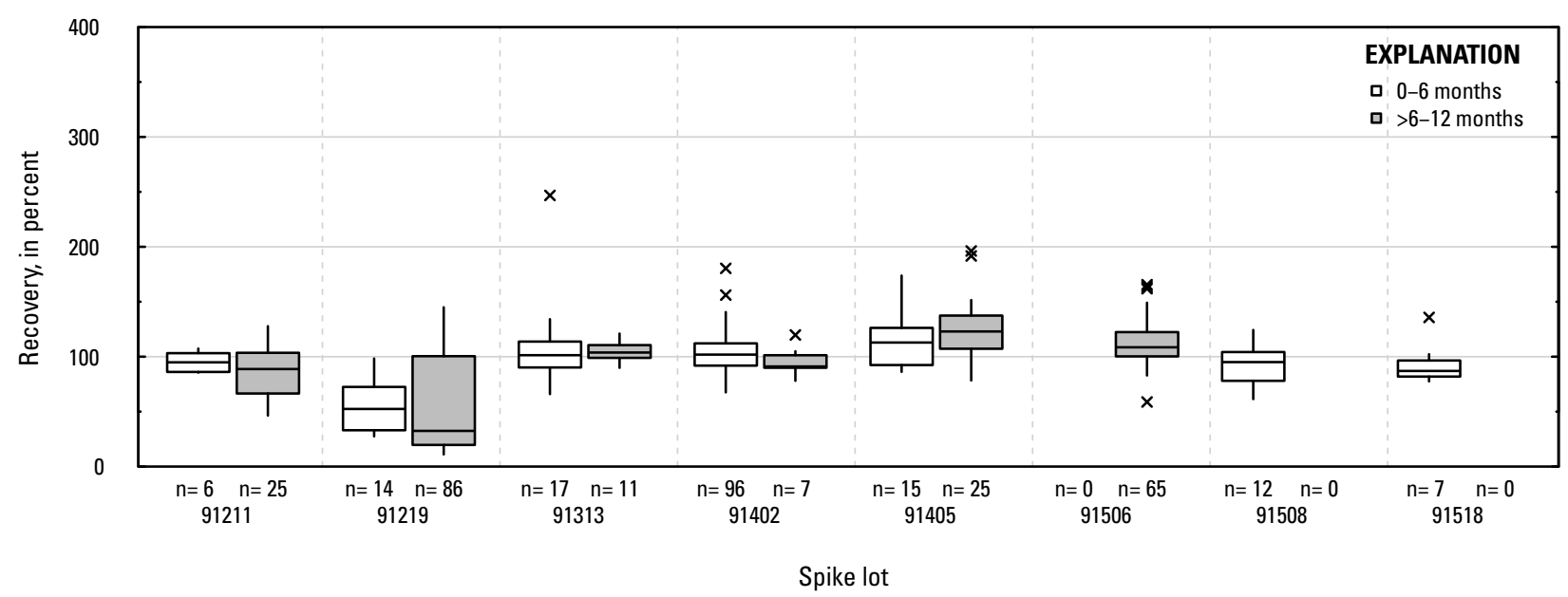

Figure 1-1. Distributions of recovery for individual pesticides in schedule 2437 by matrix, spike lot, and spike lot age. Recovery values larger than 400 percent are not shown.-Continued 
JB. Disulfoton sulfone: laboratory reagent spikes

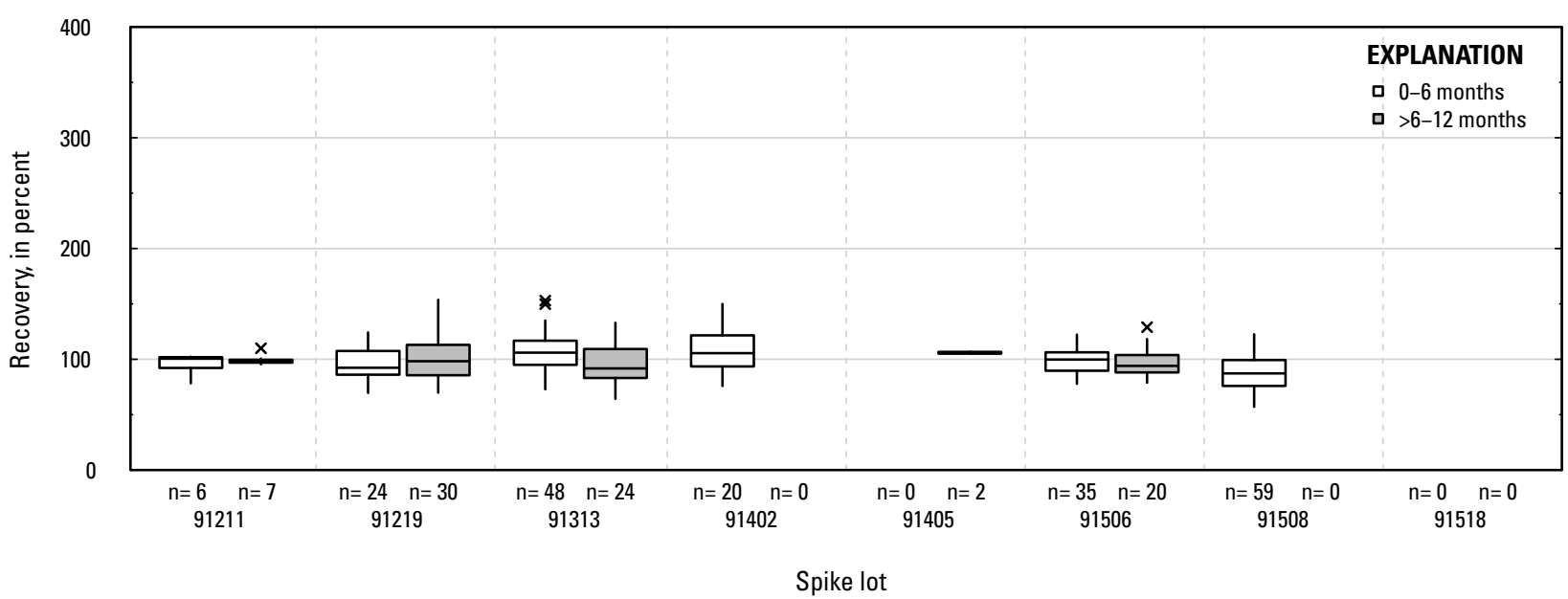

JC. Disulfoton sulfone: groundwater field matrix spikes

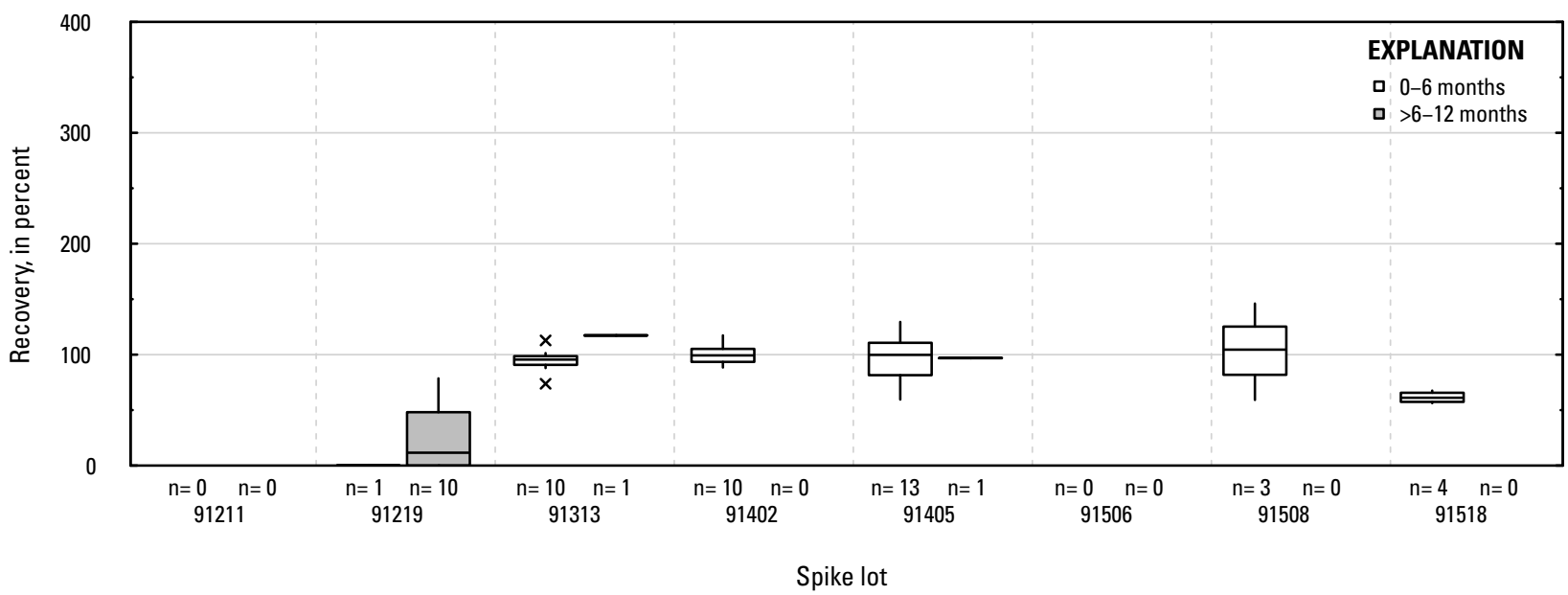

JD. Disulfoton sulfone: surface water field matrix spikes

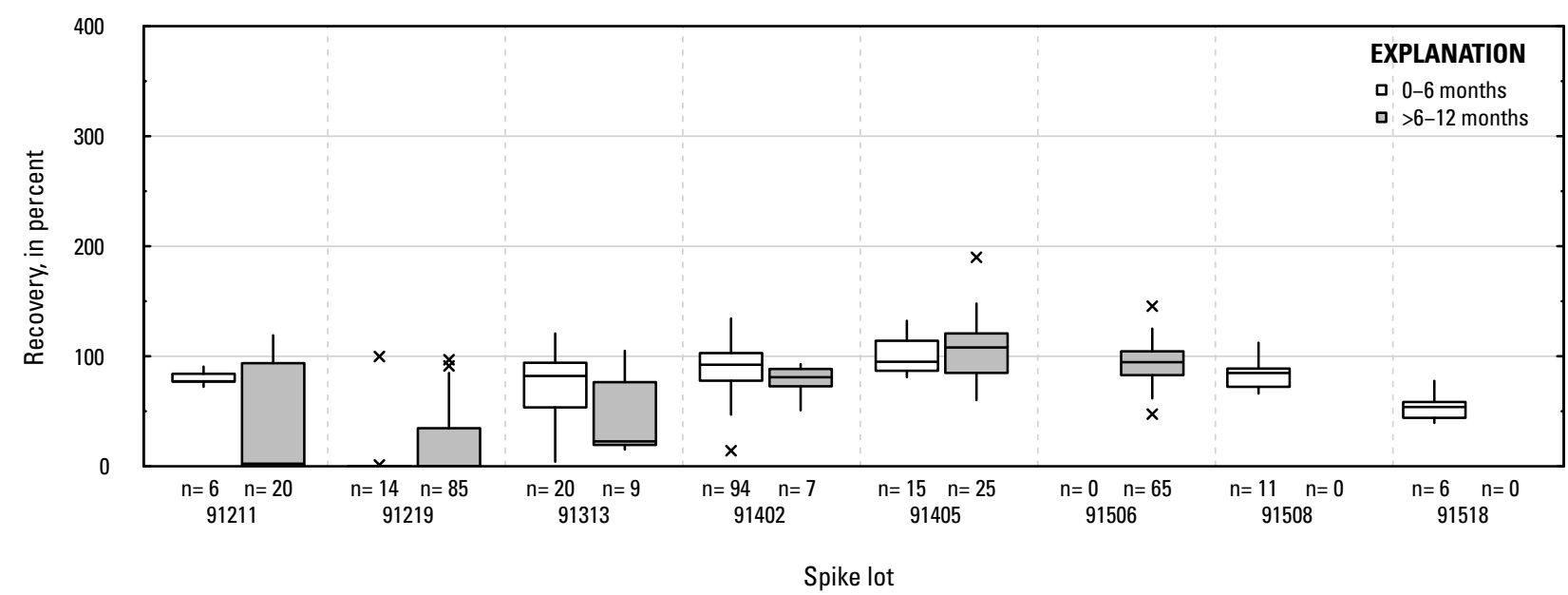

Figure 1-1. Distributions of recovery for individual pesticides in schedule 2437 by matrix, spike lot, and spike lot age. Recovery values larger than 400 percent are not shown.-Continued 


\section{JE. Disulfoton sulfoxide: laboratory reagent spikes}

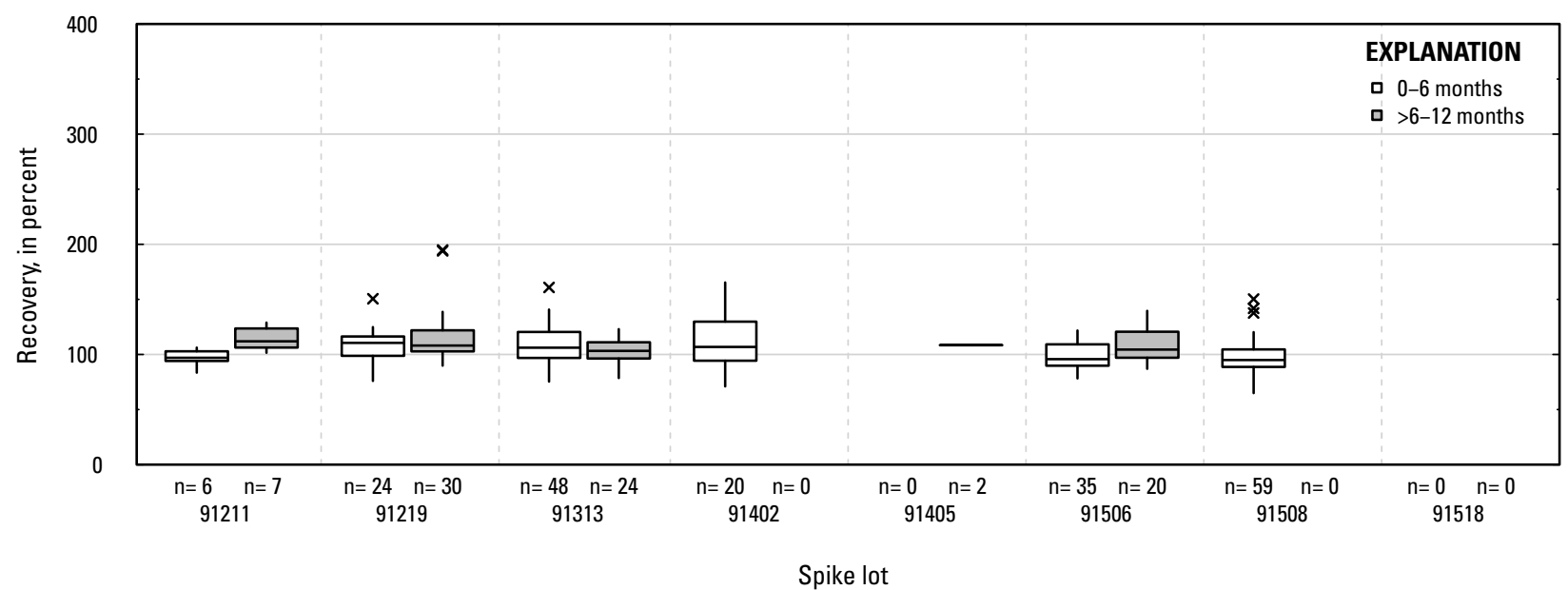

\section{JF. Disulfoton sulfoxide: groundwater field matrix spikes}

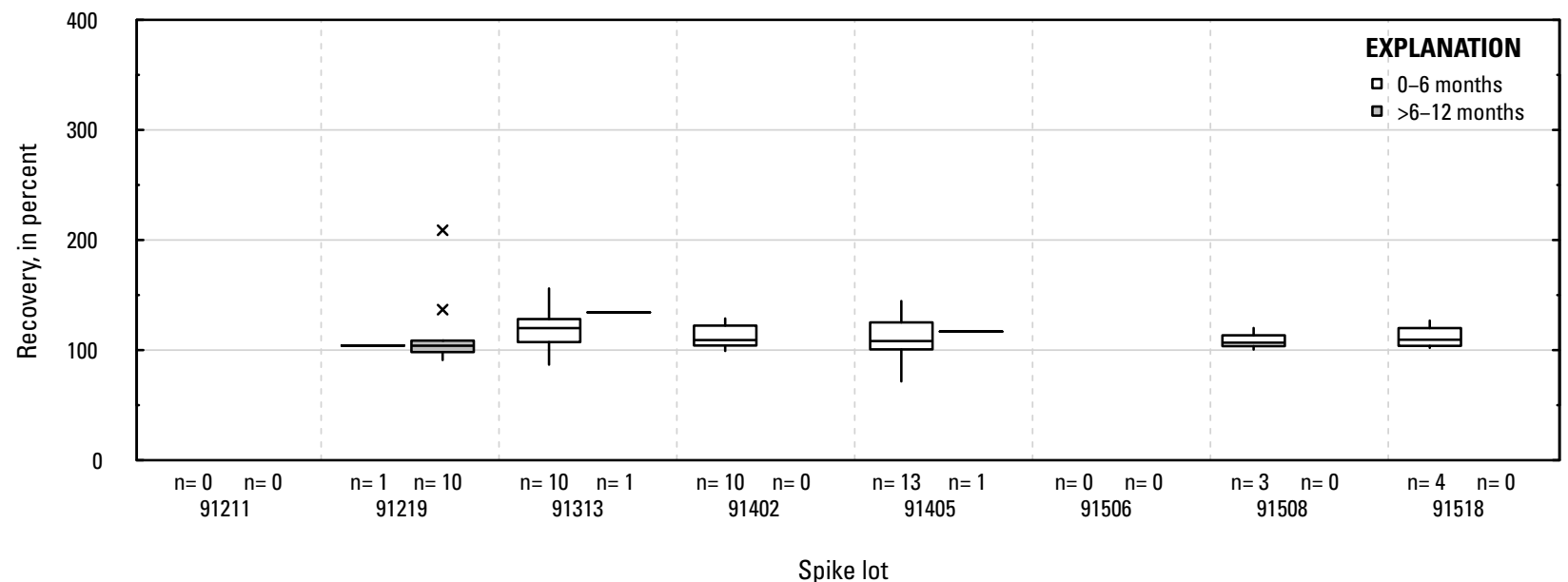

JG. Disulfoton sulfoxide: surface water field matrix spikes

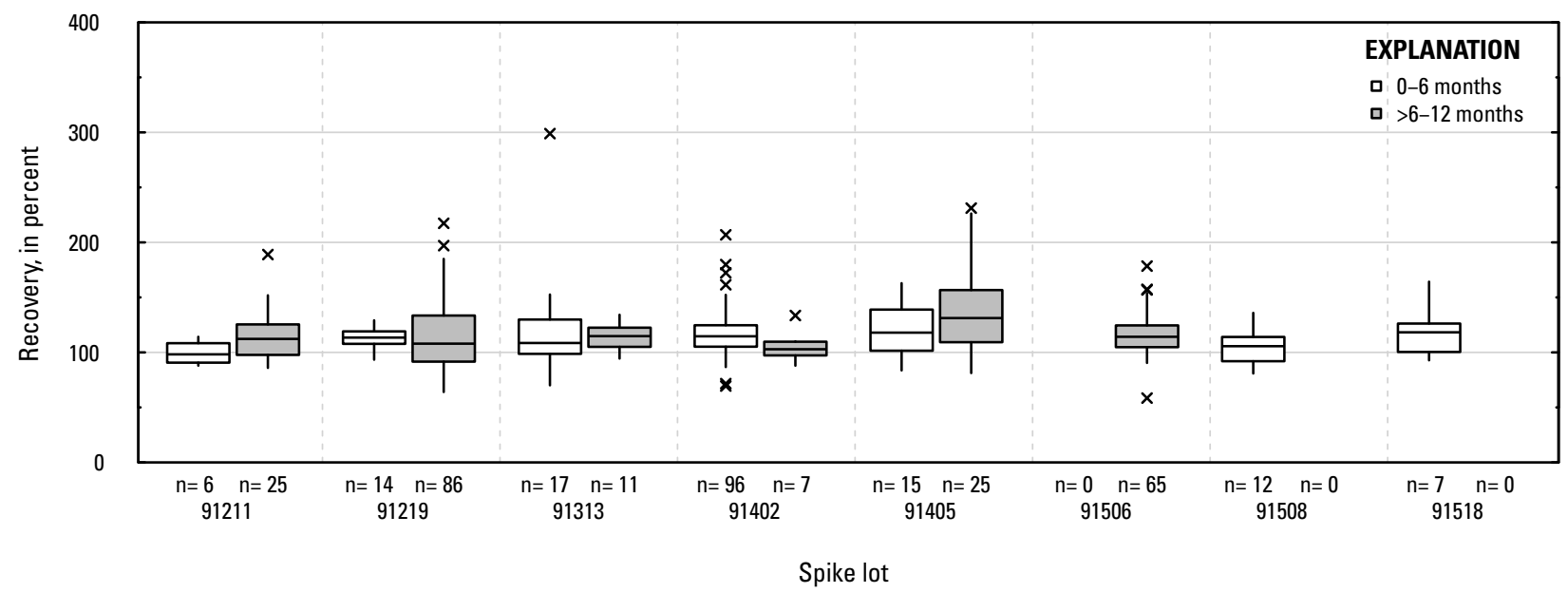

Figure 1-1. Distributions of recovery for individual pesticides in schedule 2437 by matrix, spike lot, and spike lot age. Recovery values larger than 400 percent are not shown.-Continued 


\section{$J H$. Diuron: laboratory reagent spikes}

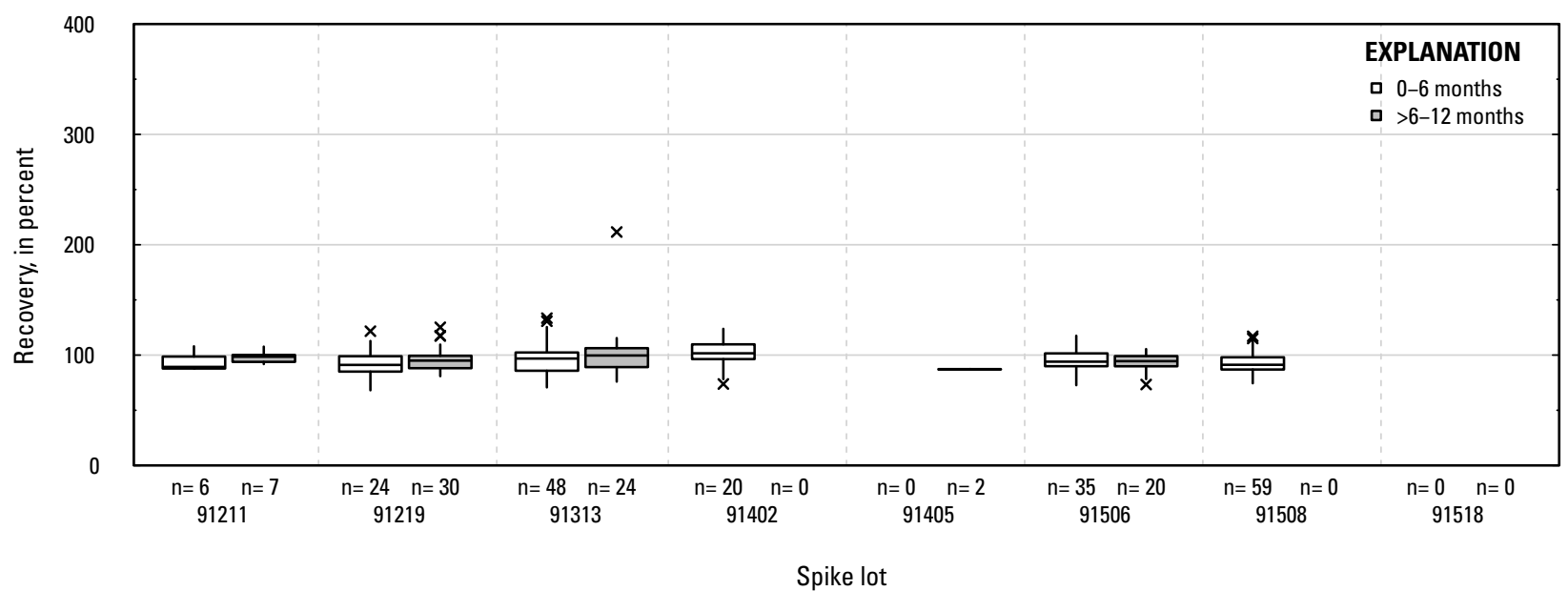

\section{Jl. Diuron: groundwater field matrix spikes}

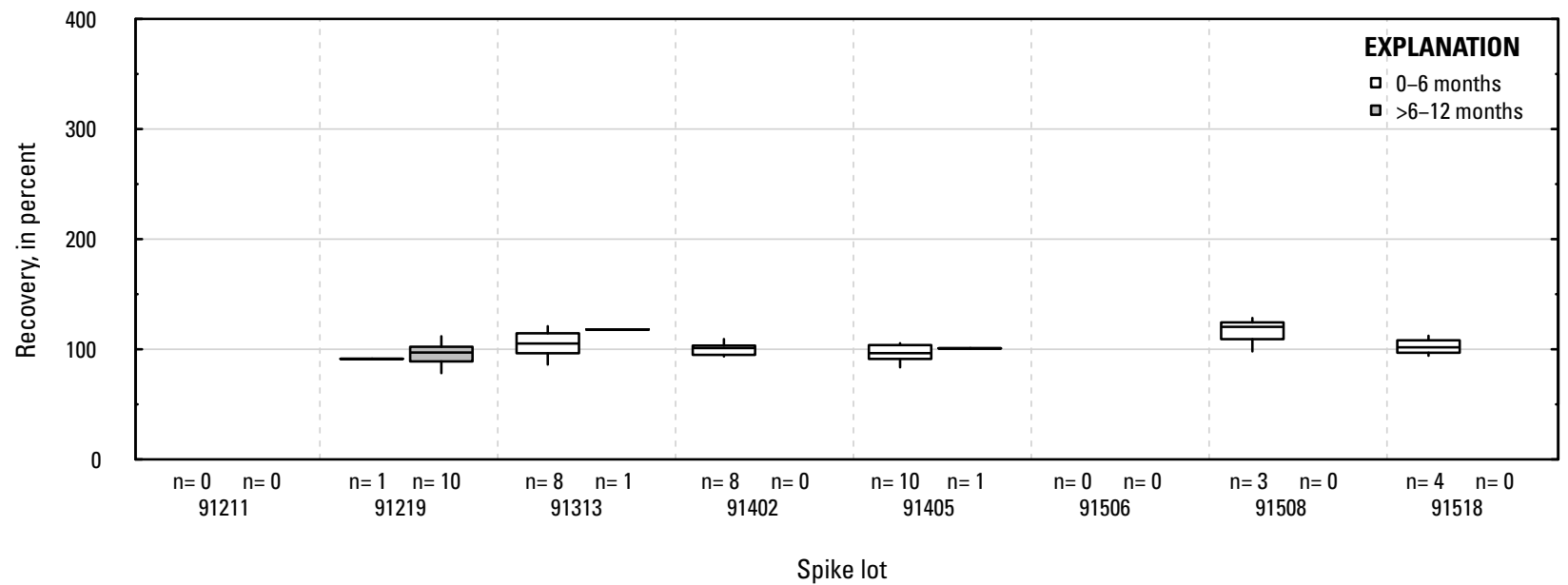

\section{JJ. Diuron: surface water field matrix spikes}

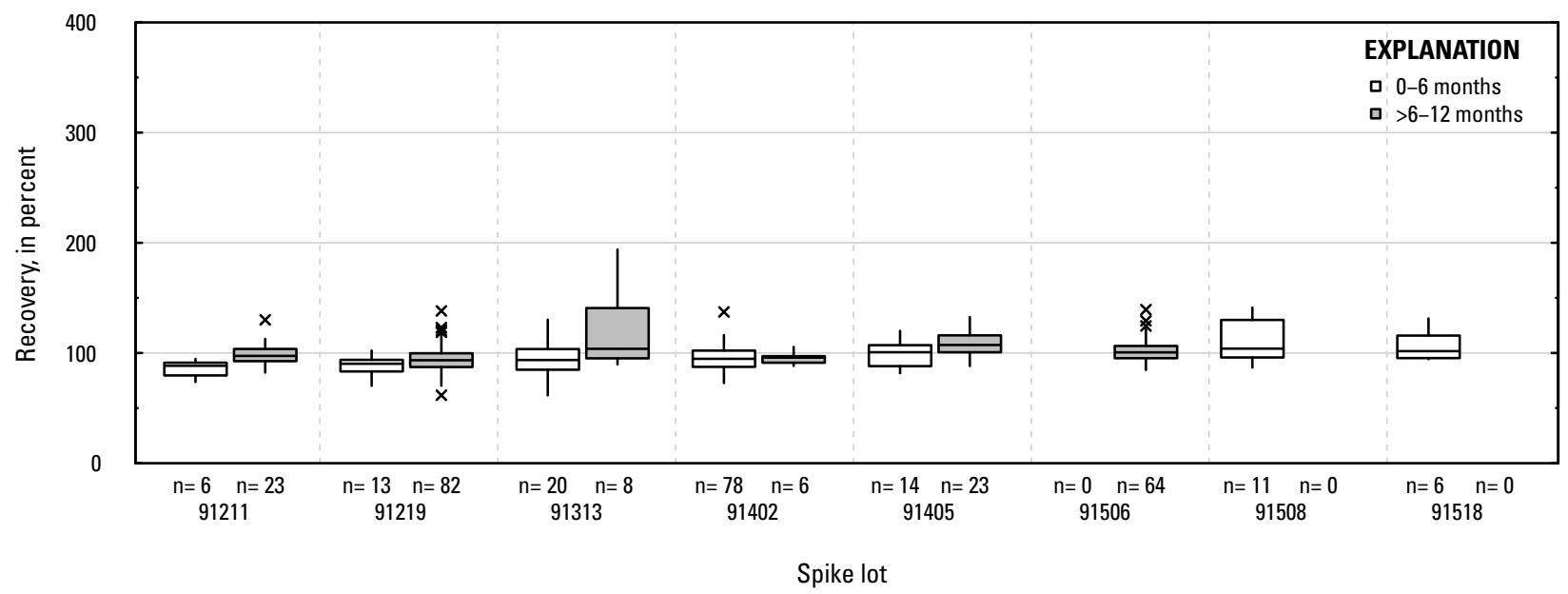

Figure 1-1. Distributions of recovery for individual pesticides in schedule 2437 by matrix, spike lot, and spike lot age. Recovery values larger than 400 percent are not shown.-Continued 


\section{JK. EPTC: laboratory reagent spikes}

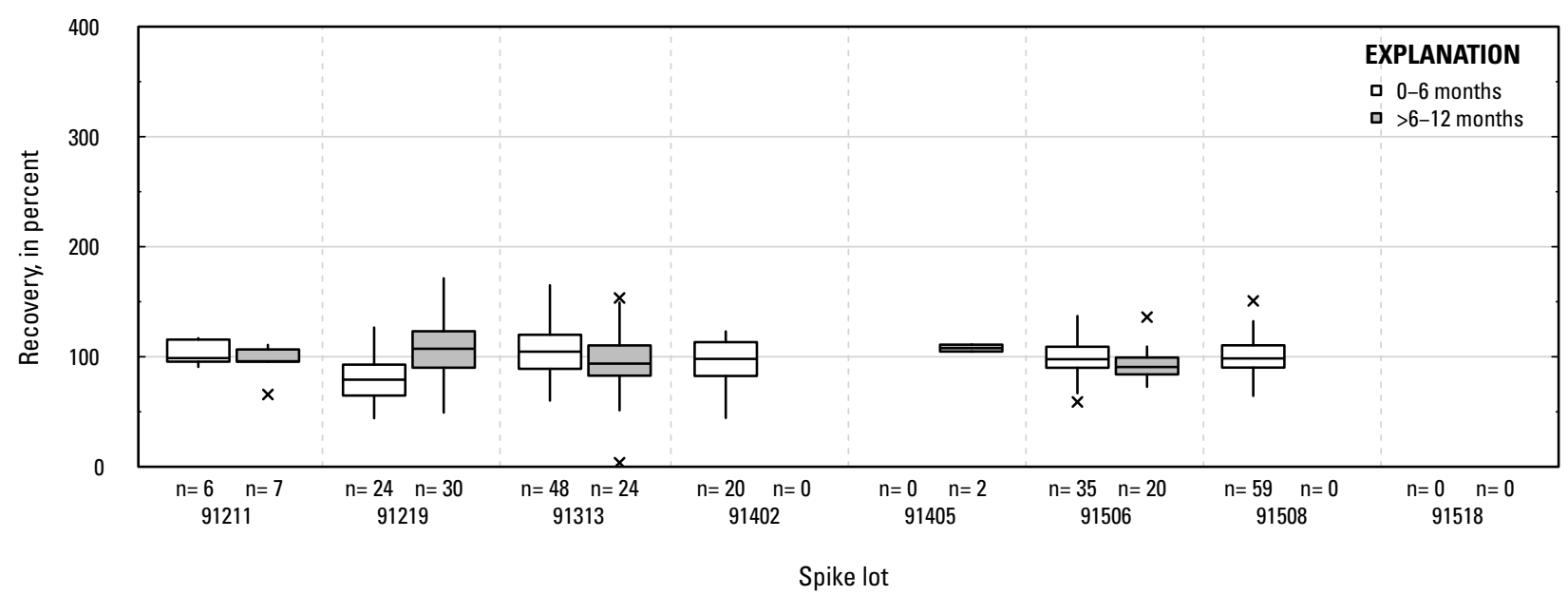

\section{JL. EPTC: groundwater field matrix spikes}

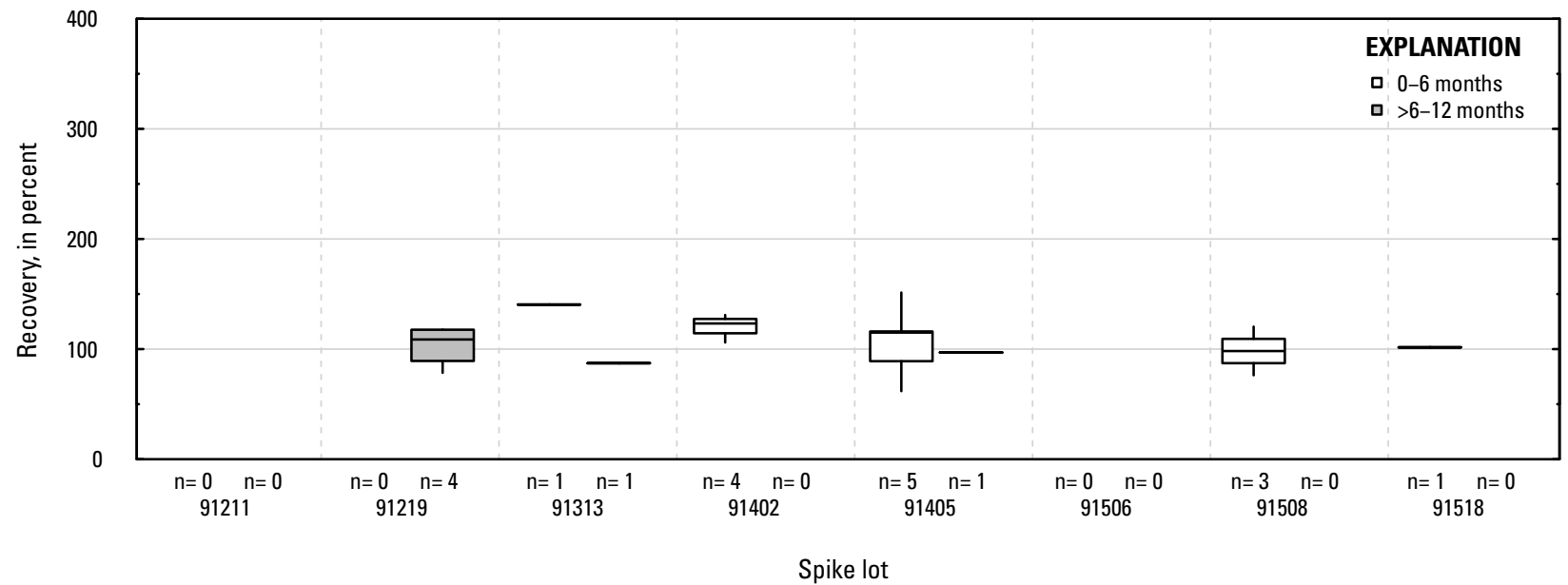

JM. EPTC: surface water field matrix spikes

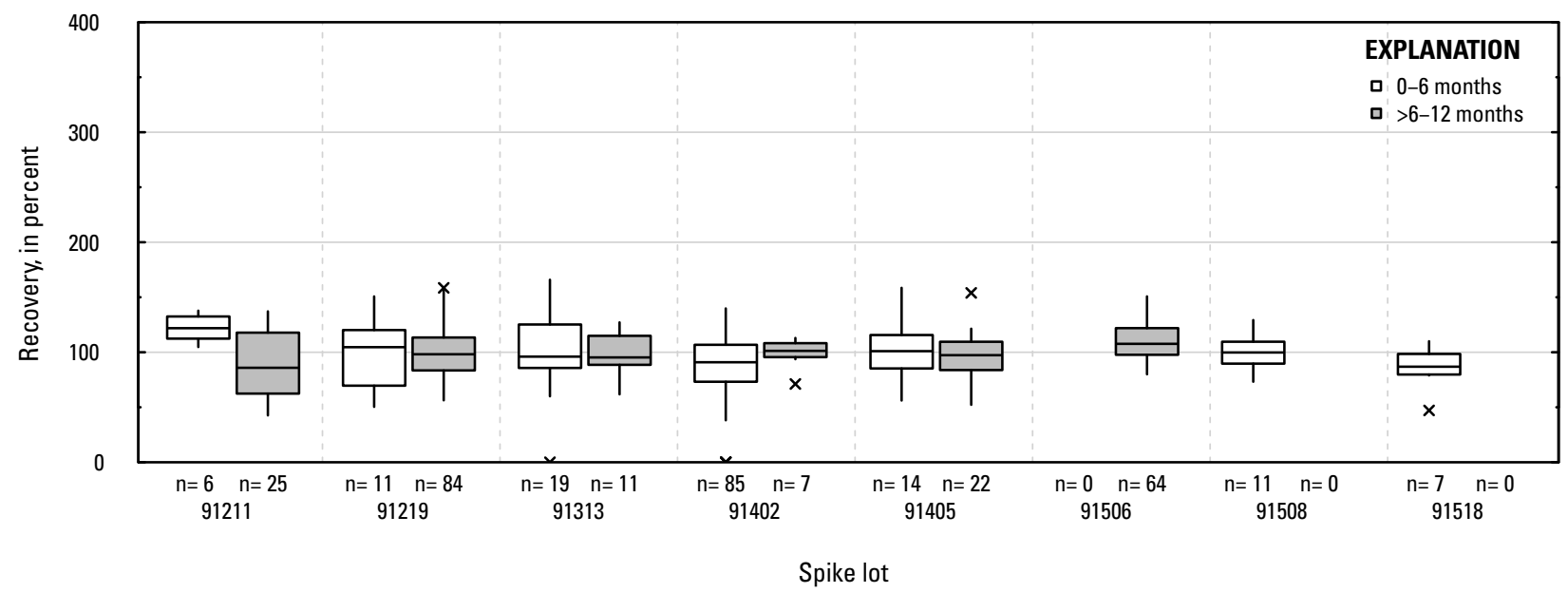

Figure 1-1. Distributions of recovery for individual pesticides in schedule 2437 by matrix, spike lot, and spike lot age. Recovery values larger than 400 percent are not shown.-Continued 
JN. EPTC degradate R248722: laboratory reagent spikes

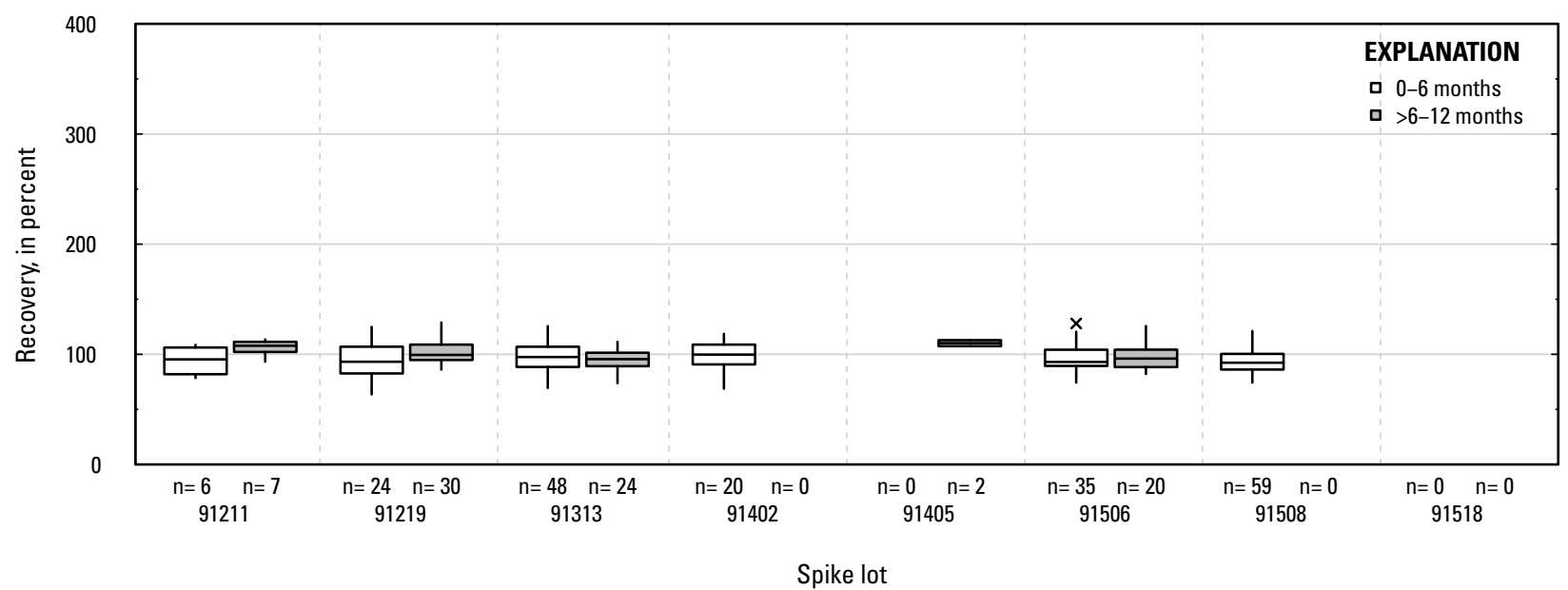

\section{JO. EPTC degradate R248722: groundwater field matrix spikes}

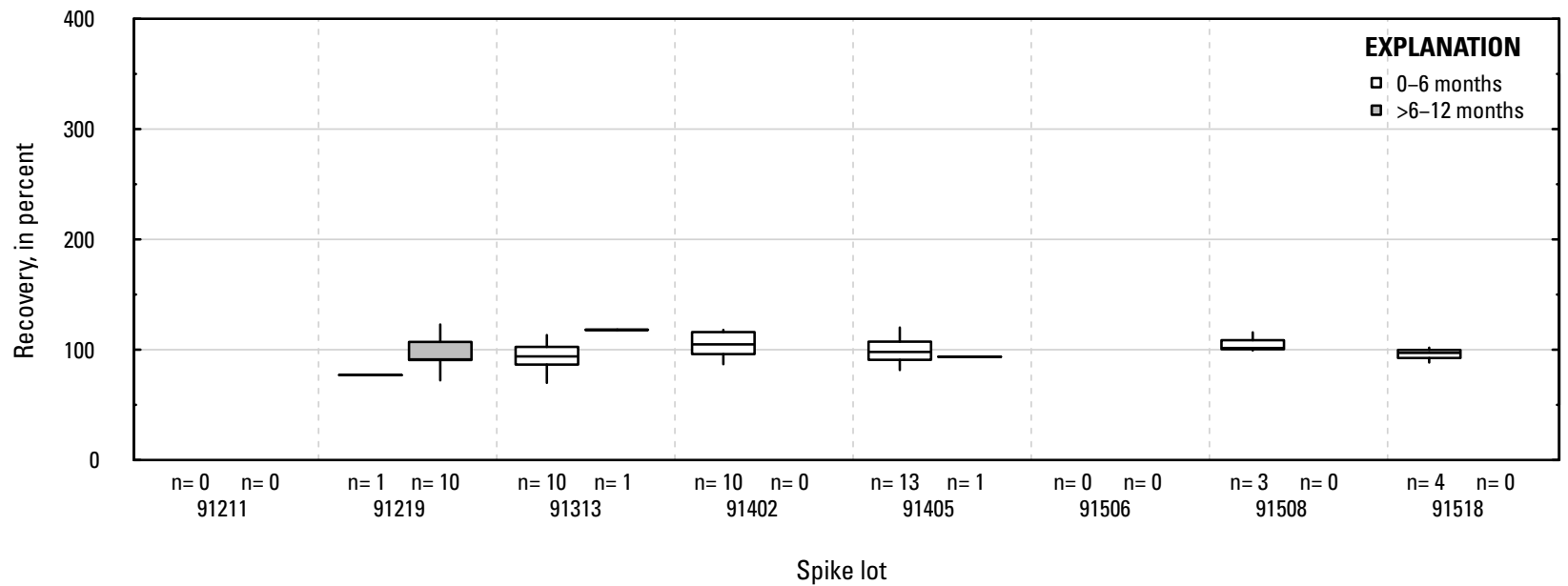

JP. EPTC degradate R248722: surface water field matrix spikes

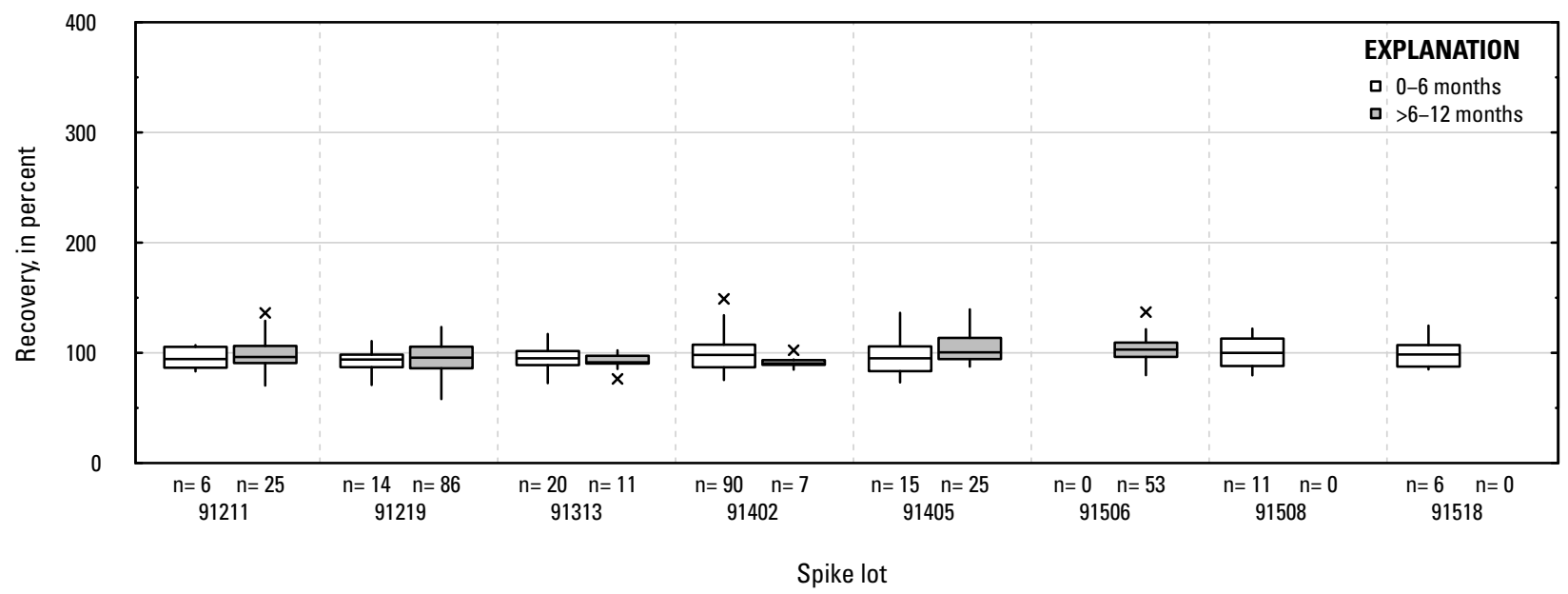

Figure 1-1. Distributions of recovery for individual pesticides in schedule 2437 by matrix, spike lot, and spike lot age. Recovery values larger than 400 percent are not shown.-Continued 


\section{JQ. Ethoprophos: laboratory reagent spikes}

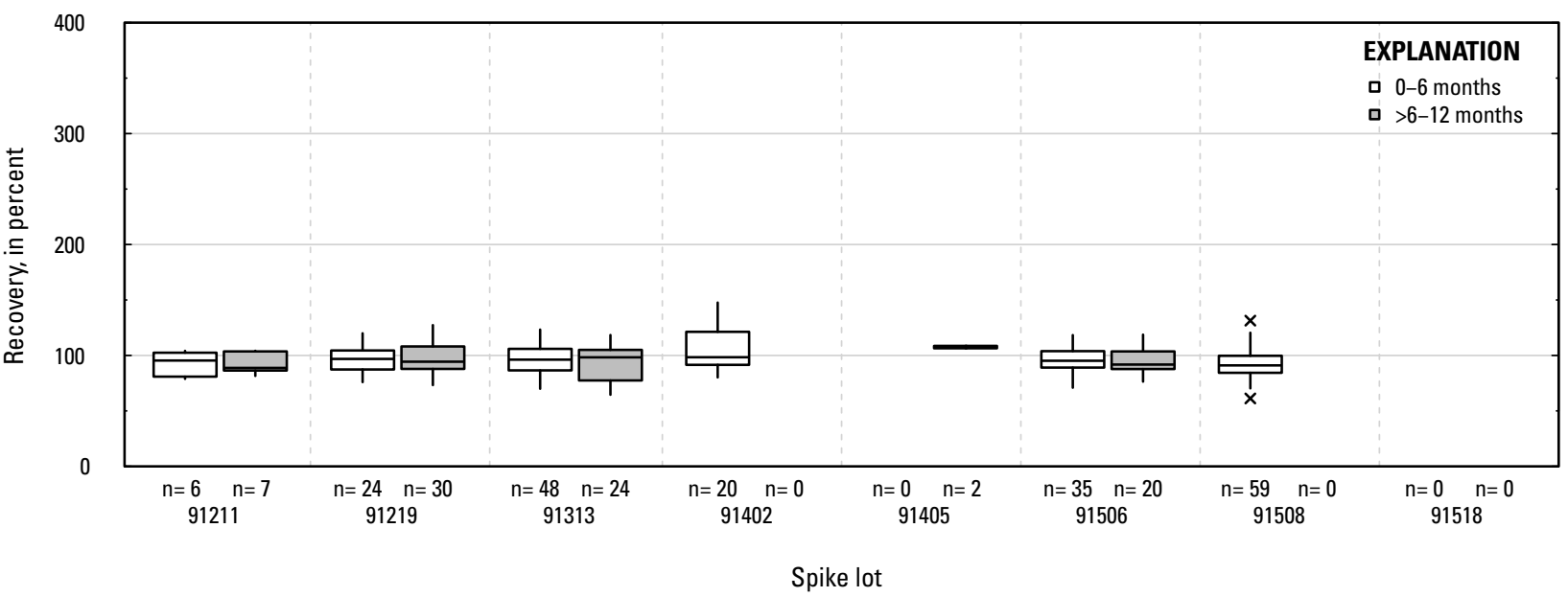

\section{JR. Ethoprophos: groundwater field matrix spikes}

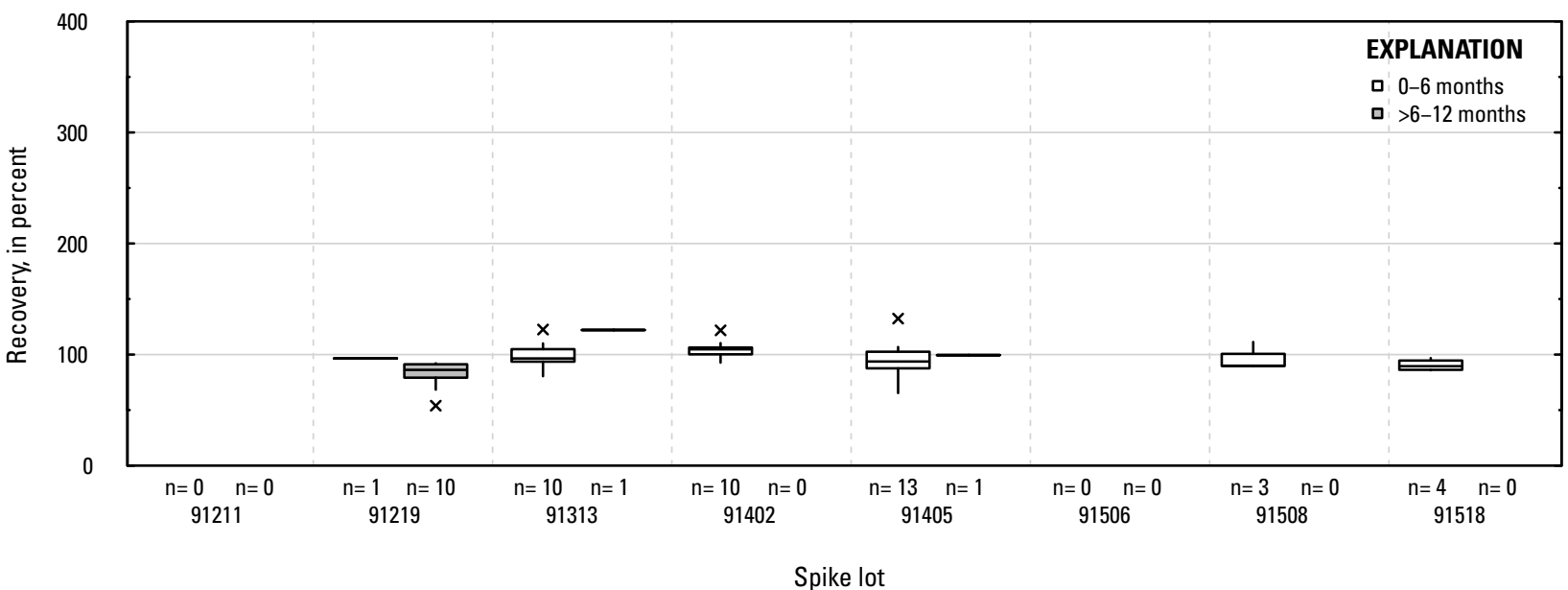

\section{JS. Ethoprophos: surface water field matrix spikes}

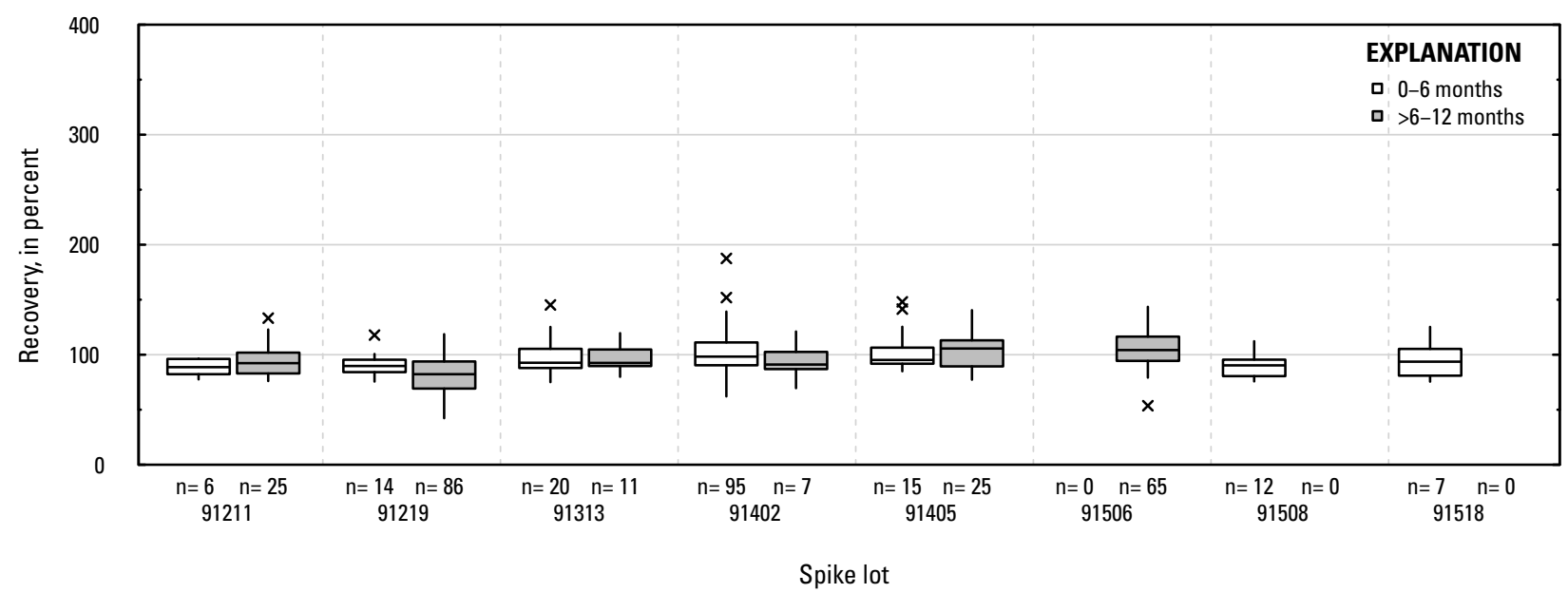

Figure 1-1. Distributions of recovery for individual pesticides in schedule 2437 by matrix, spike lot, and spike lot age. Recovery values larger than 400 percent are not shown.-Continued 


\section{JT. Etoxazole: laboratory reagent spikes}

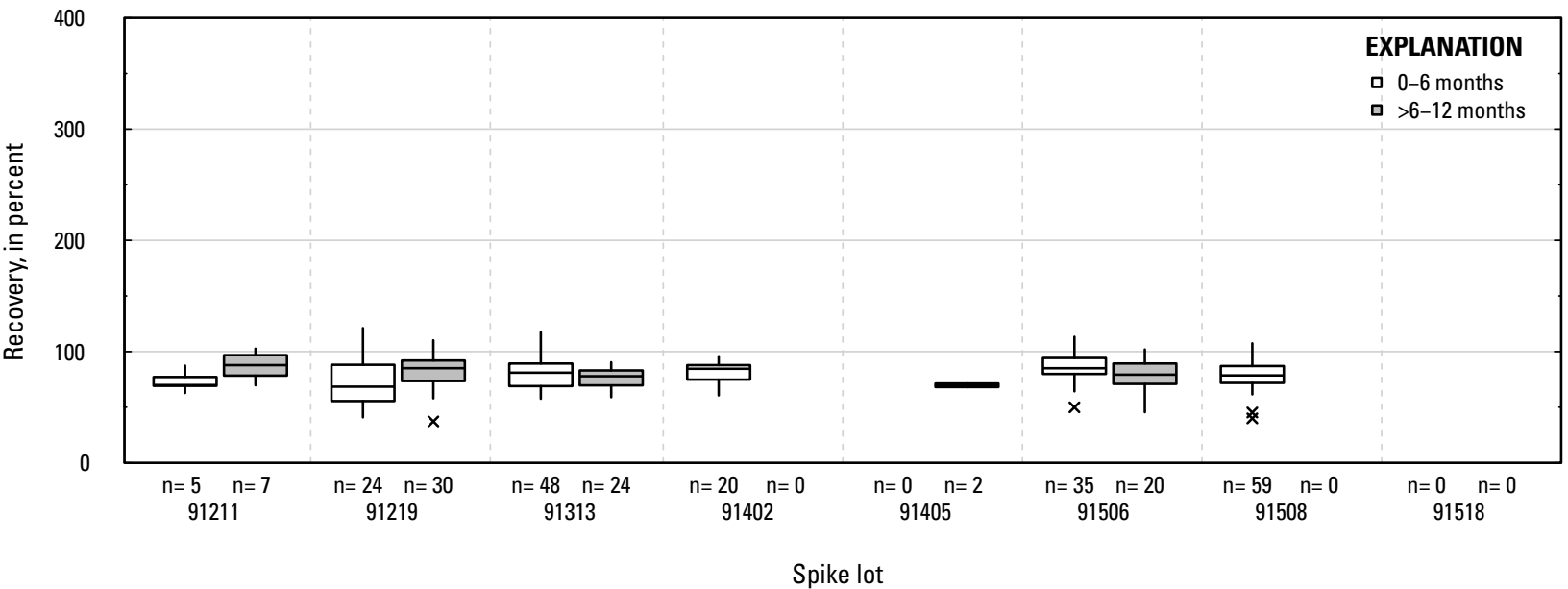

\section{JU. Etoxazole: groundwater field matrix spikes}

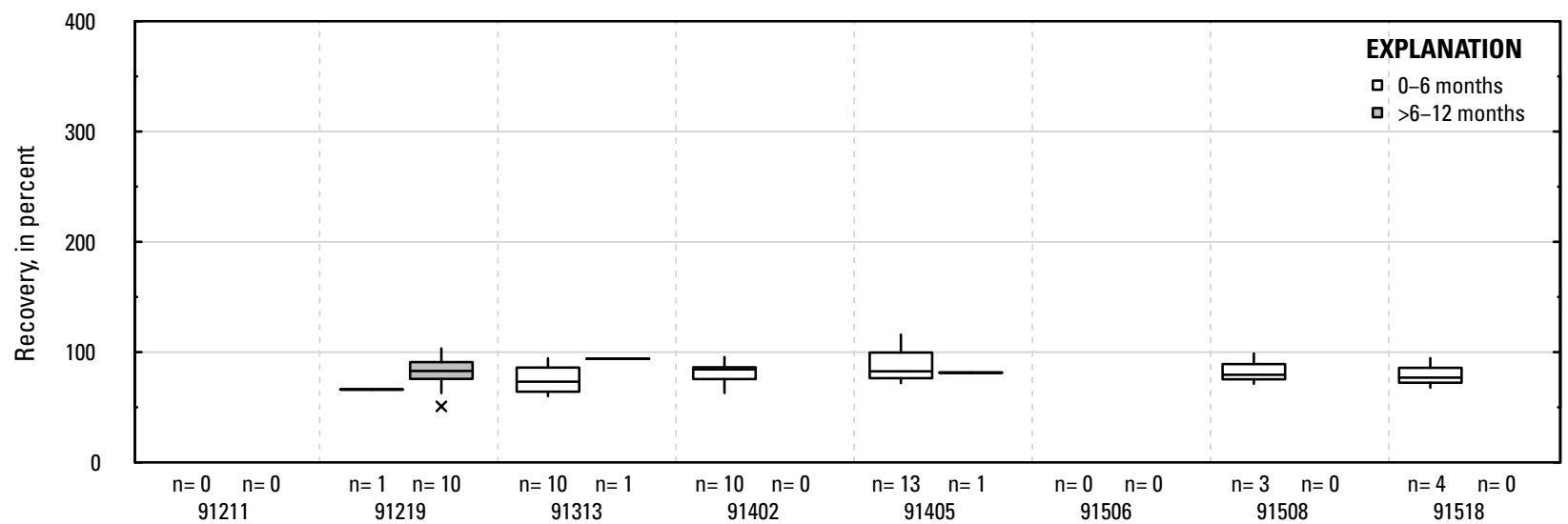

Spike lot

\section{JV. Etoxazole: surface water field matrix spikes}

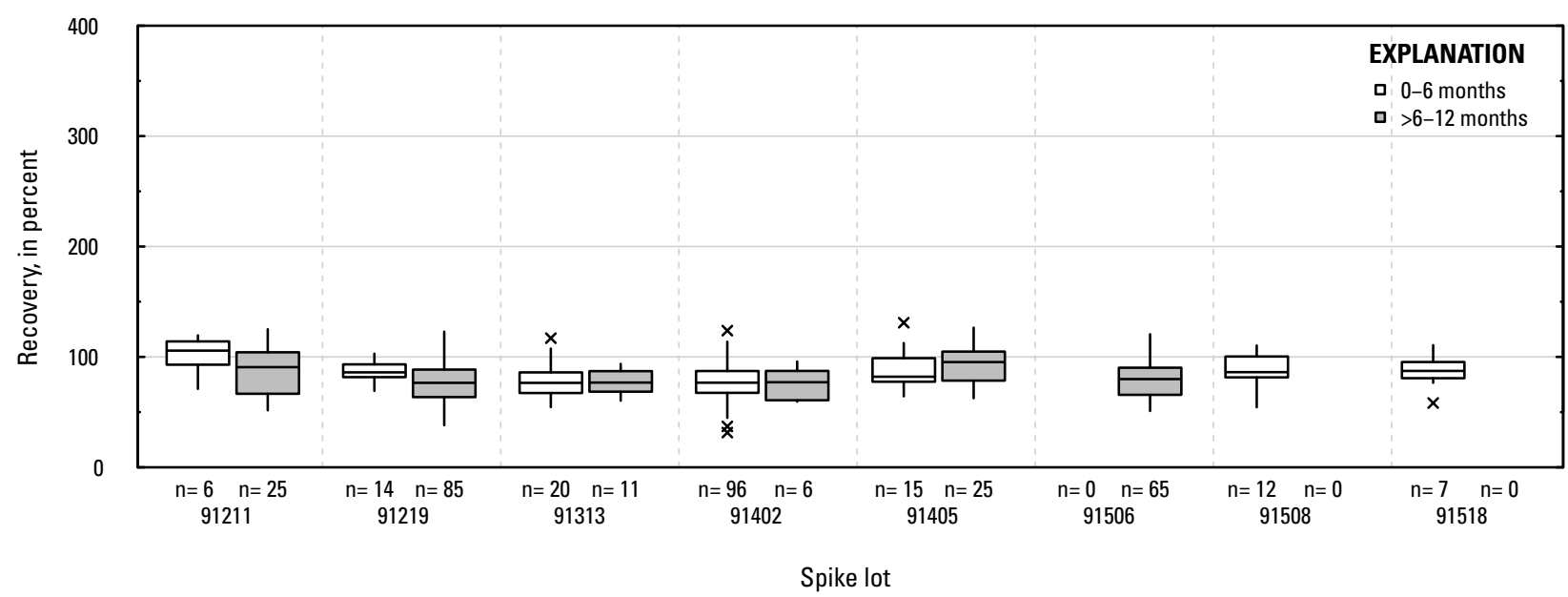

Figure 1-1. Distributions of recovery for individual pesticides in schedule 2437 by matrix, spike lot, and spike lot age. Recovery values larger than 400 percent are not shown.-Continued 
JW. Fenamiphos: laboratory reagent spikes

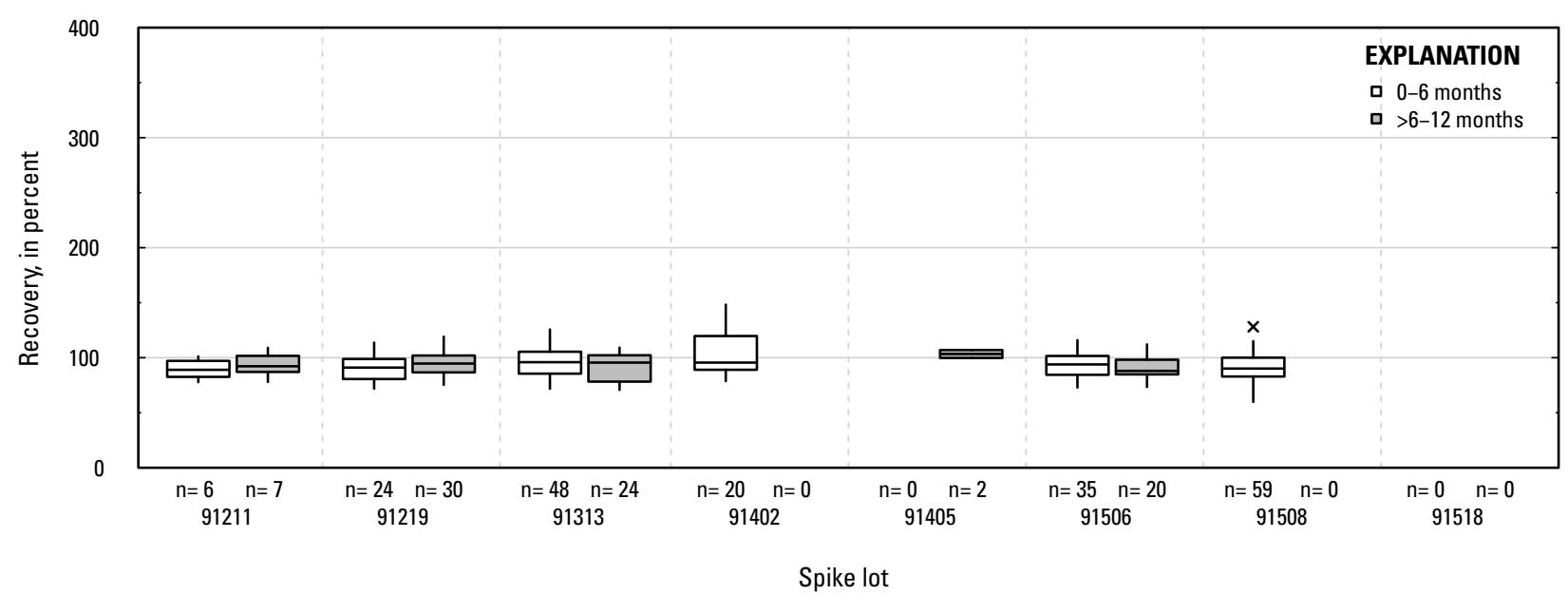

$J X$. Fenamiphos: groundwater field matrix spikes

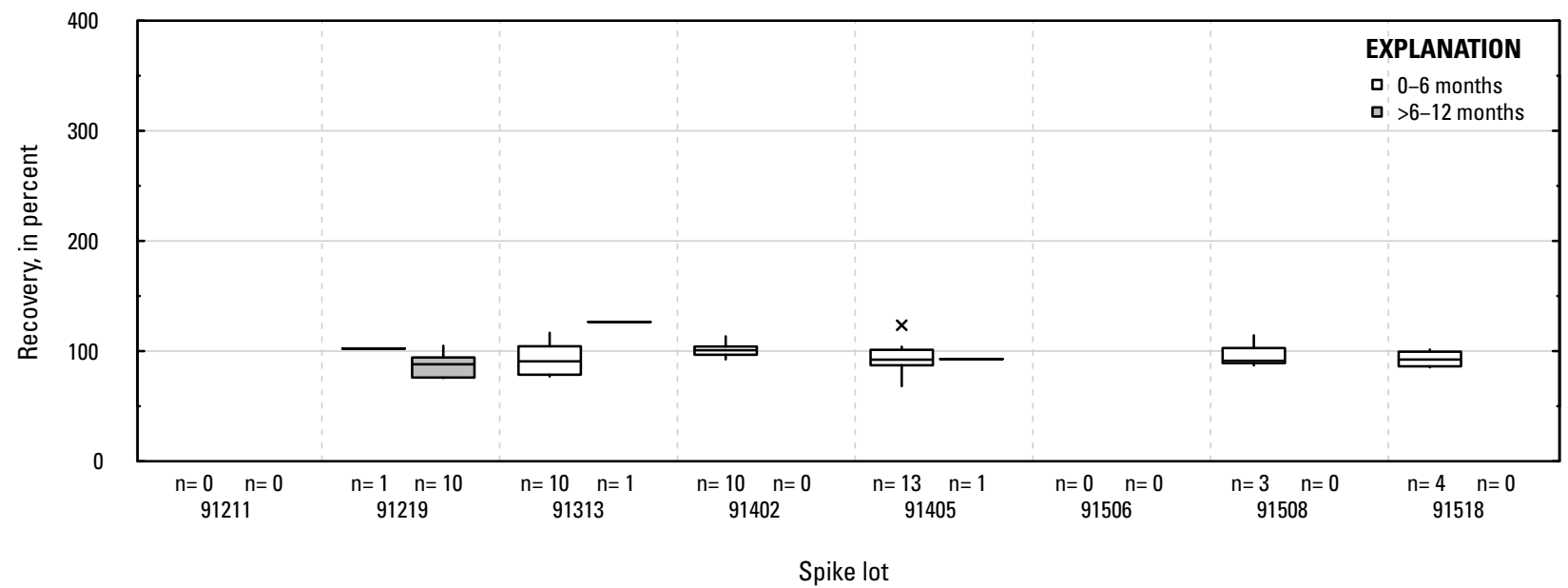

JY. Fenamiphos: surface water field matrix spikes

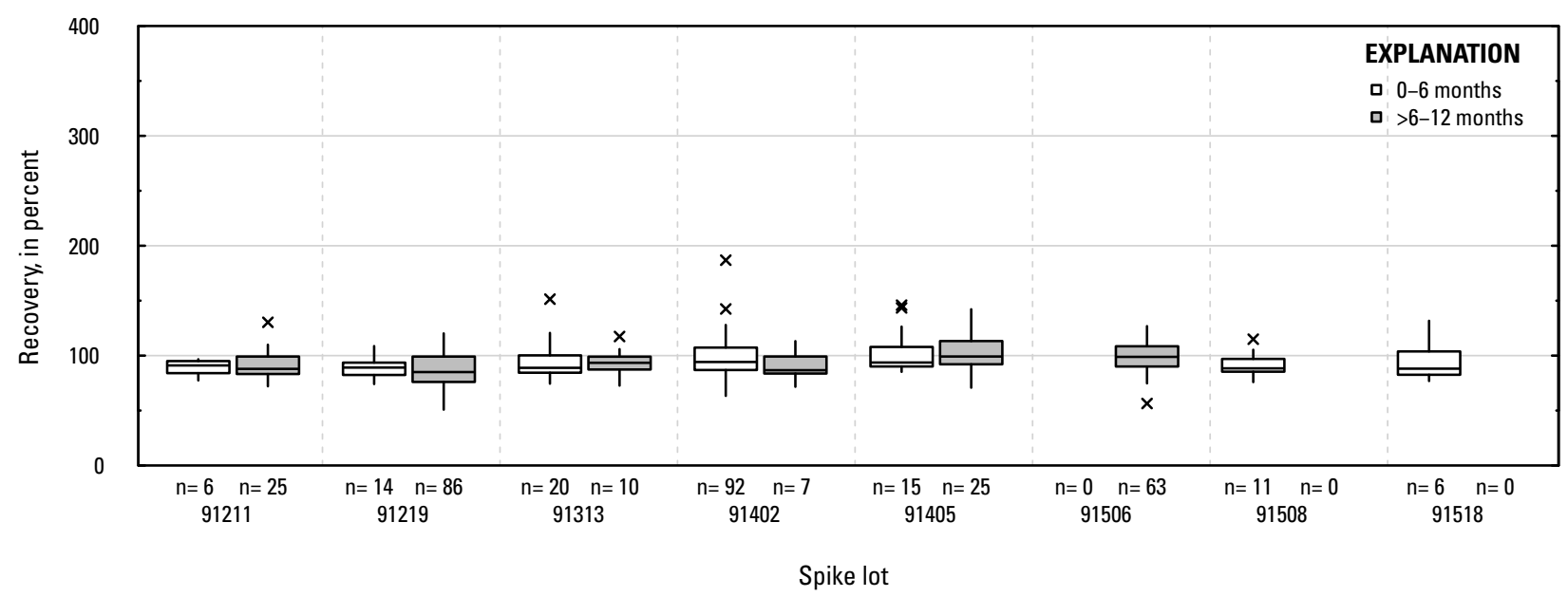

Figure 1-1. Distributions of recovery for individual pesticides in schedule 2437 by matrix, spike lot, and spike lot age. Recovery values larger than 400 percent are not shown.-Continued 
JZ. Fenamiphos sulfone: laboratory reagent spikes

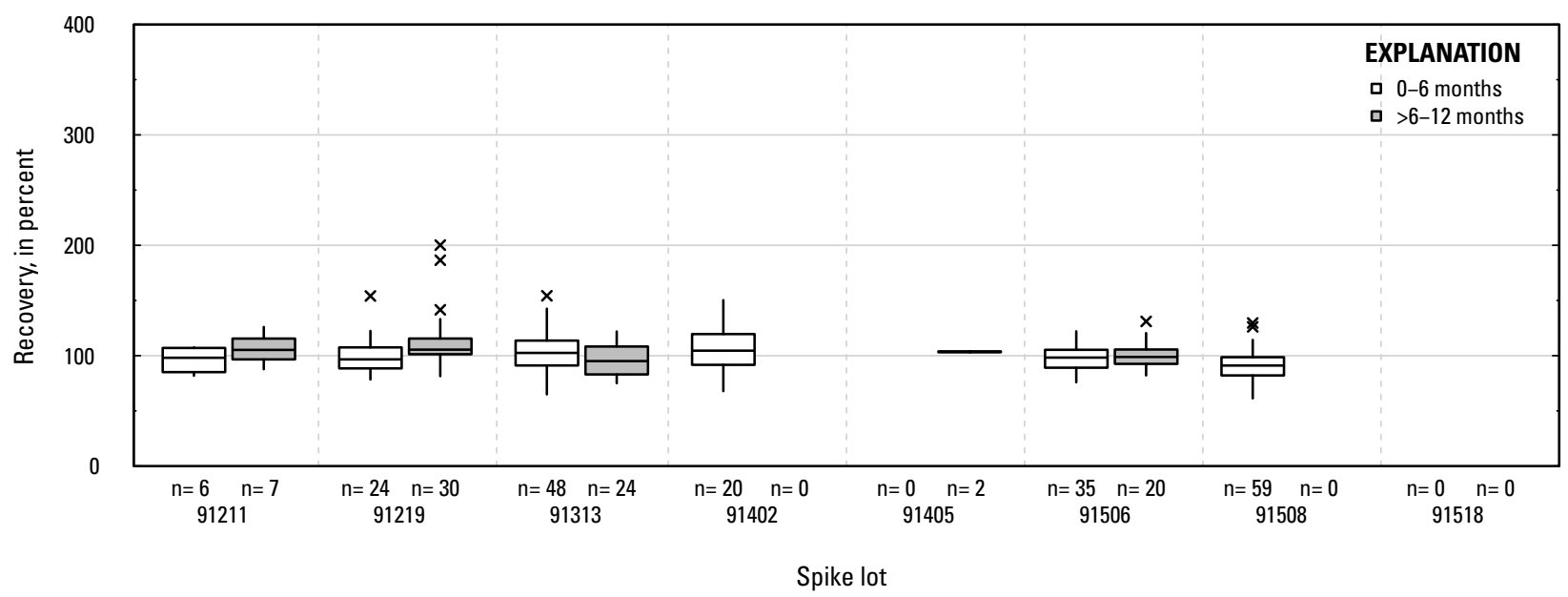

$K A$. Fenamiphos sulfone: groundwater field matrix spikes

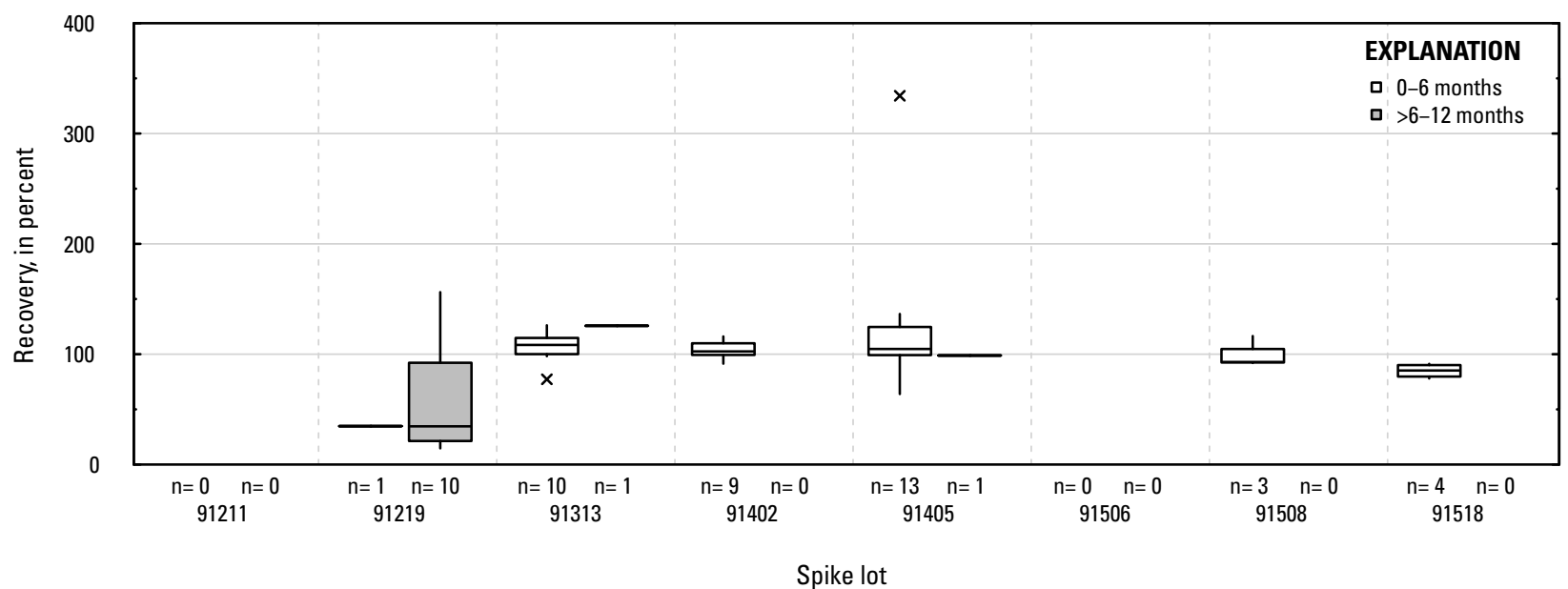

KB. Fenamiphos sulfone: surface water field matrix spikes

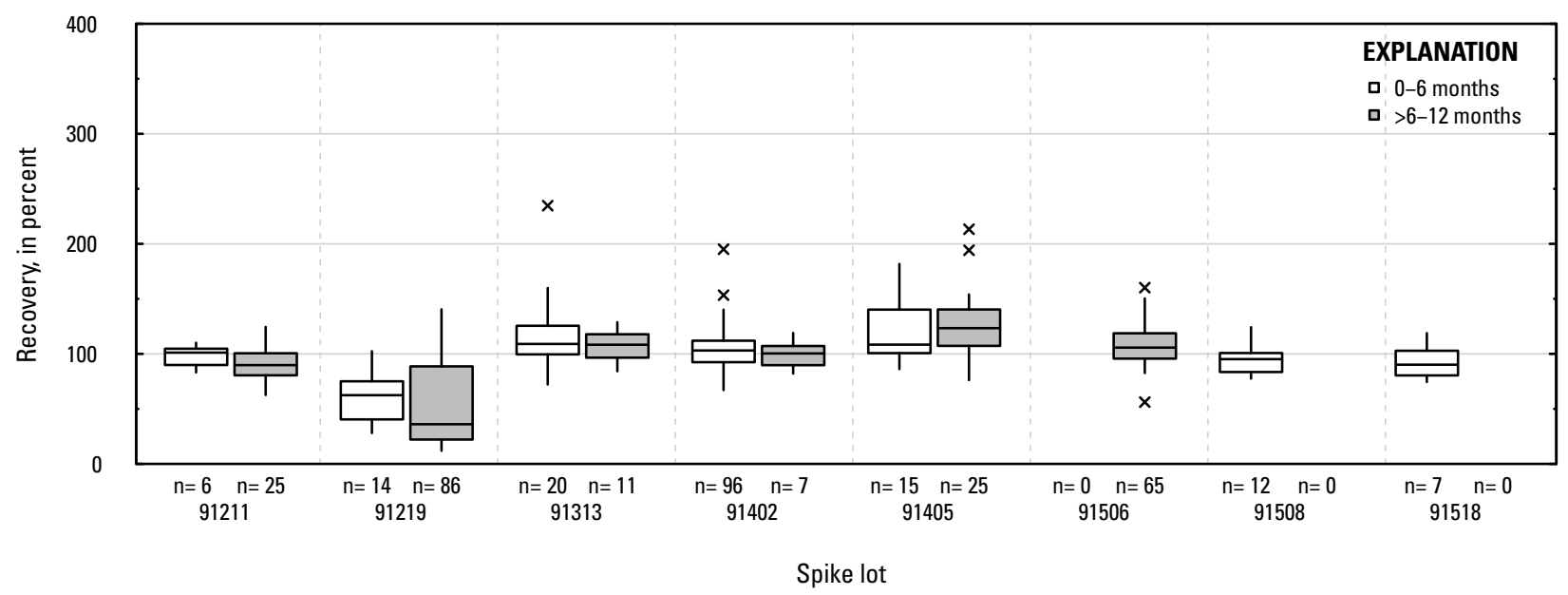

Figure 1-1. Distributions of recovery for individual pesticides in schedule 2437 by matrix, spike lot, and spike lot age. Recovery values larger than 400 percent are not shown.-Continued 


\section{KC. Fenamiphos sulfoxide: laboratory reagent spikes}

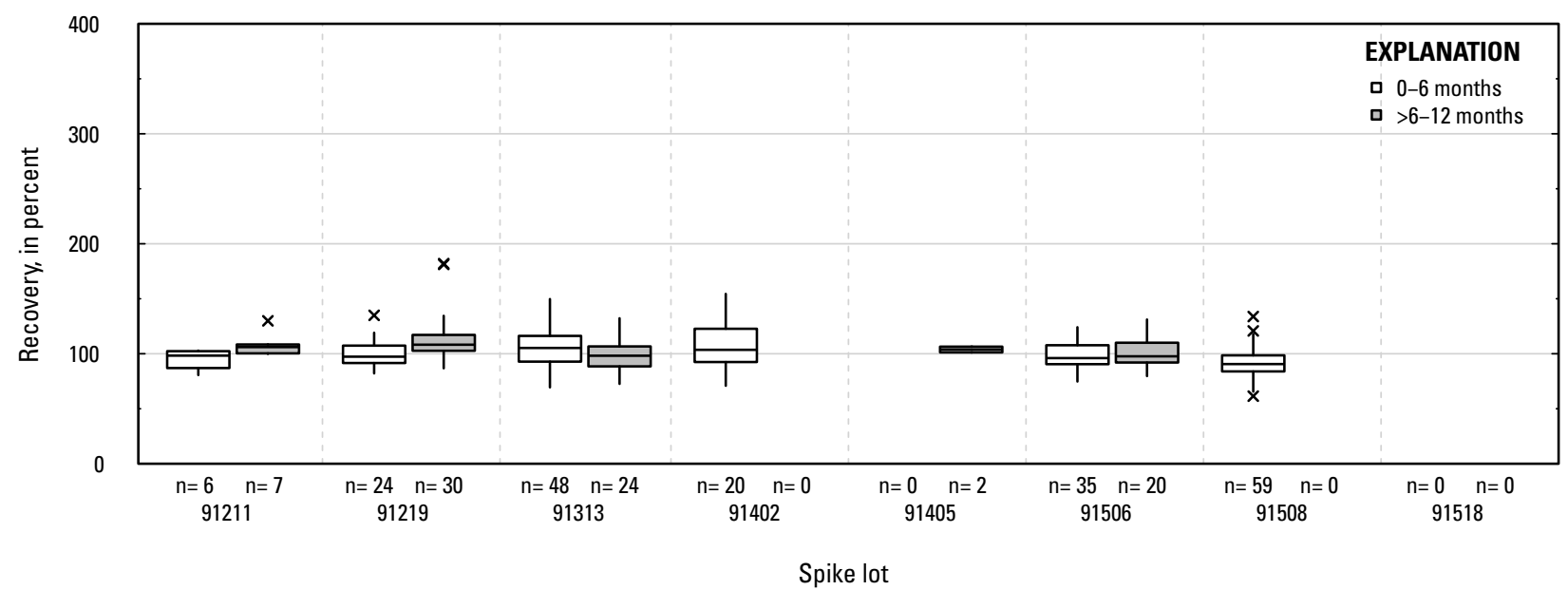

KD. Fenamiphos sulfoxide: groundwater field matrix spikes

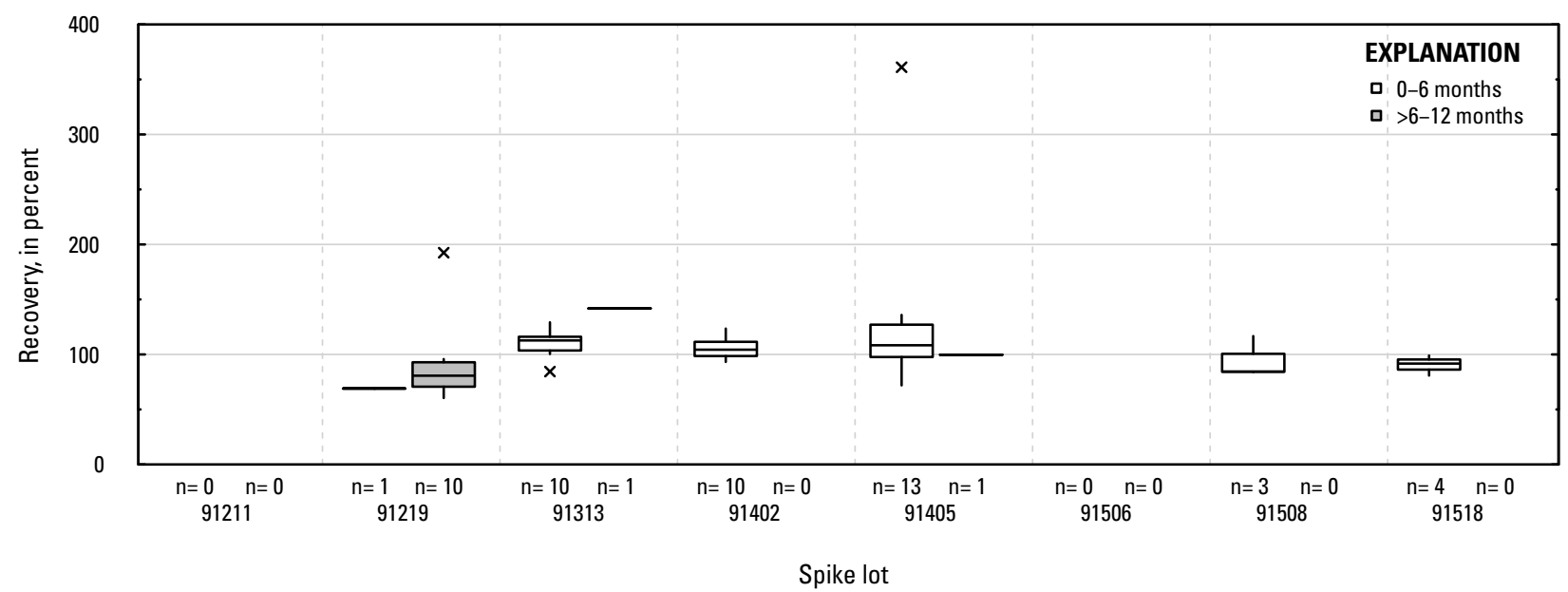

KE. Fenamiphos sulfoxide: surface water field matrix spikes

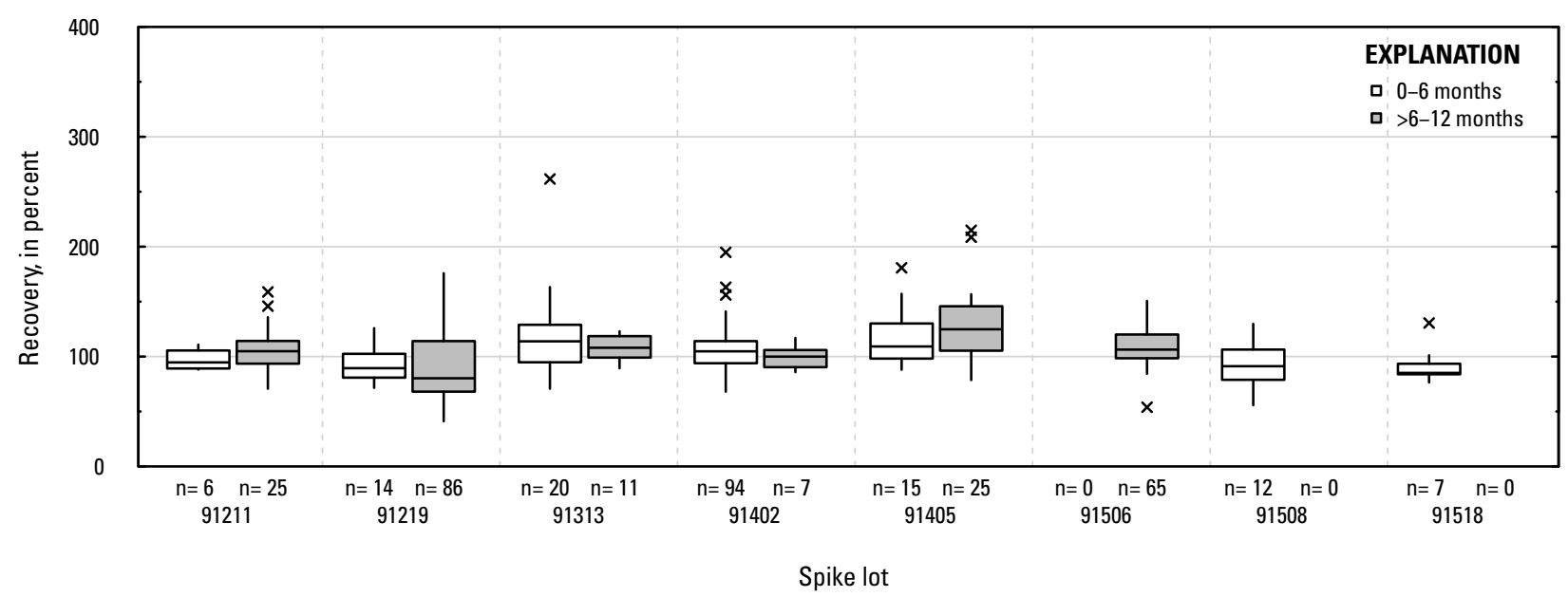

Figure 1-1. Distributions of recovery for individual pesticides in schedule 2437 by matrix, spike lot, and spike lot age. Recovery values larger than 400 percent are not shown.-Continued 
KF. Fenbutatin oxide: laboratory reagent spikes

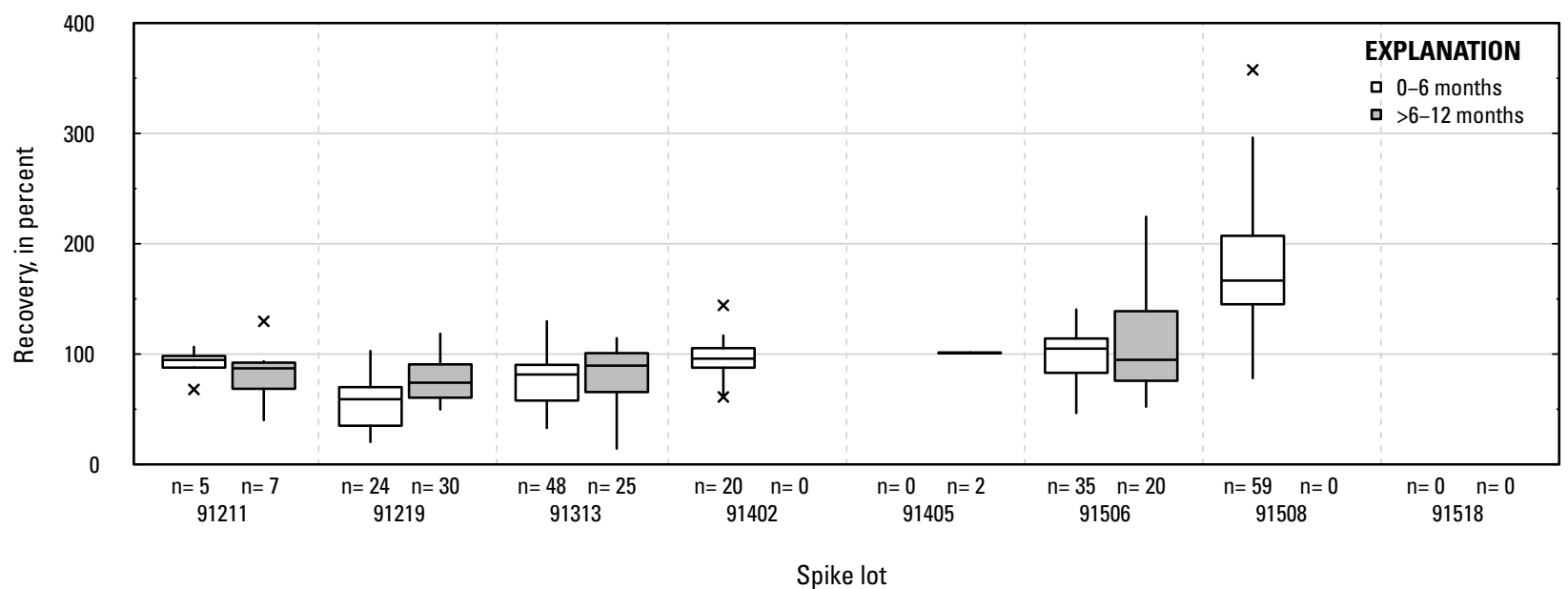

KG. Fenbutatin oxide: groundwater field matrix spikes

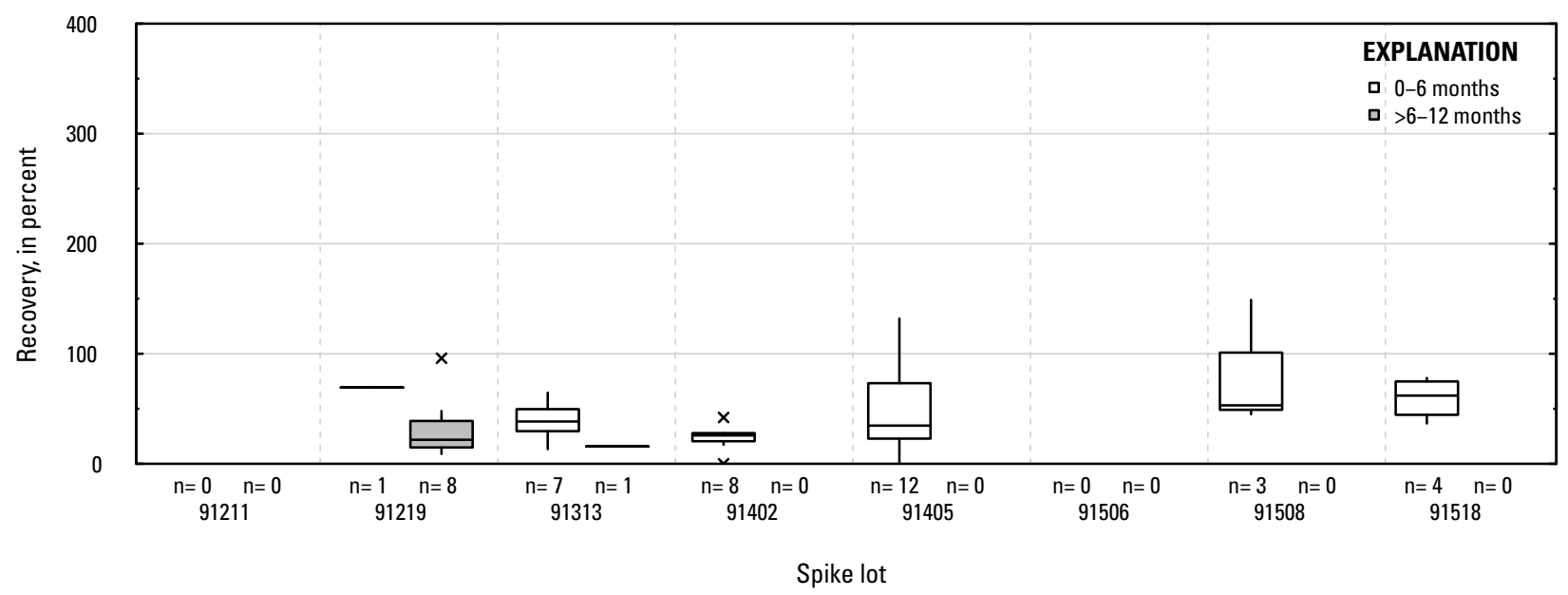

KH. Fenbutatin oxide: surface water field matrix spikes

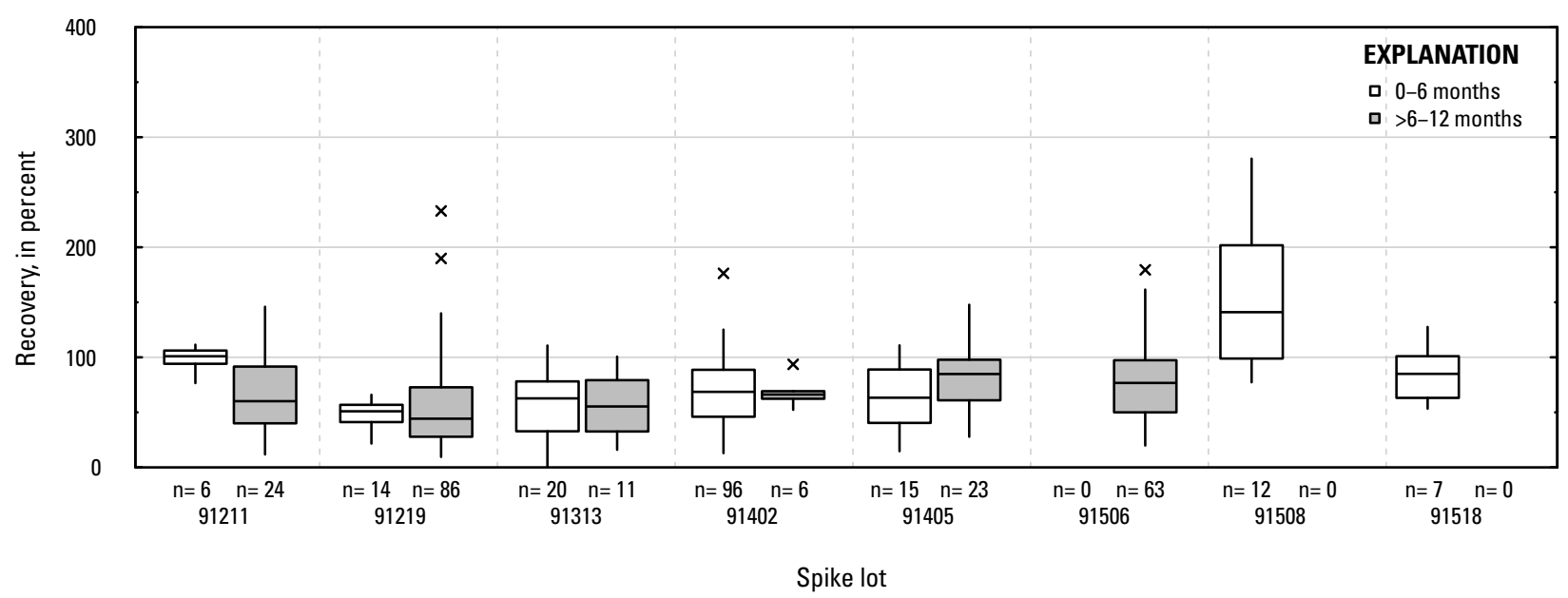

Figure 1-1. Distributions of recovery for individual pesticides in schedule 2437 by matrix, spike lot, and spike lot age. Recovery values larger than 400 percent are not shown.-Continued 


\section{KI. Fentin: laboratory reagent spikes}

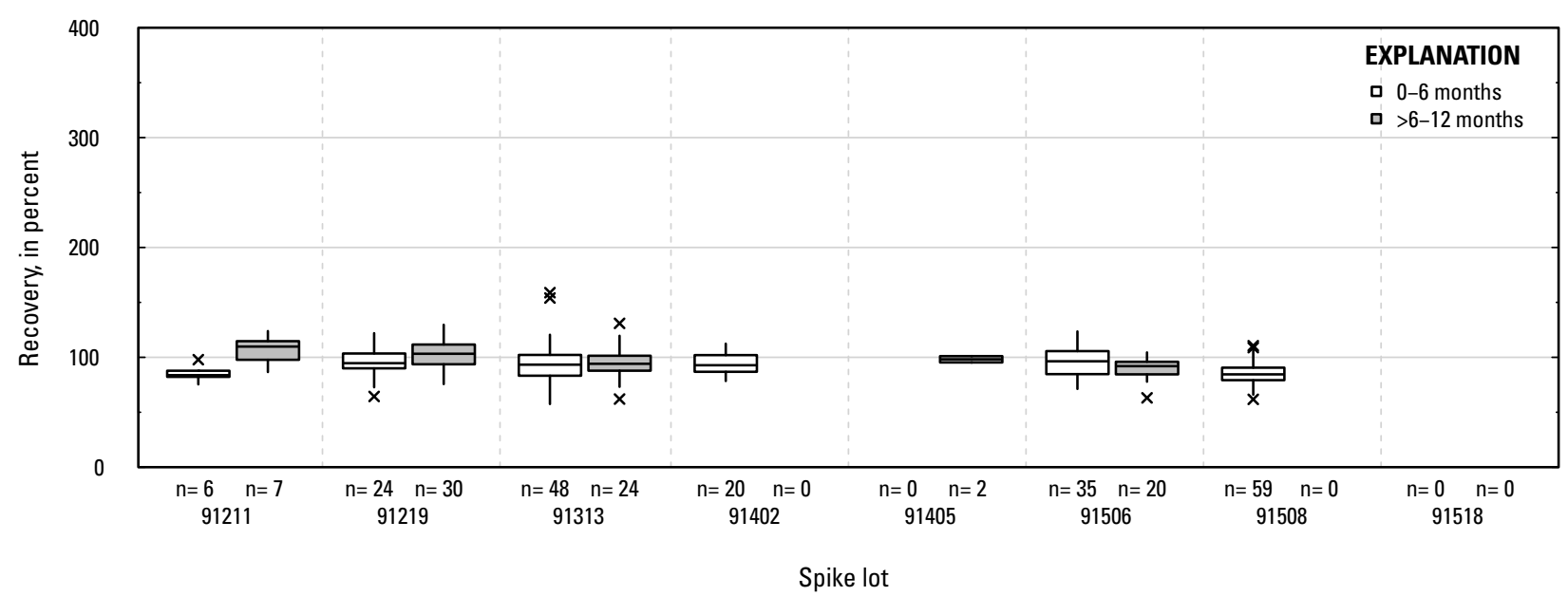

\section{$K J$. Fentin: groundwater field matrix spikes}

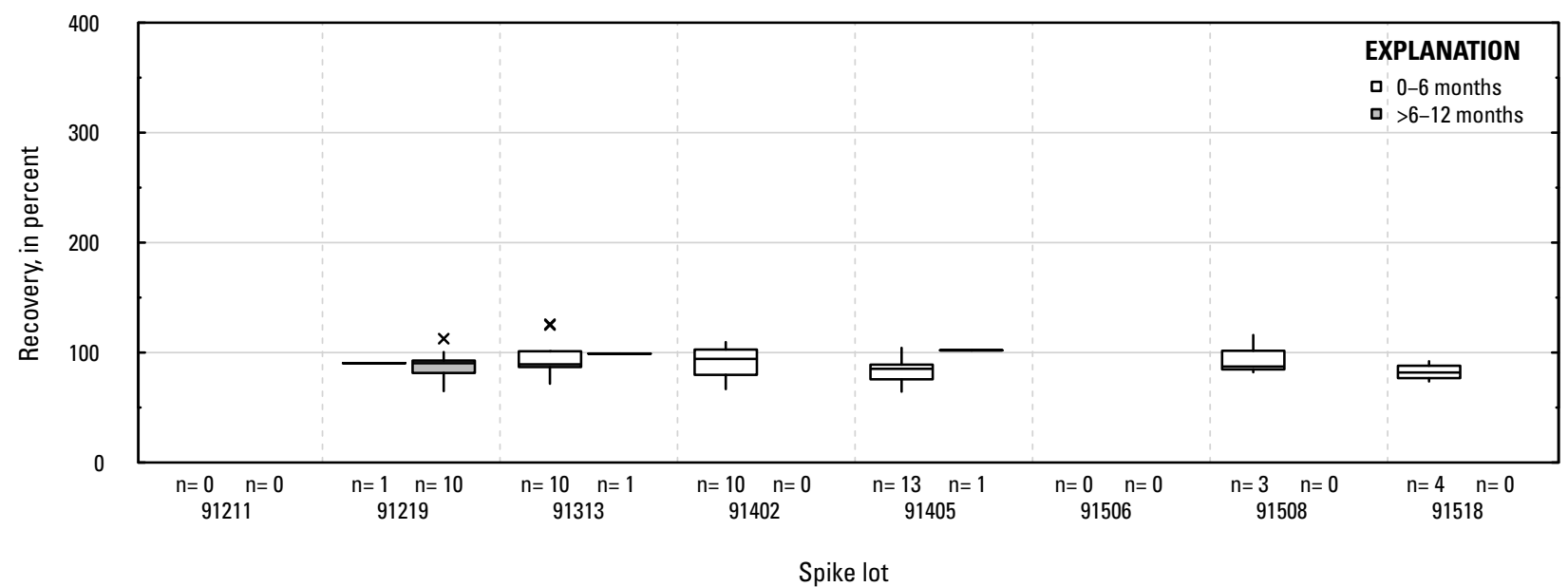

KK. Fentin: surface water field matrix spikes

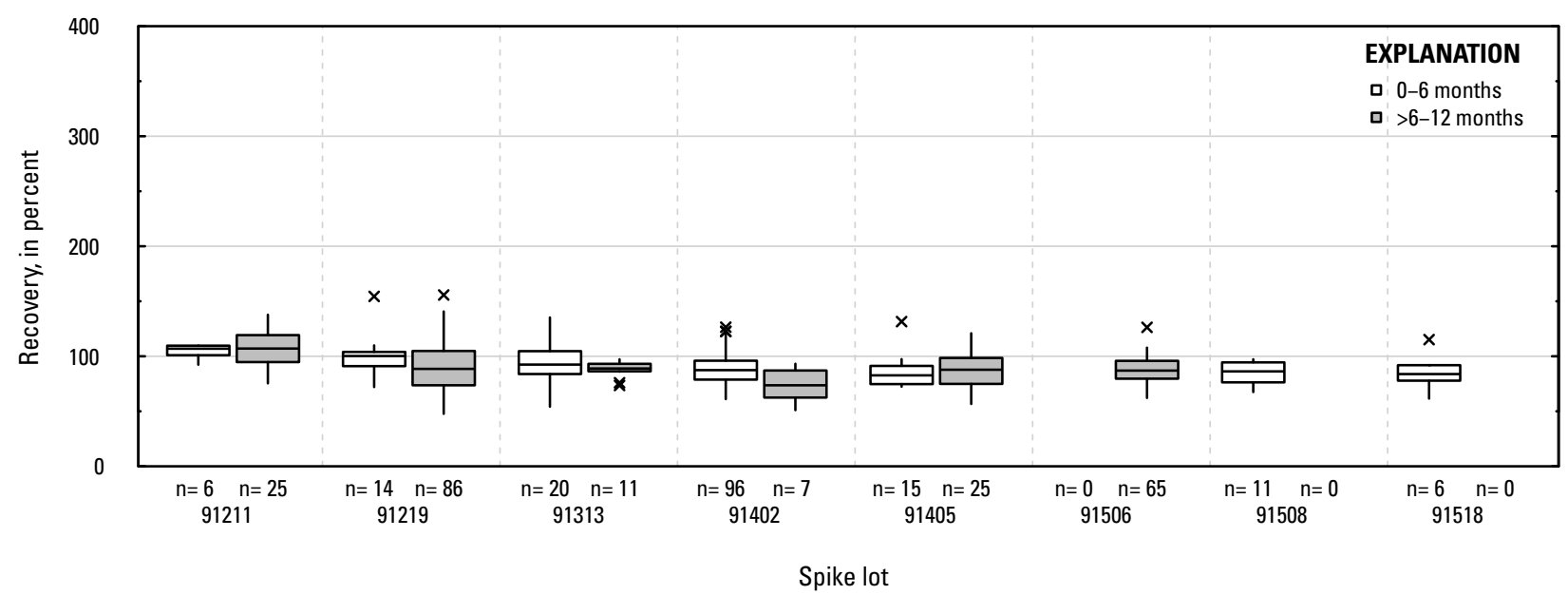

Figure 1-1. Distributions of recovery for individual pesticides in schedule 2437 by matrix, spike lot, and spike lot age. Recovery values larger than 400 percent are not shown.-Continued 


\section{KL. Fipronil: laboratory reagent spikes}

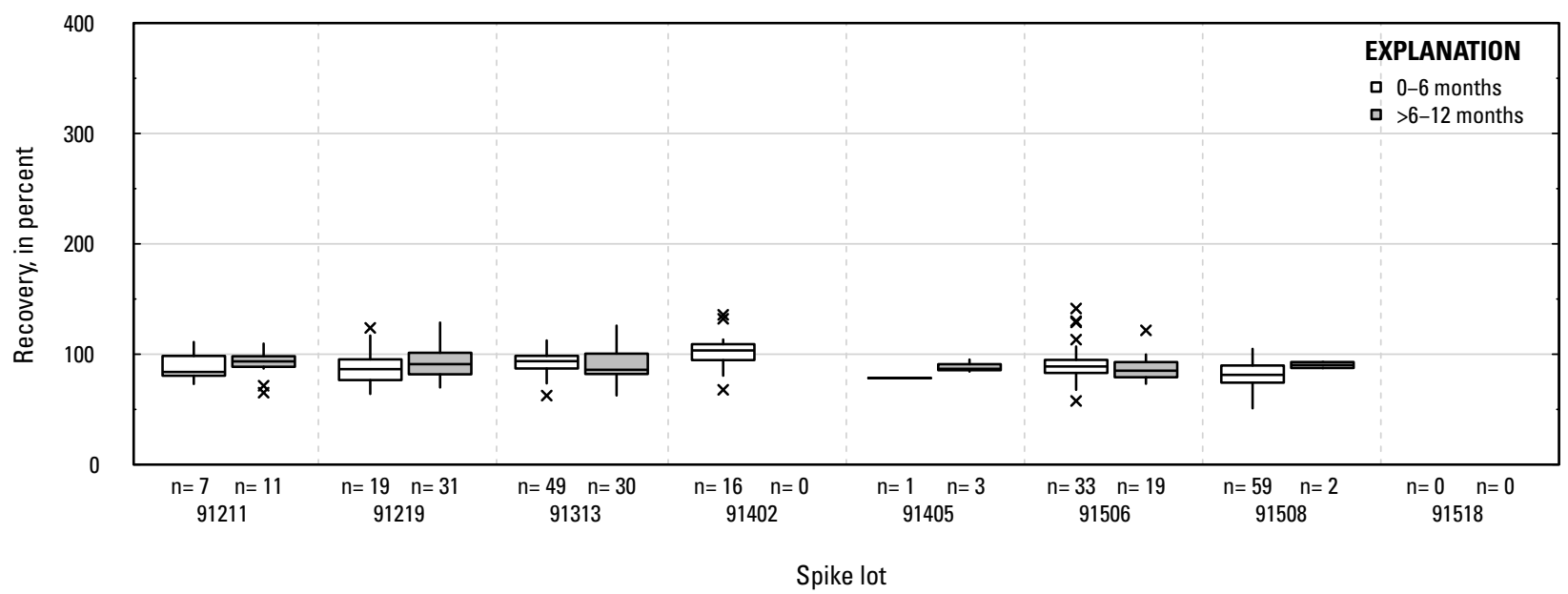

KM. Fipronil: groundwater field matrix spikes

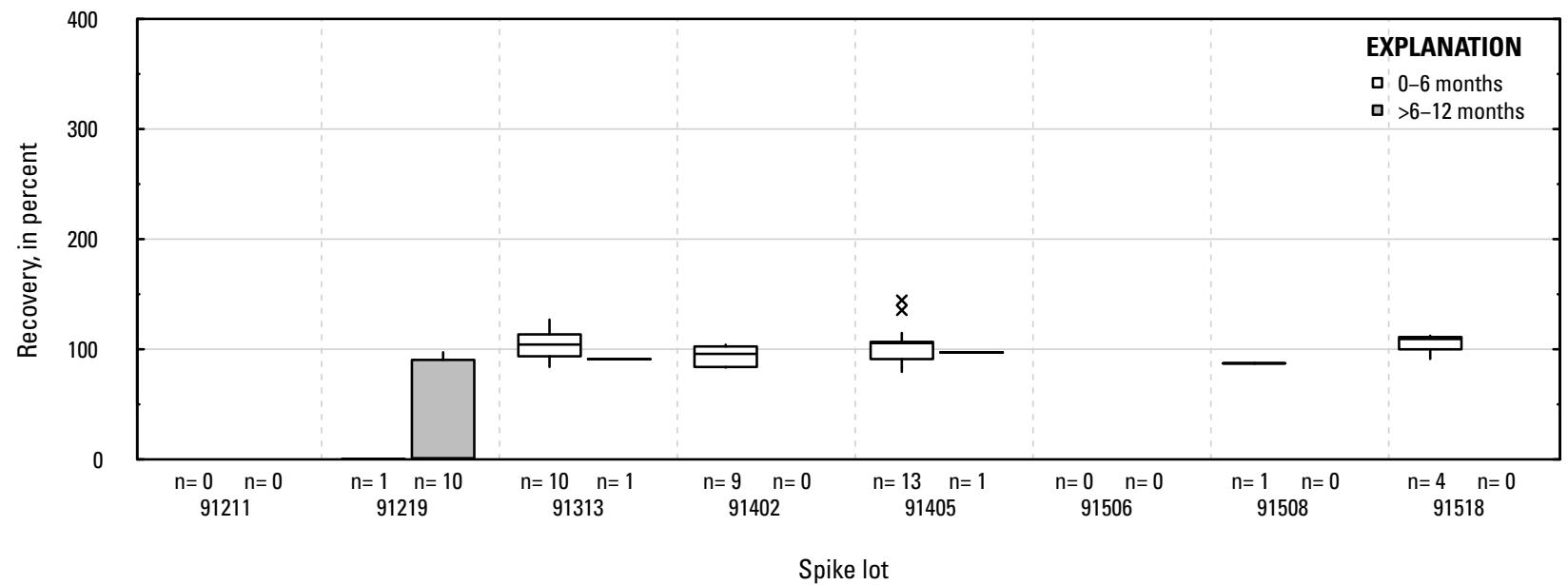

$K N$. Fipronil: surface water field matrix spikes

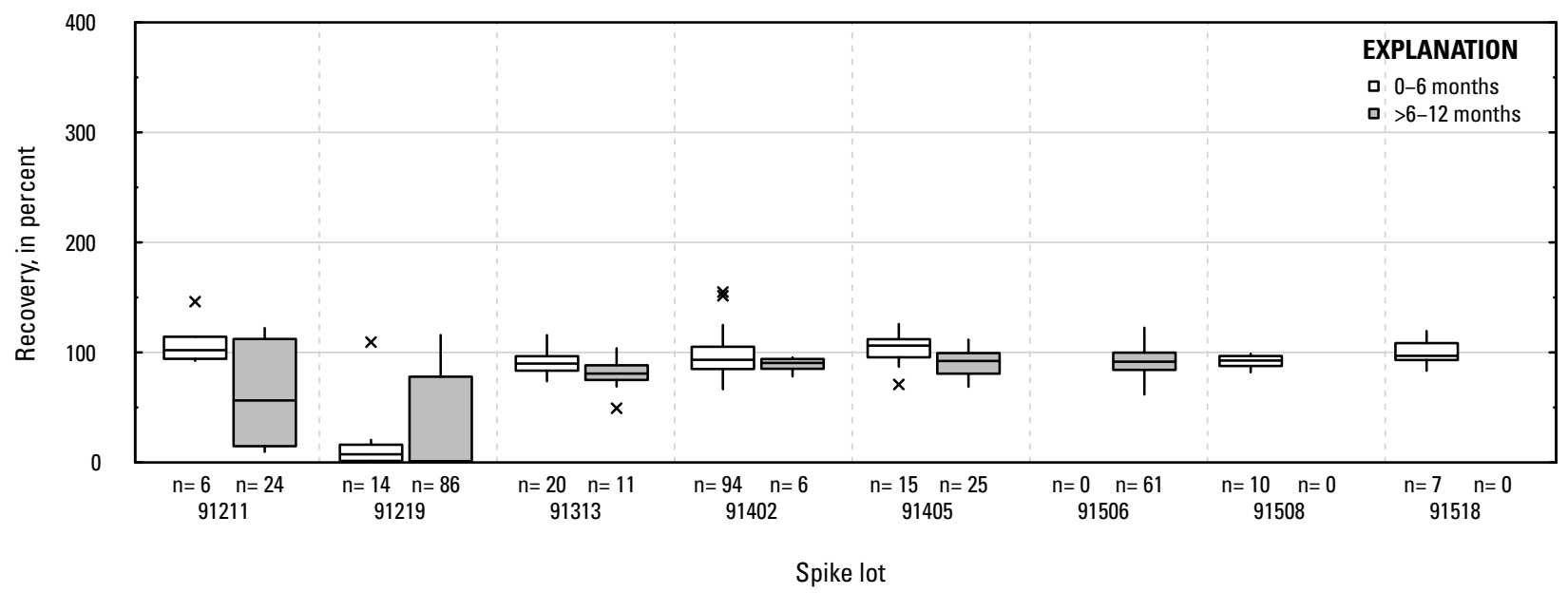

Figure 1-1. Distributions of recovery for individual pesticides in schedule 2437 by matrix, spike lot, and spike lot age. Recovery values larger than 400 percent are not shown.-Continued 
KO. Fipronil amide: laboratory reagent spikes

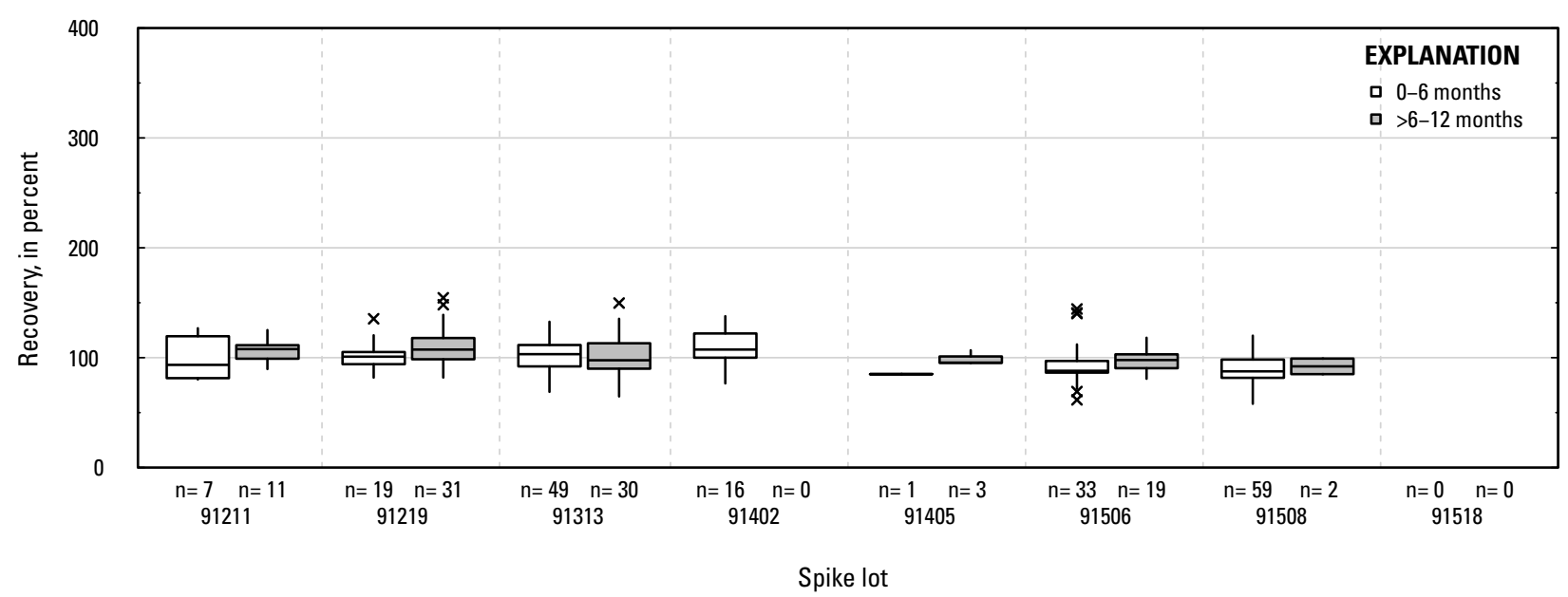

$K P$. Fipronil amide: groundwater field matrix spikes

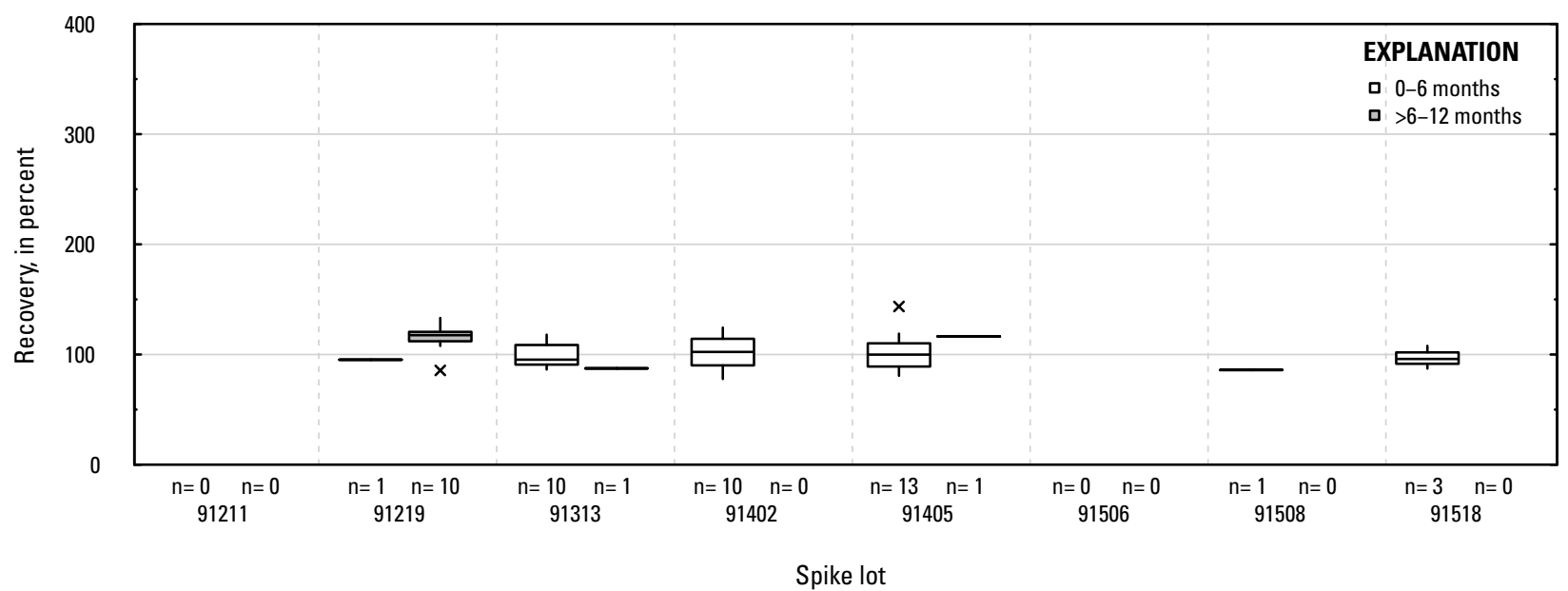

KO. Fipronil amide: surface water field matrix spikes

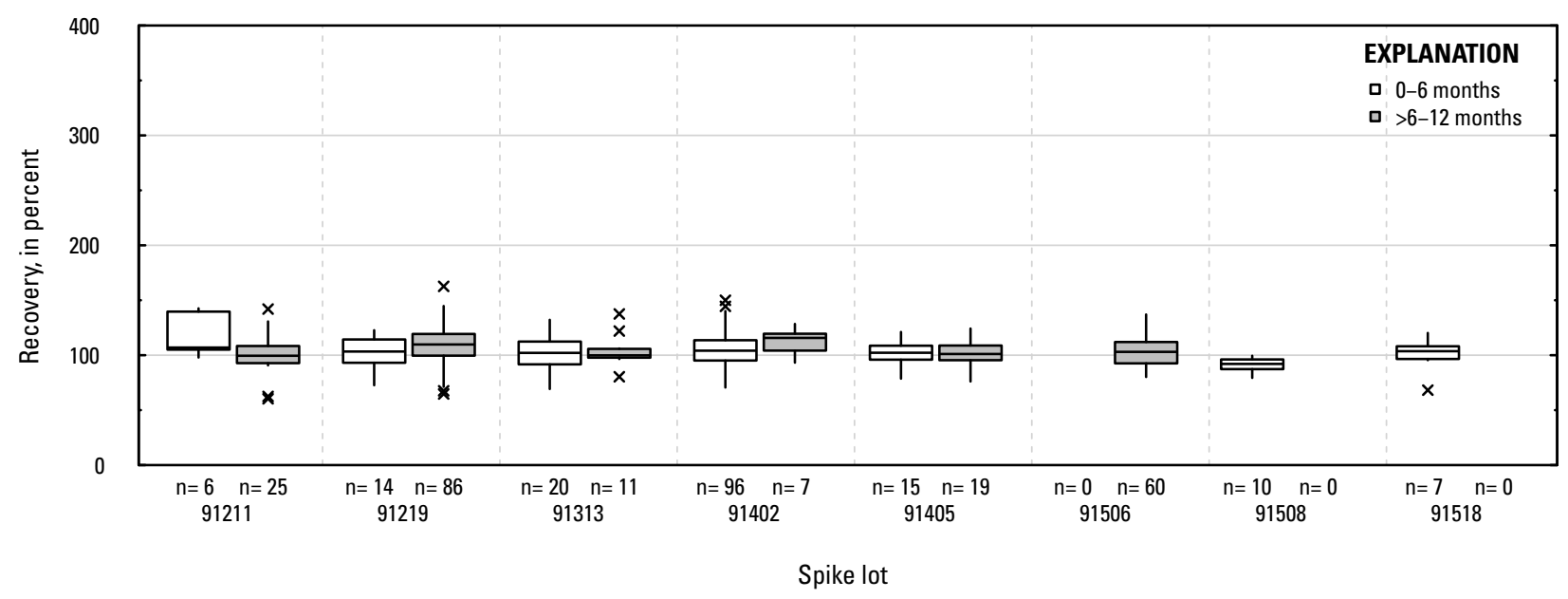

Figure 1-1. Distributions of recovery for individual pesticides in schedule 2437 by matrix, spike lot, and spike lot age. Recovery values larger than 400 percent are not shown.-Continued 
KR. Fipronil sulfide: laboratory reagent spikes

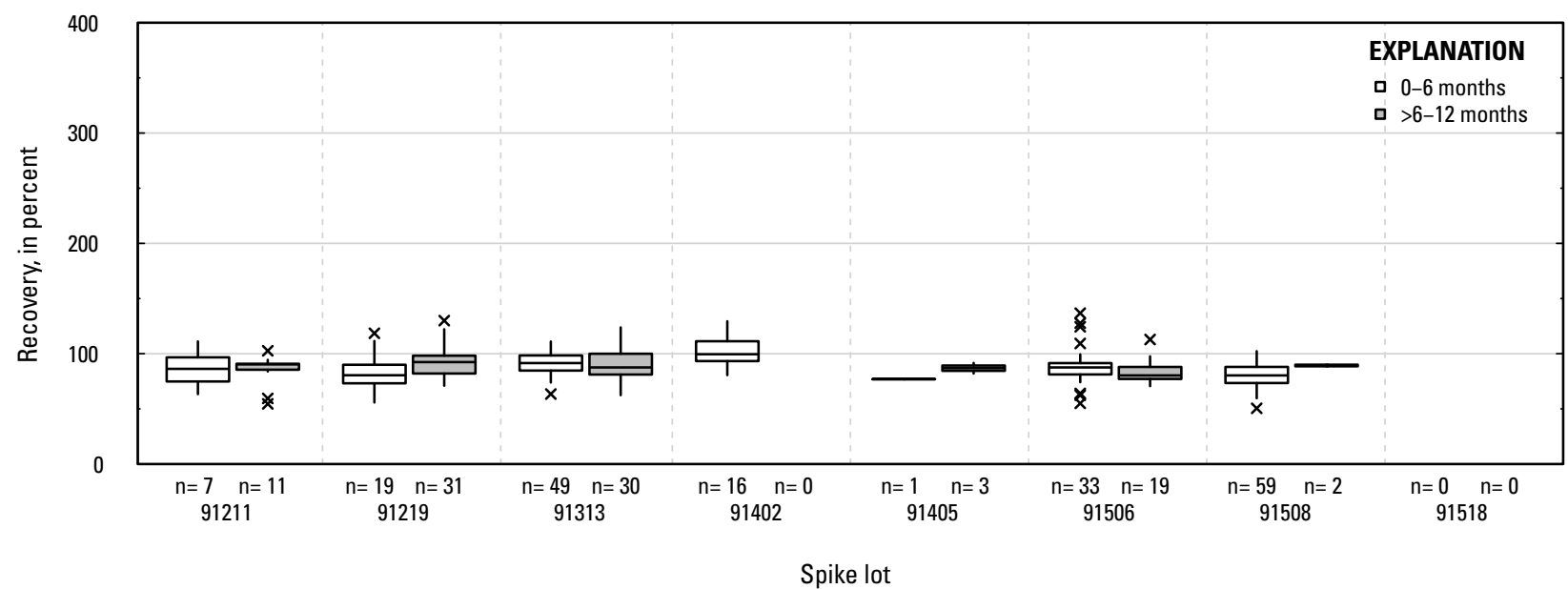

$K S$. Fipronil sulfide: groundwater field matrix spikes

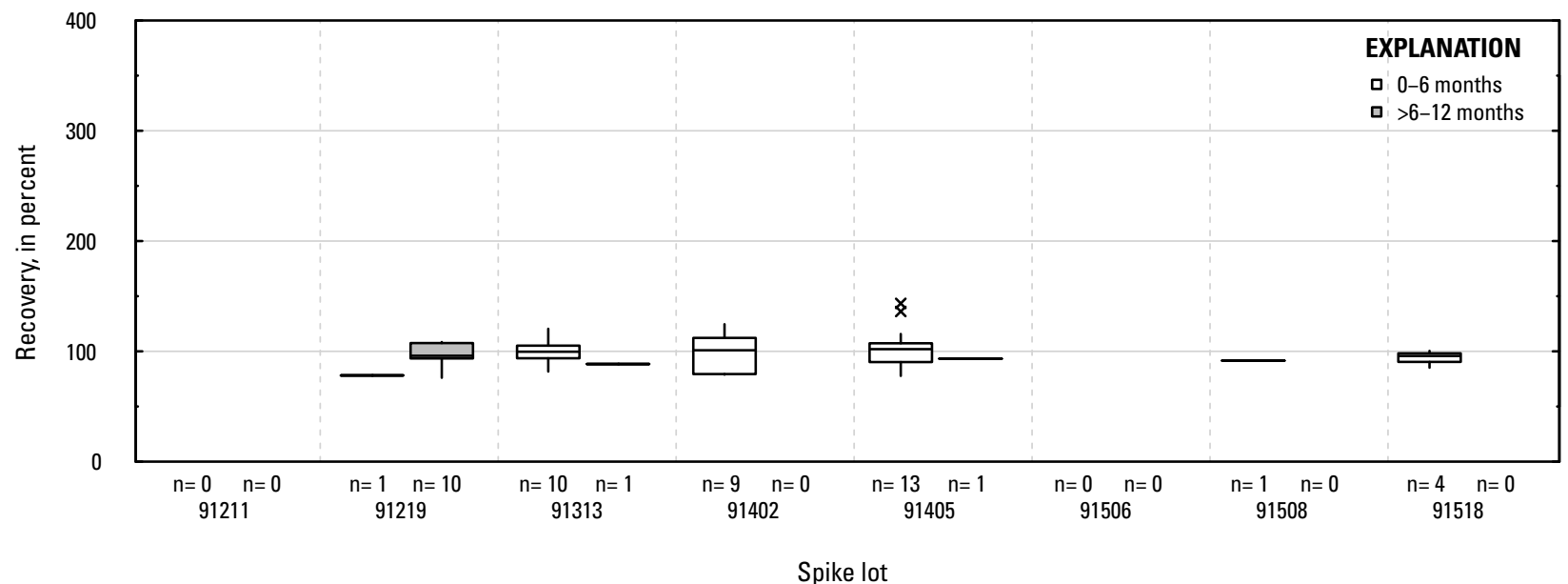

\section{$K T$. Fipronil sulfide: surface water field matrix spikes}

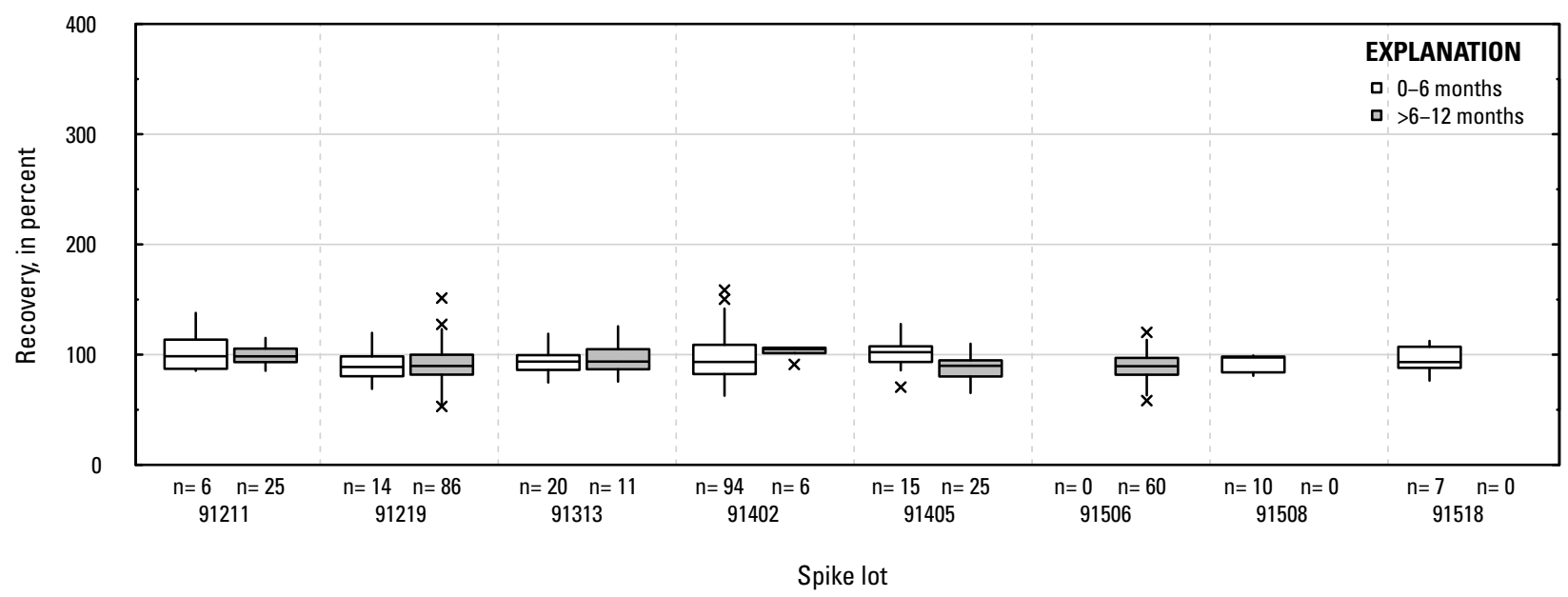

Figure 1-1. Distributions of recovery for individual pesticides in schedule 2437 by matrix, spike lot, and spike lot age. Recovery values larger than 400 percent are not shown.-Continued 


\section{$K U$. Fipronil sulfonate: laboratory reagent spikes}

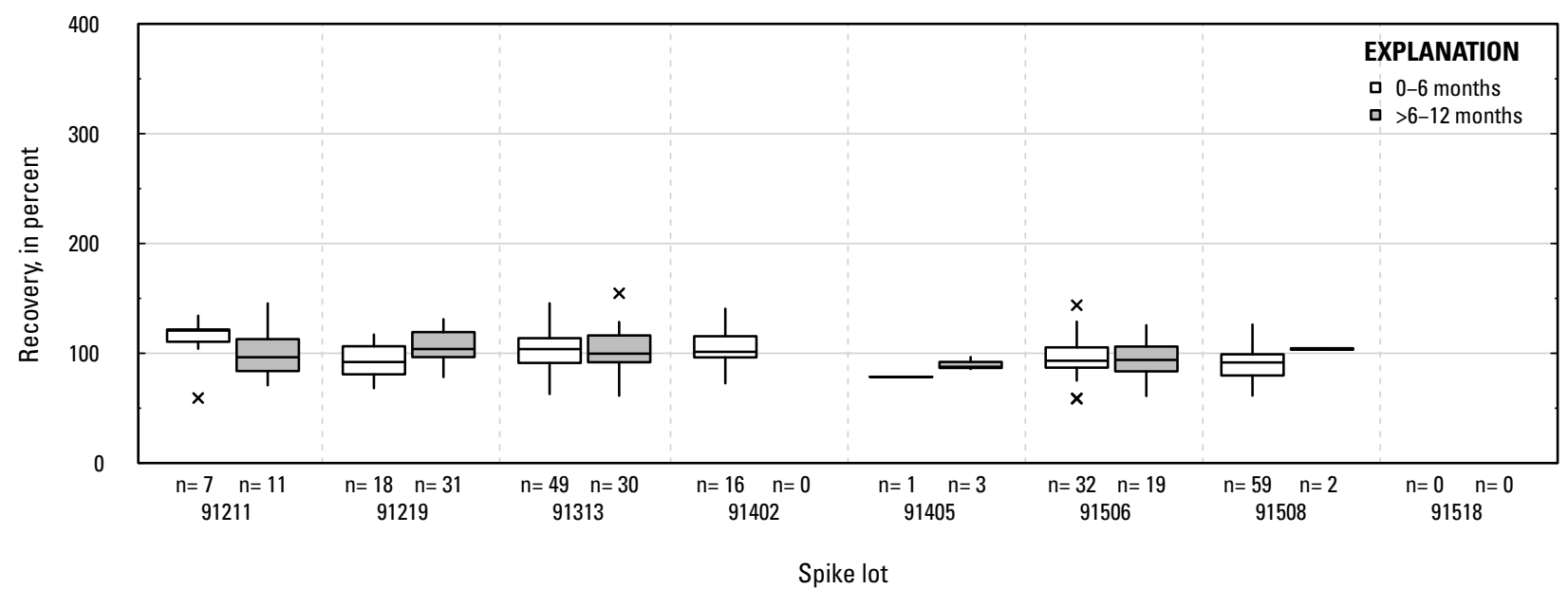

\section{KV. Fipronil sulfonate: groundwater field matrix spikes}

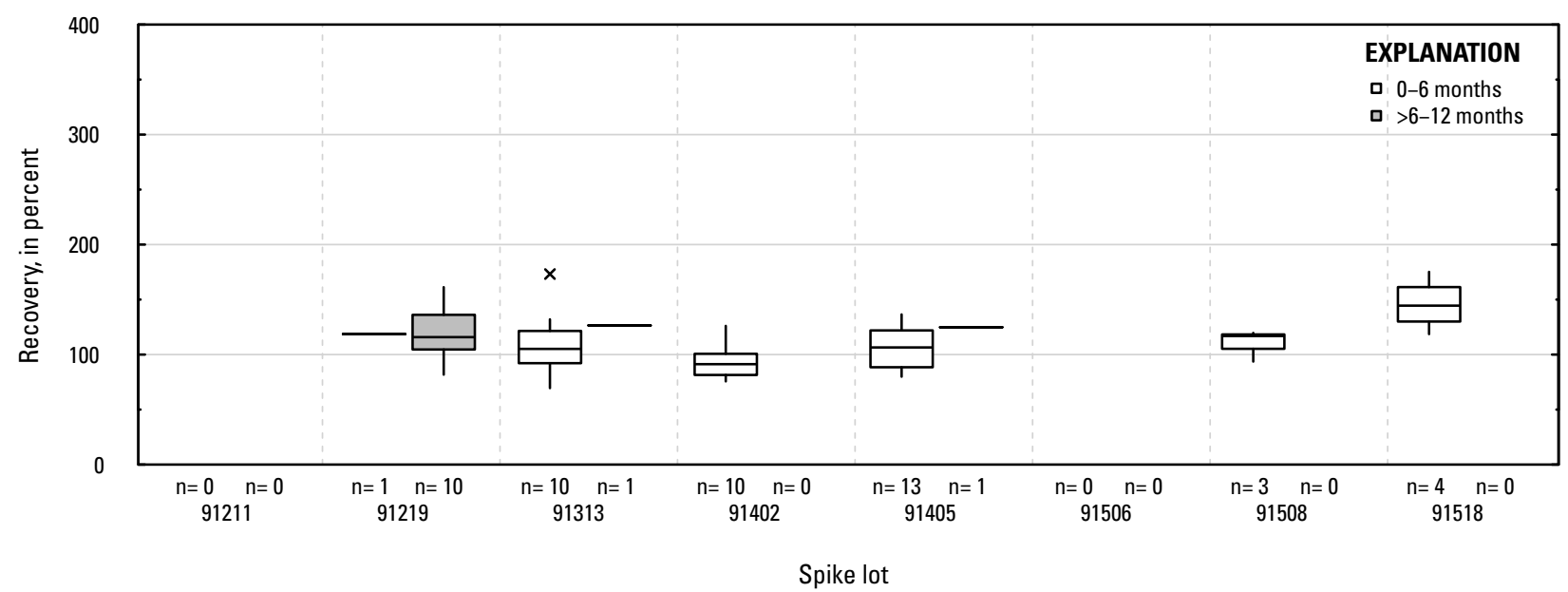

$K W$. Fipronil sulfonate: surface water field matrix spikes

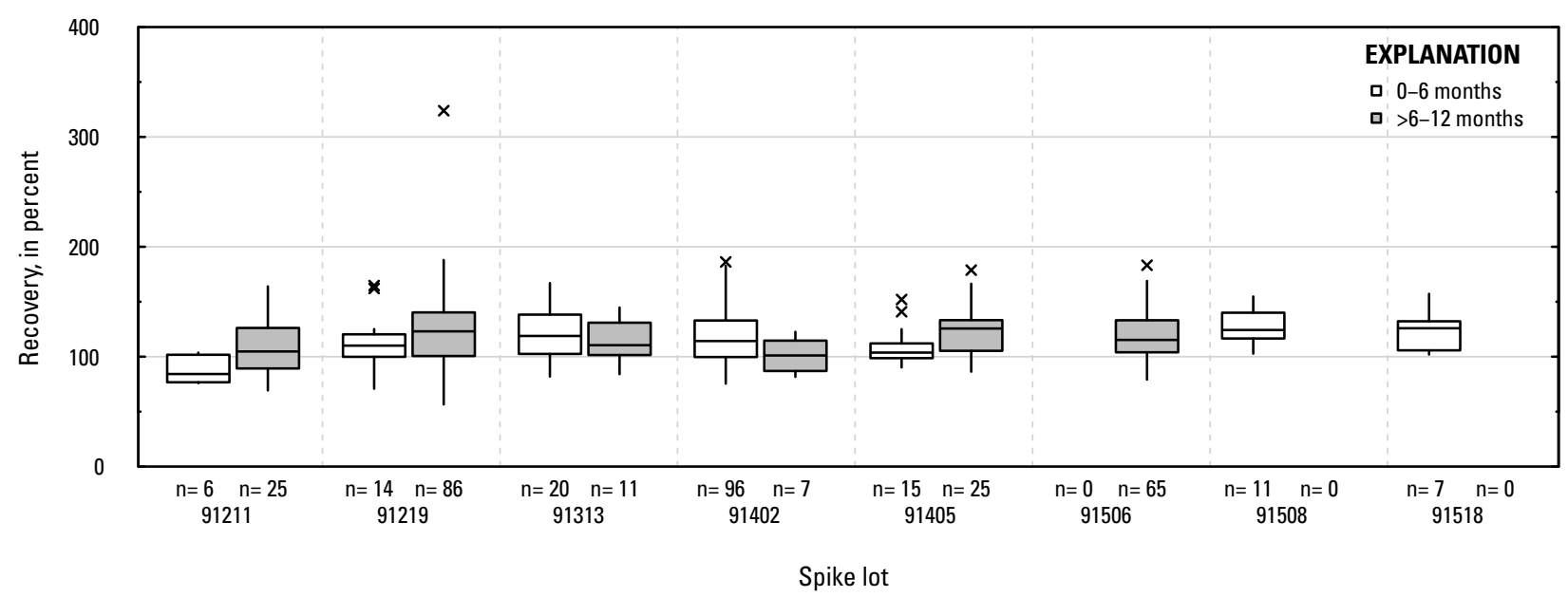

Figure 1-1. Distributions of recovery for individual pesticides in schedule 2437 by matrix, spike lot, and spike lot age. Recovery values larger than 400 percent are not shown.-Continued 
$K X$. Fipronil sulfone: laboratory reagent spikes

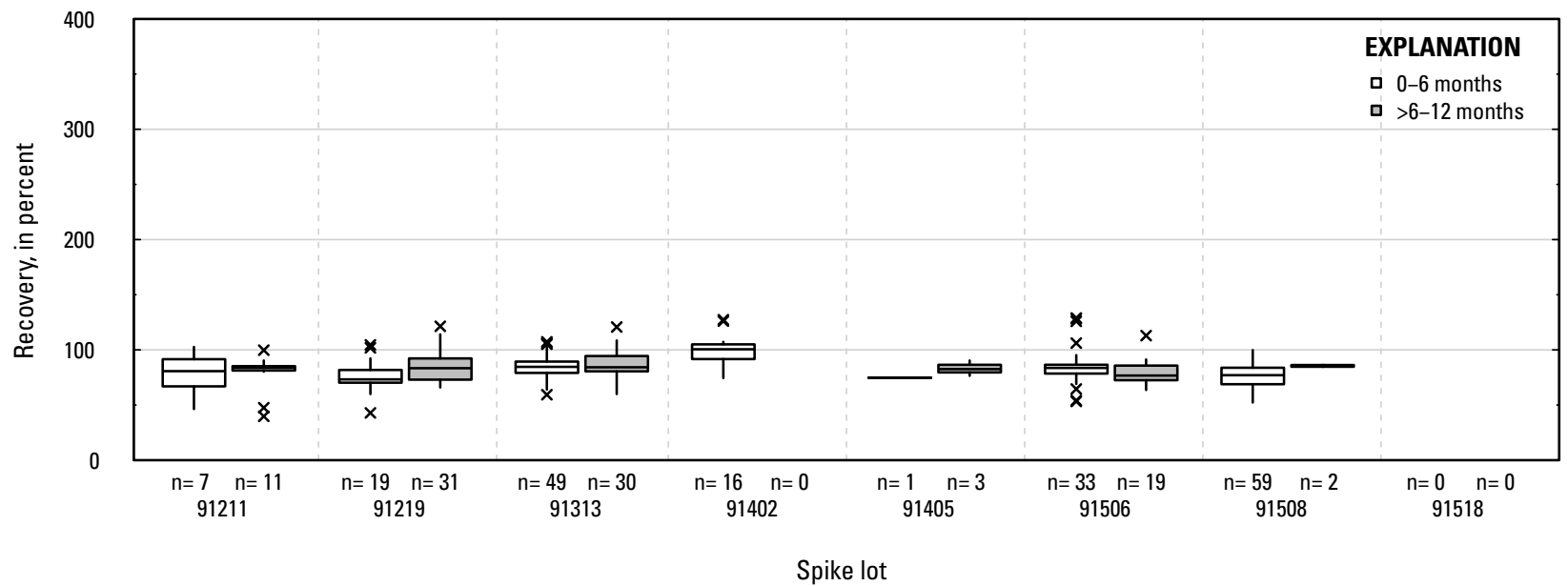

$K Y$. Fipronil sulfone: groundwater field matrix spikes

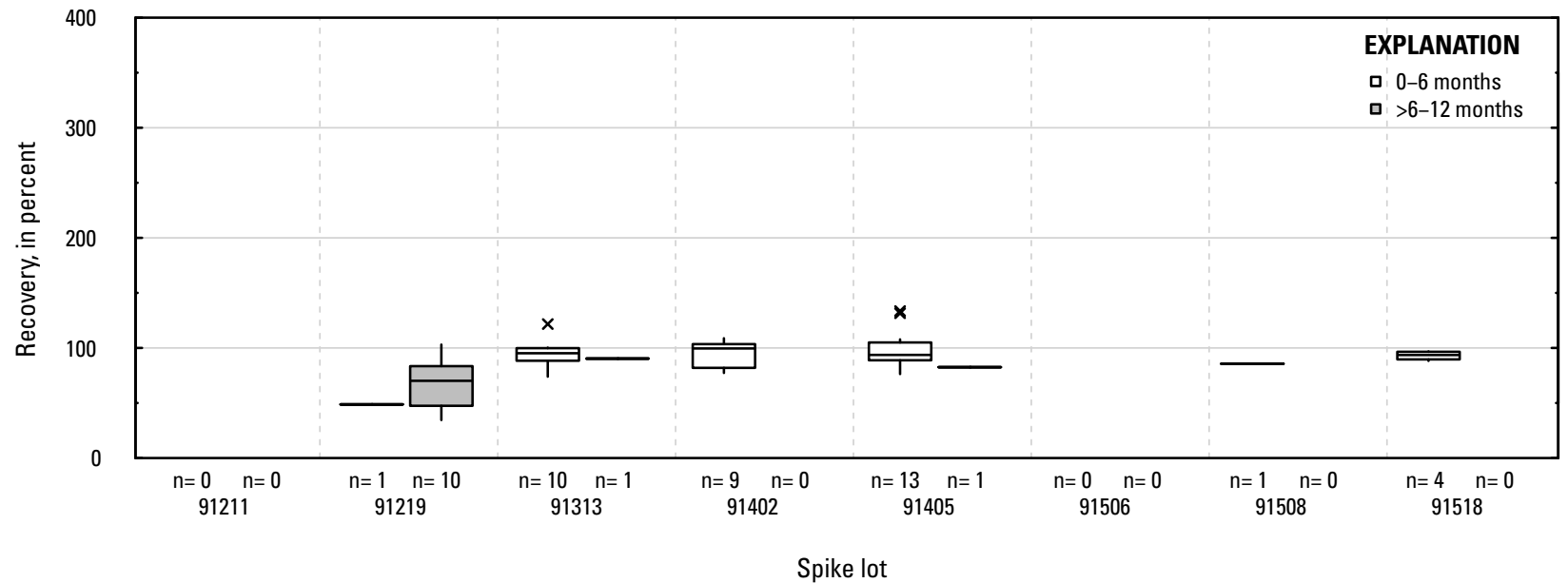

$K Z$. Fipronil sulfone: surface water field matrix spikes

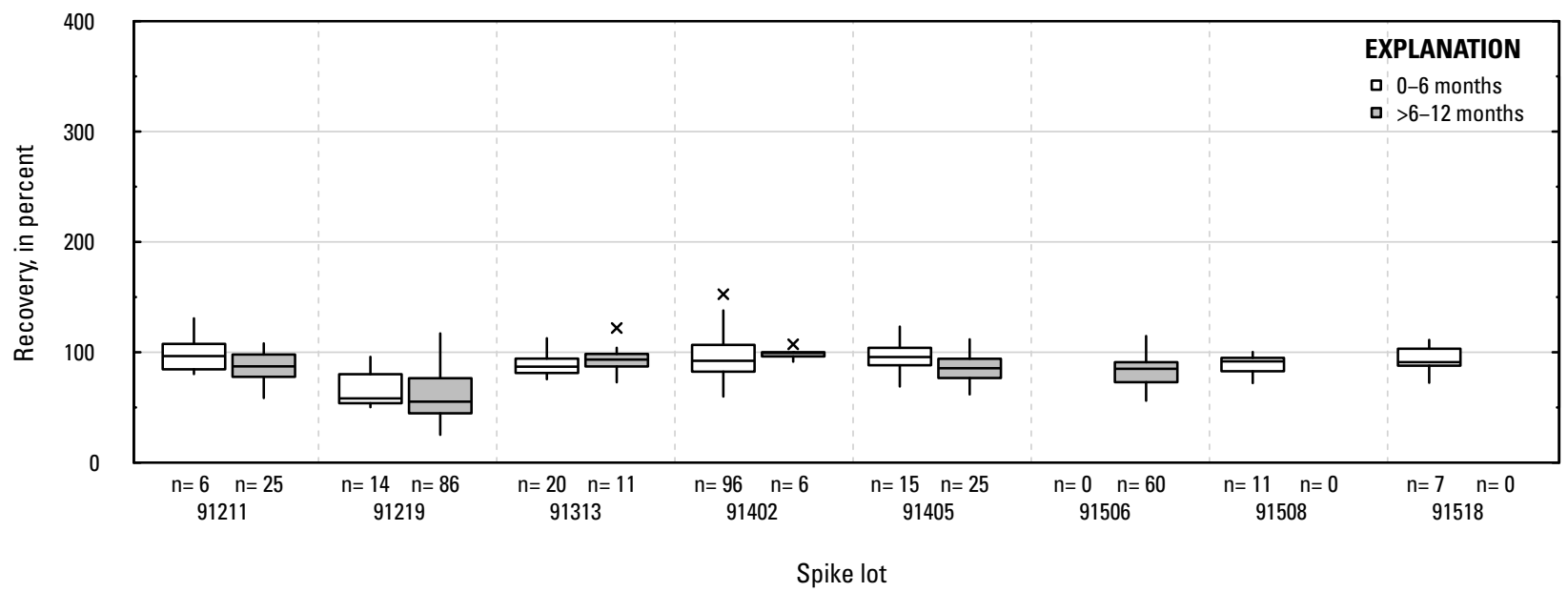

Figure 1-1. Distributions of recovery for individual pesticides in schedule 2437 by matrix, spike lot, and spike lot age. Recovery values larger than 400 percent are not shown.-Continued 


\section{LA. Flubendiamide: laboratory reagent spikes}

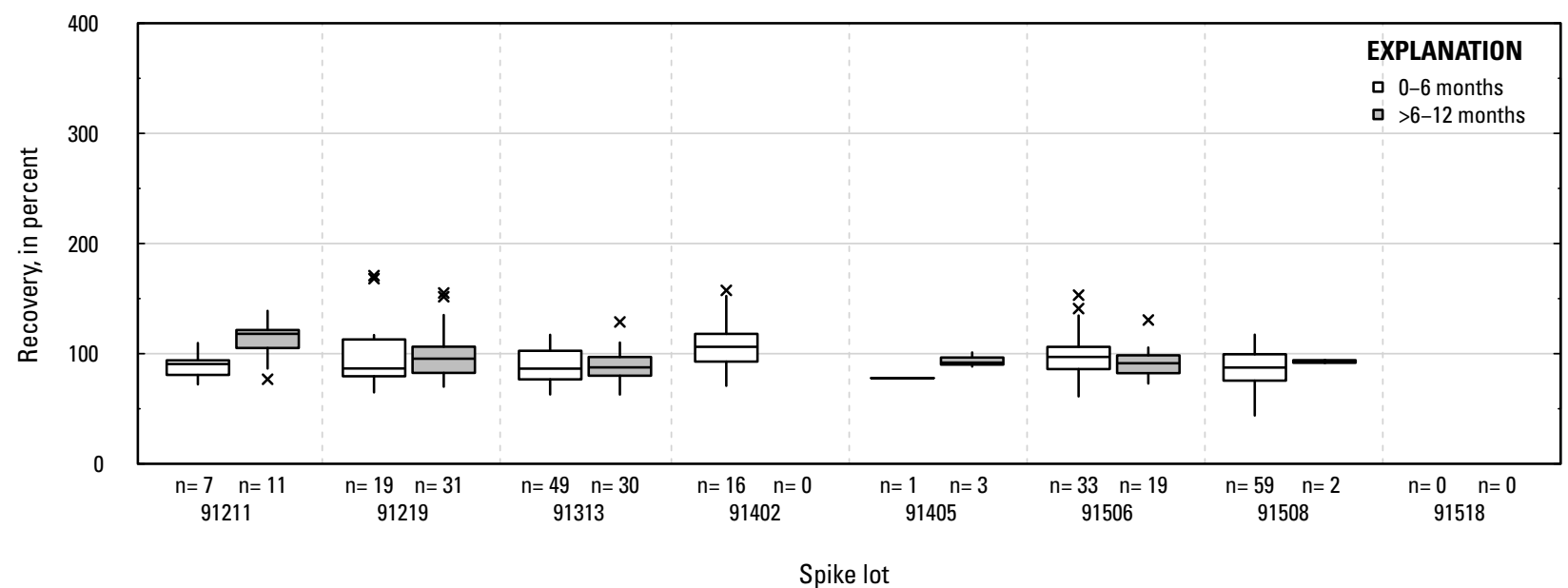

LB. Flubendiamide: groundwater field matrix spikes

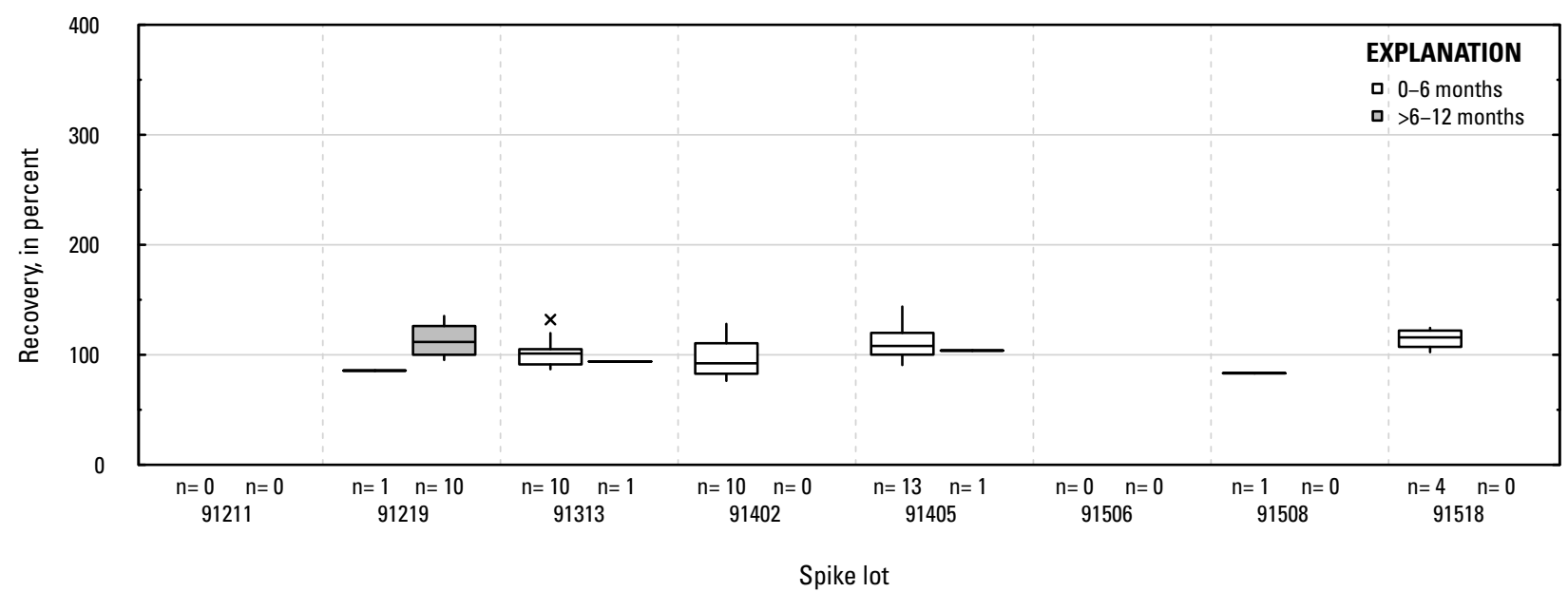

LC. Flubendiamide: surface water field matrix spikes

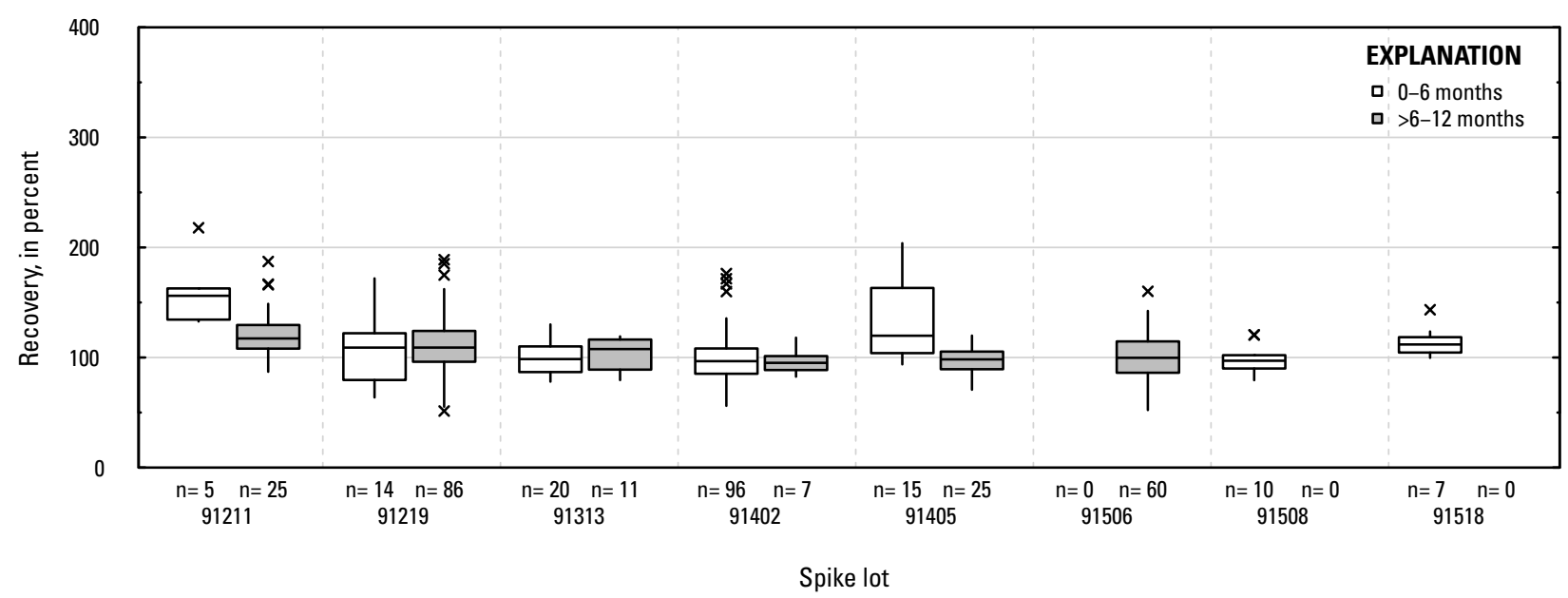

Figure 1-1. Distributions of recovery for individual pesticides in schedule 2437 by matrix, spike lot, and spike lot age. Recovery values larger than 400 percent are not shown.-Continued 


\section{LD. Flumetsulam: laboratory reagent spikes}

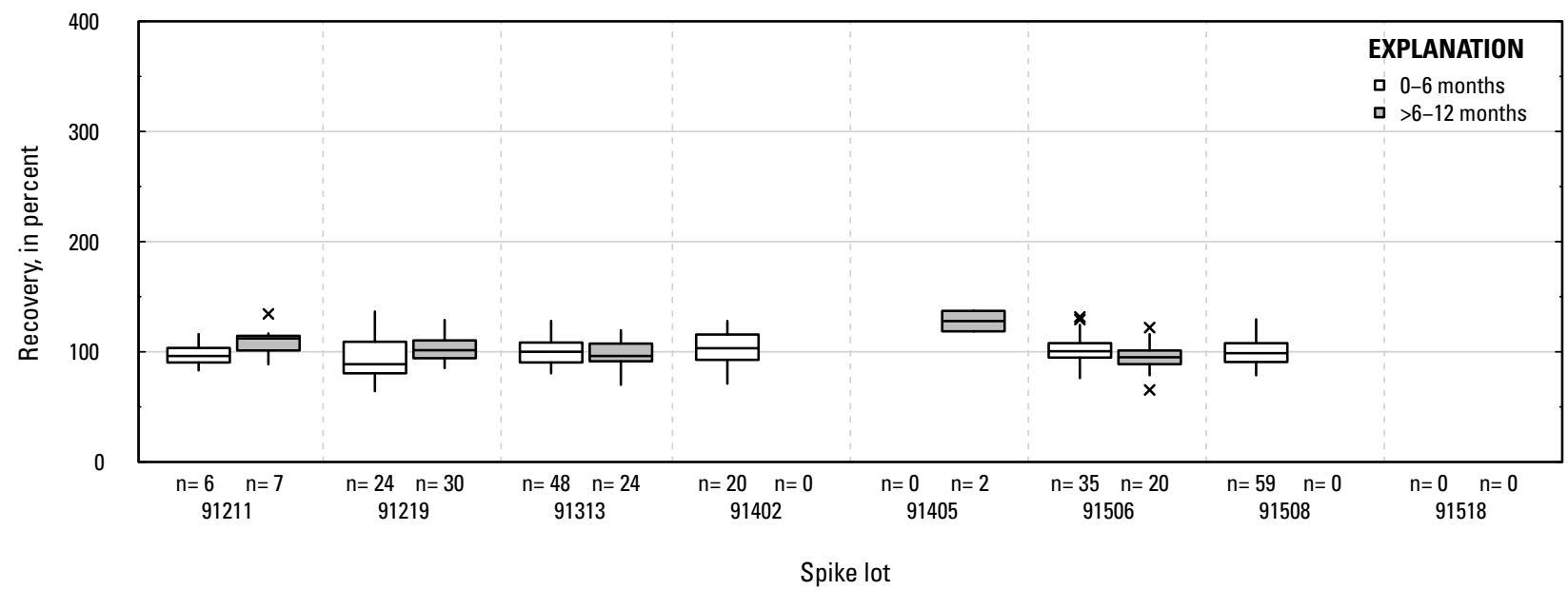

LE. Flumetsulam: groundwater field matrix spikes

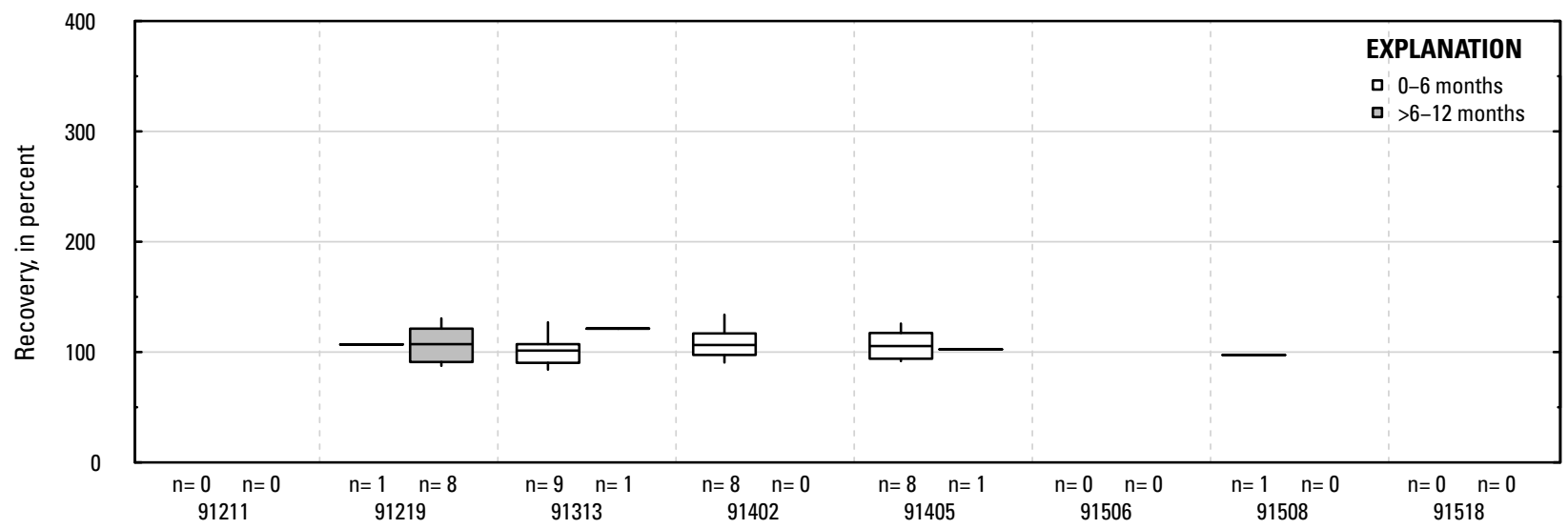

Spike lot

\section{LF. Flumetsulam: surface water field matrix spikes}

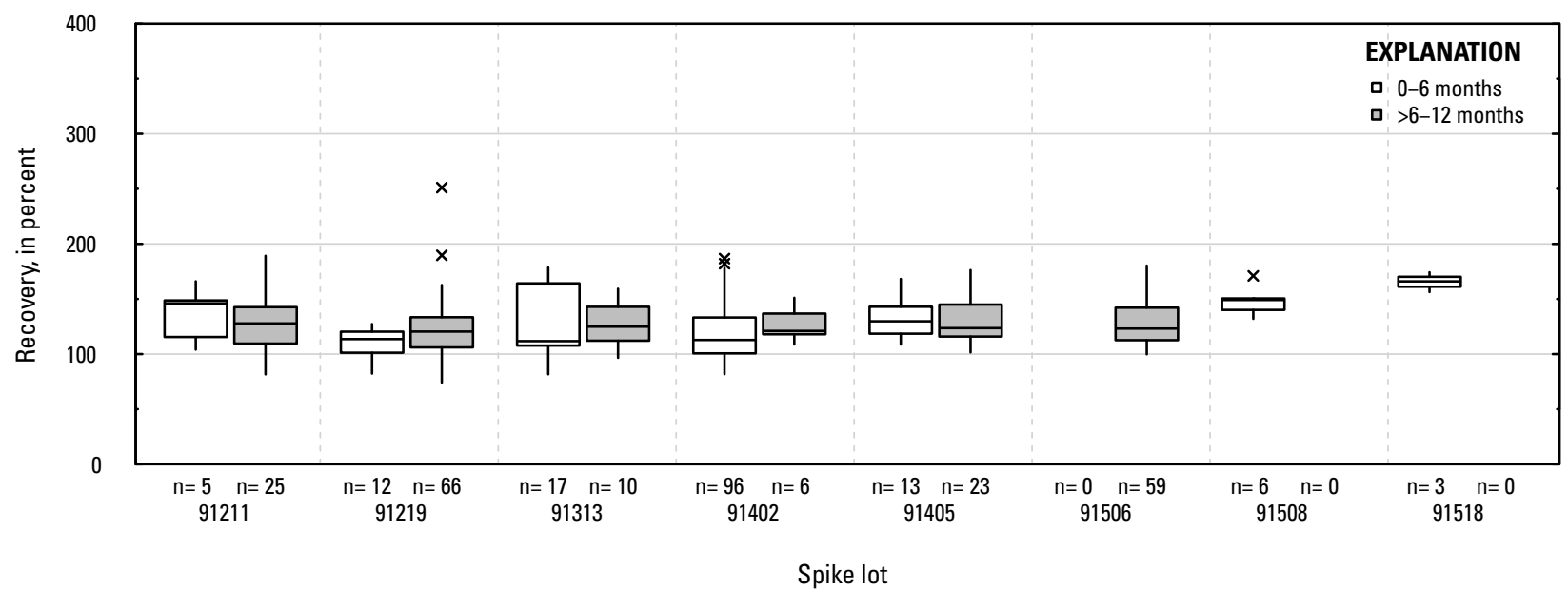

Figure 1-1. Distributions of recovery for individual pesticides in schedule 2437 by matrix, spike lot, and spike lot age. Recovery values larger than 400 percent are not shown.-Continued 


\section{LG. Fluometuron: laboratory reagent spikes}

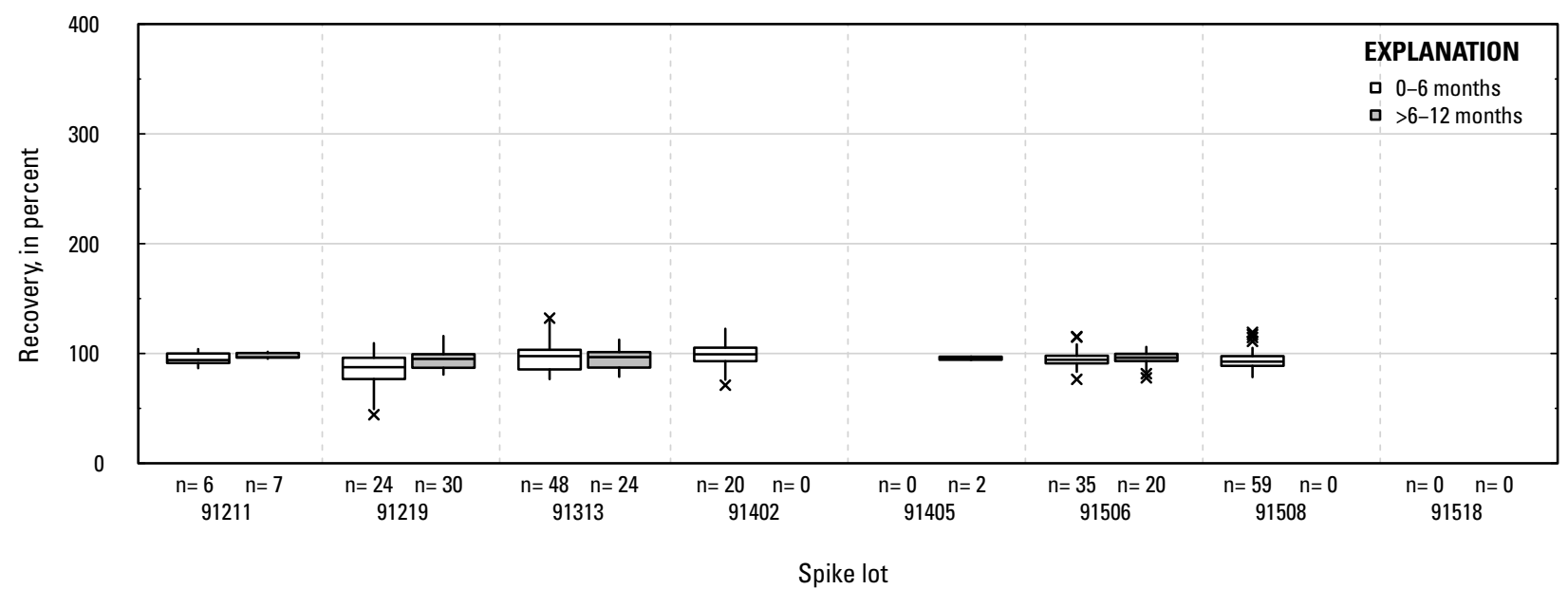

LH. Fluometuron: groundwater field matrix spikes

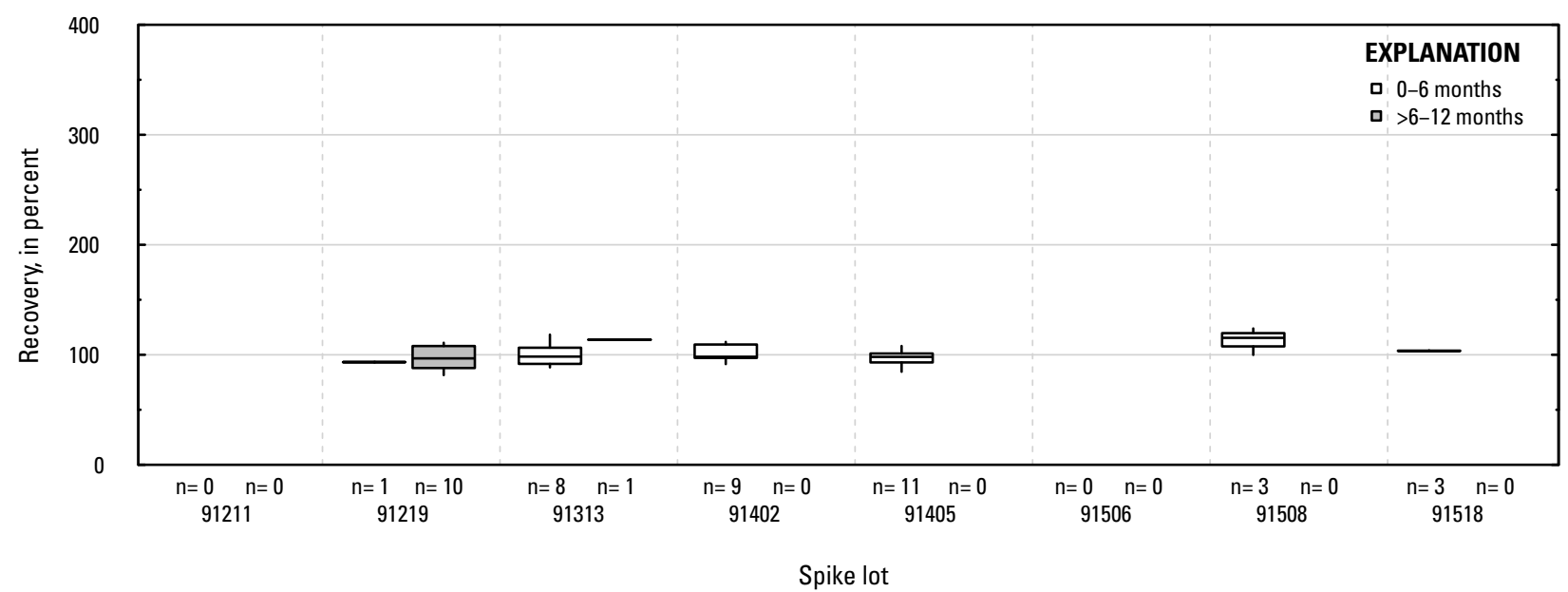

\section{Fluometuron: surface water field matrix spikes}

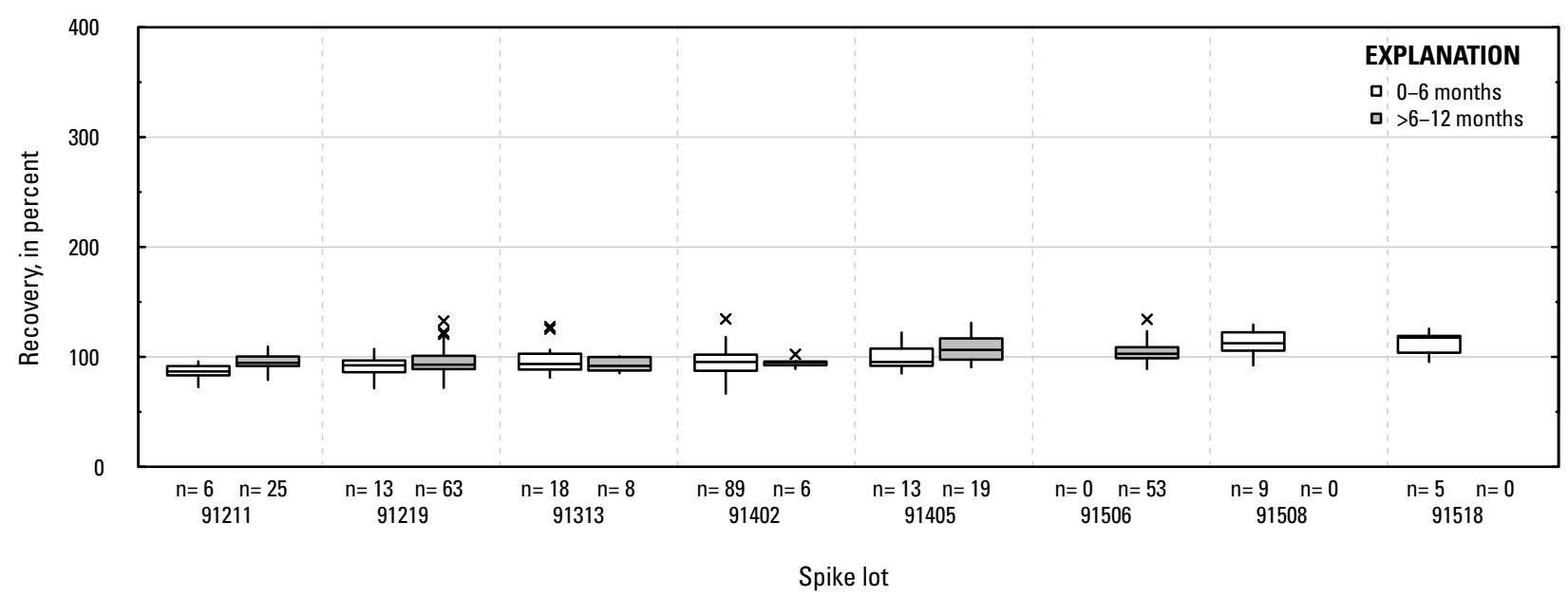

Figure 1-1. Distributions of recovery for individual pesticides in schedule 2437 by matrix, spike lot, and spike lot age. Recovery values larger than 400 percent are not shown.-Continued 


\section{LJ. Fonofos: laboratory reagent spikes}

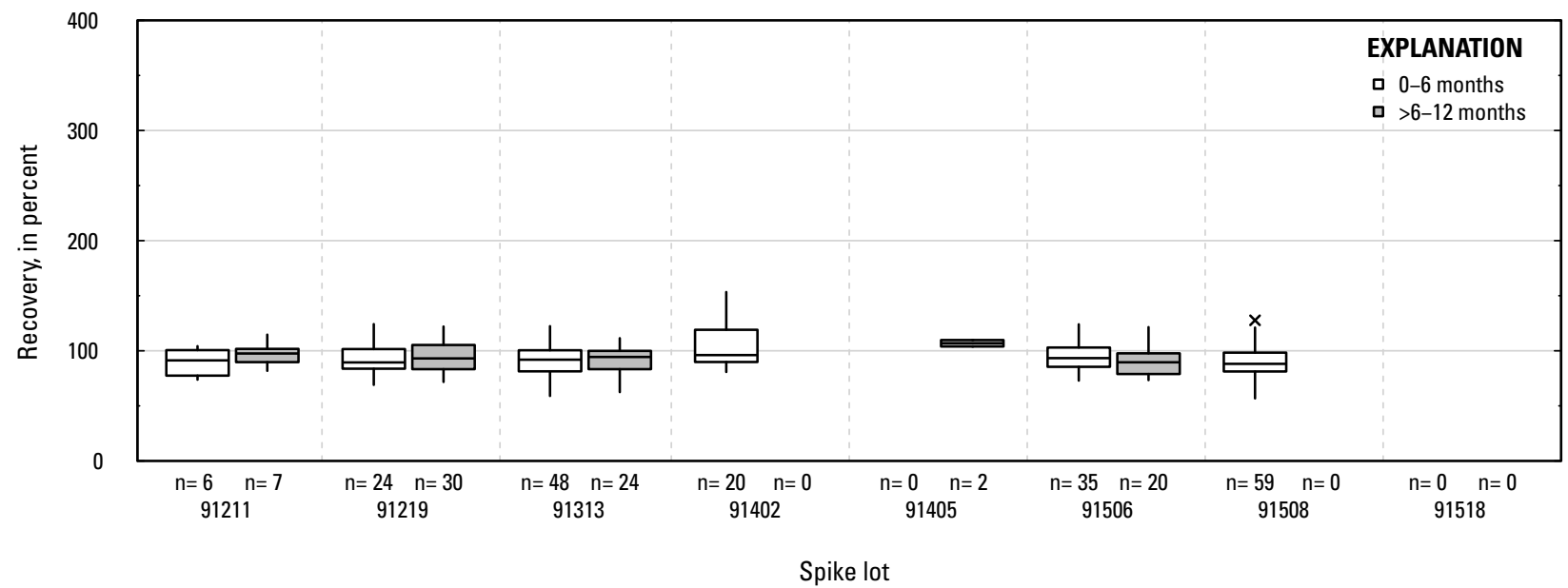

$L K$. Fonofos: groundwater field matrix spikes

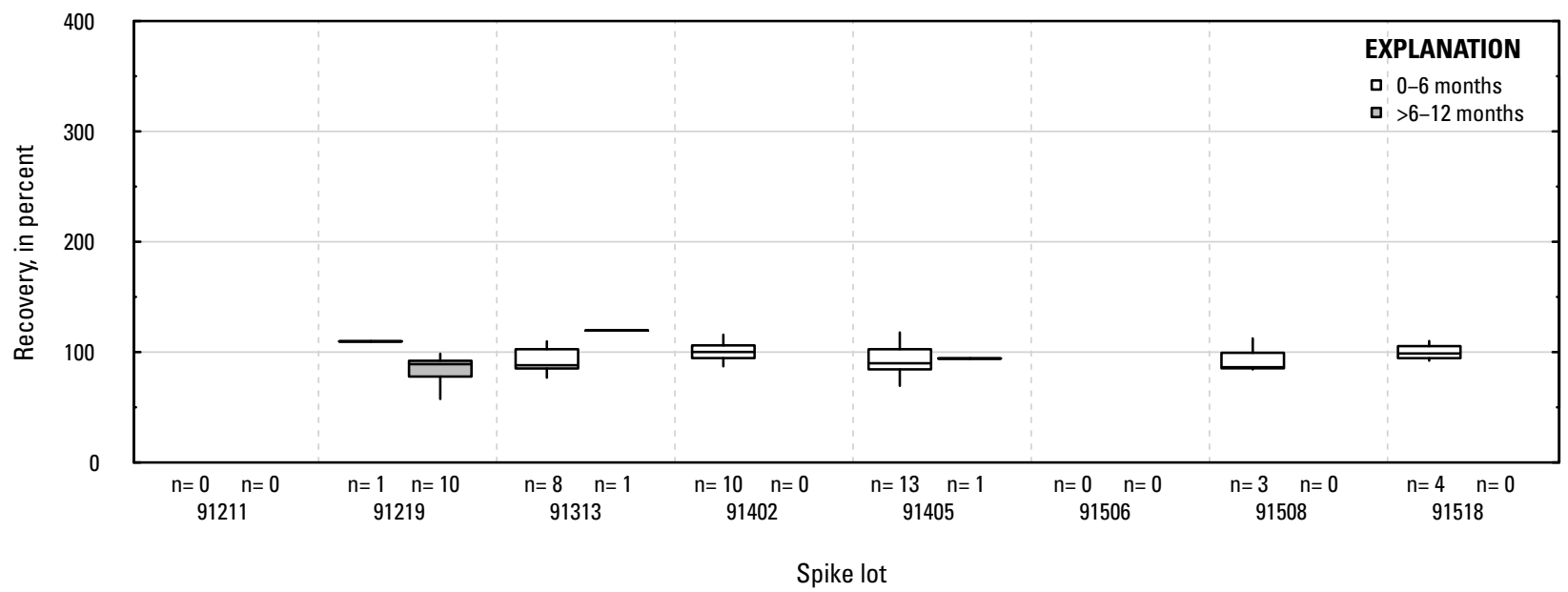

LL. Fonofos: surface water field matrix spikes

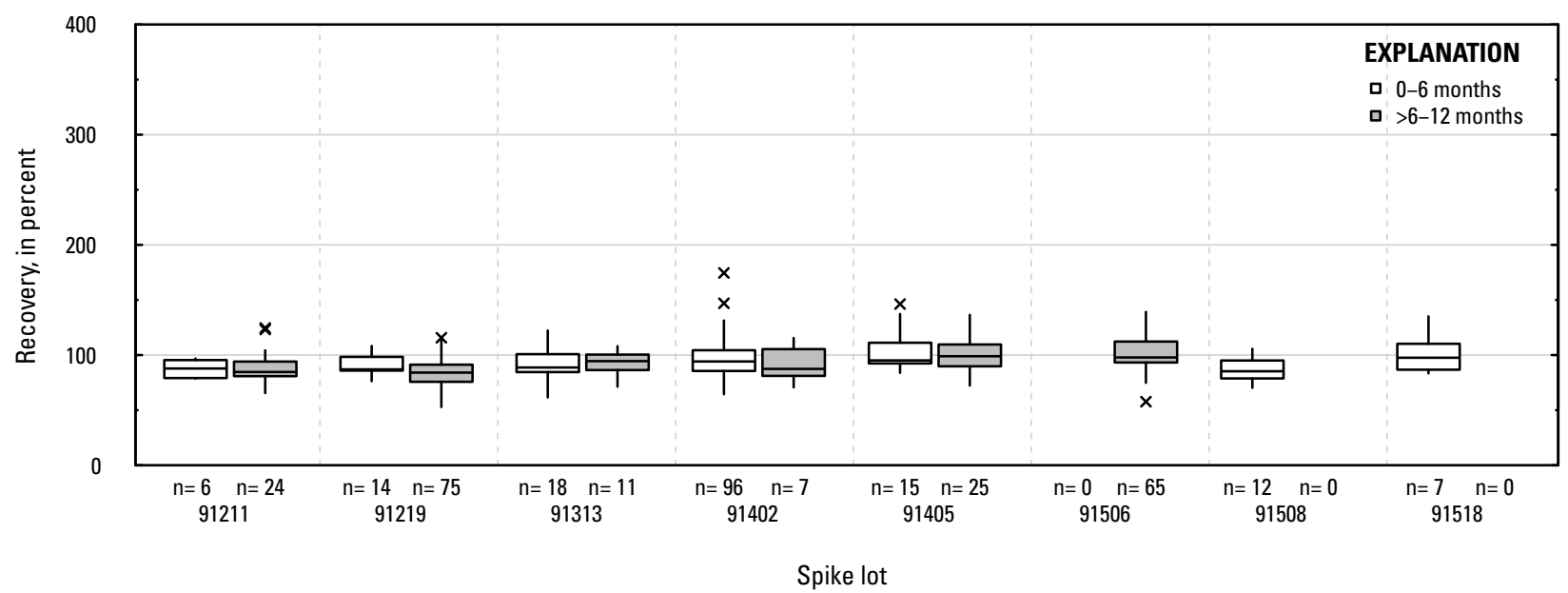

Figure 1-1. Distributions of recovery for individual pesticides in schedule 2437 by matrix, spike lot, and spike lot age. Recovery values larger than 400 percent are not shown.-Continued 
LM. Halosulfuron-methyl: laboratory reagent spikes

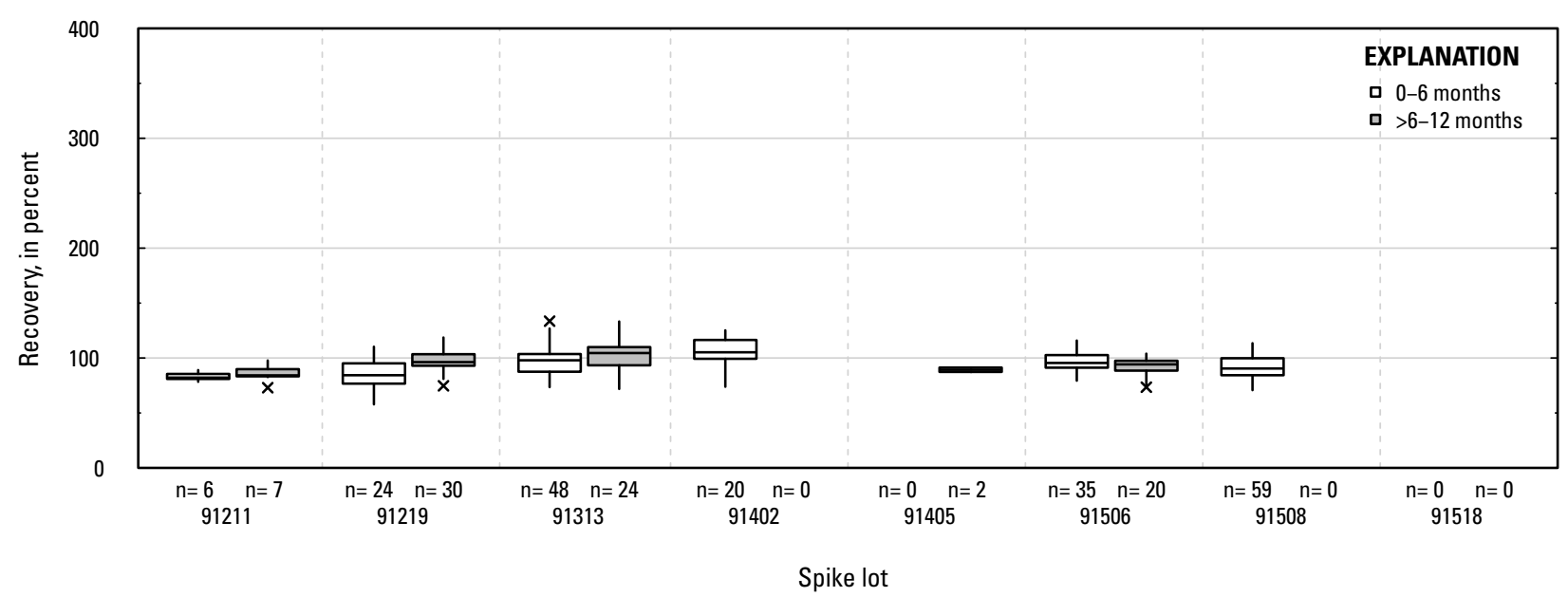

LN. Halosulfuron-methyl: groundwater field matrix spikes

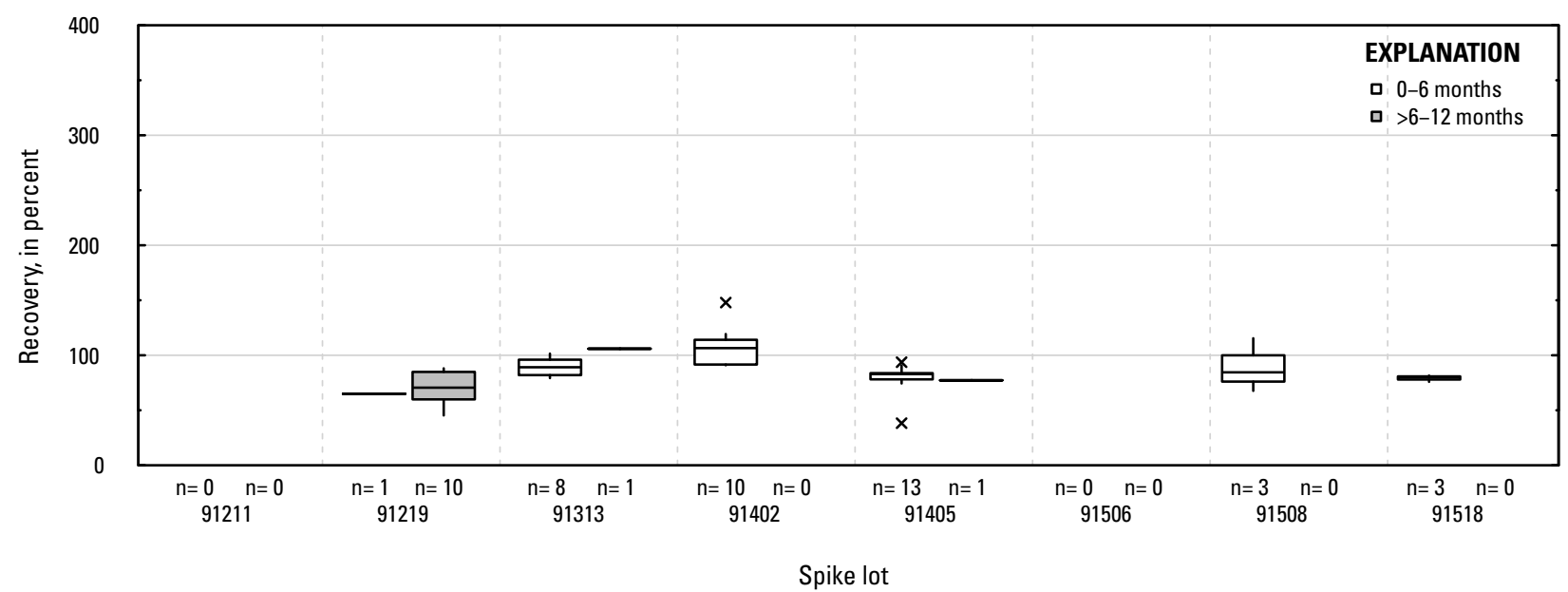

LO. Halosulfuron-methyl: surface water field matrix spikes

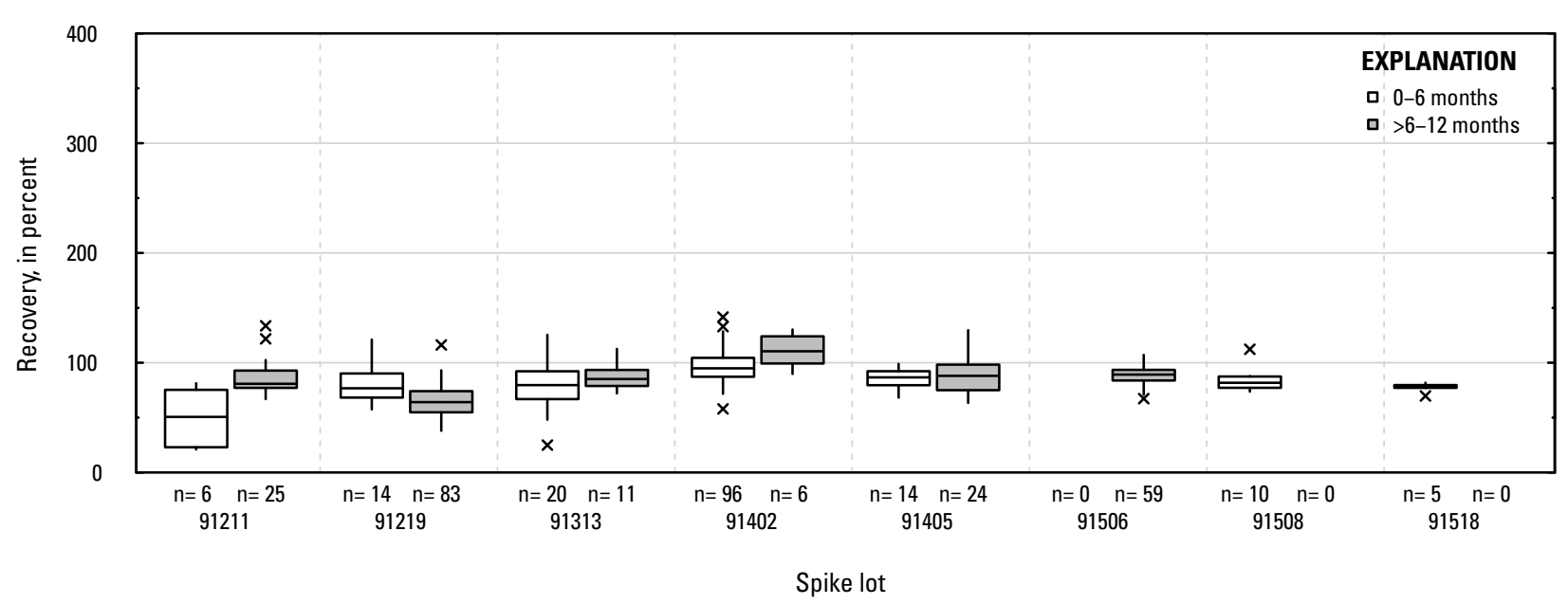

Figure 1-1. Distributions of recovery for individual pesticides in schedule 2437 by matrix, spike lot, and spike lot age. Recovery values larger than 400 percent are not shown.-Continued 


\section{LP. Hexazinone: laboratory reagent spikes}

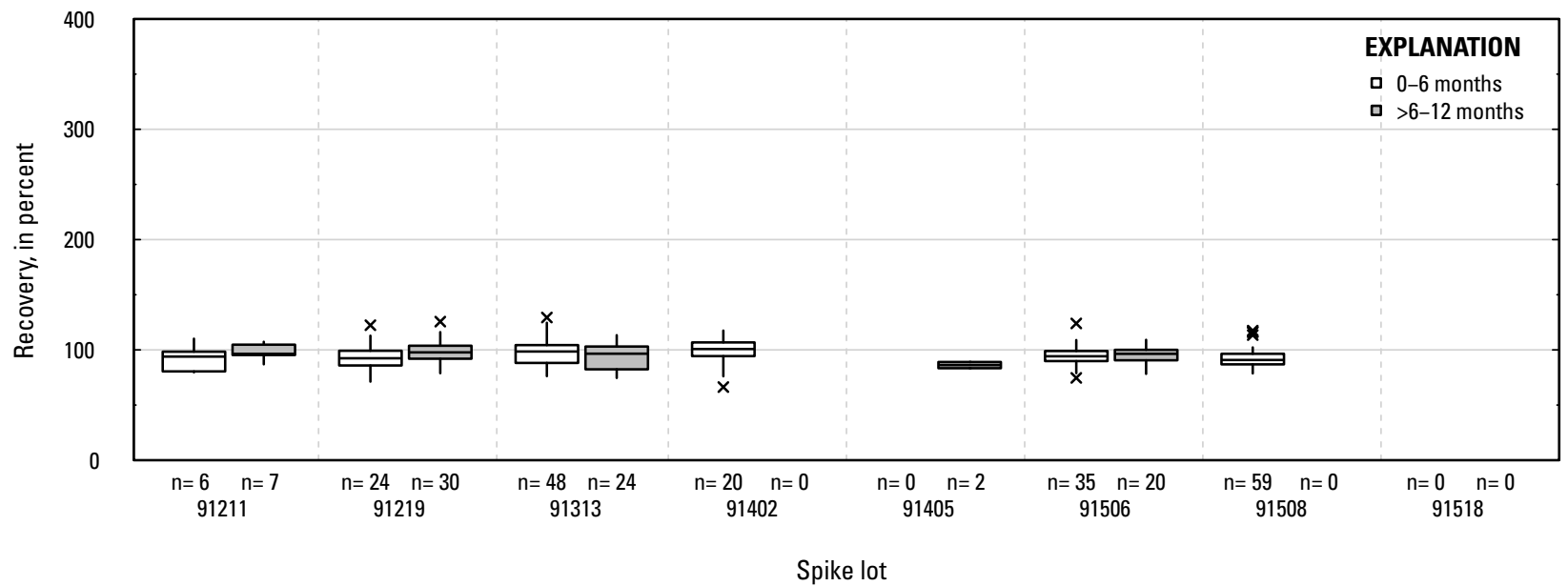

LQ. Hexazinone: groundwater field matrix spikes

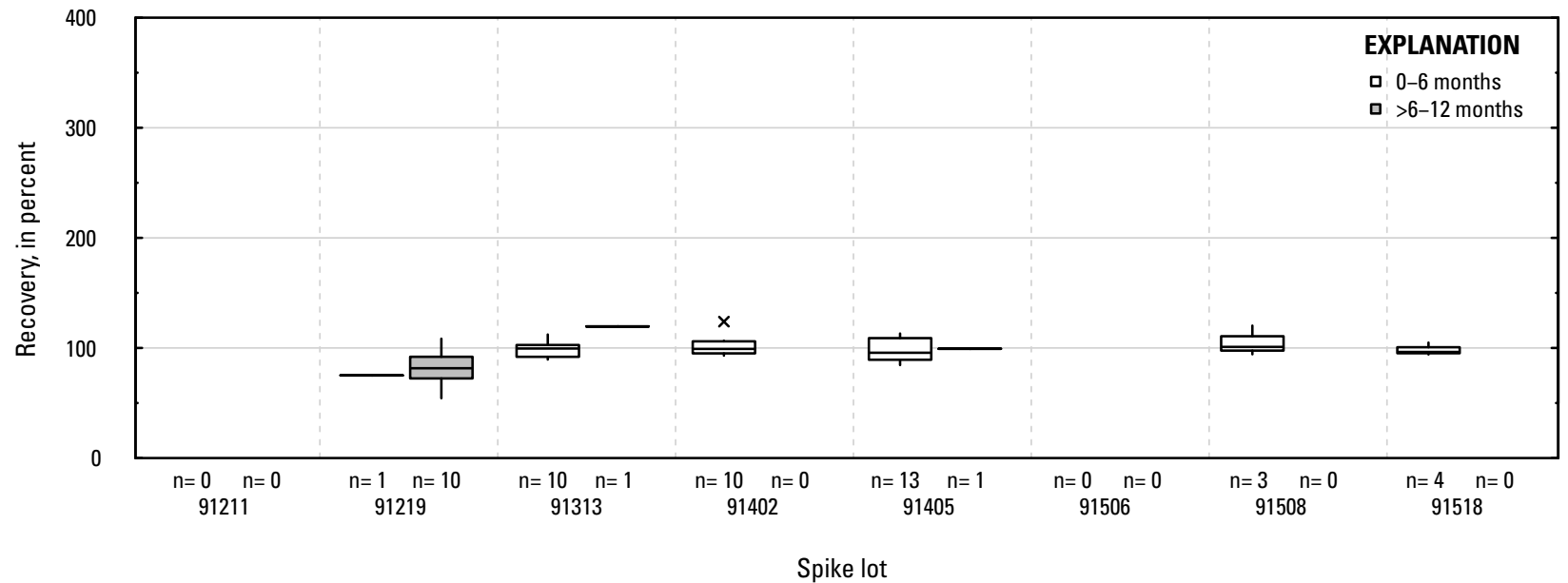

\section{LR. Hexazinone: surface water field matrix spikes}

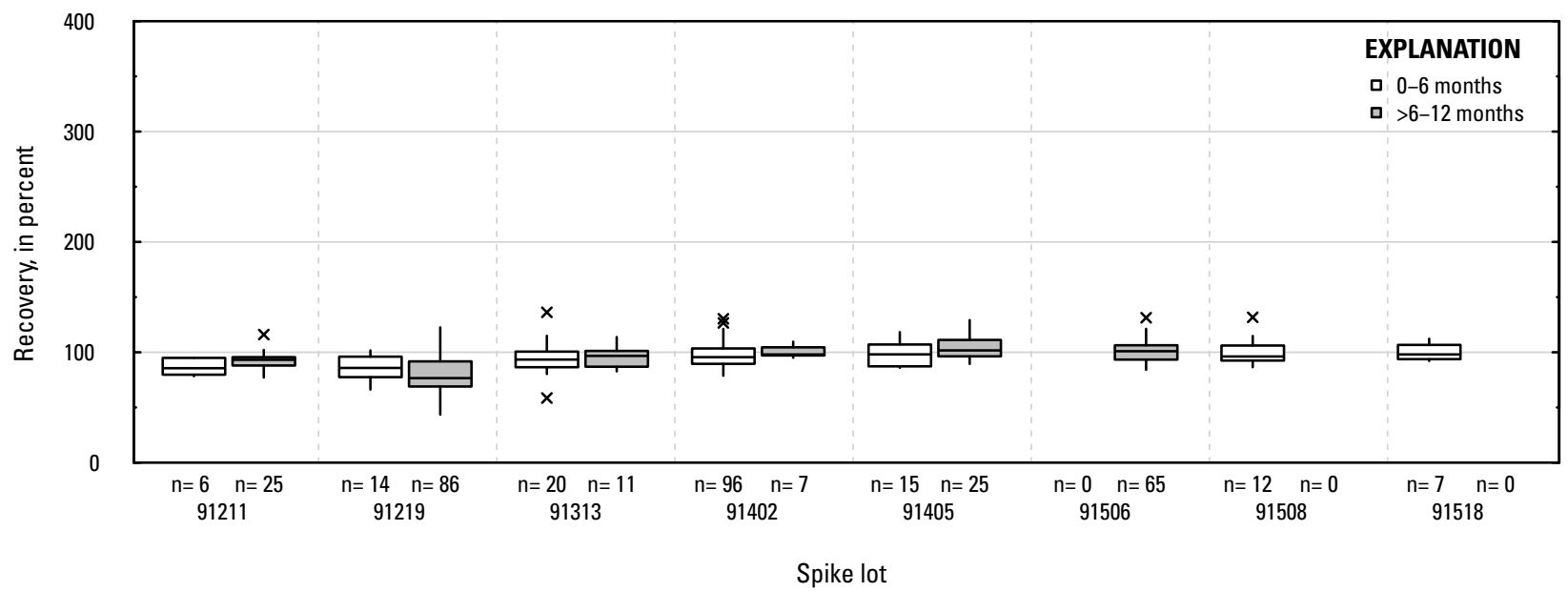

Figure 1-1. Distributions of recovery for individual pesticides in schedule 2437 by matrix, spike lot, and spike lot age. Recovery values larger than 400 percent are not shown.-Continued 


\section{$L S$. Hexazinone Transformation Product C: laboratory reagent spikes}

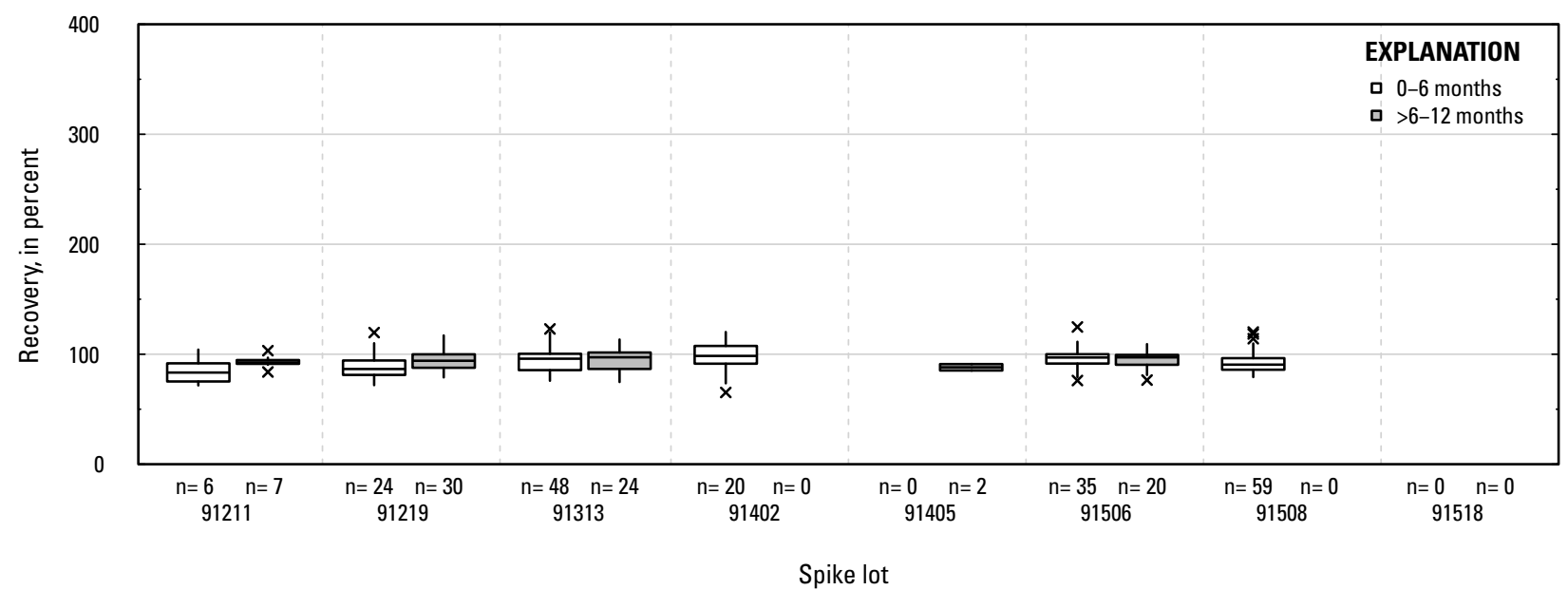

\section{LT. Hexazinone Transformation Product C: groundwater field matrix spikes}

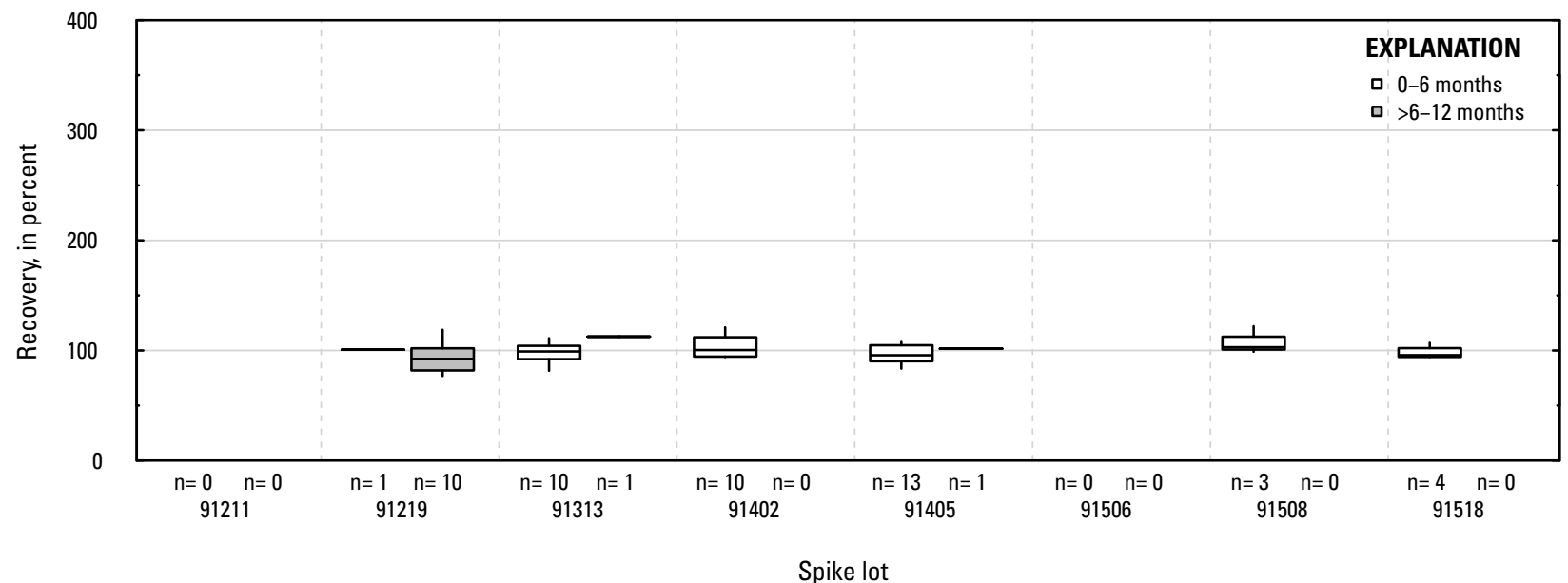

\section{LU. Hexazinone Transformation Product C: surface water field matrix spikes}

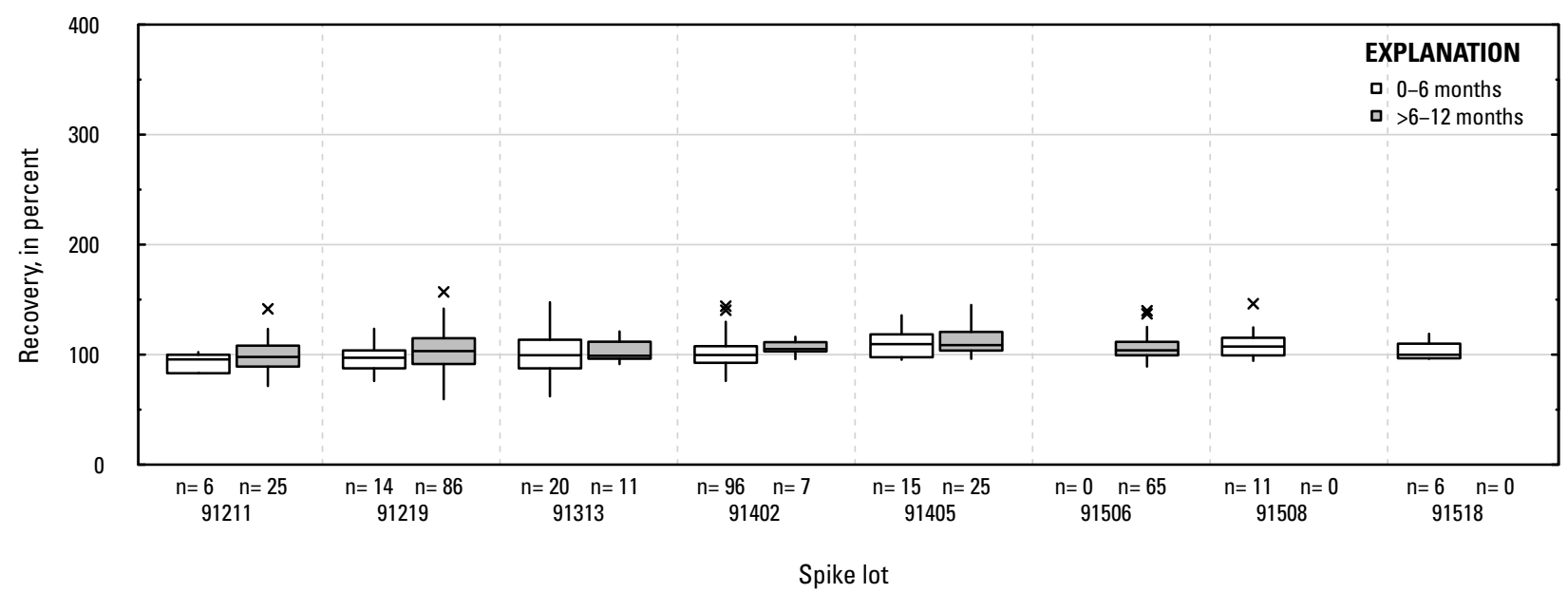

Figure 1-1. Distributions of recovery for individual pesticides in schedule 2437 by matrix, spike lot, and spike lot age. Recovery values larger than 400 percent are not shown.-Continued 


\section{Hexazinone Transformation Product D: laboratory reagent spikes}

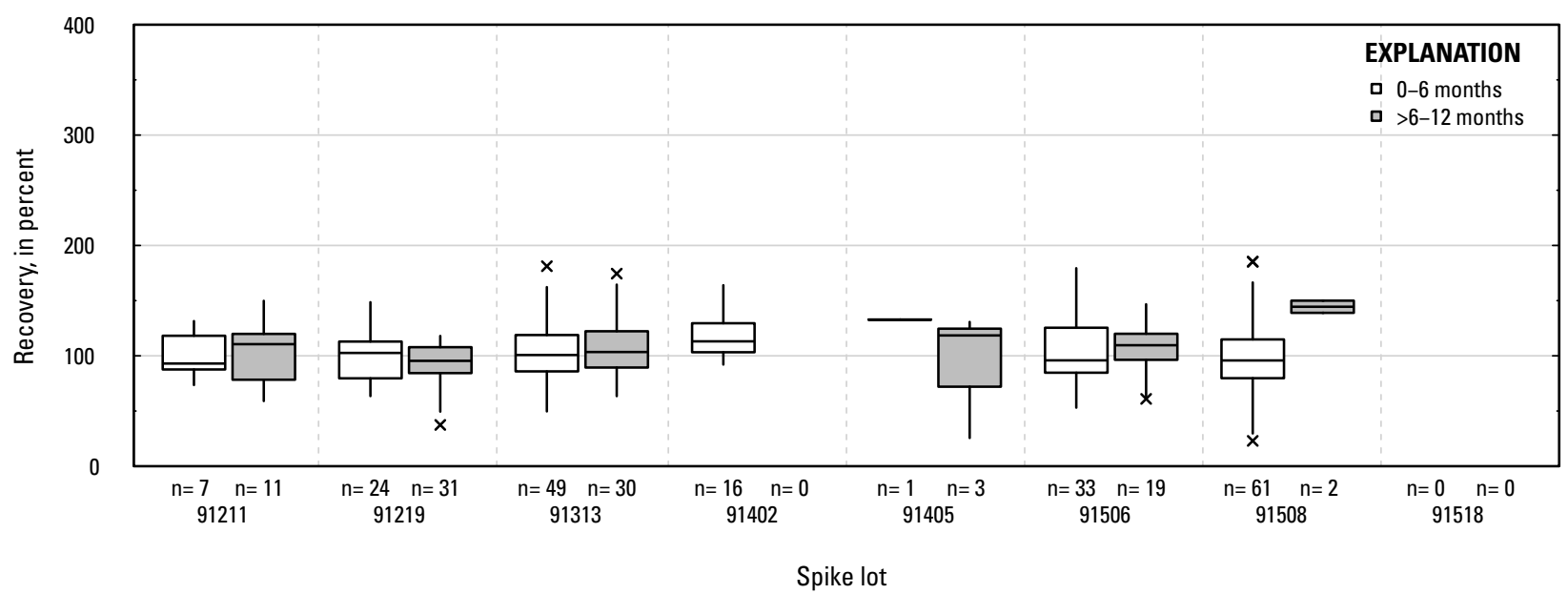

LW. Hexazinone Transformation Product D: groundwater field matrix spikes

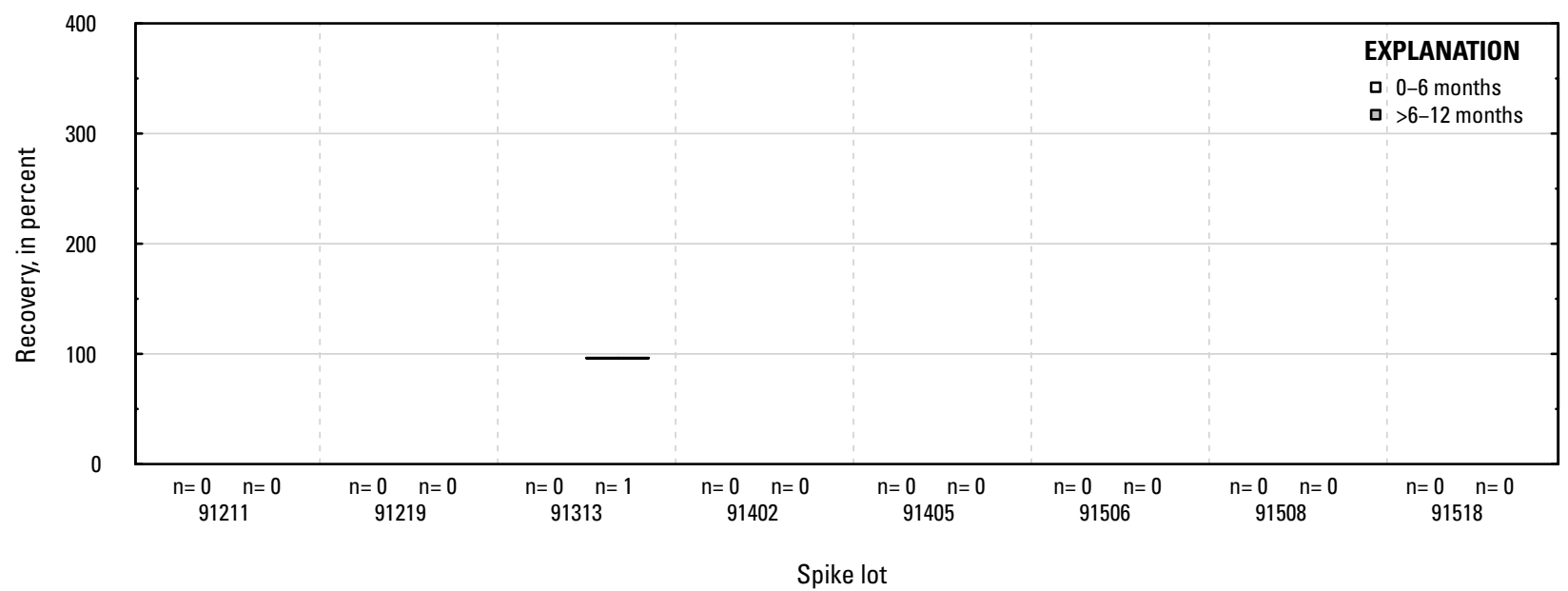

\section{$L X$. Hexazinone Transformation Product D: surface water field matrix spikes}

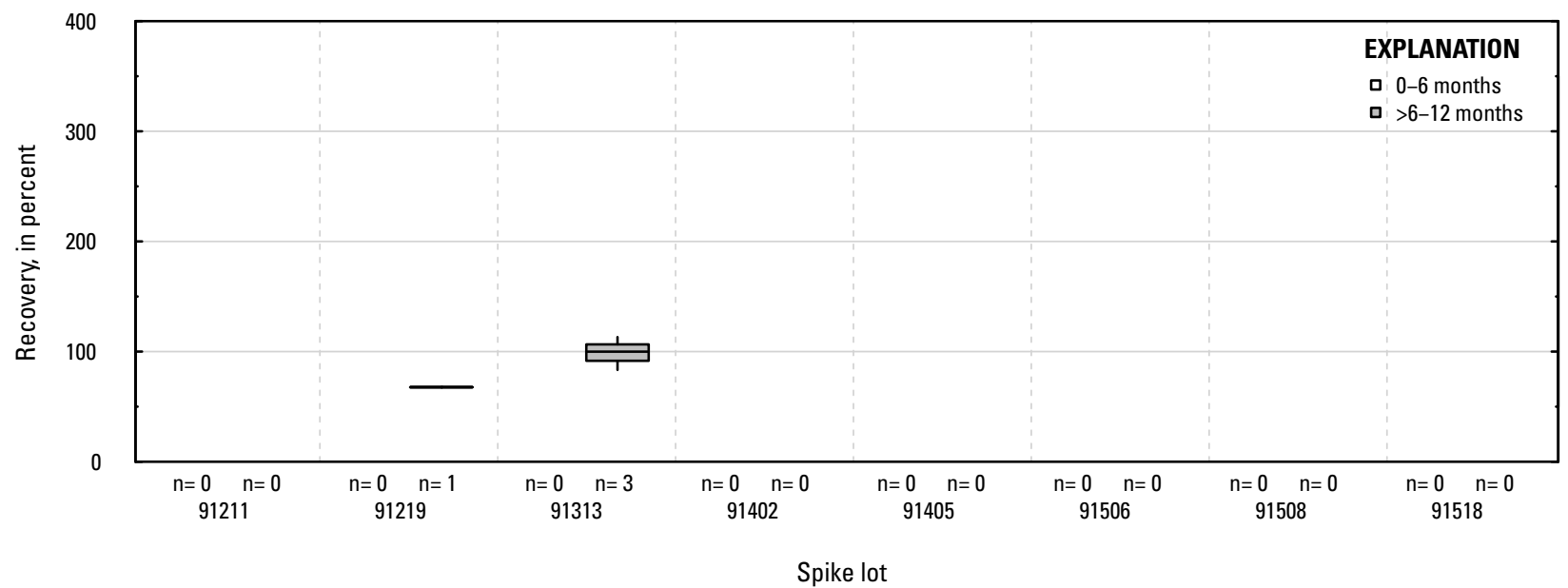

Figure 1-1. Distributions of recovery for individual pesticides in schedule 2437 by matrix, spike lot, and spike lot age. Recovery values larger than 400 percent are not shown.-Continued 


\section{LY. Hexazinone Transformation Product E: laboratory reagent spikes}

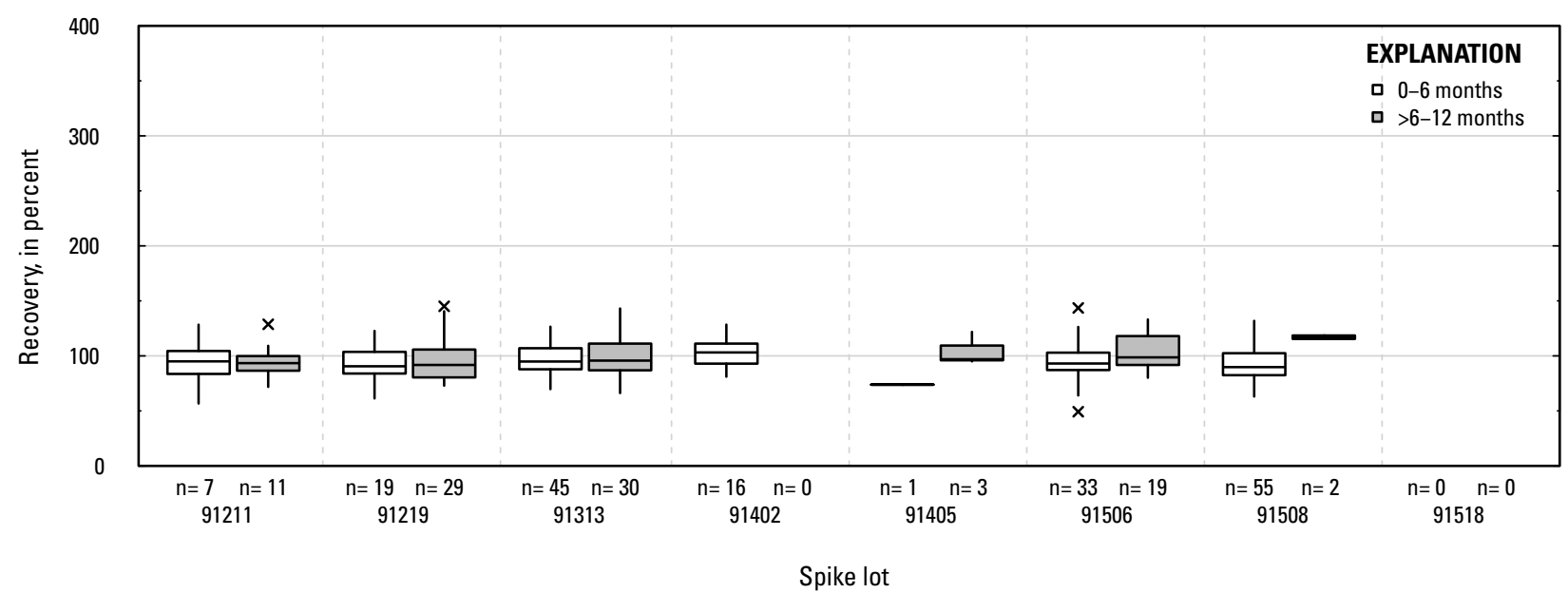

\section{LZ. Hexazinone Transformation Product E: groundwater field matrix spikes}

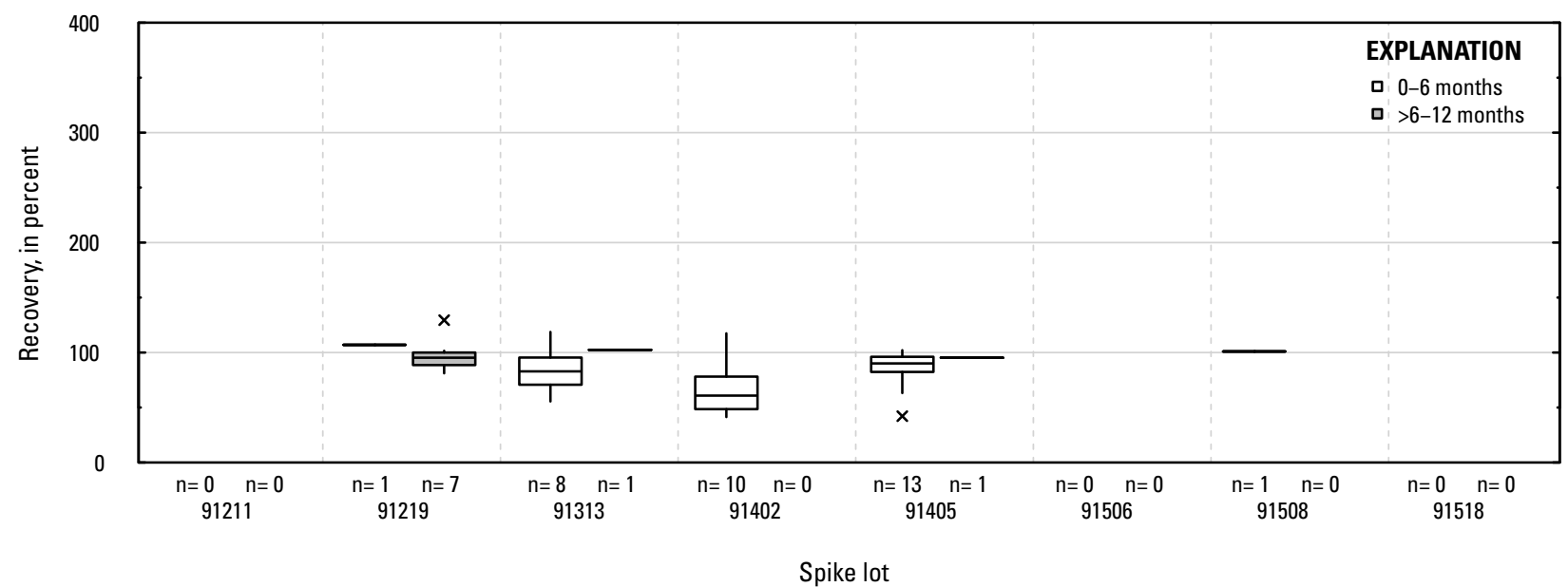

MA. Hexazinone Transformation Product E: surface water field matrix spikes

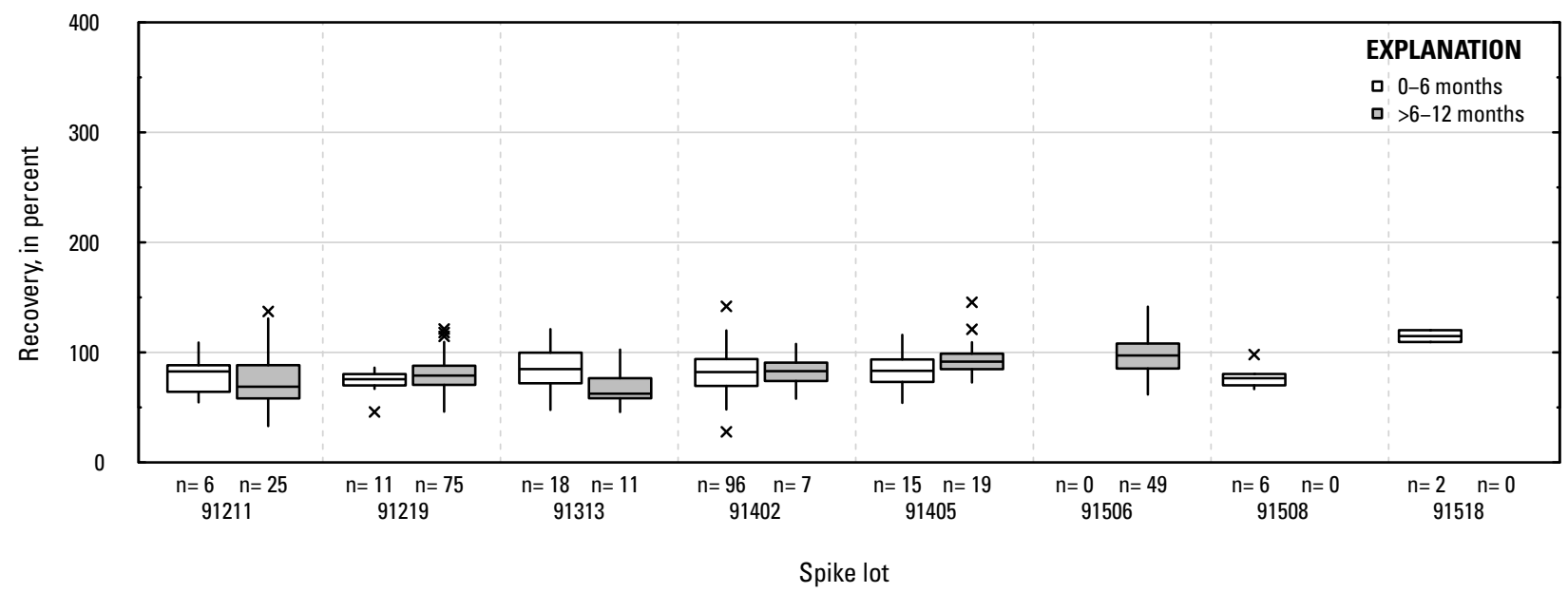

Figure 1-1. Distributions of recovery for individual pesticides in schedule 2437 by matrix, spike lot, and spike lot age. Recovery values larger than 400 percent are not shown.-Continued 
MB. Didemethyl hexazinone F: laboratory reagent spikes

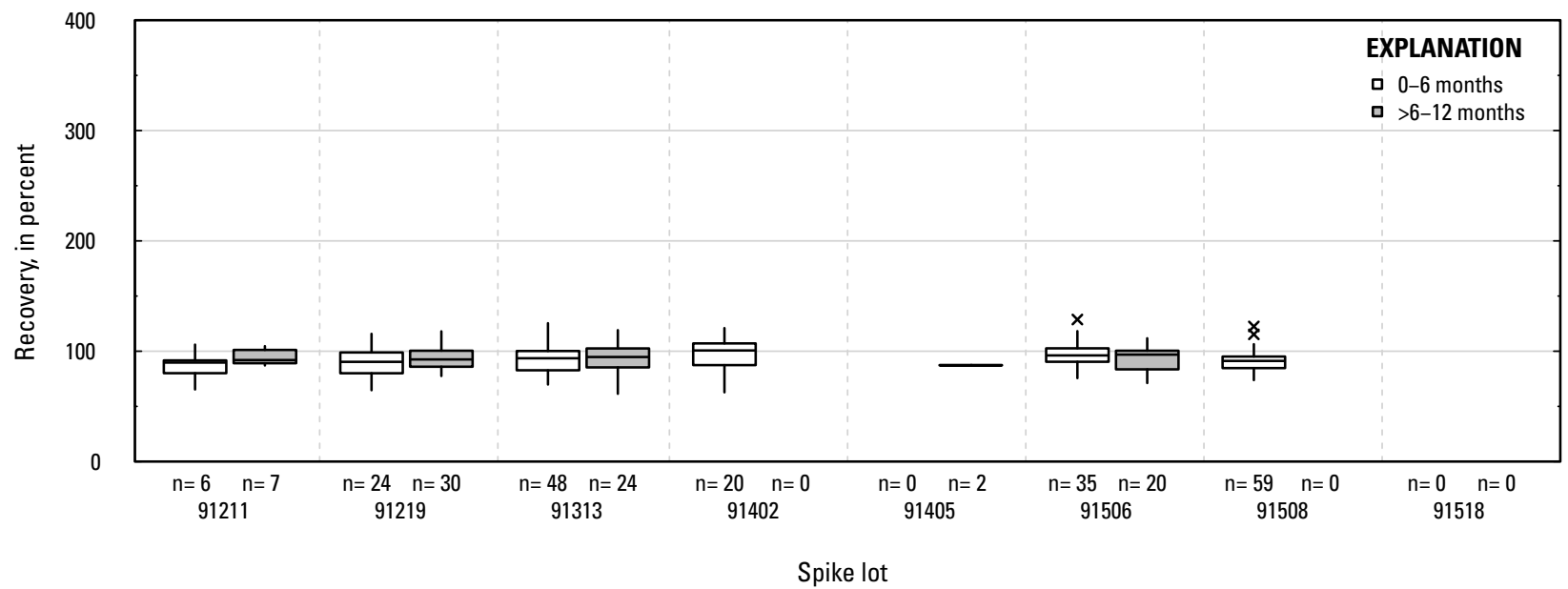

MC. Didemethyl hexazinone F: groundwater field matrix spikes

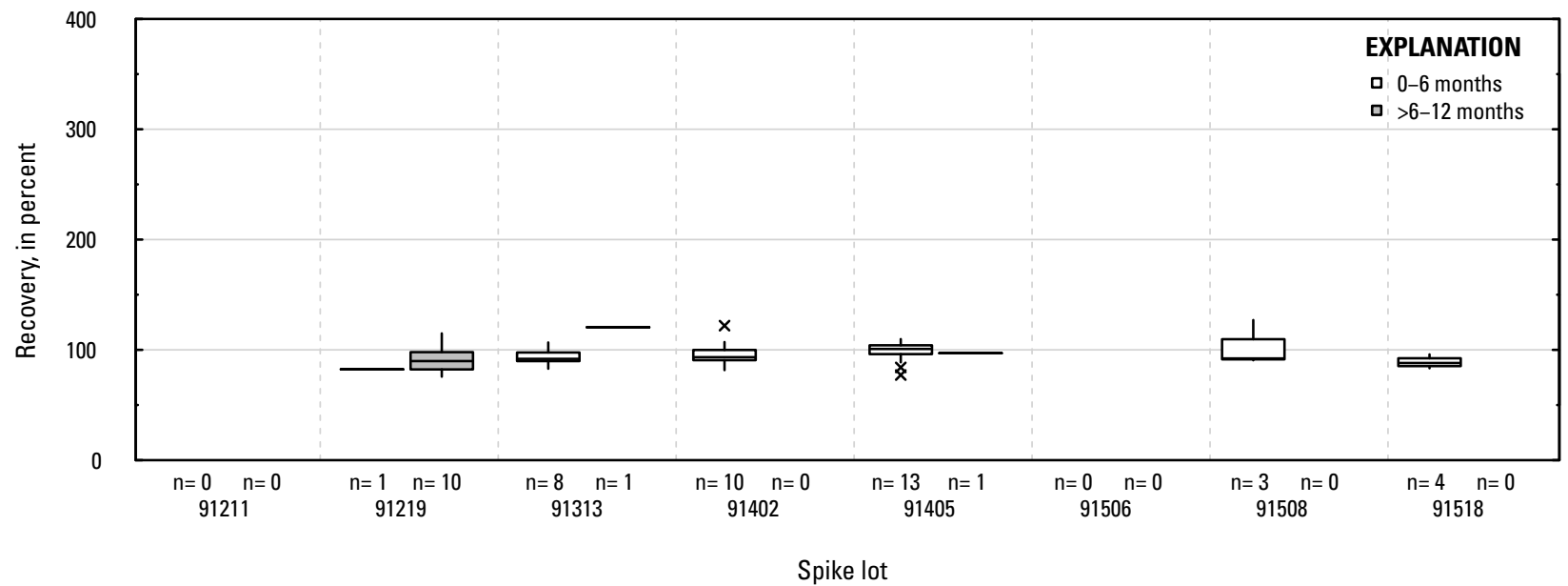

MD. Didemethyl hexazinone F: surface water field matrix spikes

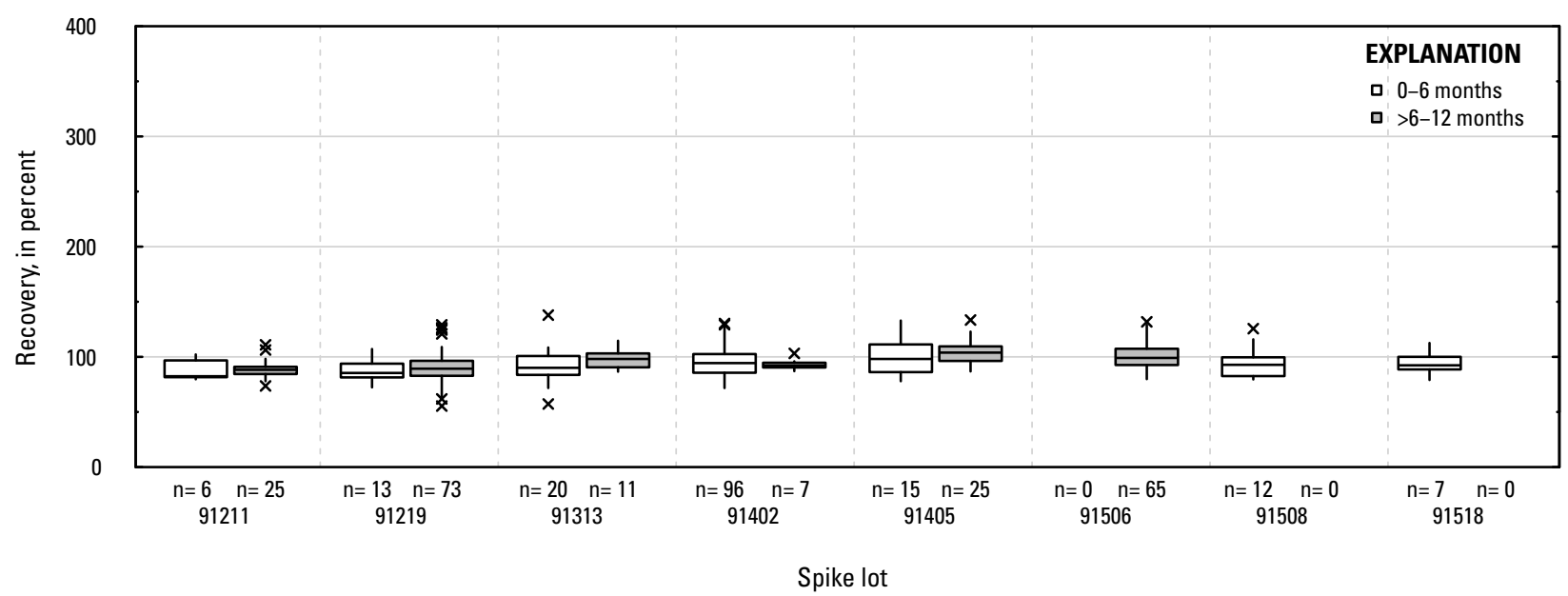

Figure 1-1. Distributions of recovery for individual pesticides in schedule 2437 by matrix, spike lot, and spike lot age. Recovery values larger than 400 percent are not shown.-Continued 
ME. Hexazinone Transformation Product G: laboratory reagent spikes

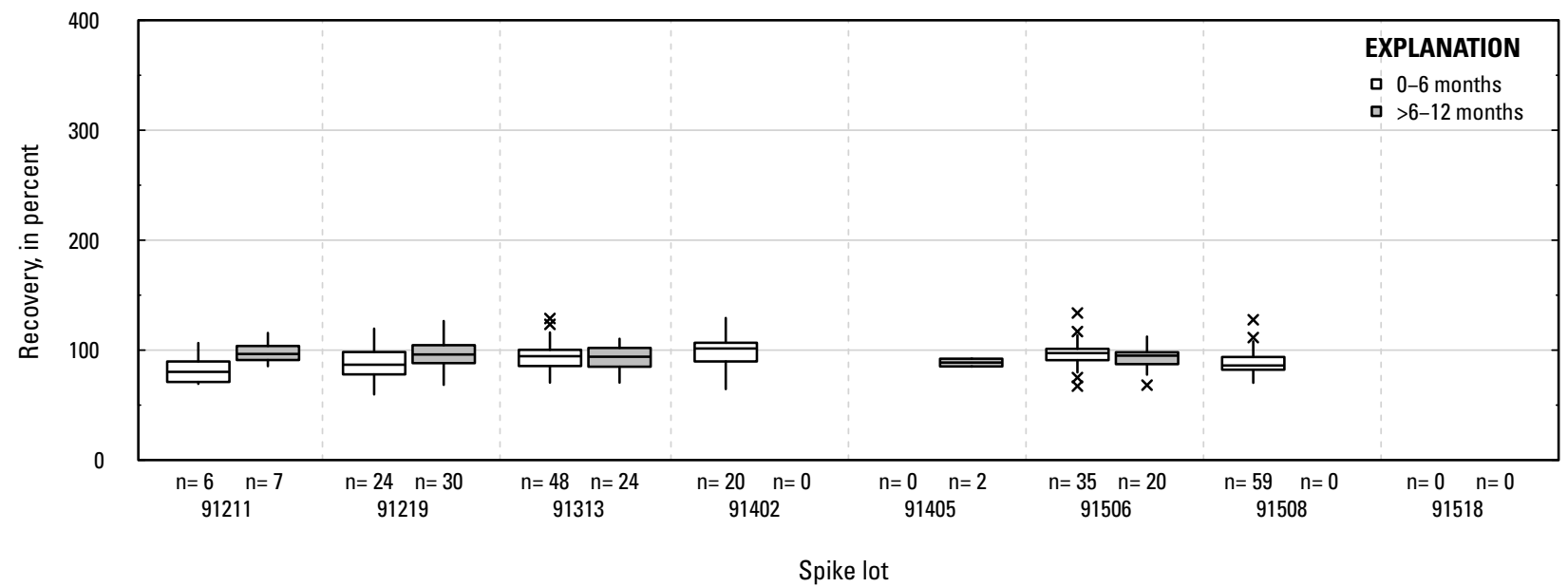

MF Hexazinone Transformation Product G: groundwater field matrix spikes

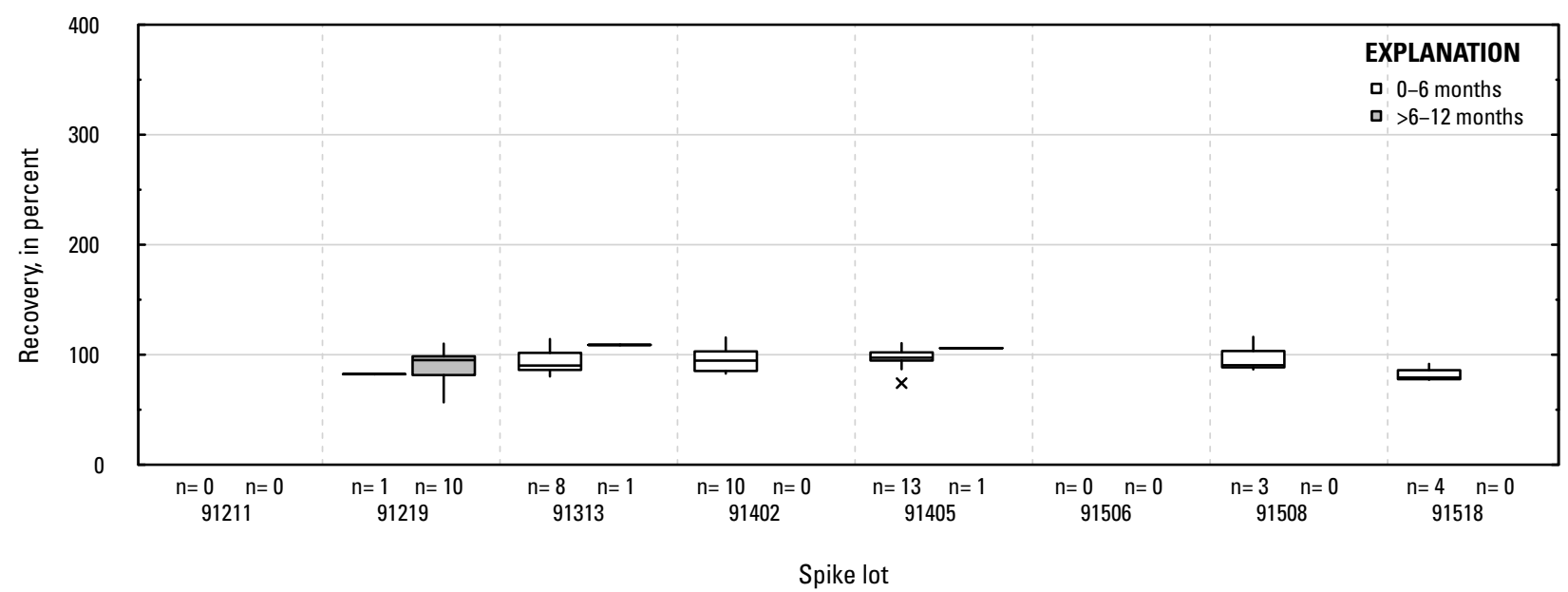

MG. Hexazinone Transformation Product G: surface water field matrix spikes

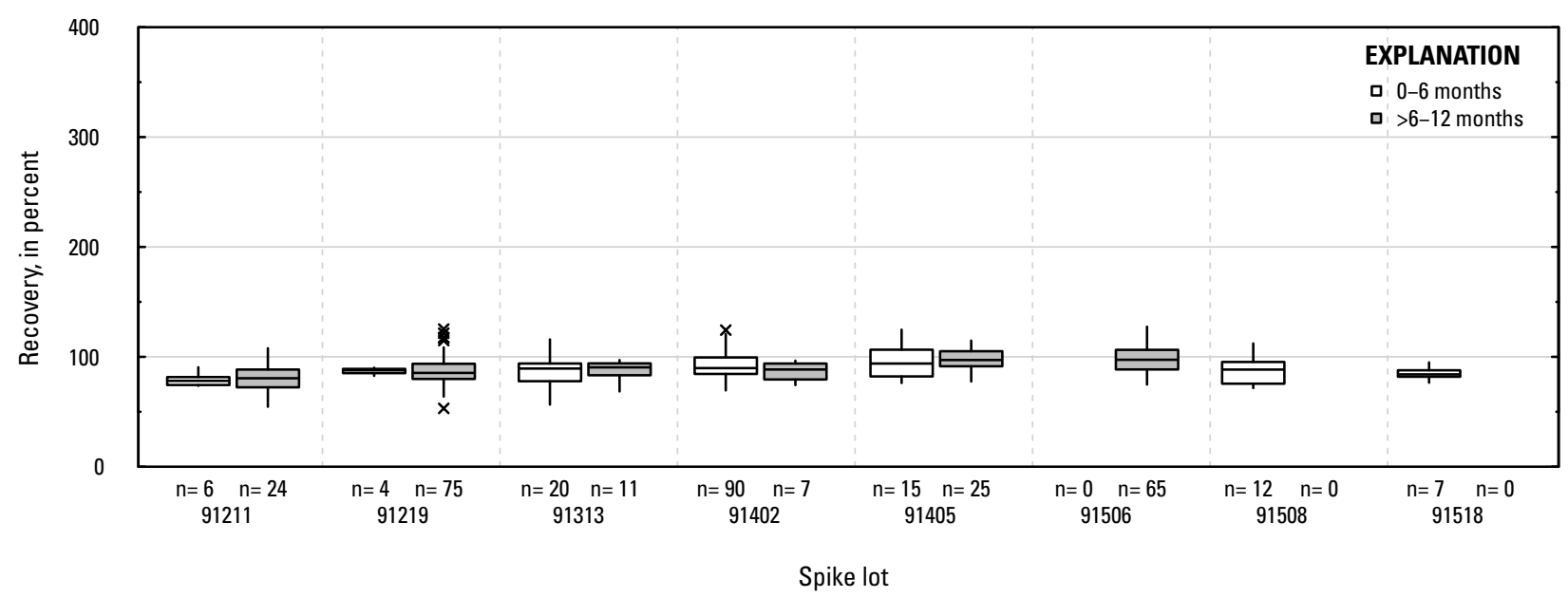

Figure 1-1. Distributions of recovery for individual pesticides in schedule 2437 by matrix, spike lot, and spike lot age. Recovery values larger than 400 percent are not shown.-Continued 
MH. Hydroxy monodemethyl fluometuron: laboratory reagent spikes

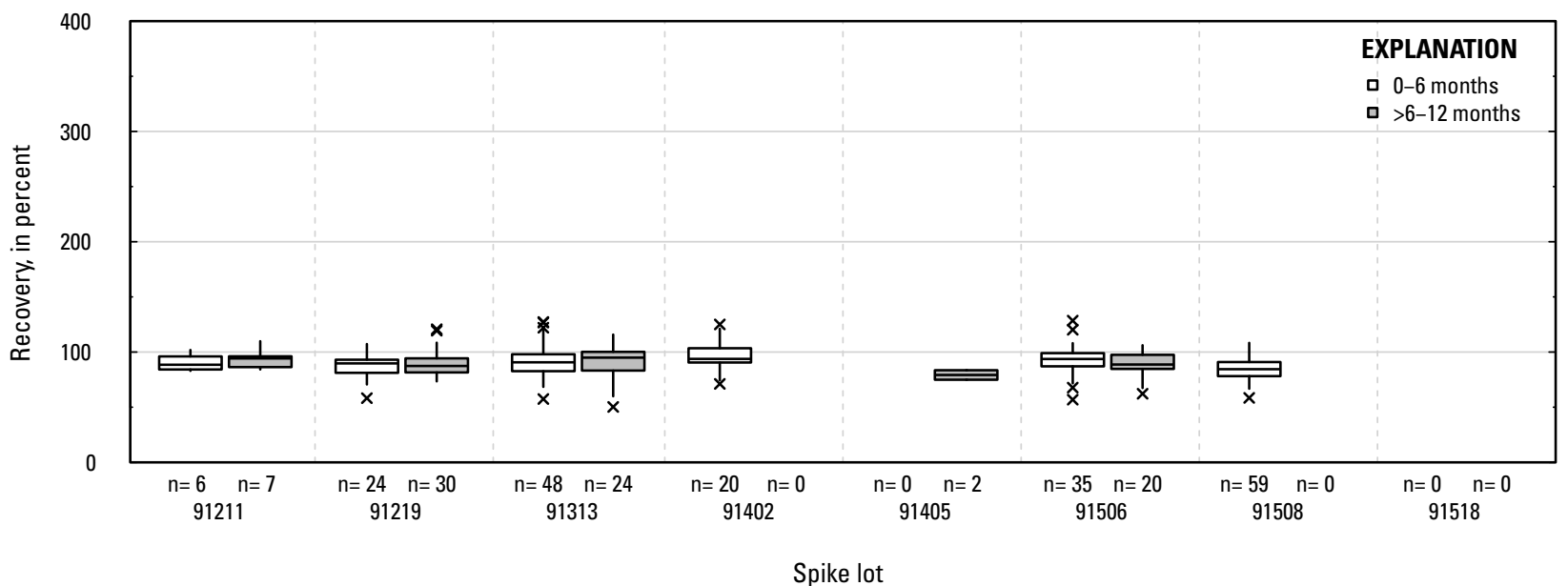

MI. Hydroxy monodemethyl fluometuron: groundwater field matrix spikes

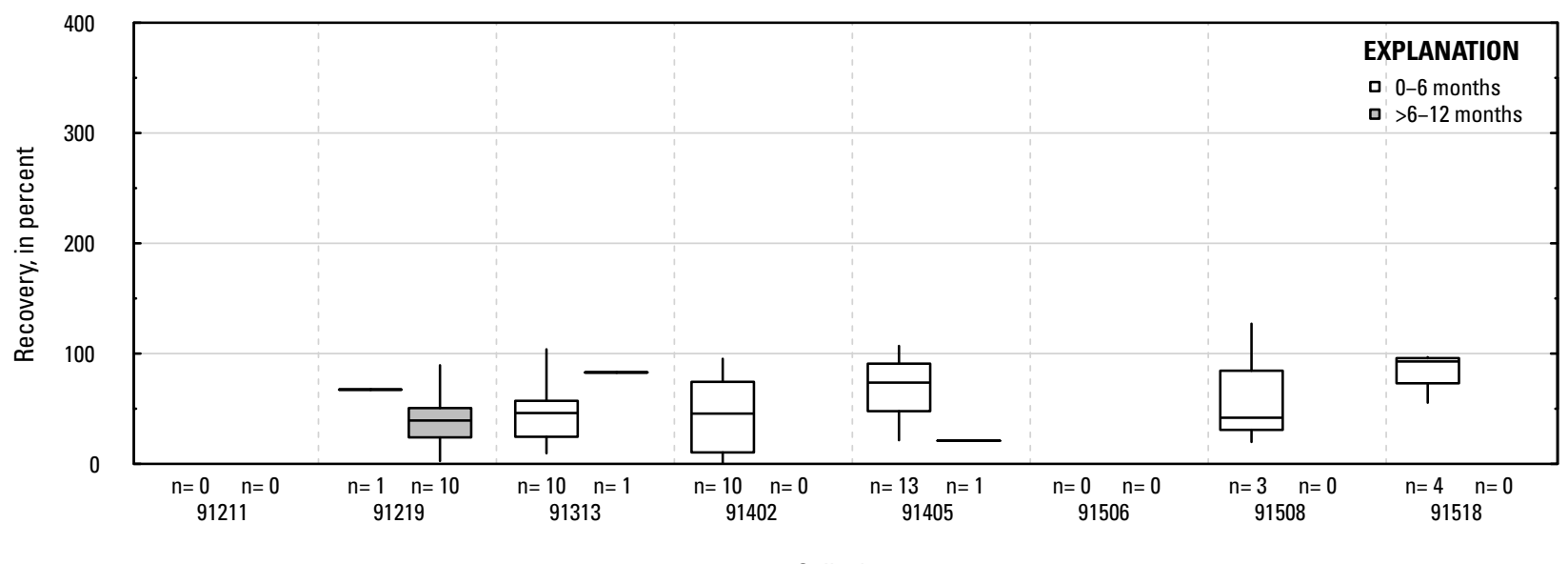

Spike lot

\section{MJ. Hydroxy monodemethyl fluometuron: surface water field matrix spikes}

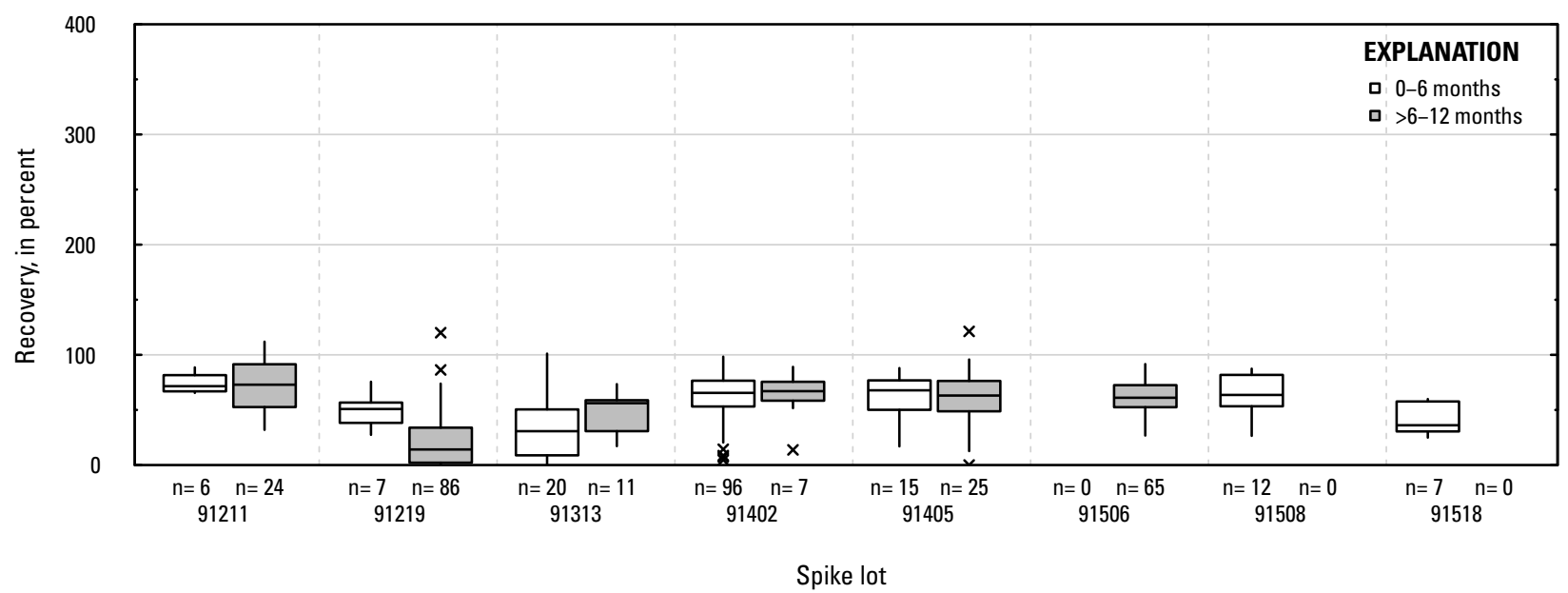

Figure 1-1. Distributions of recovery for individual pesticides in schedule 2437 by matrix, spike lot, and spike lot age. Recovery values larger than 400 percent are not shown.-Continued 
MK. Hydroxyacetochlor: laboratory reagent spikes

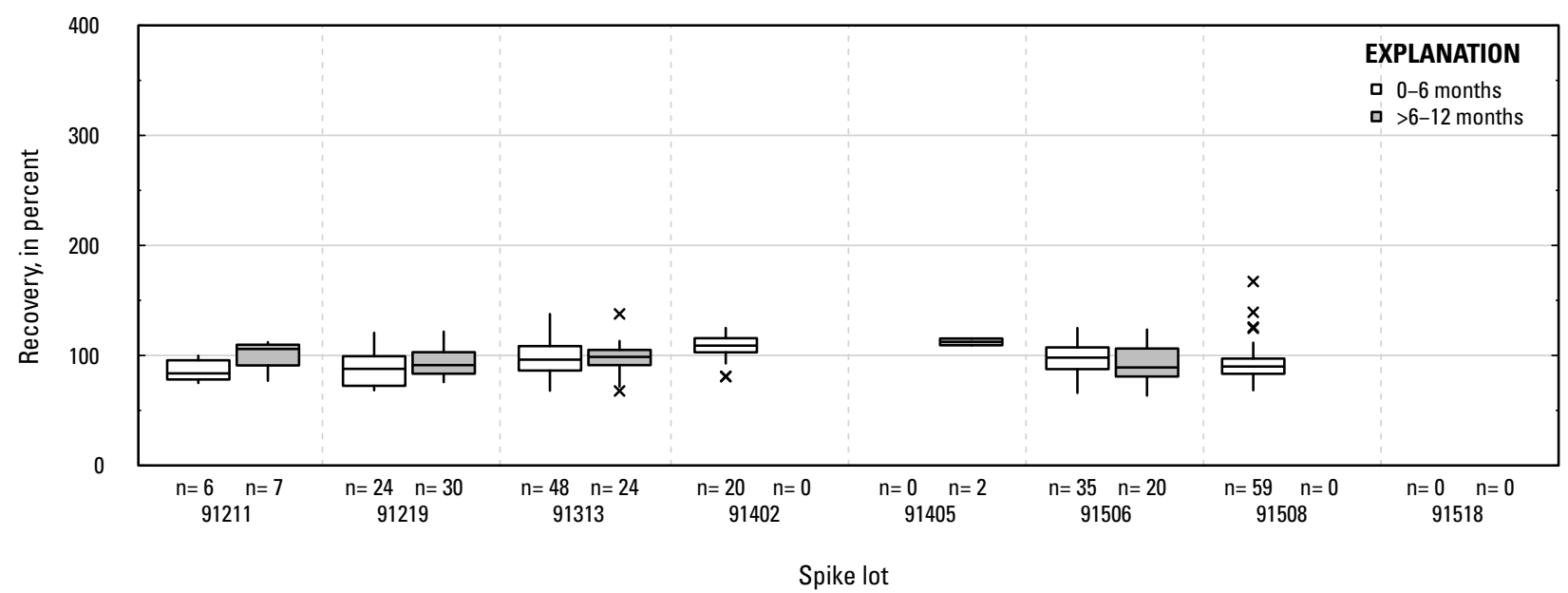

ML. Hydroxyacetochlor: groundwater field matrix spikes

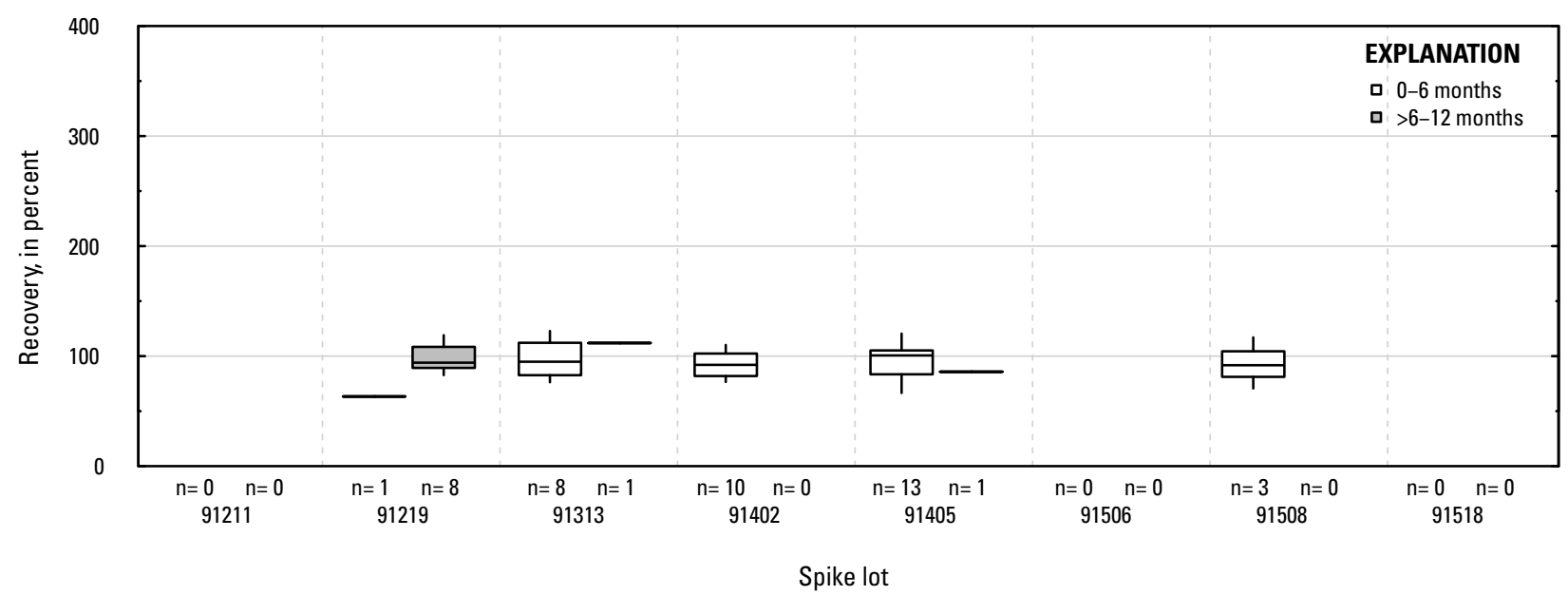

MM. Hydroxyacetochlor: surface water field matrix spikes

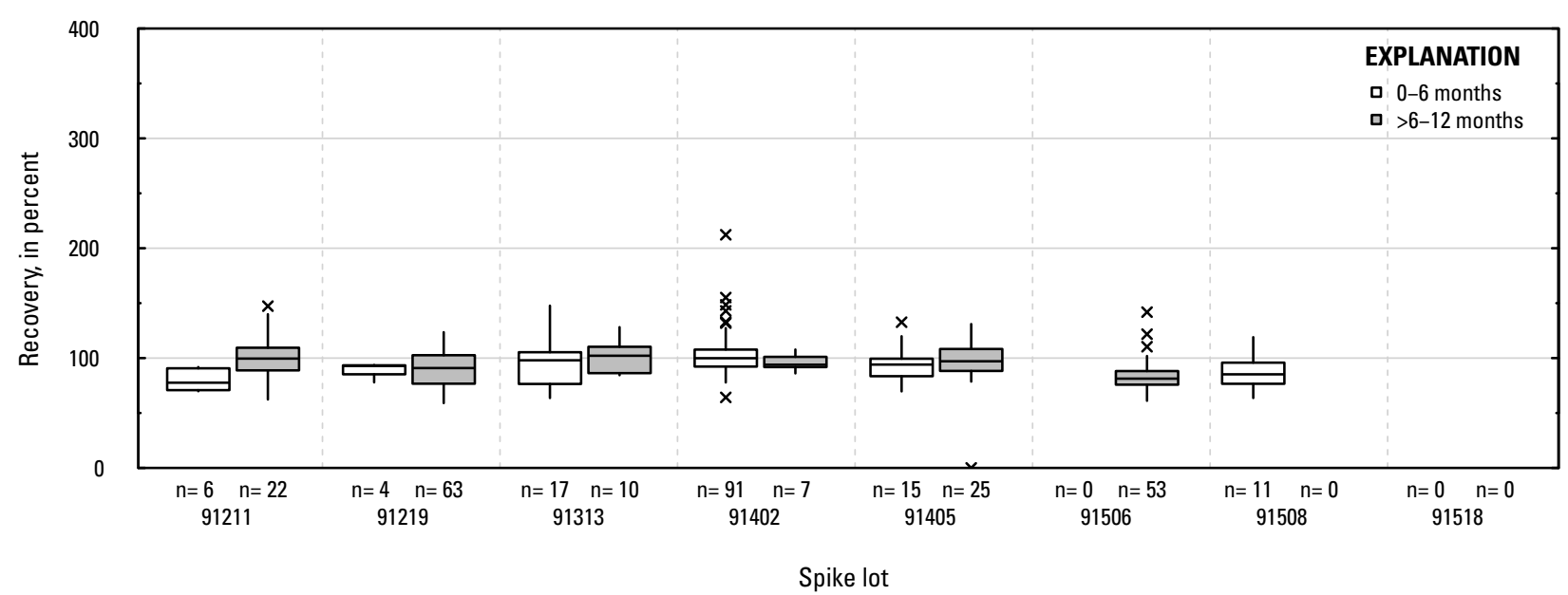

Figure 1-1. Distributions of recovery for individual pesticides in schedule 2437 by matrix, spike lot, and spike lot age. Recovery values larger than 400 percent are not shown.-Continued 
MN. Hydroxyalachlor: laboratory reagent spikes

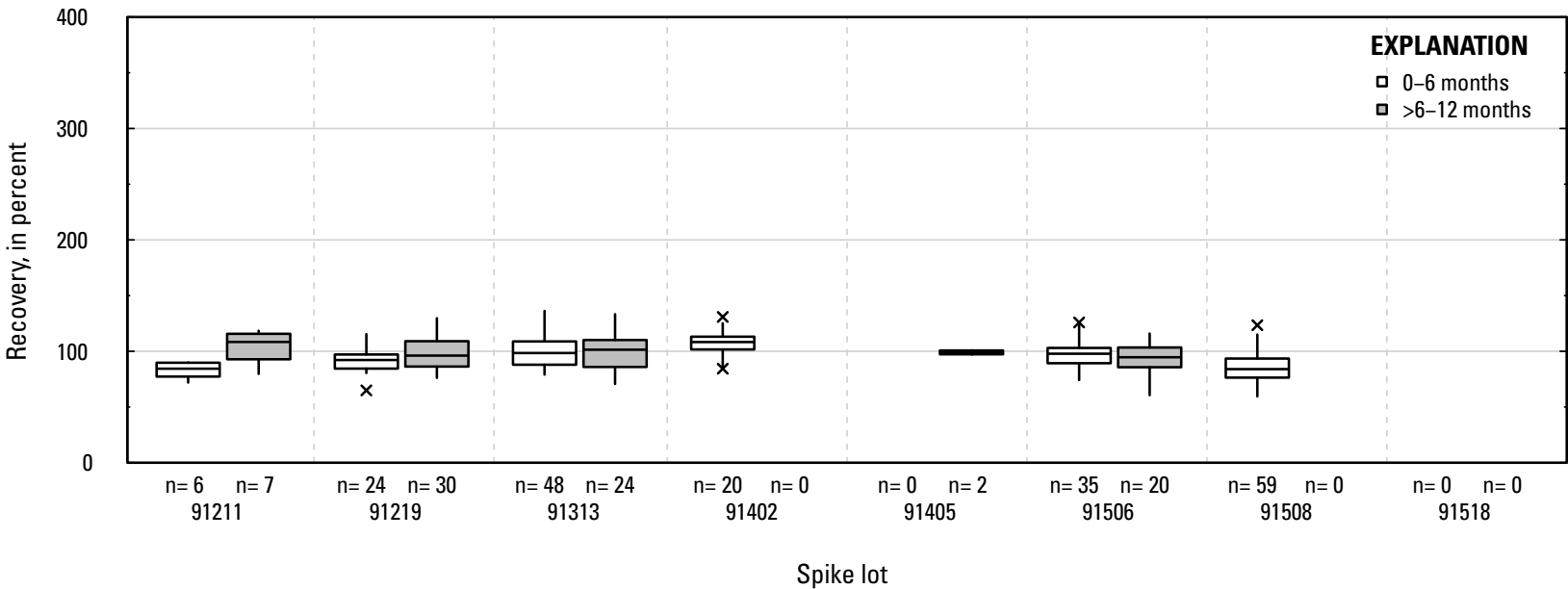

MO. Hydroxyalachlor: groundwater field matrix spikes

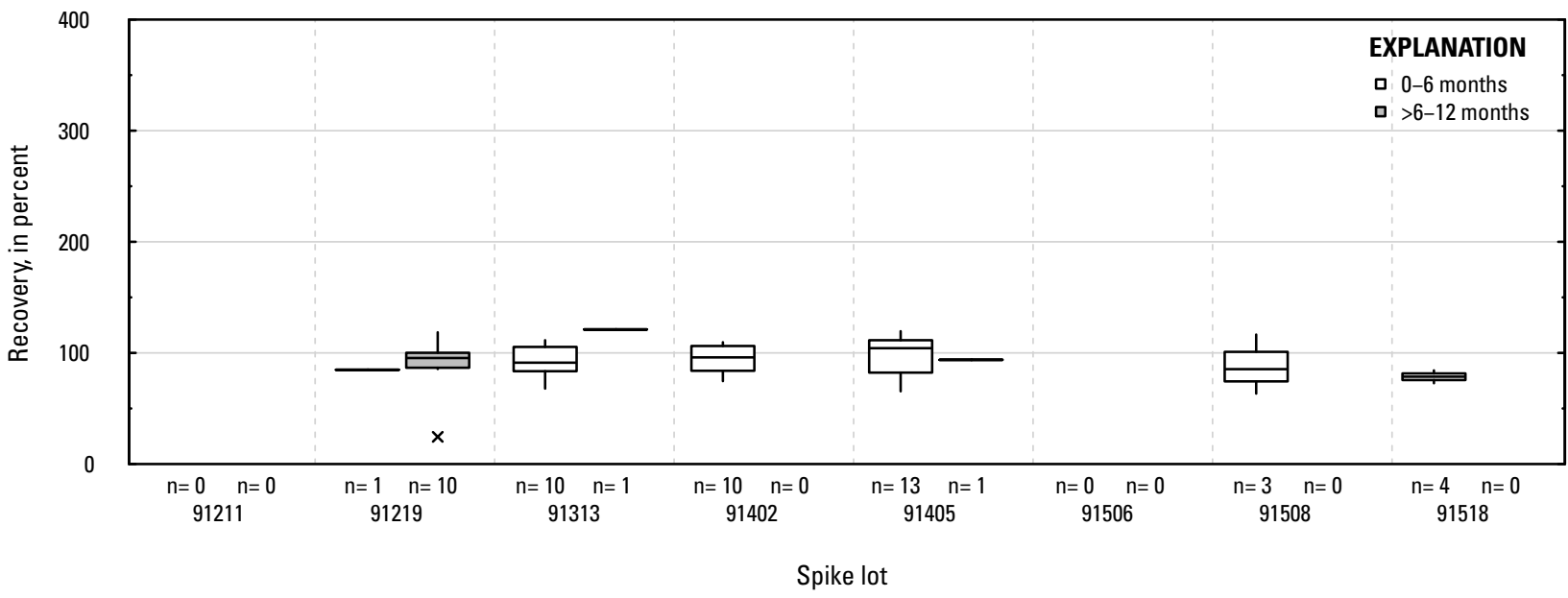

MP. Hydroxyalachlor: surface water field matrix spikes

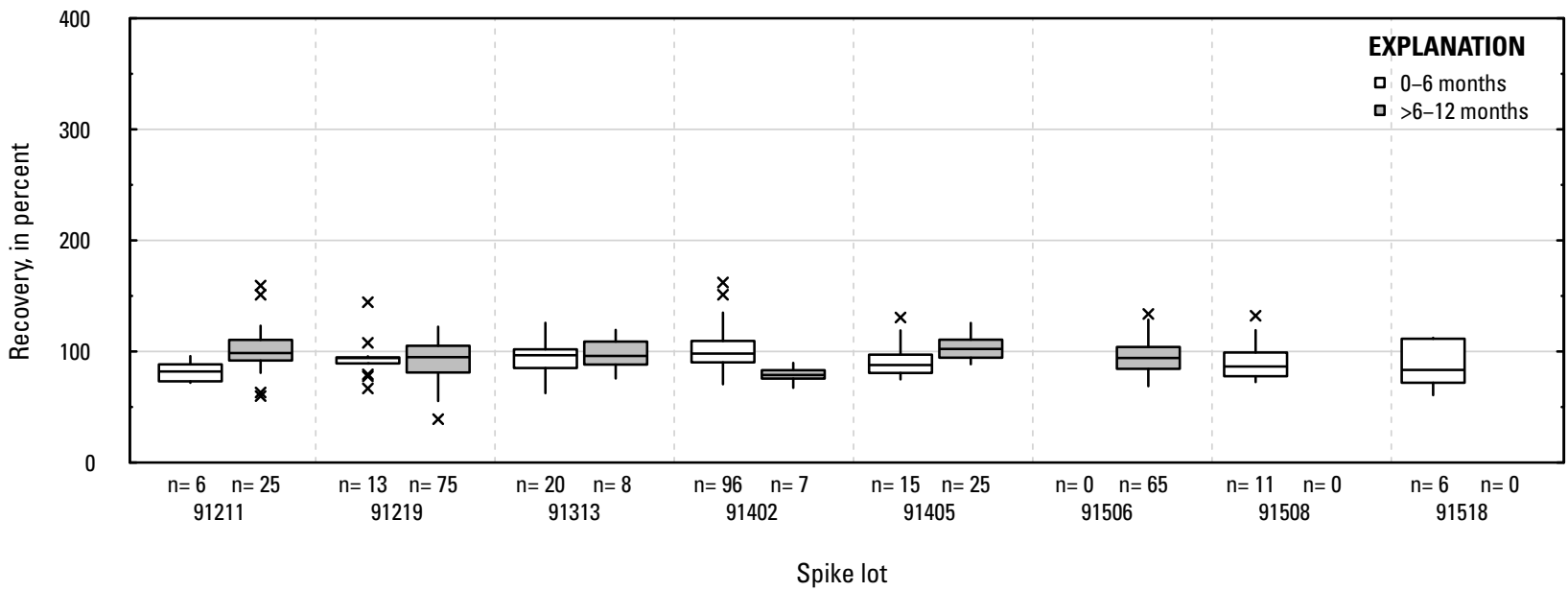

Figure 1-1. Distributions of recovery for individual pesticides in schedule 2437 by matrix, spike lot, and spike lot age. Recovery values larger than 400 percent are not shown.-Continued 
MO. Hydroxydiazinon: laboratory reagent spikes

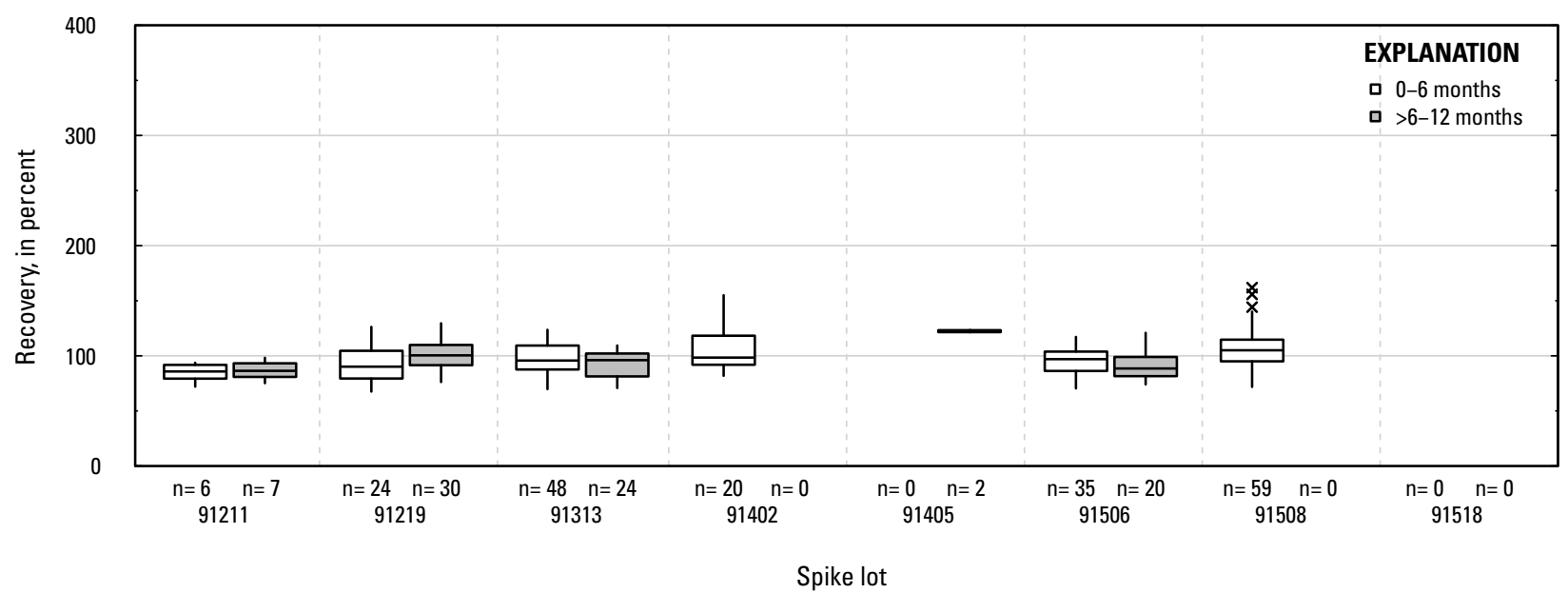

MR. Hydroxydiazinon: groundwater field matrix spikes

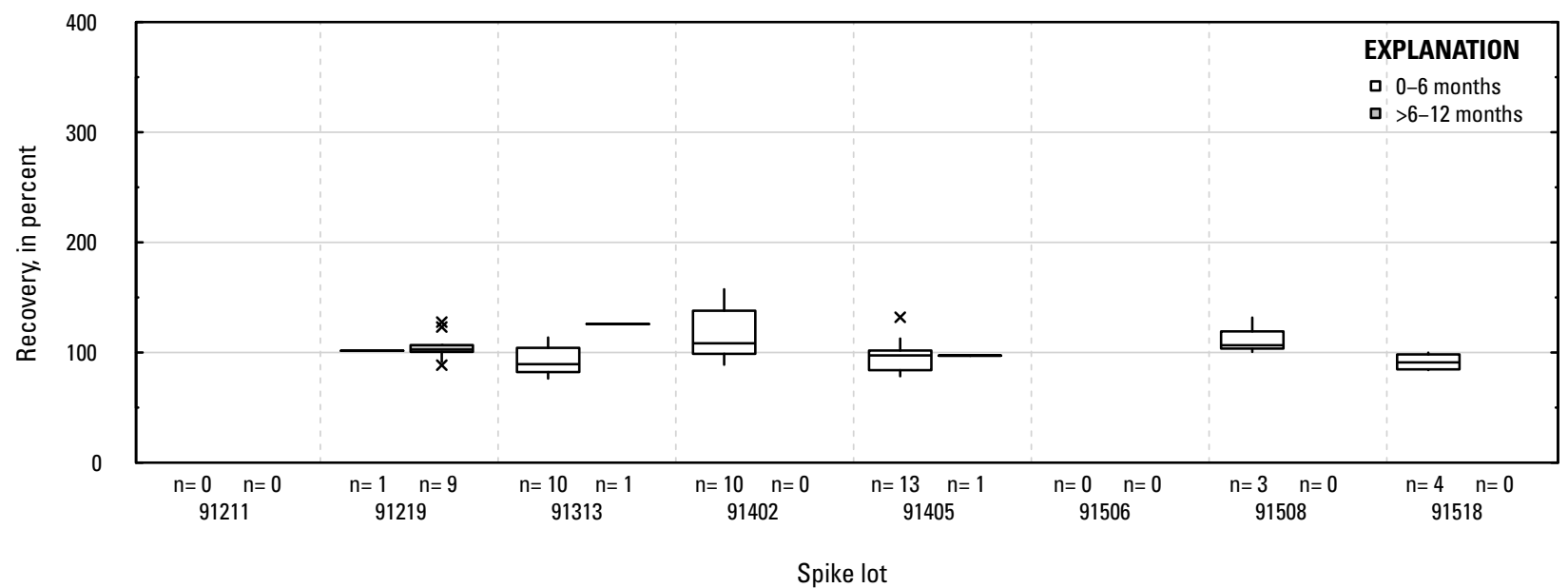

MS. Hydroxydiazinon: surface water field matrix spikes

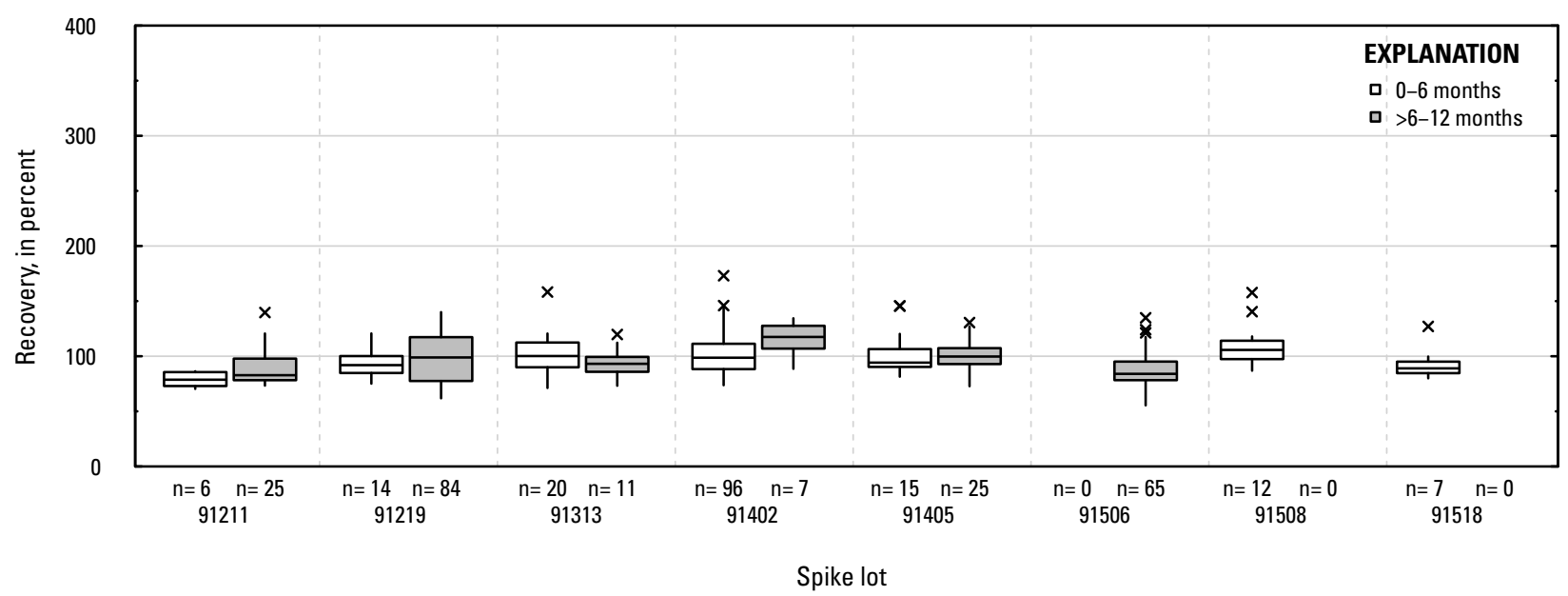

Figure 1-1. Distributions of recovery for individual pesticides in schedule 2437 by matrix, spike lot, and spike lot age. Recovery values larger than 400 percent are not shown.-Continued 
MT. Hydroxyfluometuron: laboratory reagent spikes

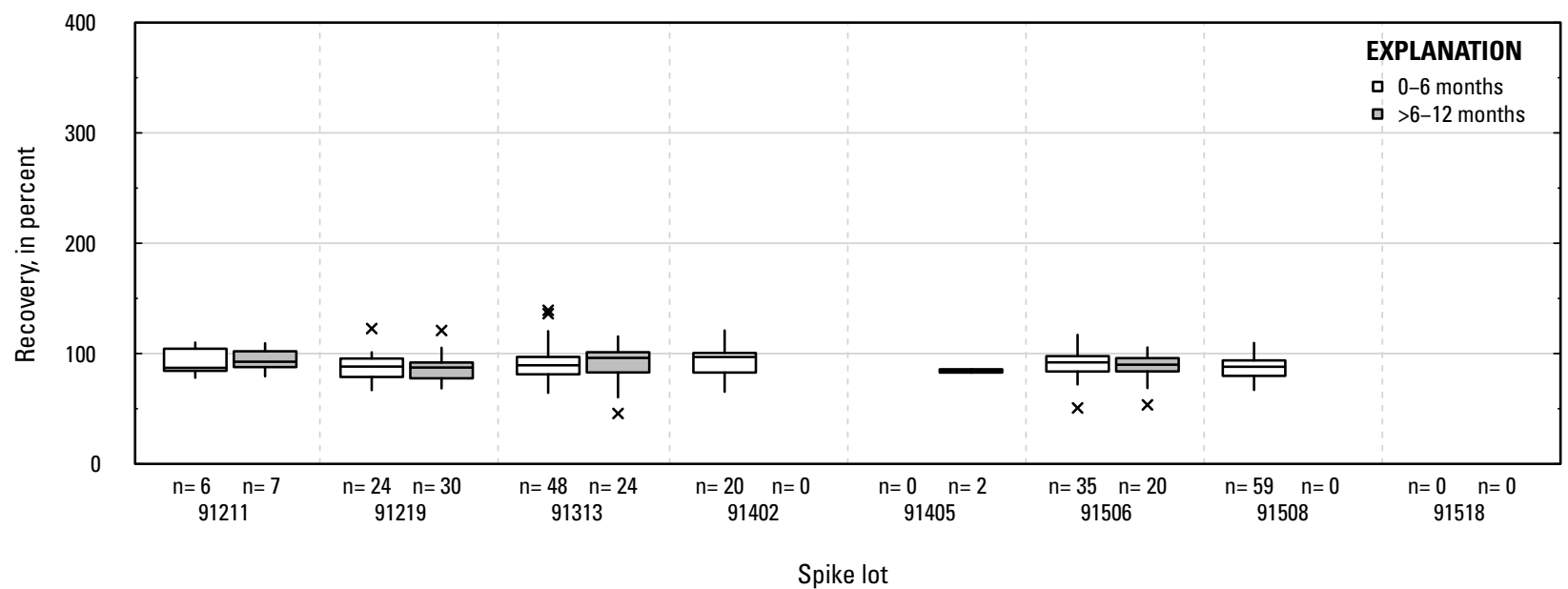

MU. Hydroxyfluometuron: groundwater field matrix spikes

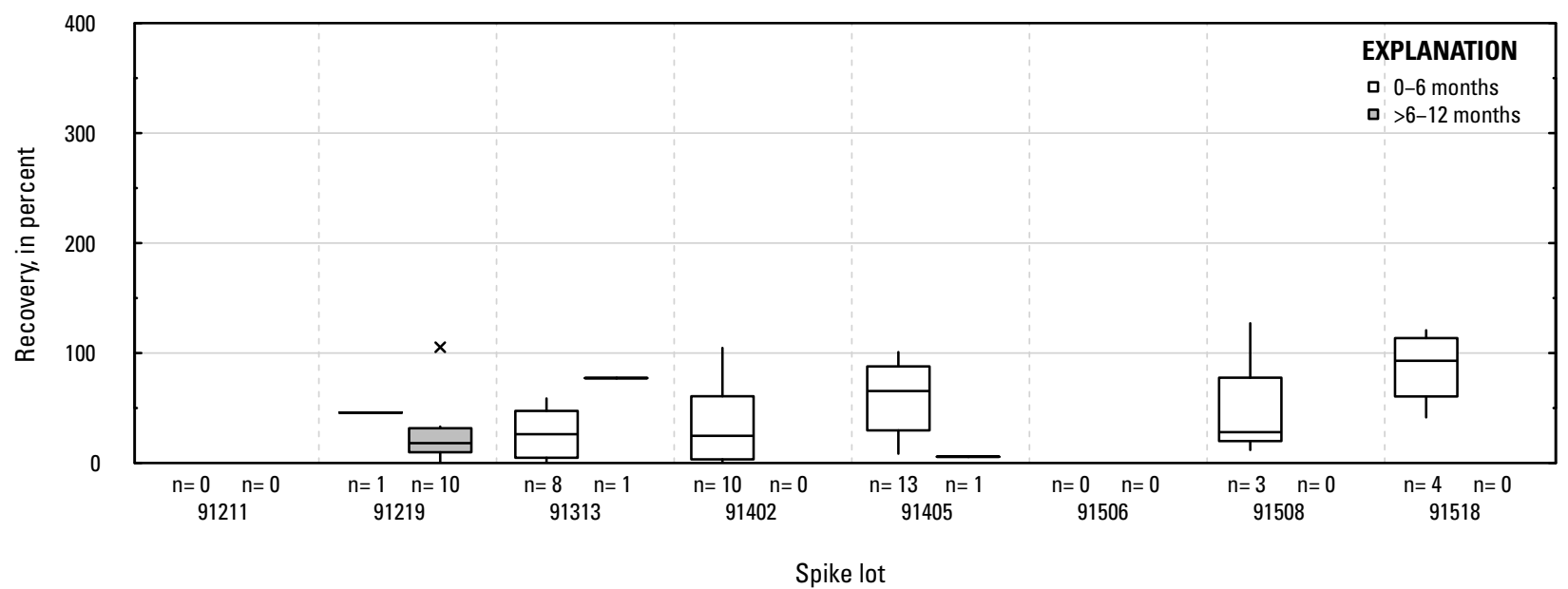

MV. Hydroxyfluometuron: surface water field matrix spikes

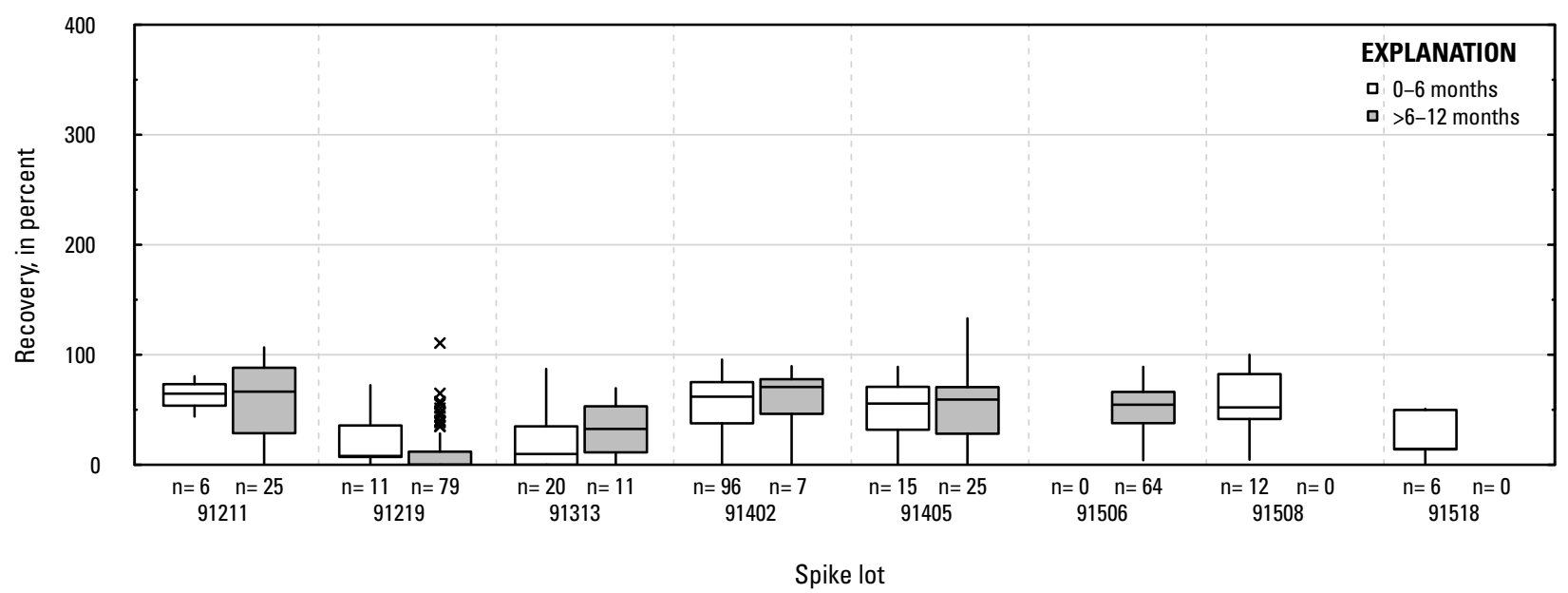

Figure 1-1. Distributions of recovery for individual pesticides in schedule 2437 by matrix, spike lot, and spike lot age. Recovery values larger than 400 percent are not shown.-Continued 
MW. Hydroxymetolachlor: laboratory reagent spikes

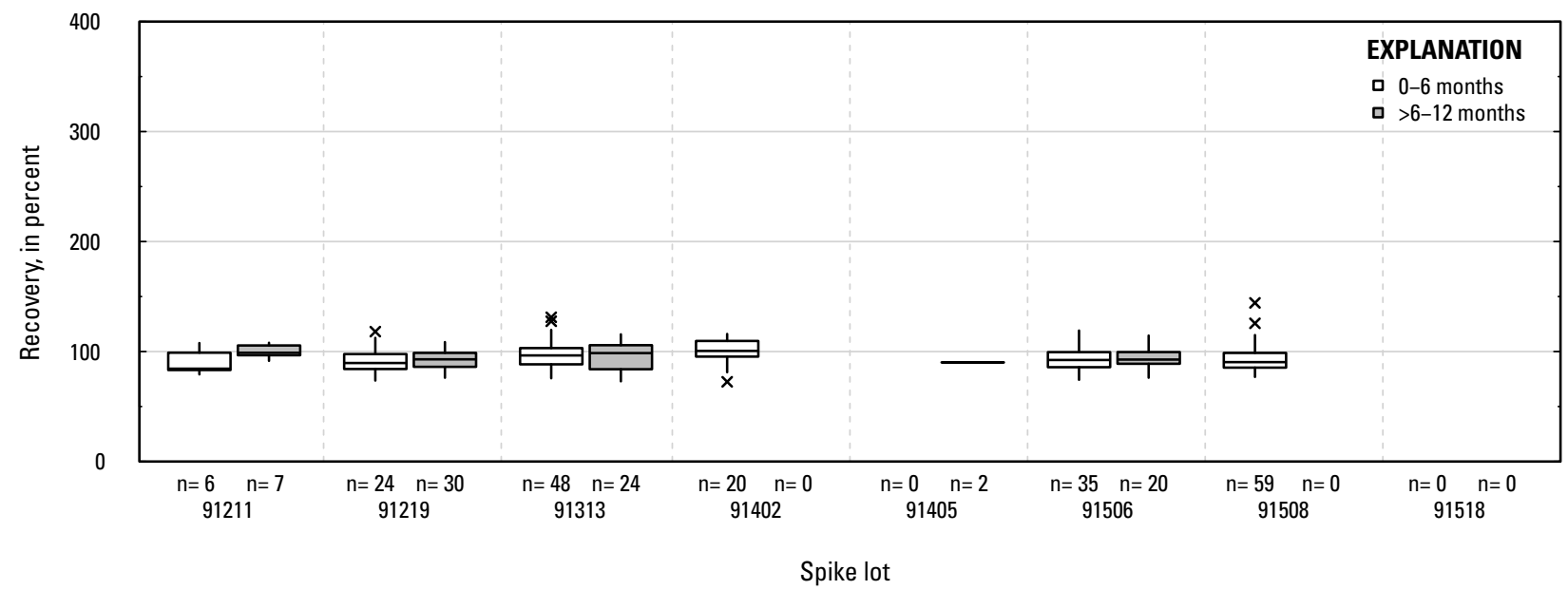

MX. Hydroxymetolachlor: groundwater field matrix spikes

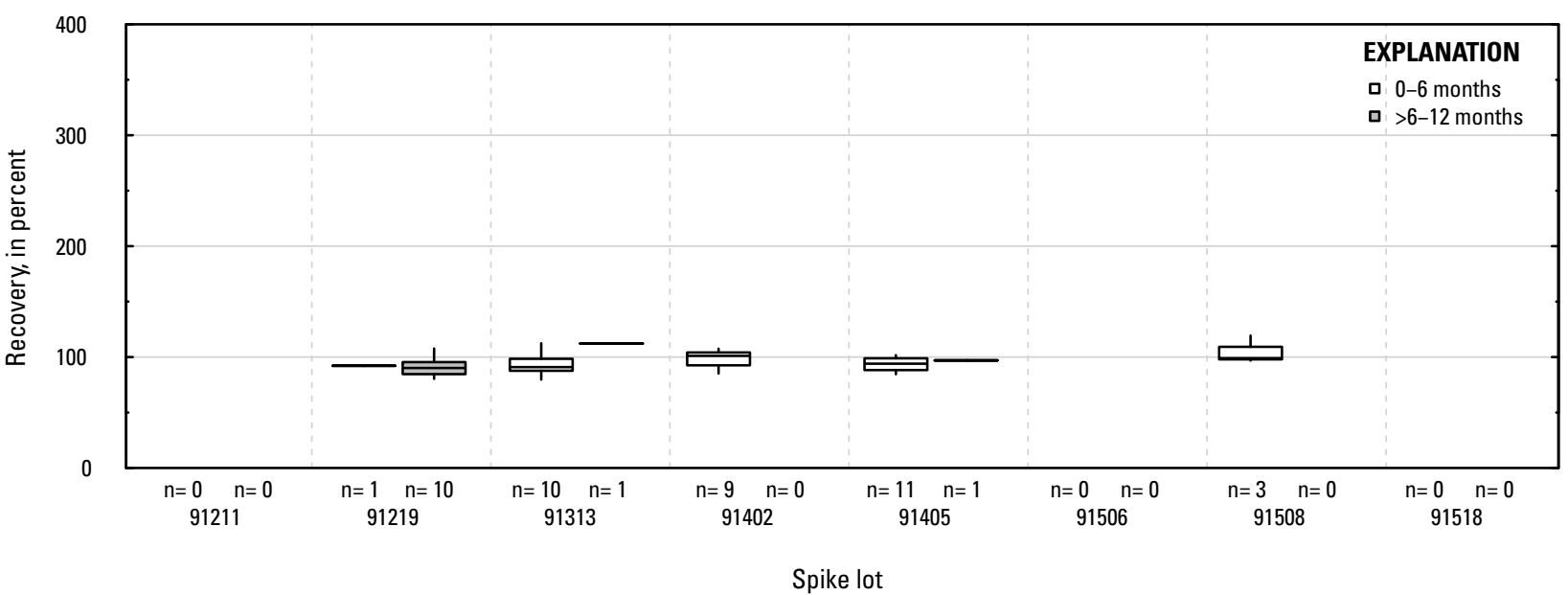

MY. Hydroxymetolachlor: surface water field matrix spikes

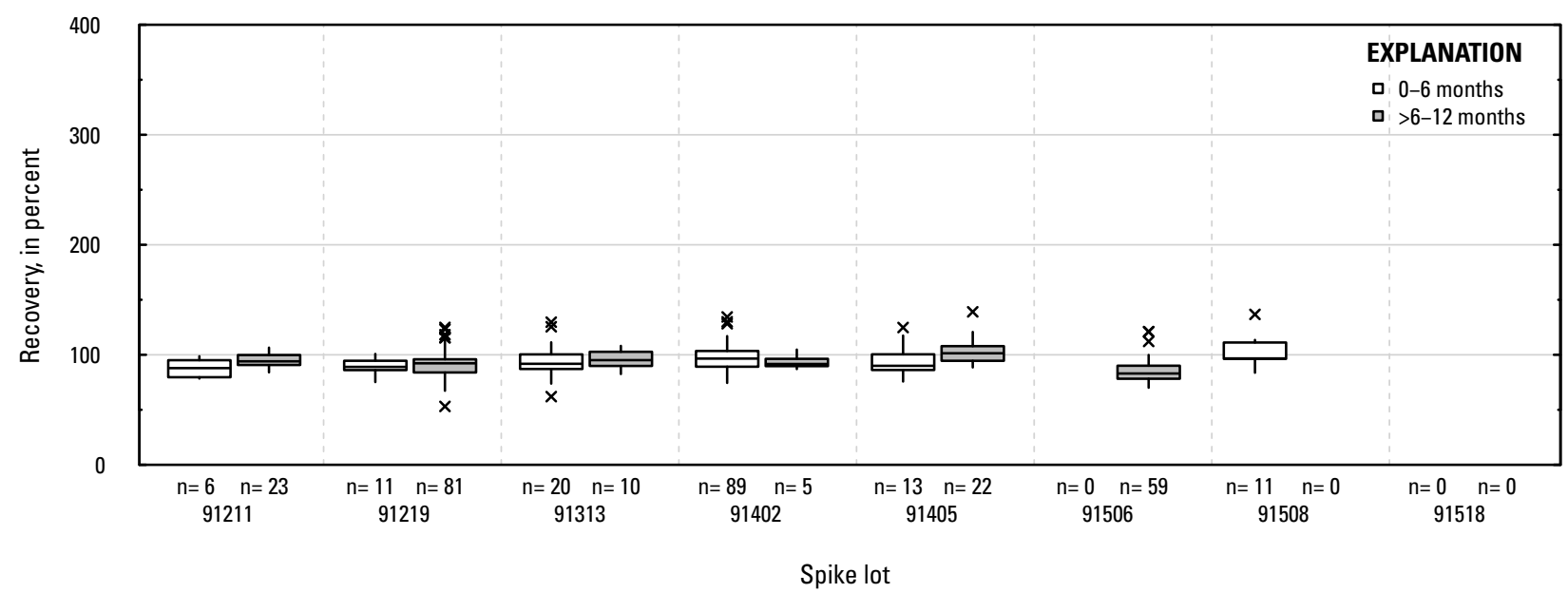

Figure 1-1. Distributions of recovery for individual pesticides in schedule 2437 by matrix, spike lot, and spike lot age. Recovery values larger than 400 percent are not shown.-Continued 
MZ. Hydroxyphthalazinone: laboratory reagent spikes

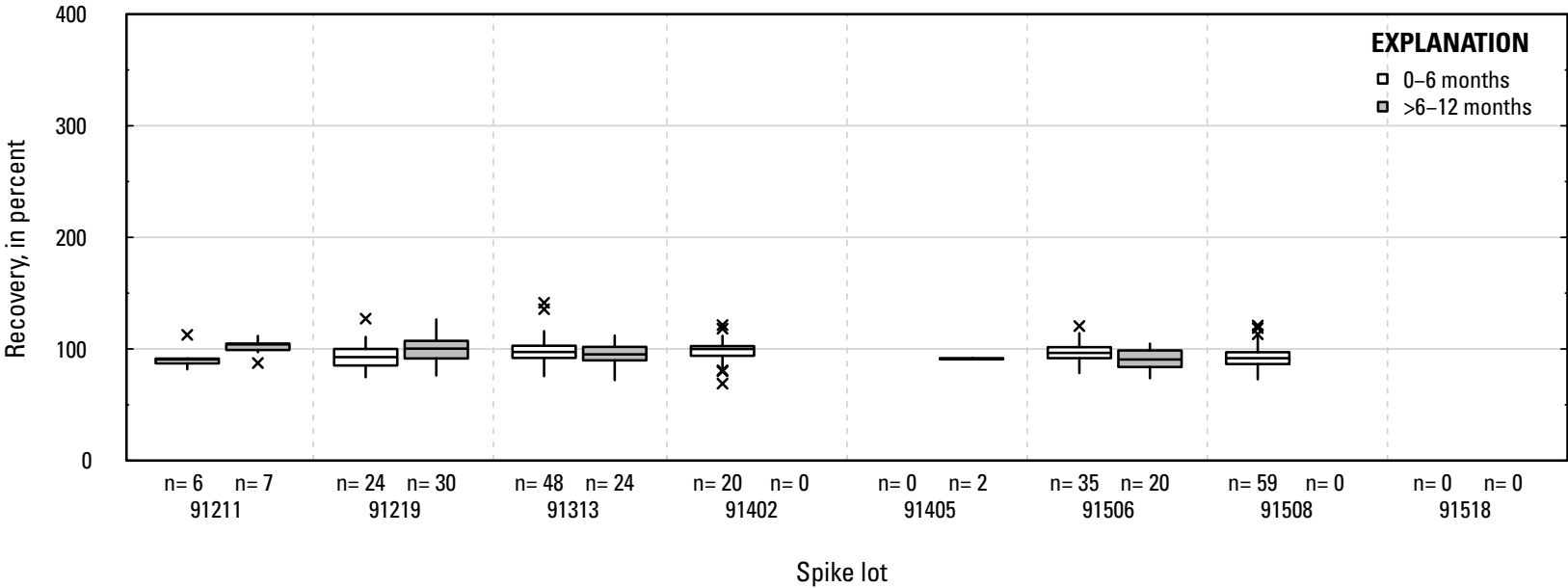

NA. Hydroxyphthalazinone: groundwater field matrix spikes

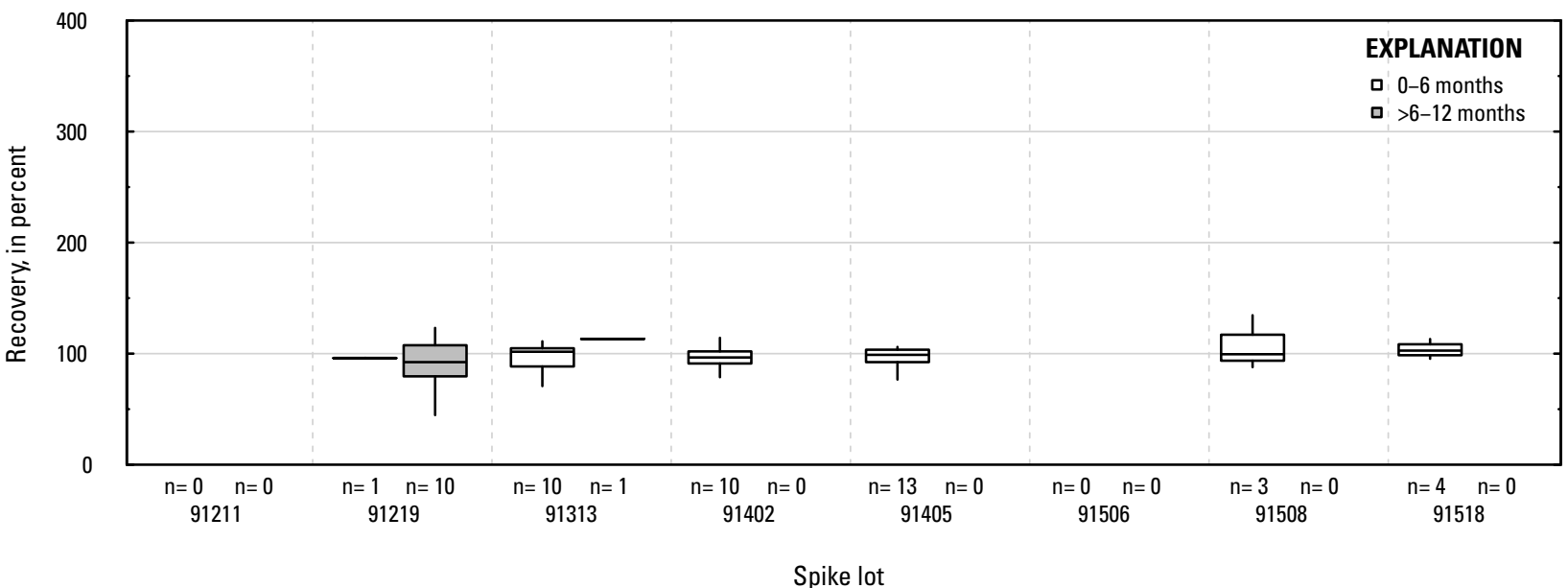

NB. Hydroxyphthalazinone: surface water field matrix spikes

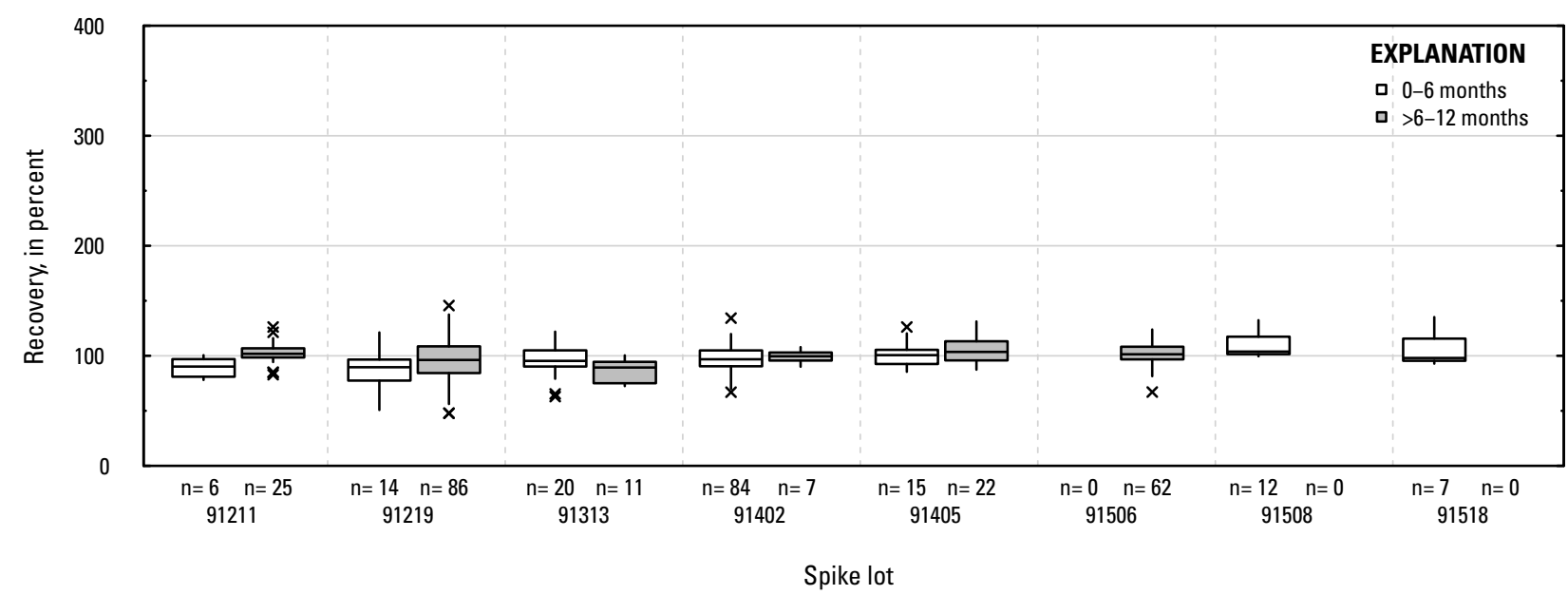

Figure 1-1. Distributions of recovery for individual pesticides in schedule 2437 by matrix, spike lot, and spike lot age. Recovery values larger than 400 percent are not shown.-Continued 
NC. Hydroxysimazine: laboratory reagent spikes

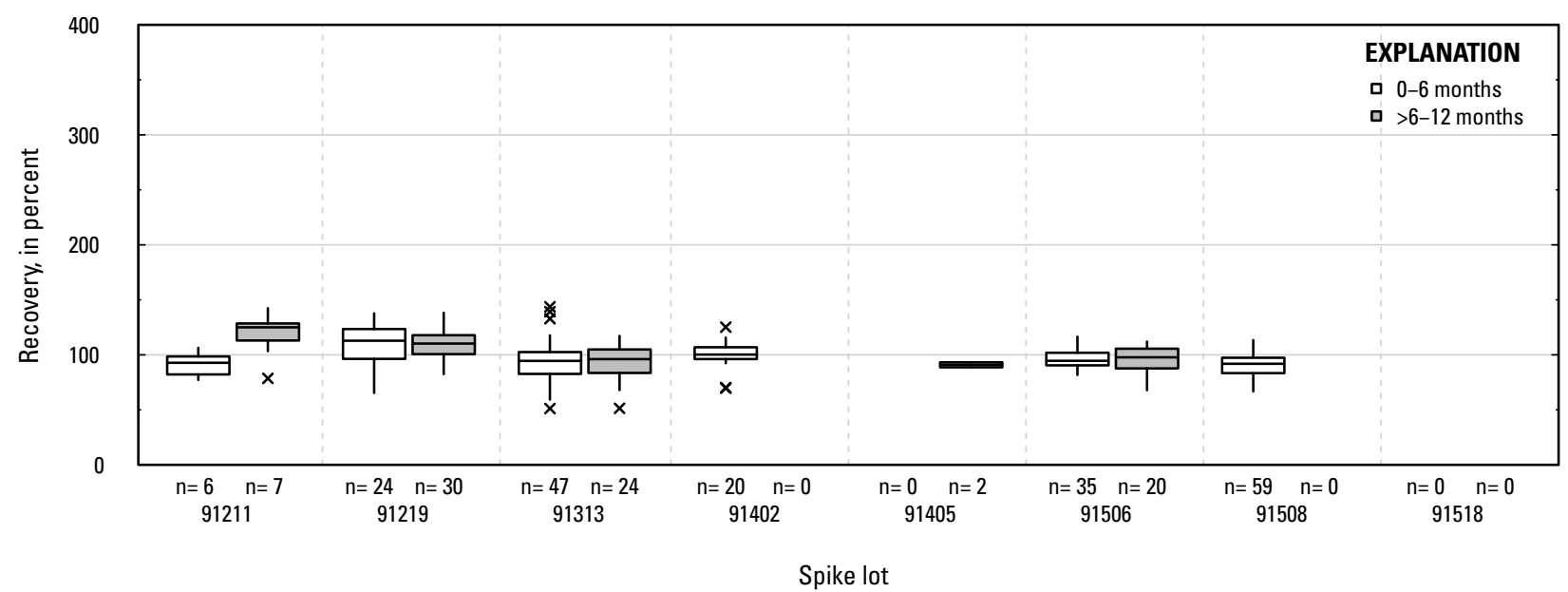

ND. Hydroxysimazine: groundwater field matrix spikes

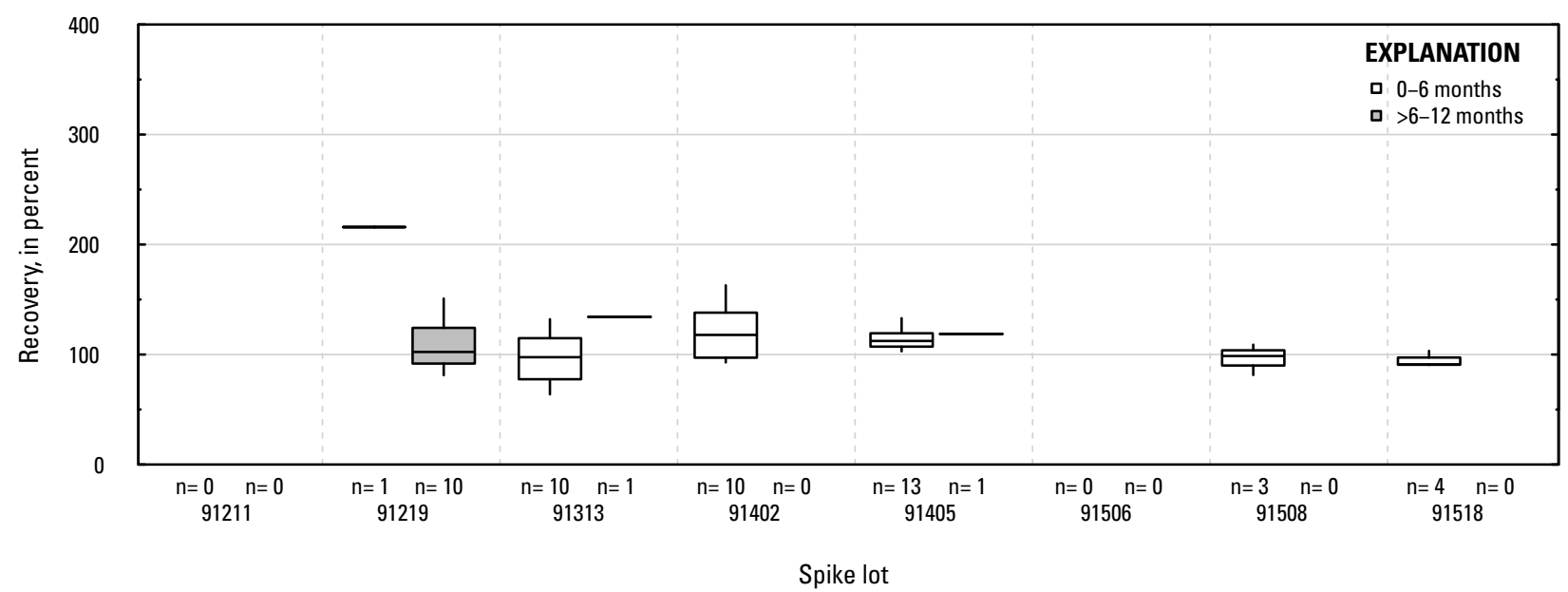

NE. Hydroxysimazine: surface water field matrix spikes

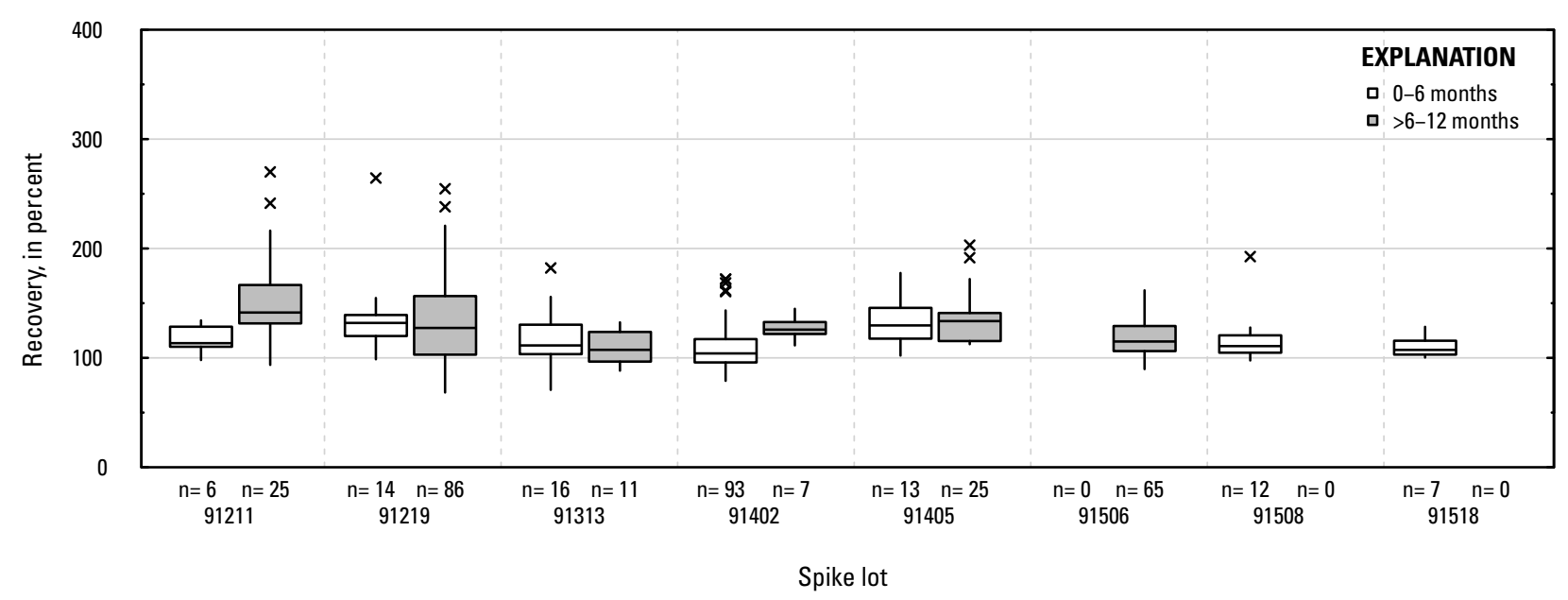

Figure 1-1. Distributions of recovery for individual pesticides in schedule 2437 by matrix, spike lot, and spike lot age. Recovery values larger than 400 percent are not shown.-Continued 
NF. Tebuthiuron TP 109: laboratory reagent spikes

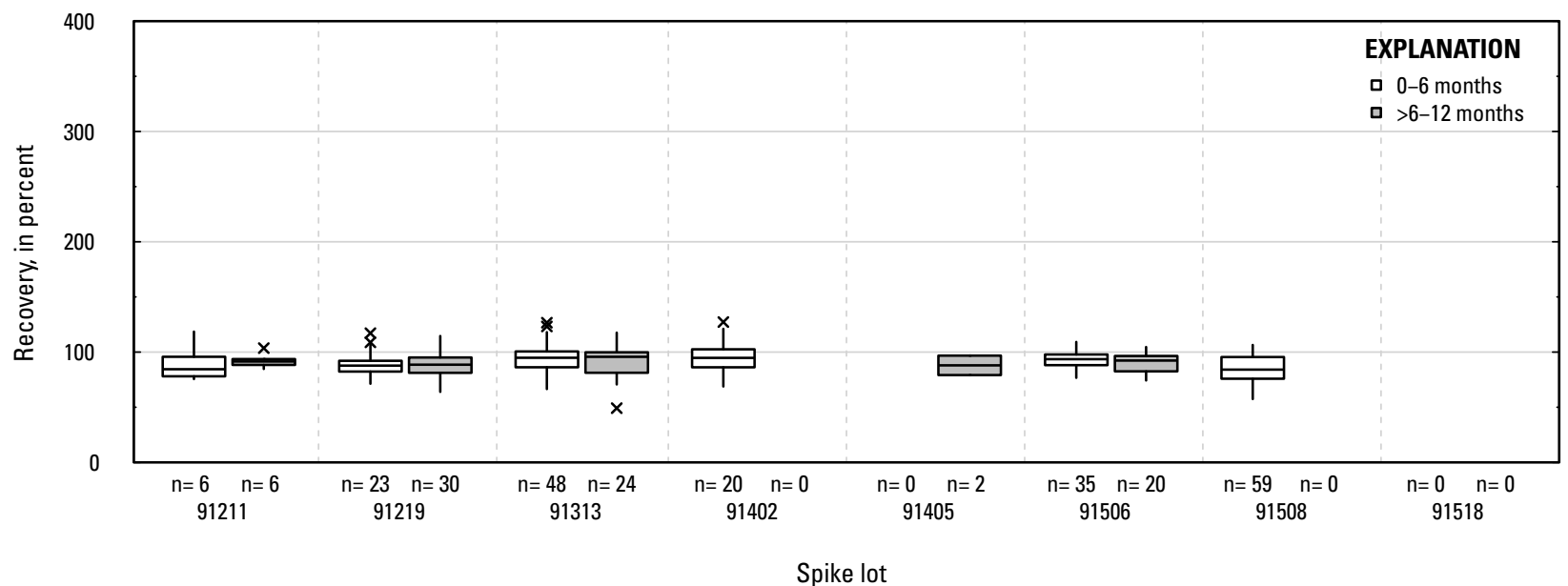

NG. Tebuthiuron TP 109: groundwater field matrix spikes

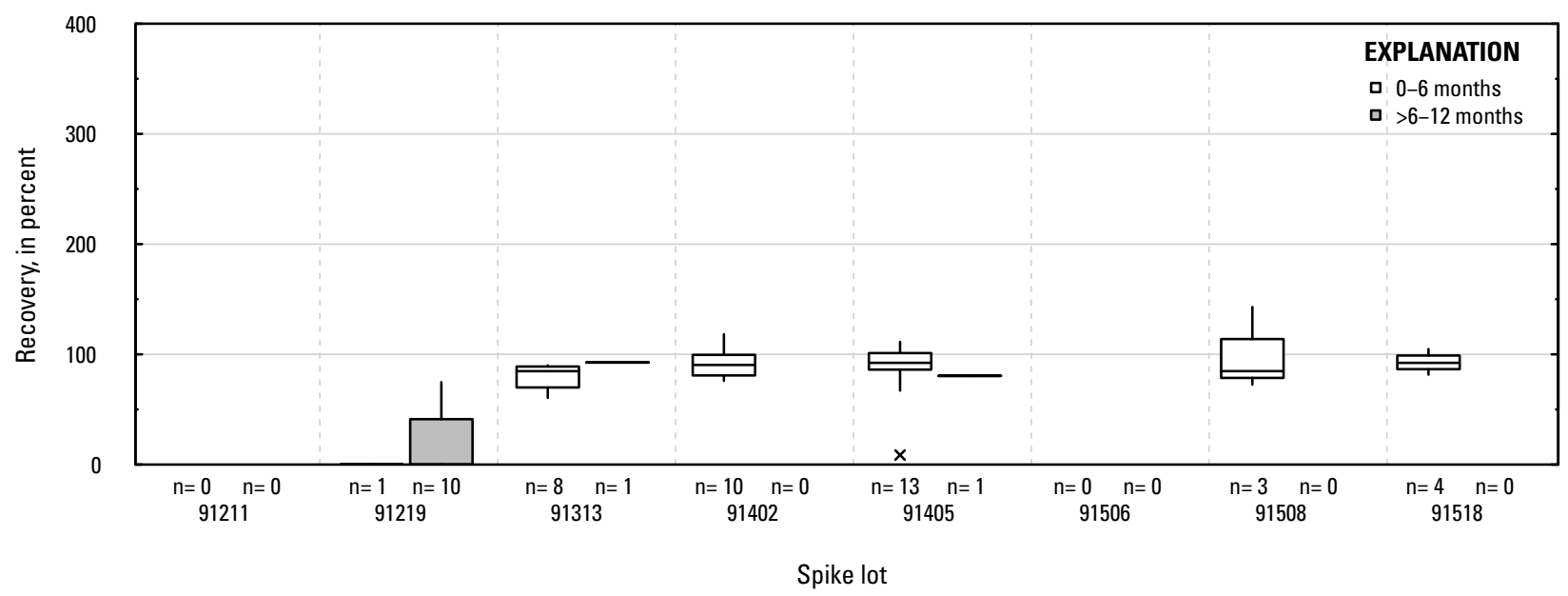

NH. Tebuthiuron TP 109: surface water field matrix spikes

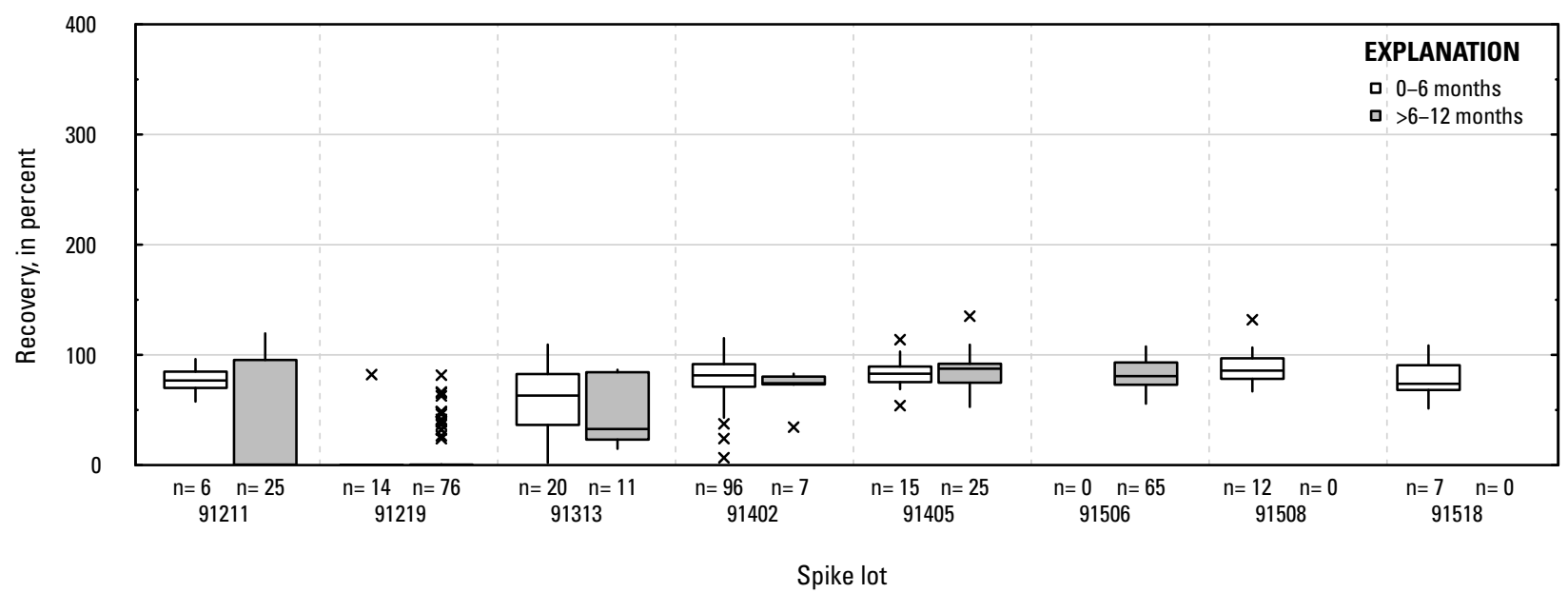

Figure 1-1. Distributions of recovery for individual pesticides in schedule 2437 by matrix, spike lot, and spike lot age. Recovery values larger than 400 percent are not shown.-Continued 


\section{NI. Imazamox: laboratory reagent spikes}

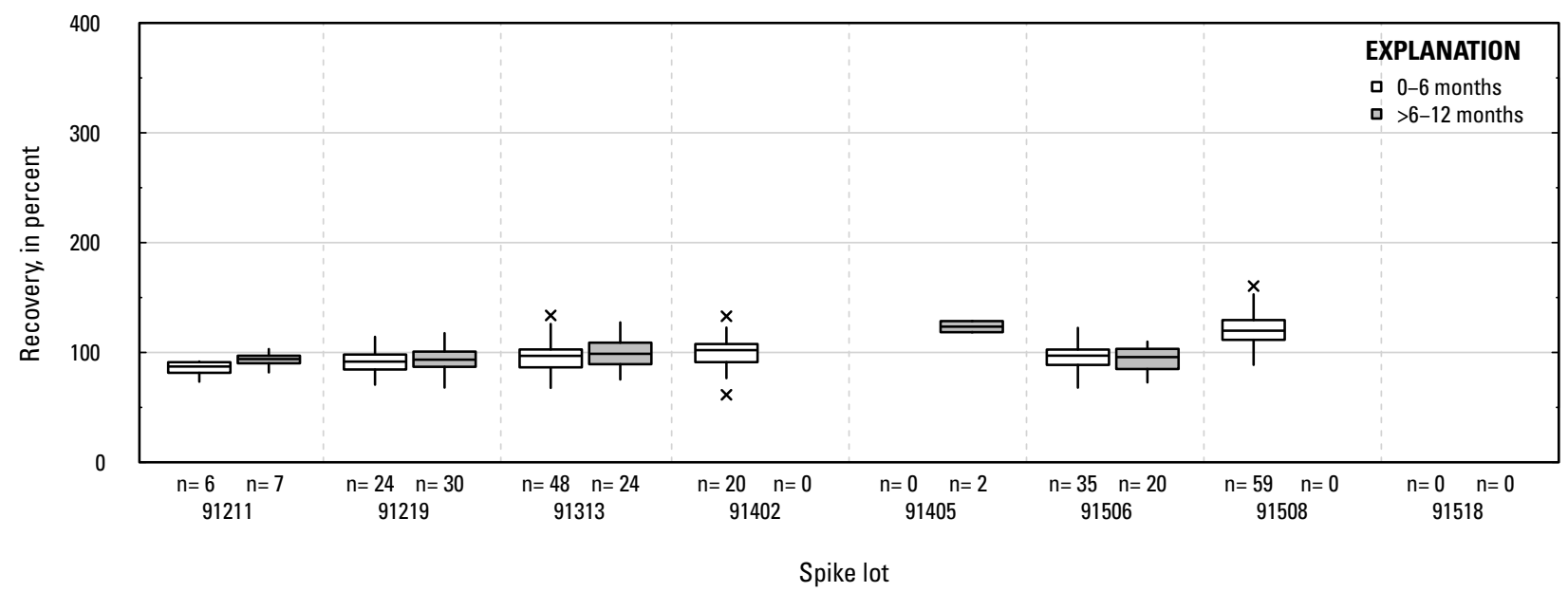

NJ. Imazamox: groundwater field matrix spikes

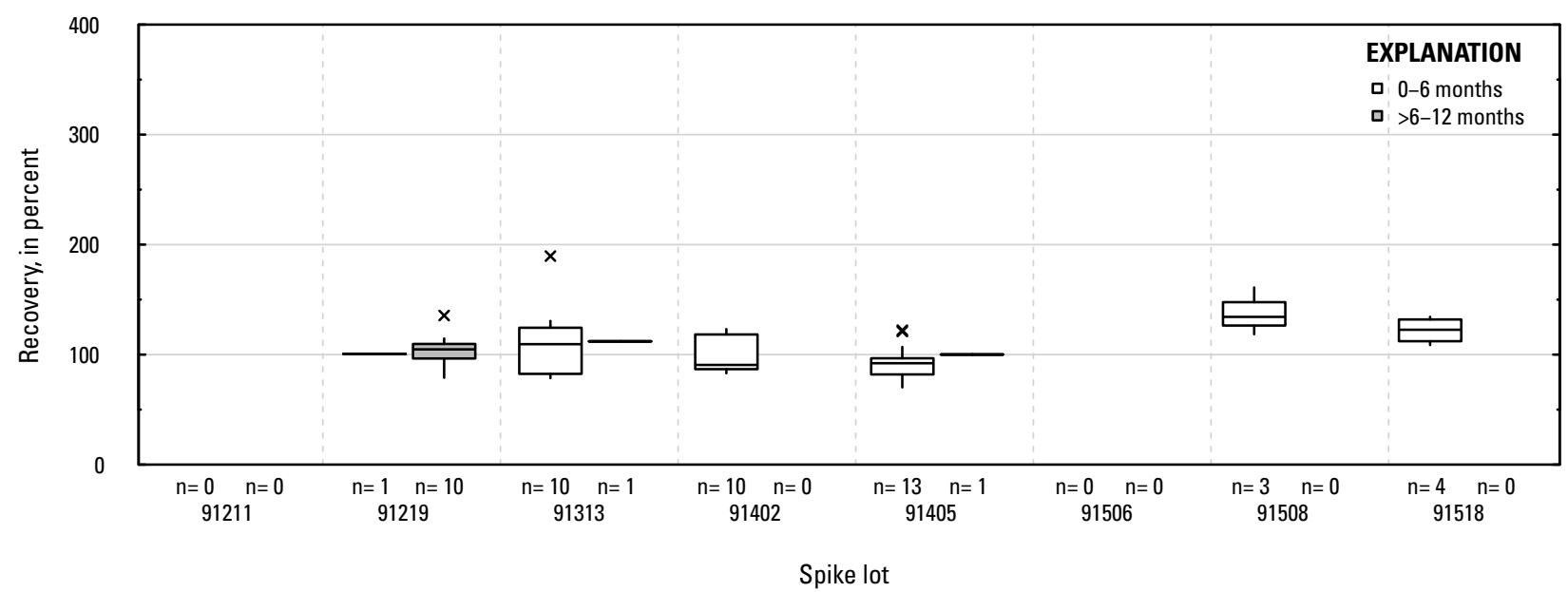

NK. Imazamox: surface water field matrix spikes

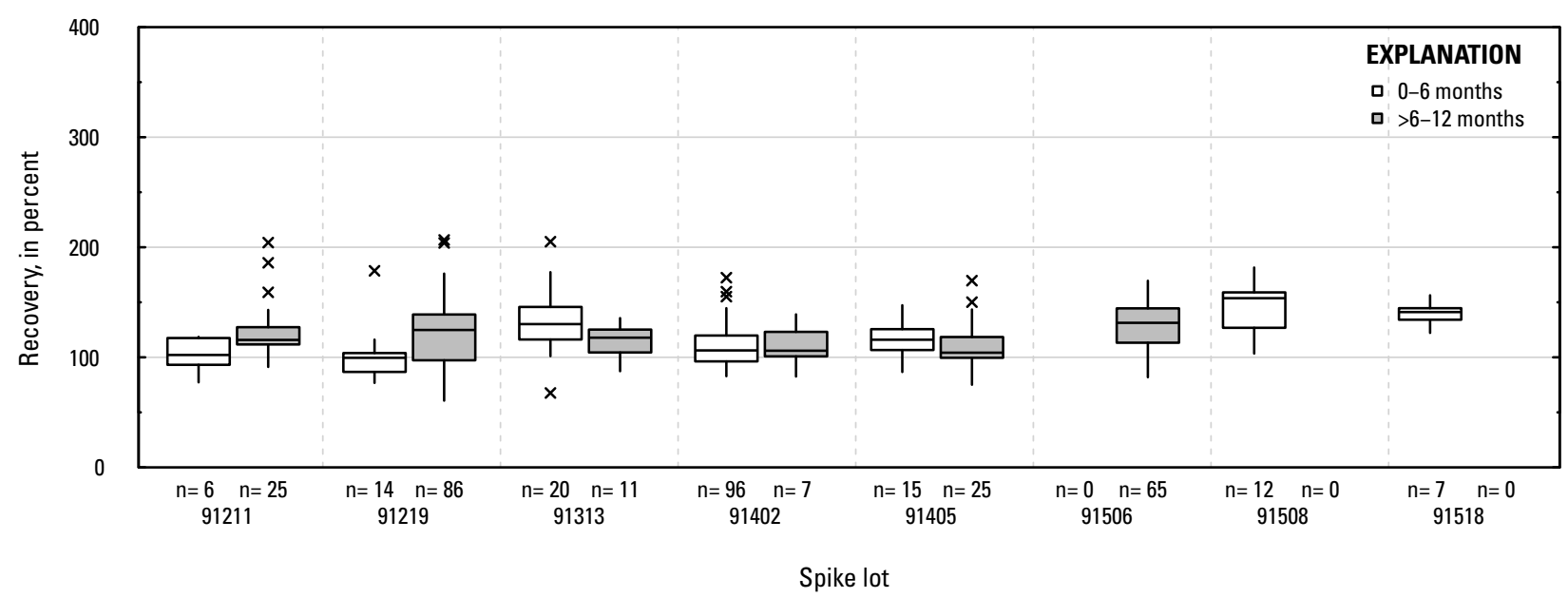

Figure 1-1. Distributions of recovery for individual pesticides in schedule 2437 by matrix, spike lot, and spike lot age. Recovery values larger than 400 percent are not shown.-Continued 
NL. Imazaquin: laboratory reagent spikes

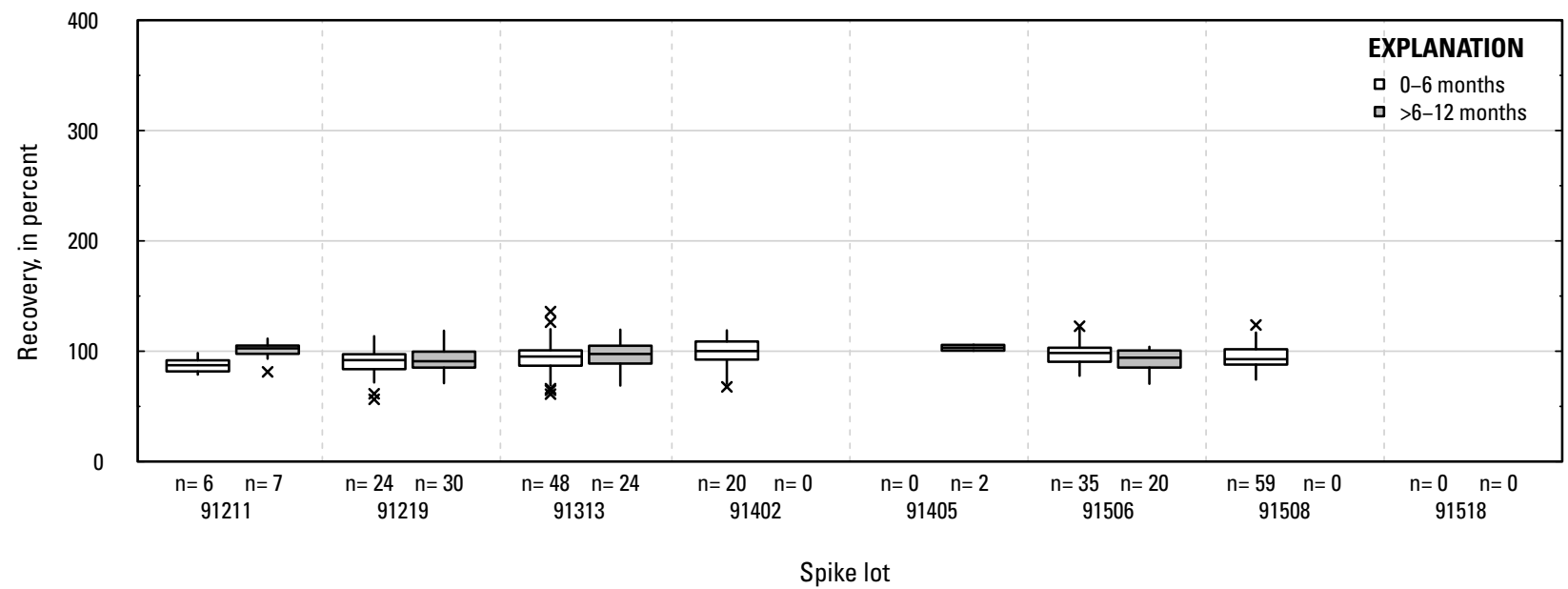

NM. Imazaquirgroundwater field matrix spikes

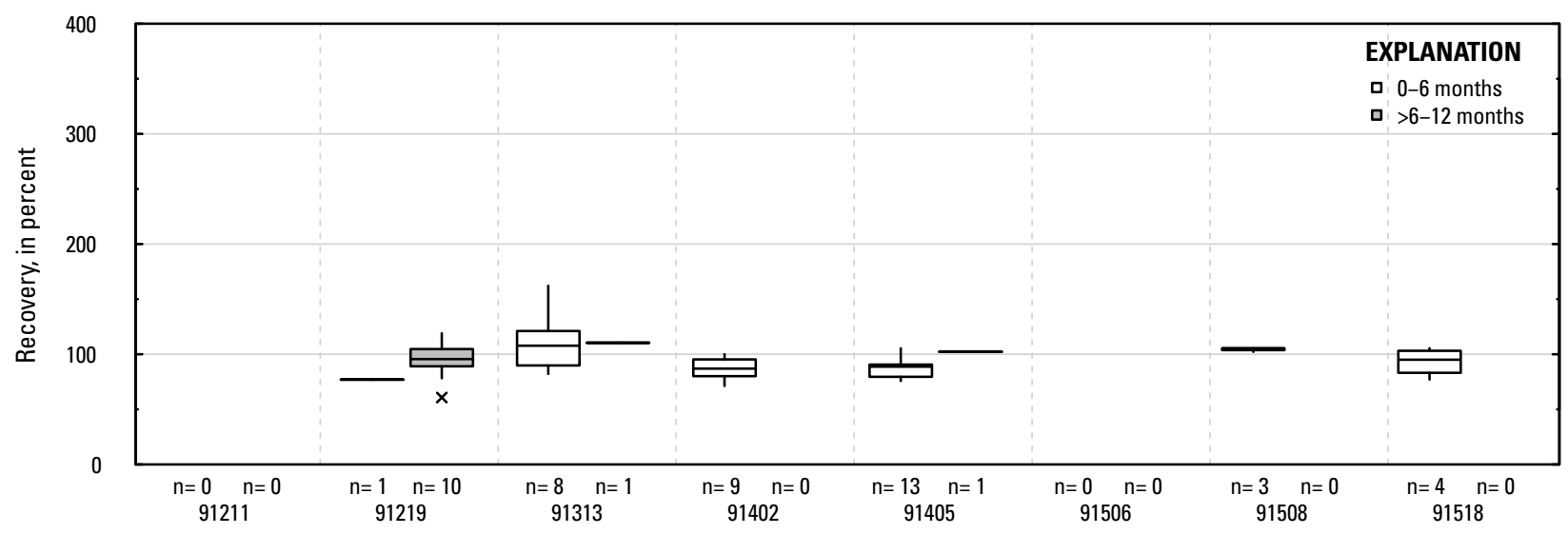

Spike lot

NN. Imazaquin: surface water field matrix spikes

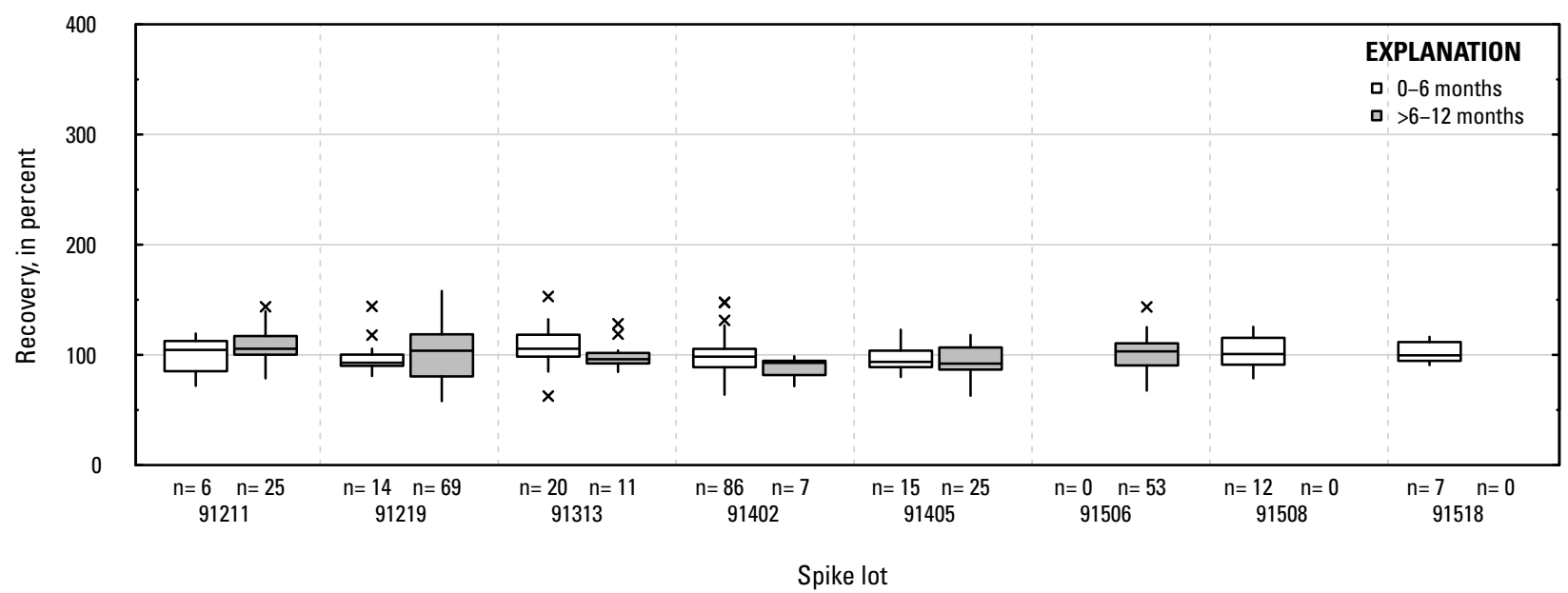

Figure 1-1. Distributions of recovery for individual pesticides in schedule 2437 by matrix, spike lot, and spike lot age. Recovery values larger than 400 percent are not shown.-Continued 
NO. Imazethapyr: laboratory reagent spikes

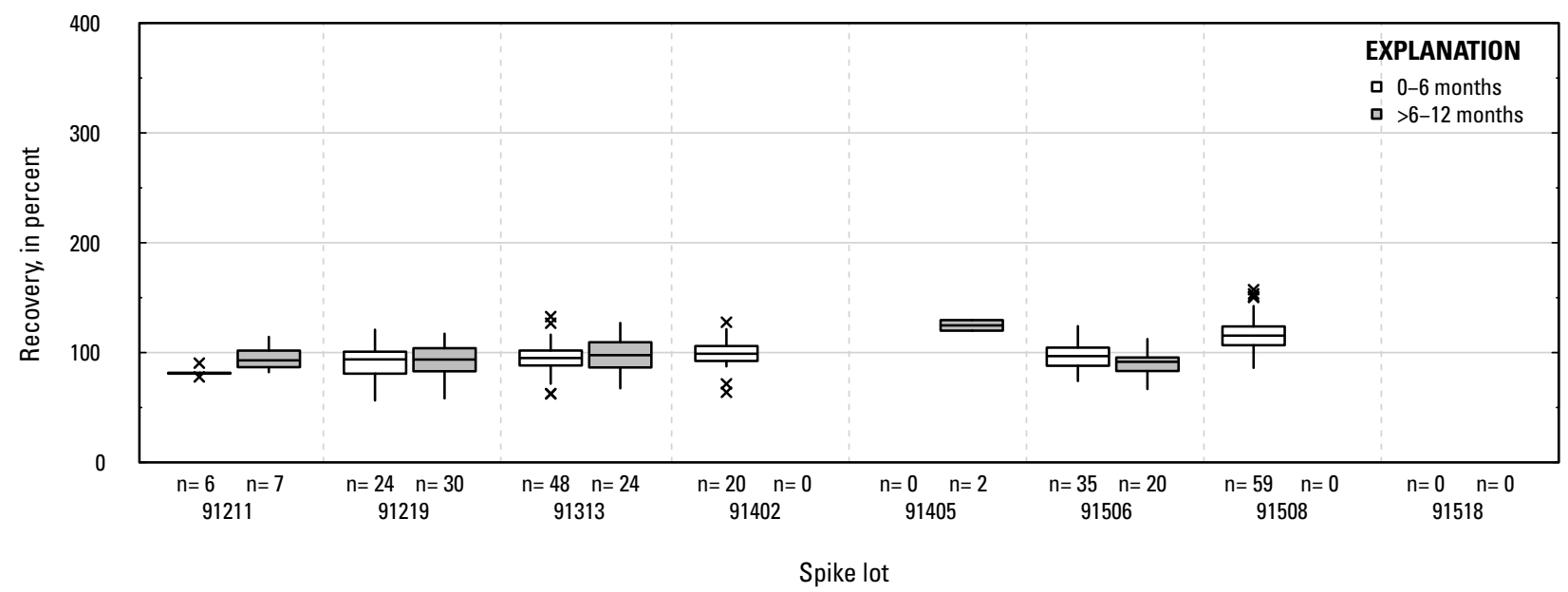

NP. Imazethapyr: groundwater field matrix spikes

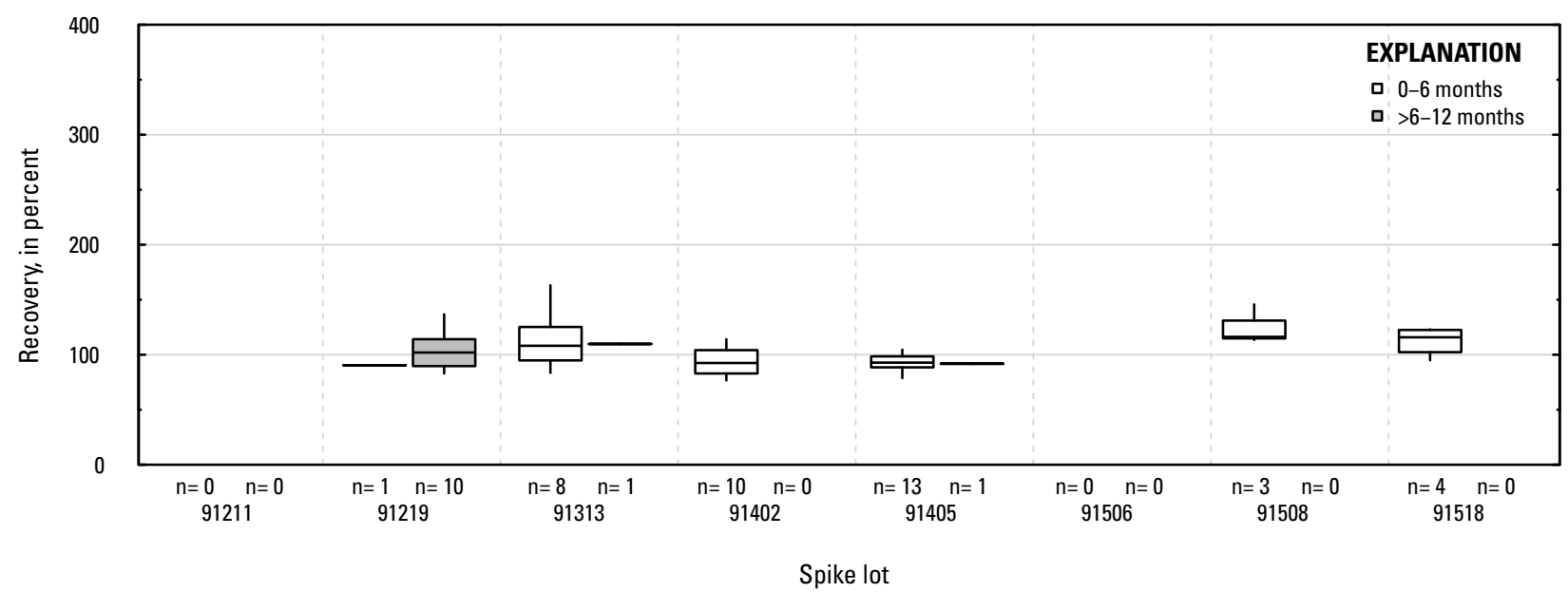

NQ. Imazethapyr: surface water field matrix spikes

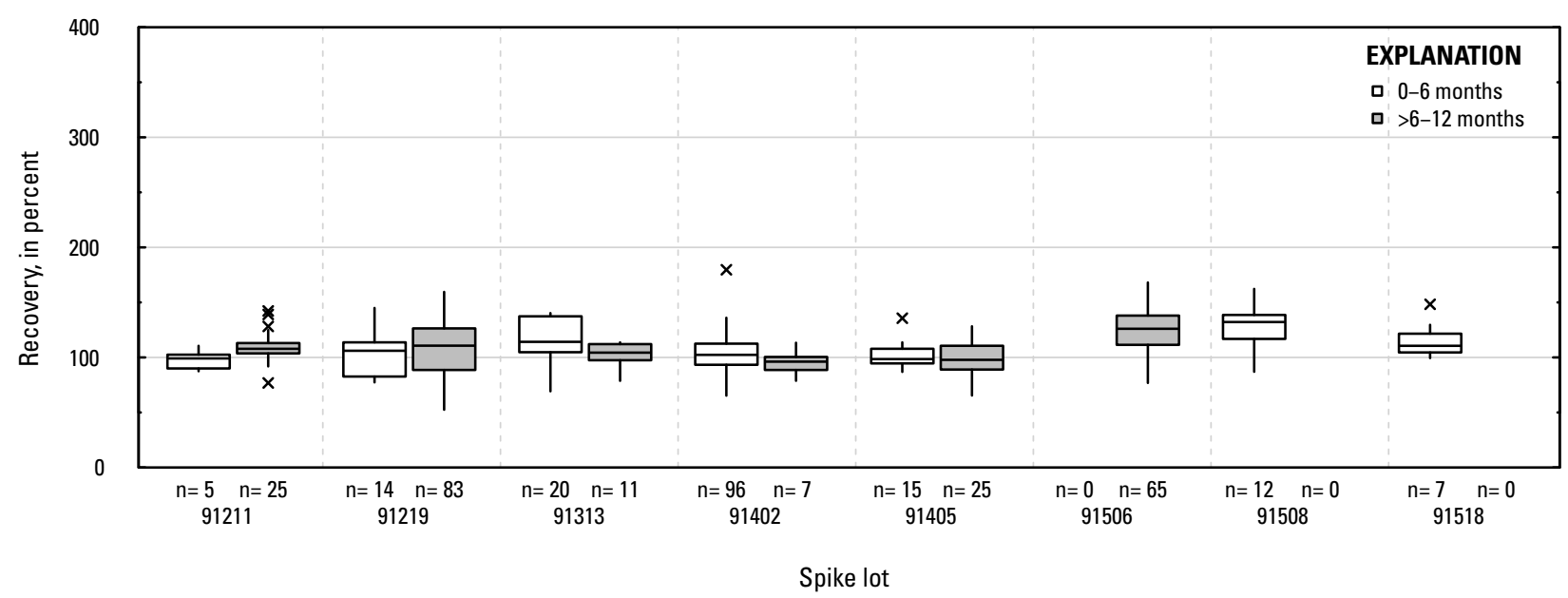

Figure 1-1. Distributions of recovery for individual pesticides in schedule 2437 by matrix, spike lot, and spike lot age. Recovery values larger than 400 percent are not shown.-Continued 
NR. Imidacloprid: laboratory reagent spikes

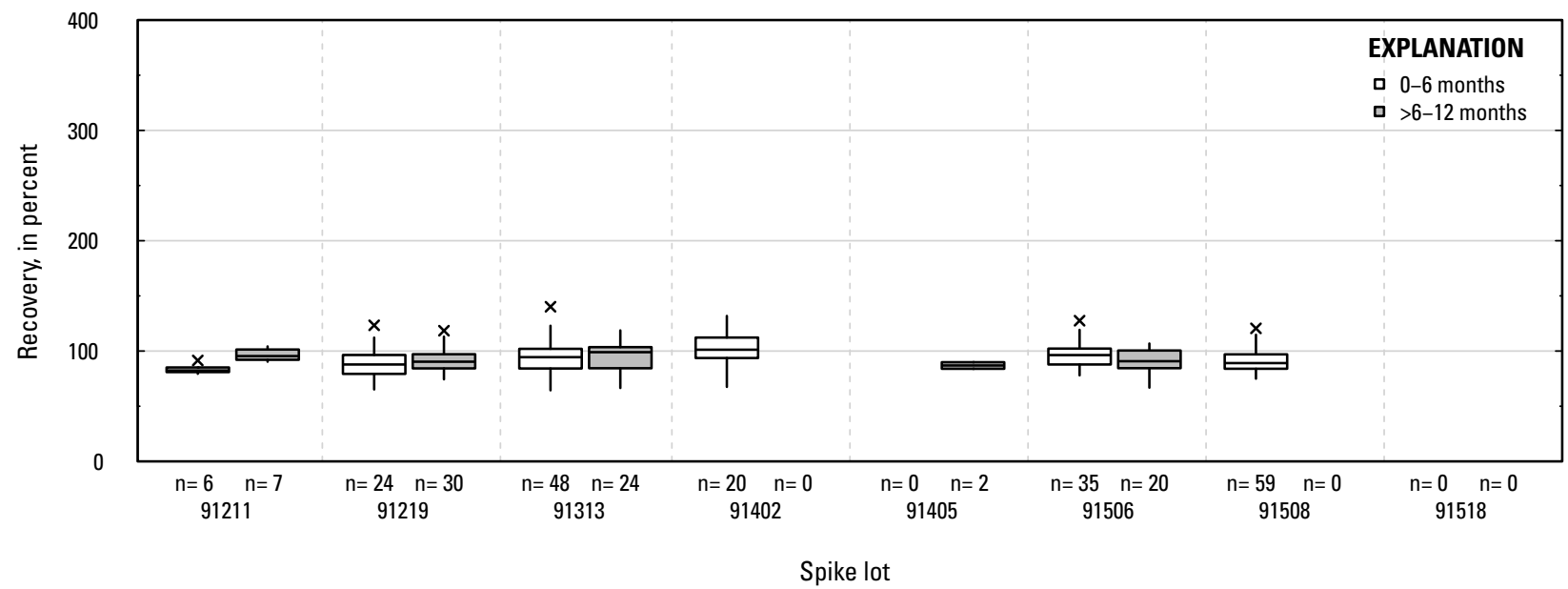

NS. Imidacloprid: groundwater field matrix spikes

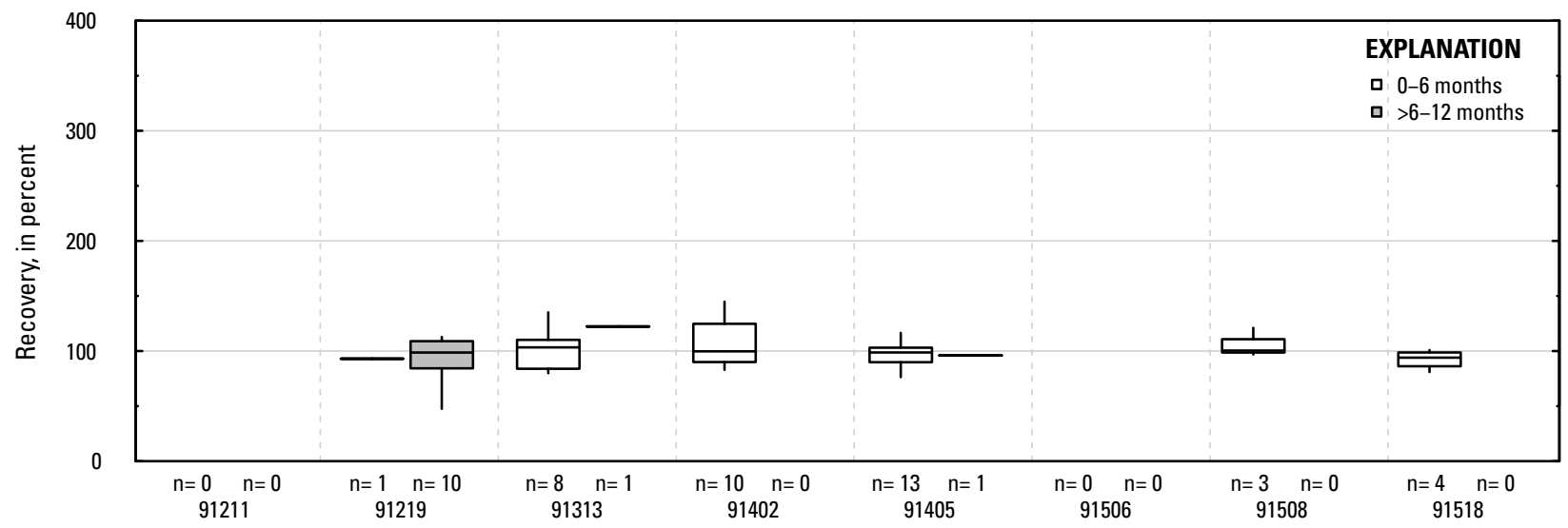

Spike lot

NT. Imidacloprid: surface water field matrix spikes

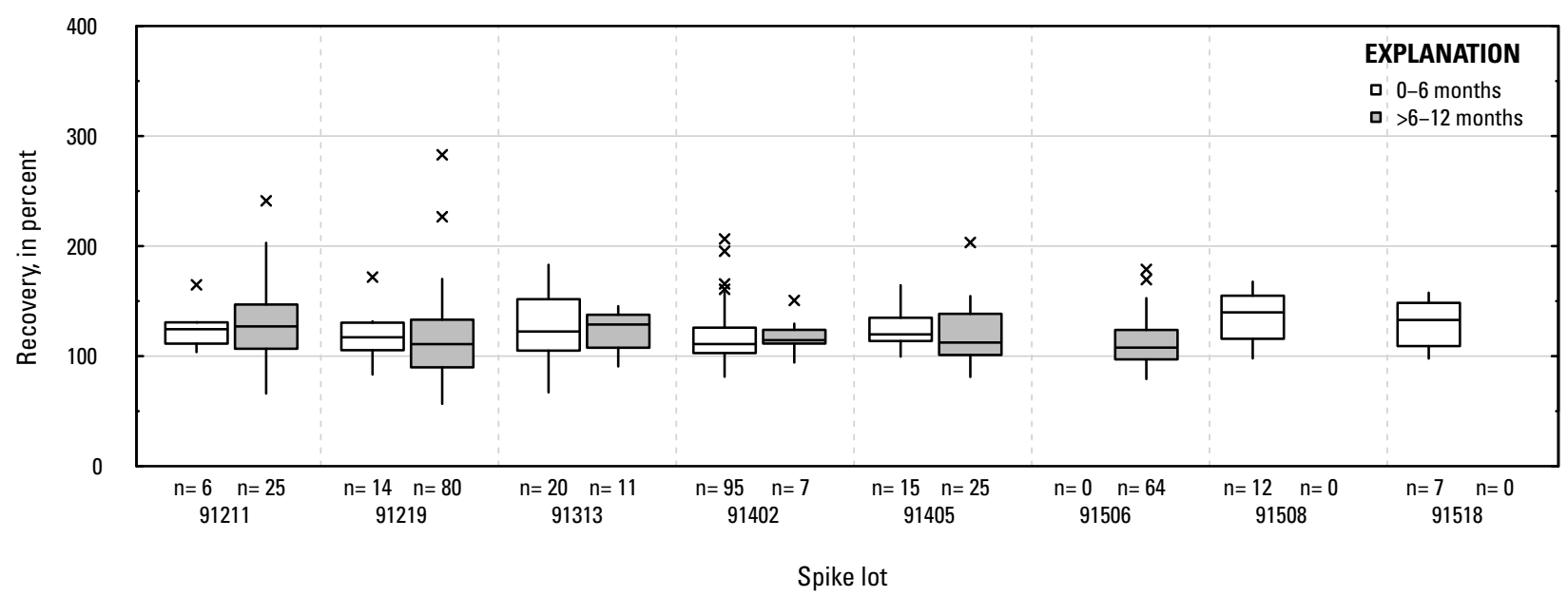

Figure 1-1. Distributions of recovery for individual pesticides in schedule 2437 by matrix, spike lot, and spike lot age. Recovery values larger than 400 percent are not shown.-Continued 
NU. Indoxacarb: laboratory reagent spikes

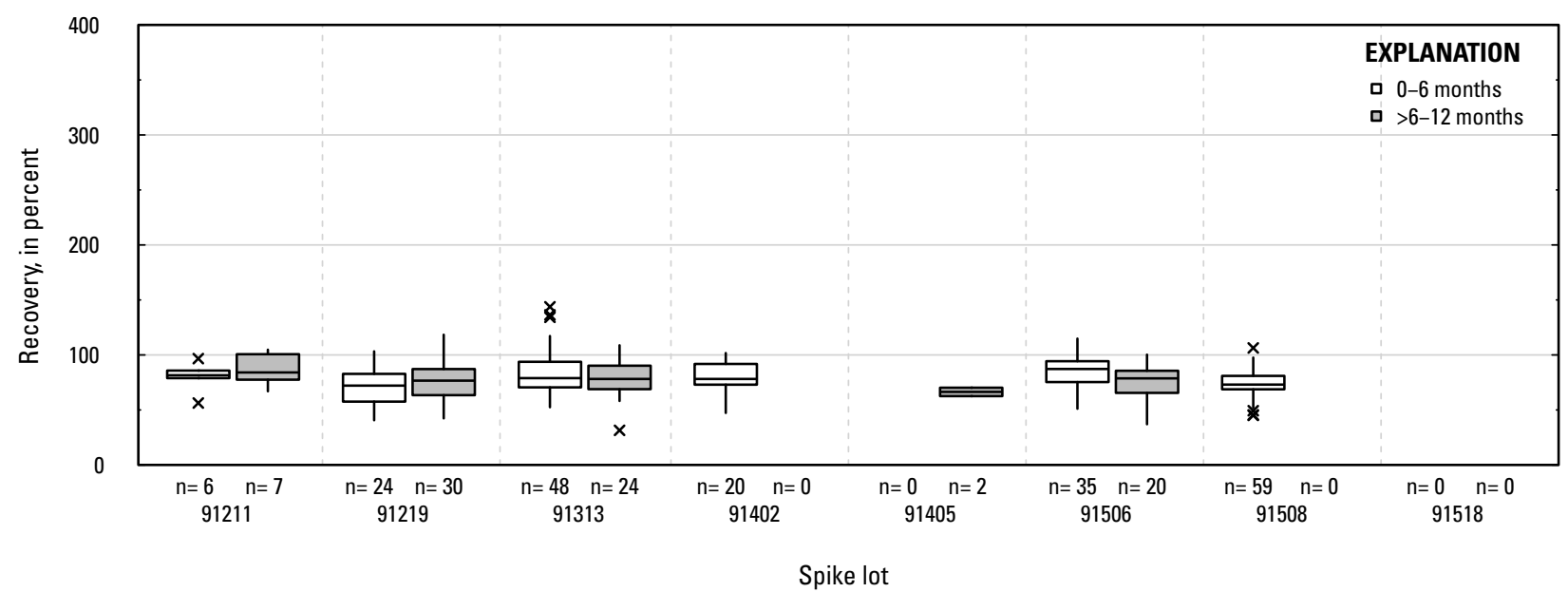

NV. Indoxacarb: groundwater field matrix spikes

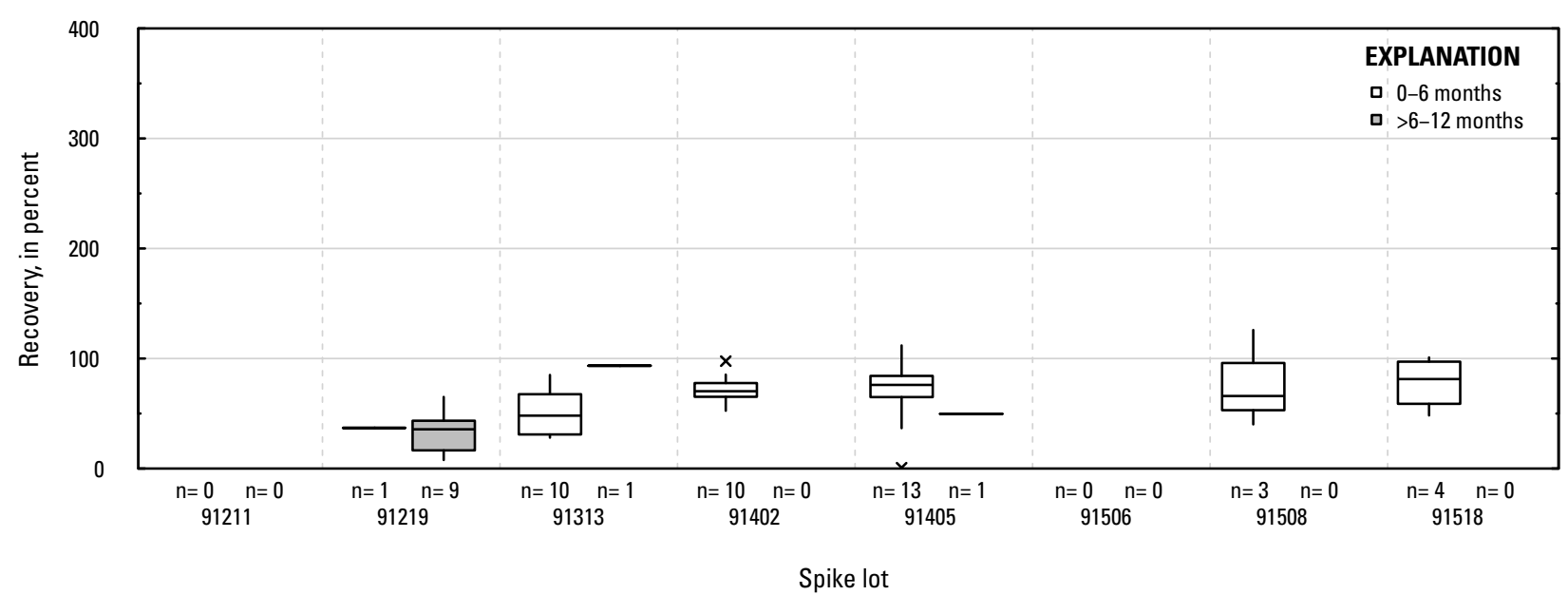

NW. Indoxacarb: surface water field matrix spikes

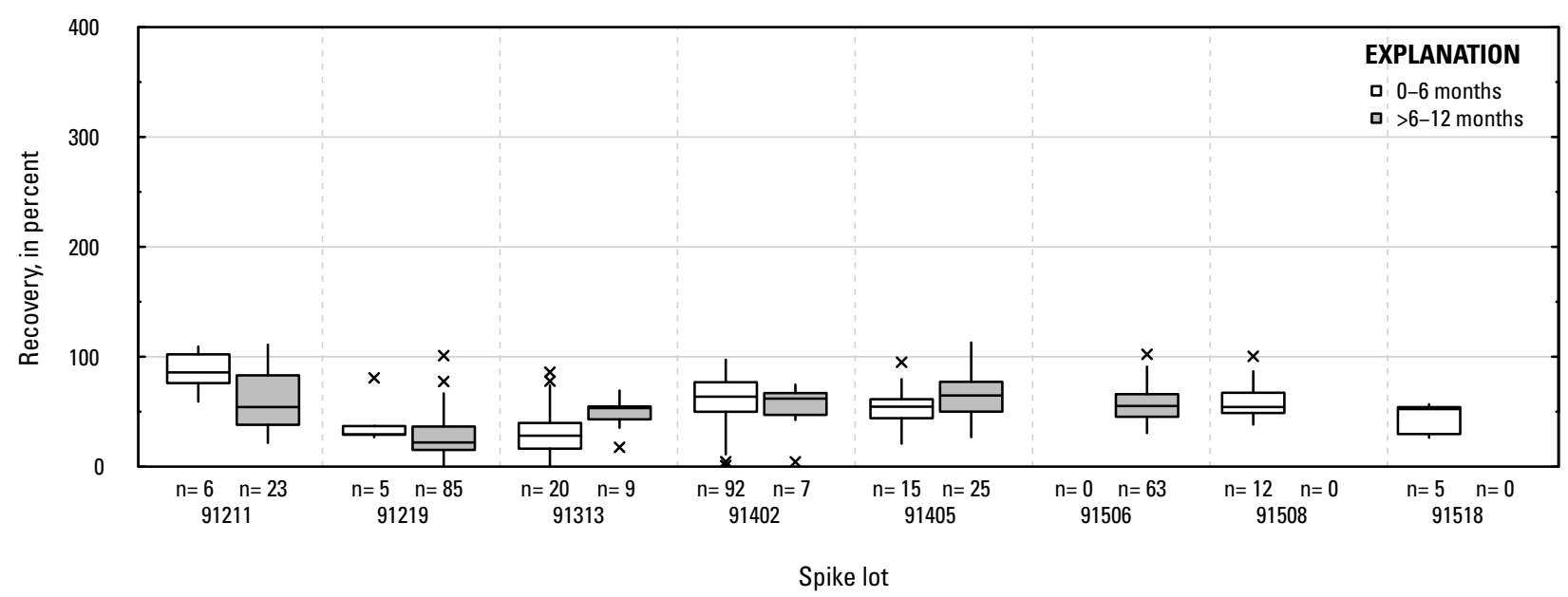

Figure 1-1. Distributions of recovery for individual pesticides in schedule 2437 by matrix, spike lot, and spike lot age. Recovery values larger than 400 percent are not shown.-Continued 
NX. Isoxaflutole: laboratory reagent spikes

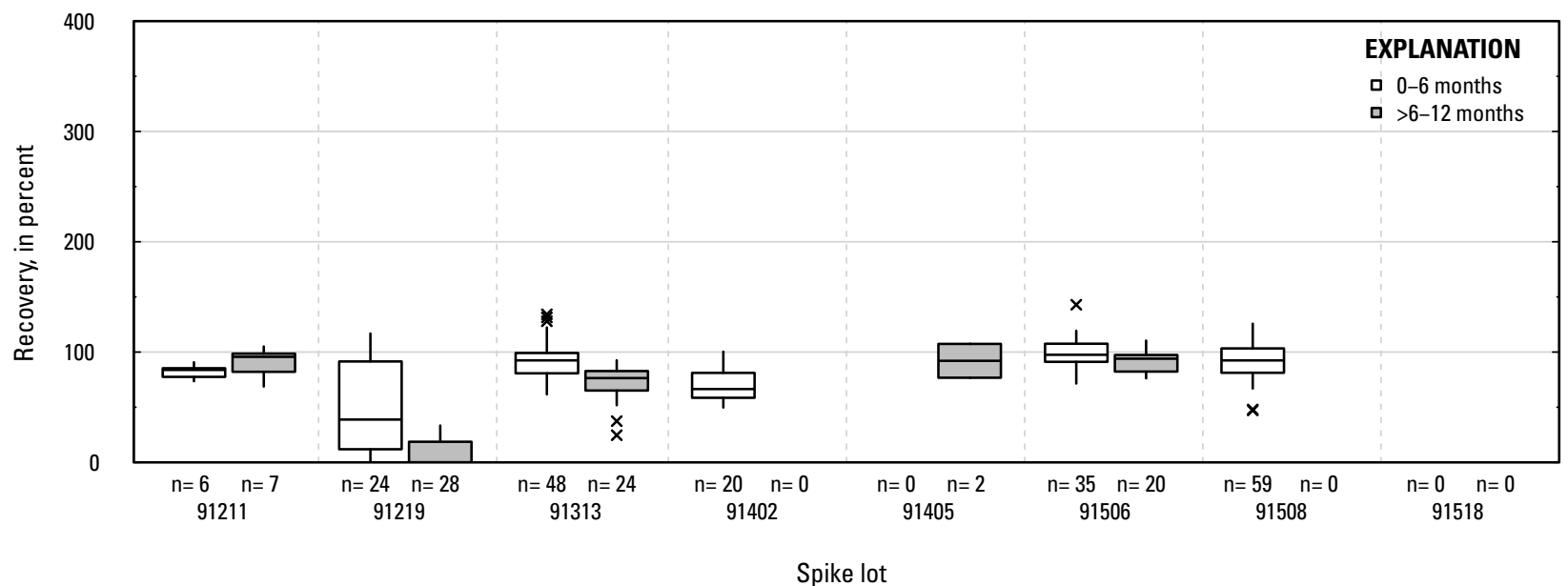

NY. Isoxaflutole: groundwater field matrix spikes

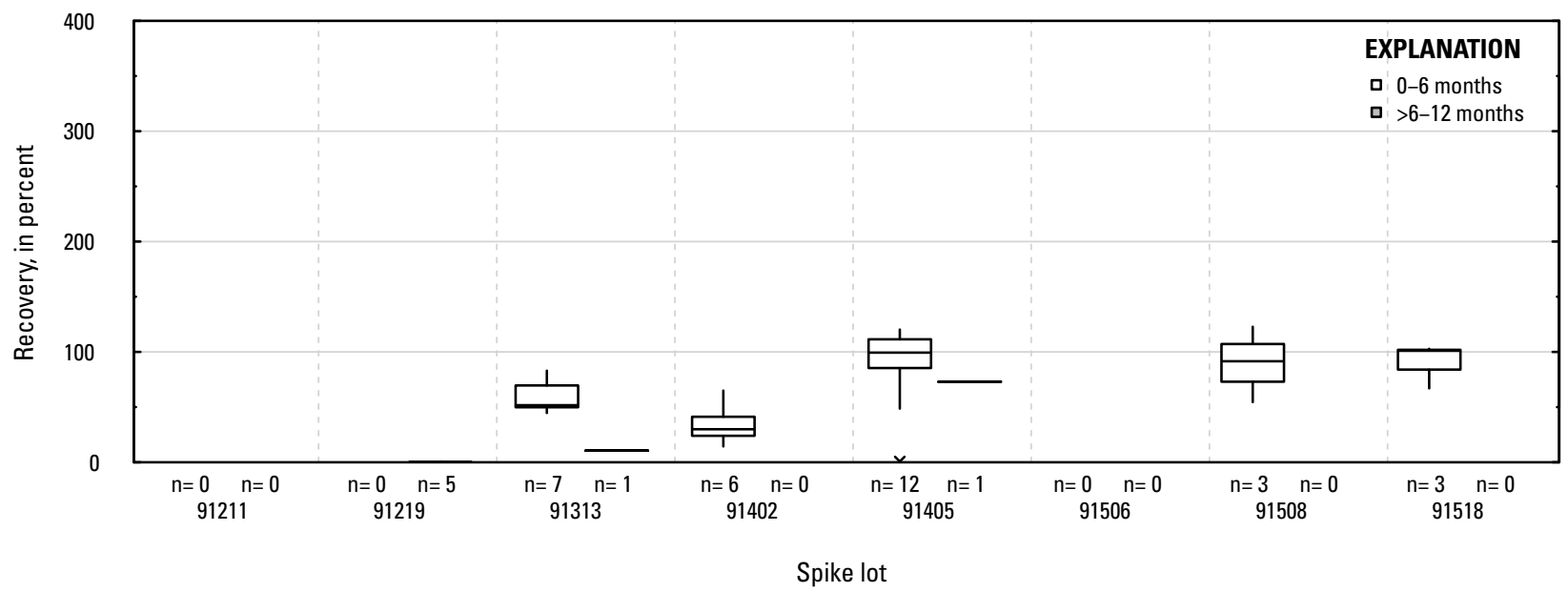

NZ. Isoxaflutole: surface water field matrix spikes

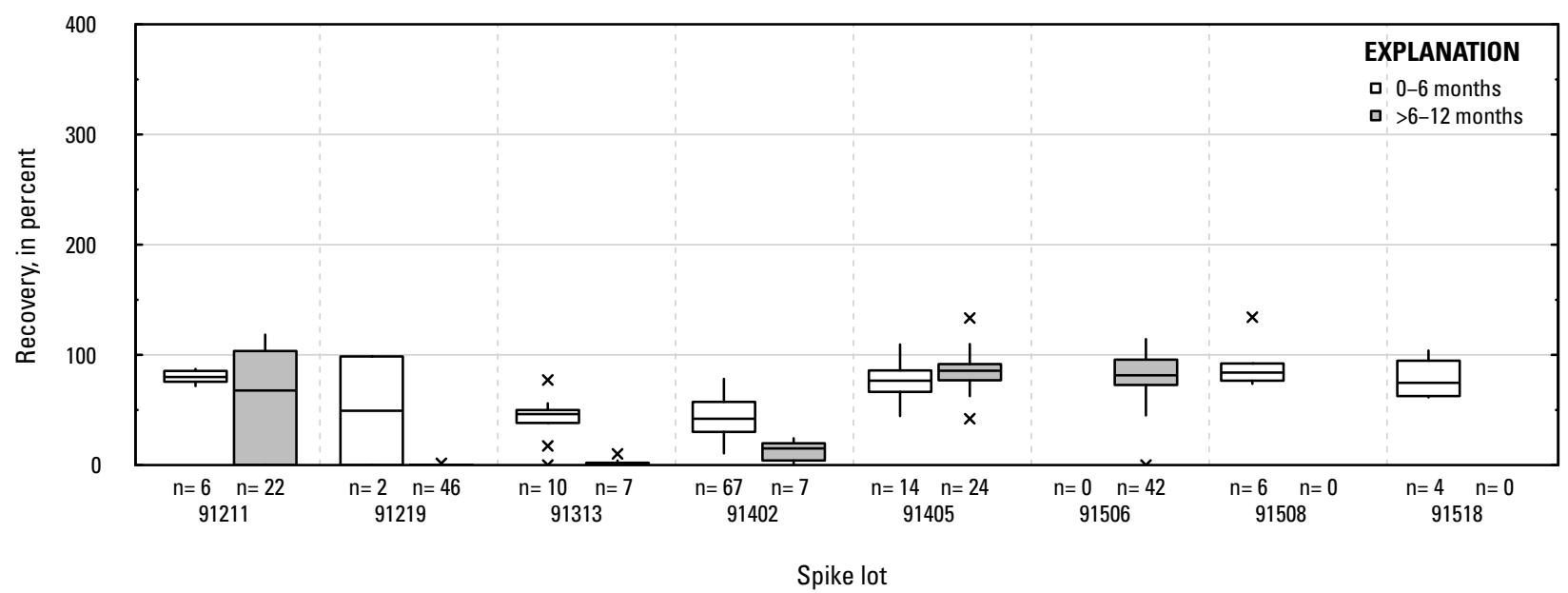

Figure 1-1. Distributions of recovery for individual pesticides in schedule 2437 by matrix, spike lot, and spike lot age. Recovery values larger than 400 percent are not shown.-Continued 
OA. Isoxaflutole acid metabolite RPA 203328: laboratory reagent spikes

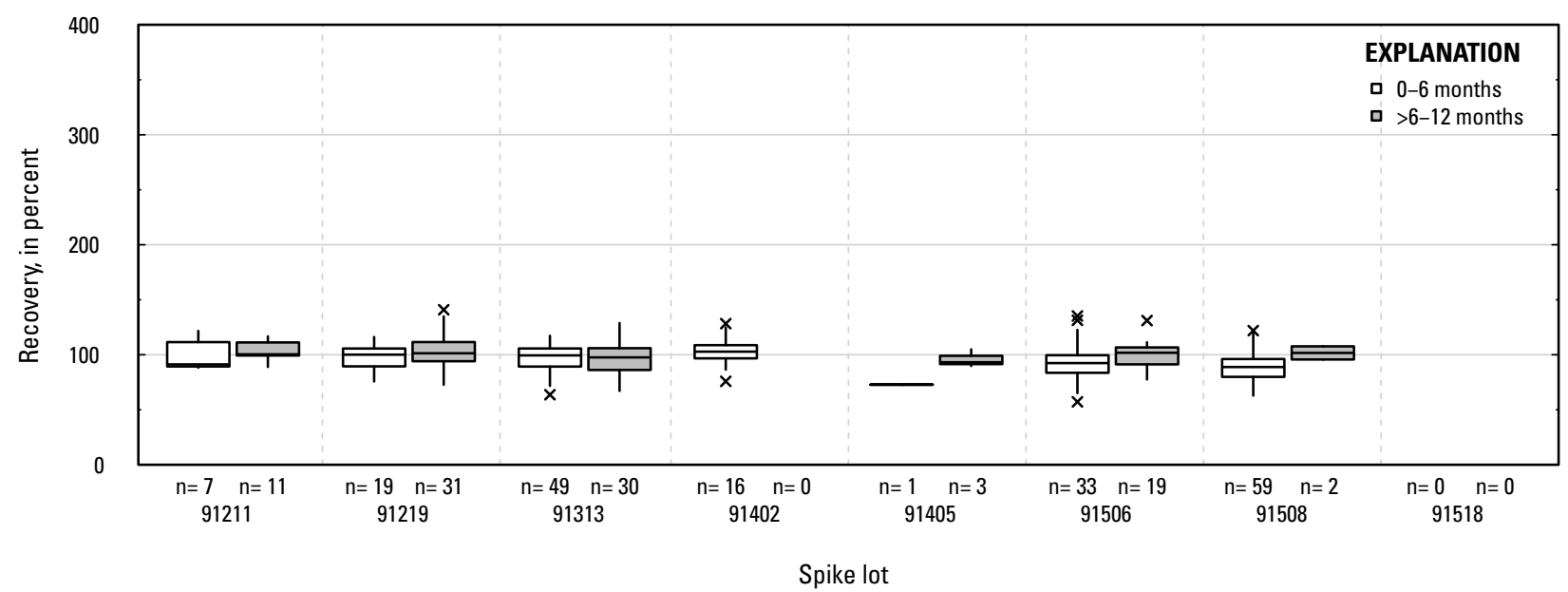

OB. Isoxaflutole acid metabolite RPA 203328: groundwater field matrix spikes

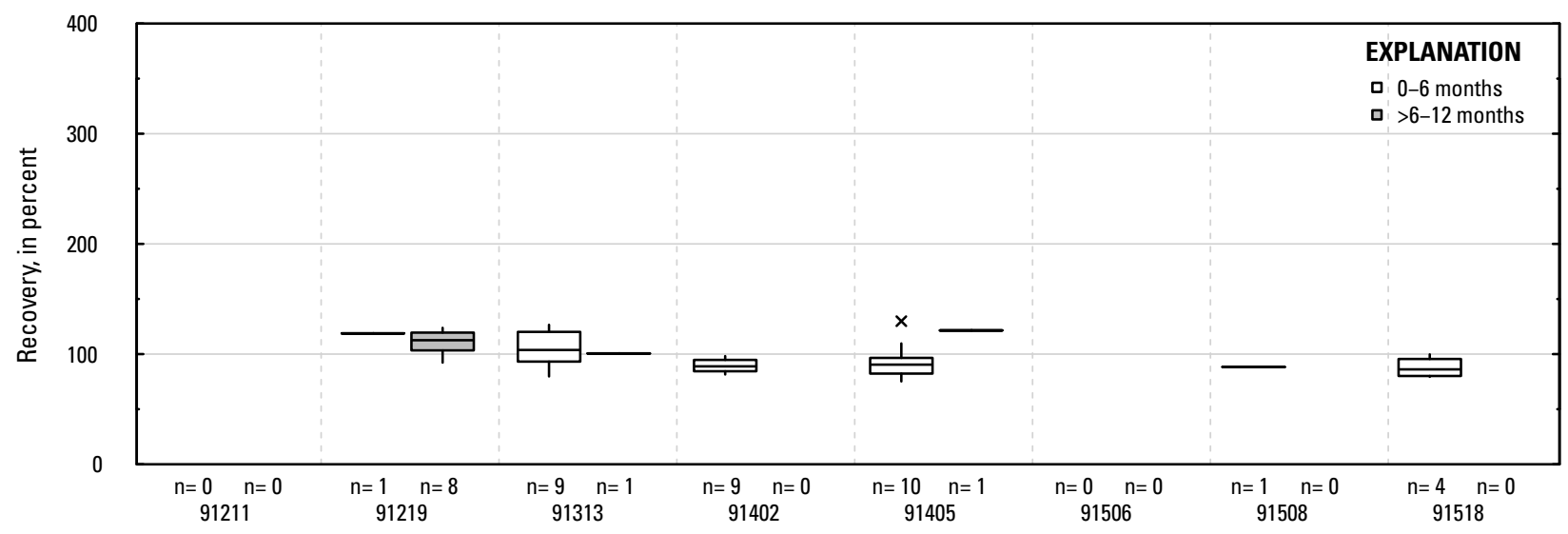

Spike lot

OC. Isoxaflutole acid metabolite RPA 203328: surface water field matrix spikes

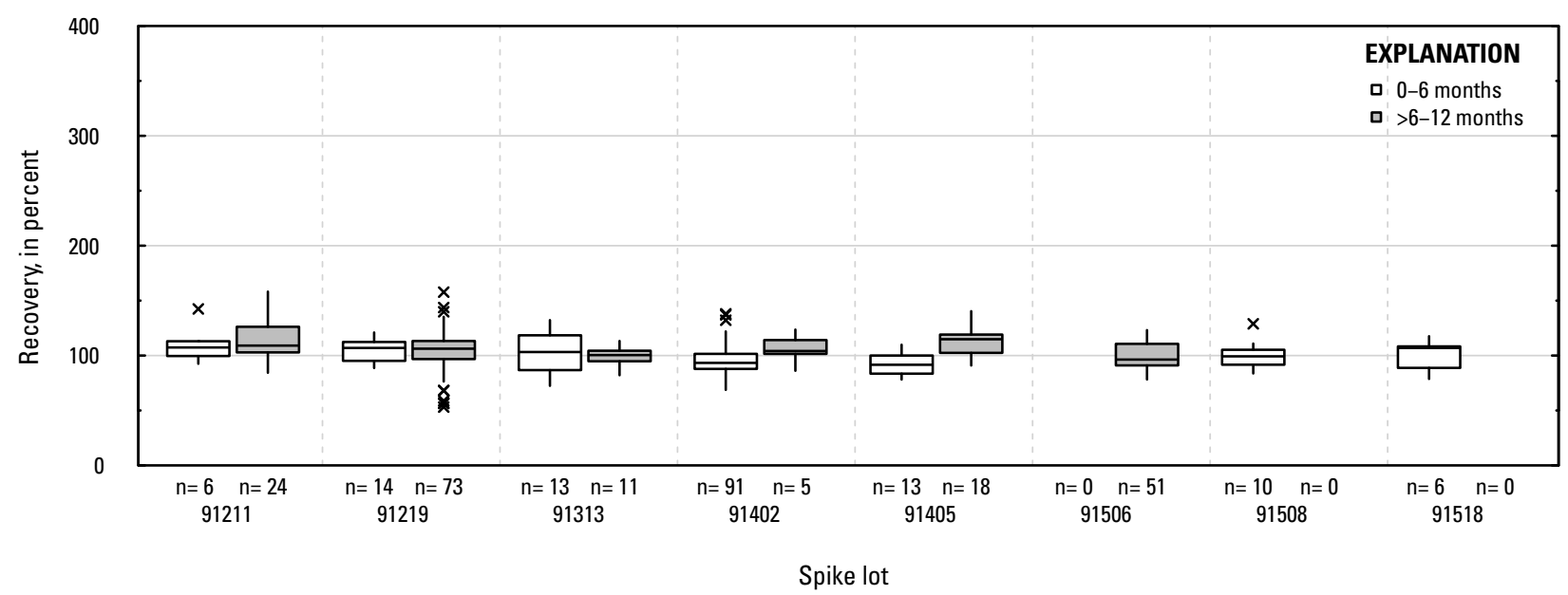

Figure 1-1. Distributions of recovery for individual pesticides in schedule 2437 by matrix, spike lot, and spike lot age. Recovery values larger than 400 percent are not shown.-Continued 
OD. Kresoxim-methyl: laboratory reagent spikes

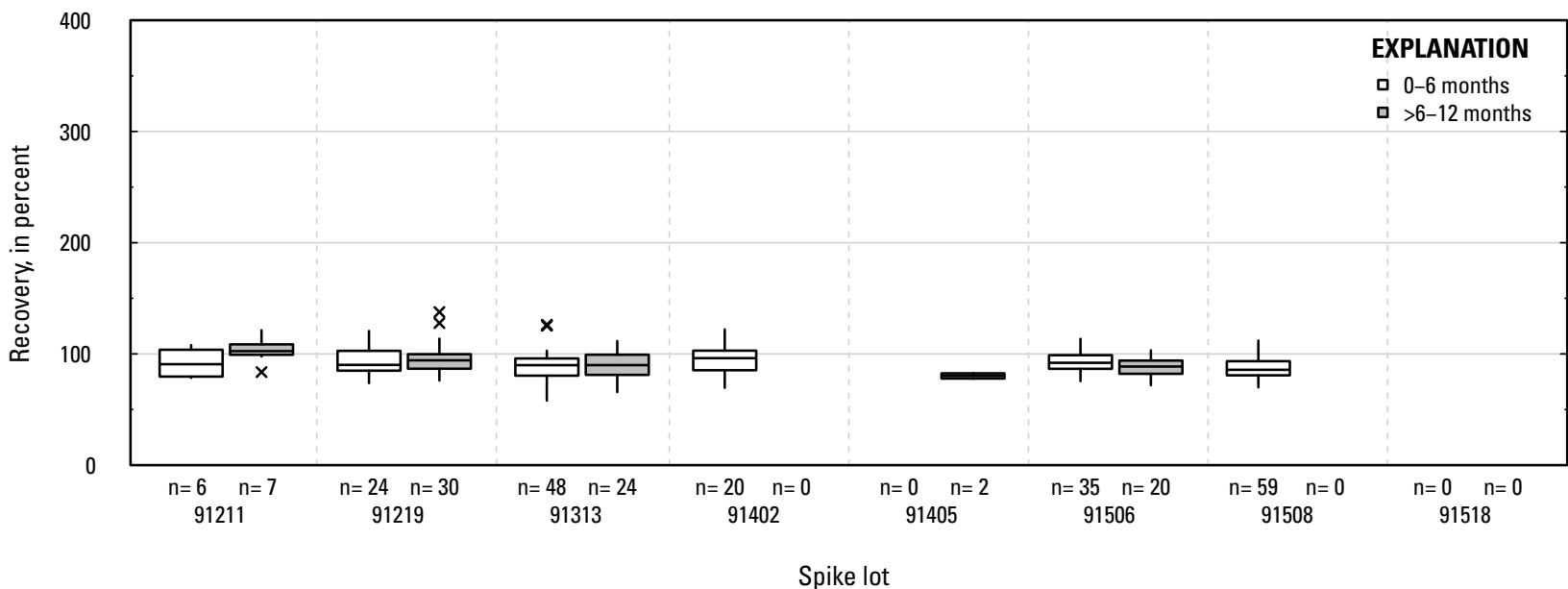

OE. Kresoxim-methyl: groundwater field matrix spikes

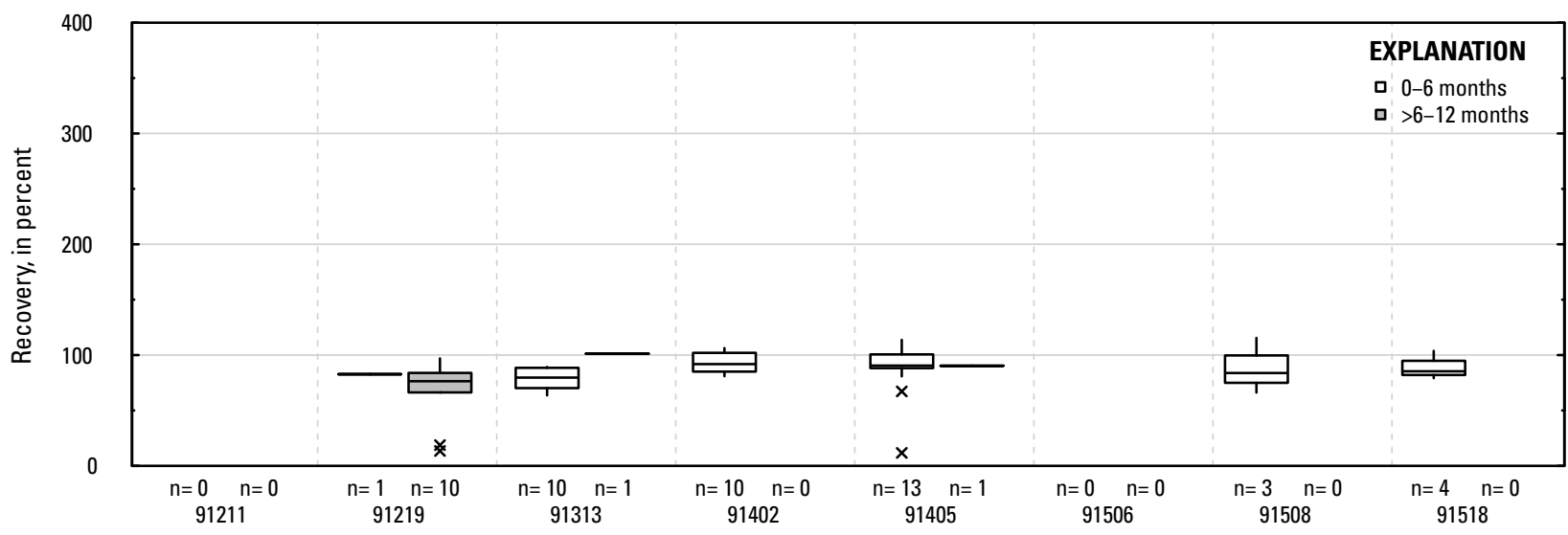

Spike lot

OF. Kresoxim-methyl: surface water field matrix spikes

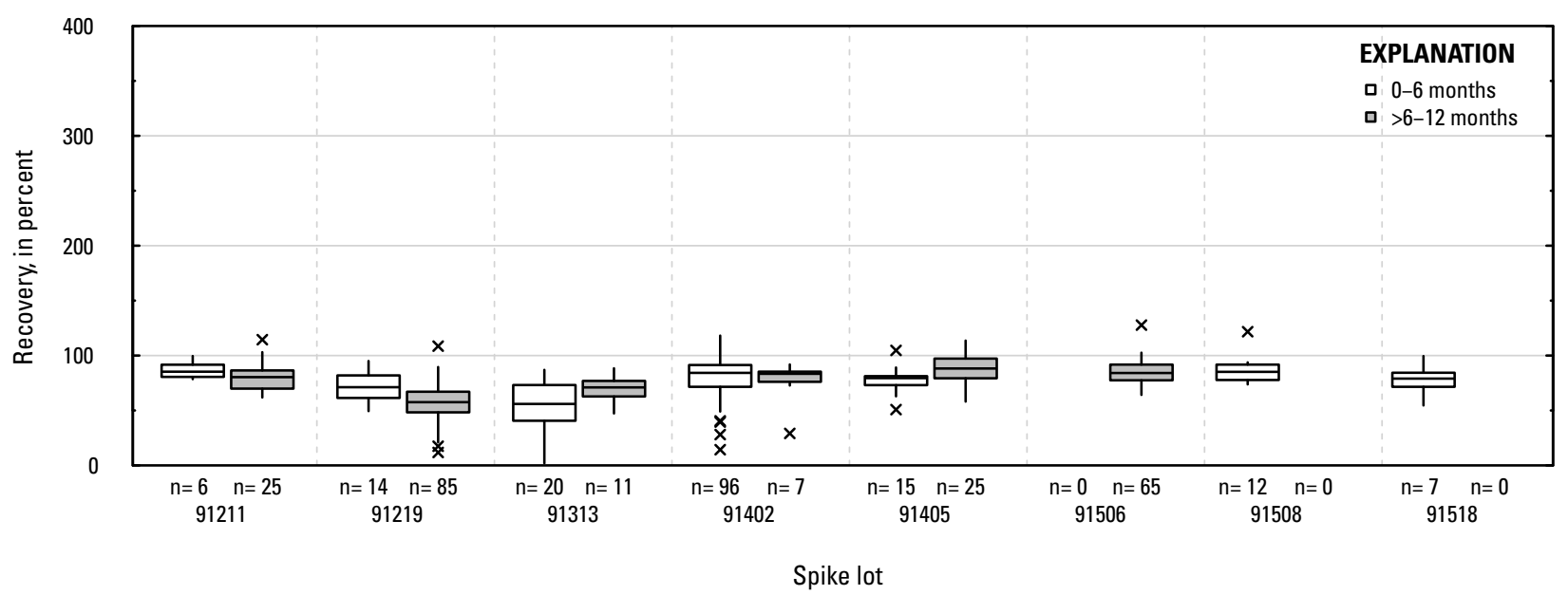

Figure 1-1. Distributions of recovery for individual pesticides in schedule 2437 by matrix, spike lot, and spike lot age. Recovery values larger than 400 percent are not shown.-Continued 


\section{OG. Lactofen: laboratory reagent spikes}

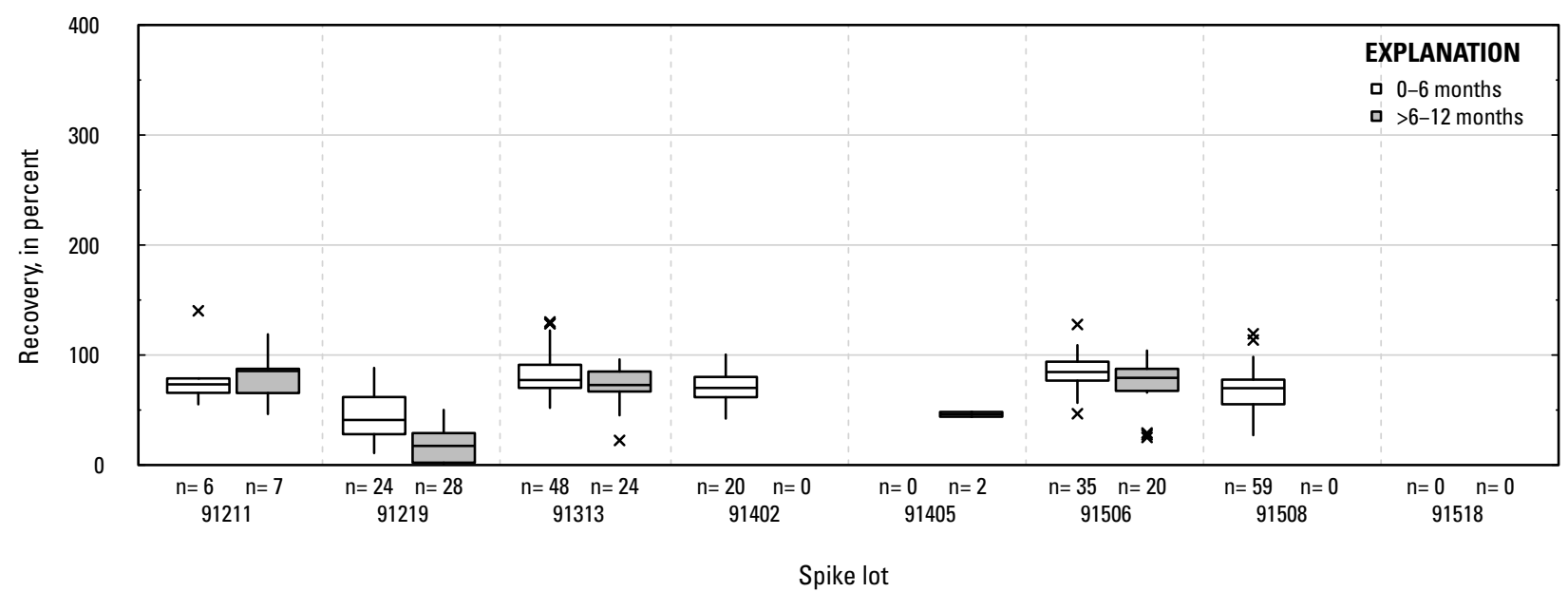

OH. Lactofen: groundwater field matrix spikes

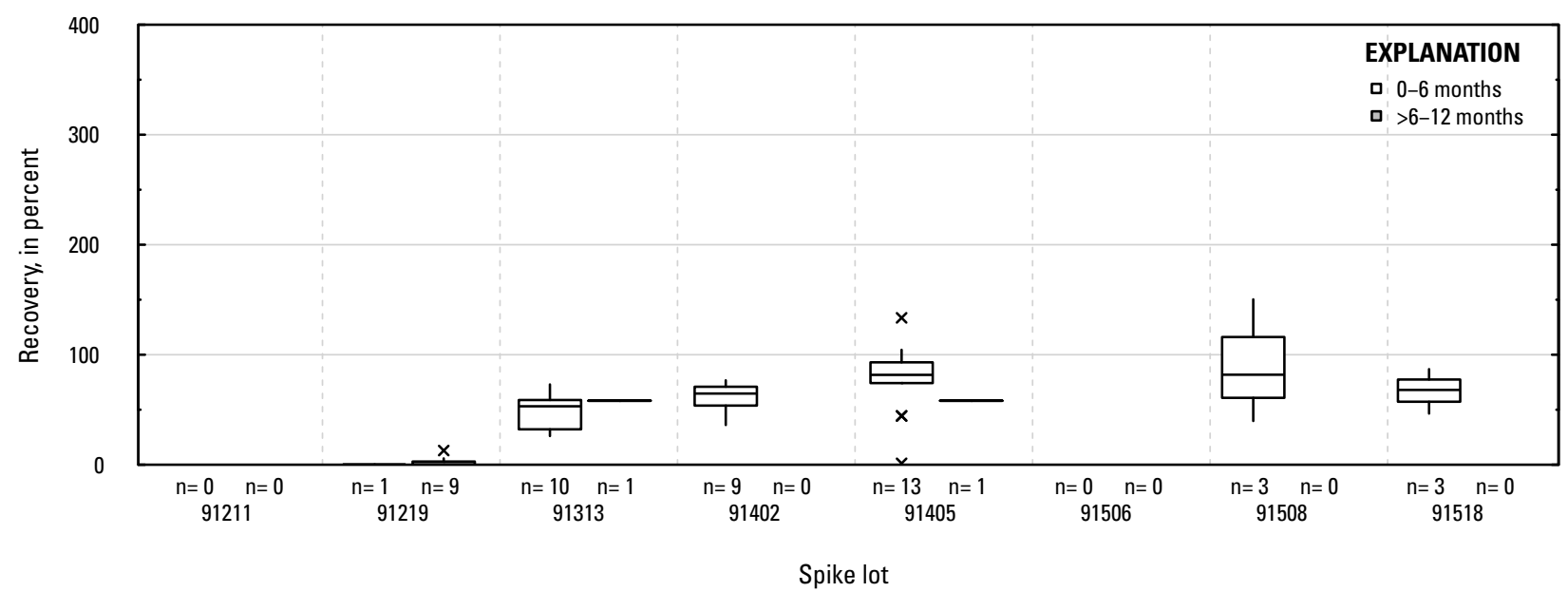

OI. Lactofen: surface water field matrix spikes

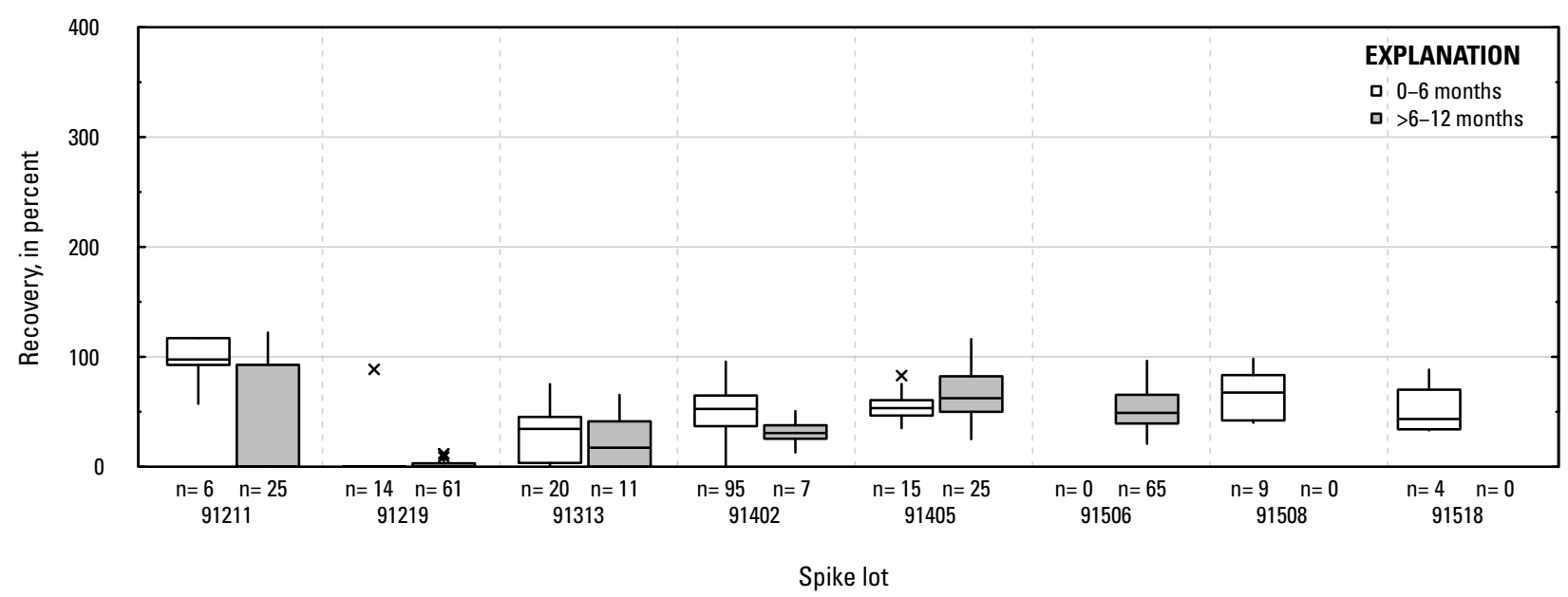

Figure 1-1. Distributions of recovery for individual pesticides in schedule 2437 by matrix, spike lot, and spike lot age. Recovery values larger than 400 percent are not shown.-Continued 


\section{OJ. Linuron: laboratory reagent spikes}

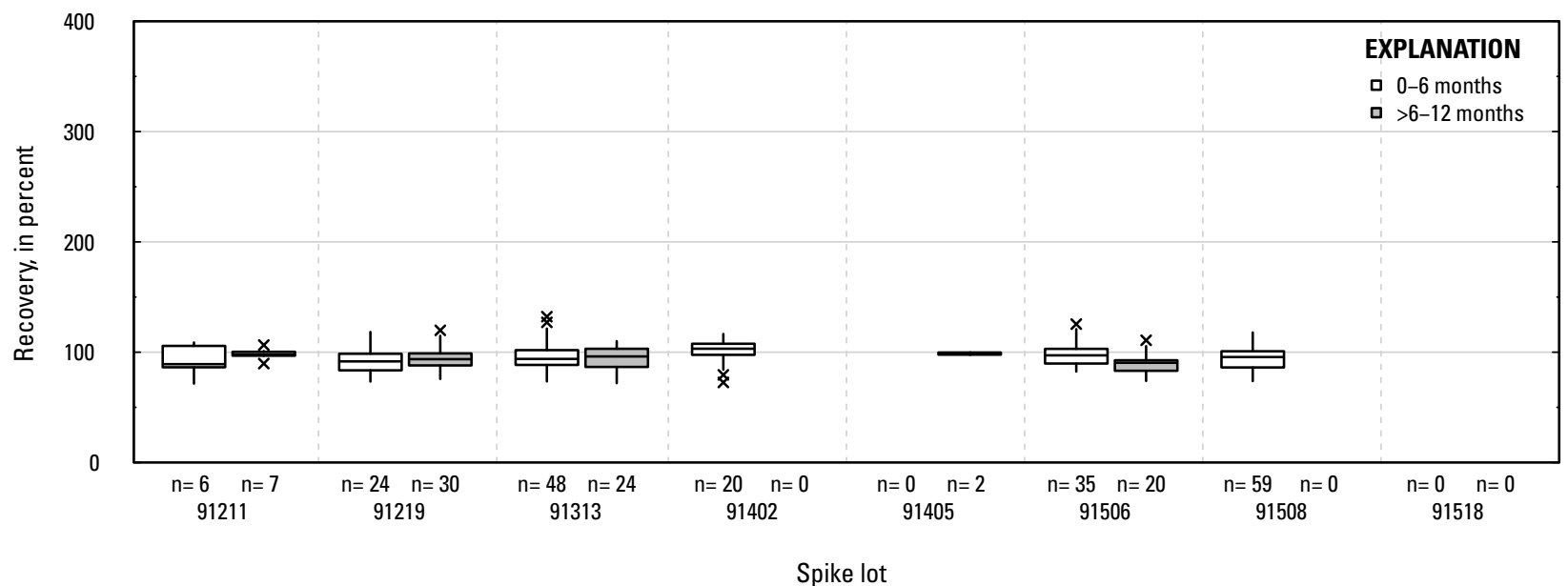

OK. Linuron: groundwater field matrix spikes

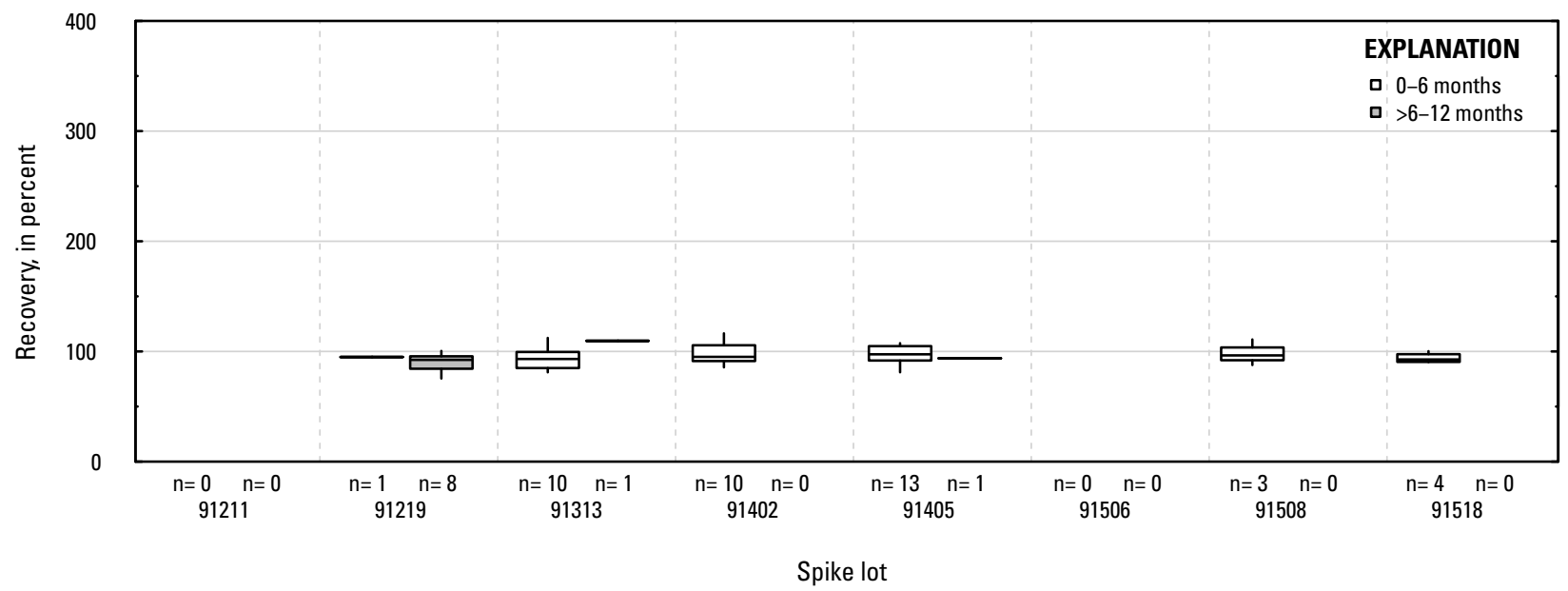

\section{OL. Linuron: surface water field matrix spikes}

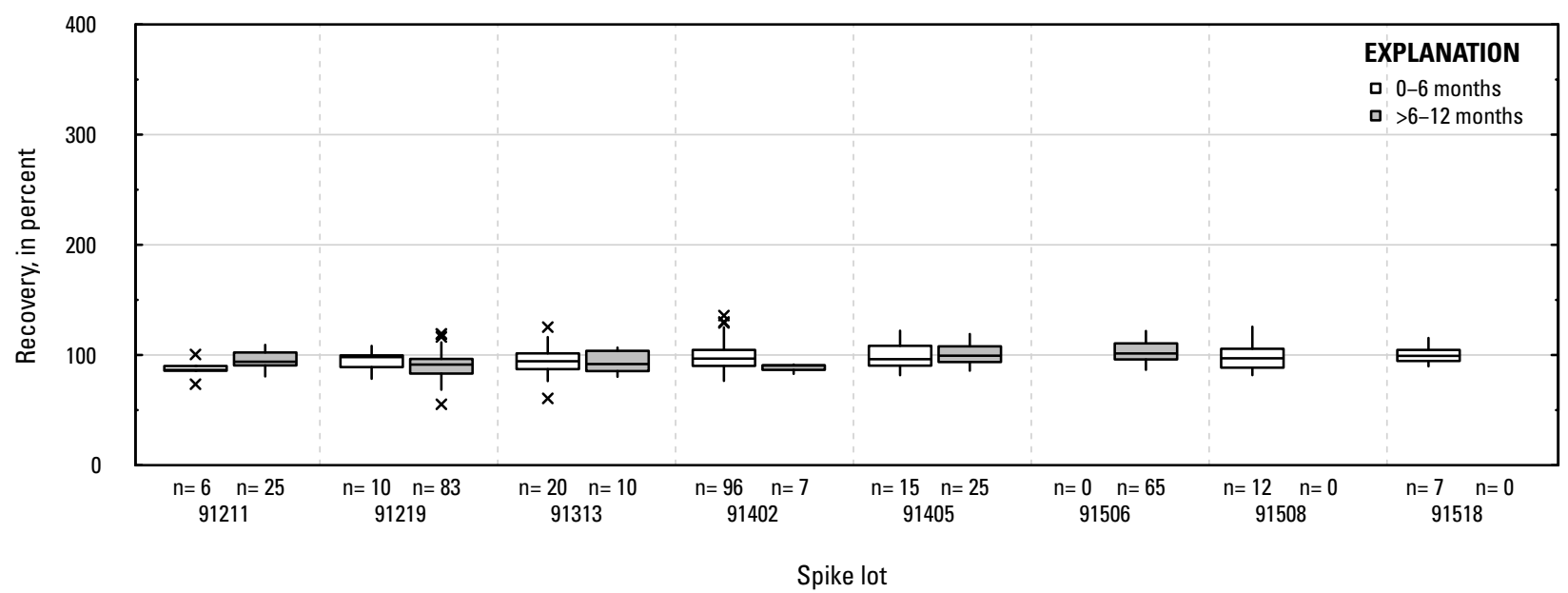

Figure 1-1. Distributions of recovery for individual pesticides in schedule 2437 by matrix, spike lot, and spike lot age. Recovery values larger than 400 percent are not shown.-Continued 
OM. Malaoxon: laboratory reagent spikes

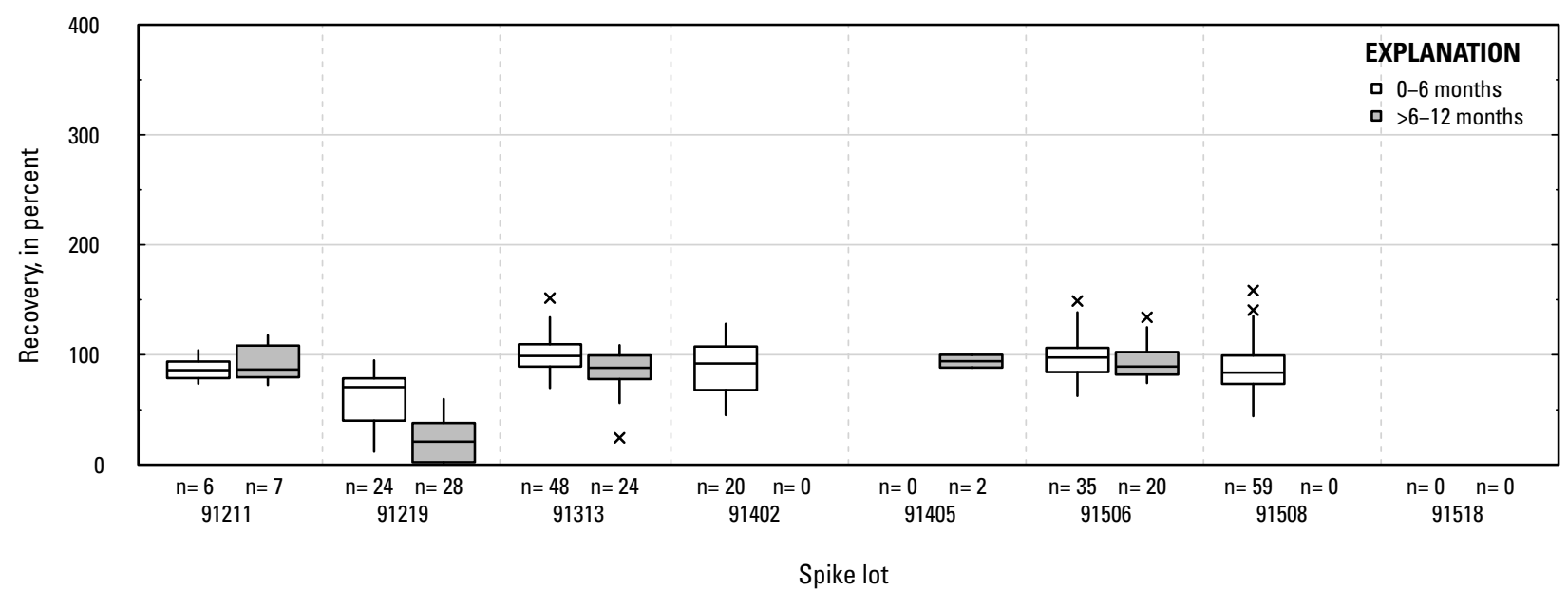

ON. Malaoxon: groundwater field matrix spikes

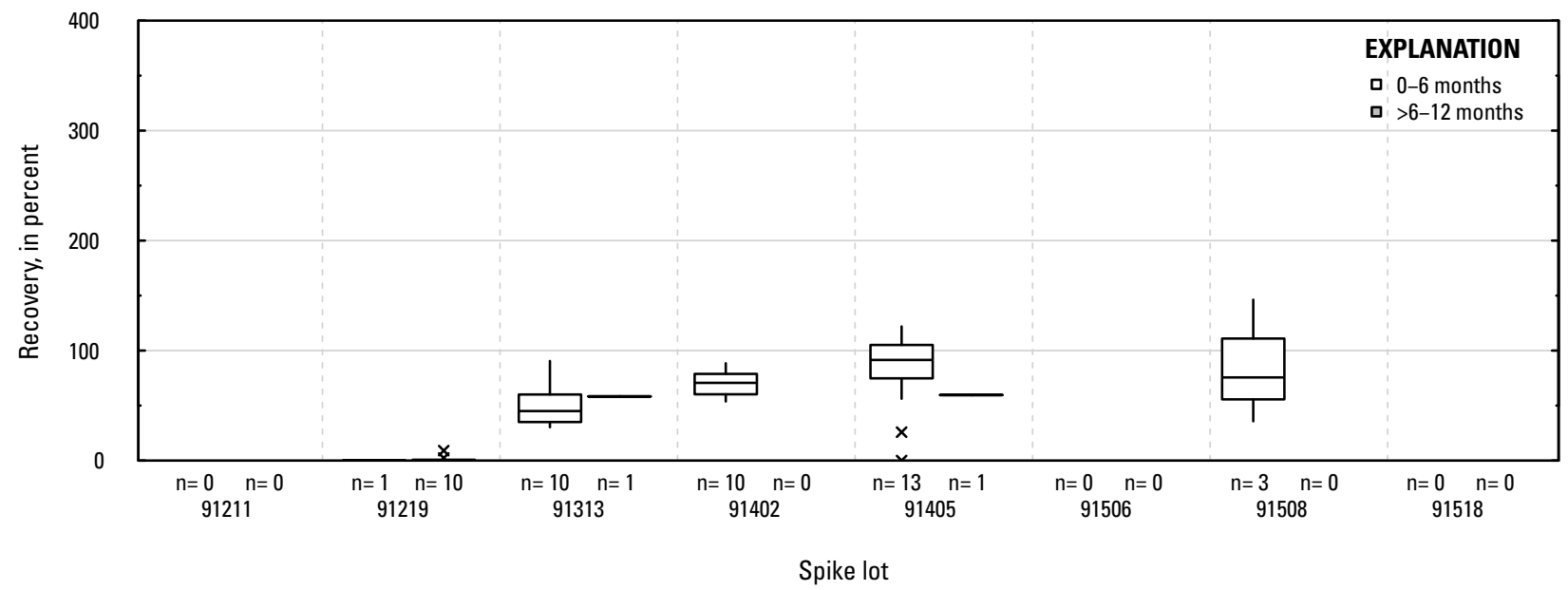

00. Malaoxon: surface water field matrix spikes

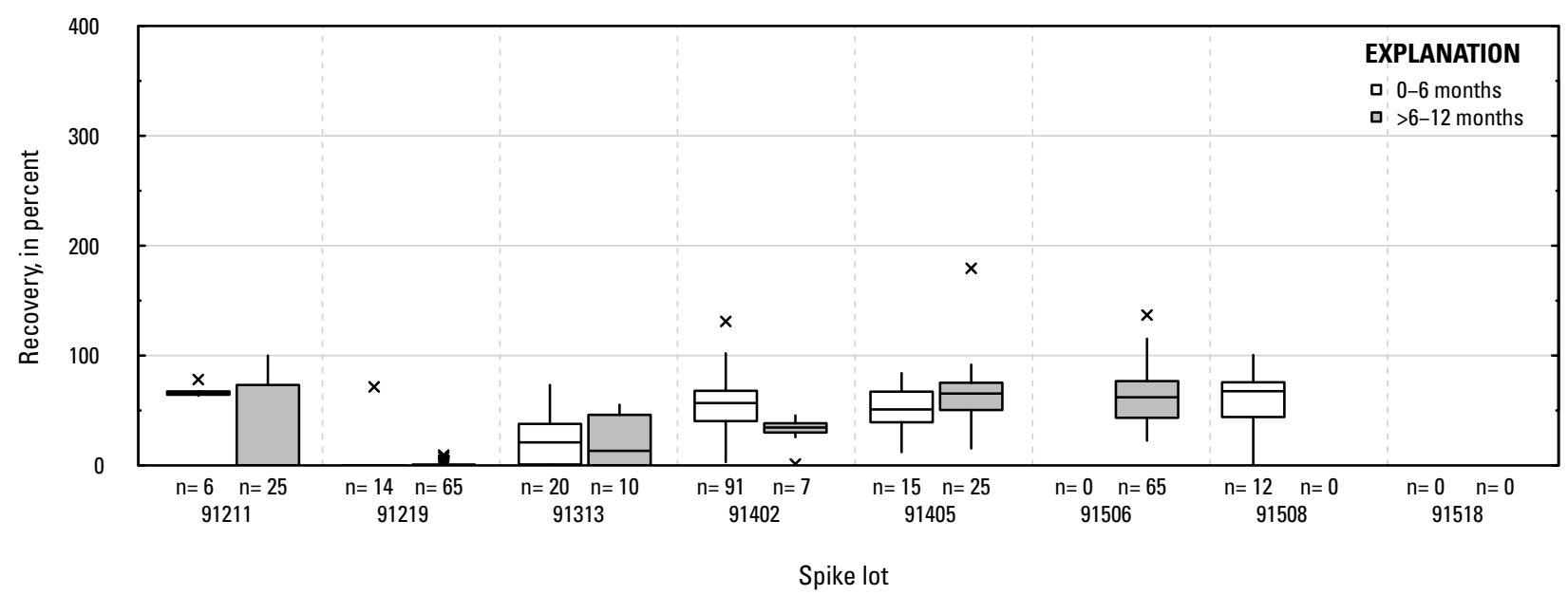

Figure 1-1. Distributions of recovery for individual pesticides in schedule 2437 by matrix, spike lot, and spike lot age. Recovery values larger than 400 percent are not shown.-Continued 
$O P$. Malathion: laboratory reagent spikes

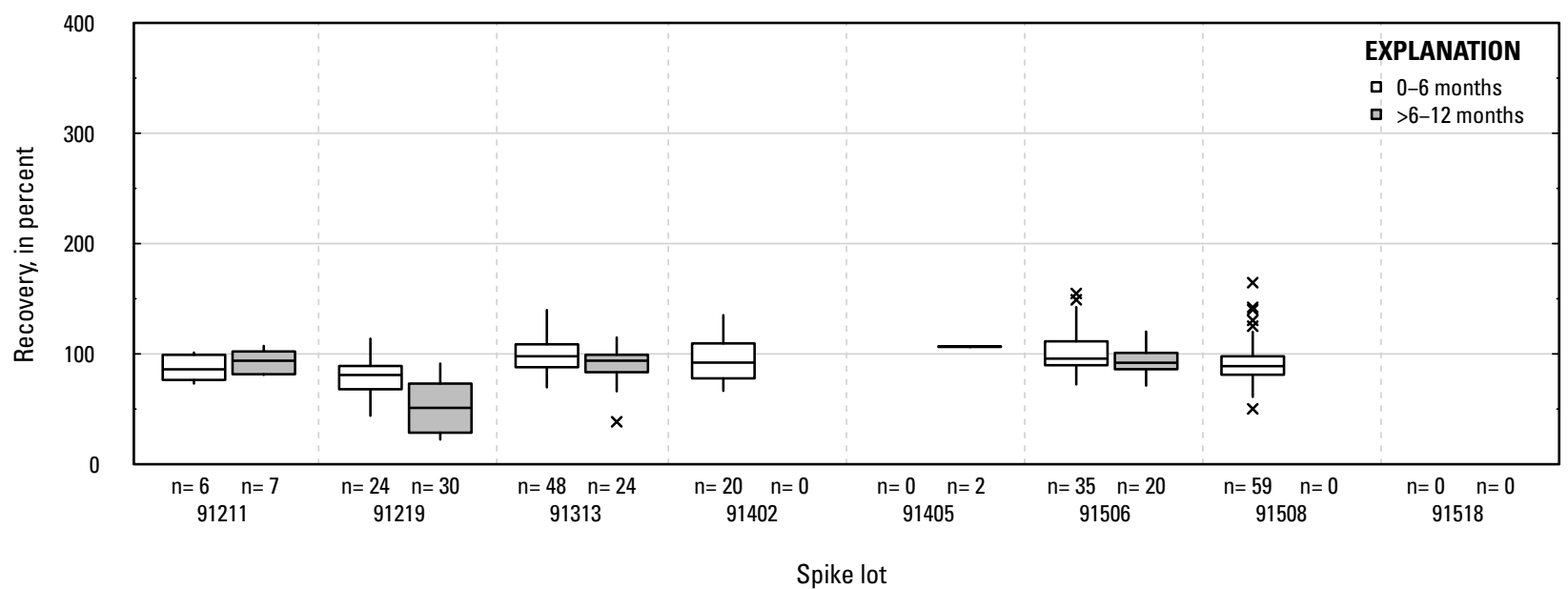

00. Malathion: groundwater field matrix spikes

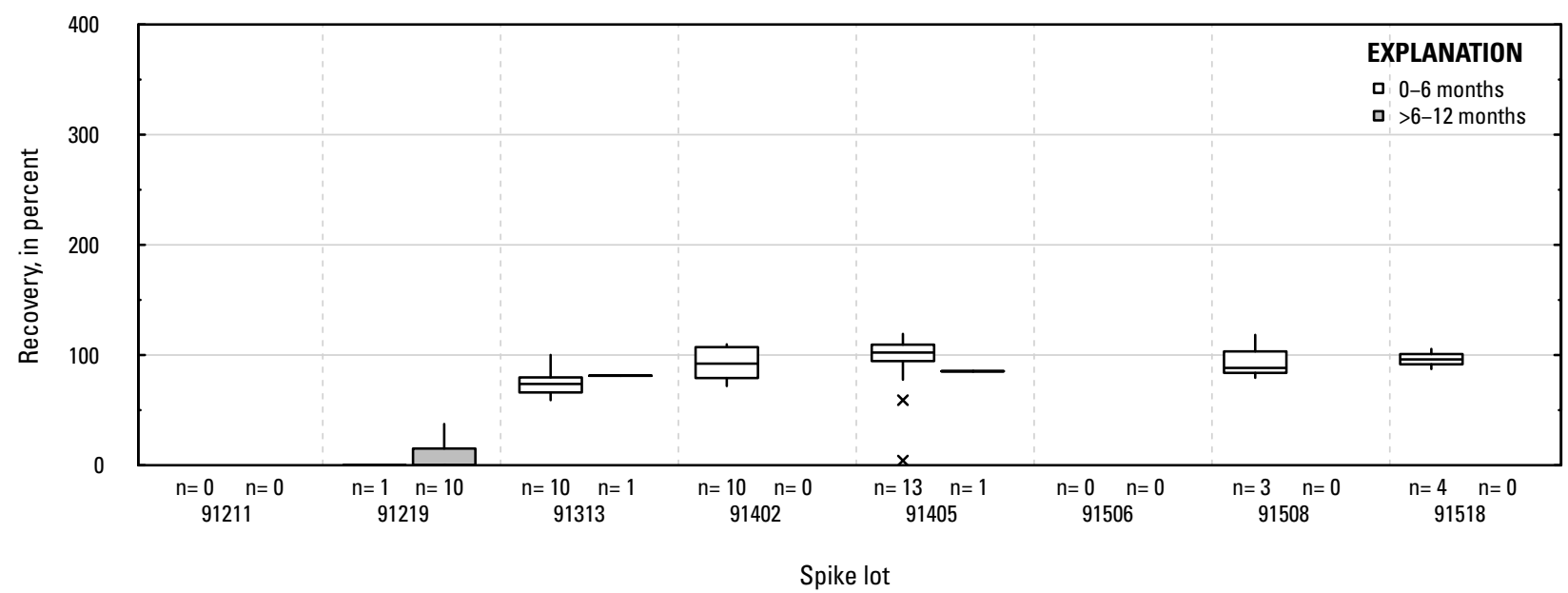

OR. Malathion: surface water field matrix spikes

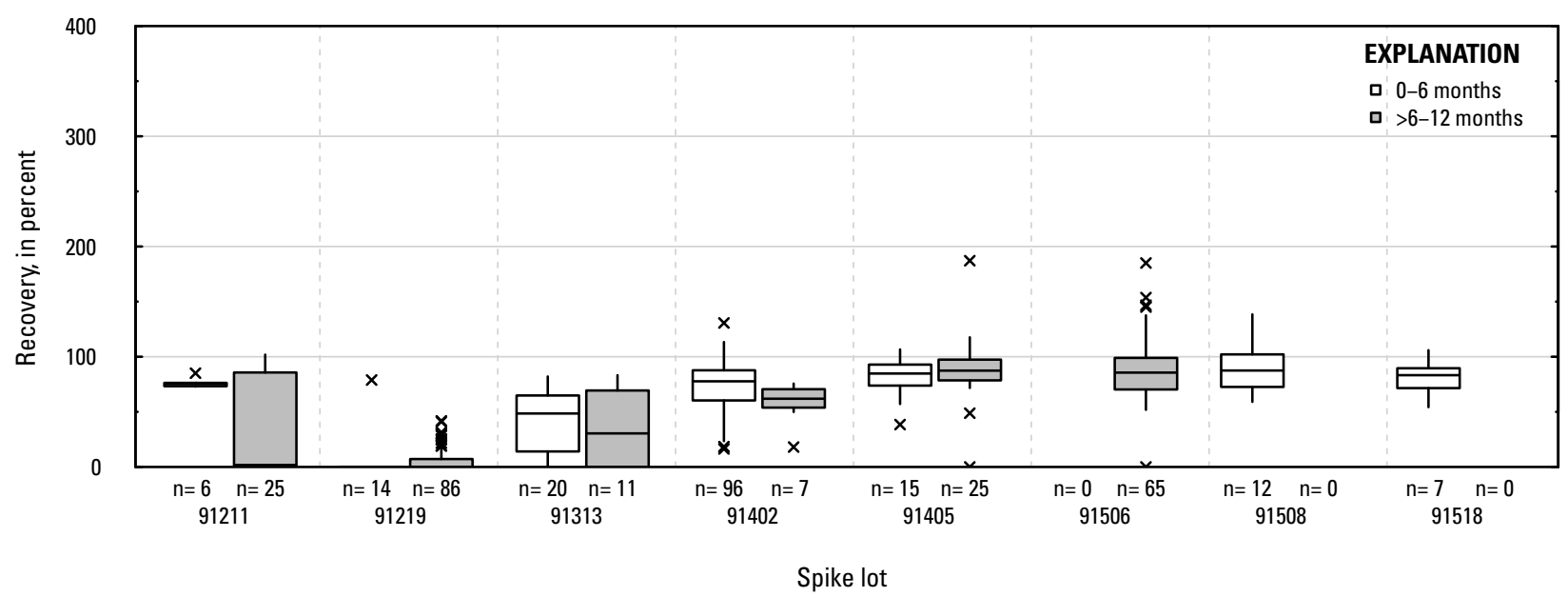

Figure 1-1. Distributions of recovery for individual pesticides in schedule 2437 by matrix, spike lot, and spike lot age. Recovery values larger than 400 percent are not shown.-Continued 


\section{OS. MCPA: laboratory reagent spikes}

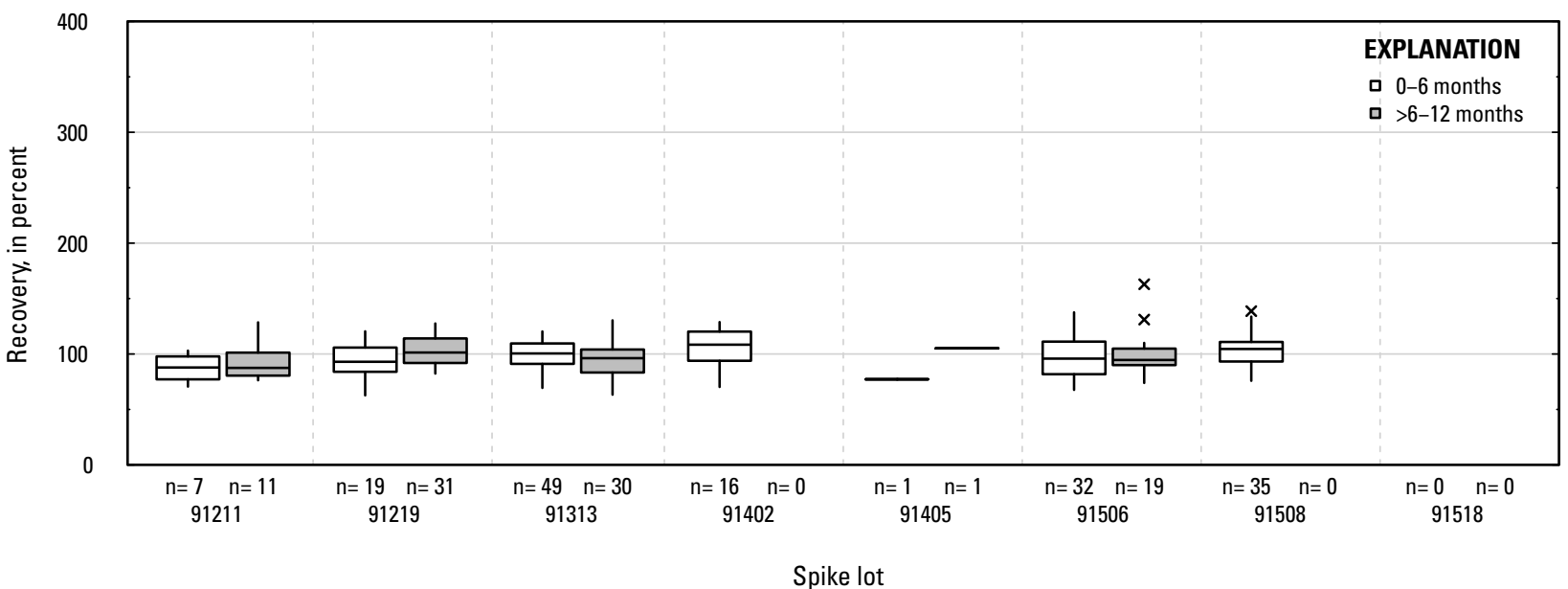

OT. MCPA: groundwater field matrix spikes

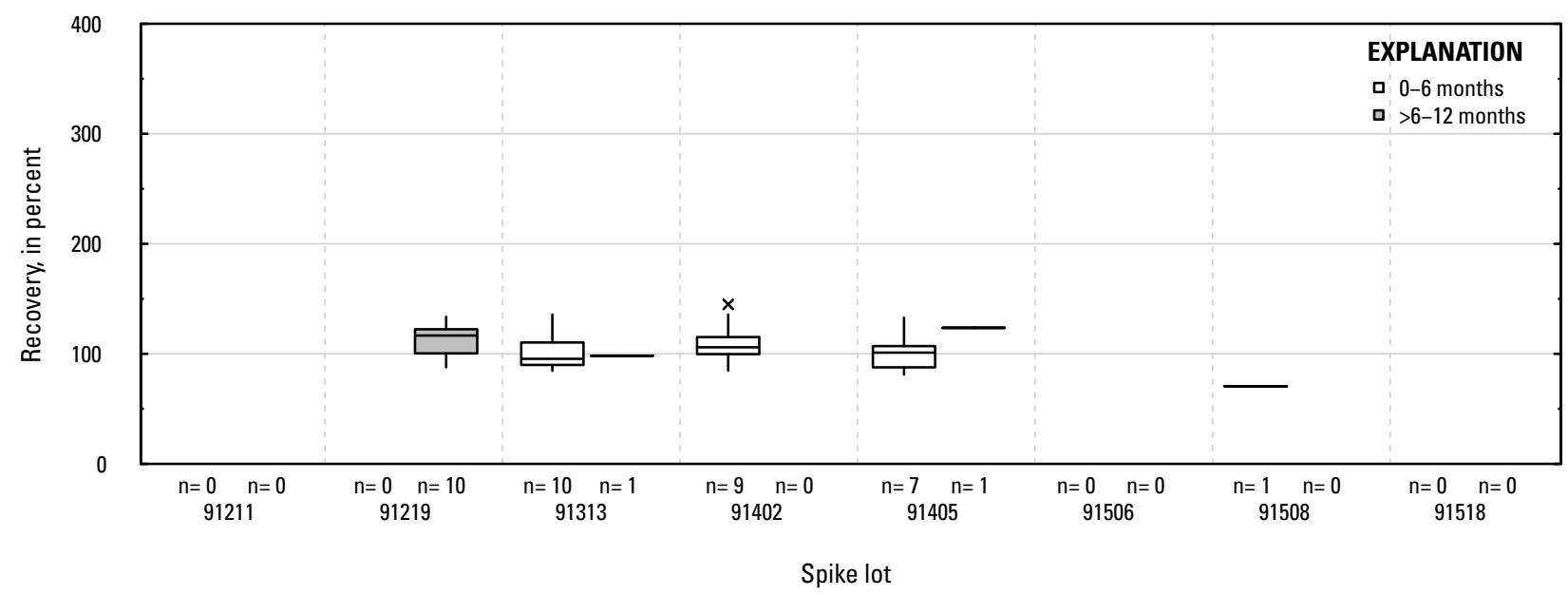

OU. MCPA: surface water field matrix spikes

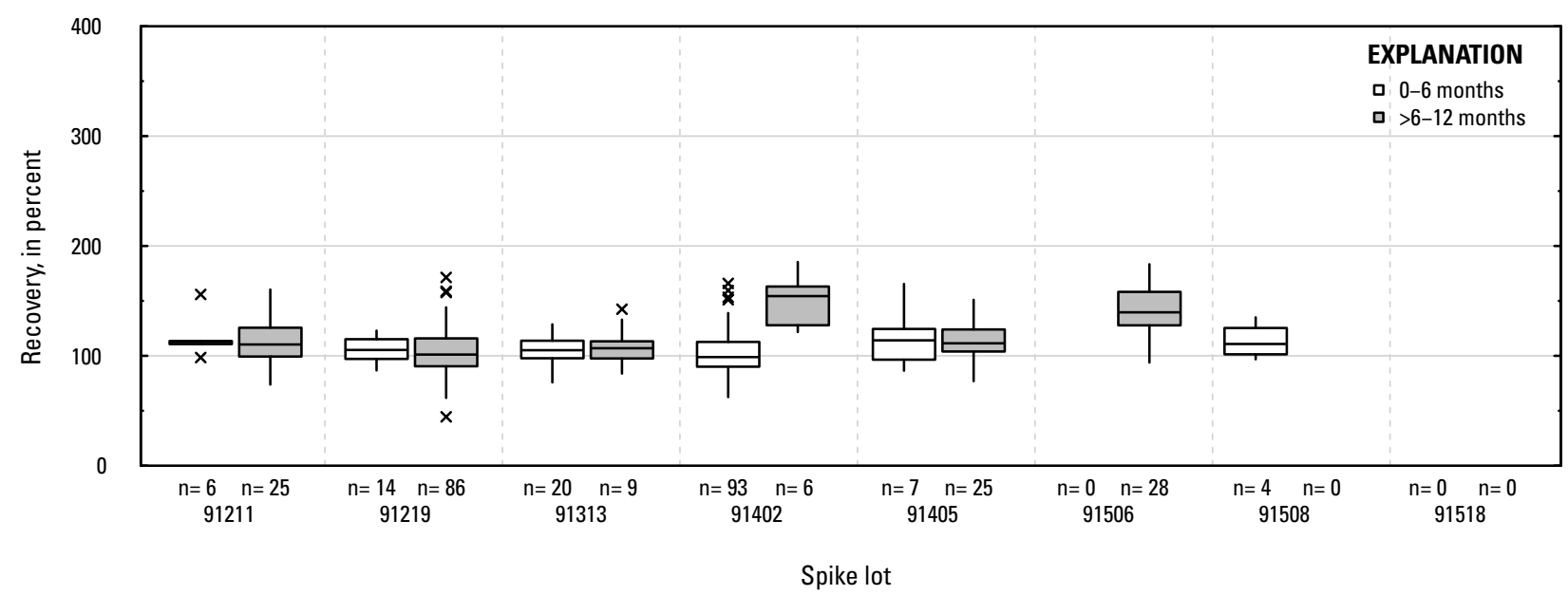

Figure 1-1. Distributions of recovery for individual pesticides in schedule 2437 by matrix, spike lot, and spike lot age. Recovery values larger than 400 percent are not shown.-Continued 
OV. Metalaxyl: laboratory reagent spikes

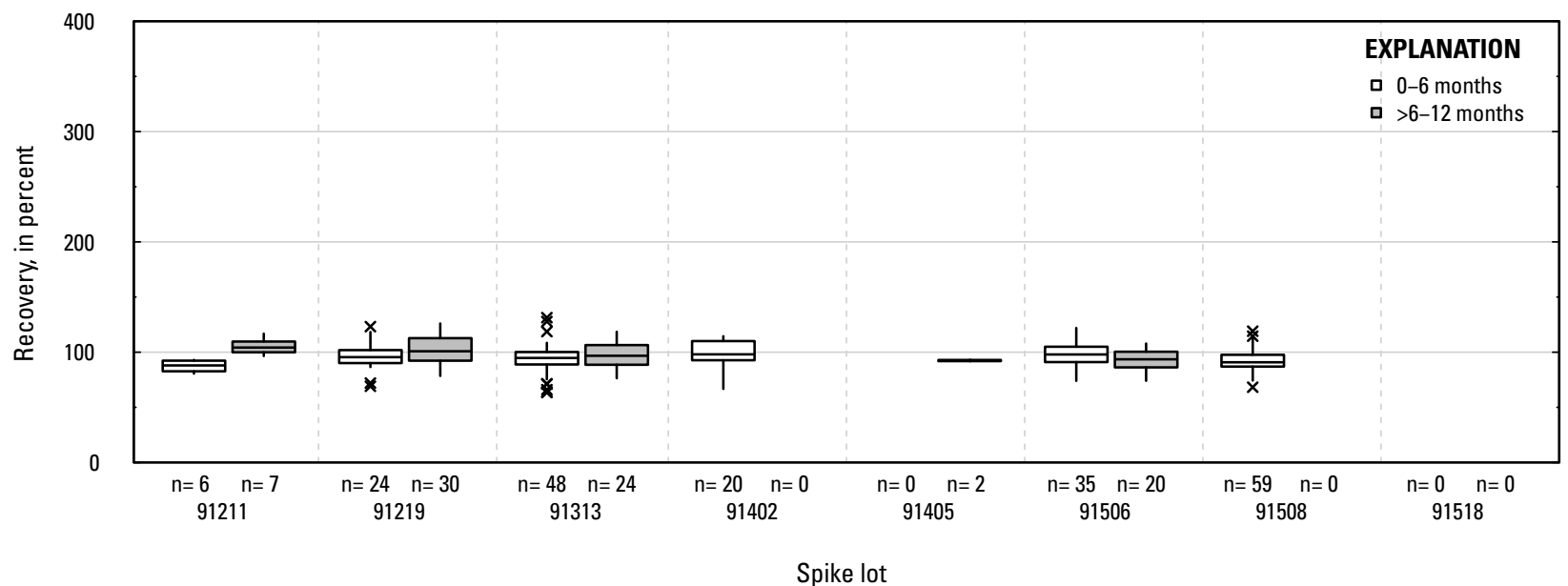

OW. Metalaxyl: groundwater field matrix spikes

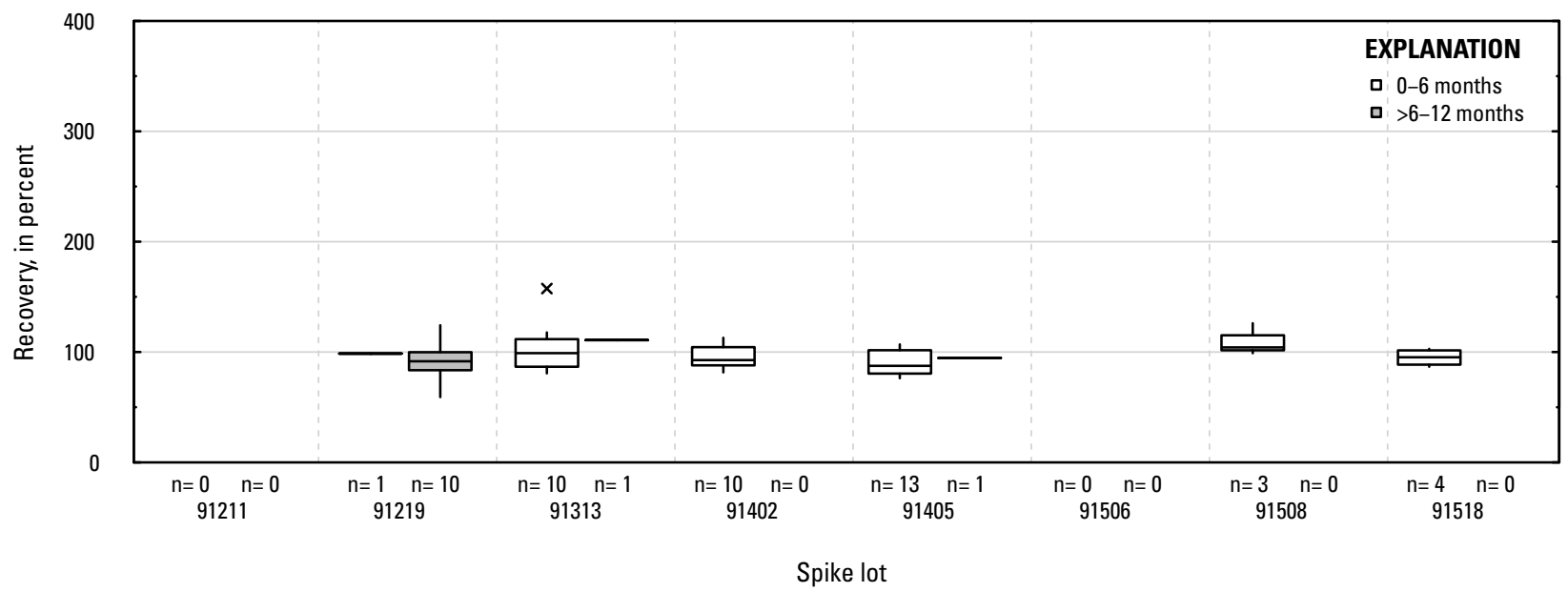

OX. Metalaxyl: surface water field matrix spikes

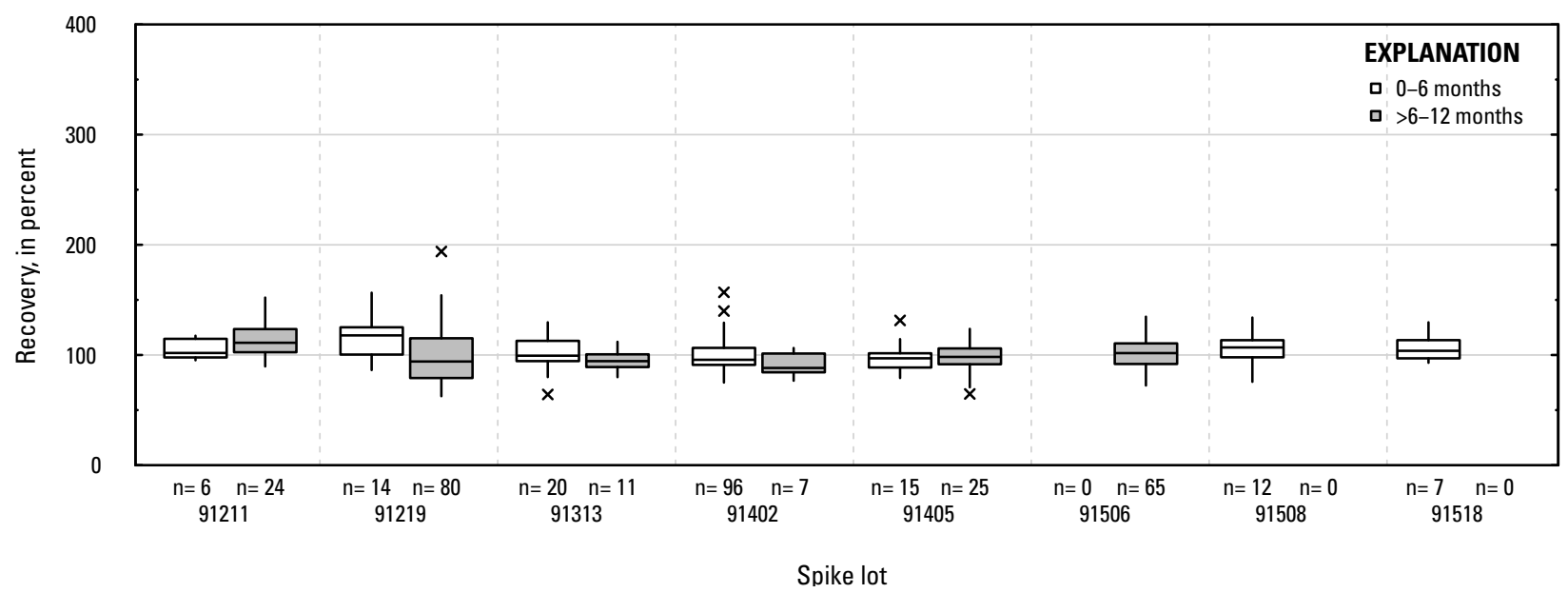

Figure 1-1. Distributions of recovery for individual pesticides in schedule 2437 by matrix, spike lot, and spike lot age. Recovery values larger than 400 percent are not shown.-Continued 
OY. Metconazole: laboratory reagent spikes

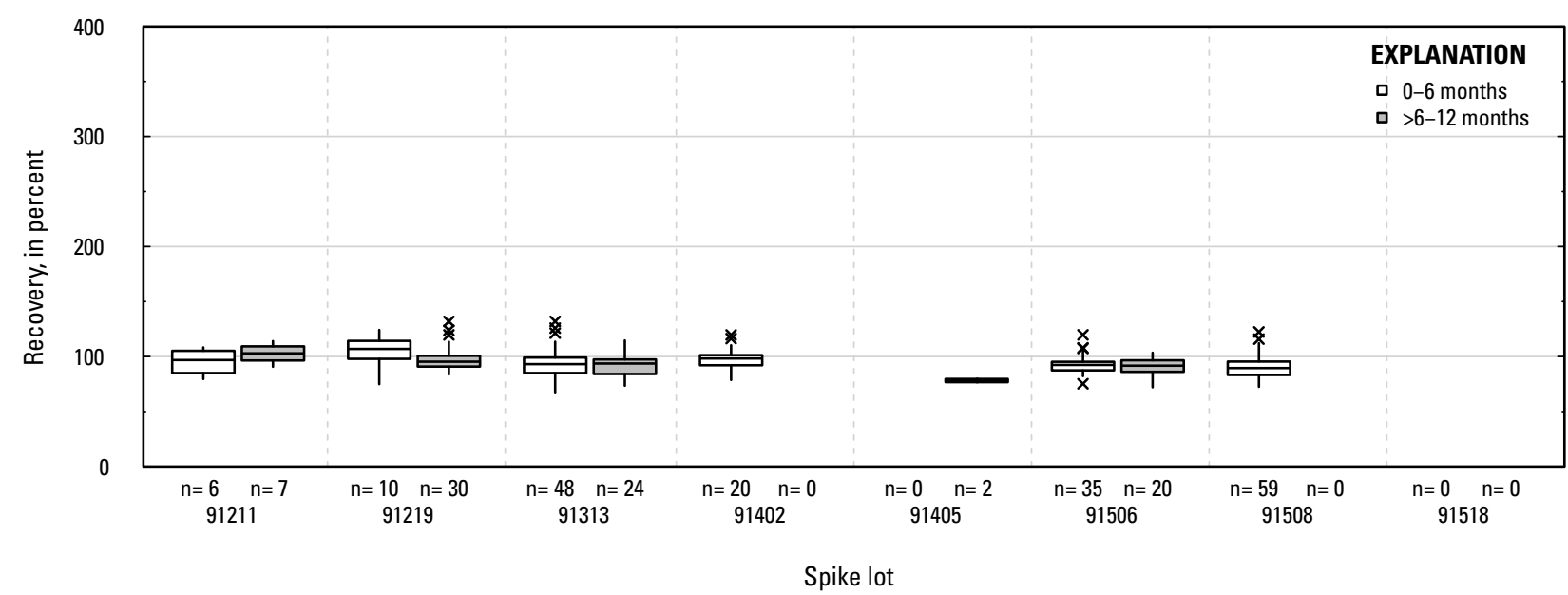

OZ. Metconazole: groundwater field matrix spikes

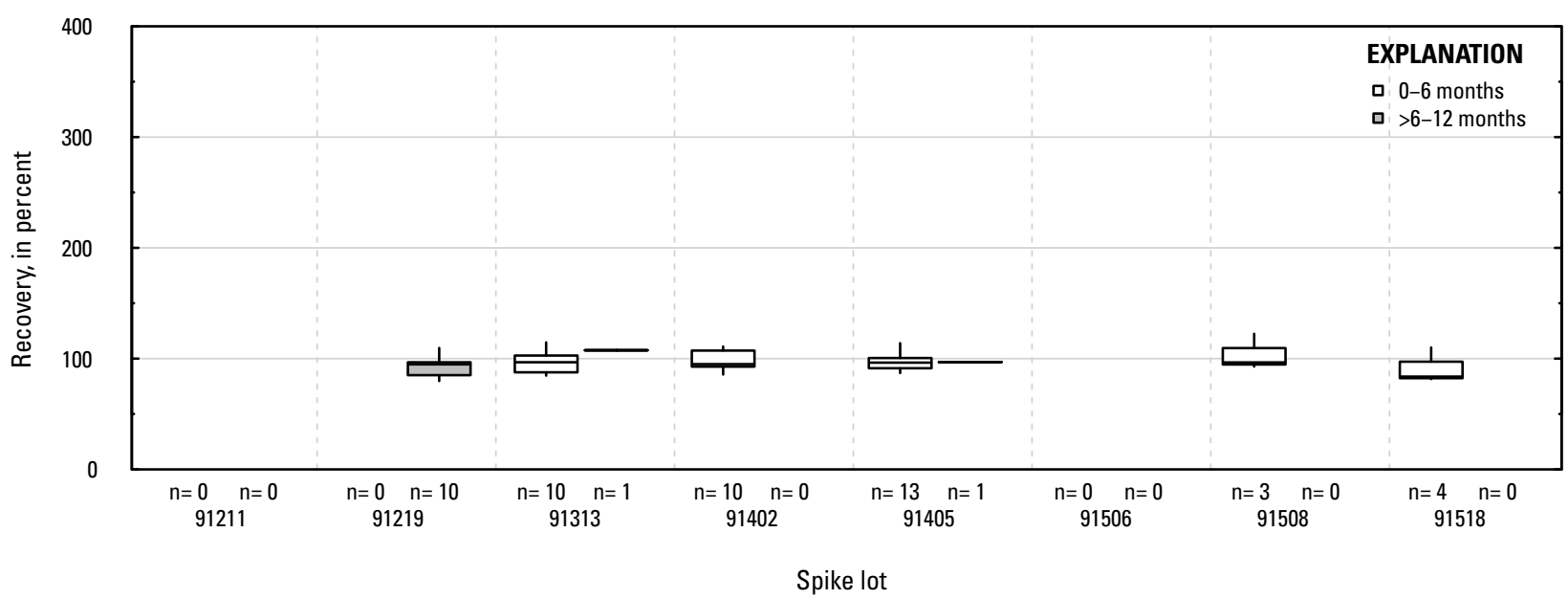

PA. Metconazole: surface water field matrix spikes

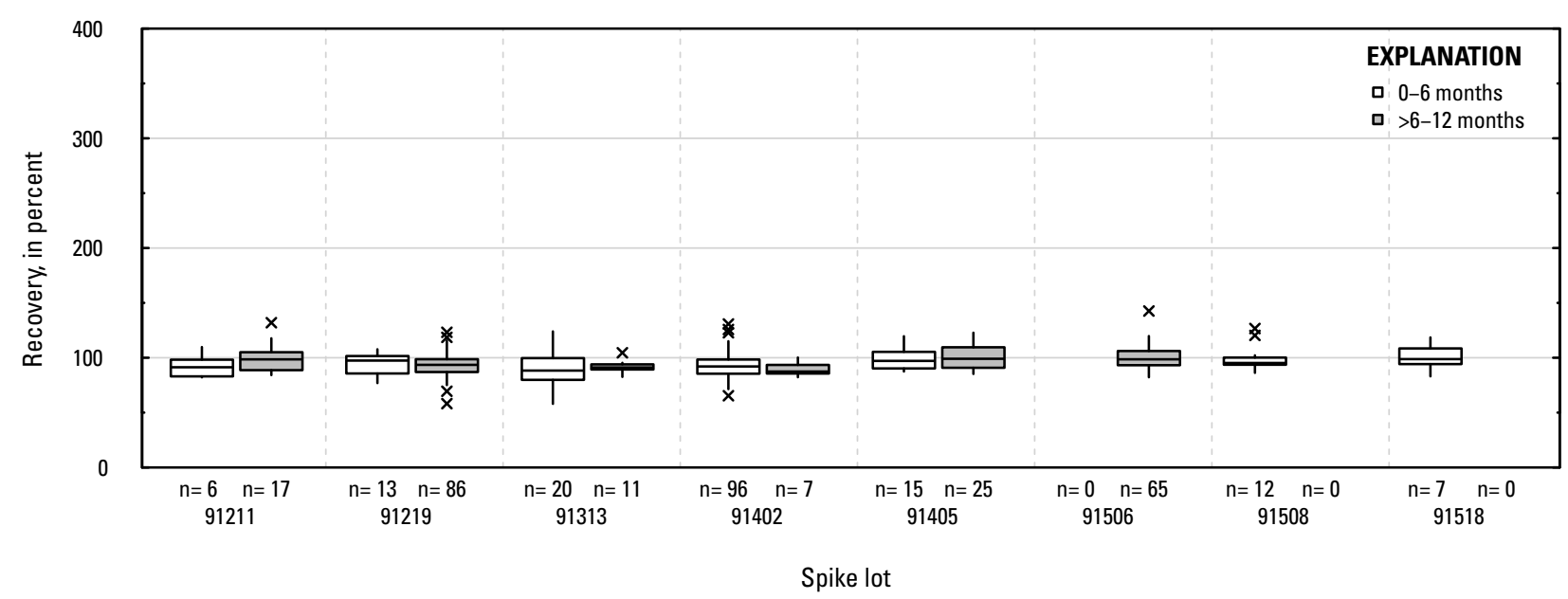

Figure 1-1. Distributions of recovery for individual pesticides in schedule 2437 by matrix, spike lot, and spike lot age. Recovery values larger than 400 percent are not shown.-Continued 
$P B$. Methamidophos: laboratory reagent spikes

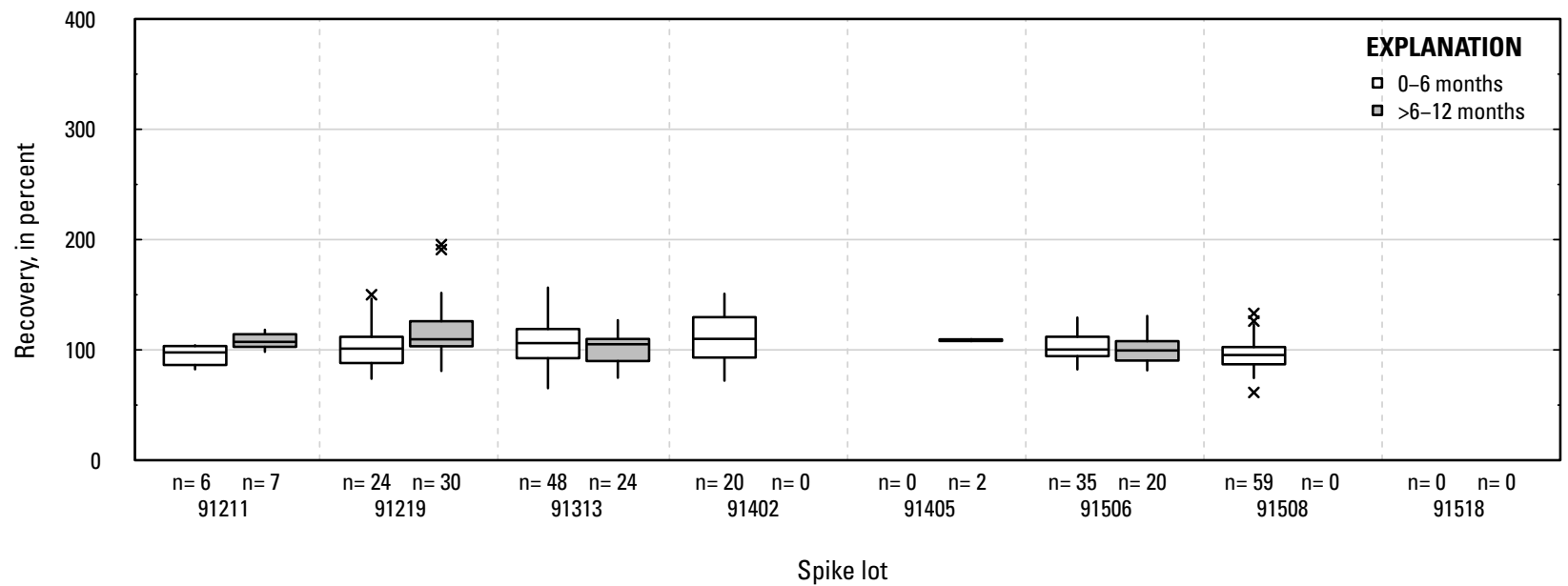

PC. Methamidophos: groundwater field matrix spikes

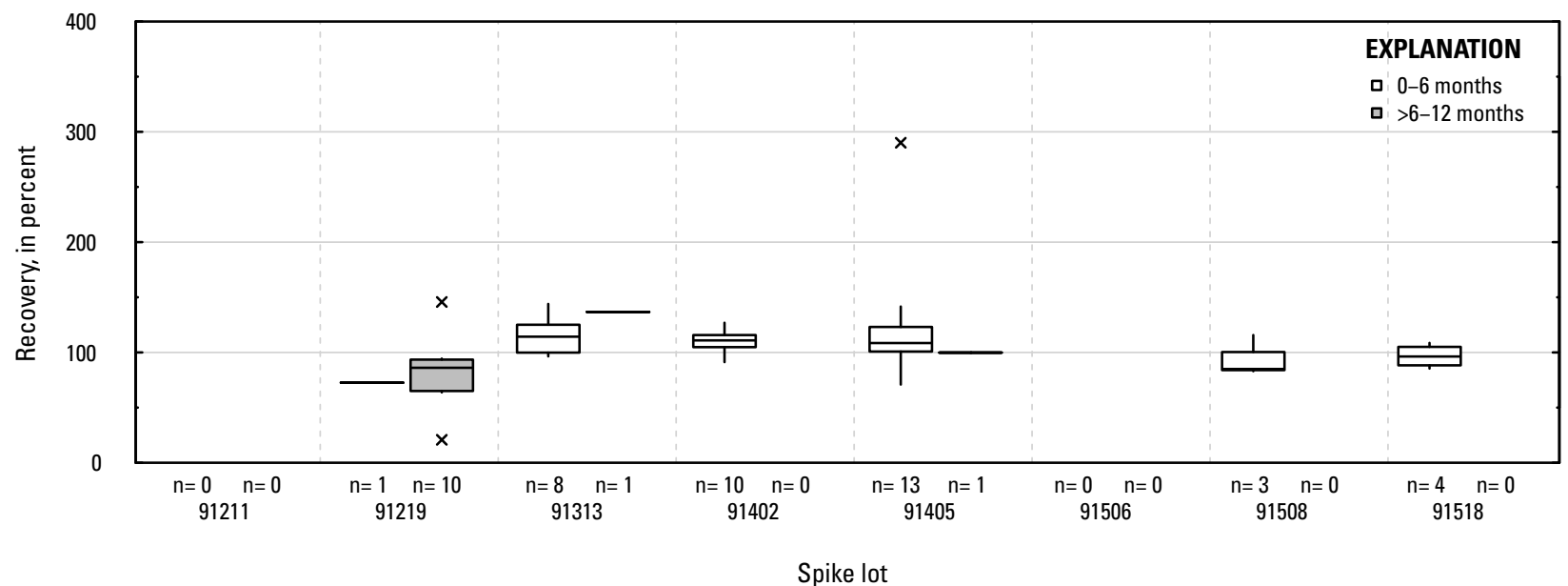

PD. Methamidophos: surface water field matrix spikes

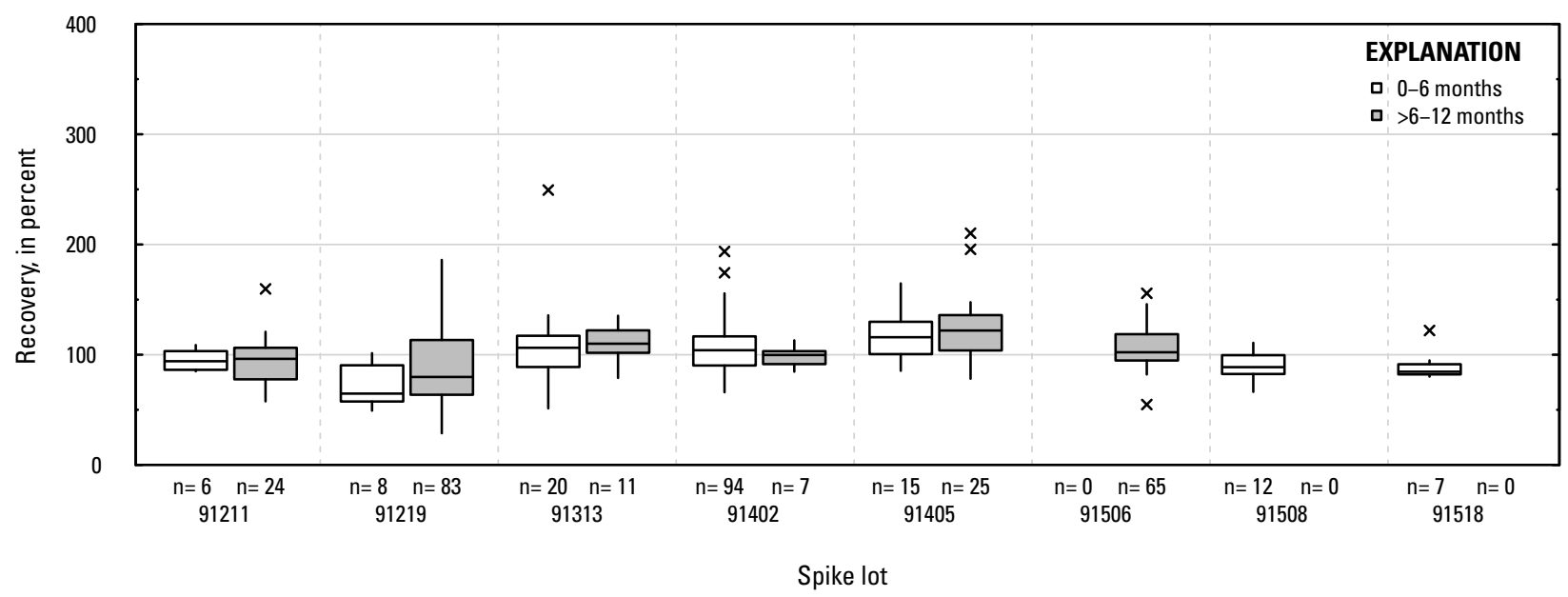

Figure 1-1. Distributions of recovery for individual pesticides in schedule 2437 by matrix, spike lot, and spike lot age. Recovery values larger than 400 percent are not shown.-Continued 
PE. Methidathion: laboratory reagent spikes

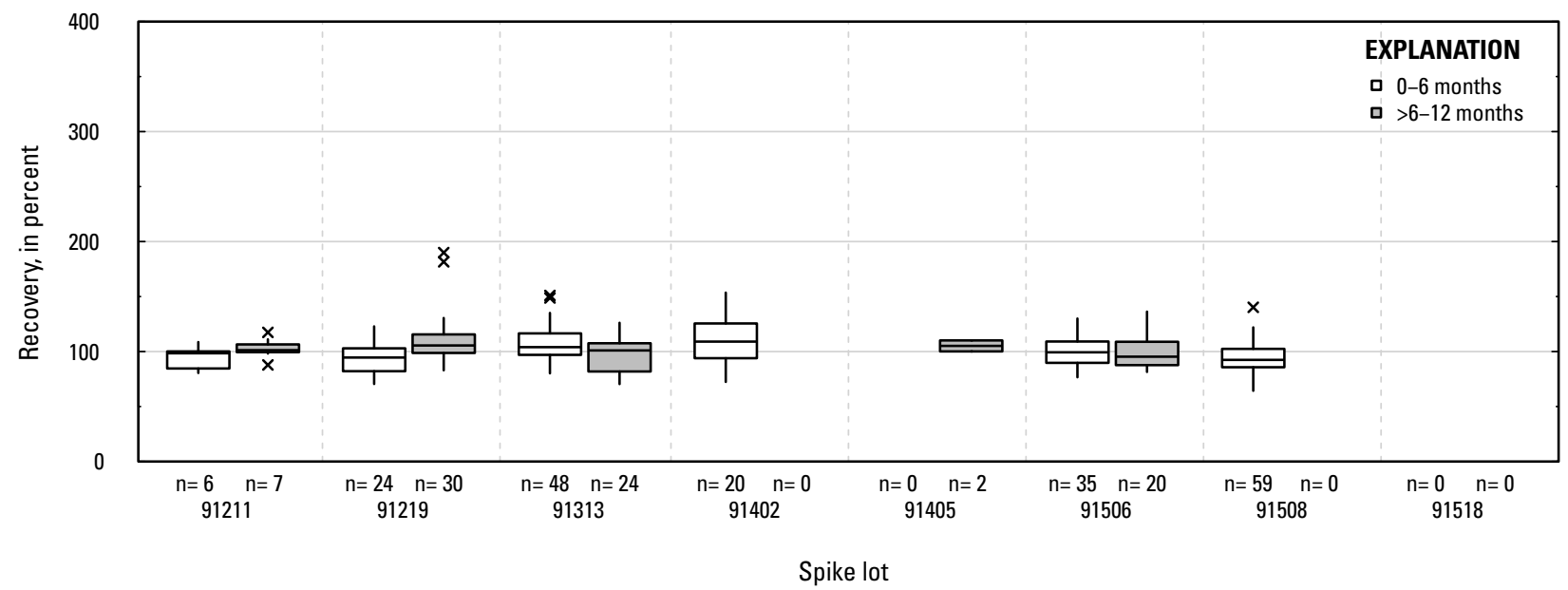

PF. Methidathion: groundwater field matrix spikes

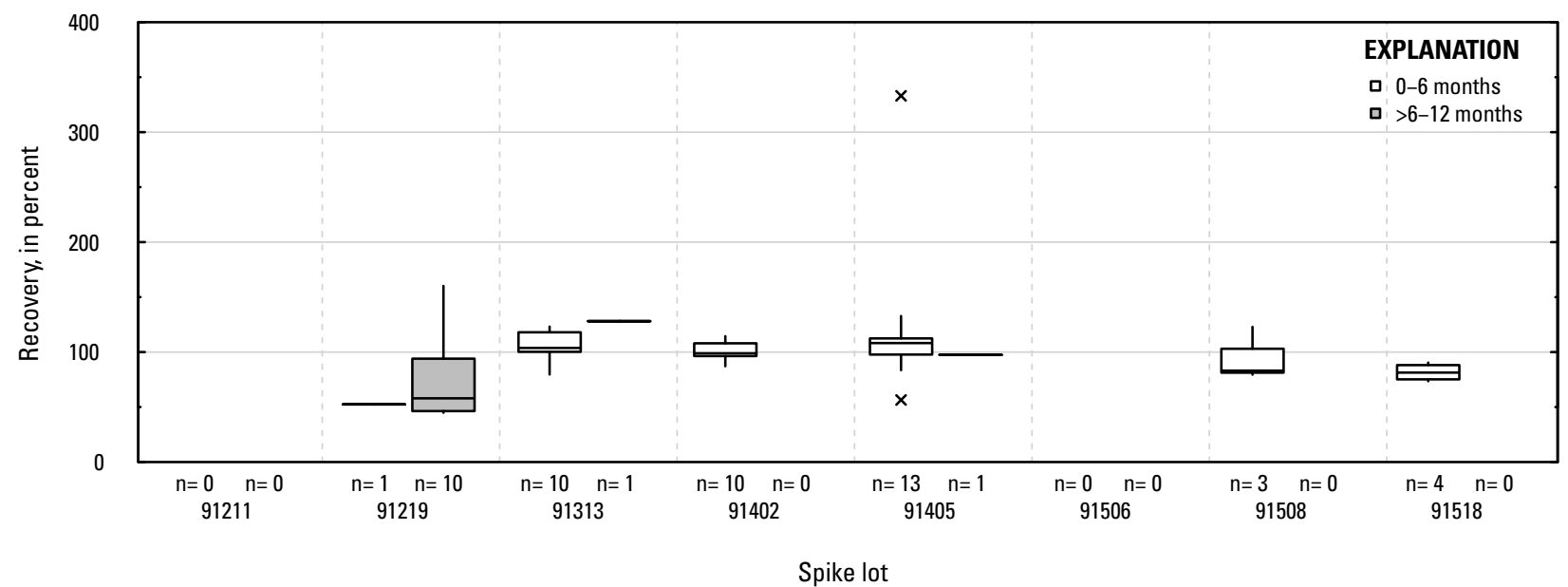

PG. Methidathion: surface water field matrix spikes

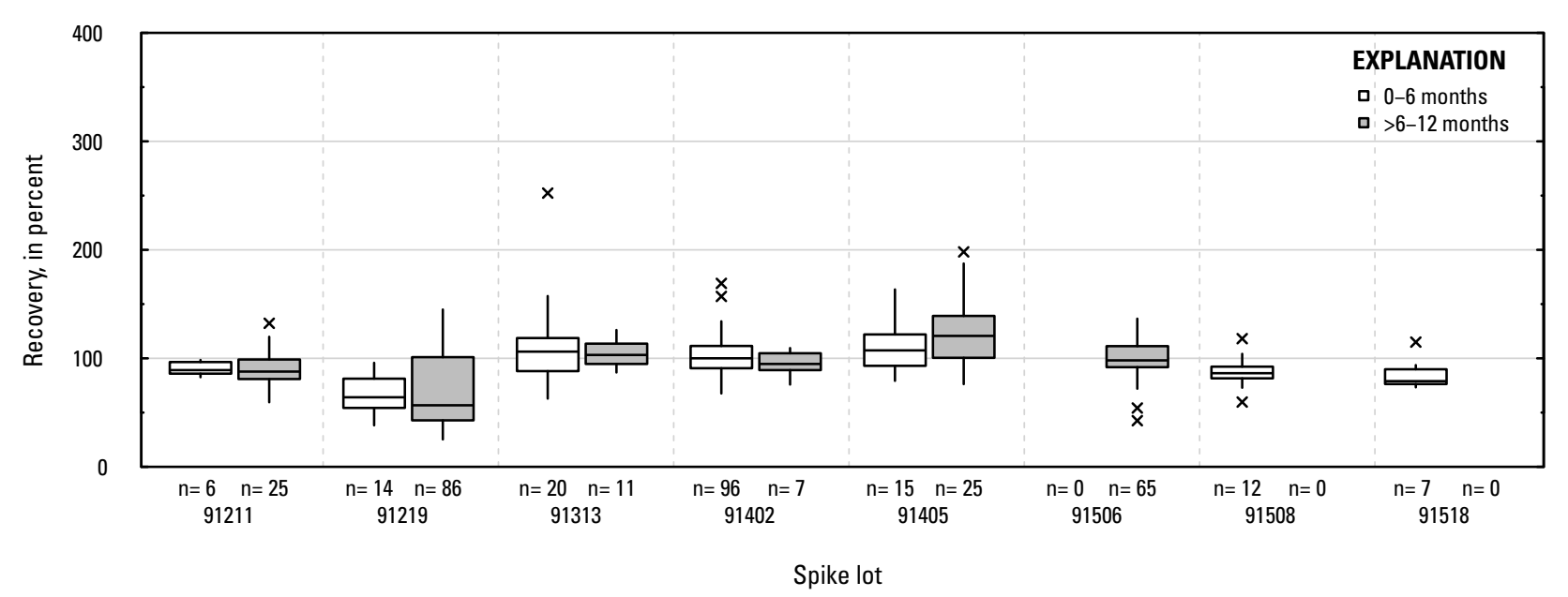

Figure 1-1. Distributions of recovery for individual pesticides in schedule 2437 by matrix, spike lot, and spike lot age. Recovery values larger than 400 percent are not shown.-Continued 
PH. Methomyl: laboratory reagent spikes

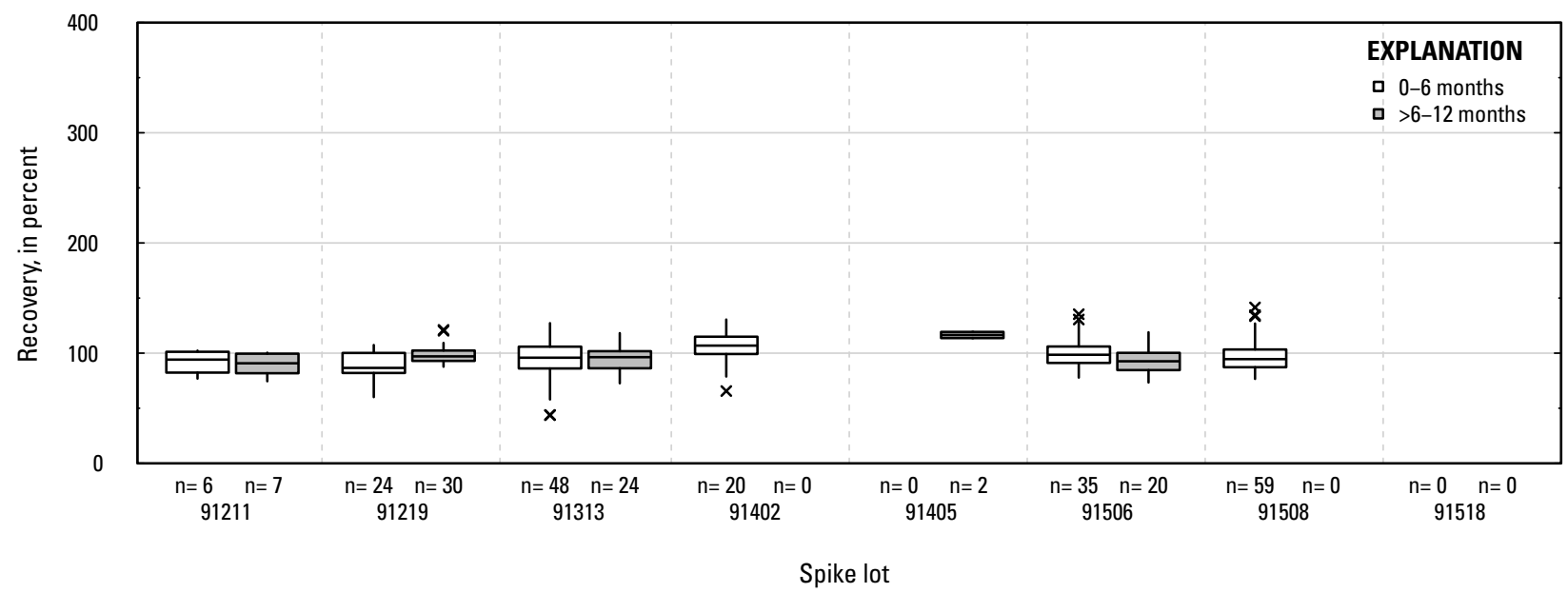

\section{PI. Methomyl: groundwater field matrix spikes}

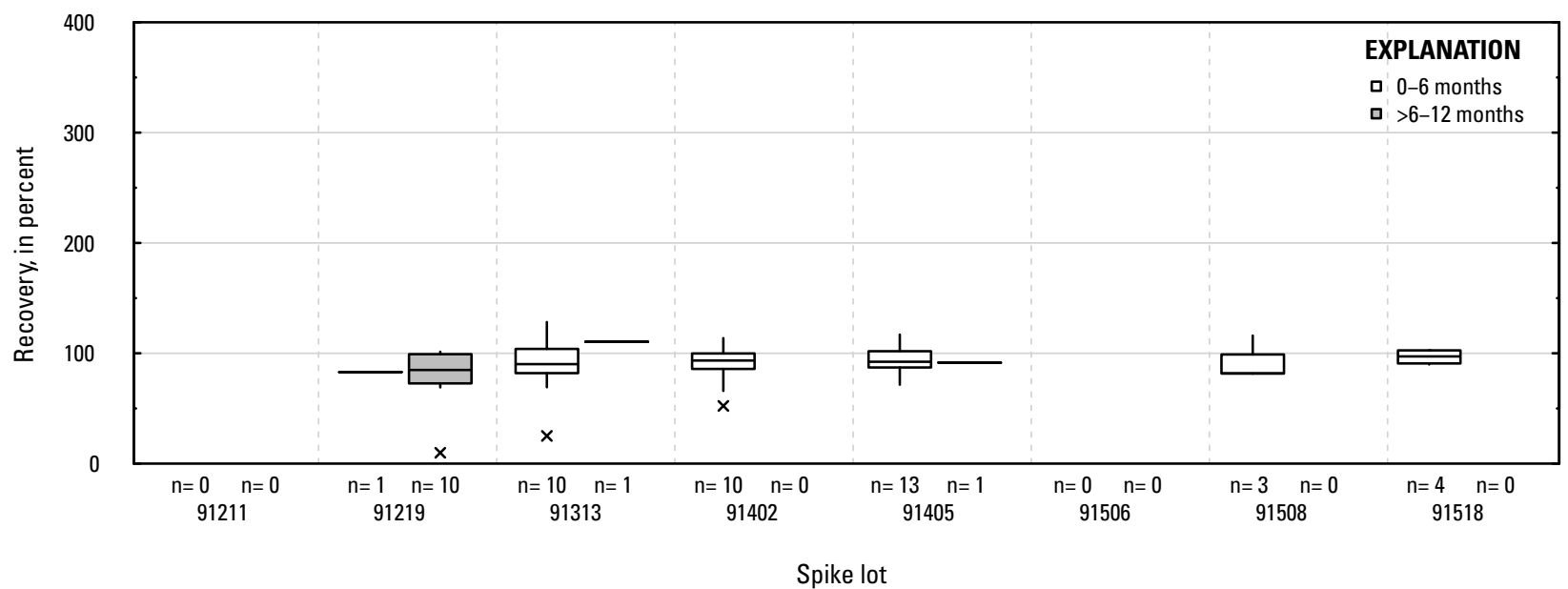

\section{PJ. Methomyl: surface water field matrix spikes}

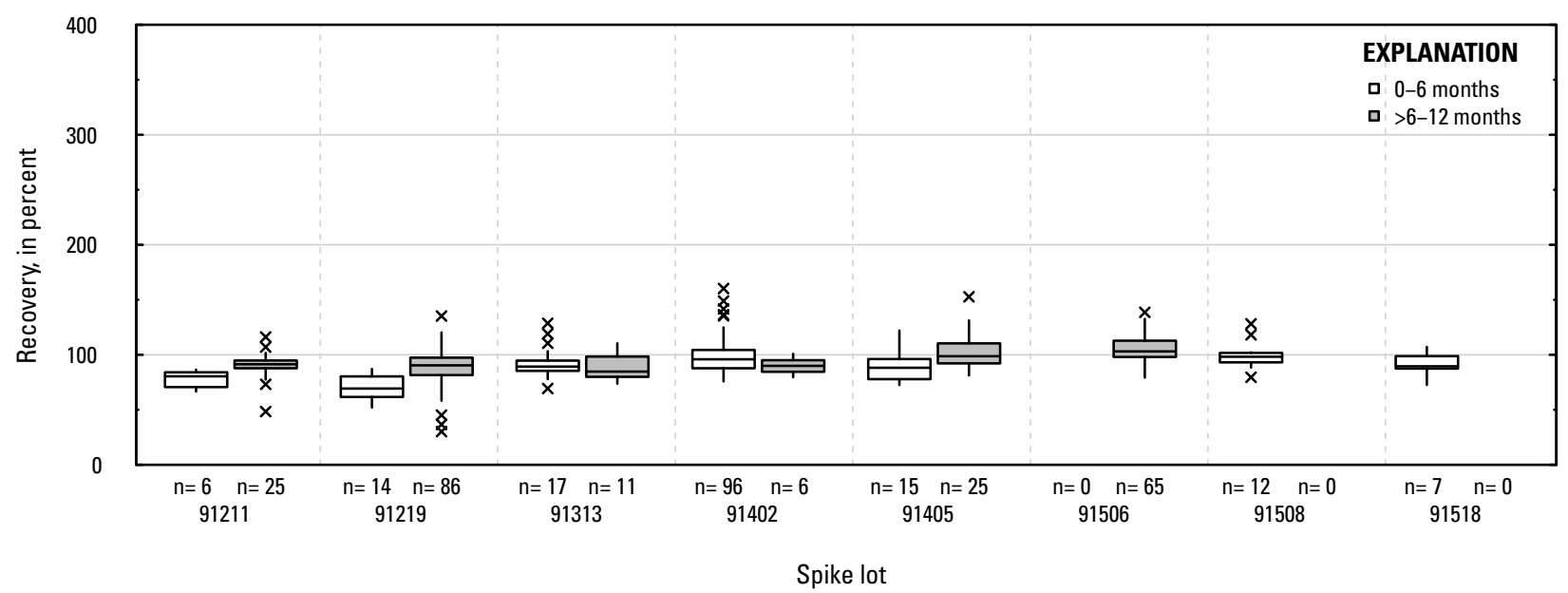

Figure 1-1. Distributions of recovery for individual pesticides in schedule 2437 by matrix, spike lot, and spike lot age. Recovery values larger than 400 percent are not shown.-Continued 
PK. Methomyl oxime: laboratory reagent spikes

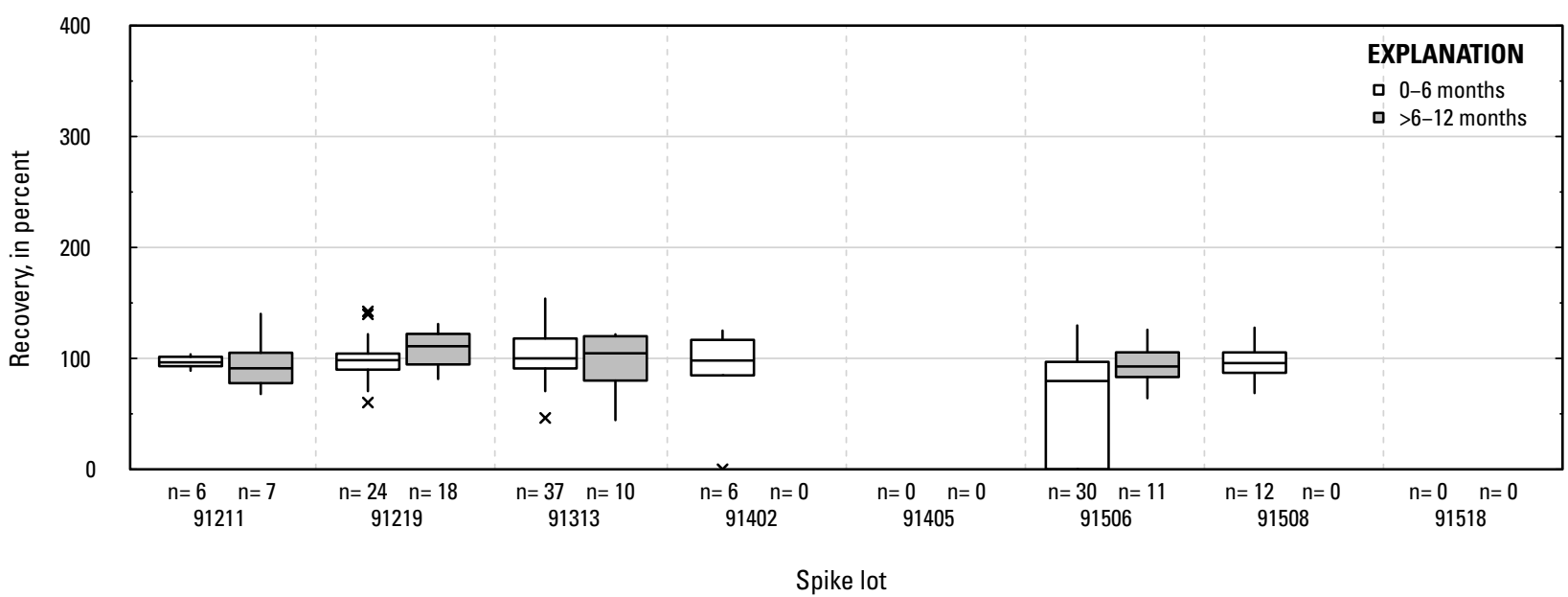

PL. Methomyl oxime: groundwater field matrix spikes

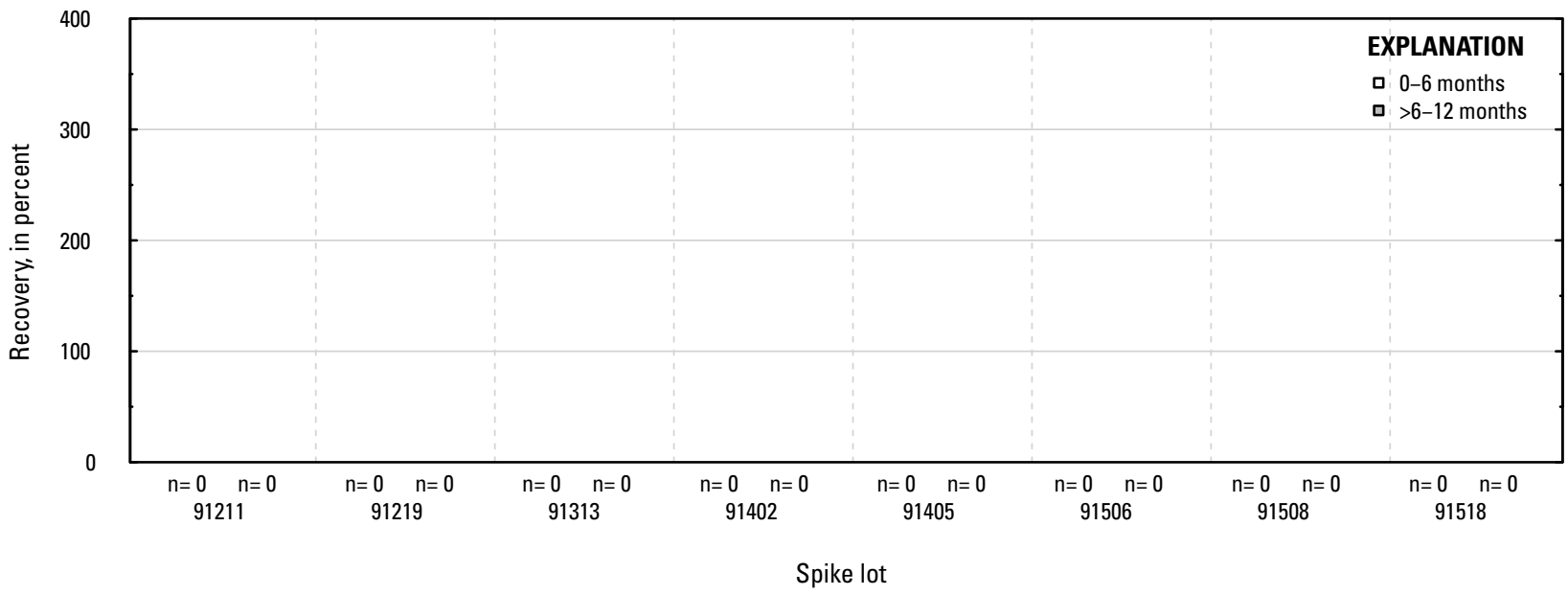

PM. Methomyl oxime: surface water field matrix spikes

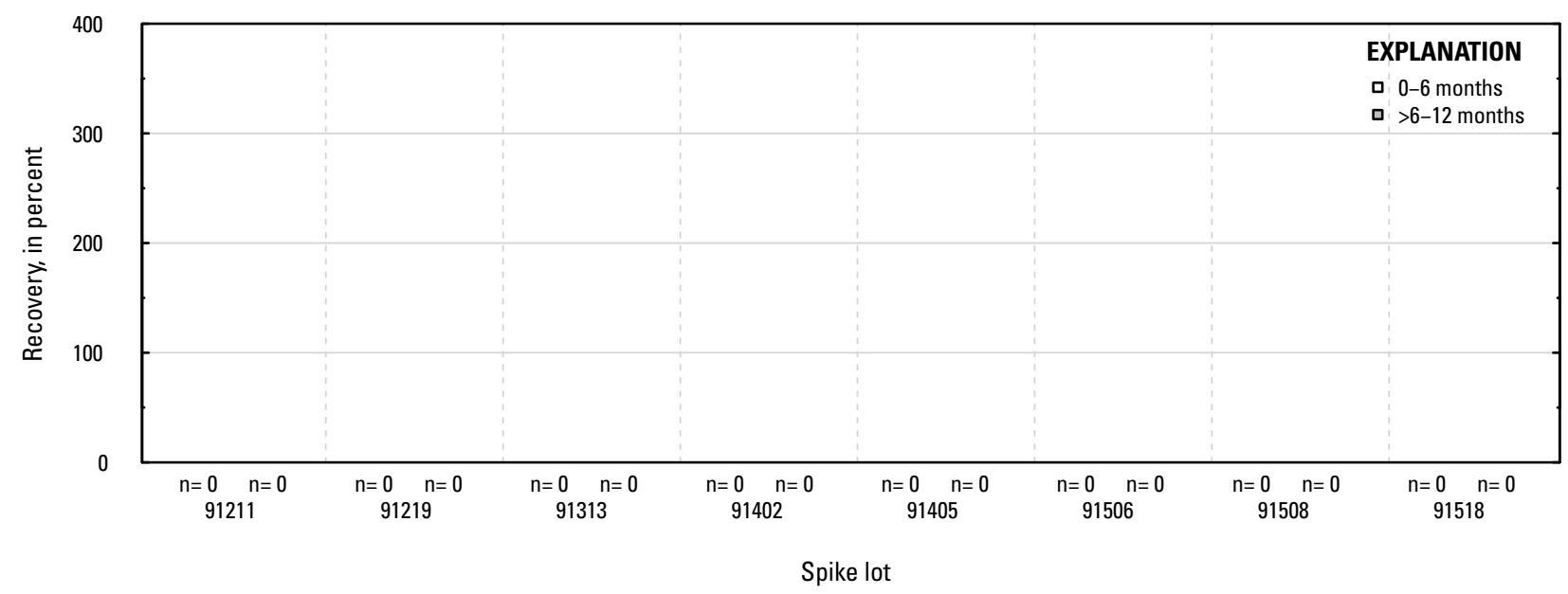

Figure 1-1. Distributions of recovery for individual pesticides in schedule 2437 by matrix, spike lot, and spike lot age. Recovery values larger than 400 percent are not shown.-Continued 
PN. Methoxyfenozide: laboratory reagent spikes

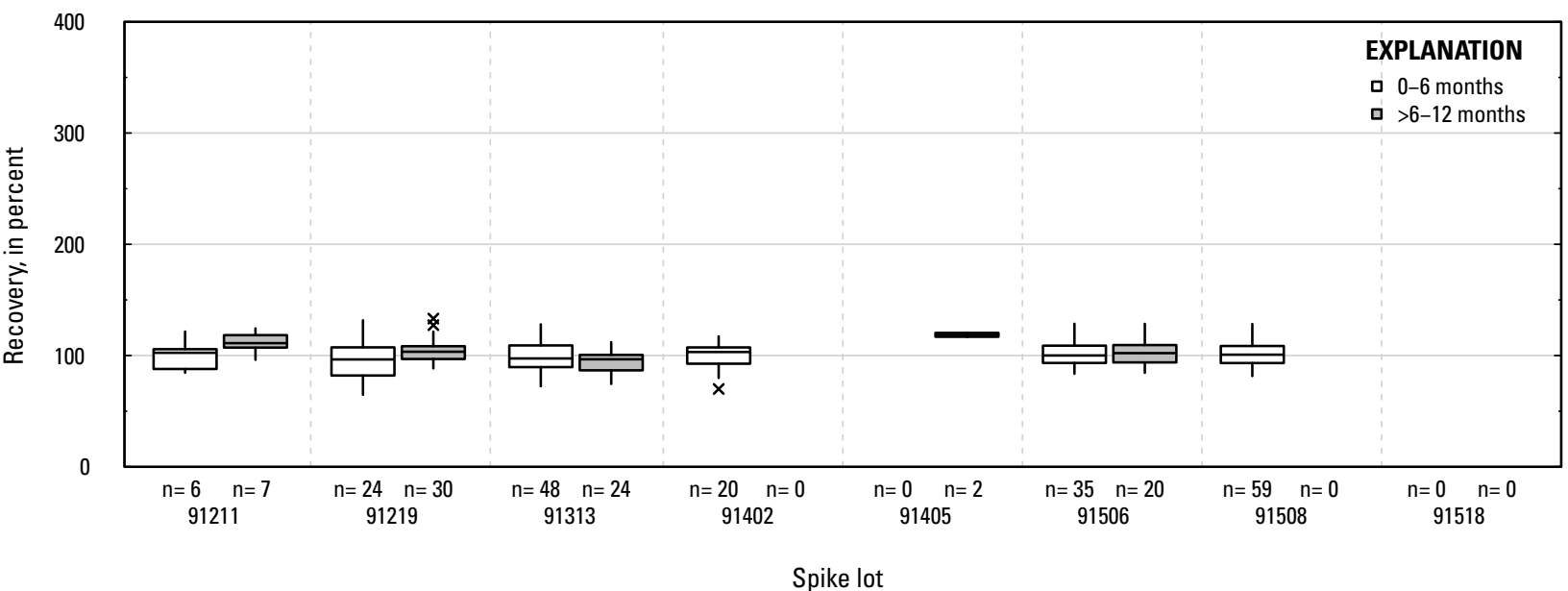

PO. Methoxyfenozide: groundwater field matrix spikes

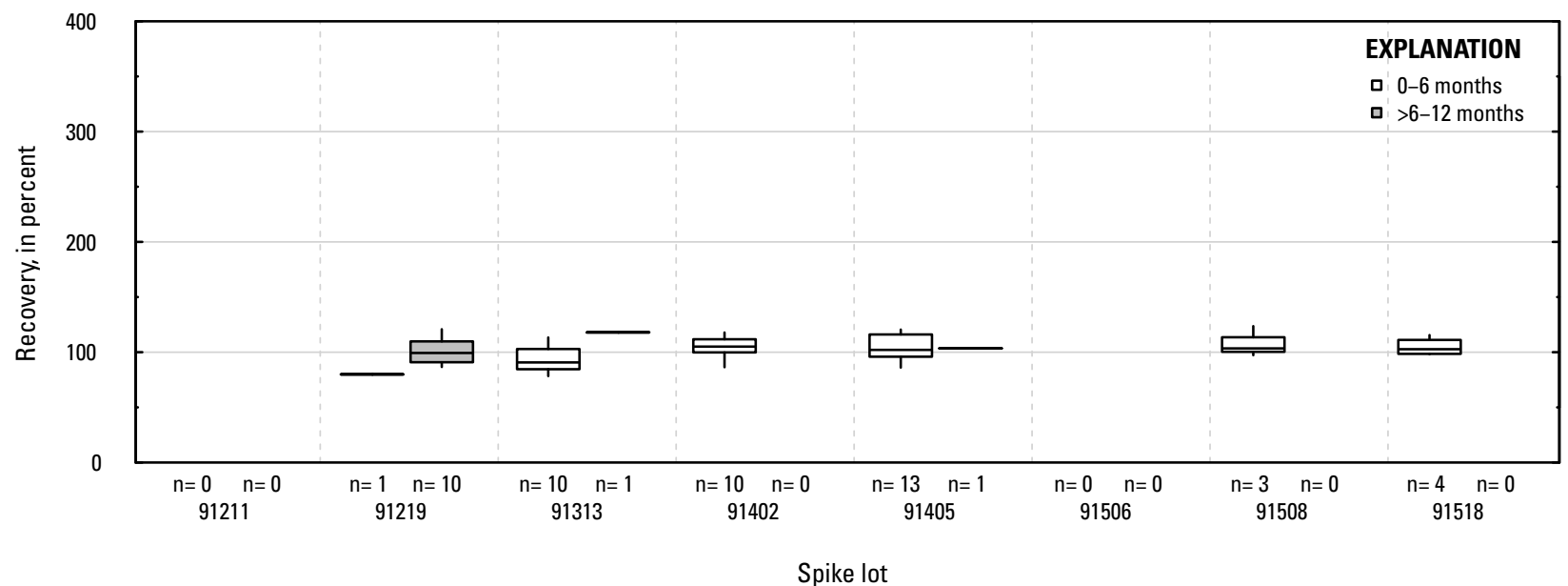

PP. Methoxyfenozide: surface water field matrix spikes

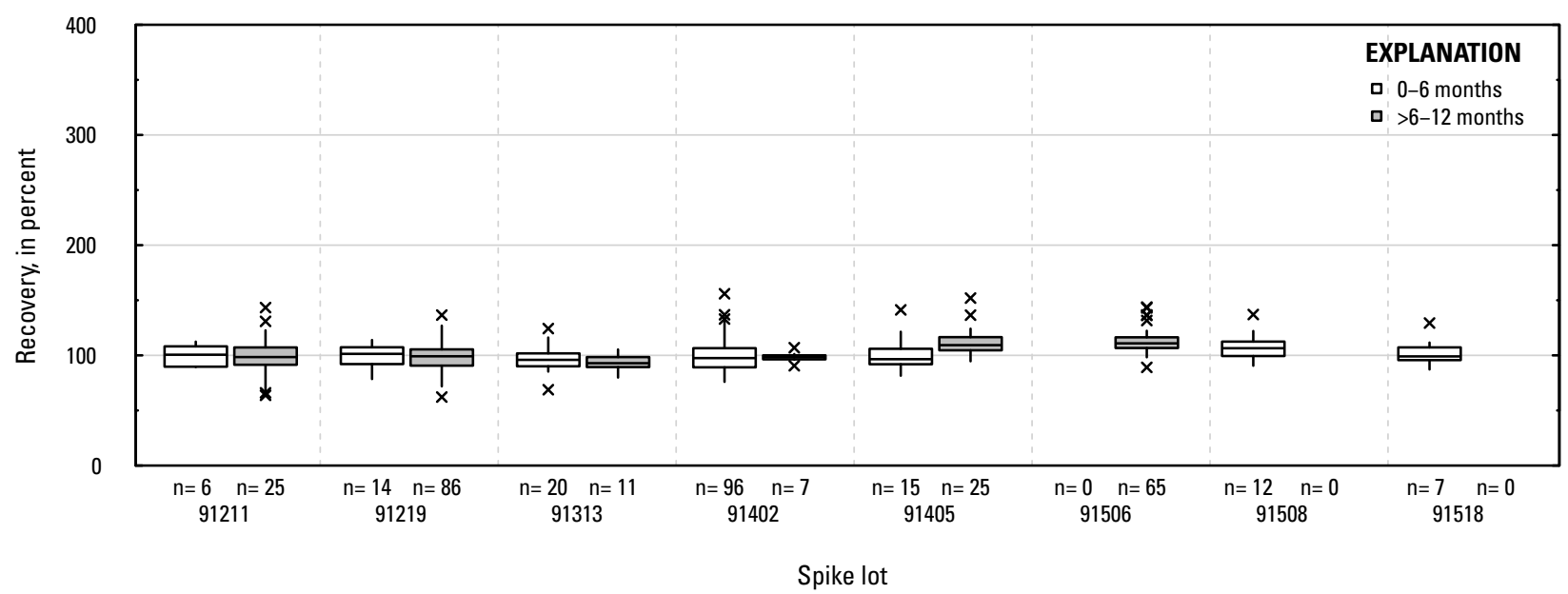

Figure 1-1. Distributions of recovery for individual pesticides in schedule 2437 by matrix, spike lot, and spike lot age. Recovery values larger than 400 percent are not shown.-Continued 
PQ. Metolachlor: laboratory reagent spikes

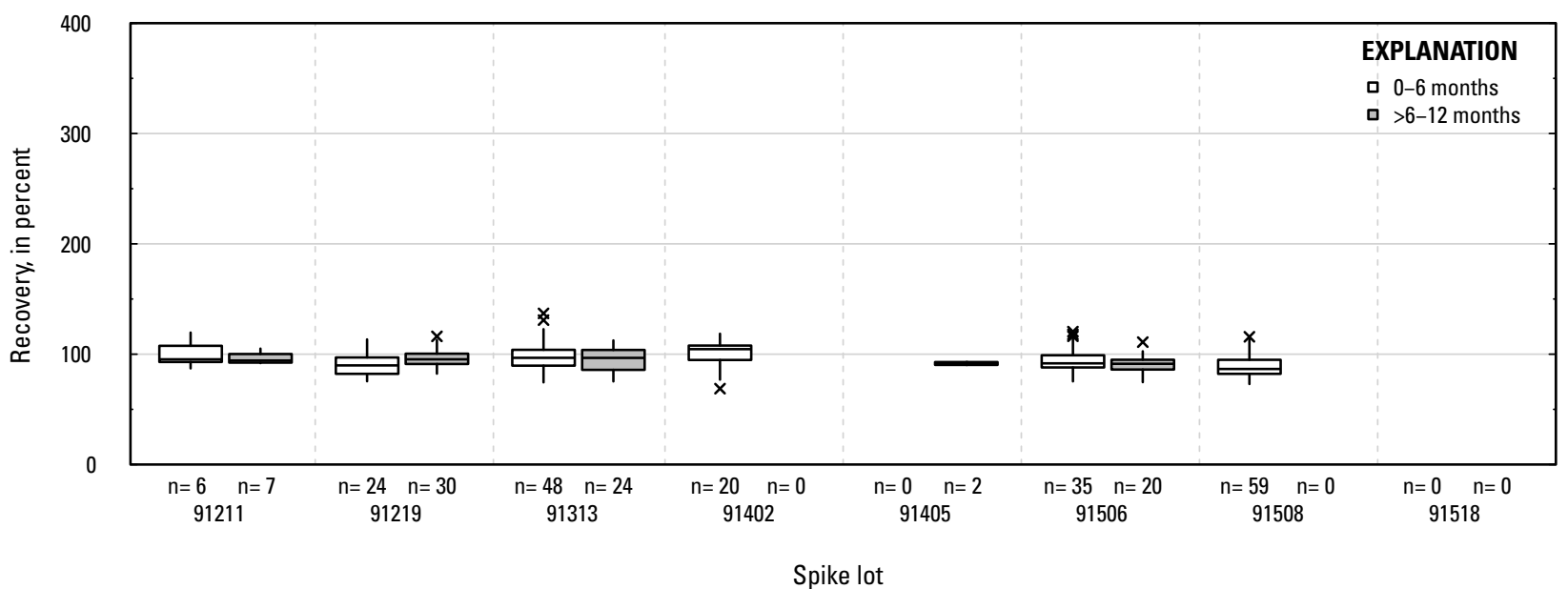

PR. Metolachlor: groundwater field matrix spikes

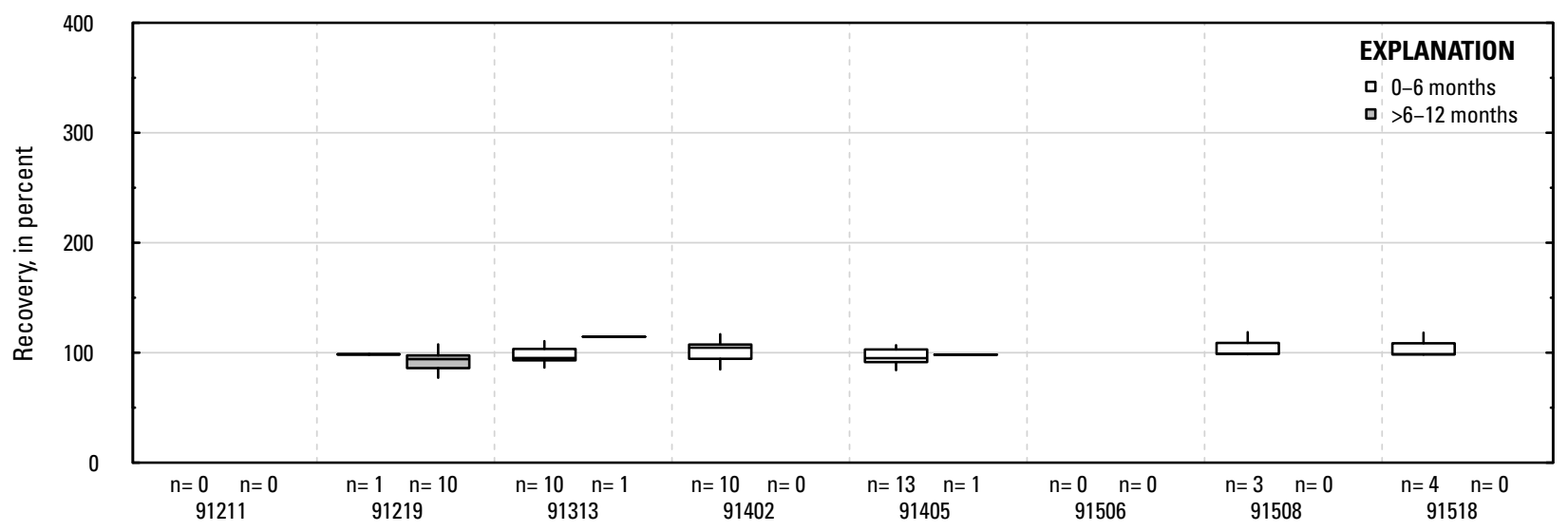

Spike lot

PS. Metolachlor: surface water field matrix spikes

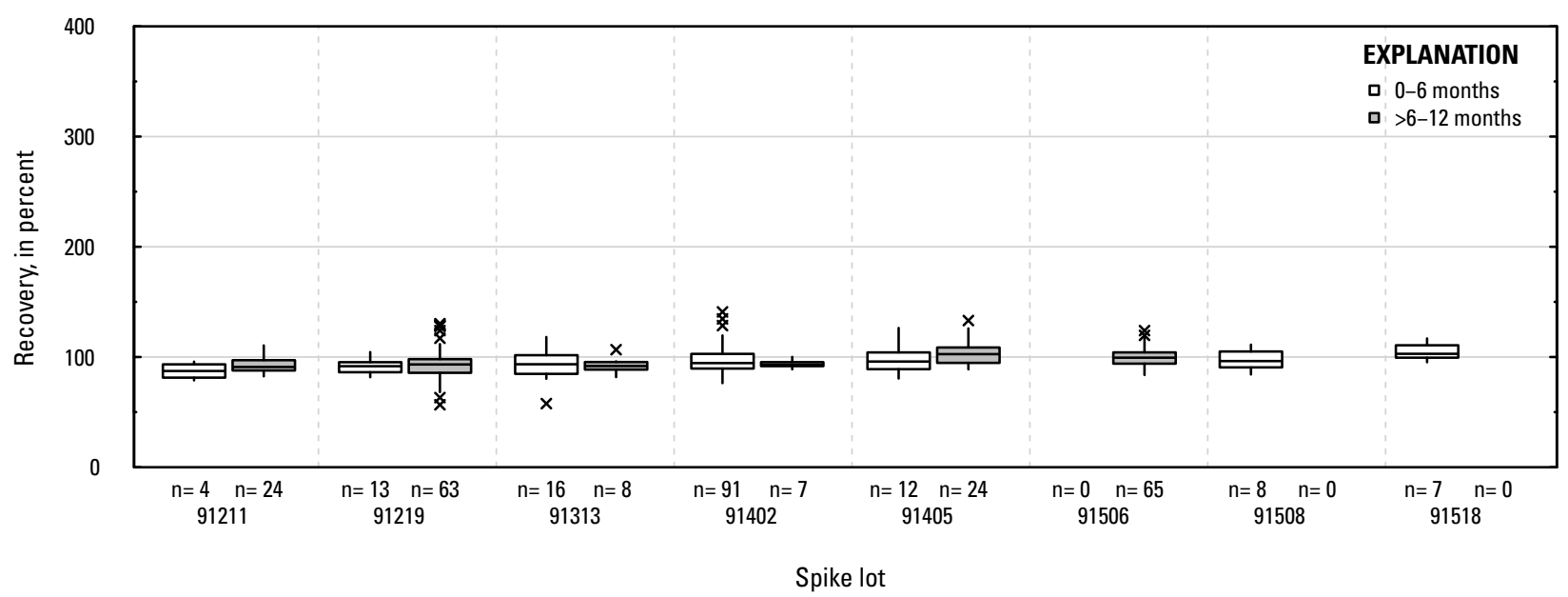

Figure 1-1. Distributions of recovery for individual pesticides in schedule 2437 by matrix, spike lot, and spike lot age. Recovery values larger than 400 percent are not shown.-Continued 
PT. Metolachlor hydroxy morpholinone: laboratory reagent spikes

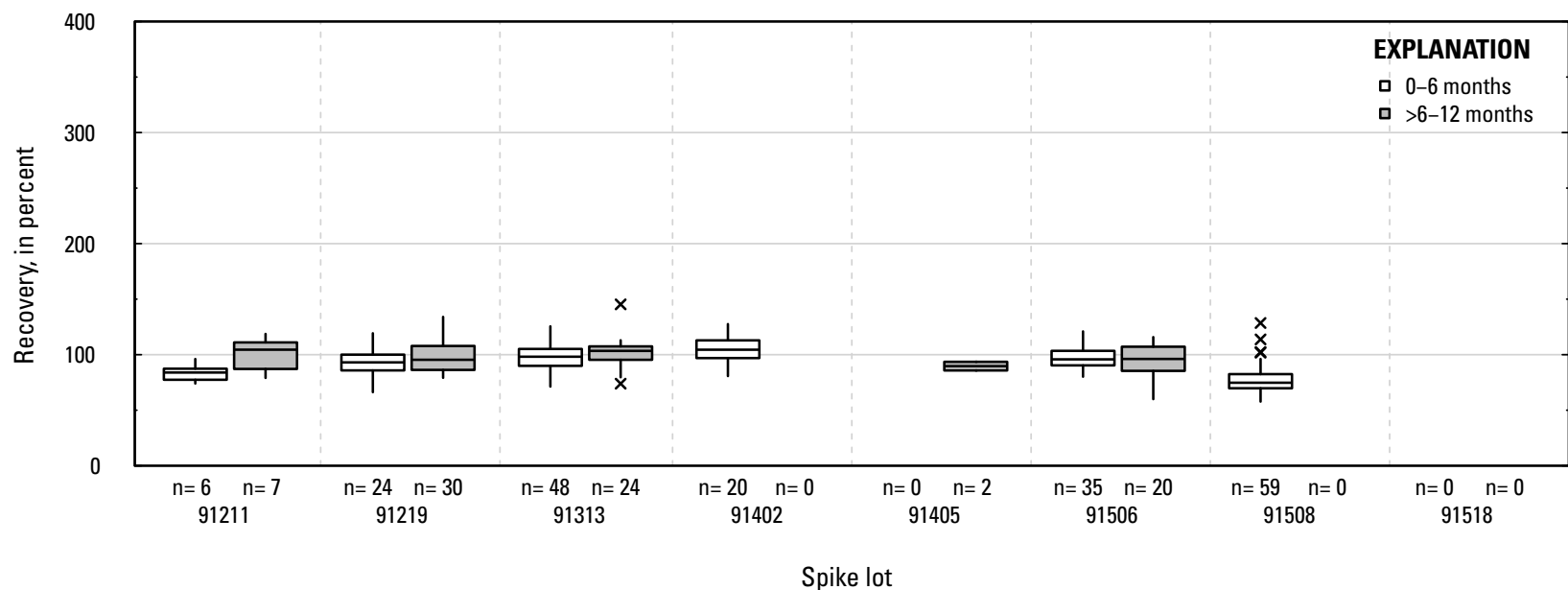

PU. Metolachlor hydroxy morpholinone: groundwater field matrix spikes

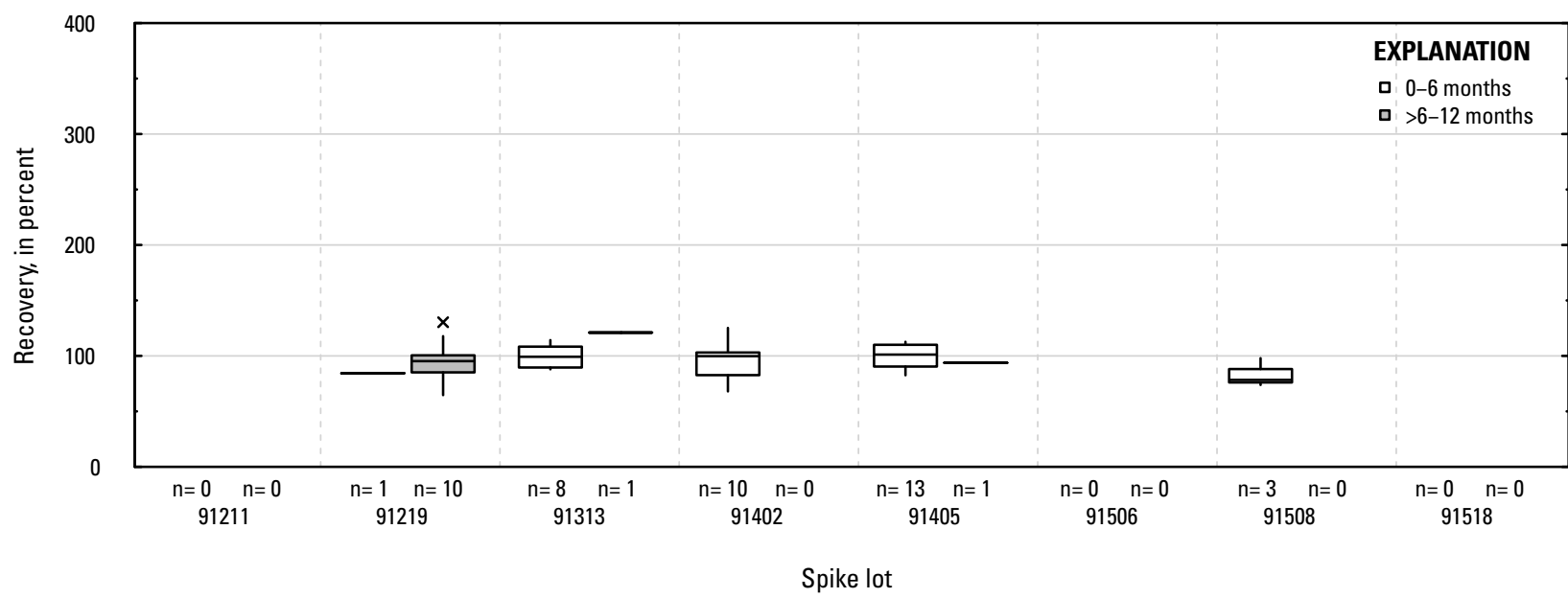

PV. Metolachlor hydroxy morpholinone: surface water field matrix spikes

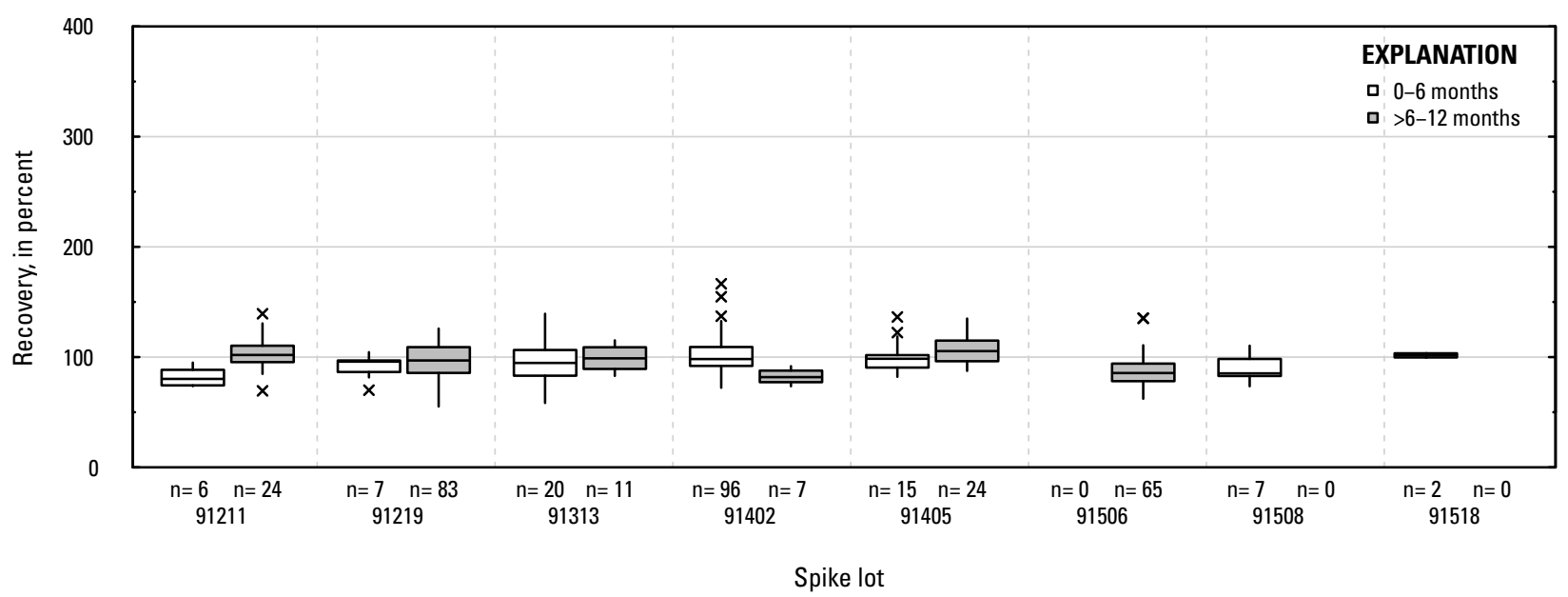

Figure 1-1. Distributions of recovery for individual pesticides in schedule 2437 by matrix, spike lot, and spike lot age. Recovery values larger than 400 percent are not shown.-Continued 
PW. Metolachlor oxanilic acid: laboratory reagent spikes

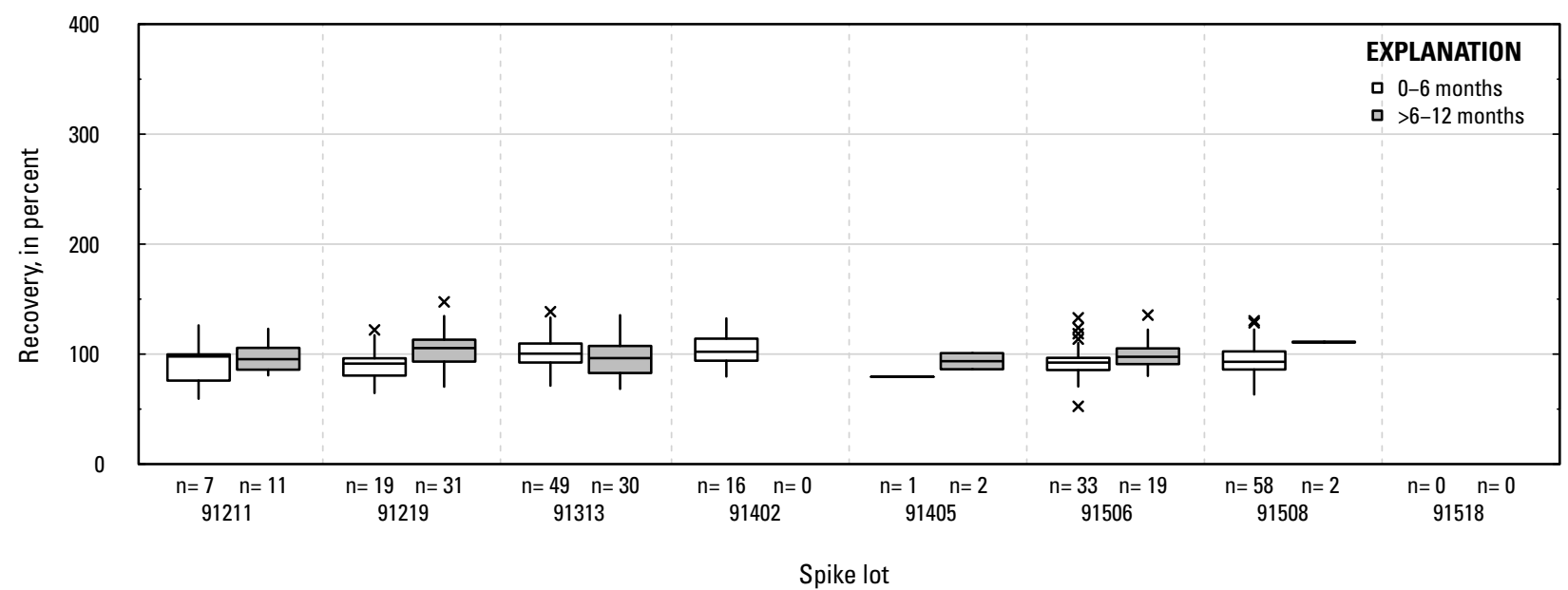

PX. Metolachlor oxanilic acid: groundwater field matrix spikes

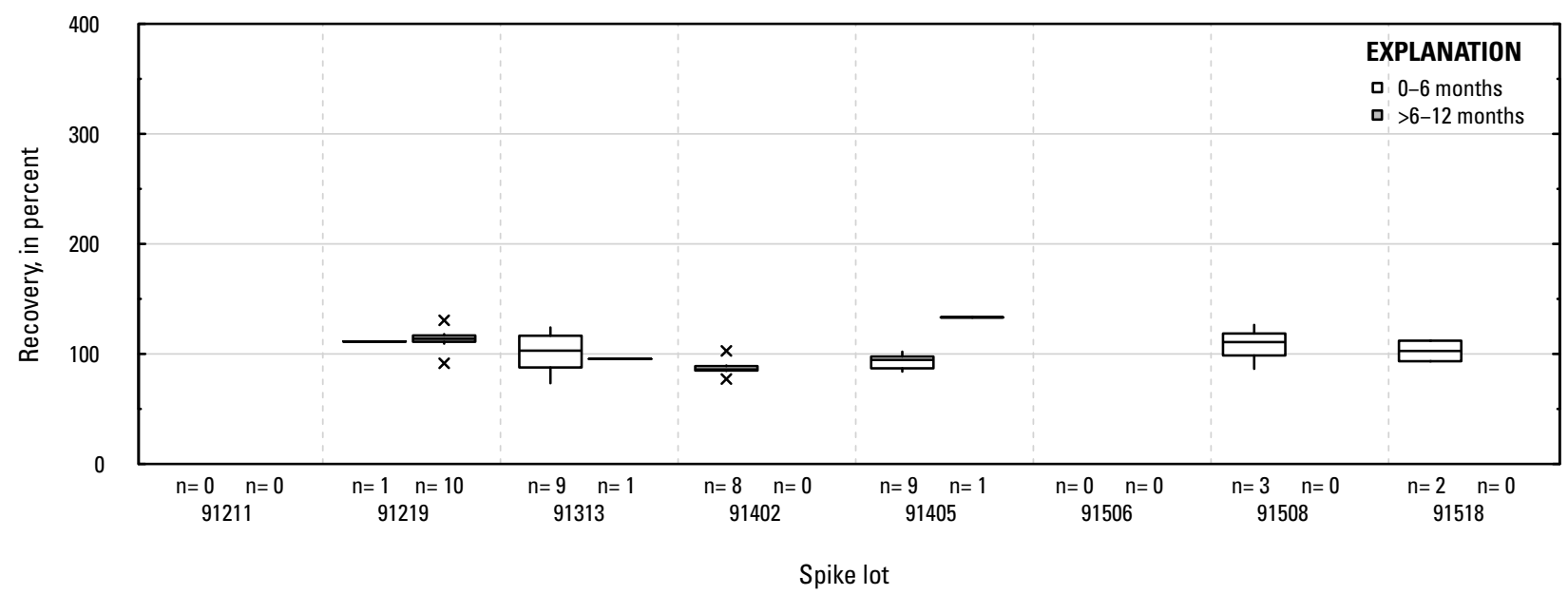

PY. Metolachlor oxanilic acid: surface water field matrix spikes

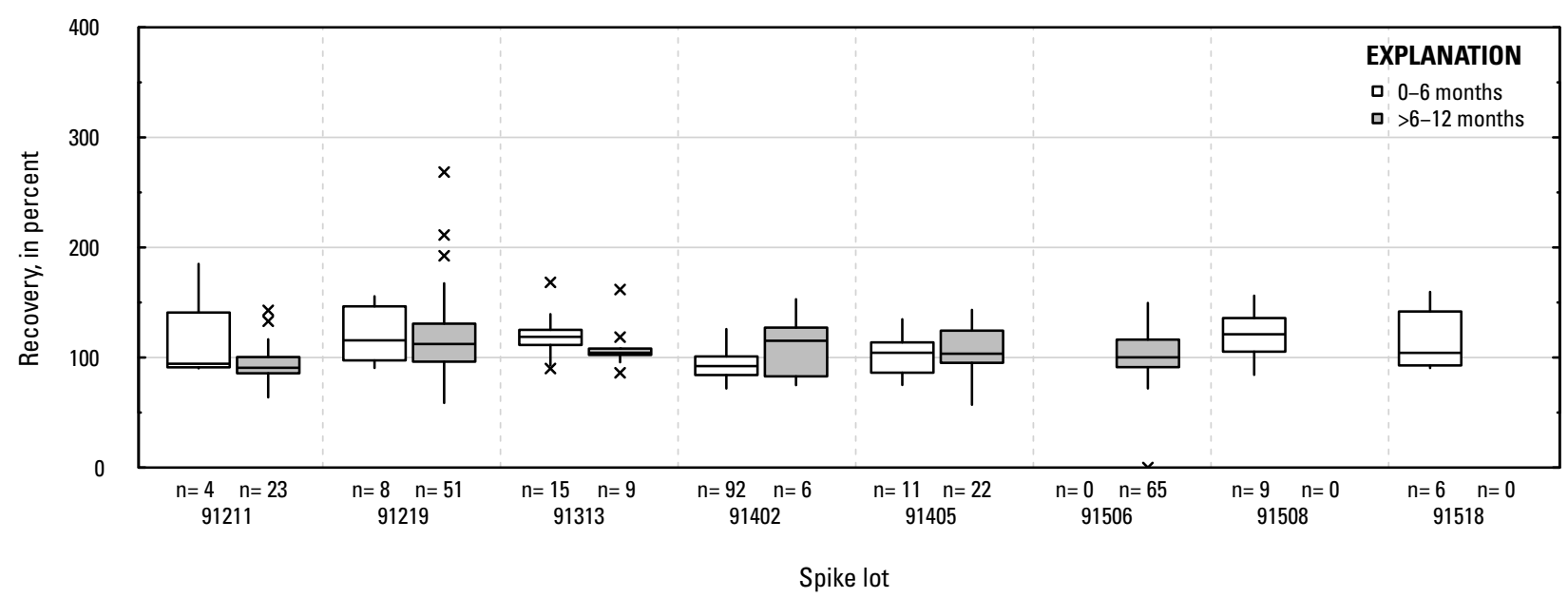

Figure 1-1. Distributions of recovery for individual pesticides in schedule 2437 by matrix, spike lot, and spike lot age. Recovery values larger than 400 percent are not shown.-Continued 
PZ. Metolachlor sulfonic acid: laboratory reagent spikes

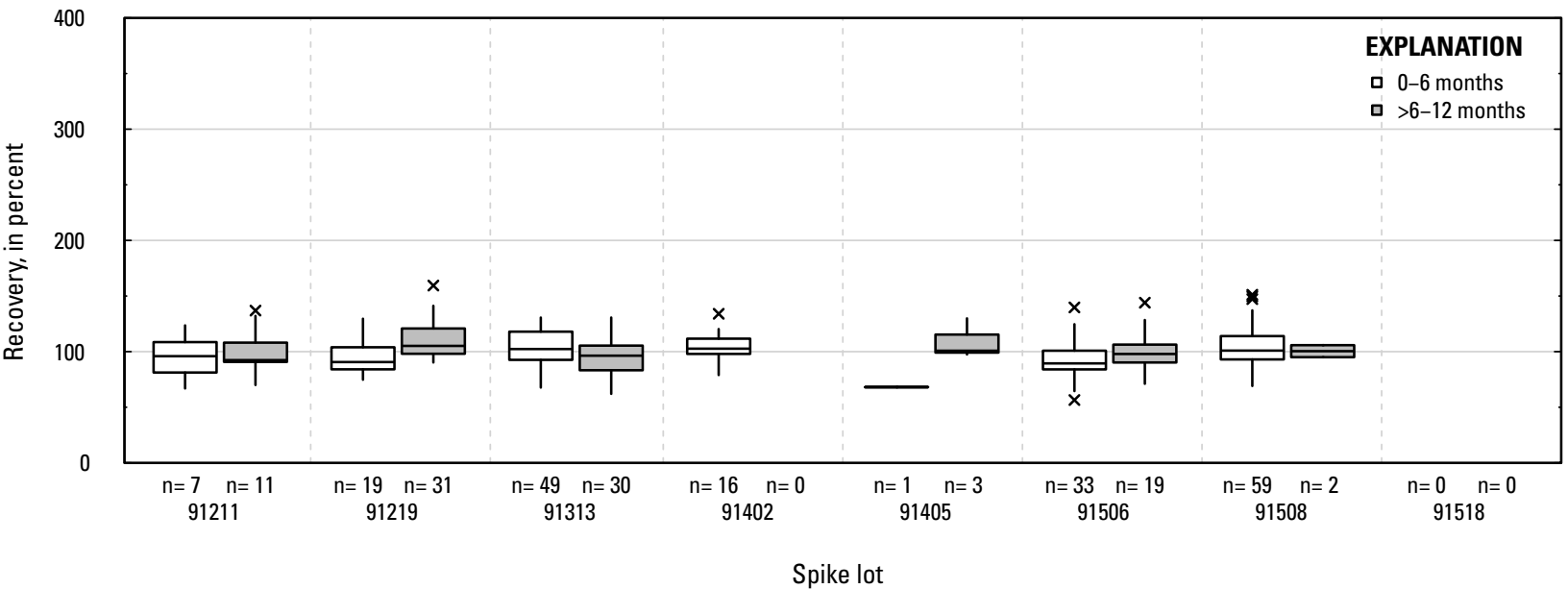

QA. Metolachlor sulfonic acid: groundwater field matrix spikes

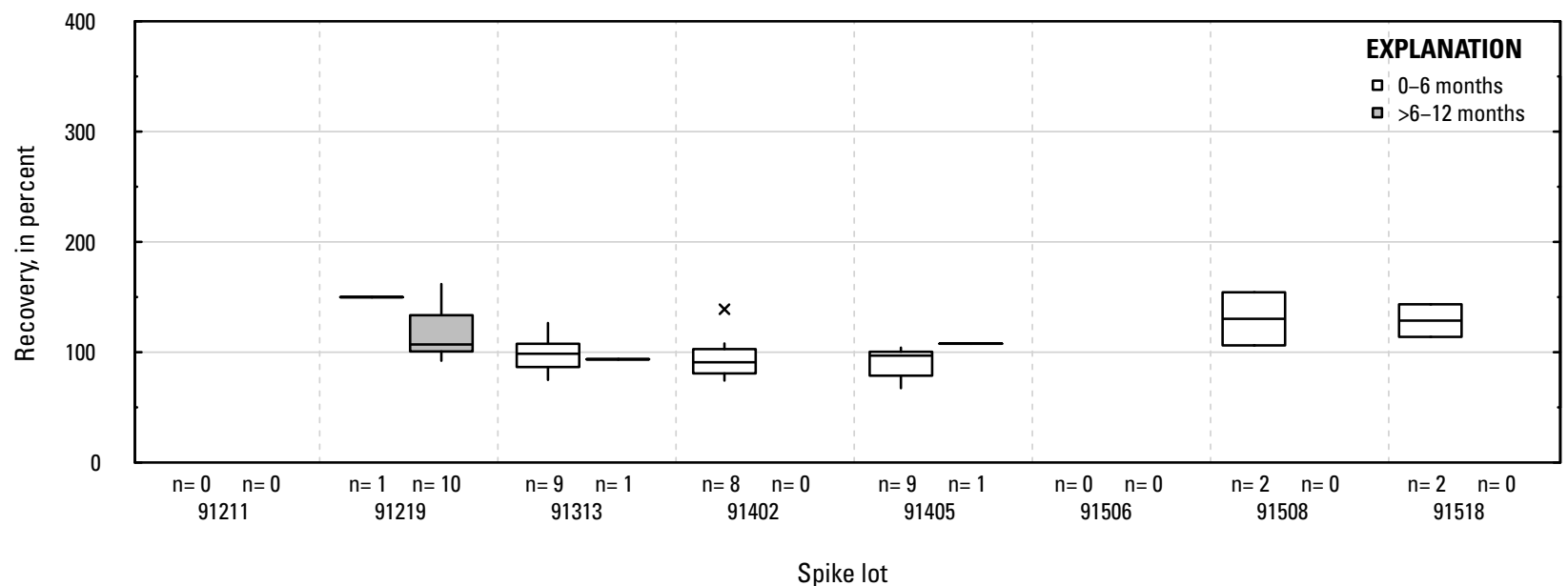

QB. Metolachlor sulfonic acid: surface water field matrix spikes

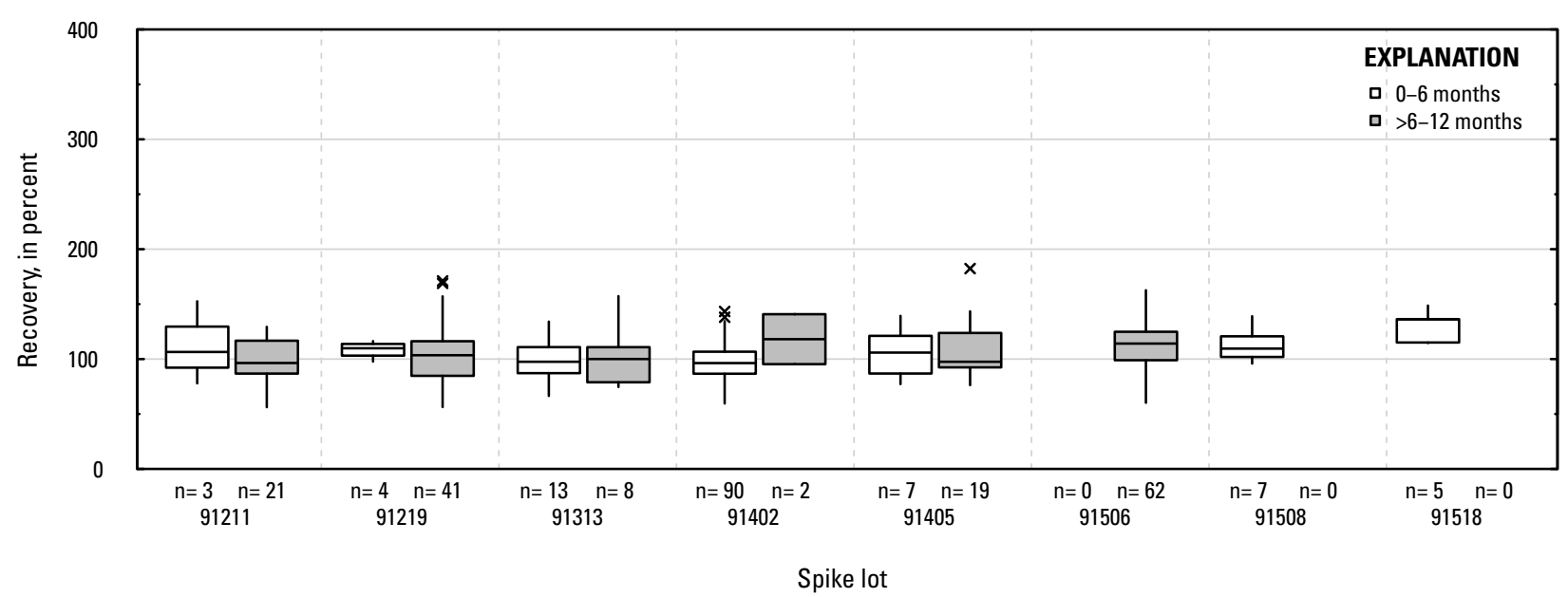

Figure 1-1. Distributions of recovery for individual pesticides in schedule 2437 by matrix, spike lot, and spike lot age. Recovery values larger than 400 percent are not shown.-Continued 
QC. Metribuzin: laboratory reagent spikes

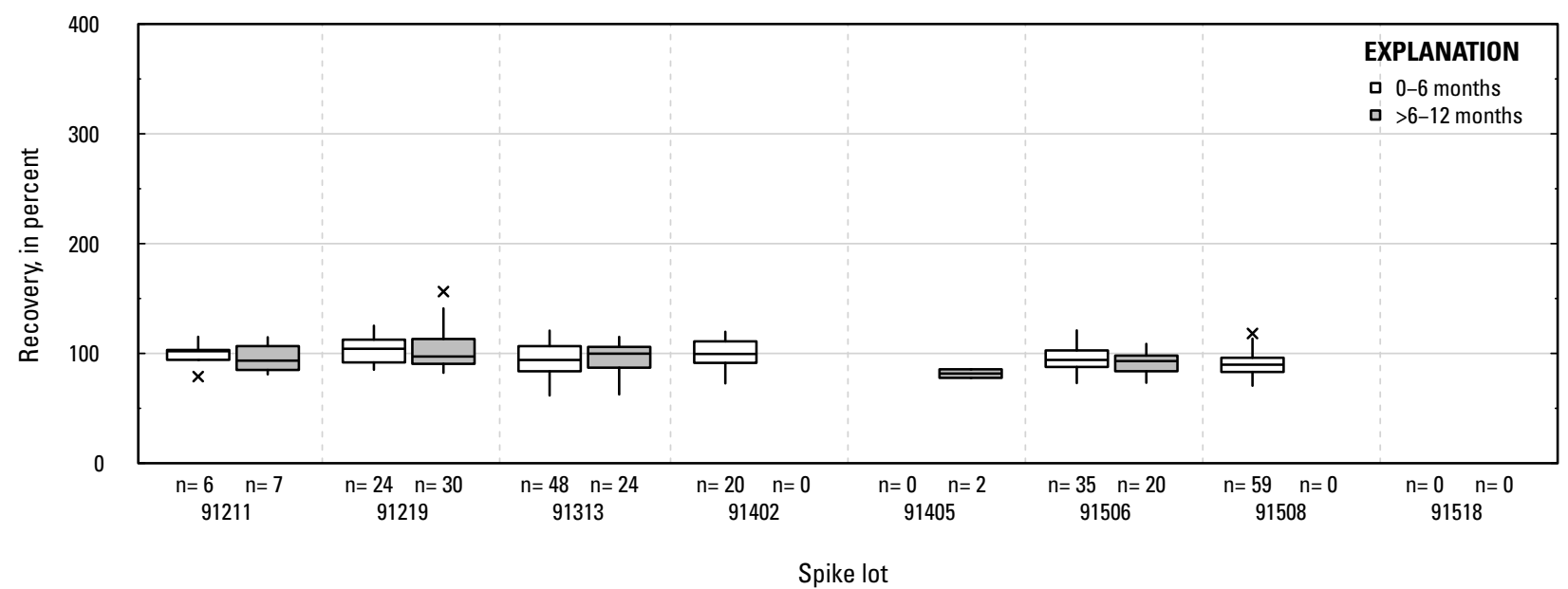

QD. Metribuzin: groundwater field matrix spikes

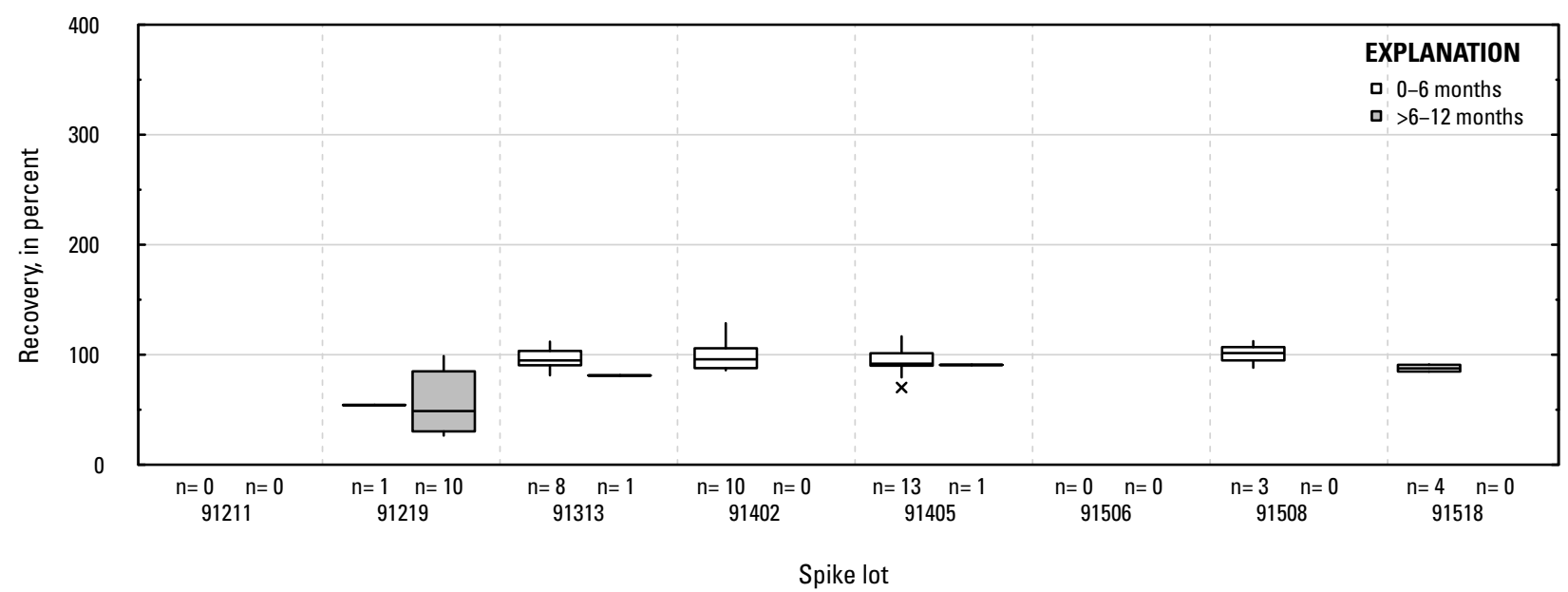

QE. Metribuzin: surface water field matrix spikes

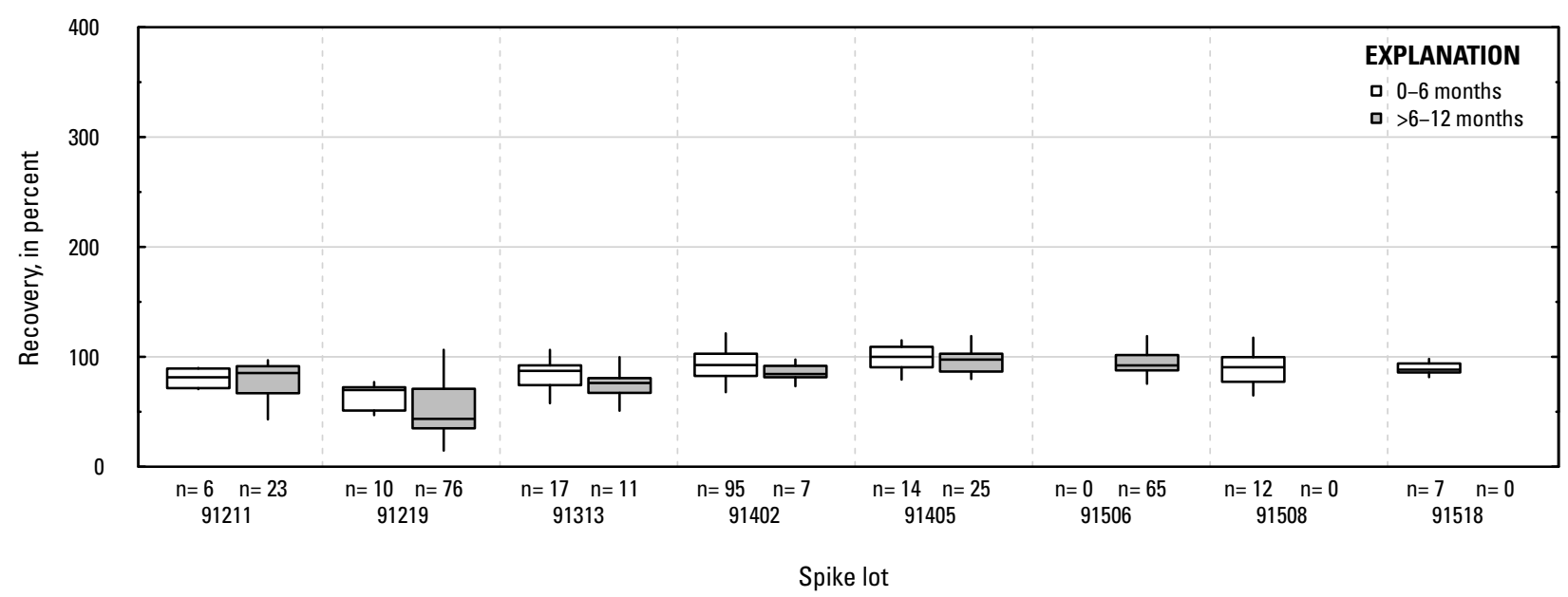

Figure 1-1. Distributions of recovery for individual pesticides in schedule 2437 by matrix, spike lot, and spike lot age. Recovery values larger than 400 percent are not shown.-Continued 
OF. Desamino metribuzin: laboratory reagent spikes

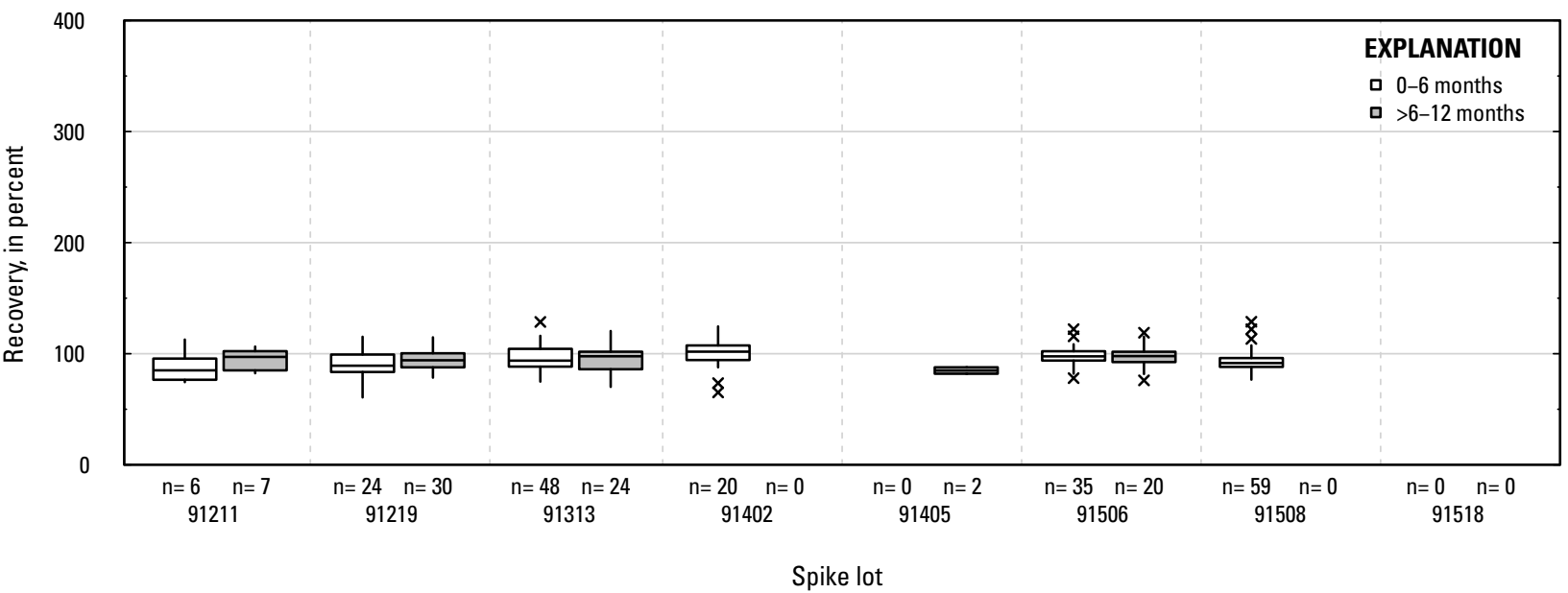

QG. Desamino metribuzin: groundwater field matrix spikes

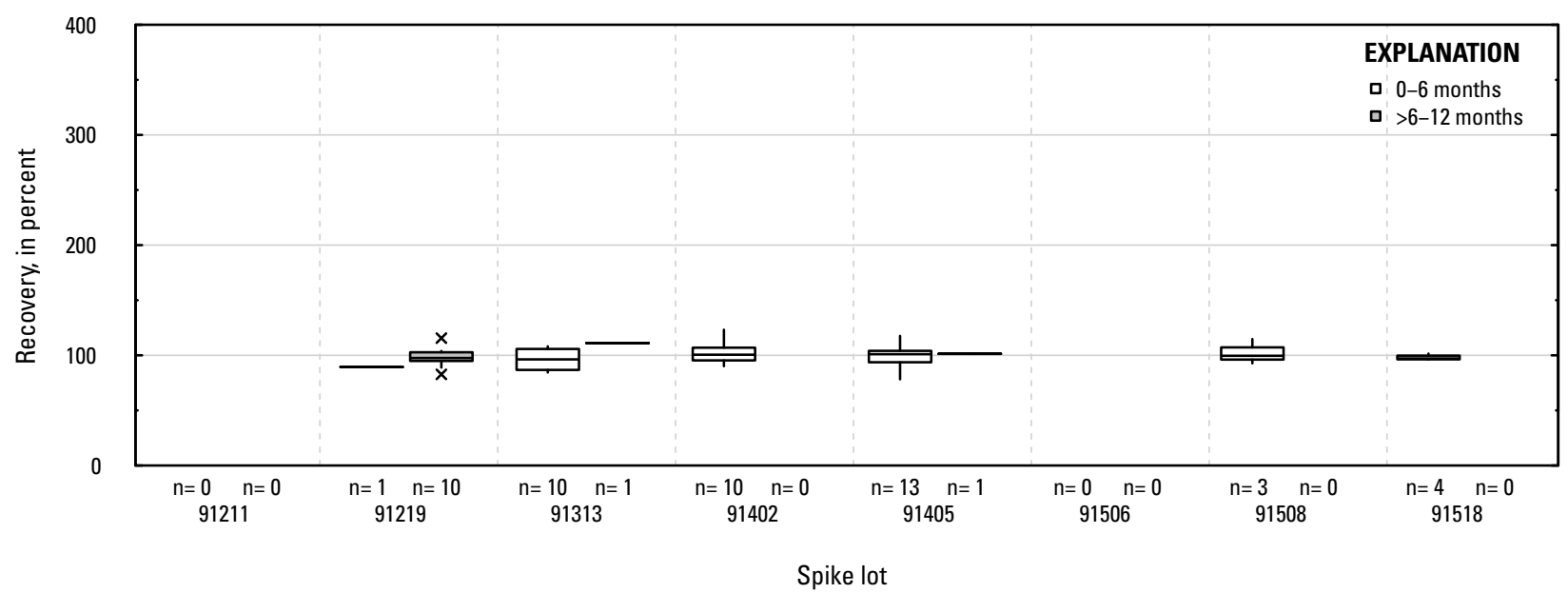

QH. Desamino metribuzin: surface water field matrix spikes

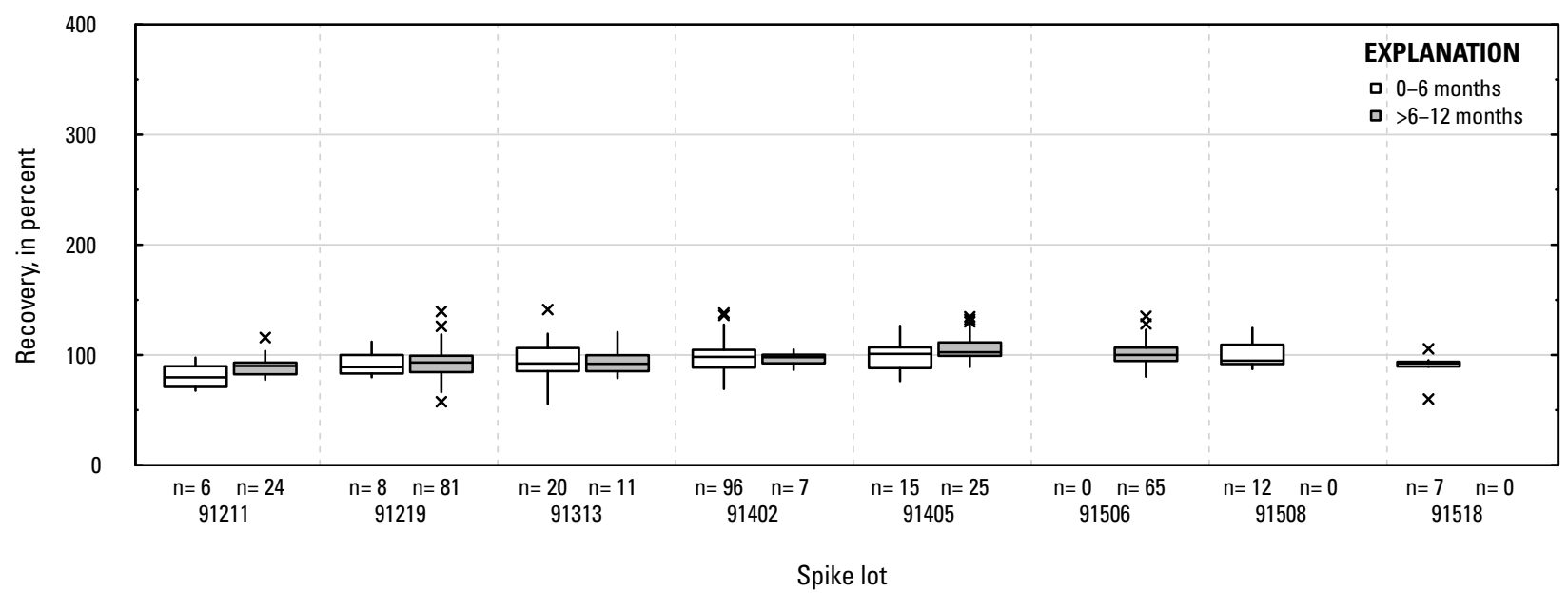

Figure 1-1. Distributions of recovery for individual pesticides in schedule 2437 by matrix, spike lot, and spike lot age. Recovery values larger than 400 percent are not shown.-Continued 


\section{QI. Desamino-diketo metribuzin: laboratory reagent spikes}

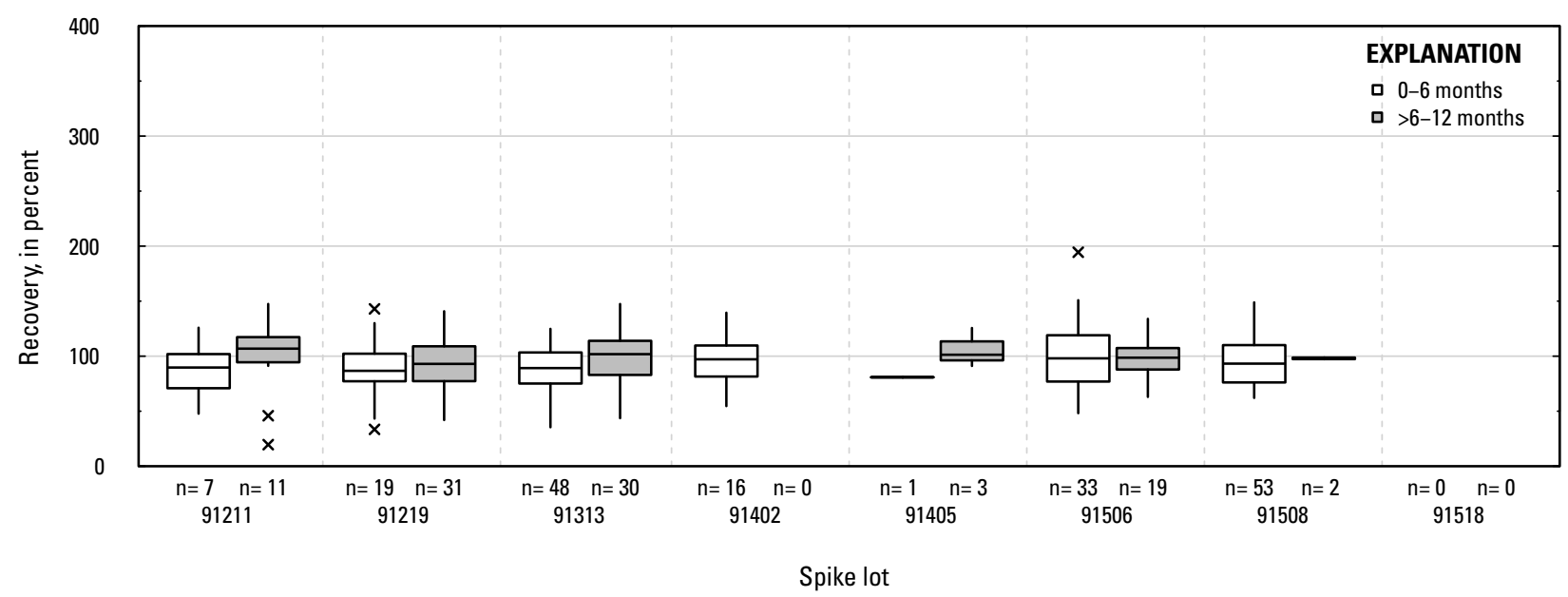

OJ. Desamino-diketo metribuzin: groundwater field matrix spikes

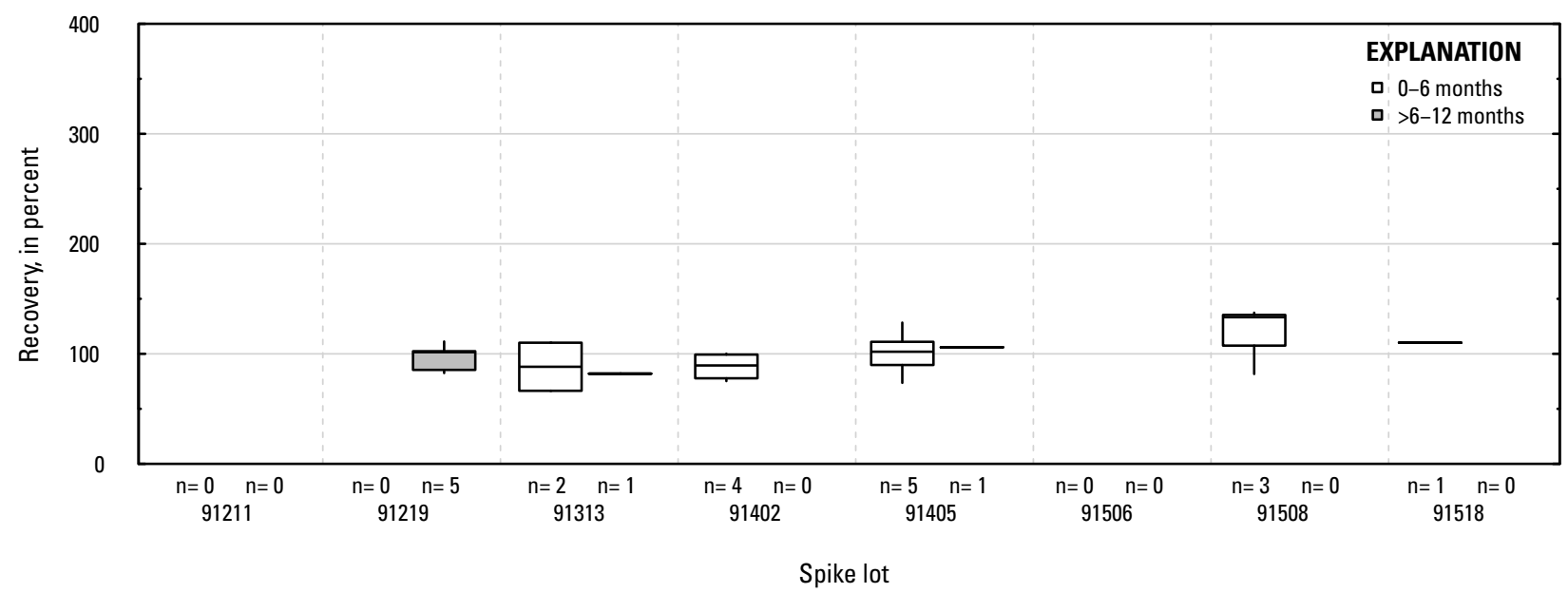

QK. Desamino-diketo metribuzin: surface water field matrix spikes

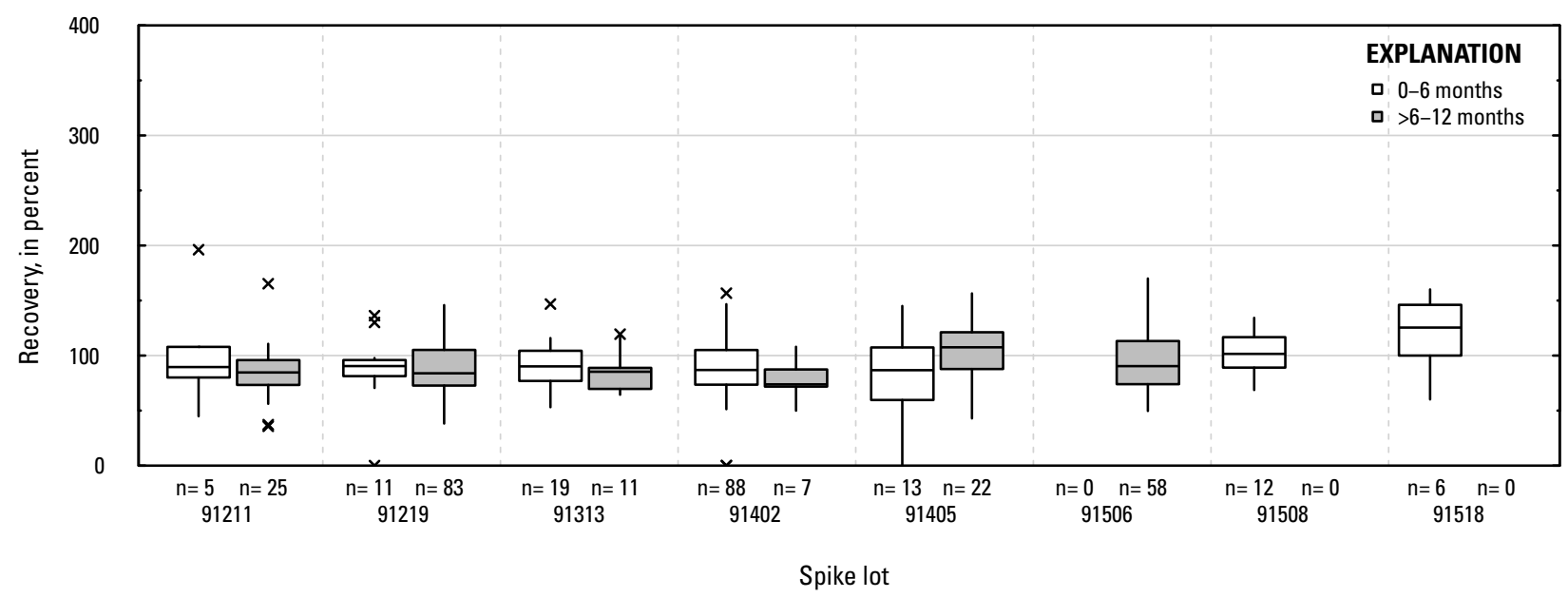

Figure 1-1. Distributions of recovery for individual pesticides in schedule 2437 by matrix, spike lot, and spike lot age. Recovery values larger than 400 percent are not shown.-Continued 


\section{QL. Metribuzin DK: laboratory reagent spikes}

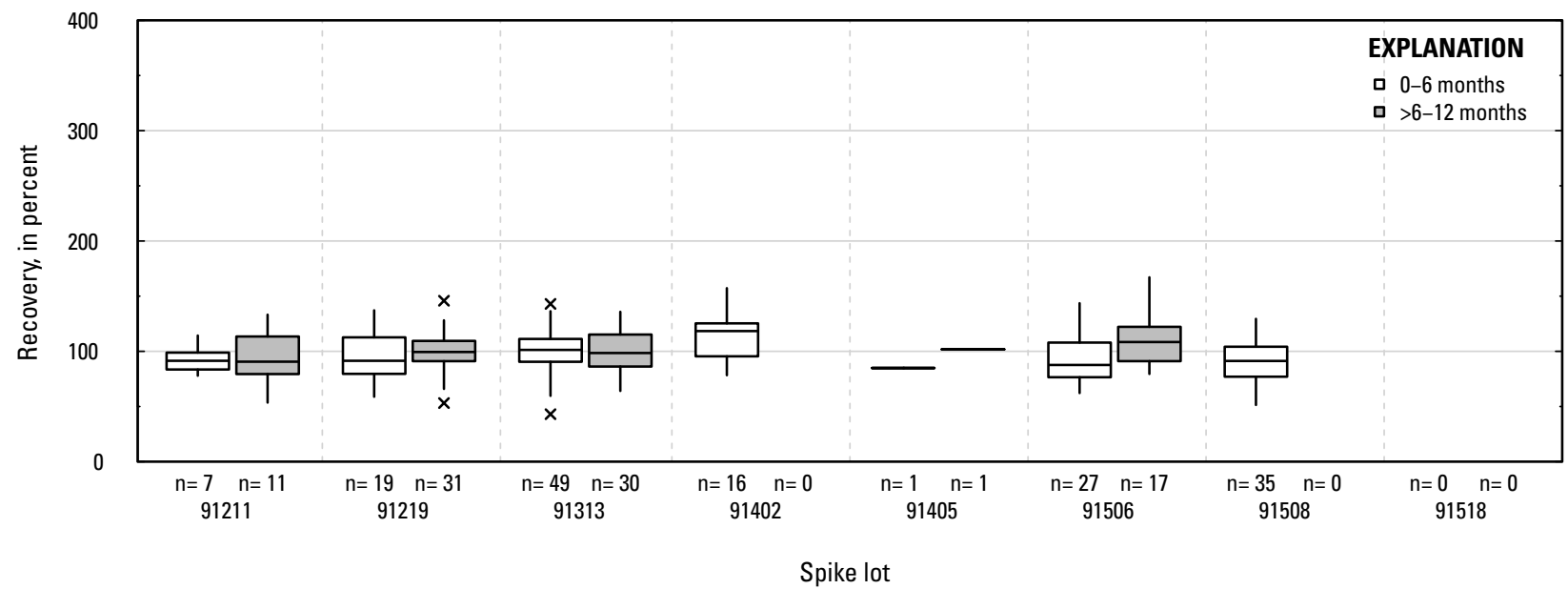

QM. Metribuzin DK: groundwater field matrix spikes

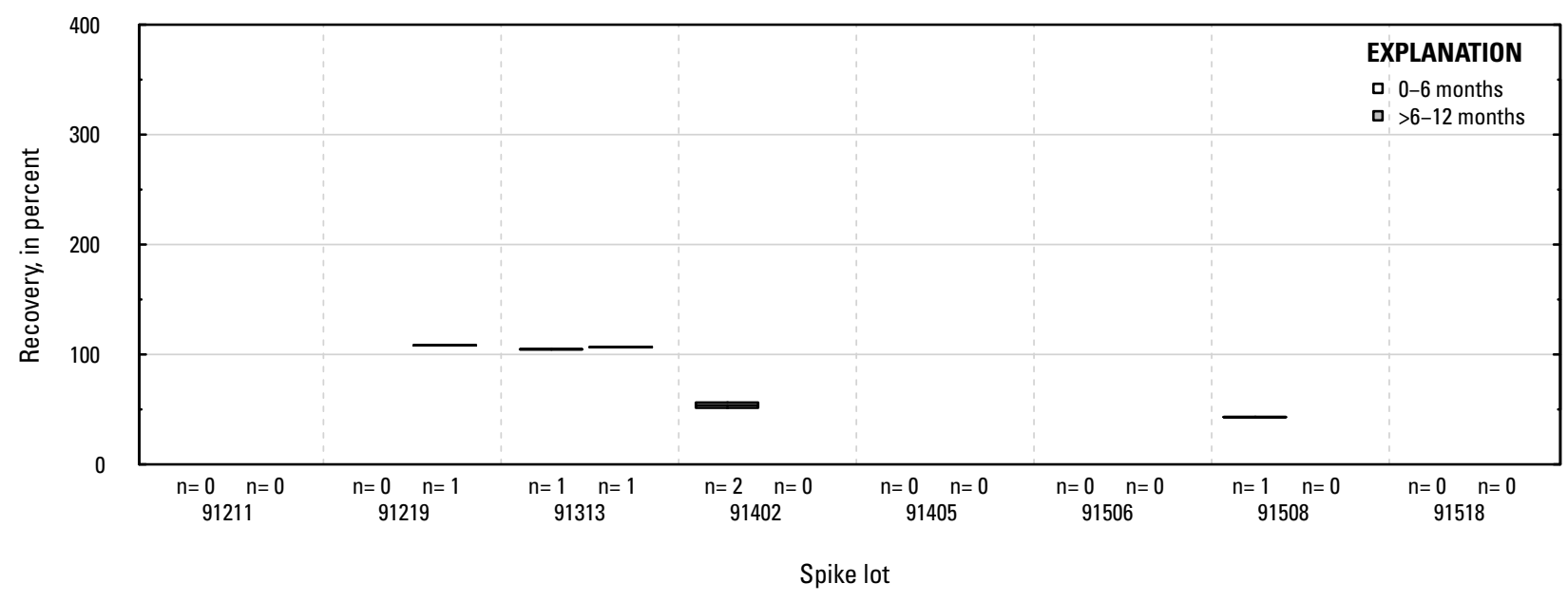

QN. Metribuzin DK: surface water field matrix spikes

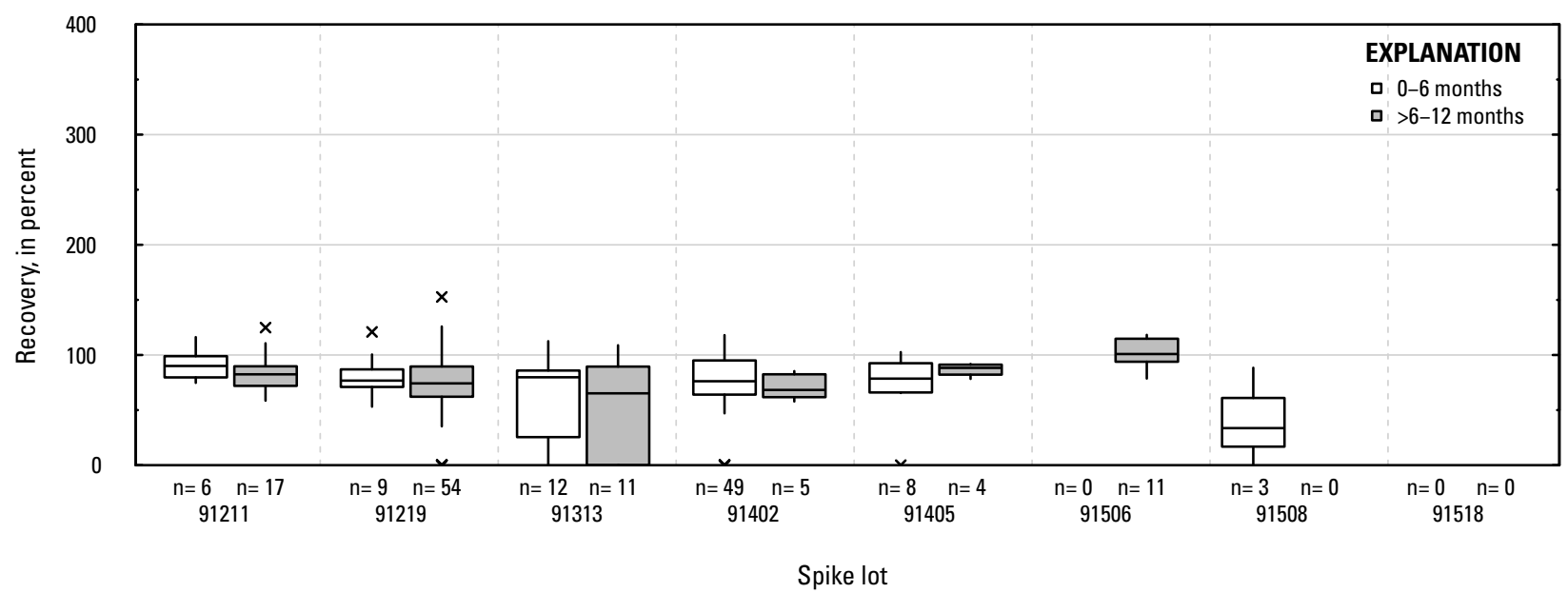

Figure 1-1. Distributions of recovery for individual pesticides in schedule 2437 by matrix, spike lot, and spike lot age. Recovery values larger than 400 percent are not shown.-Continued 
Q0. Molinate: laboratory reagent spikes

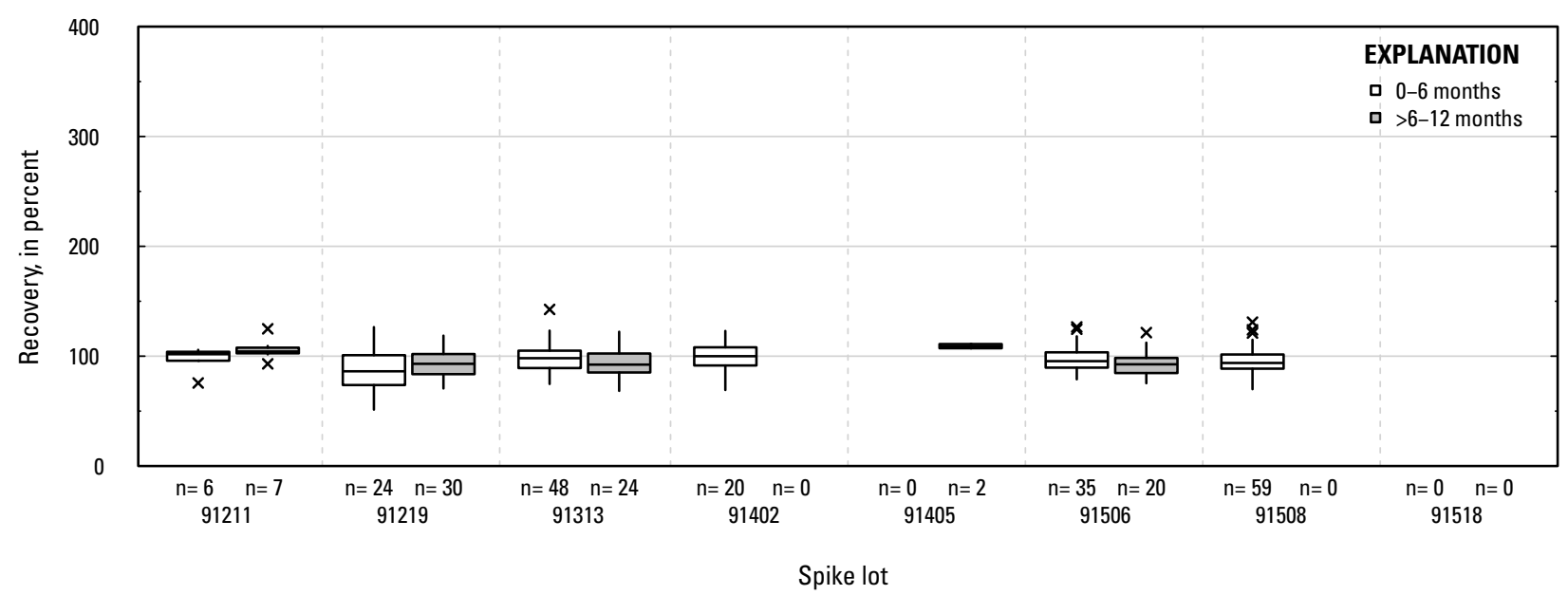

QP. Molinate: groundwater field matrix spikes

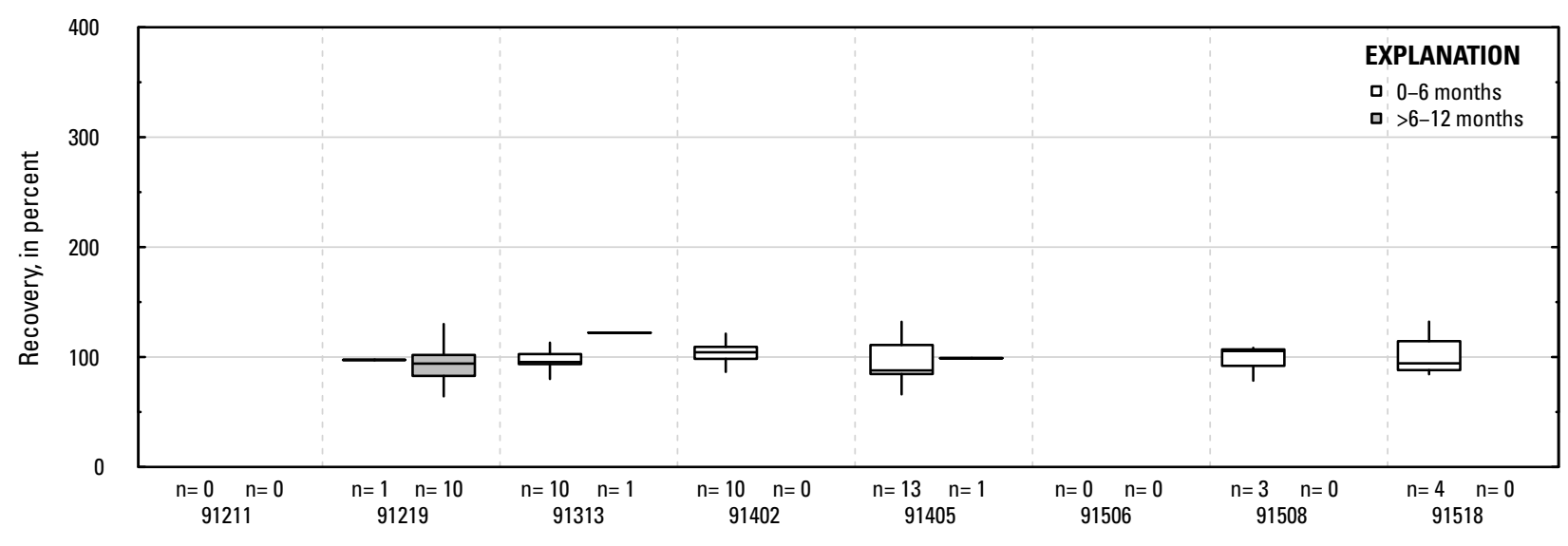

Spike lot

Q0. Molinate: surface water field matrix spikes

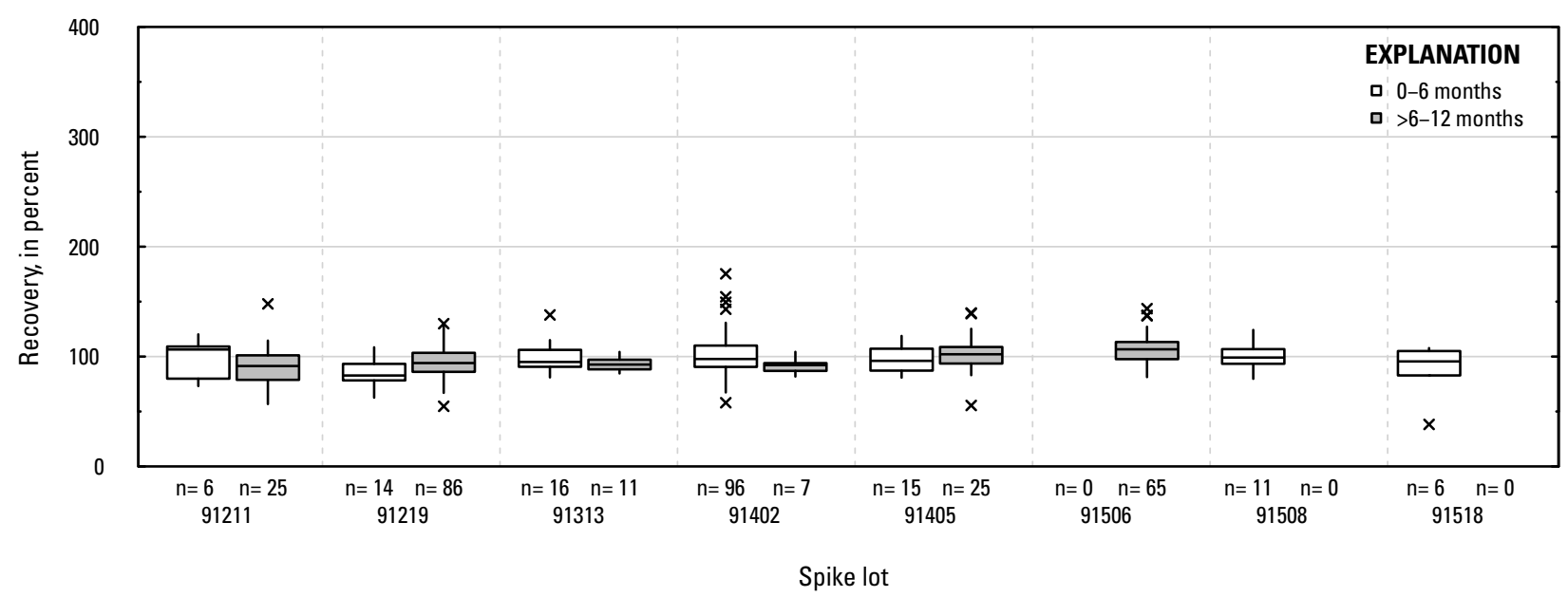

Figure 1-1. Distributions of recovery for individual pesticides in schedule 2437 by matrix, spike lot, and spike lot age. Recovery values larger than 400 percent are not shown.-Continued 


\section{QR. Myclobutanil: laboratory reagent spikes}

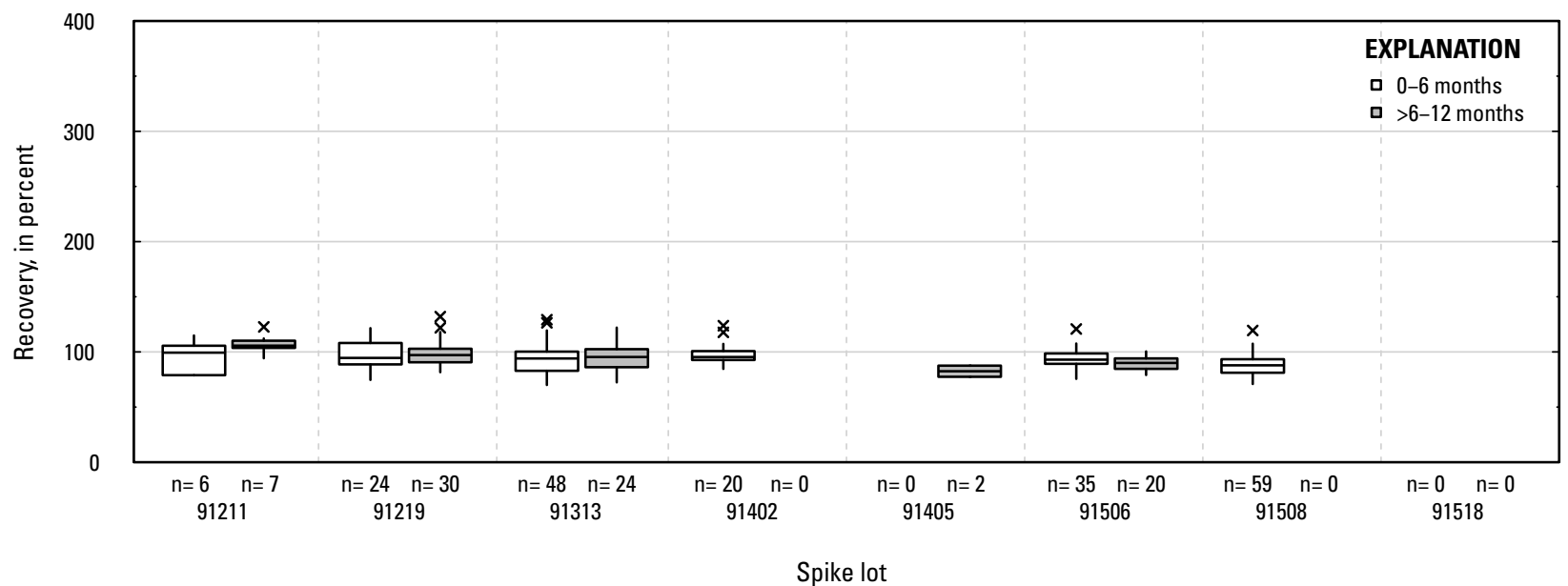

QS. Myclobutanil: groundwater field matrix spikes

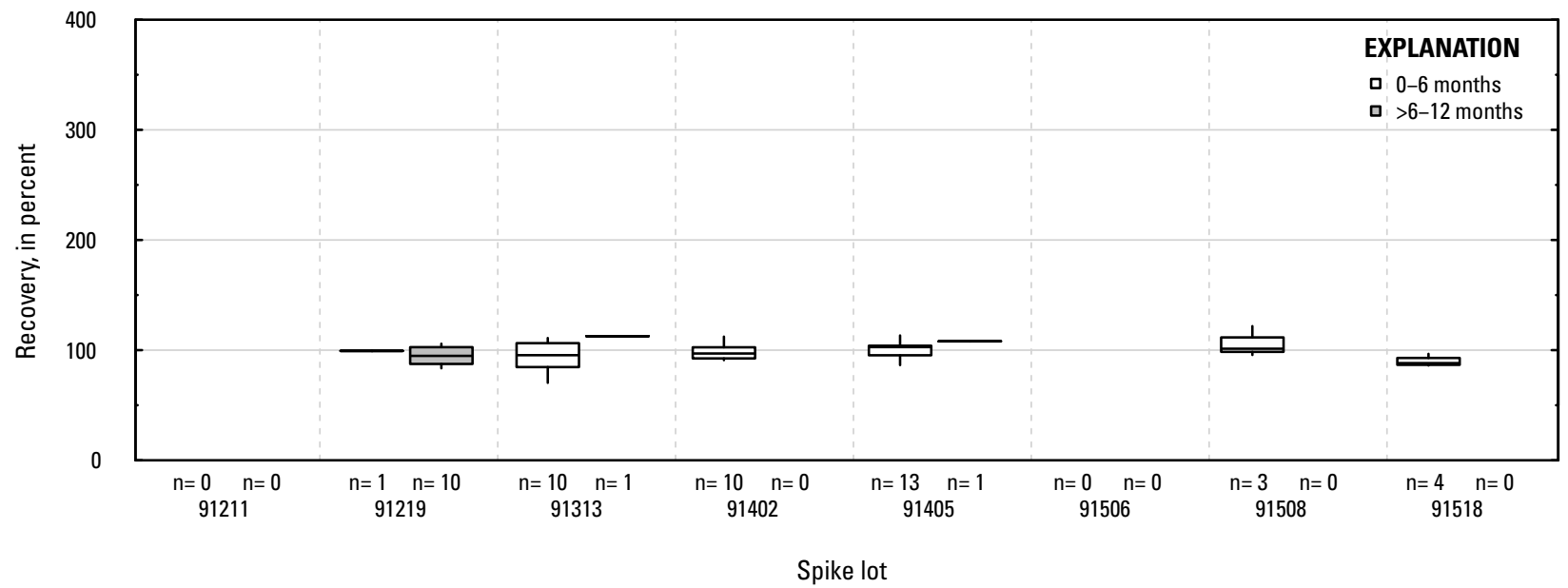

QT. Myclobutanil: surface water field matrix spikes

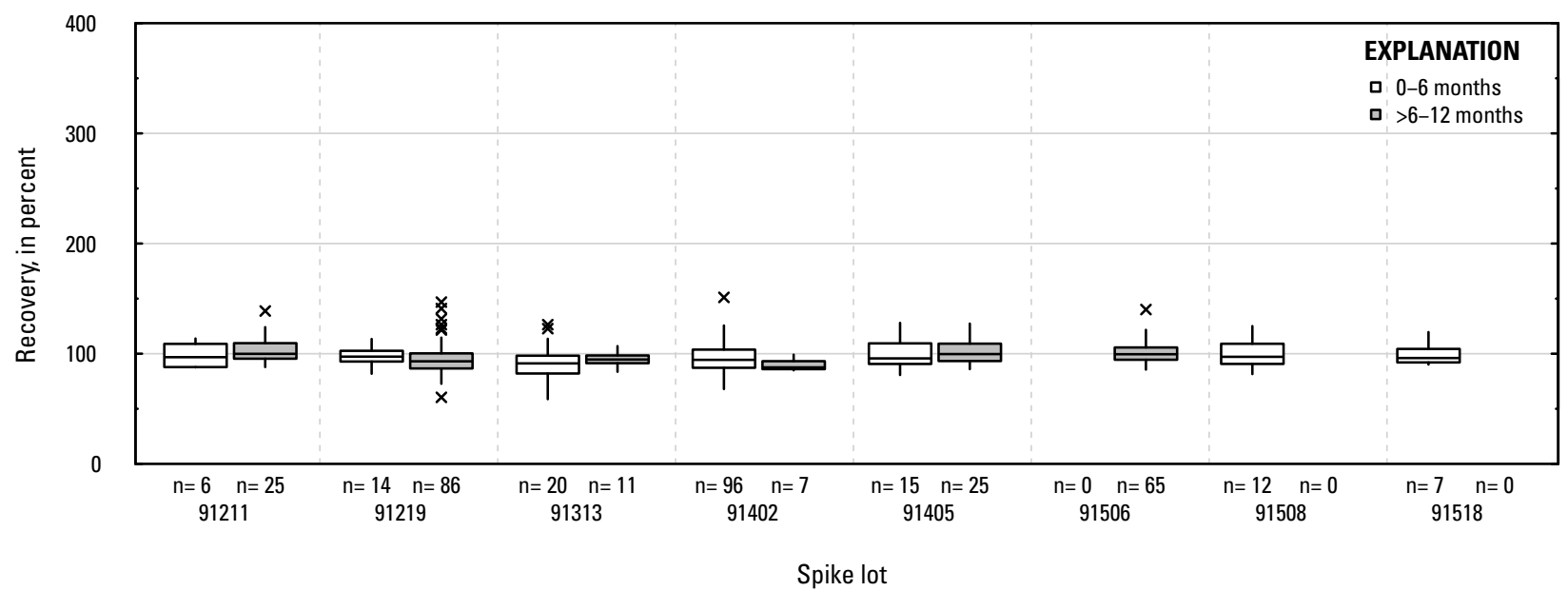

Figure 1-1. Distributions of recovery for individual pesticides in schedule 2437 by matrix, spike lot, and spike lot age. Recovery values larger than 400 percent are not shown.-Continued 
QU. N-(3,4-Dichlorophenyl)-N'-methylurea: laboratory reagent spikes

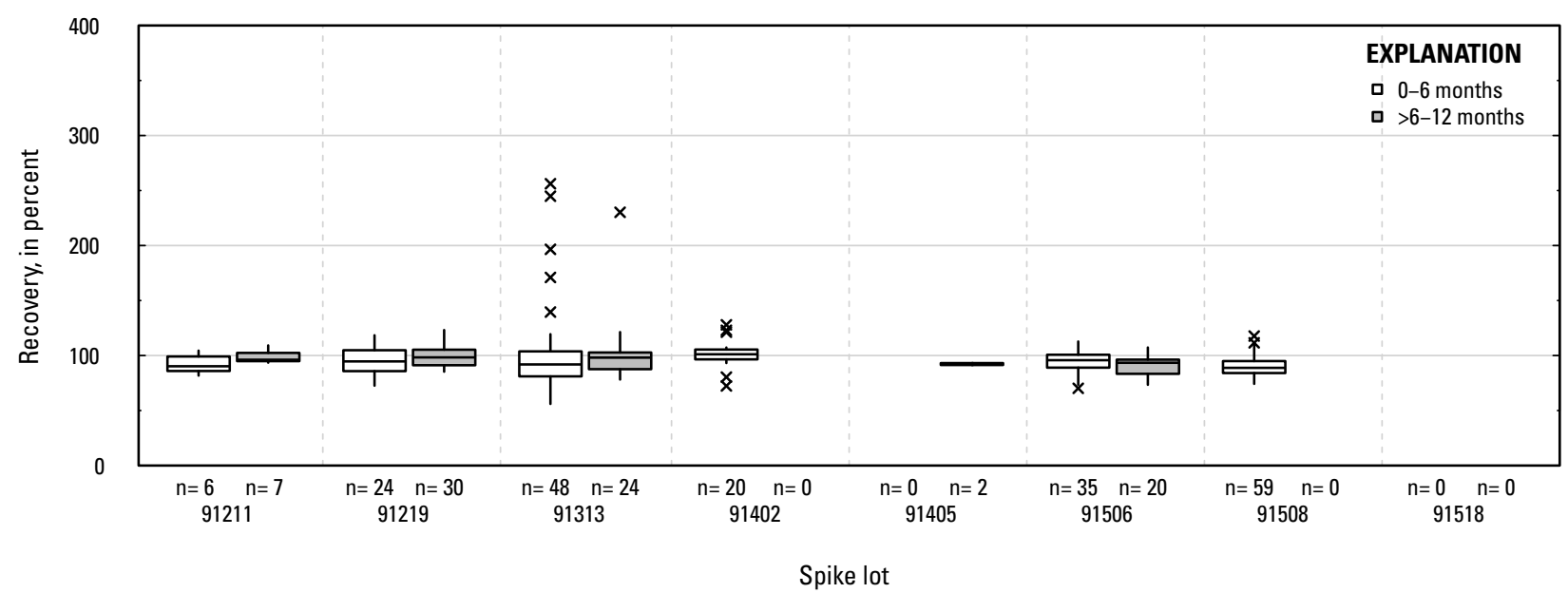

QV. N-(3,4-Dichlorophenyl)-N'-methylurea: groundwater field matrix spikes

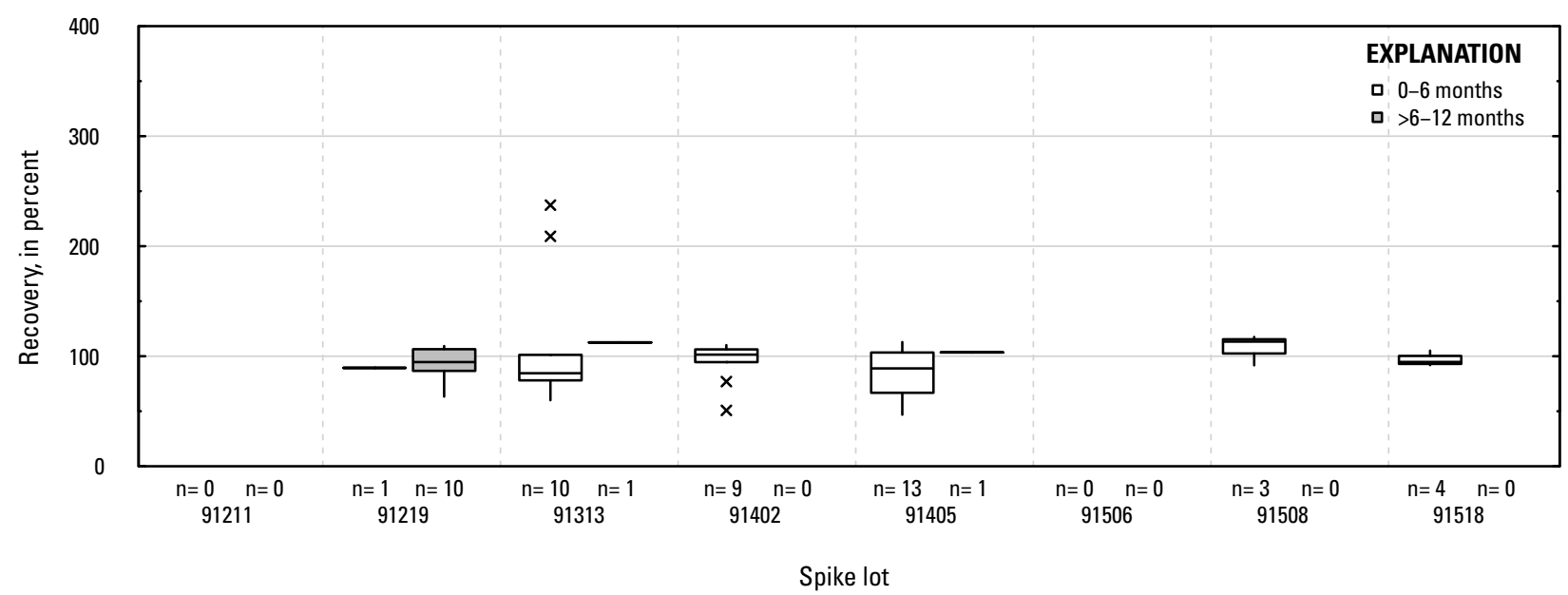

OW. N-(3,4-Dichlorophenyl)-N'-methylurea: surface water field matrix spikes

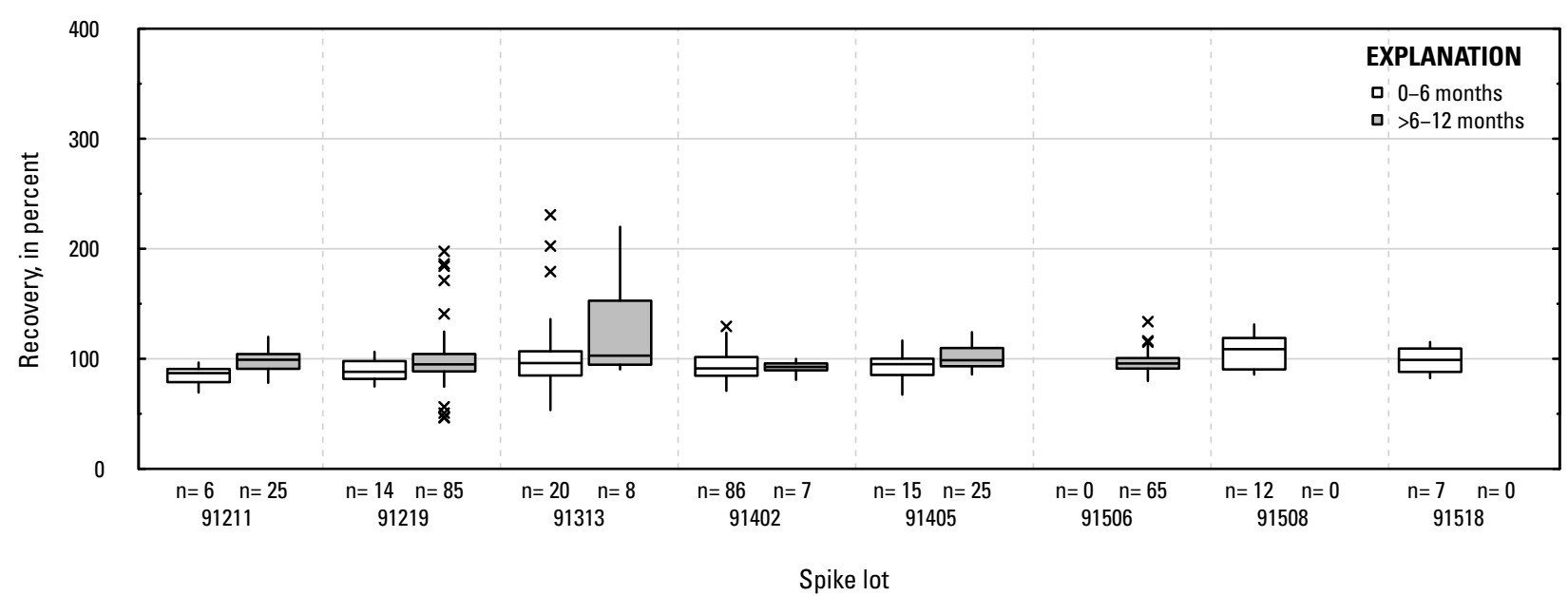

Figure 1-1. Distributions of recovery for individual pesticides in schedule 2437 by matrix, spike lot, and spike lot age. Recovery values larger than 400 percent are not shown.-Continued 


\section{QX. Naled: laboratory reagent spikes}

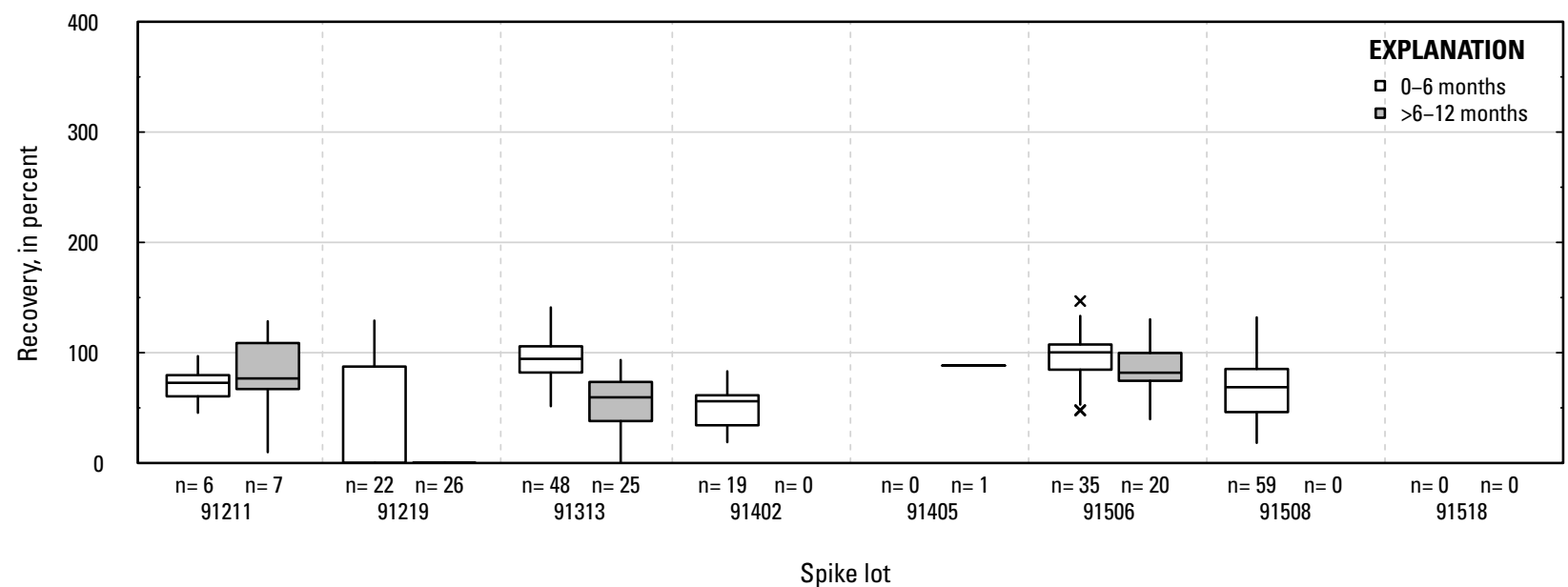

QY. Naled: groundwater field matrix spikes

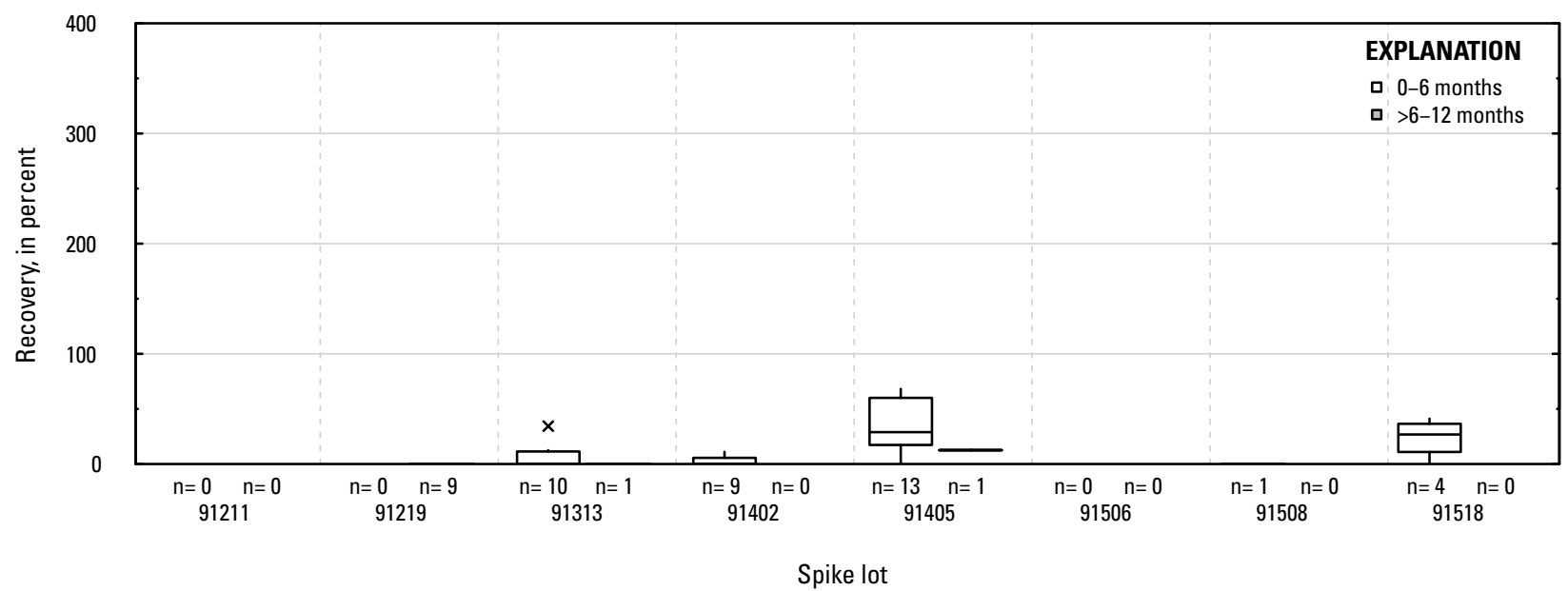

OZ. Naled: surface water field matrix spikes

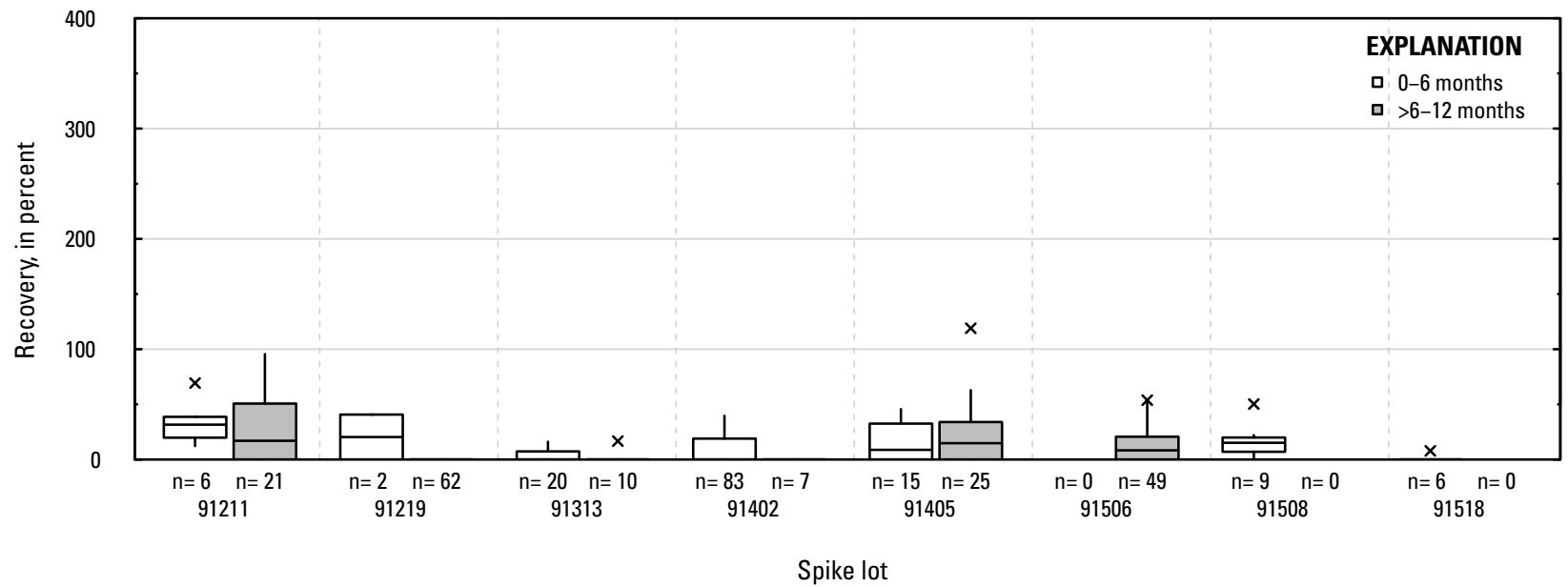

Figure 1-1. Distributions of recovery for individual pesticides in schedule 2437 by matrix, spike lot, and spike lot age. Recovery values larger than 400 percent are not shown.-Continued 


\section{RA. Nicosulfuron: laboratory reagent spikes}

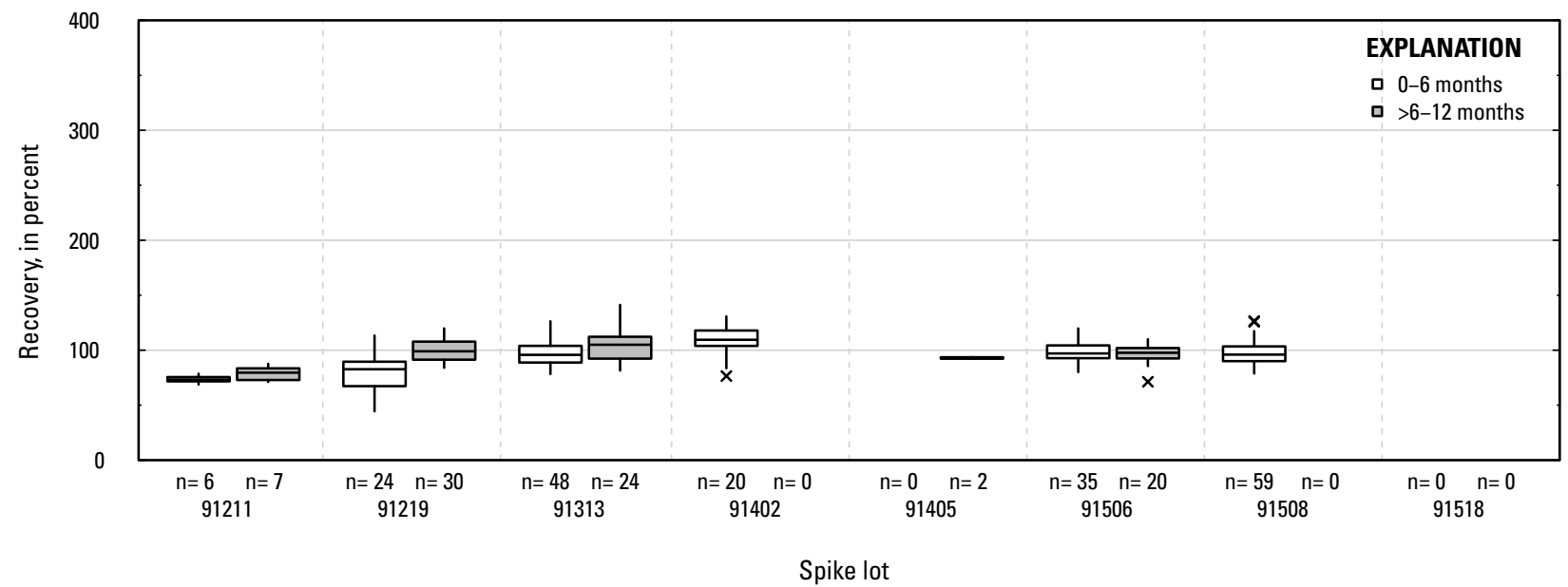

RB. Nicosulfuron: groundwater field matrix spikes

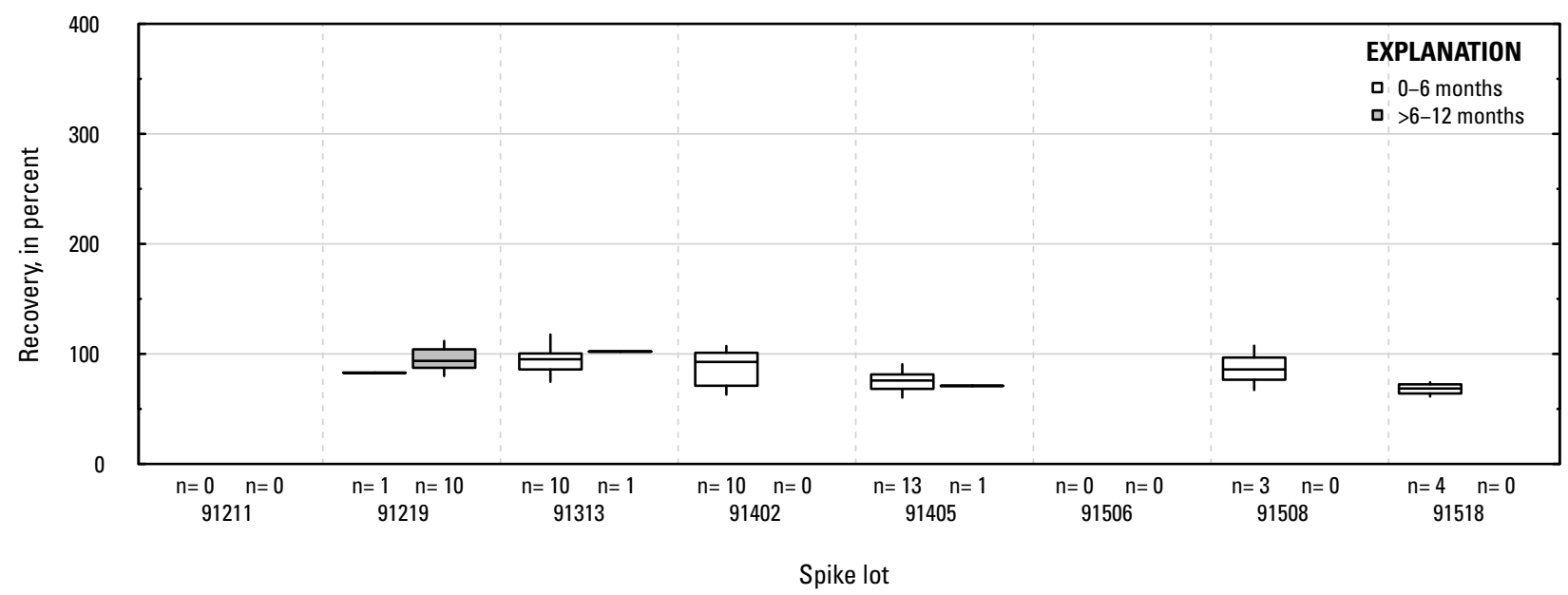

RC. Nicosulfuron: surface water field matrix spikes

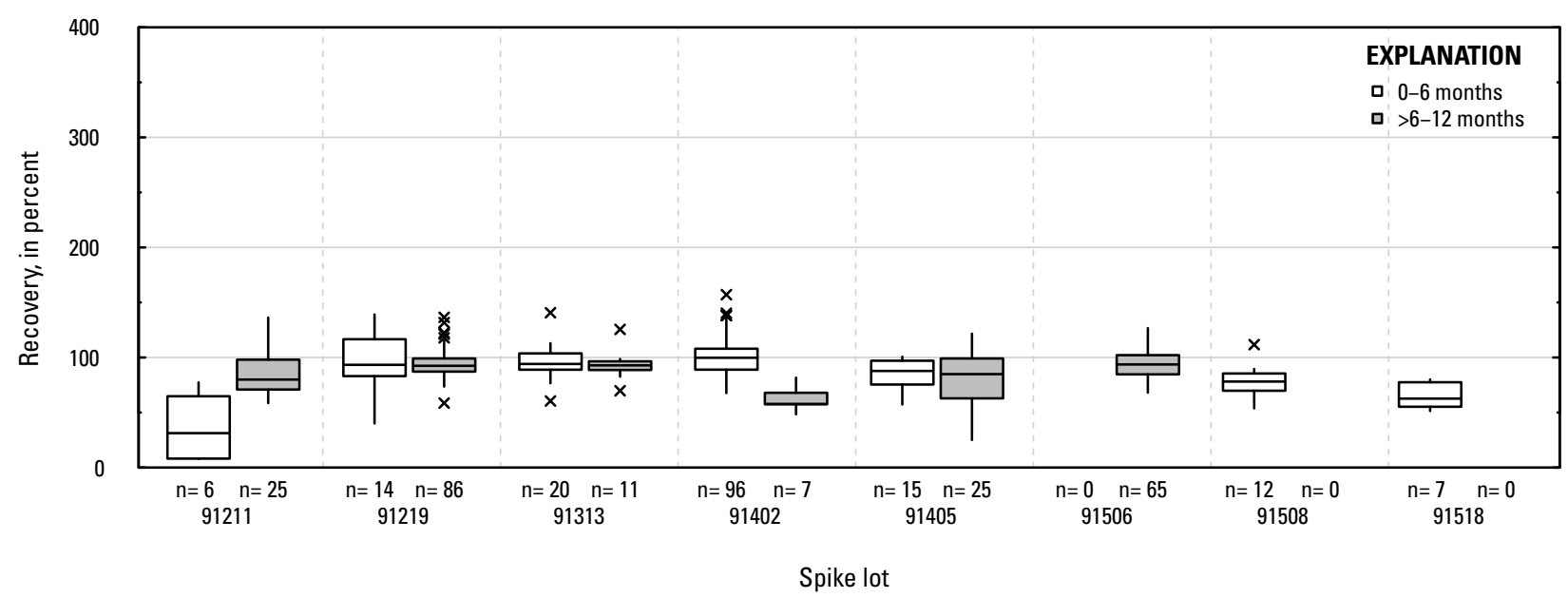

Figure 1-1. Distributions of recovery for individual pesticides in schedule 2437 by matrix, spike lot, and spike lot age. Recovery values larger than 400 percent are not shown.-Continued 
RD. Norflurazon: laboratory reagent spikes

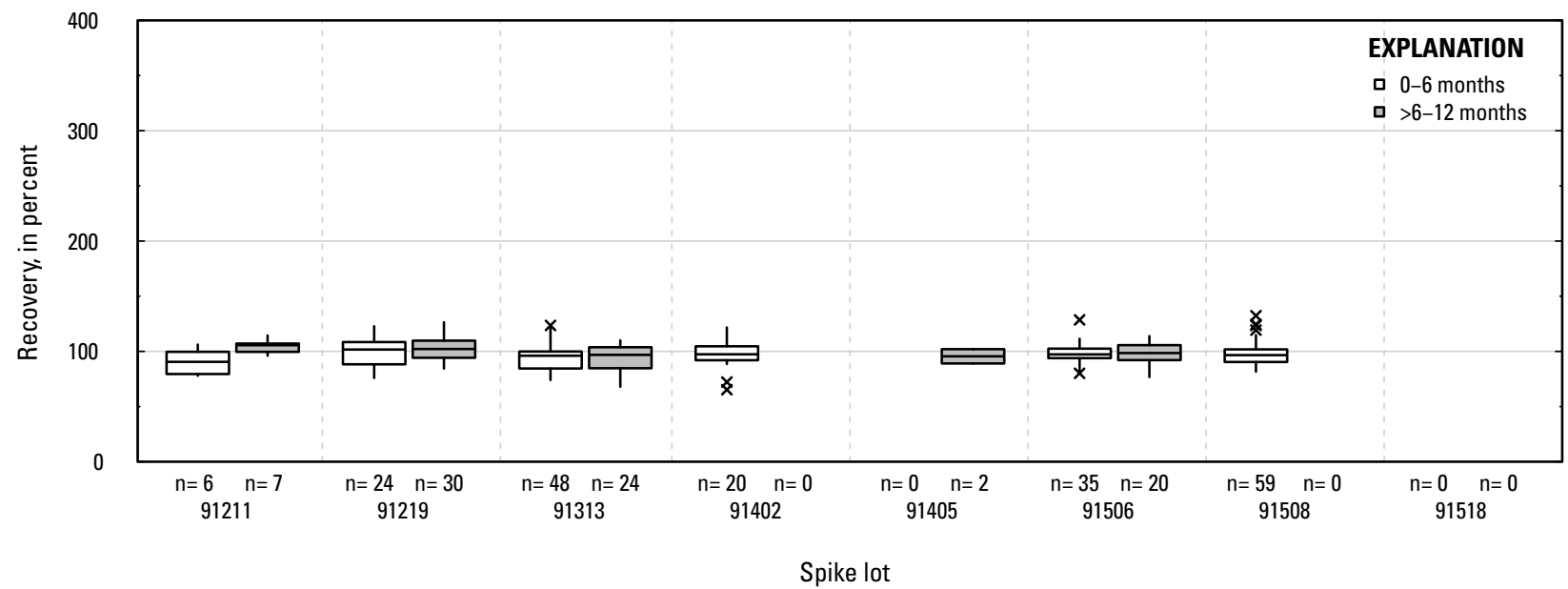

RE. Norflurazon: groundwater field matrix spikes

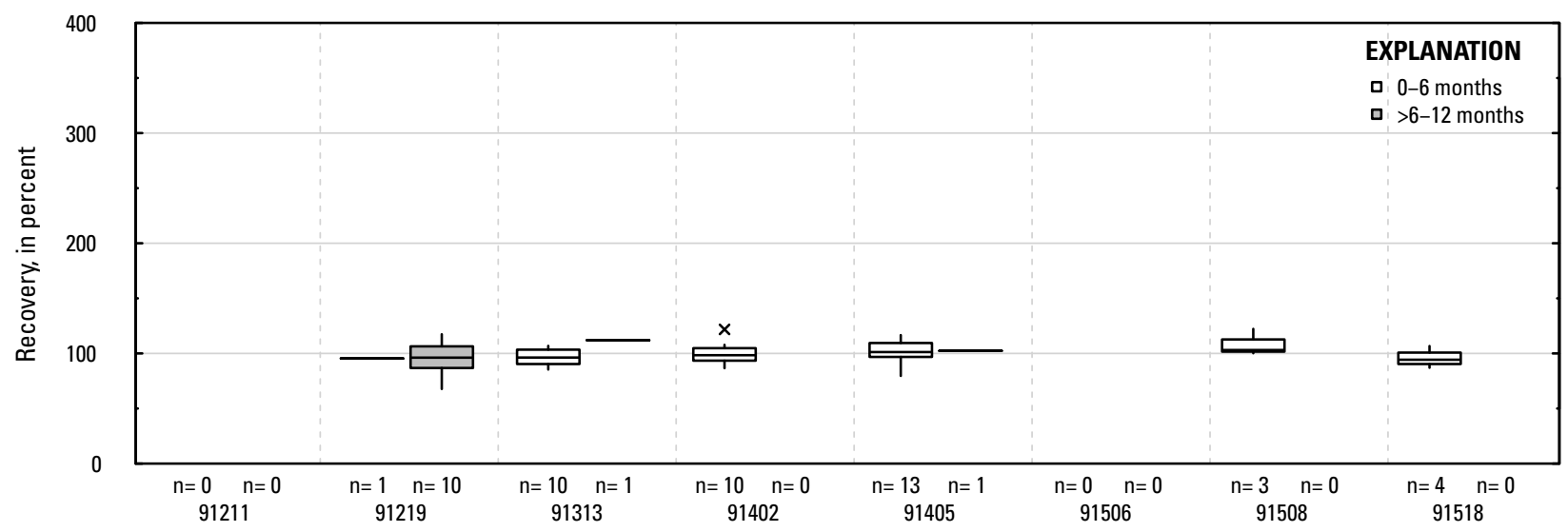

Spike lot

\section{RF. Norflurazon: surface water field matrix spikes}

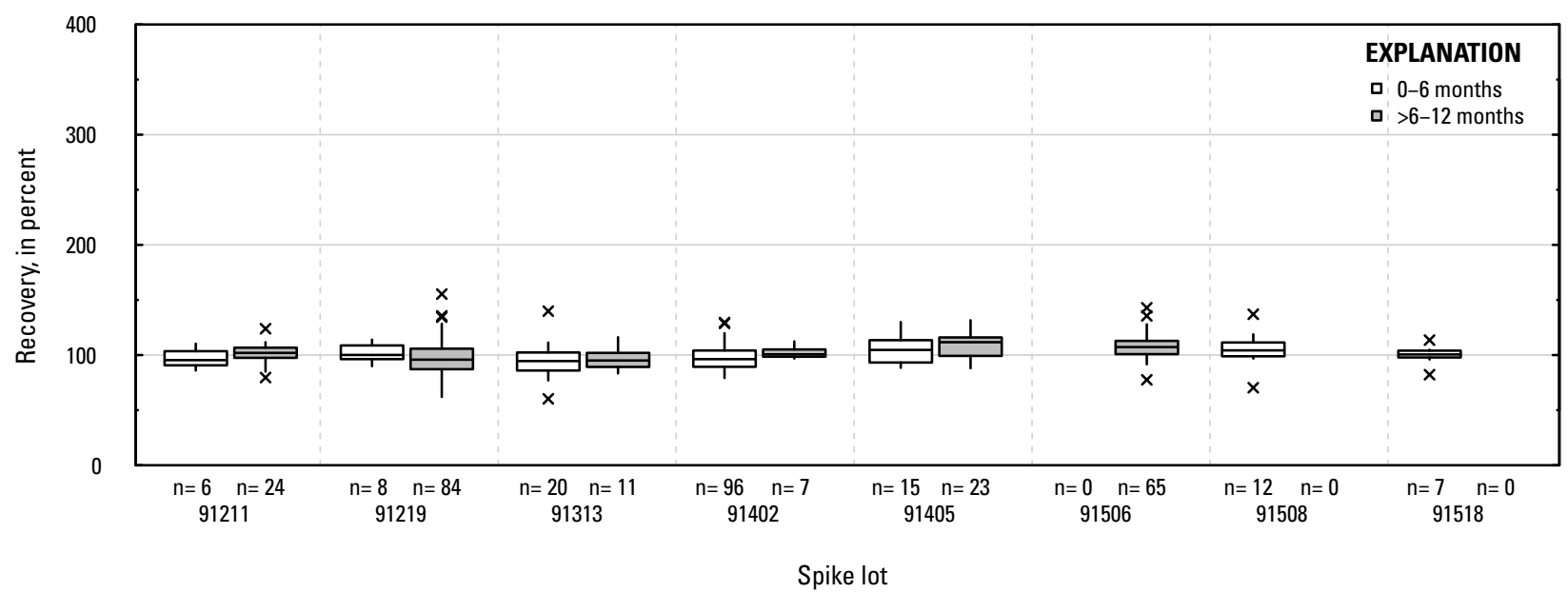

Figure 1-1. Distributions of recovery for individual pesticides in schedule 2437 by matrix, spike lot, and spike lot age. Recovery values larger than 400 percent are not shown.-Continued 
RG. Novaluron: laboratory reagent spikes

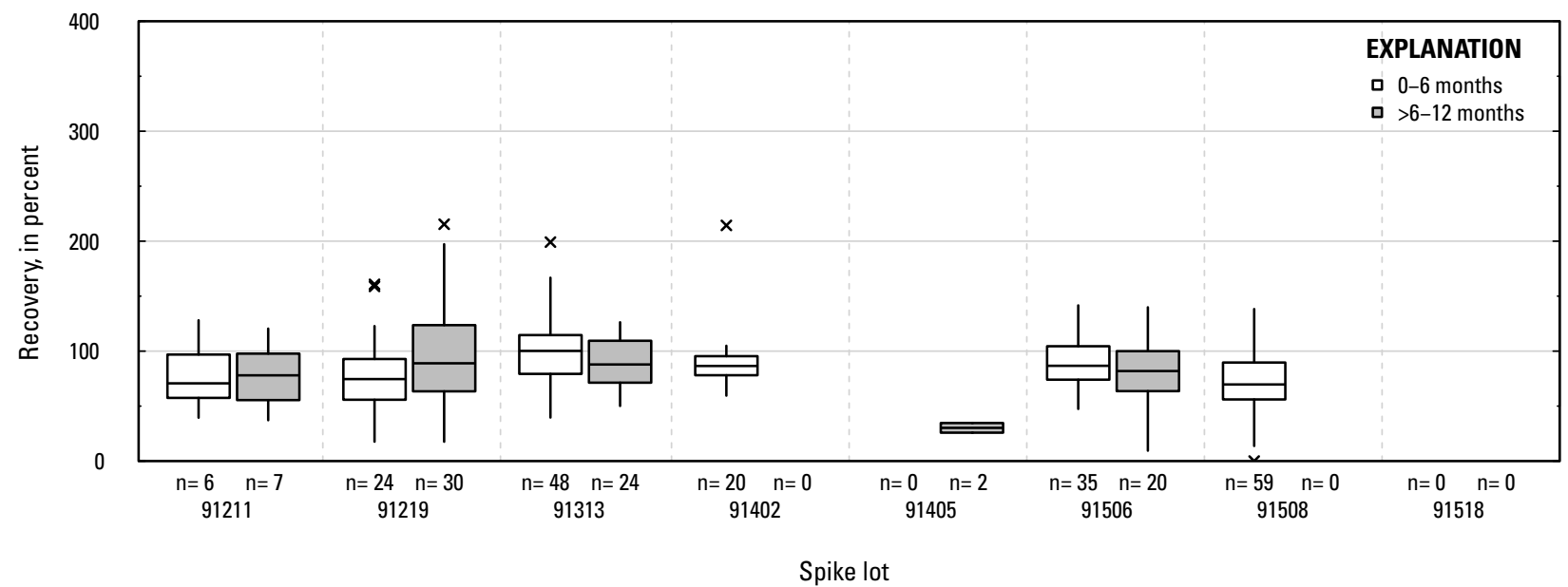

RH. Novaluron: groundwater field matrix spikes

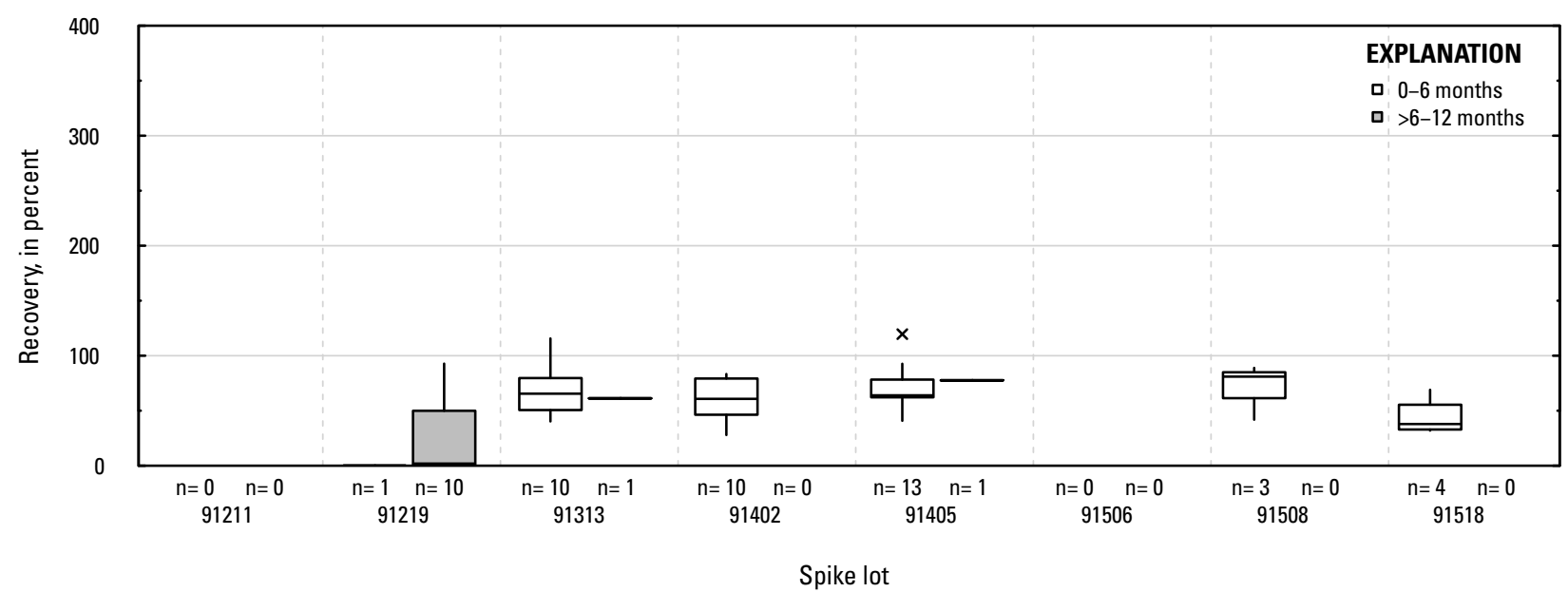

RI. Novaluron: surface water field matrix spikes

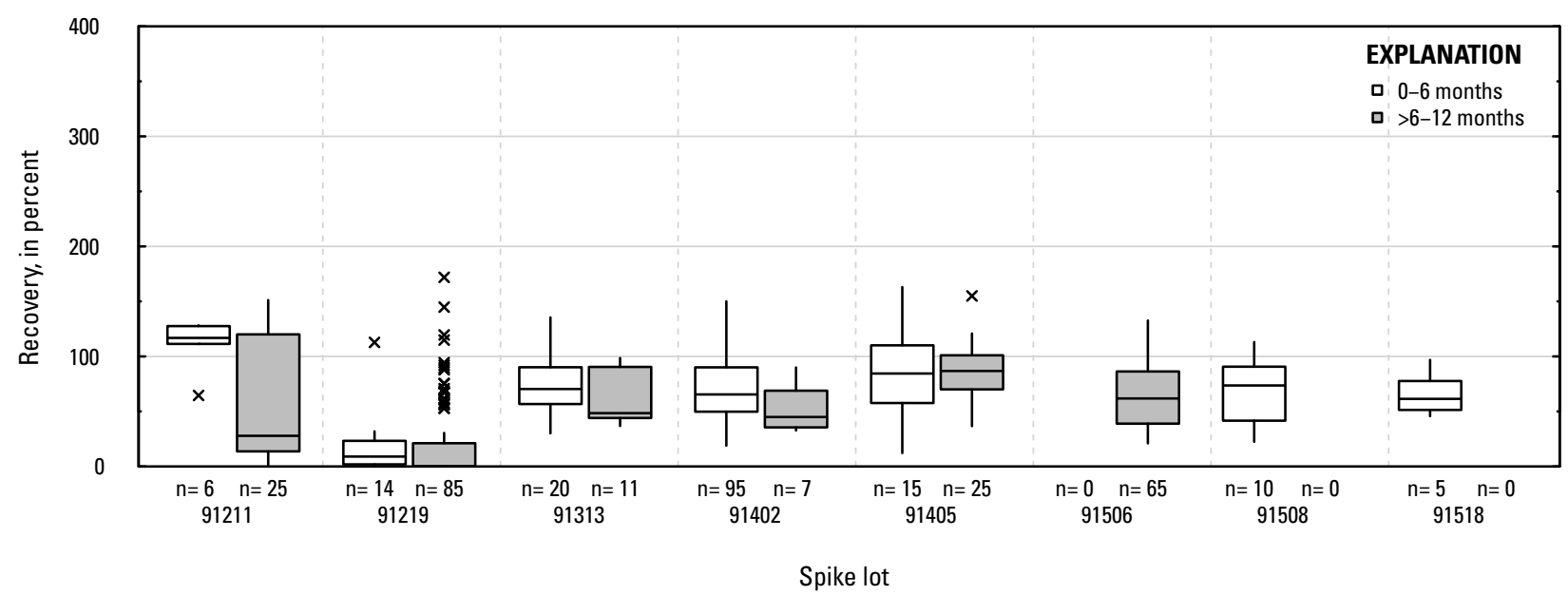

Figure 1-1. Distributions of recovery for individual pesticides in schedule 2437 by matrix, spike lot, and spike lot age. Recovery values larger than 400 percent are not shown.-Continued 
RJ. 0-Ethyl-0-methyl-S-propyIphosphorothioate: laboratory reagent spikes

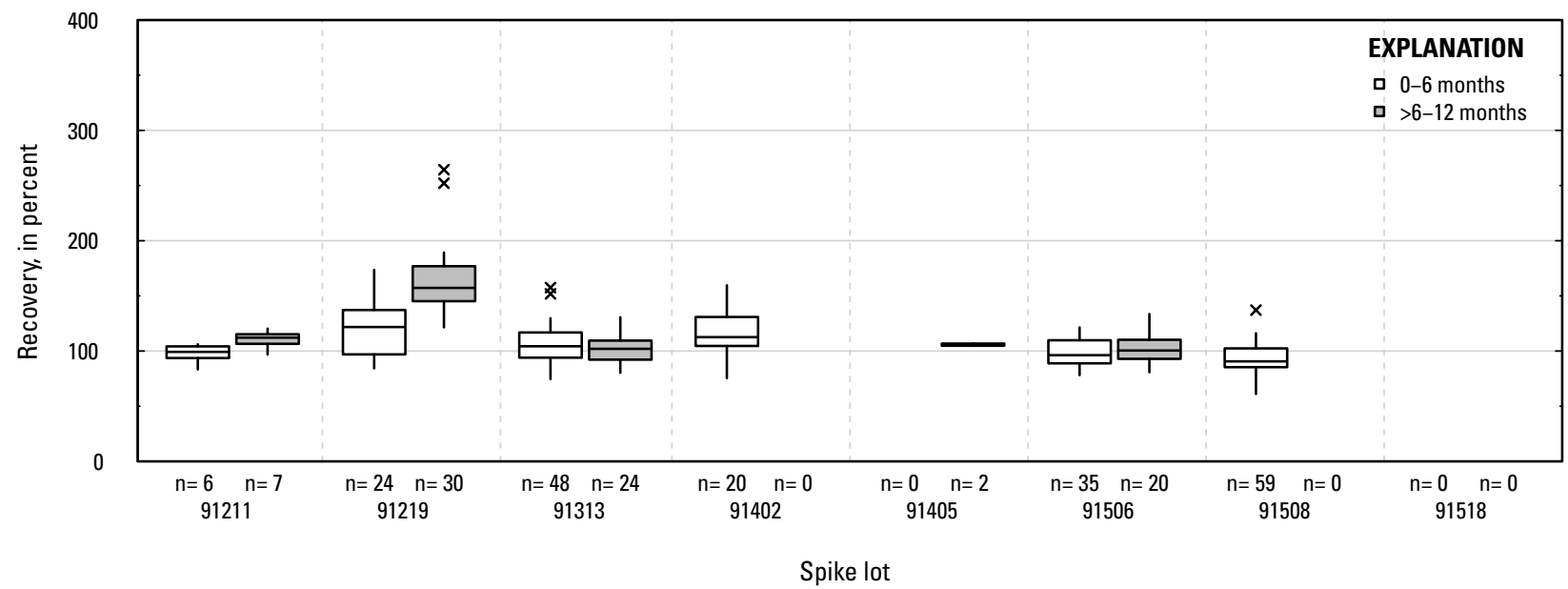

RK. 0-Ethyl-0-methyl-S-propylphosphorothioate: groundwater field matrix spikes

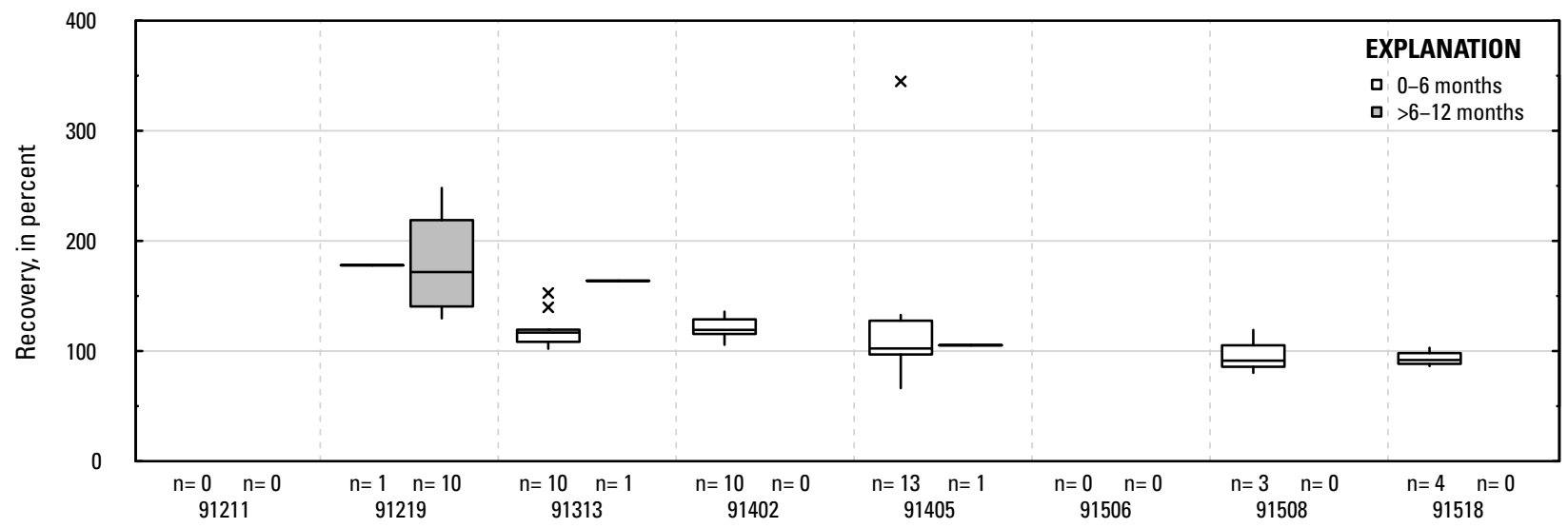

Spike lot

\section{RL. 0-Ethyl-0-methyl-S-propylphosphorothioate: surface water field matrix spikes}

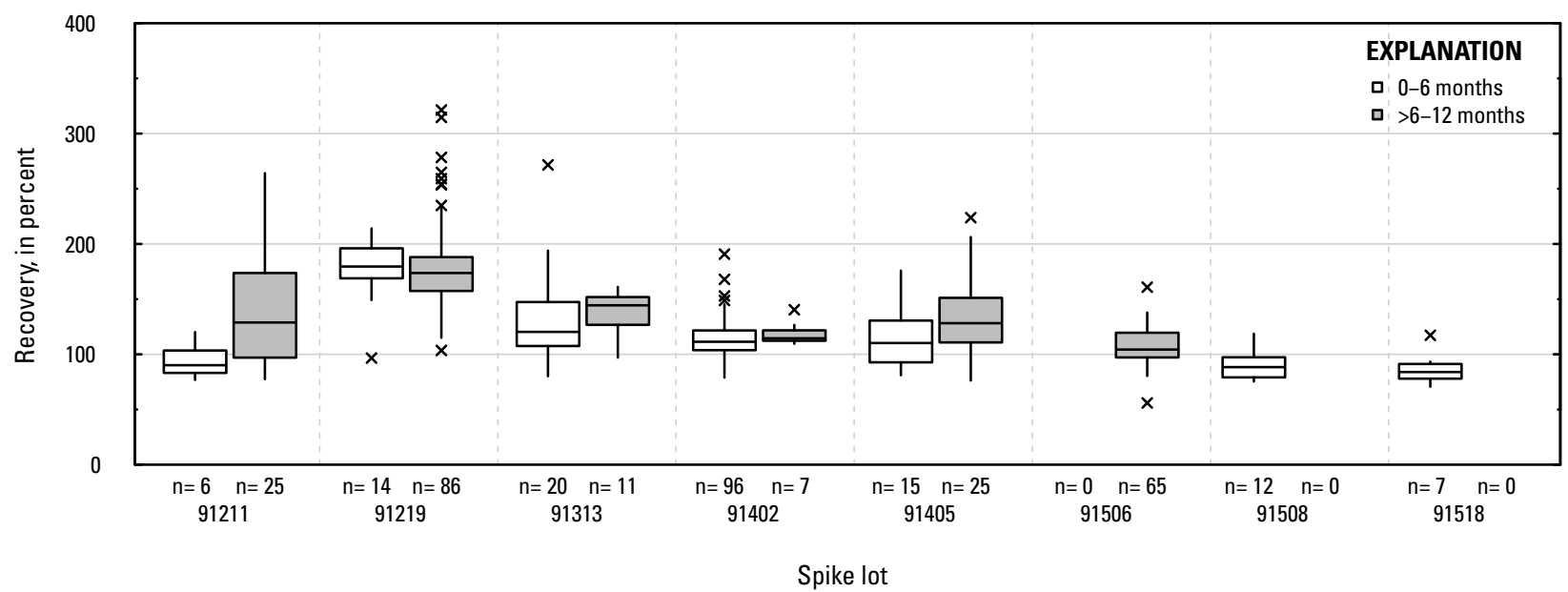

Figure 1-1. Distributions of recovery for individual pesticides in schedule 2437 by matrix, spike lot, and spike lot age. Recovery values larger than 400 percent are not shown.-Continued 
RM. 0-Ethyl-S-methyl-S-propyl phosphorodithioate: laboratory reagent spikes

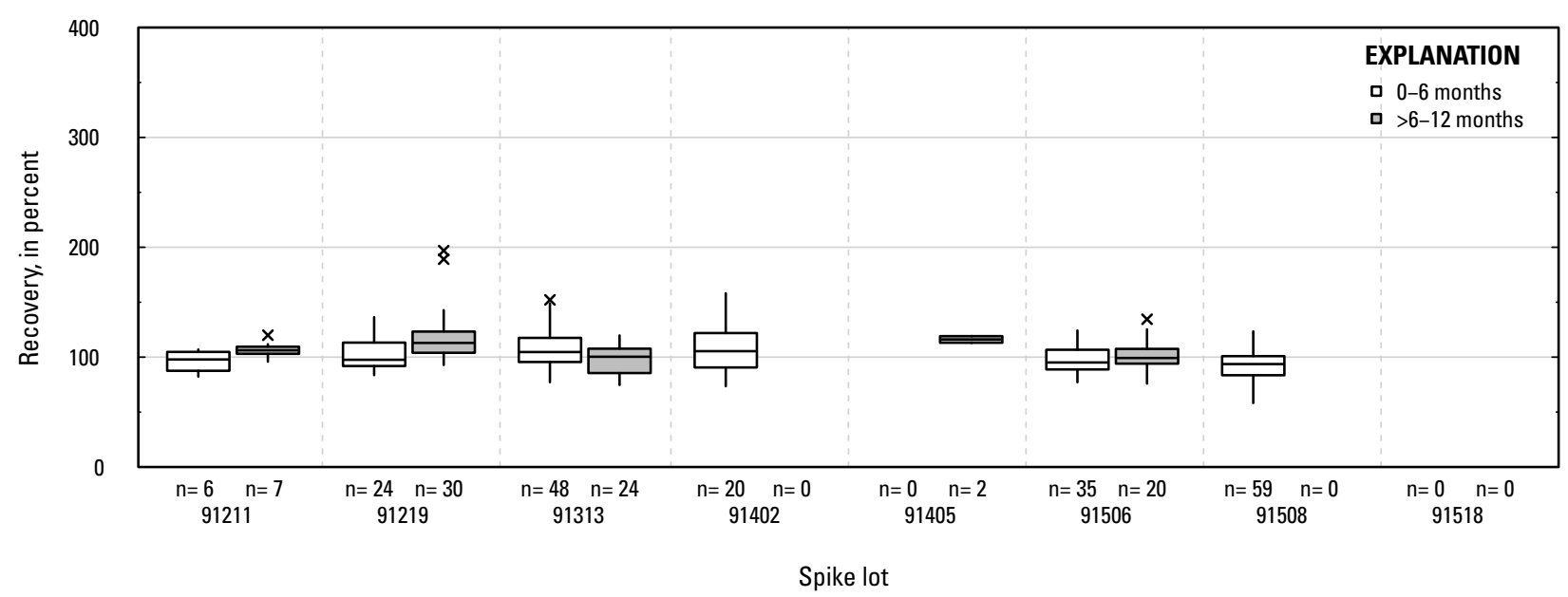

RN. 0-Ethyl-S-methyl-S-propyl phosphorodithioate: groundwater field matrix spikes

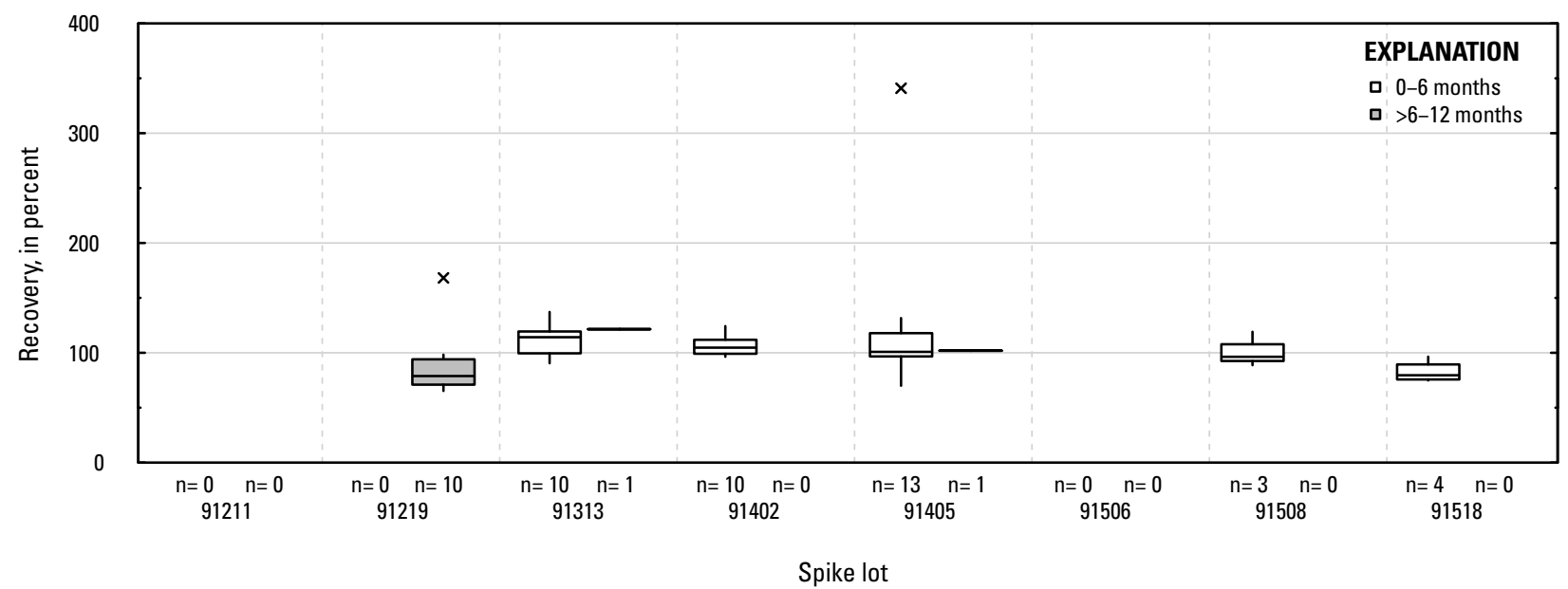

RO. 0-Ethyl-S-methyl-S-propyl phosphorodithioate: surface water field matrix spikes

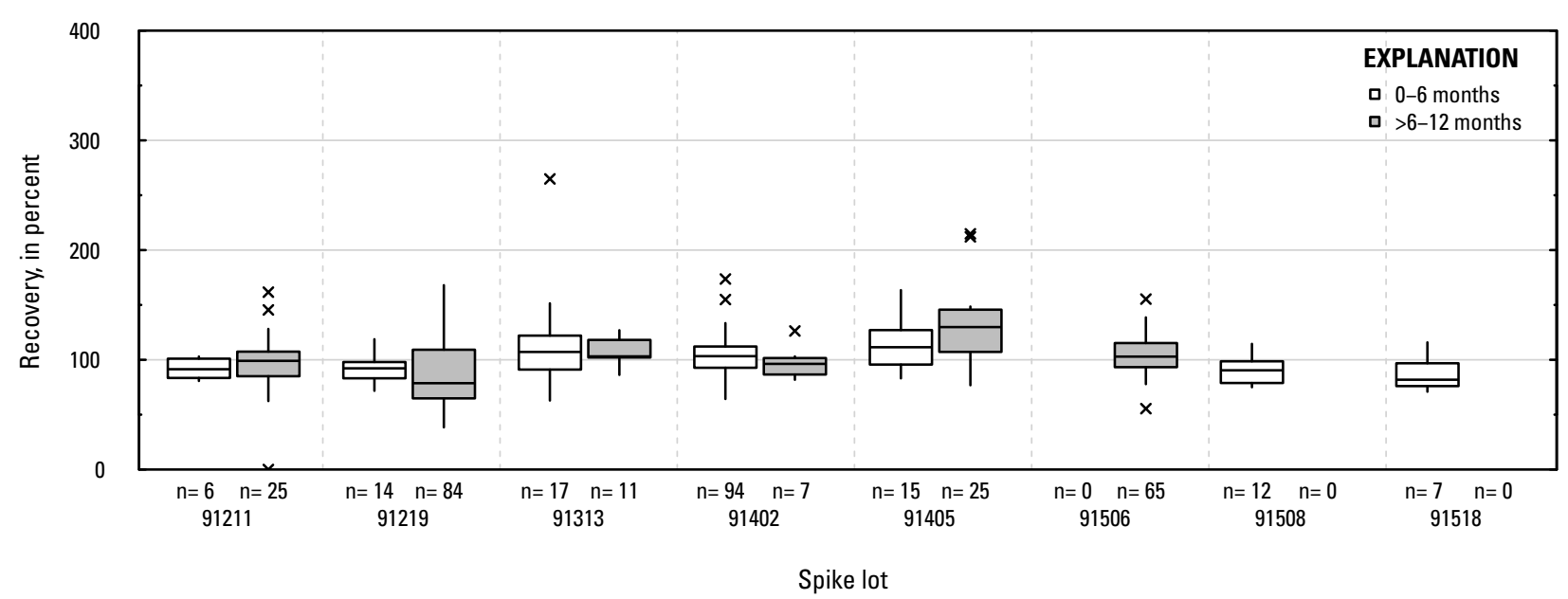

Figure 1-1. Distributions of recovery for individual pesticides in schedule 2437 by matrix, spike lot, and spike lot age. Recovery values larger than 400 percent are not shown.-Continued 
RM. 0-Ethyl-S-methyl-S-propyl phosphorodithioate: laboratory reagent spikes

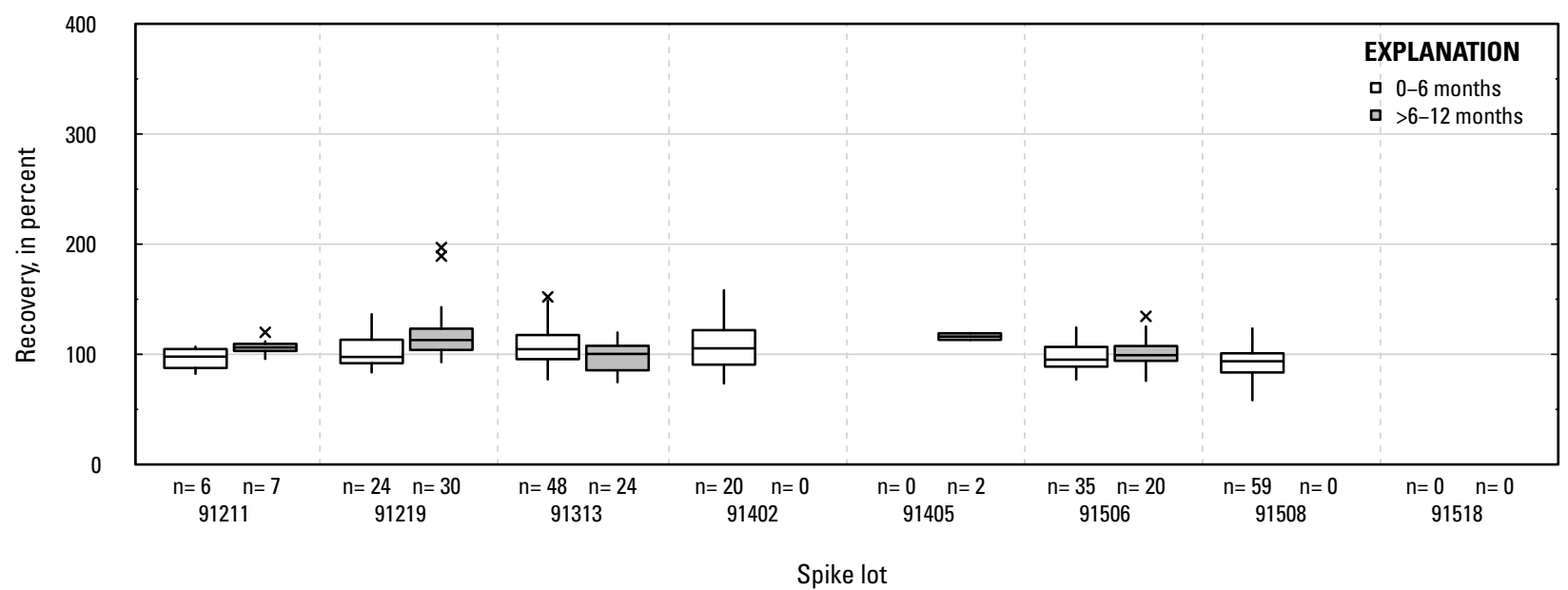

RN. 0-Ethyl-S-methyl-S-propyl phosphorodithioate: groundwater field matrix spikes

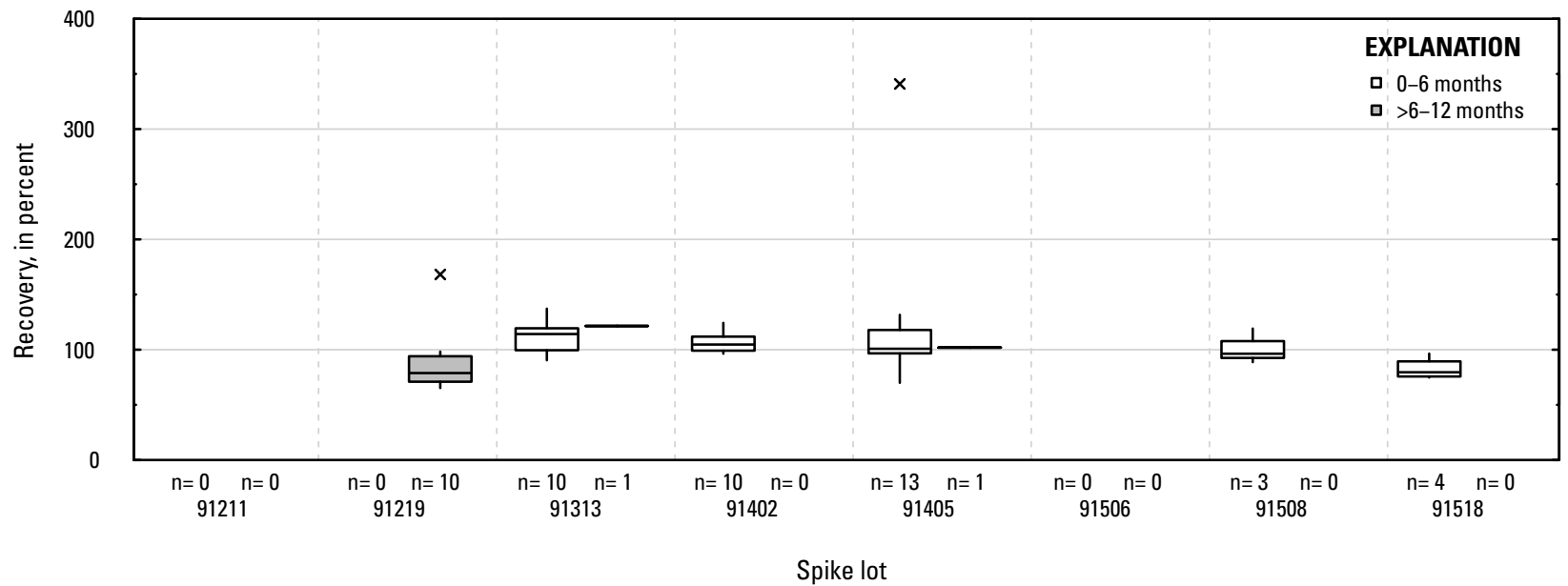

RO. 0-Ethyl-S-methyl-S-propyl phosphorodithioate: surface water field matrix spikes

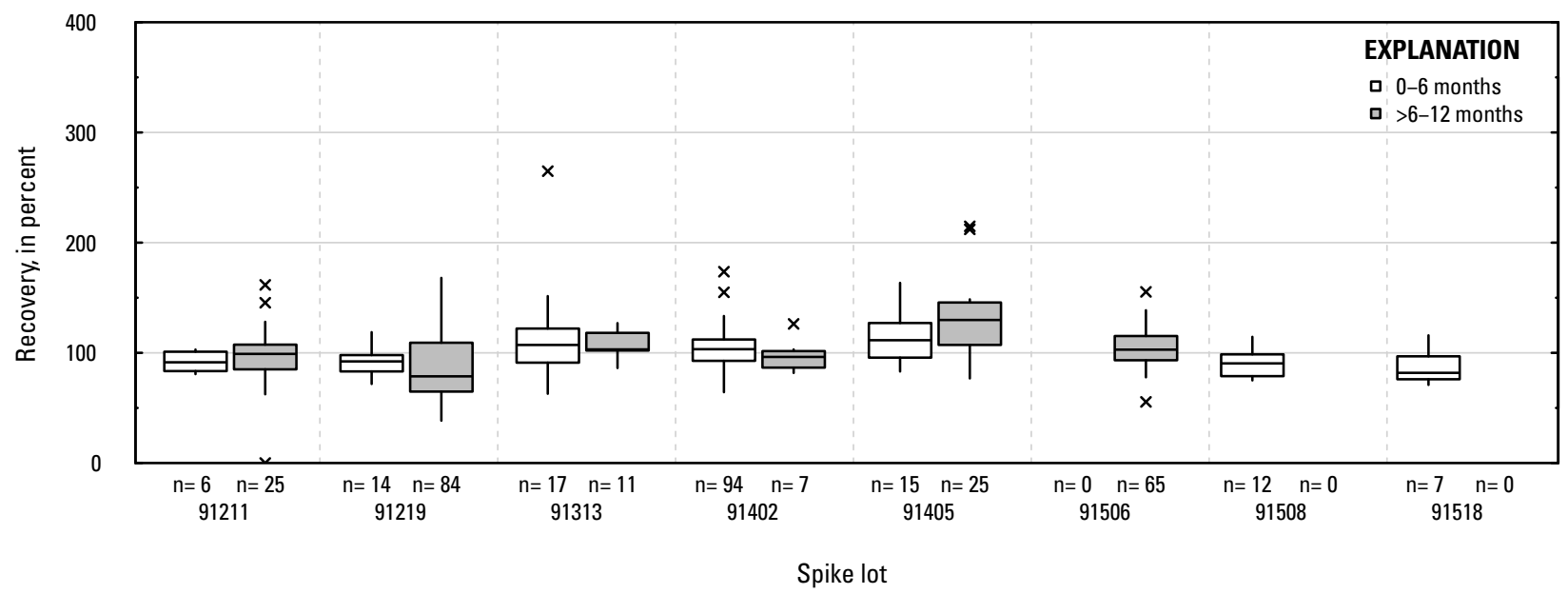

Figure 1-1. Distributions of recovery for individual pesticides in schedule 2437 by matrix, spike lot, and spike lot age. Recovery values larger than 400 percent are not shown.-Continued 
RS. Orthosulfamuron: laboratory reagent spikes

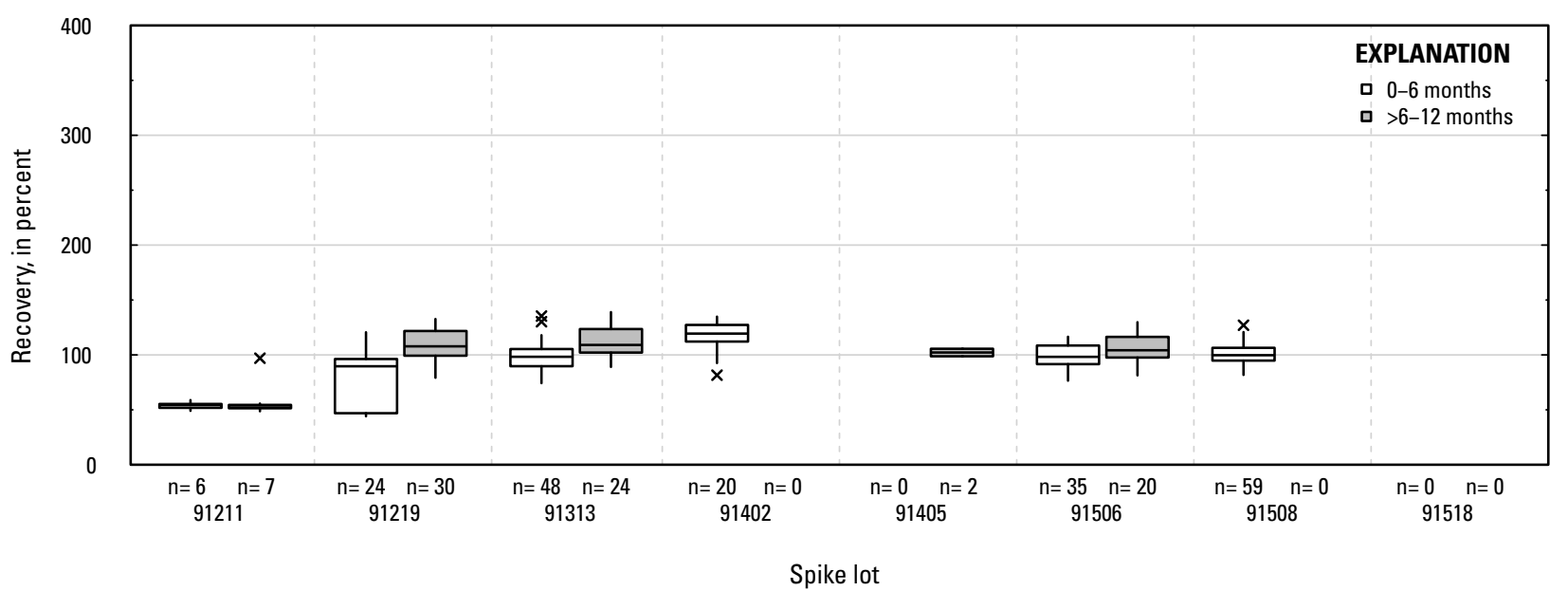

RT. Orthosulfamuron: groundwater field matrix spikes

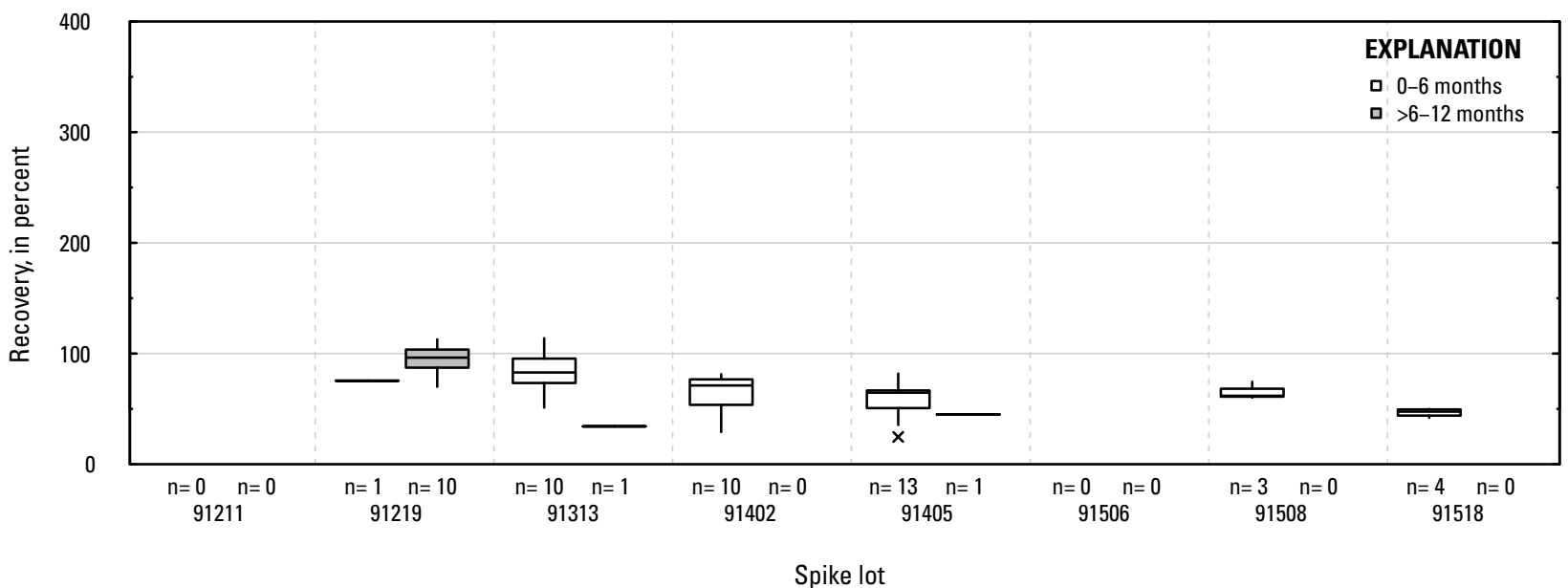

RU. Orthosulfamuron: surface water field matrix spikes

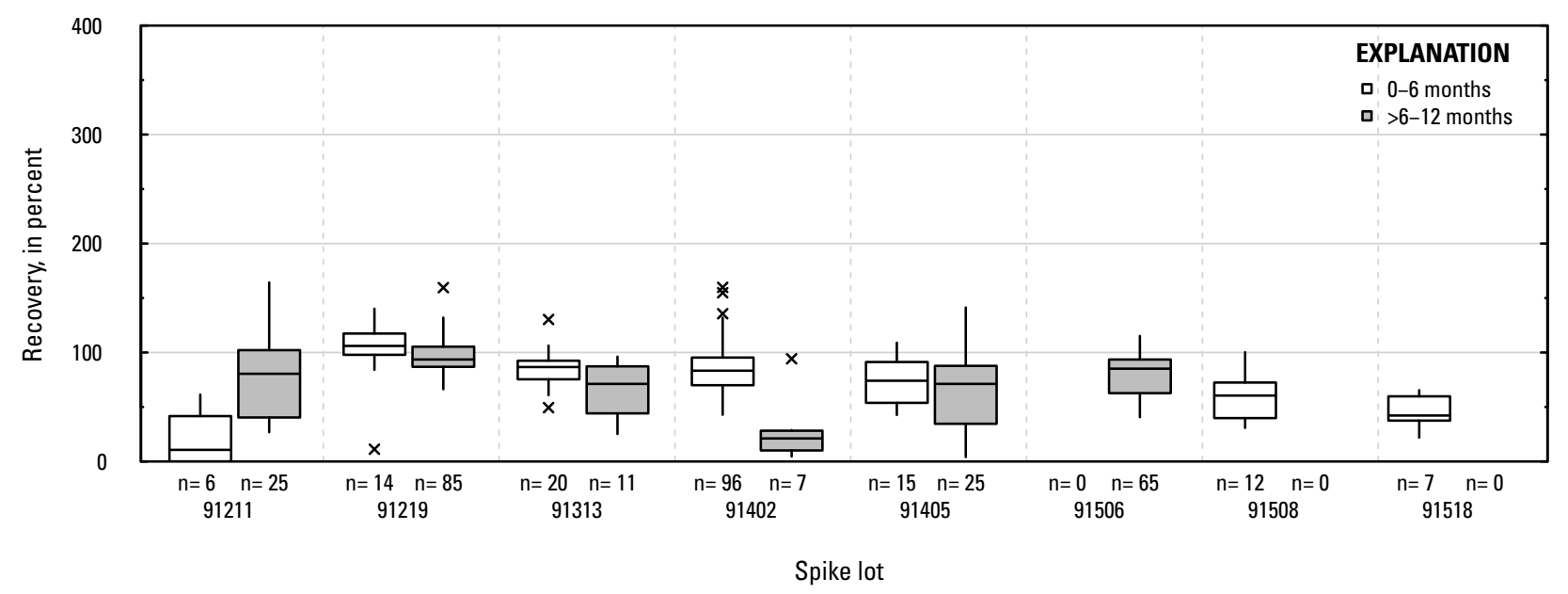

Figure 1-1. Distributions of recovery for individual pesticides in schedule 2437 by matrix, spike lot, and spike lot age. Recovery values larger than 400 percent are not shown.-Continued 
RV. Oryzalin: laboratory reagent spikes

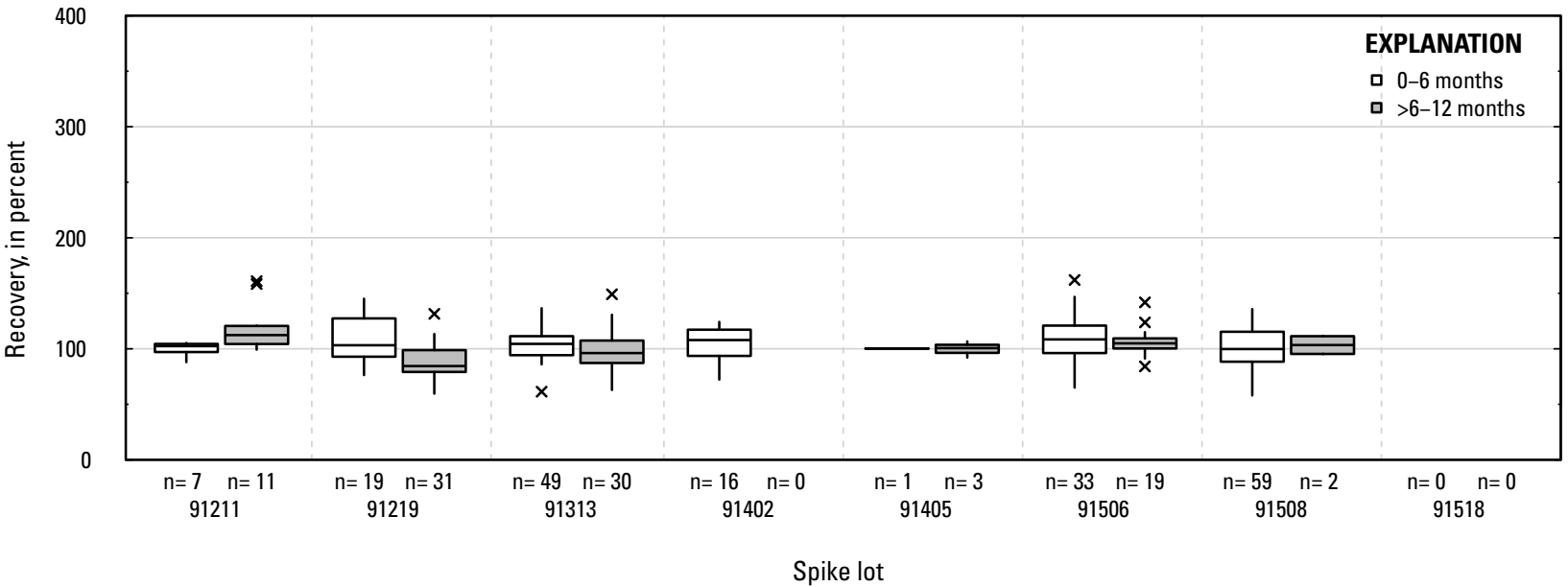

RW. Oryzalin: groundwater field matrix spikes

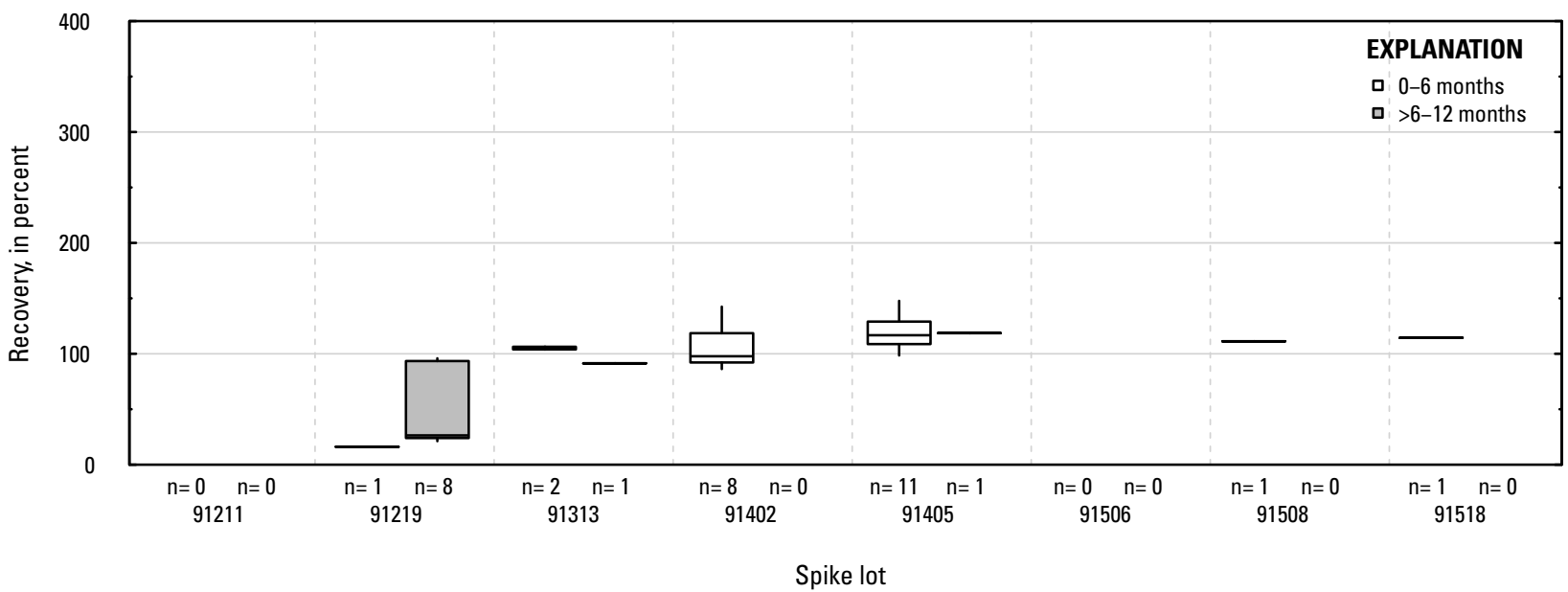

$R X$. Oryzalin: surface water field matrix spikes

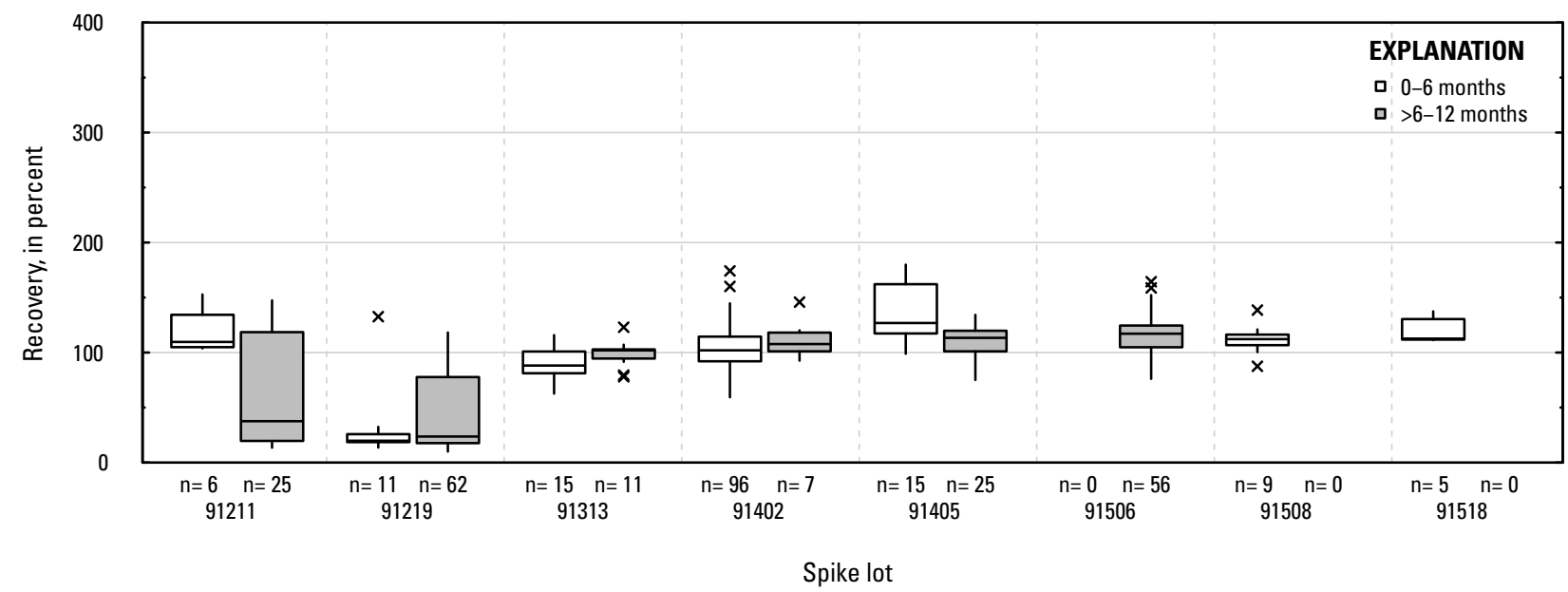

Figure 1-1. Distributions of recovery for individual pesticides in schedule 2437 by matrix, spike lot, and spike lot age. Recovery values larger than 400 percent are not shown.-Continued 


\section{RY. Oxamyl: laboratory reagent spikes}

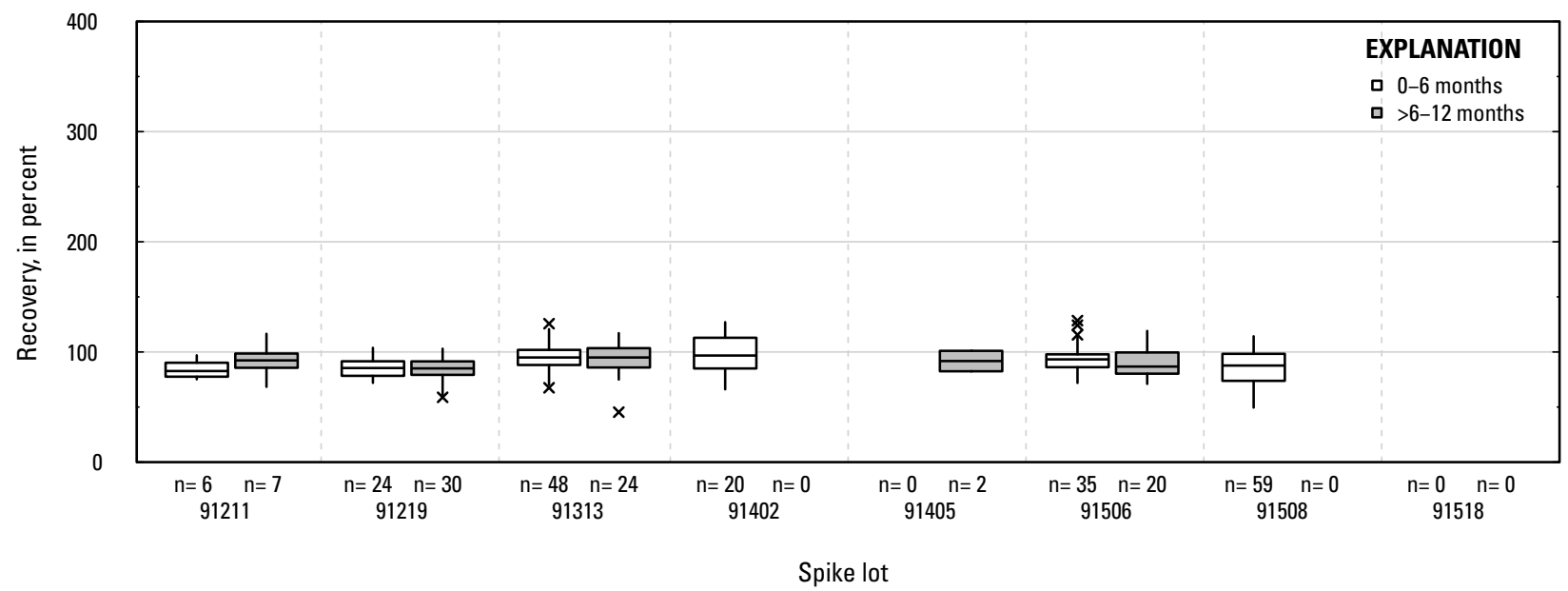

\section{RZ. Oxamyl: groundwater field matrix spikes}

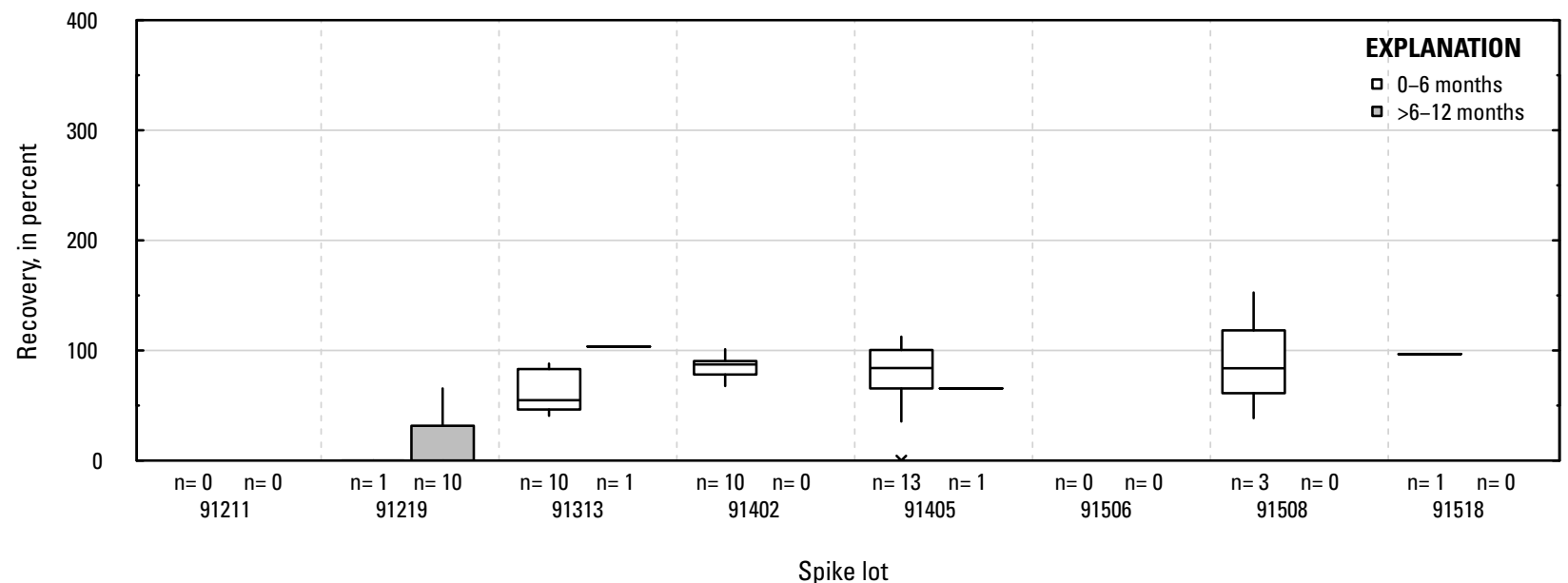

\section{SA. Oxamyl: surface water field matrix spikes}

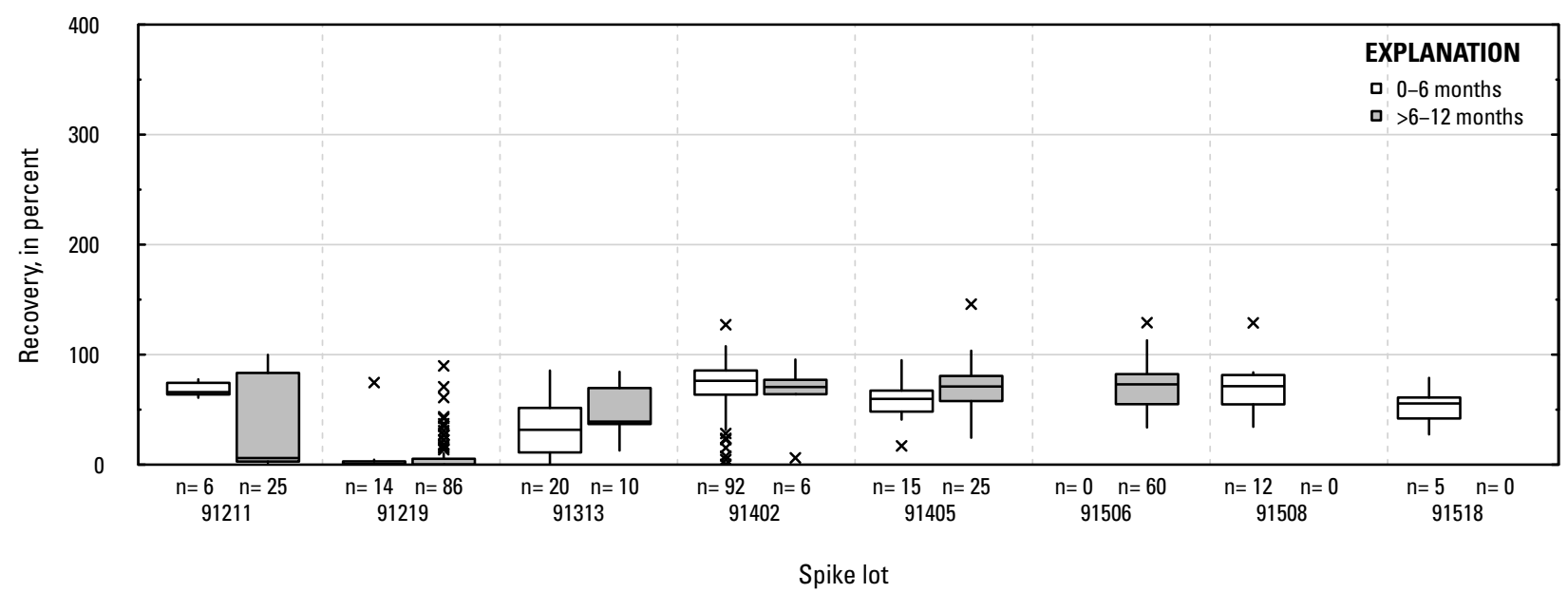

Figure 1-1. Distributions of recovery for individual pesticides in schedule 2437 by matrix, spike lot, and spike lot age. Recovery values larger than 400 percent are not shown.-Continued 
SB. Oxamyl oxime: laboratory reagent spikes

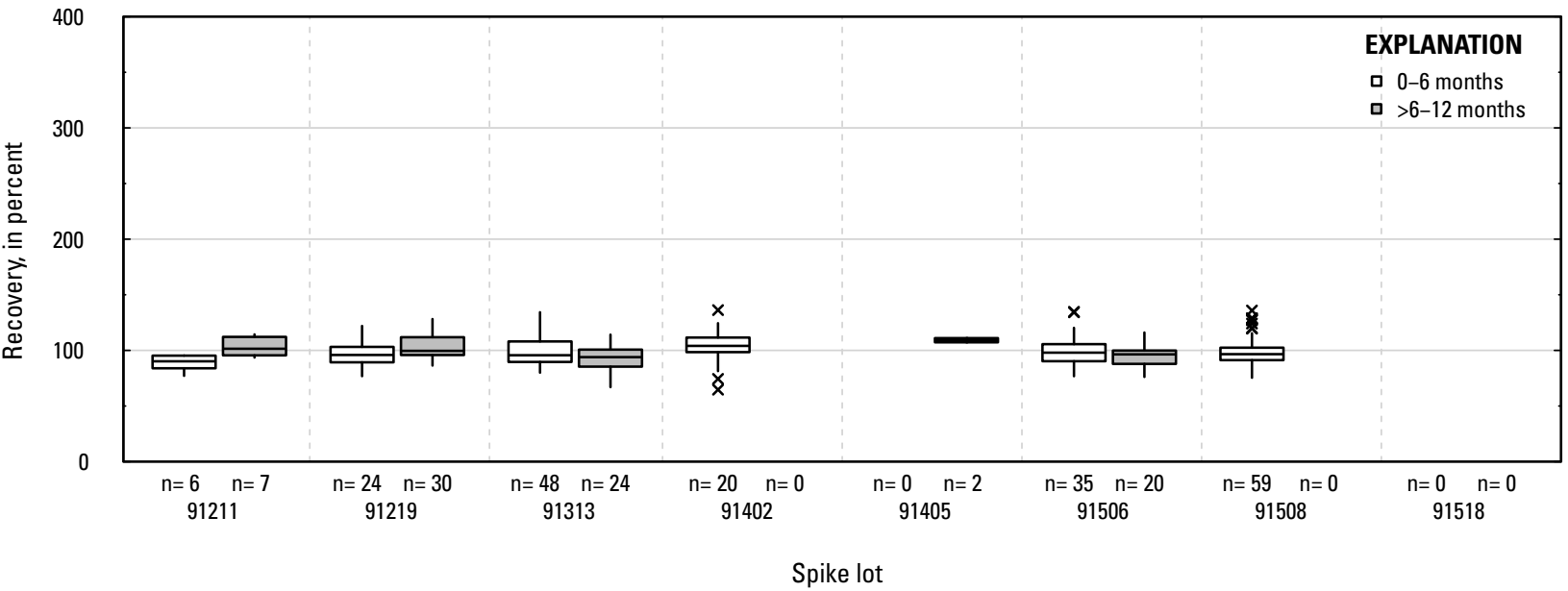

\section{SC. Oxamyl oxime: groundwater field matrix spikes}

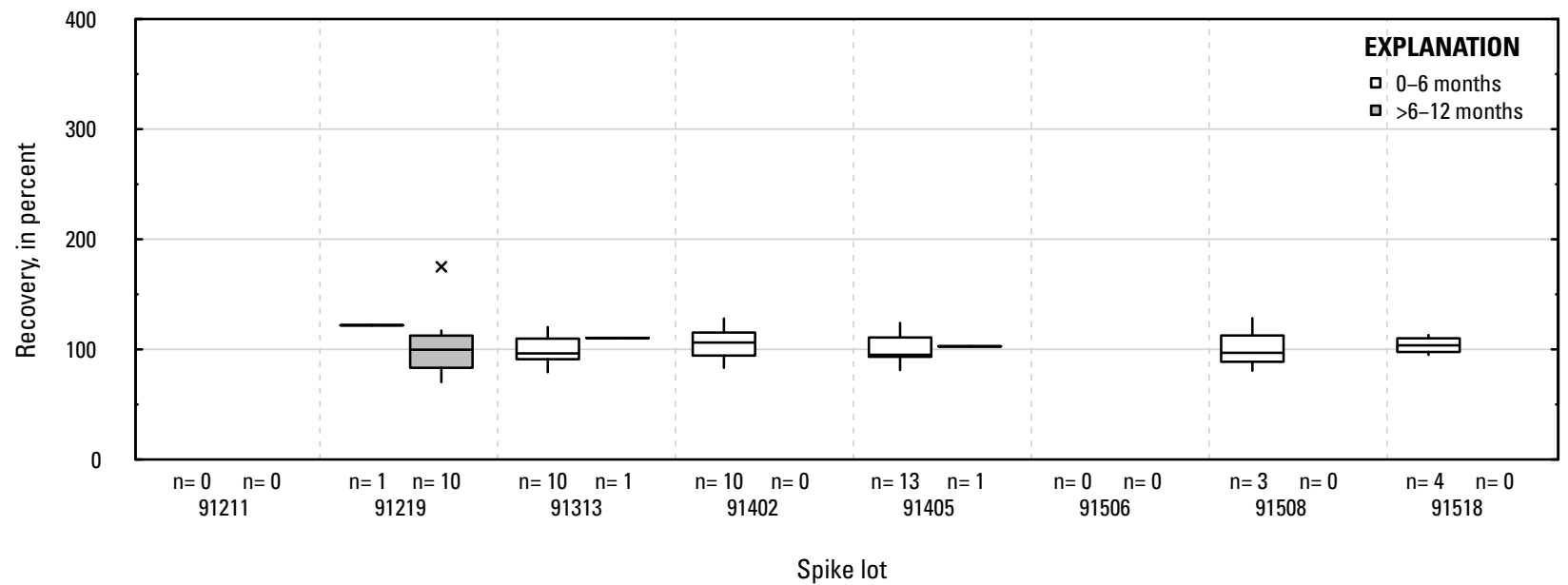

SD. Oxamyl oxime: surface water field matrix spikes

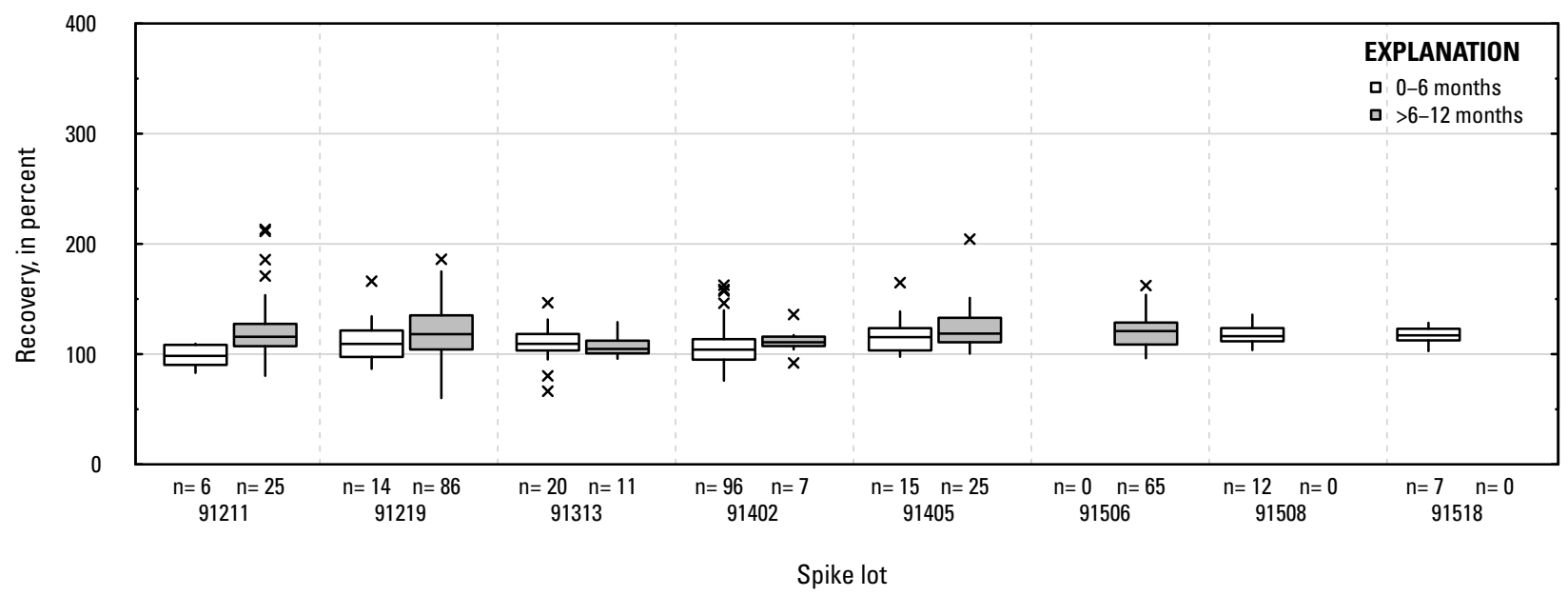

Figure 1-1. Distributions of recovery for individual pesticides in schedule 2437 by matrix, spike lot, and spike lot age. Recovery values larger than 400 percent are not shown.-Continued 
SE. Oxyfluorfen: laboratory reagent spikes

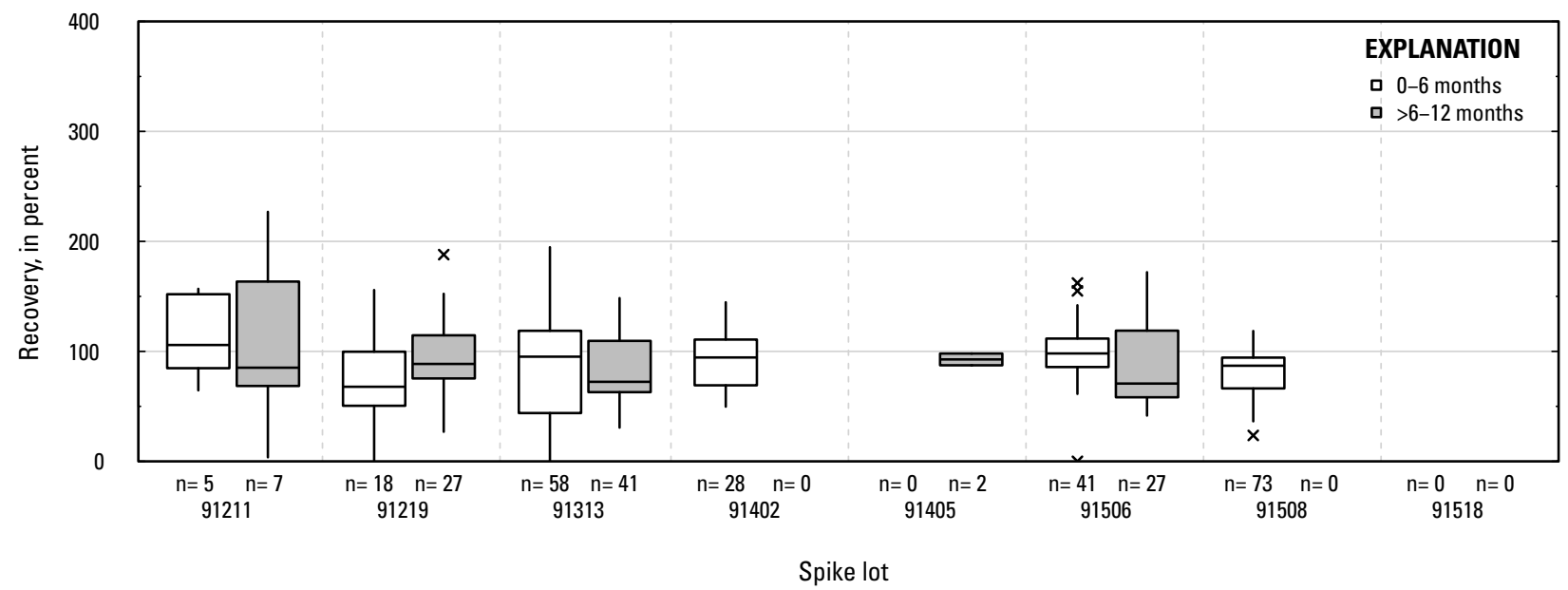

SF. Oxyfluorfen: groundwater field matrix spikes

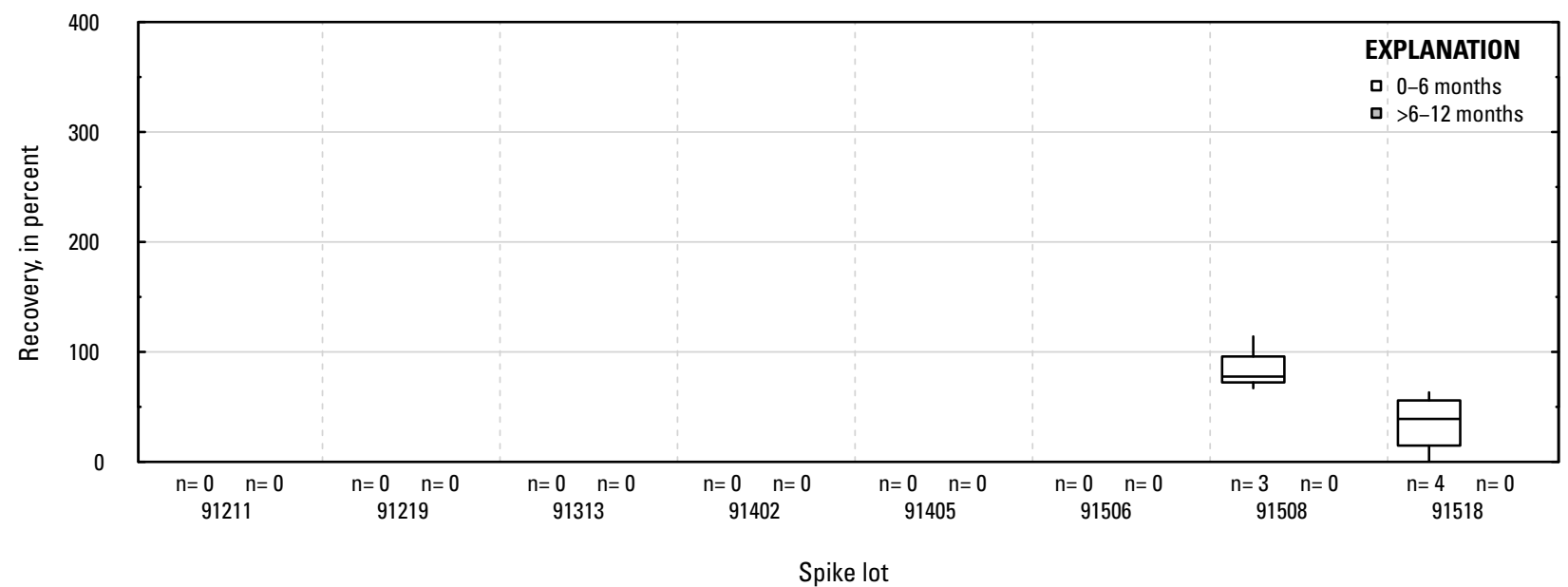

SG. Oxyfluorfen: surface water field matrix spikes

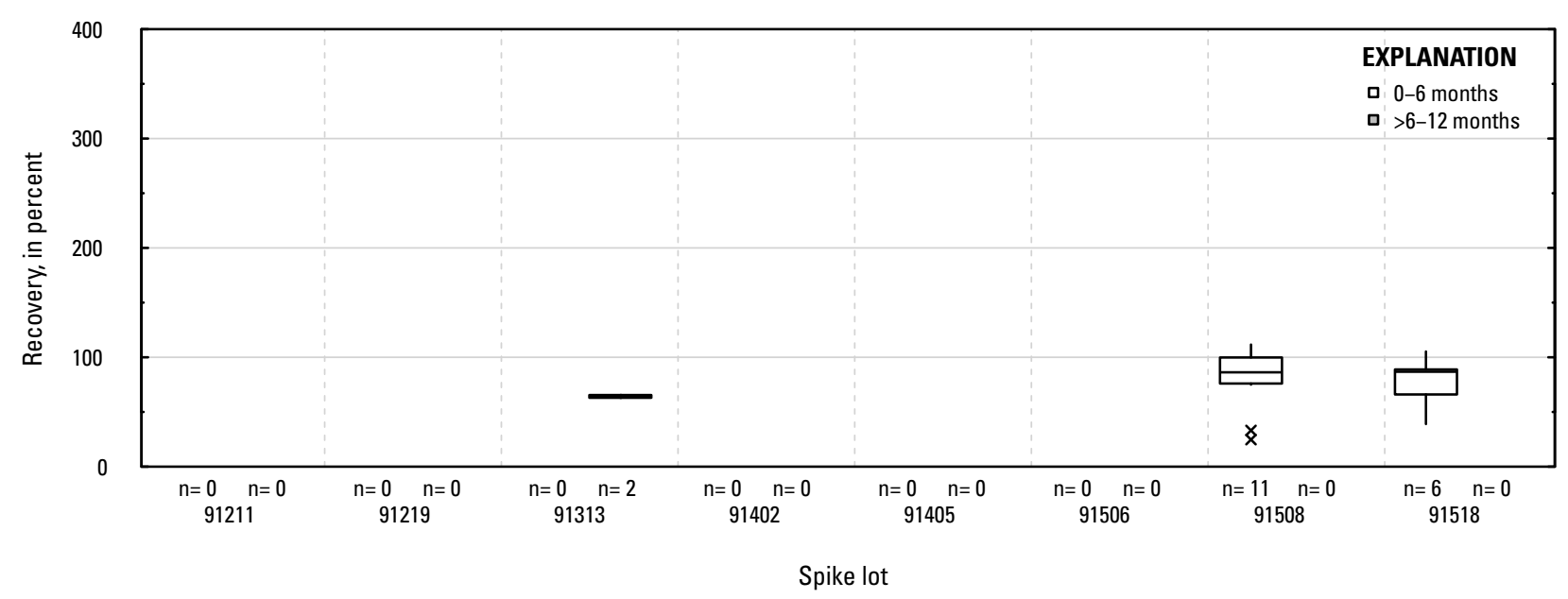

Figure 1-1. Distributions of recovery for individual pesticides in schedule 2437 by matrix, spike lot, and spike lot age. Recovery values larger than 400 percent are not shown.-Continued 
SH. Paraoxon: laboratory reagent spikes

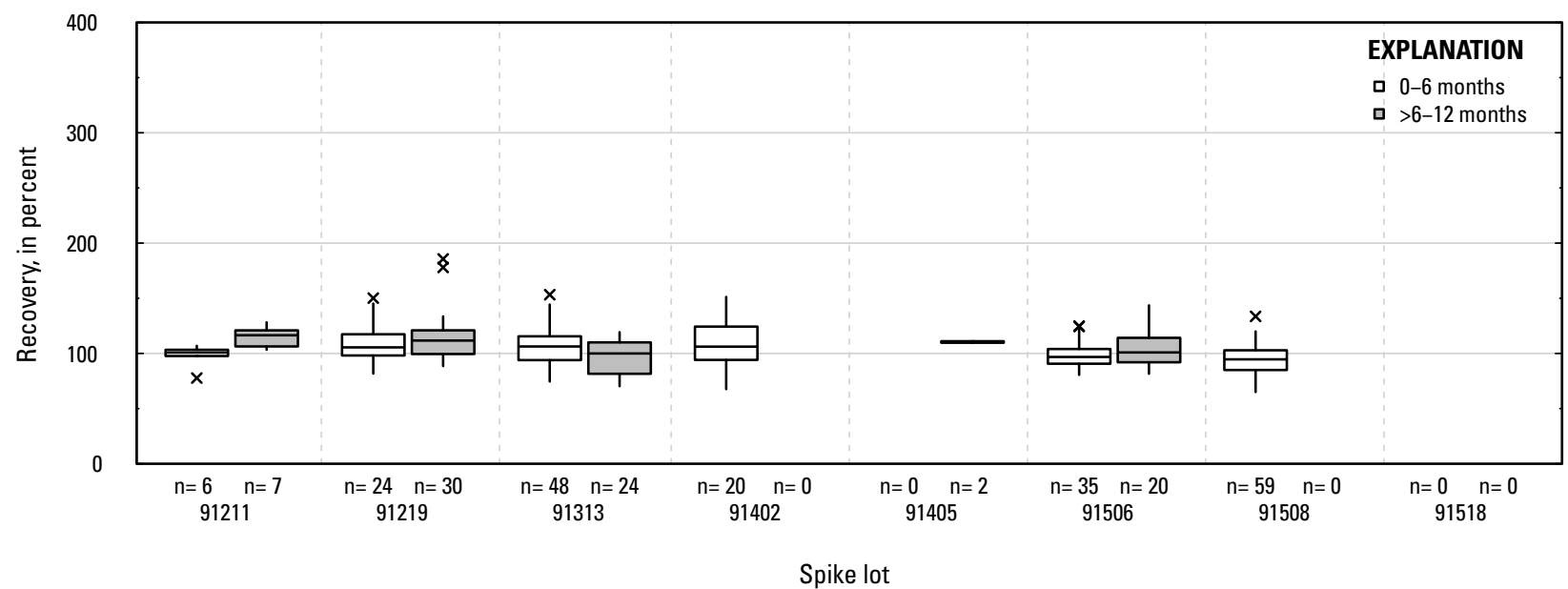

SI. Paraoxon: groundwater field matrix spikes

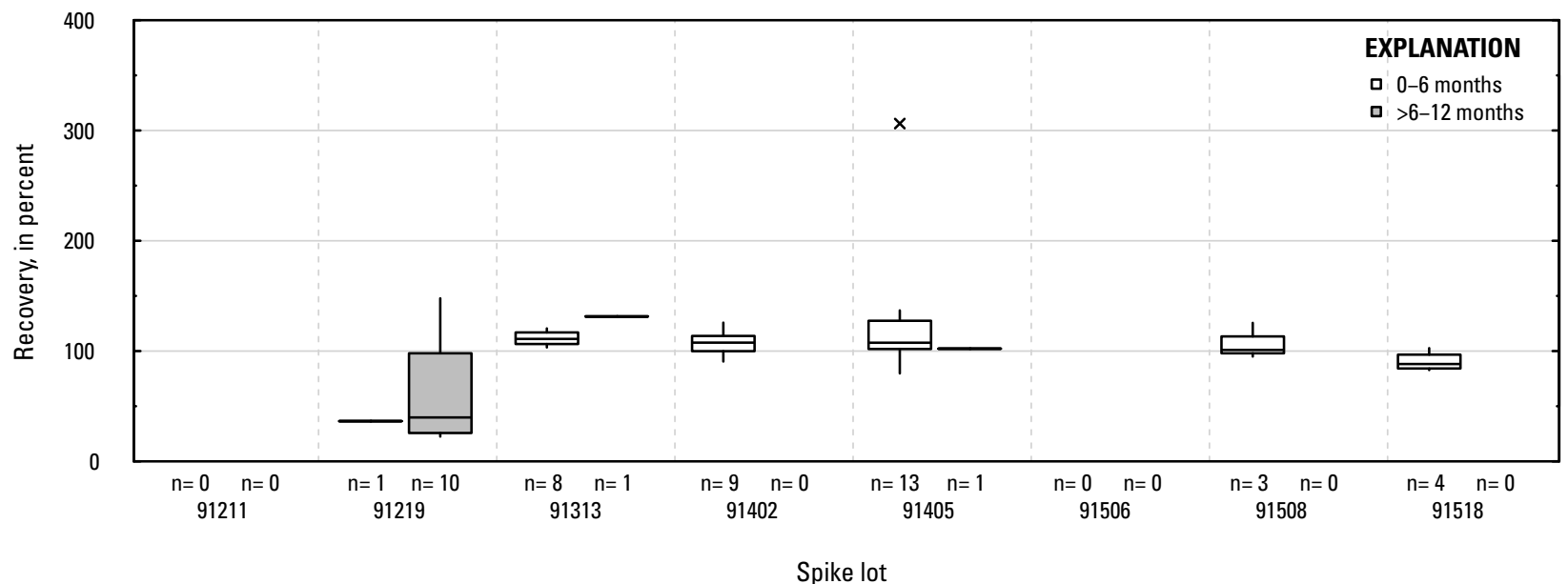

SJ. Paraoxon: surface water field matrix spikes

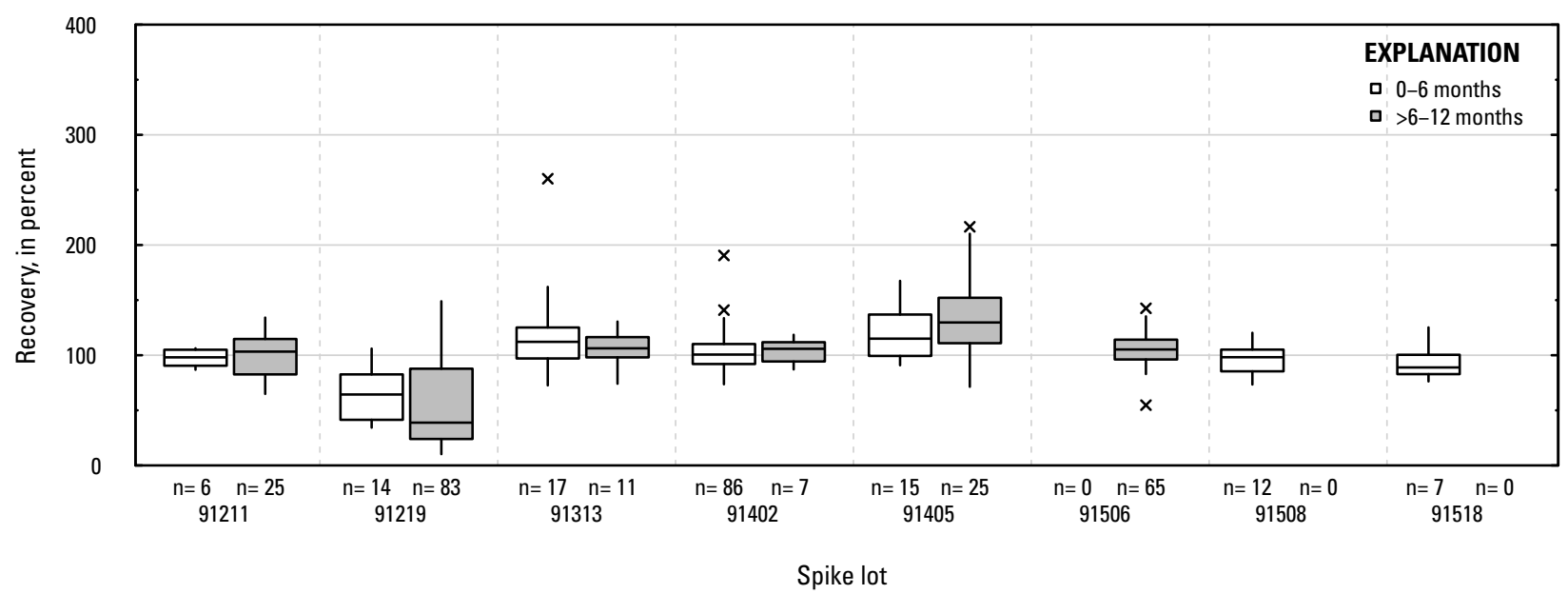

Figure 1-1. Distributions of recovery for individual pesticides in schedule 2437 by matrix, spike lot, and spike lot age. Recovery values larger than 400 percent are not shown.-Continued 


\section{SK. Methyl paraoxon: laboratory reagent spikes}

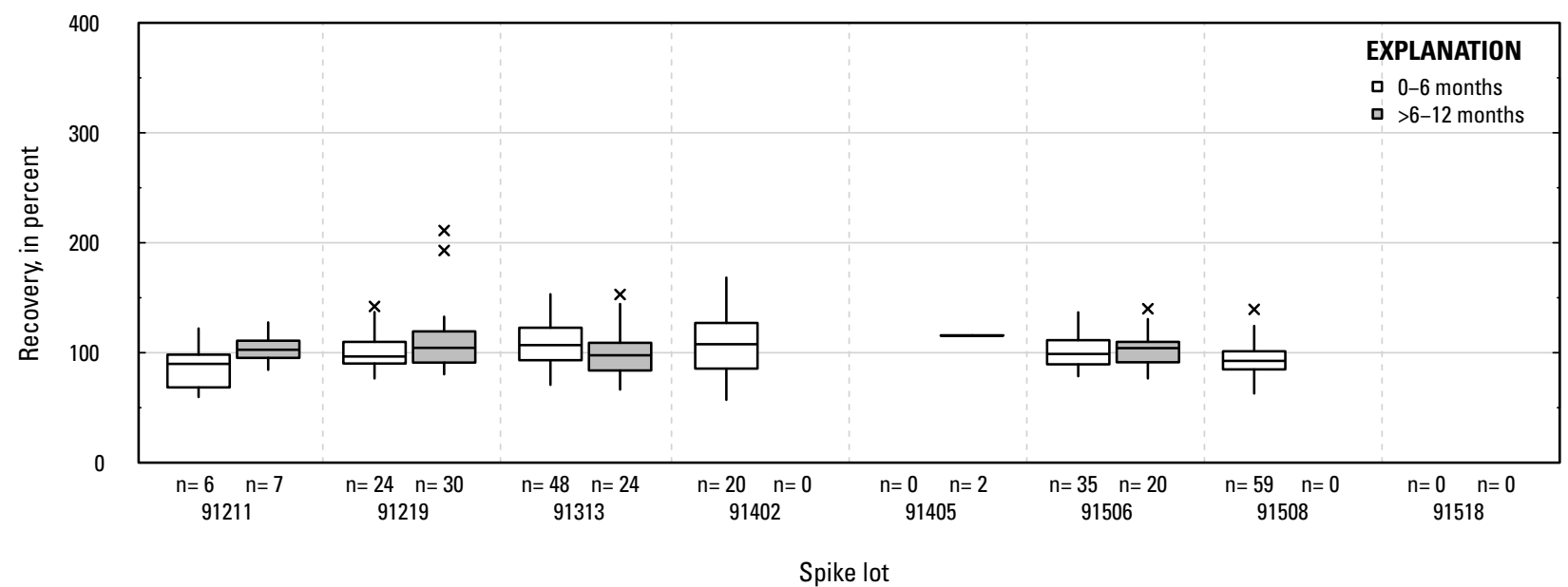

SL. Methyl paraoxon: groundwater field matrix spikes

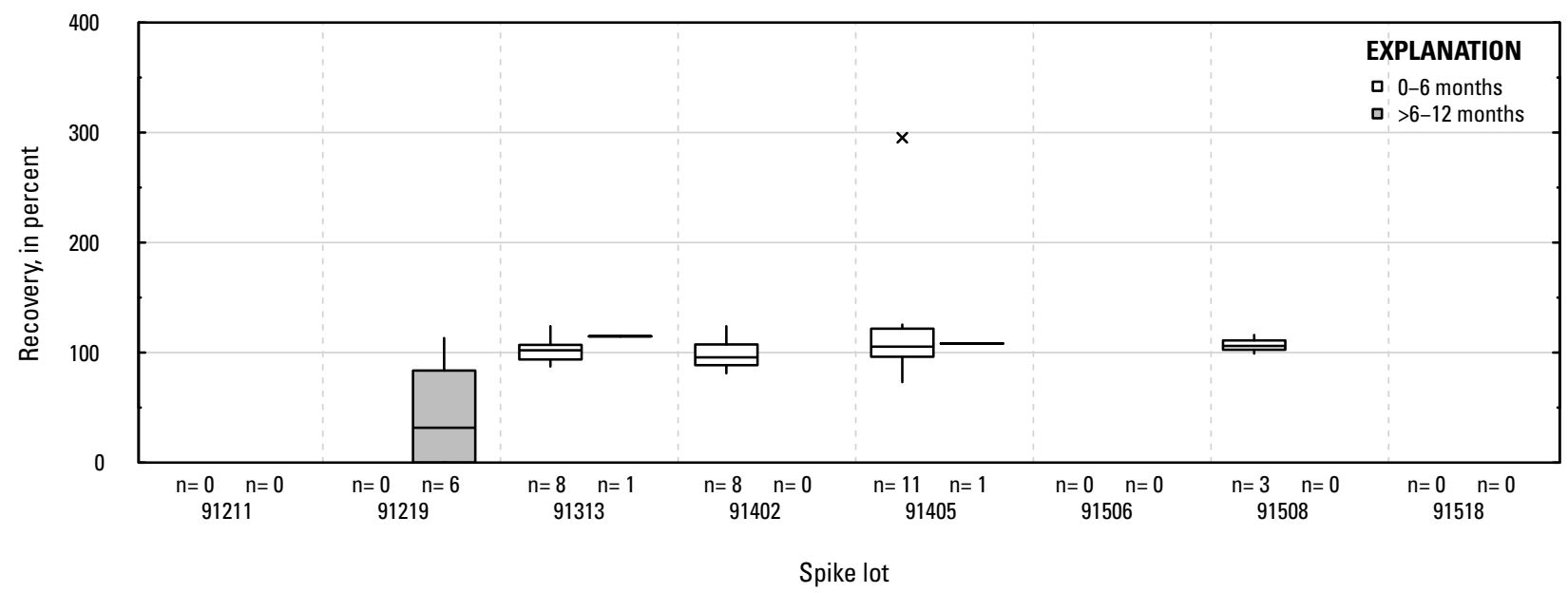

SM. Methyl paraoxon: surface water field matrix spikes

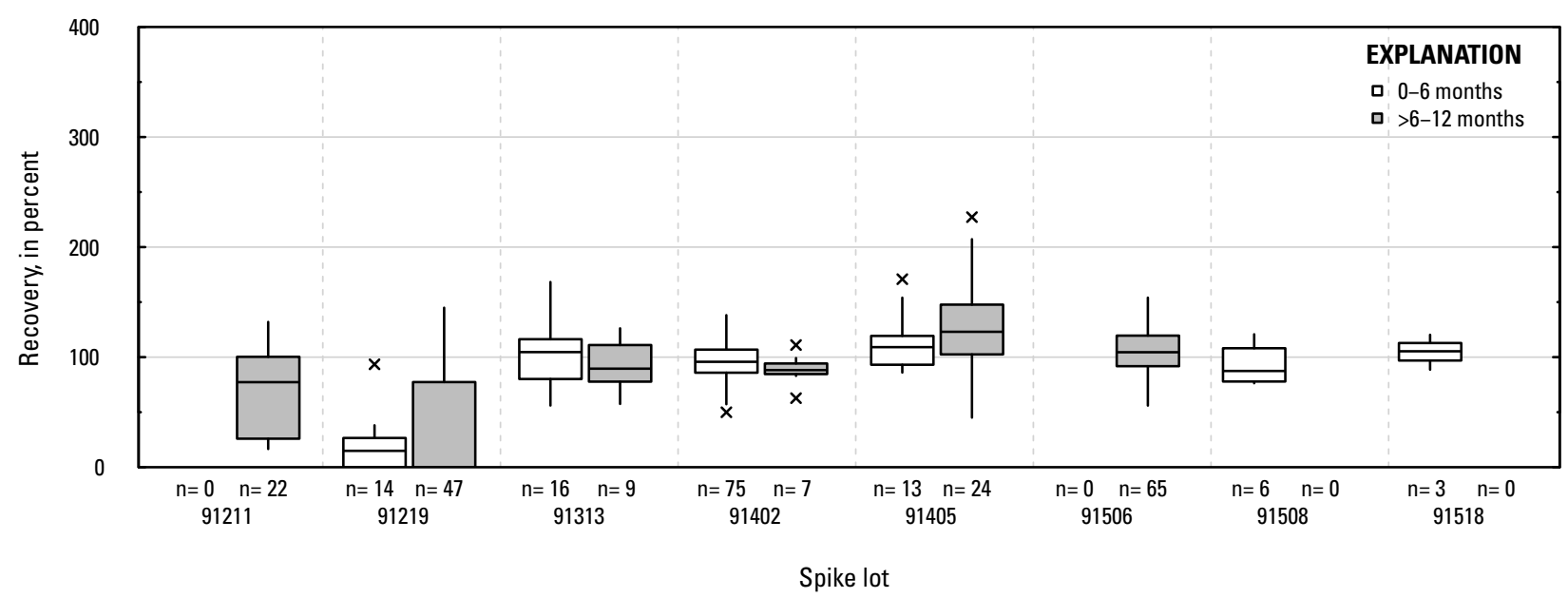

Figure 1-1. Distributions of recovery for individual pesticides in schedule 2437 by matrix, spike lot, and spike lot age. Recovery values larger than 400 percent are not shown.-Continued 
SN. Pendimethalin: laboratory reagent spikes

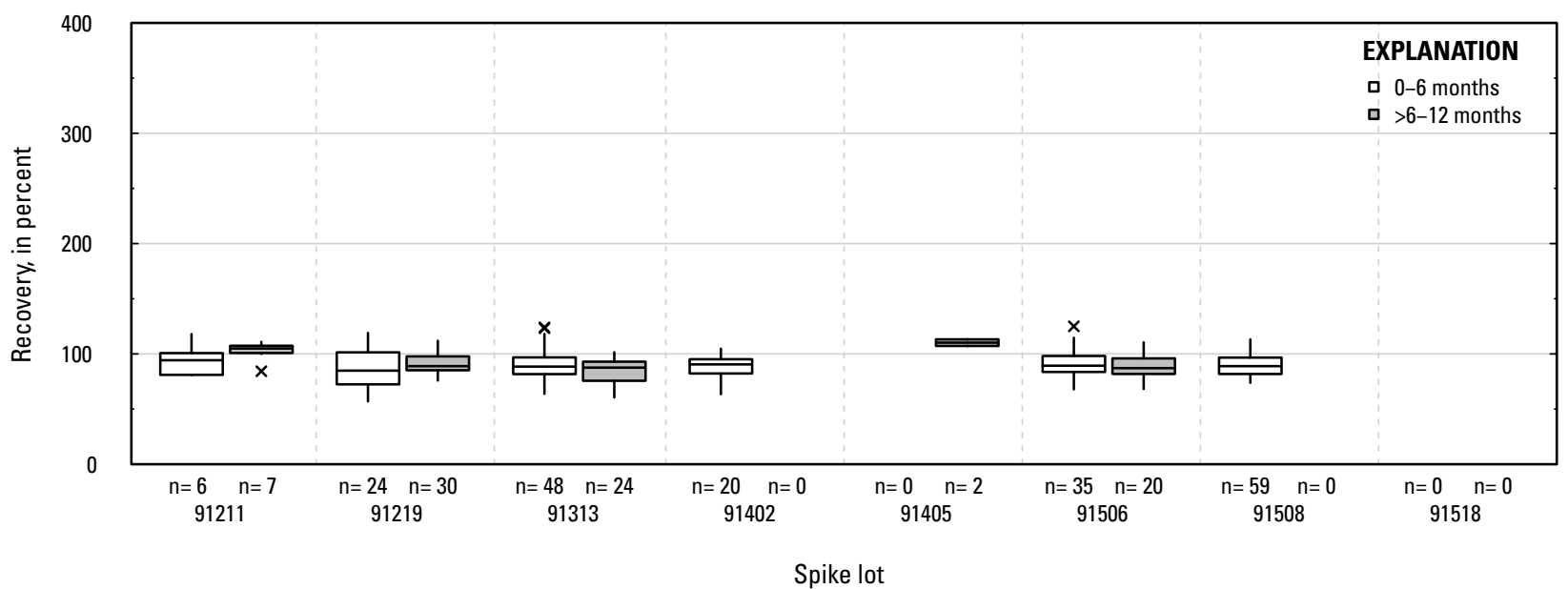

SO. Pendimethalin: groundwater field matrix spikes

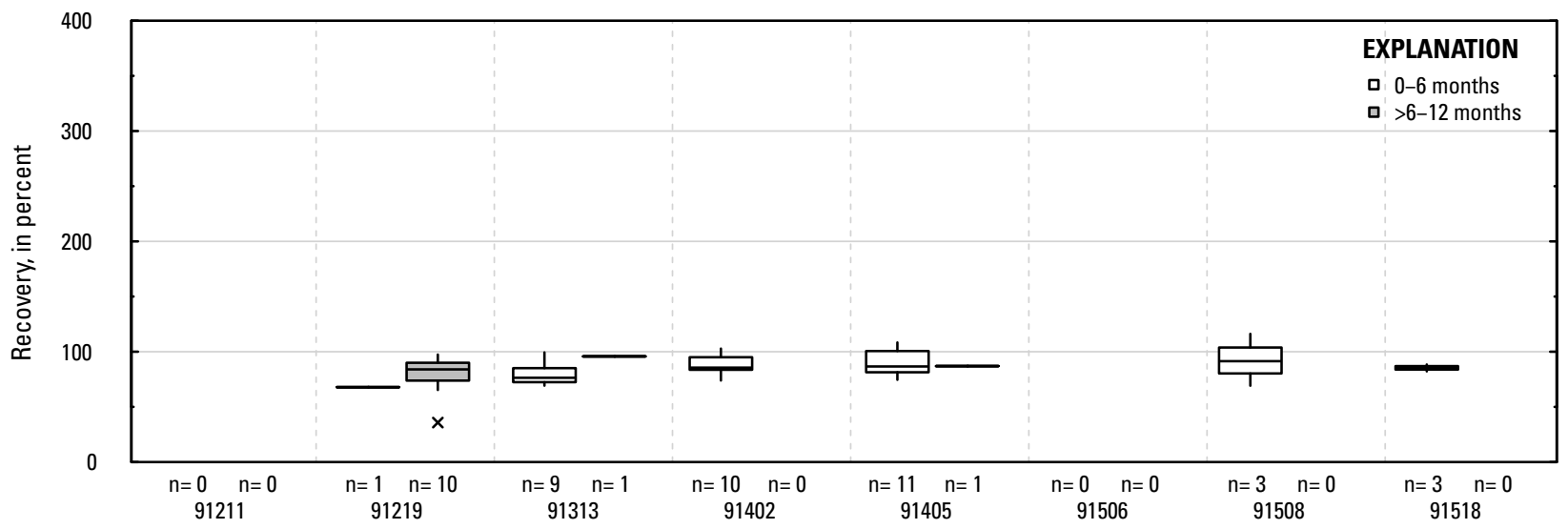

Spike lot

\section{SP. Pendimethalin: surface water field matrix spikes}

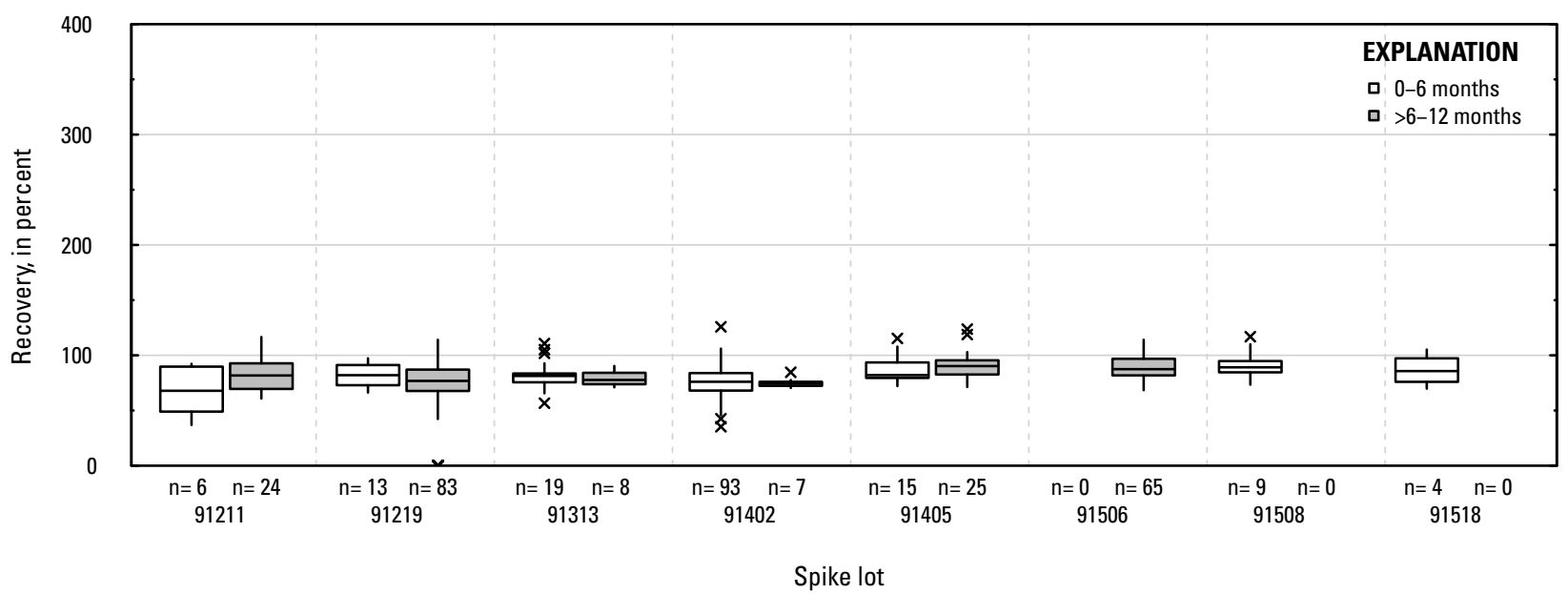

Figure 1-1. Distributions of recovery for individual pesticides in schedule 2437 by matrix, spike lot, and spike lot age. Recovery values larger than 400 percent are not shown.-Continued 


\section{SQ. Phorate: laboratory reagent spikes}

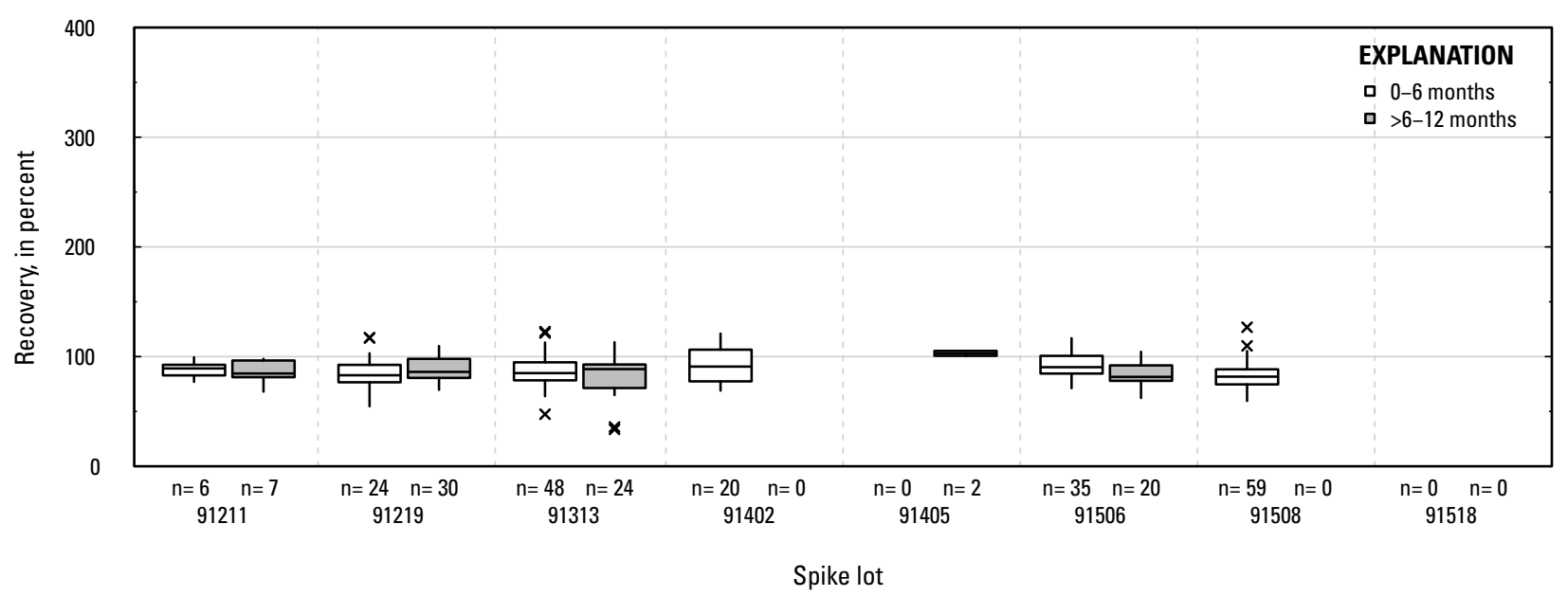

SR. Phorate: groundwater field matrix spikes

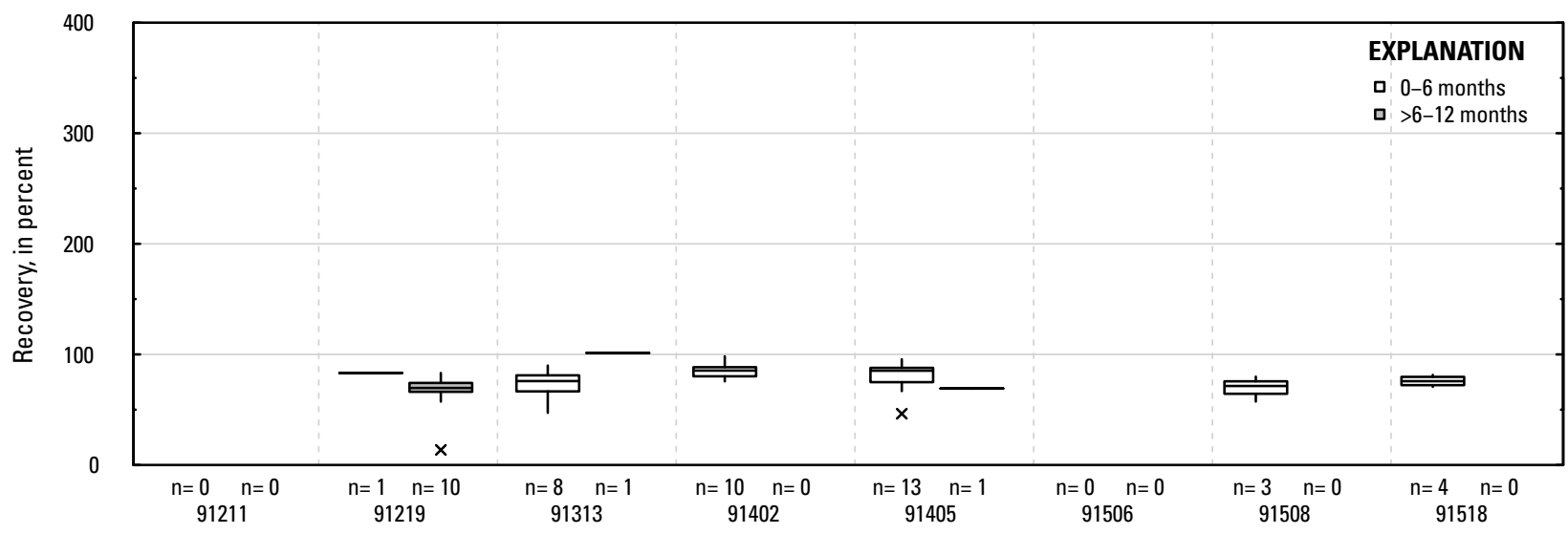

Spike lot

SS. Phorate: surface water field matrix spikes

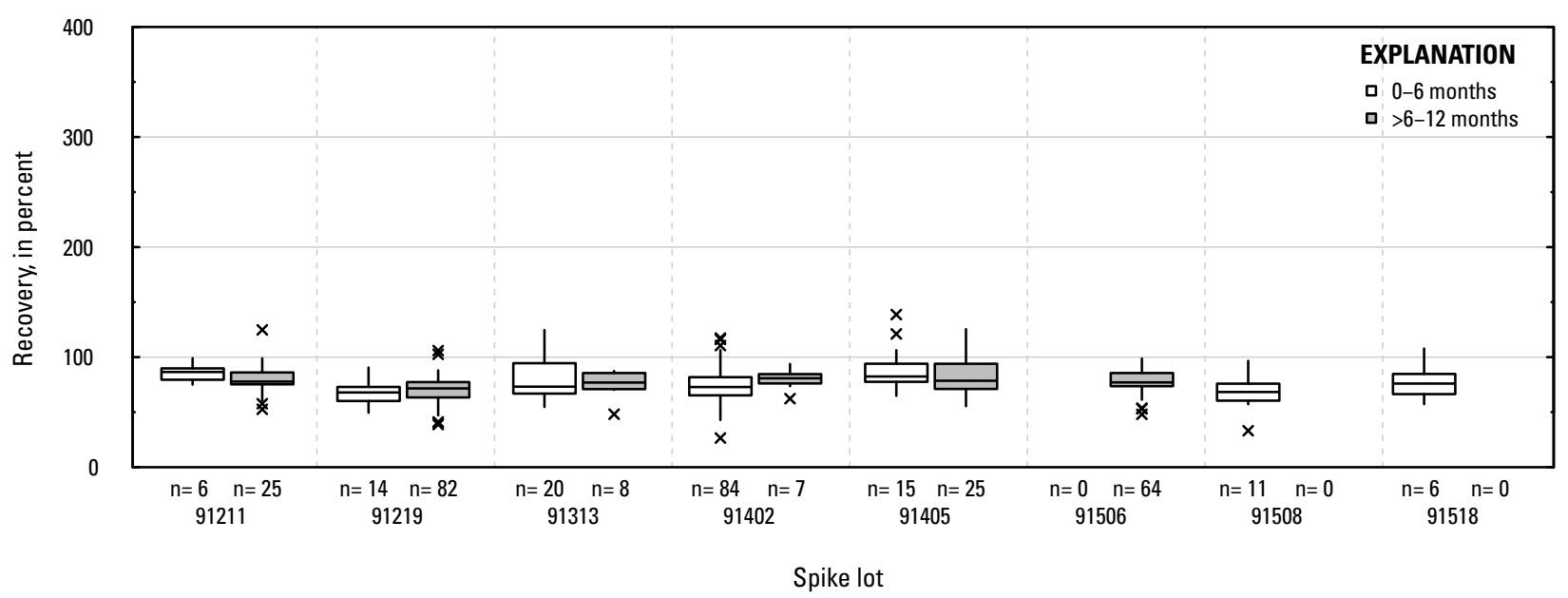

Figure 1-1. Distributions of recovery for individual pesticides in schedule 2437 by matrix, spike lot, and spike lot age. Recovery values larger than 400 percent are not shown.-Continued 
ST. Phorate oxon: laboratory reagent spikes

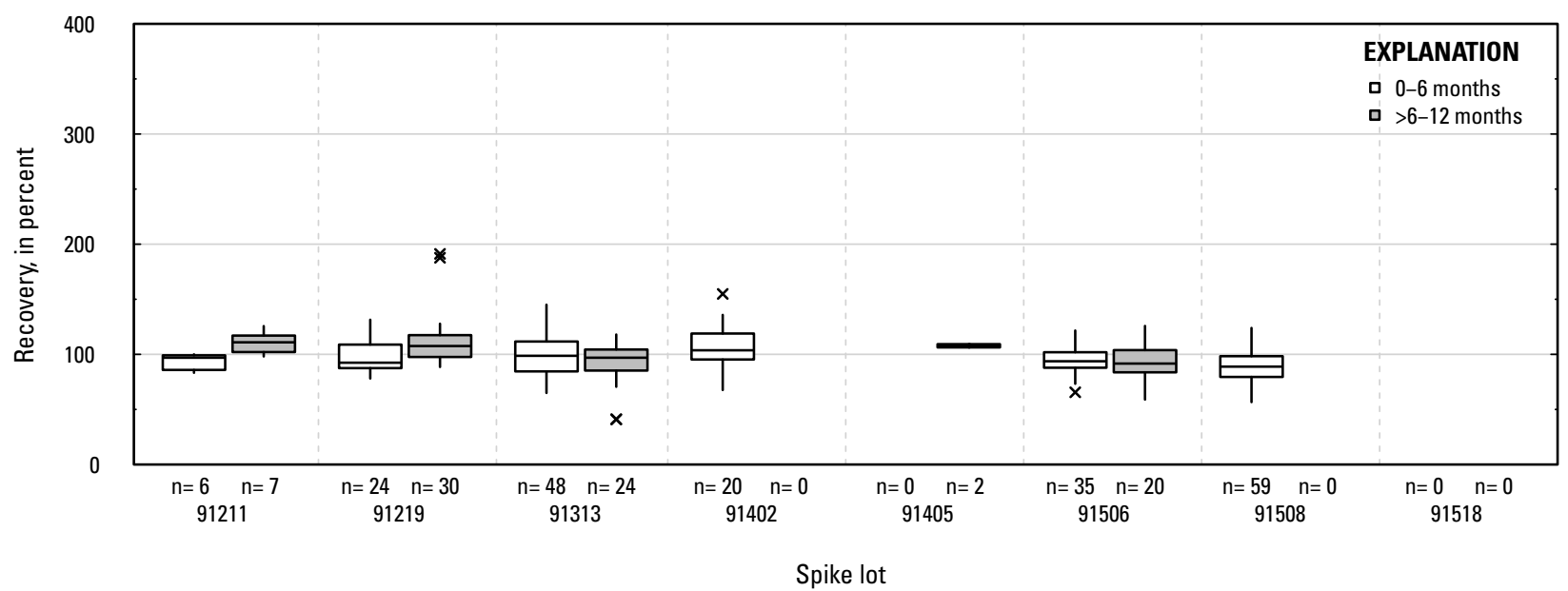

SU. Phorate oxon: groundwater field matrix spikes

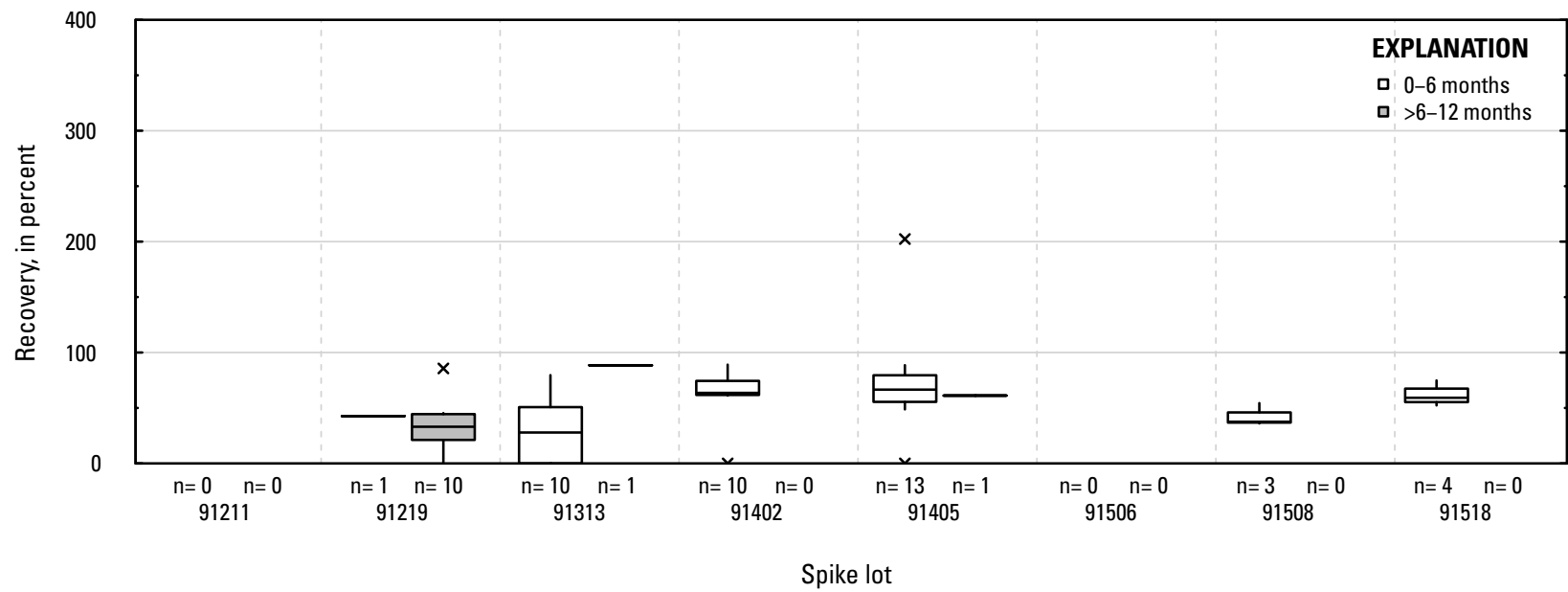

SV. Phorate oxon: surface water field matrix spikes

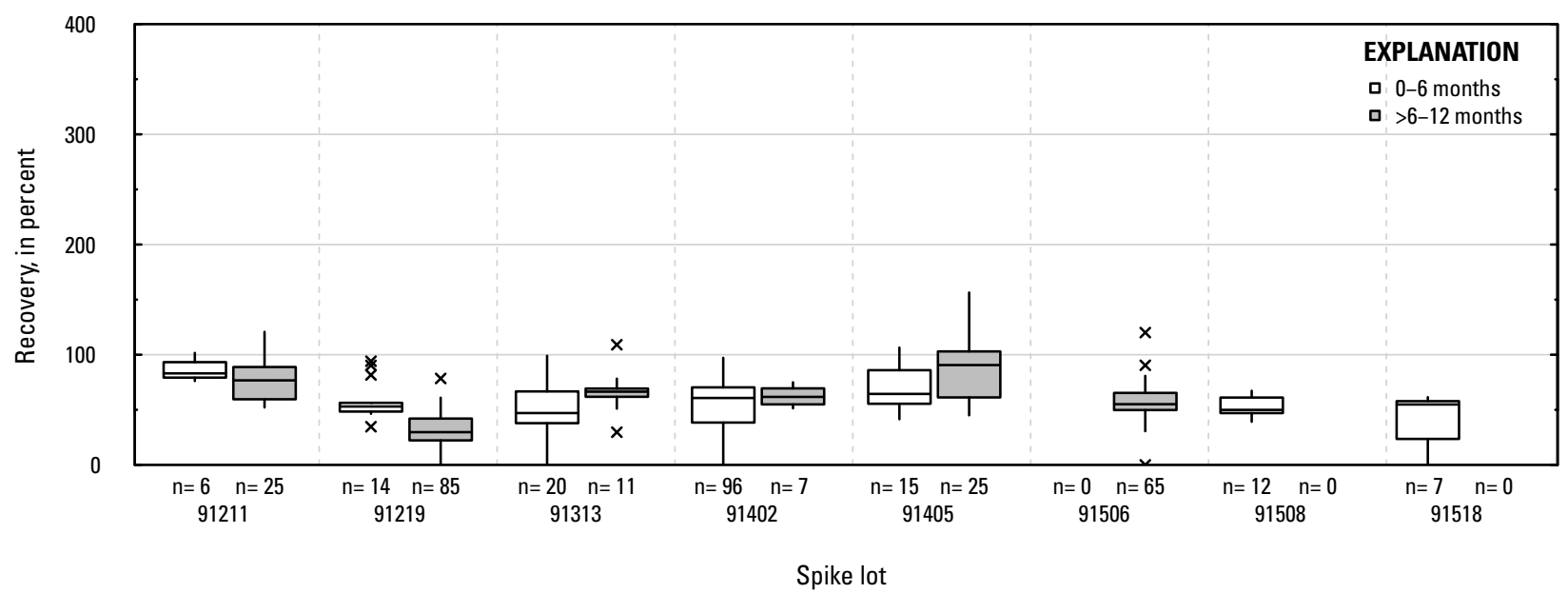

Figure 1-1. Distributions of recovery for individual pesticides in schedule 2437 by matrix, spike lot, and spike lot age. Recovery values larger than 400 percent are not shown.-Continued 
SW. Phorate oxon sulfone: laboratory reagent spikes

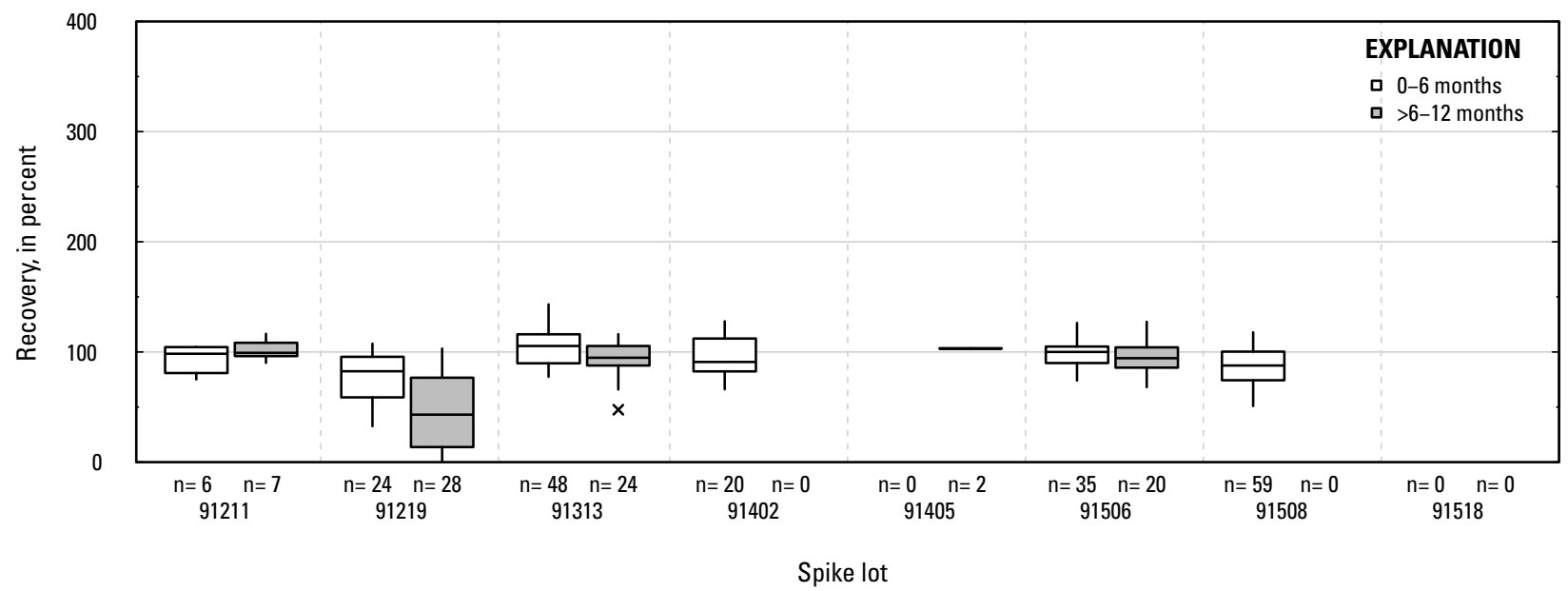

$S X$. Phorate oxon sulfone: groundwater field matrix spikes

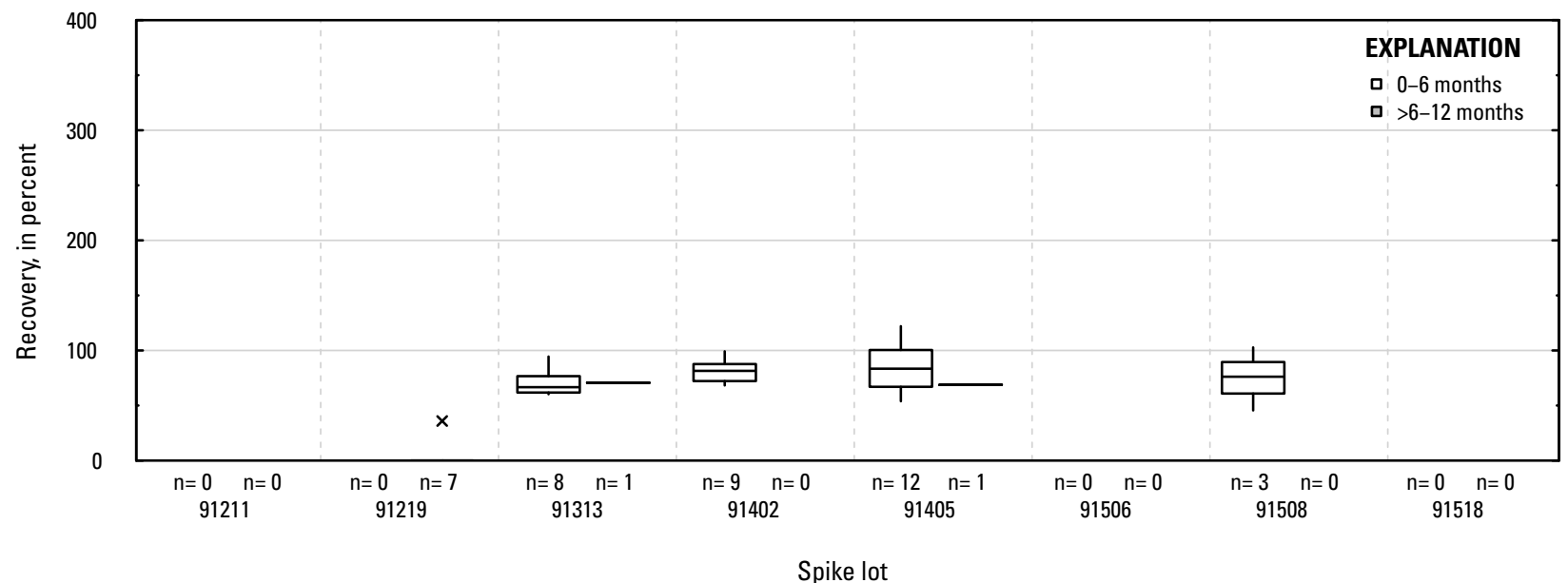

SY. Phorate oxon sulfone: surface water field matrix spikes

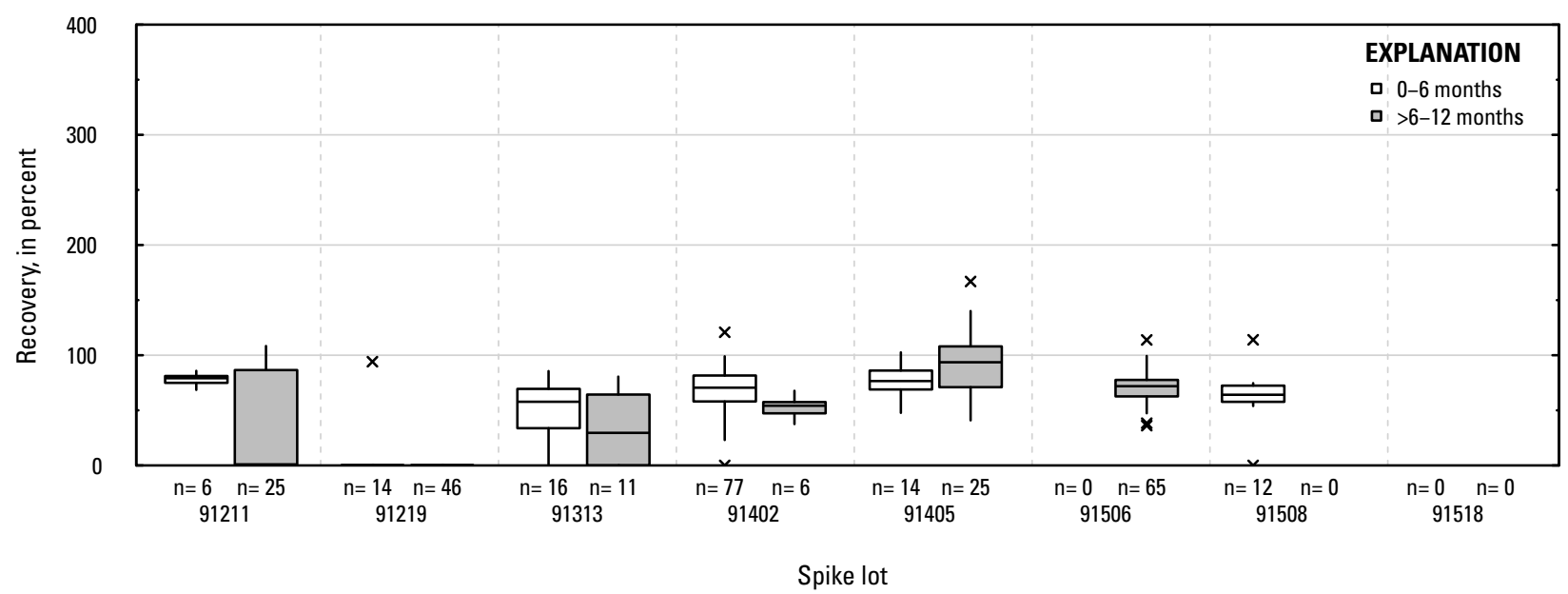

Figure 1-1. Distributions of recovery for individual pesticides in schedule 2437 by matrix, spike lot, and spike lot age. Recovery values larger than 400 percent are not shown.-Continued 
SZ. Phorate oxon sulfoxide: laboratory reagent spikes

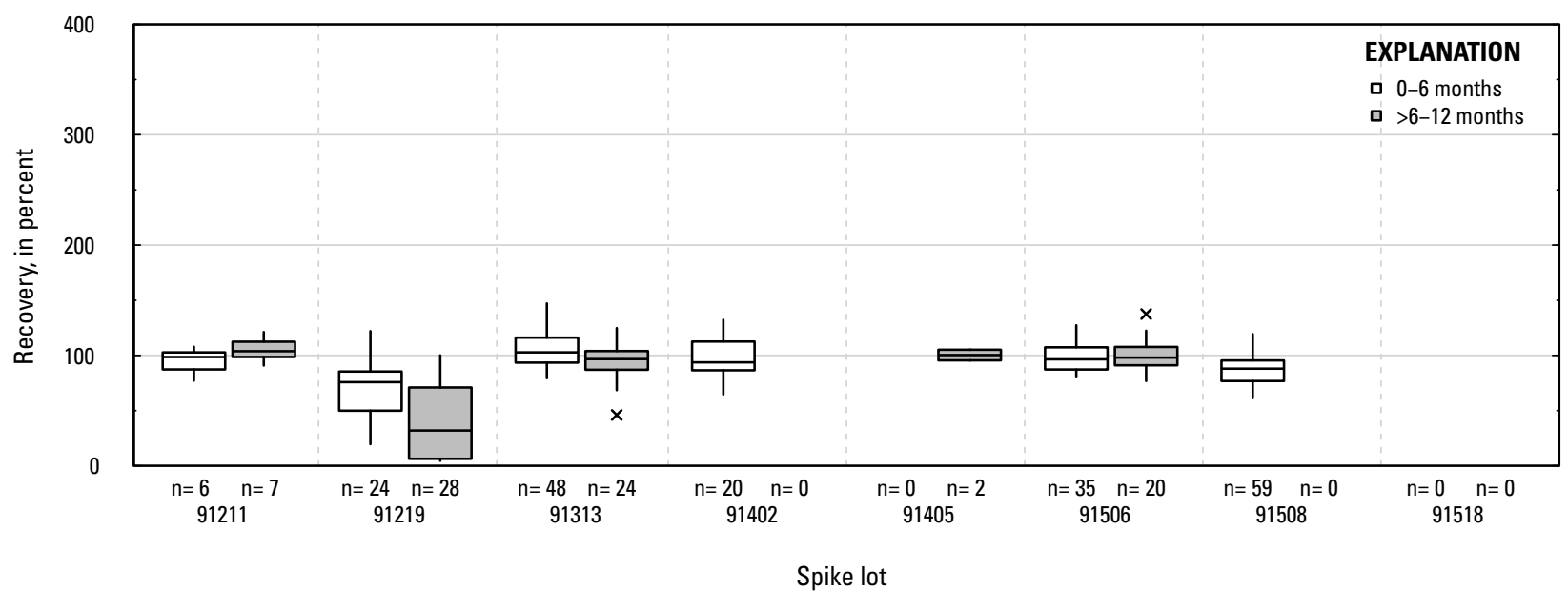

TA. Phorate oxon sulfoxide: groundwater field matrix spikes

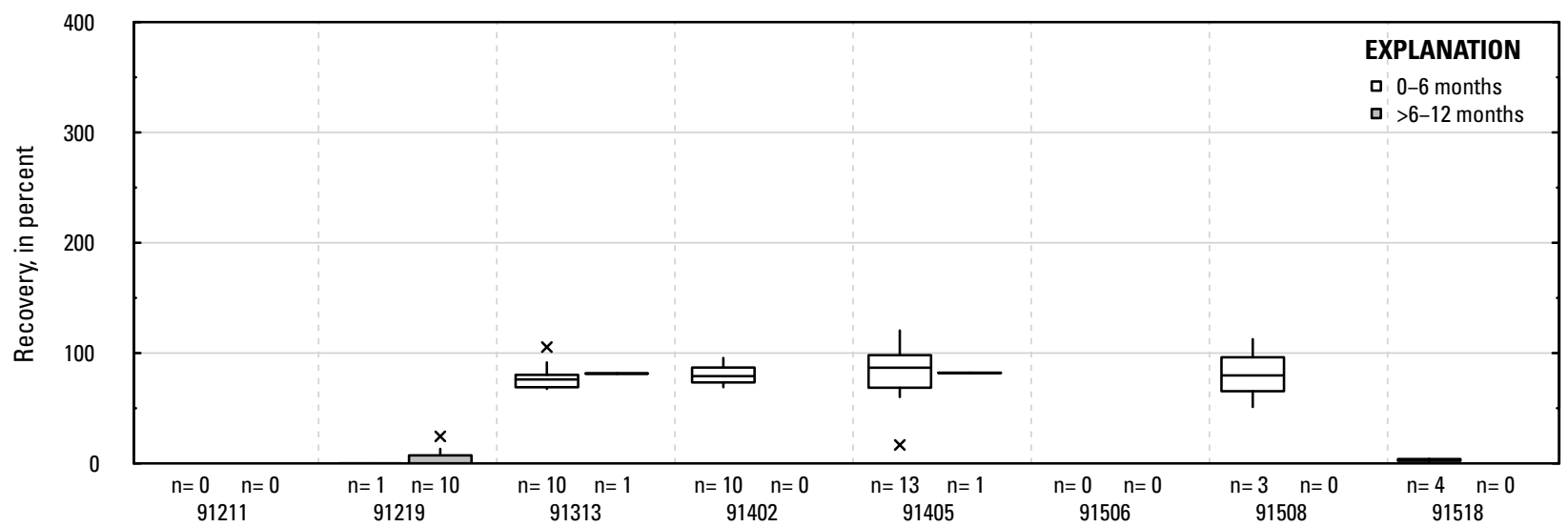

Spike lot

\section{TB. Phorate oxon sulfoxide: surface water field matrix spikes}

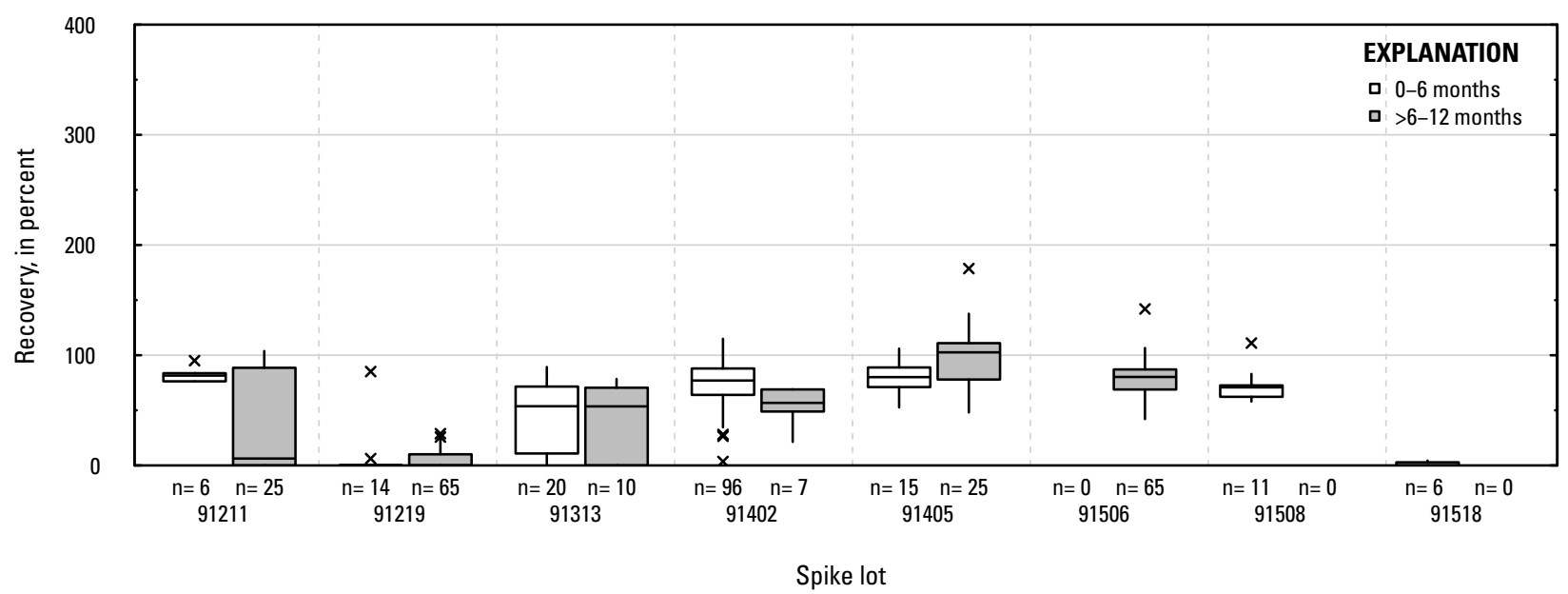

Figure 1-1. Distributions of recovery for individual pesticides in schedule 2437 by matrix, spike lot, and spike lot age. Recovery values larger than 400 percent are not shown.-Continued 
TC. Phorate sulfone: laboratory reagent spikes

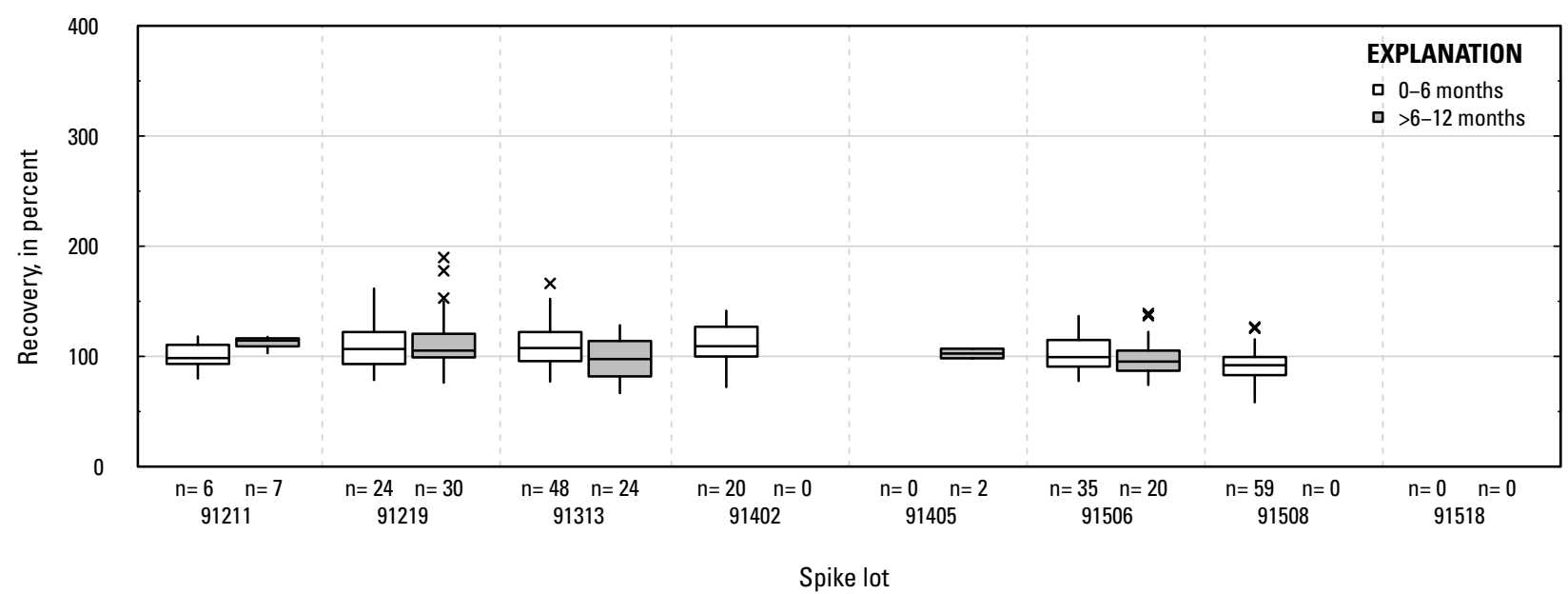

TD. Phorate sulfone: groundwater field matrix spikes

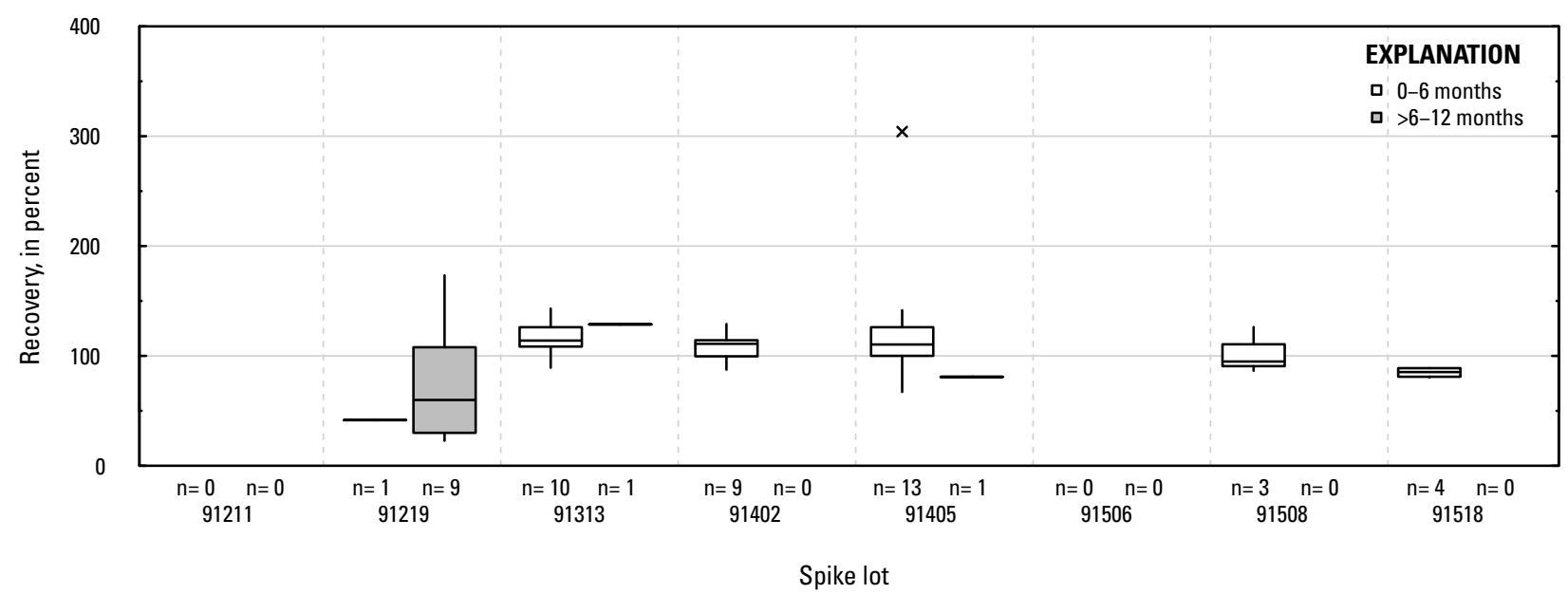

TE. Phorate sulfone: surface water field matrix spikes

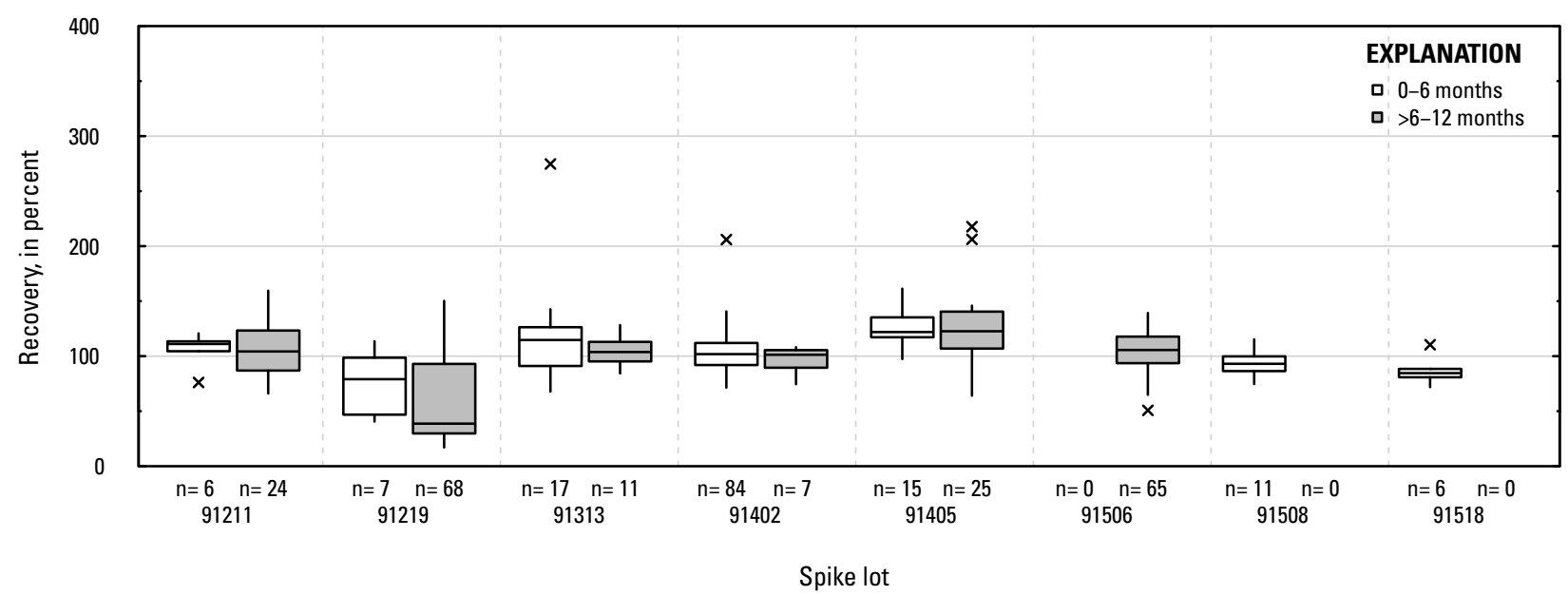

Figure 1-1. Distributions of recovery for individual pesticides in schedule 2437 by matrix, spike lot, and spike lot age. Recovery values larger than 400 percent are not shown.-Continued 
TF. Phorate sulfoxide: laboratory reagent spikes

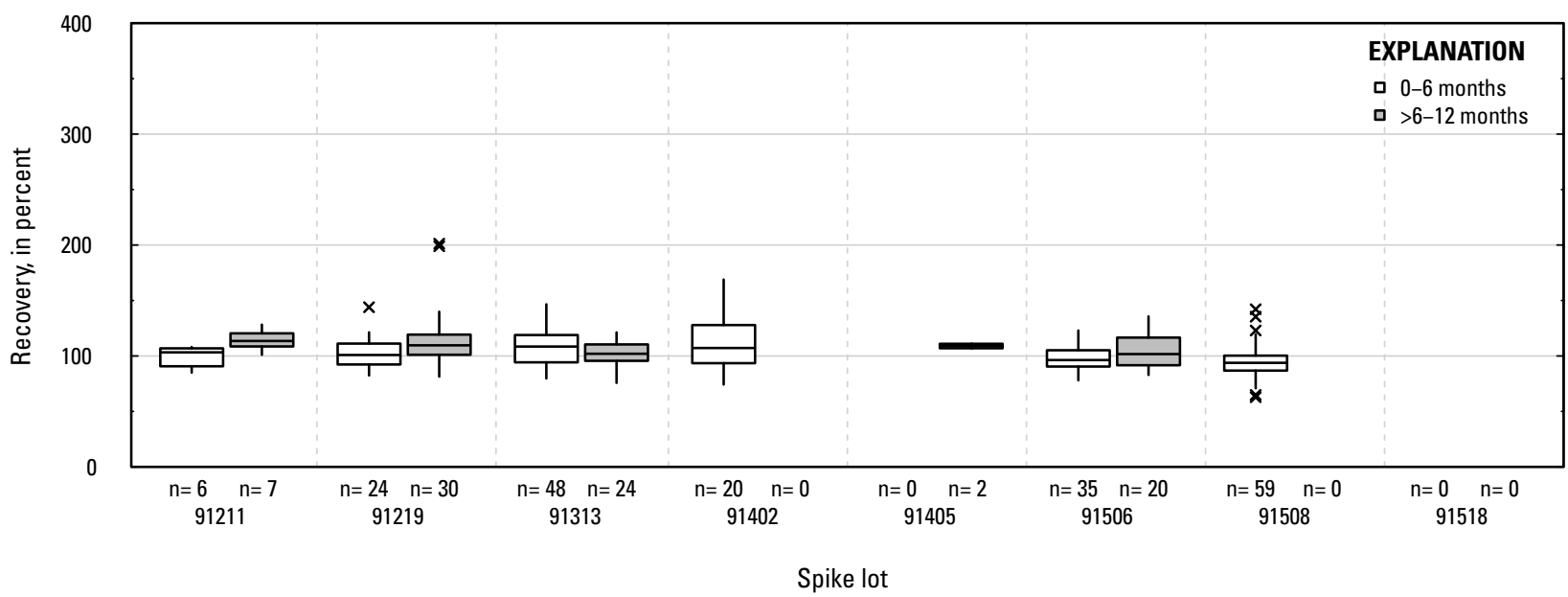

TG. Phorate sulfoxide: groundwater field matrix spikes

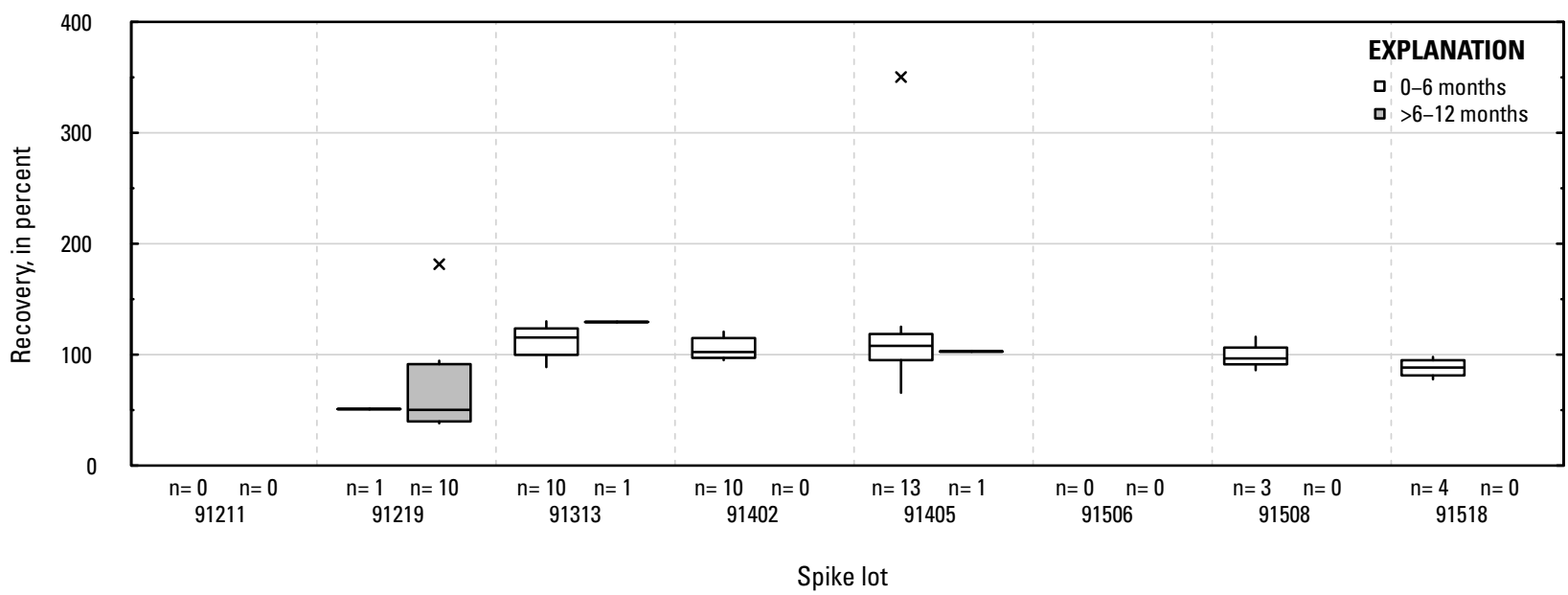

TH. Phorate sulfoxide: surface water field matrix spikes

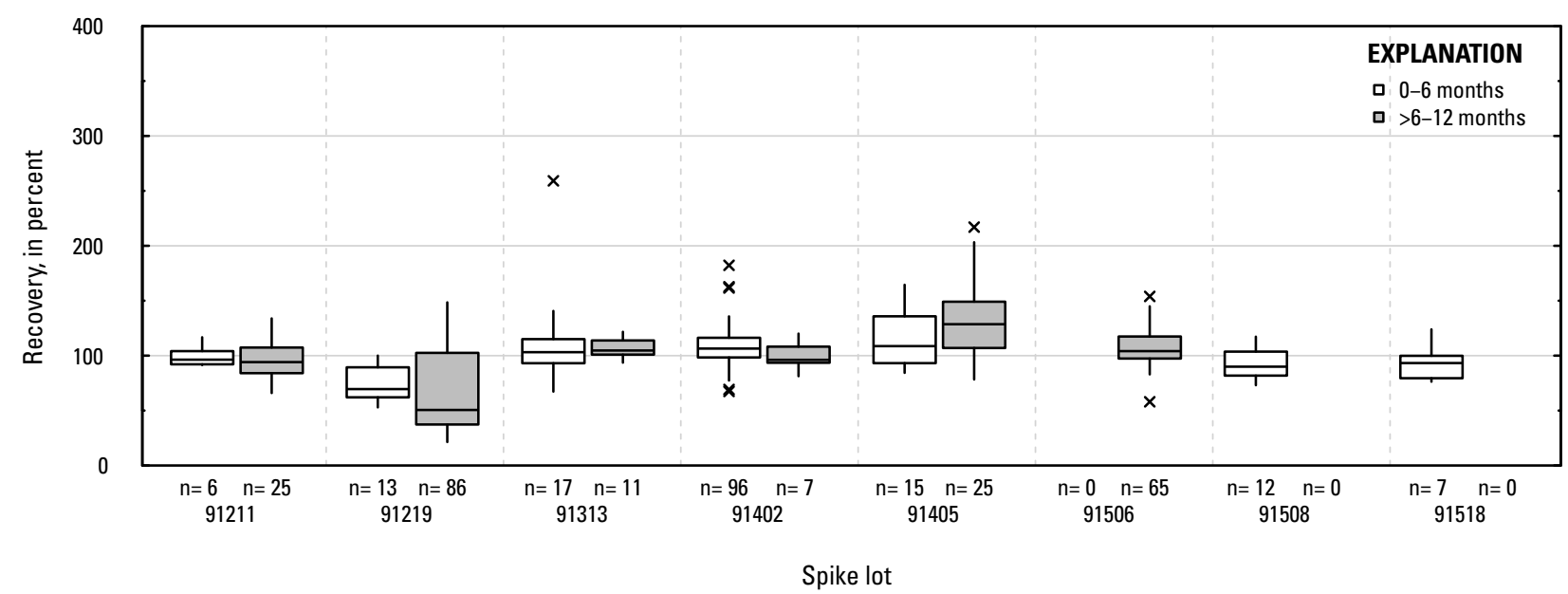

Figure 1-1. Distributions of recovery for individual pesticides in schedule 2437 by matrix, spike lot, and spike lot age. Recovery values larger than 400 percent are not shown.-Continued 


\section{TI. Phthalazinone: laboratory reagent spikes}

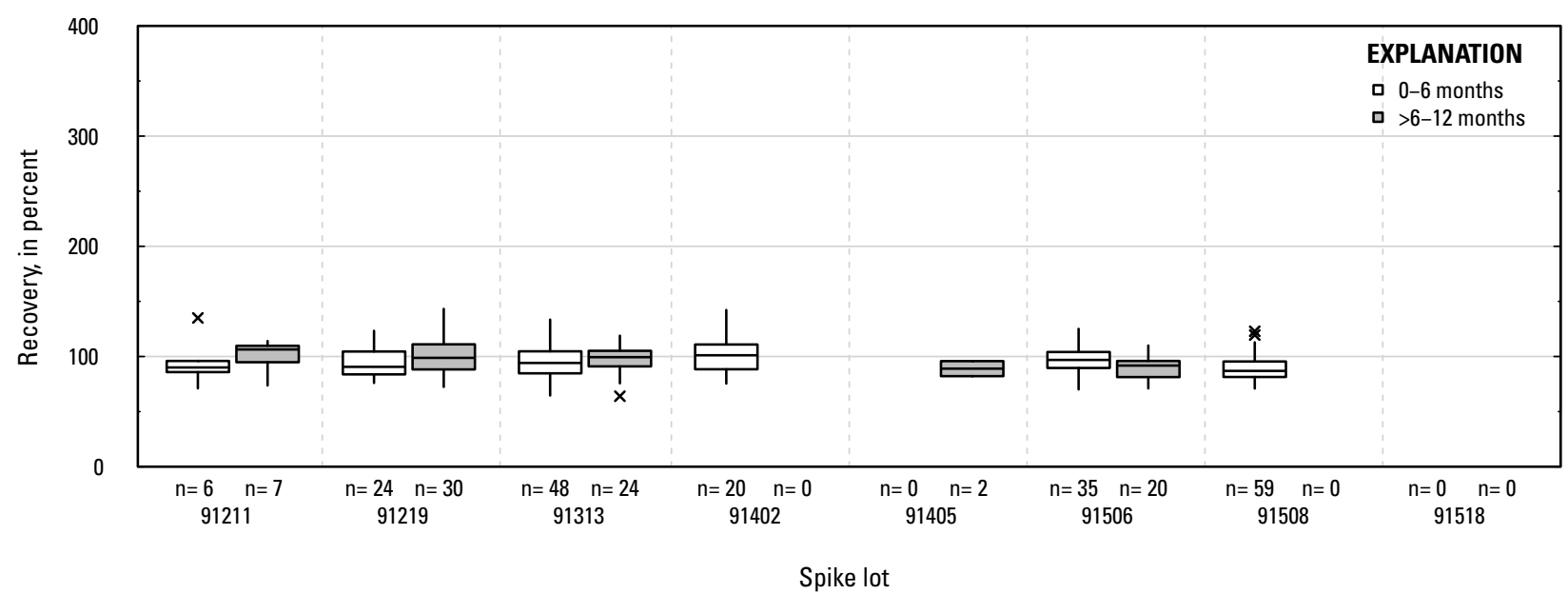

\section{TJ. Phthalazinone: groundwater field matrix spikes}

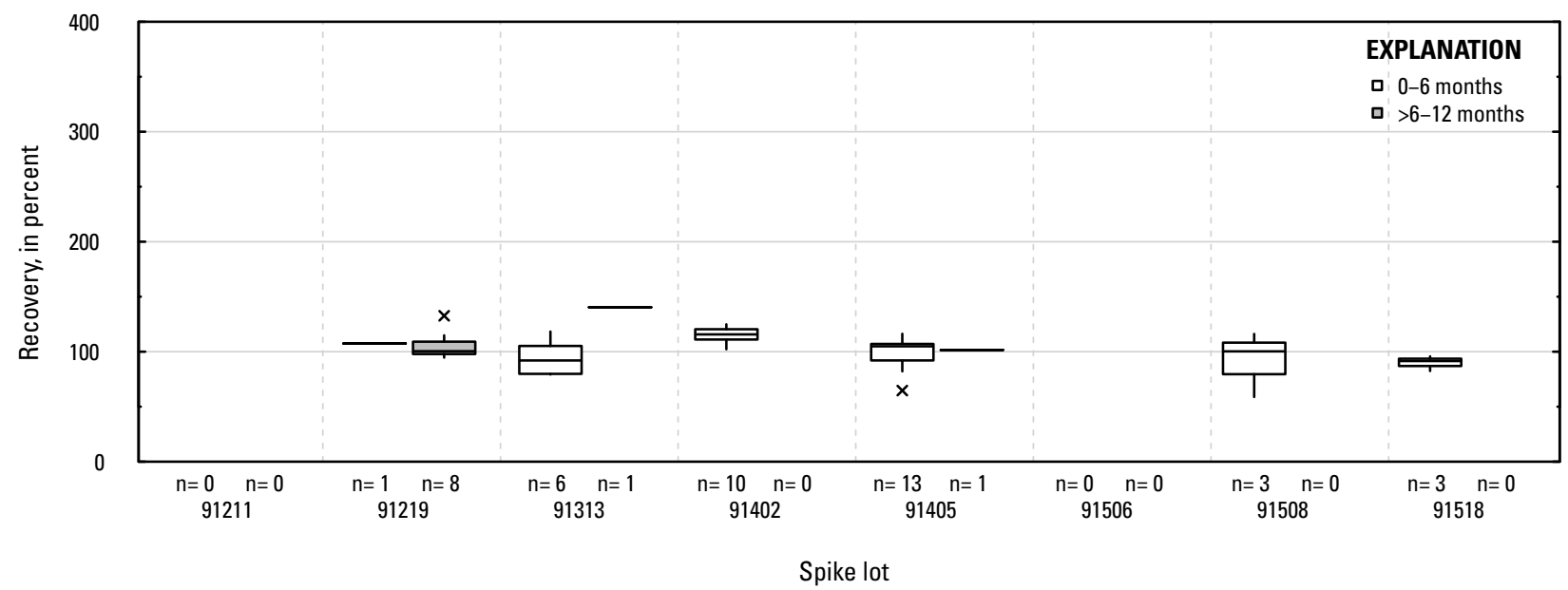

TK. Phthalazinone: surface water field matrix spikes

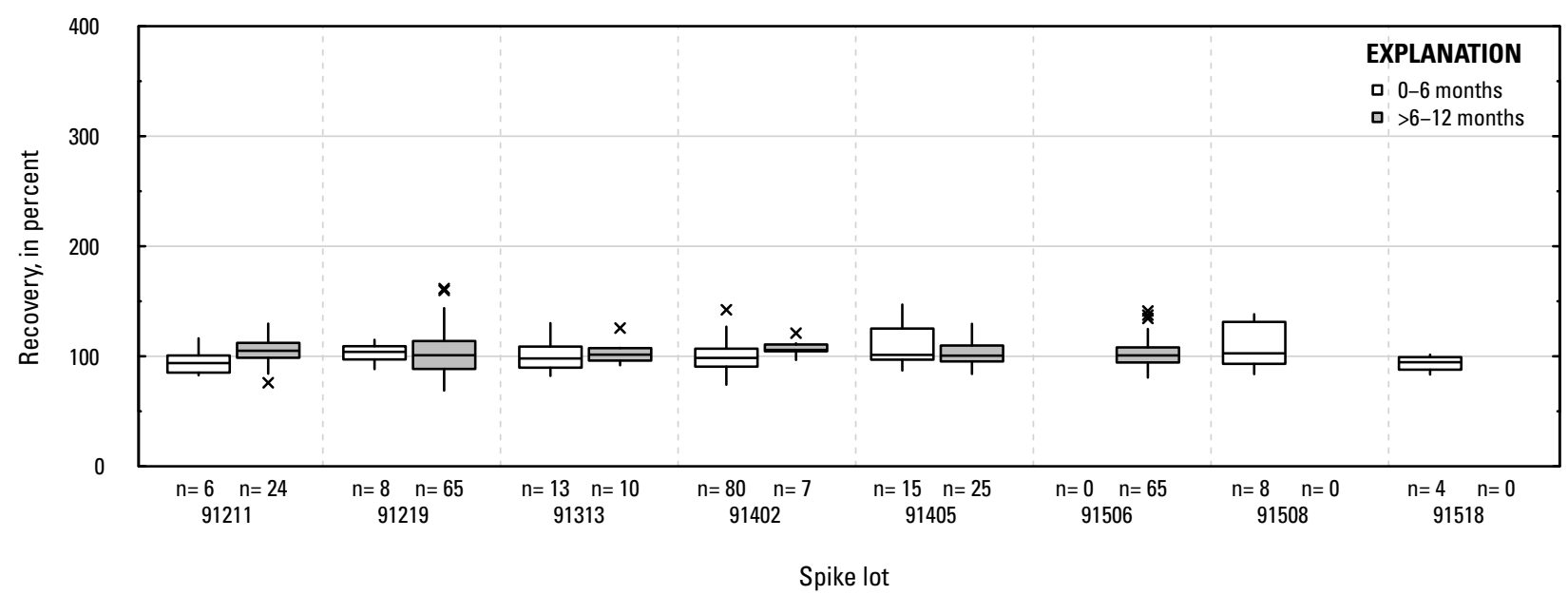

Figure 1-1. Distributions of recovery for individual pesticides in schedule 2437 by matrix, spike lot, and spike lot age. Recovery values larger than 400 percent are not shown.-Continued 


\section{TL. Piperonyl butoxide: laboratory reagent spikes}

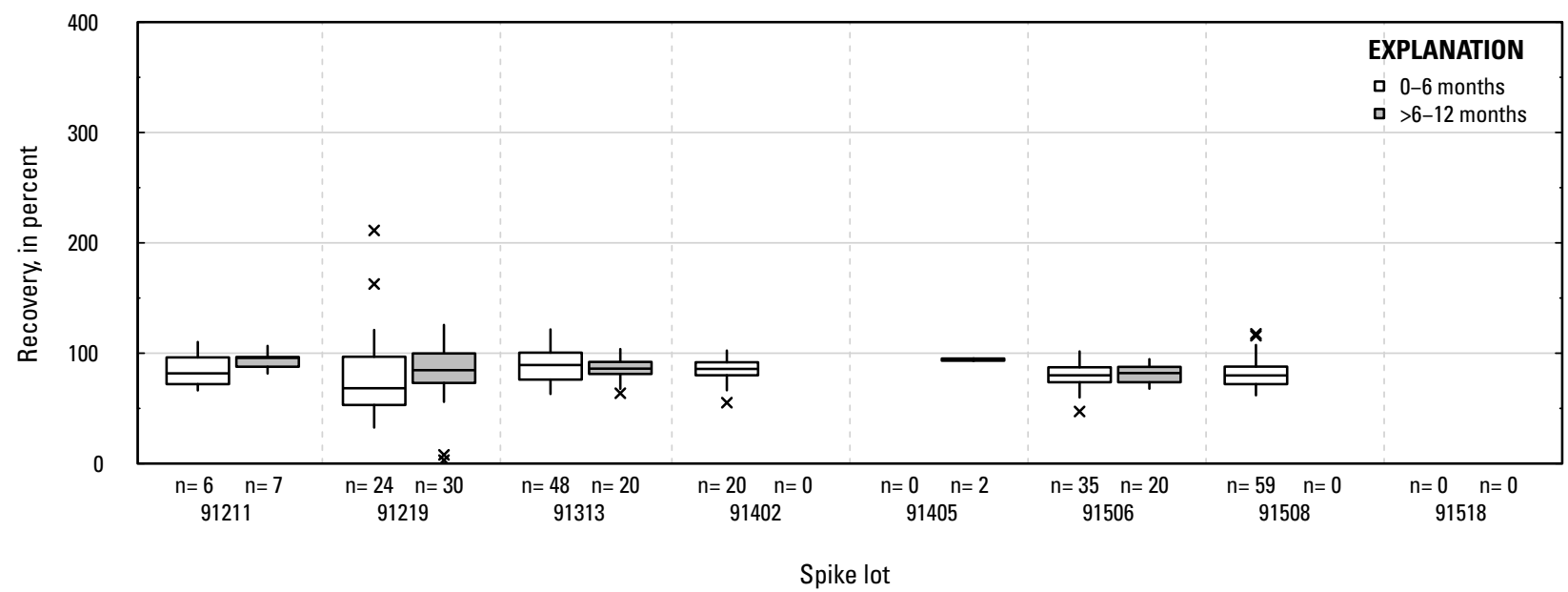

TM. Piperonyl butoxide: groundwater field matrix spikes

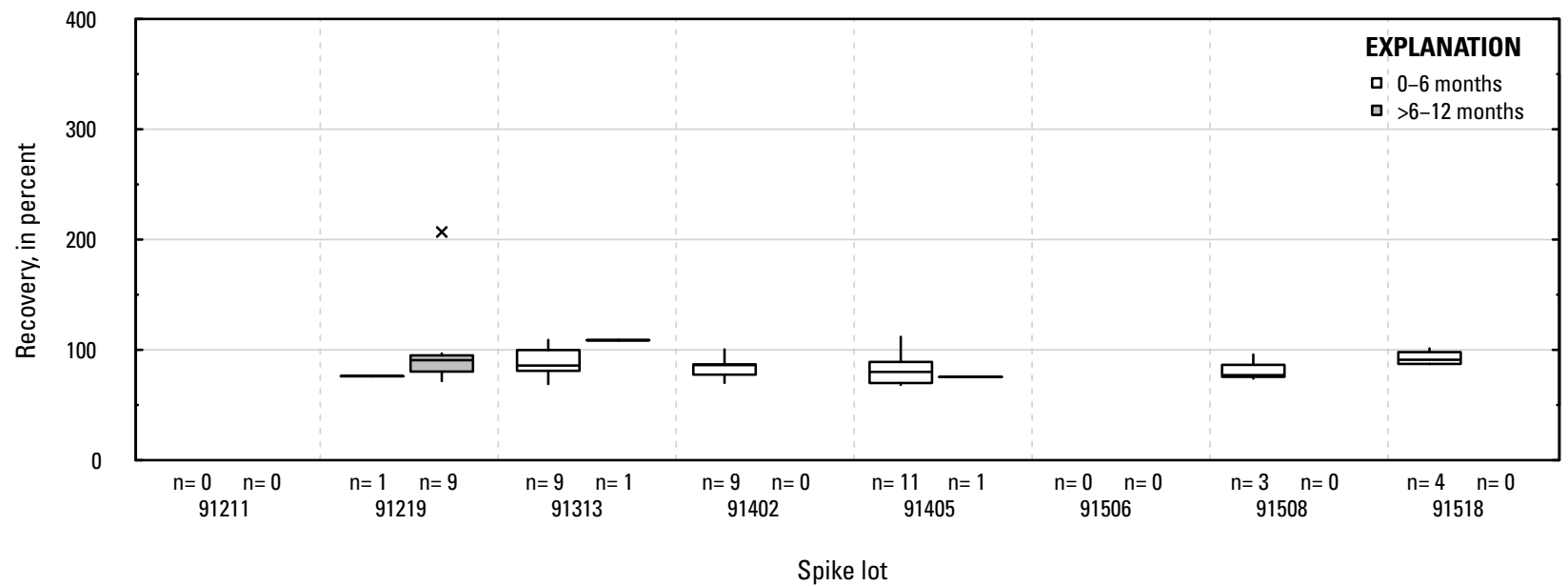

$T N$. Piperonyl butoxide: surface water field matrix spikes

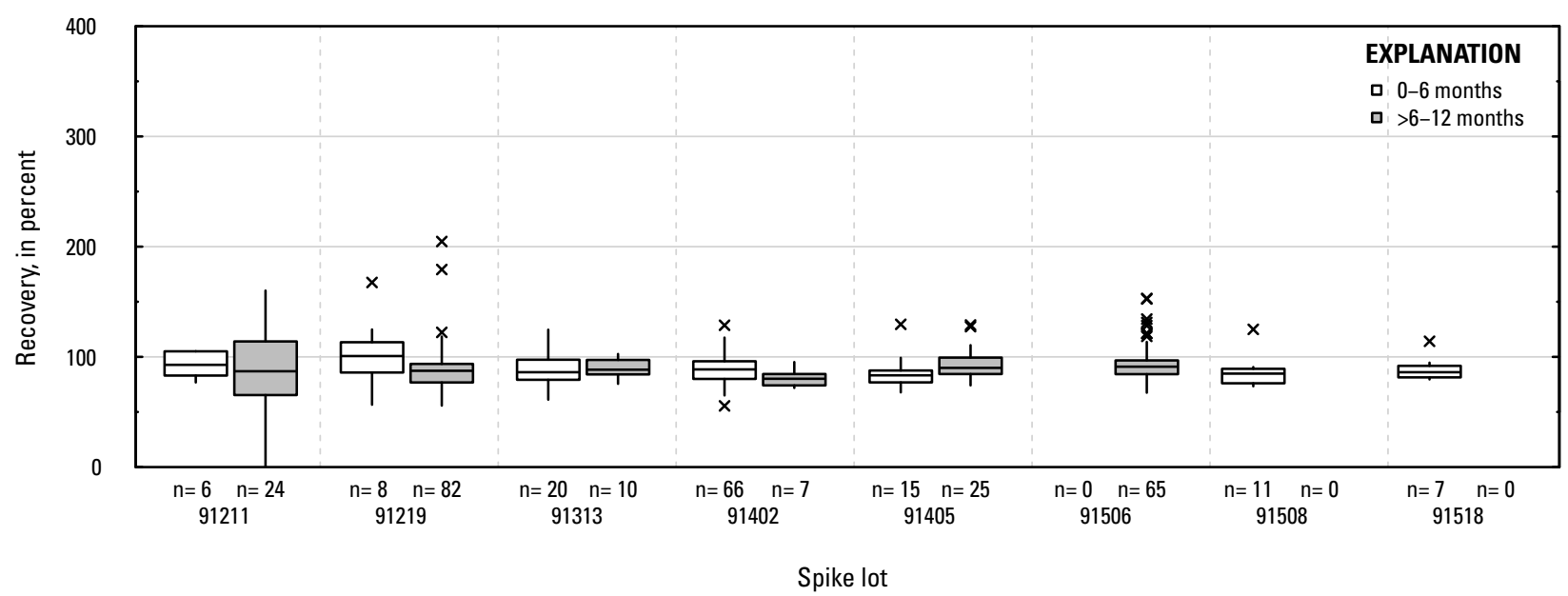

Figure 1-1. Distributions of recovery for individual pesticides in schedule 2437 by matrix, spike lot, and spike lot age. Recovery values larger than 400 percent are not shown.-Continued 
TO. Profenofos: laboratory reagent spikes

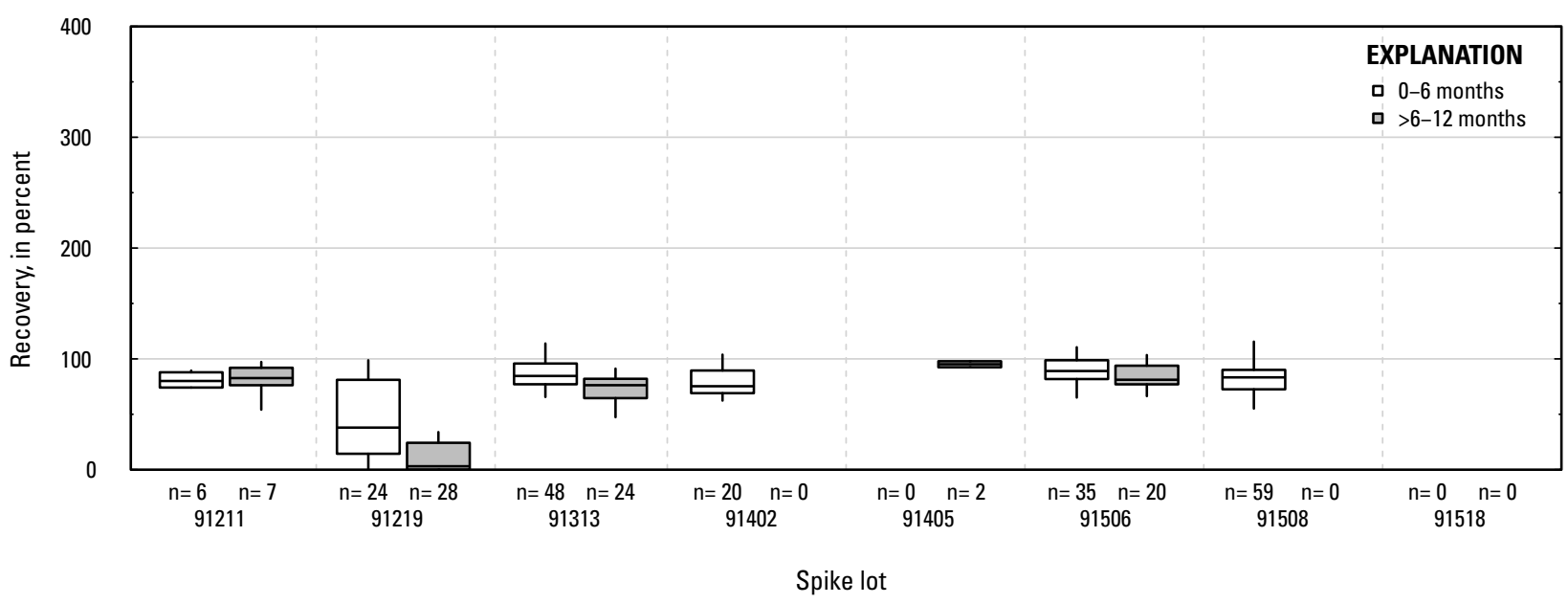

TP. Profenofos: groundwater field matrix spikes

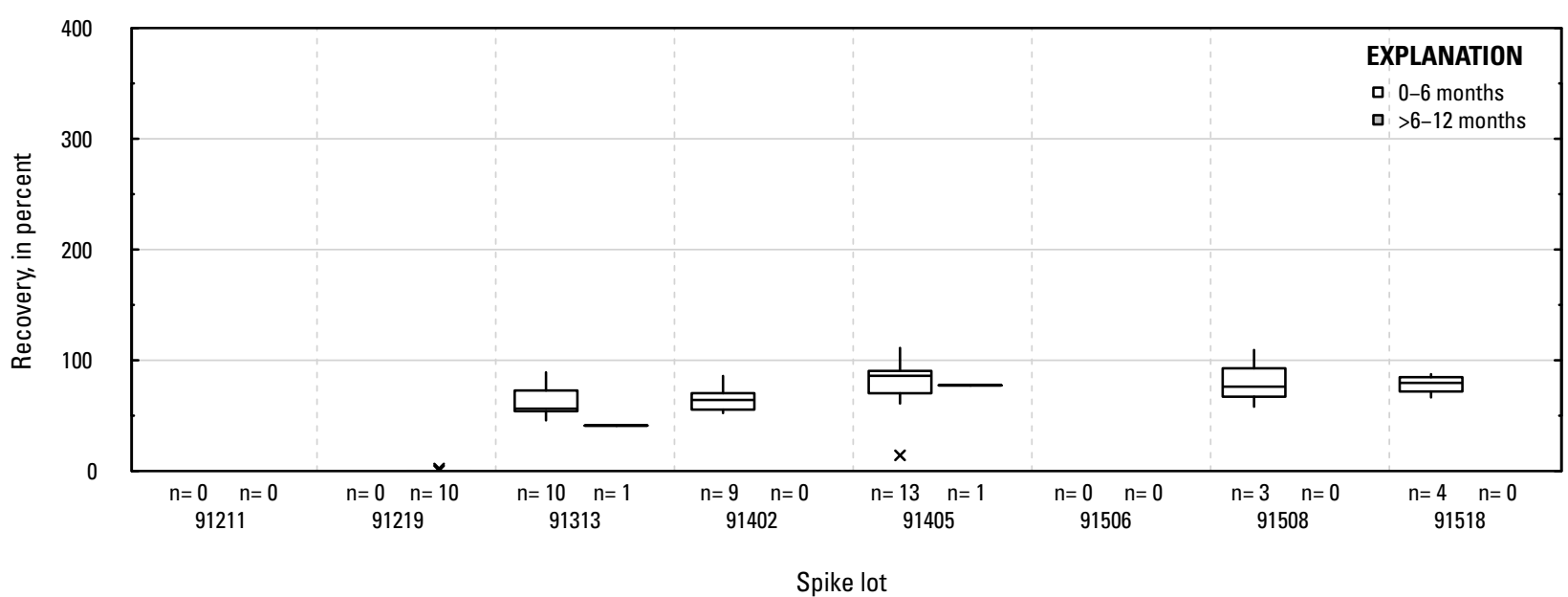

TQ. Profenofos: surface water field matrix spikes

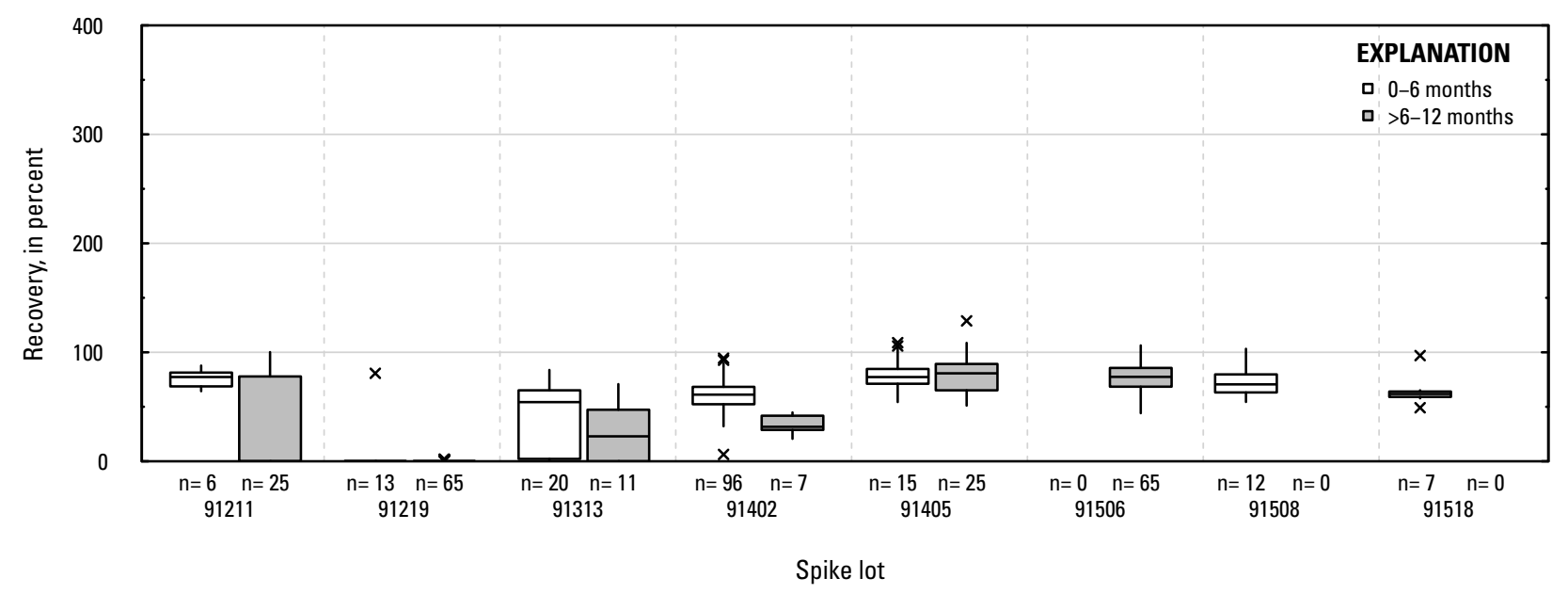

Figure 1-1. Distributions of recovery for individual pesticides in schedule 2437 by matrix, spike lot, and spike lot age. Recovery values larger than 400 percent are not shown.-Continued 
TR. Prometon: laboratory reagent spikes

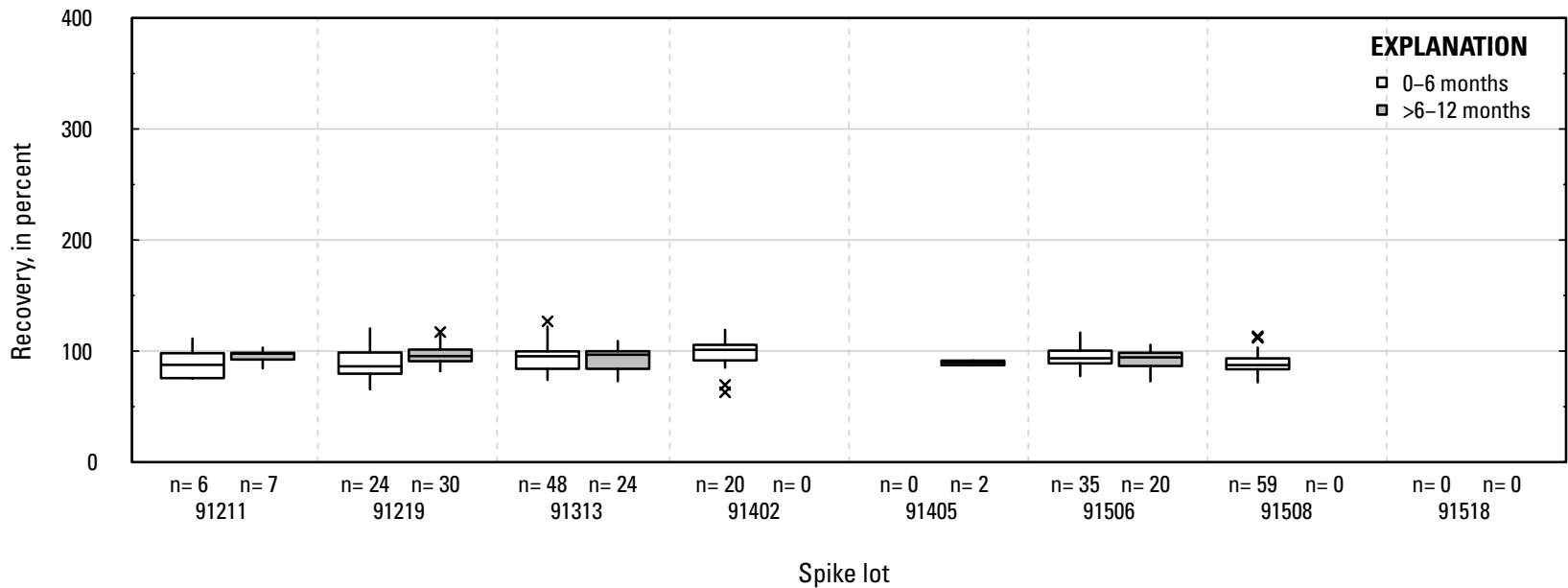

TS. Prometon: groundwater field matrix spikes

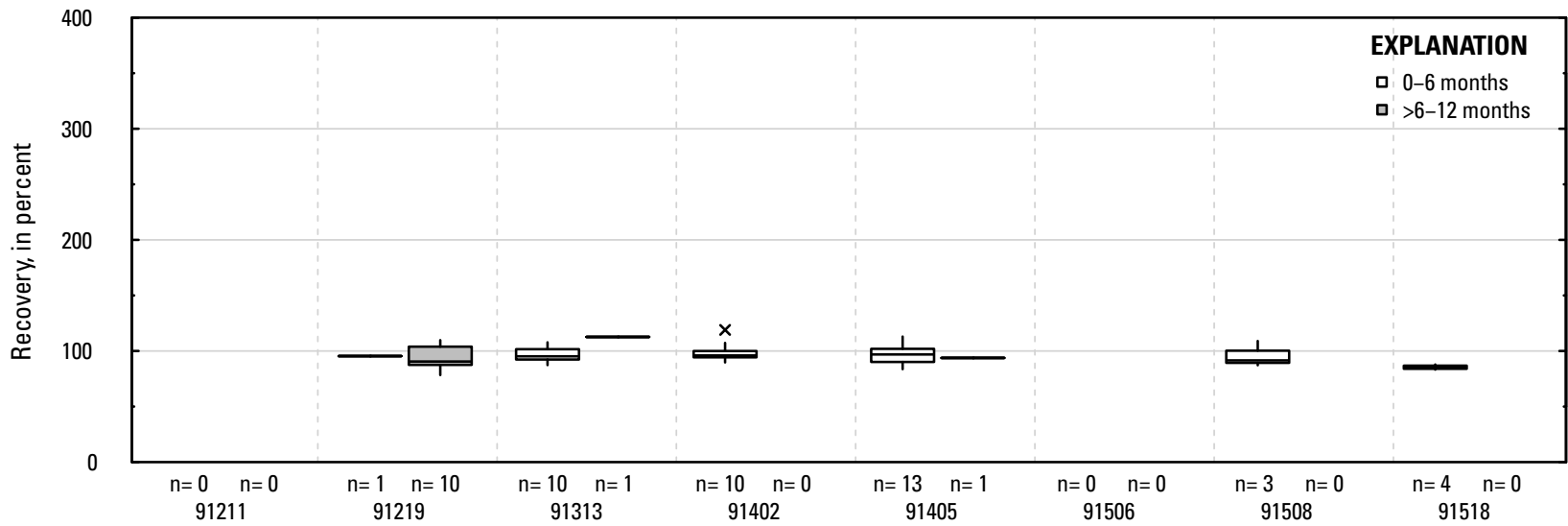

Spike lot

\section{TT. Prometon: surface water field matrix spikes}

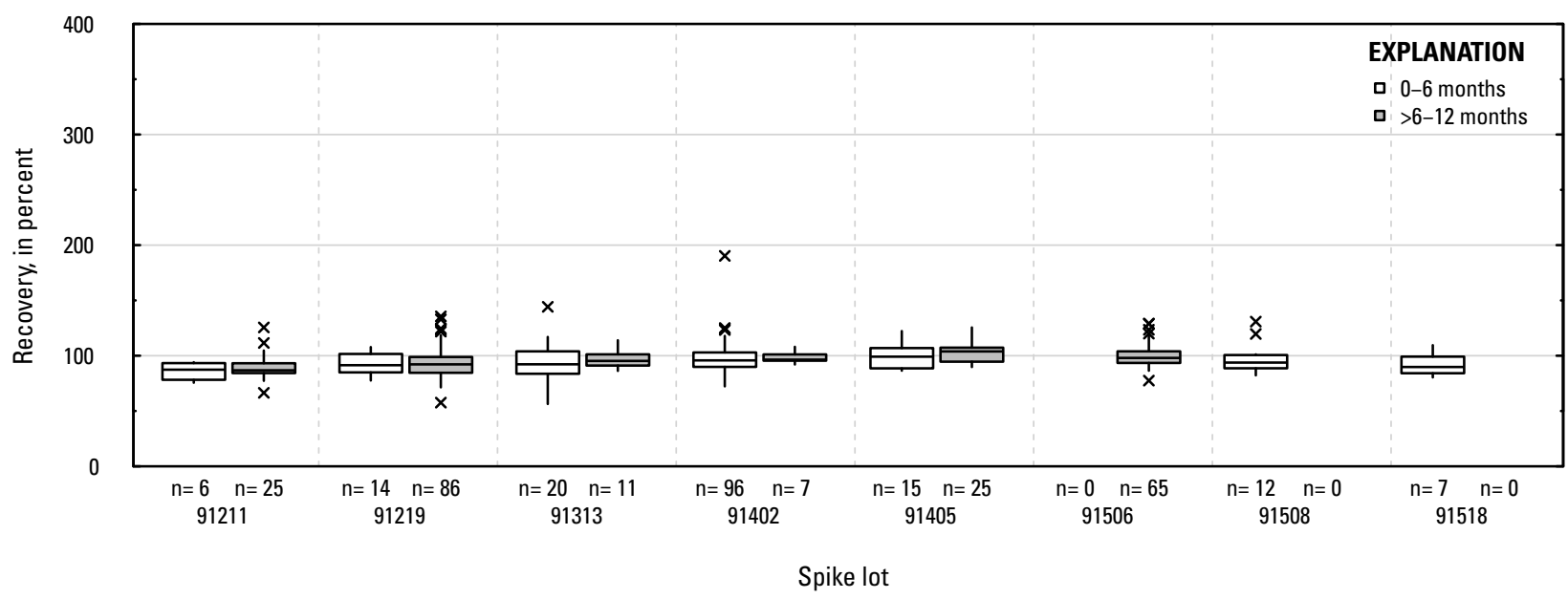

Figure 1-1. Distributions of recovery for individual pesticides in schedule 2437 by matrix, spike lot, and spike lot age. Recovery values larger than 400 percent are not shown.-Continued 


\section{TU. Prometryn: laboratory reagent spikes}

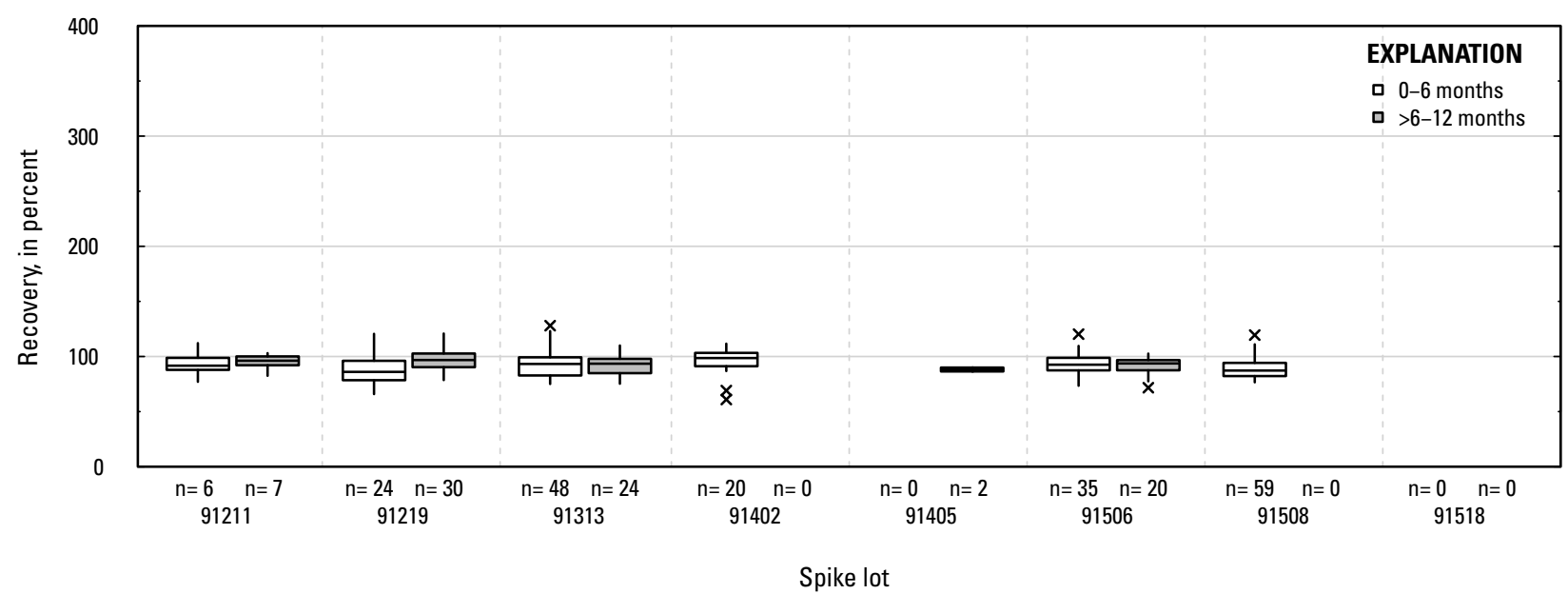

TV. Prometryn: groundwater field matrix spikes

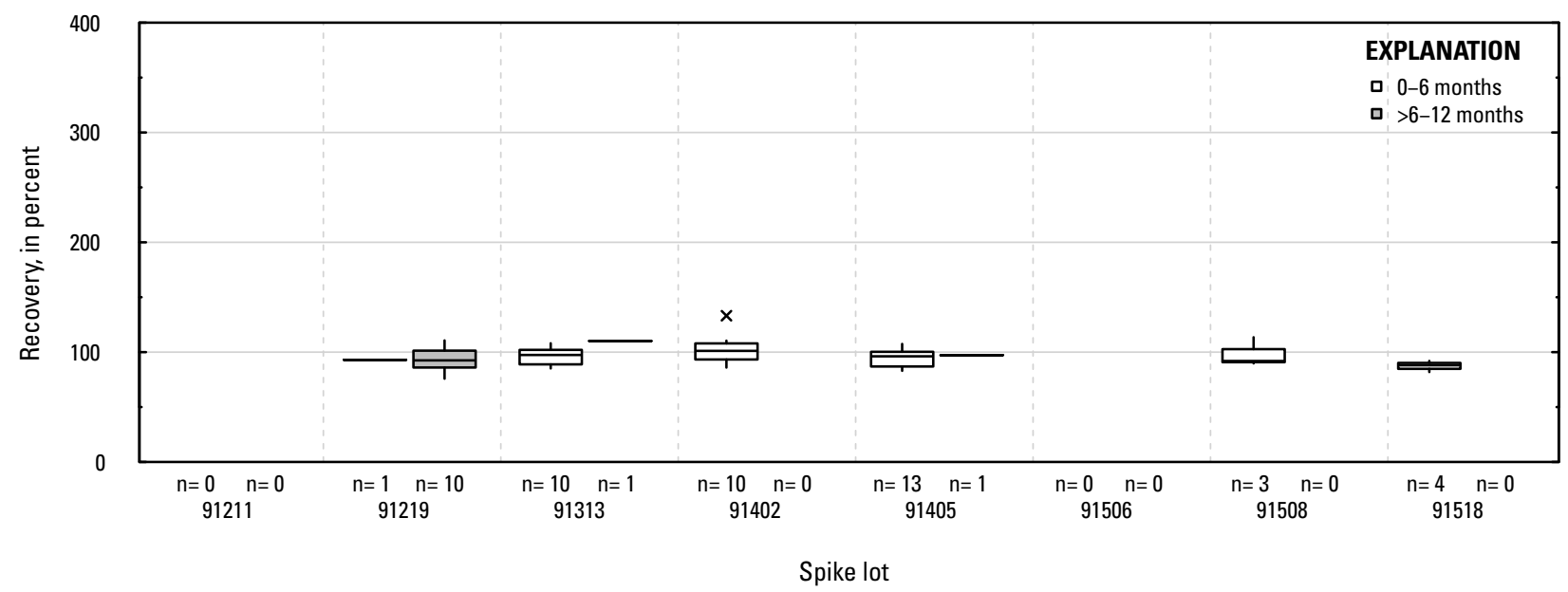

TW. Prometryn: surface water field matrix spikes

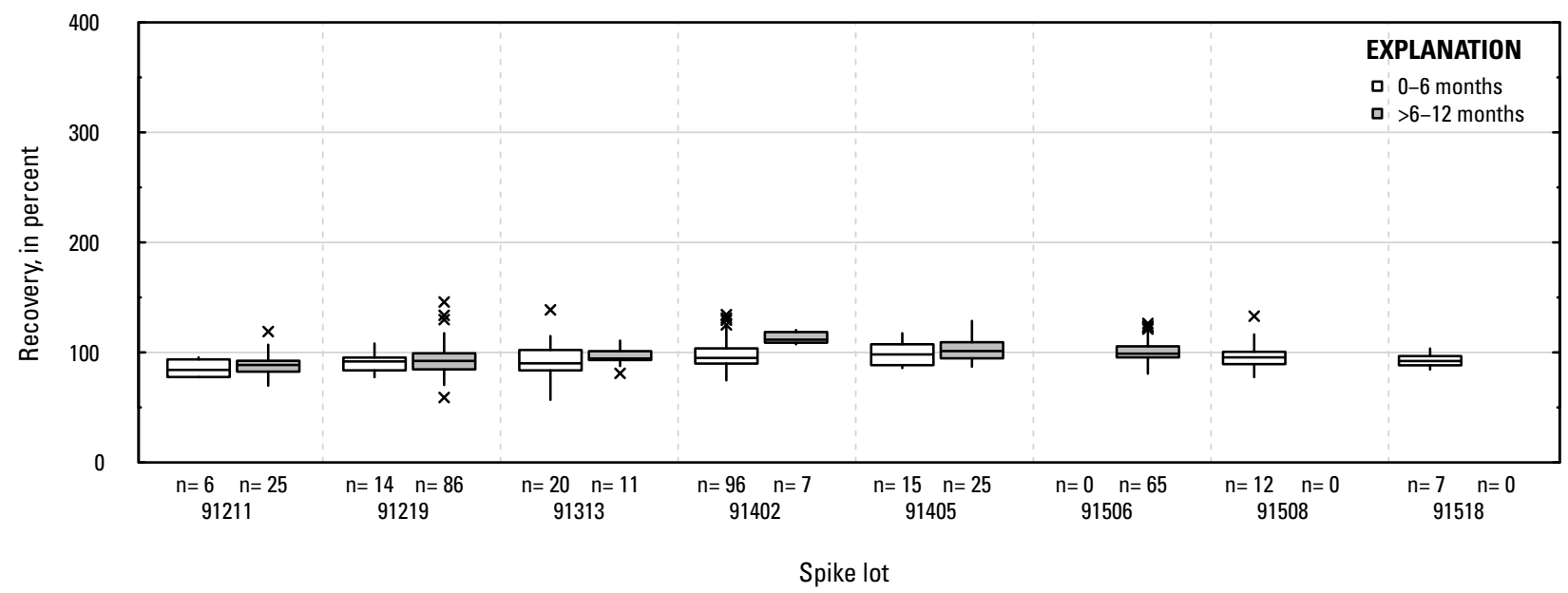

Figure 1-1. Distributions of recovery for individual pesticides in schedule 2437 by matrix, spike lot, and spike lot age. Recovery values larger than 400 percent are not shown.-Continued 
TX. Propyzamide: laboratory reagent spikes

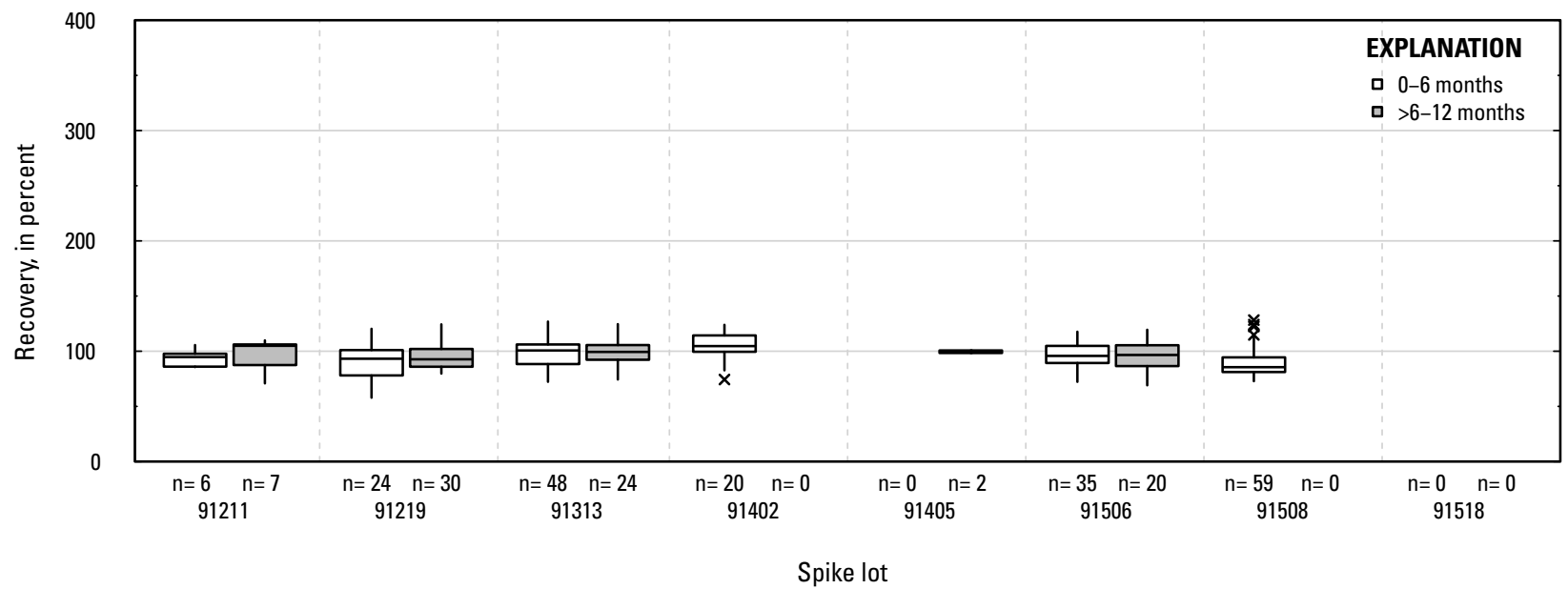

TY. Propyzamide: groundwater field matrix spikes

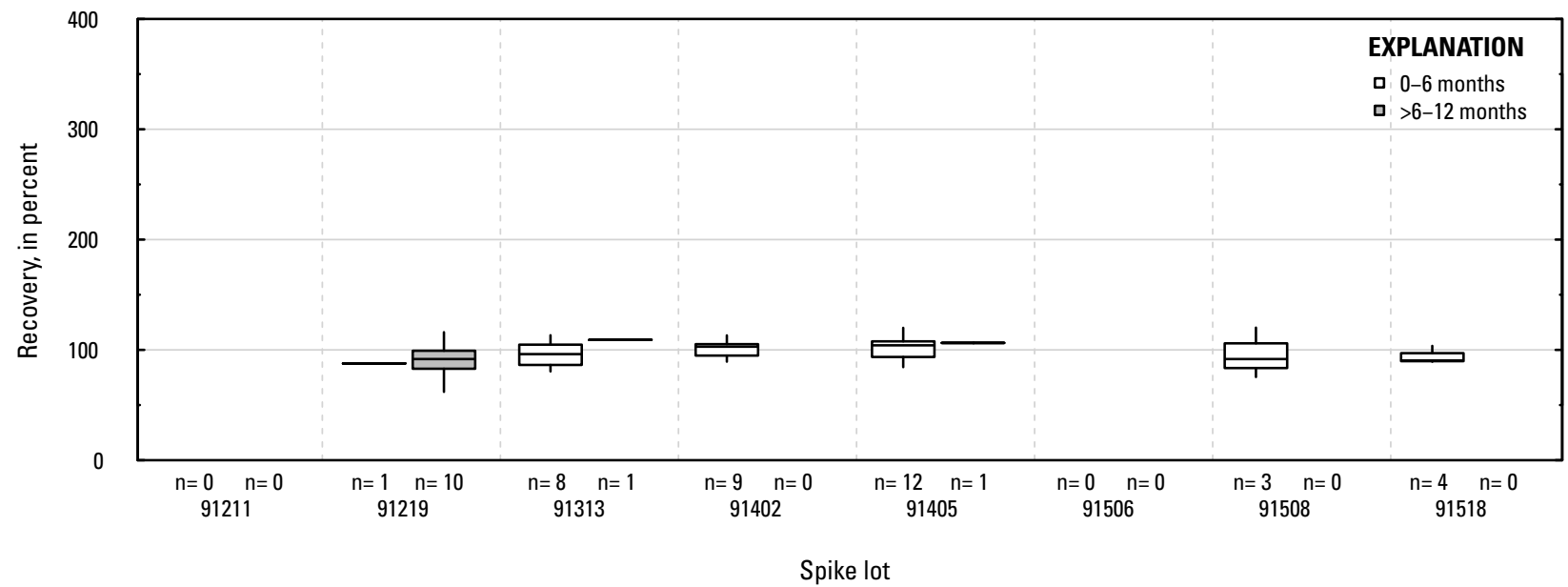

TZ. Propyzamide: surface water field matrix spikes

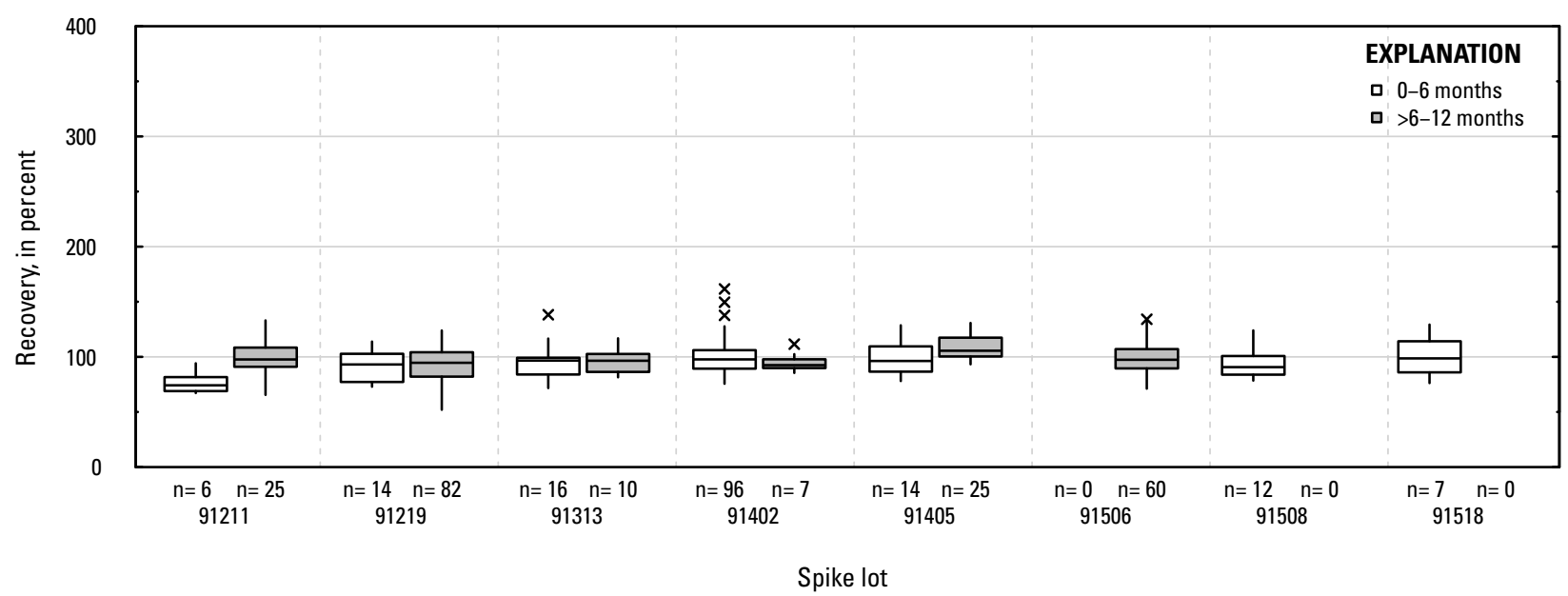

Figure 1-1. Distributions of recovery for individual pesticides in schedule 2437 by matrix, spike lot, and spike lot age. Recovery values larger than 400 percent are not shown.-Continued 
UA. Propanil: laboratory reagent spikes

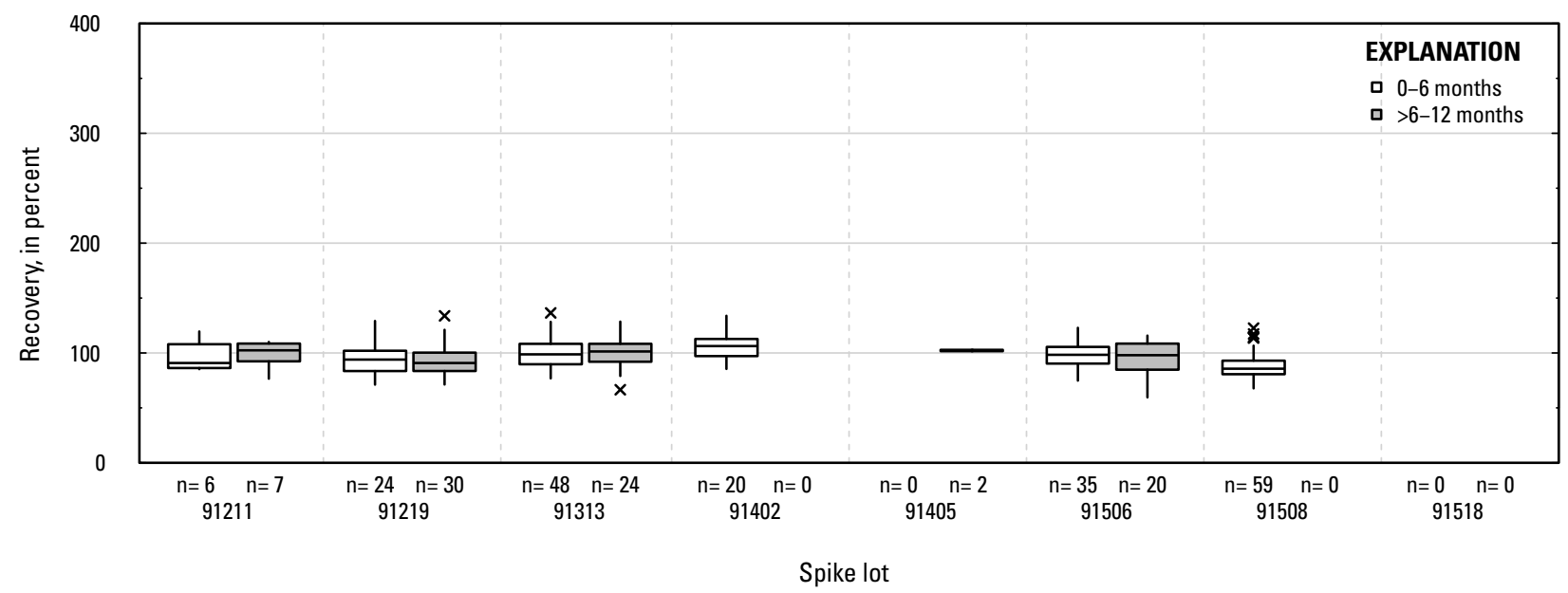

UB. Propanil: groundwater field matrix spikes

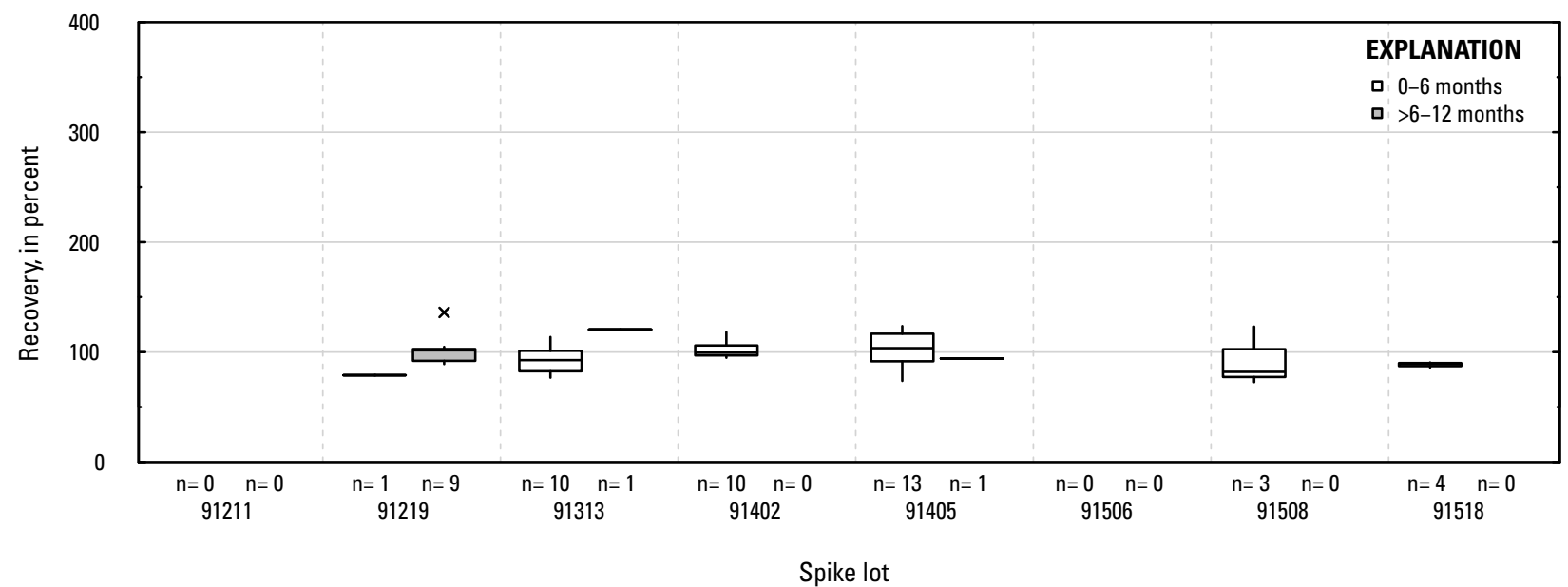

UC. Propanil: surface water field matrix spikes

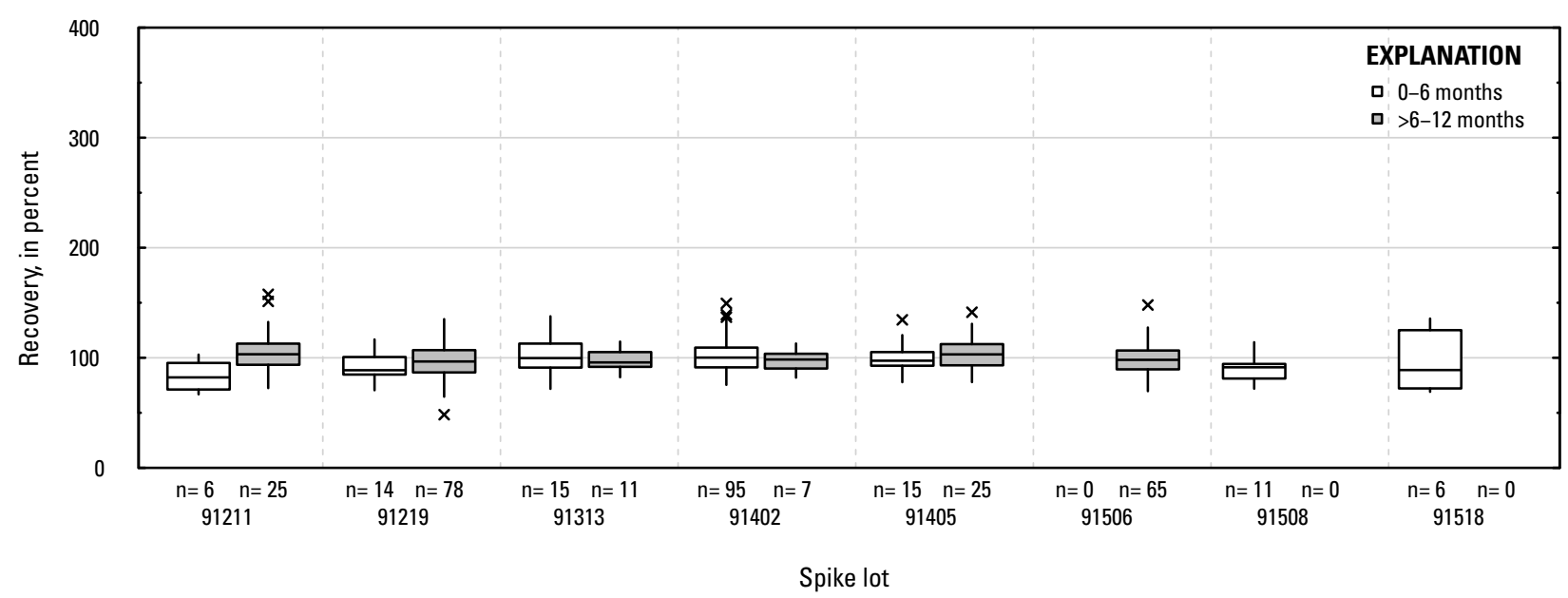

Figure 1-1. Distributions of recovery for individual pesticides in schedule 2437 by matrix, spike lot, and spike lot age. Recovery values larger than 400 percent are not shown.-Continued 
UD. Propargite: laboratory reagent spikes

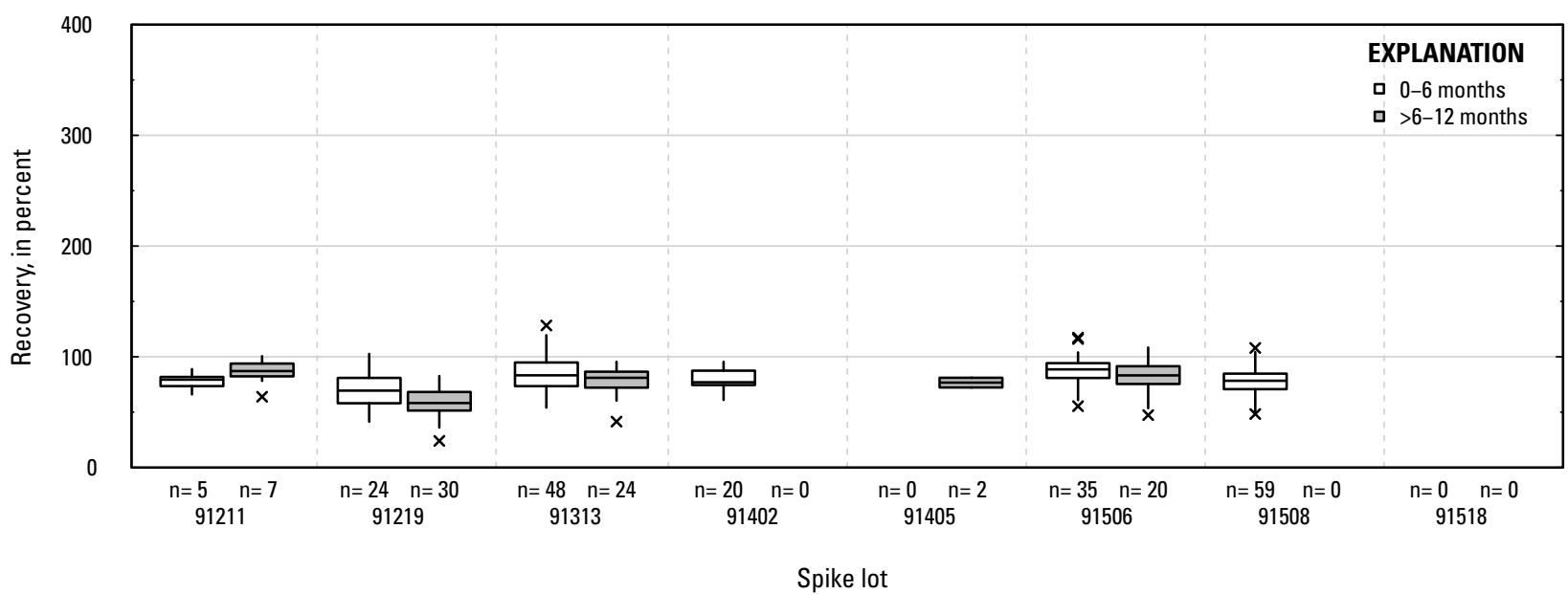

UE. Propargite: groundwater field matrix spikes

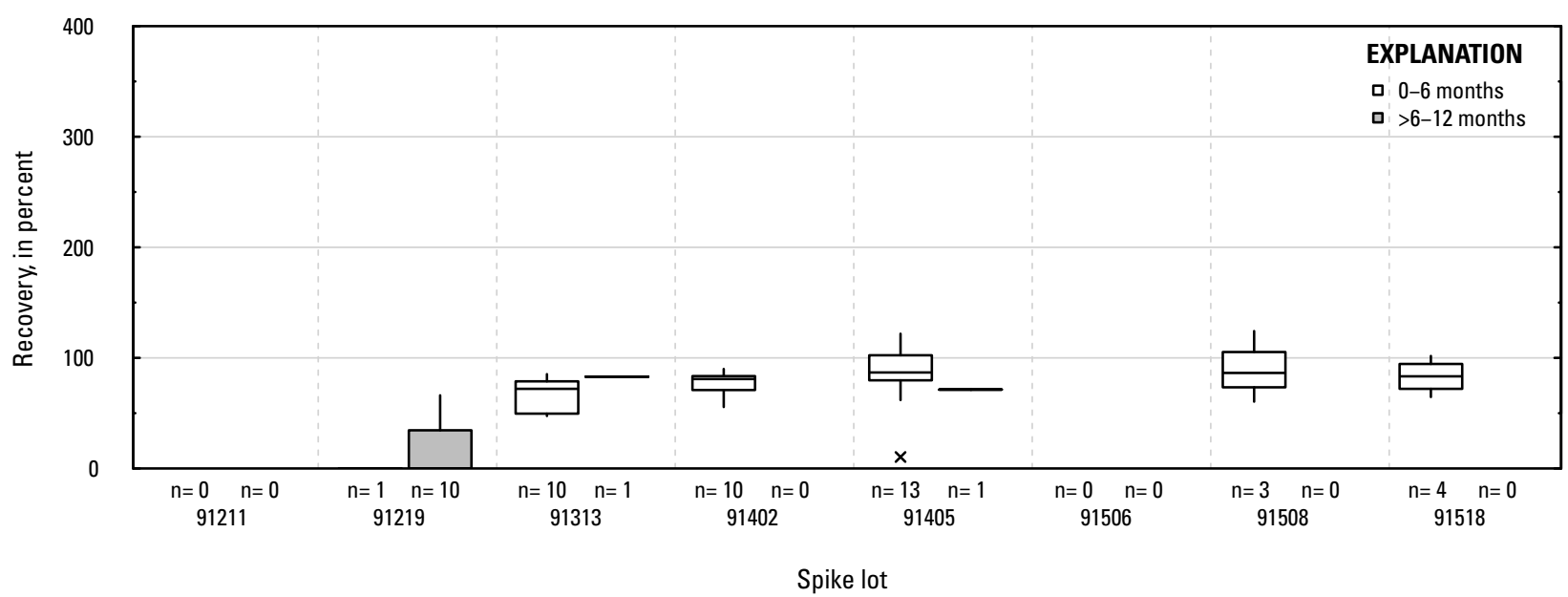

UF. Propargite: surface water field matrix spikes

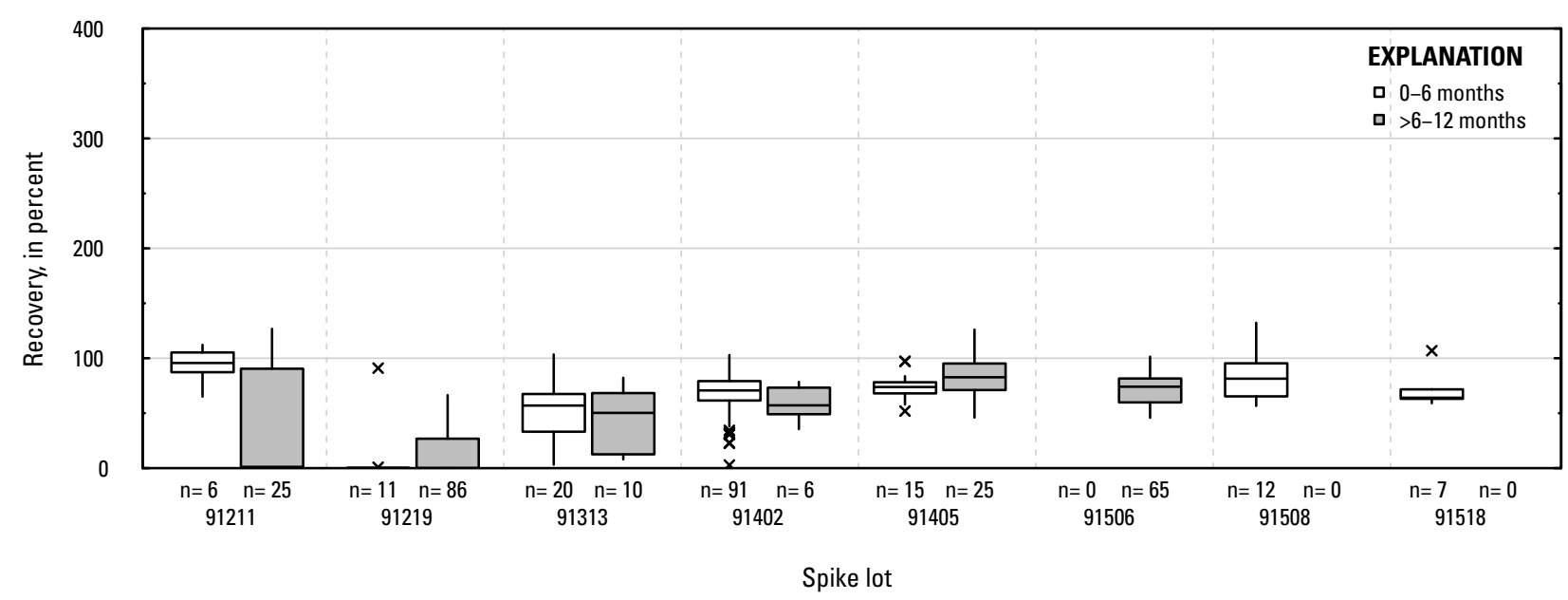

Figure 1-1. Distributions of recovery for individual pesticides in schedule 2437 by matrix, spike lot, and spike lot age. Recovery values larger than 400 percent are not shown.-Continued 
UG. Propazine: laboratory reagent spikes

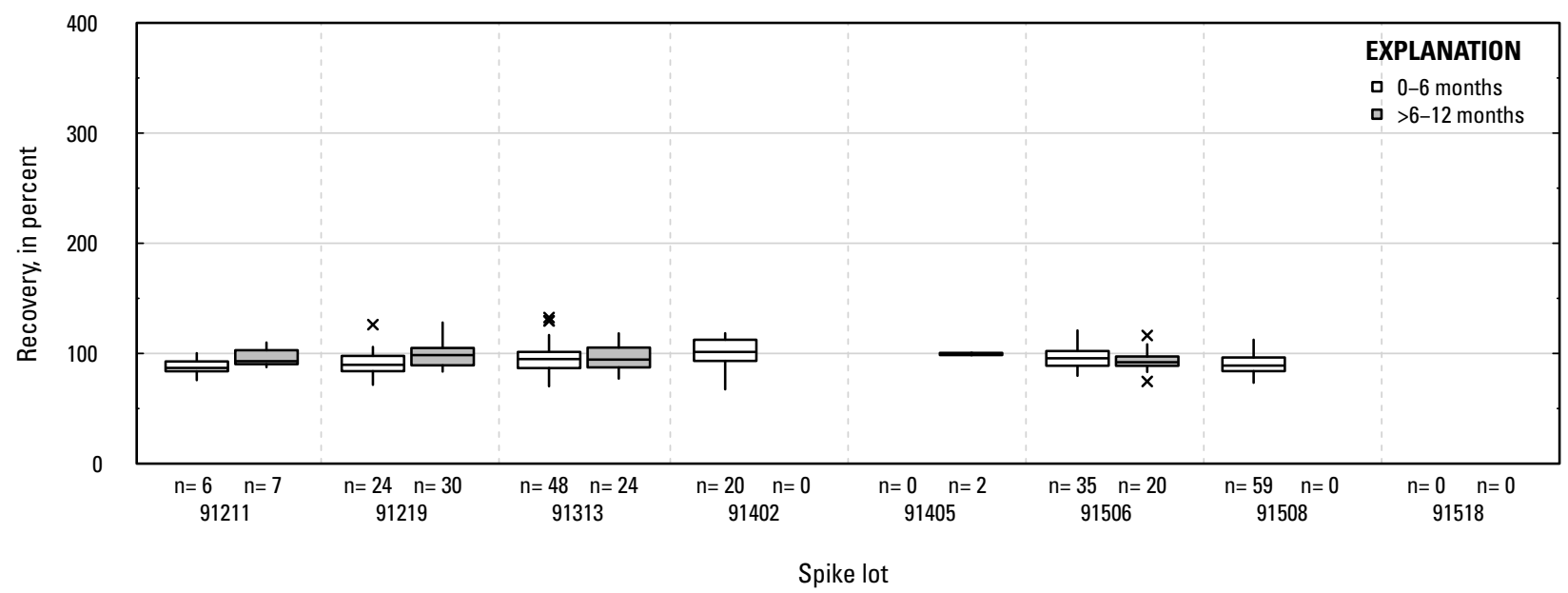

UH. Propazine: groundwater field matrix spikes

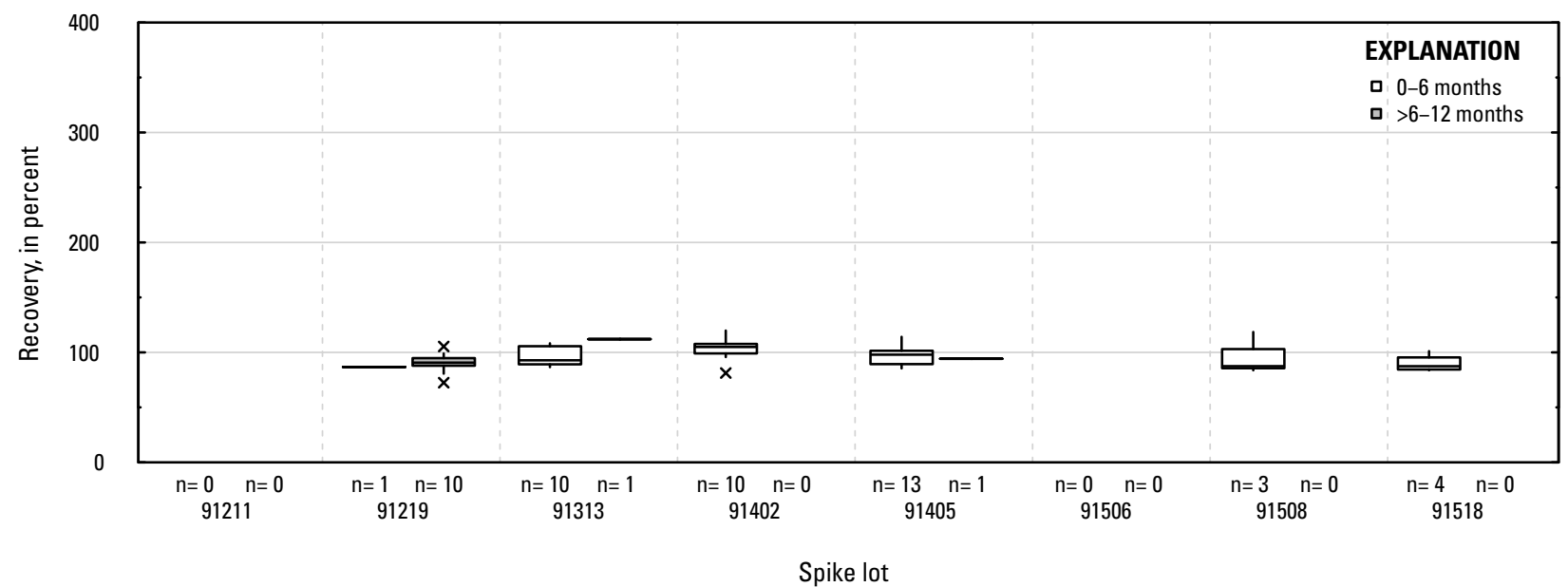

UI. Propazine: surface water field matrix spikes

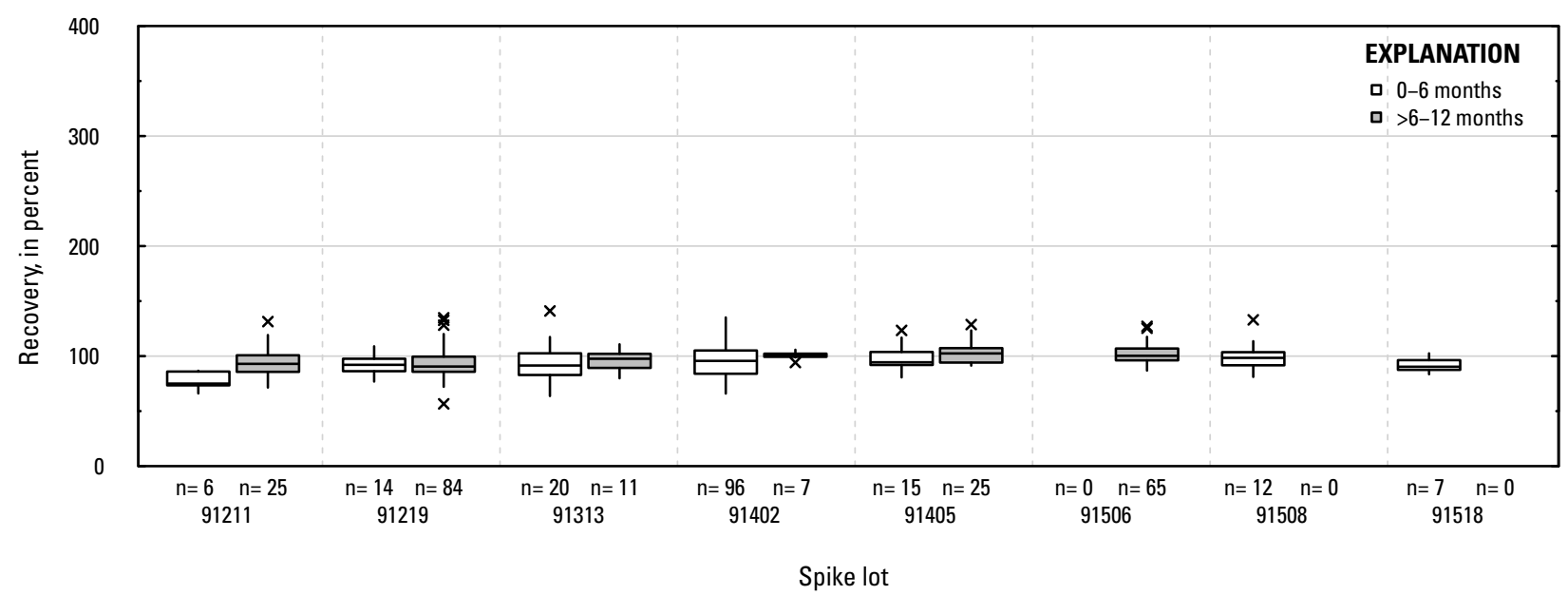

Figure 1-1. Distributions of recovery for individual pesticides in schedule 2437 by matrix, spike lot, and spike lot age. Recovery values larger than 400 percent are not shown.-Continued 
UJ. Propiconazole: laboratory reagent spikes

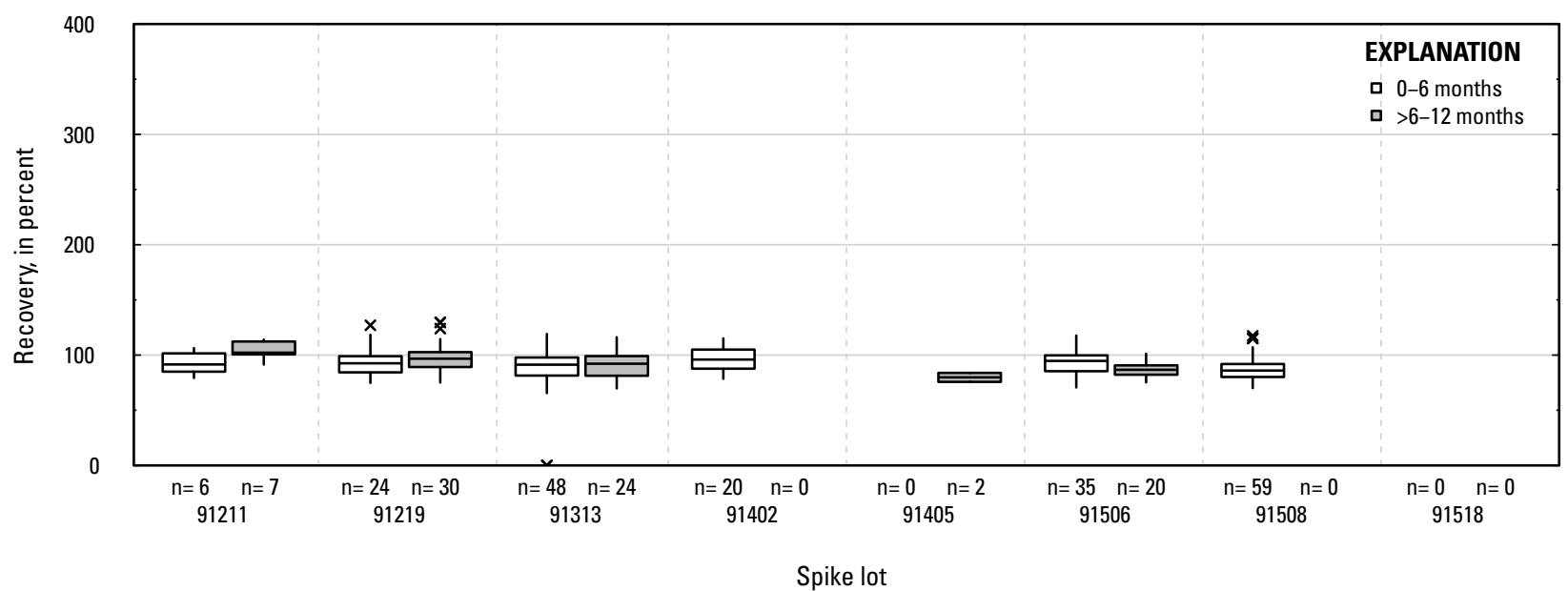

UK. Propiconazole: groundwater field matrix spikes

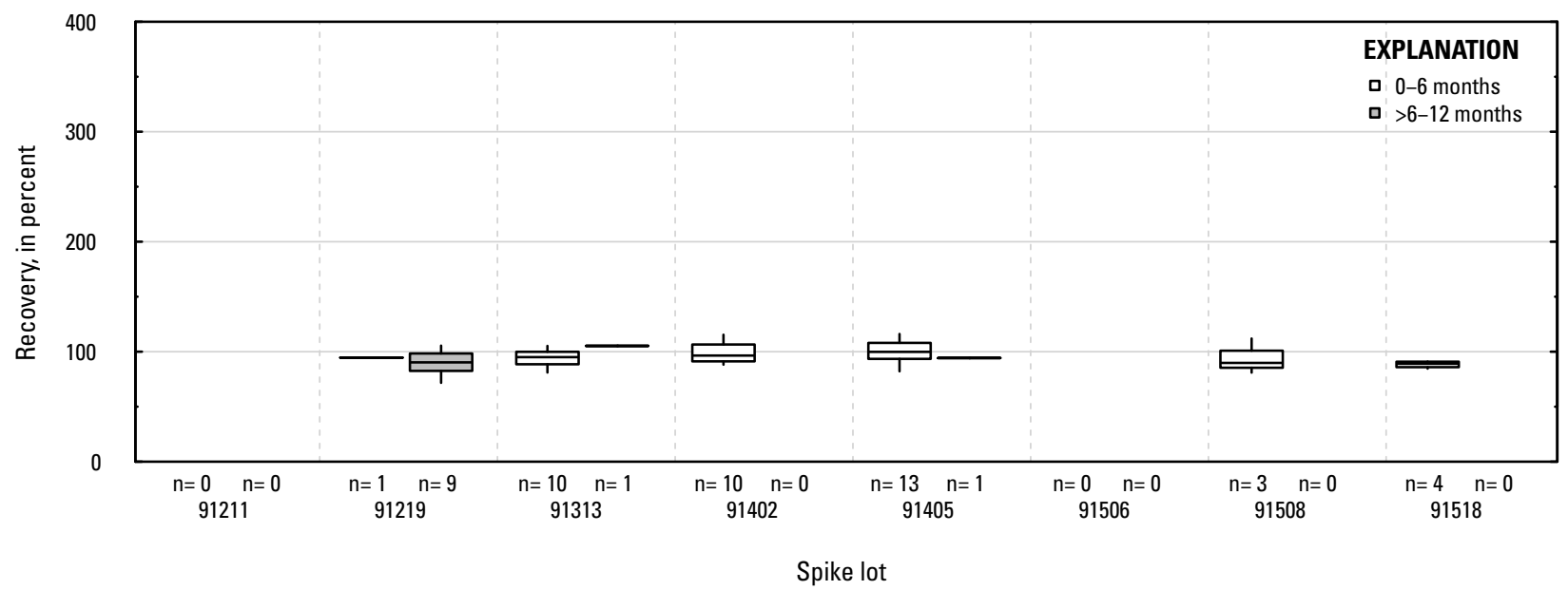

UL. Propiconazole: surface water field matrix spikes

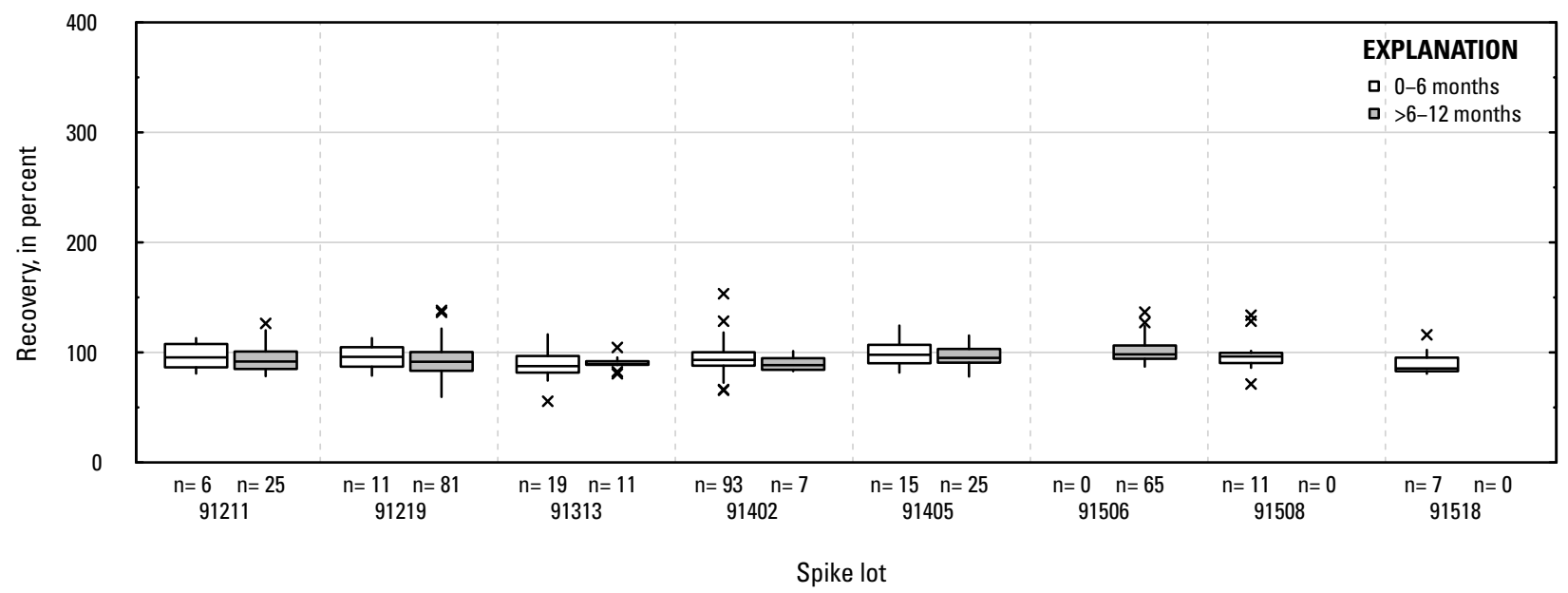

Figure 1-1. Distributions of recovery for individual pesticides in schedule 2437 by matrix, spike lot, and spike lot age. Recovery values larger than 400 percent are not shown.-Continued 
UM. Propoxur: laboratory reagent spikes

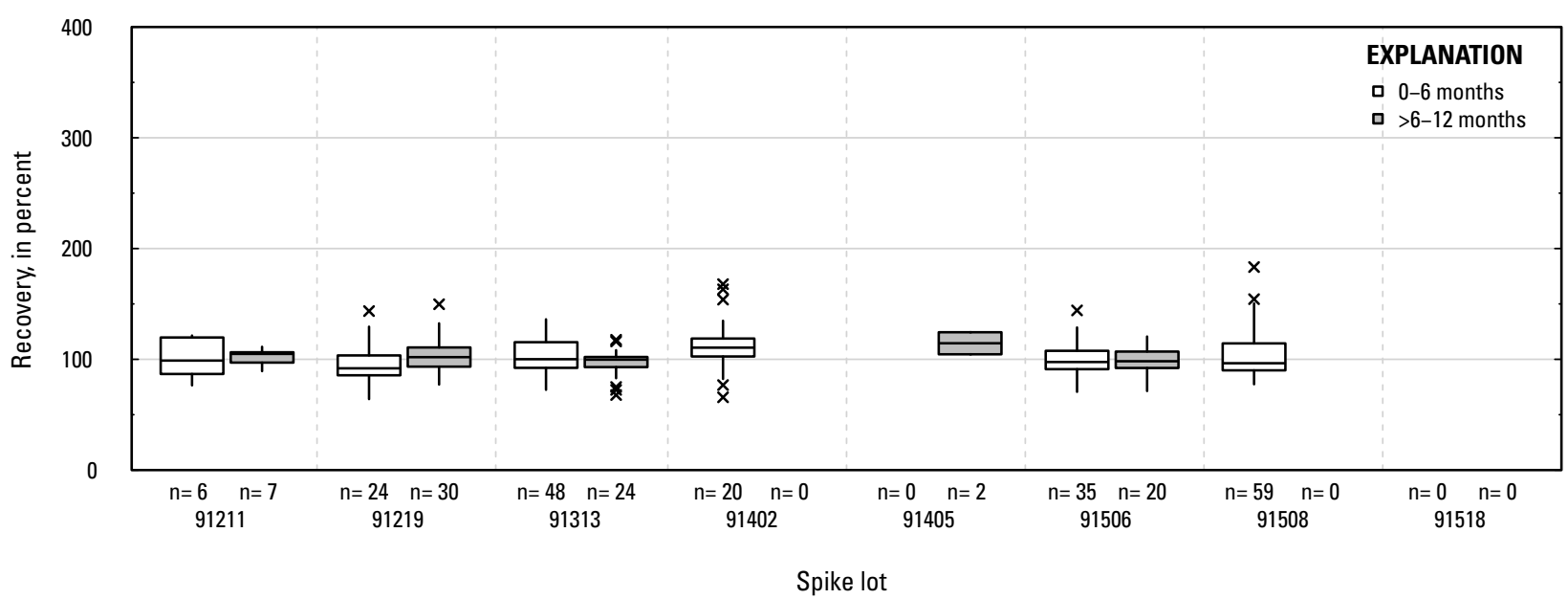

UN. Propoxur: groundwater field matrix spikes

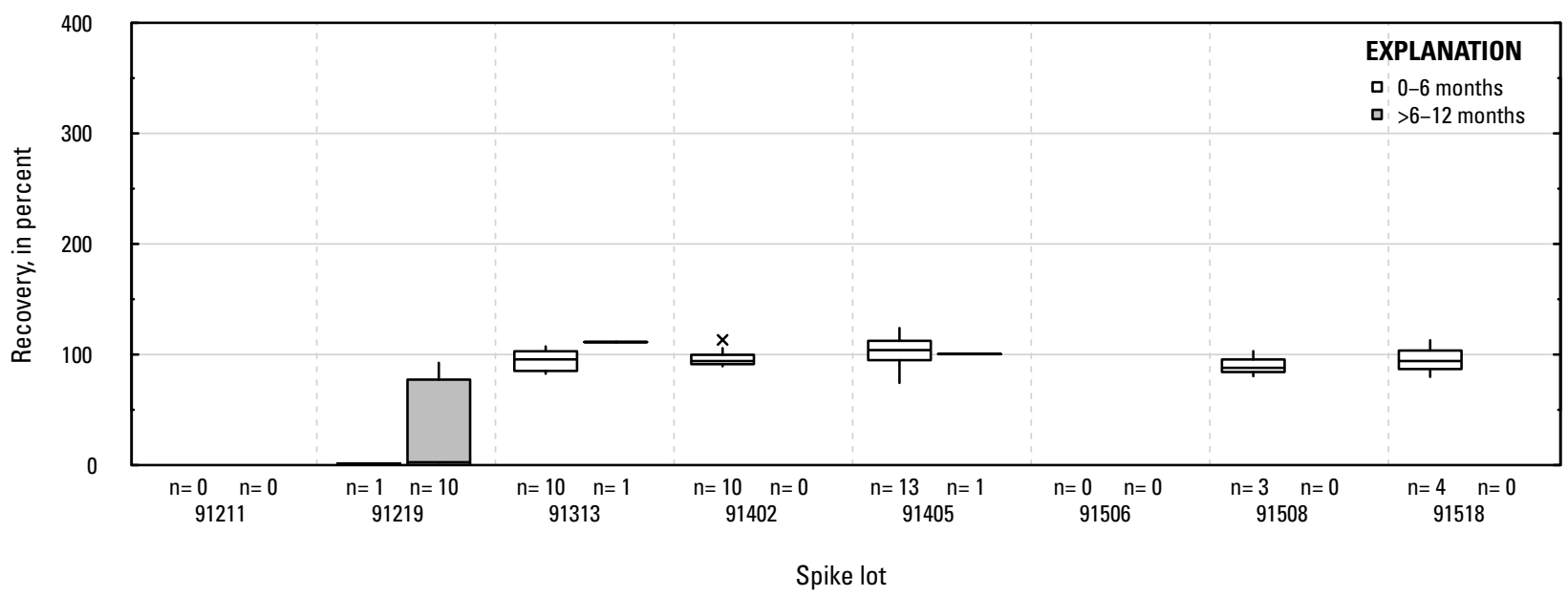

UO. Propoxur: surface water field matrix spikes

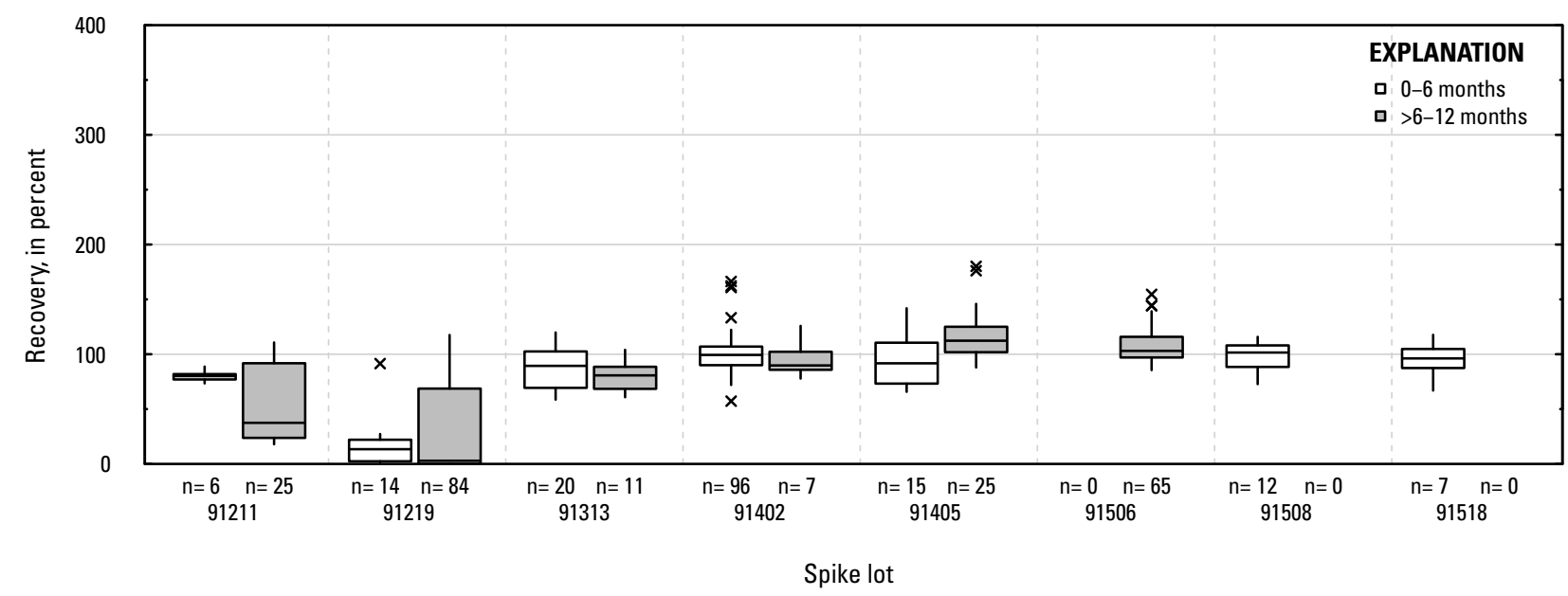

Figure 1-1. Distributions of recovery for individual pesticides in schedule 2437 by matrix, spike lot, and spike lot age. Recovery values larger than 400 percent are not shown.-Continued 
UP. Prosulfuron: laboratory reagent spikes

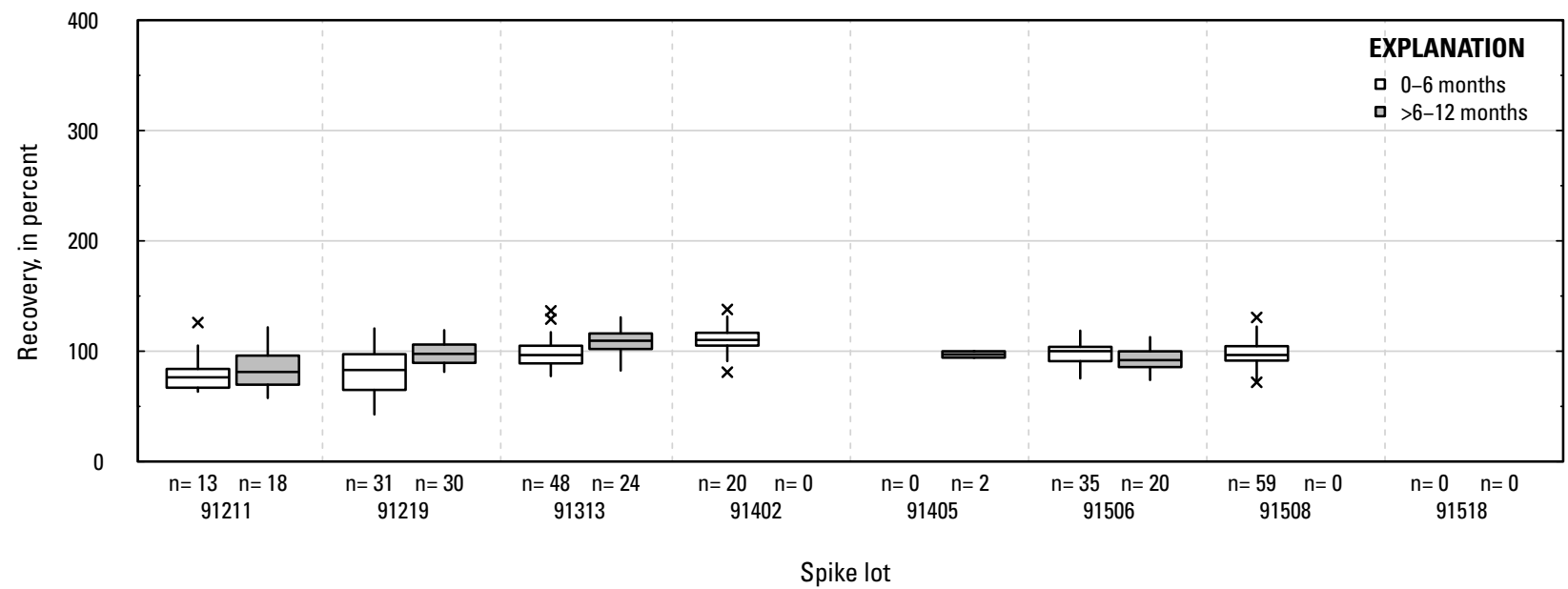

UQ. Prosulfuron: groundwater field matrix spikes

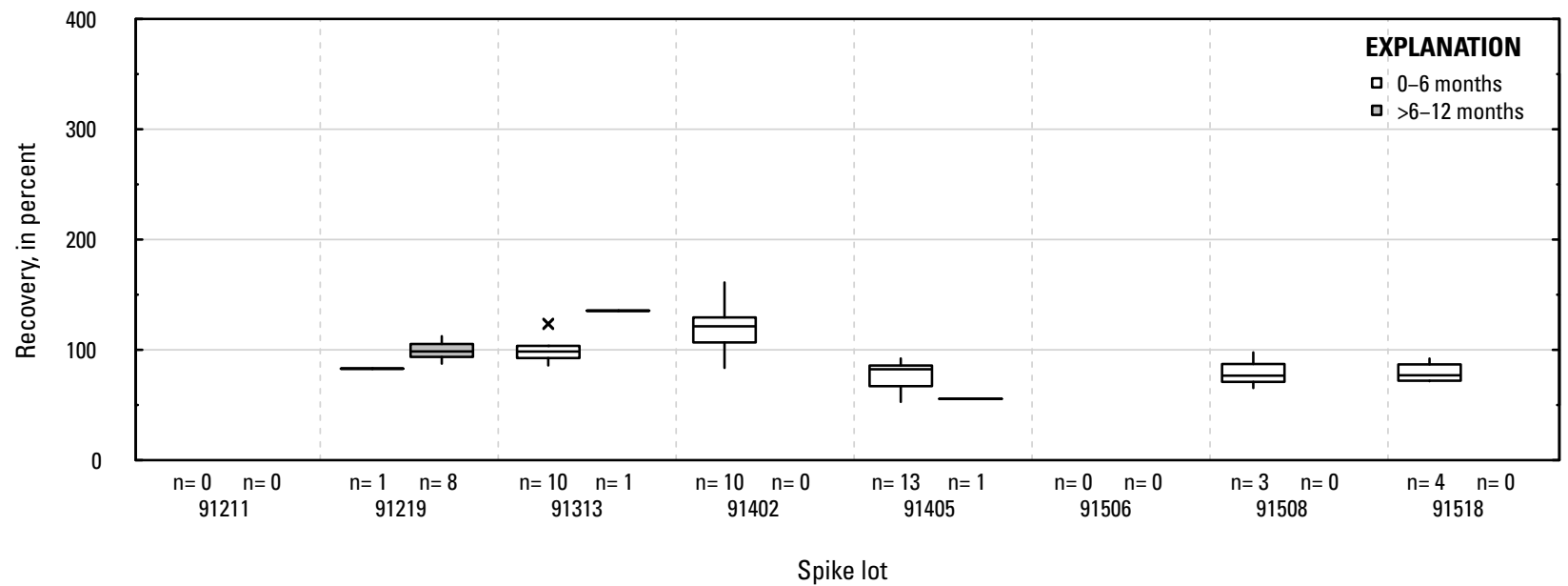

UR. Prosulfuron: surface water field matrix spikes

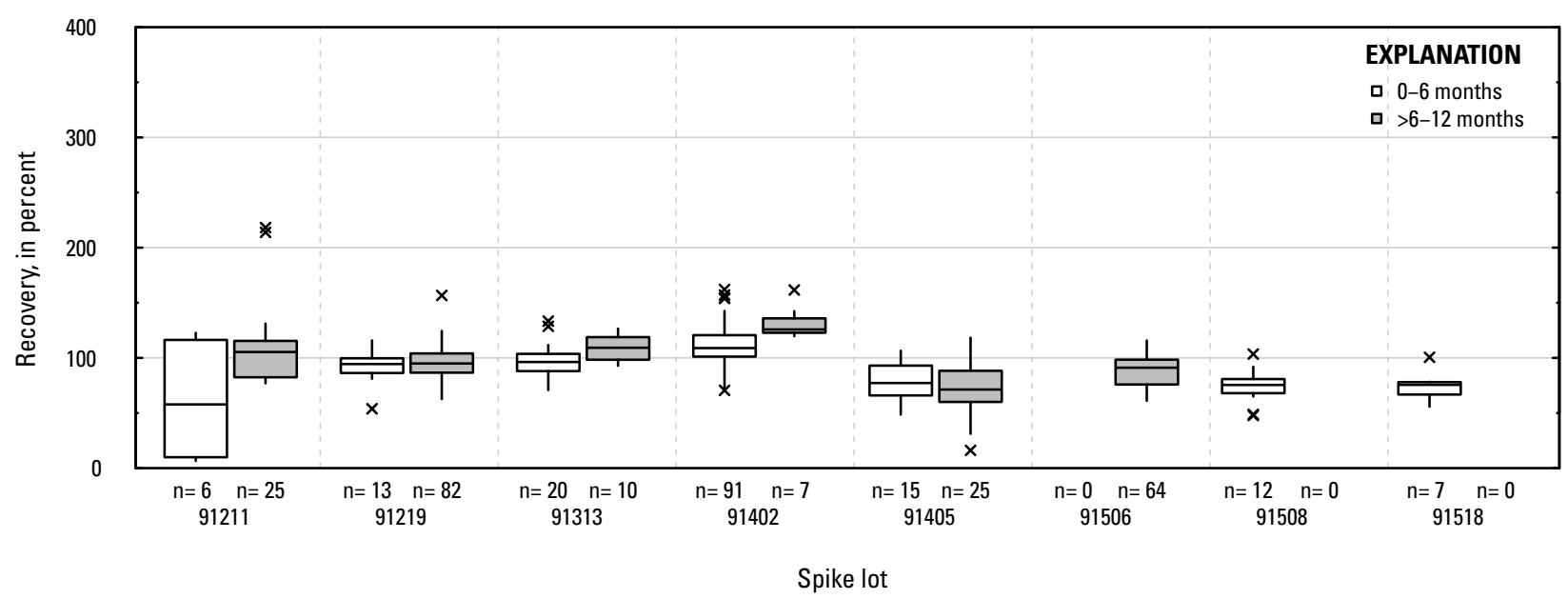

Figure 1-1. Distributions of recovery for individual pesticides in schedule 2437 by matrix, spike lot, and spike lot age. Recovery values larger than 400 percent are not shown.-Continued 
US. Pyraclostrobin: laboratory reagent spikes

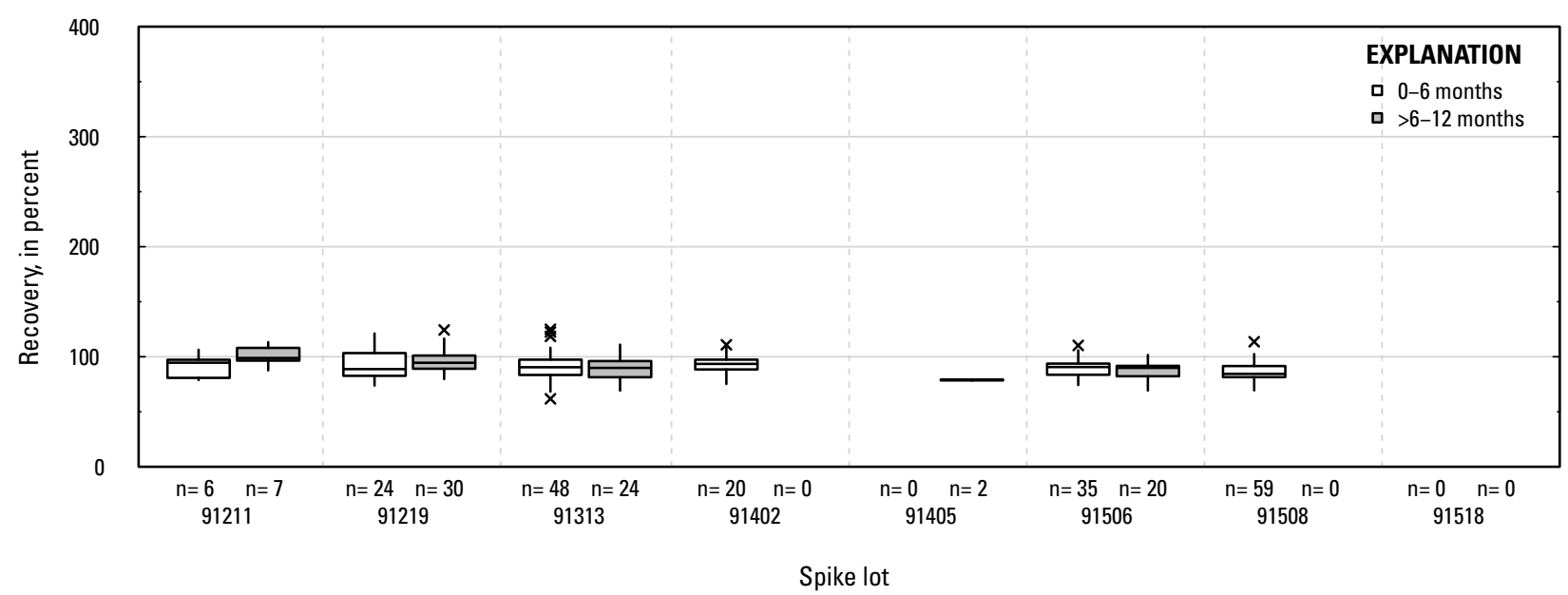

UT. Pyraclostrobin: groundwater field matrix spikes

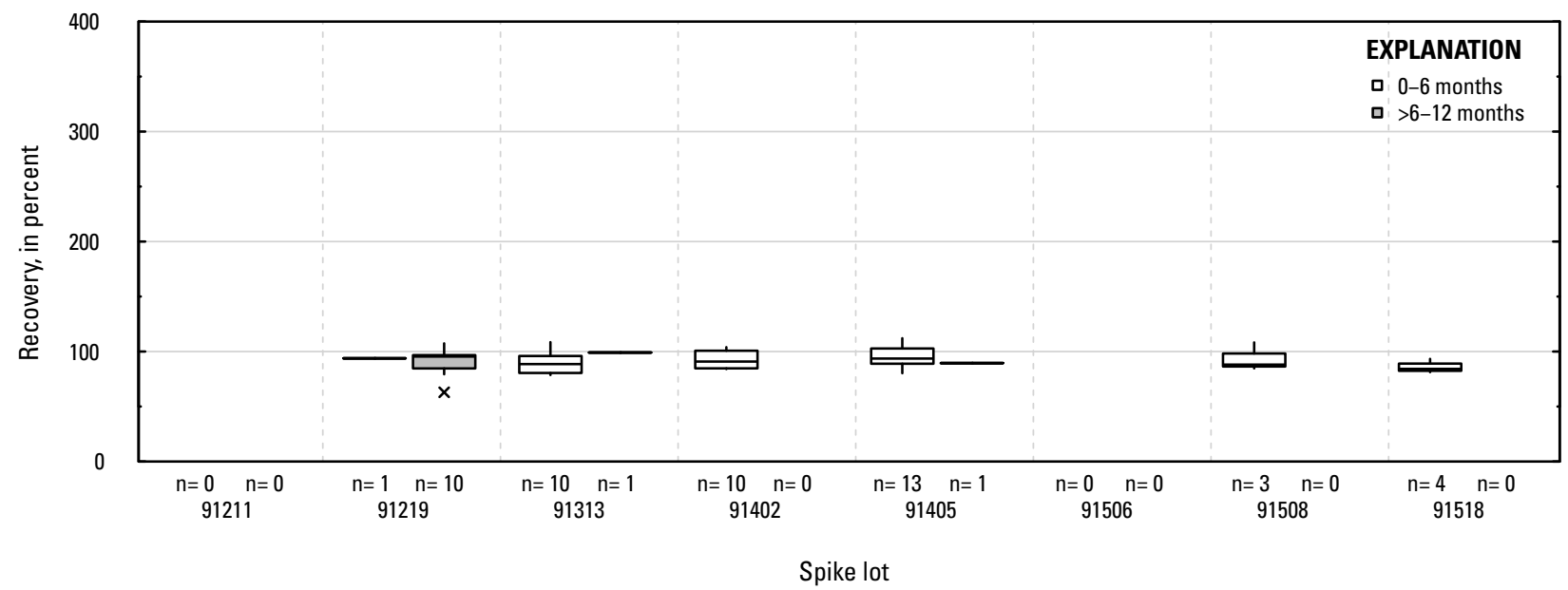

UU. Pyraclostrobin: surface water field matrix spikes

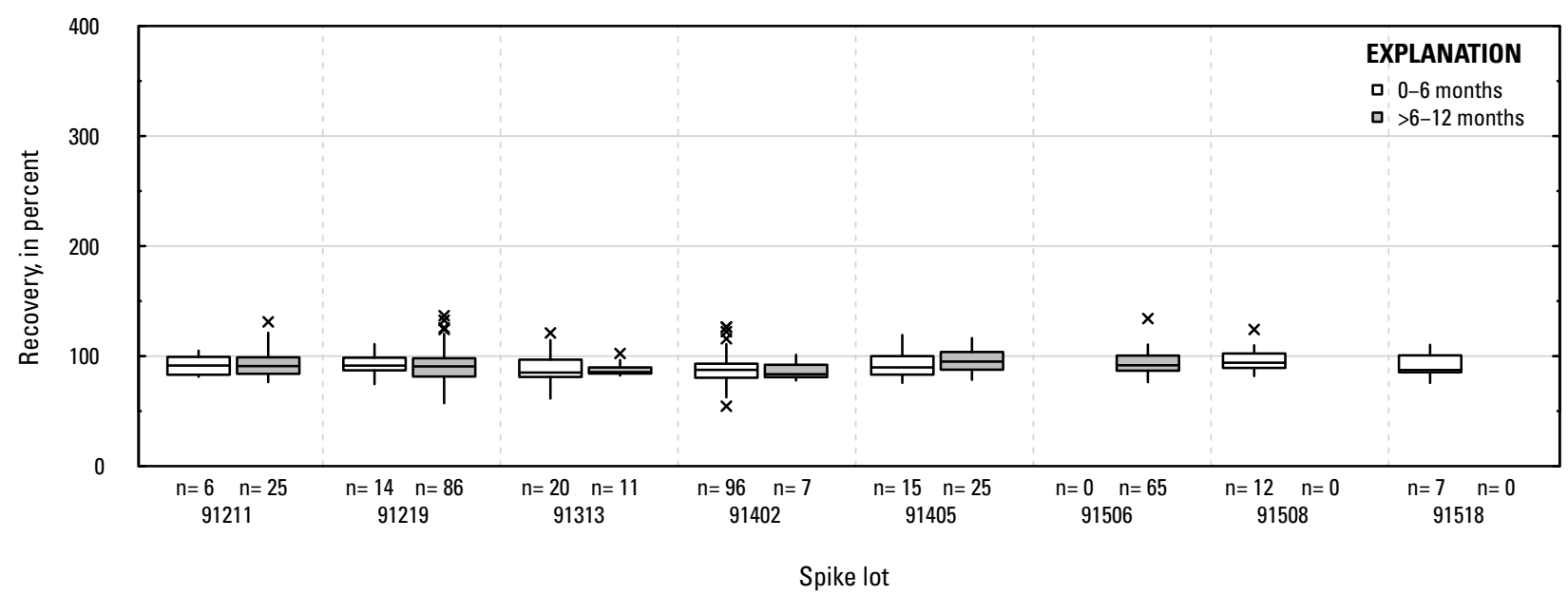

Figure 1-1. Distributions of recovery for individual pesticides in schedule 2437 by matrix, spike lot, and spike lot age. Recovery values larger than 400 percent are not shown.-Continued 
UV. Pyridaben: laboratory reagent spikes

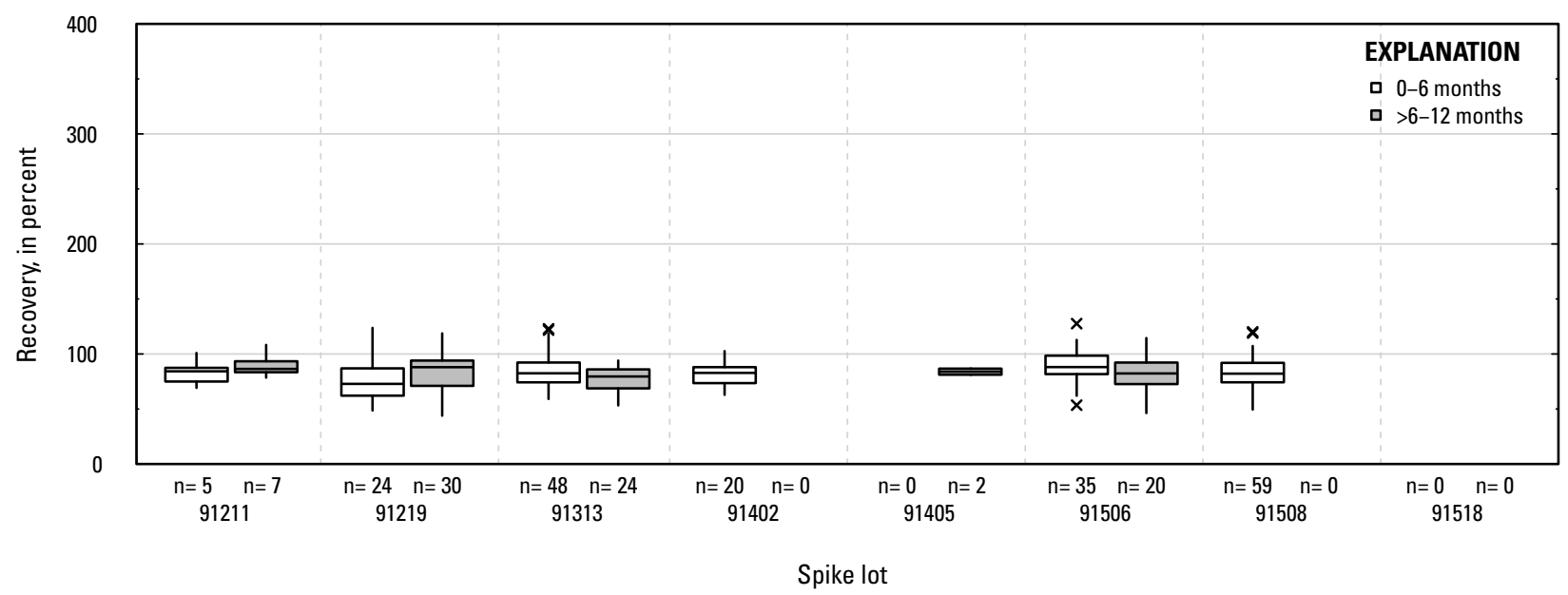

UW. Pyridaben: groundwater field matrix spikes

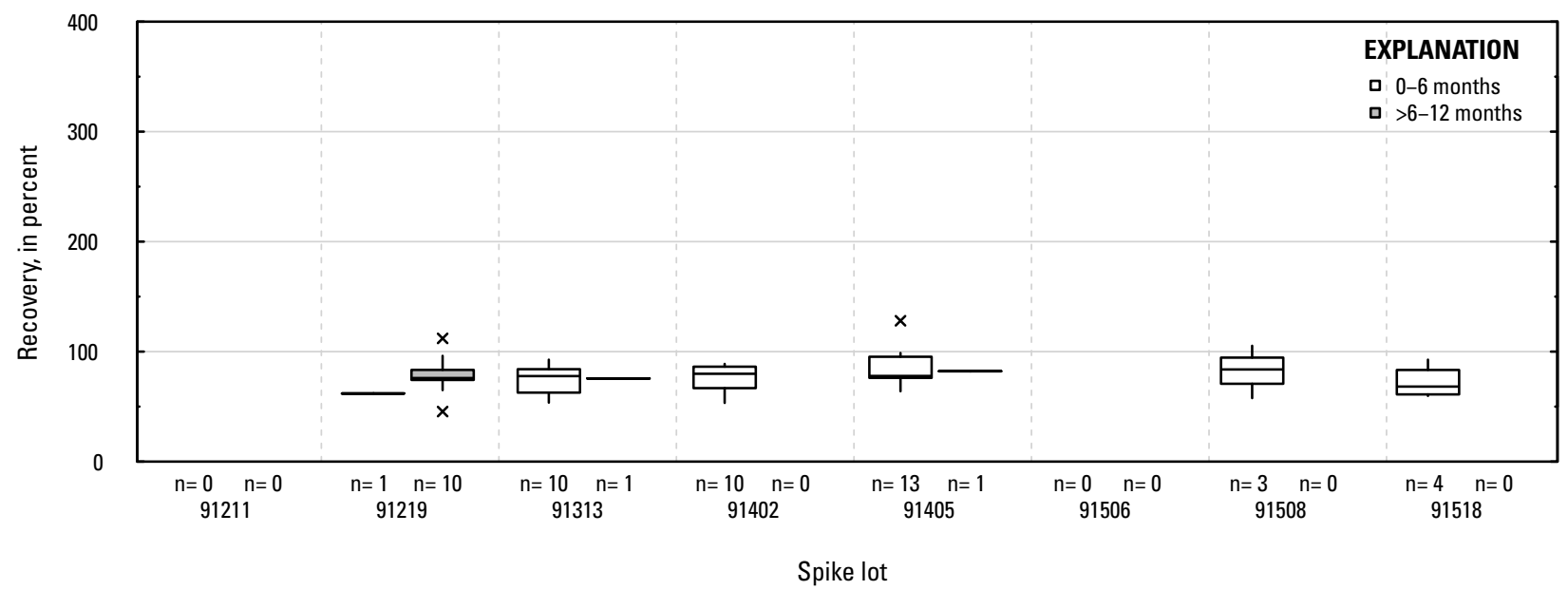

UX. Pyridaben: surface water field matrix spikes

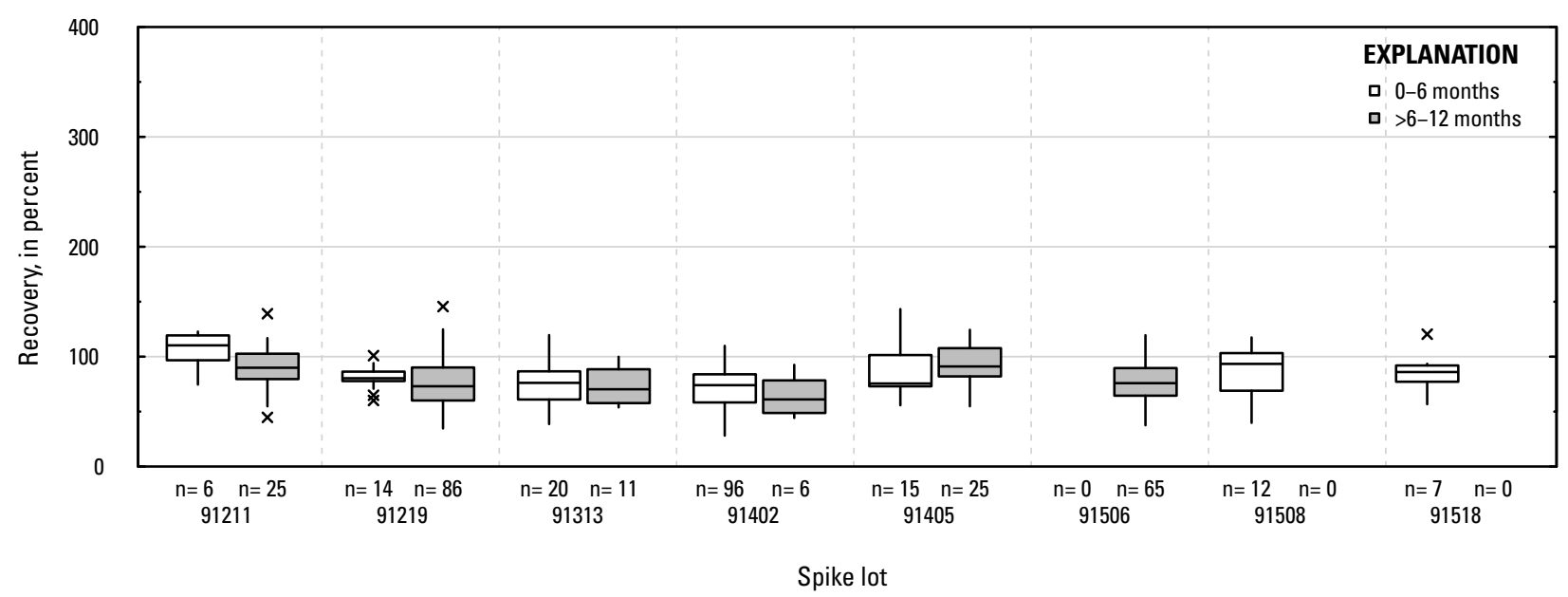

Figure 1-1. Distributions of recovery for individual pesticides in schedule 2437 by matrix, spike lot, and spike lot age. Recovery values larger than 400 percent are not shown.-Continued 
UY. 2-Isopropyl-6-methyl-4-pyrimidinol: laboratory reagent spikes

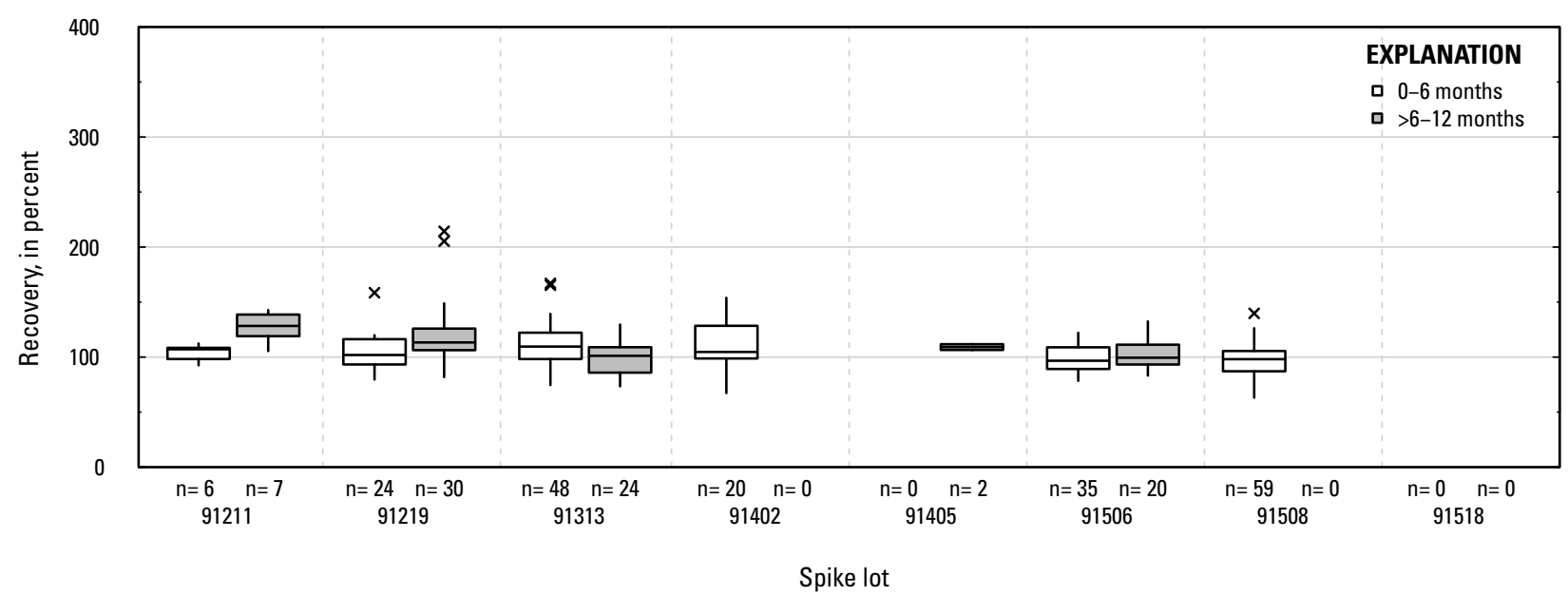

UZ. 2-Isopropyl-6-methyl-4-pyrimidinol: groundwater field matrix spikes

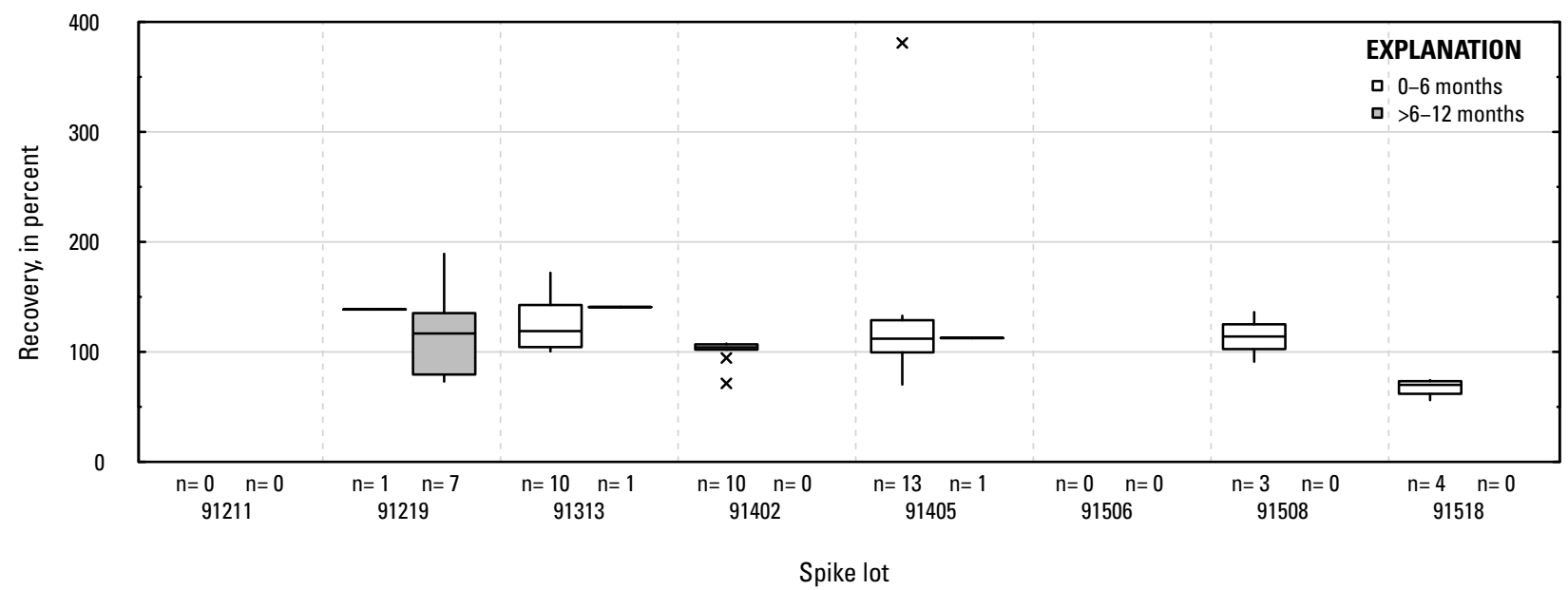

VA. 2-Isopropyl-6-methyl-4-pyrimidinol: surface water field matrix spikes

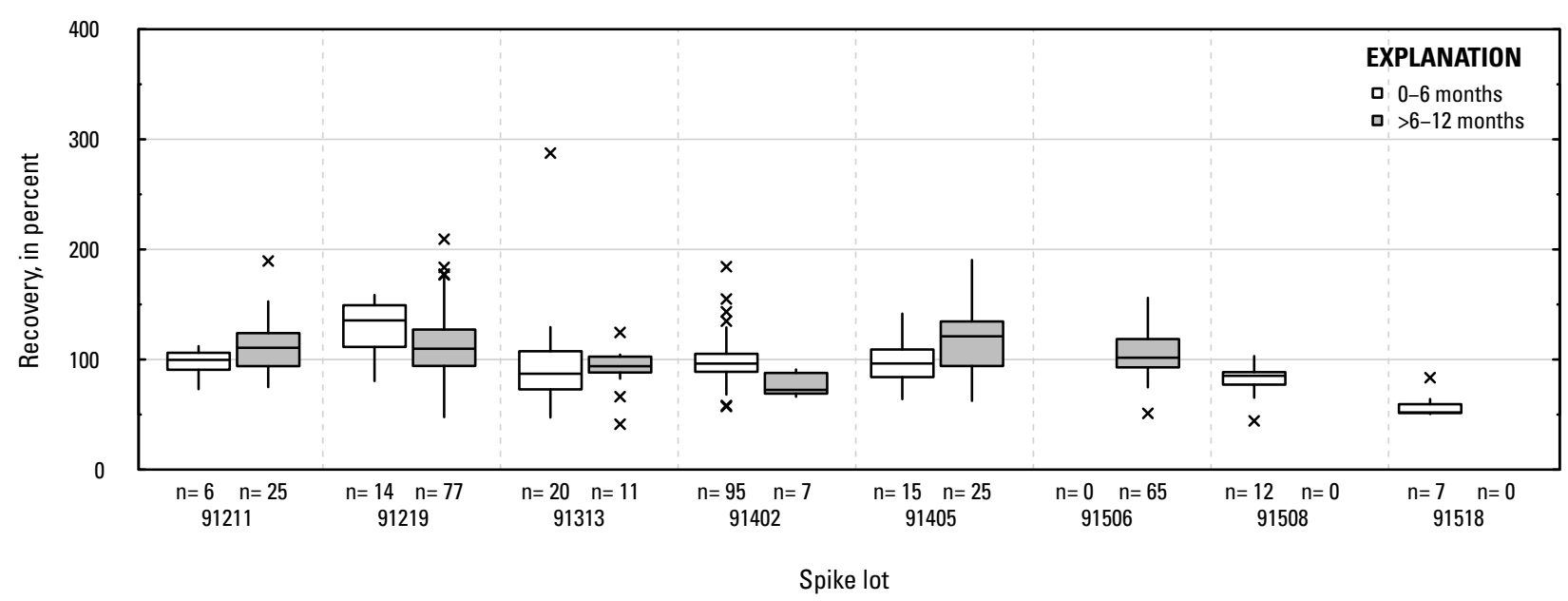

Figure 1-1. Distributions of recovery for individual pesticides in schedule 2437 by matrix, spike lot, and spike lot age. Recovery values larger than 400 percent are not shown.-Continued 
VB. Pyriproxyfen: laboratory reagent spikes

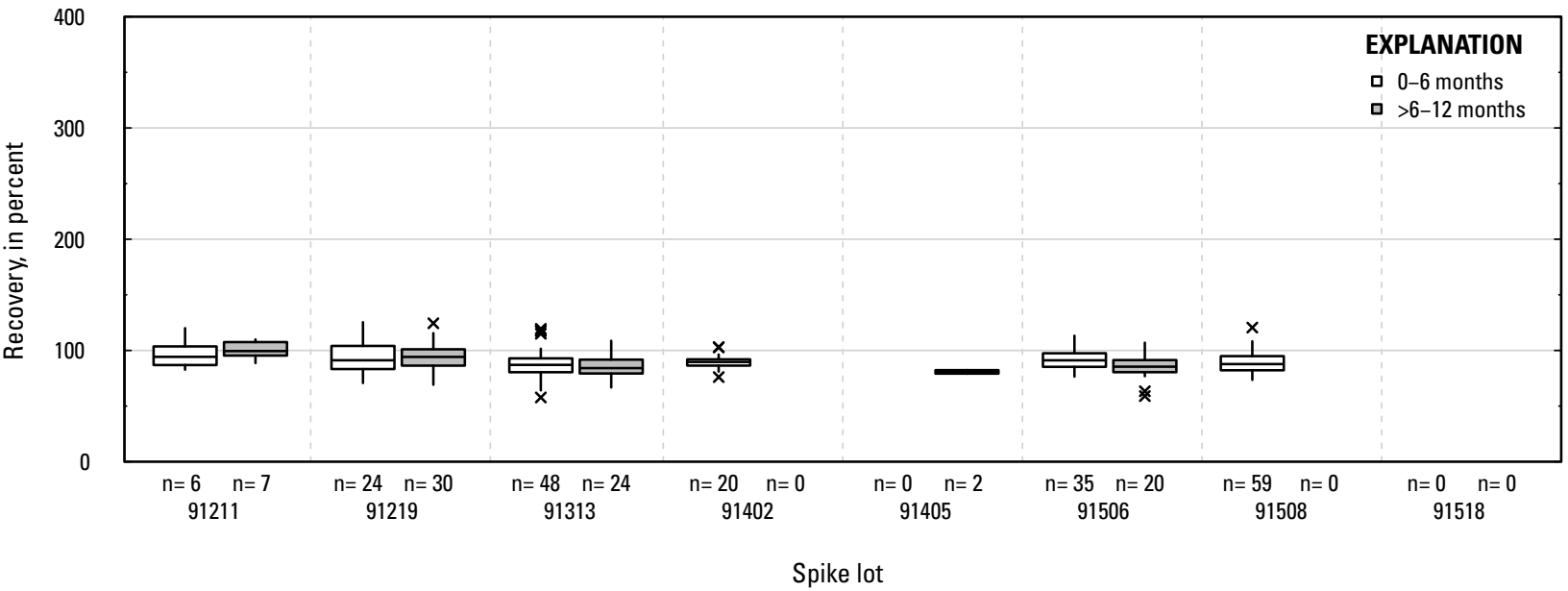

VC. Pyriproxyfen: groundwater field matrix spikes

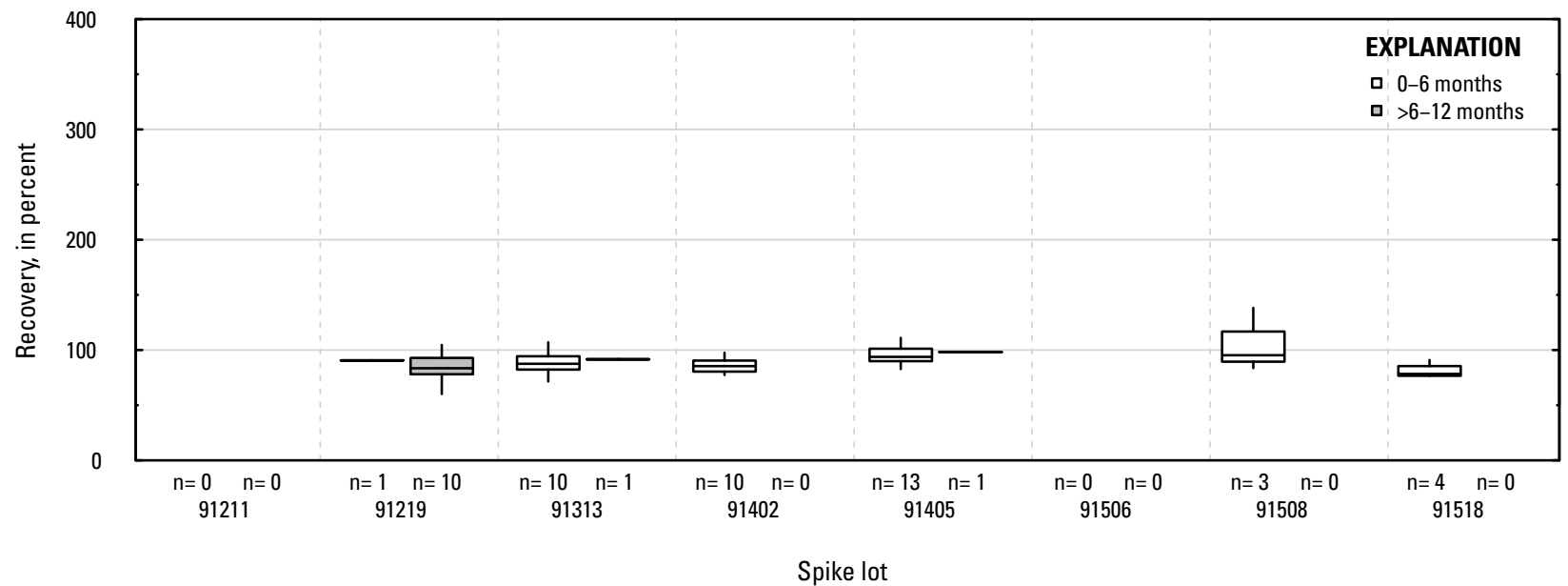

VD. Pyriproxyfen: surface water field matrix spikes

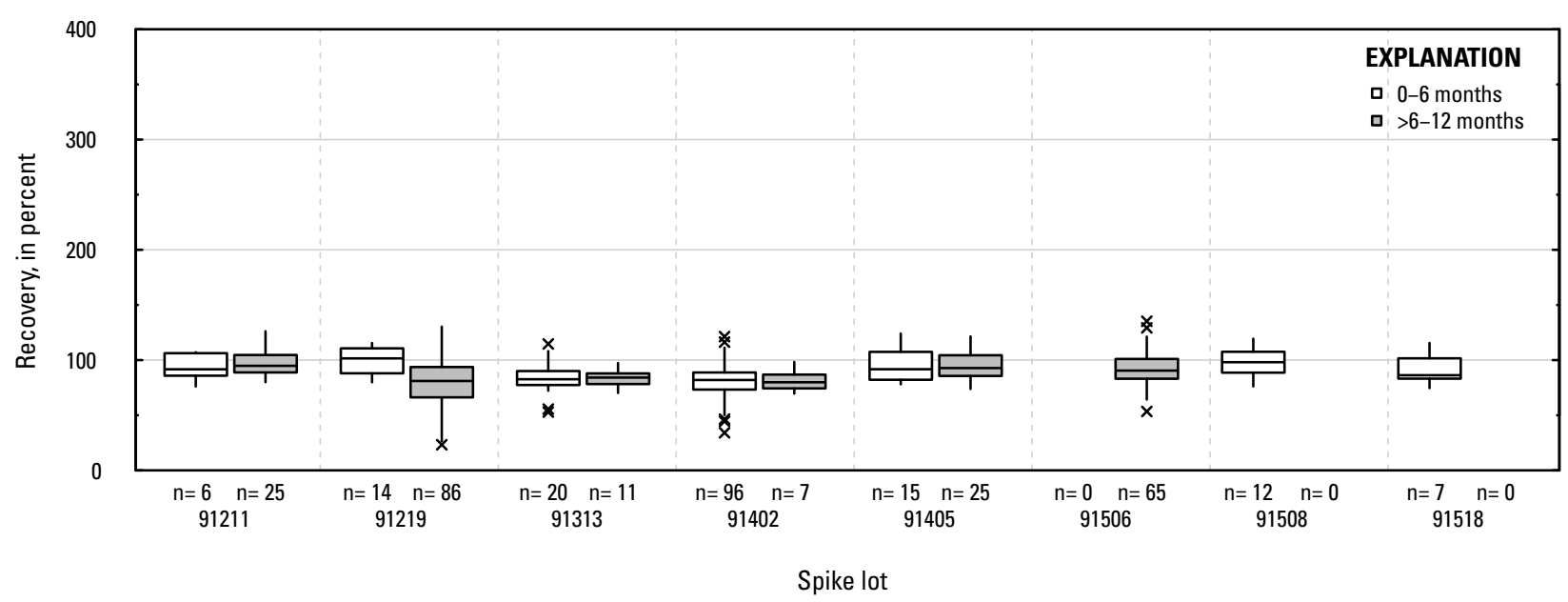

Figure 1-1. Distributions of recovery for individual pesticides in schedule 2437 by matrix, spike lot, and spike lot age. Recovery values larger than 400 percent are not shown.-Continued 
VE. sec-Acetochlor oxanilic acid: laboratory reagent spikes

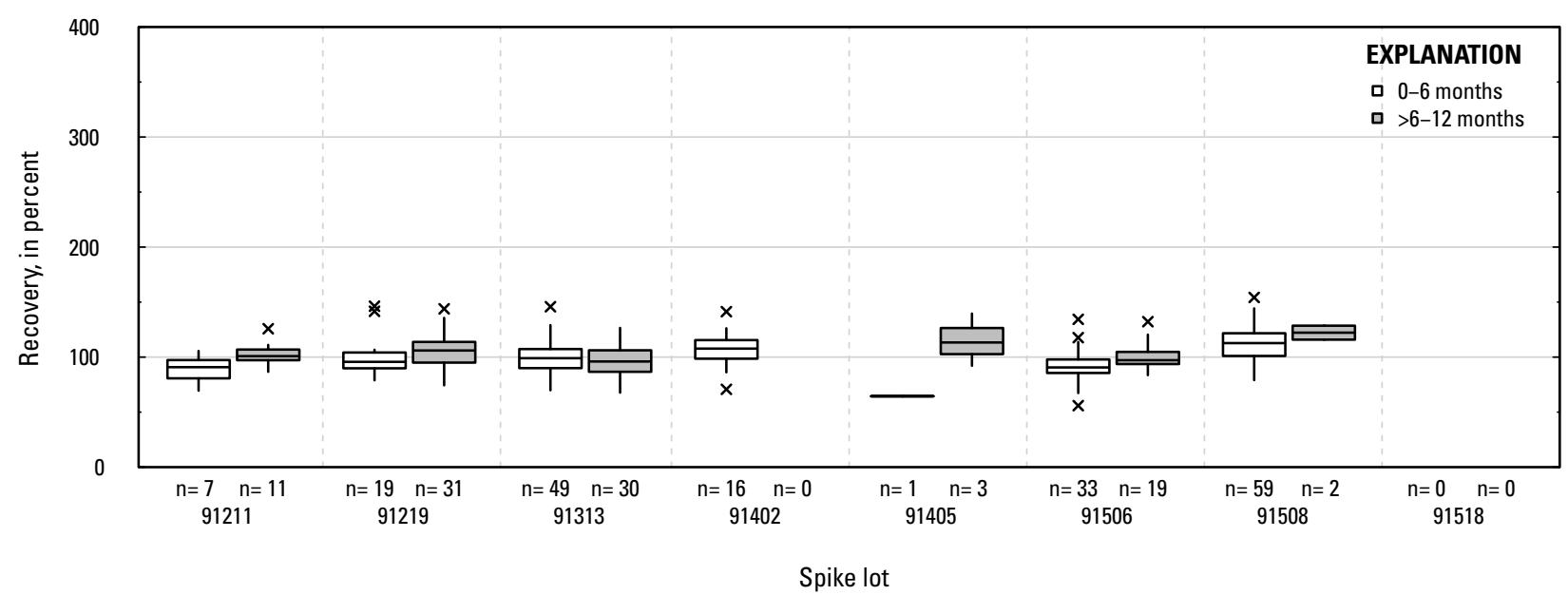

VF sec-Acetochlor oxanilic acid: groundwater field matrix spikes

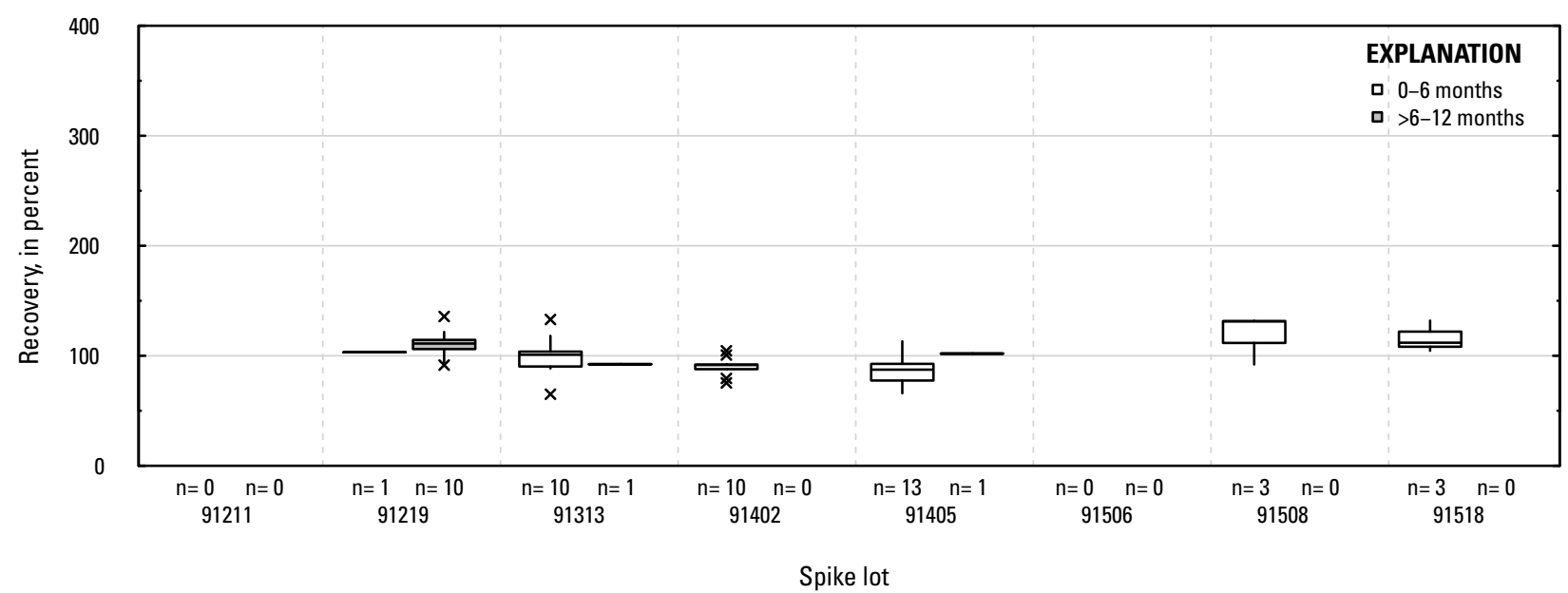

VG. sec-Acetochlor oxanilic acid: surface water field matrix spikes

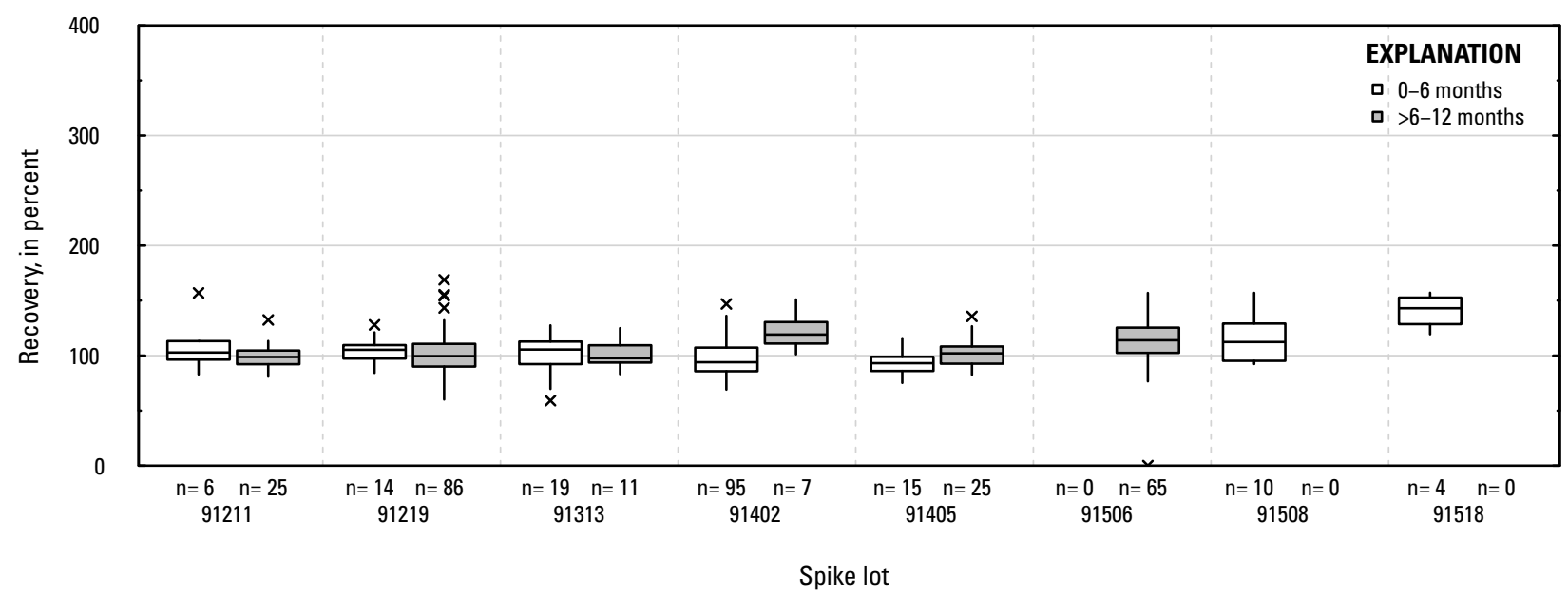

Figure 1-1. Distributions of recovery for individual pesticides in schedule 2437 by matrix, spike lot, and spike lot age. Recovery values larger than 400 percent are not shown.-Continued 
VH. sec-Alachlor oxanilic acid: laboratory reagent spikes

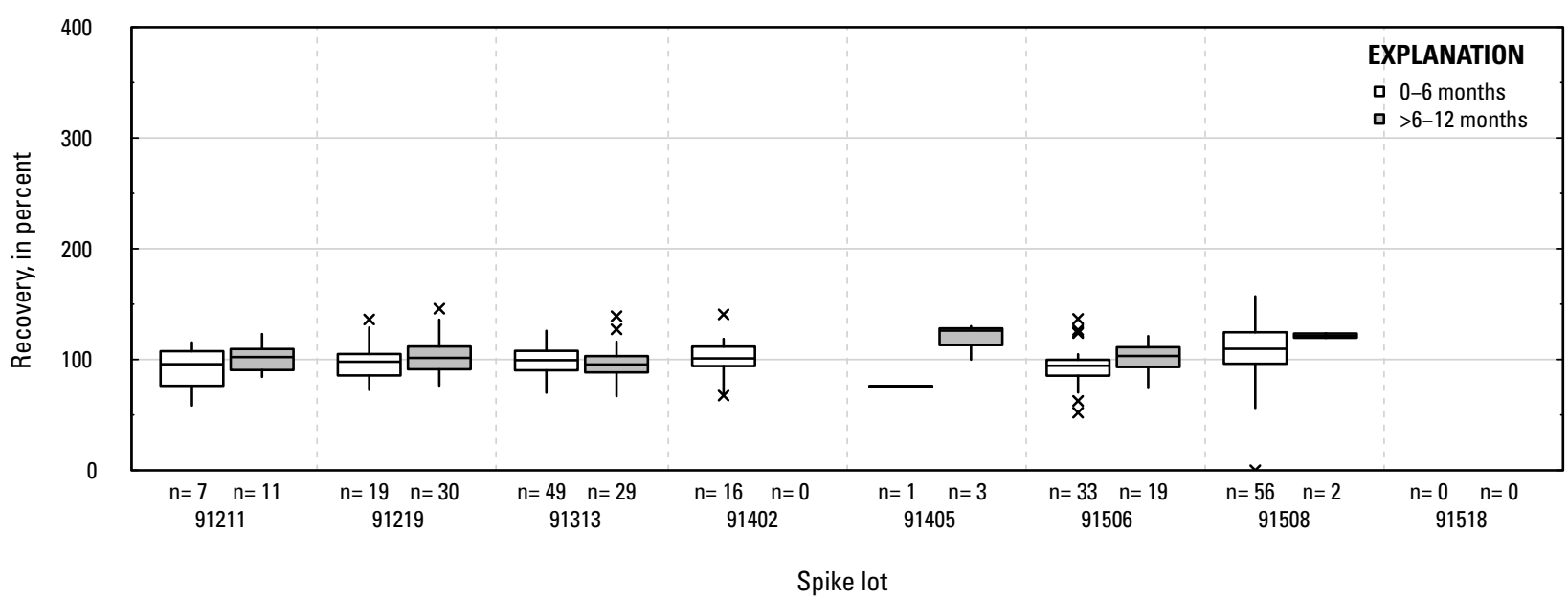

VI. sec-Alachlor oxanilic acid: groundwater field matrix spikes

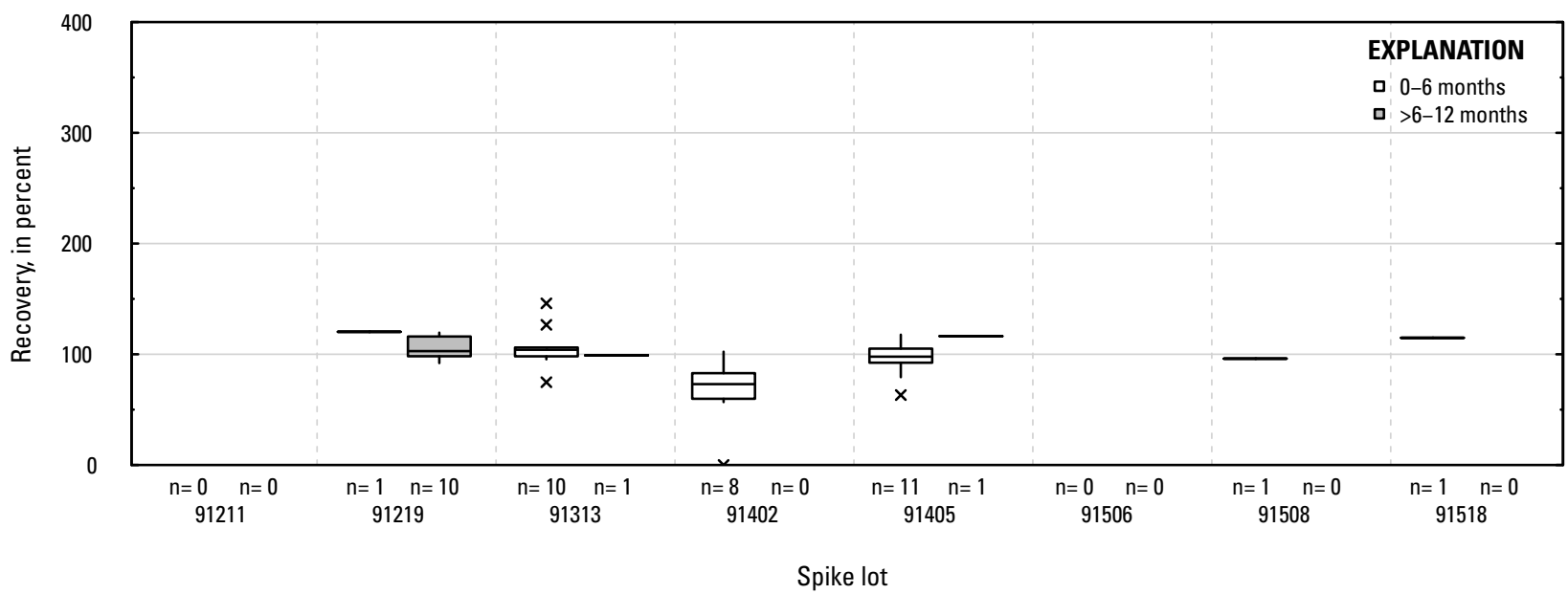

VJ. sec-Alachlor oxanilic acid: surface water field matrix spikes

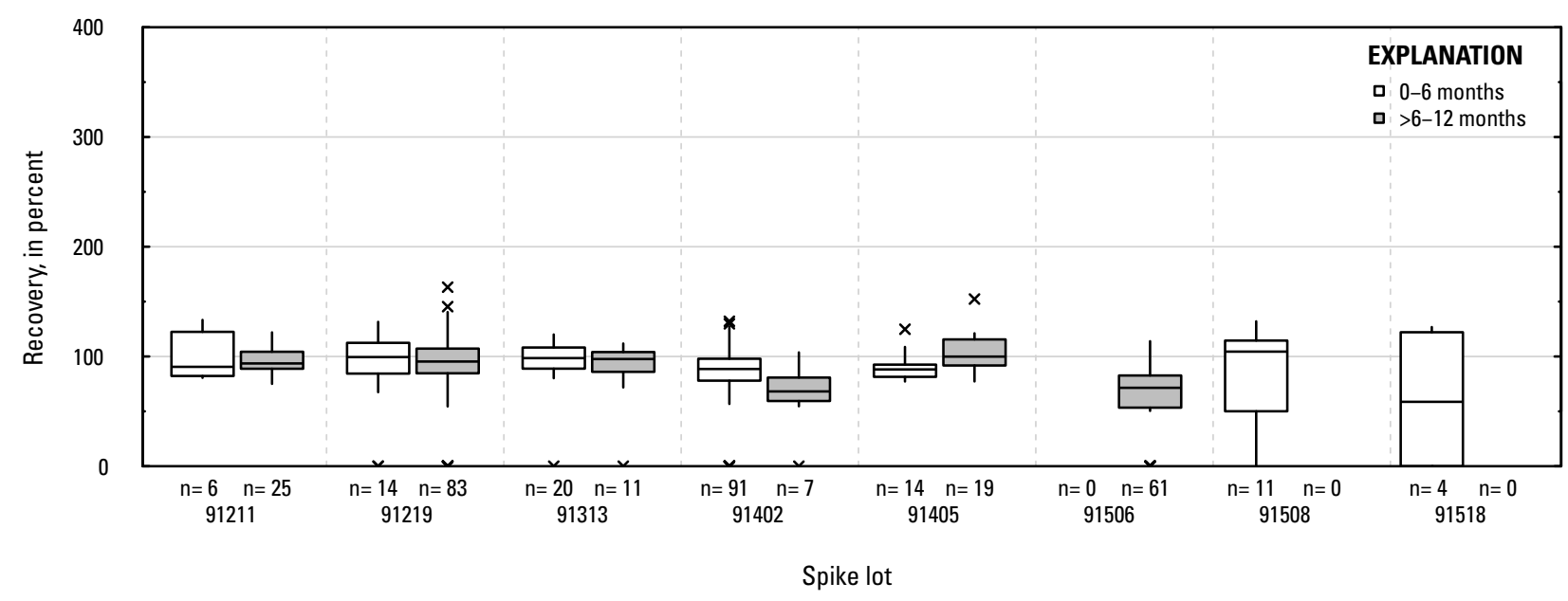

Figure 1-1. Distributions of recovery for individual pesticides in schedule 2437 by matrix, spike lot, and spike lot age. Recovery values larger than 400 percent are not shown.-Continued 
VK. Siduron: laboratory reagent spikes

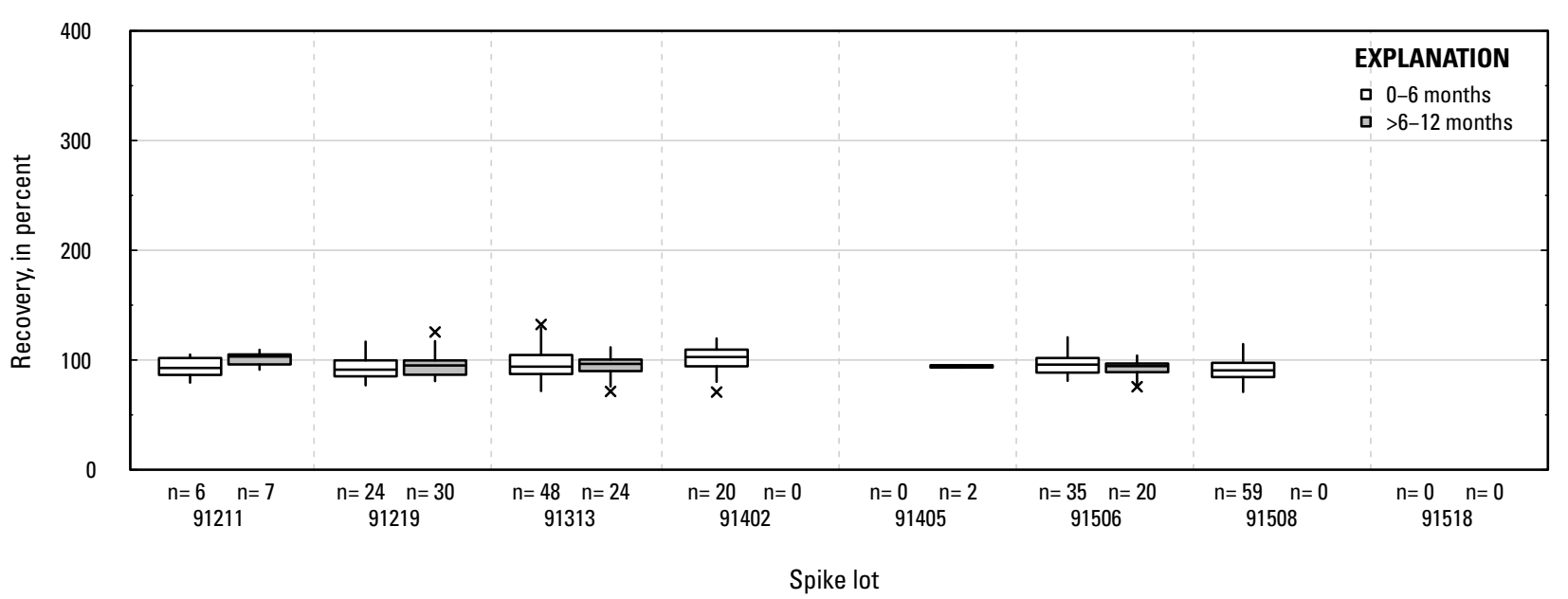

VL. Siduron: groundwater field matrix spikes

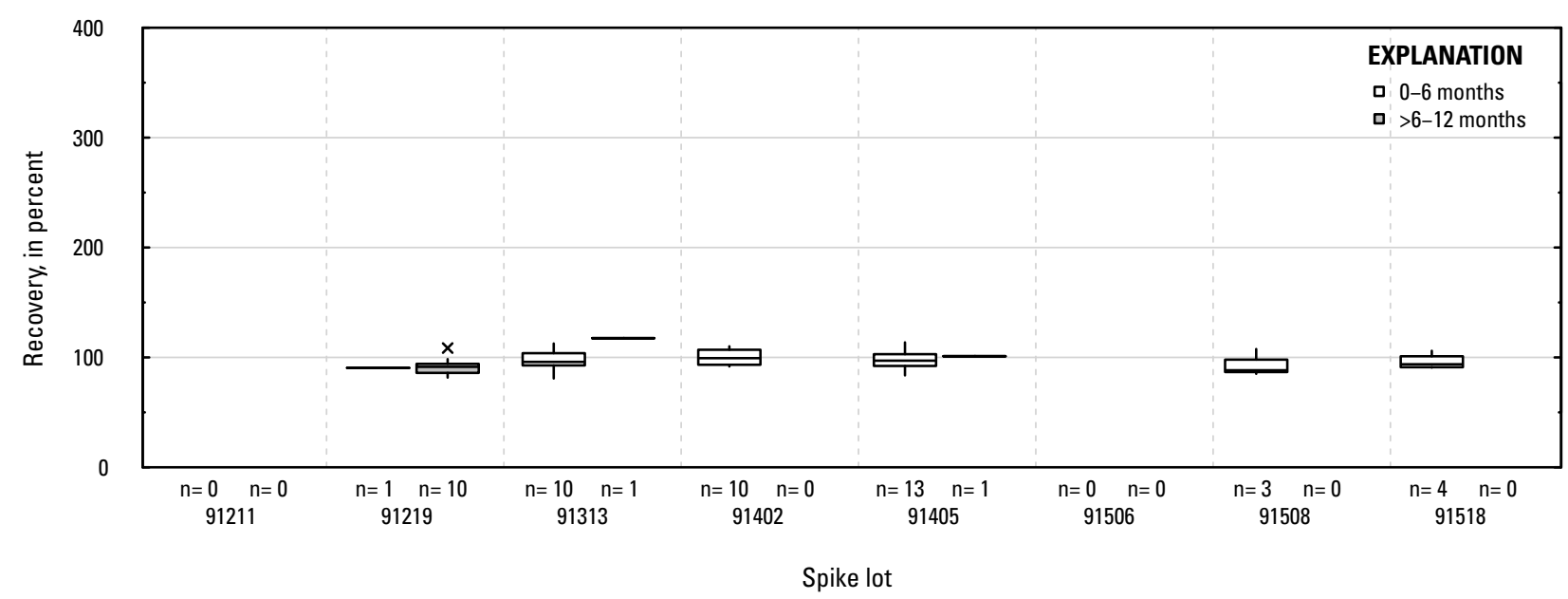

VM. Siduron: surface water field matrix spikes

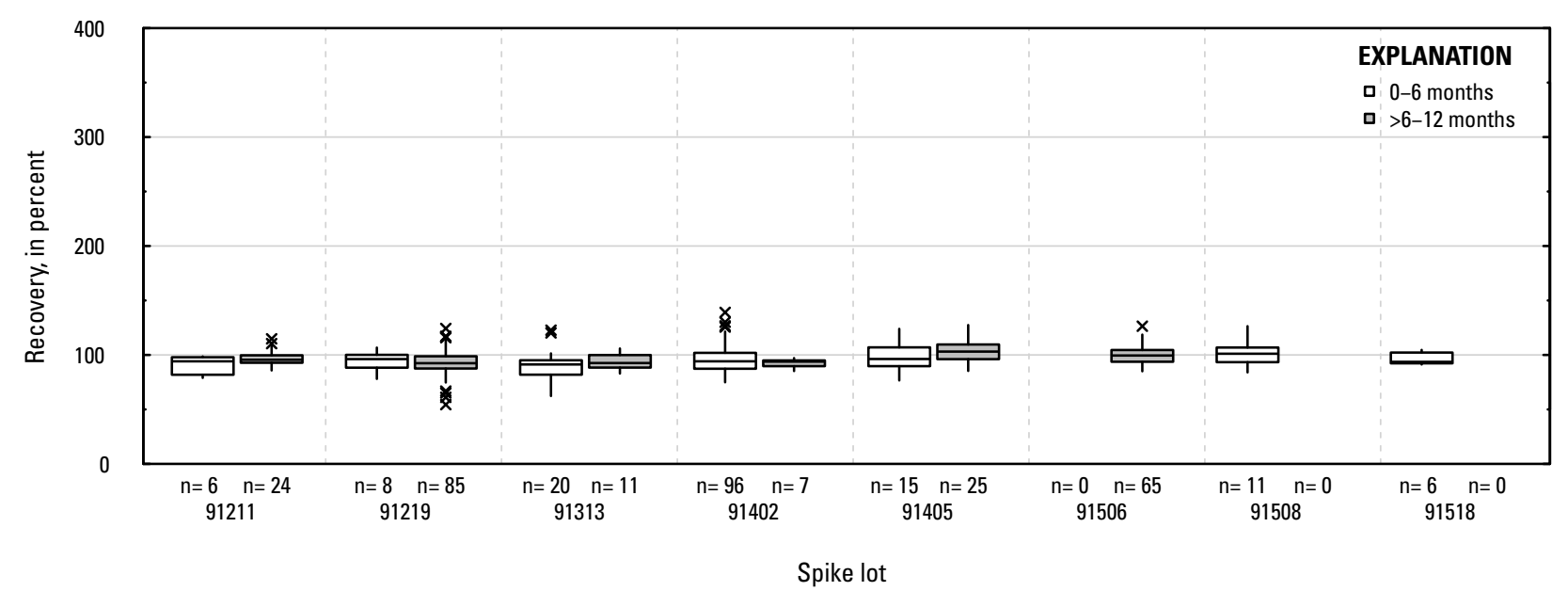

Figure 1-1. Distributions of recovery for individual pesticides in schedule 2437 by matrix, spike lot, and spike lot age. Recovery values larger than 400 percent are not shown.-Continued 
VN. Simazine: laboratory reagent spikes

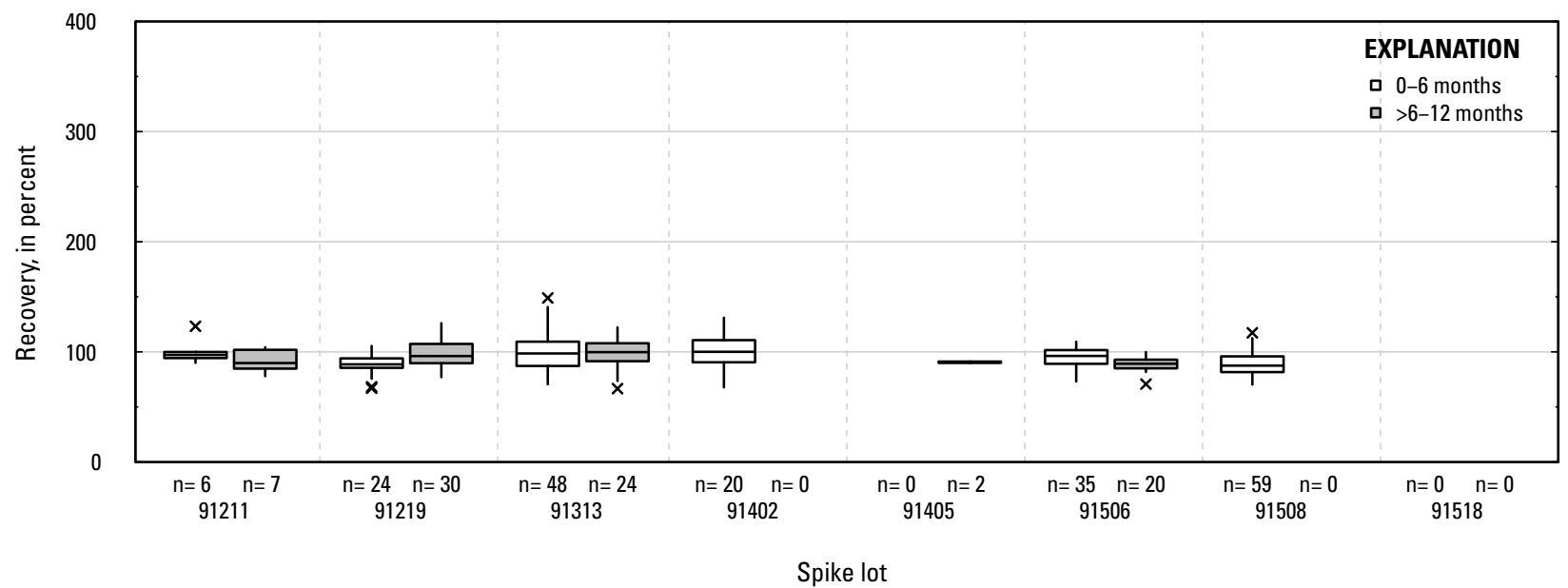

Vo. Simazine: groundwater field matrix spikes

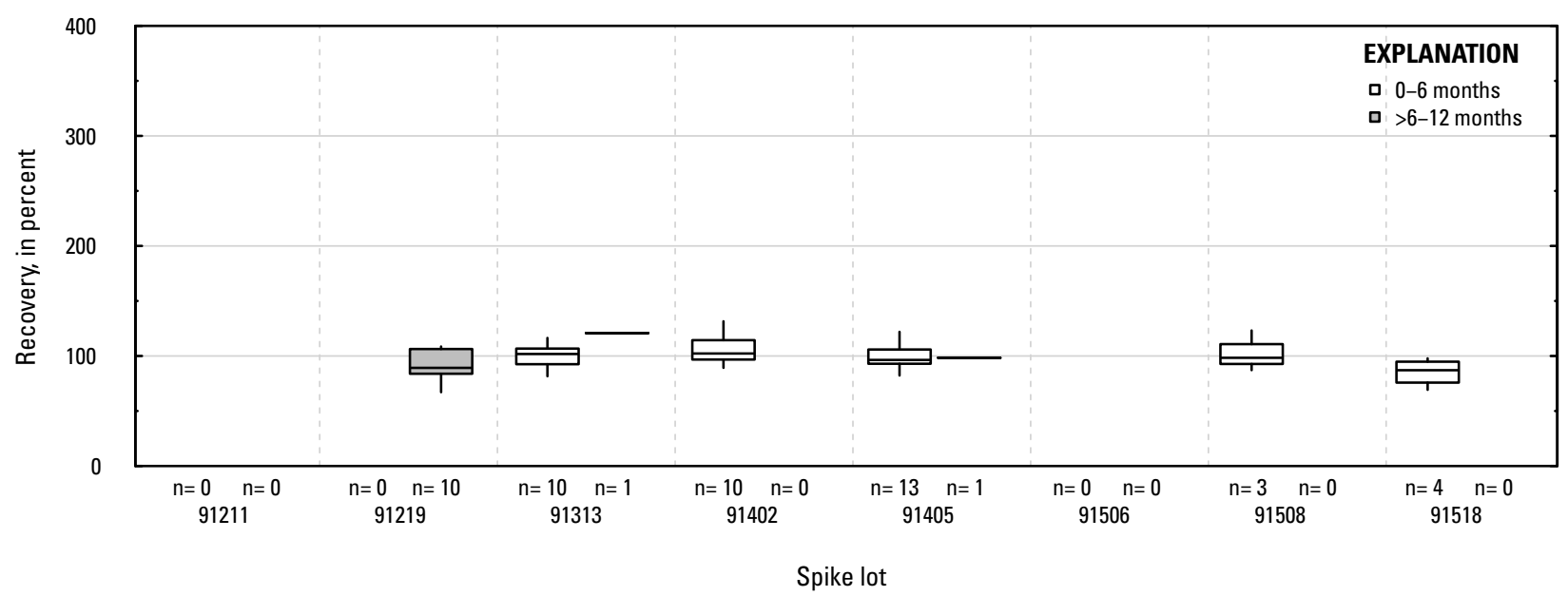

VP. Simazine: surface water field matrix spikes

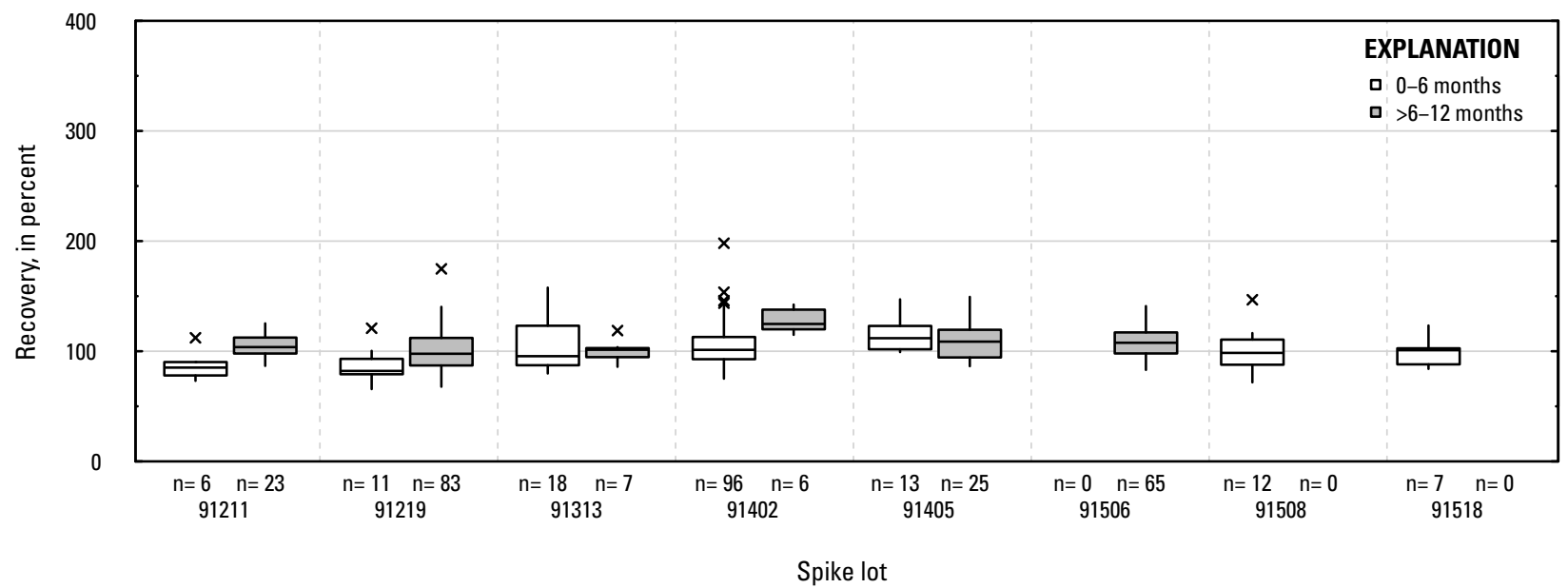

Figure 1-1. Distributions of recovery for individual pesticides in schedule 2437 by matrix, spike lot, and spike lot age. Recovery values larger than 400 percent are not shown.-Continued 
VQ. Sulfentrazone: laboratory reagent spikes

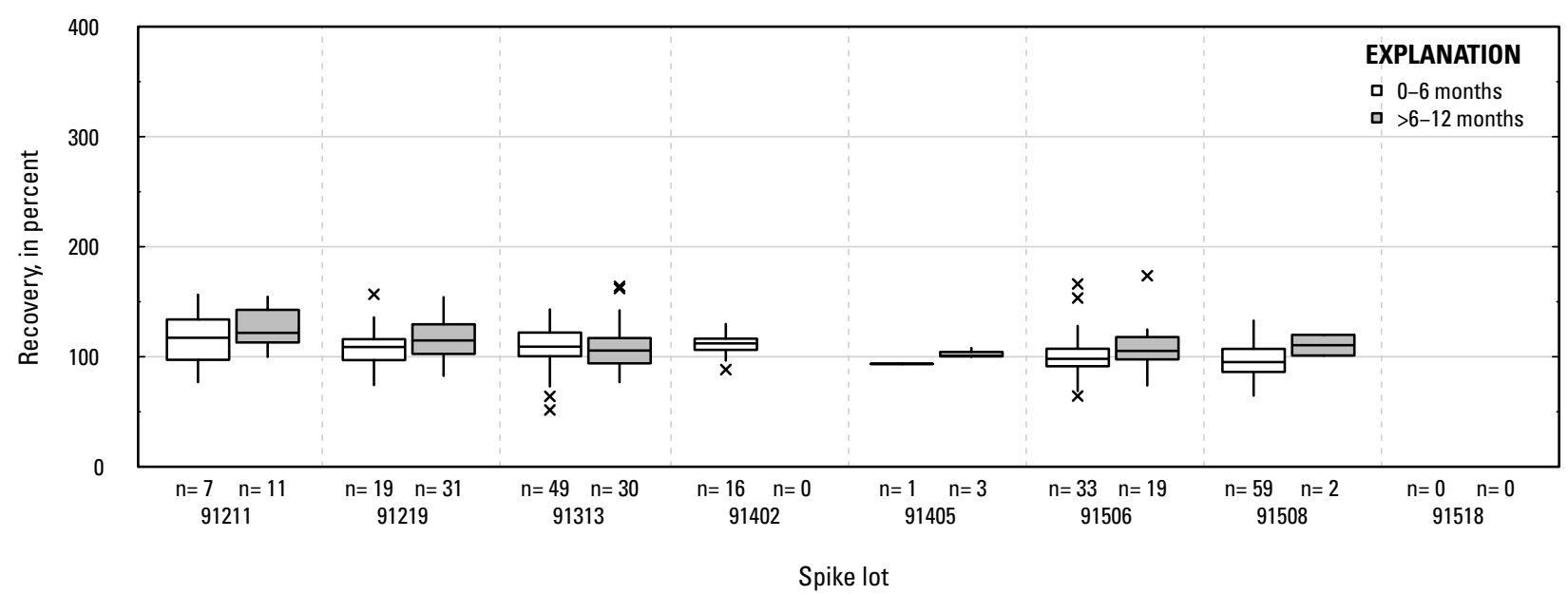

VR. Sulfentrazone: groundwater field matrix spikes

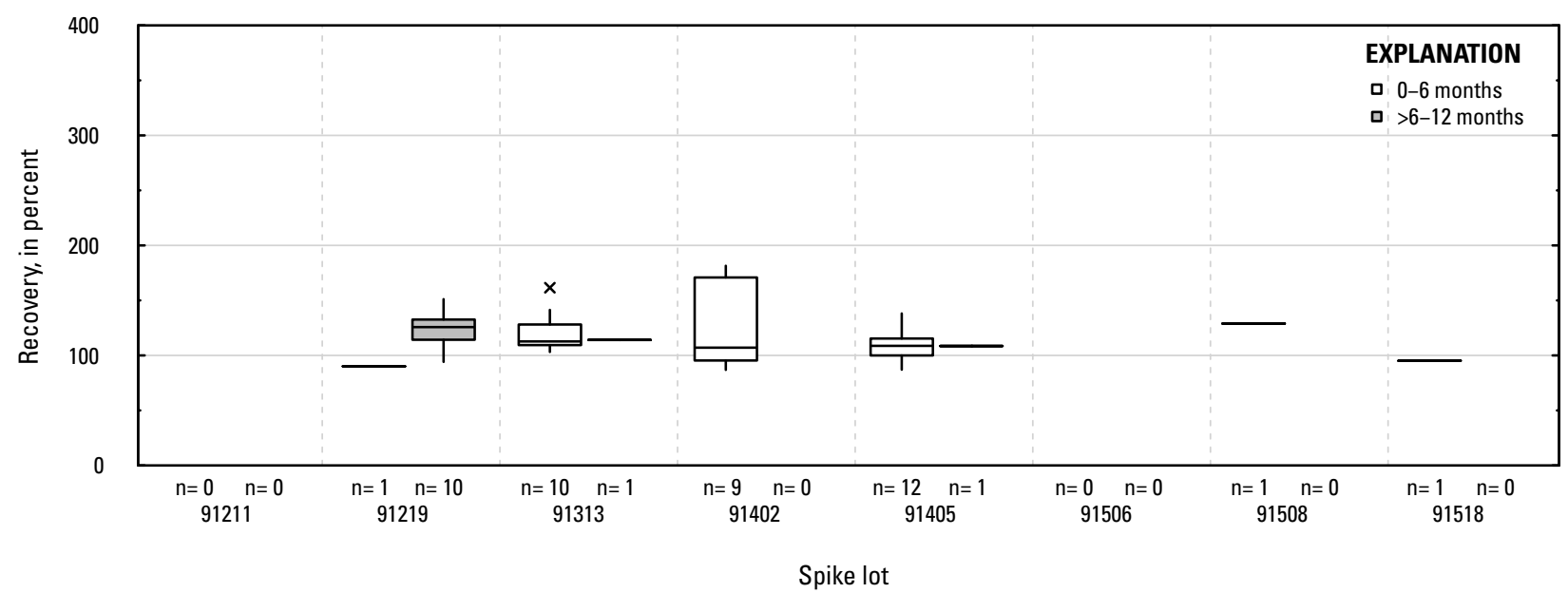

VS. Sulfentrazone: surface water field matrix spikes

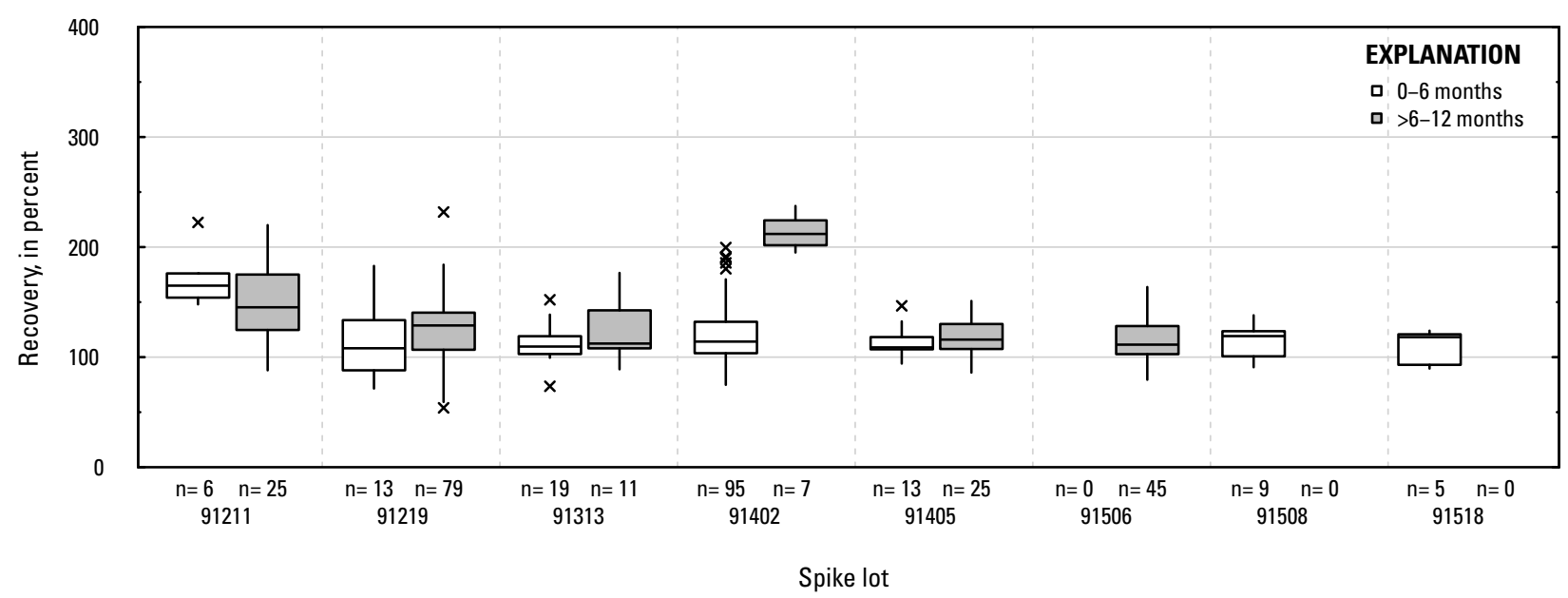

Figure 1-1. Distributions of recovery for individual pesticides in schedule 2437 by matrix, spike lot, and spike lot age. Recovery values larger than 400 percent are not shown.-Continued 
VT. Sulfometuron-methyl: laboratory reagent spikes

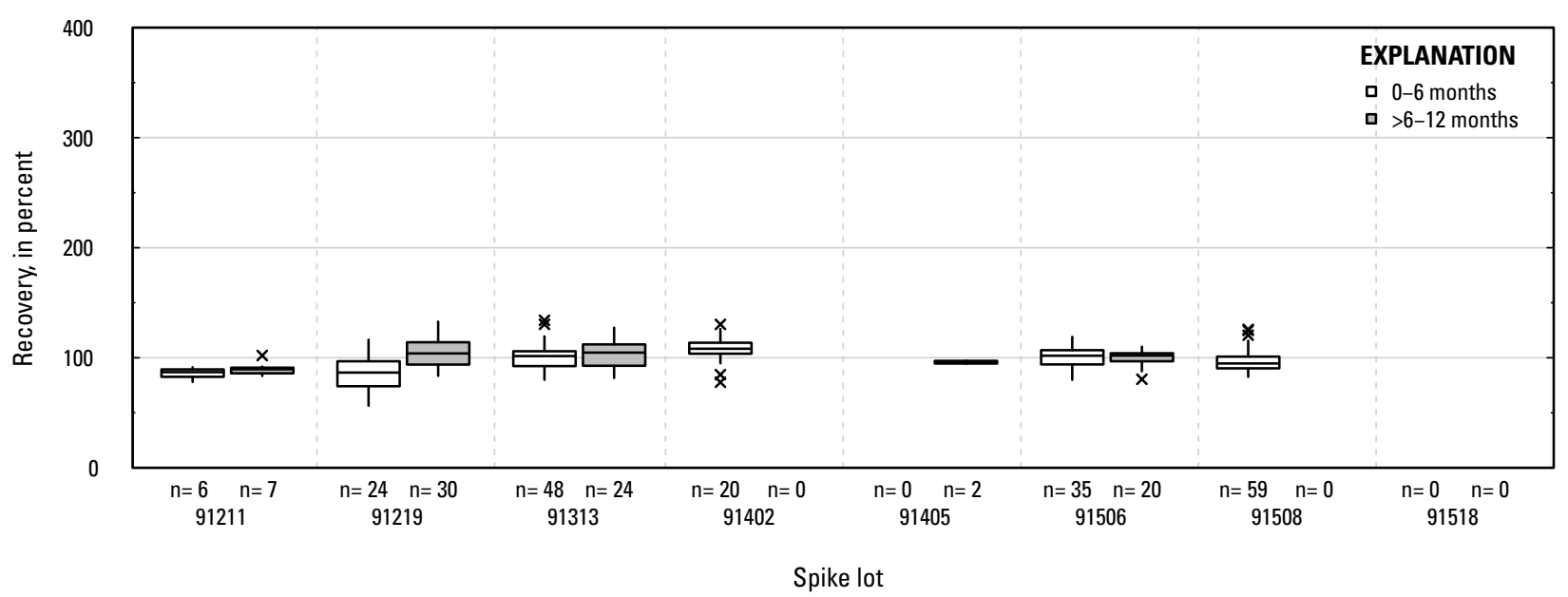

\section{VU. Sulfometuron-methyl: groundwater field matrix spikes}

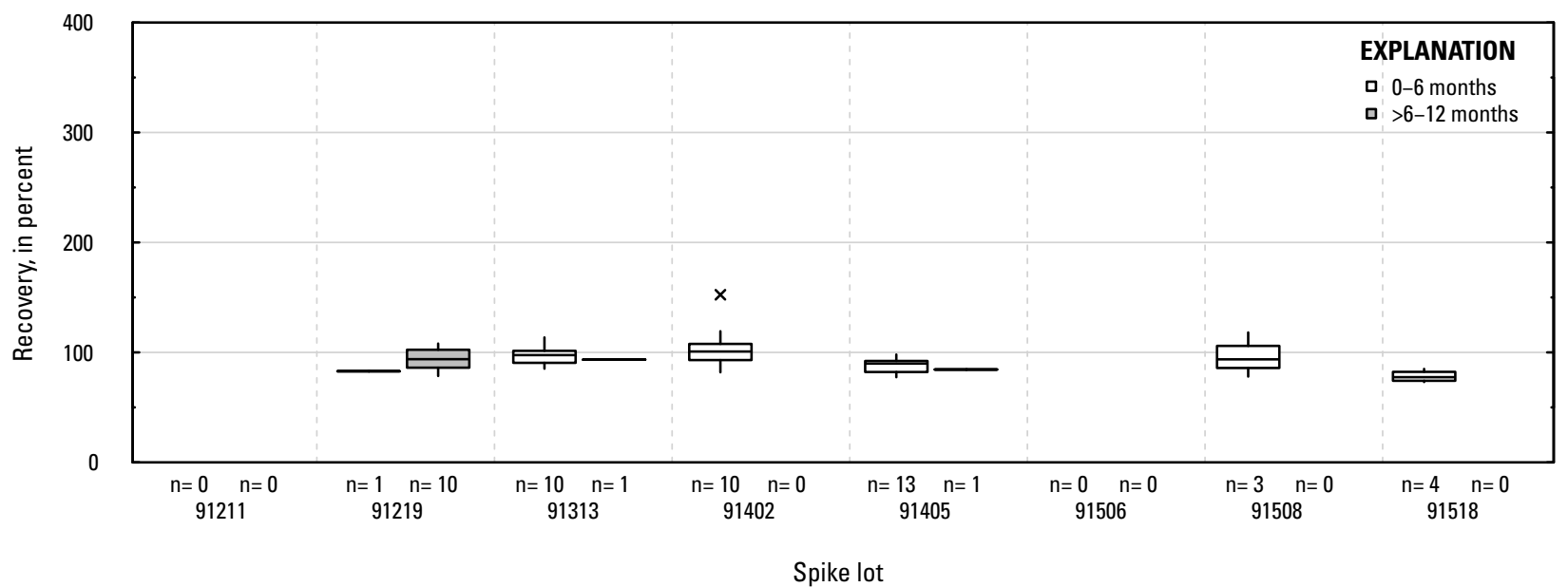

VV. Sulfometuron-methyl: surface water field matrix spikes

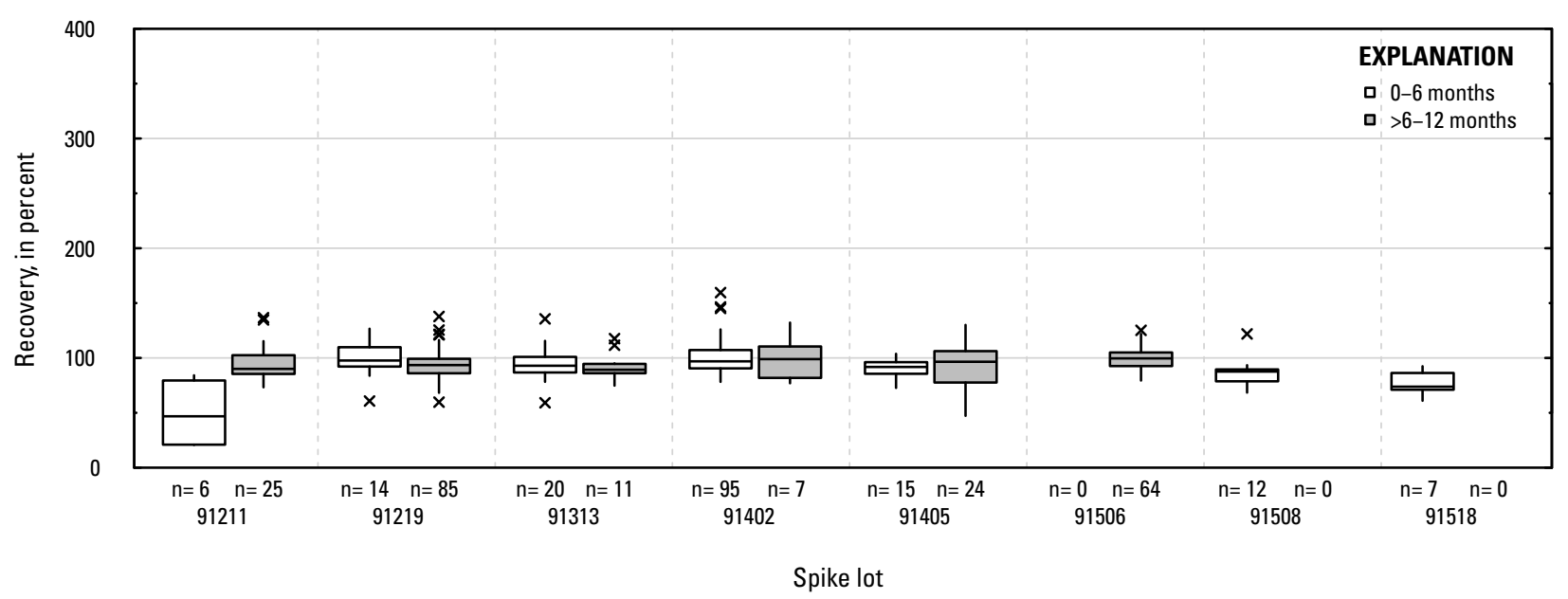

Figure 1-1. Distributions of recovery for individual pesticides in schedule 2437 by matrix, spike lot, and spike lot age. Recovery values larger than 400 percent are not shown.-Continued 
VW. Sulfosulfuron: laboratory reagent spikes

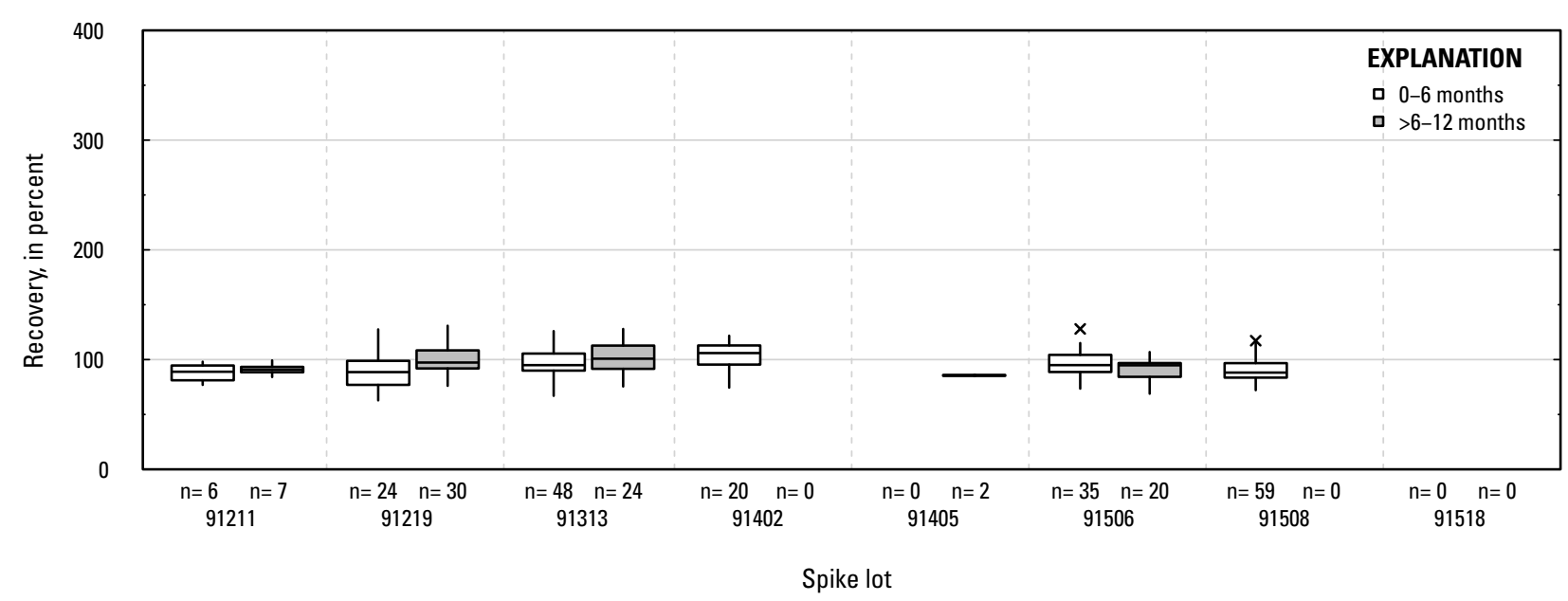

VX. Sulfosulfuron: groundwater field matrix spikes

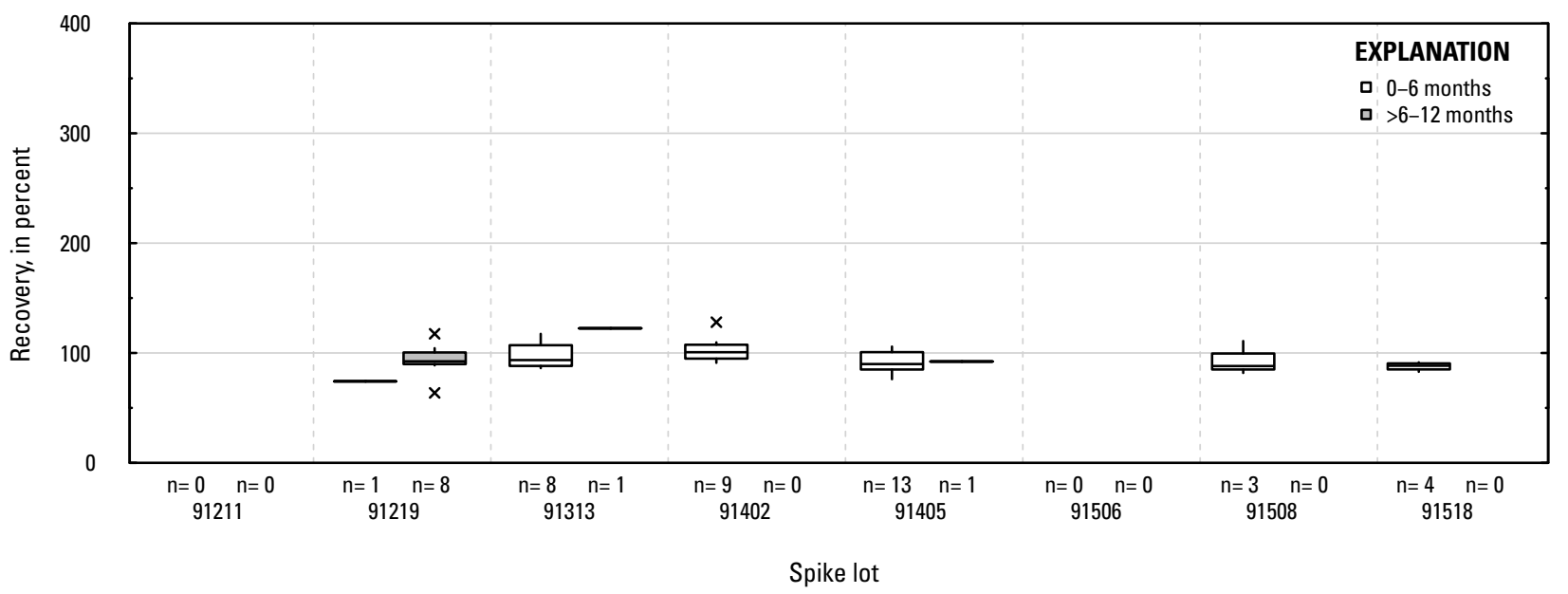

VY. Sulfosulfuron: surface water field matrix spikes

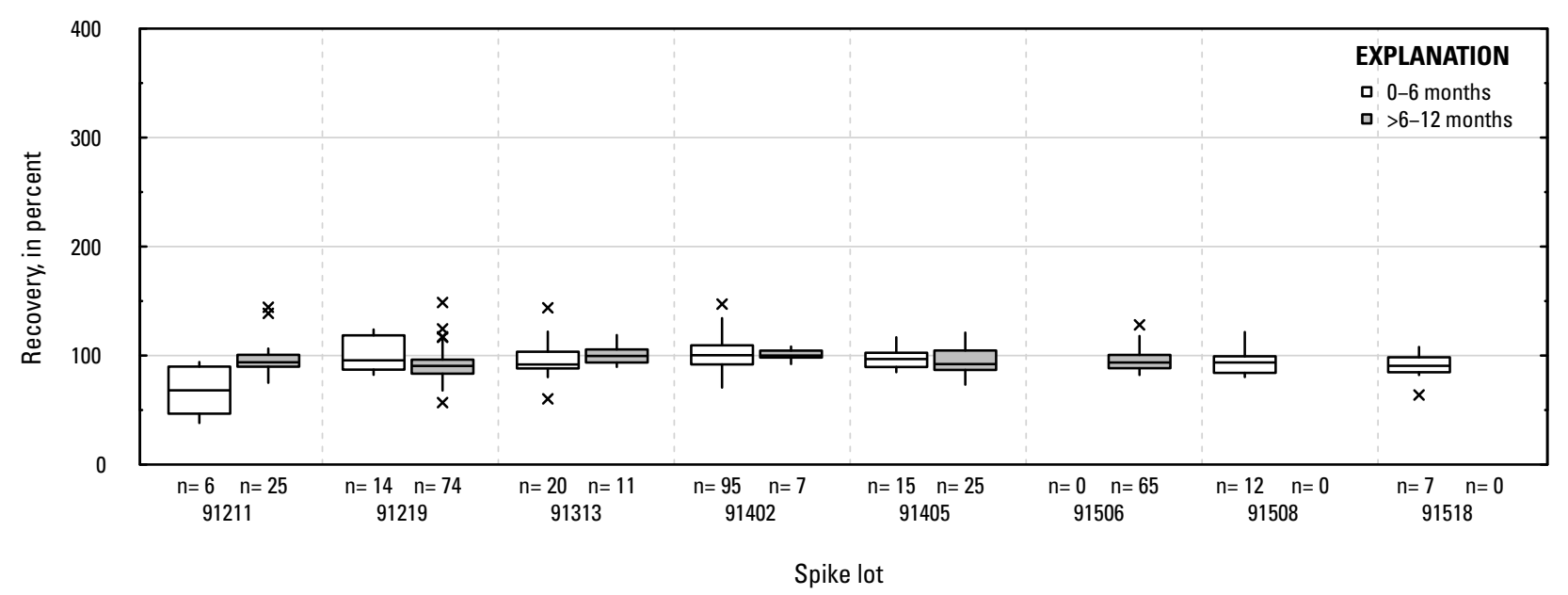

Figure 1-1. Distributions of recovery for individual pesticides in schedule 2437 by matrix, spike lot, and spike lot age. Recovery values larger than 400 percent are not shown.-Continued 
VZ. Sulfosulfuron ethyl sulfone: laboratory reagent spikes

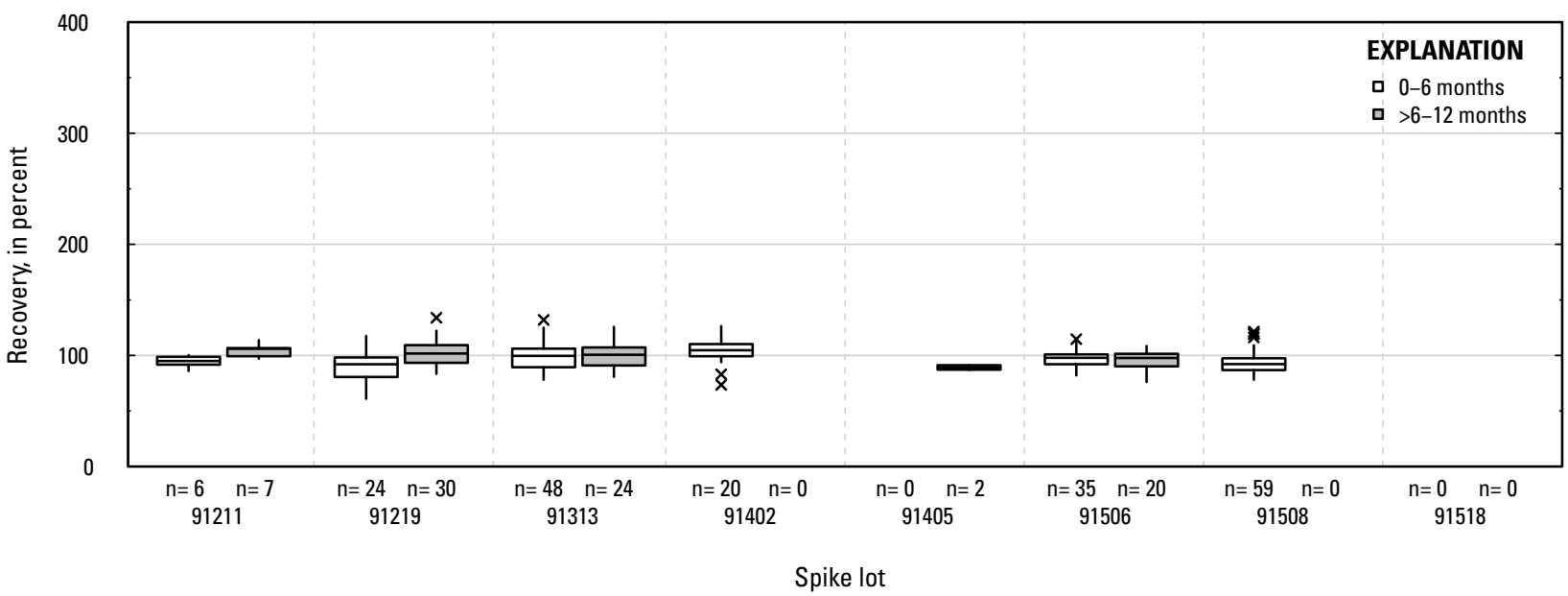

WA. Sulfosulfuron ethyl sulfone: groundwater field matrix spikes

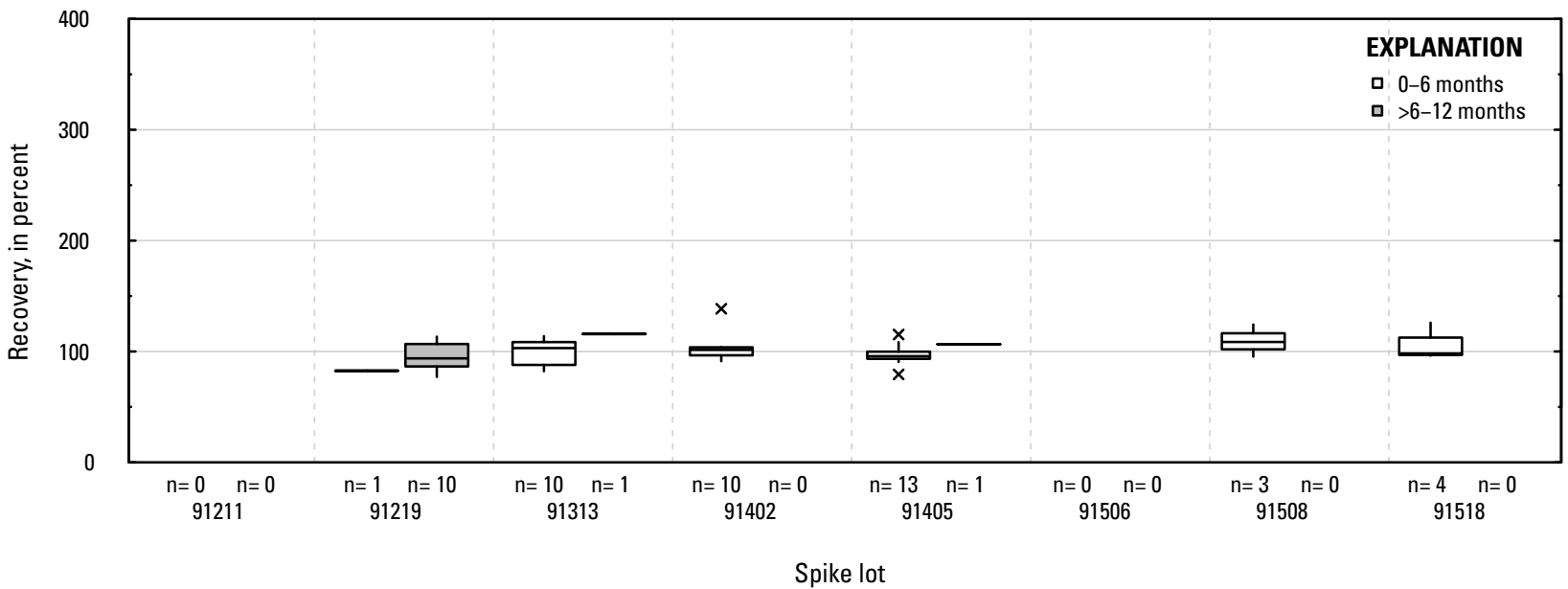

WB. Sulfosulfuron ethyl sulfone: surface water field matrix spikes

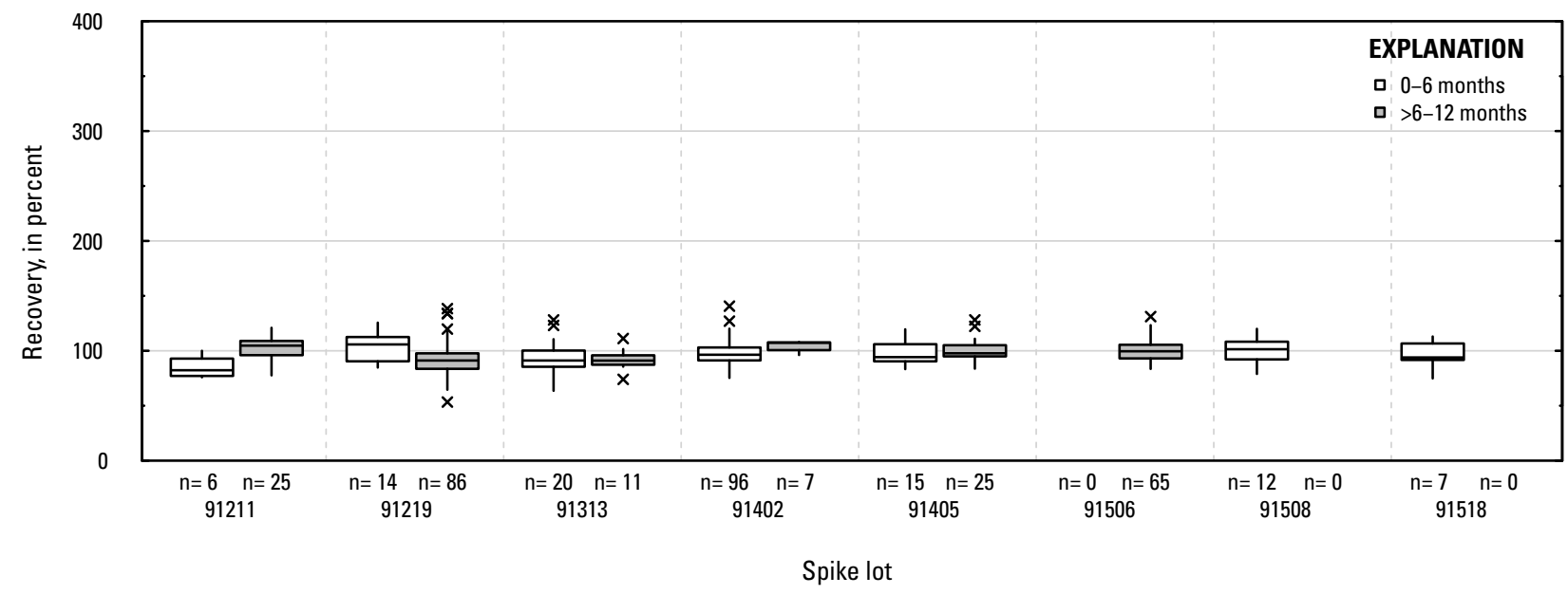

Figure 1-1. Distributions of recovery for individual pesticides in schedule 2437 by matrix, spike lot, and spike lot age. Recovery values larger than 400 percent are not shown.-Continued 
WC. Tebuconazole: laboratory reagent spikes

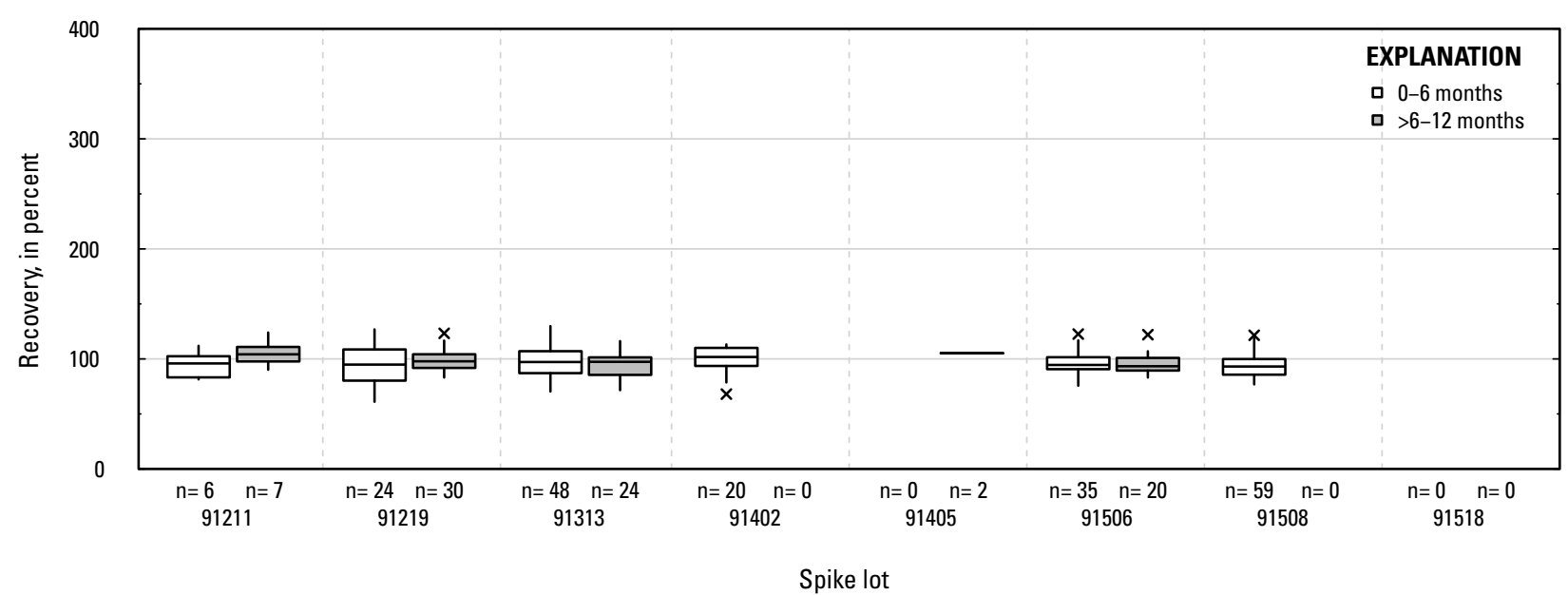

WD. Tebuconazole: groundwater field matrix spikes

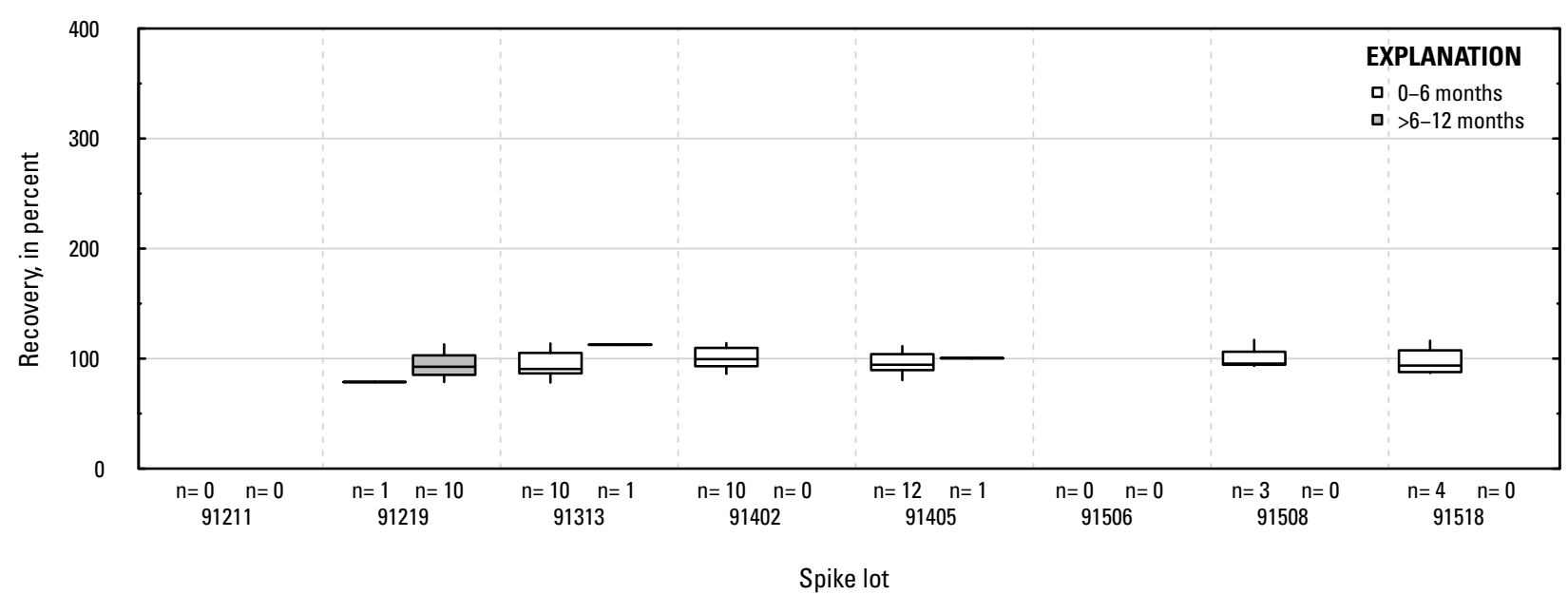

WE. Tebuconazole: surface water field matrix spikes

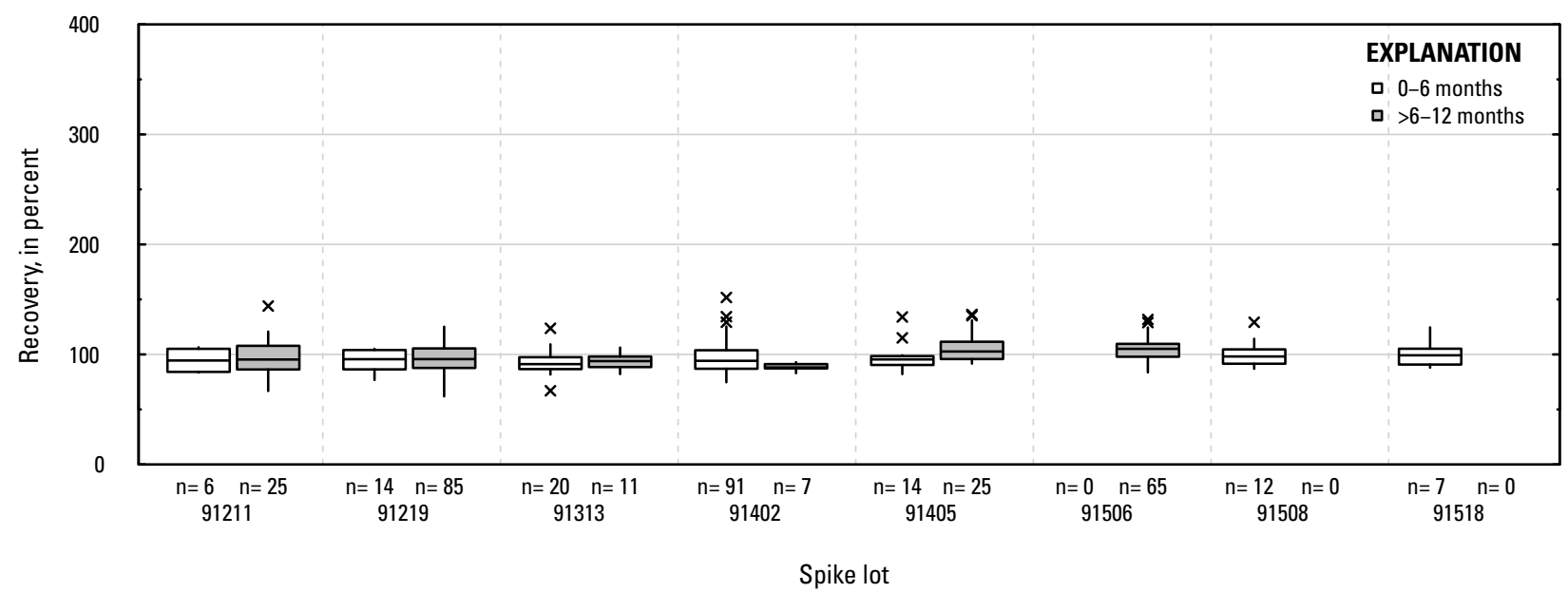

Figure 1-1. Distributions of recovery for individual pesticides in schedule 2437 by matrix, spike lot, and spike lot age. Recovery values larger than 400 percent are not shown.-Continued 
WF. Tebufenozide: laboratory reagent spikes

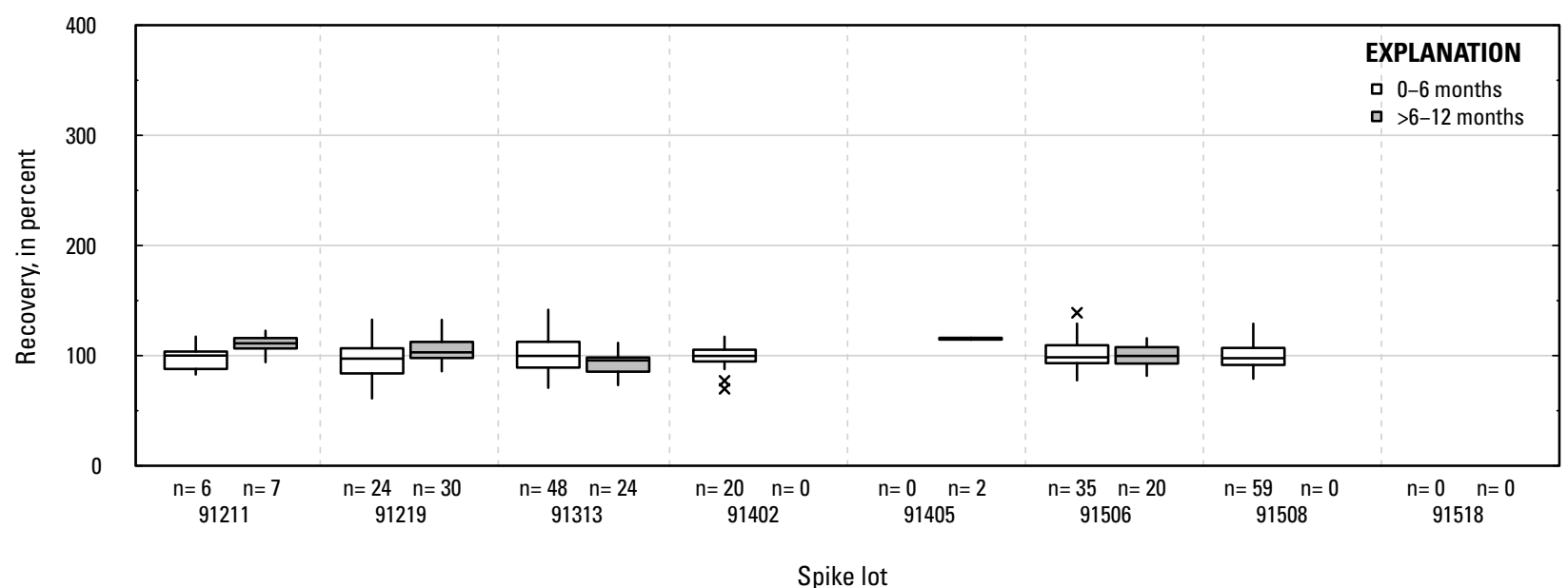

WG. Tebufenozide: groundwater field matrix spikes

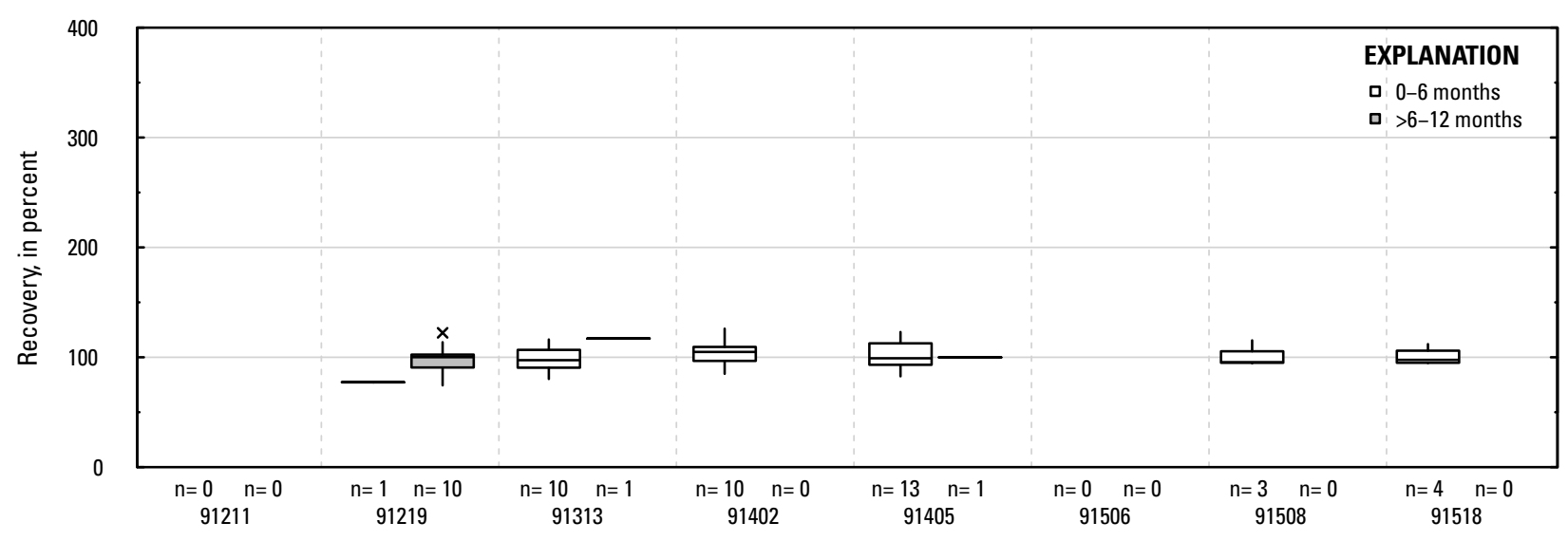

Spike lot

WH. Tebufenozide: surface water field matrix spikes

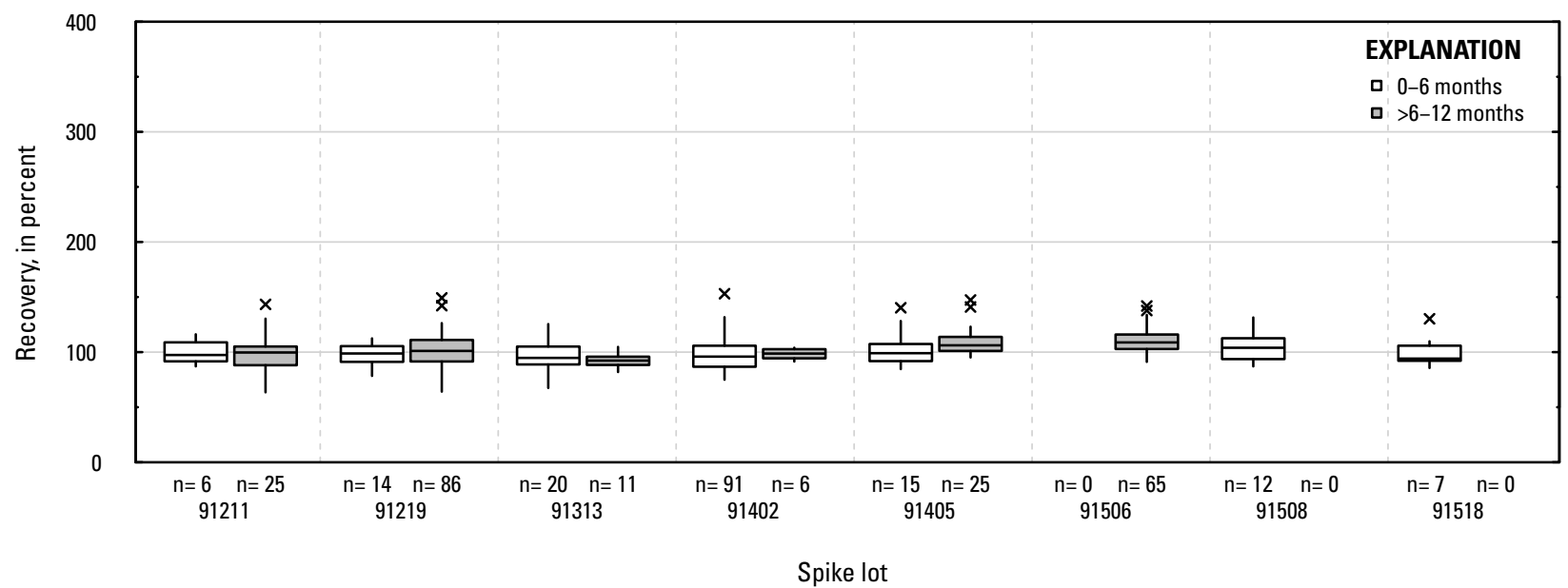

Figure 1-1. Distributions of recovery for individual pesticides in schedule 2437 by matrix, spike lot, and spike lot age. Recovery values larger than 400 percent are not shown.-Continued 
WI. Tebupirimphos: laboratory reagent spikes

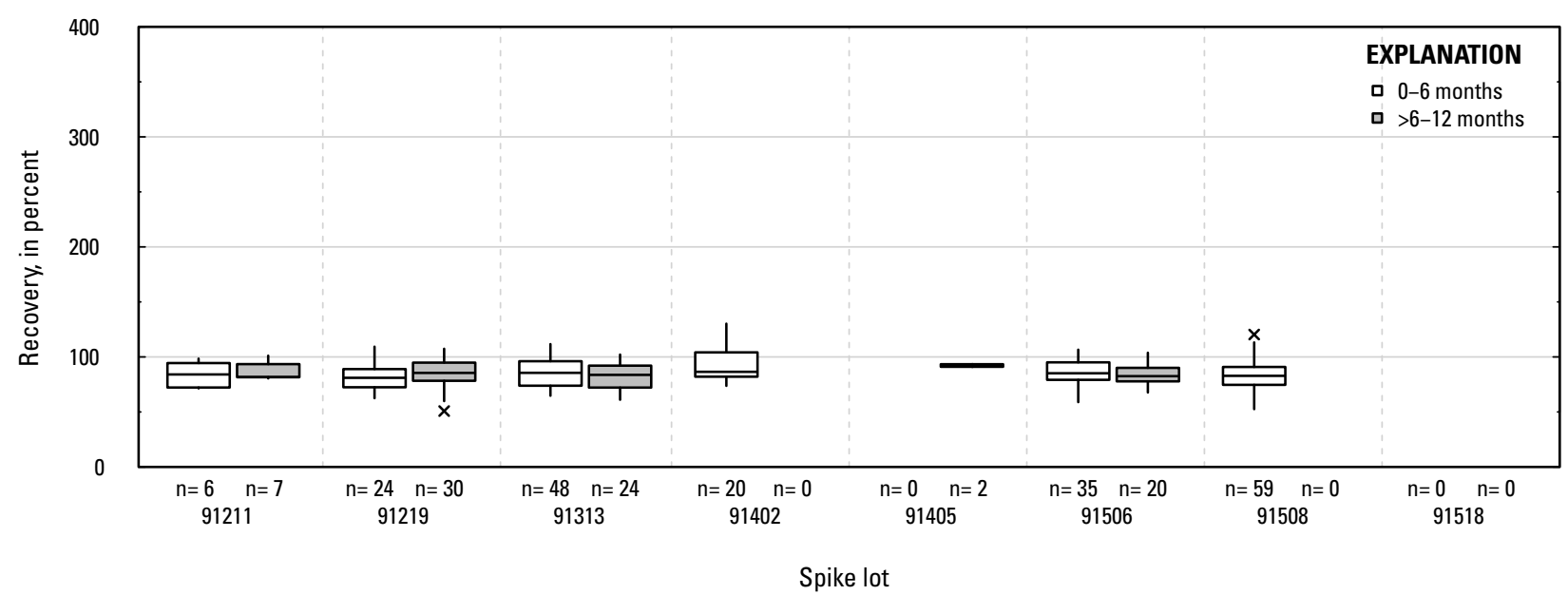

WJ. Tebupirimphos: groundwater field matrix spikes

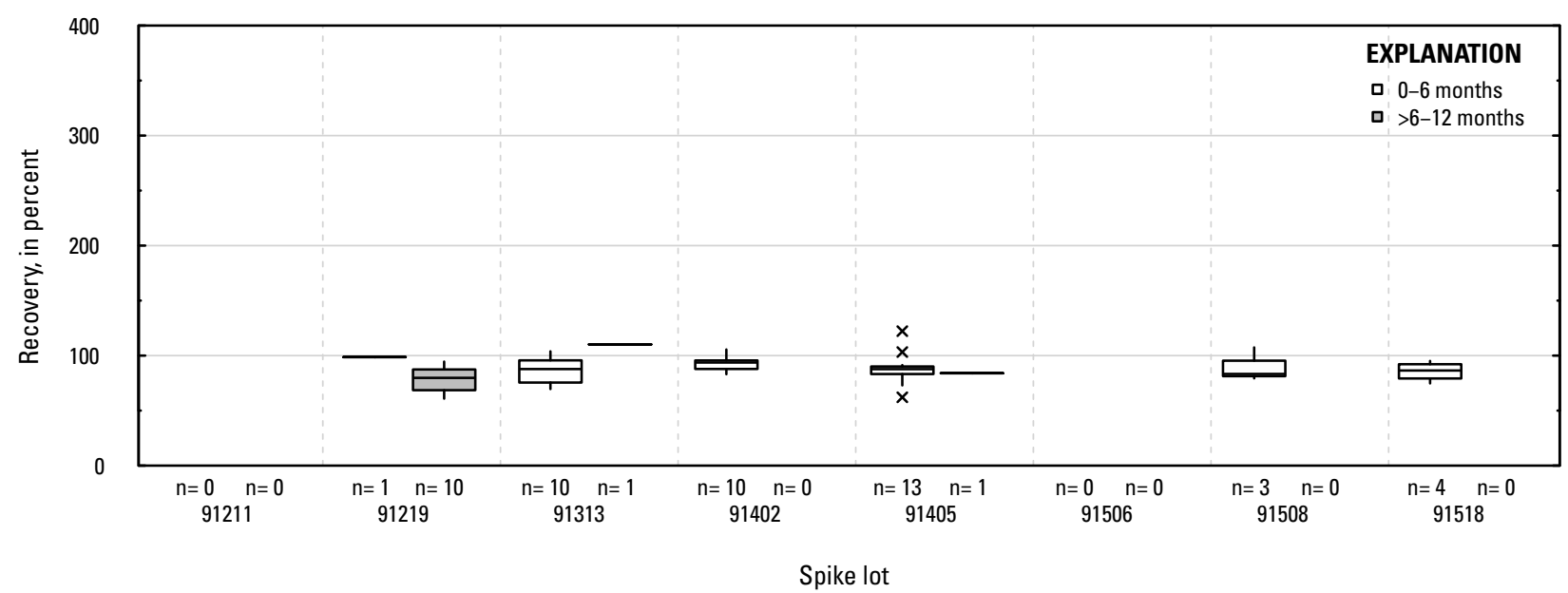

WK. Tebupirimphos: surface water field matrix spikes

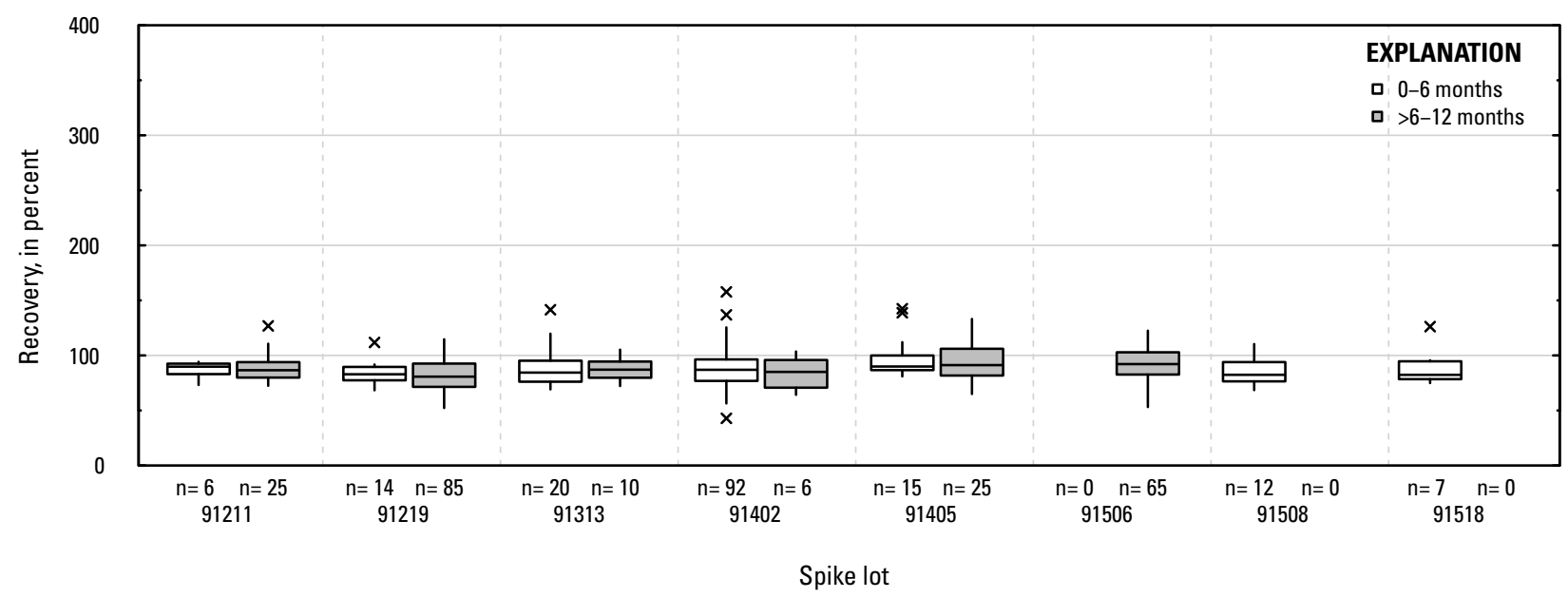

Figure 1-1. Distributions of recovery for individual pesticides in schedule 2437 by matrix, spike lot, and spike lot age. Recovery values larger than 400 percent are not shown.-Continued 
WL. Tebupirimfos oxon: laboratory reagent spikes

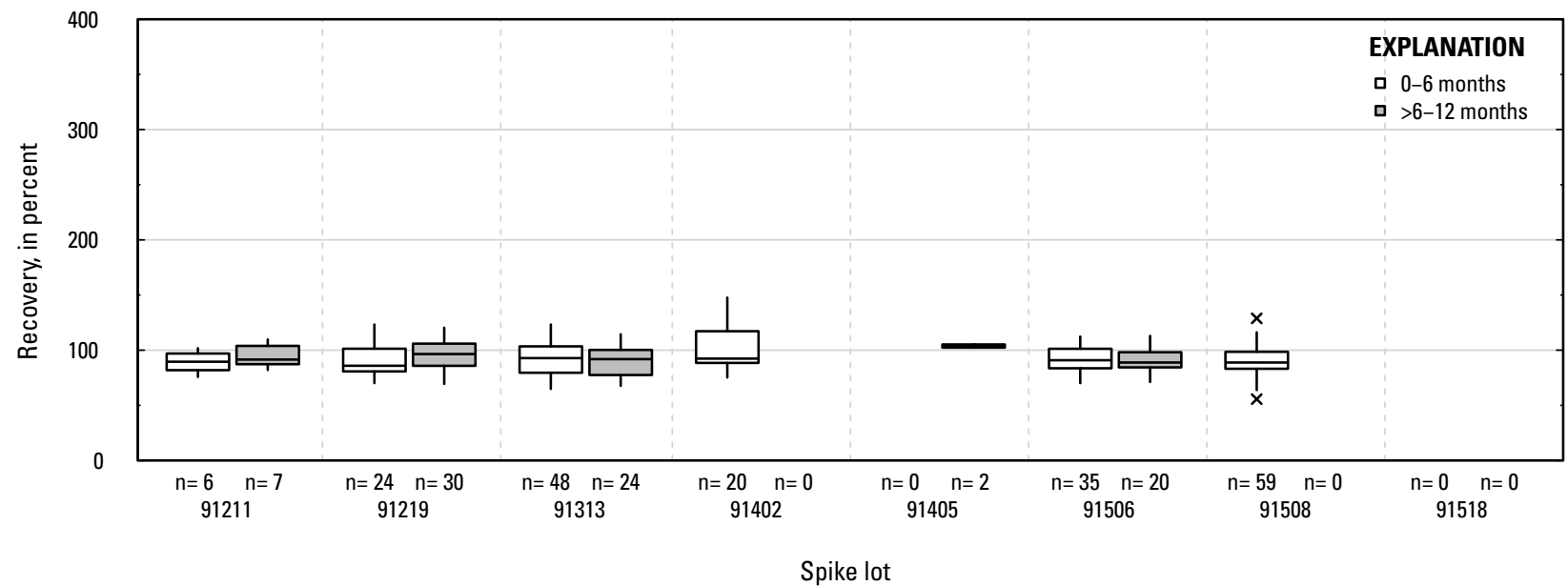

WM. Tebupirimfos oxon: groundwater field matrix spikes

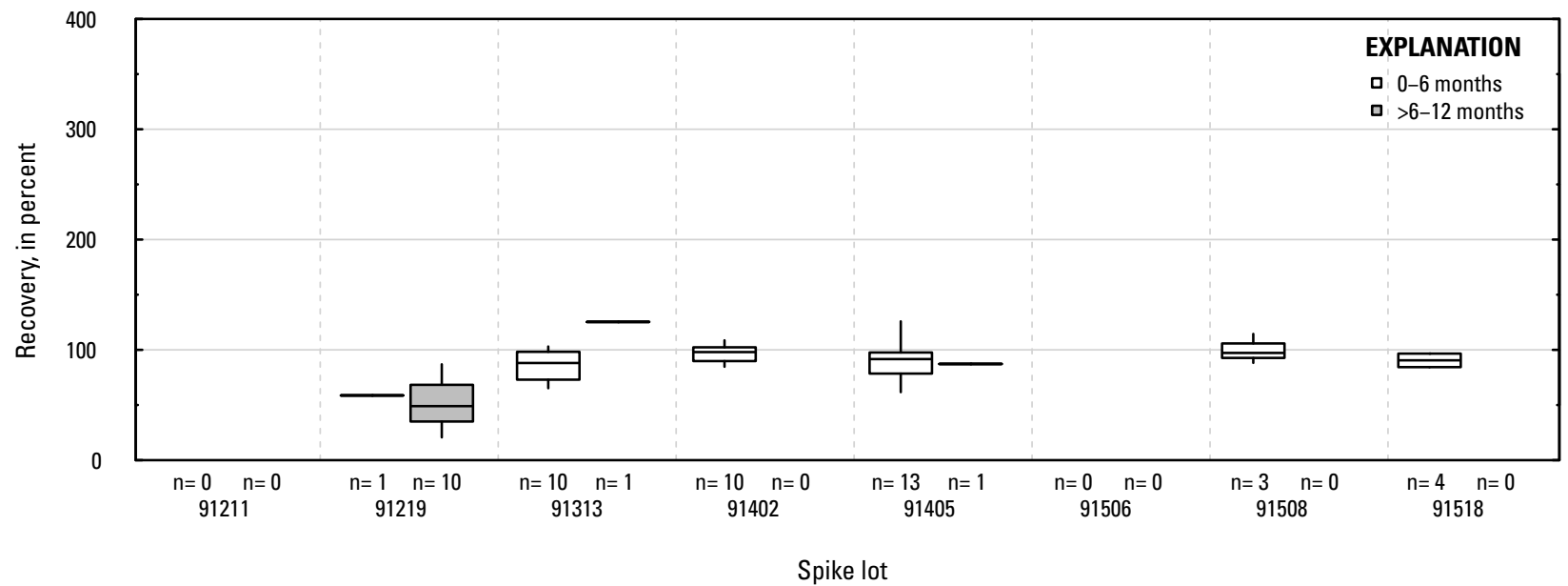

WN. Tebupirimfos oxon: surface water field matrix spikes

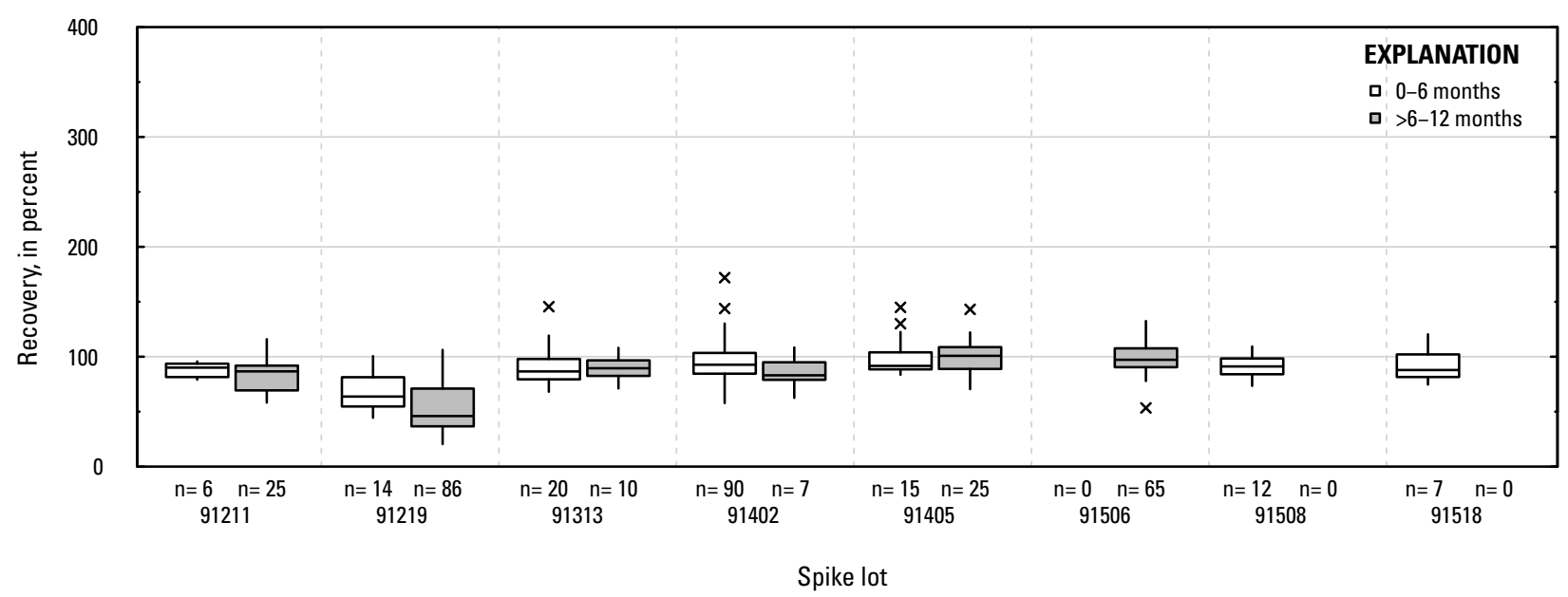

Figure 1-1. Distributions of recovery for individual pesticides in schedule 2437 by matrix, spike lot, and spike lot age. Recovery values larger than 400 percent are not shown.-Continued 
WO. Tebuthiuron: laboratory reagent spikes

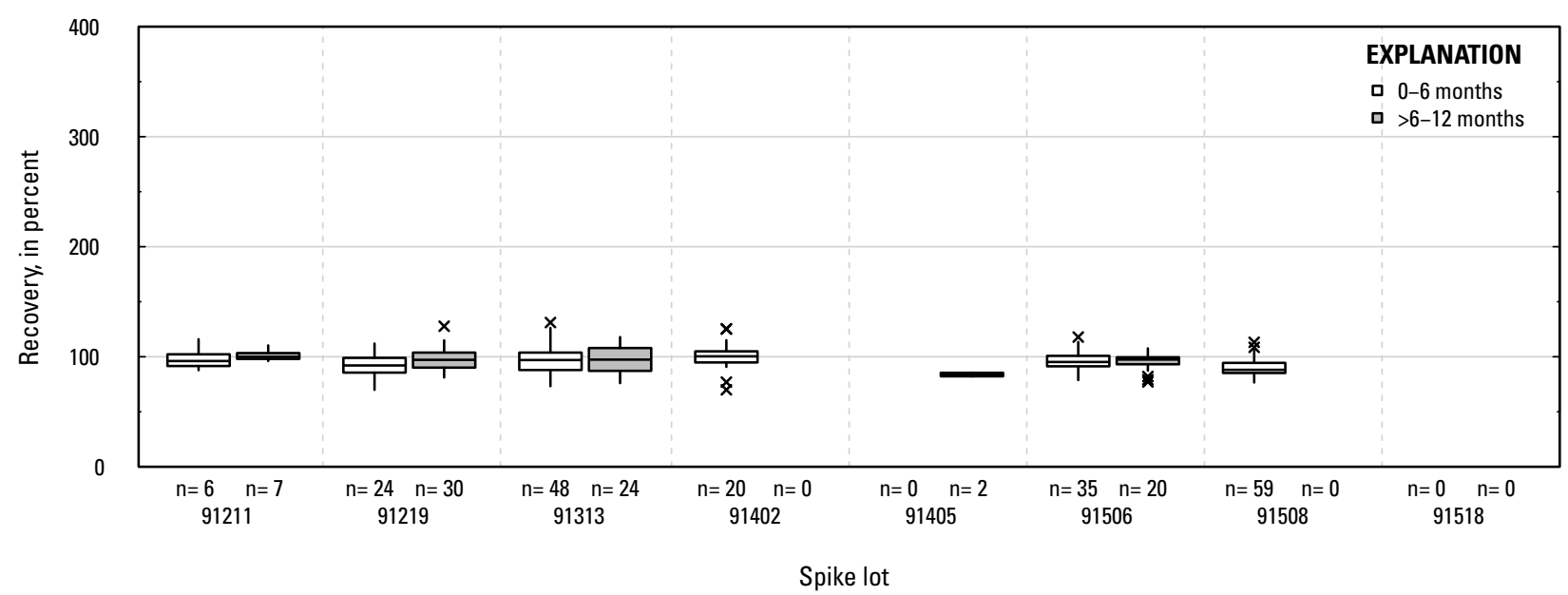

WP. Tebuthiuron: groundwater field matrix spikes

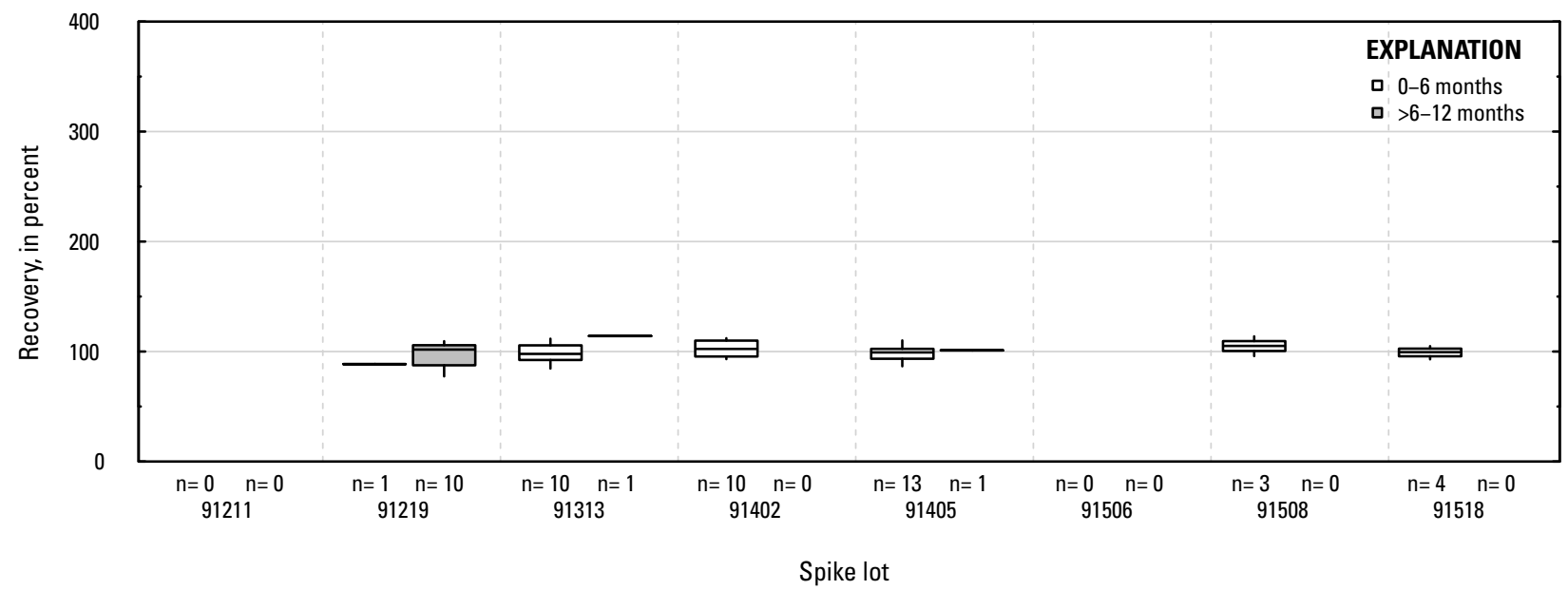

WQ. Tebuthiuron: surface water field matrix spikes

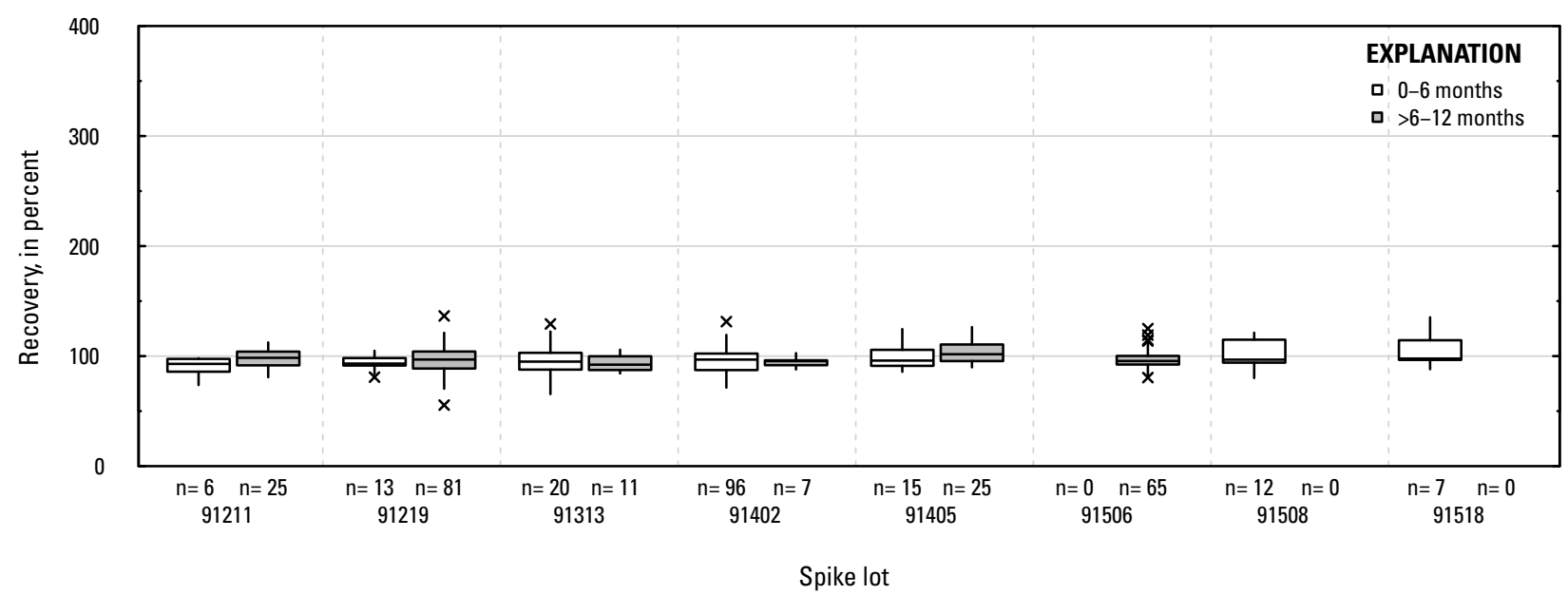

Figure 1-1. Distributions of recovery for individual pesticides in schedule 2437 by matrix, spike lot, and spike lot age. Recovery values larger than 400 percent are not shown.-Continued 
WR. Tebuthiuron TP 104: laboratory reagent spikes

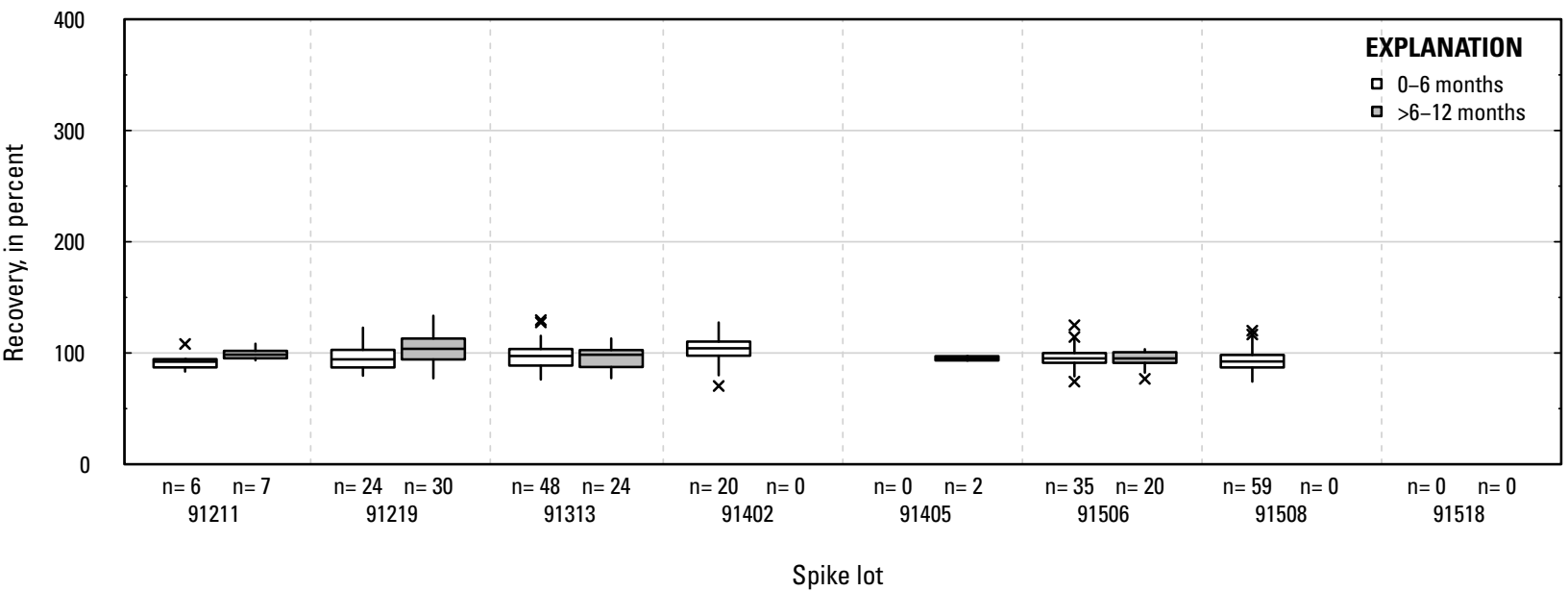

WS. Tebuthiuron TP 104: groundwater field matrix spikes

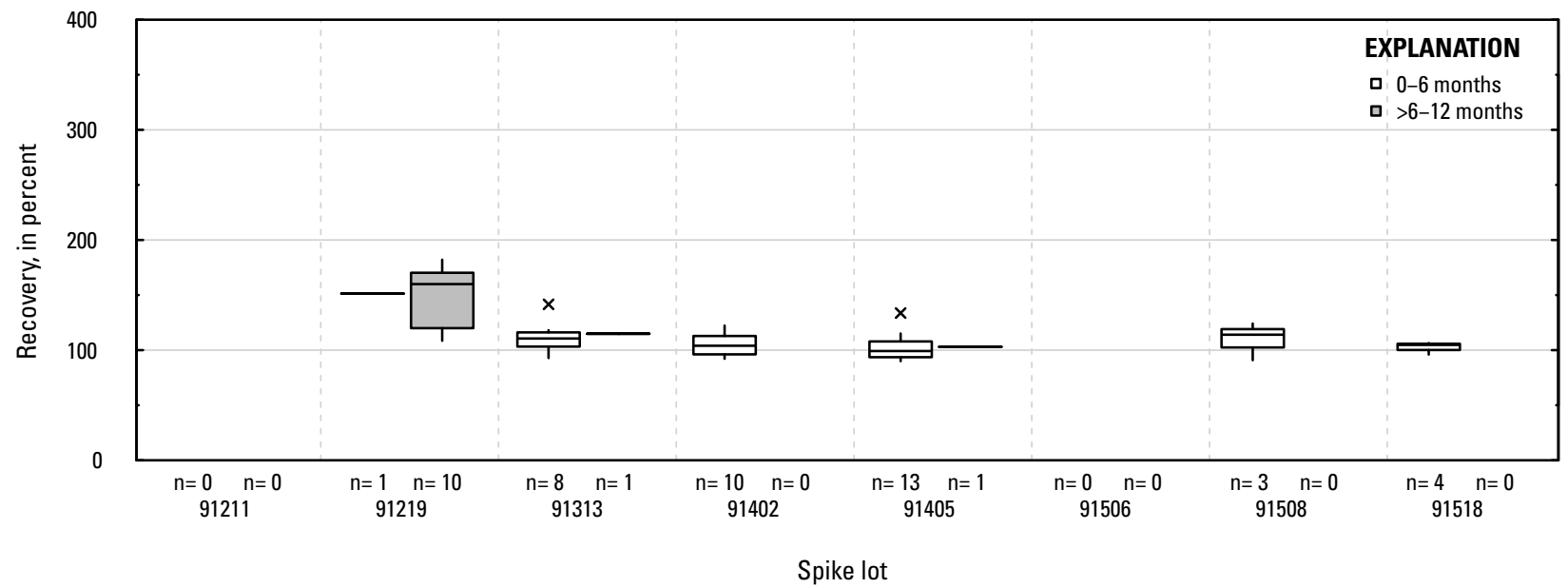

WT. Tebuthiuron TP 104: surface water field matrix spikes

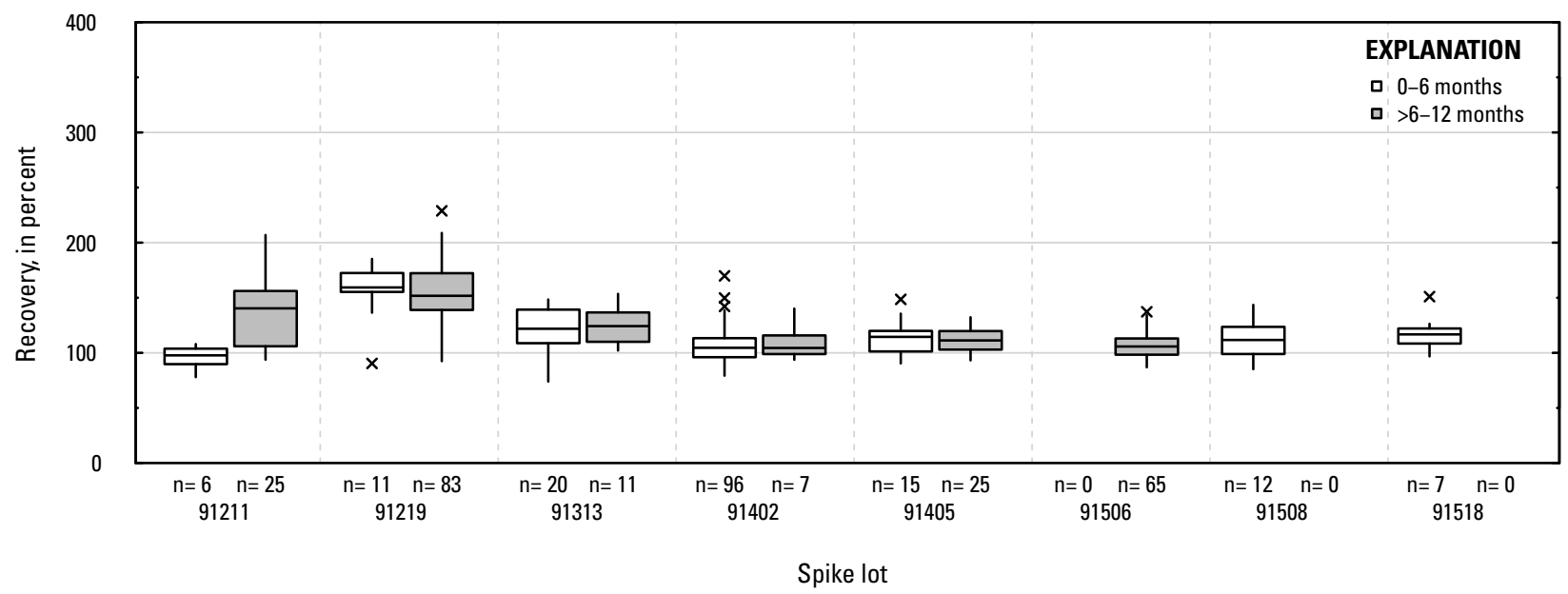

Figure 1-1. Distributions of recovery for individual pesticides in schedule 2437 by matrix, spike lot, and spike lot age. Recovery values larger than 400 percent are not shown.-Continued 
WU. Tebuthiuron Transformation Product 106: laboratory reagent spikes

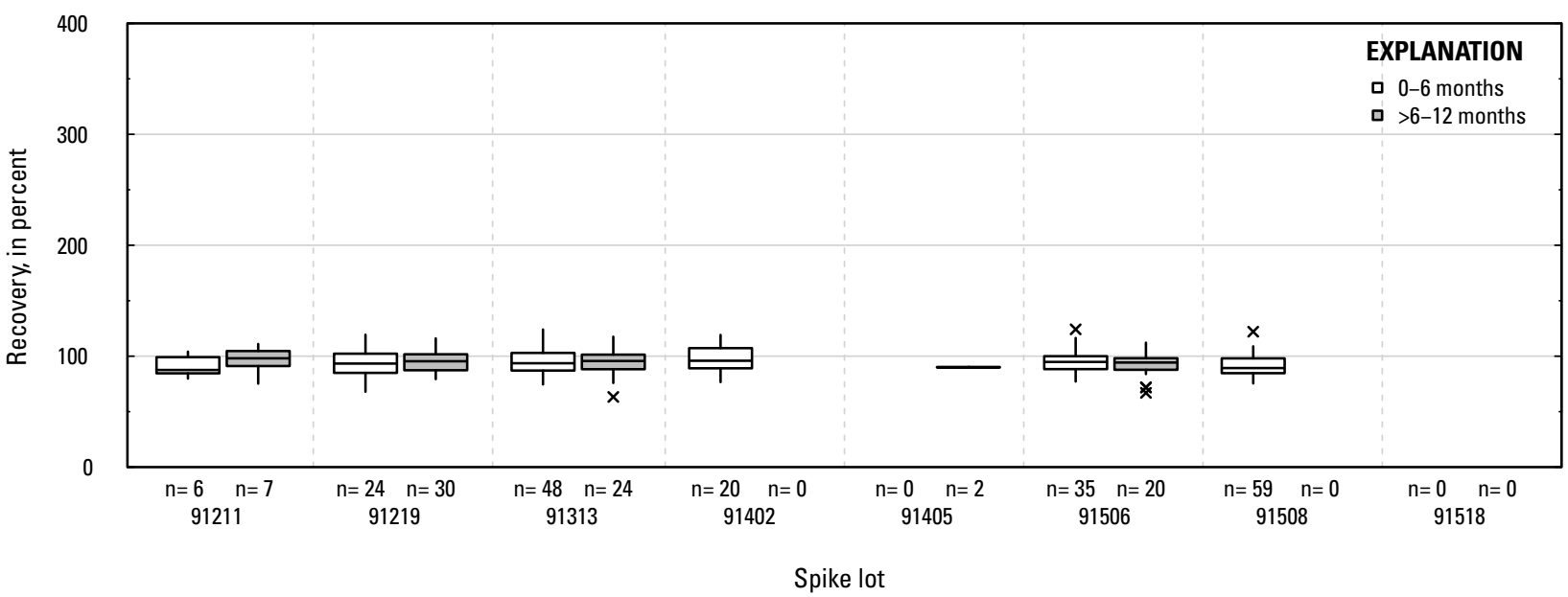

WV. Tebuthiuron Transformation Product 106: groundwater field matrix spikes

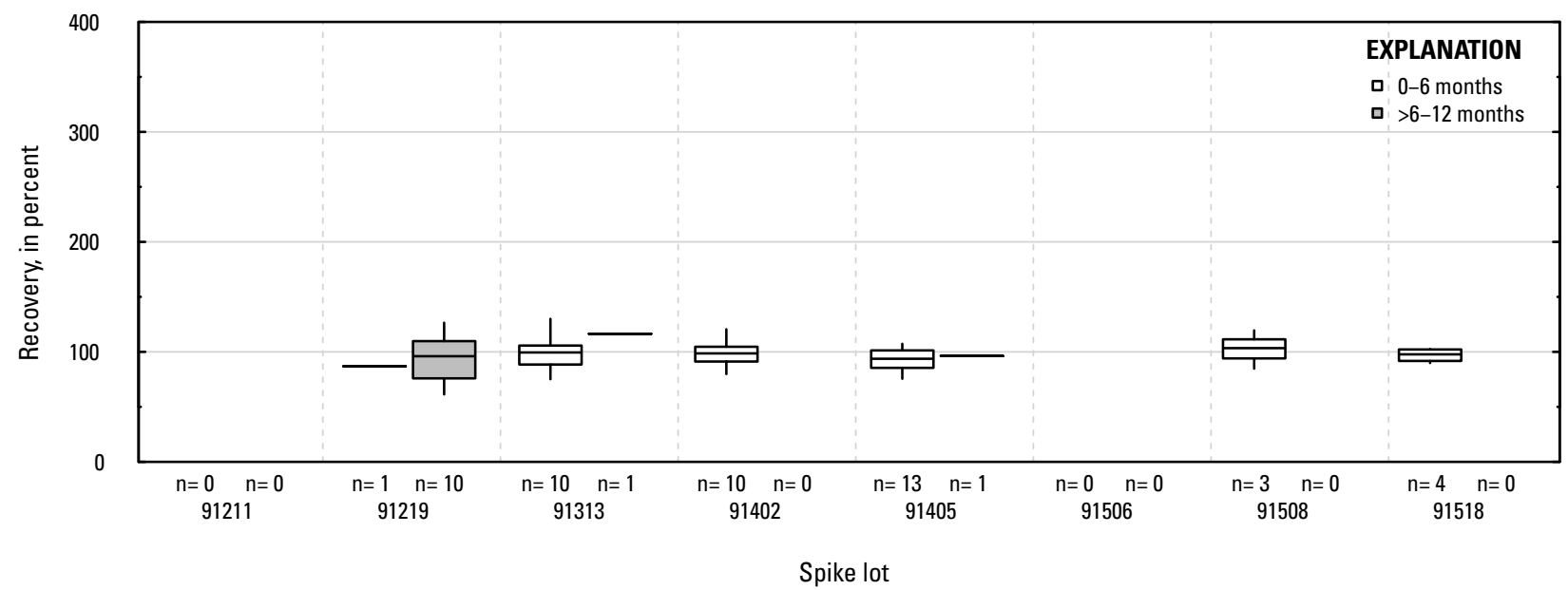

WW. Tebuthiuron Transformation Product 106: surface water field matrix spikes

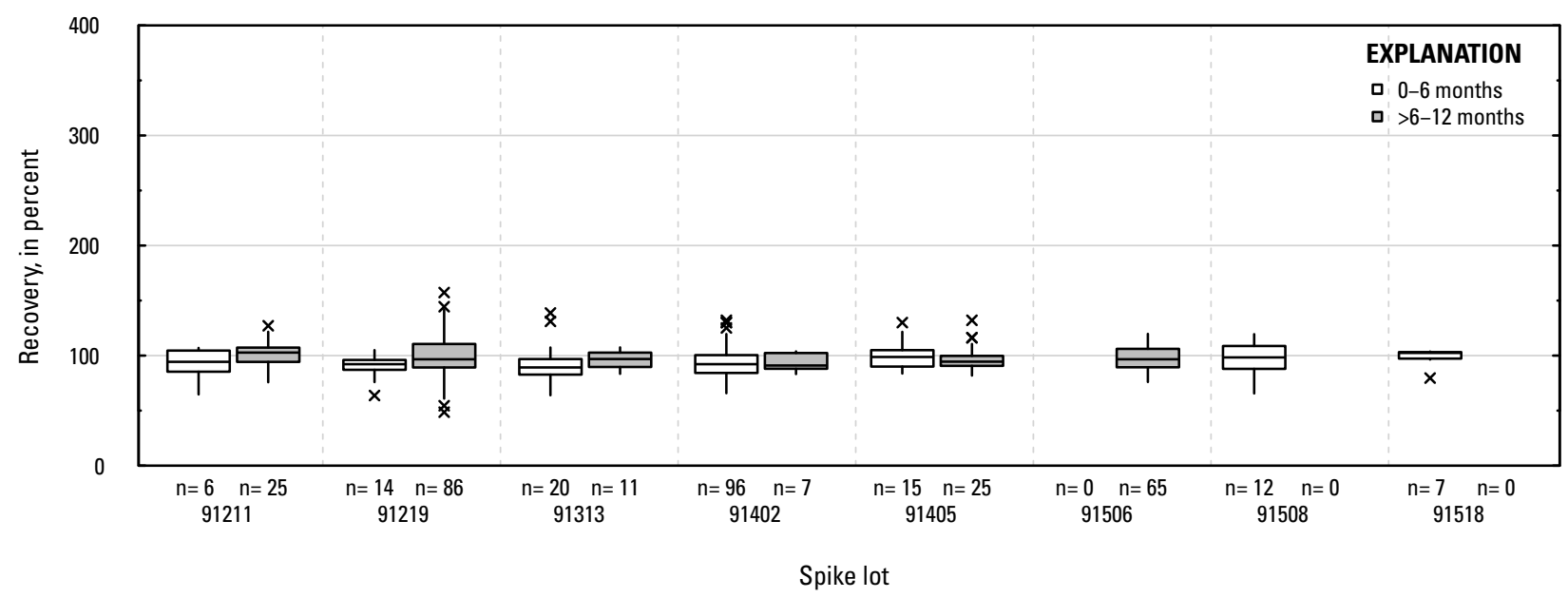

Figure 1-1. Distributions of recovery for individual pesticides in schedule 2437 by matrix, spike lot, and spike lot age. Recovery values larger than 400 percent are not shown.-Continued 
WX. Tebuthiuron TP el108: laboratory reagent spikes

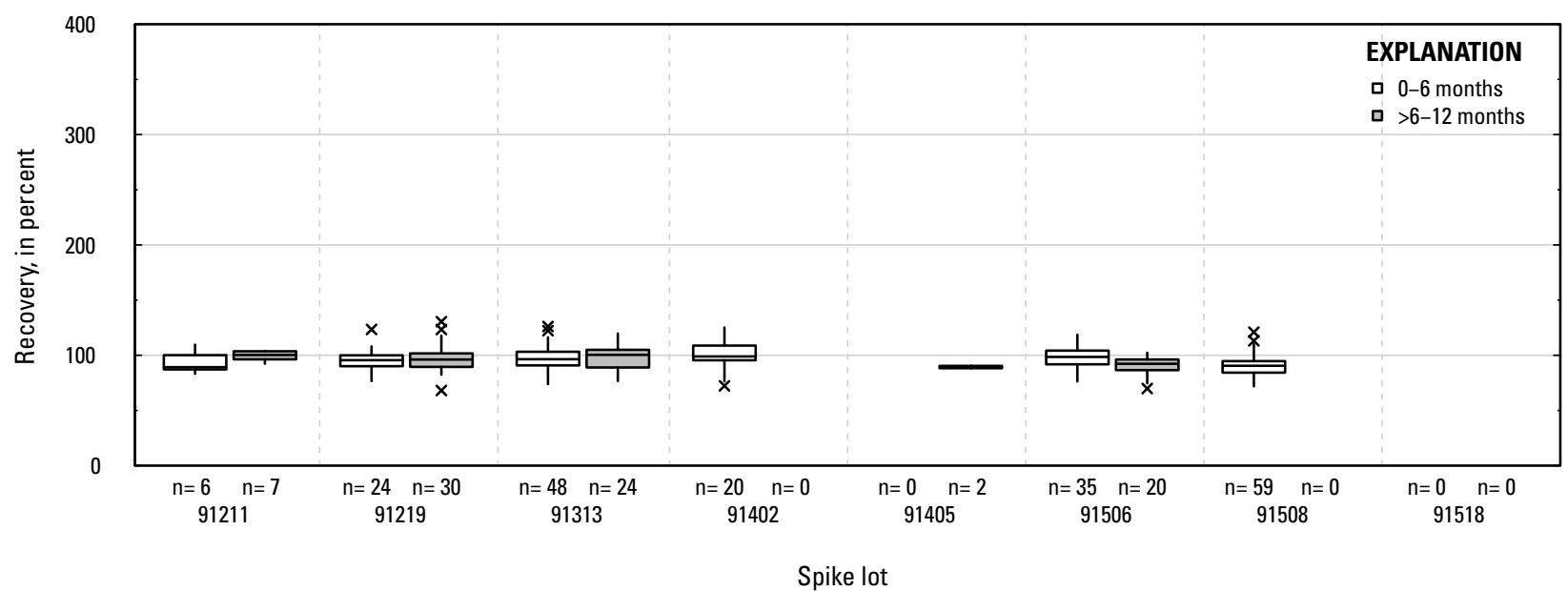

WY. Tebuthiuron TP el108: groundwater field matrix spikes

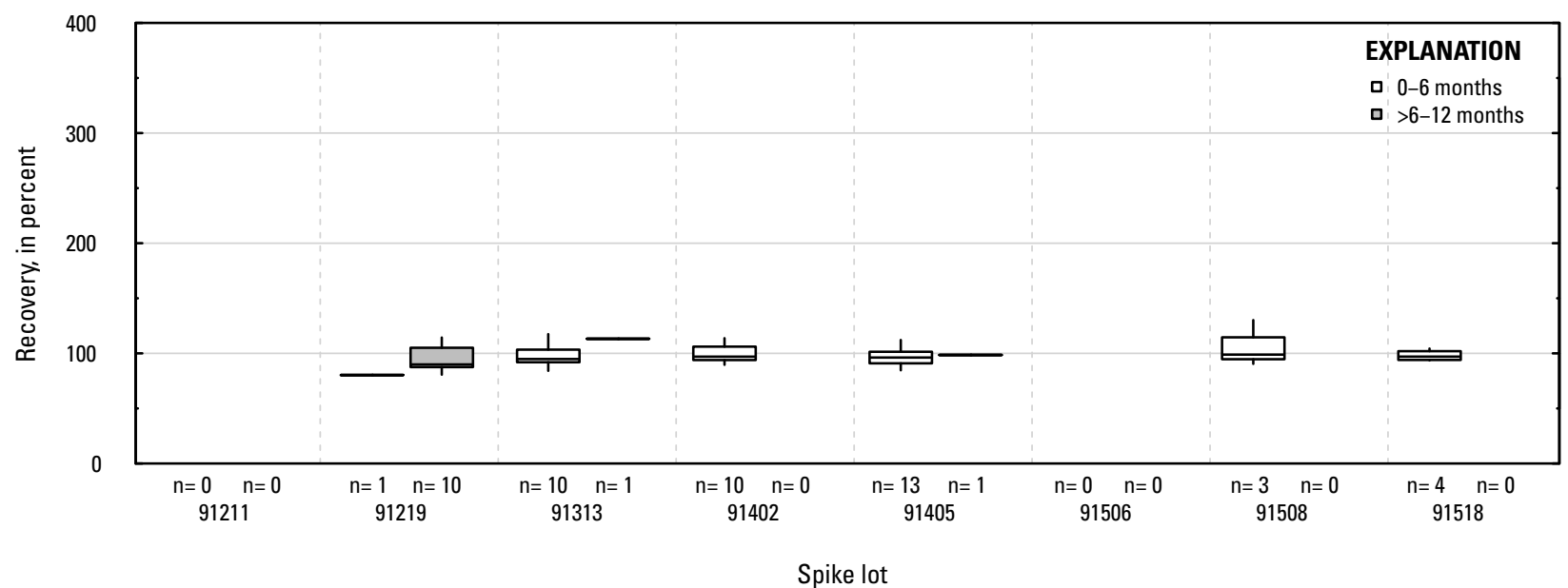

WZ. Tebuthiuron TP el108: surface water field matrix spikes

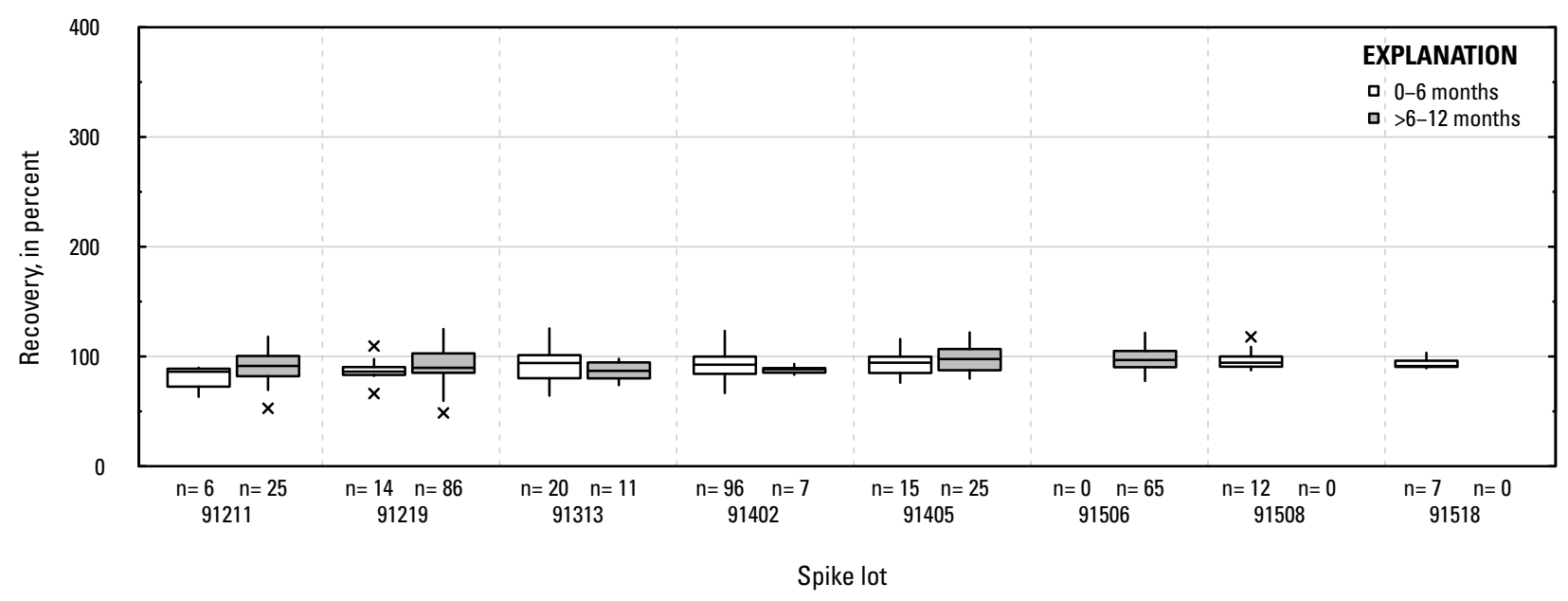

Figure 1-1. Distributions of recovery for individual pesticides in schedule 2437 by matrix, spike lot, and spike lot age. Recovery values larger than 400 percent are not shown.-Continued 
$X A$. Tebuthiuron TP $109(0 \mathrm{H})$ : laboratory reagent spikes

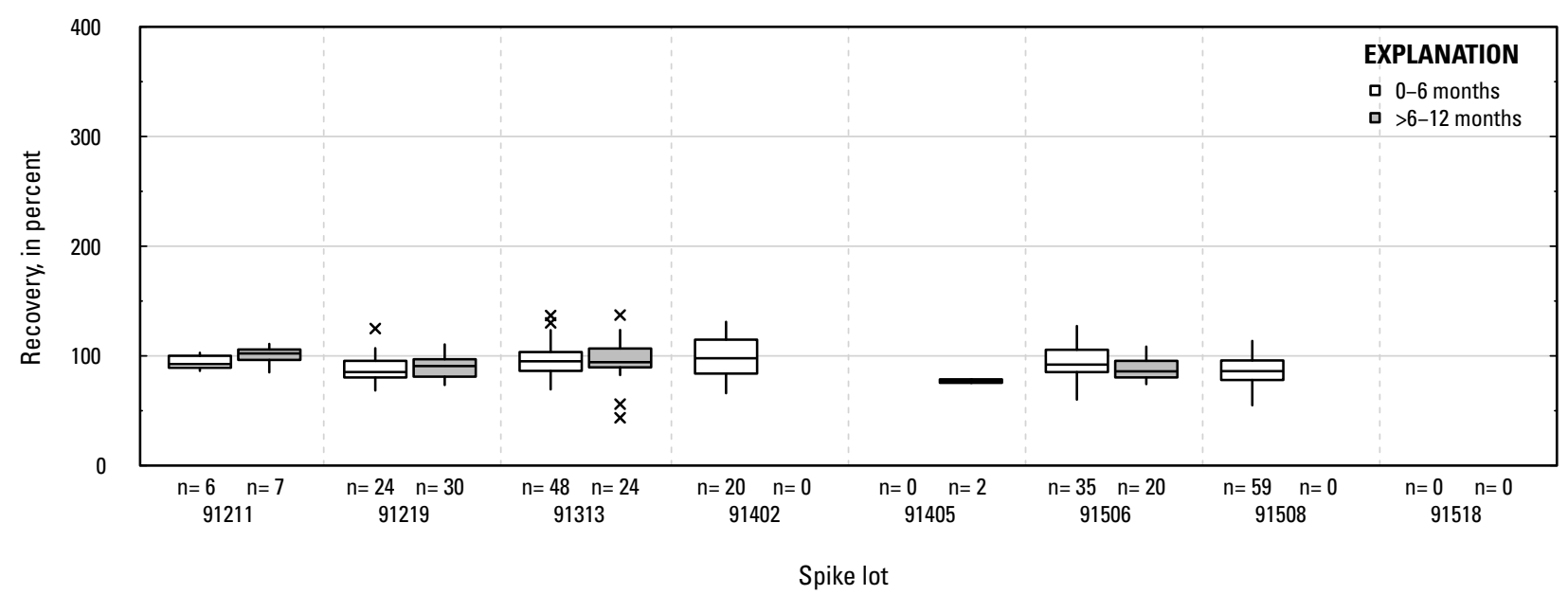

XB. Tebuthiuron TP $109(\mathrm{OH})$ : groundwater field matrix spikes

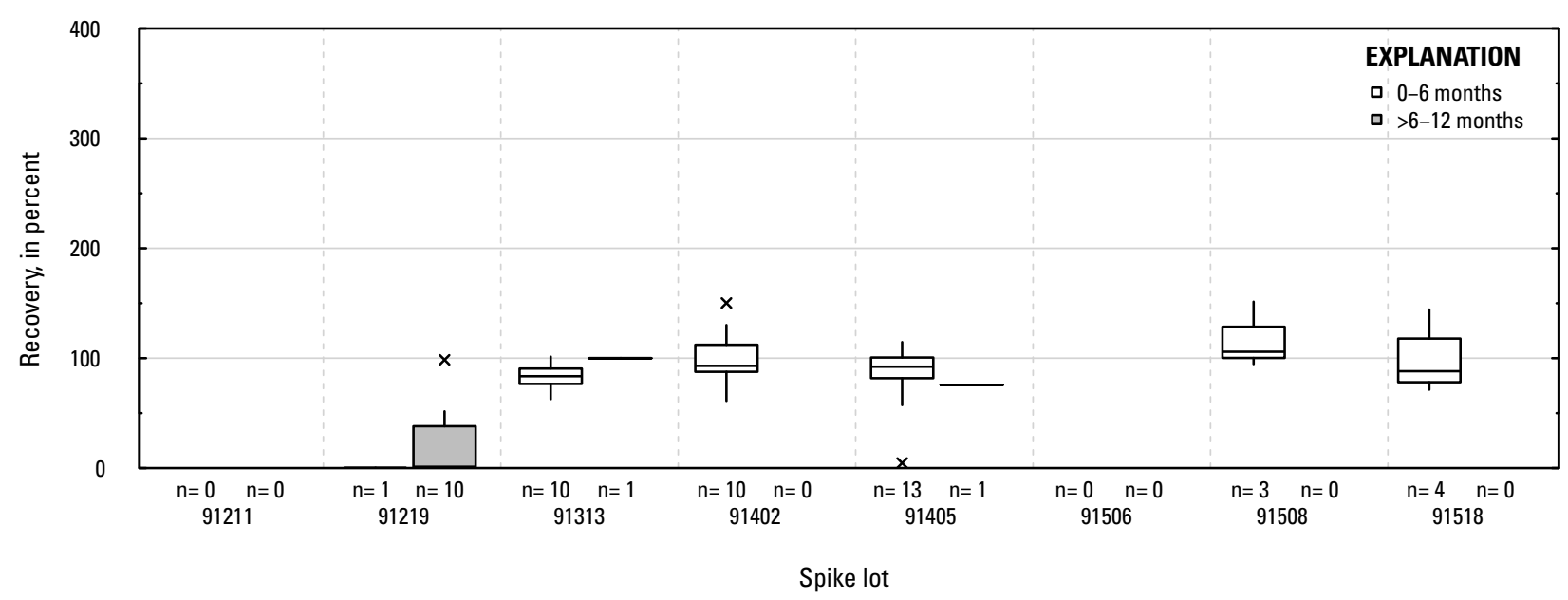

XC. Tebuthiuron TP 109 (OH): surface water field matrix spikes

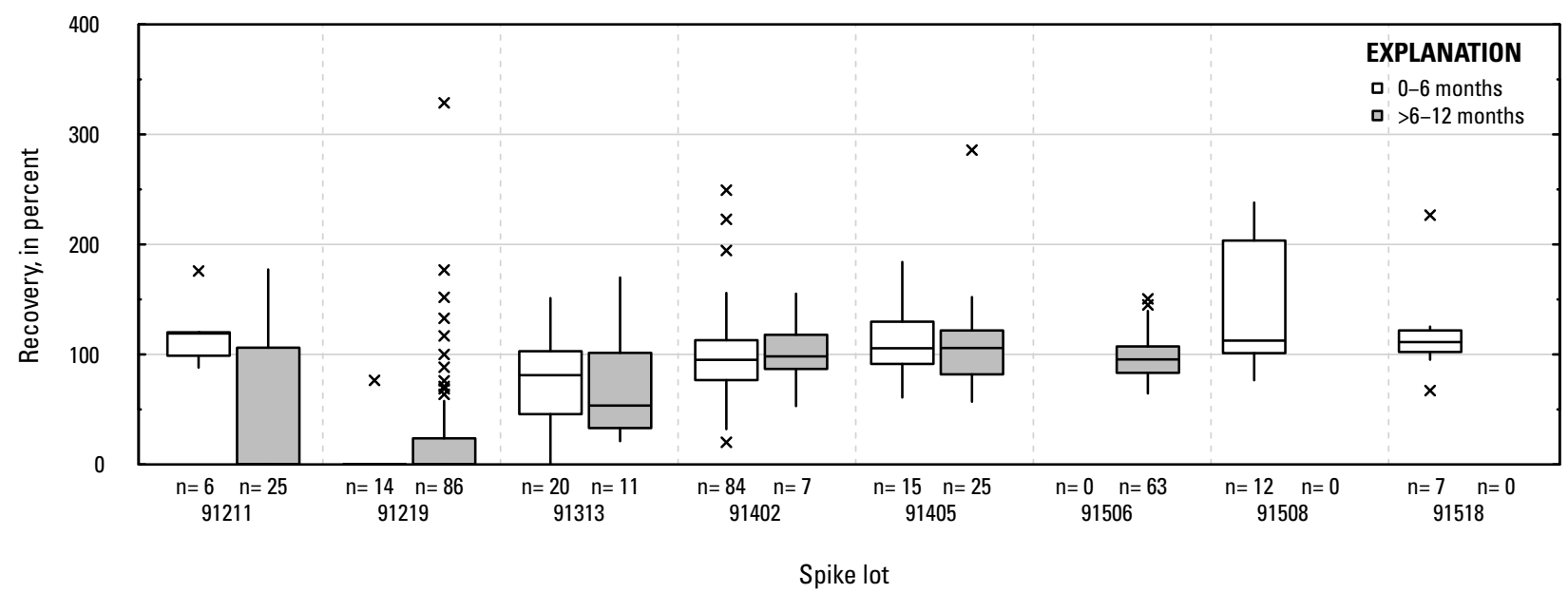

Figure 1-1. Distributions of recovery for individual pesticides in schedule 2437 by matrix, spike lot, and spike lot age. Recovery values larger than 400 percent are not shown.-Continued 


\section{$X D$. Terbacil: laboratory reagent spikes}

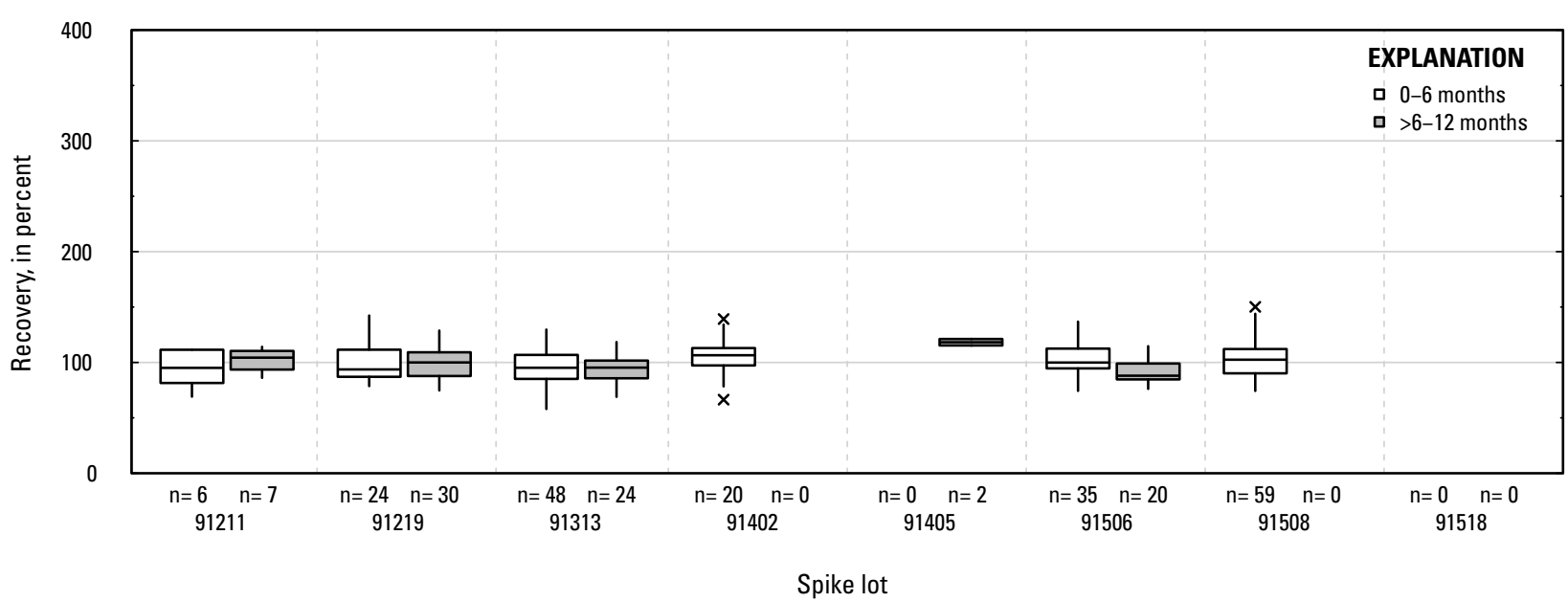

XE. Terbacil: groundwater field matrix spikes

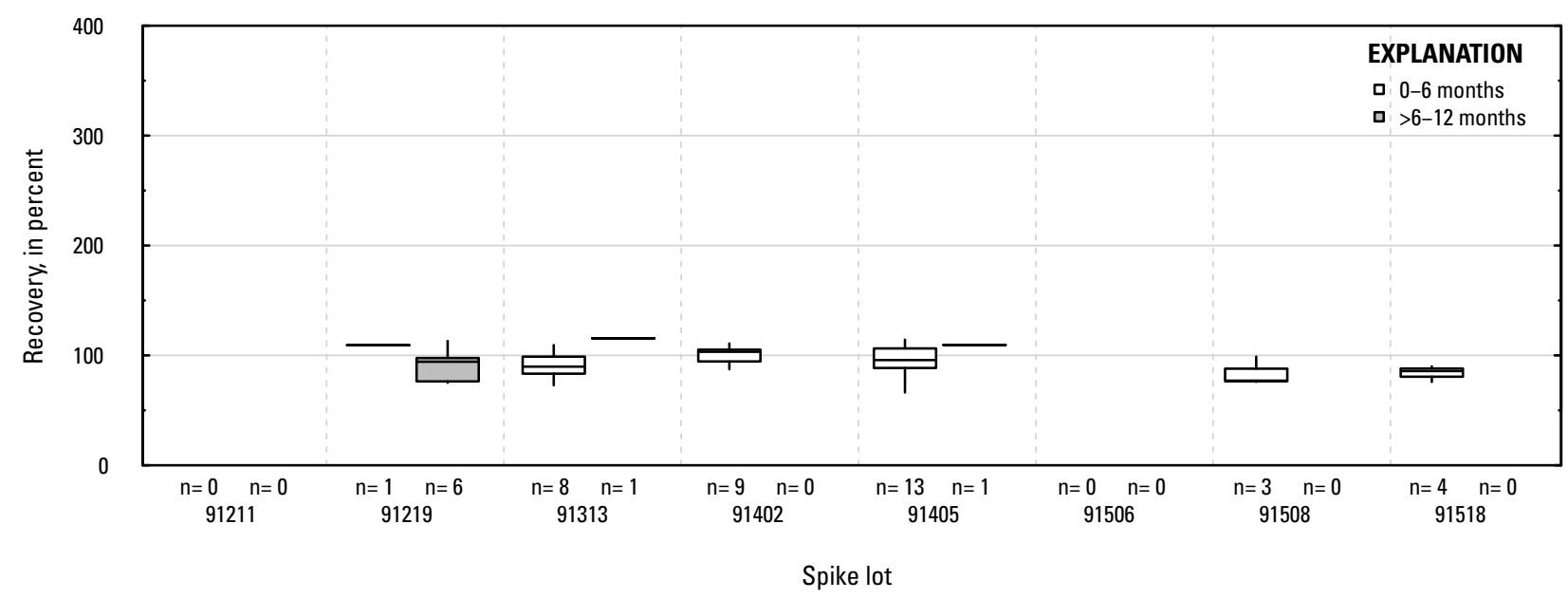

XF. Terbacil: surface water field matrix spikes

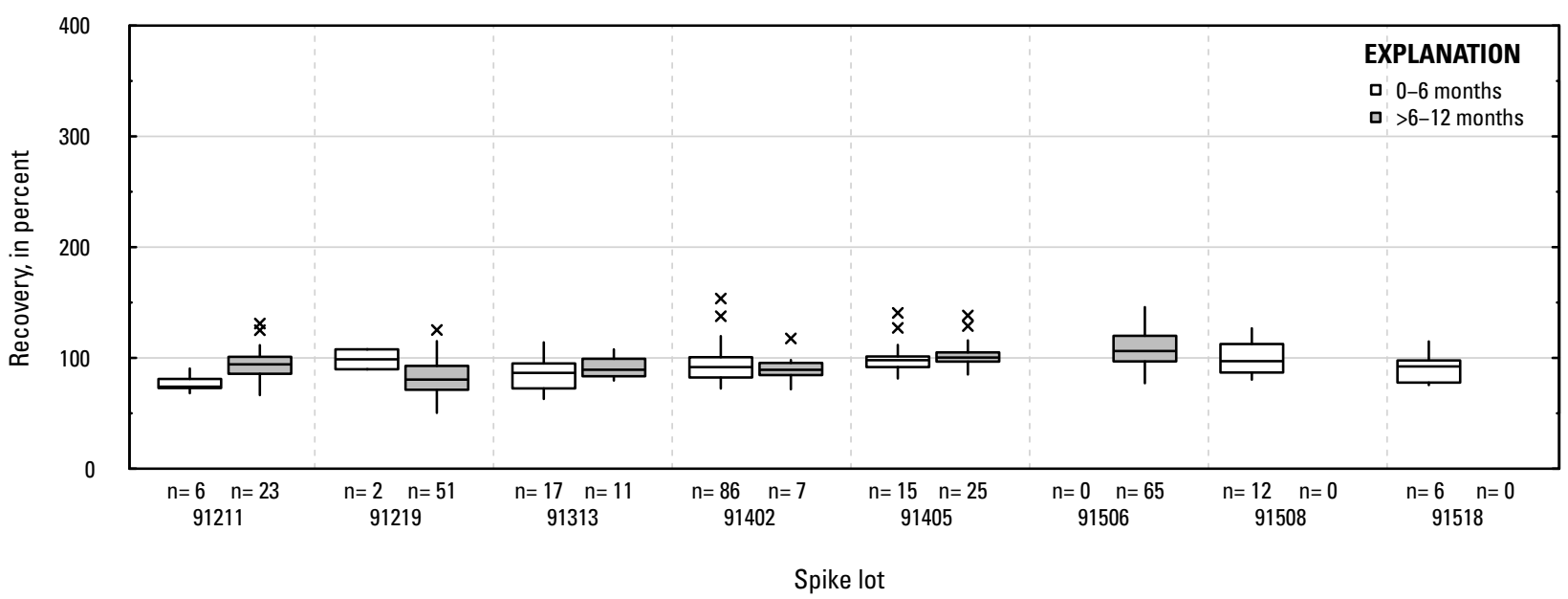

Figure 1-1. Distributions of recovery for individual pesticides in schedule 2437 by matrix, spike lot, and spike lot age. Recovery values larger than 400 percent are not shown.-Continued 
XG. Terbufos: laboratory reagent spikes

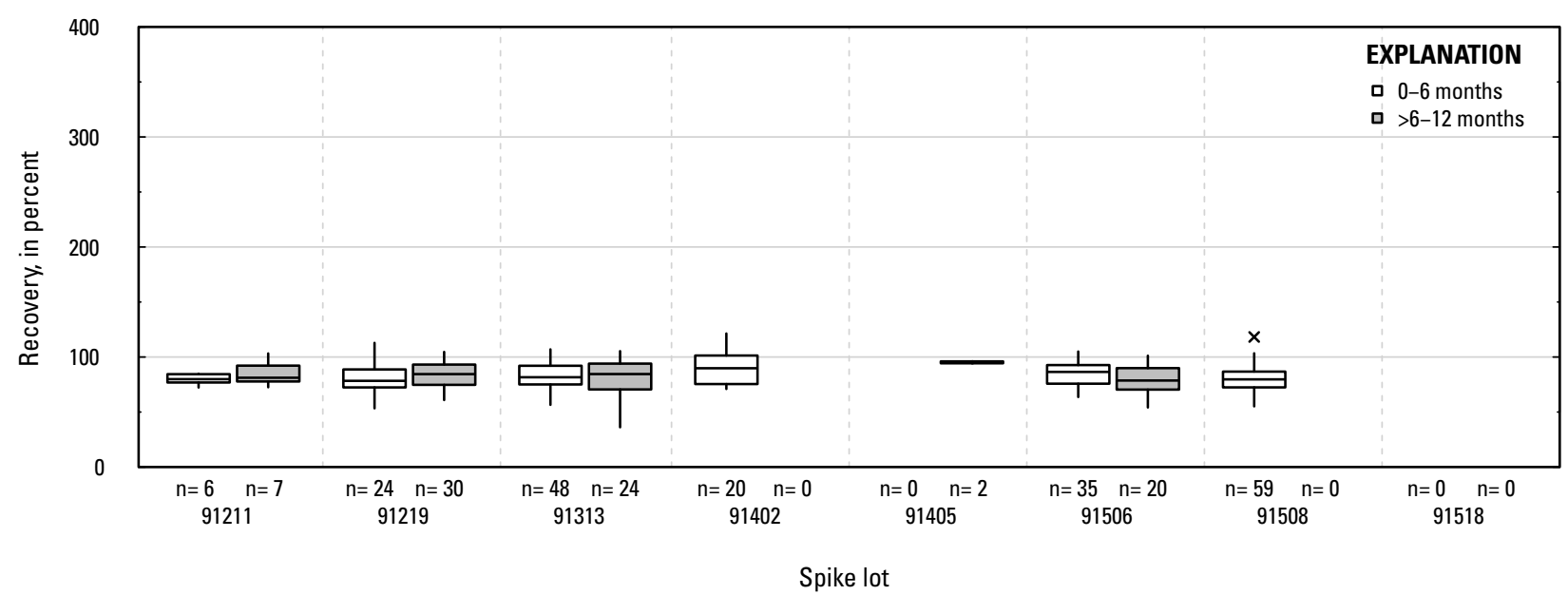

XH. Terbufos: groundwater field matrix spikes

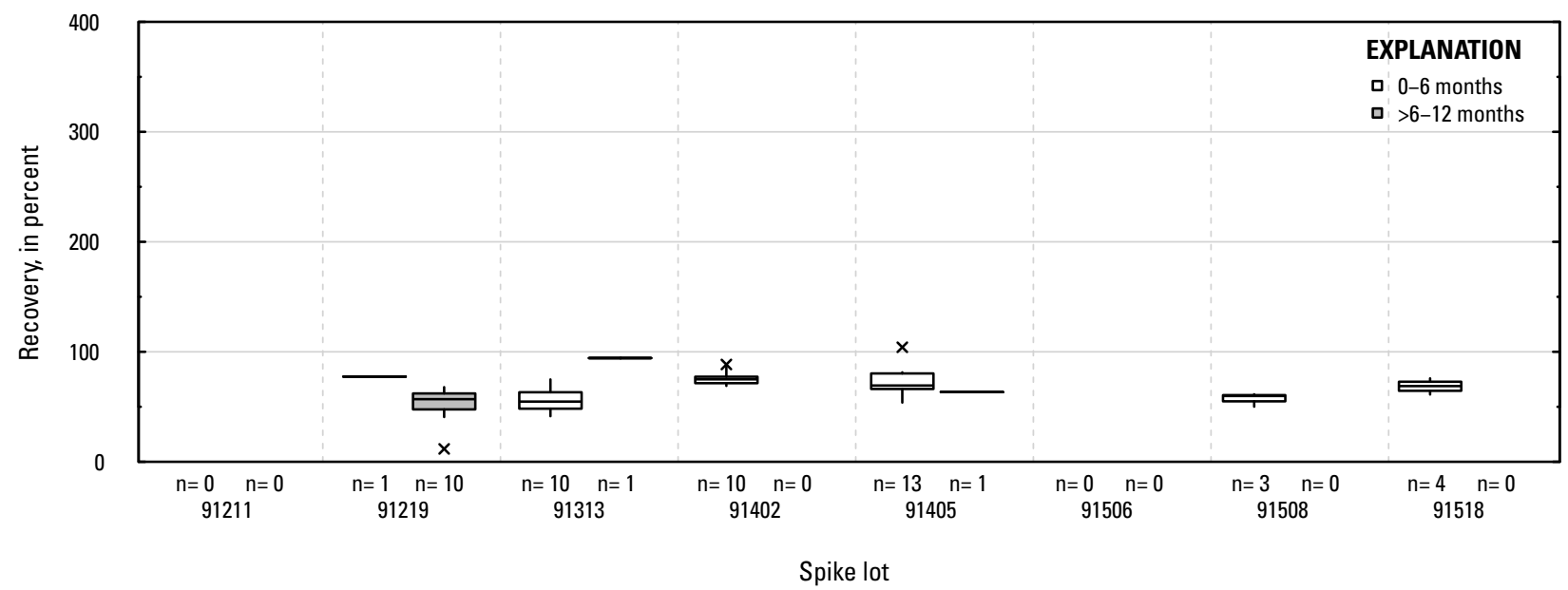

XI. Terbufos: surface water field matrix spikes

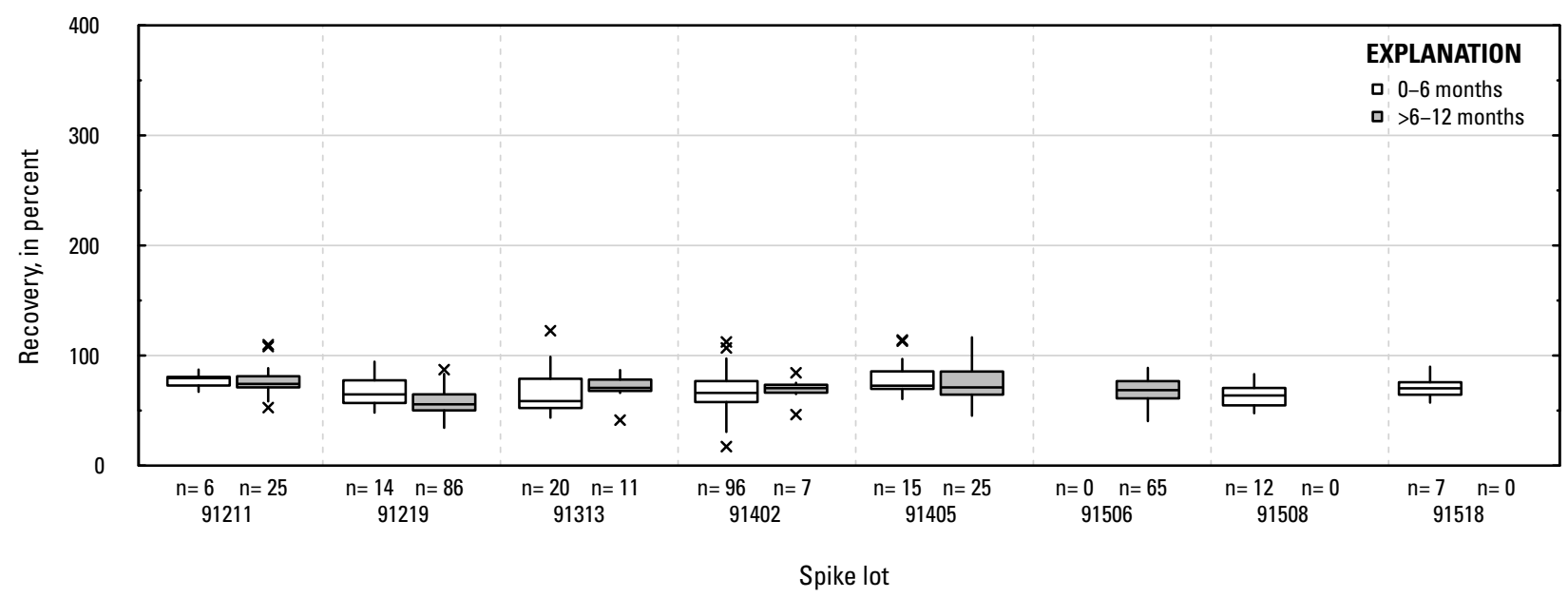

Figure 1-1. Distributions of recovery for individual pesticides in schedule 2437 by matrix, spike lot, and spike lot age. Recovery values larger than 400 percent are not shown.-Continued 


\section{$X J$. Terbufos oxon: laboratory reagent spikes}

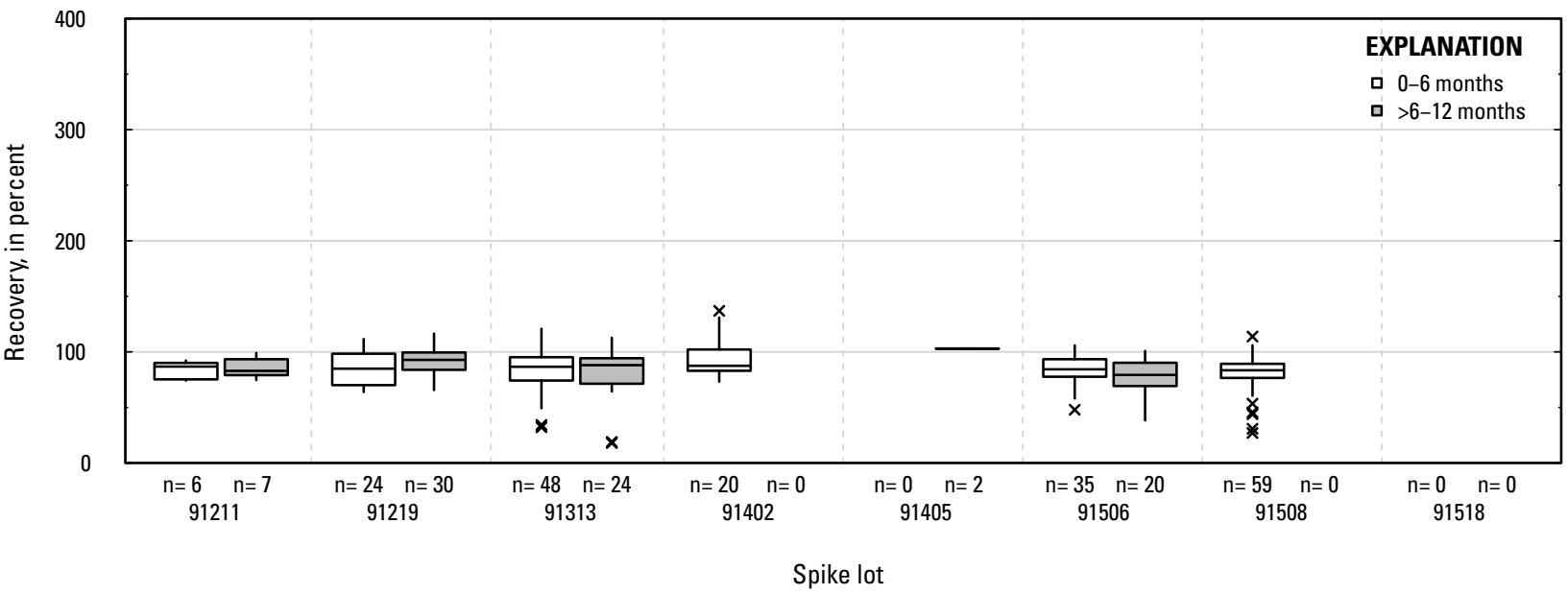

\section{$X K$. Terbufos oxon: groundwater field matrix spikes}

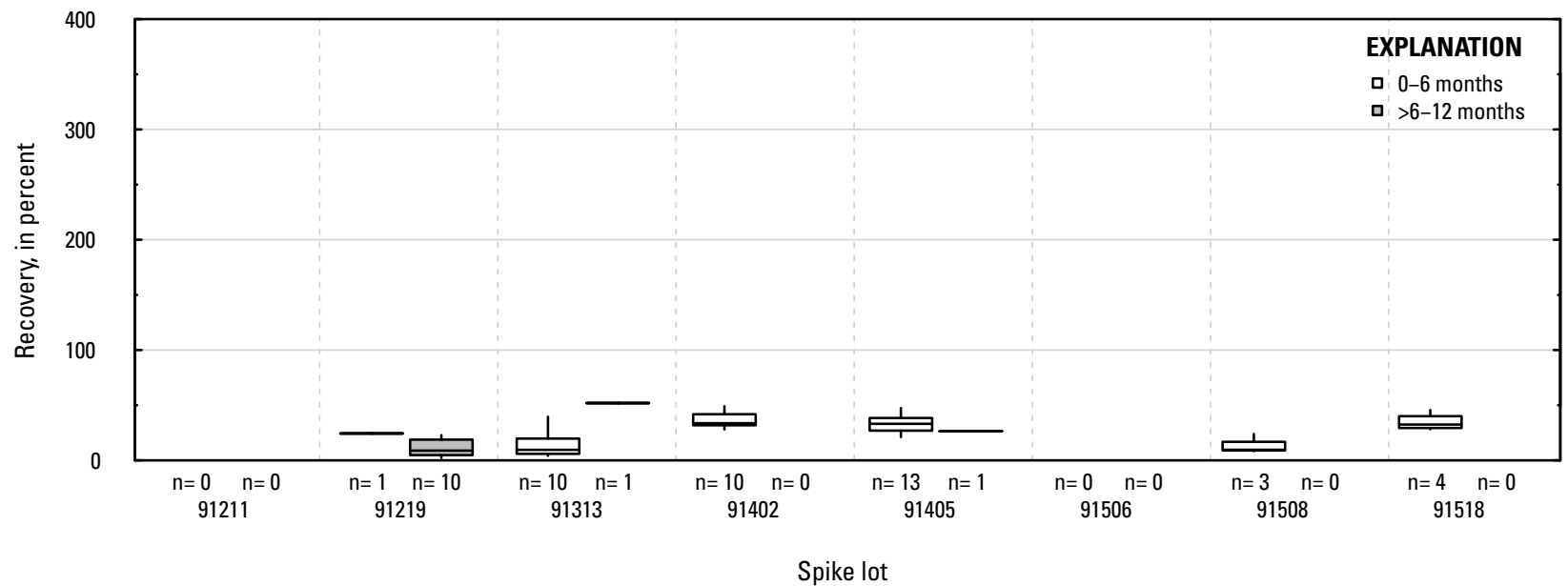

\section{$X L$. Terbufos oxon: surface water field matrix spikes}

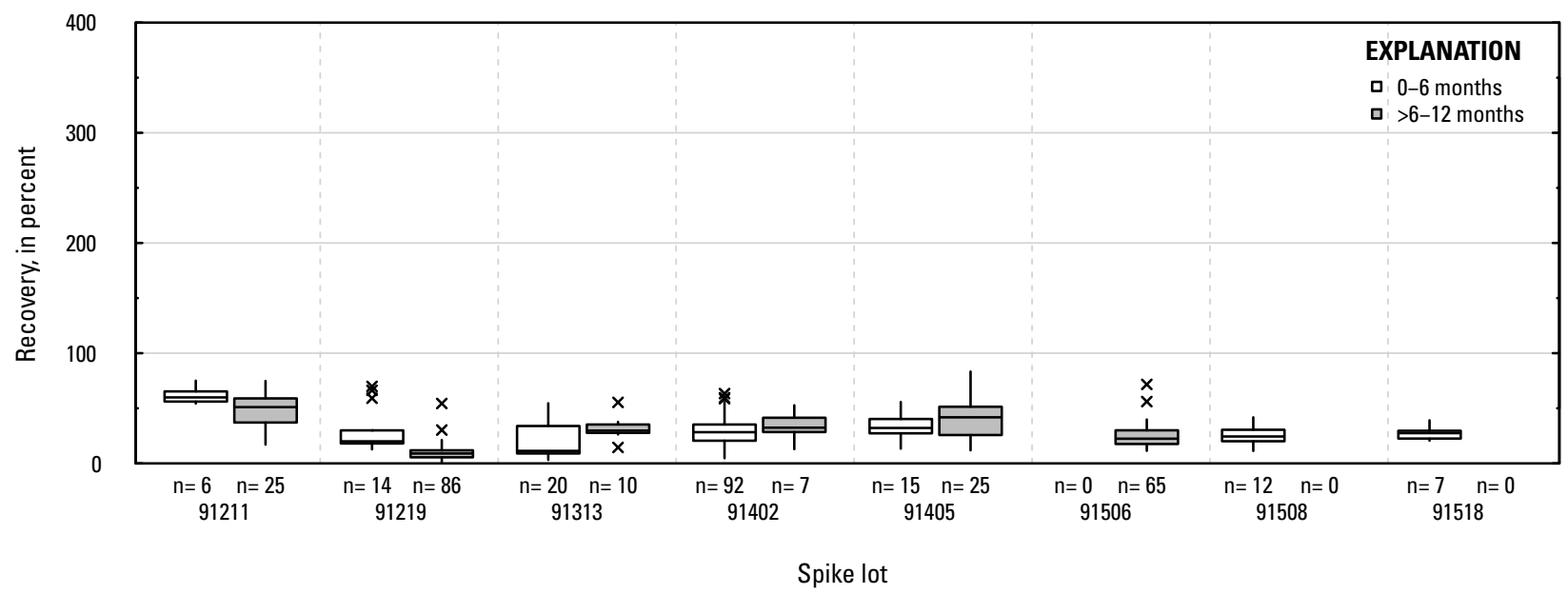

Figure 1-1. Distributions of recovery for individual pesticides in schedule 2437 by matrix, spike lot, and spike lot age. Recovery values larger than 400 percent are not shown.-Continued 
$X M$. Terbufos oxon sulfone: laboratory reagent spikes

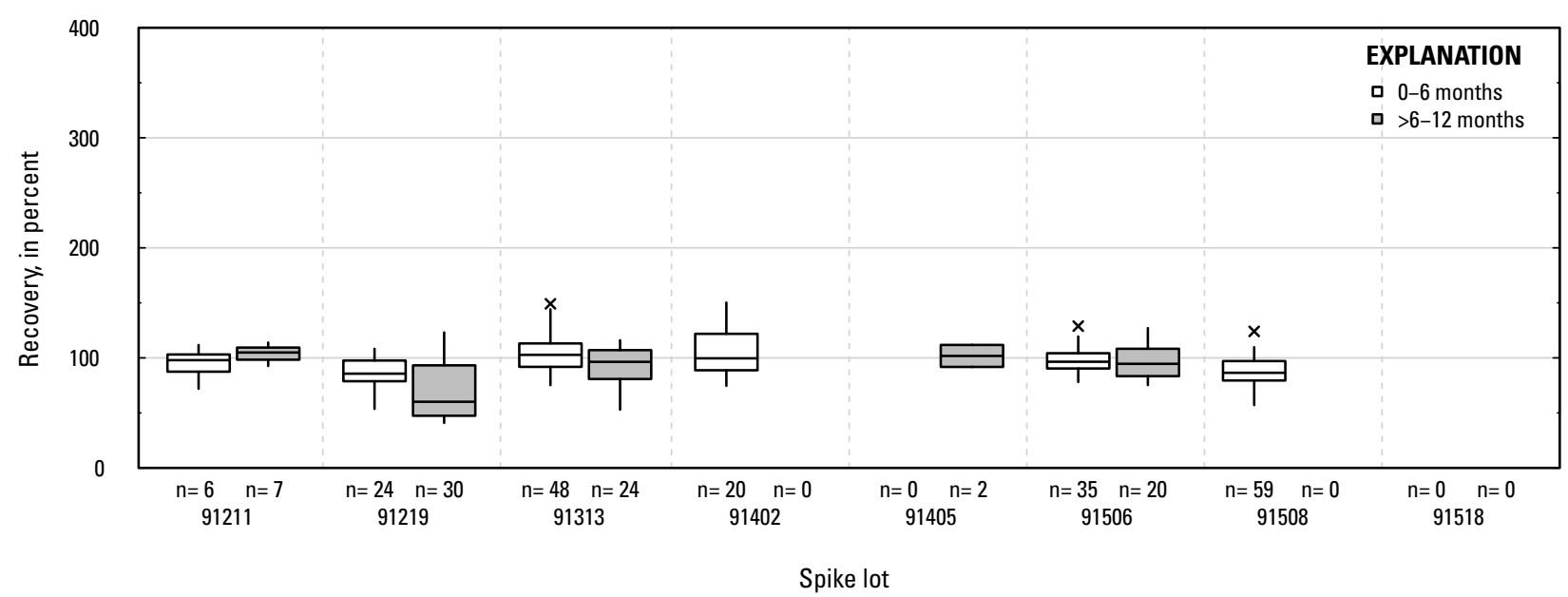

$X N$. Terbufos oxon sulfone: groundwater field matrix spikes

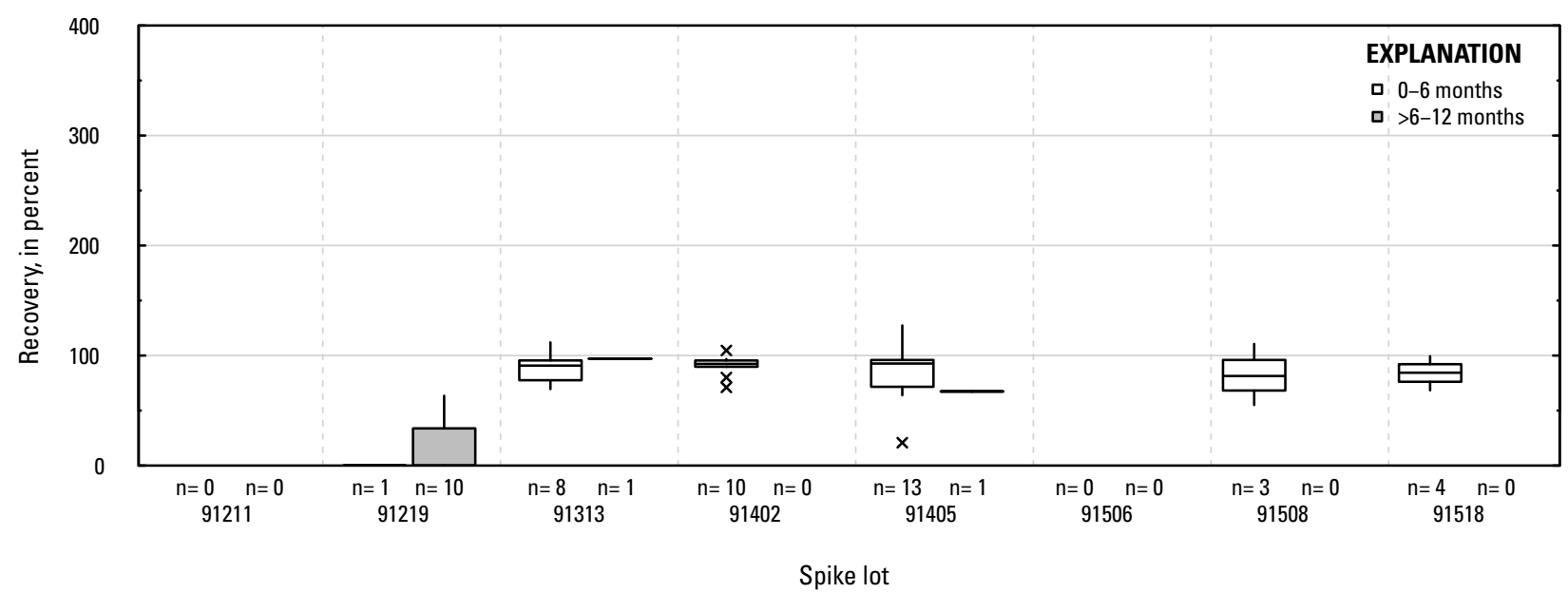

XO. Terbufos oxon sulfone: surface water field matrix spikes

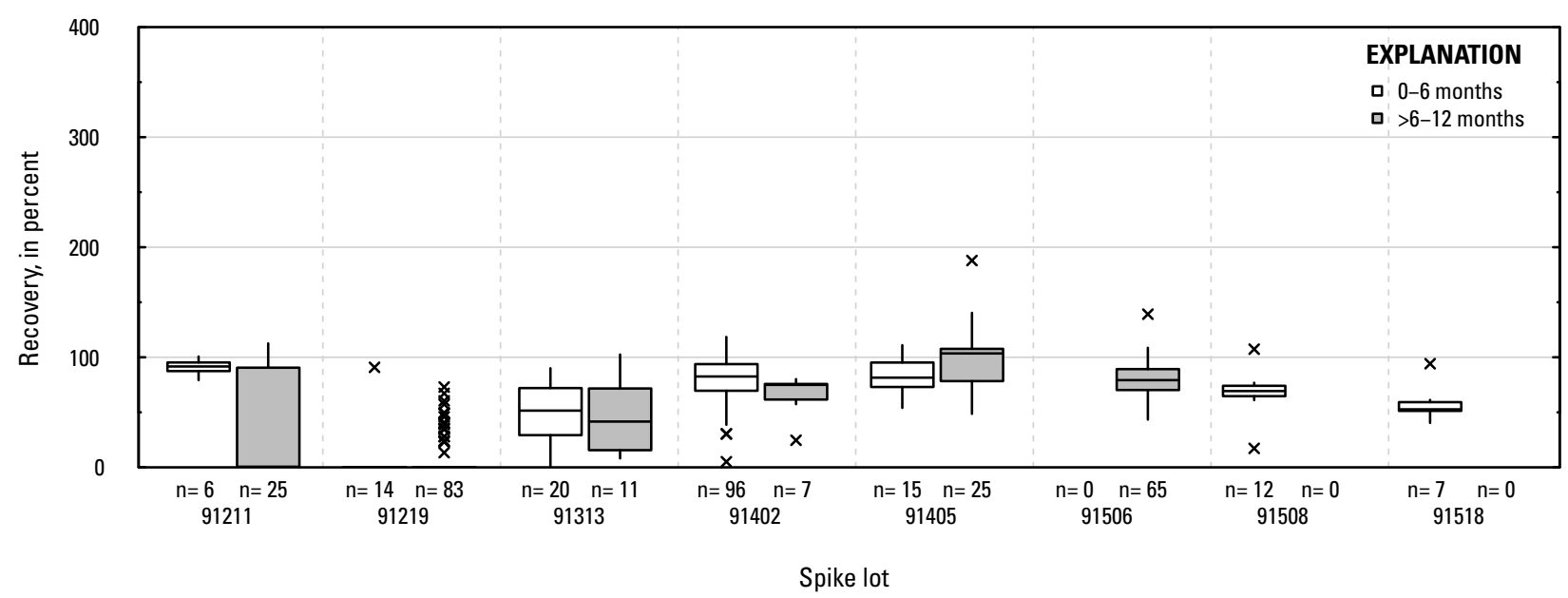

Figure 1-1. Distributions of recovery for individual pesticides in schedule 2437 by matrix, spike lot, and spike lot age. Recovery values larger than 400 percent are not shown.-Continued 
$X P$. Terbufos oxon sulfoxide: laboratory reagent spikes

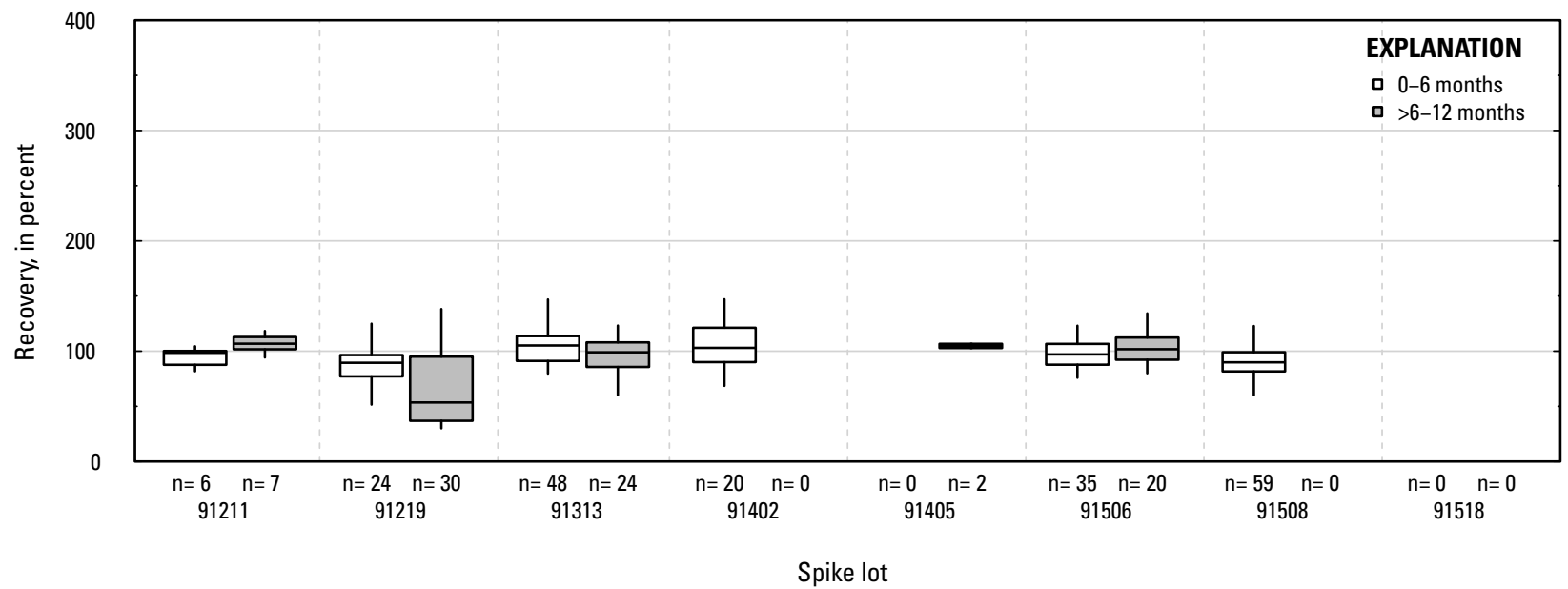

XO. Terbufos oxon sulfoxide: groundwater field matrix spikes

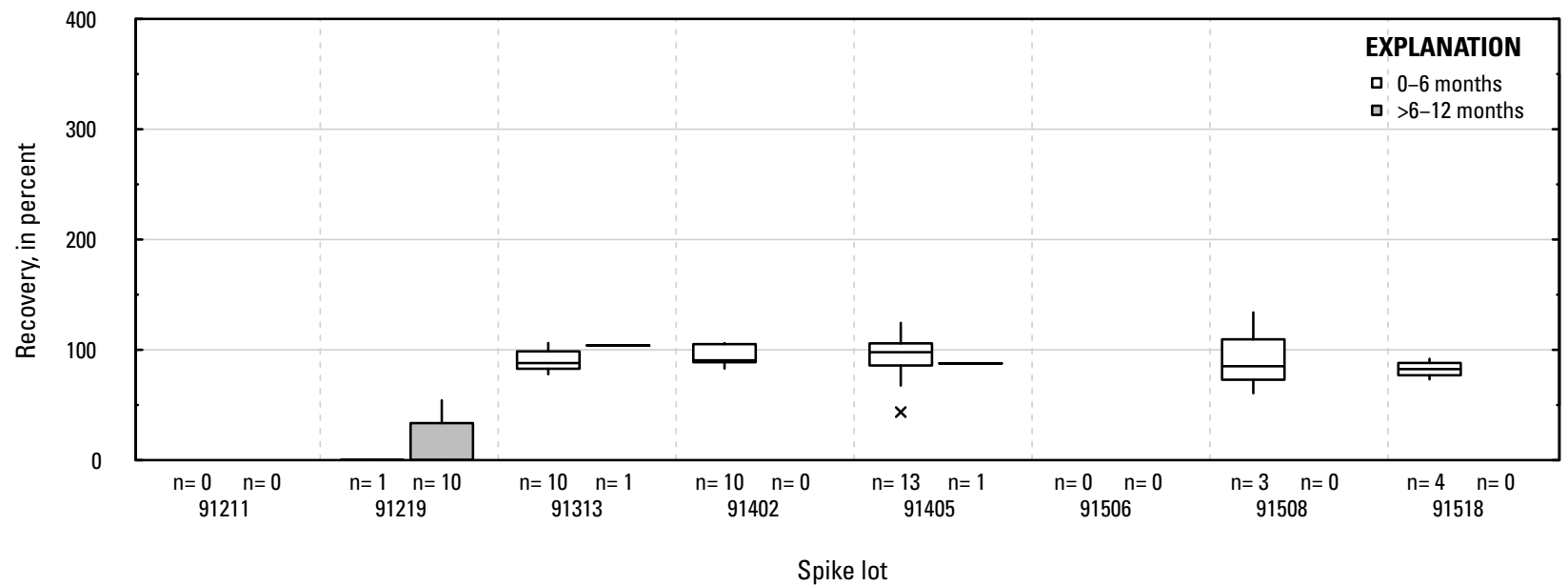

$X R$. Terbufos oxon sulfoxide: surface water field matrix spikes

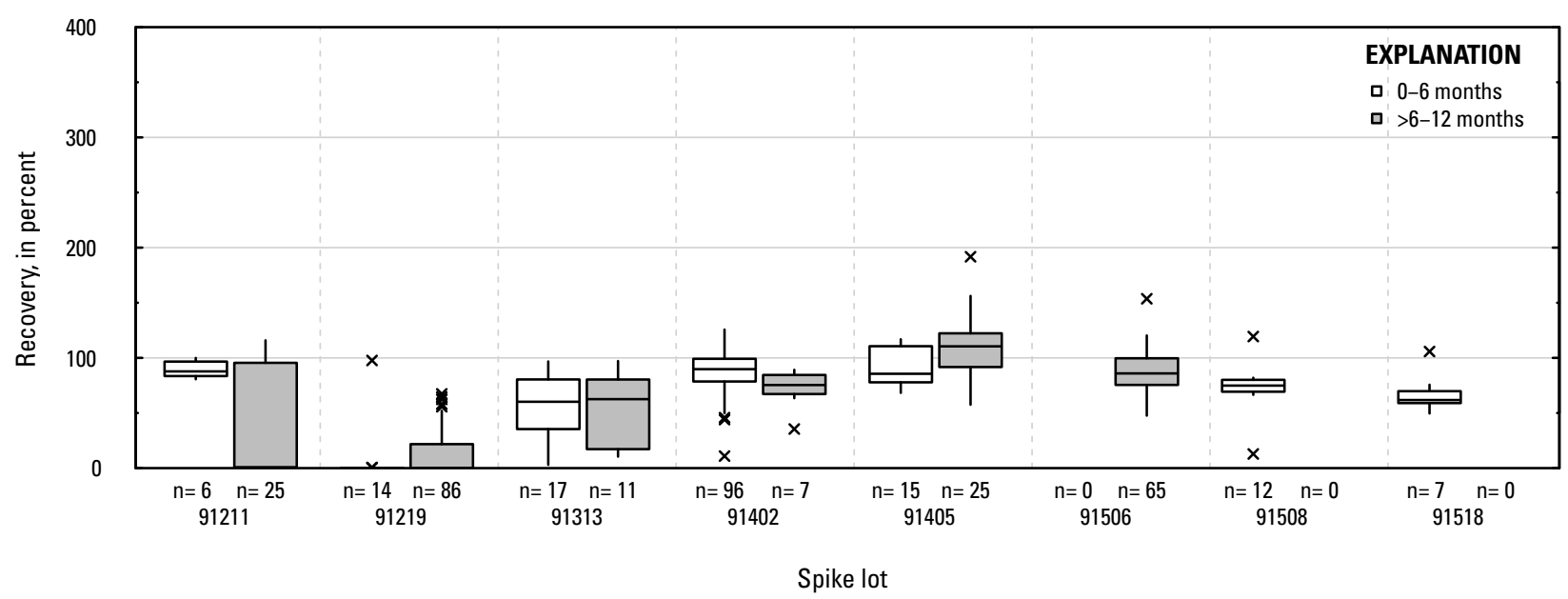

Figure 1-1. Distributions of recovery for individual pesticides in schedule 2437 by matrix, spike lot, and spike lot age. Recovery values larger than 400 percent are not shown.-Continued 


\section{$X S$. Terbufos sulfone: laboratory reagent spikes}

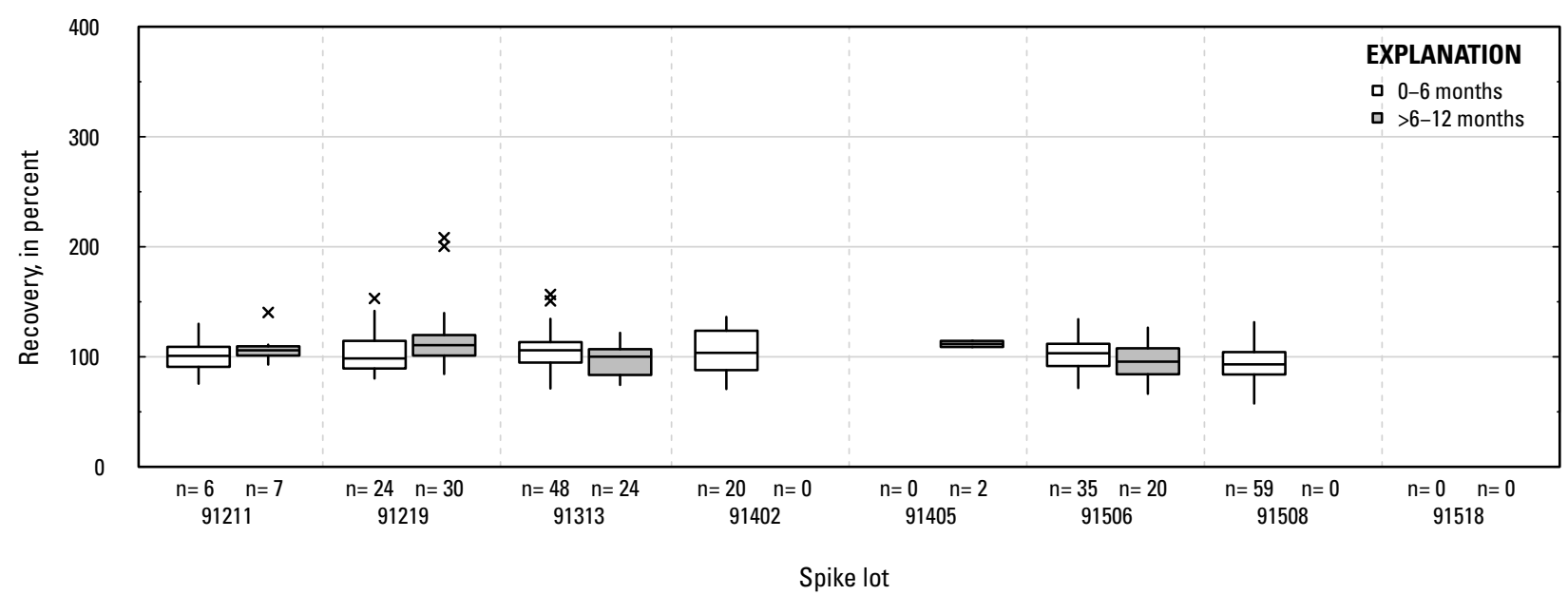

$X T$. Terbufos sulfone: groundwater field matrix spikes

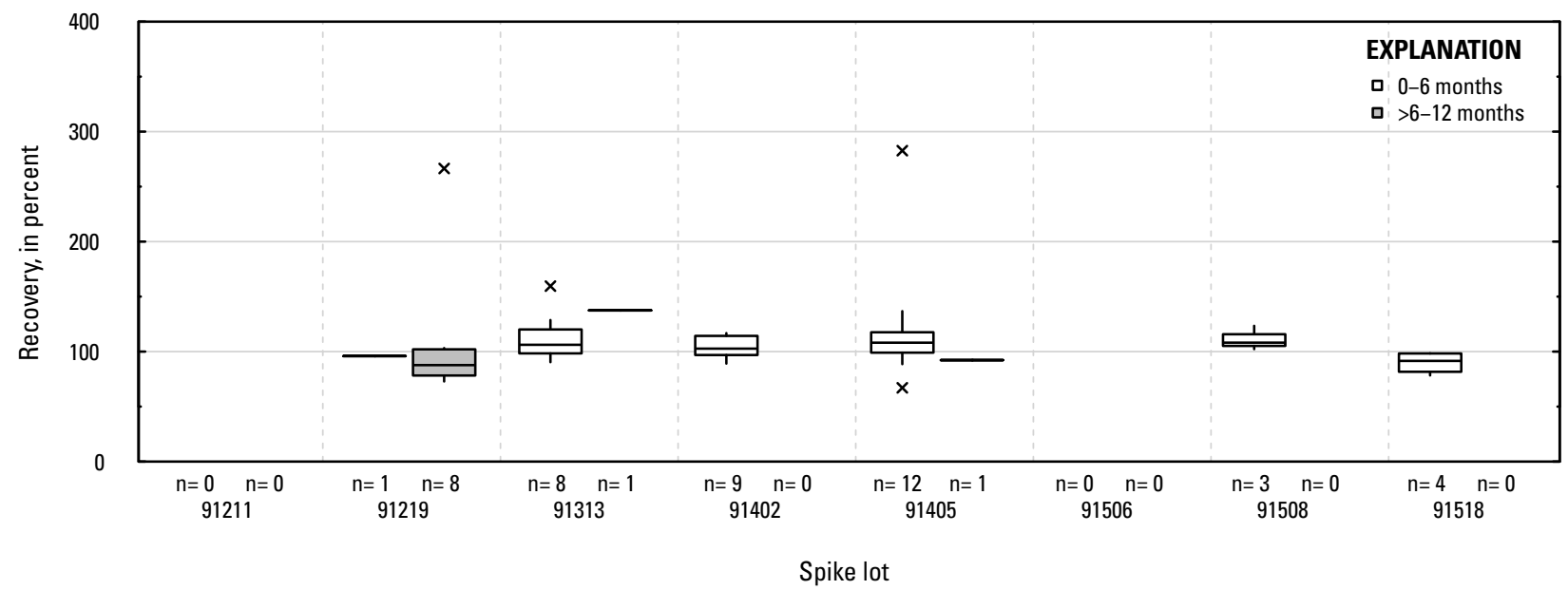

$X U$. Terbufos sulfone: surface water field matrix spikes

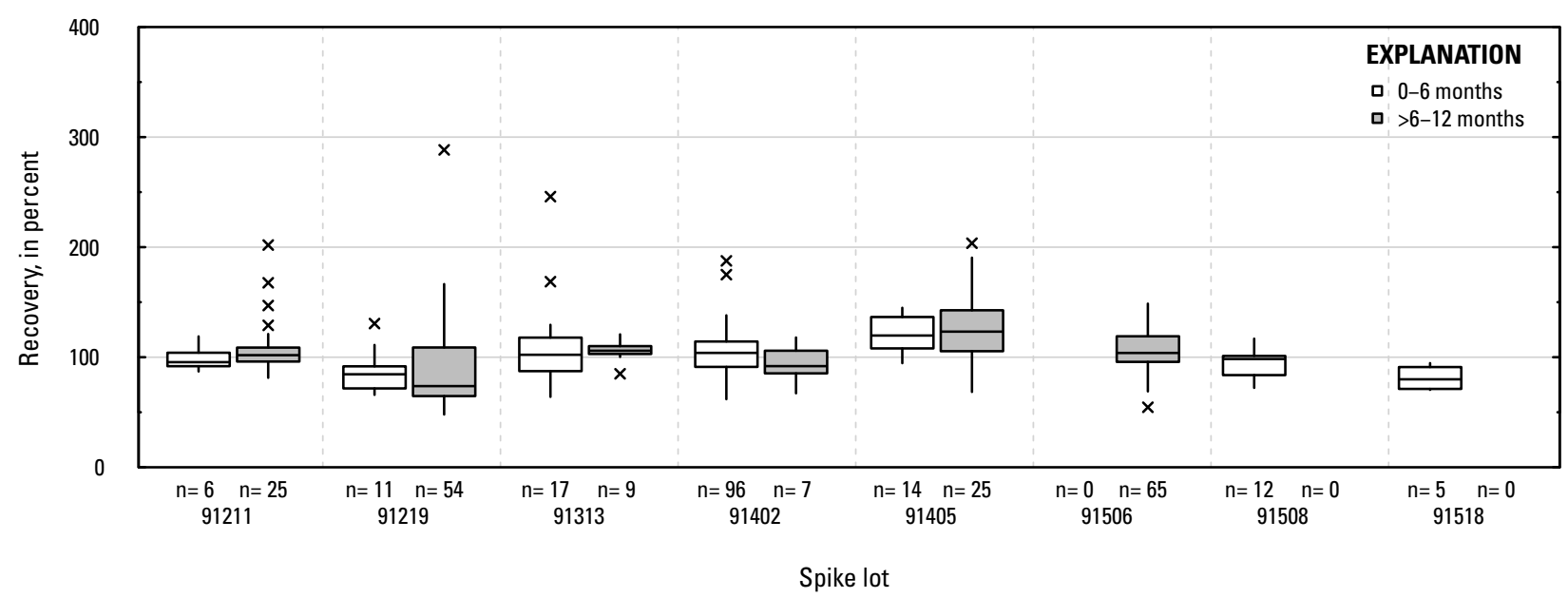

Figure 1-1. Distributions of recovery for individual pesticides in schedule 2437 by matrix, spike lot, and spike lot age. Recovery values larger than 400 percent are not shown.-Continued 
$X V$. Terbufos sulfoxide: laboratory reagent spikes

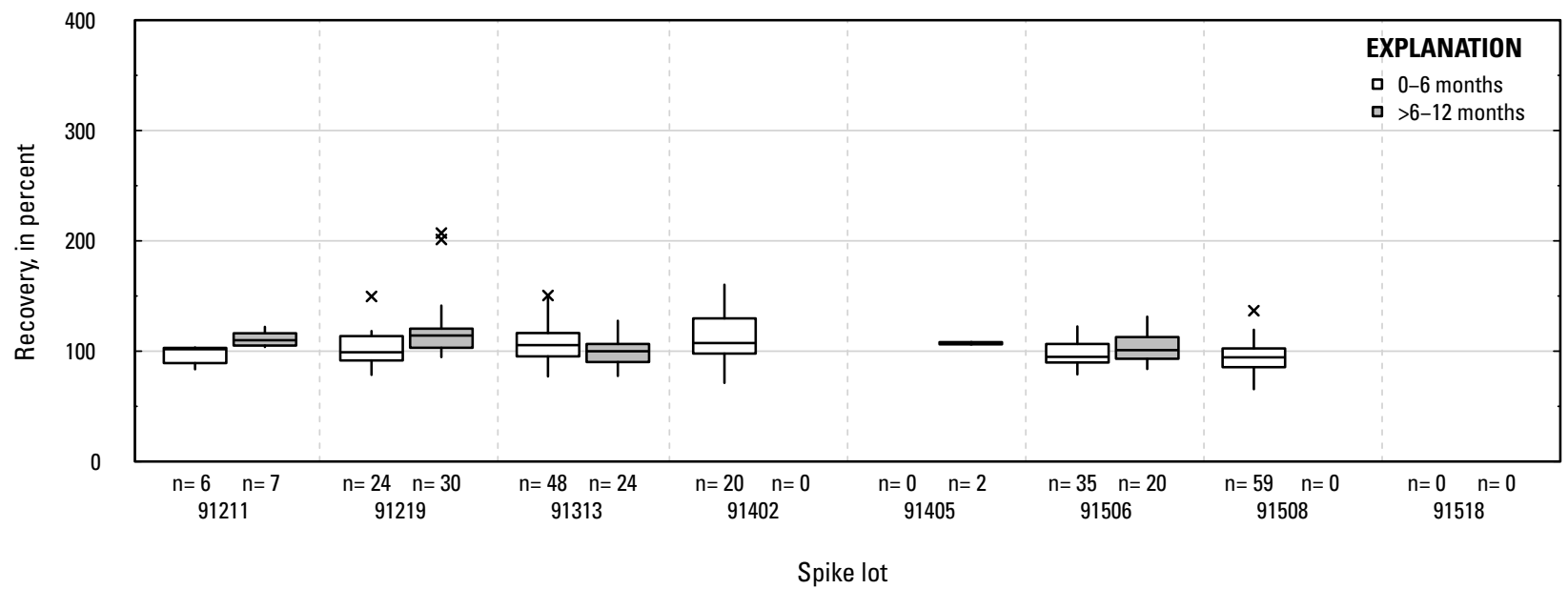

$X W$. Terbufos sulfoxide: groundwater field matrix spikes

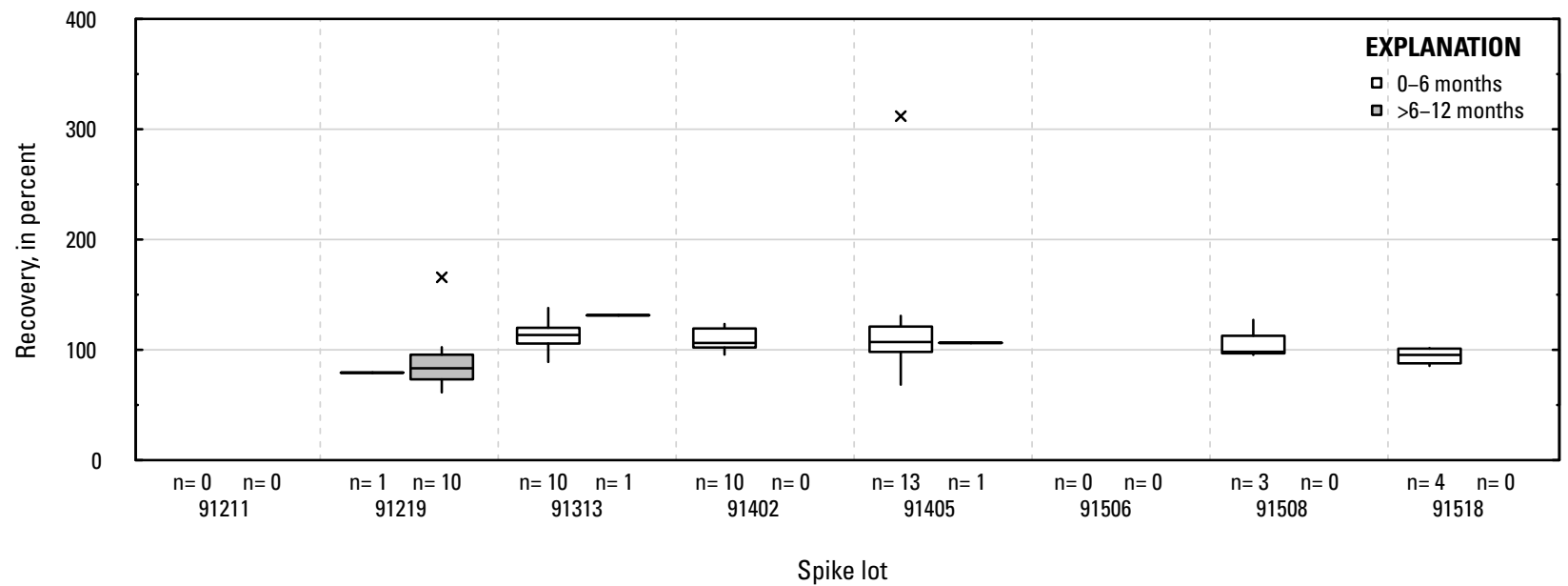

$X X$. Terbufos sulfoxide: surface water field matrix spikes

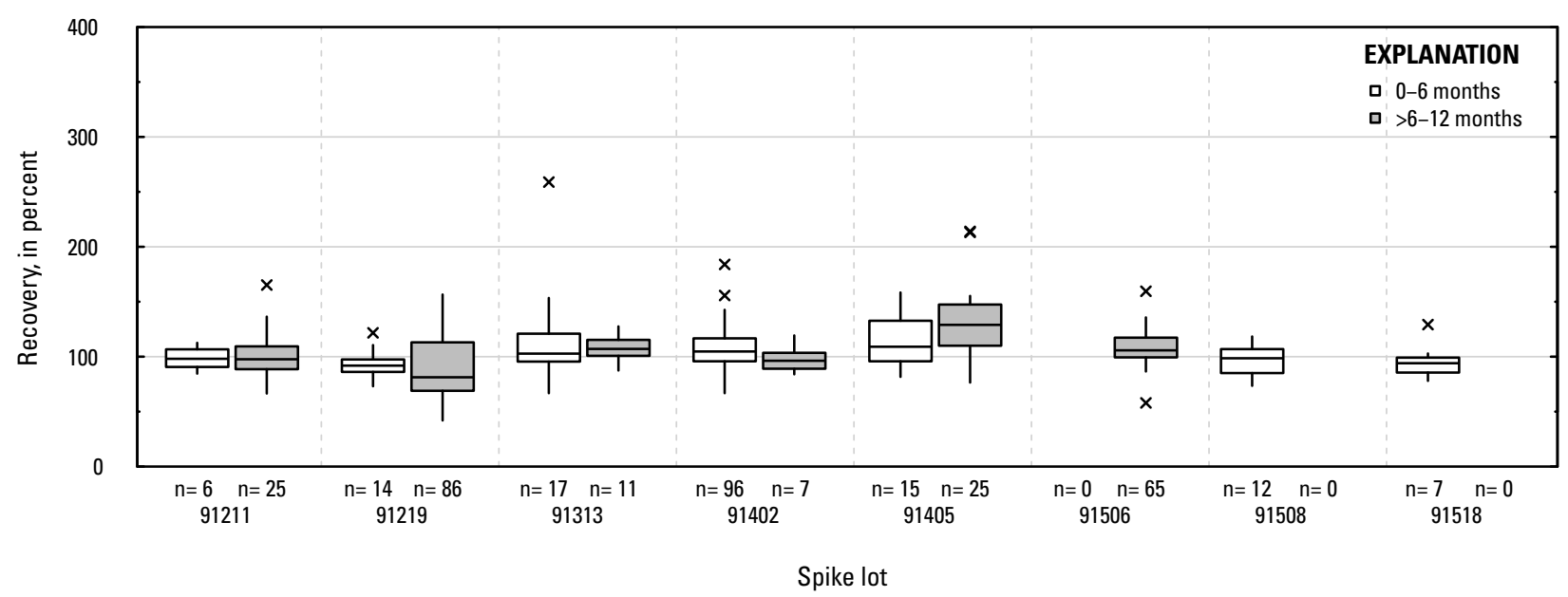

Figure 1-1. Distributions of recovery for individual pesticides in schedule 2437 by matrix, spike lot, and spike lot age. Recovery values larger than 400 percent are not shown.-Continued 


\section{XY. Terbuthylazine: laboratory reagent spikes}

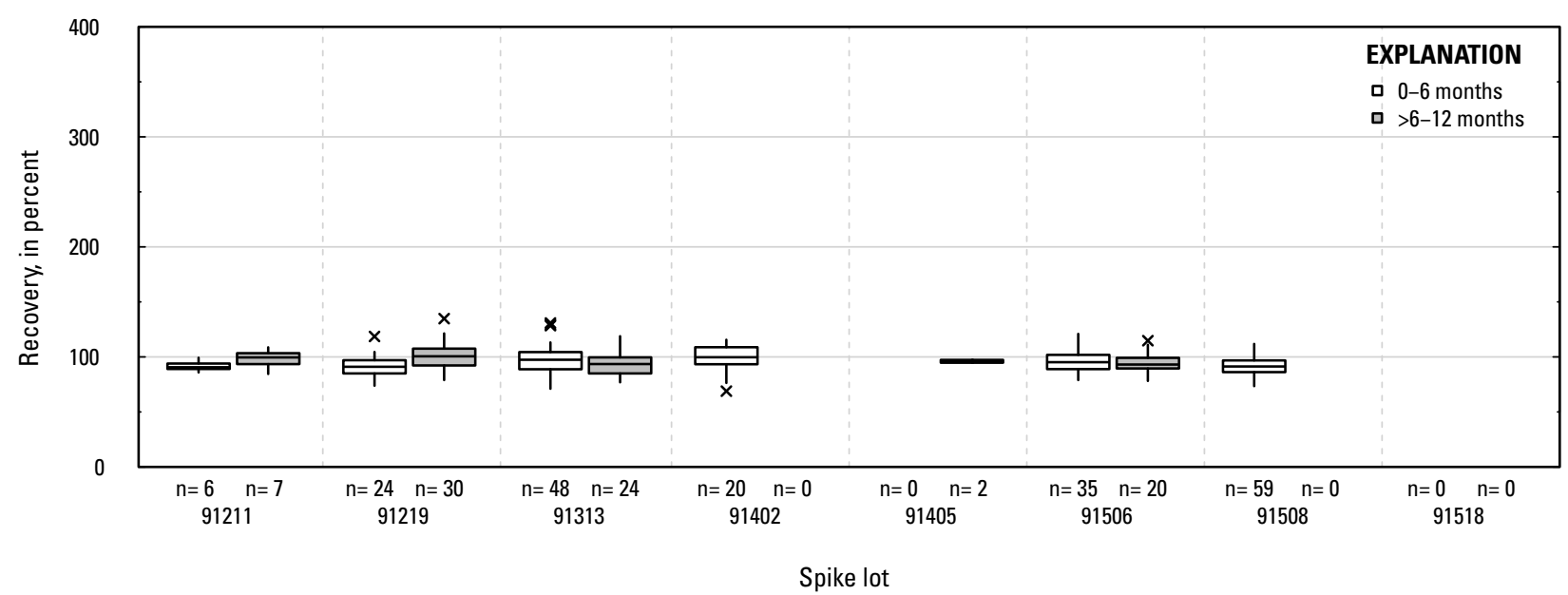

\section{$X Z$. Terbuthylazine: groundwater field matrix spikes}

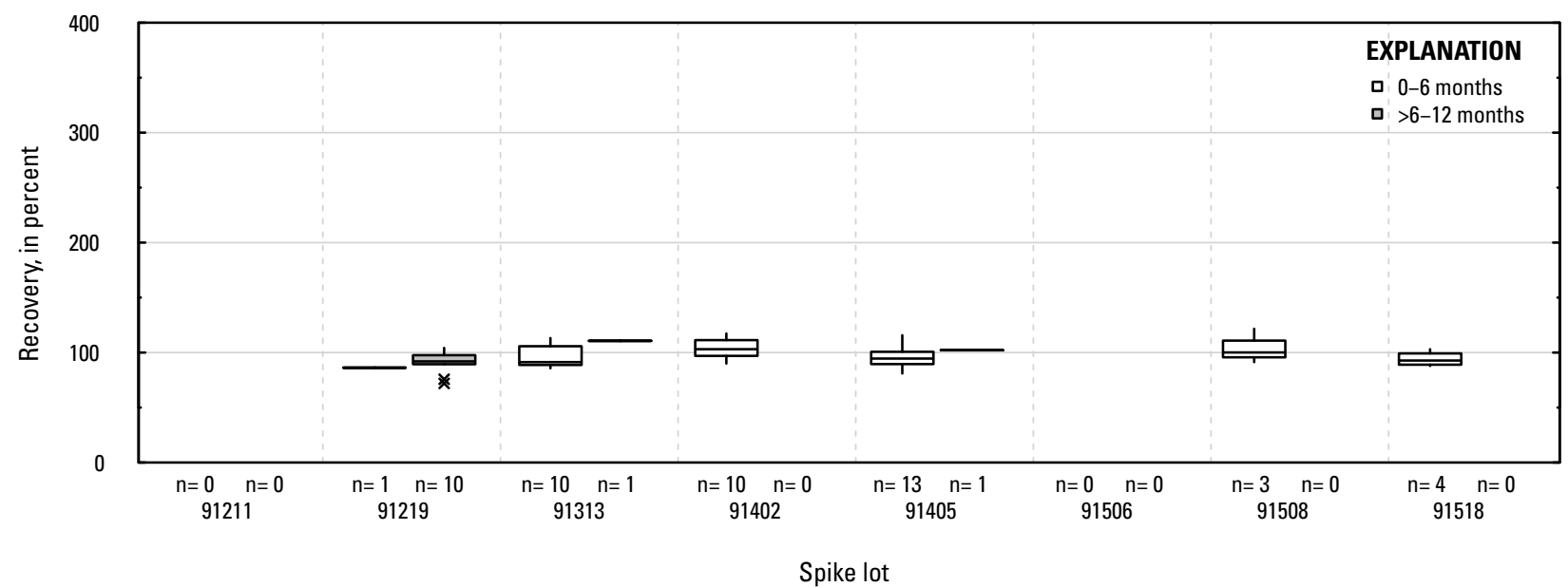

YA. Terbuthylazine: surface water field matrix spikes

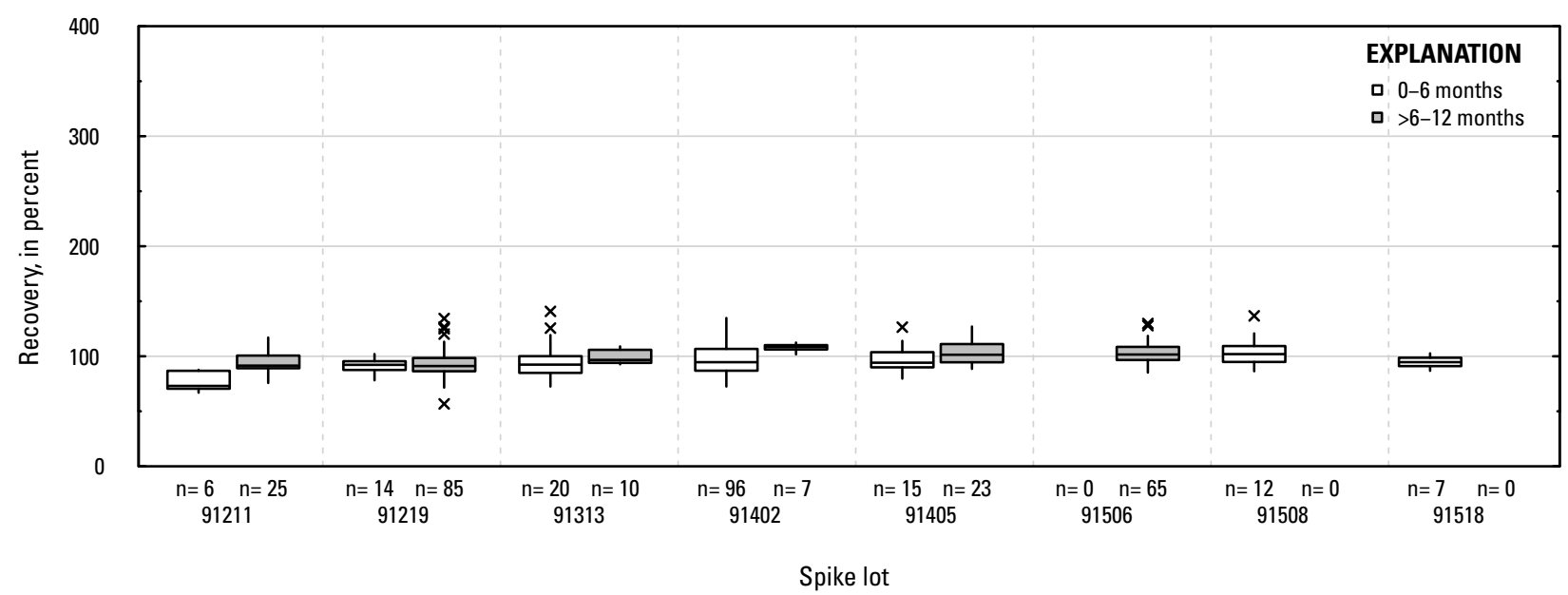

Figure 1-1. Distributions of recovery for individual pesticides in schedule 2437 by matrix, spike lot, and spike lot age. Recovery values larger than 400 percent are not shown.-Continued 
YB. Tetraconazole: laboratory reagent spikes

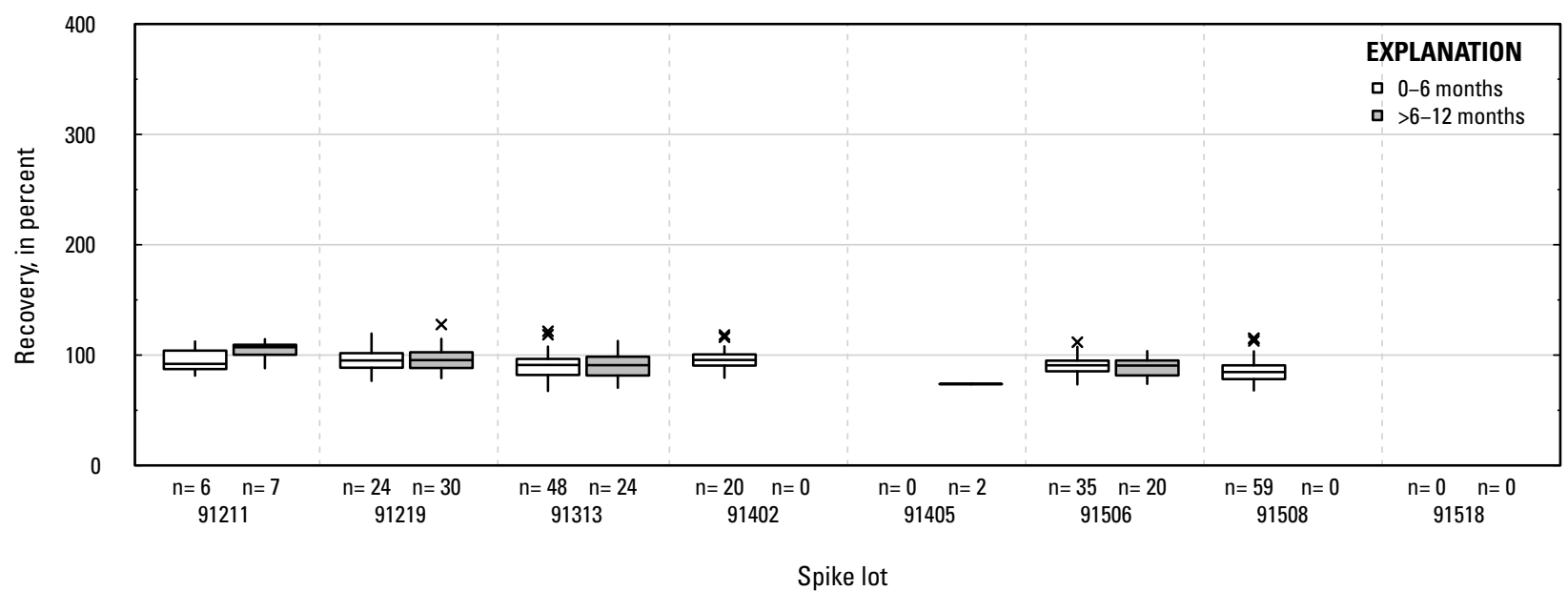

YC. Tetraconazole: groundwater field matrix spikes

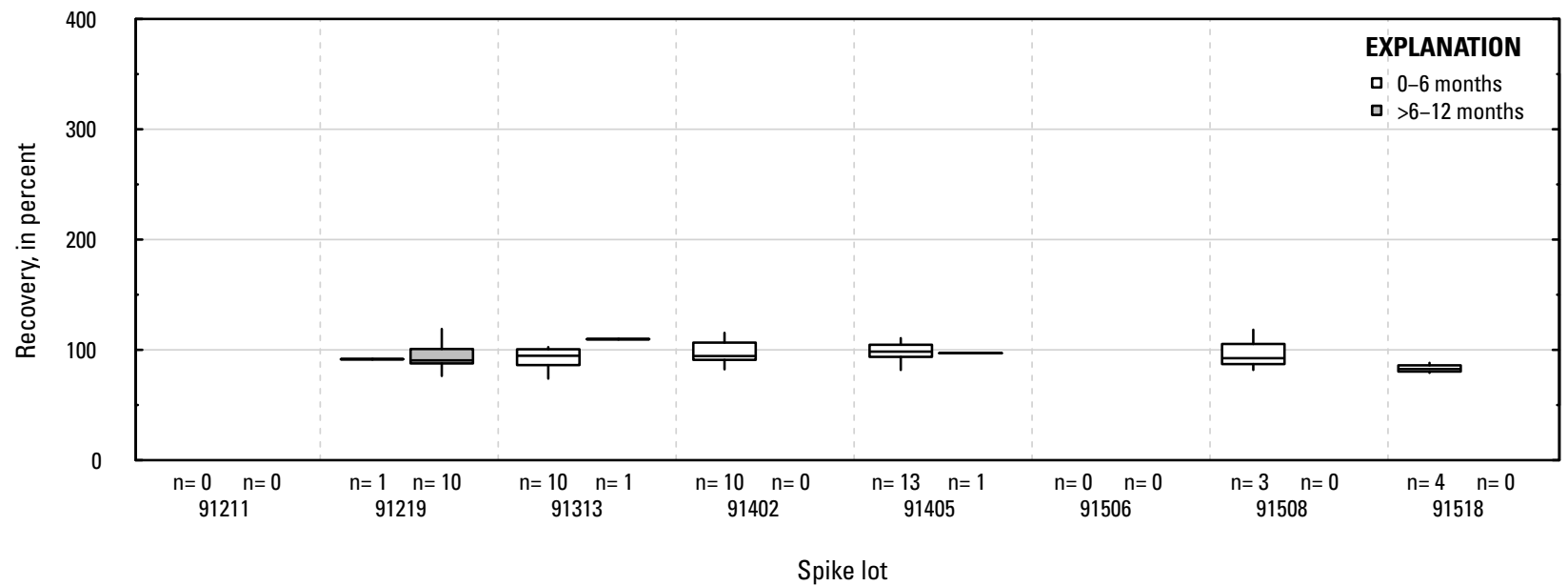

YD. Tetraconazole: surface water field matrix spikes

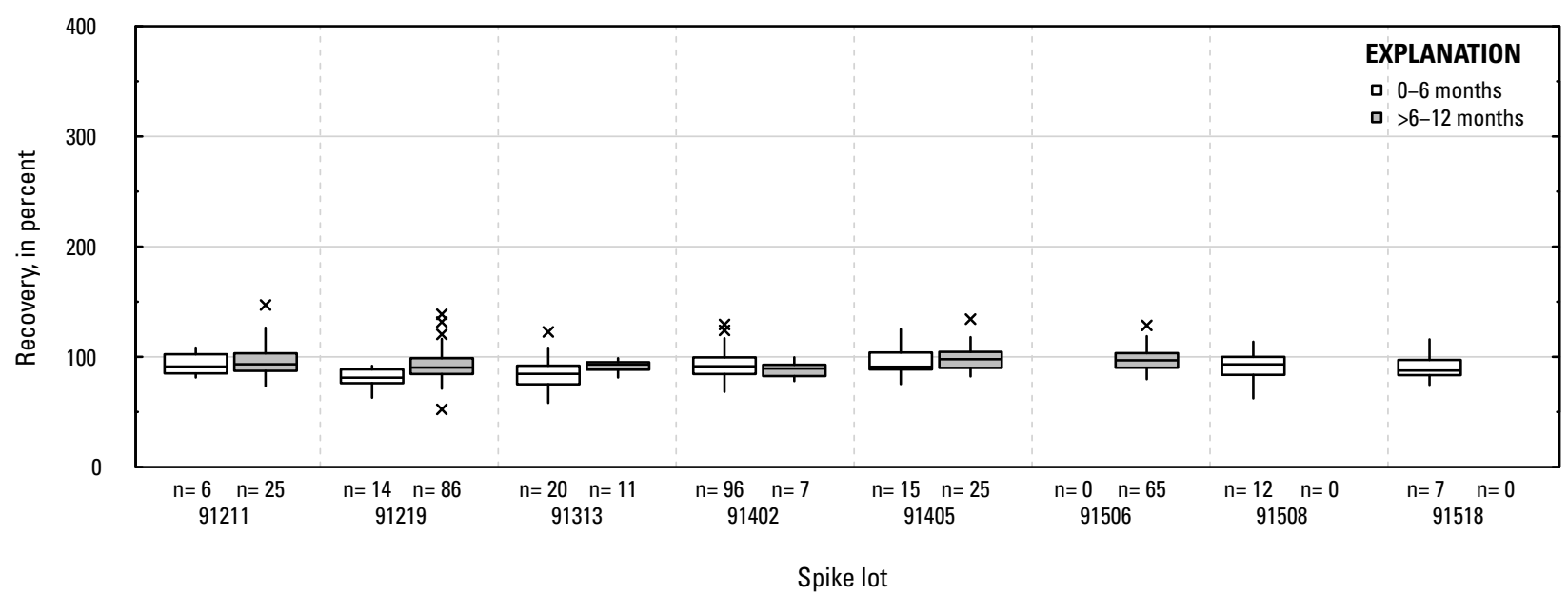

Figure 1-1. Distributions of recovery for individual pesticides in schedule 2437 by matrix, spike lot, and spike lot age. Recovery values larger than 400 percent are not shown.-Continued 
YE. Thiobencarb: laboratory reagent spikes

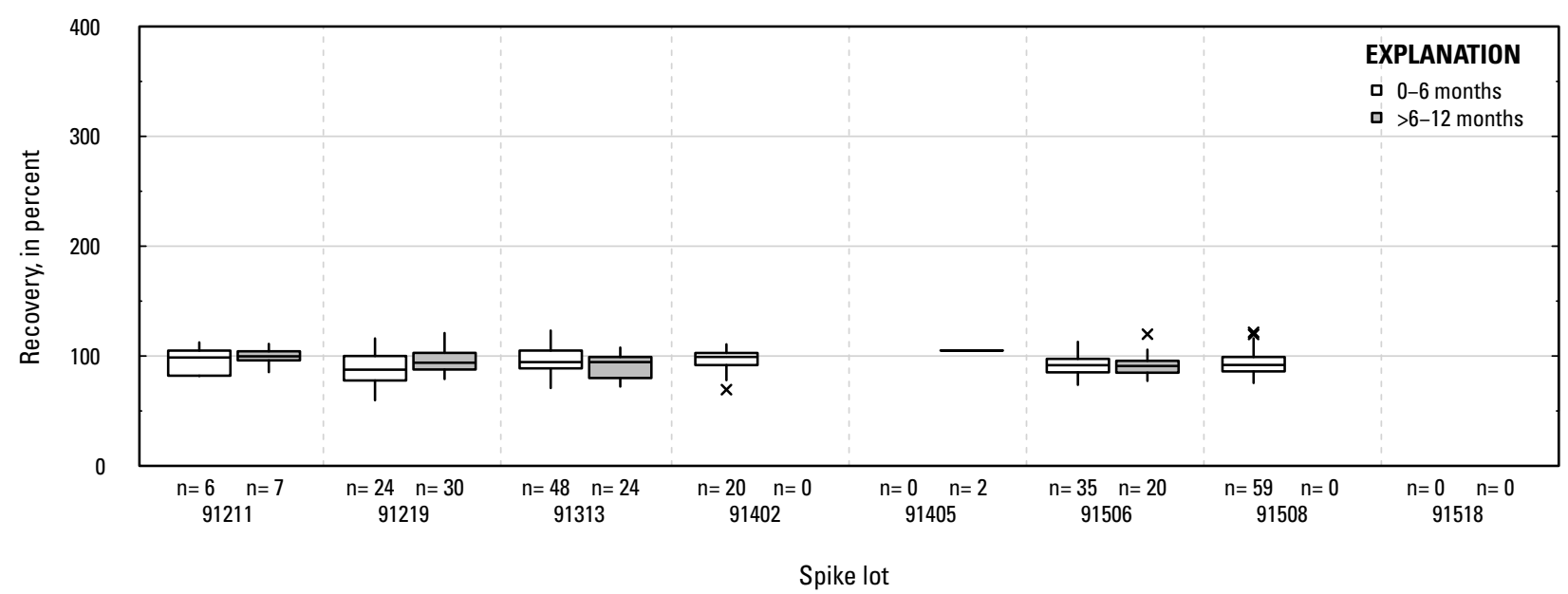

YF. Thiobencarb: groundwater field matrix spikes

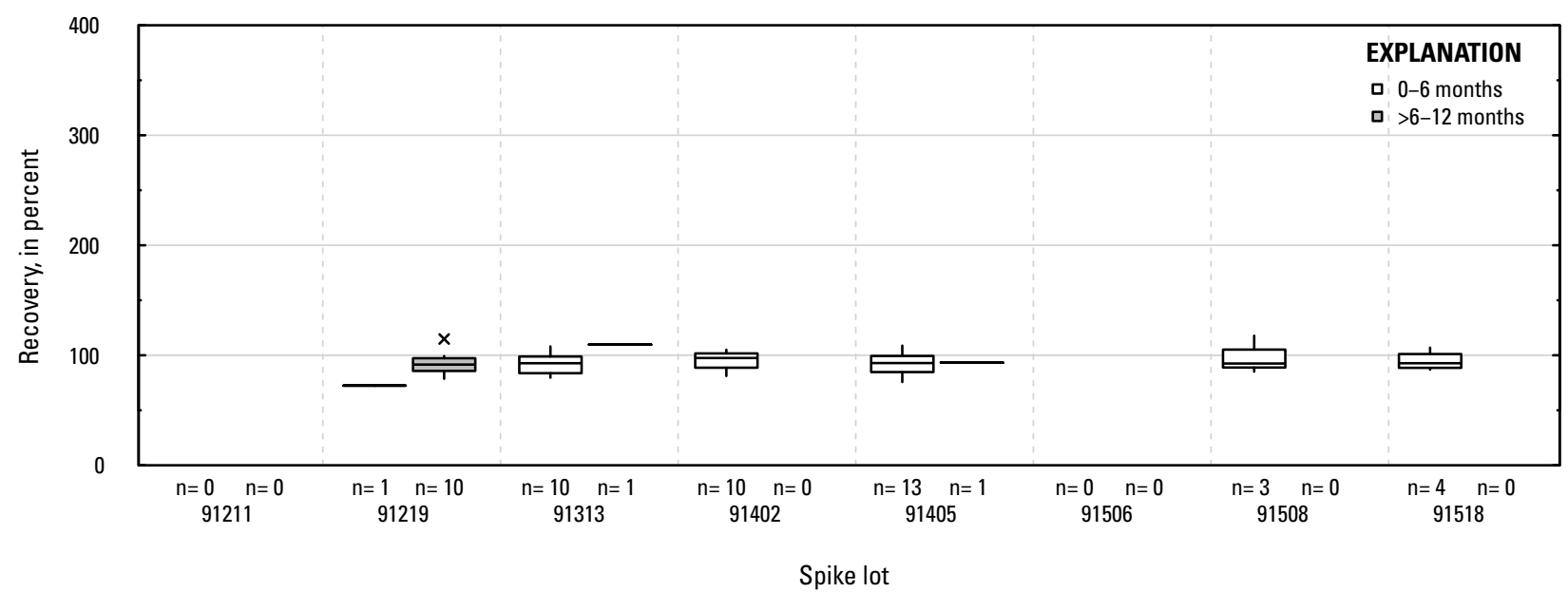

YG. Thiobencarb: surface water field matrix spikes

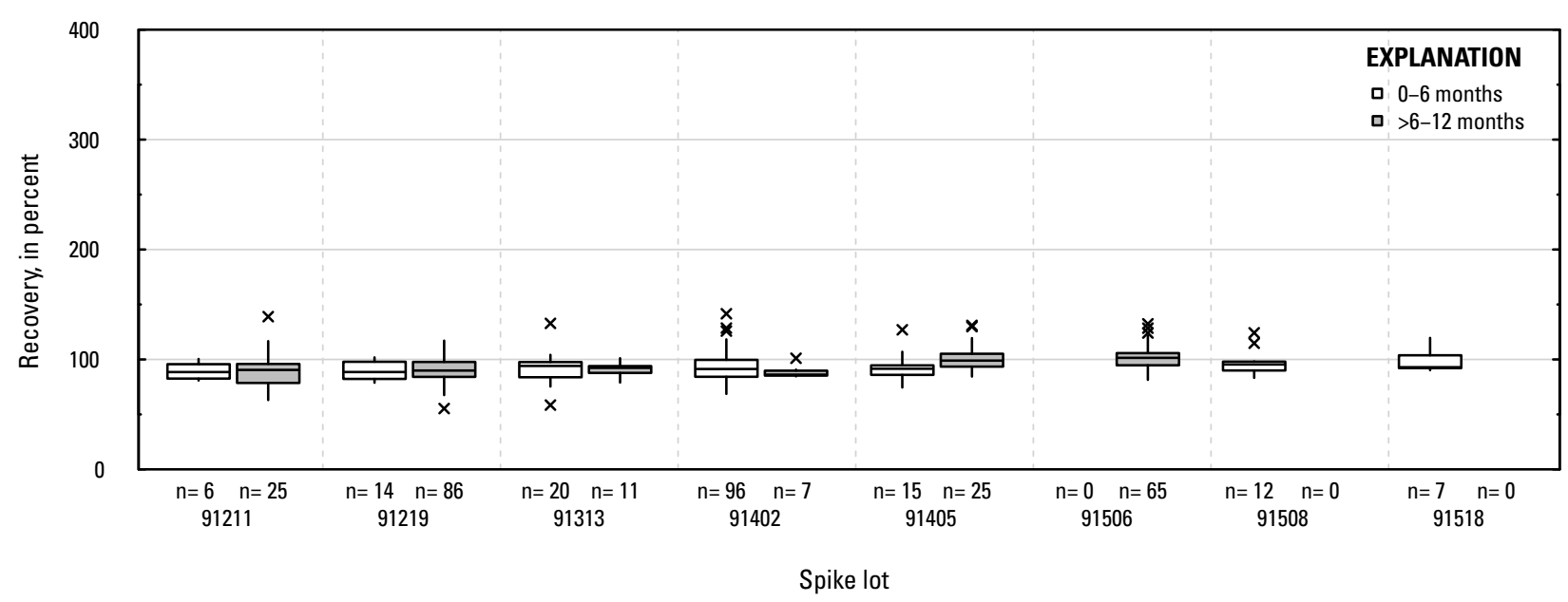

Figure 1-1. Distributions of recovery for individual pesticides in schedule 2437 by matrix, spike lot, and spike lot age. Recovery values larger than 400 percent are not shown.-Continued 
YH. trans-Permethrin: laboratory reagent spikes

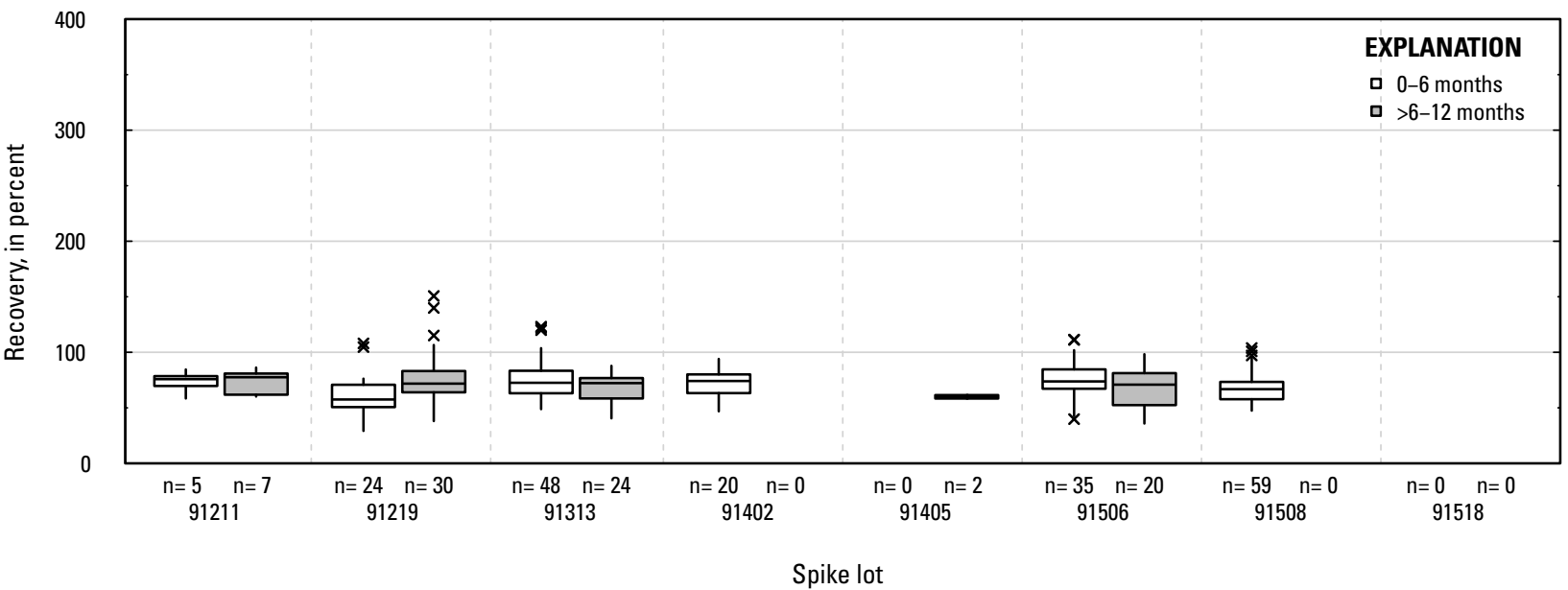

VI. trans-Permethrin: groundwater field matrix spikes

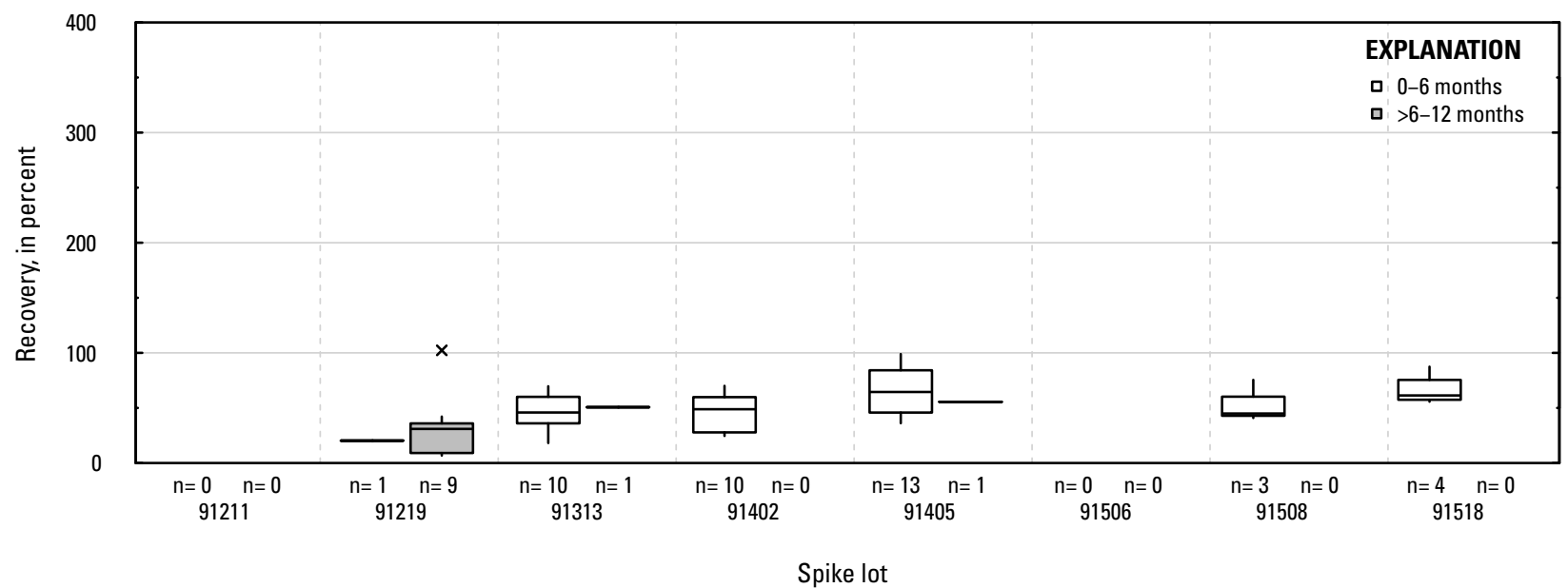

YJ. trans-Permethrin: surface water field matrix spikes

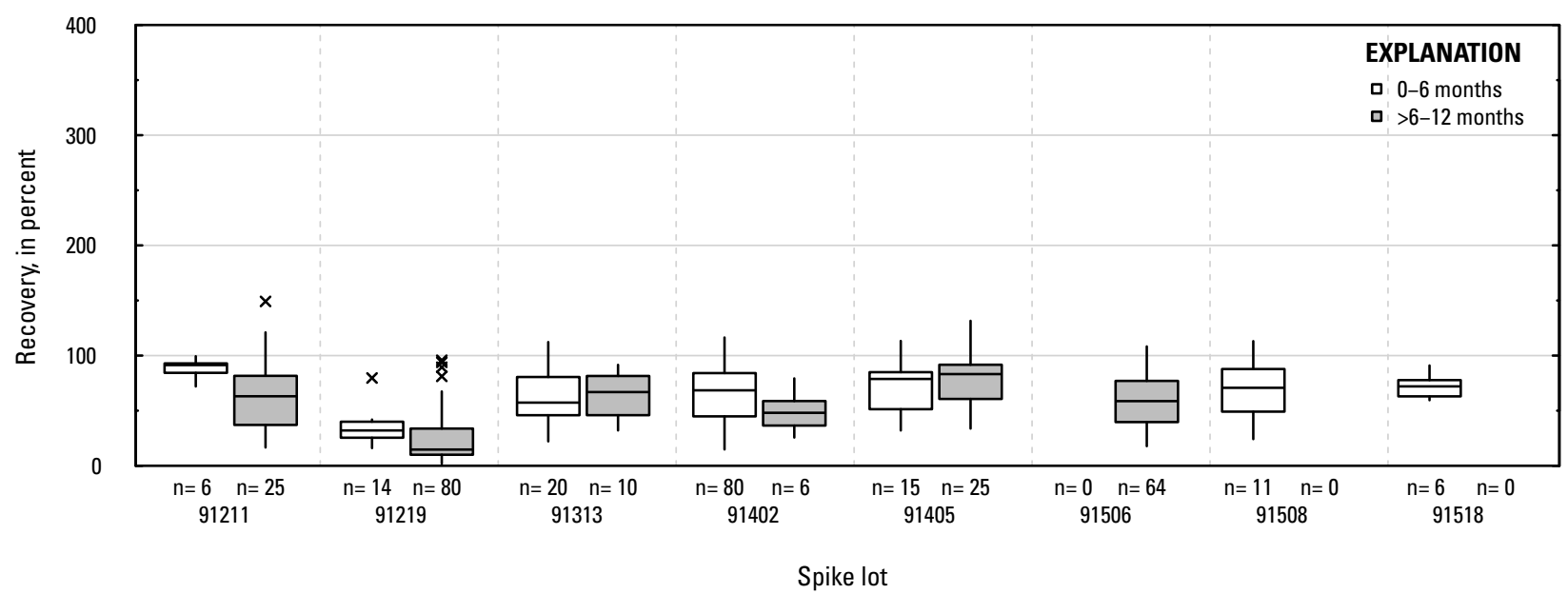

Figure 1-1. Distributions of recovery for individual pesticides in schedule 2437 by matrix, spike lot, and spike lot age. Recovery values larger than 400 percent are not shown.-Continued 
YK. Triallate: laboratory reagent spikes

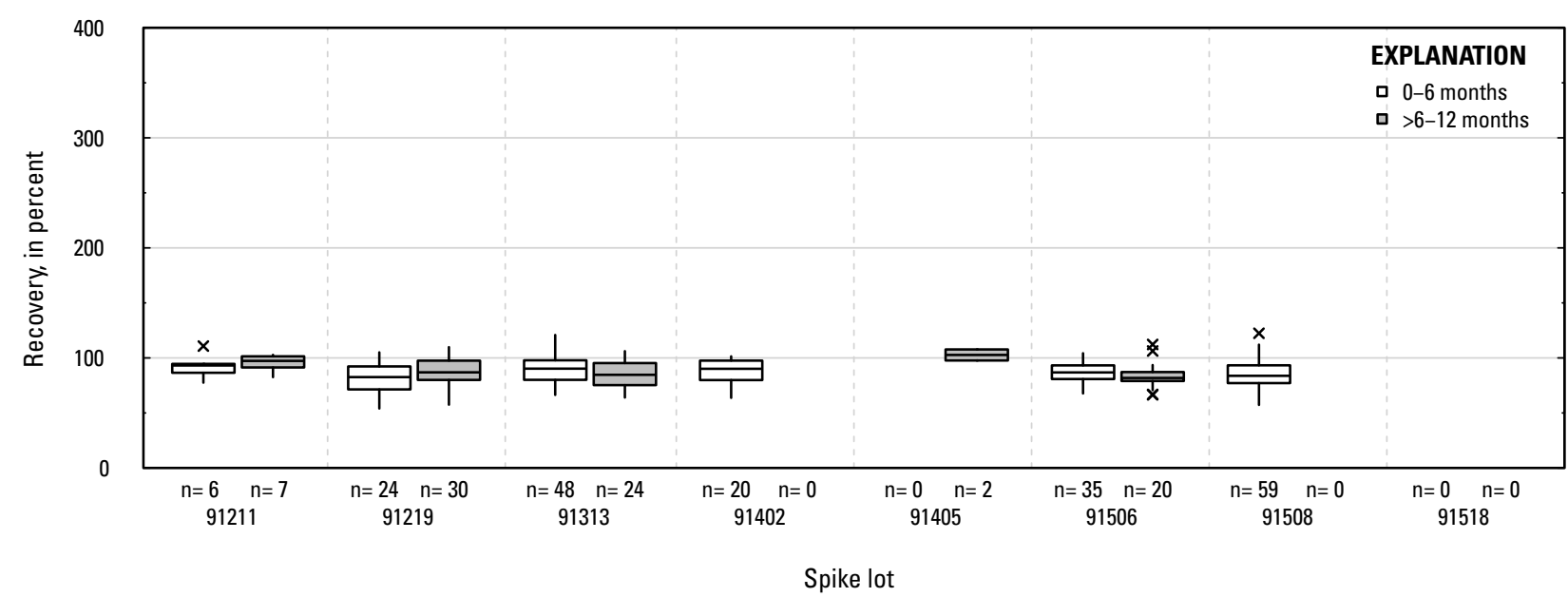

YL. Triallate: groundwater field matrix spikes

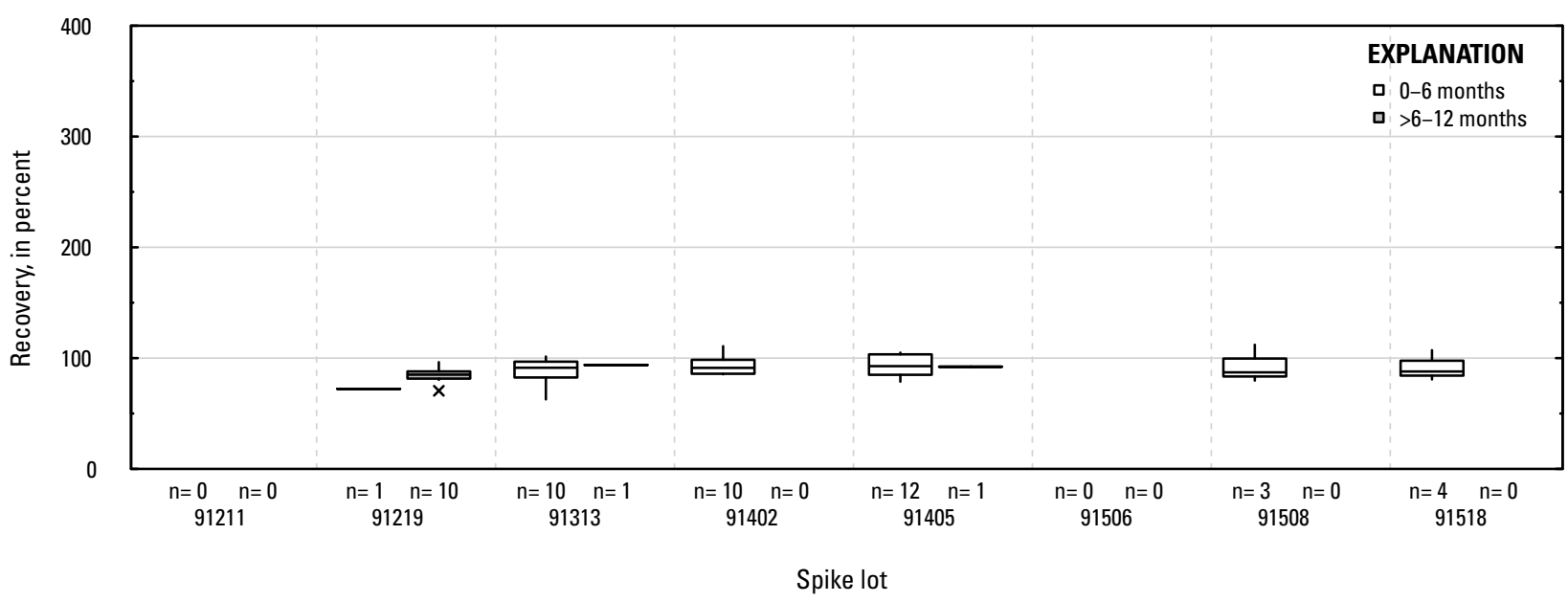

YM. Triallate: surface water field matrix spikes

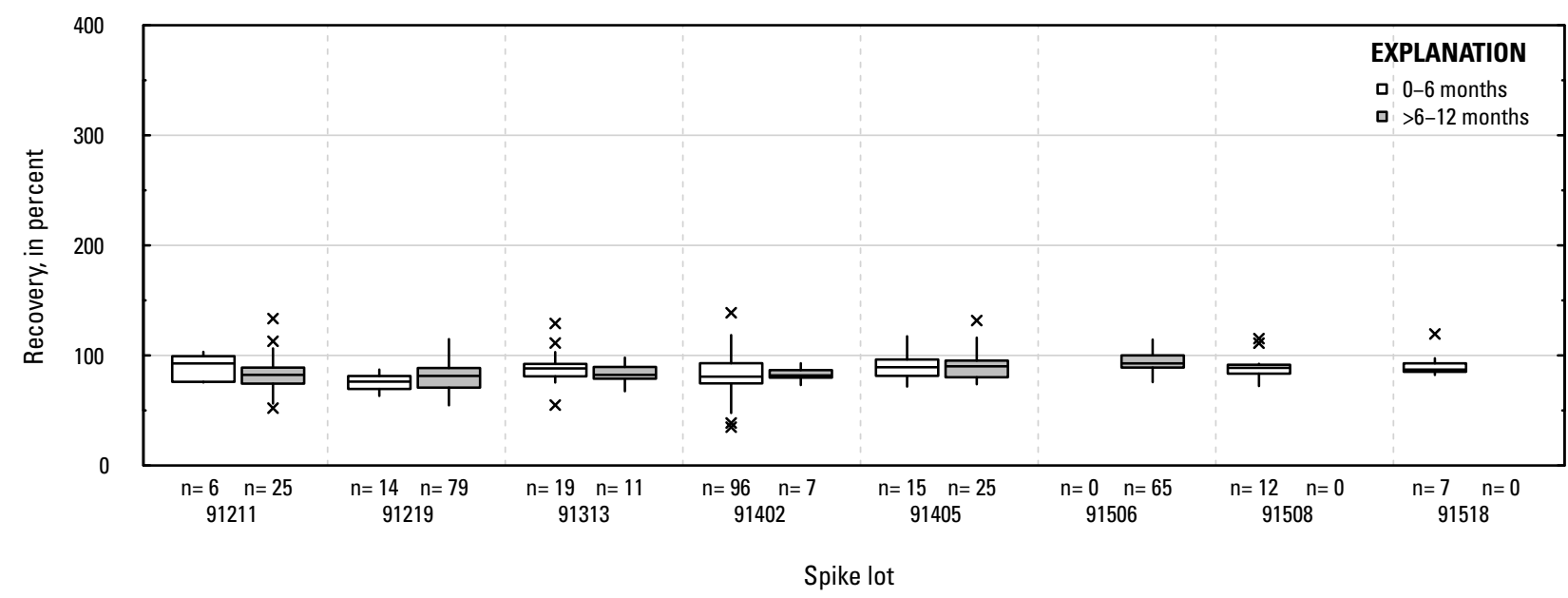

Figure 1-1. Distributions of recovery for individual pesticides in schedule 2437 by matrix, spike lot, and spike lot age. Recovery values larger than 400 percent are not shown.-Continued 
YN. Tribufos: laboratory reagent spikes

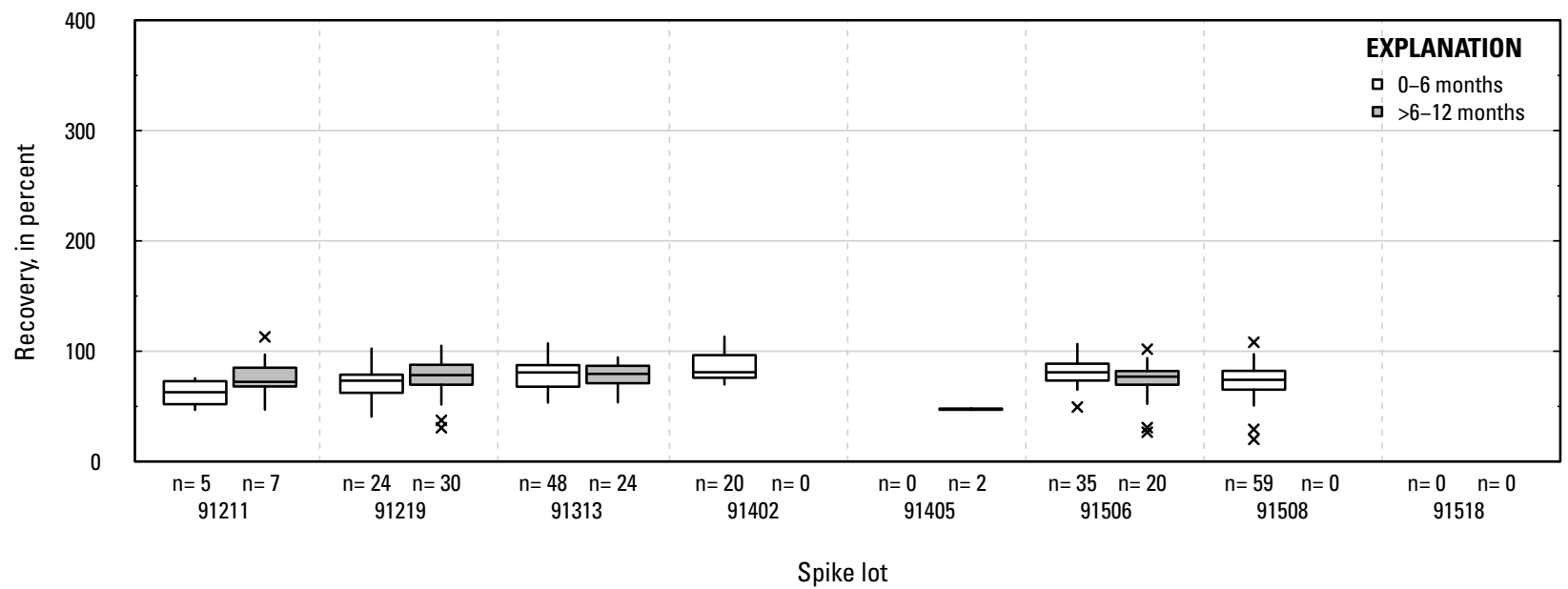

\section{Yo. Tribufos: groundwater field matrix spikes}

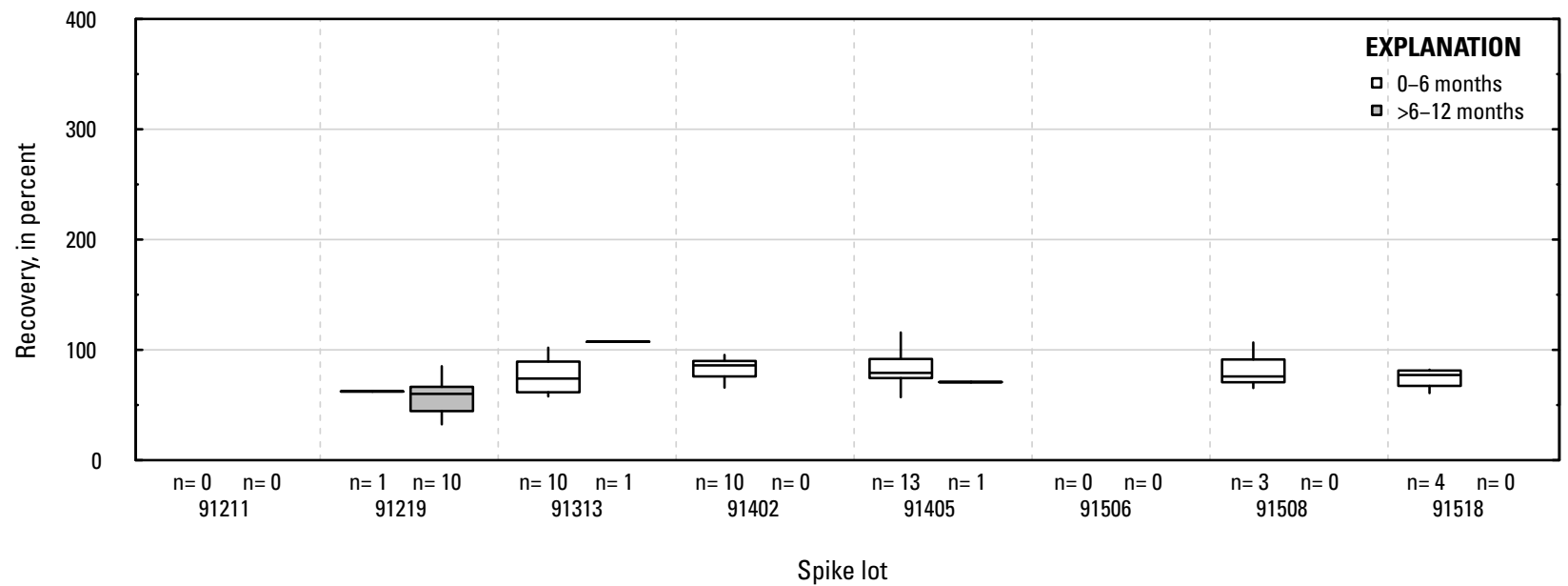

YP. Tribufos: surface water field matrix spikes

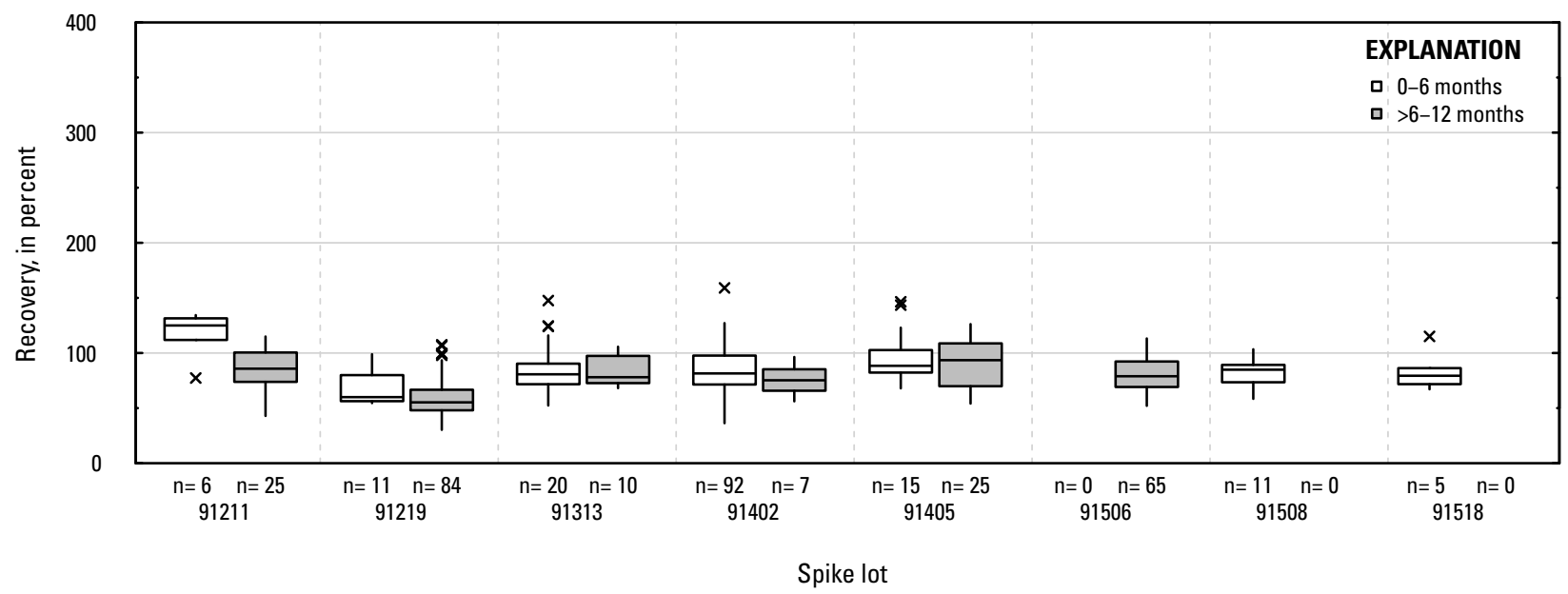

Figure 1-1. Distributions of recovery for individual pesticides in schedule 2437 by matrix, spike lot, and spike lot age. Recovery values larger than 400 percent are not shown.-Continued 
YO. Triclopyr: laboratory reagent spikes

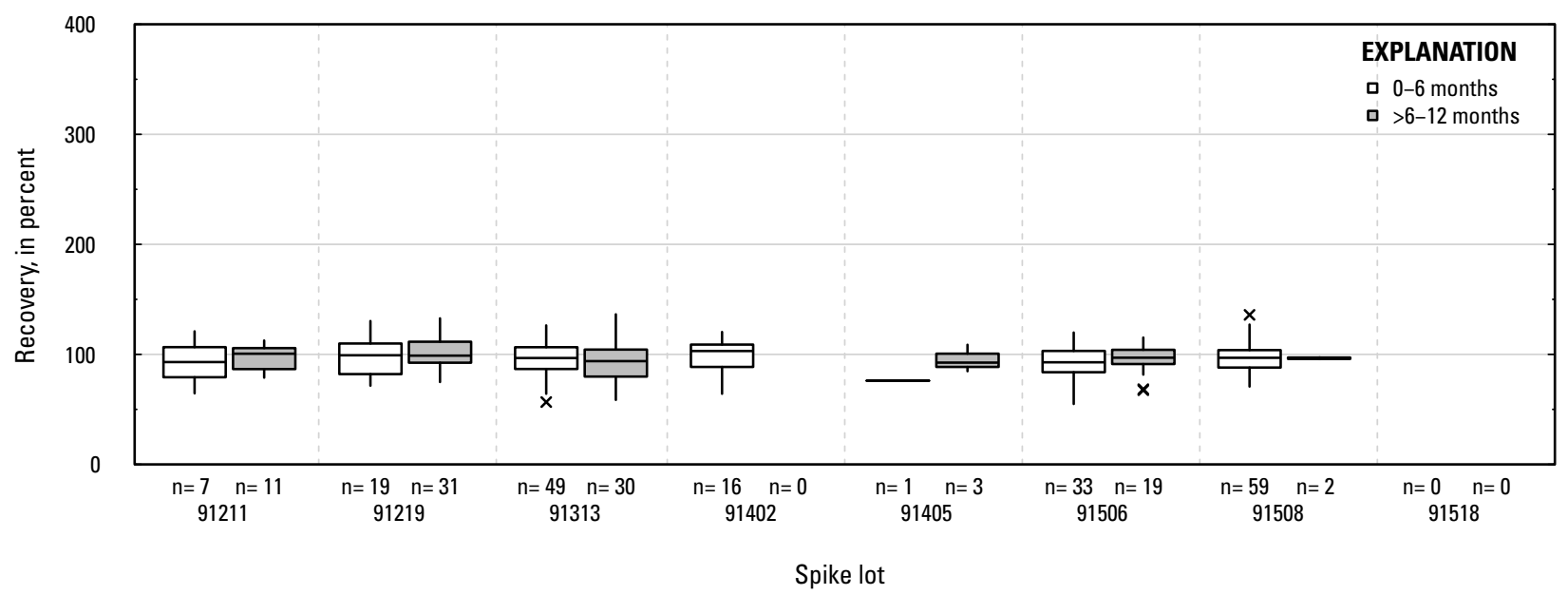

YR. Triclopyr: groundwater field matrix spikes

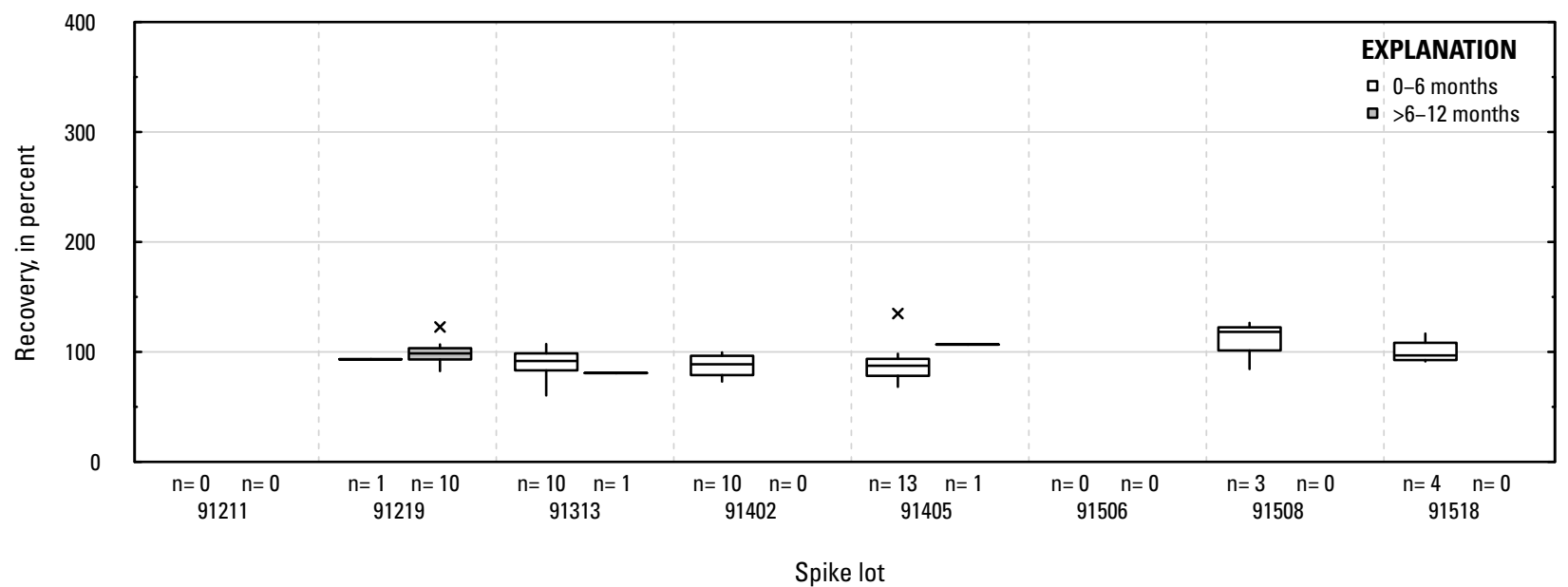

YS. Triclopyr: surface water field matrix spikes

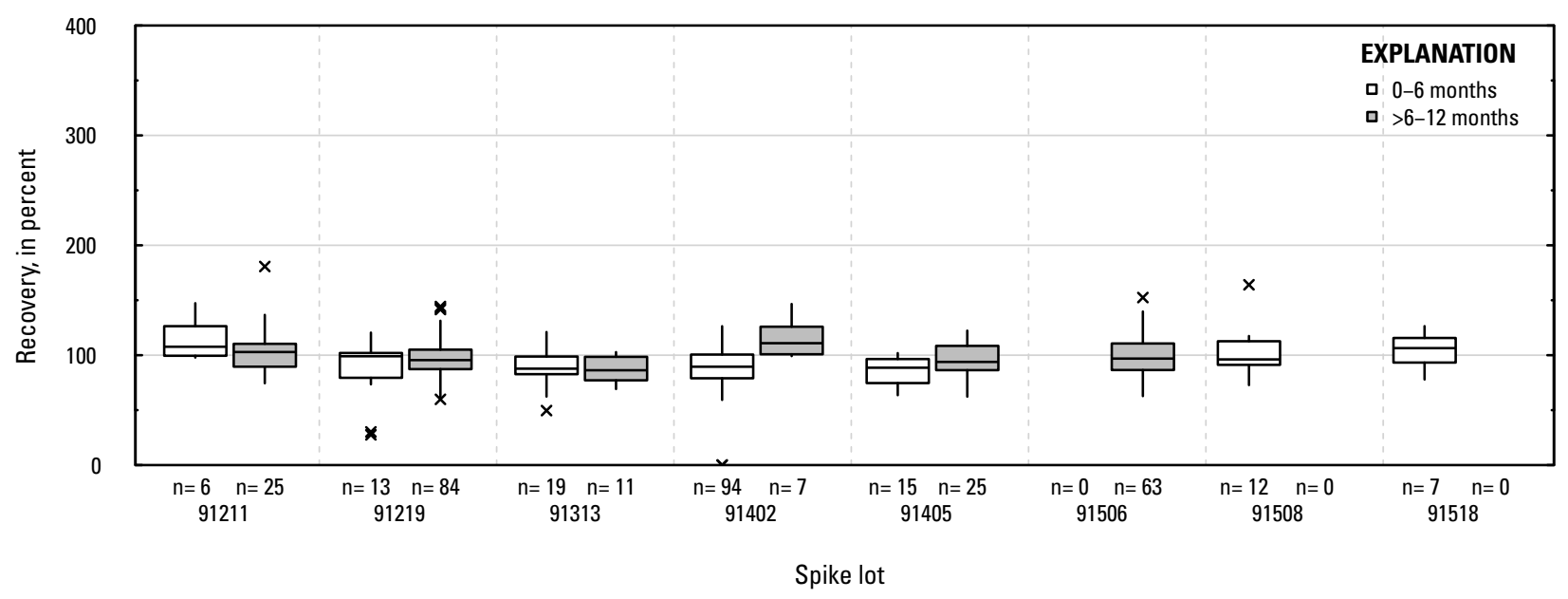

Figure 1-1. Distributions of recovery for individual pesticides in schedule 2437 by matrix, spike lot, and spike lot age. Recovery values larger than 400 percent are not shown.-Continued 
YT. Trifloxystrobin: laboratory reagent spikes

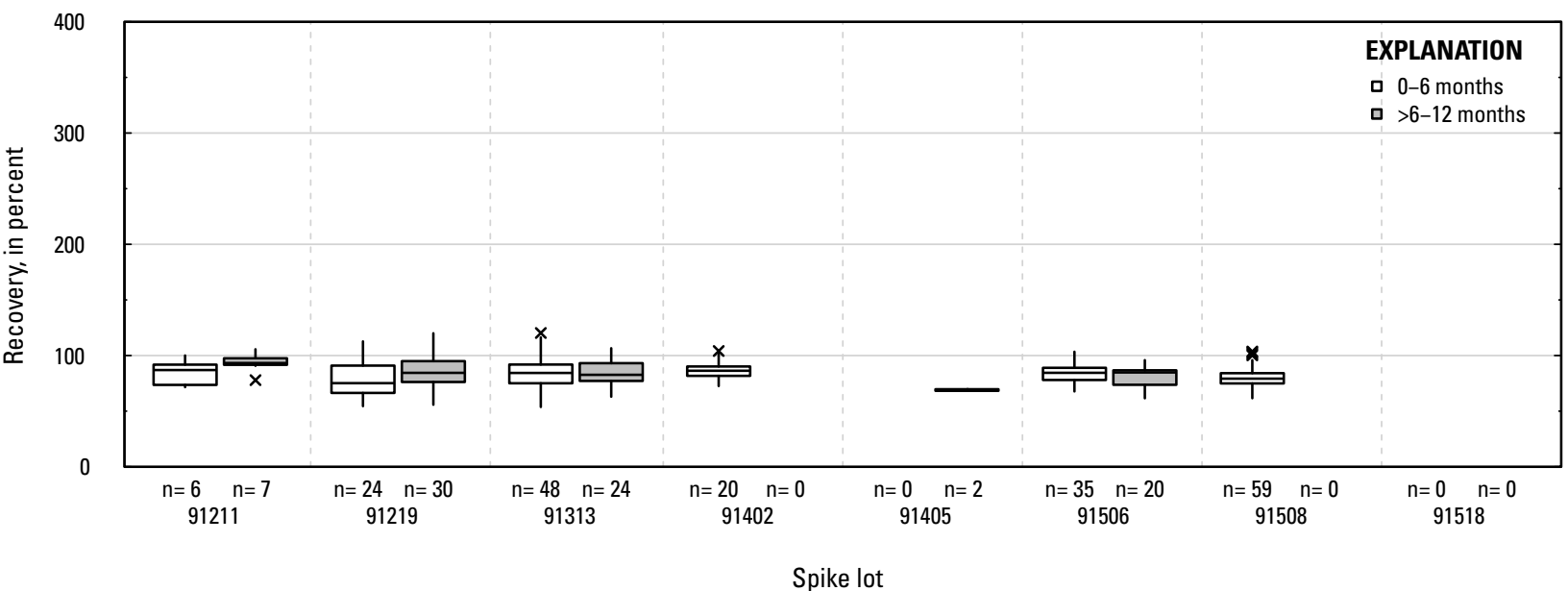

YU. Trifloxystrobin: groundwater field matrix spikes

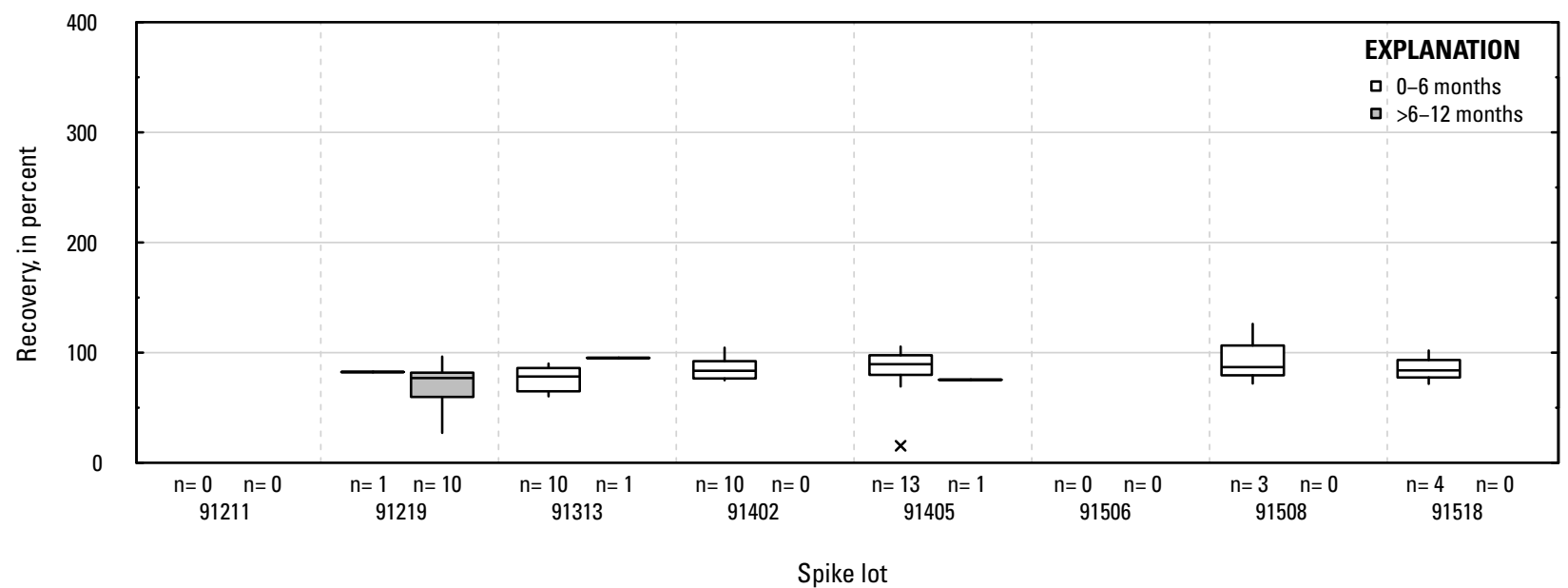

YV. Trifloxystrobin: surface water field matrix spikes

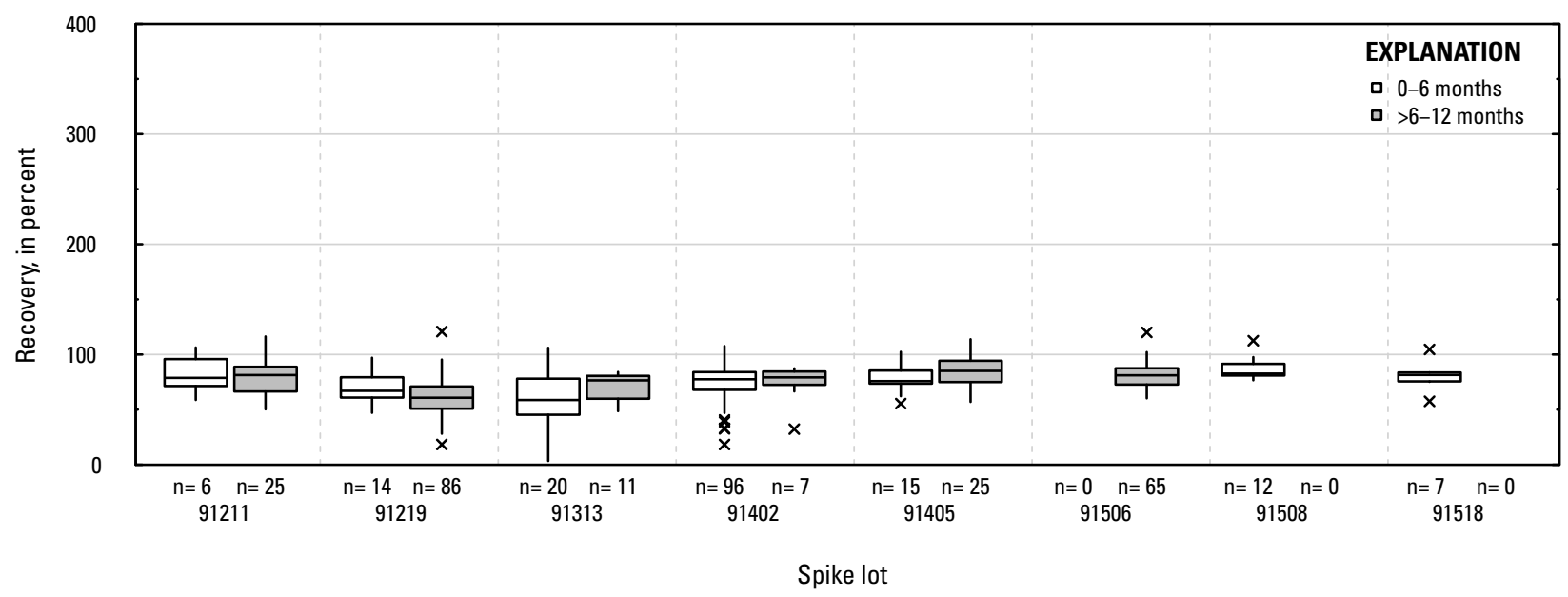

Figure 1-1. Distributions of recovery for individual pesticides in schedule 2437 by matrix, spike lot, and spike lot age. Recovery values larger than 400 percent are not shown.-Continued 


\section{A. Laboratory reagent spikes}

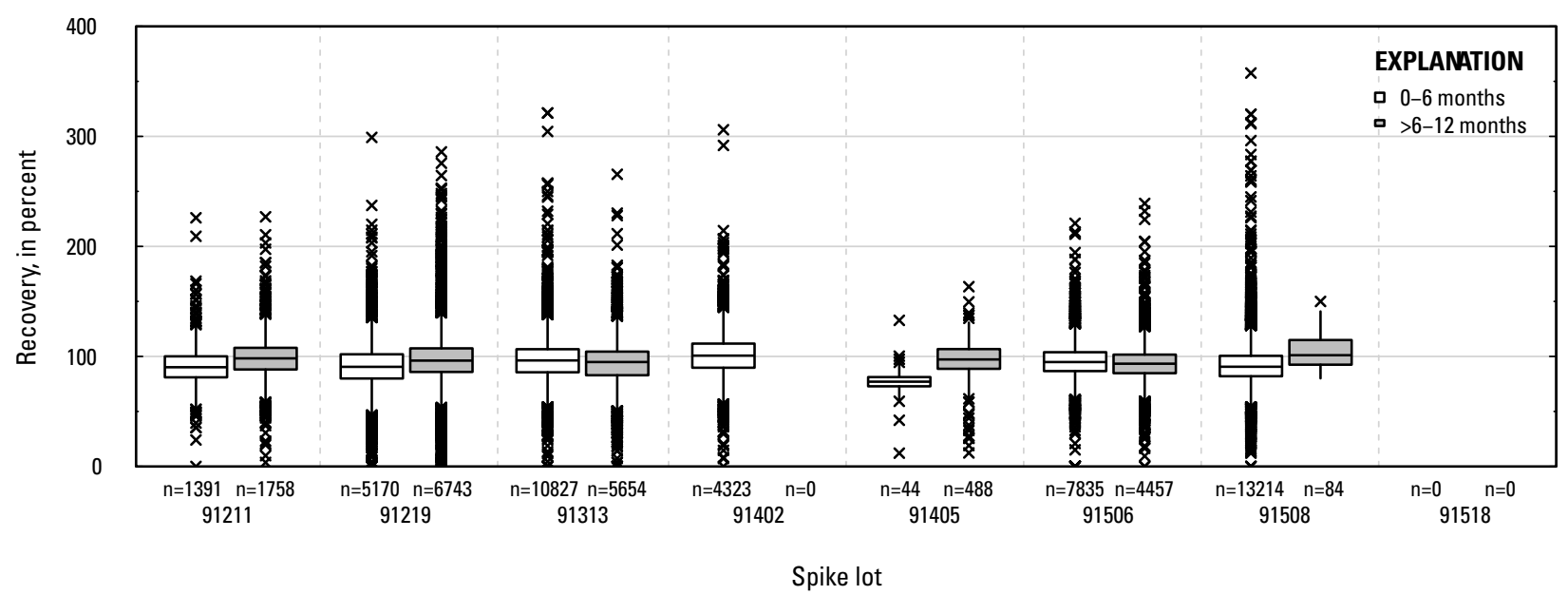

\section{B. Groundwater field matrix spikes}

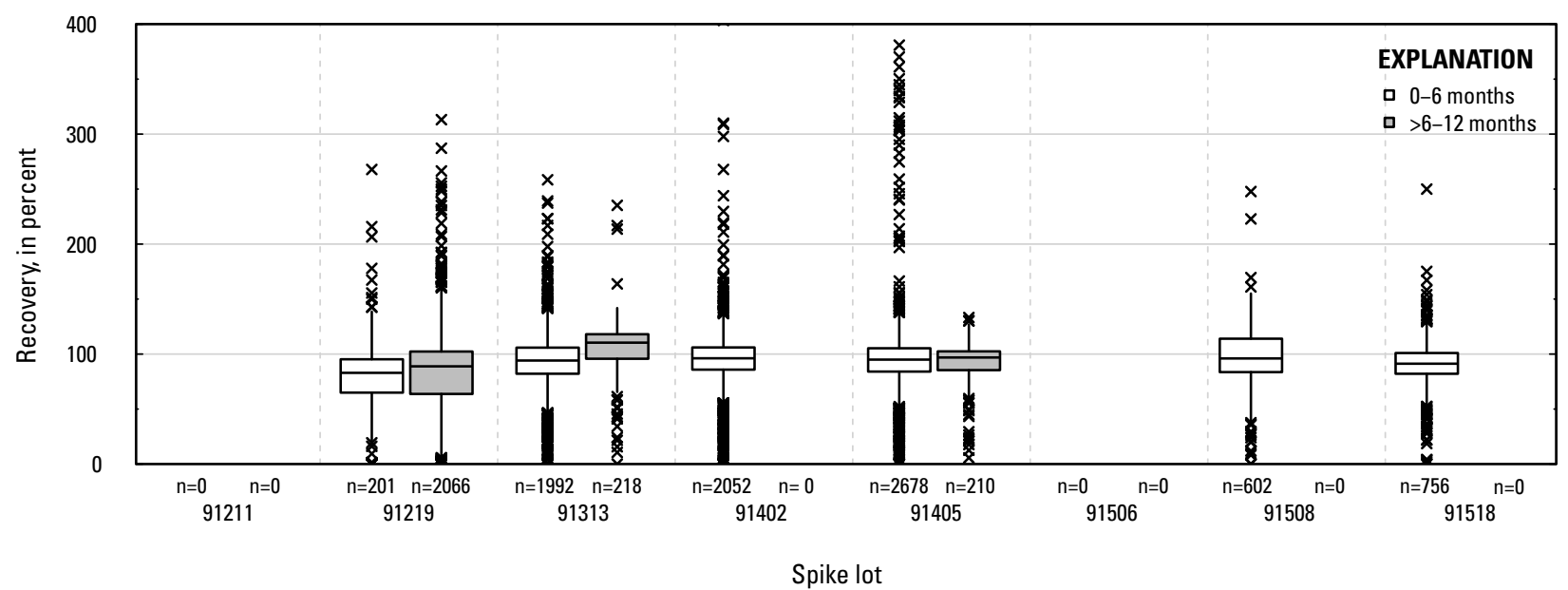

C. Surface water field matrix spikes

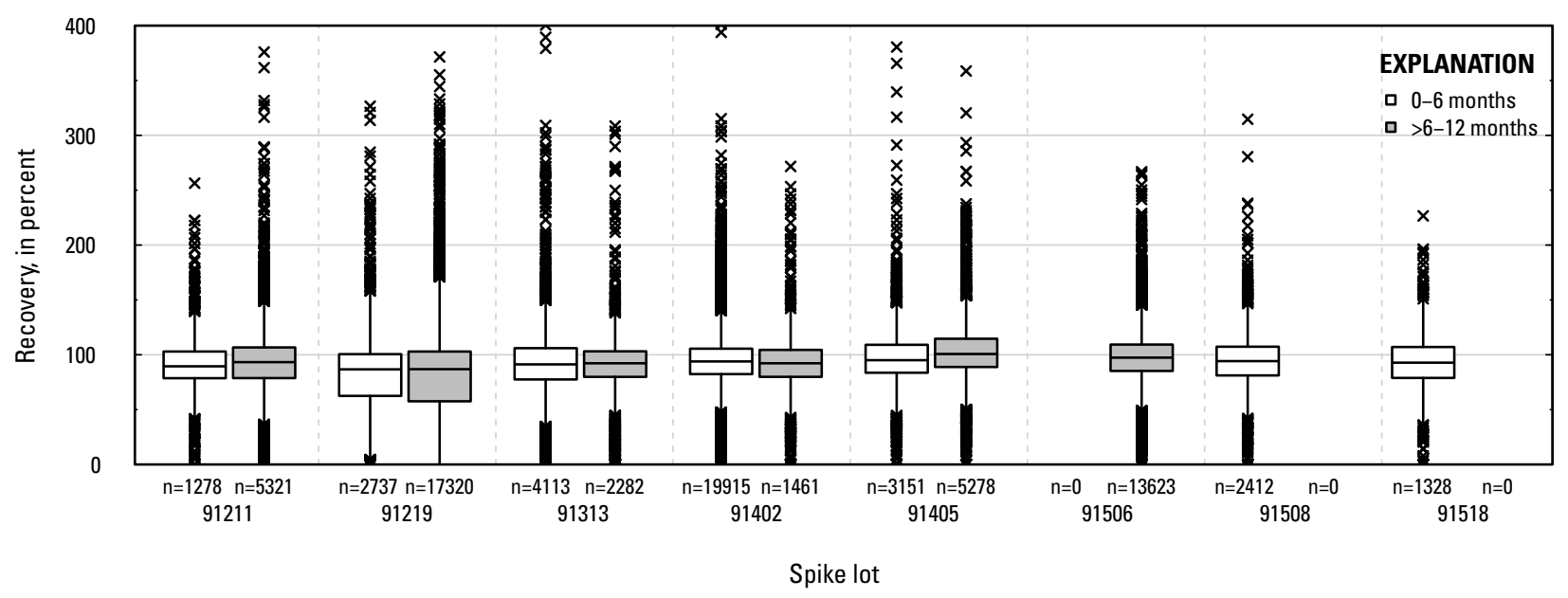

Figure 1-2. Distributions of recovery for schedule 2437 pesticides by spike lot, and spike lot age, pooled by matrix. Recovery values larger than 400 percent are not shown. 


\section{A. Organophosphate}

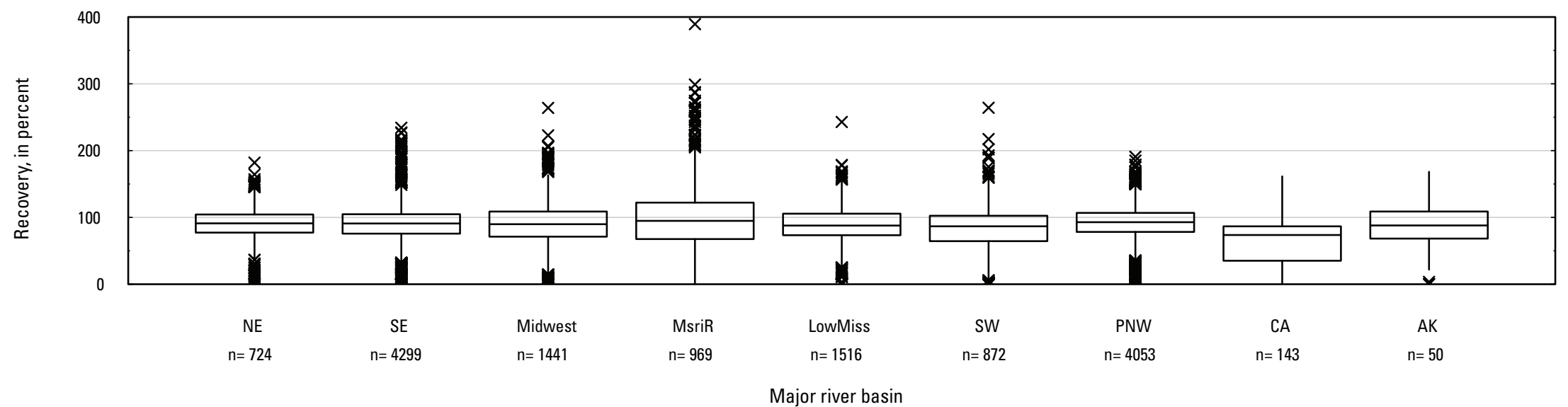

B. Carbamate and thiocarbamate

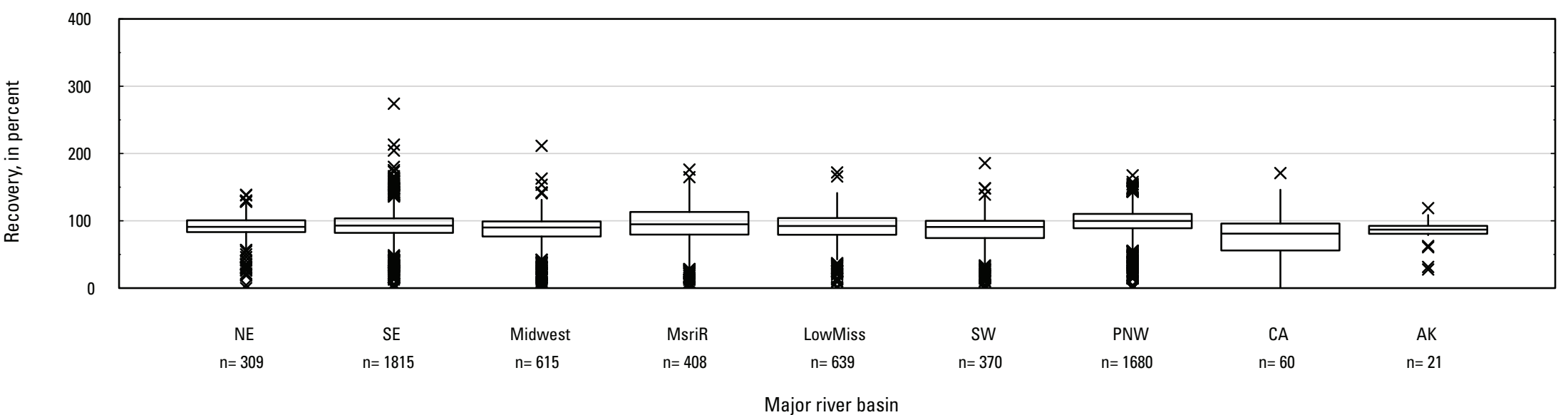

Figure 1-3. Distributions of recovery for pesticides in schedule 2437 in surface waterby analytical method group and Major River Basin. Recovery values larger than 400 percent are not shown. 


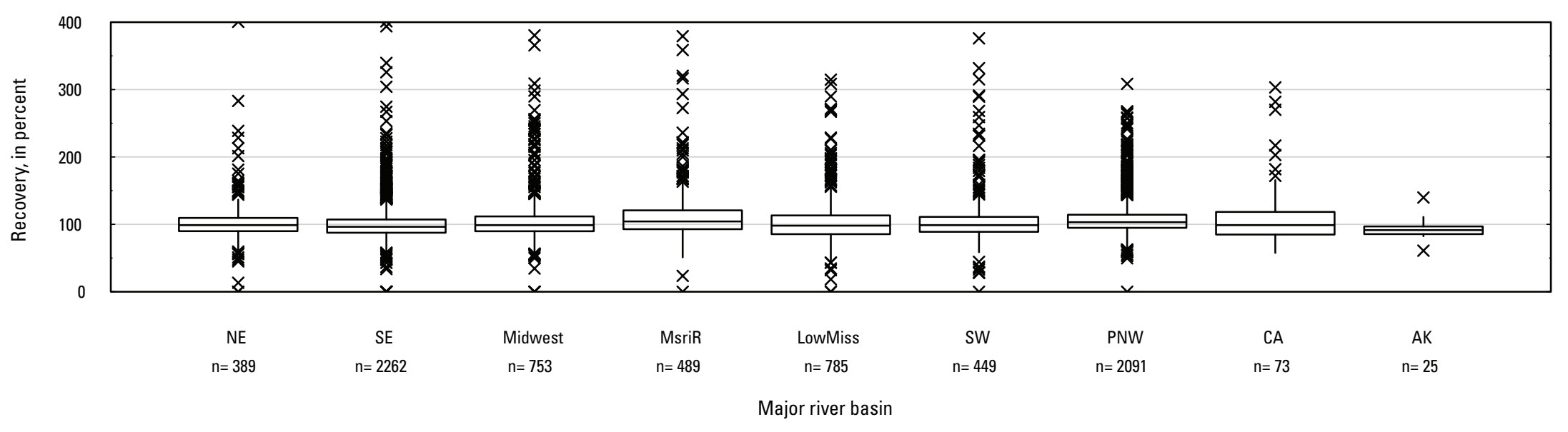

D. Sulfonylurea and urea

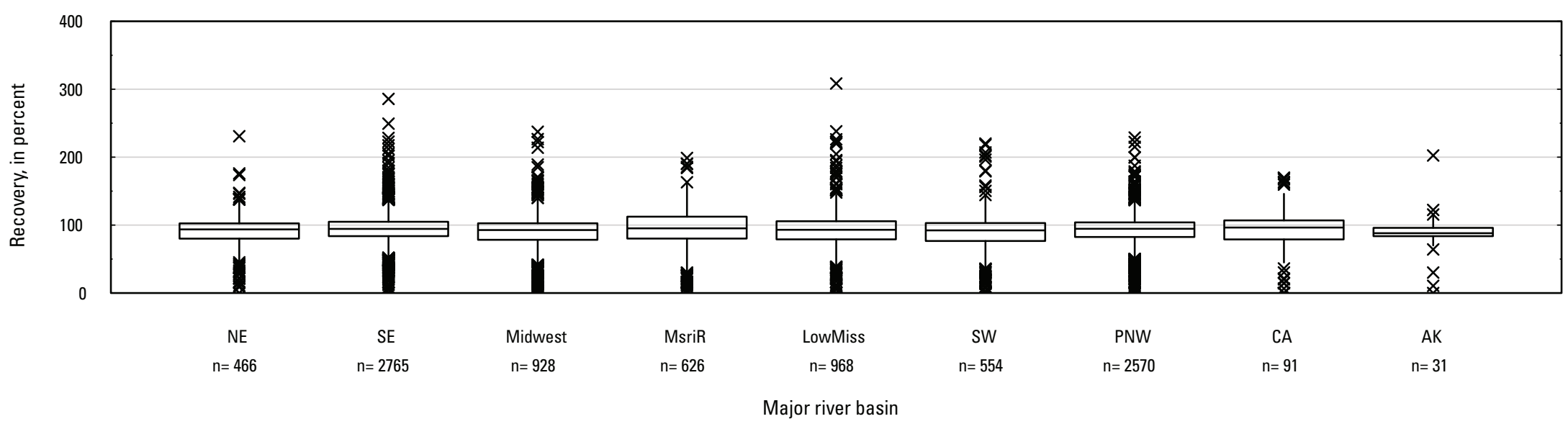

Figure 1-3. Distributions of recovery for pesticides in schedule 2437 in surface waterby analytical method group and Major River Basin. Recovery values larger than 400 percent are not shown. - Continued 


\section{E. Acetanilide and amide}

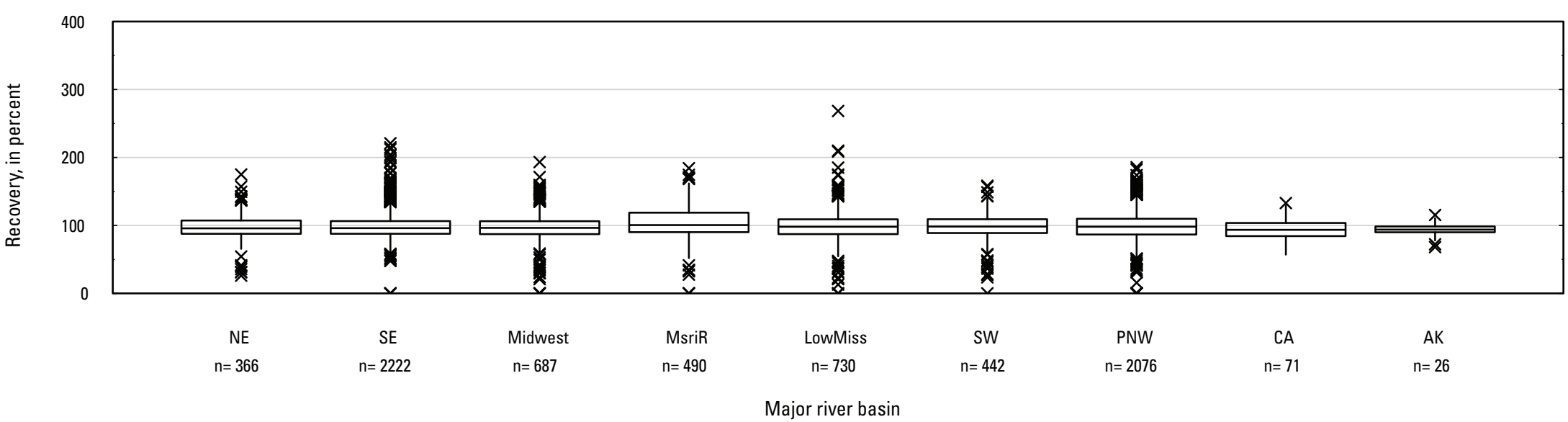

F. Pyrethroid, organochlorine and phenylpyrazine

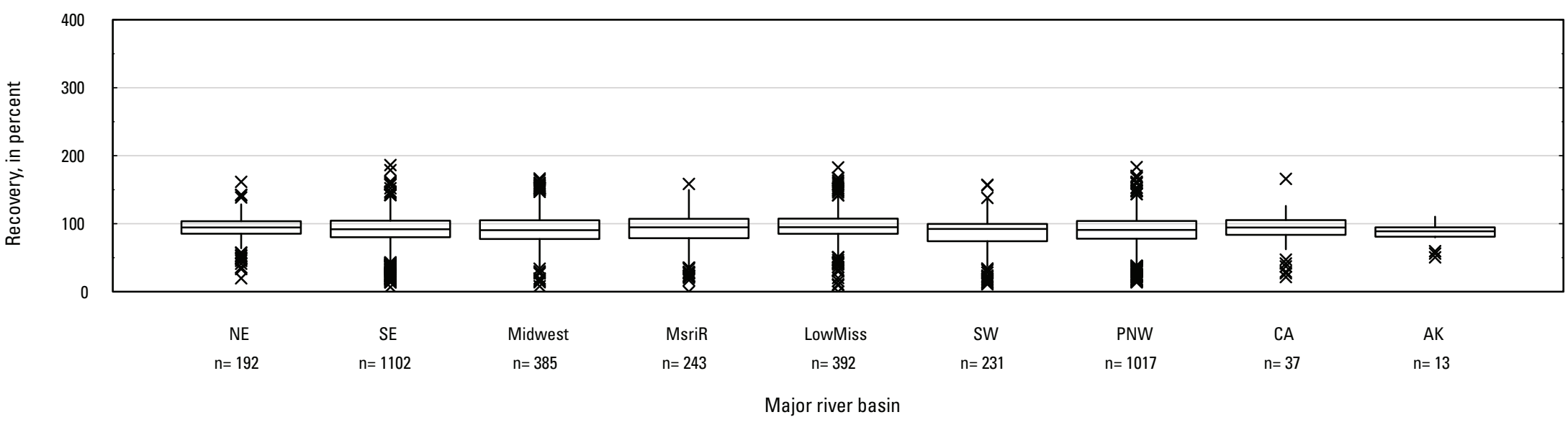

Figure 1-3. Distributions of recovery for pesticides in schedule 2437 in surface waterby analytical method group and Major River Basin. Recovery values larger than 400 percent are not shown. - Continued 


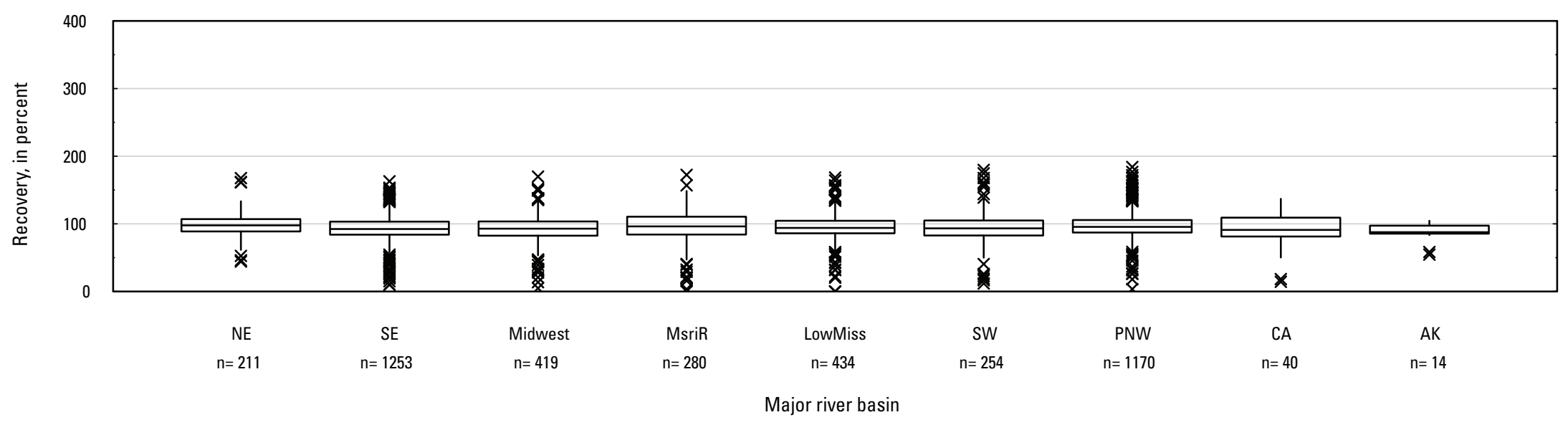

H. Acid

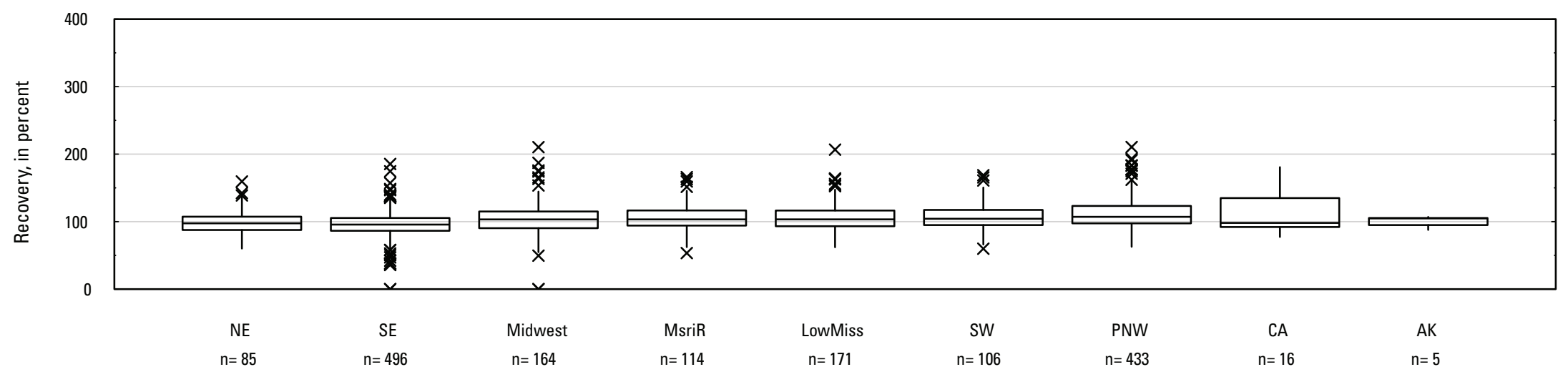

Major river basin

Figure 1-3. Distributions of recovery for pesticides in schedule 2437 in surface waterby analytical method group and Major River Basin. Recovery values larger than 400 percent are not shown. - Continued 
I. Miscellaneous

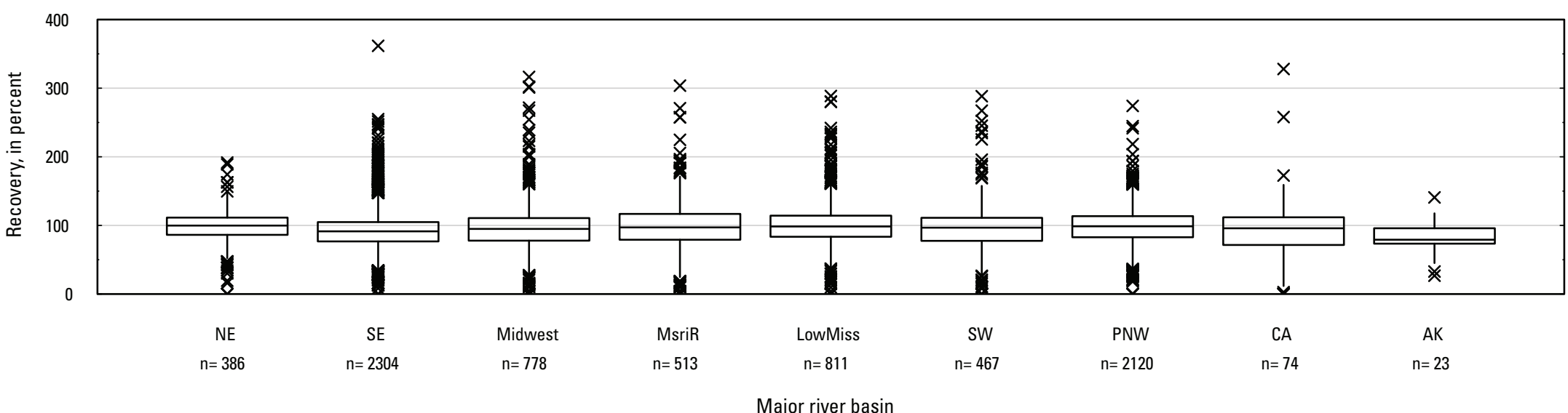

Figure 1-3. Distributions of recovery for pesticides in schedule 2437 in surface waterby analytical method group and Major River Basin. Recovery values larger than 400 percent are not shown. - Continued 
Table 1-2. Pesticides that have at least one result reported with a value qualifier code (VOC) of "m."

\begin{tabular}{ll}
\hline Pcode & \multicolumn{1}{c}{ Pesticide Name } \\
\hline 61679 & Flumetsulam \\
65067 & Bifenthrin \\
65093 & Oxyfluorfen \\
68236 & Diazinon oxon \\
68498 & 1H-1,2,4-Triazole \\
68508 & 3-Hydroxycarbofuran \\
68536 & Asulam \\
68545 & Butralin \\
68548 & Carbendazim \\
68551 & Chlorosulfonamide acid \\
68560 & Dacthal monoacid \\
68517 & Dicamba \\
68602 & Fenbutatin oxide \\
68611 & 2-(1-Hydroxyethyl)-6-methylaniline \\
68613 & Hexazinone Transformation Product D \\
68638 & Lactofen \\
68654 & Naled \\
68655 & Novaluron \\
68871 & Alachlor sulfonic acid \\
\hline
\end{tabular}


For additional information contact:

Program Coordinator, National Water Quality Program U.S. Geological Survey, 413 National Center

12201 Sunrise Valley Drive, Reston, Virginia 20192 https://water.usgs.gov/nawqa/

Publishing support provided by the Madison Publishing Service Center 


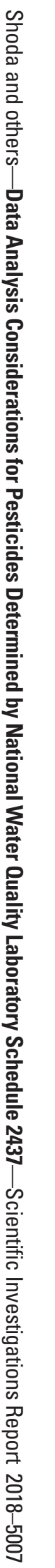

